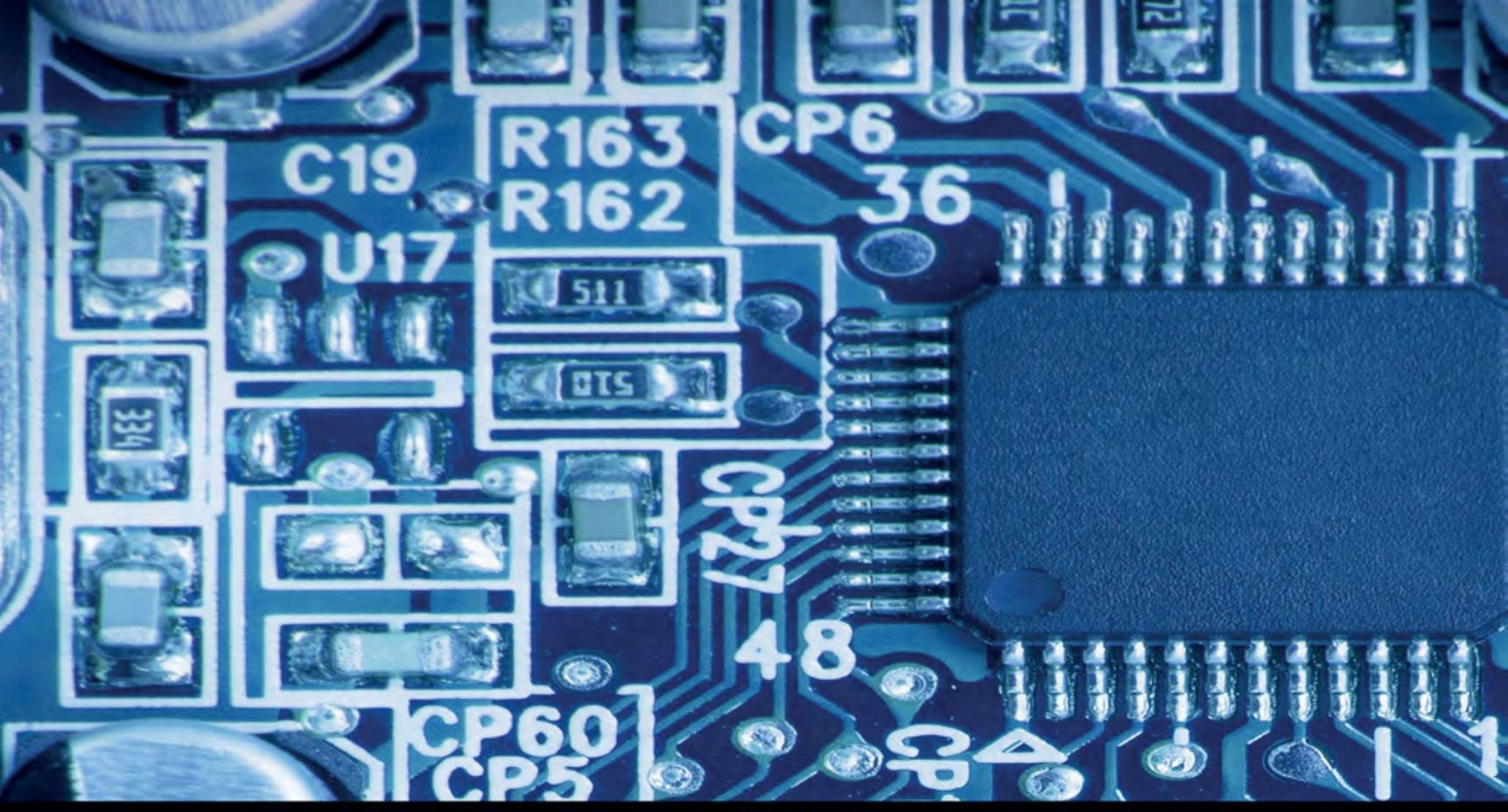

\title{
INTELLIGENT CIRCUITS AND SYSTEMS
}

Edited by

Rajesh Singh and Anita Gehlot

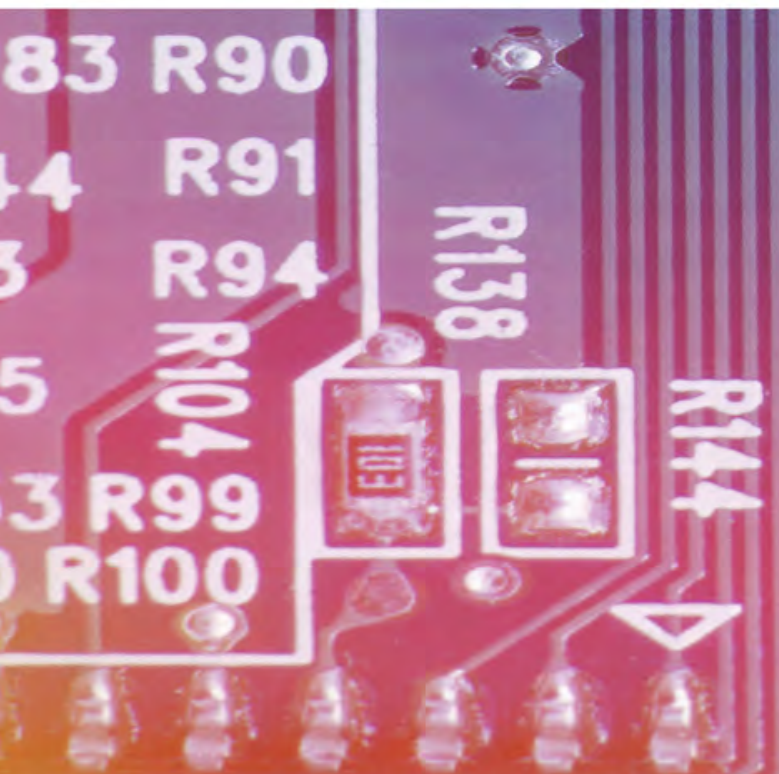

1015 (CRC $\underset{\text { Taylor \& Francis Group }}{C R C \text { Press }}$ 


\section{Intelligent Circuits and Systems}




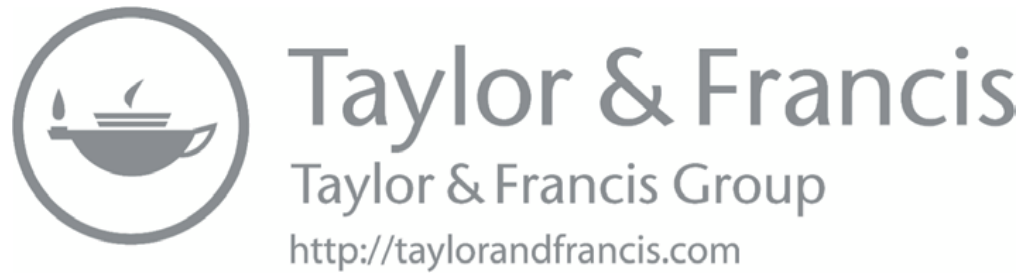




\section{Intelligent Circuits and Systems}

Proceedings of the 3rd International Conference on Intelligent Circuits and Systems (ICICS 2020)

$$
\begin{gathered}
\text { June 26-27, } 2020 \\
\text { Punjab, India }
\end{gathered}
$$

Edited by

Rajesh Singh and Anita Gehlot 
MATLAB $^{\circledR}$ is a trademark of The MathWorks, Inc. and is used with permission. The MathWorks does not warrant the accuracy of the text or exercises in this book. This book's use or discussion of MATLAB ${ }^{\circledR}$ software or related products does not constitute endorsement or sponsorship by The MathWorks of a particular pedagogical approach or particular use of the MATLAB ${ }^{\circledR}$ software.

First edition published 2022

by CRC Press

2 Park Square, Milton Park, Abingdon, Oxon OX14 4RN

and by CRC Press

6000 Broken Sound Parkway NW, Suite 300, Boca Raton, FL 33487-2742

(C) 2022 Taylor and Francis

CRC Press is an imprint of Informa UK Limited

The right of Rajesh Singh and Anita Gehlot to be identified as the author of the editorial material, and of the authors for their individual chapters, has been asserted in accordance with sections 77 and 78 of the Copyright, Designs and Patents Act 1988.

All rights reserved. No part of this book may be reprinted or reproduced or utilised in any form or by any electronic, mechanical, or other means, now known or hereafter invented, including photocopying and recording, or in any information storage or retrieval system, without permission in writing from the publishers.

The Open Access version of this book, available at www.taylorfrancis.com, has been made available under a Creative Commons Attribution-Non Commercial-No Derivatives 4.0 license

Trademark notice: Product or corporate names may be trademarks or registered trademarks, and are used only for identification and explanation without intent to infringe.

ISBN: 978-1-00-312910-3 (ebk)

DOI: $10.1201 / 9781003129103$

Typeset in Sabon

by Newgen Publishing UK 


\section{Contents}

Editors

xiii

Organizing Committee $\quad$ Xv

Technical Committee xvii

Preface xxi

1 Post PV integration protection issues 1

B. KOTI REDDY, AMIT KUMAR SINGH

2 Case study on renewable energy integration (solar) challenges with captive/cogeneration plants and optimization of plant operation ANKEM V.R.N.B. MANIKYALA RAO, AMIT KUMAR SINGH, A. SAI MRUDULA

3 Control of unmanned surface vehicles with randomized probabilistic approach

RUPAM SINGH, BHARAT BHUSHAN

4 Islanding classification and low-voltage ride through for grid connected transformerless inverter

MOHAMMED ALI KHAN, V.S. BHARATH KURUKURU, AHTESHAMUL HAQUE

5 Machine learning classifier for fault classification in photovoltaic system

V.S. BHARATH KURUKURU, MOHAMMED ALI KHAN,

AHTESHAMUL HAQUE, ARUN KUMAR TRIPATHI

6 Performance analysis and comparison of 4-bit adder architectures AISHANI MISRA, SHILPI BIRLA, NEHA SINGH

7 Improved image compression using lifting wavelet SANDEEP SHELKE, GOVIND SINGH PATEL

8 A single cycle low latency bypass router based on network on chip 
9 Proximal and remote sensing of abiotic stress in crops

10 Energy management strategy for plug-in electric vehicle with DFIG system

NARESH KUMAR GOLLA, SURESH KUMAR SUDABATTULA

11 Energy harvesting cognitive radio: A review

YOGITA THAREJA, KAMAL KUMAR SHARMA

12 Impact of geographical locations on the PV inverter lifetime considering mission profile and uncertainties

SAINADH SINGH KSHATRI, JAVED DHILLON, SACHIN MISHRA

13 Feasibility analysis of smart grid distributed generation using RETScreen: A case study of industrial building HARPREET SHARMA, SACHIN MISHRA

14 Mathematical modelling of semiconductor devices and circuits: A review SANJAY KUMAR ROY, MANWINDER SINGH, KAMAL KUMAR SHARMA, BRAHMADEO PRASAD SINGH

15 Small signal III-V high gain MOSFET

MANOJ SINGH ADHIKARI, MAYANK PUNETHA, YASHVIR SINGH, YOGESH KUMAR VERMA

16 Impact of solar grid-based virtual power plant on grid flexibility HARPREET SHARMA, SACHIN MISHRA

17 Oxide thickness variation effects in MOS AlGaN/GaN HFET YOGESH KUMAR VERMA, LAXMAN SINGH, VARUN MISHRA, ROHIT GURJAR, PRATEEK KISHOR VERMA, SUDHNIT KAUR, AKHIL SEVDA, MRITYUNJAY KUMAR JHA, SANTOSH KUMAR GUPTA

18 SAR based best designs for wearable antennas: A review DANVIR MANDAL, S.S. PATTNAIK

19 Design and development of electronic precipitator for particulate matter air purification processes HARLEEN KOUR, DEEPTI SHARMA, SACHIN GUPTA

20 A critical survey on vertical handoff algorithms 
21 A circular monopole ultra-wideband antenna with a notch band at $5.8 \mathrm{GHz}$

GURPREET KUMAR, DALJEET SINGH, RAJEEV KUMAR

22 A comprehensive anatomy for handover probability in heterogeneous wireless networks

KESHAV JHA, AKHIL GUPTA

23 Secure communication channel for grid users using AES Encryption ADITYA BAKSHI, AKHIL GUPTA

24 SIC scheduling algorithm based MIMO technique for mobile ad-hoc networks KRISHAN KUMAR

25 Detection of nitrous oxide gas using silicene nanoribbons GURLEEN KAUR WALIA, DEEP KAMAL KAUR RANDHAWA, KANWALPREET SINGH, PARULPREET SINGH, HARMANDAR KAUR

26 Placement of solar DGs and capacitors in distribution network using combined approach SHAIK AARIF, SURESH KUMAR SUDABATTULA

27 iPlanty: A smart agriculture approach ANCHIT SINHA, AYUSHMAN TRIVEDI, DEBALEENA DAS, PRAVEEN REDDY, SANDEEP BANSAL

28 Electrostatically actuated graphene NEMS switch with different bridge structures REKHA CHAUDHARY, PRASANTHA R. MUDIMELA

29 Pre- and post-filter selection for image segmentation MOHIT SRIVASTAVA, MANOJ KURI

30 Impact of temperature on $14 \mathrm{~nm}$ FINFET with high-K different oxide material

SHEKHAR VERMA, SUMAN LATA TRIPATHI

31 Violation of independence assumption in ICA and its consequences BHAVESHKUMAR C. DHARMANI

32 Architecture, key technologies and emerging applications of 5G cellular networks 


\section{viii Contents}

33 Exploration of recent medium access control (MAC) protocols for efficient transmission of data in wireless body area network THOTA SRUTHI, MANWINDER SINGH

34 A review paper on improving the network efficiency of IEEE 802.11e networks HARPREET BEDI, KAMAL KUMAR, GUPTA RAGHAV

35 Cluster head selection with dynamic clustering technique using mobility and stability parameters

MANOJ SINDHWANI, CHARANJEET SINGH

36 Optimization of fire disaster management technique using fuzzy tools

ANIL KUMAR RAWAT, KAMAL KUMAR SHARMA, MANWINDER SINGH

37 Plant species recognition using CNN

ROSEPREET KAUR BHOGAL, AJMER SINGH

38 Multilevel coding scheme with trellis codes as component code in $5 \mathrm{G}$ scenario

SHAKTI RAJ CHOPRA, AKHIL GUPTA

39 Optimal control of renewable energy system integrated with electric vehicle

MUZAFAR AHMAD SHAH, MOHAMMAD IMRAN MIR, JAVED DHILLON

40 Energy efficient concurrent MAC transmission based UWASN for tsunami warning

VIKRAM NAYYAR, KRISHAN KUMAR, MONIKA AGRAWAL

41 Comparative analysis of congestion pricing using application of PTDF method and Bialek's method in Indian Utility 146-Bus system

TANUJ MISHRA, AMIT KUMAR SINGH, VIKRAM KUMAR KAMBOJ

42 Motion detection and tracking of surveillance videos under distorted environments

CHINTHAKINDI KIRAN KUMAR, GAURAV SETHI, KIRTI RAWAL

43 An effective strategy for denoising of MRI images to detect brain tumour

BANDANA BALI, BRIJ MOHAN SINGH, KAMAL KUMAR

44 Wideband MIMO antenna with improved isolation 
45 BBO based location optimization of target nodes using single mobile anchor node in WSNs

PARULPREET SINGH, GURLEEN KAUR WALIA, GAGANDEEP SINGH WALIA

46 Smart queue management system for public transportation

ROHITH SAI VEEDULA, PRANEETH ATAGARA METI, SRI GAYATHRI PABBAREDDY, TOWFEEQ FAYAZ, GURJOT SINGH GABA, LAVISH KANSAL

47 Proposal of new performance measures for ICA BHAVESHKUMAR C. DHARMANI

48 WSN sensor deployment and sampling interval: Role of dynamics AARTI KOCHHAR, NARESH KUMAR

49 Recognition and categorization of plant from leaf image using visual traits PARUL MITTAL

50 Fast method of face recognition in real time using Raspberry-Pi and Intel Movidius NCS

NAVJOT RATHOUR, RAJESH SINGH, ANITA GEHLOT

51 Design of an automatic on-load tap changing distribution transformer KRISHAN ARORA, TARUN DHANDHEL

52 An advanced driver assistance system for autonomous vehicles: Design and test in virtual environment

RAHUL KUMAR MAHATO, AVINASH KUMAR, JYOTI PRASAD KURMI, SANABOINA SNIGDHA, GURJOT SINGH GABA, RAJAN MIGLANI

53 Evaluation of additive manufacturing using Marlin firmware JONNADA SAI ROHIT, NITIN KUMAR

54 Analysis of $5 \mathrm{G}$ channel parameters using mmWave channel simulator NYUSIM

AKHIL GUPTA, MEGHANA TRIPATHI, PRODDATUR LOK PAVAN KUMAR, ALAMURU VENKATA HARSHA VARDHAN

55 Raspberry Pi-based smart waste management system using Internet of Things SHAIK VASEEM AKRAM, RAJESH SINGH, ANITA GEHLOT

56 Comparative performance analysis of service discovery protocol and interference management schemes for device to device communication in $5 \mathrm{G}$ network 
$\mathrm{x}$ Contents

57 Raspberry-Pi and Intel Movidius Assisted Framework for Fruit Quality Monitoring

NAVJOT RATHOUR, PRANAB KUMAR MISHRA, KARTIK GAUR,

ABHISHEK MADDHESHIYA

58 Control scheme of doubly-fed induction generators applied in wind energy conversion systems

BHAGWAN SHREE RAM, NARBADA PRASAD GUPTA

59 Stability assessment of single-area thermal automatic generation control system using eigenvalue analysis

MEGHA KHATRI, S. HAREESH REDDY, HIMANSHU AGGARWAL

60 Design and analysis of a shared charged dynamic latch comparator TEJENDER SINGH, SUMAN LATA TRIPATHI

61 Load flow studies of LV microgrid using ETAP CHANDAN KAMBLE, JAVED DHILLON

62 A universal memory that never runs out of steam URVASHI SHARMA, SACHIN MISHRA, GULSHAN KUMAR, REJI THOMAS

63 Fault simulation algorithm for transistor single stuck short faults DURGESH ADDALA, P. TEJA, SOBHIT SAXENA

64 Qualitative performance analysis of greyscale image denoising techniques

AMANDEEP SINGH, GAURAV SETHI, G.S. KALRA

65 A novel hybrid GWO-PS based solution approach for combinatorial unit commitment problem

MEENAKSHI GUPTA, VIKRAM KUMAR KAMBOJ, R.K. SHARMA

66 Power quality based comparative analysis of resonant inverters SYED IQRA, HIMANSHU SHARMA

67 A comprehensive analysis of biosensor and performance parameters PAWANDEEP KAUR, AVTAR SINGH BUTTAR, ASHUTOSH SHARMA, G.P. SINGH

68 A review: Building fire detection and emergency evacuation system G.S. BIRAJDAR, RAJESH SINGH, ANITA GEHLOT

69 Statistical evaluation of colour image denoising techniques AMANDEEP SINGH, GAURAV SETHI, G.S. KALRA

70 Smart water quality analysis system using datapine 
71 Design of MPPT based controller for BLDC motor drive AARYAN RAJ, AMAN GANESH

72 Hybrid channel estimation via fuzzy method over Nakagami-m fading in MIMO-OFDM system HARMANDAR KAUR, MAMTA KHOSLA, R.K. SARIN, GURLEEN KAUR WALIA

73 Analysis of IoT sensors for monitoring the oil pipeline parameters CHAVALA LAKSHMI NARAYANA, RAJESH SINGH, ANITA GEHLOT

74 Crop survival analysis using machine learning and Internet of Things

CH SAI DINESH REDDY, SWAPNIL BAGWARI

75 A review on video forensics system using soft computing techniques SUNPREET KAUR NANDA, DEEPIKA GHAI

76 A new method for calculation of permittivity of spacer in gas insulated busduct JANAKI PAKALAPATI, N. KARTHICK, G.V. NAGESH KUMAR

77 A performance study and PAPR assessment of OFDM system for the diverse modulation schemes PRABAL GUPTA, H. PAL THETHI

78 Power system security of distributed network using contingency analysis

MOHAMMAD IMRAN MIR, AMIT KUMAR SINGH, MUZAFAR AHMAD SHAH

79 4K HDMI USB3 capture card reference design YASHWANT REDDY KEESARA, RASHI VATSA, SOMEET SINGH

80 A comparative review of different TMOs for HDR images N. NEELIMA, Y. RAVI KUMAR

81 Classification of P2P file-sharing traffic using heuristic based and statistical based techniques MAX BHATIA, VIKRANT SHARMA

82 A review of crop disease and pest forewarning using machine learning SACHIT DUBEY, RAJU BARSKAR, ANJNA JAYANT DEEN

83 Modelling of inverters using $\mathrm{MoS}_{2}$ based transistors MURALI KRISHNA M, PRASANTHA R. MUDIMELA

84 Hardware based design of smart warehouse monitoring system MOHIT CHARAN MULLAPUDI, MANASIJ CHAKRABORTI, AKHIL KUMAR PANDA, GARV SEHGAL, S.K. SINHA 
85 Design of battery management and monitoring system for electric vehicles

B. MANISH REDDY, ANOOP KUMAR VERMA, SIDDHANT TREASURE, VIJAY KUMAR TAYAL

86 Stewart Platform: The 6 DOF parallel manipulator MANAN LUTHRA, S.K. SINHA

87 IoT security algorithms: A performance comparison ADNAN MUKHTAR, P.M. TIWARI, H.P. SINGH

88 COVID-19 ramifications during pregnancy RYDHM BERI, MITHILESH K.R. DUBEY, ANITA GEHLOT, RAJESH SINGH

89 Frame selection based video watermarking technique based on DWT, K-SVD, rail fence to address quality loss of data CHIRAG SHARMA, AMANDEEP

90 Deep learning inspired continuous estimation framework for Scrum projects 


\section{About the editors}

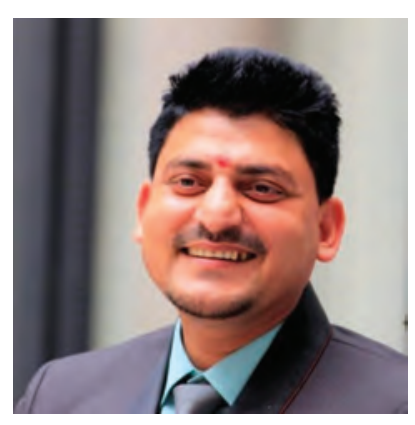

Dr. Rajesh Singh is currently associated with Lovely Professional University as Professor with more than sixteen years of experience in academics. He has been awarded as gold medalist in M.Tech from RGPV, Bhopal (M.P) India and honours in his B.E from Dr. B.R. Ambedkar University, Agra (U.P), India. His area of expertise includes embedded systems, robotics, wireless sensor networks and Internet of Things. He has organized and conducted a number of workshops, summer internships and expert lectures for students as well as faculty. He has been honoured as keynote speaker and session chair to international/national conferences, faculty development programs and workshops. He has twenty-seven patents in his account. He has published more than a hundred research papers in referred journals/ conferences and twenty-six books in the area of Embedded Systems and Internet of Things with reputed publishers like CRC/Taylor \& Francis, Narosa, NIPA, River Publishers, and Bentham Science.

Under his mentorship students have participated in national/international competitions including "Innovative Design Challenge competition" by Texas and DST and Laureate award of excellence in robotics engineering, Madrid, Spain, in 2014 and 2015. His team has been the winner of "Smart India Hackathon-2019" hardware version conducted by MHRD, Government of India, for the problem statement of Mahindra and Mahindra. Under his mentorship the students team got "InSc award 2019" under the students projects program. He has been awarded with "Gandhian Young Technological Innovation (GYTI) Award", as Mentor to "On Board Diagnostic Data Analysis System-OBDAS", Appreciated under "Cutting Edge Innovation" during the Festival of Innovation and Entrepreneurship at Rashtrapati Bahawan, India, in 2018.

He has been awarded as "Best Researcher Award" at MANTHAN (Awards of Excellence), organized by University of Petroleum and Energy Studies, 2017, and "Research Appreciation Award-2019" for contribution in research from Lovely Professional University. Twice in four years he has been awarded with "certificate of appreciation" from University of Petroleum and Energy Studies for exemplary work for conducting multi-disciplinary workshops for the students. He got "certificate of appreciation" for mentoring the projects submitted to Texas Instruments Innovation challenge India design contest, from Texas Instruments in 2015and 2018. He has been honoured with "Certificate of Excellence" from 3rd faculty branding awards-15, organized by EET CRS research wing, for excellence in professional education and industry, for the category "Award for Excellence in Research", 2015, and young investigator award at the International Conference on Science and Information in 2012. 


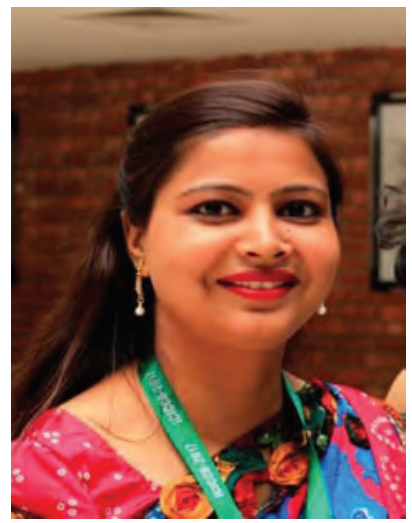

Dr. Anita Gehlot is currently associated with Lovely Professional University as Associate Professor with more than thirteen years of experience in academics. Her area of expertise includes embedded systems, wireless sensor networks and Internet of Things. She has organized and conducted a number of workshops, summer internships and expert lectures for students as well as faculty. She has been honoured as keynote speaker and session chair to international/national conferences, faculty development programs and workshops. She has twenty-four patents in her account. She has published more than seventy research papers in referred journals/conferences and twenty-five books in the area of Embedded Systems and Internet of Things with reputed publishers like CRC/Taylor \& Francis, Narosa, NIPA, River Publishers, and Bentham Science.

She has been awarded with "Certificate of Appreciation" from the University of Petroleum and Energy Studies for exemplary work. Under her mentorship the students team got "InSc Award 2019" under the students projects program. She has been awarded with "Gandhian Young Technological Innovation (GYTI) Award", as Mentor to "On Board Diagnostic Data Analysis System-OBDAS", Appreciated under "Cutting Edge Innovation" during the Festival of Innovation and Entrepreneurship at Rashtrapati Bahawan, India, in 2018. 


\section{Organizing Committee}

\section{Chief Patron}

Mr. Ashok Mittal

Mrs. Rashmi Mittal

\section{Patron}

Prof. Rameshwar Kanwar

Er. H.R. Singla

Conference General Chair

Prof. Lovi Raj Gupta

Program Chair

Dr. R.P. Saini

Publication Chair and Editor

Dr. Rajesh Singh

Dr. Anita Gehlot

Conference Chair

Prof. Bhupinder Verma

Conference Co-Chair

Dr. Gaurav Sethi

\section{Convenor}

Dr. Sachin Mishra

Dr. Prasantha Reddy

\section{Co-Convenor}

Dr. Amit Kumar Singh

Dr. Akhil Gupta
Chancellor, Lovely Professional University

Pro-Chancellor, Lovely Professional University

Vice-Chancellor, Lovely Professional University

Director General, Lovely Professional University

Executive Dean, LFTS, Lovely Professional University

Professor, Indian Institute of Technology Roorkee

School of Electronics and Electrical Engineering

School of Electronics and Electrical Engineering

School of Electronics and Electrical Engineering, Lovely Professional University

School of Electronics and Electrical Engineering, Lovely Professional University

School of Electronics and Electrical Engineering, Lovely Professional University

School of Electronics and Electrical Engineering, Lovely Professional University

School of Electronics and Electrical Engineering, Lovely Professional University

School of Electronics and Electrical Engineering, Lovely Professional University 


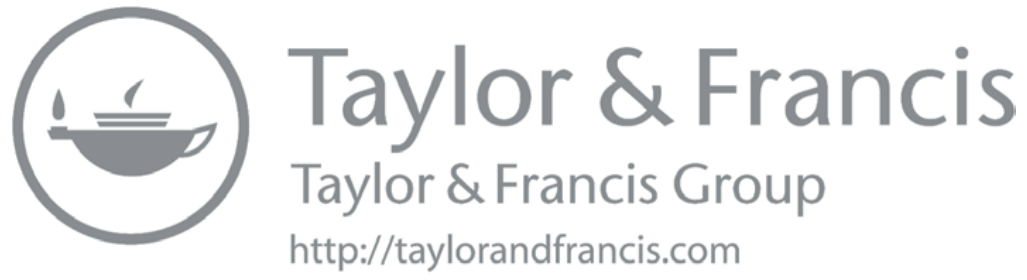




\section{Technical Committee}

\section{International Advisory Board Members}

- Dr. Shaurya Agarwal, Advisor, Electrical \& Computer Engineering, Calstatela University

- Dr. Francesca Iacopi, Professor, Computing \& Communications, School of Electrical and Data Engineering, University of Technology Sydney, Australia

- Dr. Apel Mahmud Lecturer, Faculty of Sci Eng \& Built Env, School of Engineering, Deakin University, Australia

- Dr. Hojjat Adeli (Fellow IEEE), Professor, The Ohio State University, USA

- Dr. Shwartz Adam, Professor, Faculty of Electrical Engineering, Technion - Israel Institute of Technology, Israel

- Dr. Hany Mohamad Amin Elghazaly, Cairo University

- Dr. Jamal Rammal, Technology Department, MUC University, Lebanon

- Dr. Saulius Japertas, Department of Telecommunications, Kaunas University of Technology, Lithuania

- Dr. Higmet Kamberaj, Professor, International Balkan University, Macedonia

- Dr. A. Taheri, Professor, University of Zanjan, Iran

- Dr. Sandor M. Veres, University of Sheffield

- Dr. Cuneyt Suheyl Ozveren, Sr. Lecturer, School of Engineering, Abertay University

- Dr. P. Barry Butler, Provost, The University of Iowa, USA

- Dr. A. Moffat, Bradford Professor, University of Melbourne, Australia

- Dr. Ranu Jung, Professor, Florida International University, USA

- Dr. Atif Iqbal, Professor, Qatar University

- Dr. Charles Malpas, Professor, University of Melbourne, Australia

- Dr. Kamal Bechkoum, Professor, The University of Northampton, UK

- Dr. Ellen L. Walker, Professor, Hiram College, Ohio, USA

- Dr. Nalin Sharda, Professor, University of Western Australia

- Dr. Harpreet Singh, Professor, Wayne State University, USA

- Dr. Ali Kaveh, Professor IUST, Narmak, Tehran, Iran

- Dr. Madhu Chandra, Professor, Chemnitz University of Technology, Germany

- Dr. Shiva Kumar Sastry, Associate Professor, The University of Akron, USA

- Dr. Swarup Bhunia, Associate Professor, CWR University, USA

- Dr. Taha Selim Ustun, Assistant Professor, Carnegie Mellon University, USA

- Dr. Seema Arora, Assistant Professor, Waljat College of Applied Sciences, Muscat, Oman

- Dr. Saurabh Aggrawal, WWB Development Manager, Geneva, Switzerland

- Dr. Rahul Upneja, McMaster University, Canada

- Dr. Ateet Dutt, Postdoc Fellow, UNAM Mexico 


\section{National Advisory Board Members}

- Dr. D.P. Kothari, Director General, J B Group of Institutions, Nagpur

- Dr. R.S. Khandpur, Director General, Pushpa Gujral Science City, Kapurthala (Punjab)

- Dr. S.N. Singh, Chair Professor, IIT, Kanpur

- Dr. T.S. Kamal, Principal, RIET, Abohar, Punjab

- Dr. A.A. Godbole, Professor, All India Shri Shivaji Memorial Society's College of Engineering, Pune, Maharashtra

- Dr. Naqui Anwer, Associate Professor, Department of Energy and Environment, TERI University, New Delhi

- Dr. Dheeraj Kumar Khatod, Associate Professor, EE Department, IIT Roorkee

- Dr. Gauri Sankar, Assistant Professor, EE Department, IIT (ISM) Dhanbad

- Dr. Manoj Panda, Associate Professor, EE Department, GBPEC, Pauri

- Dr. Vinay Pant, Assistant Professor, EE Department, IIT Roorkee

- Dr. B. Das, Professor, EE Department, IIT Roorkee

- Dr. Ghatak Chaudhary, Assistant Professor, EE Department, IIT Roorkee

- Dr. S.K. Sinha, Professor, EE Department, Amity University

- Dr. Anurag Chauhan, Assistant Professor, EE Department, REC Banda

- Dr. Muhammed Shanir PP, Department of Electrical \& Electronics Engineering, TKM College of Engineering, Karikodu, Kollam (Kerala)

- Dr. V.K. Tayal, Associate Professor, EE Department, Amity University

- Dr. P.M. Tiwari, Associate Professor, EE Department, Amity University

- Dr. Anwar Shahzad Siddiqui, Professor, Department of Electrical Engineering, Faculty of Engineering \& Technology, Jamia Millia Islamia

- Dr. Ravi Kumar, Associate Professor, Department of Electronics Engineering, Thapar University

- Dr. Gowari Ramaswamy, Professor, Department of Electronics \& Instrumentation Engineering, College of Engineering Studies, UPES, Dehradun

- Dr. H.P. Singh, Professor, EE Department, Amity University

- Dr. Amit Kumar Singh, Assistant Professor, EE Department, NIT Jalandhar

- Mr. M.G. Soni, Associate Professor, Department of Electrical Engineering, Faculty of Engineering, Jai Narain Vyas University, Jodhpur

- Dr. Anil Kumar, Assistant Professor, Shoolini University

- Dr. Yusuf U Khan, Professor, EE Department, AMU

- Dr. M.L. Singh, Dean Faculty of Technology and Engineering, G.N.D.U. Amritsar

- Dr. Jyotish Malhotra, Associate Dean, GNDU Regional Campus, Jalandhar

- Dr. Deepak Garg, Professor and Head, Bennett University, Greater Noida

- Dr. Y.R. Sood, Professor and Head, EED, NIT Hamirpur

- Dr. Lillie Dewan, Professor, NIT, Kurukshetra

- Dr. Anwar Shahzad Siddiqui, Professor, FET Jamia Millia Islamia, New Delhi

- Dr. R. Jha, Professor, NIT Jalandhar

- Dr. Manoj Duhan, Professor, DCRUS \& T Murthal, Haryana

- Dr. Aditya Goel, Professor, MANIT Bhopal

- Dr. Ashwini Kumar, Professor, NIT Kurukshetra

- Dr. K.S. Sandhu, Professor, NIT Kurukshetra.

- Dr. Prerna Gaur, Professor, NSIT, Secretary IEEE, Delhi Section

- Dr. S.S. Gill, Professor, GNDEC, Ludhiana

- Dr. Smarjit Ghosh, Professor, Thapar University, Patiala

- Dr. J.S. Ubhi, Associate Professor and Head, SLIET Longowal 
- Dr. Ikbal Ali, Associate Professor, FET Jamia Millia Islamia, New Delhi

- Dr. Ajaypal Singh, Associate Professor, SLIET Longowal

- Dr. Dilbagh Singh, Associate Professor, NIT Jalandhar

- Dr. B.S. Saini, Associate Professor, NIT Jalandhar

- Dr. Harsh Kumar Verma, Associate Professor, NIT Jalandhar

- Dr. Arun Khosla, Associate Professor, NIT Jalandhar

- Dr. Kulbir Singh, Associate Professor, Thapar University

- Dr. R.K. Jarial, Associate Professor, NIT Hamirpur

- Dr. Mandeep Singh, Associate Professor, Thapar University, Patiala

- Dr. Vineet Dhaliwal, Associate Professor, G.N.D.U., Regional Campus Jalandhar

- Dr. Manoj Taleja, Associate Professor, GGSIP, New Delhi

- Dr. Tirupathiraju Kanumuri, Assistant Professor and Head, NIT Delhi

- Dr. Indu Saini, Assistant Professor, NIT Jalandhar

- Dr. Balwinder Raj, Assistant Professor, NIT Jalandhar

- Dr. Prashant Kumar Tiwari, Assistant Professor, NIT Silchar

- Dr. Paresh G. Kale, Assistant Professor, NIT Rourkela, Odisha

- Dr. Ankur Dumka, Assistant Professor, University of Petroleum and Energy Studies, Dehradun

- Dr. Tushar Kanti Bera, Assistant Professor NIT Durgapur

\section{Corporate Advisory Board}

- Dr. Vivek Jain, Senior Research Engineer, Wireless Research and Technology Center Robert Bosch LLC, USA

- Dr. Manjit Singh, Director, TBRL, DRDO, Chandigarh

- Mr. Gopal Kumar Rai, Engineers India Limited, New Delhi

- Mr. Jaswinder Ahuja, Vice-President and MD, Cadence, New Delhi

- Dr. Amod Kumar, Scientist, 'G' CSIO, Chandigarh

- Mr. N.K. Jain, GM, Electronics Dev Corp. Ltd., Ambala, Haryana

- Dr. Prabhjot Kaur, Deputy Director, Reliance IITM Telecom, Chennai

- Mr. Prashant Gupta, Assistant Director (Technical), NIELIT, Delhi

- Mr. Rahul Ranjan, SMD, Qualcomm

- Mr. Jagbir Singh Nirwan, MD, Edivoyage Services

- Mr. Navdeep Singla, DGM, Ambuja Cement Limited, Chandigarh

- Mr. Surender Dhariwal, DGM, Jagatjit Industries Ltd, Behror, Alwar

- Ms. Sheetal Dhiman, Manager, Maruti Suzuki (I) Ltd, Gurgaon

- Mr. Gaurav Goel, Manager, Nokia Siemens Network, Gurgaon

- Mr. Ravinder Yadav, Manager, NISG, New Delhi

- Mr. Surendar Kalyan, Manager, NEOKRAFT Global Pvt. Ltd., Noida

- Mr. Shikhar Sahni, Group Manager (Operations), GreyB, Chandigarh

- Mr. Amit Gupta, Associate at MARKIT, Noida 


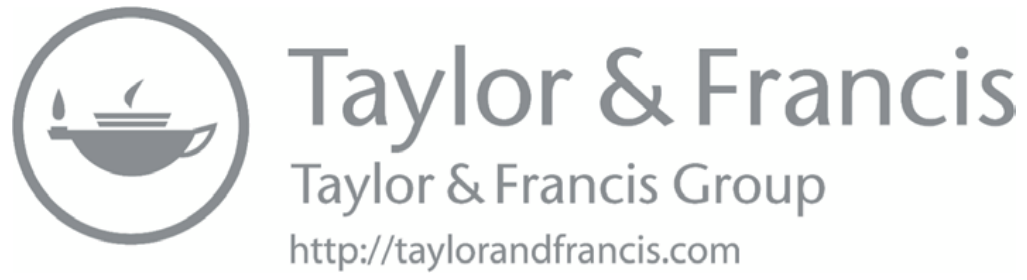




\section{Preface}

The 3rd International Conference on Intelligent Circuits and Systems was organized by the School of Electronics and Electrical Engineering of Lovely Professional University, Punjab, India, during June 26-27, 2020. Due to the ongoing pandemic this conference was held virtually through Google Meet.

The conference aims to promote, encourage and bring together researchers, scientists and academicians to share knowledge on recent advances made in the field of smart and green technologies in the fields of Energy, Electronics, Communication, Computers and Control.

A total of 306 research papers were submitted to the conference and after peer review around $30 \%$ of total papers (91 papers) were accepted for the presentation.

The presenters highlighted the challenges posed by COVID-19. It needs non-contact and smart systems using intelligent circuits and systems to realize solutions.

The two-day conference witnessed renowned keynote speakers from academicians and industrialists from different corners of the world, including Professor (Dr.) D.P. Kothari, IEEE Fellow, ex-VC VIT Vellore and ex-Director (I/C); Dr. Albert Nasibulin, Professor, Center for Photonics and Quantum Materials, Skolkovo Institute of Science and Technology, Russia; Dr. Ishak Bin Aris, Professor, Department of Electrical and Electronic Engineering, Universiti Putra Malaysia; Dr. Dushanta Nalin Jayakody, Head, Infocomm Lab, School of Computer Science and Robotics, National Research Tomsk Polytechnic University, Russia; Dr. Rajendra Pratap, Head, Design Center, Arrow Electronics India, Noida, India; and Mr. Siddharth Vasishat from Siemens Limited.

The conference covered the topics relevant to the pursuit of Intelligent Circuits \& Intelligent Systems and Emerging Technologies in Engineering, such as Image and Video Processing, Computational Electromagnetics, RF, Microwave \& Antenna, Sensors \& Sensor Technology, Industry 4.0, Industry IoT, ICT for Agriculture and Social Welfare, Machine Learning, Renewable Energy, Electrical Machines, Power Electronics and Industry Applications, Modelling and Simulation of Grid Environments and Markets, Robotics and Control Applications, Electrical Markets, Predictive Maintenance through Digitization, etc.

The Editors

MATLAB $^{\circledR}$ is a registered trademark of The MathWorks, Inc.

For product information please contact:

The MathWorks, Inc.

3 Apple Hill Drive

Natick, MA, 01760-2098 USA

Tel: 508-647-7000

Fax: 508-647-7001

E-mail: info@mathworks.com

Web: www.mathworks.com 


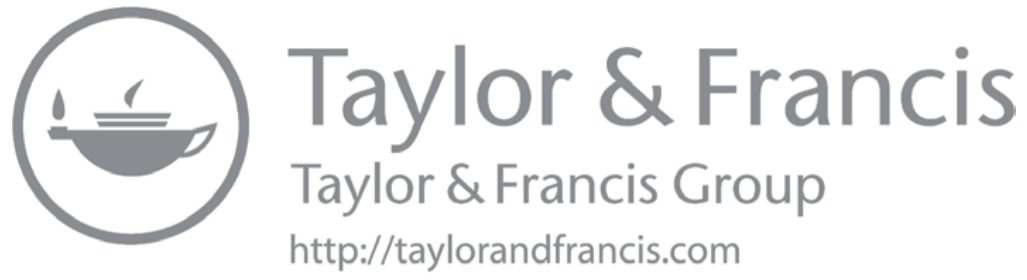




\title{
1 Post PV integration protection issues
}

\author{
B. Koti Reddy, *, Amit Kumar Sing ${ }^{2, *}$ \\ ${ }^{1} \mathrm{PhD}$ Scholar, Lovely Professional University, India \\ ${ }^{2}$ Associate Professor, Lovely Professional University, Phagwara, \\ Jallandhar, Punjab (India)
}

\section{Introduction}

The ongoing demand for continuous qualitative electrical power from consumers as well as electricity in remote areas opened the doors for de-regulated electricity markets and many innovations. Microgrids with small to medium distributed generators is one of the ideas developed to meet these demands. Even though it is at an infant stage, it will be a quick alternative to the existing power system problems of reliability, stability and efficiency (RSE). A microgrid is a group of interconnected loads and distributed energy resources (DER) within a clearly defined electrical boundary that acts as a single controllable entity with respect to the grid [1]. It can connect or disconnect from the grid to enable it to operate in both grid-connected or island (standalone) mode. A simple model of microgrid is shown in Figure 1.1 [2], which is self-explanatory. With the advances in power electronics, microgrids are set to grow in a fast phase in coming years [3].

There are many benefits with microgrids:

- Useful to serve remote places.

- Reduced transmission and distribution losses.

- Improve stability of existing grids.

- Meet mandatory requirements of DERs.

- Offers different service options to customers.

- Reduces grid congestion and peak load shedding.

Microgrids (MG) introduce many operational challenges, viz. technical, financial and legal issues, which must be addressed by utilities while adopting microgrids. The main technical issues are synchronism, voltage stability, fault currents and protection [4]. The other main challenges are the cost of DERs, cost of new protection and communication equipment, storage issues, advanced infrastructure like SCADA costs etc. The scope of this paper is restricted to protection issues, which will be discussed in subsequent paragraphs. The future DERs, storage devices and loads can be added without any hurdles like adding RAM or hard disks to the existing system in a desktop.

\section{Description and methodology}

The industry under study is the chemical industry, whose power schematic diagram before PV integration is shown in Figure 1.2, which is self-explanatory. With the addition of a 2 MWp solar photo voltaic (SPV) power plant (SPP), the same has become as a microgrid,

*Emails: kotireddy1965@gmail.com, amit.20267@lpu.co.in

DOI: $10.1201 / 9781003129103-1$ 


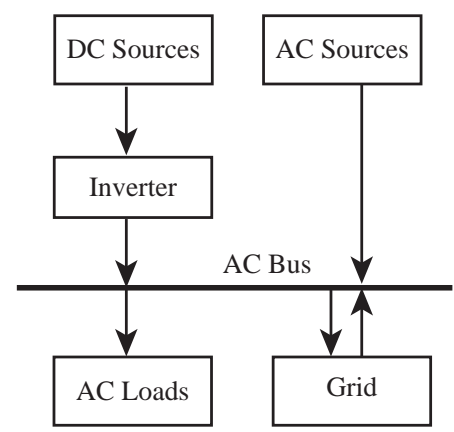

Figure 1.1 Microgrid concept.

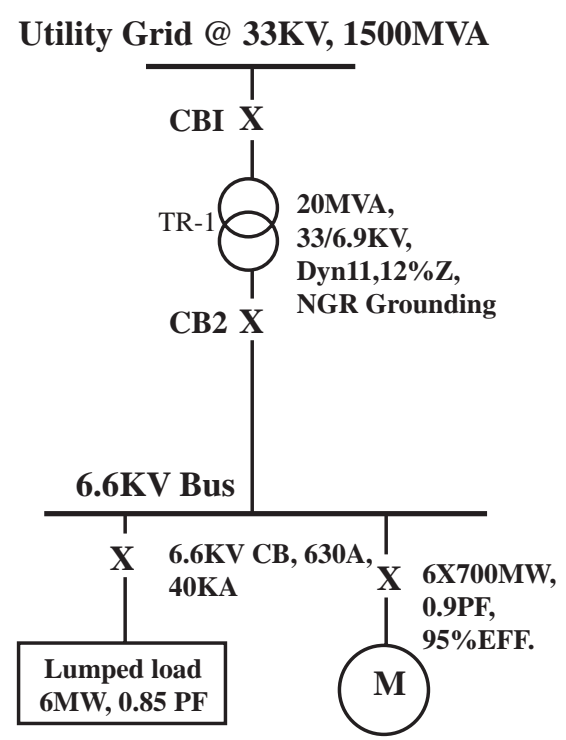

Figure 1.2 Pre-integration schematic.

as shown in Figure 1.3. At point of common coupling (PCC), the CB-3 with its protective relay isolates the DER from the utility grid in the case of fault on either side. The brief technical particulars of transformers are shown in Figure 1.4.

The protection system management (PSM) in microgrids is very challenging due to twoway or bi-directional power flow and device adequacy. The protection system has to isolate the microgrid from the utility grid in the case of fault on either side. It should protect the existing system and devices and grid when a fault occurs on either side. Hence, a properly co-ordinated protection system is required in a microgrid network with strict control of isolations. The protective relays shall be time-graded for over-current and earth faults [5]. Even though the total electrical network is large in size, the present study is restricted to $6.6 \mathrm{kV}$ switchgear (popularly known as SWGR) i.e., PCC and its power "in" and "out" connections for fault current calculations and simulations. The total load of the industries was 10.2 MW which was totally met from the utility grid and SPP as and when it is available. 


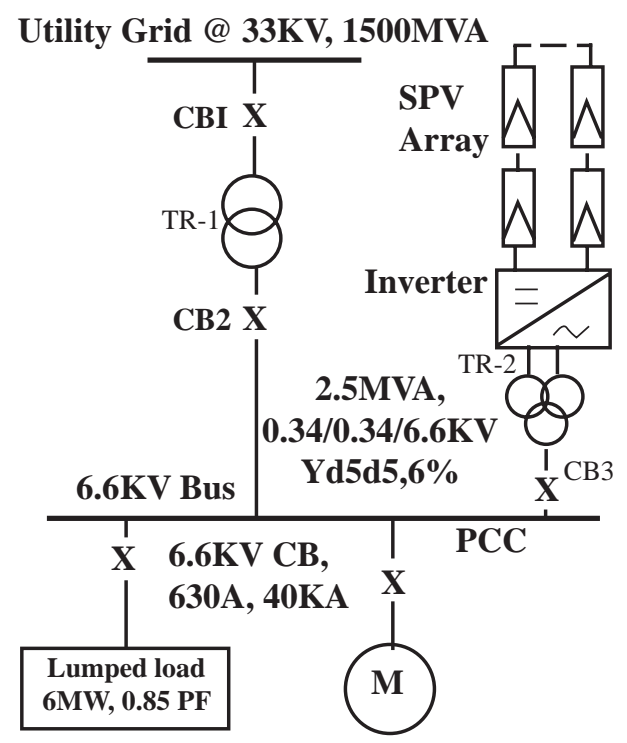

Figure 1.3 Post-integration schematic.

\begin{tabular}{|l|l|l|}
\hline Parameter & TR-1 & TR-2 \\
\hline MVA rating & 20 & 2.5 \\
\hline Primary voltage-KV & 33 & $0.34 / 0.34$ \\
\hline Sec. Voltage-KV & 6.6 & 6.6 \\
\hline$\%$ Z & 12 & 6 \\
\hline Amps-Primary & 340 & $2123 / 2123$ \\
\hline Amps-Secondary & 1749 & 209 \\
\hline Vector group & Dynll & Yd5d5 \\
\hline Grounding & 3.80 hmis resistor & Solid \\
\hline
\end{tabular}

Figure 1.4 Transformers details.

The details of the PV equipment are given below:

Each PV module: $255 \mathrm{~W}, 30.5 \mathrm{~V}$ (Vmp), 37.6 V (Voc), $8.42 \mathrm{~A}$ (Imp), 8.95 A (Isc). Each string: 21 nos of modules in series to give $340 \mathrm{~V}, 5.355 \mathrm{~kW}$. Total strings in parallel: 2 arrays X3927nos to give $630 \mathrm{~V}, 2 \times 1 \mathrm{MWp}$.

The annual average solar irradiance of the area is $5 \mathrm{kWH} / \mathrm{m}^{2}$.

Inverter: $800 \mathrm{~V} \mathrm{DC} / 340 \mathrm{~V} \mathrm{AC}, 50 \mathrm{~Hz}, 3$-phase with a maximum fault contribution of 1.5 times the rated current.

Power system analysis reviews [6] the study of the various fault currents, rating of equipment [6] to with stand the faults [7] and protective relay settings accordingly. The protective device provides a form of insurance [8] for vital electrical equipment which reduces the equipment damage and danger to humans in the vicinity. Whenever an equipment or distributed generation is added, proper relay coordination [9] is to be ensured to protect the entire network as intended and to meet the overall objective of coordination of protection and switching devices. The PV integration may cause loss of sensitivity of 


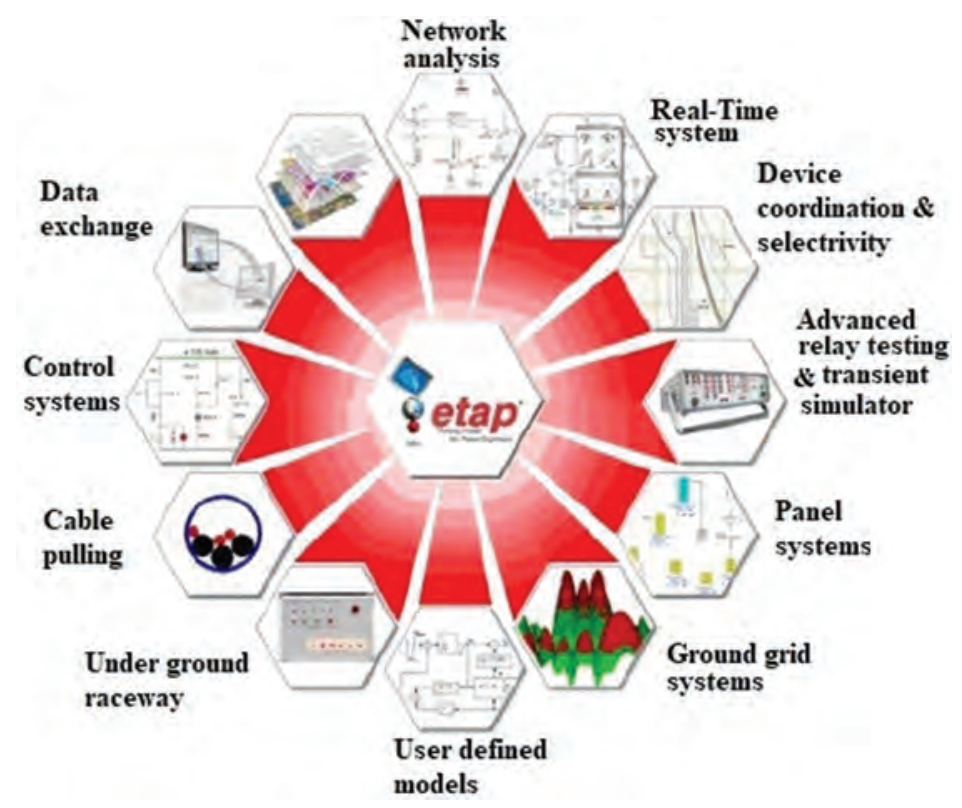

Figure 1.5 E-Tap components.

over-current and earth fault relays [10]. Hence, a detailed analysis is required to avoid mis-operation and unwanted trips. When a fault occurs in the grid, the fault is additionally supplied by distributed generators and may change the operating sequence of circuit breakers [11]. Hence, it is to be ensured that during any fault in the system, the distributed generators like PV generation should be isolated as quickly as possible so that the conventional protection system will isolate the faults as intended. It is proposed to estimate the fault currents and the relay operation time for both pre and post-integration scenarios with the help of e-tap software. The ETAP power station is the most sophisticated power system analysis tool on the market now which supports around 10,000 IEEE bus projects. The main work models are shown in Figure 1.5.

\section{Simulations before PV intergration}

Simulations are done with the help of e-tap software for the following scenarios:

A. Pre-integration load flow: the screenshot of simulation result is shown in Figure 1.6.

B. Pre-integration fault current for a 3-phase bolted fault at PCC: The screenshot of simulation is shown in Figure 1.7.

C. Pre-integration relay coordination for above fault: the screenshot of result is shown in Figure 1.8 .

\section{Simulations post-PV intergration}

A. Post-integration load flow: the simulation result is shown in Figure 1.9.

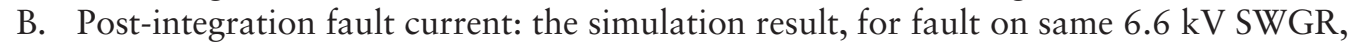
is shown in Figure 1.10.

C. Post-integration relay coordination: the screenshot of result is shown in Figure 1.11. 


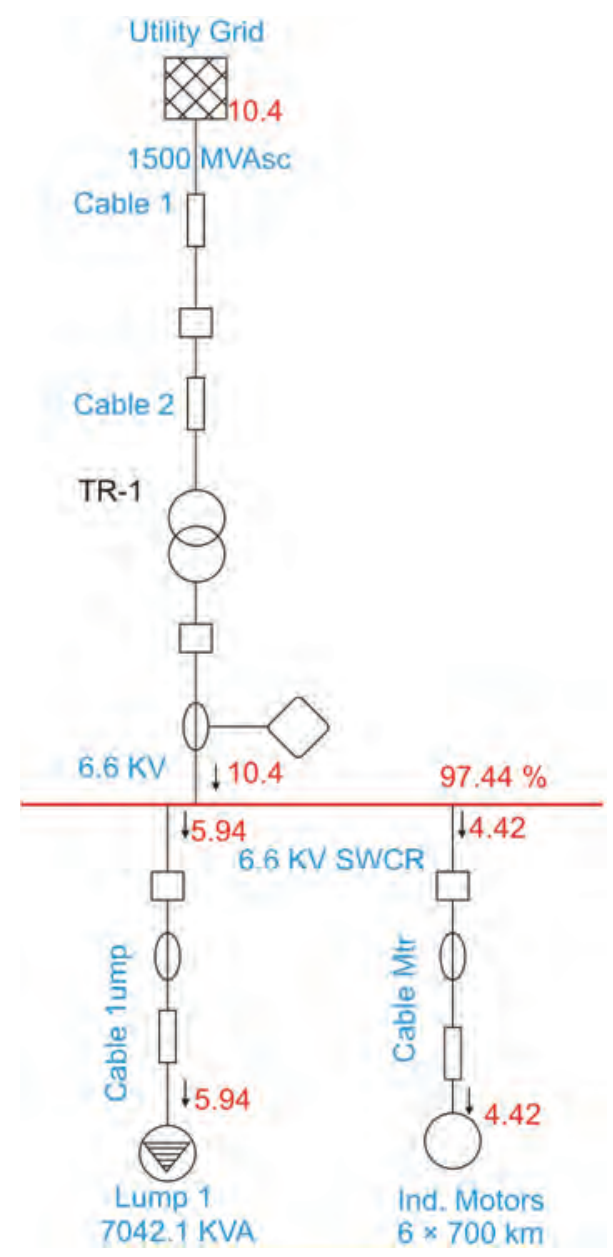

Figure 1.6 Pre-integration load flow.

\section{Inference of results}

All the simulation results are given in Table 1.1.

The protection simulation results of relays operation time are shown in Table 1.2 for fault at PCC.

\section{Inference}

The impact of DER at PCC is to be checked with the help of stiffness ratio (SR) [12], which is used to evaluate the impact of DERs on system fault levels. As a general rule, if SR of more than 50, DERs are less likely to create any voltage problem on grids. In our case study, $\mathrm{SR}=19.979 / 0.212=94.24$, which is reasonable and easily controllable. It is confirmed that there is not much variation in fault currents post PV integration since their contribution to fault is an insignificant value of around $150 \%$ of their normal rating, unlike the $1000 \%$ to $2500 \%$ of conventional system's fault current. Hence there is no need to go for retrofitting and renovation of switchgear equipment [13]. Also, there is 


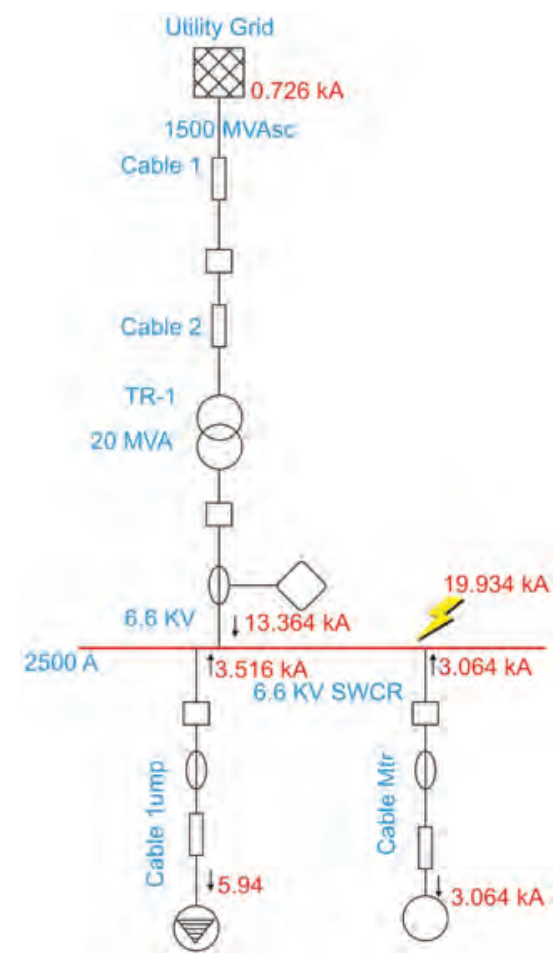

Figure 1.7 Pre-integration fault currents.

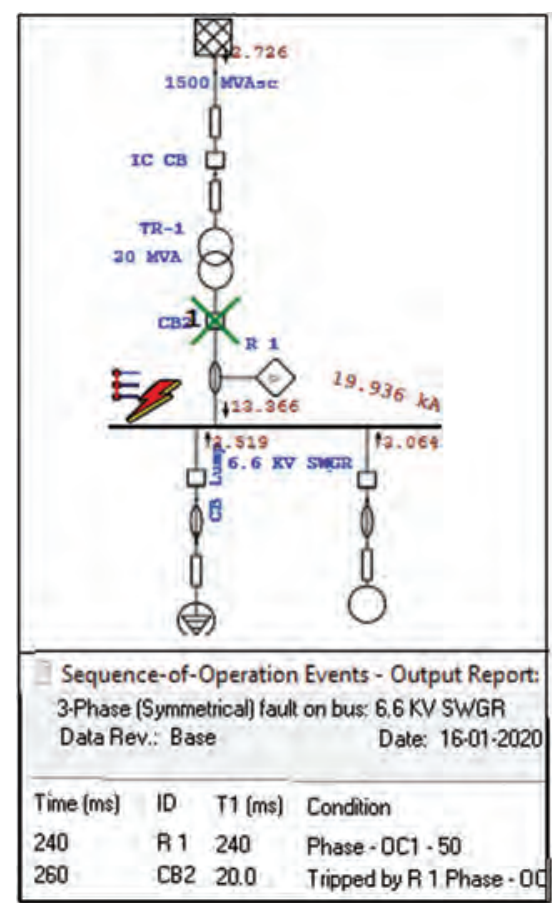

Figure 1.8 Pre-integration relay coordination. 


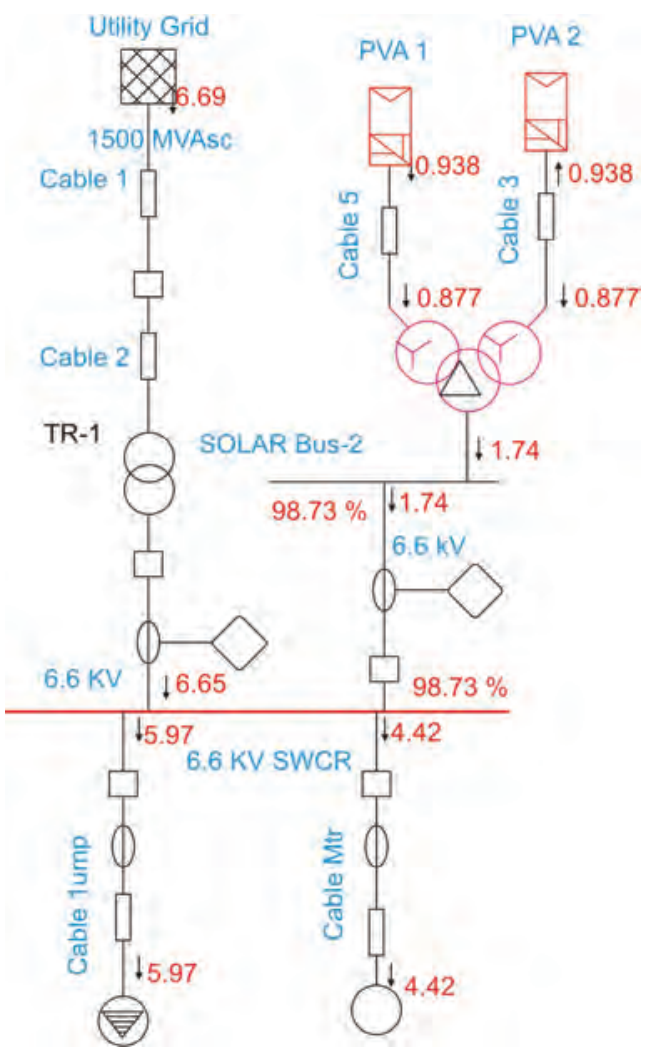

Figure 1.9 Post-integration load flow.

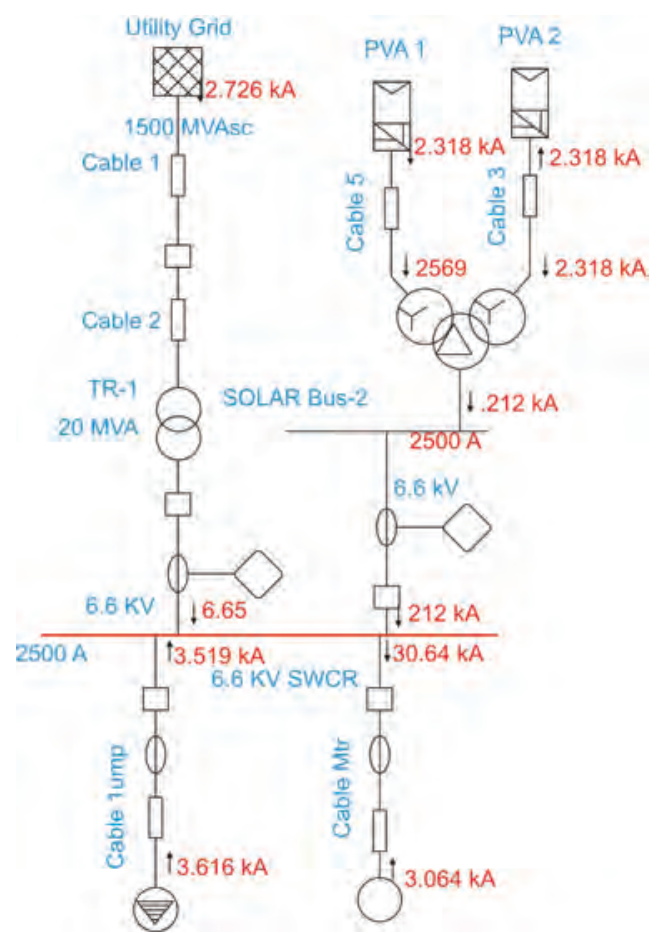

Figure 1.10 Post-integration fault current. 


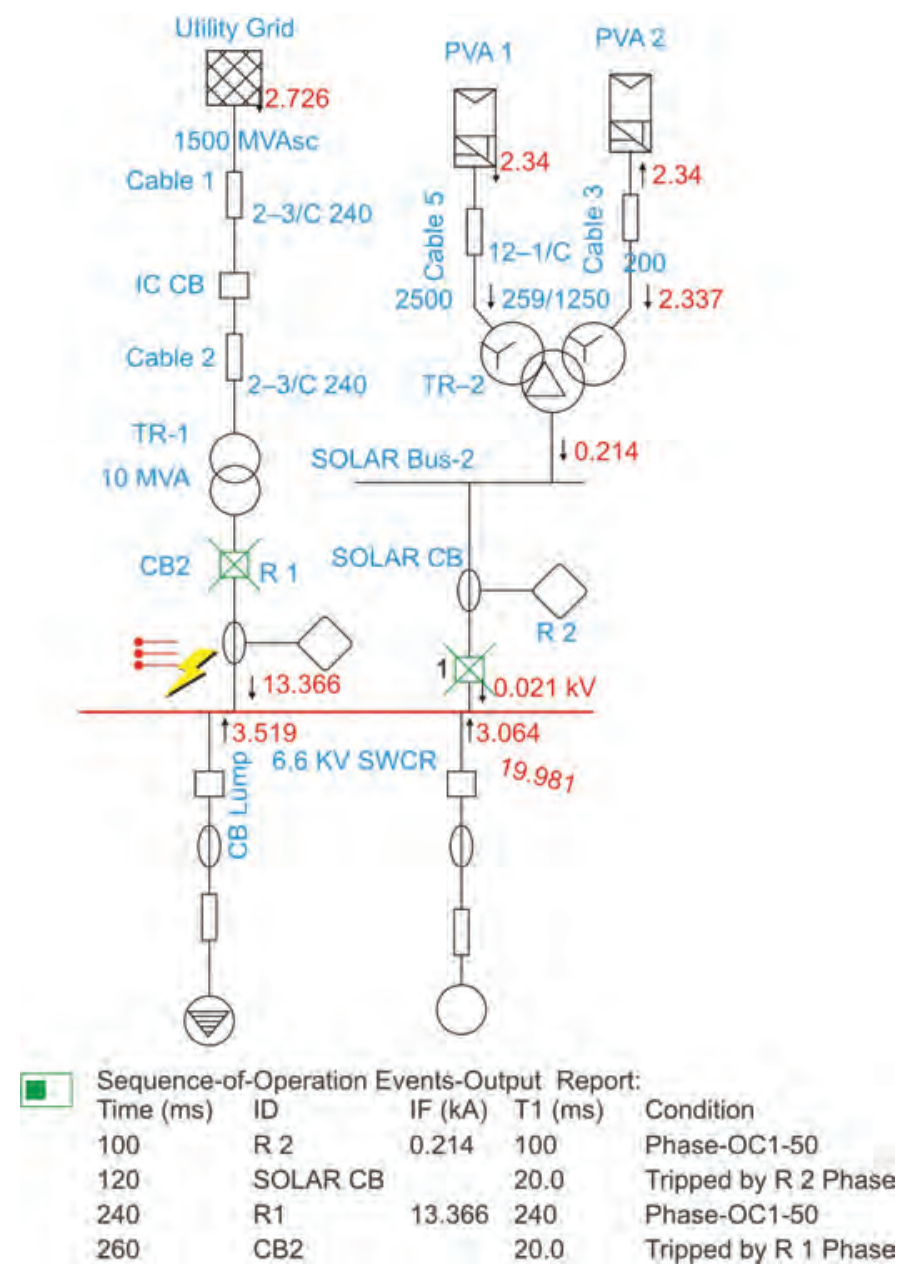

Figure 1.11 Post-integration relay.

no considerable effect on relay coordination since the faulty part is isolated very quickly including tripping of SPP on faults at grid side. Hence, this case study confirms that there is no need to think much about review of power system analysis after DG integration and a close setting of DGs is only required.

\section{Conclusions}

As the world is moving towards renewable and/or clean energy, all the utilities are making efforts to adopt distributed generation mostly by installing solar photo voltaic power plants but there are certain issues which they are concerned with. The major challenges are the increased fault currents and the relay coordination. In this paper, the same has been studied in detail with a case study. For the industry selected as a sample, various protection issues that arise due to integrating of PV system into the existing grid are discussed. Equipment rating for fault currents post PV integrating and the relay coordination are also checked and simulated for their adequacy and all the results are tabulated. From the results, it is concluded that there are no significant changes in the equipment rating and time-current 
Table 1.1 Simulation results

\begin{tabular}{|c|c|c|c|c|}
\hline \multirow[t]{2}{*}{ Simulation } & \multirow{2}{*}{$\begin{array}{l}\text { Pre-microgrid } \\
\text { at PCC }\end{array}$} & \multicolumn{2}{|c|}{ Post-microgrid } & \multirow[t]{2}{*}{ Remarks } \\
\hline & & $\begin{array}{l}\text { From grid } \\
\text { at PCC }\end{array}$ & $\begin{array}{l}\text { From } S P V \\
\text { at PCC }\end{array}$ & \\
\hline Load flow (MW) & 10.40 & 8.65 & 1.74 & Load is shared \\
\hline Bus voltage (\%) & 97.44 & 98.73 & & Improved Bus voltage \\
\hline Fault current (KA) & 13.364 & 13.364 & 0.212 & $\begin{array}{c}\text { Total }=13.576 \mathrm{kA} \text { i.e. } \\
\text { marginal increase. }\end{array}$ \\
\hline \multicolumn{5}{|c|}{ Relay operation for fault at (CB relay time in milliseconds) } \\
\hline PCC & CB2- R1- 260 & & $\begin{array}{l}\text { Solar CB- } \\
\text { R2-120 } \\
\text { CB2-R1-260 }\end{array}$ & $\begin{array}{l}\text { No change in relay } \\
\text { operation and } \\
\text { coordination is }\end{array}$ \\
\hline Lump load & CB2- R1- 260 & & $\begin{array}{l}\text { Solar CB- } \\
\text { R2-120 } \\
\text { CB2-R1-260 }\end{array}$ & maintained \\
\hline Motor & CB2- R1- 260 & & $\begin{array}{l}\text { Solar CB- } \\
\text { R2-120 } \\
\text { CB2-R1-260 }\end{array}$ & \\
\hline
\end{tabular}

Relay over current settings: R1 (Grid side): pick-up=0.71 with 0.15 TMS, instantaneous = 4.9 times @ 0.24 s. R1 (Grid side): pick-up=0.71 with 0.15 TMS, instantaneous=4.9 times@0.24 s.

For R2 (DG side), a close setting as given below, chosen for not disturbing the existing relay coordination: R2 (SPP side): pick-up =0.82 with 0.15 TMS, instantaneous =0.82 times@ $0.1 \mathrm{~s}$.

Table 1.2 Protection simulation results for L-L-L fault at PCC

\begin{tabular}{llc}
\hline & Pre-PV integration & Post-PV integration \\
\hline Fault current (kA) & 19.934 & 19.979 \\
Relay-R1 operation (s) & 0.240 & 0.240 \\
Relay-R2 operation (s) & - & 0.100 \\
Stiffness ratio (SR)* & - & 94.24 \\
\hline
\end{tabular}

*SR = fault current including DER/DER fault current.

characteristics (TCC) of protection systems. This study implies that the electrical utilities, industries or consumers need not put much attention on their electrical systems adequacy post distributed generator integration especially PV generators into their grid since their contribution during faults has a marginal impact and can be tackled very easily.

\section{References}

[1] A. Keyhani et al., "Integration of green and renewable energy in electric power system", Wiley, 2010.

[2] B. K. Reddy, B. S. Bindu, "Recent challenges in electrical engineering and the solution with IT", International Journal of Recent Technology and Engineering, vol. 8, 2S11, 2019.

[3] N. Hadsaid, J. Claude, “Smart Grids”, Wiley, 2012.

[4] P. Sudhakar et al., "Protection issues of power system with PV system based distributed generation", IOSR Journal of Electrical Engineering, vol. 9, 3, 2014.

[5] B. Ramakoti et al., "Study of fault currents and relay coordination of a chemical industry after integrating with PV generation and simulation with a software", International Journal of Engineering Trends and Technology, vol. 42, 5, 2016. 


\section{Intelligent Circuits and Systems}

[6] S. S. Rao, "Switchgear protection and power system", Khanna Publications, 2008.

[7] B. Ram, D.Vishwakarma, Power System Protection and Switchgear, Tata McGraw-Hill,2011.

[8] ABB Ltd, Distribution Automation Handbook, Section 8.2, Relay Coordination, IMRS 757285, 2011, Finland.

[9] Recommended practice for protection and coordination of industry and commercial power system, IEEE std: 242-1975, part 7.5.2.

[10] A.Canova et al., "Electrical impact of photo voltaic plant is distributed network", IEEE Trans. on Industry Applications, vol. 45, 1, 2009.

[11] P. Rodrignez, A. Ddrianvtimbus, "Flexible active power control of distributed power generation system during grid faults", IEEE Trans. on Industrial Electronics, vol. 54, 5, pp. 2583-2592, 2007.

[12] IEEE P1547-2018, IEEE Standard for Interconnection and Interoperability of Distributed Energy Resources with Associated Power Systems Interfaces.

[13] B. K. Reddy, "Retrofit of MOCB with VCB”, International Journal of Research in Engineering, Science, and Management, vol. 2, 6, 2019. 


\title{
2 Case study on renewable energy integration (solar) challenges with captive/cogeneration plants and optimization of plant operation
}

\author{
Ankem V.R.N.B. Manikyala Rao ${ }^{1, *}$, Amit Kumar Singh ${ }^{2, *}$, \\ A. Sai Mrudula
}

${ }^{1}$ PhD Scholar, LPU, Phagwara, Punjab

${ }^{2}$ Associate Professor, LPU, Phagwara, Punjab

${ }^{3} A E$, TSNPDCL, Telangana

\section{Introduction}

Cogeneration or combined heat and power (CHP) is the simultaneous generation of heat and power, both of which are simultaneously used [1]. Normally the electricity generated by the cogeneration plants is used for internal consumption, hence saving in transmission and distribution losses. The efficiency of the cogeneration plants can go up to around $90 \%$ compared to the conventional power plants where maximum efficiency is only $30-38 \%$ [1]. Therefore, the industries that require both heat and electrical energies have adopted cogeneration plants as this makes the industries independent in meeting the energy requirements with added advantages of improved reliability in power supply and no revenue drain in the name of grid electricity charges. Worldwide electricity reforms further encouraged industries to set up cogeneration plants by adopting the standard designs available to suit heat and electrical load requirements for the efficient operation of process plants. However, rapid industrialization growth and an increase in energy demand caused a threat to the environment and fossil fuel availability, hence government policies are aimed at going for a certain percentage of generation from renewable energy. But the integration of renewable energy with cogeneration systems introduced operational constraints and system inefficiencies. As the problem is newly developed due to various developments in the energy sector much of literature reviews are not available, hence data is collected from the industry and practical study is made to arrive at the optimal operational strategies and suggested ways to utilize the renewable energy to the full extent and to develop the alternative future energies for sustainable energy security.

\section{Case study: Steam turbine topping system}

As a case study, the steam turbine topping cogeneration system in the chemical process industry is considered as shown in Figure 2.1.

As the electrical power requirement of the process is higher than the thermal energy obtained, in the case of a backpressure turbine system with opted steam generation parameters, the system is designed for maximum energy required i.e. a system based on electrical load matching is adopted to ensure more reliability instead of depending on

*Emails: amr854@rediffmail.com, amit20267@lpu.co.in, ankemmrudula@gmail.com

DOI: $10.1201 / 9781003129103-2$ 


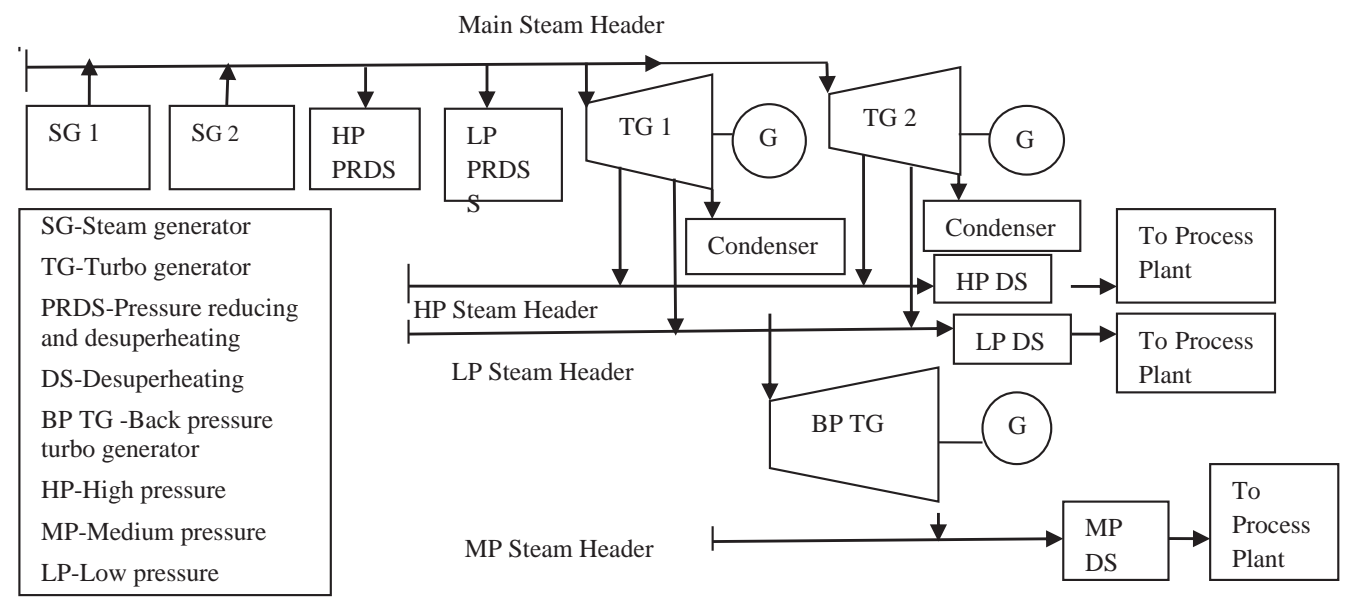

Figure 2.1 Block diagram of the cogeneration system.

Table 2.1 Plant operation data collected prior to solar plant installation

\begin{tabular}{lll}
\hline S. No & Description & Data \\
\hline i) & 2 Steam generators with main steam generation & $330 \mathrm{MT} / \mathrm{h}$ \\
ii) & 2 Turbo generators with total power generation & $31 \mathrm{MW}$ \\
iii) & Back pressure turbo generator power generation & $4.0 \mathrm{MW}$ \\
iv) & Power export/import & $\mathrm{Nil}$ \\
v) & HP/MP/LP PRDS opening & Nil \\
vii) & HP steam consumption before/after de-superheating & $210 / 245 \mathrm{MT} / \mathrm{h}$ \\
vii) & LP steam consumption & $25 \mathrm{MT} / \mathrm{h}$ \\
viii) & Condensate flow & $85 \mathrm{MT} / \mathrm{h}$ \\
\hline
\end{tabular}

the grid. Hence accordingly the double extraction cum condensing type turbine system is selected. Even though the system is designed for standalone, operational strategy is grid support based to absorb transient variations caused due to constraints from process and cogeneration systems. Two units each consisting of pulverized coal steam generator and double extraction cum condensing type turbines cater for electrical load, high pressure (HP), medium pressure (MP) and low pressure (LP) steam requirements for process plant. Even though each TG is designed to produce a maximum of $30 \mathrm{MW}$, design criteria are to operate both the units at $80 \%$ of the maximum continuous rating (MCR). However, over a period various energy conservation measures adopted like variable frequency drives, trimming of pump/fan impellers, reduction of stages in pumps and energy-saving measures in thermal systems, etc. brought down the electrical and thermal energy requirements. A backpressure turbine added to produce medium pressure (MP) steam from HP steam which was earlier produced by throttling enhanced the electrical generation by $3.5 \mathrm{MW}$. The above measures resulted in the thermal requirement of around $62 \%$ and an electrical load of around $35 \mathrm{MW}$. Accordingly, plant operations are optimized by optimizing the HP and LP extractions and load on generators and the values are presented in Table 2.1. 


\section{Control mechanisms}

For turbine control mechanism purposes a single casing condensing turbine is divided internally with the steam gland into two parts, i.e. HP and LP parts; in each part steam flow is regulated through a set of control valves. The amount of opening of each valve is controlled by the governing system. Speed/power and extraction pressure controllers in combination with ratio modules decide the signal to control valve servo motors. The speed/power controller generates the secondary oil pressure signal to control in such a way that change in the amount in steam flow through HP and LP parts of the turbine is the same. This does not affect the HP steam flow and pressure. The HP extraction pressure controller acts in such a way that upon a decrease in extraction pressure due to an increase in demand, the HP control valve opens more, and the LP control valve moves in the close direction. Valve movement through the secondary oil pressure is designed such that the increase in power in the HP part of the turbine is equal to the decrease in power in the LP part of the turbine, hence no effect on the total power developed. LP extraction control is uncontrolled and controlled externally through a control valve. Normally the speed/frequency controller is active during island mode and power control mode during operation with the grid. In addition to the above an import/export controller is provided to control the import/export of power from the grid or to the grid.

Similarly boilers are provided with various controllers like a main steam pressure controller, oxygen/air flow controller, furnace draft controller, combustion controller, drum level controller (3-element) etc. These controllers' function is similar to controllers in conventional power plants.

\section{Necessity of the integration of renewable energy}

An increase in an installed generation to meet enhanced demand caused adverse effects on the environment and vacuum on availability of the fuels like coal, oil and natural gas, reducing the reserves to production $(\mathrm{R} / \mathrm{P})$ ratio at a faster rate, hence becoming a threat to energy security. To obviate these problems government policies are aimed at encouraging renewable energy generation through incentives and regulations. As a part of these policies, most of the state regulatory commissions made mandatory that at least $5 \%$ of the total generation should be from renewable energy resources, out of which at least $0.25 \%$ should be from solar, or equivalent renewable energy certificates (RECs) should be produced. With this most of the captive/cogeneration plants have gone for renewable energy generation, mainly either solar or wind. However, the integration of renewable energy with conventional energy posed many challenges to the cogenerators.

\section{Integration of solar plant}

To meet the statutory requirement of renewable power purchase obligation (RPPO), solar generation based on the environmental conditions is added and the capacity of the plant is selected as follows

Electrical power consumption of plant $=35 \mathrm{MW} * 24 \mathrm{~h}=840 \mathrm{MWh} /$ day

$5 \%$ of consumption/day is $42000 \mathrm{kWh} /$ day; hence $5 \%$ of consumption/year becomes $15330 \mathrm{MWh} /$ year. Considering the degradation of the PV cell performance with time and variation in the solar irradiation data, weather parameters, etc., an 
additional $10 \%$ capacity over and above the calculated value is considered for evaluating the plant capacity.

Accordingly, the plant energy generating capacity is worked out as $1.1 \times 15330=16863$ $\mathrm{MWh} /$ year.

Hence, energy generating capacity in MW $=16863 / 365 \times 24=1.92 \mathrm{MW}$.

Considering $16 \%$ of solar plant efficiency, the capacity of the solar plant $=1.92 /$ $0.16=11.7 \mathrm{MW}$ (peak).

Accordingly, a $12 \mathrm{MW}$ (peak) solar power plant was set up based on photovoltaic technology and the generated energy is integrated into the existing electric network of the plant.

\section{Post-effects of operational constraints with integration of renewable energy}

With solar generation power reduction from TGs takes place due to constant electrical load, resulting in a reduction of HP and LP extraction flows leading to system inefficiencies as balance steam supply is met through HP and LP pressure-reducing and desuperheating stations. The above leads to boiler load reduction as part of the electrical load is met by solar and whenever going below a minimum level of $60 \%$ load additional oil support is essential as per original design, to avoid furnace disturbances. All these resulted in inefficiencies leading to an increase in the cost of generation.

Hence, to obviate these problems a power export agreement of $12 \mathrm{MW}$ was made. But even though an export agreement was made for $12 \mathrm{MW}$ the following effects on the system were noticed as the solar energy is variable.

a) The balance of the power when solar is not available or solar generation below $12 \mathrm{MW}$ is augmented by increasing the corresponding generation through TGs as condensing power as process heat load requirement is constant. But condensing mode power is more costly than extraction mode. This inefficient operation has become common during the non-solar period, i.e. almost $50 \%$ of duration, resulting in an expected reduction of benefits from solar addition.

b) If there is an increase in solar insolation, solar power generation increases and so the power export/import controller generates a control signal to reduce the power generation from the selected TGs by reducing the steam input. This process involves many variations of TG inlet flows, pressures, power generation, HP and LP pressures along with solar plant generation levels. The reverse phenomenon happens with a sudden reduction of solar insolation levels. The continuous variation of parameters leads to system inefficiencies and system instability along with thermal stresses.

c) If the TG inlet flow comes down due to increase in the solar generation the main steam header pressure increases above the set value and to maintain constant steam header pressure the controller generates a control signal to reduce the selected coal feeder speed and in turn reduce the steam generated from the boiler. Hence there are continuous variations of fuel and air to the furnace, causing disturbances and an increase in unburnt residues, increasing system inefficiencies and furnace stability problems.

d) Even though $12 \mathrm{MW}$ export agreement exists with the grid, the state load dispatch centre (SLDC) gives schedules based on the demand requirement and insists on $25 \%$, $50 \%, 75 \%$ backdowns. Sometimes during non-peak hours or the rainy season, there is $100 \%$ backdown also from the grid, i.e. no power requirement from the grid, resulting in zero export condition. 
All the variations are VII. in closed cycle, i.e. increasing solar generation varies the TG parameters which vary boiler parameters and this, in turn, varies TG parameters. The variations are continuous and will not become steady as solar energy is variable and does not allow the controller to stabilize. These variations are appreciable during large variations in solar generation. Thus, the cogeneration plant which was at steady and stable operating conditions before the integration with the solar plant has become unstable and inefficiencies were introduced after integration.

Hence to obviate these deficiencies, the cogeneration plant switches off the solar panels to the tune of the excess generation, i.e. whenever there is no export requirement from the grid side it reverts to the normal operation of the plant. But this is also an inefficient way as the prime reason for setting up the solar plant is defeated.

\section{Remedial solutions to the present scenario}

To come out of the above situations raised due to the integration of solar energy the feasibility of the following options is explored.

Case I: In this case, it is considered full solar generation will be utilized, by reducing correspondingly the generation on TGs with the corresponding reduction of load on the steam generators. This requires oil support. The following strategies are assumed

a) To minimize the effect on both steam generators with a reduction in TG load, one SG is assumed kept on fixed load, i.e. around 60\% MCR, and the entire steam flow variation due to solar has to be absorbed by the other boiler. By this only one boiler requires the oil support and a maximum of 4 burners are required for coal mills on light load operation.

b) Similarly, the generation of one TG is assumed to be maintained constant i.e. at $60 \%$ MCR, i.e. $18 \mathrm{MW}$, so that extractions from at least one TG will not get affected and the other TG generation has to be reduced depending on the solar generation. The extraction steam that comes down has to be compensated through PRDS. An experiment is conducted practically by simulating the above conditions and the results are tabulated in Table 2.2.

Table 2.2 Experimental results (data) when solar generation is $12 \mathrm{MW}$ (peak) and nil power export/ import

\begin{tabular}{|c|c|c|c|}
\hline S. No & Description & Solar $12 \mathrm{MW}$ & Remarks \\
\hline i) & $\begin{array}{l}2 \text { Steam generators with main steam } \\
\text { generation }\end{array}$ & $300 \mathrm{MT} / \mathrm{h}$ & $\begin{array}{l}\text { Boiler } 1165 \text { MT/ h; Boiler } 2 \\
135 \text { MT/h }\end{array}$ \\
\hline ii) & $\begin{array}{l}2 \text { Turbo generators with total power } \\
\text { generation }\end{array}$ & $19 \mathrm{MW}$ & TG 115.5 MW; TG $23.5 \mathrm{MW}$ \\
\hline iii) & Back pressure TG power generation & 4.0 MW & \\
\hline iv) & Steam through HP/ PRDS & $105 \mathrm{MT} / \mathrm{h}$ & \\
\hline v) & Steam through LP PRDS & $10 \mathrm{MT} / \mathrm{h}$ & \\
\hline vi) & $\begin{array}{l}\text { HP steam consumption before/after } \\
\text { de-superheating }\end{array}$ & $210 / 245 \mathrm{MT} / \mathrm{h}$ & $\begin{array}{l}\text { TG } 1 \text { Extraction } 105 \mathrm{MT} / \mathrm{h} ; \\
\text { PRDS } 105 \mathrm{MT} / \mathrm{h}\end{array}$ \\
\hline vii) & $\begin{array}{l}\text { LP steam consumption before/after } \\
\text { de-superheating }\end{array}$ & 20/25 MT/h & $\begin{array}{l}\text { TG } 1 \text { Extraction } 10 \mathrm{MT} / \mathrm{h} ; \mathrm{LP} \\
\text { PRDS } 10 \mathrm{MT} / \mathrm{h}\end{array}$ \\
\hline viii) & Condensate flow & $70 \mathrm{MT} / \mathrm{h}$ & TG1 55MT/h; TG2 $15 \mathrm{MT} / \mathrm{h}$ \\
\hline
\end{tabular}




\section{Cost-benefit analysis}

Cost-benefit analysis is carried out with above results obtained as follows

a) Oil cost for 4 oil guns:

Each oil gun consumes $300 \mathrm{~kg} / \mathrm{h}$. Total oil consumption for 4 oil guns $=1.2 \mathrm{MT} / \mathrm{h}$. Considered cost of oil/MT $=$ Rs 30,000/-. Total oil cost/hour $=$ Rs $1.2 * 30,000=$ Rs 36,000/-.

b) MW loss due to HP PRDS opening $=105(794-747) / 860=5.74 \mathrm{MW}$.where TG inlet steam enthalpy $=794 \mathrm{kcal} / \mathrm{kg}$ and HP extraction steam enthalpy $=747 \mathrm{kcal} / \mathrm{kg}$.

c) MW loss due to LP PRDS opening $=10(794-694) / 860=1.16 \mathrm{MW}$ where LP extraction steam enthalpy $=694 \mathrm{kcal} / \mathrm{kg}$.

d) Resultant steam reduction on steam generators;

Total loss due to HP \& LP PRDS opening $=(5.74+1.16)=6.9 \mathrm{MW}$. So equivalent $\mathrm{MW}$ reduction $=(12.0-6.9) \mathrm{MW}=5.1 \mathrm{MW}$.

Equivalent steam reduction $=5.1 * 5=25.5 \mathrm{MT} / \mathrm{h}$ Assuming $5 \mathrm{~T} / \mathrm{MW}$ (condensing steam power requirement).

Or directly from the steam generation values before and after solar $=(330-$ 300) $\mathrm{MT} / \mathrm{h}=30 \mathrm{MT} / \mathrm{h}$.

e) Coal consumption / MT of steam = $5 \mathrm{MT}$.

Hence coal saved due to steam reduction on steam generators $=30 / 5=6 \mathrm{MT} / \mathrm{h}$ (considering max reduction of $30 \mathrm{MT} / \mathrm{h}$ ).

Cost of coal per MT = Rs $3500 /$-. Cost benefit due to coal saving = Rs $6 * 3500 /-$ $=$ Rs 17, 500/-.

f) Calorific value of oil $=10,000 \mathrm{kcal} / \mathrm{kg}$. Calorific value of coal $=4,000 \mathrm{kcal} / \mathrm{kg}$. Ratio of oil to coal calorific value $=2.5: 1$.

Hence 4 oil guns oil quantity of $1.2 \mathrm{MT} / \mathrm{h}$ is equivalent to $2.5 * 1.2 \mathrm{MT}$ of coal, i.e. 3 MT of coal.

Hence coal saving due to oil firing = Rs $3 * 3,500 /-=$ Rs 10,500/-.

g) Net saving due to coal $=(\mathrm{e}+\mathrm{f})=\mathrm{Rs}(17,500+10,500)=\mathrm{Rs} 28,000 /-$.

Hence total benefit by keeping all $12 \mathrm{MW}$ solar panels in service $=(\mathrm{g}-\mathrm{a})=$ Rs $(28,000-36,000)=$ Rs $(-)$ 8,000/-.

As the result is negative it indicates a loss by going full solar generation, i.e. by keeping a full solar plant, there is no financial gain.

Case II: Further study is made to find the break-even point up to which utilizing solar is beneficial. Theoretically, this break-even point will be until steam generation reduction does not demand oil support and, at the same time, HP PRDS does not start opening. Even with the LP PRDS opening, there will be a net saving. Here the load on both TGs and SGs is assumed to be varied in parallel and also both TGs with HP and LP extractions in line.

To find out the break-even point, an experiment is conducted by switching on one by one the solar panels till non-opening of HP PRDS valves and steam generators load within the minimum threshold limit of oil support requirement. With the practical experiment conducted, the break-even point is worked out as $6.3 \mathrm{MW}$, i.e. slightly more than $50 \%$ of the solar plant capacity and the parameters are recorded in Table 2.3. The LP PRDS valves got opened by around $68 \%$ which is equivalent to $7.2 \mathrm{MT} / \mathrm{h}$ as per the LP PRDS valve characteristic. 
Table 2.3 Experimental results (data) during practical simulation to find the breakeven point

\begin{tabular}{|c|c|c|c|}
\hline S. No & Description & $\begin{array}{l}\text { Solar } 6.3 \\
\text { MW }\end{array}$ & Remarks \\
\hline i) & $\begin{array}{l}2 \text { Steam generators with main steam } \\
\text { generation }\end{array}$ & $317 \mathrm{MT} / \mathrm{h}$ & $\begin{array}{l}\text { Boiler } 1158 \mathrm{~T} / \mathrm{h} \text {; Boiler } 2 \\
\text { 159MT/h }\end{array}$ \\
\hline ii) & $\begin{array}{l}2 \text { Turbo generators with total power } \\
\text { generation }\end{array}$ & $25.5 \mathrm{MW}$ & TG $113 \mathrm{MW}$ TG; $212.5 \mathrm{MW}$ \\
\hline iii) & $\begin{array}{l}\text { Auxiliary turbo generator power } \\
\text { generation }\end{array}$ & $3.5 \mathrm{MW}$ & \\
\hline iv) & Power export/import, if any & Nil & \\
\hline v) & Steam through HP PRDS & Nil & \\
\hline vi) & Steam through LP PRDS & $7.2 \mathrm{MT} / \mathrm{h}$ & \\
\hline vii) & $\begin{array}{l}\text { HP steam consumption before/after } \\
\text { de-superheating }\end{array}$ & $\begin{array}{r}210 / 245 \\
\mathrm{MT} / \mathrm{h}\end{array}$ & $\begin{array}{l}\text { TG } 1 \text { Extraction } 105 \mathrm{MT} / \mathrm{h} \text {; } \\
\text { TG } 2 \text { Extraction } 105 \mathrm{MT} / \mathrm{h}\end{array}$ \\
\hline viii) & $\begin{array}{l}\text { LP steam consumption before/after } \\
\text { de-superheating }\end{array}$ & $25 \mathrm{MT} / \mathrm{h}$ & $\begin{array}{l}\text { TG } 1 \text { Extraction } 10 \mathrm{MT} / \mathrm{h} \text {; } \\
\text { TG } 2 \text { Extraction } 8 \mathrm{MT} / \mathrm{h} \text {; } \\
\text { LPPRDS } 7 \mathrm{MT} / \mathrm{h}\end{array}$ \\
\hline $\mathrm{x})$ & Condensate flow & $70 \mathrm{MT} / \mathrm{h}$ & TG1 55MT/h; TG2 15 MT/h \\
\hline
\end{tabular}

Table 2.4 Cost-benefit analysis results

\begin{tabular}{lllll}
\hline S. No & $\begin{array}{l}\text { Solar } \\
\text { generation } \\
\text { in } M W\end{array}$ & $\begin{array}{l}\text { Total steam } \\
\text { generation } \\
\text { in MT/h }\end{array}$ & $\begin{array}{l}\text { Steam reduction } \\
\text { on boilers with } \\
\text { solar in MT/h }\end{array}$ & $\begin{array}{l}\text { Cost saving } \\
\text { due to steam } \\
\text { reduction in } R s\end{array}$ \\
\hline 1 & 6.3 & 317 & 13 & 9100 \\
2 & 6.0 & 320 & 10 & 7000 \\
3 & 5.5 & 321.6 & 8.4 & 5880 \\
4 & 5.0 & 319 & 11 & 6700 \\
5 & 4.5 & 321 & 9 & 4300 \\
6 & 4.0 & 323.8 & 6.2 & 4340 \\
\hline
\end{tabular}

From the above, it is observed that at $6.3 \mathrm{MW}$ solar, the resultant steam reduction on steam generators $=(330-317)=13 \mathrm{MT} / \mathrm{h}$.

Hence coal saved due to steam reduction on steam generators $=13 / 5=2.6 \mathrm{MT} / \mathrm{r}$ (considering 1 ton of coal generates $5 \mathrm{MT} / \mathrm{h}$ ).

Cost benefit due to coal saving = Rs $2.6 * 3,500 /-=$ Rs 9,100/-. Cost of coal per MT = Rs 3,500/-.

Further increase of solar generation results in oil support as steam generation levels of both boilers are at verge $(60 \% \mathrm{MCR})$, hence uneconomical as explained under Case I. Hence the break-even point is $6.3 \mathrm{MW}$ and operation above $6.3 \mathrm{MW}$ is not economical, and hence part-load operation of solar is economical and recommended.

Experiment is further continued to find out the cost savings below the $6.3 \mathrm{MW}$ and data recorded is presented in Table 2.4 and represented graphically in Figure 2.2.

Steam reduction is increased as LPPRDS got closed fully. But cost saving is still maximum at 6.3 MW only even though LPPRDS is partially open. 


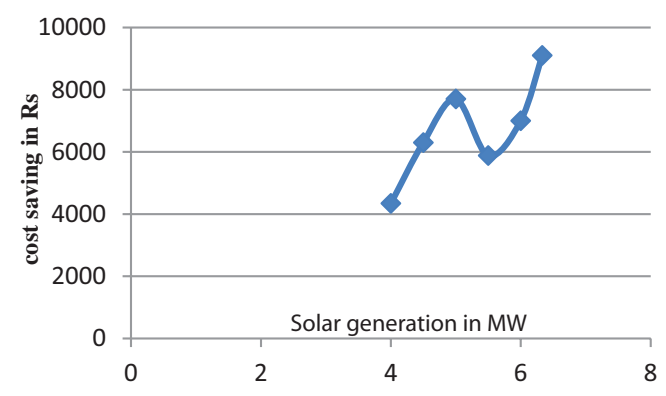

Figure 2.2 Block diagram of the cogeneration system.

Case III: The other problem of the integration of renewable energy with cogeneration plants is a continuous variation of process parameters. However, the variations can be reduced to some extent by utilizing the solar isolation level as a feed forward signal to the master pressure controller and to the import/export controller to smoothen the boiler parameters, TG generations and in turn HP and LP extraction pressure and flows.

Case IV: Another problem experienced with integration of the solar is TGs trip on under frequency depending on solar generation and grid frequency available prior to island operation. This is mainly due to solar plant isolation on auto as per the prevailing logic incorporated and leading to block out. Hence the auto-off feature shall be defeated in the case of low frequency and solar panels shall be switched off manually one by one in parallel along with plant stabilization.

\section{Options to explore further}

The above cases reveal that process parameters vary badly along with no-utilization of full solar capacity. Hence further options for future study can be (i) to search for customers who need electrical energy and agreements shall be made in such a way that nearby industries must be attracted by offering lower prices than conventional power as the solar generation cost is much lower. With this, we can get the benefit of RPPOs also.

(ii) The ideal solution appears to be the utilization of various solutions of renewable energy storage systems like battery-operated energy storage, compressed air storage, ice bank, pumped hydro storage, etc. [2]. These options shall be explored further on the techno-economic point of view so that the stored energy beyond a break-even point can be utilized in the night when solar is zero, hence helping in full utilization of solar capacity and at the same time minimizing the process parameter variations.

(iii) Installation of ozoniser: The water required normally in the process plants requires treatment before use in the process. Generally, chlorine and other chemicals are used for disinfection. Ozone is a very strong oxidizer with a powerful disinfecting property. However, the production of ozone is an energy-intensive process. Utilizing solar for ozone production may be explored. With this there are two-fold advantages, i.e. chemicals can be saved, reducing in the cost of water treatment, and solar energy utilization will be better and this will fulfill the RPPOs obligation also.

(iv) Hydrogen generation: Hydrogen is an ideal replacement for fossil fuels such as coal, oil and natural gas in furnaces, internal combustion engines, turbines, and jet engines. Hydrogen produced can be stored in fuel cells which can be utilized during peak hours or in the transport sector also as an alternative to petrol/diesel engines. A solar photovoltaic 
power cogeneration system that utilizes the electrolysis of water and solar energy to power a fuel cycle can be explored [3].

\section{Conclusion}

Challenges being faced by cogeneration plants with renewable energy integration are discussed and break-even point up to which solar is beneficial is arrived at by practically conducting experiments. However, detailed simulation models are to be developed to obtain optimal solutions for different conditions and plant ratings. Energy storage options including hydrogen generation require elaborate study which may resolve the many issues in utilizing renewable energies on a large scale. This will help the society at large in preserving the fossil fuels for future generations and at the same time minimizes environmental degradation.

\section{References}

1) Guidebook 2 on "Energy efficiency in thermal utilities" - Bureau of Energy Efficiency and National Productivity Council of India, 4th edition,2015.

2) B. Upendraroy and N. Rangarajan, "Feasibility study of an energy storage system for distributed generation system in islanding mode", Journal of Energy Resources Technology, 139, (2017) 0119011-01190116.

3) P. Nikolaidis and A. Poullikas, Renewable and Sustainable Energy Reviews", 67 (2017) 597611, www.elsevier.com/locate/rser. 


\title{
3 Control of unmanned surface vehicles with randomized probabilistic approach
}

\author{
Rupam Singh ${ }^{1,2}$, Bharat Bhushan ${ }^{1}$ \\ ${ }^{1}$ Department of Electrical Engineering, Delhi Technological University, Bawana \\ Road, Delhi, India \\ ${ }^{2}$ Institute for Intelligent System Technologies, Alpen-Adria-Universität Klagenfurt, \\ 9020 Klagenfurt, Austria
}

\section{Introduction}

In the past few years, due to the excellent development in the research field of communication navigation technology, environmental behaviours sensing technology, path planning technology, sailing intelligent control technology and trajectory tracking technology in unmanned surface vehicles (USVs) makes it a very frequent and compatible mode of transportation. These technologies mainly concentrate on the USVs balancing on water, path following and obstacle avoidance-based tasks, etc., and subjected to research findings in the area of unmanned vehicles. The completion of these difficult tasks has been a great challenge for researchers due to unpredictable circumstances. While performing these tasks the main objective is to design such controllers that can ensure path tracking efficiently, following desired position and make orientations of USVs stable. In classical control, a PID based heading controller (Banazadeh and Ghorbani 2013) has been designed where feedforward term and reference model have been added to the PID gains in order to get exact motion of USV. But switching handling may create problem with this type of controller which can be overcome by designing a fast-non-singular terminal sliding mode controller (FNTSM) (Mu et al. 2017) and multi-mode controlling becomes easy by using FNTSM. In addition, active disturbance rejection controller $(\mathrm{ADRC})$ has been designed to tune the controller as per the ability of a vessel to change its course/path ( $\mathrm{Li}$ et al. 2013). To keep vessel stationary during steering movement, an adaptive sliding mode control algorithm has been developed (Liu 2017), which is robust in nature against external disturbances. But this algorithm requires the prior knowledge of the system sign. To eliminates this, a novel adaptive nonlinear control strategy has been designed to control USVs motion and balancing problem (Du et al. 2014). Here, Lyapunov function and Nussbaum function are together employed to design an adaptive nonlinear control law in order to deal with unknown signs of control gains. This is a highly precise controller but requires model complete information. This limitation of such techniques which demands system knowledge is eliminated by advanced controllers. So, in the presence of modelling uncertainties and time-varying external disturbances various advanced control techniques also have been employed in literature, including dynamic surface control technique (Dai et al. 2018), input-output linearization technique, integrator backstepping technique (Chen and Tian 2015), and adaptive fuzzy control (Wang and Er 2016), radial function based neural network control (Zheng and Zou 2016) etc. In all these strategies, uncertainty is presented in the form of disturbances with its stochastic behaviour

\footnotetext{
*Emails: singhrupam99@gmail.com, bharat@dce.ac.in
}

DOI: $10.1201 / 9781003129103-3$ 
and the system dynamics are supposed to be unknown. The uncertainty in USVs is defined by conventions associated with the unknown wind affect, vorticity effect, external disturbances and random disruptions (Singh and Bhushan 2020).

Since the explicit relationship between the state space matrices and the uncertain parameters is available all the time, a linearization process is subjected to repetition at the time of variation in the uncertain parameters. This paper proposes an optimized probabilistic control approach to design a control algorithm with reference to randomization for gain matrix calculation and for closed-loop system analysis as well (Calafiore, Dabbene, and Tempo 2011). These randomization-based control algorithms take less computational time and are easy to implement (Dorato and Abdallah 2007) and at the same time robust boundary conditions are less conservative but at the cost of probability risk failure. For the analysis of probabilistic method based on a randomized algorithm, the two DoF ball balancer system has been considered here.

The paper is further outlined as follows: Section II describes the USV modelling using the 2DoF ball balancer model which has been used with its state space representation. Section III gives a brief overview of a randomized algorithm for probabilistic method. Further in Section IV, the controller's performance validation on ball balancer system is discussed and the research is concluded in Section V.

\section{Control of USVs}

A 2DoF ball balancer system is considered for understanding the operation of USVs. The USVs are designed in such a manner where six degrees of freedom of motion depicts the working of the system, with three of them corresponding to translation motion with respect to $\mathrm{x}, \mathrm{y}$ and $\mathrm{z}$ axis and the remaining three correspond to the rotational motions around $\mathrm{x}, \mathrm{y}$ and $\mathrm{z}$ axis. Now to perform experimental analysis a two degree of freedom ball balancer system has been taken where $\mathrm{x}$ and $\mathrm{y}$ axis translational motion is considered to show the surge and sway effect of USVs. Figure 3.1 shows the comparative three axis representation between ball balancer and USV system.

\section{Mathematical modelling of plant model}

The block representation of transfer function modelling for ball balancer system in open loop is depicted in Figure 3.2. The plant $W_{s}(s)$ represents the dynamics between servo input voltage and resulting load angle, whereas plant $W_{s s}(s)$ represents the angle of the

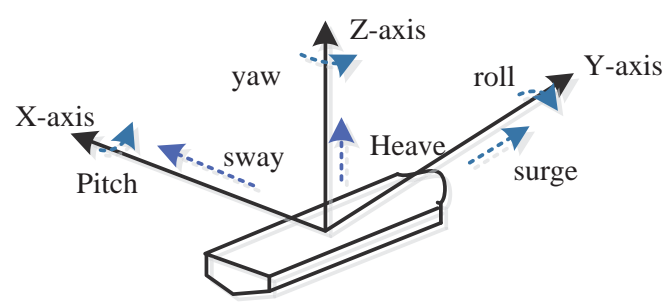

(a)

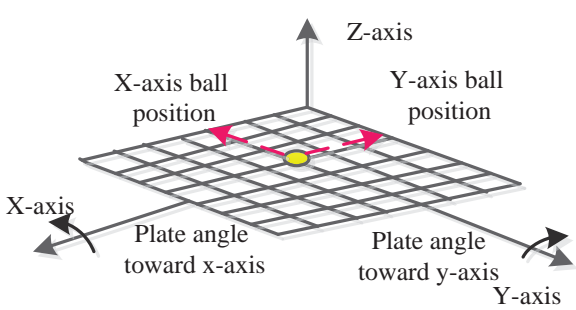

(b)

Figure 3.1 Comparative three axial dynamics of (a) USV system, (b) ball balancer. 


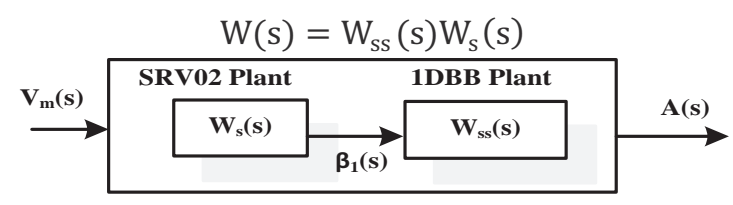

Figure 3.2 One dimensional ball balancer system in open loop.

servo load gear and dynamics of balls position. Since the system is a decoupled system, the SRV02 plant is symmetrical for both the axis and the actuator elements of $\mathrm{x}$-axis won't influence the y-axis elements. This configuration aids in modelling the system only for a particular axis. This single axis configuration can be referred as a 1-dimension ball balancer.

The overall transfer function of 1 -d ball balancer is given by

$$
W(s)=W_{s s}(s) W_{s}(s)
$$

where $W_{s s}(s)=\frac{A(s)}{\beta_{1}(s)}, W_{s}(s)=\frac{\beta_{1}(s)}{V_{m}(s)}$ final equation of movement (Singh and Bhushan 2018) for the ball balancer is given as:

$$
\ddot{A}(t)=\frac{2 M_{\text {ball }} G \beta_{1}(t) R_{\text {arm }} R_{\text {ball }}^{2}}{L_{p}\left(M_{\text {ball }} R_{\text {ball }}^{2}+j_{\text {ball }}\right)}
$$

Here the mass of the ball is given by $M_{\text {ball }}$, A depicts the displacement of the ball, $R_{\text {ball }}$ is the radius of the ball, inertia of the ball is given by $j_{\text {ball }}, \mathrm{L}_{\mathrm{p}}$ is plate length and $\mathrm{R}_{\mathrm{arm}}$ is the distance between the coupled joint and the shaft of SRV02 output gear.

\section{Randomized algorithms for probabilistic approach}

The randomized algorithm is an optimization technique where the actual system uncertainties initiate the randomization process while the rest of the deterministic decision parameters remain unconsidered. The probabilistic analysis uses classical $\mathrm{M}$ model (Calafiore, Dabbene, and Tempo 2000) and denotes the transfer matrix of the already identified part of the system. This known part contains the expanded plant and controller as shown in Figure 3.3(a). Here w represents noise with a reference signal, $z$ signifies control signal with tracking errors. Uncertainty is defined as random matrix $(\Delta)$ with support $\beta_{\mathbb{D}}$. Using $M$ configuration the measurable performance function for analysis purpose is defined as

$$
J(\Delta): \mathbb{D} \rightarrow \mathbb{R}
$$

where $\mathbb{D}$ is the uncertainty structured set with performance level as $\gamma$. The function $J(\Delta)$ considers several performance requirements. 


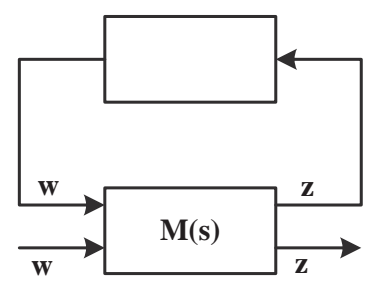

(a)

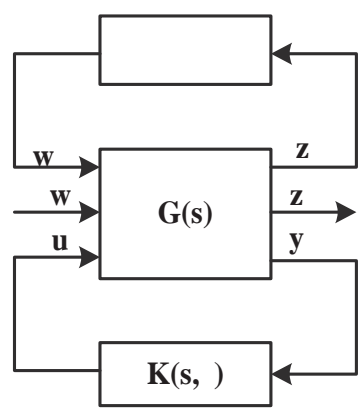

(b)

Figure 3.3 M-Delta configuration (a) for expanded plant, (b) for control design.

\section{Designing process}

The interconnection between the blocks is carried in such a way that it remains in stable condition even in the presence of uncertainty while designing the probabilistic controller $K(s, \theta)$ of a plant $\mathrm{G}(\mathrm{s})$. The block diagram is shown in Figure 3.3(b) and the design parameter vector should be like $\theta \in \mathbb{R}^{n \theta}$. The input, output, noise and control signal are denoted as $\mathrm{u}, \mathrm{y}, \mathrm{w}, \mathrm{z}$ respectively considering uncertainty $\Delta$ as a random matrix with support vector $\beta_{\mathbb{D}}$ and structure $\mathbb{D}$.

Probabilistic controller gain synthesis has been done using a randomized algorithm. The design parameter is selected by deterministic parameter while gain selection techniques are dependent on variation in random sampling when uncertainty presented in the system. During probabilistic controller design, performance function converted into the form of inequality and deliberate performance constraints associated with the system uncertainty

$$
J(\Delta, \theta) \leq \gamma
$$

The overall designing function of performance in terms of scalar valued function is denoted as

$$
J(\Delta, \theta): \mathbb{D} \times \mathbb{R}^{n \theta} \rightarrow \mathbb{R}
$$

where $J(\Delta, \theta)$, performance function is defined for fixed control parameter $(\theta)$.

\section{Result and discussion}

The transfer function of the plant is simulated using MATLAB ${ }^{\circledR} /$ Simulink and Quarc software. The Quanser 2DoF ball balancer hardware is used to connect and study the ball balancer system. The sequential randomized algorithm makes available a controller that stabilizes the uncertain system with pre-specified probability. A feedforward control is integrated with a randomized probabilistic algorithm (RPA) on providing necessary control to the system. In addition to RPA, conventional PID controller is also implemented to test the robustness of the developed approach. 


\section{Intelligent Circuits and Systems}

While performing the simulation, the contribution of integral control in PID makes the system unbalanced. This phenomenon is confirmed by observing an overshoot in the output of the system. In order to overcome this, an efficient control technique is needed. A peak overshoot of PID of $13.5 \%$ causes huge oscillations and made the ball difficult to balance on the plate. On the other side as per results observed in the graph it's observed that RPA controller has the fine response to peak overshoot $0.827 \%$ and shows the excellent balance of ball on the plate without any oscillation.

These controllers are designed by simulation of square wave response, the results are compared to validate the performance of the RPA controller by focusing on the ball movement on the plate. Lesser variation in ball movement will confirm better operation of the controller. In this simulation of the RPA controller, Figure 3.4(a), position of the ball on the $\mathrm{x}$-axis shows that the range for the position of the ball on the $\mathrm{x}$-axis is $0.01 \mathrm{~cm}$ to $3.015 \mathrm{~cm}$. Hence, initial position and final position shows the lowest difference as

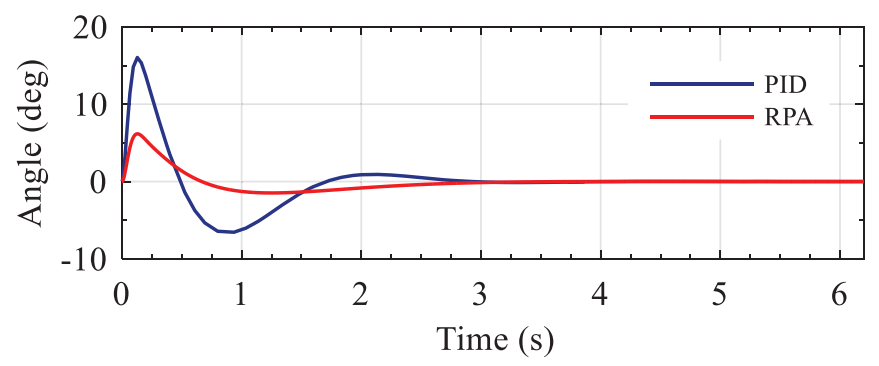

(a)

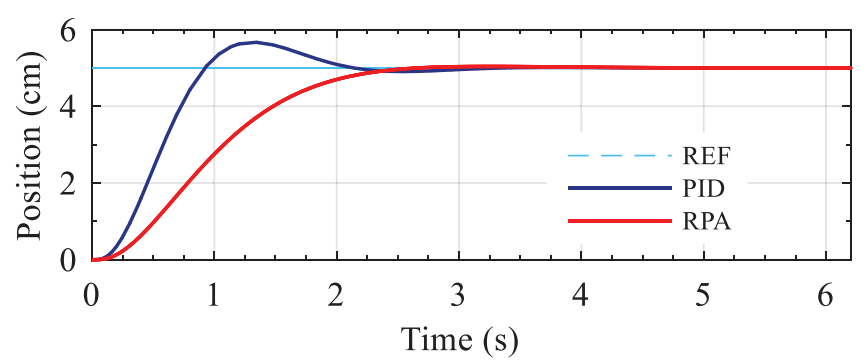

(b)

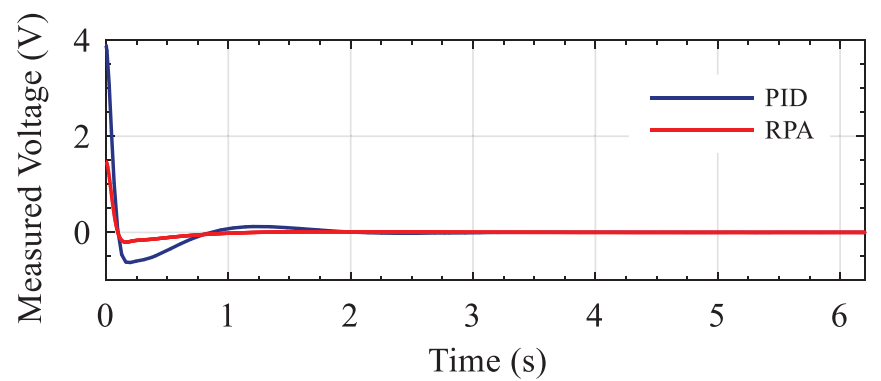

(c)

Figure 3.4 (a) Position of the ball on $\mathrm{x}$-axis, (b) servo angle response of ball on $\mathrm{x}$-axis, (c) input voltage applied to the servo motor for $\mathrm{x}$-axis. 
compared to other controllers and response, in this case, will reach its desired value in much less time. In Figure 3.4(b), the servo angle response of ball on the x-axis shows that variation in control angle provided by the servo motor is minimum in RPA, even less than a degree, due to which plate will move very slowly and show the best stable response. Figure 3.4(c) shows the servo input voltage: $0.8 \mathrm{~V}$ for RPA, $2.4 \mathrm{~V}$ for PID is the minimum in the case of RPA.

\section{Conclusion}

In this paper, a randomized probabilistic algorithm based probabilistic controller has been proposed for unmanned surface vehicles under parametric uncertainties. Analysis of the proposed controller is implemented with a $2 \mathrm{DoF}$ ball balancer system which imitates the operation of USV. To assess performance, the responses of the system for PID and RPA controller are validated based on MATLAB simulation. The results depicted that the developed controller minimizes the effect of disturbances on the system and hence depicts the robustness of the system. From the assessments of results, it was clear that the controller designed with given uncertainty bounds resulted in consistently stable closed loop response when implemented with randomized algorithm and this randomized algorithm delivers a controller which quadratically stabilizes the uncertain system.

\section{References}

Banazadeh, A., and M.T. Ghorbani. 2013. "Frequency domain identification of the Nomoto model to facilitate Kalman filter estimation and PID heading control of a patrol vessel." Ocean Engineering 72 (November): 344-55. doi:10.1016/j.oceaneng.2013.07.003.

Calafiore, Giuseppe C., Fabrizio Dabbene, and Roberto Tempo. 2011. "Research on probabilistic methods for control system design.” Automatica 47 (7): 1279-93. doi:10.1016/ j.automatica.2011.02.029.

Calafiore, Giuseppe C., Fabrizio Dabbene, and Roberto Tempo. 2000. "Randomized algorithms for probabilistic robustness with real and complex structured uncertainty." IEEE Transactions on Automatic Control 45 (12): 2218-35.

Chen, Yang-Yang, and Yu-Ping Tian. 2015. "Formation tracking and attitude synchronization control of underactuated ships along closed orbits." International Journal of Robust and Nonlinear Control 25 (16): 3023-44. doi:10.1002/rnc.3246.

Dai, Shi-Lu, Shude He, Hai Lin, and Cong Wang. 2018. "Platoon formation control with prescribed performance guarantees for USVs." IEEE Transactions on Industrial Electronics 65 (5): 4237-46. doi:10.1109/TIE.2017.2758743.

Dorato, Peter, and Chaouki T. Abdallah. 2007. Randomized algorithms for analysis and control of uncertain systems. IEEE Transactions on Automatic Control 52. doi:10.1109/ TAC.2006.889863.

Du, Jialu, Ajith Abraham, Shuanghe Yu, and Jie Zhao. 2014. "Adaptive dynamic surface control with Nussbaum gain for course-keeping of ships." Engineering Applications of Artificial Intelligence 27 (January): 236-40. doi:10.1016/j.engappai.2013.08.013.

Li, Ronghui, Tieshan Li, Renxiang Bu, Qinling Zheng, and C. L. Philip Chen. 2013. "Active disturbance rejection with sliding mode control based course and path following for underactuated ships." Mathematical Problems in Engineering 1-9. doi:10.1155/2013/743716.

Liu, Zhiquan. 2017. "Ship adaptive course keeping control with nonlinear disturbance observer." IEEE Access 5: 17567-75. doi:10.1109/ACCESS.2017.2742001.

$\mathrm{Mu}$, Dongdong, Guofeng Wang, Yunsheng Fan, and Yongsheng Zhao. 2017. "Modeling and identification of podded propulsion unmanned surface vehicle and its course control research." Mathematical Problems in Engineering 1-13. doi:10.1155/2017/3209451. 


\section{Intelligent Circuits and Systems}

Singh, Rupam, and Bharat Bhushan. 2018. "Real-time control of ball balancer using neural integrated fuzzy controller.” Artificial Intelligence Review, September. doi:10.1007/s10462-018-9658-7. . 2020. "A novel fault classification-based fault-tolerant control for two degree of freedom helicopter systems." International Journal of Adaptive Control and Signal Processing, June, acs.3121. doi:10.1002/acs.3121.

Wang, Ning, and Meng Joo Er. 2016. "Direct adaptive fuzzy tracking control of marine vehicles with fully unknown parametric dynamics and uncertainties." IEEE Transactions on Control Systems Technology 24 (5): 1845-52. doi:10.1109/TCST.2015.2510587.

Zheng, Zewei, and Yao Zou. 2016. "Adaptive integral LOS path following for an unmanned airship with uncertainties based on robust RBFNN backstepping." ISA Transactions 65 (November): 210-19. doi:10.1016/j.isatra.2016.09.008. 


\title{
4 Islanding classification and low-voltage ride through for grid connected transformerless inverter
}

\author{
Mohammed Ali Khan, V.S. Bharath Kurukuru, \\ Abteshamul Haque
}

\author{
Advance Power Electronics Research Laboratory, Department of Electrical \\ Engineering, Jamia Millia Islamia (A Central University), New Delhi, India
}

\section{Introduction}

The presence of distributed generation (DG) in power systems has grown rapidly in recent years. Traditionally, energy is produced in large power plants and transmitted to remote customers. In contrast to that approach, distributed energy is a decentralized energy source, and it usually locates at the consumer side or the network's distribution side (Ackermann, Andersson, and Söder 2001). A large number of DG units use renewable energy sources, such as solar, wind, hydro and geothermal power (Ackermann, Andersson, and Söder 2001). It's one of the potential solutions to the environmental issues caused by traditional coal and oil energy power plants. Furthermore, it can be predicted that the distributed generation will be very popular in the future grid.

However, as much distributed generation is connected to the main grid, several issues arise. Islanding is one of the typical problems which may occur when DG is present. It is the event when a part of the grid is continuously powered by the distributed generation while it is already separated from the rest of the network (Khamis et al. 2013). In other words, islanding creates a self-powered electrical island. The consequences of unintentional islanding are reduced power quality, endangerment of personnel, potentially unsafe and unstable voltage and frequency control, and equipment damage due to unsynchronized reclosing (Khamis et al. 2013).

Islanding is generally classified into intentional islanding and unintentional islanding. Intentional islanding is the islanding event initiated on purpose during, e.g., a system disturbance (Khamis et al. 2013). Intentional islanding can ensure a reliable power supply for critical loads if well designed (Balaguer et al. 2011). During a sudden power outage case, if several DG units are out of step from each other, it's better to form intentional islanding in the place where the mismatch of power between load and generation is minimum (Noor, Arumugam, and Vaziri, n.d.). In contrast, unintentional islanding is an islanding event which is not expected. Since it is not supposed to happen, the formation of unintentional islanding can cause unexpected personnel electric shock, because people are likely to touch the line which is supposed not to have electricity but can be powered due to islanding. Also, the severe consequences include electrical device damage and for the uncontrolled voltage and frequency operation many islanding detection techniques have been established, and they can be categorized into local and remote detection methods, out of which local methods can be further classified as active, passive and hybrid methods.

The principle of passive methods is to monitor system variables (e.g., voltage magnitude, frequency, phase shift, rate of change of frequency) present at the point of common coupling (PCC) of a DG. If the system variable is out of predetermined range, then this

DOI: $10.1201 / 9781003129103-4$ 
would indicate islanding. Active methods base their operation on the introduction of small perturbations and observing the system's output. It is expected that in the operation of grid connected mode, the response to perturbations would be negligible. Conversely, in islanded mode, the perturbations would cause more significant changes in the system's response (Khamis et al. 2013). Hybrid methods are the combination of active and passive methods. For instance, in the study led by W. Chang (2010), a two-step-based hybrid method was proposed to obtain higher effectiveness: the passive method was applied as the primary detection mechanism, while the active method was used subsequently to detect the islanding event again.

Communication between DGs and utility helps in establishing remote detection technique. It usually uses a central processor to check the status of circuit breakers to determine the islanding event with the help of method algorithm (Mulhausen et al. 2010). Remote methods are usually more dependable than the local detection methods, but more expensive to implement (Khamis et al. 2013).

Among all the islanding detection methods, passive detection method has the demerit of large NDZ, but it's simple to implement in practice. Active detection method has smaller NDZ problem, but it introduces additional disturbance into the power system, and usually it costs a lot to implement (Khan et al. 2019). Then, the hybrid detection method is discovered to combine passive and active detection methods. But it's not simple enough. Thus, in one way, the current development of islanding detection methods gives people various options to choose from. In the other way, it's hard to select one best detection method and make a balanced decision in terms of different considerations.

This research focuses on the control aspects of grid connected PV systems considering the grid codes and requirements. The aim of this paper is to continuously monitor the output characteristics of GCPV at PCC, and to develop a control algorithm which attempts to clear the fault within the specified time as defined by grid code. Further sections of the paper are arranged as follows: Section 2 presents a overview of grid connected system, Section 3 showed the islanding scheme implemented whereas Section 4 present low voltage ride through scheme, and in Section 5 results are discussed.

\section{Grid connected control}

The layout of a grid connected PV system is illustrated in Figure 4.1(b) and the layout of the transformerless inverter implemented in Figure 4.1(a). PV based grid connected system is mainly used for residential load. The presence of DC-DC converter helps in stabilizing the output of solar panel and make it suitable for inverter input. For operating the panel at peak efficiency at any given point of time maximum power point tracking is implemented (Haque 2014). An H5 based transformerless inverter topology is proposed in our research for reducing inverter-based losses and get maximum efficiency at conversion. Operation of H5 inverter topology can be deduced from Figure 4.1(a). In this topology, four different modes of operation are performed by five switches (Khan, Haque, and Kurukuru 2020a, $2020 \mathrm{~b})$. A filter is used to remove the harmonics from the system

During grid fault it is required that the grid is disconnected from the system at the PCC (Yang et al. 2016). For stabilizing the grid in faulty condition, a reactive power injection strategy needs to be implemented. The D-q frame-based control scheme is designed for reactive power control (Khajehoddin and Karimi-ghartemani 2013). The reference value of $i_{q}$ determines the current component active and reactive power injection. 

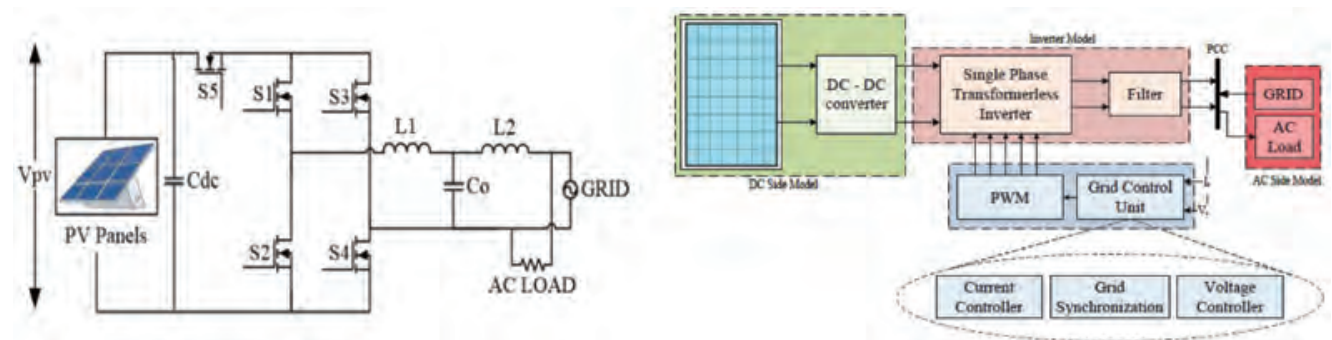

Figure 4.1 (a) H5 topology representation, (b) control configuration for grid connected PV system.

\section{Proposed islanding detection}

The various islanding scenarios that can effect the operation of a grid connected singlephase PV system are implemented and their effect on various electrical characteristics are identified at the PCC. Once the data at the PCC is obtained, the feature extraction process is performed to differentiate the effect of various islanding scenarios (Khan, Haque, and Kurukuru 2019). The feature extraction helps in obtaining new values from initial data and reduces the dimensionality of a dataset to form a feature vector. This may cause rapid classification along with improved performance.

In this paper, wavelet theory (Silva 2015) is used, where the physical 1-d signals are analysed in time and frequency domain to extract features that will assist with fault diagnosis (Kurukuru et al. 2020). A detailed overview of wavelet transform and its application for 1-d signals is given in Dremin, Ivanov and Nechitailo (2001). In general, discrete wavelet transform (DWT) is implemented for feature extraction (Haque et al. 2019; Bharath et al. 2019). The DWT (Discrete and Transform, n.d.) calculates the wavelet transform utilizing a filter bank with low- and high-pass filters.

Further, the feature vectors are arranged as per their corresponding classes and trained with a classifier to develop the classification algorithm (Fatama, Haque, and Khan 2019; Khan et al. 2020). Different machine learning classifiers are discussed in Kotsiantis, Zaharakis, and Pintelas (2006). In this research the support vector machine classifiers are used to develop the islanding classifier due to its advantage with high dimensional feature mapping and high classification efficiency. In general, the SVMs are a supervised learning method used for classification of samples into two or more classes. Here, the input vectors are mapped into a very high dimensional feature space. The algorithm constructs parallel hyperplanes, one on each side of the separating class and the hyperplane with the largest separation margin between the training points of the two classes is chosen. SVM then creates a model trained with input examples to predict the class of a new sample (Cortes and Vapnik 1995).

\section{Low voltage ride through}

The abnormalities encountered at the grid end (voltage sag) will result in fluctuation of DC link voltage. In the case of voltage sag, the value of DC voltage will increase because of the power mismatch between the PV and PCC terminals. For balancing the DC link voltage and limiting its secure range an FRT scheme is proposed. 


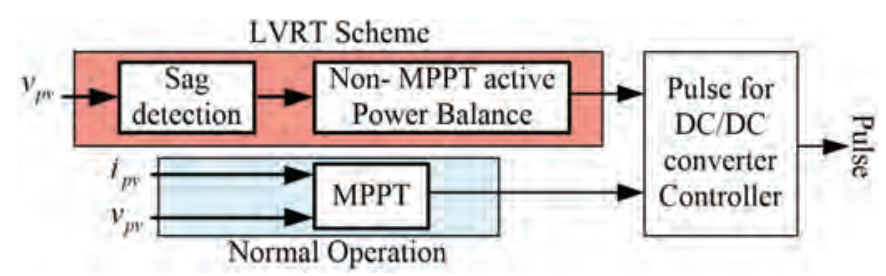

Figure 4.2 Proposed LVRT scheme.

The proposed scheme generally operates in a modified formation considering the cost for system remains the same. In the case of normal operation, the PV system operates along with MPPT for maximum power generation out of the solar. When a fault occurs at the grid end and a voltage sag is detected, the PV array will switch to a non MPPT mode of operation and depending on the voltage sag the value of active power is recalibrated to provide a power stable condition to the system.

From Figure 4.2 it can be observed that there are two possibilities of LVRT fault to take place.

\section{Results}

A 4 kW GCPVS is designed using MATLAB/Simulink software. Initially, islanding classifier is developed as discussed. Once an islanding scenario is detected, the system attempts to recover from the fault before the trip signal can be generated. The process of recovery has been explained in the previous sections. National grid code and IEC 62112 (International Electrotechnical Commission 2014) are considered to identify the range of operation at which the system will operate smoothly and in case of fault, the control will make attempts to recover the system. In case of failure to clear fault within a prescribed time threshold, a trip is created separating the PV from the utility.

In Figure 4.3(a), a line to ground fault occurs at the PCC during $t=1 \mathrm{~s}$, and $V_{P C C}$ decreases from a steady state value of $230 \mathrm{~V}$ to $50 \mathrm{~V}$. The average value of $P_{P C C}$ drops from $4100 \mathrm{~W}$ to $1320 \mathrm{~W}$ and $Q_{P C C}$ increases to an average value of $1 \mathrm{pu}$. The solar power plant is riding through the fault as required by FRT requirements and supporting the grid with $\mathrm{Q}$ injection. Considering the trip signal, as per the grid codes explained above, a voltage fall beyond $50 \%$ of nominal value results in trip generation after $0.2 \mathrm{sec}$, a trip signal is observed at $1.2 \mathrm{~s}$ in the figure 4 as fault was injected at $1 \mathrm{~s}$ and fault was not cleared within the time span. The trip disconnects the PV from the grid, hence drop in reactive and active power is observed, and $V_{d c}$ starts to act as an open circuit.

In Figure 4.3(b), a line to ground (LG) fault occurs at the PCC at $t=1 \mathrm{~s}$ and $V_{P C C}$ decreases from a steady state value of $230 \mathrm{~V}$ to $200 \mathrm{~V}$. The $P_{P C C}$ and $Q_{P C C}$ experience high-frequency oscillations because of negative sequence components, and the PV system is exchanging power with an unbalanced grid system. The average value of $P_{P C C}$ drops from $4020 \mathrm{~W}$ to $2800 \mathrm{~W}$ and $Q_{P C C}$ increases to an average value of $1 \mathrm{pu}$. The solar power plant is riding through the fault as required by FRT requirements and supporting the grid with $\mathrm{Q}$ injection. Considering trip signal, as per the grid codes explained above, a voltage drop beyond $6 \%$ of nominal value results in trip generation after $2 \mathrm{~s}$. Hence, a trip signal is observed at $3 \mathrm{~s}$ in the figure, 14 as fault was injected at $1 \mathrm{~s}$, and fault was not cleared within the time span. The trip isolates PV generation from the grid, hence drop in reactive and active power is observed, and $V_{d c}$ starts to act as an open circuit. 


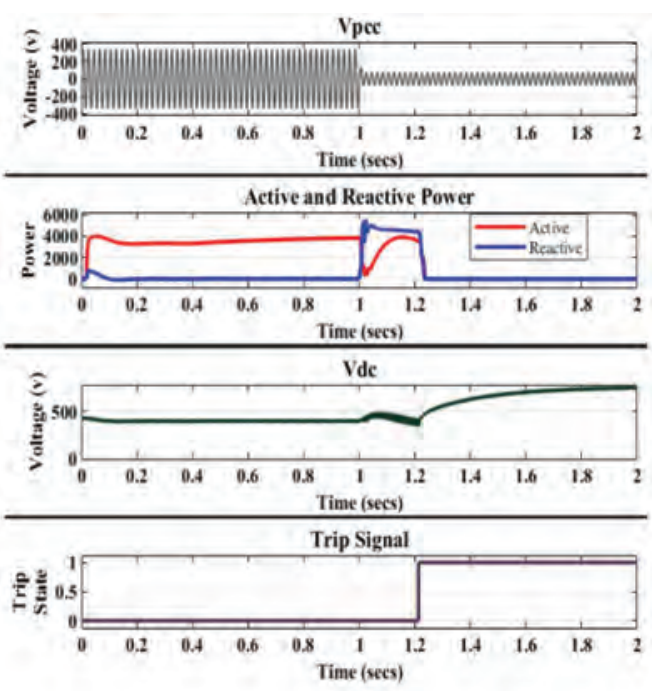

(a)

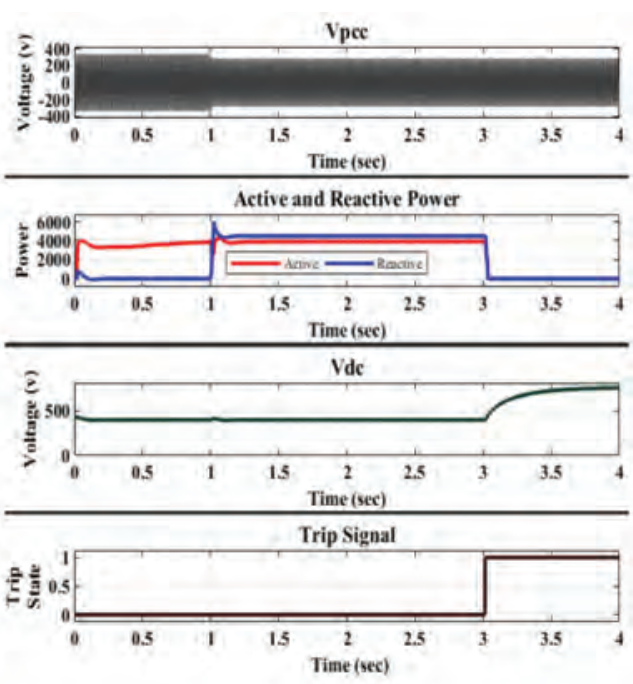

(b)

Figure 4.3 (a) 50\% voltage sag at PCC, (b) 6\% voltage sag at PCC.

\section{Conclusion}

A detailed analysis and verification of the FRT capability, as a part of the supervisory control, for a grid integrated PV system is carried out. The developed control strategy was able to effectively ride through the faults from the PCC. As a fault is observed, the controller successfully provides reactive power control, and provides FRT capability by optimally allocating $I_{q_{r f}}$. The controller proposed is capable to fulfil FRT requirement for PV system and enables grid to stay linked during the fault condition as per the grid code requirement. The PV system regularly injects reactive current $(>2 \%)$ for change in PCC voltage $(1 \%)$ within $7 \mathrm{~ms}$ of fault detection. If under any condition the reactive power injection fails to restore the system to normal operating state as per the grid code, the controller disconnects PV systems. The results present that the proposed controller operates efficiently and was tested with $4 \mathrm{~kW}$ grid connected single phase PV system to conclude that it presents a better accuracy and under specified time as defined by grid code.

\section{References}

Ackermann, Thomas, Göran Andersson, and Lennart Söder. 2001. "Distributed generation: a definition.” Electric Power Systems Research 57 (3): 195-204. doi:10.1016/S0378-7796 (01)00101-8.

Balaguer, Irvin J., Qin Lei, Shuitao Yang, Uthane Supatti, and Fang Zheng Peng. 2011. "Control for grid-connected and intentional islanding operations of distributed power generation." IEEE Transactions on Industrial Electronics 58 (1). IEEE: 147-57. doi:10.1109/ TIE.2010.2049709.

Bharath Kurukuru, V. S., Ahteshmaul Haque, and Mohammed Ali Khan. 2019. "Fault classification for photovoltaic modules using thermography and image processing." In 2019 IEEE Industry Applications Society Annual Meeting, 1-6. IEEE. doi:10.1109/ IAS.2019.8912356. 
Chang, Wen-Yeau. 2010. "A hybrid islanding detection method for distributed synchronous generators." In The 2010 International Power Electronics Conference - ECCE ASIA, 132630. IEEE. doi:10.1109/IPEC.2010.5544559.

Cortes, Corinna, and Vladimir Vapnik. 1995. "Support-vector networks." Machine Learning 20 (3): 273-97. doi:10.1023/A:1022627411411.

Dremin, I. M., O. V. Ivanov, and V. A. Nechitailo. 2001. "Wavelets and Their Use." doi:10.1070/ PU2001v044n05ABEH000918.

Fatama, Al-zoya, Ahteshamul Haque, and Mohammed Ali Khan. 2019. "A multi feature based islanding classification technique for distributed generation systems." In 2019 International Conference on Machine Learning, Big Data, Cloud and Parallel Computing (COMITCon), 160-66. IEEE. doi:10.1109/COMITCon.2019.8862442.

Haque, Ahteshamul. 2014. "Maximum power point tracking (MPPT) scheme for solar photovoltaic system.” Energy Technology \& Policy 1 (1): 115-22. doi:10.1080/ 23317000.2014.979379.

Haque, Ahteshamul, Kurukuru Varaha Satya Bharath, Mohammed Ali Khan, Irshad Khan, and Zainul Abdin Jaffery. 2019. "Fault diagnosis of photovoltaic modules." Energy Science \& Engineering, no. 7 (3): ese3.255. doi:10.1002/ese3.255.

International Electrotechnical Commission. 2014. "IEC 62116-2014."

Khajehoddin, Sayed Ali, and Masoud Karimi-ghartemani. 2013. "A Power control method with simple structure and fast dynamic response for single-phase grid-connected DG systems." IEEE Transactions on Power Electronics 28 (1): 221-33.

Khamis, Aziah, Hussain Shareef, Erdal Bizkevelci, and Tamer Khatib. 2013. "A review of islanding detection techniques for renewable distributed generation systems." Renewable and Sustainable Energy Reviews 28 (December): 483-93. doi:10.1016/j.rser.2013.08.025.

Khan, Mohammed Ali, Ahteshamul Haque, and V S Bharath. Kurukuru. 2019. "Enhancement of fault ride through strategy for single-phase grid-connected photovoltaic systems.” In 2019 IEEE Industry Applications Society Annual Meeting, 1-6. IEEE. doi:10.1109/IAS.2019.8911895.

Khan, Mohammed Ali, Ahteshamul Haque, and V. S. Bharath Kurukuru. 2020. "Intelligent control of a novel transformerless inverter topology for photovoltaic applications." Electrical Engineering 102 (2): 627-41. doi:10.1007/s00202-019-00899-2.

Khan, Mohammed Ali, Ahteshamul Haque, and V.S. Bharath Kurukuru. 2020. "Performance Assessment of Stand-alone Transformerless Inverters." International Transactions on Electrical Energy Systems 30 (1). doi:10.1002/2050-7038.12156.

Khan, Mohammed Ali, Ahteshamul Haque, and Varaha Satya Bharath Kurukuru. 2019. "Machine learning based islanding detection for grid connected photovoltaic system." In 2019 International Conference on Power Electronics, Control and Automation (ICPECA), 1-6. IEEE. doi:10.1109/ICPECA47973.2019.8975614.

Khan, Mohammed Ali, V. S. Bharath Kurukuru, Ahteshamul Haque, and Saad Mekhilef. 2020. "Islanding classification mechanism for grid-connected photovoltaic systems." IEEE Journal of Emerging and Selected Topics in Power Electronics 9 (2): 1966-1975. doi:10.1109/ JESTPE.2020.2986262.

Kotsiantis, S. B., I. D. Zaharakis, and P. E. Pintelas. 2006. "Machine learning: a review of classification and combining techniques." Artificial Intelligence Review 26 (3): 159-90. doi:10.1007/ s10462-007-9052-3.

Kurukuru, Varaha Satya Bharath, Frede Blaabjerg, Mohammed Ali Khan, and Ahteshamul Haque. 2020. "A novel fault classification approach for photovoltaic systems." Energies 13 (2): 308. doi:10.3390/en13020308.

Mulhausen, John, Joe Schaefer, Mangapathirao Mynam, Armando Guzman, and Marcos Donolo. 2010. "Anti-islanding today, successful islanding in the future." In 2010 63rd Annual Conference for Protective Relay Engineers, 1-8. IEEE. doi:10.1109/ CPRE.2010.5469490.

Noor, F., R. Arumugam, and M. Vaziri. n.d. "Unintentional islanding and comparison of prevention techniques." In Proceedings of the 37th Annual North American Power Symposium, 2005., 90-96. IEEE. doi:10.1109/NAPS.2005.1560507. 
Silva, Andre A. 2015. "Wavelet-based feature extraction methodology for pattern classification in engineering applications (FOG).” Master’s Thesis. 818. https://opencommons.uconn.edu/gs_ theses/818.

Sundararajan, D. 2015. Discrete Wavelet Transform: A Signal Processing Approach. Wiley.

Yang, Yongheng, Frede Blaabjerg, Huai Wang, and Marcelo Godoy Simões. 2016. "Power control flexibilities for grid-connected multi-functional photovoltaic inverters." IET Renewable Power Generation 10 (4): 504-13. doi:10.1049/iet-rpg.2015.0133. 


\title{
5 Machine learning classifier for fault classification in photovoltaic system
}

\author{
V.S. Bharath Kurukuru ${ }^{1}$, Mohammed Ali Khan ${ }^{1}$, \\ Abteshamul Haque ${ }^{1}$, Arun Kumar Tripathi ${ }^{2}$
}

\author{
${ }^{1}$ Advance Power Electronics Research Laboratory, Department of Electrical \\ Engineering, Faculty of Engineering and Technology, Jamia Millia Islamia, \\ New Delhi, India \\ ${ }^{2}$ National Institute of Solar Energy, Haryana, India
}

\section{Introduction}

Faults in PV systems impact the output performance of the system and may lead to critical and possibly hazardous situations. Protection from faults in PV system is important and challenging for researchers. In the conventional approach of fault detection and protection, relays, fuses and circuit breakers are added and controlled for different components of PV system (Vargas, Goss, and Gottschalg 2015; Ground-fault 1999). The inability of these protection devices to trip and isolate only for large fault currents is considered to be a major concern (Zhao et al. 2013, 2011). This phenomenon is observed, mainly due to current-limiting nature and non-linear I-V characteristics of PV arrays. Also, fault analysis in PV may become more complicated, with the effect of varying environmental conditions and ageing of modules. The effects of maximum power point tracking (MPPT) and commutation failure in PV converters can also influence the fault currents (Nayak, Mohapatra, and Mohanty 2017). Considering all the effects mentioned above, the need to continuously monitor the PV system is necessary.

An overview of the work presented in the literature related to various approaches for fault analysis was given in Jaffery et al. (2017), Haque et al. (2019) and Kurukuru et al. (2020). Fault classification approaches, which relied on the use of Fourier transform to catch signals' attributes, were introduced in Gopakumar, Reddy and Mohanta (2015) and Hessine, Jouini and Chebbi (2014). Gopakumar et al .(2015) relied on a combination of fast Fourier transform (FFT) and support vector machine to classify faults in transmission lines, where FFT was utilized to catch the signal's features (Kurukuru et al. 2019) from the phasor measurement unit (PMU) data, which were then used as an input to support vector machine (SVM). In a combination, discrete Fourier transform (DFT) and the artificial neural networks (ANNs) were applied to categorize the faults (Hessine, Jouini, and Chebbi 2014).

From the above literature, it's observed that the problem in hand is to get much better and enhanced techniques over widespread procedures to categorize faults for a single-phase PV system. The objective of this paper is to identify various faults on singlephase photovoltaic systems which consist of PV array, DC-DC converter along with MPPT, DC-AC converter, and LCL filter. Further, different faults under various operating conditions were identified and divided into 4 different classes. The novelty of the developed approach lies in its feature extraction process, where the wavelet transform

DOI: $10.1201 / 9781003129103-5$ 
is adapted to enhance the performance of feature extraction. Further, the choice of support vector machine for training the classifier provides various advantages by handling unstructured fault data and scaling it relatively well to high dimensional data. The work presented in the paper is structured as follows: Section 2 explains the requirements for developing a fault classification approach. Section 3 depicts the development of fault classification algorithms using MATLAB/Simulink software and the discussion is concluded in Section 4.

\section{Methodology}

The key objective of the proposed fault classification methodology is to determine the suitable wavelet coefficients in order to detect and classify the PV system faults accurately.

\subsection{Wavelet transform}

Generally, the wavelet transform represents a signal-processing tool that was applied to capture the features contained in the signal. There are many methods for applying wavelet transform, namely Wigner-Ville distribution, Hilbert-Huang transform, Stationary Wavelet Transform, Discrete Wavelet Transform, and Continuous Wavelet Transform. It is considered that DWT (Mallat 1989) offers an exact representation for any given signal, providing frequency sub-bands at different resolutions. This phenomenon provides a great advantage for discrete wavelets over the continuous wavelet transform. It also provides a significant reduction in the computational complexity by just computing the wavelet coefficients at the frequency sub-bands. Therefore, the computational complexity for the discrete wavelets is only $O(n)$, which is significantly less compared to the continuous wavelets, with $n$ as data size. The major advantage of DWT is its extensive library of the wavelet functions, making it adaptable for transient analysis. This provides different resolutions for the time-frequency spectrum. Depending upon the pattern of the signal the mother wavelets are chosen to perform signal decomposition, reconstruction, and feature extraction.

Once the wavelet coefficients and level of decomposition and reconstruction are determined, the feature extraction process can be initiated. During this process, the most relevant information from different signals is extracted and represented in a lowerdimensional space. This relevant information regarding the signals helps in discriminating and distinguishing different objects or faults (Kountchev and Nakamatsu 2016).

The spectral analyser is widely used in the first step of the feature detection process. The spectrogram can be useful in gathering information about a signal and in determining which features can be extracted from a signal. The spectrogram is used for defining statistical features of the signal, such as mean value, standard deviation, skewness and kurtosis. These features build a feature vector to retrieve a similar signal from the database. Some of the other features that can be extracted are peak to peak value, power, energy, entropy, total harmonic distortion and signal to noise ratio. The process of feature extraction using wavelets is explained in Ahmad et al. (2018).

\subsection{Machine learning techniques}

Machine learning (Hale 1981) is a procedure of data analysis that programs systematic model structure using techniques that iteratively learn from data. These techniques include the decision trees, k-nearest neighbours, non-deterministic classification techniques such as the support vector machine (SVM) and naïve Bayes (NB) classifier, which relies on 
probability theory. In general, these learning methods can be categorized into two categories: the eager learning method and the lazy learning method. In the eager learning method, the classification model is constructed using labelled training data: the support vector machine is commonly described as an eager learner. On the other hand, in the lazy learning method, the process of model development is delayed and the classification process is performed by observing all the relatively similar training and testing instances. Therefore, K-nearest neighbour is commonly described as a lazy learner. In this paper, SVM classifiers (Chapelle 2007), which are an eager learner, are used to automate the fault classification process due to its training time and pre-calculated algorithm.

In general, the SVM algorithm is based on constructing an optimal hyperplane with the maximal margin between support vectors. The selection of support vector is performed by training samples in the boundary region of multiple classes. Another important thing is that the number of the used support vectors is small in comparison to the size of the training data set. If the input data are linearly separable, then the SVM algorithm searches for the optimal hyperplane in the unchanged feature space of the input data. However, in the case the input data are linear non-separable, SVM maps the input data using a so-called kernel function to the higher dimensional feature space. The used kernel function must satisfy Mercer's theorem (Mercer 1909) and correspond to some type of inner product in the constructed high dimensional feature space. One of the advantages of SVM is its universality as the algorithm allows using different kernel functions, which depend on the researcher's assumptions and problem domain. Another advantage of the SVM algorithm is its effectiveness in the high dimensional feature spaces.

\section{Fault classification algorithm}

The methodology adopted for fault classification is structured as depicted in Figure 5.1. In this research, the fault classification technique is used to learn a model called a classifier. Data linking to various faults and normal operational conditions of the PV system is bifurcated into two groups; testing and training set. During the training phase, the training set of data is fed to the classifier for labeled data set into one of the classes depending on the target output. Fivefold cross-validation is applied to validate the trained data. During the testing phase, depending upon the target output test sample is verified. Once essential features have been identified, the classification of a fault condition is straightforwardly performed. The SVM technique is used in this work for fault classification due to its advantages with nonlinear data classification as mentioned in the section above. For simulation evaluation, the values of input data (i.e. the extracted features) are tabulated and imported to the classification program.

To observe the performance of the proposed methodology, a $4 \mathrm{~kW}$ two-stage PV system for a fixed load is simulated for varying climatic and load conditions. For experimental purposes, four different modes of operation (Normal Mode, Module Fault, Converter Fault, Inverter Fault) of the proposed system are considered. The voltage, current and frequency measurements of the system under different modes of operation for determined time period are logged. A sample of waveforms recorded for forming the fault classification algorithm are illustrated in Figure 5.2. The process of feature vector representation and applying DWT through HSA for the purpose of feature extraction is carried out. A feature set matrix $2656 * 6$ is obtained from the feature extraction process. Further, the extracted features are divided randomly into two sets of data: testing and training data set. Among 2656 samples, 1860 samples are selected as a training and the remaining 796 samples are selected as a testing data set. Different classes are arranged to the training samples as depicted in Table 5.1. 


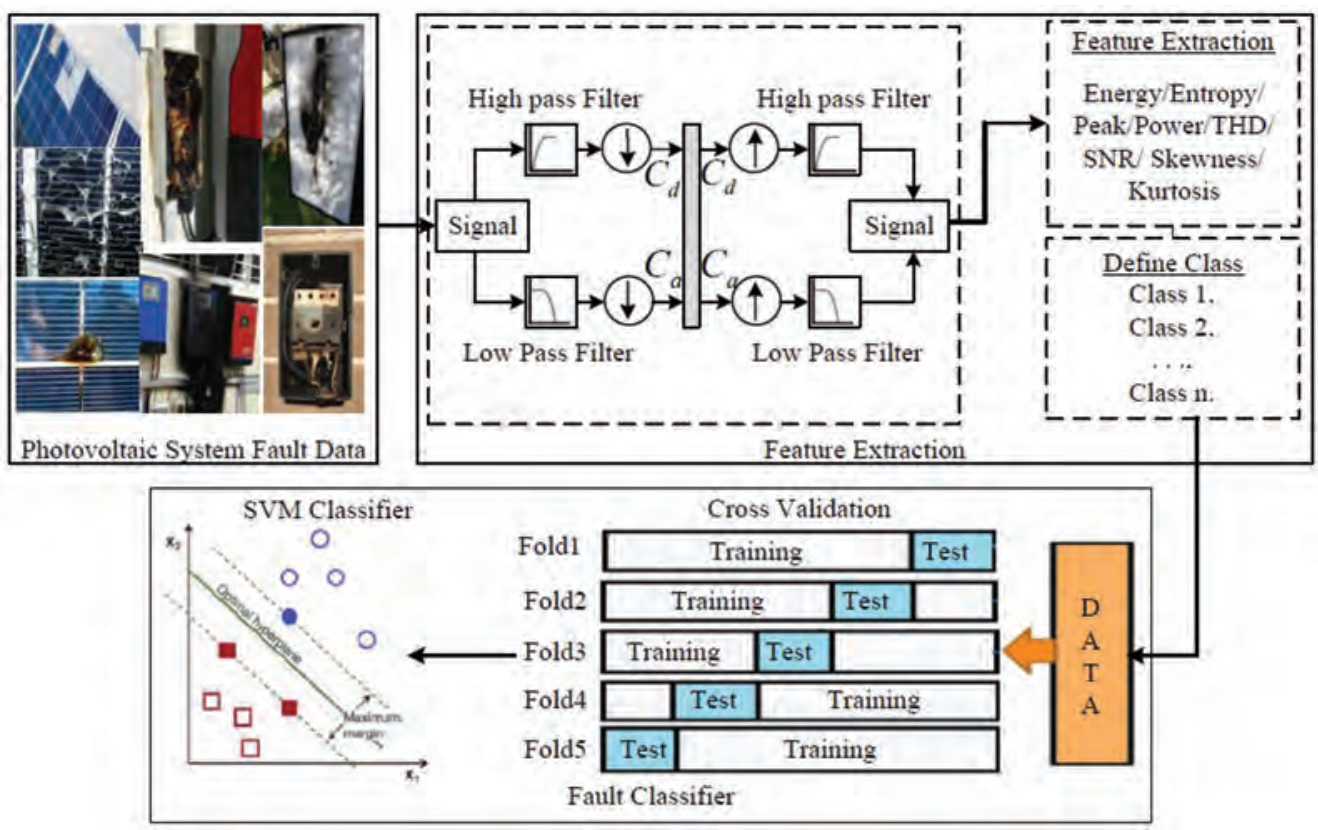

Figure 5.1 Block diagram for the wavelet based fault detection technique.
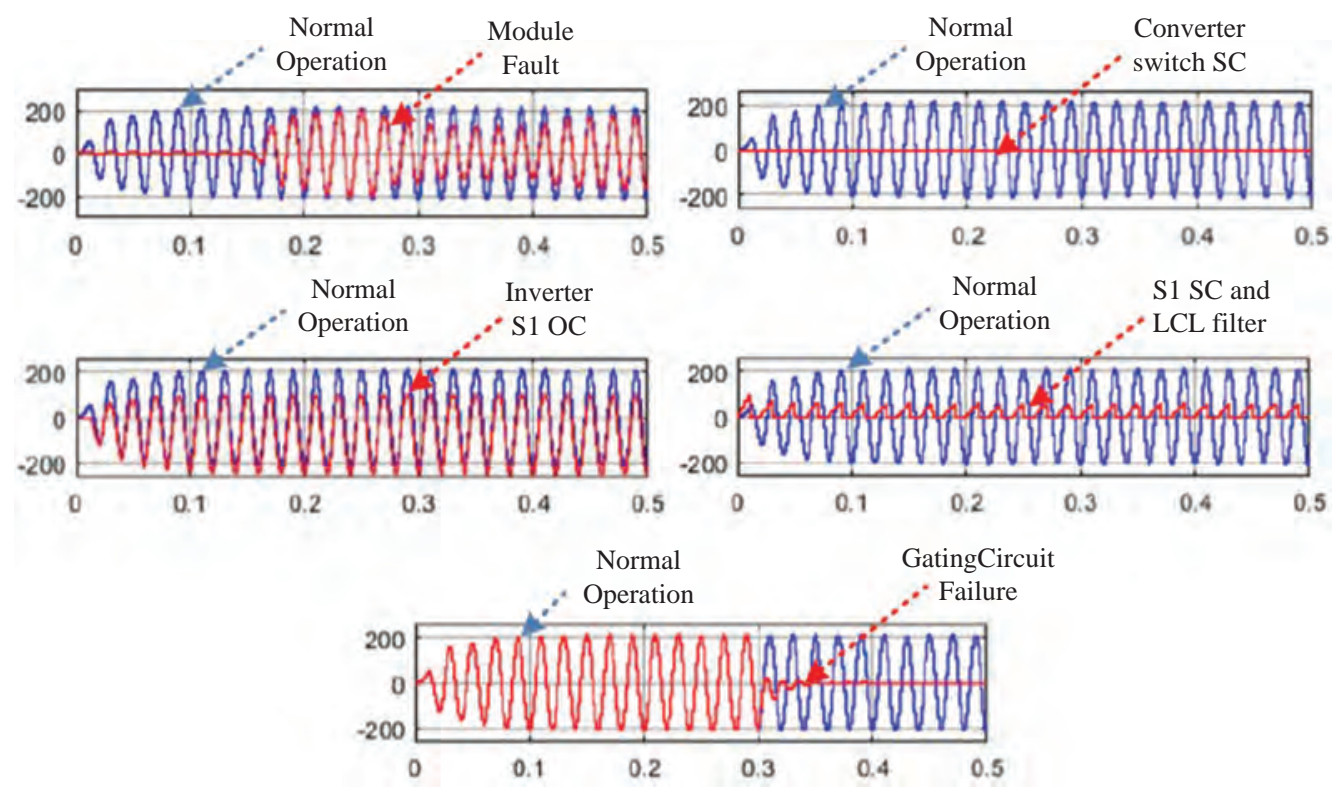

Figure 5.2 Various operating and fault conditions of PV system. 
Table 5.1 Distribution of samples

\begin{tabular}{lcc}
\hline Operating condition & \multicolumn{2}{c}{ Condition data } \\
\cline { 2 - 3 } & Training samples & Testing samples \\
\hline Normal operation & 465 & 199 \\
Module fault & 465 & 199 \\
Converter fault & 465 & 199 \\
Inverter fault & 465 & 199 \\
Total & 1860 & 796 \\
\hline
\end{tabular}
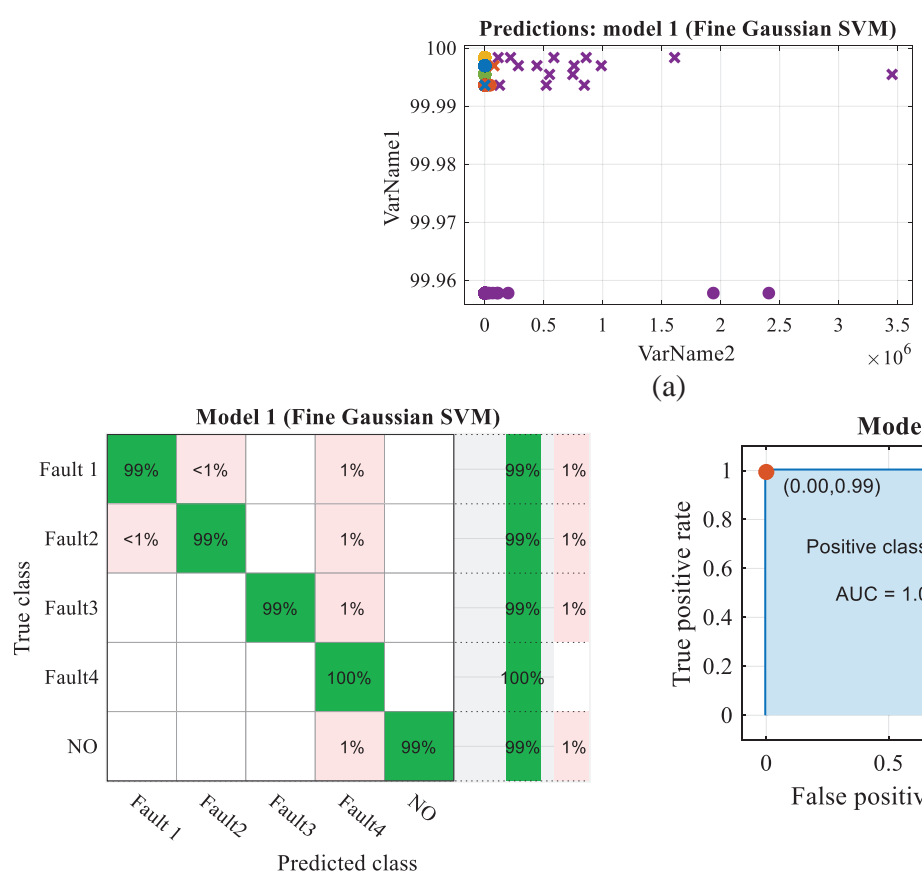

(a)

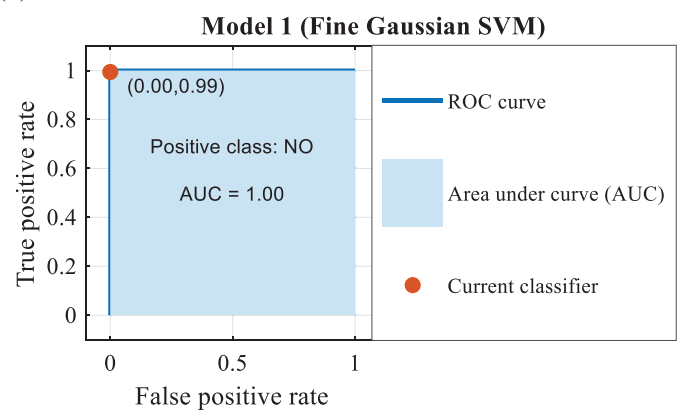

(c)

(b)

Figure 5.3 Trained data: (a) scatter plot (b) confusion matrix (c) ROC.

The classification accuracy is defined as the ratio of correctly classified samples to the total no of samples in the test data set. The corresponding equation is given as.

$$
\text { Accuracy of classification }=\frac{\text { Totalno. of samples classified correctly }}{\text { Total no. of samples in the data set }} * 100 \%
$$

To confirm the developed classifier data, a set of input data not used within the training stage is given to the trained data. This technique improves the average accuracy about $99.12 \%$ and $97.6 \%$ in training and testing, respectively, after the epoch equal to 1000 . The corresponding results are depicted in Figure 5.3(a-c). 
Table 5.2 Comparative analysis of training accuracy

\begin{tabular}{|c|c|c|c|}
\hline \multirow[t]{2}{*}{ Parameters } & $\begin{array}{l}\text { Optimized } \\
\text { wavelet-SVM } \\
\text { classifitcation }\end{array}$ & SVM classifier & $\begin{array}{l}\text { Artificial neural } \\
\text { network }\end{array}$ \\
\hline & Training process & Training process & Training process \\
\hline No. of samples & 1860 & 1860 & 1860 \\
\hline Epochs & - & - & 1000 \\
\hline No. of unclassified samples & 14 & 480 & 213 \\
\hline Kernel function & Fine Gaussian & Fine Gaussian & - \\
\hline Average accuracy of classification & $99.12 \%$ & $74.19 \%$ & $88.4 \%$ \\
\hline
\end{tabular}

The scatter plot in Figure 5.3(a) shows the scattering of feature data (two features) in XY plane. The scattered data help in identifying important predictors, outliners and visual patterns for training the classifier. To assess the performance of the classifier while training the feature matrix, a confusion matrix is plotted between the truly classified samples and the falsely classified samples as shown in Figure 5.3(b). The diagonal elements in the confusion matrix contribute to the percentage of features that are truly classified and the elements above and below the diagonal correspond to the falsely classified features. By plotting the features between true class and predicted class, it is observed that all the features were classified with their corresponding classes with precision. To support the classification accuracy plotted using confusion matrix, the receiver operating characteristic (ROC) of the data are plotted as shown in Figure 5.3(c). The plot resembles the data of two classified classes between the true positive rate (sensitivity of the data) and false positive rate (1 - specificity of the data) for various cut points in a training network. From the figure it can be identified that the corresponding class data lie above the diagonal region and close to the top left border of the ROC space, substantiating that the training process is most accurate. Table 5.2 summarizes the results by comparing with conventional classification methods.

\section{Conclusion}

This research developed a fault classification algorithm for condition monitoring of PV systems. The developed algorithm performed signal preprocessing by discrete wavelet transform for noise removal, feature extraction and region of interest segmentation. Various types of features were extracted once the signal preprocessing was completed. The extracted features are assigned towards corresponding classes and randomly divided as training and test data for the purpose of evaluation of the classifier. SVM is used to classify the fault conditions of PV systems into normal and faulty status. A fivefold cross validation is performed to measure the performance of the classifier with input data. On validation, the developed approach showed a training accuracy of $99.12 \%$, which is highly improved when compared to $96.72 \%$ in the literature.

\section{References}

Ahmad, Sameen, Nabeel Hasan, V S Bharath Kurukuru, Mohammed Ali Khan, and Ahteshamul Haque. 2018. "Fault classification for single phase photovoltaic systems using machine learning techniques." In 2018 8th IEEE India International Conference on Power Electronics (IICPE) 1-6. IEEE. doi:10.1109/IICPE.2018.8709463. 
Chapelle, Olivier. 2007. "Training a support vector machine in the primal.” Neural Computation 19 (5): 1155-78. doi:10.1162/neco.2007.19.5.1155.

Gopakumar, Pathirikkat, Maddikara Jaya Bharata Reddy, and Dusmanta Kumar Mohanta. 2015. "Adaptive fault identification and classification methodology for smart power grids using synchronous phasor angle measurements." IET Generation, Transmission \& Distribution 9 (2): 133-45. doi:10.1049/iet-gtd.2014.0024.

Ground-fault, O F, Protection Devices, F O R Photovoltaic, and Power Systems. 1999. Ward Bower, Sandia National Laboratones, Photovoltaic Systems Applications, Albuquerque, NM 87185-0753 John Wiles, Southwest Technology Development Institute, New Mexico State University, Las Cruces, NM 88003, 6 (d).

Hale, Robert L. 1981. "Cluster analysis in school psychology: an example." Journal of School Psychology 19 (1): 51-56. doi:10.1016/0022-4405(81)90007-8.

Haque, Ahteshamul, Kurukuru Varaha Satya Bharath, Mohammed Ali Khan, Irshad Khan, and Zainul Abdin Jaffery. 2019. "Fault diagnosis of photovoltaic modules." Energy Science \& Engineering, September (March): ese3.255. doi:10.1002/ese3.255.

Hessine, Moez Ben, Houda Jouini, and Souad Chebbi. 2014. "Fault detection and classification approaches in transmission lines using artificial neural networks." Proceedings of the Mediterranean Electrotechnical Conference - MELECON, no. April: 515-19. doi:10.1109/ MELCON.2014.6820588.

Jaffery, Zainul Abdin, Ashwani Kumar Dubey, Irshad, and Ahteshamul Haque. 2017. "Scheme for predictive fault diagnosis in photo-voltaic modules using thermal imaging." Infrared Physics \& Technology 83 (June): 182-87. doi:10.1016/j.infrared.2017.04.015.

Kountchev, Roumen, and Kazumi Nakamatsu. 2016. New Approaches in Intelligent Image Analysis 108. doi:10.1007/978-3-319-32192-9.

Kurukuru, V. S. Bharath, Ahteshamul Haque, Mohammed Ali Khan, and Arun Kumar Tripathy. 2019. "Fault classification for photovoltaic modules using thermography and machine learning techniques." In 2019 International Conference on Computer and Information Sciences (ICCIS), 1-6. IEEE. doi:10.1109/ICCISci.2019.8716442.

Kurukuru, V.S. Bharath, Frede Blaabjerg, Mohammed Ali Khan, and Ahteshamul Haque. 2020. "A novel fault classification approach for photovoltaic systems." Energies 13 (2): 308. doi:10.3390/en13020308.

Mallat, Stephane G. 1989. "A theory for multiresolution signal decomposition: the wavelet representation.” IEEE Transactions on Pattern Analysis and Machine Intelligence 11 (7): 674-93. doi:10.1109/34.192463.

Mercer, J. 1909. "Functions of positive and negative type, and their connection with the theory of integral equations." Philosophical Transactions of the Royal Society A: Mathematical, Physical and Engineering Sciences 209 (441-458): 415-46. doi:10.1098/rsta.1909.0016.

Nayak, Byamakesh, Alivarani Mohapatra, and Kanungo Barada Mohanty. 2017. "Selection criteria of Dc-Dc converter and control variable for MPPT of PV system utilized in heating and cooking applications." Cogent Engineering 4 (1): 1-16. doi:10.1080/23311916.2017.1363357.

Vargas, J. P., B. Goss, and R. Gottschalg. 2015. "Large scale PV Systems under non-uniform and fault conditions.” Solar Energy 116: 303-13. doi:10.1016/j.solener.2015.03.041.

Zhao, Ye, Brad Lehman, Jean François De Palma, Jerry Mosesian, and Robert Lyons. 2011. "Challenges to overcurrent protection devices under line-line faults in solar photovoltaic arrays." IEEE Energy Conversion Congress and Exposition: Energy Conversion Innovation for a Clean Energy Future, ECCE 2011, Proceedings, 20-27. doi:10.1109/ECCE.2011.6063744.

Zhao, Ye, J de Palma, J Mosesian, R Lyons, and B Lehman. 2013. "Line fault analysis and protection challenges in solar photovoltaic arrays." Industrial Electronics, IEEE Transactions On 60 (9): 3784-95. doi:10.1109/TIE.2012.2205355. 


\title{
6 Performance analysis and comparison of 4-bit adder architectures
}

\author{
Aishani Misra ${ }^{1, *}$, Shilpi Birla, ${ }^{2, *}$ Neha Singh ${ }^{3, *}$ \\ ${ }^{1}$ Dept. of Electronics \& Comm. Eng., Manipal University Jaipur, \\ Jaipur, Rajasthan, India \\ ${ }^{2}$ Dept. of Electronics \& Comm. Eng., Manipal University Jaipur, \\ Jaipur, Rajasthan, India \\ ${ }^{3}$ Dept. of Electronics \& Comm. Eng., Manipal University Jaipur, \\ Jaipur, Rajasthan, India
}

\section{Background}

Adder is one of the most significant and widely used arithmetic operators in arithmetic logic unit (ALUs), microprocessors, DSP processors, etc. Adders, besides being used to design adder circuits, can also be adopted to design subtractor, multiplier and divider circuits as well. Efficient performance of resident adders is essential for the overall performance of a VLSI system. The parameters that determine the performance of a system are delay, power dissipation, area and leakage current. Optimum adders can have effect in reducing the area, power, leakage current and increasing the speed, i.e. the constraints of a system. A single adder cannot optimally operate meeting all these constraints, since there is trade-off among them. The adders are thus selected for application based on the constraints for each specific application.

There are several reports of work that has been done on adders. A comparison of 100-bit RCA and a CSA has been done by [1]. By using a poly biasing technique the PMOS/NMOS strength ratio was balanced [2] and was compared by 32-bit and 16-bit adders respectively, thus showing their proposal was more energy efficient. Speed and power efficient 4-bit CLA has been designed by [3] using pass transistor logic (PTL), on $180 \mathrm{~nm}$ CMOS technology, because of which power consumption, delay and energy have been reduced. Various 32-bit carry-skip-adders were designed and compared [4] on $45 \mathrm{~nm}$ CMOS technology and as per results concatenation and incrementation carry-skip adder has $51 \%$ improvement in the critical path delay and energy, compared with that of conventional carry-skip adder. Ultra-low power and low voltage adders were designed and analysed [5] with an extended body bias voltage to improve the functional yield and balance the pull-up and pull-down networks on 22nm FDSOI technology. Harikrishnan and Sethi [6] optimally designed Manchester carry chain adder and reduced the delay by $19 \%$ using linear and El-more delay model. Numerous studies $[7,8]$ have looked at various adders namely parallel prefix adders (PPA), MCC etc.

We have implemented, compared and evaluated four types of adders based on delay, area, power dissipation and leakage current. The adders implemented and simulated were 4-bit RCA, CLA, MCC and CSK. Cadence Virtuoso has been used for implementation of

\footnotetext{
*Emails: aisha4ni@gmail.com, shilpi.birla@jaipur.manipal.edu,neha.singh@jaipur.manipal.edu
}

DOI: 10.1201/9781003129103-6 
the adder circuits and ELDO was used for simulation and analysis. All the work has been carried out on $28 \mathrm{~nm}$ technology within the supply voltage range of $0.6-1.0 \mathrm{~V}$.

\section{Designs}

For a particular combination of inputs $\mathrm{A} j$ and $\mathrm{B} j$, the propagate signal $\mathrm{P} j$ determines whether the carry-in to the $j$ th block would propagate to the output, whereas the generate signal $\mathrm{G} j$ determines if a carry-out would be set from inside the block independently from the inputs. The expression for $\mathrm{G} j$ and $\mathrm{P} j$ with two input binary operands, $\mathrm{A} j$ and $\mathrm{B} j$ as given by $[9,10]$ can be expressed as follows:

$$
\begin{aligned}
& \mathrm{G} j=\mathrm{A} j \bullet \mathrm{B} j \\
& \mathrm{P} j=\mathrm{A} j \oplus \mathrm{B} j
\end{aligned}
$$

The sum and carry Boolean expression for $i$ th stage of an adder are:

$$
\begin{aligned}
& \mathrm{S} j=\mathrm{P} j \oplus \mathrm{C} j \\
& \mathrm{C}_{j+1}=\mathrm{G} j+\mathrm{P} j \bullet \mathrm{C} j
\end{aligned}
$$

The signals are created as per (I), for each bit position in CLA. Further the sum and carry are given by eq. (III) and (IV).

In order to optimize the adder operation further we use (MCC), given as:

$$
A n j=\overline{A j+B j}
$$

The above-mentioned designs, namely RCA, CLA, MCC and CSK, are all 4-bit implemented adders.

The CSK uses (I)-(IV) along with a skip logic block which consists of a 5-input AND gate and a 2-input OR gate.

\section{Performance evaluation and results}

The simulation of the re-designed RCA, CLA, MCC and CSK is carried out using ELDO tool on $28 \mathrm{~nm}$ technology. The simulation results for Delay parameter at supply voltages ranging from 0.6 to $1 \mathrm{~V}$ for different adder architecture are presented in Figure 6.1.

It can be seen here that the adders follow an increasing linear trend as we lower the voltage. At $0.6 \mathrm{~V}$, CLA displays minimum delay of $243.02 \mathrm{ps}$, thereby implying that CLA is $27.9 \%$ faster than CSK, $8.2 \%$ faster than MCC and $26.46 \%$ faster than RCA, while CSK displays maximum delay of 337.3 ps. But as we increase the supply voltage, i.e. towards $1 \mathrm{~V}$, RCA produces maximum delay.

The power dissipation from $0.6-1 \mathrm{~V}$ for all the adder architectures is presented in Figure 6.2. It shows RCA gives minimum power dissipation of $15.531 \mathrm{pW}$ at $0.6 \mathrm{~V}$. MCC produces maximum power dissipation for supply voltage ranging from 0.6 to $1 \mathrm{~V}$ among the 4-bit architectures.

The third aspect which is evaluated is the area or the number of transistors used. Figure 6.3 shows the number of transistors used for different adders. CSK uses minimum number of transistors, i.e. 98, whereas CLA and MCC utilize 136 transistors each, thereby consuming maximum area. 


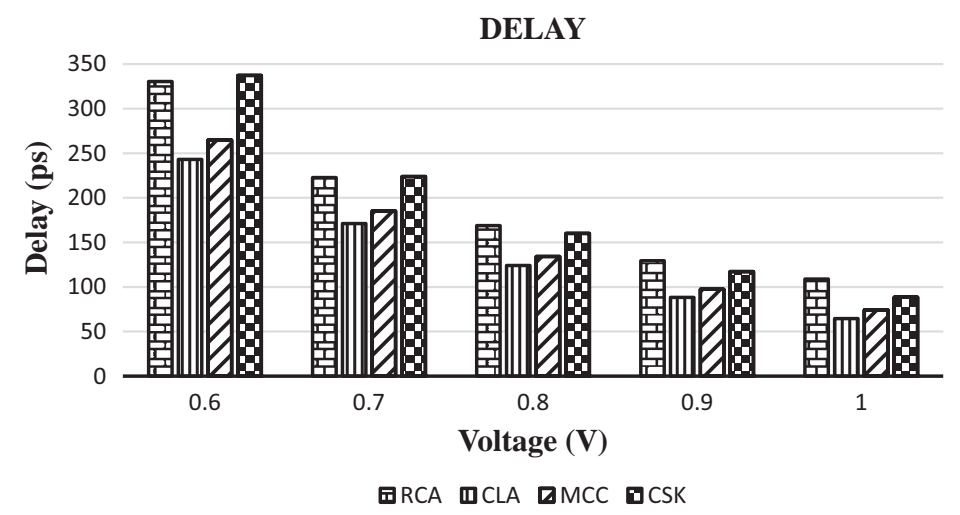

Figure 6.1 Delay of adders from 0.6 to $1 \mathrm{~V}$.

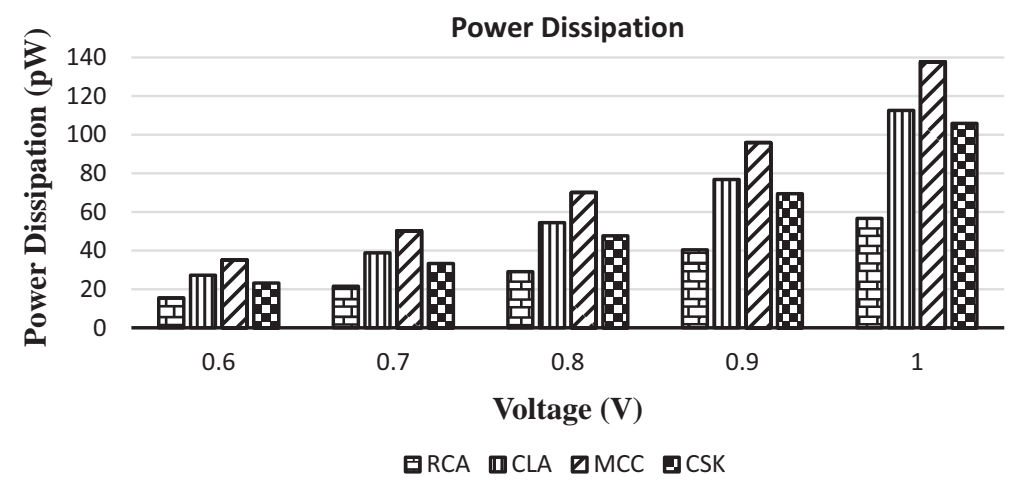

Figure 6.2 Power dissipation of adders from 0.6 to $1 \mathrm{~V}$.

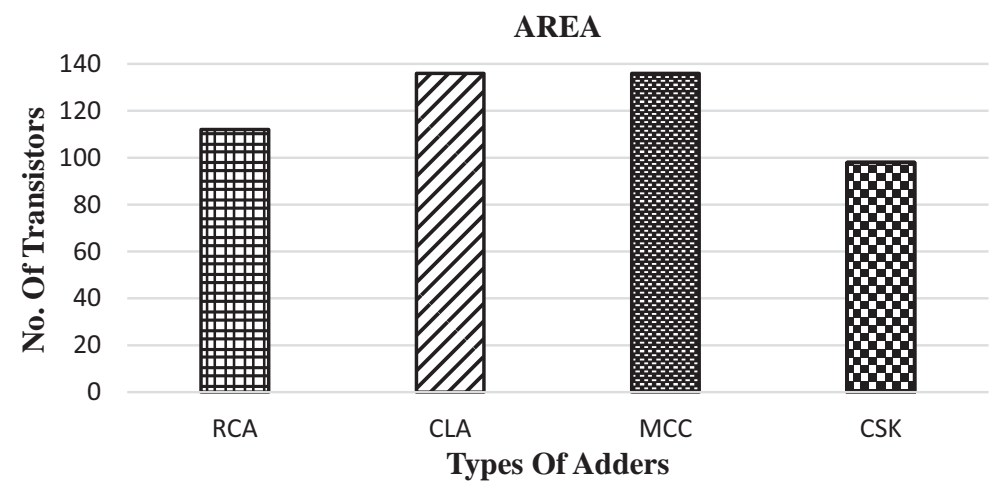

Figure 6.3 Area utilized (no. of transistors). 


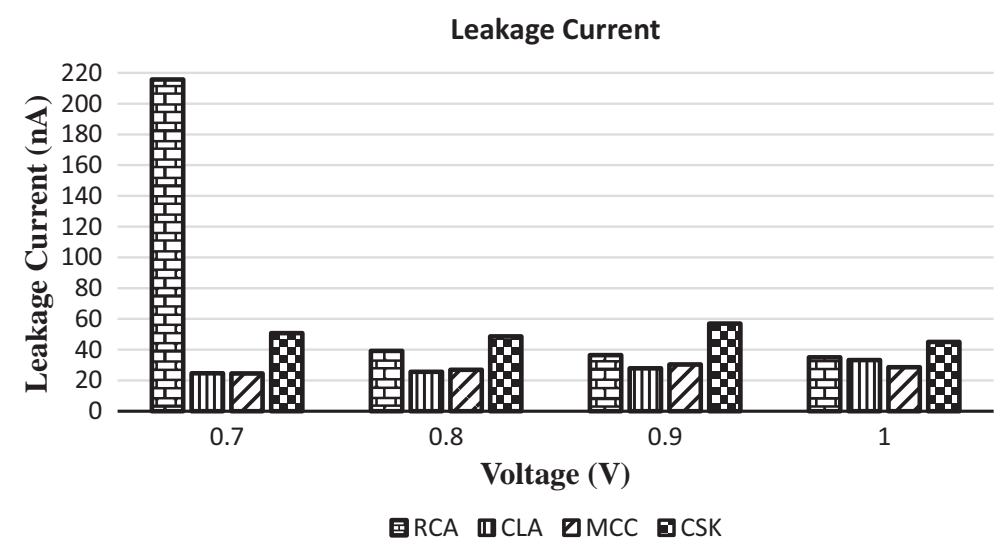

Figure 6.4 Leakage current of adders from 0.7 to $1 \mathrm{~V}$.

Figure 6.4 presents a plot of leakage current at supply voltages ranging from 0.7 to 1 $\mathrm{V}$. The leakage current at $0.7 \mathrm{~V}$ shows that MCC is minimum and maximum is that of RCA, but as we increase the voltage CSK gives maximum leakage current. The leakage current at $0.6 \mathrm{~V}$ for RCA, CLA, MCA and CSK is very high, i.e. greater than $100 \mathrm{nA}$, and therefore has not been taken into consideration for plotting.

\section{Conclusion}

In this paper, we have implemented 4-bit adder architectures, namely RCA, CLA, MCC and CSK, on Cadence Virtuoso and for simulation the ELDO tool has been used on $28 \mathrm{~nm}$ technology.

The comparative analysis show that at $0.6 \mathrm{~V}$, CLA produces minimum delay of 243.02 ps, making CLA faster than CSK by $27.9 \%$, MCC by $8.2 \%$ and RCA by $26.46 \%$. In terms of power dissipation, RCA provides the least for all supply voltages ranging from 0.6 to 1 V. CSK utilizes minimum area of 98 transistors, whereas MCC produces minimum leakage current for lower supply voltages as compared to the rest of the 4-bit adder architectures.

The adder design can further be optimized to reduce the delay, power dissipation, leakage current and area. They can later be used to create a library consisting of these adder cells, which can be utilized by designers for any specific application.

\section{References}

[1] Bedrij, Orest J. “Carry-select adder." IRE Transactions on Electronic Computers 3 (1962): 340-346.

[2] Vatanjou, Ali Asghar, Even Låte, Trond Ytterdal, and Snorre Aunet. "Ultra-low voltage and energy efficient adders in $28 \mathrm{~nm}$ fdsoi exploring poly-biasing for device sizing." Microprocessors and Microsystems 56 (2018): 92-100.

[3] Khan, Burhan, and Suraj Pattanaik. "Design a 4-bit carry look-ahead adder using pass transistor for less power consumption and maximization of speed." In Advances in Data Science and Management, pp. 531-542. Springer, Singapore, 2020.

[4] Sanjana, B., and K. Ragini. "Design of a novel high-speed-and energy-efficient 32-bit carry-skip adder." In Innovations in Electronics and Communication Engineering, pp. 335-343. Springer, Singapore, 2019. 
[5] Zadeh, Somayeh Hossein, Trond Ytterdal, and Snorre Aunet. "Comparison of ultra low power full adder cells in $22 \mathrm{~nm}$ fdsoi technology." In 2018 IEEE Nordic Circuits and Systems Conference (NORCAS): NORCHIP and International Sourabh Sethi Symposium of Systemon-Chip (SoC), pp. 1-5. IEEE, 2018.

[6] Harikrishnan, A., and Sourabh Sethi. "Sizing techniques for delay optimisation of 4-bit dynamic CMOS Manchester carry chain." In 2019 International Conference on Vision Towards Emerging Trends in Communication and Networking (ViTECoN), pp. 1-4. IEEE, 2019.

[7] Das, P., A. L. Bhalerao, A. Mane, A. A. Angeline, and V. S. K. Bhaaskaran. "Design of Manchester carry chain adder using high speed domino logic." In IOP Conference Series: Materials Science and Engineering 561, no. 1, p. 012125. IOP Publishing, 2019.

[8] Pinto, Rohan, and Kumara Shama. "Low-power modified shift-add multiplier design using parallel prefix adder." Journal of Circuits, Systems and Computers 28, no. 02 (2019): 1950019.

[9] Seng, Yeo Kiat, and Kaushik Roy. "Low voltage, low power VLSI subsystems." McGraw-Hill, New York, 2009.

[10] Misra, Aishani, Shilpi Birla, and Neha Singh. "Comparative analysis of various adder architectures on 28nm CMOS technology." 2020 7th International Conference on Computing for Sustainable Global Development (INDIACom), pp. 73-36. IEEE, 2020. 


\title{
$7 \quad$ Improved image compression using lifting wavelet
}

\author{
Sandeep Shelke*, Govind Singh Patel \\ School of Electronics and Electrical Engineering, Lovely Professional University, \\ Phagwara, Punjab, India
}

\section{Introduction}

Image processing is increasingly used in several application fields, such as medical $[1,2]$, aerospace [3] or automotive [4]. The complete image processing system is composed of three blocks as image filtering, image compression and image cryptography, with the image compression block being one of the most vital blocks in the image processing system.

An efficient image compression is critical phase in image processing system, in ordered to ensure better throughput so as to have image frame transmission at faster rates. The basic block diagram of image compression system is given in Figure 7.1.

As shown in Figure 7.1, the input image is converted into text form image using image to text converter. This text form image is compressed using FPGA compression module so that at its output compressed image in text form is received; this text form image is then converted into image form using text to image converter. For the purpose of decompression, the de-compression module is used so that at its output, the de-compressed image is achieved but this de-compressed image is in text form; this text form decompressed image is converted into image form using text to image converter in order to get de-compressed image.

\section{Methodology}

In order to compress the image, the image is converted into hexadecimal code using MATLAB as:

$03071016141103-018686868585868788$ 89 A9 89 8A 8A 8B 89 8B 9C 8C 8B 8C 8C 8C 8B 88 B2 85 8E 919093 9B 8398 9D 9E 65 9D 9C 9C 9F A4 A9 AC B0 B1 B1 .................... continued, each of the values indicates value of a pixel on grey scale. This sequence is further decomposed into two different sequences. For demonstration, first eight pixel values are considered as decomposed into two sequences as shown in Figure 7.2.

The first decomposed sequence is obtained by adding two successive pixel values and dividing by 2 , whereas second sequence is obtained by subtracting two successive pixel values and dividing by 2 . The architecture and system function diagram for above process are given in Figure 7.3.

Now the obtained sequence after decomposition needs less space than original sequence hence it is clear the compression is achieved with this process. Now in order to reconstruct

\footnotetext{
* Email: sandeepshelke18@gmail.com
}

DOI: $10.1201 / 9781003129103-7$ 


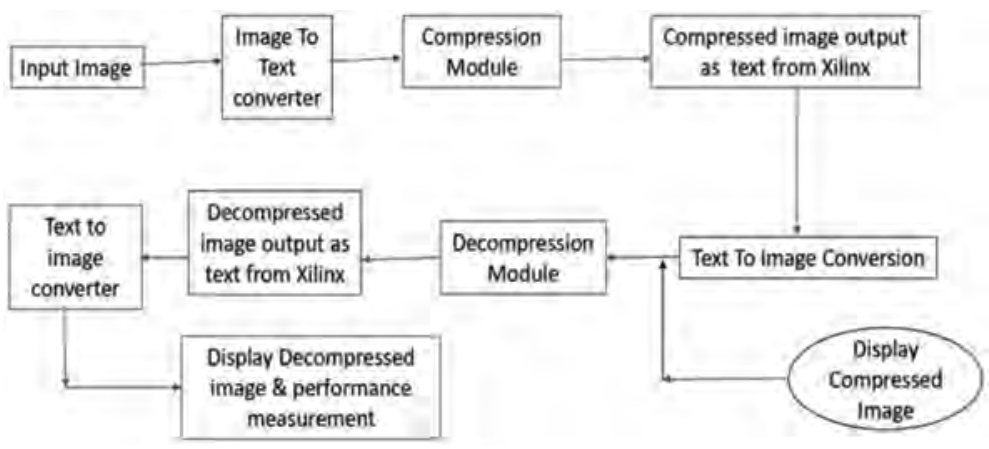

Figure 7.1 Image compression system.

\section{Original Sequence $\left[\mathrm{y}_{n}\right]$}

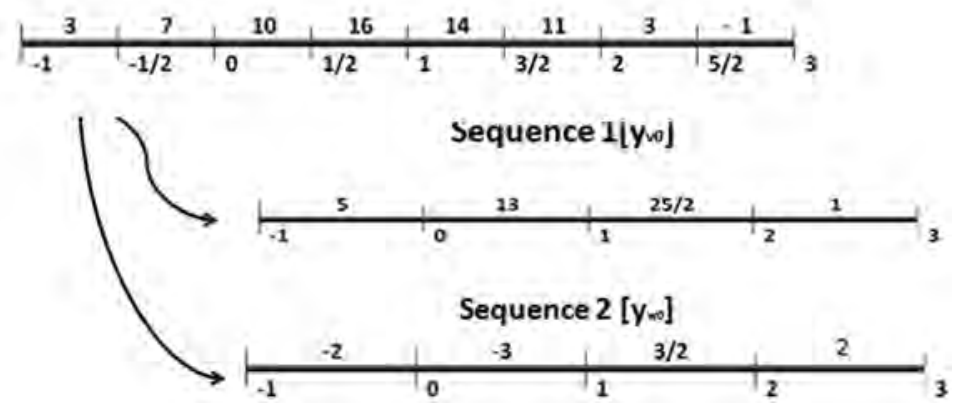

Figure 7.2 Text sequence decomposition.
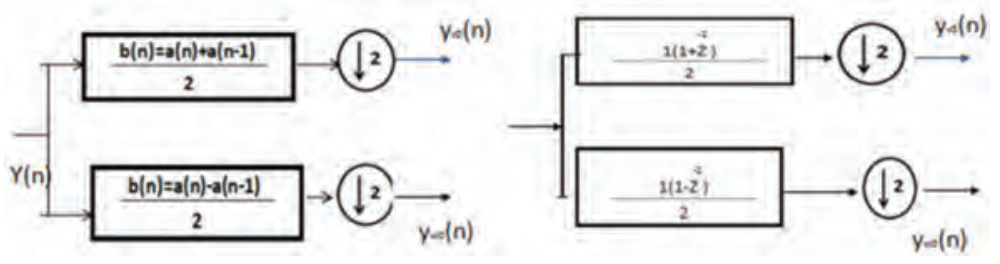

Figure 7.3 Architecture and system function for decomposition.

original sequence, both the sequences are first up-sampled and latter alternate addition and subtraction of the same indexed value of both sequences will give the original sequence. This is illustrated in Figure 7.4.

The complete structure for the above process is given in Figure 7.5.

Here this structure is reconstructed with the delay of one sample. The above architecture is processed using poly-phase matrices to have the lifting structure as given in Figure 7.6. 


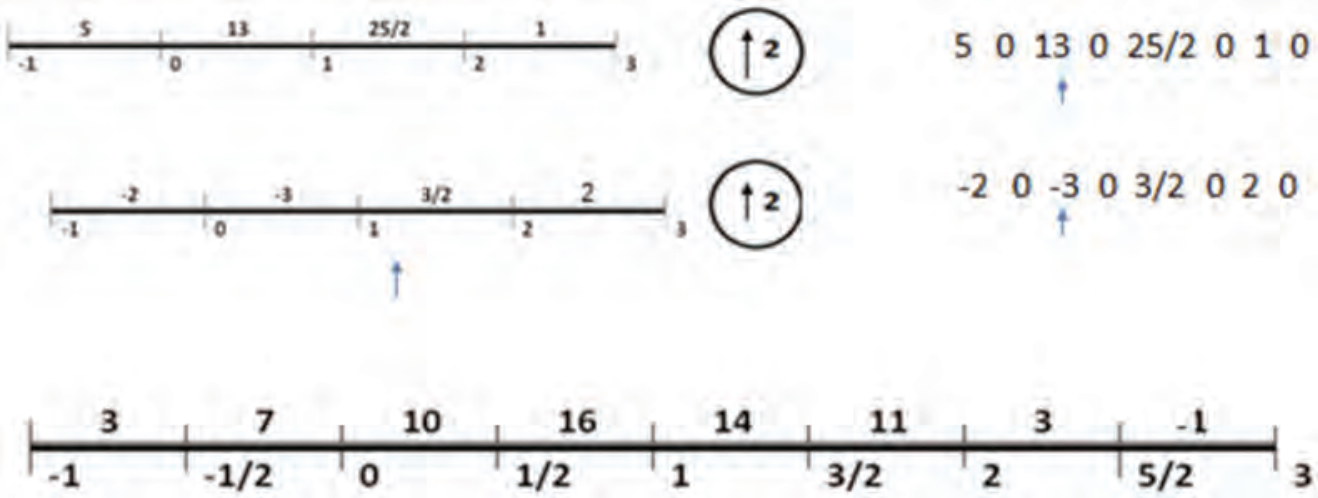

Figure 7.4 Reconstruction of original sequence.

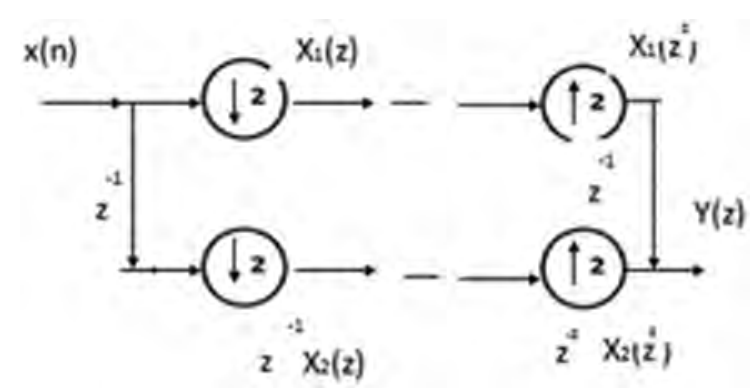

Figure 7.5 Complete compression and decompression structure.

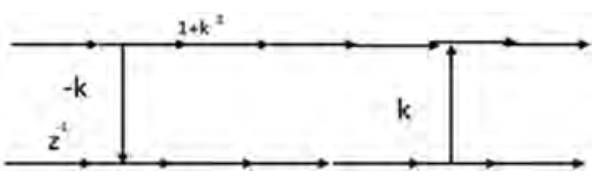

Figure 7.6 Lifting structure for compression.

Table 7.1 Result comparison

\begin{tabular}{lllllll}
\hline & $\begin{array}{l}\text { Panigrahy } \\
\text { et al. [5] }\end{array}$ & $\begin{array}{l}\text { Haque } \\
\text { et al. [6] }\end{array}$ & $\begin{array}{l}\text { Jackson } \\
\text { et al. [7] }\end{array}$ & $\begin{array}{l}\text { Samvi } \\
\text { et al. [8] }\end{array}$ & $\begin{array}{l}\text { Saad } \\
\text { et al. [9] }\end{array}$ & $\begin{array}{l}\text { Proposed } \\
\text { method }\end{array}$ \\
\hline Platform & FPGA & GPU & FPGA & FPGA & FPGA & FPGA \\
Image size & $256 \times 256$ & $1024 \times 1024$ & $512 \times 512$ & $256 \times 256$ & $1024 \times 1024$ & $512 \times 512$ \\
PSNR of image(dB) & 32.7 & NA & 33.1 & 25.3 & 30.3 & 36.77 \\
Compression ratio & 5.1 & 4.1 & 12.8 & 26 & 34.1 & 46.37 \\
\hline
\end{tabular}


This structure eliminates the delay of one sample as the upper and lower braches modify alternately, which is not the case in conventional compression technique.

\section{Result}

The result for compression ratio and PSNR is given in Table 7.1.

\section{Conclusion}

The alternate modification of upper and lower branches in lifting structure eliminates the delay that is available more prominently in conventional approach; hence the compression performance is improved to great extent with lifting architecture. Because of this architecture for compression PSNR is improved $11.05 \%$ and compression ratio is improved by $35.89 \%$.

\section{References}

[1] Li, Chao, Souleymane Balla-Arabé, and Fan Yang. "Embedded multi-spectral image processing for real-time medical application." Journal of Systems Architecture 64 (2016): 26-36.

[2] Pal, Chandrajit, Avik Kotal, Asit Samanta, Amlan Chakrabarti, and Ranjan Ghosh. "An efficient FPGA implementation of optimized anisotropic diffusion filtering of images." International Journal of Reconfigurable Computing (2016): 1.

[3] Taher, Fatma, Amal Zaki, and Hamed Elsimary. "Design of low power FPGA architecture of image unit for space applications.” In 2016 IEEE 59th International Midwest Symposium on Circuits and Systems (MWSCAS), pp. 1-4. IEEE, 2016.

[4] Schwiegelshohn, Fynn, Lars Gierke, and Michael Hübner. "FPGA based traffic sign detection for automotive camera systems." In 2015 10th international symposium on reconfigurable communication-centric systems-on-chip (ReCoSoC), pp. 1-6. IEEE, 2015.

[5] Panigrahy, Mamata, Indrajit Chakrabarti, and Anindya Sundar Dhar. "Low-delay parallel architecture for fractal image compression." Circuits, Systems, and Signal Processing 35, no. 3 (2016): 897-917.

[6] Haque, M, Abdullah Al Kaisan, Mahmudur R. Saniat, and Aminur Rahman. "GPU accelerated fractal image compression for medical imaging in parallel computing platform." arXiv preprint arXiv: 1404.0774 (2014).

[7] Jackson, D. J., H. Ren, X. Wu, and K. G. Ricks, "A hardware architecture for real-time image compression using a searchless fractal image coding method," J. Real-Time Image Processing 1, no. 3 (2007): 225-237.

[8] Samavi, Shadrokh, Mehdi Habibi, Shahram Shirani, and Narges Rowshanbin. "Real time fractal image coder based on characteristic vector matching." Image and Vision Computing 28, no. 11 (2010): 1557-1568.

[9] Saad, Abdul-Malik HY, and Mohd Z. Abdullah. "High-speed fractal image compression featuring deep data pipelining strategy." IEEE Access 6 (2018): 71389-71403.

[10] Mukhedkar, M. M., and U. Kolekar. E-TDGO: An encrypted trust-based dolphin glowworm optimization for secure routing in mobile ad hoc network. International Journal of Communication Systems, e4252.

[11] Mukhedkar, M., and Uttam D. Kolekar, "Development of optimized and secure routing algorithm using AODV, ACO and LSB steganography for mobile ad-hoc network", Journal of Advanced Research in Dynamical and Control Systems (JARDCS) 11, no. 9 (2019): 560-568.

[12] Mulajkar A. A. "3D torus router architecture for efficient NoC design”, Think India Journal 22, no. 16 (2019). 


\title{
$8 \quad$ A single cycle low latency bypass router based on network on chip
}

\author{
Ashish A. Mulajkar*, Govind Singh Patel \\ School of Electronics and Electrical Engineering, Lovely Professional University, \\ Phagwara, Punjab, India
}

\section{Introduction}

Network on chip (NoC) is a feasible communication architecture [1] which handles issues like flexibility, reliability and scalability. Consumption of power in NoC architecture is a major concern which could affect the performance of a multi-core processor. Network on chip consumes a total of $18-33 \%$ of the entire power of a chip [2]. At heavy network usage due to a greater number of components in a router, power leakage becomes a major factor that consumes about $74 \%$ of entire chip power at reduced scalability. Thus power gating optimizations and buffer-less routing are the two major techniques to save power at various levels like subnet, router and buffer level. Generally NoC framework is divided depending upon its execution results and cost. Average packet latency and throughput are included in execution parameters whereas peak temperature, area overhead and power consumption [7] are costing parameters in NoC router design.

Latency in a NoC is dependent upon the communication channels and the routers. Packet latency is calculated on basis of clock cycle using the following equation [7]

$$
T=H \cdot t_{r}+H \cdot t_{w}+T_{c}+L / b
$$

where $\mathrm{H}=$ hop counts, $\mathrm{t}_{\mathrm{r}}=$ router delay, $\mathrm{t}_{\mathrm{w}}=$ delay among communication links, $\mathrm{T}_{\mathrm{c}}=$ congestion delay and $\mathrm{L} / \mathrm{b}=$ delay among tail and body flits. The router proposed in this paper transfers flits within a single attempt to many destinations. The performance of the router architecture is enhanced by mechanisms like anti-flit packets, multiple destinations in a single attempt and less delay in transfer of packets [3]. The data flow architecture is efficient by $3.6 \times$ as compared to conventional routers. The major parameters considered in router design are less delay, higher rate of injection and manifold destination.

\section{A. Generic router architecture}

The basic problem of the generic router shown in Figure 8.1 based on application is traffic levels which are uneven at various locations [4]. As the load is unbalanced congestion in network takes place due to virtual channel (VC) and no load appears on ideal ports.

\footnotetext{
* Email: ashishmulajkar86@gmail.com
}

DOI: $10.1201 / 9781003129103-8$ 


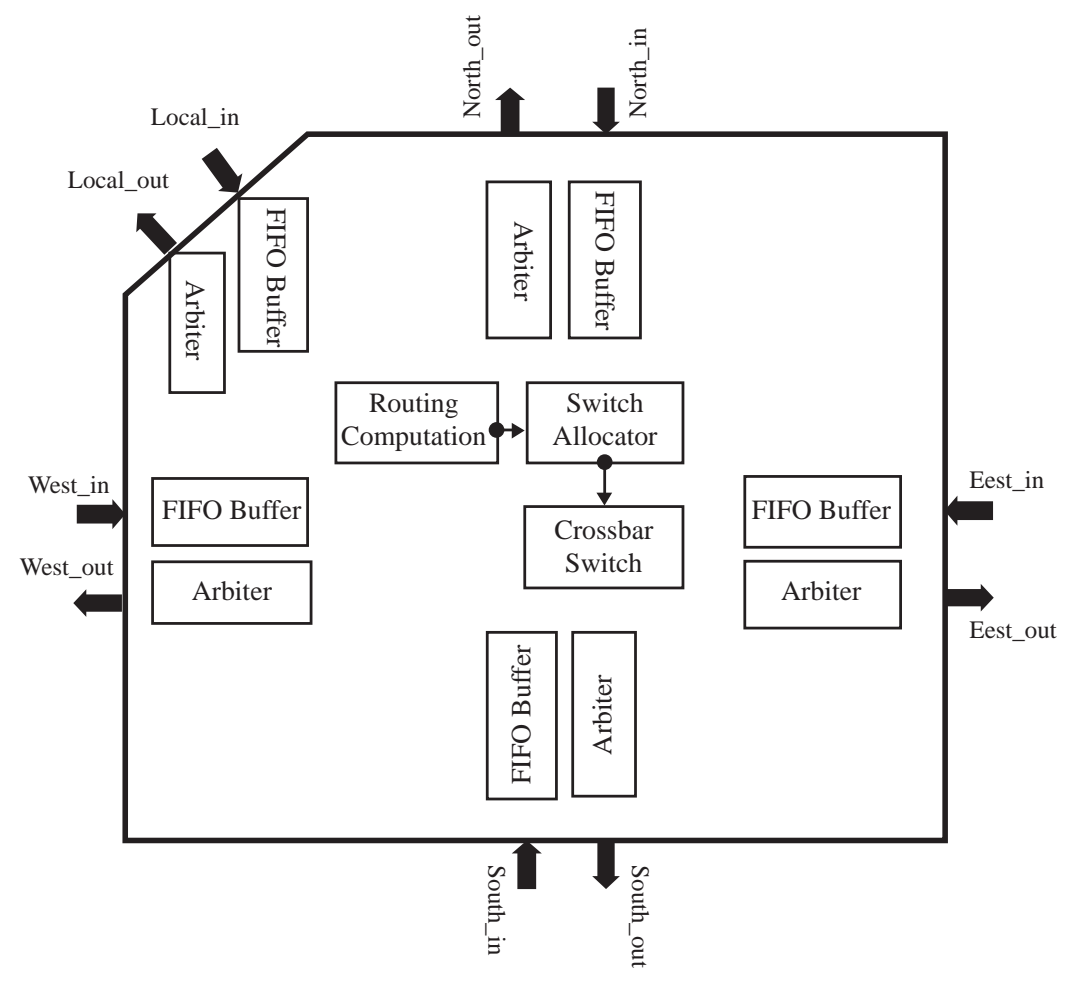

Figure 8.1 Generic router architecture.

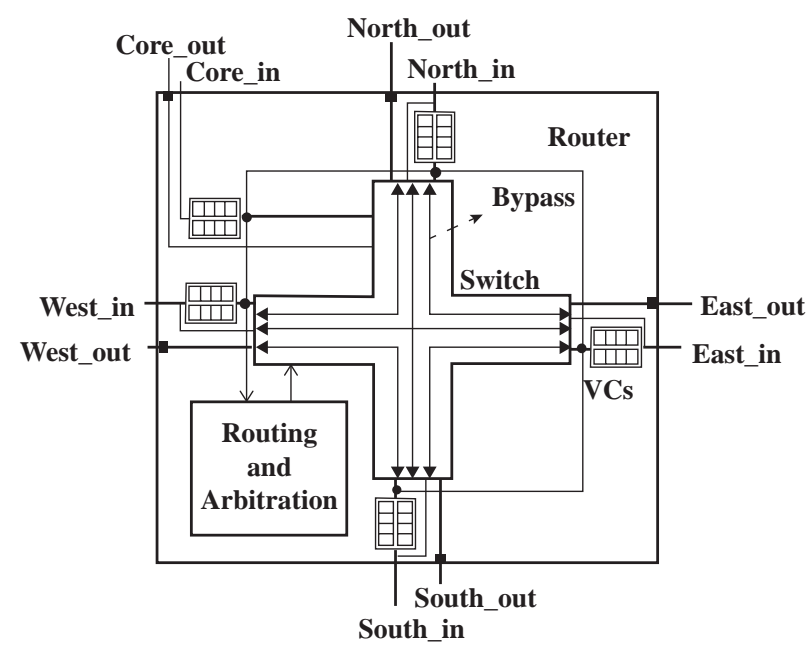

Figure 8.2 Bypass router.

\section{Bypass router description}

The router shown in Figure 8.2 consists of 5 ports (E, W, N, S and IP). The main target of the router is to forward the flits towards links. In the proposed by-pass router flits are passed among the routers without any latch. 


\section{Routing algorithm}

We propose a modified deadlock free routing algorithm called Standard Adaptive routing algorithm. This router can be designed using standard XY as well as Adaptive routing algorithm. In proposed algorithm flits are transferred through the bypass channel and are transferred among the routers excluding latching. As the number of intermediate routers in path reduces, the latency overhead at lower injection rates and power consumed by routers reduce.

\section{Evaluation}

Noxim and Orion 2.0 simulators are used to implement proposed bypass router. The parameters to be configured are shown in Table 8.1 and Table 8.2.

Table 8.1 One-dimension V/S baseline router design parameters

\begin{tabular}{llll}
\hline Parameter & Topology & Algorithm & Results \\
\hline $\begin{array}{l}\text { One dimension } \\
\text { Bypass router }\end{array}$ & $4 \times 4,8 \times 8,16 \times 16 \mathrm{Mesh}$ & Proposed & Latency reduces \\
Baseline router & $4 \times 4,8 \times 8,16 \times 16 \mathrm{Mesh}$ & Adaptive & Latency increases \\
\hline
\end{tabular}

Table 8.2 Two-dimension V/S baseline router design parameters

\begin{tabular}{llll}
\hline Parameter & Topology & Algorithm & Results \\
\hline $\begin{array}{l}\text { Two dimension } \\
\text { Bypass router }\end{array}$ & $8 \times 8 \mathrm{Mesh}$ & Semi-adaptive & Latency reduces \\
Baseline router & $8 \times 8 \mathrm{Mesh}$ & Adaptive & Latency increases \\
\hline
\end{tabular}

\section{Simulation results}

Energy and area consumption by proposed network are better than SMART, as shown in Figure 8.3 as SSR wiring is used in SMART. The results obtained considering single and two dimension bypass router can be compared with baseline routers to reduce latency with network area minimized.

\section{Conclusion}

The bypass router implemented in this paper provides path for forwarding flits without using latching technique at the router end. As compared with SMART, there is improvement in latency of the proposed router without adding control link over the network. Also proposed standard adaptive routing algorithm provides lower latency as compared to turning bypass. 


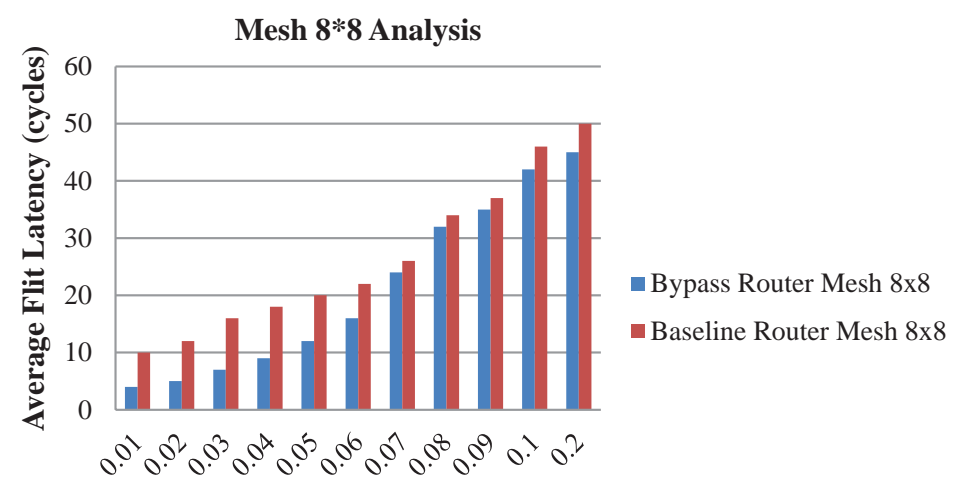

\section{Injection Rates (flits/nodes/cycle)}

Figure 8.3 Mesh $8 \times 8$ analysis of two-dimensional bypass router.

\section{References}

[1] Zoni, Davide, Federico Terraneo, and William Fornaciari. "A dvfs cycle accurate simulation framework with asynchronous noc design for power-performance optimizations." Journal of Signal Processing Systems 83, no. 3 (2016): 357-371.

[2] Wu, Ji, Dezun Dong, Xiangke Liao, and Li Wang. "Energy-efficient NoC with multi-granularity power optimization.” The journal of Supercomputing 73, no. 4 (2017): 1654-1671.

[3] Shen, Xiao-Wei, Xiao-Chun Ye, Xu Tan, Da Wang, Lunkai Zhang, Wen-Ming Li, Zhi-Min Zhang, Dong-Rui Fan, and Ning-Hui Sun. "An efficient network-on-chip router for dataflow architecture." Journal of Computer Science and Technology 32, no. 1 (2017): 11-25.

[4] Su, Nan, Huaxi Gu, Kun Wang, Xiaoshan Yu, and Bowen Zhang. "A highly efficient dynamic router for application-oriented network on chip." The Journal of Supercomputing 74, no. 7 (2018): 2905-2915.

[5] Beechu, Naresh Kumar Reddy, Vasantha Moodabettu Harishchandra, and Nithin Kumar Yernad Balachandra. "Energy-aware and reliability-aware mapping for NoC-based architectures." Wireless Personal Communications 100, no. 2 (2018): 213-225.

[6] Furhad, Hasan, Mohammad A. Haque, Cheol-Hong Kim, and Jong-Myon Kim. "An analysis of reducing communication delay in network-on-chip interconnects architecture." Wireless Personal Communications 73, no. 4 (2013): 1403-1419.

[7] Noghondar, Amir Fadakar, and Midia Reshadi. "A low-cost and latency bypass channel-based on-chip network." The Journal of Supercomputing 71, no. 10 (2015): 3770-3786.

[8] Ramani, S., and J. Sundararajan. "A case study on NoC router architecture for optimizing the latency." In 2013 International Conference on Advanced Computing and Communication Systems, pp. 1-4. IEEE, 2013.

[9] Sandeep Shelke, G.Patel, and Bhupinder Verma "Analysis of complete image processing system and design of improved image compression system." Think India Journal 22, no. 16 (2019): 1161-1169. 


\title{
9 Proximal and remote sensing of abiotic stress in crops
}

\author{
Harpinder Singh, *, Ajay Roy ${ }^{1}$, R.K. Setia ${ }^{1}$, \\ Brijendra Pateriya ${ }^{1}$
}

\author{
${ }^{1}$ Punjab Remote Sensing Centre, Ludhiana, India \\ ${ }^{2}$ Lovely Professional University, Phagwara, Punjab, India
}

\section{Introduction}

Food insecurity is a complex problem and millions of people worldwide are affected by it. Decades of research have focussed on the study of various factors affecting it. Abiotic and biotic stress factors are a major threat to food security. Biotic stress happens as a consequence of harm done to the crop by other living things like virus, fungus, insects, bacteria, weeds etc. It is unlike abiotic stress which is the harmful effect of non-living factors on living organisms in a particular environment and it results in reducing crop yield below optimum levels. Examples of abiotic stressors are: nutrient deficiency, salinity, floods, drought, high and low temperature, chemicals and radiation. It is usually caused by human activity. These stress factors are accountable for the major decline in the crop yield (Jackson 1986). Yield decline is critical for countries which are largely dependent on agriculture.

Effect of stress on crop is usually identified only when it is noticeable and visible. Hence, continuous monitoring of plant/crop conditions using proximal and remote sensing technologies can assist in the early diagnosis of the problem and then timely measures can be applied to curb it.

Proximal sensing techniques involve a lot of resources, time and manpower to identify stress in crops. The process is also very intricate and location specific. On the other hand, remote sensing provides a continuous coverage over a larger area (Ullah et al. 2012). Proximal sensing systems provide better quality (spatial, spectral and radiometric resolution) data as compared to remote sensing. Proximal sensor data have an upper hand primarily due to less interference of gases, air particles and water vapour in the atmosphere (Rumpf et al. 2010; Van Beek et al. 2015). Examples of various instruments used in the proximal techniques are the field spectroradiometer, camera, thermometer, SPAD etc., while the remote sensing instruments are various sensors (optical and non-optical) on board a satellite, airplane or an unmanned aerial vehicle (UAV). These sensors sense the electromagnetic waves in the visible, infrared and microwave regions.

Proximal sensors usually output hyperspectral data while remote sensors output both multi and hyperspectral datasets. A multispectral sensor senses the electromagnetic energy in few, broad bands; on the other hand the hyperspectral sensor data is composed of numerous narrow bands. Narrow bands of hyperspectral dataset are extremely sensitive and responsive to even small changes in the energy wavelengths. As a result hyperspectral

\footnotetext{
*Email: harpinder13@gmail.com
}

DOI: $10.1201 / 9781003129103-9$ 
data have a vast possibility for analysing crop stress as compared to the multispectral datasets. Prasad S. Thenkabail and John G. Lyon (2016) and Im and Jensen (2016) reviewed the use of hyperspectral remote sensing for agriculture and vegetation studies. They have explained important modelling and classification algorithms which are being used to estimate and predict crop type using biophysical factors. Sahoo, Ray and Manjunath (2015) reviewed a range of hyperspectral crop data analysis techniques. Hyperspectral dataset is huge and intricate therefore its analysis techniques are also different from multispectral data. Jones (2011) listed various stressors which can affect the crop and several sensors to identify crop stress like spectral, LIDAR, thermal, microwave and fluorescence.

This research reviews the application of remote and proximal sensing methods to detect abiotic stress in crops.

\section{Review}

Abiotic stress is detected in crops using the following proximal and remote sensing techniques: reflectance, thermal and fluorescence. This paper will focus more on the reflectance techniques.

Thermal sensors measure the temperature of the objects under study. For crop stress research these sensors are generally used to study water related stress. Probability of water stress is more if the temperature of the crops is higher. According to a study by Irmak, Haman and Bastug (2000), the crop water stress index (CWSI) is extremely useful to compute and check water related stress in the corn crop. Proximal sensors have been employed to sense the vapour pressure and canopy and air temperature. These parameters were used to create the index. CSWI has also been successfully used by Nielsen (1990) to schedule the irrigation of soybean crop. They have measured the air, plant canopy and vapour pressure using various proximal sensors. They initiated the irrigation of the crop when a particular CSWI threshold value was reached. They found that if the value of CSWI threshold was higher then the grain yield was less. Jones (2011) used a combination of thermal and optical sensing datasets to monitor the water shortage in horticultural crop. According to the research a multi-sensor approach has a great potential to sense water stress and further use it for scheduling the irrigation. Thermal data from remotely sensed satellite imagery are also used for identification of water stress. Leroux et al. (2016) have computed various vegetation and thermal indices from MODIS satellite data of crops in West Africa. They combined the generated indices with crop yield models and found that the combined data give better results of yield assessment than using the indices alone.

A second sensing technique is fluorescence. During photosynthesis, plant leaves absorb solar radiation with the help of chlorophyll. Fluorescence is the process of re-emission of absorbed solar radiation at longer wavelengths. It is used to detect the nutrient stress by monitoring the photosynthetic efficiency of a crop. Chaerle et al. (2009) detected plant stress in early stages and developed a stress catalogue by combining thermal, fluorescence and video imagery. The research shows that fluorescence is useful to detect nitrogen, pollutants, heavy metal and water related stress in crops. According to them use of multiple sensors in a study is more beneficial. Zarco-Tejada et al. (2009) collected fluorescence data of crops using a narrow band multispectral airborne camera. The research conducted on the olive and peach plantations established the possibility to detect water stress using remotely sensed fluorescence data.

The third sensing technique is reflectance. Das, Mahajan and Singh (2018) reviewed various researches in which the crop reflectance data were used to identify nutrient and water related stress. Major bands which are useful to sense crop stress are green, red, red 
edge and near infrared. Rossini et al. (2013) confirmed the possibility of using indices computed from hyperspectral imagery (airborne) for the mapping of water related stress in maize crop. For this research 126 spectral bands were sensed in the near infrared and visible region of the spectrum (400-992 nm). Wang et al. (2015) used plant canopy reflectance data $(350-2500 \mathrm{~nm})$ to estimate water stress. Parameters which were measured or computed in the research are: (1) hyperspectral reflectance, (2) soil water potential, (3) leaf area index, (4) soil moisture, (5) chlorophyll content, (6) canopy water content and (7) various environmental factors. Relationship between the water content and reflectance of the crops was studied and it was found that reflectance information could be very useful to detect stress in crops. Li et al. (2014) estimated the nitrogen values of wheat by simulating the reflectance values. For this purpose the indices computed from the hyperspectral field dataset were compared with the partial least square regression (PLSR) model results. They found that for the estimation of N, PLRS model results are better than the spectral indices. Pimstein et al. (2011) also experimented on the use of PLS and spectroscopy data for calculation of phosphorus $(\mathrm{P})$ and potassium $(\mathrm{K})$ for wheat. The results concluded that as compared to the traditional broadband indices, PLS and narrowband indices give better results. Leaf area index is another parameter which is very effective to calculate stress in crops. It is the projected plant leaf area over a unit of land. It is significant as it helps to monitor crop/vegetation properties like photosynthesis, evaporation and transpiration. Zongyao Sha et al. (2018) studied various methods to estimate the LAI of plants using data from a range of remote sensors. Machine learning algorithms were reviewed by Singh et al. (2016) to detect stress in crops. Support vector machine, artificial neural network (ANN), K-means, self organizing map (SOM) etc. were utilized to identify, classify, predict and quantify the crop stress.

\section{Conclusion}

The three techniques, reflectance, thermal and fluorescence, are being employed globally to detect abiotic stress in crops. Remote sensing techniques output multi and hyperspectral data while proximal sensing usually yields hyperspectral data. Many studies have combined both the techniques for crop stress identification. Research is still required to simulate the multispectral data (low cost and easily available) from the hyperspectral data (costly, requires many resources). A lot of researchers have analysed the proximal and remote sensing data using traditional statistical methods. Although few researchers have used machine and deep learning algorithms for crop stress studies, still research is required to analyse the voluminous and complex hyperspectral data for classification, regression or prediction problems. Also, research is still required to discriminate the stress factors when more than one stress affects a crop as the signatures of the stress are usually similar.

\section{References}

Beek, Jonathan Van, Laurent Tits, Ben Somers, Tom Deckers, Wim Verjans, Dany Bylemans, Pieter Janssens, and Pol Coppin. 2015. "Temporal dependency of yield and quality estimation through spectral vegetation indices in pear orchards." Remote Sensing 7 (8): 9886-903.

Chaerle, Laury, Sándor Lenk, Ilka Leinonen, Hamlyn G. Jones, Dominique Van Der Straeten, and Claus Buschmann. 2009. "Multi-sensor plant imaging: towards the development of a stresscatalogue.” Biotechnology Journal 4 (8): 1152-67. https://doi.org/10.1002/biot.200800242.

Das, Bappa, Gopal R. Mahajan, and Ronald Singh. 2018. "Hyperspectral remote sensing: use in detecting abiotic stresses in agriculture." Advances in Crop Environment Interaction, 31735. https://doi.org/10.1007/978-981-13-1861-0_12. 
Im, Jungho, and John R Jensen. 2016. "Hyperspectral remote sensing of vegetation.” Hyperspectral Remote Sensing of Vegetation 6: 1943-61. https://doi.org/10.1201/b11222.

Irmak, S., D. Z. Haman, and R. Bastug. 2000. "Determination of crop water stress index for irrigation timing and yield estimation of corn." Agronomy Journal 92 (6): 1221-27. https://doi. org/10.2134/agronj2000.9261221x.

Jackson, R D. 1986. "Remote sensing of biotic and abiotic plant stress." Annual Review of Phytopathology 24 (1): 265-87. https://doi.org/10.1146/annurev.py.24.090186.001405.

Jones, H. G. 2011. "Remote detection of crop water 'stress' and distinguishing it from other stresses." Acta Horticulturae 922: 23-34. https://doi.org/10.17660/ActaHortic.2011.922.2.

Leroux, Louise, Christian Baron, Bernardin Zoungrana, Seydou B. Traore, Danny Lo Seen, and Agnes Begue. 2016. "Crop monitoring using vegetation and thermal indices for yield estimates: case study of a rainfed cereal in semi-arid West Africa." IEEE Journal of Selected Topics in Applied Earth Observations and Remote Sensing 9 (1): 347-62. https://doi.org/ 10.1109/JSTARS.2015.2501343.

Li, Fei, Bodo Mistele, Yuncai Hu, Xinping Chen, and Urs Schmidhalter. 2014. "Reflectance estimation of canopy nitrogen content in winter wheat using optimised hyperspectral spectral indices and partial least squares regression." European Journal of Agronomy 52: 198-209. https://doi.org/10.1016/j.eja.2013.09.006.

Nielsen, D. C. 1990. "Scheduling irrigations for soybeans with the crop water stress index (CWSI)." Field Crops Research 23 (2): 103-16. https://doi.org/10.1016/0378-4290(90)90106-L.

Pimstein, Agustin, Arnon Karnieli, Surinder K. Bansal, and David J. Bonfil. 2011. "Exploring remotely sensed technologies for monitoring wheat potassium and phosphorus using field spectroscopy." Field Crops Research 121 (1): 125-35. https://doi.org/10.1016/j.fcr.2010.12.001.

Prasad S. Thenkabail, John G. Lyon, and Alfredo Huete. 2016. "Hyperspectral remote sensing of vegetation.” Hyperspectral Remote Sensing of Vegetation. https://doi.org/10.1201/b11222.

Rossini, M., F. Fava, S. Cogliati, M. Meroni, A. Marchesi, C. Panigada, C. Giardino, et al. 2013. "Assessing canopy PRI from airborne imagery to map water stress in maize." ISPRS Journal of Photogrammetry and Remote Sensing 86: 168-77. https://doi.org/10.1016/ j.isprsjprs.2013.10.002.

Rumpf, T., A.-K. Mahlein, U. Steiner, E.-C. Oerke, H.-W. Dehne, and L. Plümer. 2010. “Early detection and classification of plant diseases with support vector machines based on hyperspectral reflectance." Computers and Electronics in Agriculture 74 (1): 91-99.

Sahoo, R. N., S. S. Ray, and K. R. Manjunath. 2015. "Hyperspectral remote sensing of agriculture." Current Science 108 (5): 848-59.

Singh, Arti, Baskar Ganapathysubramanian, Asheesh Kumar Singh, and Soumik Sarkar. 2016. "Machine learning for high-throughput stress phenotyping in plants." Trends in Plant Science 21 (2): 110-24. https://doi.org/10.1016/j.tplants.2015.10.015.

Ullah, Saleem, Yali Si, Martin Schlerf, Andrew K. Skidmore, Muhammad Shafique, and Irfan Akhtar Iqbal. 2012. "Estimation of grassland biomass and nitrogen using MERIS data." International Journal of Applied Earth Observation and Geoinformation 19: 196-204.

Wang, Xiaoping, Chuanyan Zhao, Ni Guo, Yaohui Li, Shenqi Jian, and Kai Yu. 2015. "Determining the canopy water stress for spring wheat using canopy hyperspectral reflectance data in Loess Plateau semiarid regions.” Spectroscopy Letters 48 (7): 492-98. https://doi.org/10.1080/ 00387010.2014 .909495$.

Zarco-Tejada, P. J., J. A. J. Berni, L. Suárez, G. Sepulcre-Cantó, F. Morales, and J. R. Miller. 2009. "Imaging chlorophyll fluorescence with an airborne narrow-band multispectral camera for vegetation stress detection." Remote Sensing of Environment 113 (6): 1262-75. https://doi. org/10.1016/j.rse.2009.02.016.

Zongyao Sha, Yuwei Wang, Yongfei Bai, Yujin Zhao, Hua Jin, Ya Na, and Xiaoliang Meng. 2018. "Comparison of leaf area index inversion for grassland vegetation through remotely sensed spectra by unmanned aerial vehicle (UAV) and field-based spectroradiometer." Journal of Plant Ecology 12 (3): 395-408. 


\title{
10 Energy management strategy for plug-in electric vehicle with DFIG system
}

\author{
Naresh Kumar Golla1, Suresh Kumar Sudabattula ${ }^{2}$ \\ ${ }^{1}$ Assistant Professor, Department of Electrical and Electronics Engineering, \\ B.V. Raju Institute of Technology, Medak, Telangana, India \\ ${ }^{2}$ Associate Professor, School of Electronics and Electrical Engineering, Lovely \\ Professional University, Phagwara, Punjab, India
}

\section{Introduction}

Energy is described as a key resource that can be used in rural and regional areas as a tool for economic growth. Power is also among one of the most vital building blocks in human development and also therefore is an important factor in establishing the economic development of any kind of country [1]. In an effort to satisfy the demands of an emerging nation, rapid growth and development are witnessed by the power sector. It is necessary to note that the standard sources of energy are considerably depleted by human usage, whereas sustainable resources are rapidly increasing day by day, which can maintain uncertain human exploitation [2].

In the last few years, the electrical industry has actually begun to improve increasingly. Power industry development has been affected by relevant issues such as global warming, power costs, the power industry, and also expanding need for energy. Development of smaller rated distributed power resources for load demand, the same as the remote area power supply network, has enhanced over the past couple of years. Thus, renewable resources of energy like wind, solar, biomass, and also geothermal are playing an important role in the power field [3]. The requirement for cleaner, greener energy has resulted in progress in making the power harnessed by renewable energies. Typically, a lot of energy is harnessed and also distributed to loads by means of high-voltage line, resulting in high operational as well as day losses.

Wind power has become one of the most commonly used technologies for the generation of renewable energy among renewable energy choices. Wind energy is highlighted in this study because it can be used to generate electricity in remote areas where there is no grid available or electricity cannot be transmitted economically via grid [4]. Automotive companies have recently moved towards electric vehicles. Through reducing carbon emissions, researchers are offering electric vehicles as a solution for eco-friendly environmental transport. Most countries are making incentive programmes for EVs, resulting in increased use of EVs. This paper focuses on the electric vehicles plug-in battery where wind serves as a significant source of energy for remote locations $[5,6]$. In this paper, energy management approach is presented with three case studies on real time, such as how the EVs are used as an energy source as well as a charging solution for EVs [7]. Finally, the MATLAB Simulink validates the findings. 


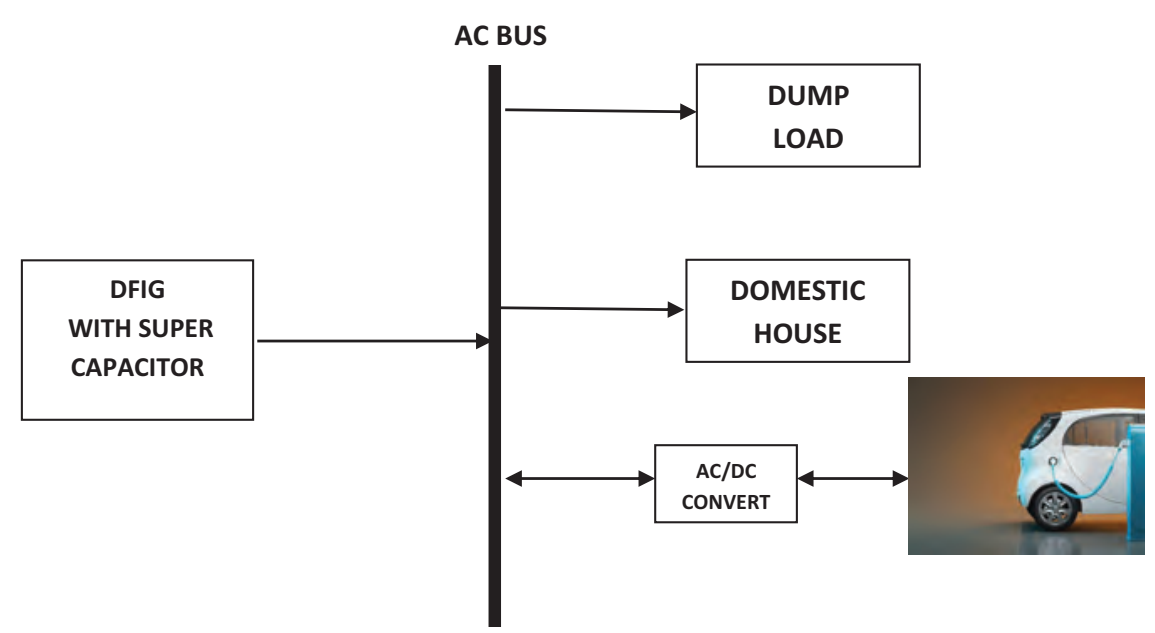

Figure 10.1 Proposed block diagram.

\section{Wind energy}

The growth of wind energy started in the 1980s, without much control on wind turbines with just a couple of $10 \mathrm{~s}$ of $\mathrm{kW}$ which were integrated to power grids. To manage and send reactive power between wind turbines and primary grids, substantial improvements have been made in the generation of wind energy and the usage of power electronic devices tools to the system today.

When wind energy is compared with other sources, it has the largest share of renewable energy sources. Rapid wind technology developments have reduced the cost of wind energy to such a level that it is affordable with traditional power.

\section{Electric vehicles}

EVs are a promising technology that is becoming increasingly popular on the global market. From the perspective of the power system, they present new challenges as their unregulated charging will lead to increased reserve requirements, increased electricity generation, possibly network strengthening, and higher market prices [8]. Nevertheless, their ability to store and supply electricity back to the vehicle-to-grid (V2G) network positions them as mobile storage systems providing distributed storage for power grids [9]. In this regard, they are considered to be an appropriate means of stabilizing the network and promoting the incorporation of renewable resources [10].

\section{Simulation results}

The proposed model is designed in MATLAB Simulink tool. The proposed system consists of DFIG [11-13], domestic house, EV and dump load that are connected to load. A total of three real time cases are studied in this paper.

Case 1: If power supply > load demand:

If power supply > load demand, then EV battery is allowed to charge. 


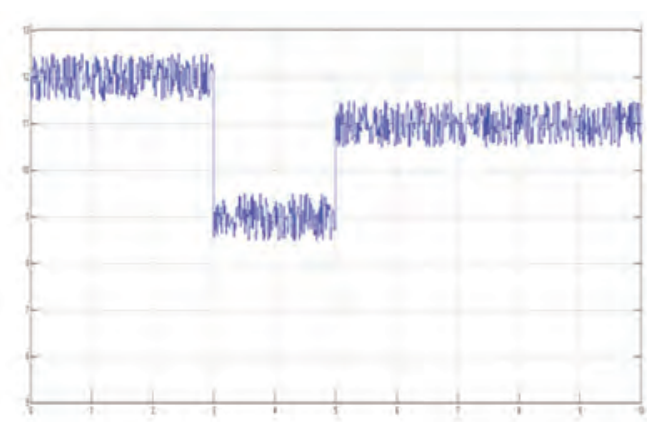

Fig: 2(a) Wind Speed in $\mathrm{m} / \mathrm{s}$ vs Time in Sec

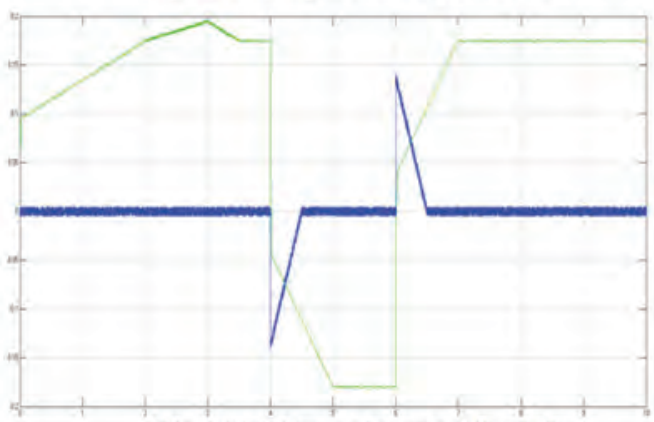

Fig: 2 (c) EV Battery Storage vs Time in Sec

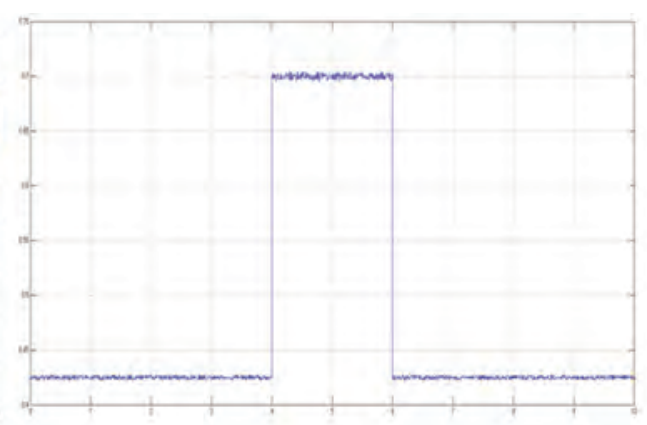

Fig: 2(b) Load Demand in P.U. vs Time in Sec

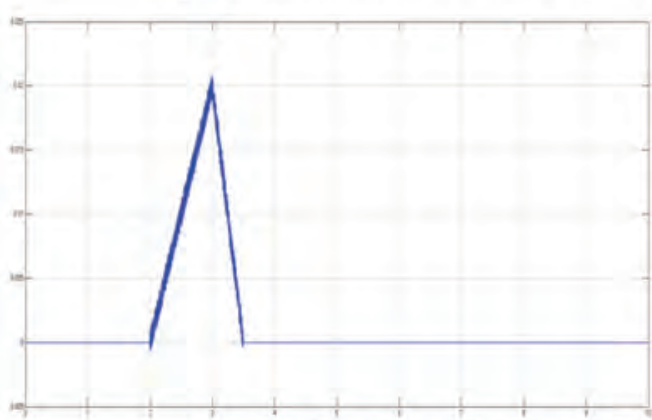

Fig: 2 (d) Dump Load in P.U. vs Time in Sec

Figure 10.2 (a) Wind speed in $\mathrm{m} / \mathrm{s}$ vs time in s. (b) Load demand in PU vs time in s. (c) EV battery storage vs time in s. (d) Dump load in PU vs time in s.

Case 2: If power supply < load demand:

If power supply < load demand, then supply is taken from the EV

Case 3: If power supply > load demand and EV battery is full:

If power supply $>$ load demand and EV battery is full then dump load will be switched ON.

The wind condition under which the system has been proposed is presented in Figure 10.2 (a). It can be seen that the wind rate is set originally at $12 \mathrm{~m} / \mathrm{s}$. At $\mathrm{t}=3 \mathrm{~s}$, the wind rate goes down to $9 \mathrm{~m} / \mathrm{s}$, and also it rises to $11 \mathrm{~m} / \mathrm{s}$ at $\mathrm{t}=7 \mathrm{~s}$. As seen in Figure $10.2(\mathrm{~b})$, first load demand is switched $\mathrm{ON}$ at $0.425 \mathrm{PU}$ and afterwards it is increased to a value of $0.7 \mathrm{PU}$ at $\mathrm{t}=4 \mathrm{~s}$ and also the included additional demand (i.e. 0.275 PU) is switched OFF from the system at $t=6 \mathrm{~s}$. It can be seen from Figure 10.2(c) that the battery begins discharging at $t=4 \mathrm{~s}$ to $6 \mathrm{~s}$. Before $\mathrm{t}=4 \mathrm{~s}$ the generation is greater than the demand and the battery is in complete full charging condition and dump load is activated before $t=4 \mathrm{~s}$.

From Figure 10.3 it is noted that even though there is variable generation the load side voltage is constant; this is because of the energy management with the EV's battery.

\section{Conclusion}

In this paper energy management strategy is proposed for plug-in battery electric vehicles where wind is acting as a major energy source which is applicable for remote locations. Three real time case studies, i.e. if the power supply is greater than the load demand, if the 


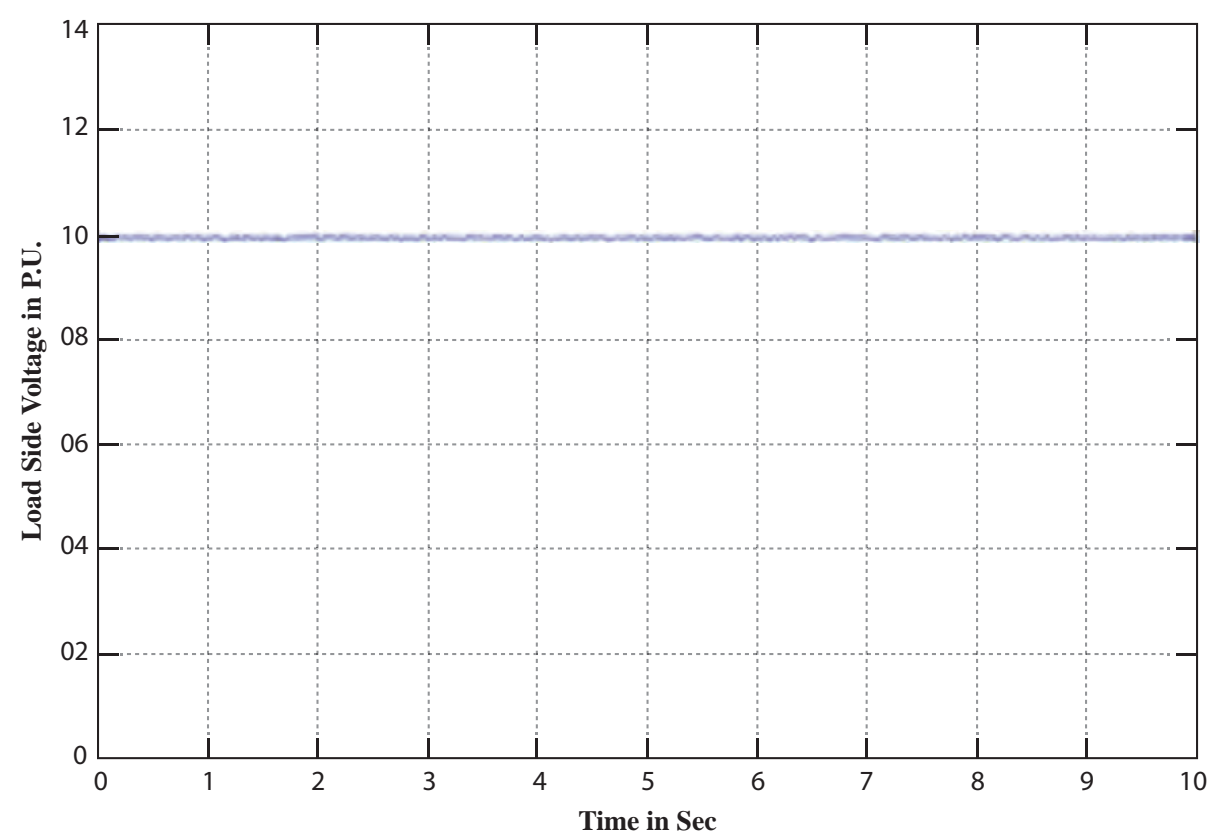

Figure 10.3 Load side voltage in PU vs time in s.

power supply is less than the load demand, if the power supply is greater than the load demand and the EV battery is full, are considered for the energy management strategy. This paper gives the solution of EVs as an energy source as well as a charging purpose of EVs. Proposed energy management strategy for EV applications is simulated in MATLAB environment. In all the three real time case studies the load voltage is constant. Hence this shows the effectiveness of the proposed energy management strategy.

\section{References}

[1] S. Bhattacharyya (ed.). Rural electrification through de-centralized off-grid systems in developing countries. Green Energy and Technology, doi:10.1007/978-1-4471-4673-5_2, Springer-Verlag, London, 2013.

[2] Global EV Outlook 2018-Towards cross-modal Electrification. International Energy Agency (IEA); May 2018.

[3] M. Beaudin, H. Zareipour, A. Schellenberglabe and W. Rosehart. Energy storage for mitigating the variability of renewable electricity sources: An updated review. Energy Sustainable Development 2010;14(4):302-314.

[4] N. Mendis and K.M. Muttaqi. Management of low- and high-frequency power components in demand-generation fluctuations of a DFIG-based wind-dominated RAPS system using hybrid energy storage. IEEE Transactions on Industry Applications 2014;50(3).

[5] D. Leskarac et al. PEV charging technologies and V2G on distributed systems and utility interfaces. Vehicle-to-Grid: Linking Electric Vehicles to the Smart Grid 2015;79:157-209.

[6] Z. Wan et al. Model-free real-time EV charging scheduling based on deep reinforcement learning. IEEE Transactions on Smart Grid 2018.

[7] W. Li, G. Joos and J. Belanger. Real-time simulation of a wind turbine generator coupled with a battery supercapacitor energy storage system. IEEE Trans. Ind.Electron.2010;57(4):1137-1145. 
[8] K. Sahay and B. Dwivedi. Design and analysis of supercapacitors energy storage system for energy stabilization of distribution network. Electrical Power Quality and Utilisation Journal 2009; XV(1).

[9] L. Hua, J. Wang and C. Zhou. Adaptive electric vehicle charging coordination on distribution network. IEEE Transactions on Smart Grid 2014;5(6):2666-2675.

[10] M.C. Kisacikoglu, M. Kesler and L.M. Tolbert. Single-phase on-board bidirectional PEVcharger for V2G reactive power operation. IEEE Transactions on Smart Grid 2015; 6(2):767-75.

[11] D. Santos-Martin, S. Arnaltes and J.L.R. Amenedo. Reactive power capability of doubly fed asynchronous generators. Journal of Electric Power Systems Research 2008;78(11):1837-1840.

[12] Literature review paper on doubly fed induction generator wind turbine technology Ajay Kushwaha1, Inderpreet Singh2 1,2EIED, Thapar University, Patiala, Punjab, India. ISSN: 23197463. vol. 2, issue 9, September 2013.

[13] K. Leban. Doubly fed induction generator fault simulations. PhD thesis, Institute of Energy Technology, Aalborg University, Alborg, Denmark, 2009. 


\title{
11 Energy harvesting cognitive radio A review
}

\author{
Yogita Thareja ${ }^{1, *}$, Kamal Kumar Sharma ${ }^{2}$
}

\author{
${ }^{1}$ Research Scholar, SEEE, Lovely Professional University, Phagwara, India \\ ${ }^{2}$ Professor, SEEE, Lovely Professional University, Phagwara, India
}

\section{Introduction}

Wireless sensor networking is a rising innovation that has a wide scope of potential applications. Such a system ordinarily comprises an enormous number of conveyed nodes that sort themselves out into a multi-hop remote network. Every node has at least one sensor, implanted processors and low-control radios, and is ordinarily battery operated [1]. A cognitive radio (CR) is a radio that can be modified and designed progressively to utilize the best remote diverts in its region to stay away from client obstruction and blockage. The cognitive procedure is the way toward knowing through recognition, arranging, thinking, acting, and ceaselessly refreshing and updating with a background marked by learning. CR can know the unutilized range in a permit and unlicensed range band, and use the unused range sharply. The primary users (PU) reserve the option to utilize the range whenever secondary users (SU) can use the range just when the PU isn't utilizing it [3]. Wireless sensor networks (WSN) can be made to work in either authorized or unlicensed groups. In any case, to work in authorized range, the channels must be rented, which is costly and have organization limits. Consequently, including cognitive radio abilities in WSNs through CR helped remote system will enable sensor nodes to use the benefits of dynamic range [3]. With the utilization of cognitive radio in WSN directing, the likelihood of node information conveyance corresponds to the accessibility of empty authorized channels [4]. In fact, a WSN composed of sensor nodes equipped with cognitive radio may benefit from the potential advantages of the salient features of dynamic spectrum access [5]. CR-WSNs may have a wide scope of utilization areas. Without a doubt, CR-WSN can be sent any place instead of WSNs. A few instances of planned zones where CR-WSNs can be conveyed are as per the following: medicine and health, object following, telemetries, security, activation and upkeep of complex frameworks, monitoring of indoor and outdoor environments, military and public security applications, health care and real-time surveillance applications.

\section{Overview of RF energy harvesting CRNS}

\section{Sources of RF energy harvesting}

There are mainly two types of RF energy sources: unwitting or ambient sources and witting, deliberate or dedicated sources.

\footnotetext{
* Email: yogitat26@gmail.com
} 


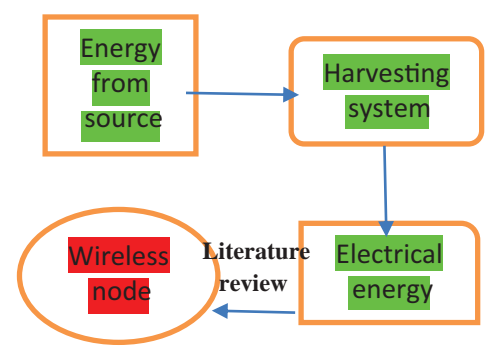

Figure 11.1 Harvest use architecture.

(a) Unwitting RF sources: RF sources like TV towers, radio towers, cell frameworks, WiFi frameworks and cell phones are the surrounding RF sources which are not implied for energy movement and the energy radiated by these encompassing sources is a complete waste and disseminated as some other type of energy.

(b) Witting RF sources: These are the energy sources which are sent to control the wireless nodes. These sorts of sources are utilized where unsurprising stockpile of energy is required. The utilization of expected RF sources hoists the use of a correspondence organization and these sorts of sources ought to be conveyed distinctly in systems having quality of service constraints.

\section{Architecture of energy harvesting}

Energy harvesting can be classified into two architecture: harvest use architecture and harvest use store architecture

Harvest use architecture. In this sort, the energy being collected is utilized legitimately to give power to the wireless nodes with no storage and it is shown in Figure 11.1. The harvesting system gathers the energy from the available sources with the help of harvesting antennas. These harvesting antennas might be intended to work either on a single frequency or on different frequencies so that the gathering framework can collect energy from a solitary RF energy source or from multiple RF energy sources separately. After this the harvesting energy is converted into electrical energy and this electrical energy is provided to wireless nodes and the nodes use this energy for playing out its tasks.

Harvest use and store architecture. There is an energy storage component to store the converted electrical energy in case the energy is greater than the energy consumption of the wireless node and this stored energy can be used in future (Figure 11.2).

\section{Literature review}

Energy harvesting community (EHN) is a trending subject matter: in a few of the current researches, EH-CRN is going through huge demanding situations associated with technical design [8]. Bhowmick et al. in [19] make use of non-RF strength harvesting as a supplementary of energy harvesting from RF signal of PU, motivated by using the work done in [19]. Static sources, for example, TV towers and radio towers, emanate moderately consistent power with time though unique RF sources such as Wi-Fi frameworks and cell phones transmit fluctuating force with time [27]. The energy putting away component might be single stage or two phases where the subsequent stage goes about as a reinforcement arrangement which is utilized when the energy put away in the first stage is devoured [28]. 


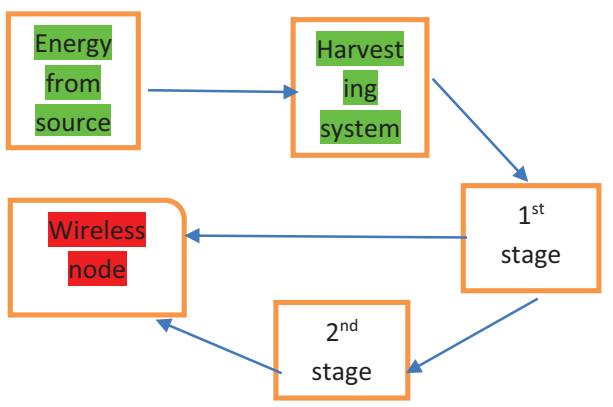

Figure 11.2 Harvest use and store architecture.

Summary of EHCRN

\begin{tabular}{|c|c|c|}
\hline Energy sources & Objectives & Constraints \\
\hline Ambient / linear [9-11] & $\begin{array}{l}\text { Optimization and utilization of } \\
\text { harvesting energy }\end{array}$ & Battery-free nodes \\
\hline WPT[13] & $\begin{array}{l}\text { Optimal transmission strategy to } \\
\text { achieve different trade-offs for } \\
\text { maximal information rate versus } \\
\text { energy transfer }\end{array}$ & Improvise information rate \\
\hline $\begin{array}{l}\text { Ambient light- RF power / } \\
\text { photodiode [14] }\end{array}$ & \begin{tabular}{|l} 
Energy harvesting \\
Battery free devices \\
\end{tabular} & Improvise range \\
\hline $\begin{array}{l}\text { Natural resources / } \\
\text { primary RF } \\
\text { transmission [15] }\end{array}$ & $\begin{array}{l}\text { Improvising throughput for both PU } \\
\text { and SU }\end{array}$ & \\
\hline RF energy [16] & $\begin{array}{l}\text { Minimizes the false alarm } \\
\text { probability } \\
\text { Energy harvesting } \\
\text { Improve the global detection } \\
\text { probability }\end{array}$ & Energy consumption \\
\hline $\begin{array}{l}\text { Non-RF and RF energy } \\
\text { [17] }\end{array}$ & $\begin{array}{l}\text { Detection performance, harvested } \\
\text { energy, } \\
\text { Energy penalty during harvesting } \\
\text { Throughput }\end{array}$ & $\begin{array}{l}\text { Wideband sensing - multiple } \\
\text { PU scenario }\end{array}$ \\
\hline PU [21] & $\begin{array}{l}\text { Resource allocation } \\
\text { Maximize sum throughput }\end{array}$ & Transmission power \\
\hline PU [22] & $\begin{array}{l}\text { Improve security of primary and } \\
\text { secondary network. }\end{array}$ & $\begin{array}{l}\text { Power minimization } \\
\text { Security }\end{array}$ \\
\hline Primary transmitter [23] & $\begin{array}{l}\text { Derived optimal and zero forcing } \\
\text { solutions }\end{array}$ & $\begin{array}{l}\text { The channel quality of SU } \\
\text { to the primary transmitter } \\
\text { should be good }\end{array}$ \\
\hline
\end{tabular}

\section{Conclusion}

A survey of EHCRN is presented in this paper. Based on the existing literature, the stateof-the-art of EH-CRNs with emphasis on throughput maximization, resource allocation, energy minimization, power intake and secure facts switch is offered. New techniques 
should be used to maintain the network in power saving mode with high energy retaining capacities. Different techniques should be used to transfer data with low energy in a battery-free system. More research needs to be done to decrease the brownout probability in battery-free cognitive nodes making them interference free, noise free and pollution free.

\section{References}

1. Shaikh FK, Zeadally S. Energy harvesting in wireless sensor networks: A comprehensive review. Renewable and Sustainable Energy Reviews 2016;55:1041-54.

2. Raza M, Aslam N, Le-Minh H, Hussain S, Cao Y, Khan NM. A critical analysis of research potential, challenges, and future directives in industrial wireless sensor networks. IEEE Communications Surveys \& Tutorials 2017;20(1):39-95.

3. Görgü L, Kroon B, O'Grady MJ, Yılmaz Ö, O'Hare GM. Sensor discovery in ambient IoT ecosystems. Journal of Ambient Intelligence and Humanized Computing 2018;9:447-58.

4. Carie A, Li M, Marapelli B, Reddy P, Dino H, Gohar M. Cognitive radio assisted WSN with interference aware AODV routing protocol. Journal of Ambient Intelligence and Humanized Computing 2019;10:4033-42.

5. Akan OB, Karli OB, Ergul O. Cognitive radio sensor networks. IEEE Network 2009; 23(4):34-40.

6. Ali A, Hamouda W. Advances on spectrum sensing for cognitive radio networks: Theory and applications. IEEE Communications Surveys \& Tutorials 2016;19(2):1277-304.

7. Darsena D, Gelli G, Verde F. An opportunistic spectrum access scheme for multicarrier cognitive sensor networks. IEEE Sensors Journal 2017;17(8):2596-606.

8. Alfaqawi, M.I., Habaebi, M.H., Siddiqi, M.U., Islam, M. R., Zyoud, A., Al-Shibly, M. (2006). Survey on energy harvesting cognitive radio network.

9. S. Sudevalayam and P. Kulkarni. Energy harvesting sensor nodes: Survey and implications. Commun. Surveys Tuts., 13, 3, 443-461, 2011.

10. N. Shinohara. Power without wires. IEEE Microw. Mag., 12, 7, 64-73, 2011.

11. RF energy harvesting and wireless power for low-power applications. Available: www. powercastco.com/PDF/powercastoverview.

12. A. N. Parks, A. Liu, S. Gollakota and J. R. Smith. Turbocharging ambient backscatter communication. Proc. ACM Conf. SIGCOMM, 619-630, 2014.

13. R. Zhang and C. K. Ho.MIMO broadcasting for simultaneous wireless information and power transfer. IEEE Trans. Wireless Commun., 12, 5, 1989-2001, 2013.

14. V. Talla, B. Kellogg, S. Gollakota and J. R. Smith. Battery-free cellphone. PACM Interact. Mob. Wearable Ubiquitous Technol., 1, Article 25, 20 pages, 2017.

15. A. E. Shafie, M. Ashour, T. Khattab and A. Mohamed. On spectrum sharing between energy harvesting cognitive radio users and primary users. Proc. Int. Conf. Comput., Netw. Commun., Garden Grove, CA, USA, pp. 214-220, 2015.

16. Gao, Y., He, H., Deng, Z., and Zhang, X. Cognitive radio network with energy-harvesting based on primary and secondary user signals. IEEE Access, 6, 9081-9090, 2018.

17. A. Bhowmick, S. Roy, and S. Kundu. Throughput of a cognitive radio network with energyharvesting based on primary user signal. IEEE Wireless Commun. Lett., 5, 2, 136-139, 2016.

18. A. Bhowmick, S. D. Roy and S. Kundu. Cognitive radio network with continuous energyharvesting. International Journal of Communication Systems, 30, 6, 2017.

19. A. Bhowmick, K. Yadav and S. D. Roy. Throughput of an energy harvesting cognitive radio network based on prediction of primary user. IEEE Transactions on Vehicular Technology, 66, 9, 8119-8128, 2017.

20. Huynh, V. V., Nguyen, H. S., Hoc, L. T. T., Nguyen, T. S., and Voznak, M. Optimization issues for data rate in energy harvesting relay-enabled cognitive sensor networks. Computer Networks, 157, 29-40, 2019.

21. Yi. Wang, Yu. Wang, F. Zhou, Y. Wu and H. Zhou. Resource allocation in wireless powered cognitive radio networks based on a practical non-linear energy harvesting model. IEEE Access, 5, 17618-17626, 2017. 
22. Y. Huang, Z. Li, F. Zhou and R. Zhu. Robust AN-aided beamforming design for secure MISO cognitive radio based on a practical nonlinear EH model. IEEE Access 5, 14011-14019, 2017.

23. G. Zheng, Z. Ho, E. A. Jorswieck and B. E. Ottersten. Information and energy cooperation in cognitive radio networks.” IEEE Trans. Signal Processing, 62, 9, 2290-2303, 2014.

24. Singla, J., Mahajan, R., and Bagai, D. (2018, November). A survey on energy harvesting cognitive radio networks. In 2018 6th Edition of International Conference on Wireless Networks \& Embedded Systems (WECON) (pp. 6-10). IEEE.

25. L. Mohjazi, M. Dianati, G. K. Karagiannidis, S. Muhaidat and M. AlQutayri. RF-powered cognitive radio networks: Technical challenges and limitations. IEEE Communications Magazine, 53, 94-100, 2015.

26. X. Lu, P. Wang, D. Niyato, D. I. Kim and Z. Han. Wireless networks with RF energy harvesting: A contemporary survey. IEEE Communications Surveys \& Tutorials, 1, 757-789, 2014.

27. C. Mikeka and H. Arai. Design issues in radio frequency energy harvesting system. Sustainable Energy Harvesting Technologies Past, Present and Future, InTech, 235-256, 2011.

28. S. Sudevalayam and P. Kulkarni. Energy harvesting sensor nodes: Survey and implications. IEEE Communications Surveys \& Tutorials, 13, 443-461, 2010 


\title{
12 Impact of geographical locations on the PV inverter lifetime considering mission profile and uncertainties
}

\author{
Sainadh Singh Kshatri ${ }^{1}$, Javed Dhillon ${ }^{2}$, Sachin Mishra
}

\author{
${ }^{1}$ Research Scholar, School of Electronics and Electrical Engineering, \\ Lovely Professional University, Phagwara, Punjab, India \\ Assistant Professor, B. V. Raju Institute of Technology, Narsapur, Medak, \\ Telangana, India, sainadhsingh@gmail.com \\ ${ }^{2}$ School of Electronics and Electrical Engineering, Lovely Professional \\ University, Phagwara, Punjab, India
}

\section{Introduction}

It is reported that at least $99.9 \mathrm{GW}$ of PV systems have been installed and commissioned globally last year. Global PV installed capacity crosses half TW at the end of 2018. China leads globally in the total PV installation capacity with $176.1 \mathrm{GW}$. India is listed by the top ten global PV markets with total installed capacity of $32.9 \mathrm{GW}$ and places at second position in top PV market for the year 2018 with $10.8 \mathrm{GW}$ of installed capacity. Five Asian Pacific nations, China, India, Japan, Australia and Korea, are listed in the top ten global PV markets. The next three are the Netherlands, Germany and Turkey, then the USA and Mexico for 2018. The top 10 nations share about $87 \%$ of total PV installed capacity. Now several countries are generating more electricity than the demand with the PV systems. About $2.6 \%$ of the global electricity demand is shared by the PV systems [1]. The above statistics show the evidence for the exponential rise in the PV systems. Figure 12.1 shows the global annual PV cumulative capacity; annual PV installed capacity is shown in Figure 12.2.

\section{Lifetime assessment methodology}

As the PV inverter is cited as the most unreliable component of the entire PV system, lifetime assessment is needed. Several lifetime assessment methodologies are currently available. For the more realistic approach, a mission profile based lifetime assessment is considered. In this paper temperature (ambient) and irradiance of the sun (solar) for top ten countries are considered for lifetime assessment [2-4].

\section{Mission profile of top 10 countries}

Performance of the PV system mainly depends on the environmental conditions, i.e. temperature (ambient) and irradiance of the sun (solar), called as mission profile. Mission profile data of one year with one-hour sample is taken from the NASA website for all the top 10 countries. The temperature (ambient) and irradiance of the sun (solar) of the top 10 countries are shown in Figure 12.3 and Figure 12.4 [5-7].

DOI: 10.1201/9781003129103-12 


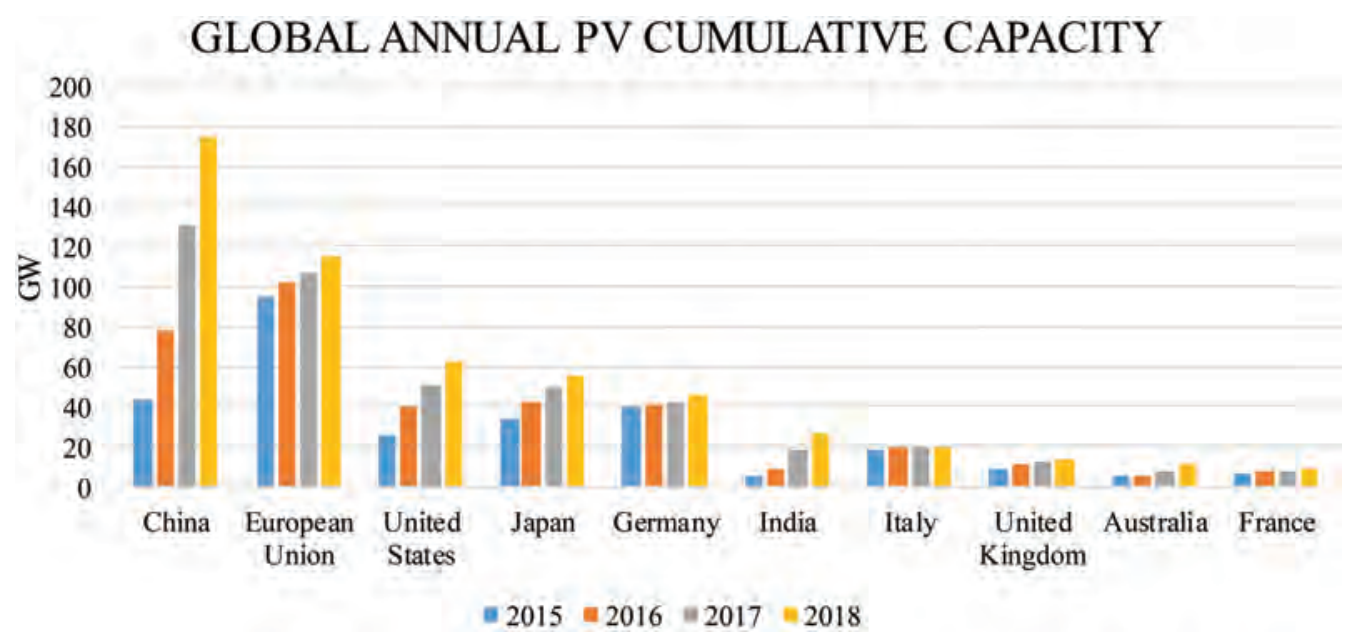

Figure 12.1 Global annual PV cumulative capacity.

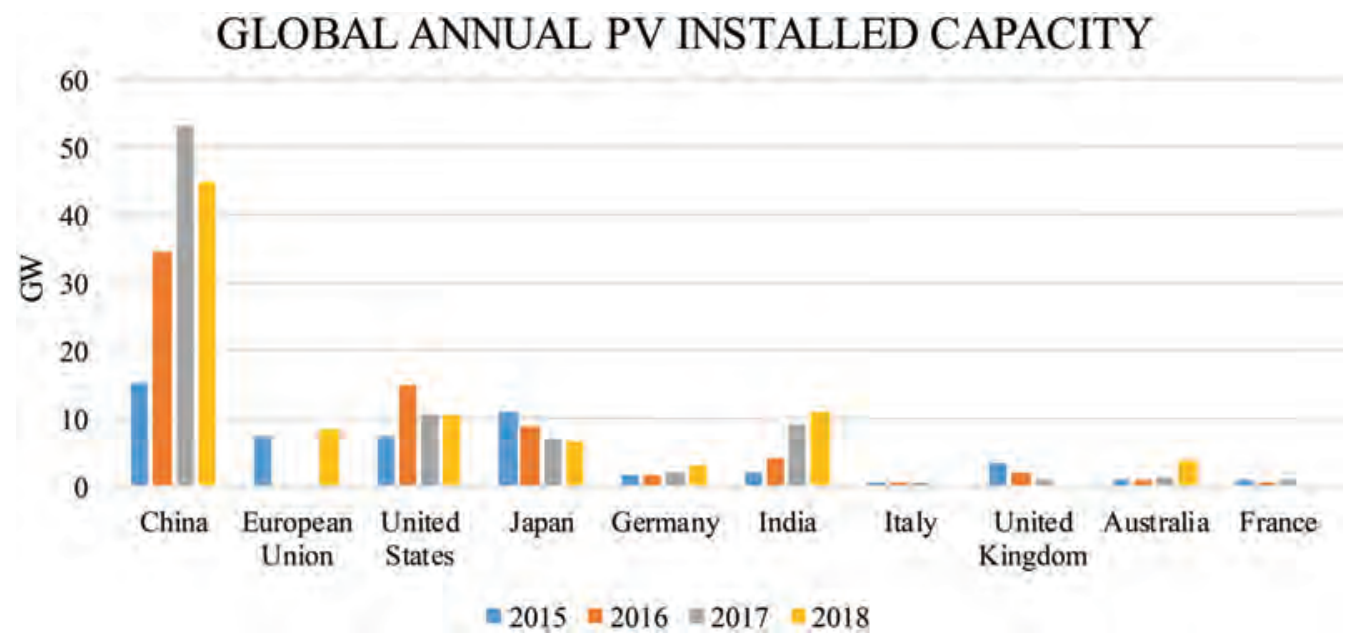

Figure 12.2 Global annual PV installed capacity.

\section{Results and discussion}

For the lifetime assessment of the PV inverter a 3-kW full bridge PV inverter for a 1- $\phi$ system is considered. A full bridge PV inverter consists of four IGBTs. The entire system is simulated in the PLECS simulation software. Junction temperature ( $\mathrm{Tj}$ ) of an individual IGBT is calculated for top ten countries with the help of the PLECS. Figure 12.5 shows the junction temperature $(\mathrm{Tj})$ for top 10 countries of PV markets.

With the help of the rainflow counting algorithm number of cycles $n_{i}$, cycle period $t_{o n}$, mean junction temperature $T_{j}$, cycle amplitude $\Delta T_{j}$ can be calculated and with the help of the Bayes' lifetime equation life consumption (LC) and lifetime (LT) are calculated. For considering the uncertainties in the system, Monte Carlo simulation with 10,000 samples is considered and lifetime is calculated at component level and system level. Figure 12.6 

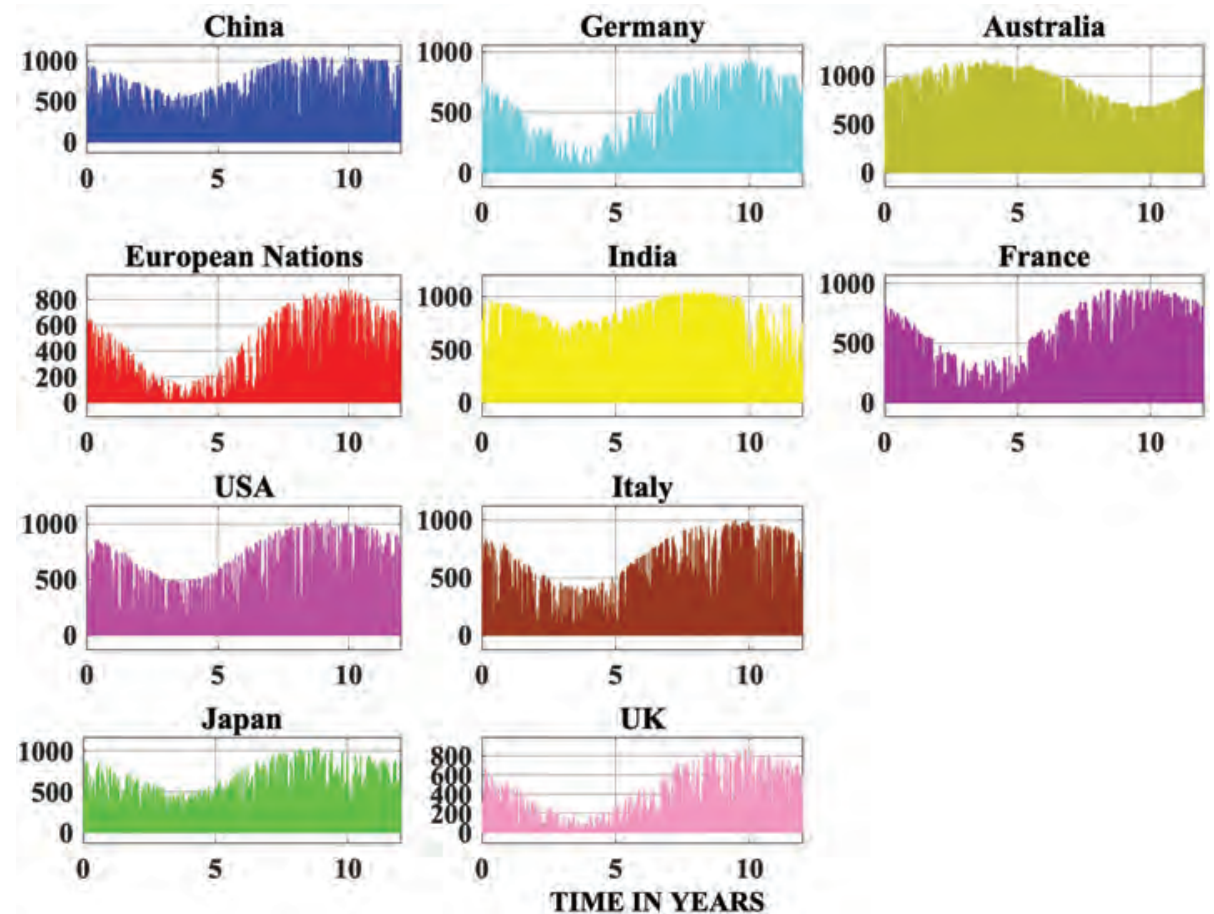

Figure 12.3 One-year solar irradiance of the top 10 countries of PV market.
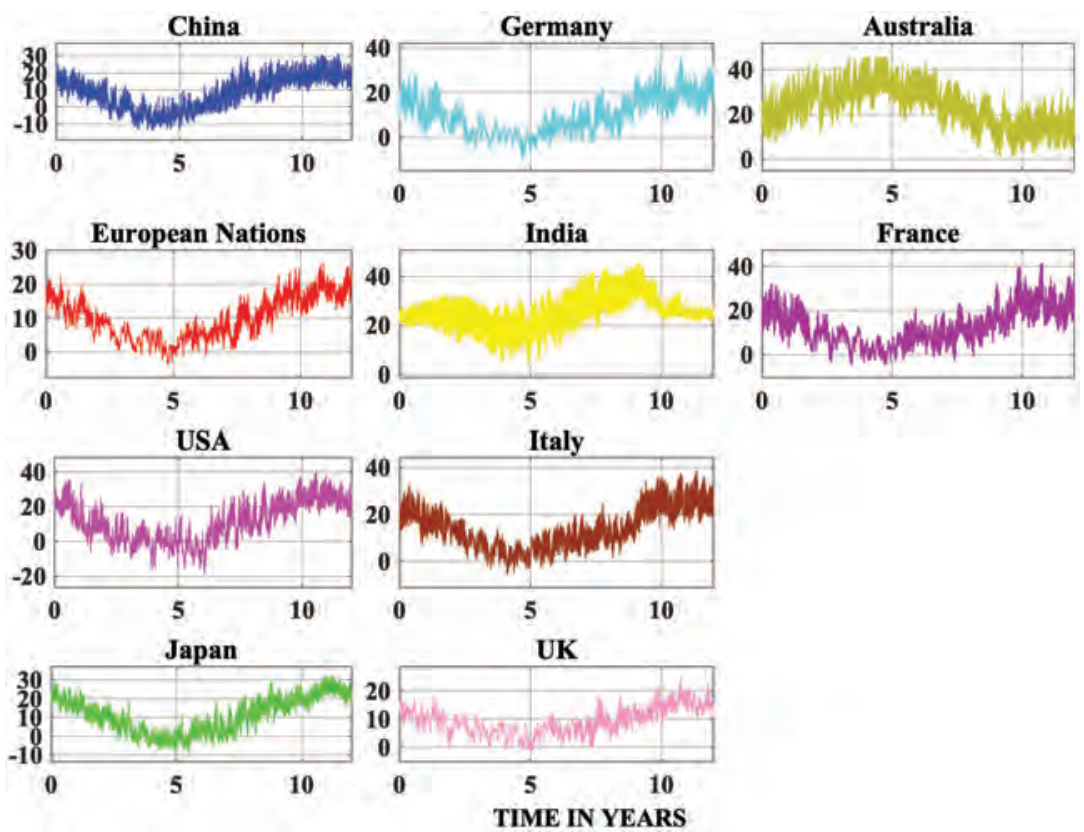

Figure 12.4 One-year ambient temperature of the top 10 countries of PV market. 

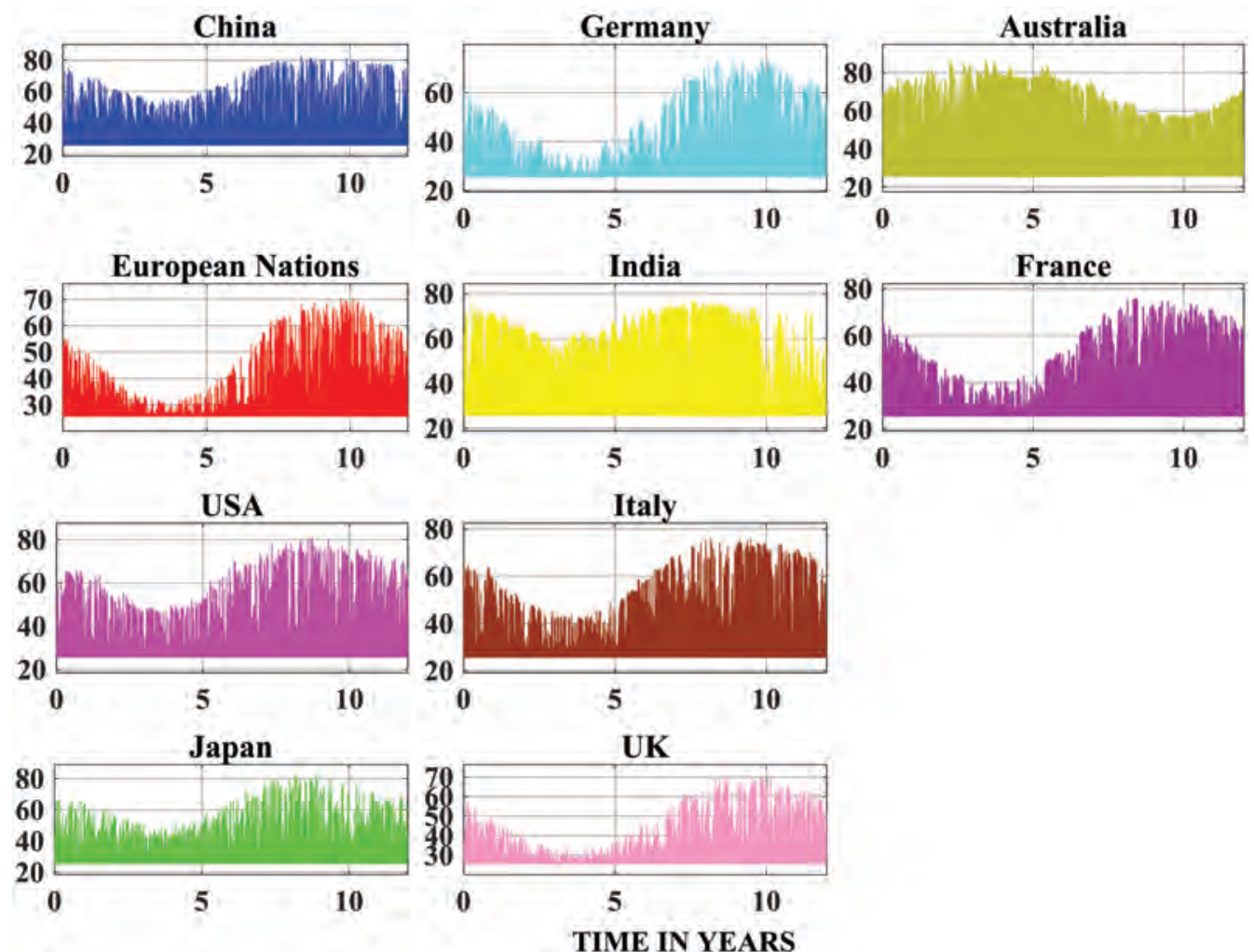

Figure 12.5 Junction temperature of the top 10 countries of PV market.

\section{Life Consumption}

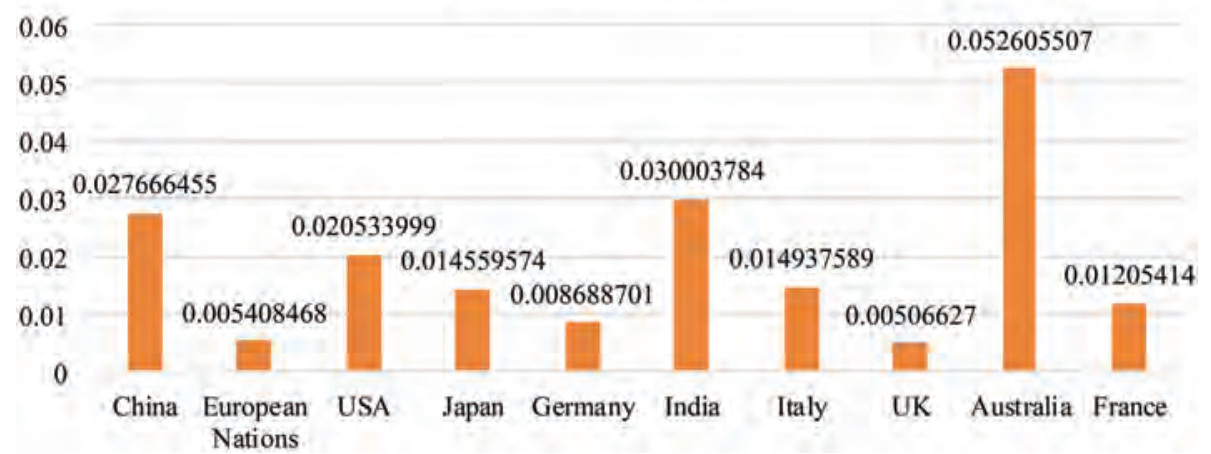

Figure 12.6 PV inverter life consumption (LC) of top 10 countries at component level. 


\section{Life Time}

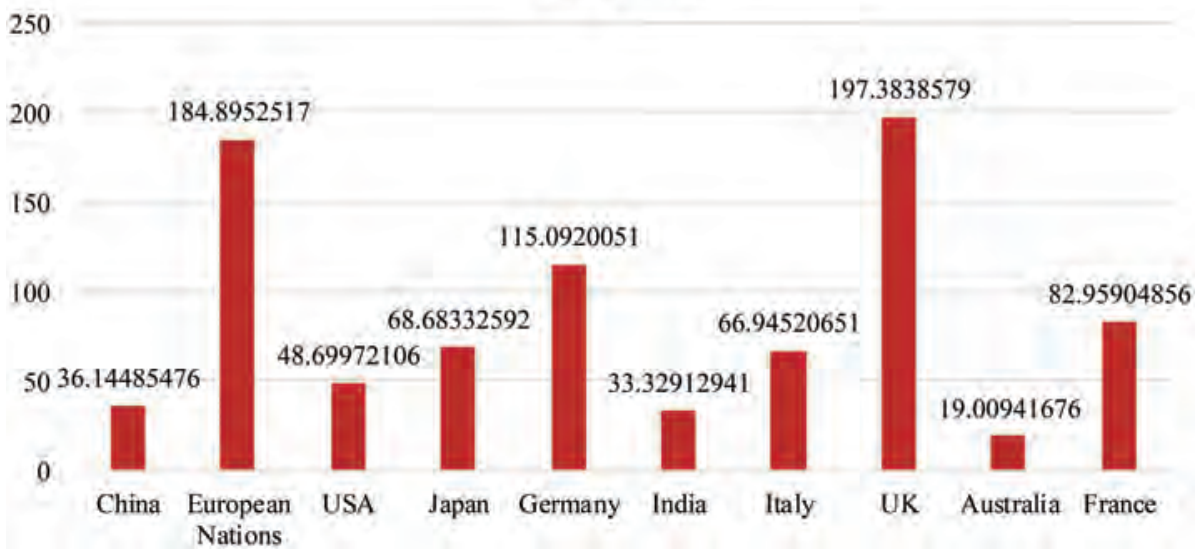

Figure 12.7 PV inverter lifetime (LT) of top 10 countries at component level.

\section{Life Consumption}

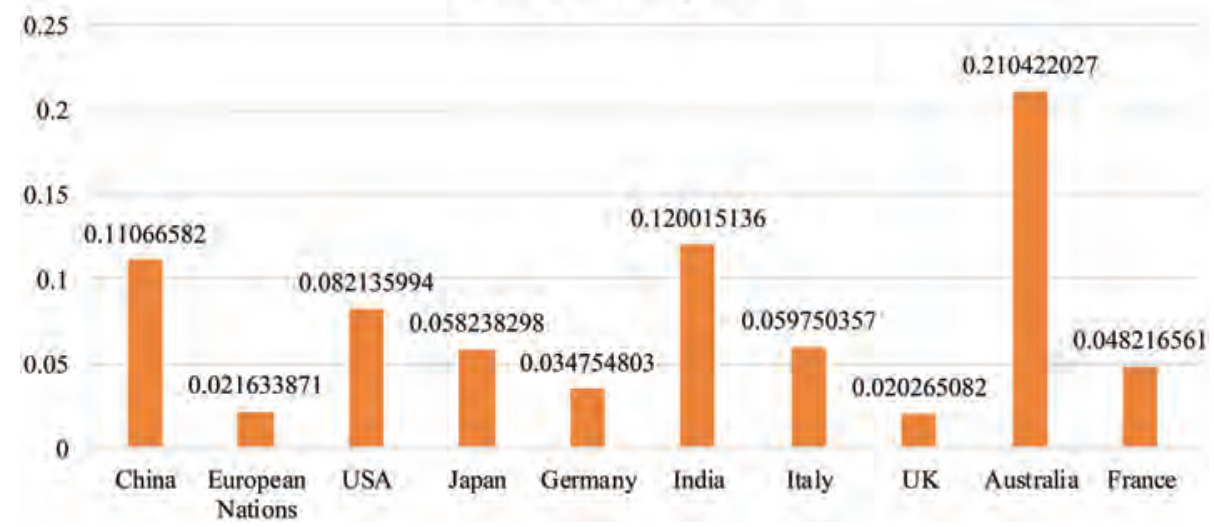

Figure 12.8 PV inverter life consumption (LC) of top 10 countries at system level.

shows the PV inverter life consumption (LC) of top 10 countries at component level; Figure 12.7 shows the PV inverter lifetime (LT) of top 10 countries at component level. Similarly, Figure 12.8 shows the PV inverter life consumption (LC) of top 10 countries at system level; Figure 12.9 shows the PV inverter lifetime (LT) of top 10 countries at system level.

From the above statistics it is clear that lifetime of the PV inverter varies with respect to the geographical locations.

\section{Conclusion}

This paper presents the impact of geographical location on the PV inverter lifetime. In the paper top ten countries for the PV markets are identified and mission profile, i.e. temperature (ambient), irradiance of the sun (solar), for one year is logged from the NASA website 


\section{Life Time}

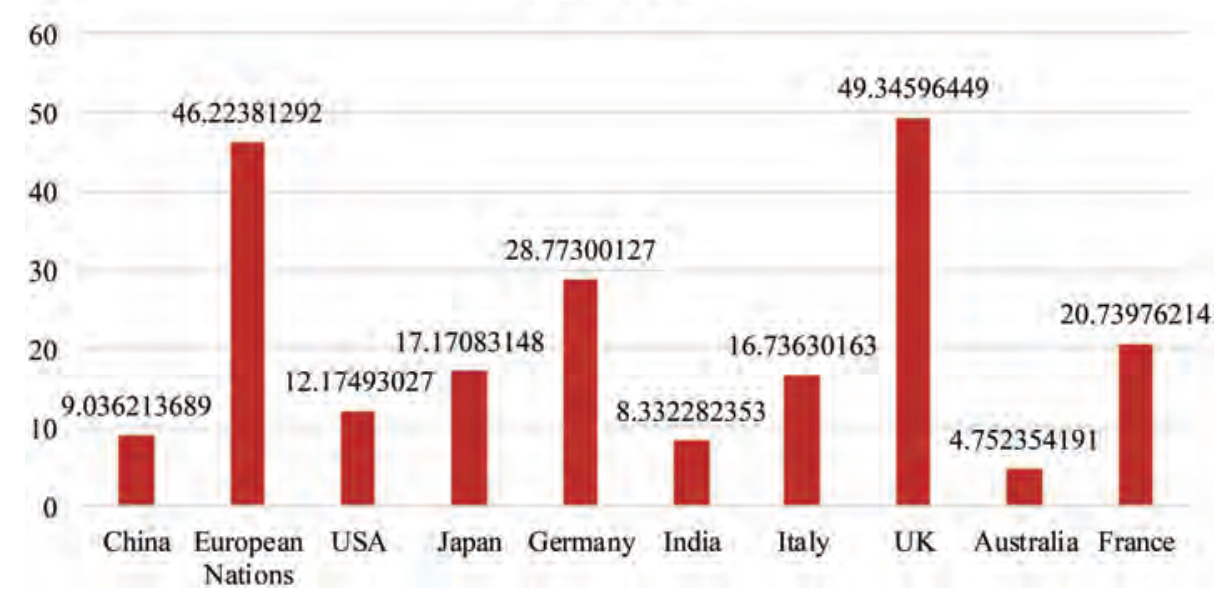

Figure 12.9 PV inverter lifetime (LT) of top 10 countries at system level.

for all the top ten countries. A single phase 3-kW full bridge PV inverter for 1- $\phi$ system is considered for the case study and simulated in the PLECS software. Junction temperature is calculated for all the countries, and using rainflow counting algorithm and Bayerer's lifetime equation life consumption and lifetime are calculated. For considering the uncertainties Monte Carlo simulation is used. Finally, results shows that lifetime of the PV inverter varies with respect to the geographical locations.

\section{References}

[1] IEA PV Snapshot 2019. International Energy Agency. Retrieved 2 June 2019.

[2] R. Kumar Gatla et al. Lifetime comparison of IGBT modules in grid-connected multilevel PV inverters considering mission profile. 2019 10th International Conference on Power Electronics and ECCE Asia (ICPE 2019-ECCE Asia). IEEE, 2019.

[3] S. S. Kshatri, J. Dhillon, Reliability assessment methodologies for grid connected PV inverter. Think India, vol. 22, no. 17, pp. 908-917, 2019.

[4] Y. Yang, A. Sangwongwanich, F. Blaabjerg, Design for reliability of power electronics for gridconnected photovoltaic systems, CPSS Trans. Power Electron. Appl., vol. 1, pp. 92-103, 2016.

[5] G. Ranjith Kumar, G. R. Zhu, J. Lu, W. Chen, B. Li, Thermal analysis and reliability evaluation of cascaded H-bridge MLPVI for grid-connected applications, The Journal of Engineering, vol. 2017, no. 13, pp. 1595-1599, 2017.

[6] L. R. Gopi Reddy, L. M. Tolbert, B. Ozpineci, J. Pinto, Rainflow algorithm based lifetime estimation of power semiconductors in utility applications, IEEE Trans. Ind. Appl., vol. 51, no. 4, pp. 3368-3375, 2015.

[7] Ranjith Kumar Gatla, Chen Wei, Zhu G.R, Zeng Dingjun, Nirudi Ramchander, Lifetime estimation of modular cascaded H-bridge MLPVI for grid-connected PV systems considering mission profile, Microelectronics Reliability, vol. 88-90, pp. 1051-1056, 2018. 


\title{
13 Feasibility analysis of smart grid distributed generation using RETScreen
}

\section{A case study of industrial building}

\author{
Harpreet Sharma ${ }^{1,2}$, Sachin Mishra ${ }^{2}$
}

\author{
${ }^{1}$ Junior Engineer, Punjab State Power Coorporation Limited, Jalandhar, India \\ ${ }^{2}$ School of Electronics and Electrical Engineering, Lovely Professional University \\ Phagwara, India
}

\section{Introduction}

The smart grid revolutionizes the power grid infrastructure with its concept of information technology and distributed generation. The DG reduces the power loss of the utility as it generates the power locally [1,2] and improves the reliability of the network by acting as an alternative source of energy. The idea of DG is now implemented on a large scale all over the world especially in countries like India, where the carbon emissions and fuel prices are major issues. The paper describes the role of the smart grid while implementing the DG [3] concept in the industrial building. In this industry, the die casting procedure is done with a load of heating, cooling and electrical. One of the popular energy tools RETScreen is utilized for determining the feasibility of the DG in the case study of industrial building [4].

The literature survey [5-8] reveals the use of different simulation tools and models to design the DG integrating microgrid with various load profiles. Those studies revealed that there is a significant reduction in energy costs with DG. However, the studies do not consider sufficient technical parameters and no study was conducted which includes the industrial load profile.

The study is classified into four sections. Section 2 describes the case study including its location and resource data of the site selected for the case study. The DG and load data are briefly described in Section 3. In Section 4, the RETScreen model is described which includes PV as DG and interconnected with the grid during operation. The results are also discussed in this section and the feasibility of a smart grid is determined. Section 5 concludes the overall results of the study.

\section{Case study}

The site selected for investigation is an industrial building located in Jalandhar, Punjab (India). There is an abundance of solar radiation available throughout the year with a clear sky. The location and solar radiation data are shown in Figure 13.1

The data clearly show that the month of May received high radiation, but at the same time the high temperature reduces the efficiency of solar cells. 


\begin{tabular}{lcc} 
& Unit & Climate data location \\
\hline Name & & India - Punjab - Jalandhar \\
Latitude & ${ }^{\circ}$ & 31.3 \\
Longitude & ${ }^{\circ} \mathrm{E}$ & 75.6 \\
Climate zone & & $2 \mathrm{~A}-$ Hot - H umid \\
Elevation & $\mathrm{m}$ & 250
\end{tabular}

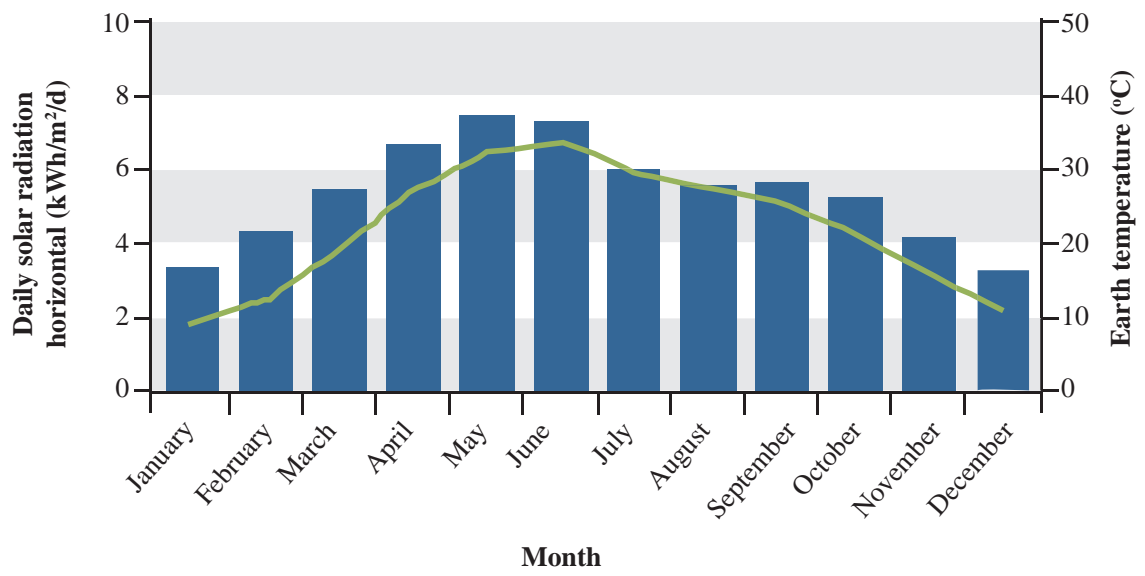

L egend

Daily solar radiation - horizontal

$\sim$ Earth temperature

Figure 13.1 Location and solar radiation data.

Table 13.1 Demand profile

\begin{tabular}{lcc}
\hline Load type & Maximum demand & Consumption \\
\hline Lighting & $38.5 \mathrm{~kW}$ & $180675 \mathrm{kWh}$ \\
Electrical equipment & $6.25 \mathrm{~kW}$ & $17598 \mathrm{kWh}$ \\
Mechanical load (electrical motors) & $22.38 \mathrm{~kW}$ & $544811.2234 \mathrm{kWh}$ \\
Furnace load & $7.46 \mathrm{~kW}$ & $350306.92 \mathrm{kWh}$ \\
\hline
\end{tabular}

\section{Load and generation data}

The annual demand for various equipment including electric motors, lights and water heating apparatus is tabulated in Table 13.1.

The DG in the form of solar PV is utilized in this study and the technical parameters are shown in Table 13.2.

The cost of solar PV varies with different countries and regions and its price is expected to reduce further in the coming future with technology advance. 
Table 13.2 DG profile

Power capacity $(\mathrm{kW})$

Initial cost $(\$ / k W)$

Total cost (\$)

Daily solar radiation horizontal $\left(\mathrm{kWh} / \mathrm{m}^{2}\right)$
58

800

46800

5.39

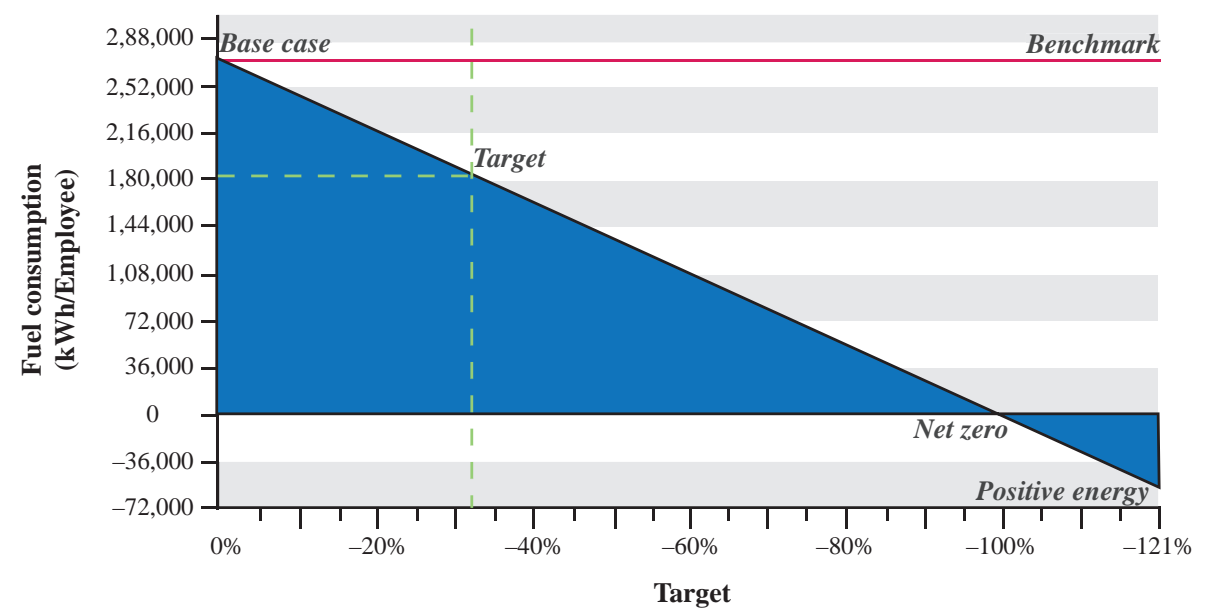

Figure 13.2 Fuel consumption.

\section{Results of RETScreen feasibility analyses}

The feasibility of DG installed industrial building is studied with the help of RETScreen and various parameters of both base case and proposed case are briefly discussed.

\subsection{Fuel consumption}

The fuel consumption of the base case and the proposed case is illustrated in Figure 13.2. The fuel-saving target is set in the RETScreen model and the benchmark is created.

The comparison of fuel consumption of both cases are determined and summarized in Table 13.3.

The target of fuel saving of $32.1 \%$ is set with total fuel saving of $8,711,952 \mathrm{kWh}$.

\subsection{Energy saving}

With DG integration the energy is significantly saved with the combined dispatch of the grid and solar PV. Figure 13.3 and Table 13.4 show the complete details of energy-saving between the two cases.

The fuel saved from the DG dispatch is found to be $12.6 \%$ including energy consumption in heating, cooling and electricity. The fuel is saved from the different appliances utilized in the industry and sharewise details of this equipment are shown in Table 13.5 . 
Table 13.3 Comparison of fuel consumption

\begin{tabular}{lll}
\hline Facility size & 100 & Employee \\
Benchmark & $2,71,326$ & $\mathrm{kWh} /$ employee \\
Minimum - average & $1,26,607$ & $\mathrm{kWh} /$ employee \\
Maximum - average & $4,42,068$ & $\mathrm{kWh} /$ employee \\
Base case & $2,71,325$ & $\mathrm{kWh} /$ employee \\
Target & $-32.1 \%$ & \\
Proposed case & $1,84,206$ & $\mathrm{kWh} / \mathrm{employee}$ \\
Facility - plan & & \\
Fuel consumption & Annual & $\mathrm{kWh}$ \\
Base case & $2,71,32,500$ & $\mathrm{kWh}$ \\
Proposed case & $1,84,20,548$ & $\mathrm{kWh}$ \\
Fuel saved & $87,11,962$ & \\
\hline
\end{tabular}

\subsection{Carbon emission reduction}

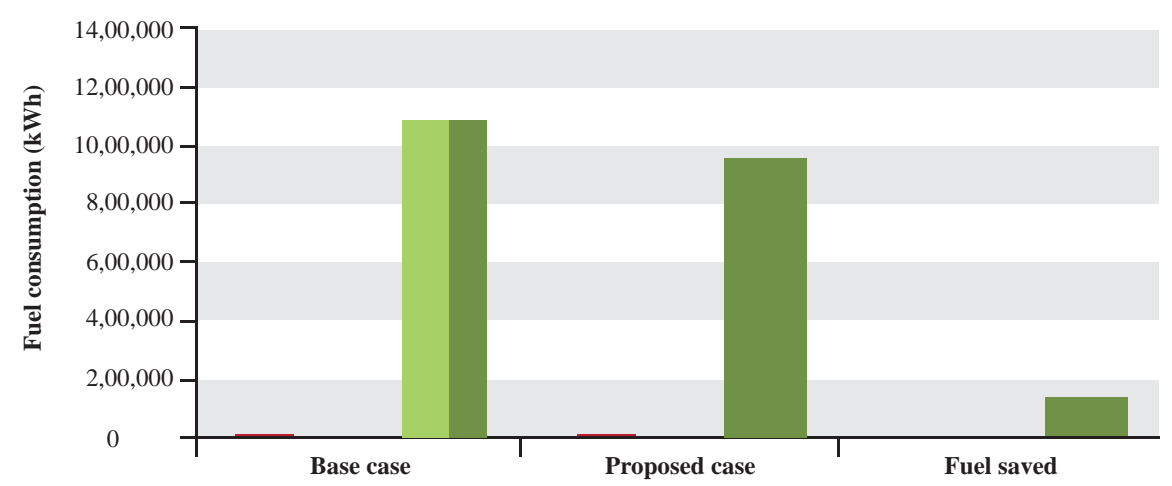

Heating 미 Cooling || Electricity

Figure 13.3 Energy saving (kWh).

Table 13.4 Fuel saved

\begin{tabular}{lllll}
\hline Fuel consumption & $\begin{array}{l}\text { Heating } \\
k W h\end{array}$ & $\begin{array}{l}\text { Cooling } \\
k W h\end{array}$ & $\begin{array}{l}\text { Electricity } \\
k W h\end{array}$ & $\begin{array}{l}\text { Total } \\
k W h\end{array}$ \\
\hline Base case & 8,548 & 0 & $10,93,391$ & $11,01,940$ \\
Proposed & 7,469 & 0 & $9,56,217$ & $9,63,687$ \\
Fuel saved & 1,079 & 0 & $1,37,174$ & $1,38,253$ \\
Fuel saved - percent & $12.6 \%$ & $0 \%$ & $12.5 \%$ & $12.5 \%$ \\
\hline
\end{tabular}

The DG not only reduces energy consumption but also decreases the carbon emission, which helps us to have a clean and sustainable future. The reduction in carbon emission is illustrated in Figure 13.4.

The carbon dioxide emission is reduced by 126 tons, which is equivalent to 23 cars not used in the complete year. 
Table 13.5 Equipment-wise fuel saving

\begin{tabular}{lrl}
\hline Section & $k W h$ & $\begin{array}{l}\text { Fuel saved } \\
\%\end{array}$ \\
\hline Mechanical equipment & 81,068 & $58.6 \%$ \\
Light & 52,195 & $37.8 \%$ \\
Miscellaneous & 4,990 & $3.6 \%$ \\
Electrical equipment & 3,911 & $2.8 \%$ \\
Hot water & 1,079 & $0.78 \%$ \\
\hline
\end{tabular}

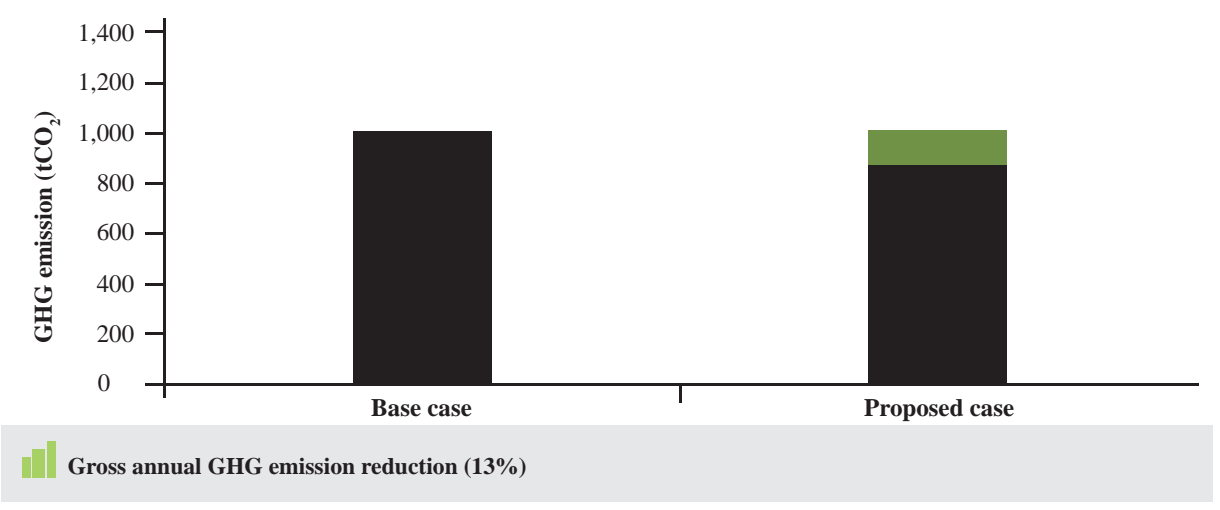

Figure 13.4 Carbon emission reduction.

\subsection{Economic analyses}

The financial viability needs to be verified before the implementation of the smart grid concept. The various financial parameters are analysed and the results are illustrated in Table 13.6. The total annual savings come about $\$ 1,33,722$ and the payback period is coming 6.4 years, which is quite attractive.

\section{Conclusion}

In this study, the feasibility analysis of the smart grid distributed generation is discussed. It was found that implementing a smart grid at the industrial building is feasible and cost-effective as the annual cost of energy is reduced significantly. RETScreen simulates detailed configuration and its recommendations are given against different parameters for the best option. Fuel consumption and carbon emission are reduced by $32.1 \%$ and $13 \%$ respectively.

The estimations of parameters utilized right now is explicit to that district and can be differed with area, government arrangements, framework power rates and hardware cost. The investigation shows that introducing PV boards according to housetop approach is monetarily gainful and will guarantee a spotless, reasonable future. The power is legitimately bought from the network when there is no or less PV power as per request. During PV power abundance period the power can be sold back to the matrix according to the tax plan with the assistance of brilliant meter. 
Table 13.6 Economic analyses

\begin{tabular}{|c|c|c|c|}
\hline $\begin{array}{l}\text { Total initial costs } \\
\text { Annual costs and debt payments } \\
\text { O\&M costs (savings) } \\
\text { Fuel - proposed payments }\end{array}$ & $100 \%$ & $\begin{array}{l}\$ \\
\$\end{array}$ & $\begin{array}{r}1,11,400 \\
-620 \\
1,16,945\end{array}$ \\
\hline $\begin{array}{l}\text { Total annual costs } \\
\text { Annual savings and revenue } \\
\text { Fuel cost - case } \\
\text { Total annual savings and revenue }\end{array}$ & & $\begin{array}{l}\$ \\
\$\end{array}$ & $\begin{array}{l}1,16,325 \\
1,33,722 \\
1,33,722\end{array}$ \\
\hline $\begin{array}{l}\text { Financial viability } \\
\text { Pre - tax IRR - assets } \\
\text { Sample payback } \\
\text { Equity payback }\end{array}$ & & $\begin{array}{l}\% \\
\mathrm{yr} \\
\mathrm{yr}\end{array}$ & $\begin{array}{c}16.9 \% \\
6.4 \\
6\end{array}$ \\
\hline
\end{tabular}

The fate of this situation is splendid as the expense of a PV board is required to decrease with innovation headway. The legislatures additionally have stepped toward clean vitality and give different impetuses and sponsorships.

\section{Acknowledgements}

The authors like to thank the developer of the RETScreen model for providing their simulation tool for feasibility analysis

\section{References}

[1] F. Guzzi, D. Neves, and C. A. Silva, "Integration of smart grid mechanisms on microgrids energy modelling," Energy, 2017.

[2] T. Adefarati and R. C. Bansal, "Reliability, economic and environmental analysis of a microgrid system in the presence of renewable energy resources," Applied Energy, vol. 236, no. August 2018, pp. 1089-1114, 2019.

[3] R. K. Mathew, "Impact of rooftop solar PV based DG on reliability of distribution systems," 2016.

[4] A. Raghoebarsing and A. Kalpoe, "Performance and economic analysis of a $27 \mathrm{~kW}$ gridconnected photovoltaic system in Suriname," IET Renewable Power Generation, vol. 11, no. 12, pp. 1545-1554, 2017.

[5] Y. V. P. Kumar and R. Bhimasingu, "Renewable energy based microgrid system sizing and energy management for green buildings," vol. 3, pp. 1-13, 2015.

[6] P. Taylor, "Optimization of grid tied hybrid power system in smart premises," International Journal of Green Energy, no. February 2015, pp. 37-41, 2014.

[7] J. Jung and M. Villaran, "Optimal planning and design of hybrid renewable energy systems for microgrids," Renewable and Sustainable Energy Reviews, October, pp. 1-12, 2016.

[8] H. Hassanzadehfard and A. Jalilian, "Optimal sizing and siting of renewable energy resources in distribution systems considering time varying electrical / heating / cooling loads using PSO algorithm," International Journal of Green Energy, pp. 1-16, 2018. 


\title{
14 Mathematical modelling of semiconductor devices and circuits
}

\author{
A review
}

\author{
Sanjay Kumar Roy ${ }^{1}$, Manwinder Singh ${ }^{2}$, Kamal Kumar \\ Sharma ${ }^{3}$, Brahmadeo Prasad Singh ${ }^{4}$
}

${ }^{1} \mathrm{PhD}$ Scholar, Head (Power Distribution), OMQ Division, TATASTEEL

Ltd., India

${ }^{2}$ Associate Professor, Electronics and Communication Engineering, Lovely

Professional University, Phagwara, Punjab, India

${ }^{3}$ Professor, Electronics and Communication Engineering, Lovely Professional

University, Phagwara, Punjab, India

${ }^{4}$ Professor Adjunct, Netaji Subhas University of Technology, New Delhi, India

\section{Introduction}

Mathematical modelling should form the basis of any educational system, especially the engineering education, because it indicates the proper place with suitable tools to achieve the desired result for a set of given input variables. Thus, it plays a very big role in the engineering education considering the views of all stake holders to improve the quality of the outcome. It is needless to say that stake holders are students, guardians, faculty positions, management groups, and very importantly the industrialist as employers and others. These variables are properly set in the mathematical model to achieve the desired outcome. If the desired result is not achieved, a relook in the mathematical modelling with fine tuning of increasing or decreasing any one or more variables is done to achieve the set goal. Thus, the variables of mathematical modelling should be tuned such that the set goal should be achieved very easily and neatly.

There are numerous methods of mathematical modelling available in the literature based on equivalent circuit approach [1-9]. Chirlian [1] suggested very general approach; wherein any three terminal devices can fit in it. Mitra [5], Gray et al. [7] and Millman et al. $[3,9]$ have provided more particular equivalent circuit approaches for BJTs and MOSFETs. One has to select the proper mathematical modelling scheme in a given constraint to achieve the best result with ease. As an instance, the transfer function of linear, timeinvariant, differential equation system is best suited for the Laplace transform method. The nullor $[10,11]$ and admittance matrix [12] methods have been used in the symbolic form extensively in the past. We have suggested an elegant mathematical modelling approach for both active devices and passive circuits and components, called the floating admittance matrix model. As the word spelt floating, it does not have any reference terminal in the analysis and design of any circuit whether active or passive or mixed of the active devices and passive components. The floating admittance matrix (FAM) model has been developed for the BJT or MOSFET to demonstrate the beauty of the method over other conventional techniques. The outcome of the developed mathematical models has been

DOI: $10.1201 / 9781003129103-14$ 
tested on any amplifier configuration that corroborates the result obtained in the available literature and overrides in the simplicity.

The floating admittance matrix model presented here is so simple that even a pure mathematician without the knowledge of electronic devices can work and analyse all transfer functions of any circuits, provided the parameters of devices are known to him. The analysis and design of any circuit using floating admittance matrix model is based on pure mathematical manoeuvring of matrices. The transfer functions are expressed as ratio of minors with proper signs, called cofactors of first and or second order. The mathematical modelling using FAM approach provides a leverage to the designer to adjust their style of design comfortably.

The conventional approach to mathematical model of the actives devices such as BJT and FET/MOSFET [1-9] uses its equivalent circuit as per the requirement of (a) either large signal or small signal models, (b) low frequency or high frequency modes so and so forth. For cascaded or cascoded connections of many devices (BJTs and MOSFETs) or the combinations of both BJTs and MOSFETs, in any circuit, the conventional method of equivalent circuit approach becomes very cumbersome. All types of transfers functions such as voltage gain, current gain, input resistance (impedance), output resistance (impedance) and power gains of any complicated circuit are obtained very easily based on the matrix partitioning method using the floating admittance matrix approach. This FAM technique very well satisfies the superposition theorem. The computer can very well be used for complicated networks, because the method uses only cofactors of the developed FAM.

The analysis becomes lucid and corroborates the transfer functions obtained in the literature. These transfer functions depend only on the cofactors of the FAM of any circuit: active and or passive or a combination of both. The outstanding merit of the floating admittance matrix is that it can be written by inspection for simple circuits.

\section{Concept of nullor}

The circuit symbols of the active devices 'nullator' and 'norator' [5] are shown in Figure 14.1. It is evident from Figure 14.1 that the nullator $[10,11]$ is a two-pole network with no current and voltage, whereas norator is also a two-pole network without any restriction on its voltage and current.

The nullor is an active two-port network consisting of nullator and norator. The circuit symbol of the nullor in the most basic form is depicted in Figure 14.2. Thus, the nullator is connected as the input port of the nullor whereas the norator forms its output port.

Figure 14.3 shows the approximate equivalent of ideal active devices such as BJT, MOSFET, and Op amp in the form of nullor. The use of the symbolic form of nullor is almost extinct from the analysis of active network in the current scenario. The use of symbolic form of BJT, MOSFET, and Op amp in place of the nullor, as per the circuit requirement, is the current trend in circuit analysis.

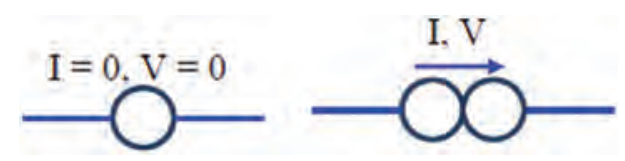

Figure 14.1 Symbolic form of nullator and norator. 


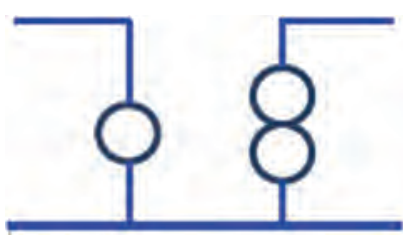

Figure 14.2 Two-port symbol of nullor.

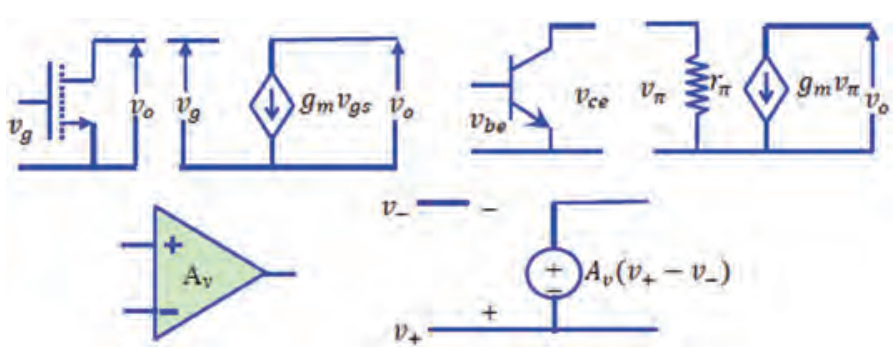

Figure 14.3 Practical nullor symbols.

Replacing each of the non-ideal Op amp or transistor as finite gain voltage controlled current source (VCCS) is a big task. This is possible only in the case when the active element falls in the category of the metal oxide field effect transistor (MOSFET) or a transconductance $\mathrm{Op}$ amp. In contrast, when an active device is supposed to function as voltage control voltage source (VCVS), or current controlled current source (CCCS), or a transresistance type of device, then Op amp and BJT are particularly suitable active devices.

The nullor based analysis and synthesis use ideal Op amp and BJT/ MOSFET as the dependent source and impedance (resistor) converter. The scaling technique of the elements of a matrix and its movement within itself is permissible provided the port equivalence is maintained.

The single four-terminal floating nullor in the form of a current conveyer can realize a single resistor controlled sinusoidal oscillator [12]. The frequency of oscillation and the condition of oscillation are independently controlled by separate resistors. The major advantage of this oscillator lies in the use of only one four-terminal floating nullor in contrast to the others using more numbers of four-terminal floating nullor. The model permits topological method of solution of the network.

A network analysis problem can be solved unequivocally [11], if an unambiguous relationship can be established between currents and voltages of the two poles forming the network. The nullator, in turn, represents two restrictions, namely, the insertion of a nullator into a real circuit makes the analysis problem redundant and the number of the possible independent Kirchhoff equations is increased by one, while the number of relationships of voltages and currents is increased by two. The insertion of a narrator into the circuit adds another independent Kirchhoff equation, leaving the number of restrictions for voltages and currents unchanged. Accordingly, the insertion of a narrator makes the problem indefinite. For an equal number of inserted nullators and narrators, the network calculation problem can be solved. 
Though a number of circuits are available in the literature for capacitance multiplier, the scheme presented [14] uses only one differential ideal Op amp to realize the frequency dependent resistance in the form of capacitance multiplier. The scheme facilitates the ondemand realization of both negative as well as positive values of resistances. The value of realized capacitance is proportional to the gain of the Op amp. The higher the value of gain of the $\mathrm{Op}$ amp, the higher will be the capacitance multiplication factor.

The ideal transistor and the ideal Op amp represent an active nullor as the small signal circuit element. The gain of the nullor tending towards infinity results in an dependent source of any of the four possible types, i.e. voltage-controlled current-source, currentcontrolled voltage-source, voltage-controlled voltage-source and current-controlled current-source. The input admittance and output impedance for a VCVS should strictly be zero.

Chen [15] stresses fundamental theory of circuit analysis for professional applications with the reinforcement by using frequent examples. The book defines and demonstrates the schematic symbol and corresponding notation of (a) resistors, (b) capacitors, (c) inductors, (d) voltage sources and (e) current sources etc. very well. The circuit schematic symbols for (a) voltage-controlled voltage-source (VCVS), (b) current-controlled voltage-source (CCVS), (c) voltage-controlled current-source (VCCS) and (d) current-controlled currentsource (CCCS) are also available along with many examples in the book. The circuit symbol for a voltage mode operational amplifier, first-order linear model of the Op amp, a voltage amplifier as the gain element, equivalent circuit of the voltage amplifier are available [15] as symbolic models.

Any value of input and output resistances (impedances) of both positive and negative magnitude can be realized, as has been demonstrated [14].

\section{Concept of floating admittance}

A very simple circuit of an admittance $\mathrm{Y}$ connected between two voltage sources $\mathrm{V}_{1}$ and $\mathrm{V}_{2}$ is shown in Figure 14.4 without any reference point.

These floating terminal voltages $V_{1}$ and $V_{2}$ result in the currents $I_{1}$ and $I_{2}$. The relationships between current $I_{1}$ and $I_{2}$ and voltages $V_{1}$ and $V_{2}$ are expressed in the $2 \times 2$ matrix form as:

$$
\left[\begin{array}{l}
\mathrm{I}_{1} \\
\mathrm{I}_{2}
\end{array}\right]=\left[\begin{array}{cc}
Y & -Y \\
-Y & Y
\end{array}\right]\left[\begin{array}{l}
\mathrm{V}_{1} \\
\mathrm{~V}_{2}
\end{array}\right]
$$

The coefficient matrix in Eq. (1) is called the floating admittance matrix. To have better sense of floating admittance matrix method of modelling [13,14,16-20], let us have a practical circuit called bridge-T attenuation [21]. The bridge-T attenuator is very versatile circuit used in communication network as shown in Figure 14.5.

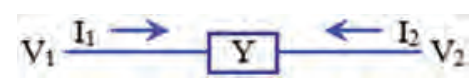

Figure 14.4 Series connected Y. 


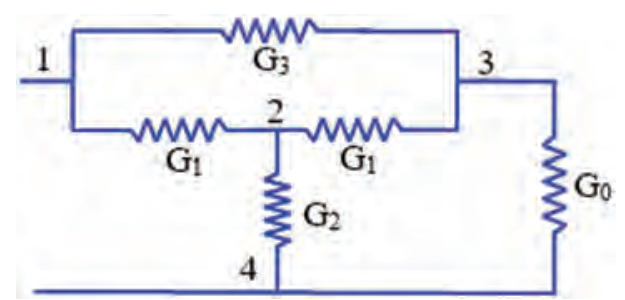

Figure 14.5 Bridge-T passive attenuator.

The coefficient floating admittance matrix of Figure 14.5 can be written by inspection very easily in the form of $4 \times 4$ matrix as:

$$
\left[\begin{array}{cccc}
1 & 2 & 3 & 4 \\
G_{1}+G_{3} & -G_{1} & -G_{3} & 0 \\
-G_{1} & 2 G_{1}+G_{2} & -G_{1} & -G_{2} \\
-G_{3} & -G_{1} & G_{1}+G_{3}+G_{0} & -G_{0} \\
0 & -G_{2} & -G_{0} & G_{2}+G_{0}
\end{array}\right] \begin{gathered}
3 \\
4
\end{gathered}
$$

The input resistance [13,16-18] of the bridge-T between its terminals 1 and 4 in Figure 14.5 is expressed as:

$$
\begin{aligned}
& R_{i n}=R_{i(14)}=\frac{\left|Y_{14}^{14}\right|}{\left|Y_{4}^{4}\right|_{g_{s}=0}} \\
& \left|Y_{14}^{14}\right|=\left(2 G_{1}+G_{2}\right)\left(G_{1}+G_{3}+G_{0}\right)-G_{1}^{2}
\end{aligned}
$$

For $G_{1}^{2}=G_{0}^{2}=G_{2} G_{3}$

$$
\begin{aligned}
& \left|Y_{14}^{14}\right|=\frac{2 G_{0}}{G_{2}}\left(G_{0}^{2}+2 G_{0} G_{2}+G_{2}^{2}\right)=\frac{2 G_{0}}{G_{2}}\left(G_{0}+G_{2}\right)^{2} \\
& \left|Y_{4}^{4}\right|=\frac{2 G_{0}^{2}}{G_{2}}\left(G_{0}+G_{2}\right)^{2} \\
& R_{\text {in }}=R_{i(14)}=\frac{\frac{2 G_{0}}{G_{2}}\left(G_{0}+G_{2}\right)^{2}}{\frac{2 G_{0}^{2}}{G_{2}}\left(G_{0}+G_{2}\right)^{2}}=R_{0}
\end{aligned}
$$

Equation (4) indicates that the input and output ports of Figure 14.5 are matched under the condition $G_{1}^{2}=G_{0}^{2}=G_{2} G_{3}$. 
The voltage transfer function between terminals 3 and 4 and 1 and 4 of the bridge-T network in Figure 14.5 is expressed as:

$$
\begin{aligned}
& \left.A_{v}\right|_{14} ^{34}=\operatorname{sgn}(3-4) \operatorname{sgn}(1-4)(-1)^{12} \frac{\left|Y_{34}^{14}\right|}{\left|Y_{14}^{14}\right|}=\frac{\left|Y_{34}^{14}\right|}{\left|Y_{14}^{14}\right|} \\
& \left|Y_{34}^{14}\right|=\frac{2 G_{0}^{2}}{G_{2}}\left(G_{0}+G_{2}\right) \\
& \left.A_{v}\right|_{14} ^{34}=\frac{v_{34}}{v_{14}}=\frac{\frac{2 G_{0}^{2}}{G_{2}}\left(G_{0}+G_{2}\right)}{\frac{2 G_{0}}{G_{2}}\left(G_{0}+G_{2}\right)^{2}}=\frac{G_{0}}{G_{0}+G_{2}}=\frac{R_{2}}{R_{2}+R_{0}}
\end{aligned}
$$

The propagation constant $(\mathrm{N})$ [21] of the bridge-T network in Figure 14.5 is described as:

$$
\begin{aligned}
& e^{\gamma}=\frac{v_{14}}{v_{34}}=1+\frac{R_{0}}{R_{2}}=N, \frac{R_{0}}{R_{2}}=N-1 \\
& R_{2}=\frac{R_{0}}{N-1}, \quad R_{3}=\frac{R_{0}^{2}}{R_{2}}=(N-1) R_{0}, R_{1}=R_{0},
\end{aligned}
$$

For a given value of propagation constant $(\mathrm{N})$ and the normal value of characteristic impedance $\left(R_{0}=600\right)$, the other components of the bridge- $T$ attenuator can be designed very easily using FAM approach.

Hence, we conclude that the design of bridge-T attenuator becomes very simple using floating admittance matrix approach in comparison to the conventional method.

\section{Conclusion}

In principle, the proposed technique can be used to model any circuit, whether active or passive or the combination of both. The proposed "floating admittance matrix" is an elegant approach to mathematical modelling of active and passive devices and circuits. The specific advantage of the floating admittance matrix approach lies in the fact that the algebraic sum of all elements of any row or of any column yielding zero provides first check that the process of analysis and design is in the correct direction of any circuit. Once the floating admittance matrix of the active device is known, the rest of the floating admittance matrix of the complete circuit containing passive components can be written by inspection without any difficulty. The rigorous equivalent circuit analysis with more active devices in any circuit can be avoided using this technique.

\section{References}

1. Chirlian, P., Electronic Circuits: Physical Principles, Analysis and Design, McGraw-Hill, New York, 1971.

2. Millman, J., and Halkias, C.C., Electronic Devices and Circuits, McGraw-Hill, New York, 1967. 
3. Millman, J., and Halkias, C.C., Integrated Electronics: Analog and Digital Circuit and System, McGraw-Hill, New York, 1972.

4. Balbanian, N., and Bickart, T.A., Electrical Network Theory, McGraw-Hill, 1969.

5. Mitra, S.K., Analysis and Synthesis of Linear Active Networks, John Wiley, 1969.

6. Ramey, R.L., and White, E., Matrices and Computers in Electronic Circuit Analysis, McGrawHill, 1971.

7. Paul R. Gray, Paul J. Hurst, Stephen H. Lewis and Robert G. Meyer, Analysis and Design of Analog Integrated Circuits, John Wiley, 2008.

8. D.A. Neamen, Semiconductor Physics and Devices - Basic Principles, McGraw-Hill, 2010.

9. Jacob Millman and Arvin Gabel, Microelectronics, Tata McGraw-Hill Education.

10. Haigh, David G., and Paul M. Radmore. "Admittance matrix models for the nullor using limit variables and their application to circuit design", IEEE Transactions on Circuits and Systems I: Regular Papers 53, no. 10 (2006): 2214-2223.

11. Vago, I., and E. Hollos. "Two-port models with nullators and norators", Periodica Polytechnica Electrical Engineering 17, no. 4 (1973): 301-309.

12. Kumar, Pragati, and Raj Senani. "Improved grounded-capacitor SRCO using only a single PFTFN", Analog Integrated Circuits and Signal Processing 50, no. 2 (2007): 147-149.

13. D.S.P. Rao, B.P. Singh and Dikshitulu Kaluri, "A note on the measurement of FET parameters", International Journal of Electronics 41, (1976): 521-524.

14. B. P. Singh, "On demand realization of a frequency-dependent negative resistance and an infinite input impedance", International Journal of Electronics Theoretical and Experimental 44, no. 3 (1978): 243-249.

15. Chen, Wai-Kai, Circuit Analysis and Feedback Amplifier Theory, CRC Press, 2005.

16. Meena Singh, Sanjay Roy and B. P. Singh, "On demand realization of input and output resistances of MOSFET amplifier", American Institute of Physics Conference Proceedings 1414, no. 1 (2011): 266-270.

17. B.P. Singh, "A null method for measuring the parameters of a FET", International Journal of Electronics 44 (1978): 251-256.

18. B.P. Singh, "Minimum sensitive FET Filter”, Indian Journal of Pure \& Applied Physics 20 (1982): 389.

19. B.P. Singh, "Null method for measuring h-parameter", Indian Journal of Pure \& Applied Physics 16 (1982): 337-339.

20. B.P. Singh, "Active Bridge for Measurements of the Admittance Parameters of the Transistor", Indian Journal of Pure \& Applied Physics 15 (1976): 783.

21. W.L. Everitt and G.E. Anner, Communication Engineering, McGraw-Hill, 1937. 


\title{
15 Small signal III-V high gain MOSFET
}

\author{
Manoj Singh Adhikari, *, Mayank Punetha ${ }^{2}$, \\ Yashvir Singh ${ }^{3}$, Yogesh Kumar Verma ${ }^{1}$
}

\author{
${ }^{1}$ SEEE Department, Lovely Professional University, Phagwara, Punjab, India \\ ${ }^{2}$ Department of ECE, Bipin Chandra Tripathi Kumaon Engineering College, \\ Dwarahat, India \\ ${ }^{3}$ Department of ECE, GB Pant Institute of Engineering \& Technology, \\ Pauri, India
}

\section{Introduction}

The III-V (i.e. InGaAs) MOSFET has become very popular in the today's market due to high performance in small signal RF, digital logic circuits and abundance in the nature of the Earth. With the benefit of high electron mobility, low energy band gap and superior injection velocity of $\mathrm{n}$-channel InGaAs semiconductor MOSFETs make these transistors a suitable candidate for logic memory, high-gain and high-speed applications [1,2]. Nowadays, researchers observed InGaAs MOSFETs as a key ingredient for future semiconductor and electronics industry [3-5]. In the past three decades, silicon is used as a popular material in semiconductor and VLSI industry for manufacturing various e-products. However, today's market requirement is changed and requires a novel and better material. The InGaAs is used as an alternative or new material. It required some advance for the interfacing with oxide, thus the development of MOS device along with InGaAs channel. Due to the higher mobility, good oxide interface, low energy band-gap, InGaAs acts as an emerging semiconductor material capable of producing high speed electronics devices in comparison to the silicon MOS devices [6-11]. For high frequency (i.e. microwave) applications, the III-V semiconductor device should exhibit high drain current, maximum transconductance, large current ratio $\left(\mathrm{I}_{\mathrm{ON}}\right.$ to $\left.\mathrm{I}_{\mathrm{OFF}}\right)$, low leakage current and high gain. The higher mole fraction of indium in InGaAs with high-k gate dielectrics exhibits maximum drain current value at small drain voltage with acceptable short channel effects (i.e. SS, DIBL). InGaAs semiconductor material consists of small band-gap so it causes a leakage current in the MOS device due to band-to-band tunnelling phenomenon [12-16]. Therefore, it is very challenging task for researchers and scientists to achieve improvement in all these parameters simultaneously. In this research paper, we propose a new architecture with III-V material by introducing trench concept that can bring significant progress in the performing parameters over the planer structure. Simulations are accomplished by using semiconductor simulator (ATLAS) [17], the performance of the InGaAs vertical trench MOSFET (VE-MOS) has been reviewed and also a comparison is accomplished with the InGaAs conventional MOSFET (CO-MOS).

\footnotetext{
* Email: manoj.23429@lpu.co.in
} 


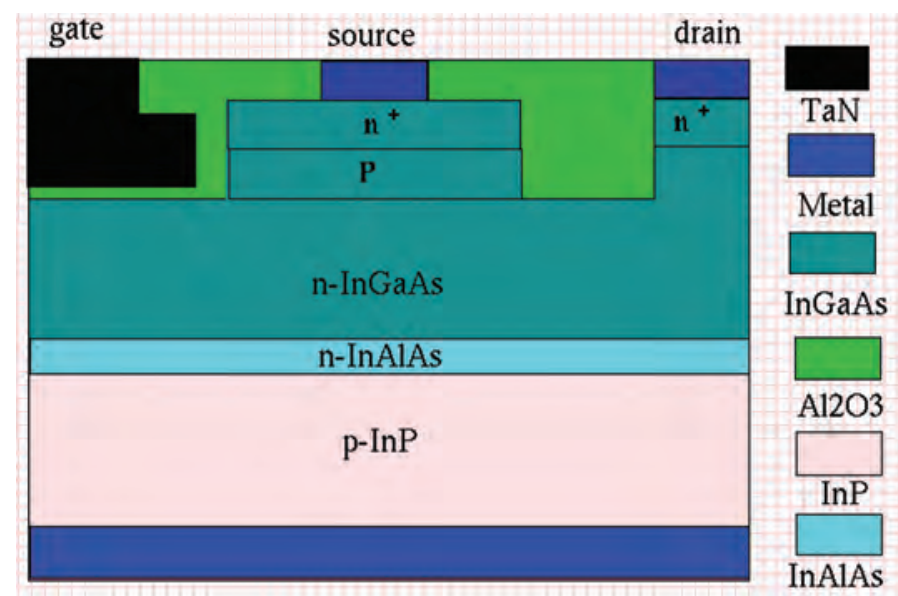

Figure 15.1 Cross-sectional view of VE-MOS.

\section{MOS architecture}

The schematic view of the trench based MOS (VE-MOS) device is shown in Figure 15.1. Device is implemented on III-V semiconductor material as $\operatorname{In}_{0.53} \mathrm{Ga}_{0.47} \mathrm{As}$. In the architecture, $\mathrm{InP}$ is considered as substrate material. The MOSFET has a $380 \mathrm{~nm} \mathrm{InP}$ layer along with $380 \mathrm{~nm} \mathrm{In}_{0.53} \mathrm{Ga}_{0.47}$ As quantum well. A high concentration of $\operatorname{In}_{0.53} \mathrm{Ga}_{0.47}$ As cap-layer having thickness $15 \mathrm{~nm}$ is used for the CO-MOS and VE-MOS structures. The source and the drain contacts are made with the help of highly concentric InGaAs layers. The P-body is realised with doping concentration of $2 \times 10^{18} \mathrm{~cm}^{-3}$ for the VE-MOS and CP-MOS. Devices consists of $40 \mathrm{~nm}$ channel length. Figure 15.1 depicts the planer MOSFET which consists of single source, gate, and drain contacts on the top of the structure. Only a single channel is formed in the p-base when the gate potential value is more than the threshold potential and a current starts to flow from drain to source terminal for positive drain potential. However, in VE-MOS, the gate contact is located in a trench made in the epi-layer, a subsequent creation of higher amount of current in the channel. Drain contact is located at the corner side of structure while source contact is placed in between the gate and drain electrodes. A high value of input supply produces conducting channels in the VE-MOS and a significant value of current drifts from drain contact to source contact via the channels.

\section{Outcome analysis}

The VE-MOS structure was implemented with the help of ATLAS software. The output characteristics of the VE-MOS structure at various input drive potentials are depicted in Figure 15.2. From the I-V curves of the device, it is noted that the current ability of the VE-MOS is considerably higher than the CO-MOS for all gate potentials. At $\mathrm{V}_{\mathrm{GS}}=1 \mathrm{~V}$, the values of the saturated output current of the VT-MOS and CO-MOS are 0.42 and 0.31 $\mathrm{mA} / \mu \mathrm{m}$, respectively. This result clearly indicates the output drive I value of the VE-MOS is 1.4 times higher in contrast to the CO-MOS. To achieve a desired output current, the VE-MOS will require less width as compared to CO-MOS and due to this the advantage there is a reduction of fabrication area. Therefore, overall there is a cost advantage of the VE-MOS over the CO-MOS. 


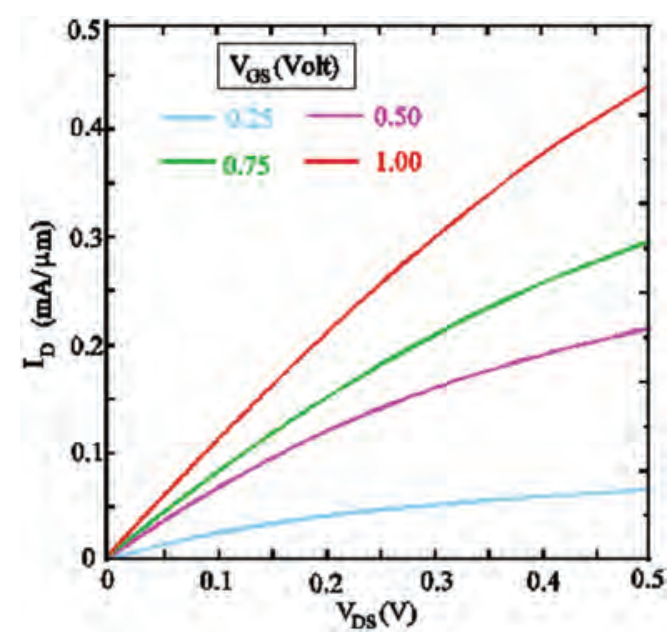

Figure 15.2 I-V characteristics of the VE-MOS.

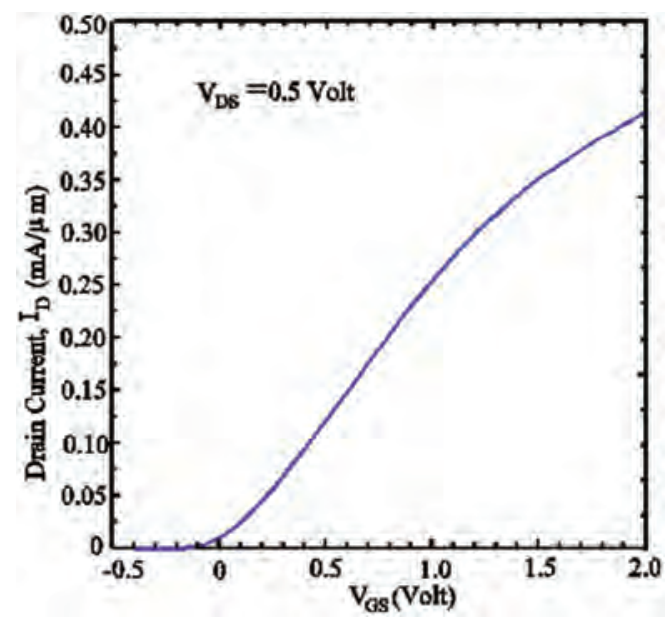

Figure 15.3 Transfer curves of the VE-MOS.

The transfer curve of VE-MOS is shown in Figure 15.3. For the extraction of threshold voltage, a tangent line is drawn at the point of the extreme slope of the curve existing with the input bias axis. The values of threshold voltage in the VE-MOS and CO-MOS are detected to be 0.03 and $0.04 \mathrm{~V}$, respectively. It signifies that the VE-MOS consists of lower threshold potential as compared to the CO-MOS, i.e. gives $33 \%$ reduction in threshold voltage.

The variation of $g_{m}$ (gain) with input potential of the VE-MOS is shown in Figure 15.4. It is observed that the VE-MOS demonstrates higher $g_{m}$ as compared to the CO-MOS. Therefore, VE-MOS represents good control of gate over the drive I. The gain value of the VE-MOS is $577 \mu \mathrm{S} / \mu \mathrm{m}$ and CO-MOS is $222 \mu \mathrm{S} / \mu \mathrm{m}$. In other words, VE-MOS gives $27 \%$ enhancement in gain. This is due to the fast current conduction and advanced structure. 


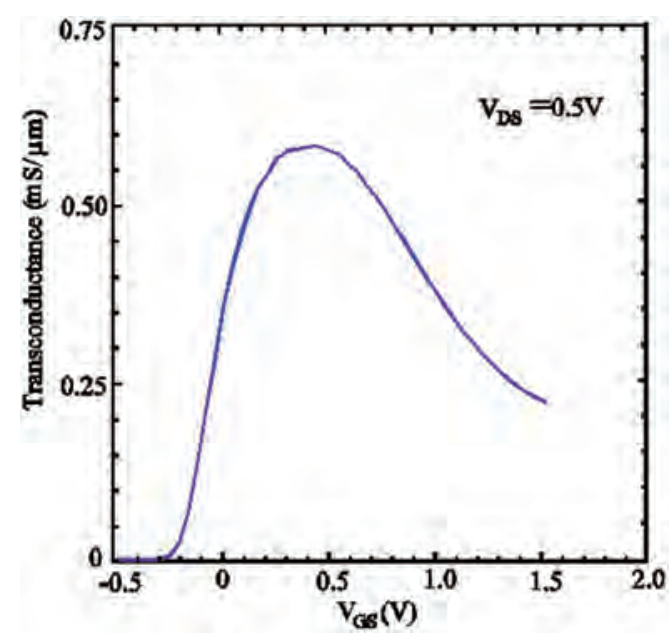

Figure 15.4 Transconductance curves of the VE-MOS.

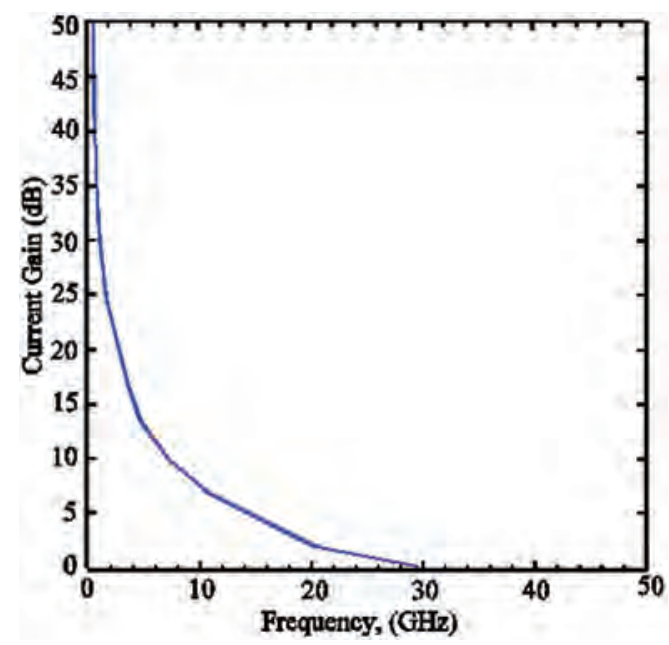

Figure 15.5 Frequency characteristics of the VT-MOS.

The RF response of VE-MOS is depicted in Figure 15.5. The cut-off frequencies $\left(\mathrm{f}_{\mathrm{t}}\right)$ of the VE-MOS and CO-MOS are $29 \mathrm{GHz}$ and $24 \mathrm{GHz}$, respectively. As a result, VE-MOS consists of $18 \%$ higher cut-off frequency. The reason behind this is due to higher value of transconductance in VT-MOS.

\section{Conclusion}

In this paper, a small signal III-V high gain MOSFET is proposed. It can be observed that InGaAs has been the material of choice for the modern semiconductor industry. A new III$\mathrm{V}$ n-channel MOSFET (VE-MOS) with trench technology is demonstrated. The InGaAs VE-MOS consists of very good current conduction in order to obtain the considerable enhancements in $g_{m}, I_{D}$, and $f_{t}$ for satellite and radio-frequency applications. With the 
help of 2D simulator analysis, the VE-MOS architecture was demonstrated to produce 1.4 times higher current, $27 \%$ enhancement in $g_{m}$, and $17 \%$ higher in cut-off frequency in comparison to the CO-MOS. All the results indicate that substantial enhancements in semiconductor device (VE-MOS) performance parameters can be achieved at the cost of some extra processing steps.

\section{References}

[1] R. S. Saxena and M. J. Kumar, "Trench gate power MOSFET: Recent advances and innovations," in Advances in Microelectronics and Photonics, pp. 1-23, 2012. Hauppauge, NY: Nova Science Publishers.

[2] A. T. M. G. Sarwar, M. R. Siddiqui, M. M. Satter, and A. Haque, "On the enhancement of the drain current in indium-rich InGaAs surface-channel MOSFETs," IEEE Trans. on Electron Devices, vol. 59, no. 6, pp. 1653-1660, Jun. 2012.

[3] N. Waldron, D. H. Kim, and J. A. D. Alamo, "A self-aligned InGaAs HEMT architecture for logic applications," IEEE Trans. on Electron Devices, vol. 57, no. 1, pp. 297-04, Jan. 2010.

[4] M. S. Adhikari and Y. Singh, "A nanoscale dual-channel trench (DCT) MOSFET for analog/ RF applications," Superlattices and Microstructures, vol. 88, pp. 567-573, Dec. 2015.

[5] M. S. Adhikari and Y. Singh, "High performance multi-channel MOSFET on InGaAs for RF amplifiers," Superlattices and Microstructures, vol. 102, pp. 79-87, Feb. 2017.

[6] Y. Singh and M. Badiyari, "Performance optimization of InGaAs power LDMOSFET," Microelectronics Journal, vol. 46, no. 5, pp. 404-409, May 2015.

[7] Y. Singh and M. S. Adhikari, "Performance evaluation of a lateral trench-gate power MOSFET on InGaAs," Journal of Computational Electronics, vol. 13, no. 1, pp. 155-160, Mar. 2014.

[8] S. Sant and A. Schenk, "Methods to enhance the performance of InGaAs/InP heterojunction tunnel FETs," IEEE Trans. on Electron Devices, vol. 63, no. 5, pp. 2169-2175, May 2016.

[9] R. S. Saxena and M. J. Kumar, "A new strained-silicon channel trench gate power MOSFET: Design and analysis," IEEE Trans. on Electron Devices, vol. 55, no. 11, pp. 32993304, Nov. 2008.

[10] R. J. E. Hueting, E. A. Hijzen, A. Heringa, A. W. Ludikhuize, and M. A. A. Zandt, "Gate-drain charge analysis for switching in power trench MOSFETs," IEEE Trans. on Electron Devices, vol. 51, no. 8, pp. 1323-1330, Aug. 2004.

[11] M. J. Kumar and R. Sithanandam, "In0.53Ga0.47As/InP trench gate power MOSFET based on impact ionization for improved performance: Design and analysis," IEEE Trans. on Electron Devices, vol. 64, no. 11, pp. 4561-4567, Nov. 2017.

[12] Yogesh Kumar Verma, Varun Mishra, Prateek Kishor Verma, and Santosh Kumar Gupta, "Analytical modelling and electrical characterisation of $\mathrm{ZnO}$ based HEMTs", International Journal of Electronics, vol. 106, no. 5, pp. 707-720, Nov 2019.

[13] Varun Mishra, Yogesh Kumar Verma, and Santosh Kumar Gupta, "Investigation of localized charges and temperature effect on device performance of ferroelectric dual material gate all around TFETs", Journal of Nanoelectronics and Optoelectronics, vol. 14, no. 2, pp. 161-168, Feb 2019.

[14] Yogesh Kumar Verma, Varun Mishra, and Santosh Kumar Gupta, "A physics based analytical model for MgZnO/ZnO HEMT", Journal of Circuits, Systems, and Computers, vol. 29, no. 1, pp. 2050009-1, Jan 2020.

[15] Yogesh Kumar Verma, Varun Mishra, and Santosh Kumar Gupta, "Linearity distortion analysis of III-V and Si quadruple gate field effect transistor (QG-FET) for analog applications”, Journal of Nanoelectronics and Optoelectronics, vol. 15, pp. 1-18, 2020.

[16] Santosh Kumar Gupta, Akash Singh Rawat, Yogesh Kumar Verma, and Varun Mishra, "Linearity distortion analysis of junctionless quadruple gate MOSFETs for analog applications", Silicon, vol. 11, no. 1, pp. 257-265, Feb 2019.

[17] ATLAS user's manual: Device simulation software, Silvaco Int., Santa Clara, CA, 2010. 


\title{
16 Impact of solar grid-based virtual power plant on grid flexibility
}

\author{
Harpreet Sharma*, Sachin Mishra \\ Lovely Professional University, Phagwara, India
}

\section{Introduction}

The modern energy system values reliability and flexibility as its main parameters while drafting energy policy to meet consumer expectations which are very high in the present scenario. The utilities are adopting VPP in the distribution network to enhance the grid flexibility in event of grid outage by supplying the high priority demand through DER locally and low priority load is curtailed or shifted to the remaining part of the day. The VPP also aggregates and coordinates the numerous grid-connected DER and storage devices into a single operating profile [1-3]. This one operating profile is much simpler and easier to control in real-time situations in comparison to managing the numerous resources at a time. In this paper, a case study of utility is evaluated in order to study the implication of VPP on grid flexibility considering the grid outage. The DER-CAM (distributed energy resources customer adoption model) is utilized in this study for finding an optimal solution, which is a far more sophisticated and accurate model than present tools. The Lawrence Berkeley National Laboratory, USA, develops the DER CAM, which is an optimization model based on mixed integer linear programming (MILP) and used to find an optimal investment solution for minimizing annual energy costs [4,5]. The modelling of combined dispatch of DER, DR, and storage has been done in DER CAM and optimal sizing of DER and storage is determined.

\section{Components of VPP}

\section{A. Distributed energy resources}

The decentralized renewable energy resources such as solar PV, wind and biomass are widely popular all over the world and these small scale generators are installed at industrial, residential and commercial buildings. The energy generation from rooftop solar PV is a well-established business in a country like India due to favourable weather conditions [6].

\section{B. DR programs}

The DR programs such as load shifting and load curtailment are used to tackle the intermittency of weather-dependent solar power. In load shifting, the low priority load is shifted from on-peak hours to off-peak hours by offering lower energy tariffs at a particular time of a day. In load shedding, the load with the least priority is shed in the period of grid outage in order to supply the essential demand by the DER $[7,8]$.

\footnotetext{
* Email: harpreetsharma1981@gmail.com
}

DOI: $10.1201 / 9781003129103-16$ 
Table 16.1 Location and climate data

Name of site

Location

Average solar radiation

Average temperature

Peak solar generation month
East Division PSPCL

31,19 $\mathrm{N}$ latitude and 75,21 E longitude

$5.17 \mathrm{kWh} / \mathrm{m}^{2} /$ day

23.9 degree Celsius

June

\section{Battery storage}

The energy storage smoothens the wide variations of solar PV generation much effectively than any other method [9]. The surplus generation from DER is stored and supplied back to the load during DER low generation or during a utility outage. The capacity of the battery strictly depends upon the capital investment and average outage duration.

\section{Case study}

The case study of Punjab State Power Corporation Limited (PSPCL) is analysed in this paper. The PSPCL is state government utility, which has a monopoly in the state of Punjab for electrical power generation and distribution. According to latest PSPCL regulations regarding rooftop PV, the customer can install solar PV capacity ranging from $1 \mathrm{~kW}$ to $1 \mathrm{MW}$; however, the maximum cap on this capacity is limited to $80 \%$ of connected load and aggregated capacity of these DERs is limited to $20 \%$ of the distribution transformer rating to limit the reverse power flow [10]. Another government agency, Punjab Energy Development Agency (PEDA), was established by the state government for providing subsidies and monetary benefits to the customers who are willing to install PV panels at their premises [11].

\section{A. Site location and weather conditions}

The geographical and weather data of the particular distribution division of the PSPCL are studied in order to access the implication of the VPP on the grid flexibility. The location and climate data of the east division are illustrated in Table 16.1.

\section{B. Demand specification}

There is a significant variation in load demand at various times of the day and there is a peak load on weekdays around 2 p.m. There is a steep decline in the demand at night and weekends due to sudden shed of inductive load with the closing of industries. The typical load profile of the month of January is shown in Figure 16.1.

\section{Techno-economic data}

The technical and financial data of various components of the VPP are visualized in Table 16.2. These include complete specifications of DER such as solar PV and battery storage.

\section{Results and discussion}

After running various configurations in DER CAM, the optimal sizing of DER and storage is determined, which is around PV capacity of $1916 \mathrm{~kW}$ and $9511 \mathrm{kWh}$. The topology of the DER CAM model is shown in Figure 16.2. 


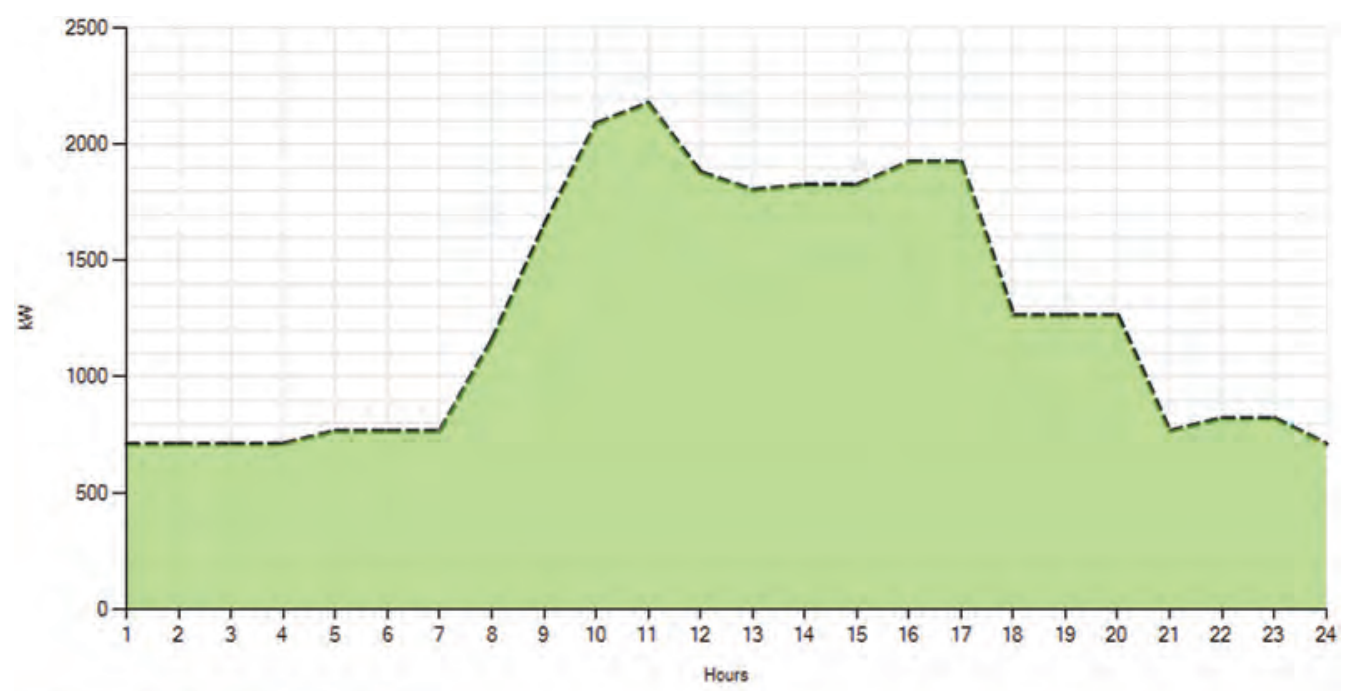

- Utility Purchase -- Total Original Electric Load

Figure 16.1 Daily load profile.

Table 16.2 Techno-economic specifications

\begin{tabular}{ll}
\hline PV technology & Cadmium telluride \\
\hline PV cost $(\$ / \mathrm{kW})$ & $\$ 985$ \\
PV lifetime & 25 years \\
PV inverter cost $100 \mathrm{~kW}$ & $\$ 271$ \\
PV maximum efficiency & $14.9 \%$ \\
Inverter efficiency & $95 \%$ \\
Battery storage cost $(\$ / \mathrm{kW})$ & $\$ 218$ \\
Charging efficiency & $90 \%$ \\
Minimum state of charge & $30 \%$ \\
Base case cost & $\$ 1282000$ \\
Simple payback $(\mathrm{yr})$ & 10 years \\
\hline
\end{tabular}

\section{A. Outage electrical dispatch}

To minimize the energy cost and to maintain the security of the distribution network, the DER CAM determines optimal electrical dispatch. The optimal electrical dispatch during the outage weekday of January is illustrated in Figure 16.3 below. The sudden loss of grid supply is compensated by DER generation, storage, load shifting and load curtailment. In the event of an outage, the DER generation is given first priority for dispatch, and storage is utilized when PV generation is limited. The load shifting and load-shedding programs are used when none of the supply resources is sufficient to meet the full demand. 


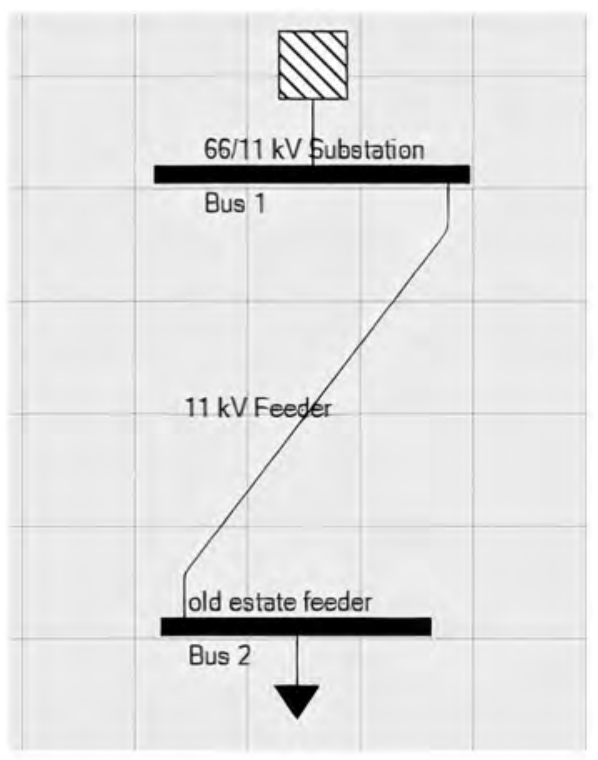

Figure 16.2 Topology of VPP.

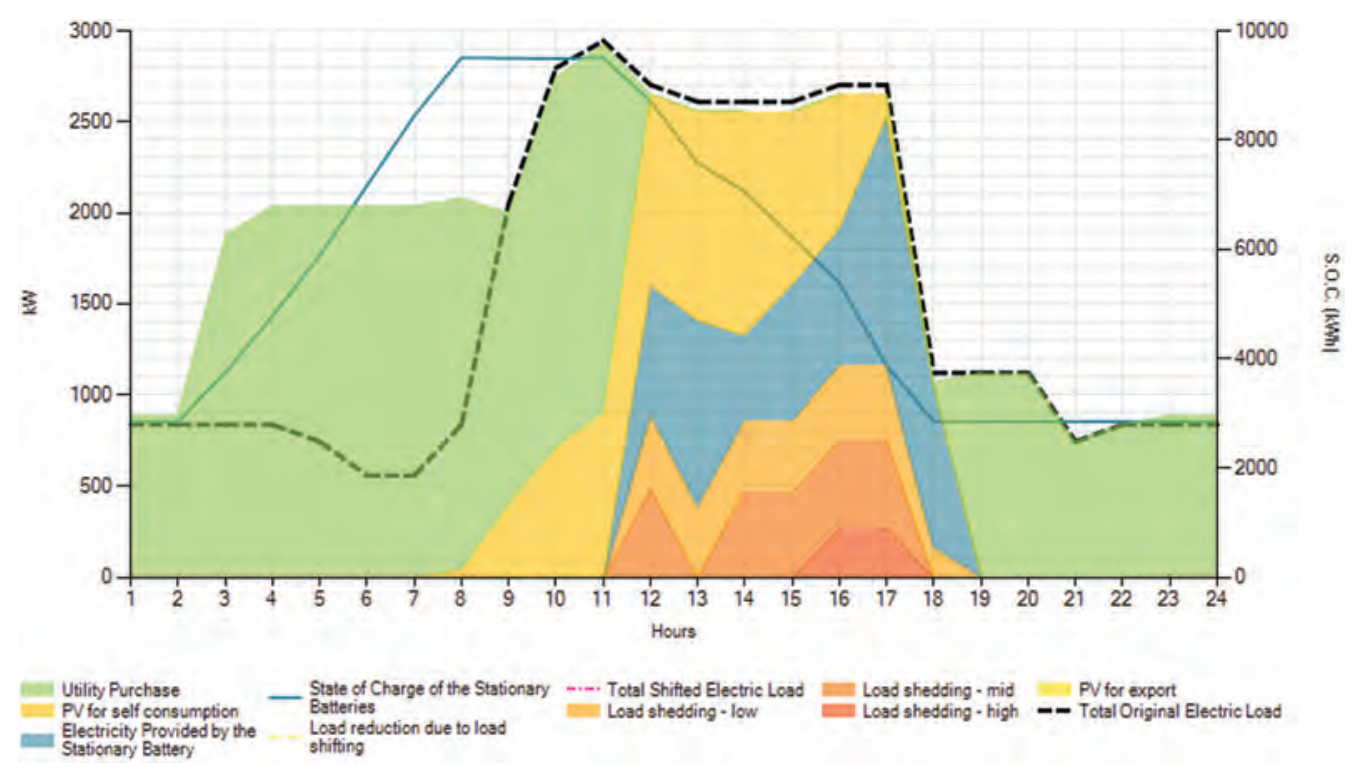

Figure 16.3 Electrical dispatch during an outage.

\section{B. Techno-economic analyses}

The technical and financial implications of the VPP integrated distribution grid are shown in Table 16.3. With the contribution of DER, total annualized energy cost is reduced by $7.1 \%$ and annual operational cost by $49 \%$ of the cost that is incurred in the base case scenario. The energy import from the grid is also reduced by $38.9 \%$ as a significant share of energy is supplied from the DER. 
Table 16.3 Techno-economic analyses

\begin{tabular}{lll}
\hline Investment & $\begin{array}{l}\text { Base case without } \\
\text { DER investment }\end{array}$ & $\begin{array}{l}\text { Base case with DER } \\
\text { investment and } \\
\text { demand response }\end{array}$ \\
\hline PV capacity & - & $1916 \mathrm{~kW}$ \\
$\begin{array}{l}\text { Electricity sales from PV } \\
\text { Total annual energy costs (incl. annualized capital } \\
\quad \text { costs and electricity sales) }\end{array}$ & $\$ 1368000$ & $4030321 \mathrm{kWh}$ \\
$\begin{array}{l}\text { Annual savings } \\
\text { Optimized operational cost }\end{array}$ & - & $\$ 1271000$ \\
Total electric costs & $\$ 1368000$ & $\$ 667305$ \\
Total annual electricity purchase & $\$ 1367645$ & $\$ 514380$ \\
\hline
\end{tabular}

\section{Conclusion}

In this paper, the impact of technical VPP on the utility grid flexibility is analysed for a particular case of the state power utility. The DER-CAM model is utilized in this study, which simulates various configurations and determines the optimal capacity of DER and storage. In the optimal configuration, the annual operational cost is reduced by $49 \%$, which makes the VPP implementation completely beneficial for both consumers and grid operators. The DR programs such as load shifting and load curtailments also play an important role in grid security as balance between supply and demand is maintained by it in the event of a grid outage. The design of this VPP can be improved further with the inclusion of different types of DERs and storage devices.

\section{Acknowledgement}

The authors wish to thank Punjab State Power Corporation Limited, India, for providing relevant data and their cooperation for the conduct of this study.

\section{References}

[1] Saboori H, Mohammadi M, Taghe R. Virtual power plant (VPP), definition, concept, components and types. Asia-Pacific Power and Energy Engineering Conference, APPEEC 2011. doi:10.1109/ APPEEC.2011.5749026.

[2] Mashhour E, Moghaddas-Tafreshi SM. A review on operation of micro grids and virtual power plants in the power markets. ICAST 2009 - 2nd International Conference on Adaptive Science and Technology 2009:273-7. doi:10.1109/ICASTECH.2009.5409714.

[3] Adu-kankam KO, Camarinha-matos LM. Towards collaborative virtual power plants: trends and convergence. Sustainable Energy, Grids and Networks 2018. doi:10.1016/ j.segan.2018.08.003.

[4] Schittekatte T, Stadler M, Cardoso G, Mashayekh S, Sankar N. The impact of short-term stochastic variability in solar irradiance on optimal microgrid design. IEEE Transactions on Smart Grid 2018;9:1647-56. doi:10.1109/TSG.2016.2596709.

[5] Sreedharan P, Farbes J, Cutter E, Woo CK, Wang J. Microgrid and renewable generation integration: University of California, San Diego. Applied Energy 2016;169:709-20. doi:10.1016/ j.apenergy.2016.02.053.

[6] Koussa DS, Koussa M. A feasibility and cost benefit prospection of grid connected hybrid power system (wind-photovoltaic) - Case study: An Algerian coastal site. Renewable and Sustainable Energy Reviews 2015;50:628-42. doi:10.1016/j.rser.2015.04.189. 
[7] Eid C, Codani P, Perez Y, Reneses J, Hakvoort R. Managing electric flexibility from Distributed Energy Resources: A review of incentives for market design. Renewable and Sustainable Energy Reviews 2016;64:237-47. doi:10.1016/j.rser.2016.06.008.

[8] Kakran S, Chanana S. Smart operations of smart grids integrated with distributed generation: A review. Renewable and Sustainable Energy Reviews 2018;81:524-35. doi:10.1016/ j.rser.2017.07.045.

[9] Setlhaolo D, Xia X. Combined residential demand side management strategies with coordination and economic analysis. International Journal of Electrical Power and Energy Systems 2016;79:150-60. doi:10.1016/j.ijepes.2016.01.016.

[10] Sharma H, Mishra S. Techno-economic analysis of solar grid-based virtual power plant in Indian power sector: A case study. International Transactions on Electrical Energy Systems 2019. doi:10.1002/2050-7038.12177.

[11] Sharma H. Hybrid optimization model for smart grid distributed generation using HOMER. 2019 3rd International Conference on Recent Developments in Control, Automation \& Power Engineering (RDCAPE). IEEE. 2019:94-9. 


\title{
17 Oxide thickness variation effects in MOS $\mathrm{AlGaN} / \mathrm{GaN}$ HFET
}

\author{
Yogesh Kumar Verma, *, Laxman Singh ${ }^{1}$, Varun Mishra ${ }^{3}$, \\ Rohit Gurjar, Prateek Kishor Verma ${ }^{5}$, Sudhnit Kaur 6 , \\ Akhil Sevda ${ }^{6}$, Mrityunjay Kumar Jha, \\ Santosh Kumar Gupta
}

\author{
1,6SEEE, Lovely Professional University, Punjab, India \\ ${ }^{2}$ Department of ECE, NIET, Greater Noida, India \\ ${ }^{3}$ Department of ECE, Graphic Era (Deemed to be University), Dehradun, \\ Uttarakhand, India \\ ${ }^{4}$ Department of ECE, PSIT College of Engineering, Kanpur, UP, India \\ ${ }^{5}$ Department of ECE, MNNIT, Allahabad, UP, India
}

\section{Introduction}

AlGaN/GaN HFETs are used for power amplification applications [1,3,5,8,11,12,14,2935]. The wide band-gap of $\mathrm{GaN}$ in comparison to silicon enhances the breakdown voltage of the AlGaN/GaN AlGaN/GaN HFET [2,4,6,7,9,10,13]. AlGaN/GaN heterostructurefield effect transistor (HFET) has emerged as an outstanding wide band-gap semiconductor device since the last decade due to its inherent properties over conventional AlGaAs/GaAs and Si based devices of narrow band-gap [15-25]. The impact of the different thickness of oxide layer on the performance parameters of the MOS AlGaN/GaN HFET is analysed using the numerical computations which can be further extended for circuit applications, similar to $[26,27]$. The work in the present paper consists of four sections.

\section{Model description}

Figure 17.1 represents the structure of MOS-AlGaN/GaN HFET. The structure comprises three layers: oxide layer, $\mathrm{AlGaN}$ and $\mathrm{GaN}$. There is one semiconductor layer of each type constituted by AlGaN and GaN material respectively. The oxide thickness is $2 \mathrm{~nm}$, channel length is $20 \mathrm{~nm}$, channel height is $20 \mathrm{~nm}$, mole fraction of $\mathrm{Al}$ is 0.28 , similar to [28].

\section{Results and discussion}

The calculations of linearity distortion parameters for different values of gate voltage $(=0$, $0.2,0.4,0.6,0.8$, and $1 \mathrm{~V}$ ) are presented. Figure 17.2 (a) represents the calculation of drain current for $t_{\mathrm{ox}}=1,1.5,2$ and $2.5 \mathrm{~nm}$. Figure 17.2(b) represents the calculation of $g_{\mathrm{m} 1}$ for $t_{\mathrm{ox}}=1,1.5,2$ and $2.5 \mathrm{~nm}$; where $g_{m n}=(1 / \mathrm{n} !)\left[\partial^{n} I_{D S} / \partial V_{G S}^{n}\right]\left(n=1\right.$ for $\left.g_{\mathrm{m} 1}\right)$.

Figure $17.3(\mathrm{a})$ represents the calculation of $g_{\mathrm{m} 2}$ for $t_{\mathrm{ox}}=1,1.5,2$ and $2.5 \mathrm{~nm}$. Figure 17.3(b) represents the calculation of $g_{\mathrm{m} 3}$ for $t_{\mathrm{ox}}=1,1.5,2$ and $2.5 \mathrm{~nm}$; where $g_{m n}=(1 / n !)\left[\partial^{n} I_{D S} / \partial V_{G S}^{n}\right]\left(n=2\right.$ and 3 for $g_{\mathrm{m} 2}$ and $g_{\mathrm{m} 3}$ respectively). It is evident from

\footnotetext{
*Email: yogesh.25263@lpu.co.in
}

DOI: 10.1201/9781003129103-17 


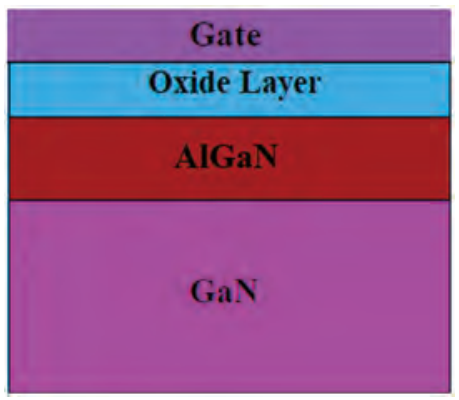

Figure 17.1 Structure of MOS-AlGaN/GaN HFET.

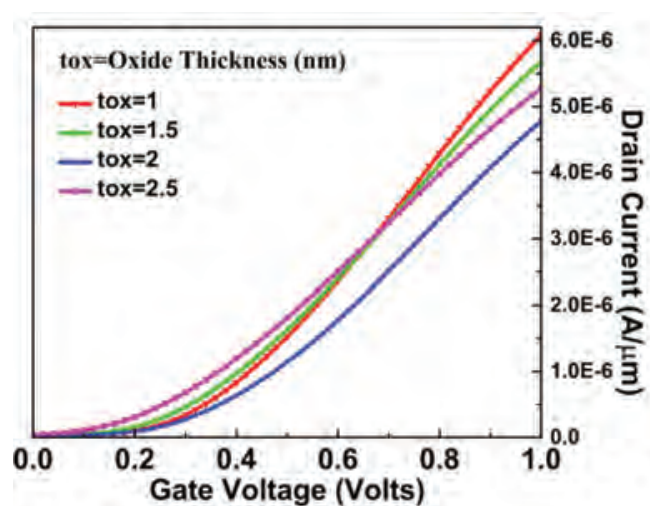

(a)

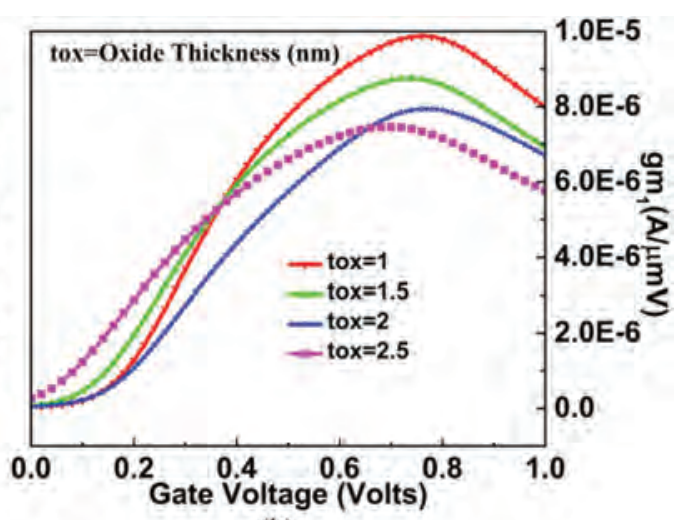

(b)

Figure 17.2 Calculation of (a) drain current, (b) $g_{\mathrm{m} 1}$.

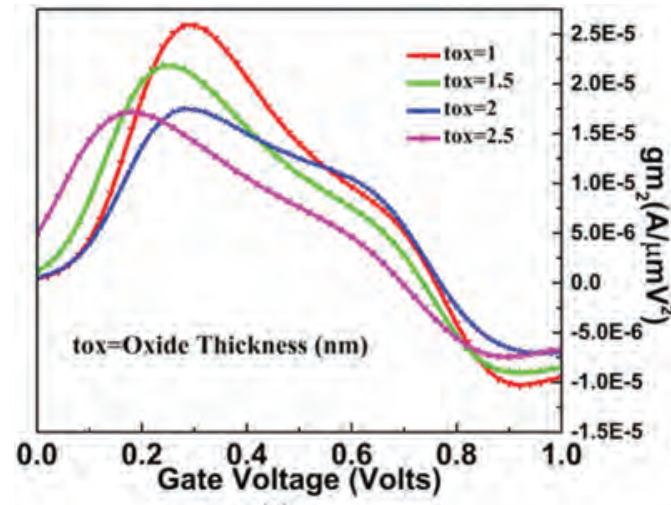

(a)

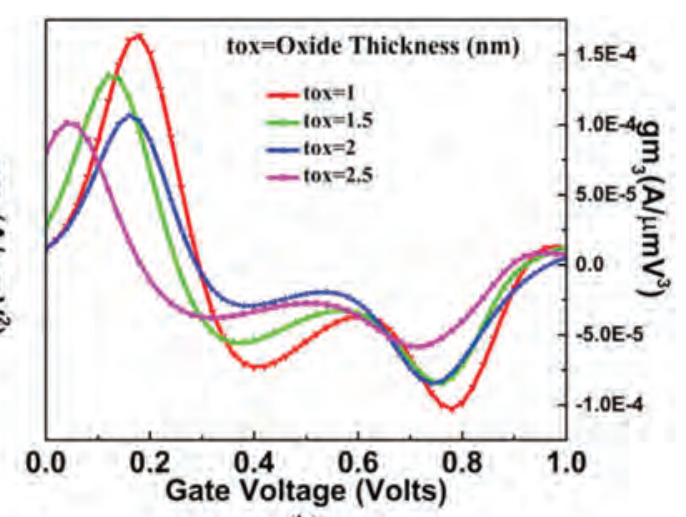

(b)

Figure 17.3 Calculation of (a) $g_{\mathrm{m} 2}$, (b) $g_{\mathrm{m} 3}$. 


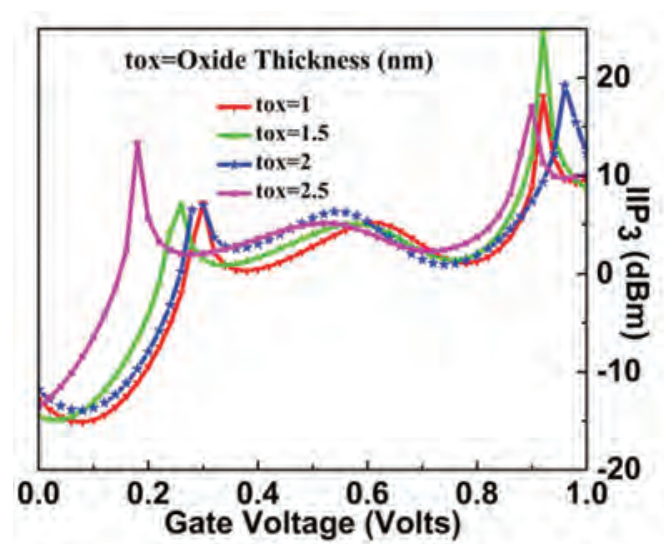

(a)

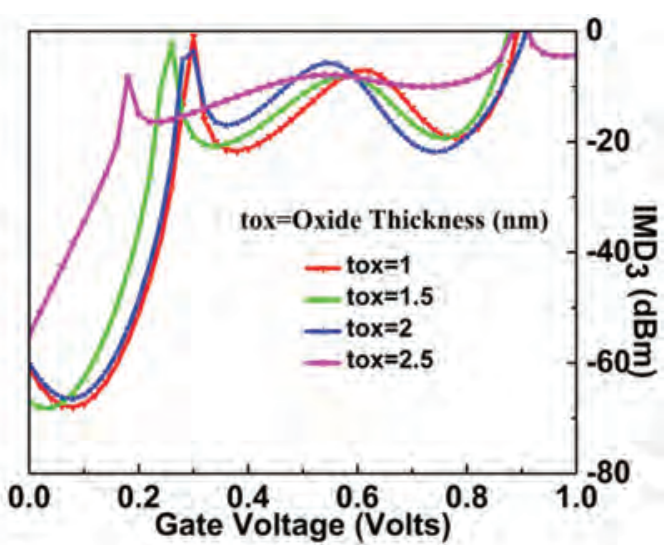

(b)

Figure 17.4 Calculation of (a) $\mathrm{IIP}_{3}$, (b) $\mathrm{IMD}_{3}$.

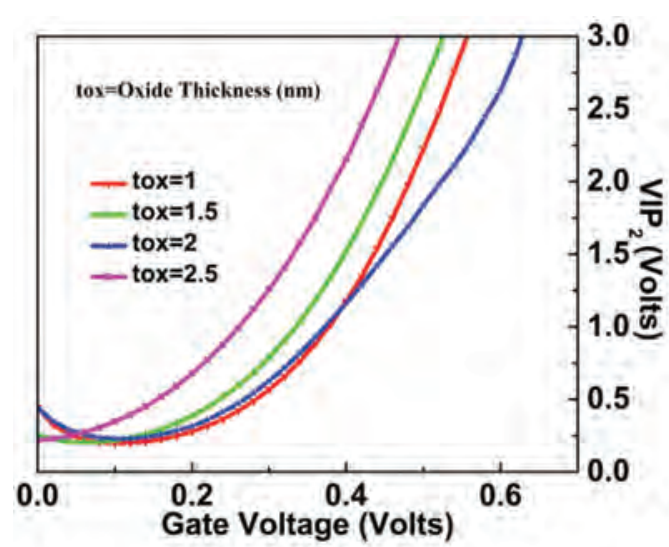

(a)

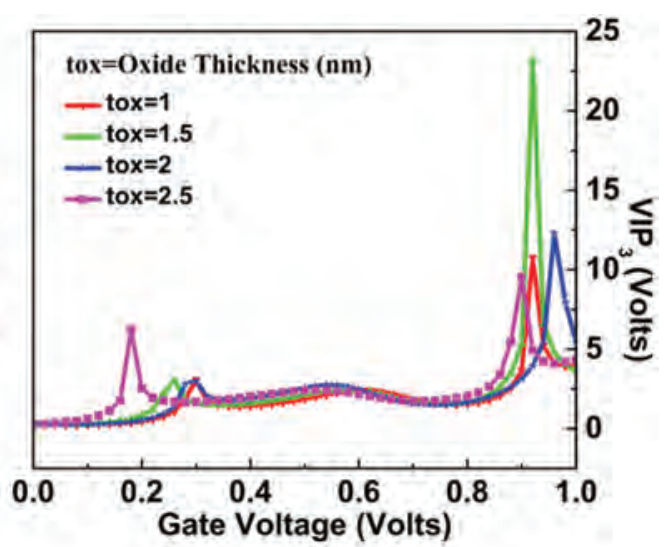

(b)

Figure 17.5 Calculation of (a) $\mathrm{VIP}_{2}$, (b) $\mathrm{VIP}_{3}$.

Figure 17.3(a) and (b) that the peak magnitude of $g_{\mathrm{m} 2}$ and $g_{\mathrm{m} 3}$ exists at lower values of gate voltage.

Figure 17.4(a) represents the calculation of $\mathrm{IIP}_{3}$ for $t_{\mathrm{ox}}=1,1.5,2$ and $2.5 \mathrm{~nm}$. Figure 17.4(b) represents the calculation of $\mathrm{IMD}_{3}$ for $t_{\mathrm{ox}}=1,1.5,2$ and $2.5 \mathrm{~nm}$. It is evident from Figure 17.4(a) and (b) that there are two peaks of $\mathrm{IIP}_{3}$ and $\mathrm{IMD}_{3}$ occurring at lower and higher magnitude of gate voltage.

Figure 17.5(a) represents the calculation of $\mathrm{VIP}_{2}$ for $t_{\mathrm{ox}}=1,1.5,2$ and $2.5 \mathrm{~nm}$ considering different values of gate voltage $(=0,0.2,0.4,0.6,0.8$ and $1 \mathrm{~V}) . \mathrm{VIP}_{2}$ represents the extended value of input voltage for second- and third-order harmonic voltages to be equal ( $\mathrm{VIP}_{2}$ $\left.=4 \cdot g_{\mathrm{m} 1} / g_{\mathrm{m} 2}\right)$. Figure $17.5(\mathrm{~b})$ represents the calculation of $\mathrm{VIP}_{3}$ for $t_{\mathrm{ox}}=1,1.5,2$ and 2.5 $\mathrm{nm}$. VIP ${ }_{3}$ represents the extended input voltage for first and third harmonic voltages to be equal $\left(\mathrm{VIP}_{3}=\sqrt{ } 24 \cdot g_{\mathrm{m} 1} / g_{\mathrm{m} 3}\right)$. 


\section{Conclusion}

The drain current, transconductance, first and second order derivatives of transconductance, input intercept point, intermodulation distortion, and linearity distortion parameters are calculated for different values of oxide layer thickness. The structure in the present work is surrounded by gates from all sides hence improves the control of the gate over the channel and thus reduces the short channel effects (SCEs). The present device can be used in applications including low noise amplifiers and high frequency.

\section{References}

[1] Yogesh Kumar Verma, Varun Mishra, Prateek Kishor Verma, Santosh Kumar Gupta, "Analytical modelling and electrical characterisation of $\mathrm{ZnO}$ based HEMTs", International Journal of Electronics, vol. 106, no. 5, pp. 707-720, November 2019, doi:10.1080/ 00207217.2018.1545931.

[2] Manoj Singh Adhikari, Yashvir Singh, "Implementation of trench-based power LDMOS and small signal MOSFET on InGaAs", IETE Technical Review, vol. 36, no. 3, pp. 234-242, 2019, doi:10.1080/02564602.2018.145065.

[3] Yogesh Kumar Verma, Varun Mishra, Santosh Kumar Gupta, "Analog/RF and linearity distortion analysis of $\mathrm{MgZnO} / \mathrm{CdZnO}$ quadruple-gate field effect transistor (QG-FET)", Silicon, 2020, doi:https://doi.org/10.1007/s1263.

[4] Namrata Mendiratta, Suman Lata Tripathi "A review on performance comparison of advanced MOSFET structures below $45 \mathrm{~nm}$ technology node" Journal of Semiconductor, vol. 41, pp.1$10,2020$.

[5] S. Khandelwal, N. Goyal, T. A. Fjeldly, "A physics-based analytical model for 2DEG charge density in AlGaN/GaN HEMT devices," IEEE Trans. Electron Devices, vol. 58, no. 10, pp. 3622-3625, 2011.

[6] Santosh Kumar Gupta, Akash Singh Rawat, Yogesh Kumar Verma, Varun Mishra, "Linearity distortion analysis of junctionless quadruple gate MOSFETs for analog applications", Silicon, vol. 11, no. 1, pp. 257-265, February 2019, doi:https://doi.org/ 10.1007/s12633-018-9850-z.

[7] Varun Mishra, Yogesh Kumar Verma, Santosh Kumar Gupta, "Surface potential based analysis of Ferroelectric Dual Material Gate All Around (FE-DMGAA) TFETs”, International Journal of Numerical Modelling, 2020, doi:https://doi.org/10.1002/jnm.2726.

[8] Yogesh Kumar Verma, Varun Mishra, Santosh Kumar Gupta, "Linearity distortion analysis of III-V and Si quadruple gate field effect transistor (QG-FET) for analog applications”, Journal of Nanoelectronics and Optoelectronics, vol. 15, pp. 1-18, 2020, doi:https://doi.org/10.1166/ jno.2020.2741.

[9] Varun Mishra, Yogesh Kumar Verma, Prateek Kishor Verma, Santosh Kumar Gupta, "EMA Based modeling of surface potential and drain current of dual material gate all around TFETs", Journal of Computational Electronics, vol. 17, no. 4, pp. 1596-1602, December 2018, doi:https://doi.org/10.1007/s10825-018-1250-5.

[10] Varun Mishra, Yogesh Kumar Verma, Santosh Kumar Gupta, "Investigation of localized charges and temperature effect on device performance of ferroelectric dual material gate all around TFETs", Journal of Nanoelectronics and Optoelectronics, vol. 14, no. 2, pp. 161-168, February 2019, doi:https://doi.org/10.1166/jno.2019.2462.

[11] Yogesh Kumar Verma, Varun Mishra, Santosh Kumar Gupta, "A physics based analytical model for MgZnO/ZnO HEMT", Journal of Circuits, Systems, and Computers, vol. 29, no. 1, pp. 2050009-1, January 2020, doi:http://doi.org/10.1142/S0218126620500097.

[12] Yogesh Kumar Verma, Varun Mishra, Santosh Kumar Gupta, "Electrical characterization of AlGaN/GaN quadruple gate heterostructure field effect transistor for analog applications", Journal of Nanoelectronics and Optoelectronics, vol. 14, no. 10, pp. 1491-1502, October 2019, doi:https://doi.org/10.1166/jno.2019.2655. 
[13] Varun Mishra, Yogesh Kumar Verma, Santosh Kumar Gupta, "Investigation of localized charges on linearity and distortion performance of ferroelectric dual material gate all around TFETs", Journal of Nano and Electronic Physics (JNEP), vol. 11, no. 04, 04014 (6pp), 2019.

[14] Y. K. Verma, S. K. Gupta, V. Mishra, P. K. Verma, "Surface potential based analysis of $\mathrm{MgZnO} / \mathrm{ZnO}$ high electron mobility transistors," IEEE International Students' Conference on Electrical, Electronics and Computer Science (SCEECS), MANIT, Bhopal, pp. 1-4, 2018.

[15] Yogesh Kumar Verma, Varun Mishra, Prateek Kishor Verma, Santosh Kumar Gupta, Rajeev Kumar Chauhan, "Effect of ionizing radiation and temperature on SiGe HBT", International Conference on Electrical, Electronics and Computer Engineering (UPCON-2018), Madan Mohan Malaviya University of Technology Gorakhpur (UP) India, November 2-4, 2018.

[16] Yogesh Kumar Verma, Varun Mishra, Prateek Kishor Verma, Santosh Kumar Gupta, Rajeev Kumar Chauhan, "Impact of extrinsic reliability issues including radiation and temperature on SiGe HBT", Computational and Characterization Techniques in Engineering and Sciences (CCTES), Integral University, Lucknow (UP) India, September 14-15, 2018.

[17] Digvijay Singh Mehta, Varun Mishra, Yogesh Kumar Verma, Santosh Kumar Gupta, “A novel dual material extra insulator layer fin field effect transistor for high-performance nanoscale applications", Advances in VLSI, Communication, and Signal Processing, 2020.

[18] Digvijay Singh Mehta, Varun Mishra, Yogesh Kumar Verma, Santosh Kumar Gupta, "A hardware minimized gated clock multiple output low power linear feedback shift register", Advances in VLSI, Communication, and Signal Processing, 2020.

[19] Varun Mishra, Yogesh Kumar Verma, Prateek Kishor Verma, Ningthoujam Qoonand Singh, Santosh Kumar Gupta, "Performance of double gate tunnel FET devices with source pocket", Advances in VLSI, Communication, and Signal Processing, 2020.

[20] Varun Mishra, Santosh Kumar Gupta, Yogesh Kumar Verma, Vishal Ramola, Abhishek Bora, "A high-gain, low-power latch comparator design for oversampled ADCs", Signal Processing and Integrated Networks (SPIN), IEEE, Amity University, Noida, February 22-23, 2018.

[21] Yogesh Kumar Verma, Santosh Kumar Gupta, Rajeev Kumar Chauhan, “Analysis of inherent properties of SiGe hetero-structure device using analytical modelling and simulation (I2CT)", IEEE, 2nd International Conference for Convergence in Technology, Pune, April 7-9, 2017.

[22] Laxman Singh, Sunil Kumar Chaudhary, Yogesh Kumar Verma, Jay Kant Pratap Singh Yadav, Rajeev Kumar, "Smart volume controller for mobile phones", International Journal of Engineering and Advanced Technology, vol. 9, no. 2, December 2019, SJR: 0.11, doi:10.35940/ ijeat.B2313.129219.

[23] Suman Lata Tripathi, Raju Patel, Vimal Kumar Agrawal, "Low leakage pocket junction-less DGTFET with bio sensing cavity region", Turkish Journal of Electrical Engineering and Computer Sciences, vol. 27, no. 4, pp. 2466-2474, 2019.

[24] Manoj Singh Adhikari, Yashvir Singh, "High performance multi-channel MOSFET on InGaAs for RF amplifiers", Superlattices and Microstructures, pp. 79-87, 102, 2017.

[25] Manoj Singh Adhikari, Yashvir Singh, "High Performance Multi-Finger MOSFET on SOI for RF Amplifiers”, Indian Journal of Physics, vol. 91, no. 10, pp. 1211-1217, 2017.

[26] D. Kumar Gautam, S.R.P. Sinha, Y. Kumar Verma, "Design a low power half subtractor using AVL technique based on $65 \mathrm{~nm}$ CMOS technology," International Journal of Advanced Research in Computer Engineering and Technology, vol. 2, pp. 2891-2897, 2013.

[27] D. Kumar Gautam, S.R.P. Sinha, Y. Kumar Verma, "Design a low power high speed full adder using AVL technique based on CMOS nano-technology", IOSR Journal of Electronics and Communication Engineering, vol. 8, no. 1, pp. 19-26, 2013.

[28] S. Baskaran et al., "Switching transient analysis and characterization of E-mode B-doped GaN capped AlGaN DH-HEMT with freewheeling Schottky barrier diode (SBD)", Journal of Electronic Materials, 2020.

[29] Yogesh Kumar Verma, Santosh Kumar Gupta, "Center potential based analysis of Si and III-V gate all around field effect transistors (GAA-FETs)", Silicon, 2020 (recently accepted).

[30] Yogesh Kumar Verma, Varun Mishra, Santosh Kumar Gupta, "Performance enhancement of AlGaN/GaN HEMT by optimization of device parameters considering nano-meter barrier layer thickness", International Journal of Nanoscience, vol. 19, no. 06, 2050011, 2020. 
[31] Prateek Kishor Verma, Yogesh Kumar Verma, Varun Mishra, Santosh Kumar Gupta, "Chargeplasma based dual metal gate recessed source/drain dopingless junctionless transistor for enhanced analog and RF performances”, Journal of Computational Electronics, 2020 (recently accepted).

[32] Varun Mishra, Yogesh Kumar Verma, Prateek Kishor Verma, Santosh Kumar Gupta, "Ferroelectric dual material gate all around TFET architecture for enhanced electrical performance", 15th IEEE India Council International Conference (INDICON), IEEE, Coimbatore (Tamil Nadu), December 16-18, 2018.

[33] Yogesh Kumar Verma, Rajeev Kumar Chauhan, "Reliability issues of SiGe HBTs", National Conference, Advances in Computer Communications and Embedded Systems (ACCES-2012), Madan Mohan Malaviya Engineering College Gorakhpur, UP, India, 2012.

[34] Yogesh Kumar Verma, Santosh Kumar Gupta, "Reliability Analysis of SiGe Heterojunction bipolar transistor", Asian Journal for Convergence in Technology (AJCT), vol. 4, no. I, 2018, http://asianssr.org/index.php/ajct/article/view/584.

[35] Yogesh Kumar Verma, Santosh Kumar Gupta, Rajeev Kumar Chauhan, Prateek Kishor Verma, "Effect of temperature on linearity and distortion parameters of gate all around AlGaN/GaN high electron mobility transistor", International Conference on Communication Computing and Signal Processing (CCSP-2020), Dr. B.R. Ambedkar National Institute of Technology, Jalandhar, Punjab (accepted). 


\title{
18 SAR based best designs for wearable antennas
}

\author{
A review \\ Danvir Mandal ${ }^{1}$, S.S. Pattnaik ${ }^{2}$ \\ ${ }^{1}$ Lovely Professional University, Phagwara, Punjab, India \\ ${ }^{2}$ National Institute of Technical Teachers Training and Research, \\ Chandigarh, India
}

\section{Introduction}

In the last two decades, wearable antennas have gained attention of the researchers. The main reason for this attention is the future technology that demands use of wearable devices, which include sensors and routine gadgets. These wearable devices require special antennas which need to be integrated in such a way that the health hazard to the wearer can be minimized. In the current scenario, antennas for firefighters, body area networks and medical applications have been designed by the researchers to fulfil the need of communication in wearable applications [1-3]. Antennas based on metamaterial structure have been presented in [4] and [5]. Different substrates like jeans and electromagnetic band-gap (EBG) material have been used for wearable antennas [6,7]. Other wearable antennas for different applications have been presented in [8-14]. Two multiband antennas fabricated on polyester cloth are designed and presented in [15] and [16]. In Section 2, different wearable antenna are considered and compared on the basis of SAR values. The work in concluded in Section 3.

\section{Wearable antennas}

Different wearable antennas have been proposed and presented in the last few years. Broadly, they can be classified into the following categories:

i. Wearable patch antennas

ii. Wearable antennas loaded with metamaterial structure

iii. Coplanar waveguide (CPW) fed wearable antennas

iv. EBG structure based wearable antennas

On the basis of classes mentioned above, each type of wearable antenna is explained further in detail.

\subsection{Wearable patch antennas}

The substrates that have been used for wearable patch antennas have a wide range. The main substrates that were employed for the designing of the wearable patch antennas are flexible foam [1], felt [4], jeans [6,9], polydimethylsiloxane [11] and polyester cloth

DOI: $10.1201 / 9781003129103-18$ 
Table 18.1 Comparison of SAR values for wearable patch antennas

\begin{tabular}{lll}
\hline Antenna & Resonant frequency $(\mathrm{GHz})$ & SAR $(\mathrm{W} / \mathrm{kg})$ \\
\hline Antenna [1] & 2.400 & $<4.00000$ (localized value) \\
Antenna [4] & 2.448 & 0.05000 \\
Antenna [6] & 5.800 & 0.00436 \\
Antenna [8] & 0.430 & 0.05000 \\
Antenna [9] & 0.900 & 0.00110 \\
& 1.800 & 0.00350 \\
Antenna [11] & 2.450 & 0.24800 \\
& 5.800 & 0.09100 \\
Antenna [15] & 1.800 & 0.12100 \\
& 2.400 & 0.41100 \\
& 3.600 & 0.87400 \\
& 5.500 & 0.95400 \\
\hline
\end{tabular}

Table 18.2 Comparison of SAR values for CPW fed wearable antennas

\begin{tabular}{lll}
\hline Antenna & Resonant frequency $(\mathrm{GHz})$ & SAR $(\mathrm{W} / \mathrm{kg})$ \\
\hline Antenna [10] & 5.200 & 0.06460 \\
& 5.800 & 0.02580 \\
Antenna [15] & 1.780 & 0.22930 \\
& 2.400 & 0.38920 \\
& 3.460 & 0.46400 \\
& 5.260 & 0.25350 \\
\hline
\end{tabular}

[15]. The wearable patch antennas are compared on the basis of their SAR values and are presented in Table 18.1.

Table 18.1 revealed that minimum SAR value of $0.0011 \mathrm{~W} / \mathrm{kg}$ at $0.9 \mathrm{GHz}$ was provided by wearable antenna presented in [9]. The patch of the wearable antenna presented in [9] has a polygon shape. To achieve the dual band operation, the patch was slotted in circular fashion and slits were introduced in the patch. Jeans substrate was used to fabricate the antenna.

\subsection{Wearable antennas loaded with metamaterial structures}

Two wearable antennas based on metamaterial structures [4,5] have been considered and compared. In [4], the designers have computed the SAR values which are presented in Table 18.1. However, in [5], SAR values are not computed by the designers.

\subsection{CPW fed wearable antennas}

In this review work, we have considered two wearable antennas which are fed by CPW. Their SAR values are compared and presented in Table 18.2. The minimum SAR values of 0.0646 and $0.0258 \mathrm{~W} / \mathrm{kg}$ at $5.2 \mathrm{GHz}$ and $5.8 \mathrm{GHz}$, respectively, were obtained by the antenna proposed in [10]. Rogers RO4003 substrate was used for the fabrication of the antenna. The antenna exhibited dual band operation. 
Table 18.3 Comparison of SAR values for EBG based wearable antennas

\begin{tabular}{lll}
\hline Antenna & Resonant frequency $(\mathrm{GHz})$ & SAR $(\mathrm{W} / \mathrm{kg})$ \\
\hline Antenna [2] & 2.450 & 0.23000 \\
Antenna [3] & 2.400 & 0.01380 \\
Antenna [7] & 1.800 & 0.31000 \\
& 2.450 & 0.04300 \\
& 5.800 & 0.09000 \\
\hline
\end{tabular}

\subsection{EBG structure based wearable antennas}

Three wearable antennas with EBG structure have been considered and their SAR values are presented in Table 18.3.

The EBG structure based antenna presented in [3] has shown minimum SAR value of $0.0138 \mathrm{~W} / \mathrm{kg}$ at $2.4 \mathrm{GHz}$. The antenna proposed in [3] was fabricated on denim material.

\section{Conclusion}

The designs of the different wearable antennas are reviewed and their corresponding SAR values have been compared and presented. The comparison of the antennas revealed that the wearable patch antennas and EBG based antennas provide smaller SAR values as compared to CPW fed wearable antennas. But it is observed that CPW fed wearable antennas can easily provide multiband operation with low SAR values.

\section{References}

[1] Hertleer, C., H. Rogier, L. Vallozzi and L. V. Langenhove. 2009. A textile antenna for offbody communication integrated into protective clothing for firefighters. IEEE Transactions on Antennas and Propagation 57, no. 4: 919-925.

[2] Gao, G. P., B. Hu, S. F. Wang and C. Yang. 2018. Wearable circular ring slot antenna with EBG structure for wireless body area network. IEEE Antennas and Wireless Propagation Letters 17, no. 3: 434-437.

[3] Ashyap, A.Y.I., Z. Z. Abidin, S. H. Dahlan, H. A. Majid, S.M. Shah, M. R. Kamarudin and A. Alomainy. 2017. Compact and low-profile textile EBG-based antenna for wearable medical applications. IEEE Antennas and Wireless Propagation Letters 16: 2550-2553.

[4] Yan, S., and G. A. E. Vandenbosch. 2016. Radiation pattern reconfigurable wearable antenna based on metamaterial structure. IEEE Antennas and Wireless Propagation Letters 15: $1715-1718$.

[5] Joshi, J. G., S. S. Pattnaik and S. Devi. 2012. Metamaterial embedded wearable rectangular microstrip patch antenna. International Journal of Antennas and Propagation, Article ID 974315: 9 pages.

[6] Ahmed, M. I., M. F. Ahmed and A. E. A. Shaalan. 2018. Novel electrotextile patch antenna on jeans substrate for wearable applications. Progress In Electromagnetics Research C 83: 255.

[7] Zhu, S., and R. Langley. 2009. Dual-band wearable textile antenna on an EBG Substrate. IEEE Transactions on Antennas and Propagation 57, no. 4: 926-935.

[8] Wang, H., Z. Zhang, Y. Li and Z. Feng. 2013. A dual-resonant shorted patch antenna for wearable application in $430 \mathrm{MHz}$ band. IEEE Transactions on Antennas and Propagation 61, no. 12: 6195-6200. 
[9] Sundarsingh, E. F., S. Velan, M. Kanagasabai, A. K. Sarma, C. Raviteja and M. G. N. Alsath. 2014. Polygon-shaped slotted dual-band antenna for wearable applications. IEEE Antennas and Wireless Propagation Letters 13: 611-614.

[10] Yang, H., W. Yao, Y. Yi, X. Huang, S. Wu and B. Xiao. 2016. A dual-band low-profile metasurface-enabled wearable antenna for WLAN devices. Progress In Electromagnetics Research C 61: 115-125.

[11] Simorangkir, R. B. V. B., Y. Yang, L. Matekovits and K. P. Esselle. 2016. Dual-band dual-mode textile antenna on PDMS substrate for body-centric communications. IEEE Antennas and Wireless Propagation Letters 16: 677-680.

[12] Liu, B., J. Han, S. Hu and L. Zhang. 2015. Novel multiband metal-rimmed antenna for wearable applications. International Journal of Antennas and Propagation 2015, Article ID 319894: 7 pages.

[13] Klemm, M., I. Z. Kovcs, G. F. Pedersen and G. Troster. 2005. Novel small-size directional antenna for UWB WBAN/WPAN applications. IEEE Transactions on Antennas and Propagation 53, no. 12: 3884-3896.

[14] Islam, M. R., and M. Ali. 2013. A 900 MHz beam steering parasitic antenna array for wearable wireless applications. IEEE Transactions on Antennas and Propagation 61, no. 9: 4520-4527.

[15] Mandal, D., and S. S. Pattnaik. 2018. Quad-band wearable slot antenna with low SAR values for $1.8 \mathrm{GHz}$ DCS, $2.4 \mathrm{GHz}$ WLAN and $3.6 / 5.5 \mathrm{GHz}$ WiMAX applications. Progress In Electromagnetics Research B 81: 163-182.

[16] Mandal, D., and S. S. Pattnaik. 2019. Wide CPW-fed multiband wearable monopole antenna with extended grounds for GSM/WLAN/WiMAX applications. International Journal of Antennas and Propagation, Article ID 4264513: 14 pages. 


\title{
19 Design and development of electronic precipitator for particulate matter air purification processes
}

\author{
Harleen Kour*, Deepti Sharma, Sachin Gupta
}

School of Electronics and Electrical Engineering, Lovely Professional University, Phagwara, Punjab, India

\section{Introduction}

Air pollution is a big problem for human kind for several decades [1]. The impurity in air is due to various sources regularly entering into it staying for too long time and sustaining for penetrating the human body too deep in body parts. The air pollutions are classified into broadly three categories. In the high level, the dust particles and toxic gasses are releasing directly from anthropogenic sources. These pollutants are very harmful to ecology where humans live [2]. To cure these issues, till now there has been a lack of engineering solutions from various research communities. They are seeking for solutions but there are a lot of challenges when the cost of the device, bio-compositional and electronic sensors for in depth challenge measurement, their installation and other factors are taken into consideration. The use of kuku-based plants and their extracts for absorption of pollution from ambient environment is a small solution to this problem, as per WHO and other pollution control boards. Even an odd system is also being implemented in Delhi city to cure the challenge [3]. As per technical considerations, due to this scheme, 6.8 metric cubic tons of pollution is getting stopped by this wonderful practice. The use of precipitation-based hoppers and electrostatic separating chambers has also been a big solution to it for a long time. Figures 19.1 and 19.2 shows the hoppers, which are extensively used only in industry where releasing is just taken into environment by chimneys. The semi platinum rods are one of the good alternates to build such high electro blunt charge which is assimilating the dust particles and sulphur ions from the caused air. The major challenge to use these technologies is installation of hoppers on economic scale [4,5]. They require minimum $600 \mathrm{kVA}$ supply to work for cleaning purpose. The other disadvantage of this challenge is to build such ground stations for generating the safety cures for humankind. The condensed hoppers are also another solution to this problem which are using economizers for such platforms [6]. A simple but effective way to treat air is a solo electric module to nourish it by such engineering marvels.

\section{Materials and methods}

To review such methods, sources are explored. The main task of the search is to explore best solutions proposed by industries for such challenges. We went through such methods which are useful and implementable. There are various electrical, mechanical and electronic

\footnotetext{
* Emails: harleenkour414@gmail.com, deeptigarg339@gmail.com, sachin.23305@lpu.co.in
}

DOI: 10.1201/9781003129103-19 


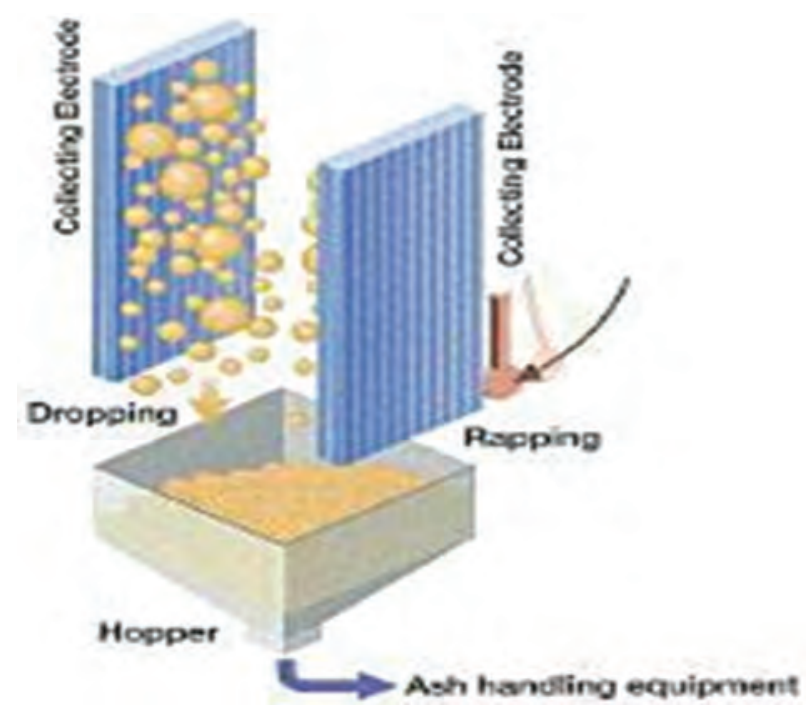

Figure 19.1 Electrostatic precipitator used in industry for treatment of dust particles.

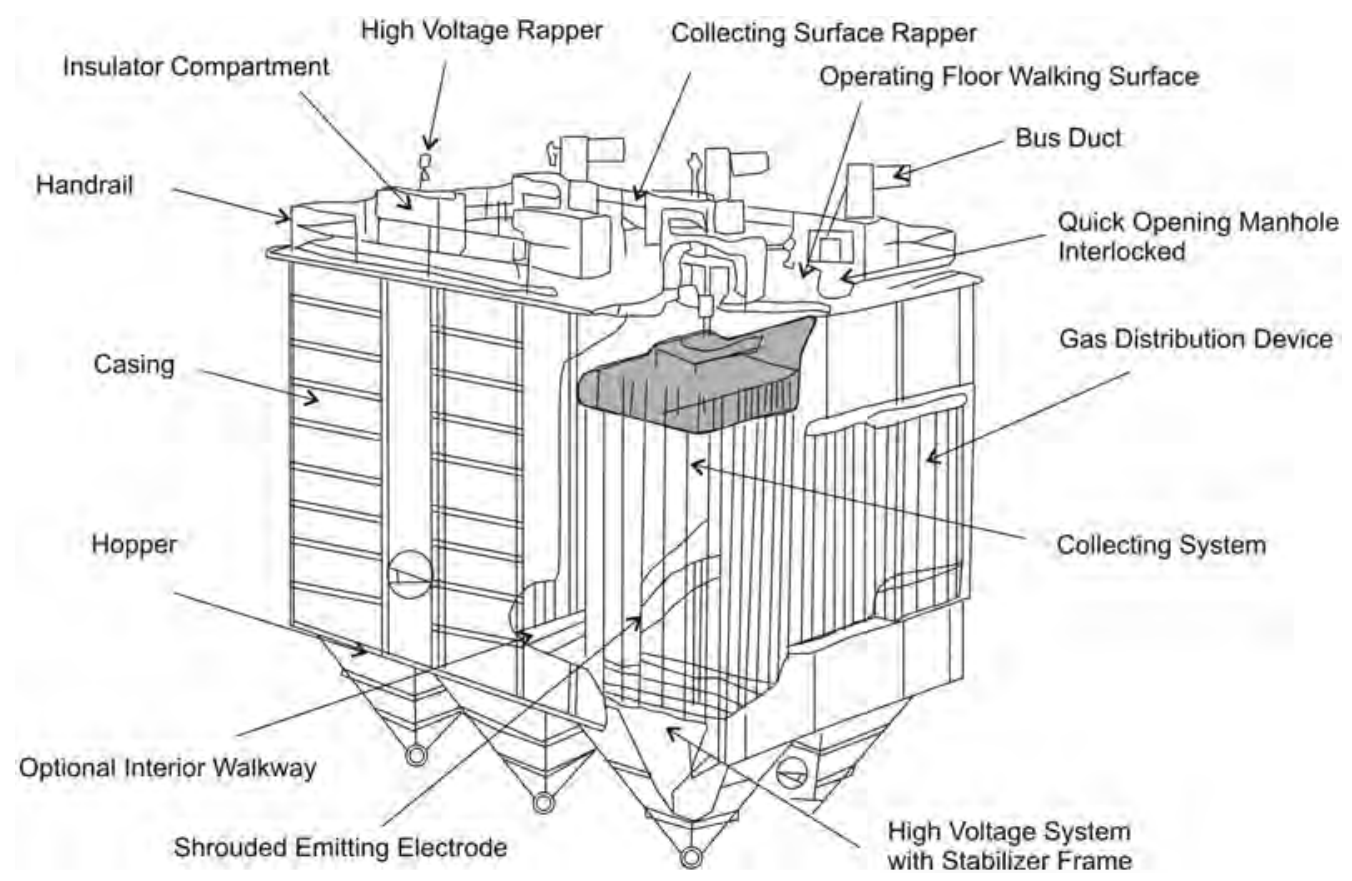

Figure 19.2 Generation of electrostatic charge in chambers for air cleaning. 


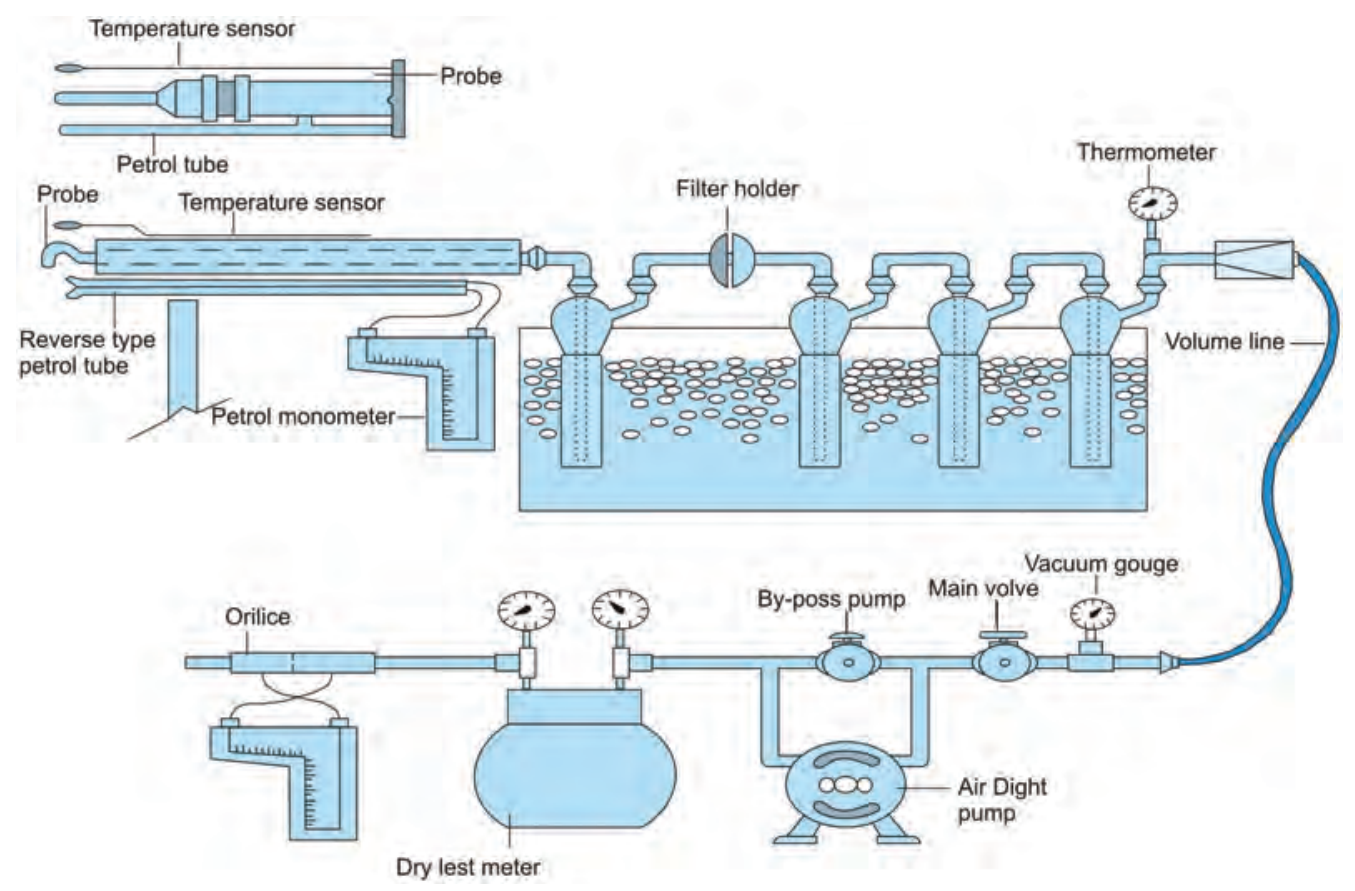

Figure 19.3 Schematic of air purification installation in industry by electronic precipitation.

precipitators which are constantly used by humans of improving contaminated pours of air. Figure 19.3 shows a basic process to treat air in the environment.

\section{Results and discussion}

In the modern time, with advances in technology, new biosensors and precipitation methods are coming into the market. The charcoal-based methods are prominently used in small and medium scale air pollution cleaners. Various methods are prominently used by consumers in their households. The electronic ion-based method which are the most advanced method given by researchers till now is a marvellous technique. Here, in such method positive and negative ions are generated. The positive ion plates are pushing darts and dust toward negative plate, whereas the negative ion plate attracts the ions forcefully sent by the positive ion selective plate toward it. The best example to showcase the example is the laptop screen. If we see the laptop screen [6,7], we can see the dust particles accumulating on it. The attraction of particles on the screen is just because of negative ions accumulating on it by forceful attraction given by ions. Figure 19.4 shows the mechanism of such medium and small-scale precipitators used extensively in medium and small-scale rooms and offices. In this method, the air has been gritted with pulp and other screen printed gets treated and then overlying on the purifying gel for maintaining of air quality [8].

As per local pollution control boards and their statistical inferences, the pollution control by these devises was a cumbersome and tedious method. With the invention of new methods of research it is now become safe to use these devices for purifications and retreating air for such purposes [7-9]. 


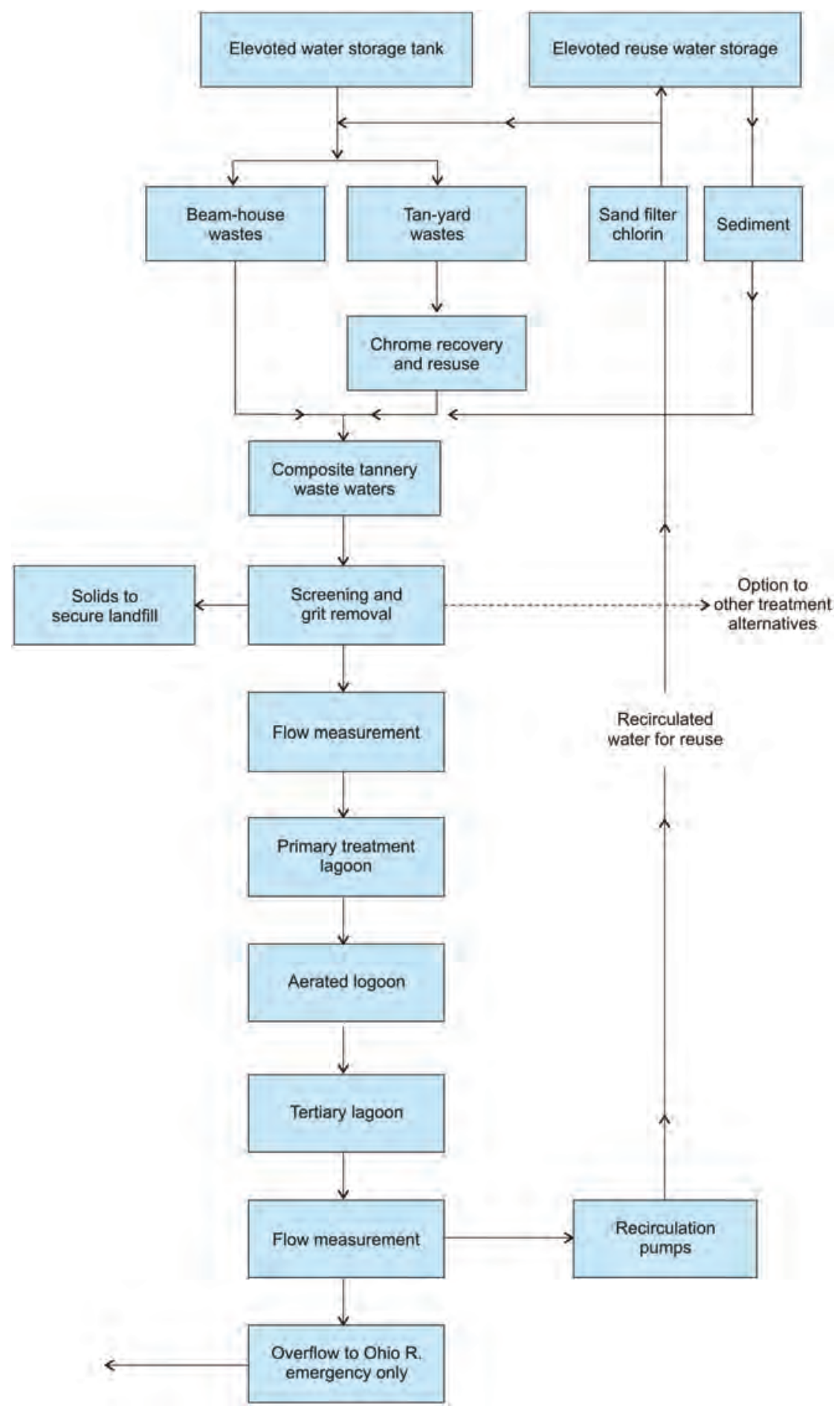

Figure 19.4 Ion based air purification methods and proposed technique. 


\section{Conclusions}

Air pollution is a big challenge for the research community and industry people for curing it and retreating it for use by ecological inhabitants. In the early days, the hoppers were too big and too noisy that the use is too difficult and the cost of such devices is very high. Electrostatic hoppers and ion based small scale hoppers are the biggest achievement by industry to cure the problem of clean air for ecological inhabitants. In future such methods will spark new ideas in young minds for originating such devices for personal use. With advances in biochemical based sensors, there is a need to embed such concepts in upcoming technologies for implementation [10].

\section{Acknowledgements}

We are very graceful to Prof. Bhupinder Verma and Prof. Neelam Verma from Lovely Professional University for their two-day workshop on biosensors, where we got the directions to explore such machines and engineering solutions for air pollution and other challenges.

\section{References}

[1] Yang, Li, and Miao Ye. "CFD simulation research on residential indoor air quality." Science of the Total Environment 472 (2014): 1137-1144.

[2] Kovats, R. Sari, Andrew Haines, Rosalind Stanwell-Smith, Pim Martens, Bettina Menne, and Roberto Bertollini. "Climate change and human health in Europe." Bmj 318, no. 7199 (1999): 1682-1685.

[3] Gammage, Richard B. Indoor Air and Human Health. CRC Press, 1996.

[4] Fuzzi, Sandro, Urs Baltensperger, Ken Carslaw, Stefano Decesari, Hugo Denier van der Gon, Maria C. Facchini, David Fowler et al. "Particulate matter, air quality and climate: lessons learned and future needs." Atmospheric Chemistry and Physics 15, no. 14 (2015): 8217-8299.

[5] Yu, B. F., Z. B. Hu, M. Liu, H. L. Yang, Q. X. Kong, and Y. H. Liu. "Review of research on airconditioning systems and indoor air quality control for human health." International Journal of Refrigeration 32, no. 1 (2009): 3-20.

[6] Duflo, Esther, Michael Greenstone, and Rema Hanna. "Indoor air pollution, health and economic well-being." SAPI EN. S. Surveys and Perspectives Integrating Environment and Society 1 , no. 1 (2008).

[7] Lave, Lester B., and Eugene P. Seskin. Air Pollution and Human Health. RFF Press, 2013.

[8] Bush, Judith, Suzanne Moffatt, and Christine Dunn. "'Even the birds round here cough' stigma, air pollution and health in Teesside." Health \& Place 7, no. 1 (2001): 47-56.

[9] Clausen, Geo, Gabriel Bekö, Richard L. Corsi, Lars Gunnarsen, W. W. Nazaroff, Bjarne W. Olesen, Torben Sigsgaard, Jan Sundell, Jørn Toftum, and Charles J. Weschler. "Reflections on the state of research: indoor environmental quality.” Indoor Air 21, no. 3 (2011): 219-230.

[10] Franceschini, F., M. Galetto, and D. Maisano. "A short survey on air quality indicators: properties, use, and (mis)use." Management of Environmental Quality: An International Journal 16, no. 5 (2005): 490-504. 


\title{
20 A critical survey on vertical handoff algorithms
}

\author{
Keshav Jha ${ }^{1, *}$, Akhil Gupta,* \\ ${ }^{1}$ Punjab University, Chandigarh, India \\ ${ }^{1,2}$ School of Electrical and Electronics Engineering, Lovely Professional \\ University, Phagwara, India
}

\section{Introduction}

At present a day's next generation remote framework is giving numerous entrance systems to the client. So the versatile client can get to a wide scope of utilizations given by various remote systems. So when the portable client moves starting with one spot then onto the next there is a need to handover the correspondence channel starting with one system then onto the next by considering its highlights and client necessities [1]. Versatility is the highest significant part of a remote cell communication structure.

\section{A. Types of handoffs}

In $5 \mathrm{G}$ systems, the handoffs are characterized into two standards.

\section{Horizontal handover}

The process of handoff between two base stations (BSs) of the same structure is known as a horizontal handoff. Horizontal handoffs include a terminal gadget to replace cells to keep the administration of a similar type of network (e.g., inside the UMTS system).

\section{Vertical handoff (VHO)}

Vertical handoff means that there is a framework centre that converts like a system to achieve a supporting foundation, which usually helps with centre mobility. For example, an intelligently organized PC may have the option of using both an accelerated remote LAN and cell development for the Internet.

\section{B. Research framework}

With the help of reviewed composition, various kinds of scholars use various kinds of terms, for example, to manage models, frameworks, and award parts. To assemble a VHO instrument for a $5 \mathrm{G}$ remote framework, it is fundamental to think about the existing VHO segments. The first type of approach relies on the "Gotten Signal Strength (GSQ)" that can meet with different approaches, for example, the composition of weights and outline costs. The second kind of process is using the framework of mechanized thinking.

\footnotetext{
*Emails: keshavjha775@gmail.com, akhilgupta112001@gmail.com
}

DOI: $10.1201 / 9781003129103-20$ 


\section{4}

Intelligent Circuits and Systems

\section{Handover management process}

The structure of the paper is as follows: Section II gives handovers to the authorities, Section III requests vertical handover estimates, for example, RSS-based, bandwidthbased, cost-based, combination-based, confirmation-based and MADV-based calculations.

\section{Handover information gathering}

First, arrange the handover, get the handover information, organize the assembly mastermind information together and besides information about the rest of the system, for example, properties, mobile phone, manner and customer trend.

\section{Make handover choice}

The second arrangement in the handover, organizing handover decisions, is one of the most fundamental methods during handover.

\section{Handover completion}

The third arrangement in the handover, this stage plays handover itself; in addition to playing handover, the stage should guarantee a smooth session progress process. The VHO shows up in solicitation, and IP boards to play together to control the particular handover function. This step is commonly known as the handover execution.

\section{Grouping of VHD calculations}

\section{A. RSS estimation calculations}

In this gathering, RSS is used as the primary handover choice foundation. The RSS-based VHD calculation looks at the RSS's current purpose of connection against others to compromise on handover options.

\section{An adaptive lifetime-based handover estimator}

The common lifetime criteria (expected range beyond which the convenient node will not have the option to have its relationship with the WLAN) or by combining RSS estimates with the range of open information is presented for handover between the $3 \mathrm{G}$ framework and the WLAN candidate. The preferred condition of this count is that it gives upgrades to open information speed limits. Its drawback is prolonged avoidance and additional questions cell .

\section{An RSS threshold-based dynamic heuristic}

The common lifetime metric (expected range beyond which the convenient terminal will not have the option to have its relationship with the WLAN) or by combining RSS estimates with the range of open information is proposed for handover between the $3 \mathrm{G}$ framework and the WLAN candidate. 


\section{A travel distance prediction based estimate}

To remove unwanted handovers in the procedure demonstrated in the last paragraph [2], a VHD computation has been developed, indicating the time when the versatile terminal is dependent on the WLAN cell to be spent. The technique takes care of the calculation of the WLAN travel time (for example the time that the compact terminal is spent inside the WLAN cell) and a duration range.

\section{B. Bandwidth estimation systems}

This area depicts existing data transfer capacity estimation procedures and begins to eliminate methods to assess the range and accessible transfer speeds in individual leaps. It is focused on four important processes: variable packet size (VPS) checking, packet pair/ train dispersion (PPTD), self-loading periodic currents (SLoPS) and trains of parcel pairs (TOPP).

\section{Packet pair check}

Through the evaluation and the point of imprisonment, the source sends a diverse bundle set to the beneficiary. The extent of dissolution experienced by the package coordinates is revealed through the beneficiary and the breaking point. Various package sets are sent to exclude the effect of cross-traffic on the estimation of the far point and authentic techniques are used to filter through mixed estimates.

\section{VPS check}

In some cases, points of imprisonment of different skips are examined, different bundles of a given size are sent. This technique can see the packs ending on a particular skip using the TTL field of the IP header. RTT sources the ICMP bot messages received from changes to make that skip.

\section{Self-loading periodic currents}

In [3] the SLOPS measures the available transmission range by all means that a course of action of similarly rated bundled test trains is sent at a specific rate. Again, if the spouting rate is less than the open move speed, the single heading delay of the test pack will not increase.

\section{Cost function estimation calculations}

These computations have a mixture of profiling and DE factors, for example, RSS, local, organization costs, open exchange speed, stable quality, battery control, security, and DE transportability models, etc., and these factors are covered. Describes a cost range subject to. Survey the performance of the target framework.

\section{Cost function-based strategies (CFBS)}

The vertical handoff choice cost limit is an estimate of the amount of bitcoin to be derived from presenting a typical framework. It is calculated for every framework that comes under the customer's organization district. The weighted range is included with unequal 
parameters. The framework that is reliably solved for the most negligible monetary policy is taken as the goal framework.

\section{Based on customer preference in dissimilar kinds of remote access networks}

The presented vertical handoff choice depends on the customer's tendency. If the customer needs to use an application in which the organization's high gauge is of yet low value, the AP that has the most outrageous APQW may not be the best, because its APCW is intolerably low (i.e., the framework is very costly).

\section{Combination estimation calculations}

For example, blend assessments combine various parameters in the handover decision, which are used in calculating cost work. These sculptures depend on fake neural structures or padding causes. A VHD assessment is performed under a simulated neural framework (ANN) [4].

\section{E. Authentication estimation calculations}

In NGN, security is considered to be one of the most frequent testing issues initiated by a convenient framework organization. Customer facilitates the presence of genuine customers for convenient illegal customers. Therefore, the need is that the handover strategy is given a similar affirmation scheme of security. Besides, it should have the option of reducing approval delays during handover techniques. The confirmation system can be discussed in the following procedure:

1. The prime time of the handover system involves picking when necessary to replace the $\mathrm{AP}$, and expecting that this is the situation, which the AP must next relate to with the STA. A few moments can be added to this phase, anyway, fortunately, most remote LAN cards can do this without tearing the relationship correctly with the APs used.

2. The handover system has a zero-confirmation step over time, which is the inheritance of the WEP (wired equivalent privacy), the security configuration principal fixed in the 802.11 standards. This step typically provides no protection, and it takes an incredibly brief time.

3. The simultaneous stage connection is organized, in which the STA develops an intelligent relationship with the AP. The biggest task of this phase is to propagate the wired structure in such a way that the given STA will now have the option of coming through the new AP.

4. The system of true confirmation starts after the arrangement of the alliance. In this phase, the STA confirms itself.

5. Finally, STA and AP perform a four-way handshake, by which they confirm each other learning the session key, and they extract the new key from the common session key for different purposes.

\section{F. Multiple merit decision}

Given the nature of the framework assurance issue, MADM processes address a promising game plan that can be applied to the orchestrate assurance issue. The Logical Hierarchy (AHP) of the Leadership Process, the Kushaned Mathematical Level (FAHP) of the Leadership Process, the Logical Framework Process (ANP), and Mistake Sensible 
Framework. Procedure (FANP) and (B) MADM situational processes that fuse different systems, for example, the consummate game plan (TOPSIS), Dim Social Appraisal (GRA), Divimate to Ghagra Alternative (DIA), equivalent demand for qualitative models, strategies for the trend of weighting (MEW), direct induction weight (SAW), wicker and Mahalanobis distance [1].

\section{Analysis hierarchy process (AHP)}

Such calculations depend on division and world-victory. The issue of fundamental choice is divided into sub-issues, where each sub-issue is evaluated as a choice factor. By arranging the alternative arrangement, AHP finds the most ideal system.

\section{Grey relational analysis (GRA)}

This scientific calculation creates a dark relationship between the components (systems), one of them being the best quality properties. Along these lines, the remaining components are, moreover, evaluated against the correct arrangement. The alternative is to get the most notable score in a better way with this correct arrangement.

\section{The technique for order preference for equality by ideal solution (TOPSIS)}

Similarly to the GRA calculation, the TOPSIS calculation considers, also, the perfect answer for the performance correlation, as the best option closest to a correct arrangement, and as the most noticeable from such an arrangement. Think farthest.

\section{Simple summative weight (SAW)}

SAW calculations are often used when MCDM is implemented. The method consists of scoring each option, including the trait attributes repeated by its weight, to score the common choice, the highest score of the most ideal decision.

\section{Exponent with multiplier weight (MWE)}

MWE works in line with SAW calculations. To score a, generally speaking, option, it uses a weighted result of all attributes. Since this item does not have an upper part, it is sensible to think of the score against a correct arrangement.

\section{Handoff choice framing}

VHO Basic Leadership is a process in which accessible remote access systems are assessed. The result of such kind of functionality is the choice of a system through which a mobile node must be delivered taking into account the factors assembled during the framework disclosure phase. In the accompanying section is presented a subset of the theory that presents the currently existing handover system.

\section{A. Capacity-based choice calculation}

Such a methodology depends on usability capabilities. The objective is to engage with the best accessible system that enhances the goal function which is a weighted aggregate of the nature of governance (NOE), monetary policy, trust, proximity, and propensity and 


\section{8}

Intelligent Circuits and Systems

boundary factors. Kaundourakis et al. [9] presented a target task in which all the entertainment engaged due to the process of choosing to take an interest in the social opportunity of the parameters of information.

\section{B. Client-driven choice calculation}

These methodologies are primarily concerned with the arrangement for the client. Universally, we think that clients are primarily concerned about what is more, should mark their exchange between Noe and Cost. Ormond et al. [10] introduced a clientdriven mechanism for non-persistent data traffic. Customers arrange for easy remote access and exchange fees of each of them as a final notification of information moves In [6], Calvagna et al. figure out a customer-driven choice calculation that finishes the last client control over the choice of remote access that best accommodates his (her) contradictions.

\section{Various properties choice making (VPCM)}

The same way as incapacity based methods, this handoff system is in light of the meaning of utility capacities. Here, it is figured as a VPCM issue as it intends to choose an applicant to arrange from a set of accessible ones for various criteria. The most mainstream VPCM strategies are abridged by Chai et al. [11] and portrayed in the accompanying text.

Simple Additive Weighting (SAW): the general applicant systems values are arranged by a weighted aggregate of all the considered measurements. Every up-and-comer organizes I the value arranged by including the standardized commitments of each thought to be metric rij increased by the weight it is relegated wj. The chosen system is that system that boosts this score as follows:

$$
A *_{\text {SAW }}=\arg \max \sum \mathrm{w}_{\mathrm{j}} \mathrm{r}_{\mathrm{ij}}
$$

A technique for Order Preference by Similarity to Ideal Solution (TOPSIS): the chosen applicant system is the nearest factor to the most suitable answer which is reached by thinking about the high esteem for every criterion.

Analytic Hierarchy Process (AHP): breaks down the system determination issue into sub-issues that are given loads and assessed as choice factors.

Grey Relational Analysis (GRA): assembles a grey connection between different systems and places them to choose with the highest position. The GRA condition is characterized by explaining a positive complete system with positive components. A standardization process is required to manage profit and cost measurement and a grey relational coefficient (GRC) of each system is determined. GRC is the score that is assumed to accurately reflect the simulation between each accessible contestant. The chosen system is that which is like a perfect system [5].

Multiplicative Exponent Weighting (MEW): the value score of each system is controlled by the weighted result of the considered choice measurements as follows: $S_{i}=\pi r_{i j}{ }^{w j}$. The chosen system is one that increases the ratio of this value by the positive absolute system value. The entire system is known as an attribute, with each metric having the best features. In [7], AHP and GRA join to introduce a choice tool that selects the system that provides the best trade-off for client leanings, terms of administration, and system capabilities. In a case VHO calculation that combines SAW and AHP is presented. 


\section{Markov-based choice calculation}

Markov's choice estimations are movable procedures designed to streamline the issues where the age of choice follows a probability spread. In a case, the creators introduced a $\mathrm{VHO}$ choice calculation for dissimilar kinds of remote access systems. The issue is planned as a Markov decision procedure (MDP), where the connection remuneration capability is dependent on the QoS requirements of the applications. Similarly, it has a flagging cost capability that is related to handling load and the flagging overhead of vertical handover achievement. Its purpose is to increase the limited compensation to all. The potential for the probability of a Markovian state change is obtained to understand the following state.

\section{E. Fuzzy logic-based choice calculation}

The fuzzy justification manages uncertainty and is great for dealing with choice process issues. The reverse of such a depiction is its ability to check for uncertain information, for example, the conduct of the RSS, the stack or the BER. It usually joins other choice techniques to make the best decision. In a case, Horrich et al. [8] proposed an alcoholic various channels vertical handover calculation that relies on a fuzzy logic control (FLC).

\section{F. Game theoretic-based procedure for choice handling}

The ability to interface an MT at the same time for different remote access systems is one of the most important features in state-of-the-art systems dependent on the consensus of state-of-the-art innovations. Together, we give some models from writing. In a case, Niato et al. [12] presented an acceptable transmission capacity assignment calculation dependent on the liquidation game.

\section{G. Reputation-based choice handling}

Zekri et al. [13] present the use of a more emotional measure based on previous encounters and perceptions of customers in comparative settings. The paper effectively introduces the use of notoriety of systems as a familiar fulfilment reflector. The proposed notoriety framework data relies on the experience of notorious esteem of past customers worldwide and allows portable terminals to quickly settle on VHO options In [8], Tristian et al., a notoriety-based system presented the means of choice.

\section{H. Vertical handover assessment measurements}

In this section, the most delegate VHO assessment measurements utilized in the literature is quickly portrayed. VHO choice instruments might be assessed by estimating handoff latencies, number of handovers, $\mathrm{VHO}$ cost, $\mathrm{VHO}$ blocking rate, and the general data rate of a session over a portability scenario.

Delay: Signifies the length of the vertical handover procedure thinking about its three stages: data gathering, choice-making, execution stages.

The number of handovers: Lowering the number of handovers is for the most part wanted to abstain from ping-pong impacts and protect arranged assets.

Data rate: It is typically linked to handover to systems presenting a higher data rate.

VHO failure rate: It is because of wrong choices. For example, it happens when the objective system is no longer accessible or doesn't offer enough assets (for example over-burden). 


\section{Research challenges}

Future remote frameworks will be founded on heterogeneous remote get-to innovation. To give consistent administrations many moving challenges are to be unravelled.

SoS Strength of service challenges: Versatile terminals conveying constant and nonongoing traffic ought to be overhauled with ensured SOS strength of service or quality of service. To give the best system administration a few factors need to be taken into account.

TCP Performance challenges: When changing from low transmission capacity, high information rate system to high transfer speed, low information rate arrange TCP execution ought to be considered for the clog.

Safety challenges: As a result of the wide inclusion region when the delicate information is transmitted it ought to be moved in a verified way.

\section{Conclusion}

There are different approaches to arrange VHD calculations. In this paper, we have divided the VHD calculation into six assemblies that depend on the criteria of choice of handover and the strategies used to process them. Handover calculations quickly develop late as a result of customer leaning. Here we talk about signal quality, data transfer capability, costbased, combinational, validation, and MADM dependent calculations.

\section{References}

[1] Nilakshee Rajule, Bhavna Ambudkar, A. P. Dhande, Survey of vertical handover decision algorithms. International Journal of Innovations in Engineering and Technology (IJIET) 2(1) (2013).

[2] X. Yan, N. Mani, Y.A. S_Ekercioğlu, A traveling distance prediction based method to minimize unnecessary handovers from cellular networks to WLANs. IEEE Communications Letters 12(1) (2008): 14-16.

[3] Manish Jain, Constantinos Dovrolis, End-to-end available bandwidth: measurement methodology, dynamics, and relation with TCP throughput. IEEE/ACM Trans. Networks 11(4) (2003): 537-549.

[4] N. Nasser, S. Guizani, E. Al-Masri, Middleware vertical handoff manager: a neural networkbased solution. Proceedings of the 2007 IEEE International Conference on Communications (ICC’07), Glasgow, Scotland, June 2007, pp. 5671-5676.

[5] Mrs. Chandralekha, Dr. Prafulla Kumar Behera, Use of adaptive resonance theory for vertical handoff decision in heterogeneous wireless environment. International Journal of Recent Trends in Engineering 2(3) (2009).

[6] A. Calvagna, G. Di Modica, A user-centric analysis of vertical handovers. Proceedings of the Second ACM international workshop on Wireless mobile applications and services on WLAN hotspots. WMASH '04. New York: ACM (2004): 137-146.

[7] Q. Song, A. Jamalipour, A network selection mechanism for next-generation networks, in IEEE International Conference on. Communications 2 (2005): 1418-1422.

[8] R. Trestian, O. Ormond, G.-M. Muntean, Reputation-based network selection mechanism using game theory, Physical Communication (2011)..

[9] G. Koundourakis, D. Axiotis, M. Theologou, Network-based access selection in composite radio environments, in: IEEE, Wireless Communications and Networking Conference, March 2007, pp. 3877-3883.

[10] O. Ormond, P. Perry, J. Murphy, Network selection decision in wirelessheterogeneous networks, in: IEEE 16th International Symposium on Personal Indoor and Mobile Radio, Communications, vol. 4, September 2005, pp. 2680-2684. 
[11] R. Chai, W.-G. Zhou, Q.-B. Chen, L. Tang, A survey on vertical handoff decision for heterogeneous wireless networks, in: IEEE Youth Conference on Information, Computing and Telecommunication, September 2009, pp. 279-282.

[12] D. Niyato, E. Hossain, A cooperative game framework for bandwidth allocation in $4 g$ heterogeneous wireless networks, in: IEEE International Conference on Communications, ICC '06, vol. 9, June 2006, pp. 4357-4362.

[13] M. Zekri, B. Jouaber, D. Zeghlache, in: On the Use of Network QoS Reputation for Vertical Handover Decision Making, IEEE Globecom, Workshop on Advances in Communications and Networks (ACN 2010), vol. 12, Miami, Florida, USA, 2010, pp. 2006-2011. 


\title{
21 A circular monopole ultra-wideband antenna with a notch band at $5.8 \mathrm{GHz}$
}

\author{
Gurpreet Kumar, *, Daljeet Singh ${ }^{2}$, Rajeev Kumar ${ }^{3}$ \\ ${ }^{1,2}$ School of Electronics and Electrical Engineering, Lovely Professional \\ University, Punjab, India \\ ${ }^{3}$ Chitkara University Institute of Engineering and Technology, Chitkara \\ University, Punjab, India
}

\section{Introduction}

In 2002, the FCC by allocating a band, i.e., 3.1 GHz to10.6 GHz band for UWB systems, has made it an area of extensive research in the past decade. Initially, for UWB systems the antenna design with desired bandwidth is a challenge. So, many techniques such as using monopole antenna structures, employing CPW feeding, embedding parasitic elements and bevelling techniques, etc., have been utilized by the researchers [1-3]. Further, as there are so many other licensed bands, i.e., WLAN (5.15-5.825 GHz), HIPELAN/ $2(5-6.3 \mathrm{GHz})$ and C-band $(4-8 \mathrm{GHz})$, etc., already available in the prescribed bandwidth for UWB systems, hence it is also essential to avoid the interference from these mentioned bands while achieving the UWB operation [3-7]. In [7], many techniques for achieving notch band characteristics are presented. The antenna structures have been categorized into many types. For example, the antenna structures can be classified based on a technique used for achieving band notch operation, i.e., using slots and parasitic elements, inserting fractal structures, embedding metamaterials, and EBG structures for static notch band characteristics and via switches for achieving reconfigurable band-reject characteristics. Further, the antennas can also be classified based on the number of notch bands achieved, i.e., single notch antenna, dual notch antenna, triple notch antenna and quad notch antenna $[7,8]$.

Band notch operation in the UWB antenna should not affect the other parameters essential for UWB systems, e.g., the gain of the antenna should not change drastically throughout the UWB bandwidth. In addition, the antenna should also possess an omnidirectional radiation pattern. These challenges have been addressed in the proposed design and the measured as well as simulated results show good agreement for UWB. This paper is organized into different sections. Section I represents the introduction to the UWB antennas. Section II represents the design geometry of the designed antenna. Further, Section III gives the results and discussion, and lastly Section IV presents the conclusion.

\section{Design outline}

As mentioned above, the monopole antenna structures provide higher bandwidth as associated with the traditional antenna structures. Hence, a compact planar monopole antenna has been designed as represented in Figure 21.1. The radiation properties of

\footnotetext{
* Corresponding author: Gurpreet.14632@lpu.co.in
}

DOI: $10.1201 / 9781003129103-21$ 


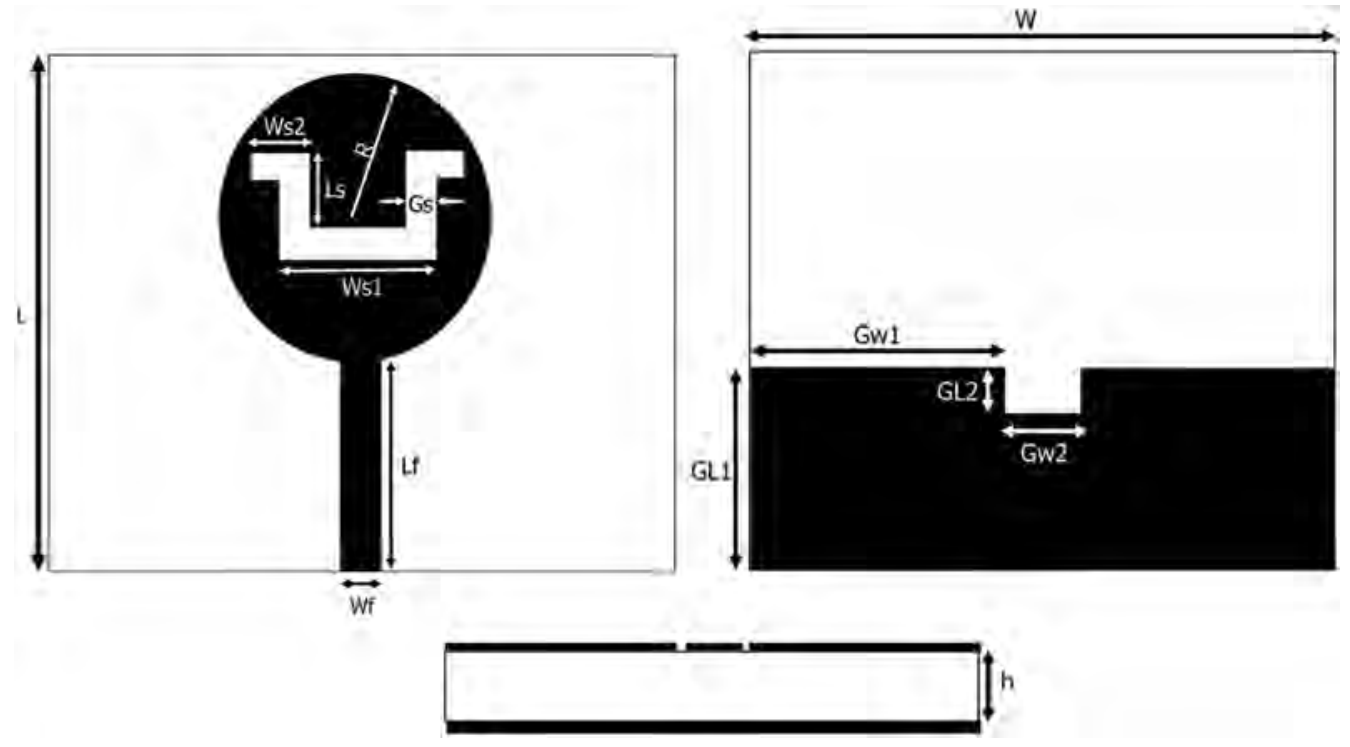

Figure 21.1 Design outline of the proposed antenna.

Table 21.1 Final design dimensions in $\mathrm{mm}$

\begin{tabular}{llllllllllllll}
\hline R & 9 & Ws2 & 3.5 & Gw1 & 12.5 & Wf & 1.6 & Lf & 9 & GL1 & 9 & Ws1 & 11 \\
Gs & 2 & Ls & 6 & Gw2 & 5 & L & 30 & GL2 & 2 & W & 30 & h & 1.6 \\
\hline
\end{tabular}

the structure have been further enhanced by using a circular radiator and modifying the ground. The radius of the circular patch has been adjusted to attain the desired bandwidth and also the ground has been modified so as to achieve enhanced radiation properties. The antenna utilizes the FR4 substrate and the overall volume of the structure is $30 \times 30 \times 1.6 \mathrm{~mm}^{3}$. For achieving the required notch band, a modified U-shape slot is engraved on the radiator. The positioning as well as the dimensions of the slot have been altered for achieving Wi-MAX $(5.8 \mathrm{GHz})$ notched band. Table 21.1 is used to present the various design parameters for the proposed design.

\section{Results and discussions}

As presented in Figure 21.2, the proposed UWB antenna bandwidth of $7.19 \mathrm{GHz}$ is achieved from $3.43 \mathrm{GHz}$ to $10.62 \mathrm{GHz}$. Further, the notch band, i.e., $5.07 \mathrm{GHz}$ to 6.54 $\mathrm{GHz}$ with a centre reject frequency of $5.78 \mathrm{GHz}$ and a small variation of $0.02 \mathrm{GHz}$, is observed in the simulated results. The radius of the circular radiator along with the dimensions of ground structure are customised so as to attain the mentioned results.

While modifying the antenna structure for notch operation, in order to get the best results, the other required parameters for UWB operation are also observed precisely. Figure 21.3 presents the surface current distribution at different frequencies for the given antenna. This can be viewed that the current concentration is maximum at the modified U-slot intended for the centre notch frequency, i.e., 5.78 GHz. Due to this, the mentioned notch 


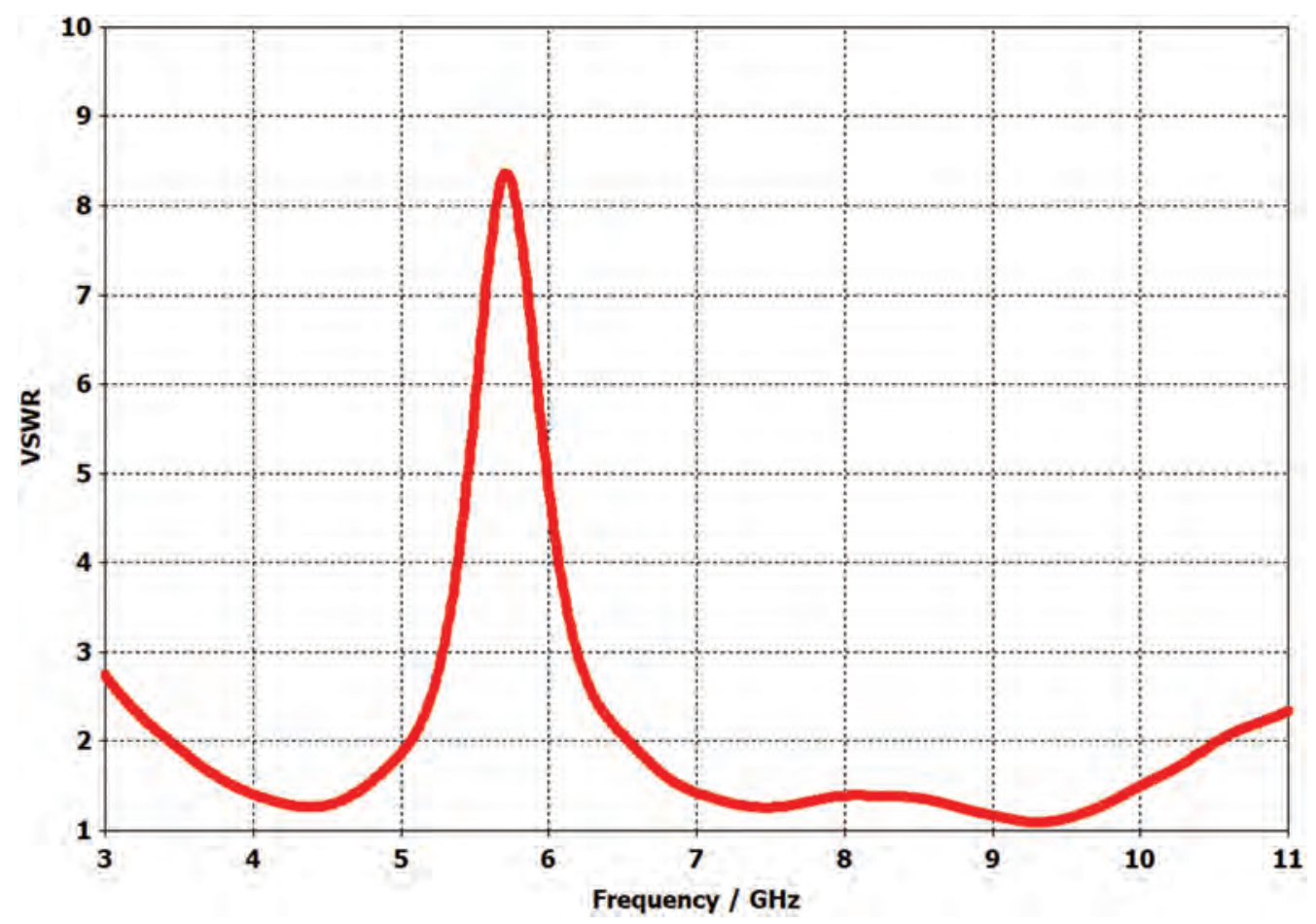

Figure 21.2 VSWR plot.

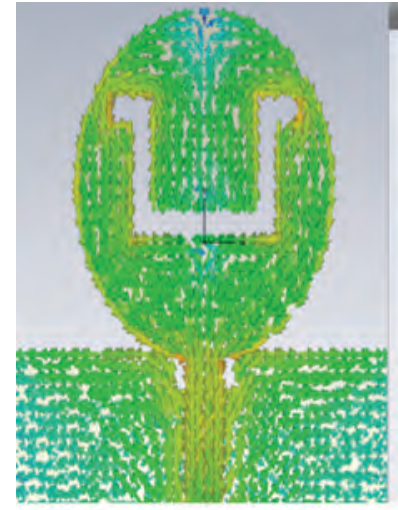

(a)

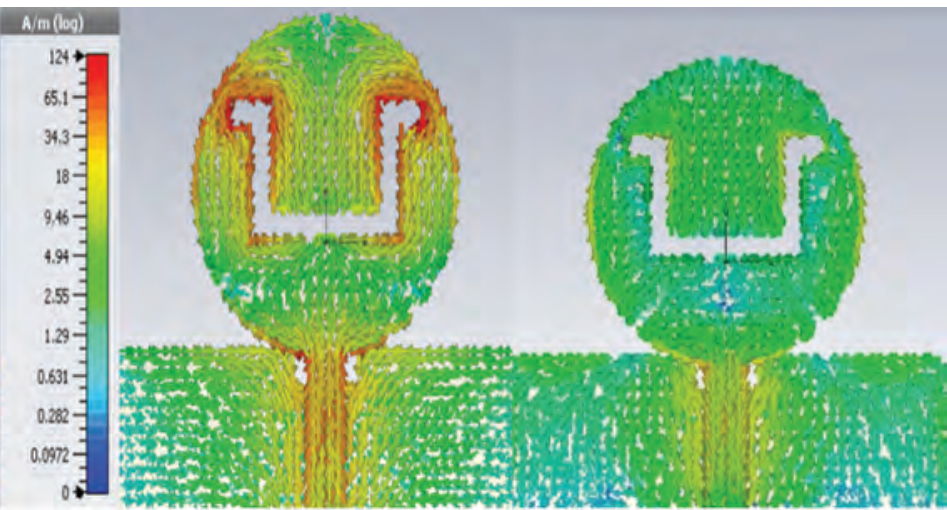

(b) (c)

Figure 21.3 Surface current distribution of the proposed antenna at different frequencies: (a) 3.7 $\mathrm{GHz}$, (b) $5.78 \mathrm{GHz}$, (c) $7.5 \mathrm{GHz}$.

frequency is completely suppressed by the engraved notch band structure. Further, for frequencies except for the notch frequency, the current distribution is similar, i.e., all over the radiator, feedline and the ground. This, in turn, gives the radiation from the antenna.

The prototype of the antenna, as well as its measurement setup, is presented in Figure 21.4. An MS46322A (Anritsu) vector network analyser having a range of $1 \mathrm{MHz}$ 


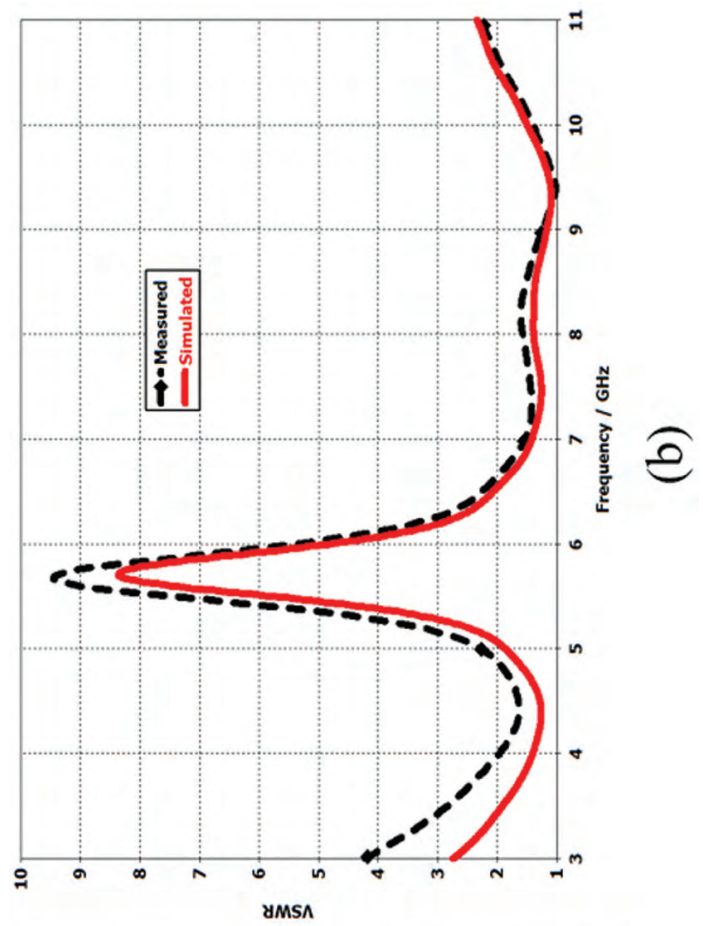

D
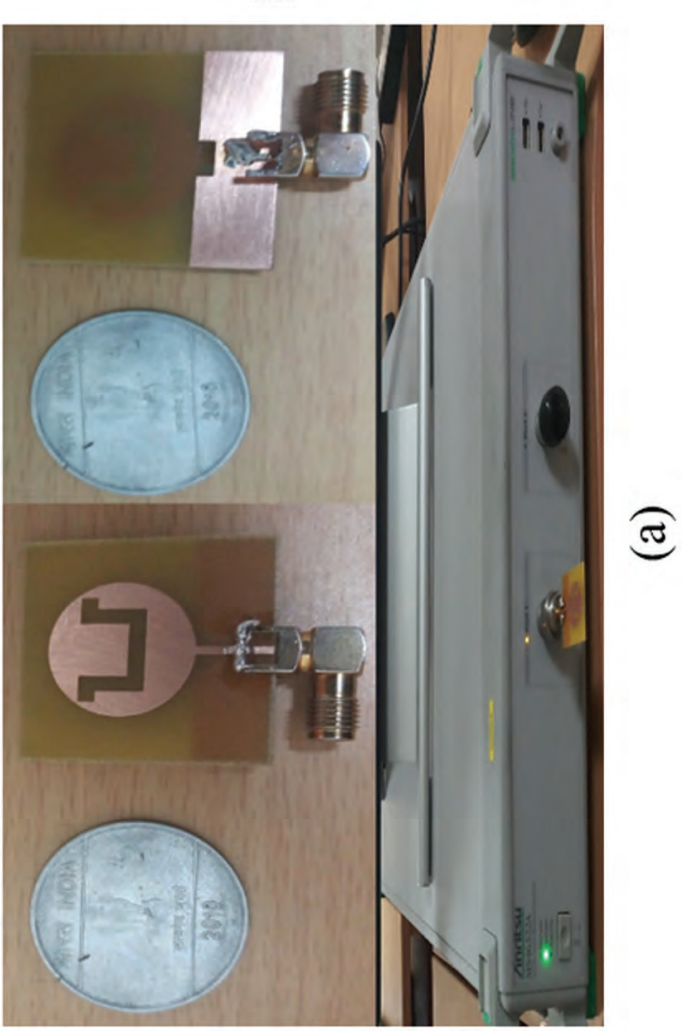

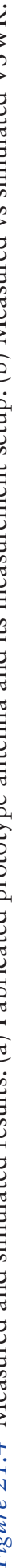



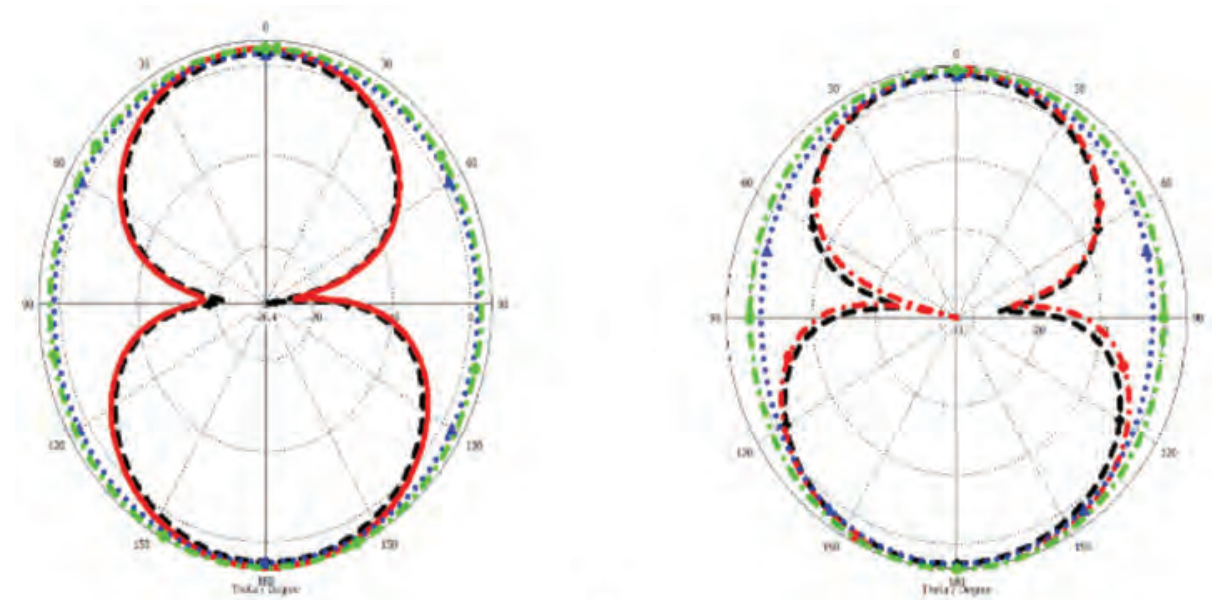

(a)

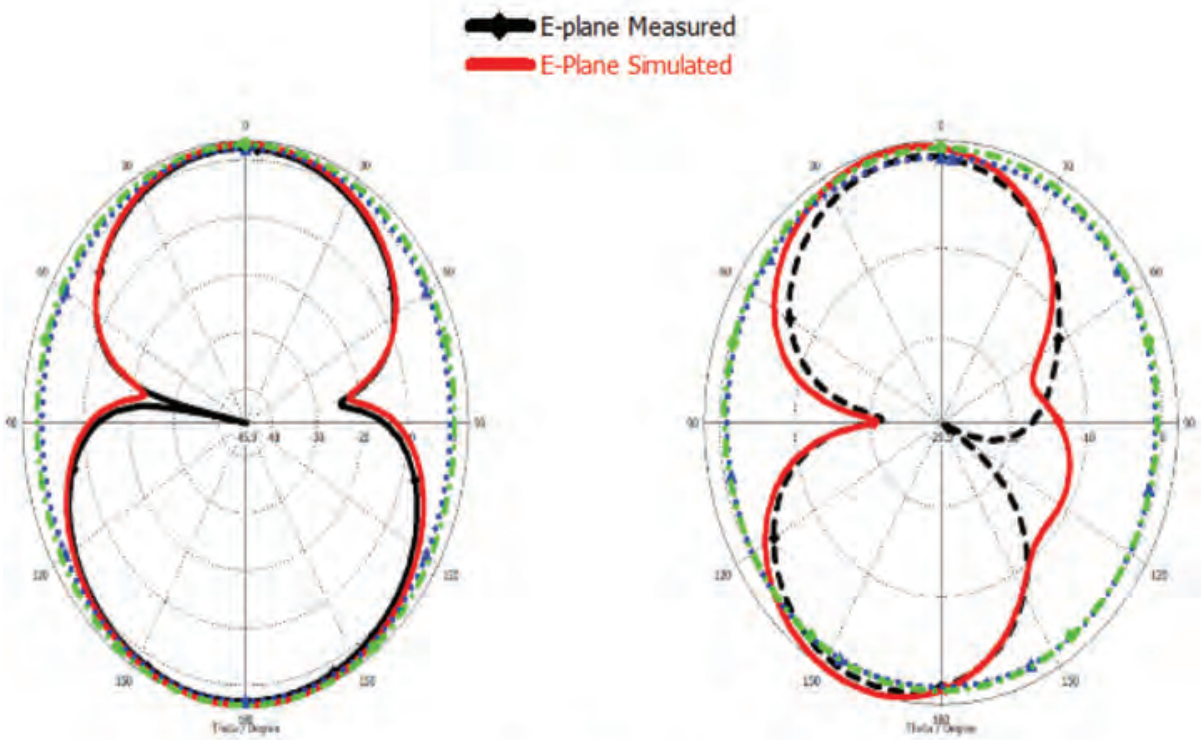

(b)

- $\mathbf{A}$ - H-Plane Measured

- H Plane Simulated

Figure 21.5 Field pattern at different frequencies. (a) $3.6 \mathrm{GHz}$, (b) $5.07 \mathrm{GHz}$, (c) $4.80 \mathrm{GHz}$, (d) $6.54 \mathrm{GHz}$.

to $20 \mathrm{GHz}$ is used for the measurement of group delay and VSWR. Further, for measurement of the gain along with radiation pattern, the antenna was positioned in the anechoic chamber with a distance which is more than the minimum far-field distance from the reference antenna.

As depicted in Figure 21.4, the measured and simulated VSWR show suitable agreement with each other. The lower and higher frequency for UWB operation is slightly shifted to 


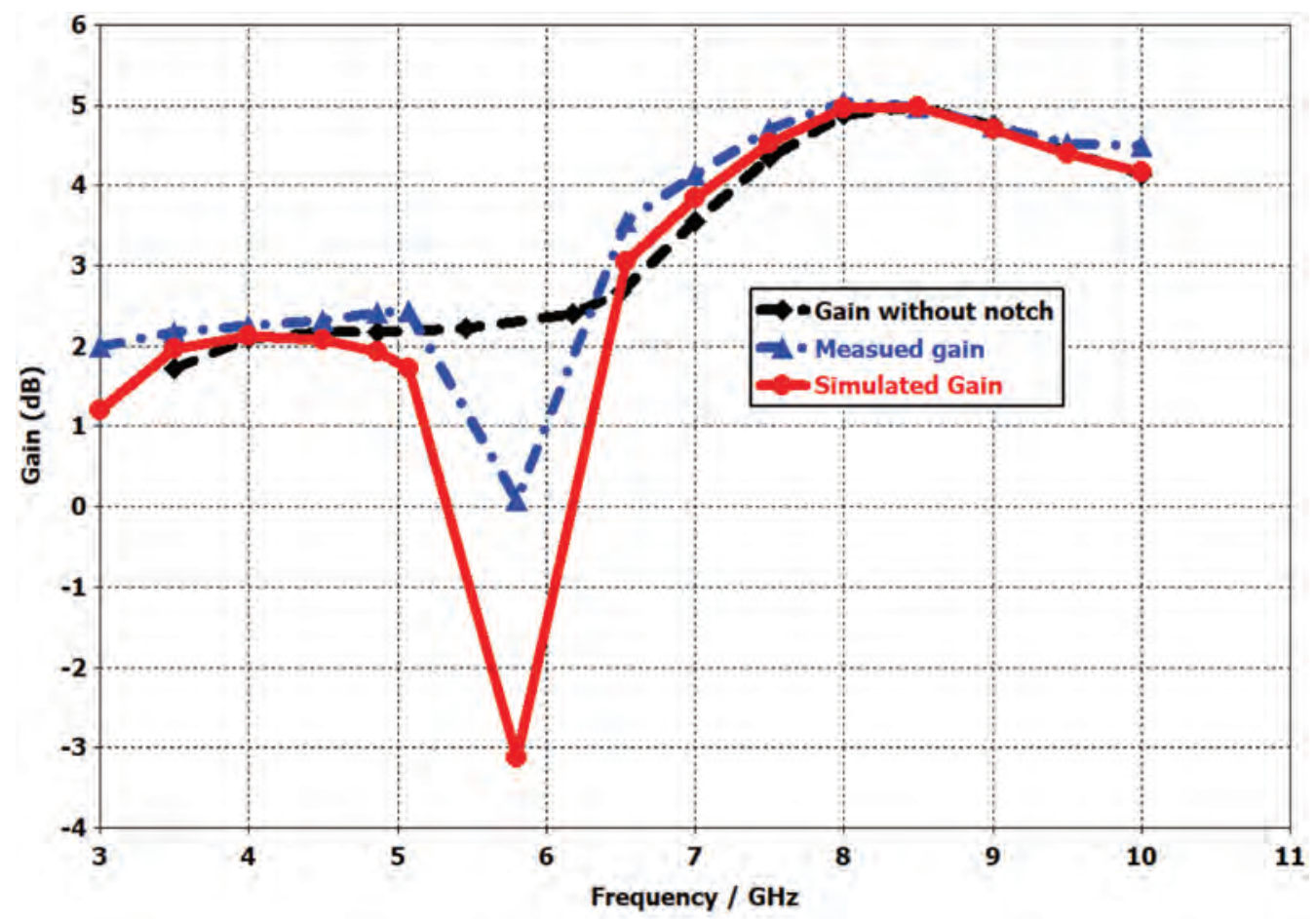

(a)

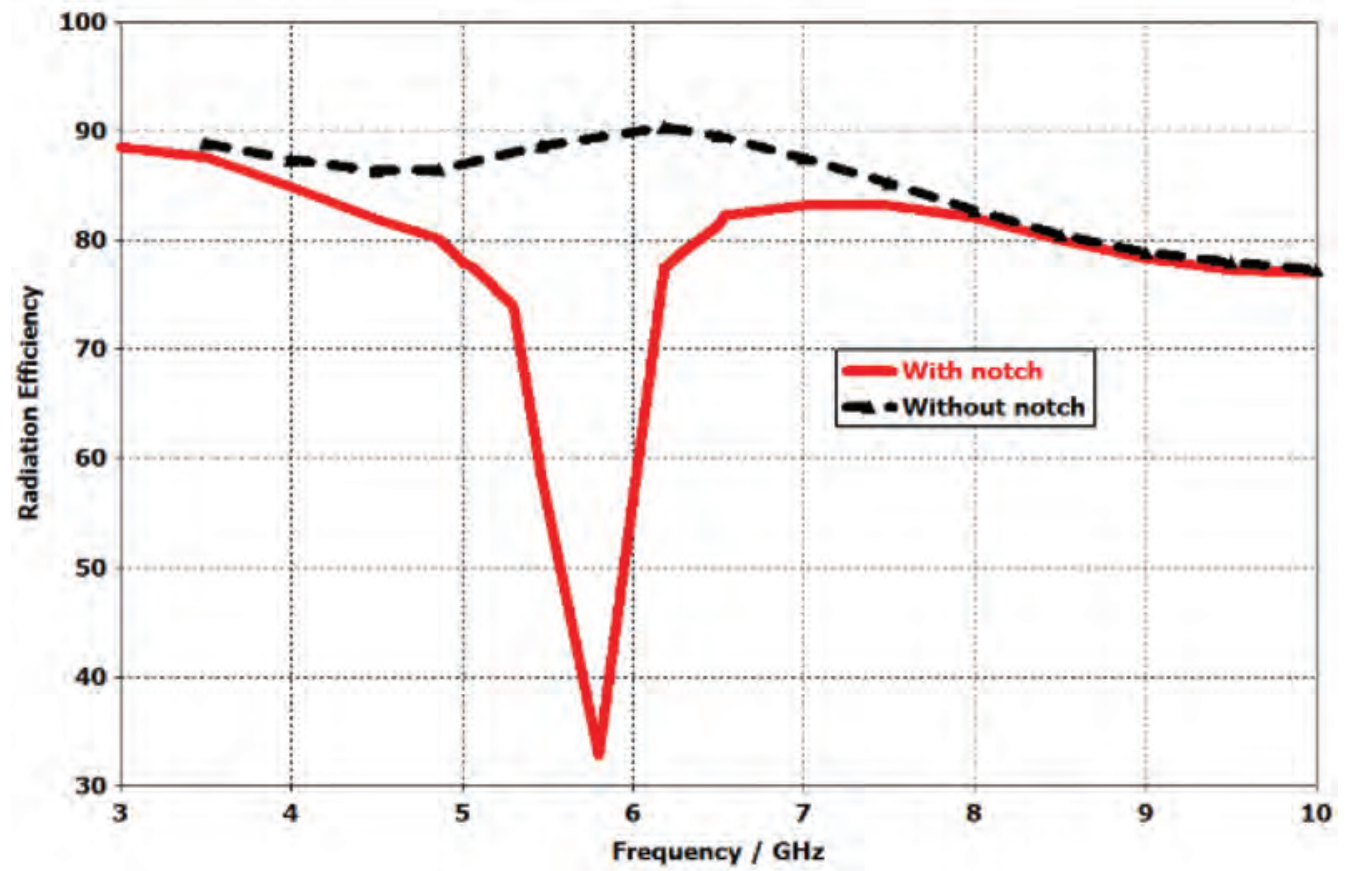

(b)

Figure 21.6 Measured vs simulated results. (a) Gain. (b) Radiation efficiency. 


\section{8}

3.96 GHz and $10.7 \mathrm{GHz}$, respectively. For notch band operation the centre frequency is shifted a little to $5.72 \mathrm{GHz}$ and a small difference of $0.06 \mathrm{GHz}$ is calculated between both the results. The measured bandwidth for the notch is a tad higher than the simulated notch bandwidth, i.e., $1.76 \mathrm{GHz}$, ranging from $4.88 \mathrm{GHz}$ to $6.64 \mathrm{GHz}$.

The E-Plane and H-plane normalized patterns at a different frequency in the UWB band are portrayed in Figure 21.5. The omnidirectional pattern is achieved in H-plane for all the mentioned frequencies. Further, the E-plane pattern is in accordance with the desired except for slight variation at frequencies greater than $6 \mathrm{GHz}$ in the UWB band, as portrayed in Figure 21.5(d).

Also, gain and radiation efficiency are presented in Figure 21.6. The gain is almost stable for the UWB range with a variation of $4 \mathrm{~dB}$ and $3 \mathrm{~dB}$ except for the notched band where the gain reduces to $-3.13 \mathrm{~dB}$ and $0.82 \mathrm{~dB}$ for measured and simulated results respectively. Similarly, the simulated radiation efficiency is in the range of $73 \%$ to $88 \%$ except for the notch band where it is reduced to $32 \%$ approximately.

\section{Conclusion}

A planar circular antenna having a reject band at $5.8 \mathrm{GHz}$ for UWB applications is presented. This antenna has a notched band bandwidth of $1.47 \mathrm{GHz}$ from $5.07 \mathrm{GHz}$ to $6.54 \mathrm{GHz}$ and a centre frequency of $5.78 \mathrm{GHz}$. All the results show befitting agreement with each other and are also suitable for the UWB operation. In the future, the notch band optimization and extensive time domain analysis can be incorporated for further validation of results. Moreover, the higher-order notch band characteristics can be achieved by embedding metamaterials and EBG structures.

\section{References}

[1] G. Kumar Sharma and R. Kumar, "Design of dual-polarized ultra-wideband antenna for microwave imaging," in Proceedings - 2nd International Conference on Intelligent Circuits and Systems, ICICS 2018, 2018, pp. 87-92.

[2] P. Kumar Malik and H. Parthasarthy, "Synthesis of randomness in the radiated fields of antenna array," Int. J. Microw. Wirel. Technol., vol. 3, no. 6, pp. 701-705, 2011.

[3] G. Kumar and R. Kumar, "Design and analysis of CPW fed planar antenna for ultra-wideband applications," in Proceedings - 2nd International Conference on Intelligent Circuits and Systems, ICICS 2018, 2018, vol. 22, no. 1, pp. 1-5.

[4] M. D. Ardakani, J. Pourahmadazar and S. O. Tatu, "A monopole antenna with notch-frequency function for UWB application," in 32nd General Assembly and Scientific Symposium of the International Union of Radio Science, URSI GASS, 2017, August, pp. 1-4.

[5] E. Jang, C. Kim, D. Yang and S. Hong, "Suppressed band characteristics of an UWB conical monopole antenna with split loops based on the equivalent circuit," Int. J. Antennas Propag., vol. 2017, 2017.

[6] X. Qu, S.-S. Zhong and W. Wang, "Study of the band-notch function for UWB circular disc monopole antenna," Microw. Opt. Technol. Lett., vol. 48, no. 1, pp. 1667-1670, 2006.

[7] G. Kumar and R. Kumar, "A survey on planar ultra-wideband antennas with band notch characteristics: principle, design, and applications," AEUE - Int. J. Electron. Commun., vol. 109, pp. 76-98, 2019.

[8] S. Das, D. Mitra and S. R. B. Chaudhuri, "Design of UWB planar monopole antennas with etched spiral slot on the patch for multiple band-notched characteristics," Int. J. Microw. Sci. Technol., vol. 2015, 2015. 


\title{
22 A comprehensive anatomy for handover probability in heterogeneous wireless networks
}

\author{
Keshav Jha ${ }^{1, *}$, Akhil Gupta,* \\ ${ }^{1}$ Panjab University, Chandigarh, India \\ ${ }^{2}$ School of Electrical and Electronics Engineering, Lovely Professional University, \\ Phagwara, India
}

\section{Introduction}

The tremendous peak in the growth of cell phones has led to the notorious development of remote data transfer capability requests that prevent maximum traffic in the system. The system of density has been proposed as an important leap for future cellular systems to stay away from destructive system barriers, where many low-power pixels are exposed to overlapping existing masseuses, it is believed. This refers to the competent cell organ (HCO) [1]. Extensive research has been done to address potential problems of system density, including bureaucratic disruptions, self-alignment and improvement, cell organization methodology, and so on [2,3]. In particular, supporting vertical handoffs that occur between different levels of base stations (BS) is another aspect and essential system utility to maintain continuous availability on HCN. Performance research on handoff potential is important for understanding the versatility of $\mathrm{HCN}$. Despite the productive results on the standard HCN performance test, this case is not done solely with customer versatility.

Writing opportunities in both classes of writing will be considered. At the top of the line assigned as a direction-based handoff, the handover occurs at the point when the moving client detects the cell limits of the distinctive BSs in its direction [4]. An examination of the handoff potential at that time involves estimating the amount of coupling between customer direction and the setting of cell limits. In the subsequent classification, handoff opportunities occur when there is a BS in the neighbourhood which currently gives a higher level of signal value than the base station [5]. To find a better base station, the customer usually has an assessment method during development. Accordingly, we interpret this meaning of handoff as an approximation-based handoff.

The two meanings of the handoff possibility can be immediately combined with the stochastic geometry hypothesis as the most built innovation tool of the year [4,5]. Handoff research utilizing direction-featured identification has toughness in exactly assessing the actual spread of cell limits required in testing [4]. Once again, the approximation-based definition introduces more direct probe architecture, although the present work [5] only deals with flat handoffs, that is, the same level of handoff between the BSs. The fact that the BS that is closest to the customer after the handover is consistently diagnosed as the novel working base station is likely to be invalidated in multi-level HCNs. Now because vertical handoff is an important utility in $\mathrm{HCN}$, the total alignment of both the level and vertical handoff in the direction of effective alignment of the HCN is required.

\footnotetext{
*Emails: keshavjha775@gmail.com, akhilgupta112001@gmail.com
} 
In the present research area, we stated that the handover test for the vertical handoff in the HCN relies on the approximation-featured identification, which can be used utilizing the same probing procedure that performs direct testing. In particular, we consider the possibility of a handoff between the objective customer's current level of service and the subjective level and minimize the vertical affiliation to a similar flat handoff opportunity by proportional separation by the cell affiliation rule. To achieve normal handoff potential, the above method is presented to each mixture of base station levels. Utilizing the suggested handover likelihood, we similarly break the inclusion likelihood into a multifunctional state. The vision gained for comprehensive performance evaluation is intelligent and helpful.

The end part of the paper will be addressed as follows. The Section II framework clarifies the model. Section III determines handoff potential and the possibility of joining a multi-level HCN versatile client. The numerical result is introduced in Section IV. Finally, completing comments are in Section V.

\section{System model}

The K-level HCN with $\{\mathrm{T}\} \mathrm{K} \mathrm{j}=1$ represents the arrangement of tire files. After homogeneous Poisson point processing (PPP), BS operates in a two-dimensional plane, with j level $\lambda \mathrm{j}$ and $\{\phi \mathrm{j}\} \mathrm{K} \mathrm{j}=1$ free PPPs. At the JH level, all BSs use the same transmission power and range extension propagation (REB) [6] individually referred to as PJ and BJ. The client is asked to sign a pre-defined limit, the Per-Impedance Ratio (SIR), which means the jth level. It is more noticeable than J. Then, the client is associated with BS, which achieves a longer running arbitrary control (BRP) [6] which is given by $P_{r, j}=P_{j} L_{0}\left(r_{j} / r_{0}\right)-\alpha_{j} B_{j}$ where L0 is given reference deflection at the process of occurrence at $\mathrm{r} 0$, $\mathrm{rj}$ is the separation between the normal subscriber and the nearest BS at the J ground level, and $\alpha \mathrm{j}$ is the pathloss type of $\mathrm{J}$ ground level. By setting bj > 1, BS can attract more clients at the jth level without physically expanding the transmission control. As far as the design of client portability is concerned, the client moves toward a path controlled by a constant speed $[7,8]$, which is the service-related point of BS before the new area is developed [9].

\section{Performance of customers}

We first established the analogy in the vertical handoff and the level handoff in the HCN. Implicit comparison is used to determine the normal handoff probability and incidence of inclusion, counting the two sorts of handoff.

\section{A. Handover likelihood}

The situation for the BS BS $S_{k}$ in the portable customer's status $L_{1}$ level $T_{k}$ [9]. $R_{k}$ separates between customers and $\mathrm{BS}_{\mathrm{k}}$. The area centred on $\mathrm{l}_{1}$ with the range $\mathrm{r}_{\mathrm{k}}$ is assumed to be $\mathrm{A}$. The position is designed to allow the client to perform deeper and higher thresholds of the report as it moves toward $L_{2}$. Let $R_{k}$ be the intersection between $L_{2}$ and $B S_{k}$ and the area centred on $\mathrm{L}_{2}$ with $\mathrm{R}_{\mathrm{k}}$. In the case of $\mathrm{L}_{2}$, the most severe BRP than the other levels, the handoff is likely to occur when the $T_{j}$ level is more marked. From $T_{k}$, or an equally given position.

$$
r_{j}<w_{j} \cdot r_{k}^{-\alpha j}, w_{j}=\left(P_{j}^{\prime} B_{j}^{\prime}\right)^{1 / \alpha j}
$$

where $\mathrm{P}^{\prime}{ }_{\mathrm{j}}=\mathrm{P}_{\mathrm{j}} / \mathrm{P}_{\mathrm{k}}, \mathrm{B}_{\mathrm{j}}{ }_{\mathrm{j}}=\mathrm{B}_{\mathrm{j}} / \mathrm{B}_{\mathrm{k}}, \alpha^{\prime}{ }_{\mathrm{j}}=\alpha_{\mathrm{j}} / \alpha_{\mathrm{k}}$, and $\mathrm{r}_{0}=1$ for effortlessness. From (1), we have the accompanying perceptions 
(i), Put the $r_{j}=Y_{j}$ attribute raj into $R$, the levels in the Objective BS represent some very good practices from $B_{J}$, where the target is the client's vertical handoff from $T_{K}$ to $T_{j}$. We consider $\mathrm{R}$ to be a proportional division of $r_{k}$.

(ii) $r^{\prime}{ }_{j}$ is the weight ratio of $r_{k}$.

(iii) Not a close BS servicing BS after a0.

For example, when rk> rj> r $\mathrm{j}$, the level BS at level $\mathrm{Tj}$ (separation $\mathrm{rj}$ ) is at the level $\mathrm{T}_{\mathrm{k}}$ (separation from the separation), the vertical handoff between these two levels is not in the light. This neglects (a). From the above dialogues, the test for vertical handoff likelihood is based on the fact that the presence of the near BS cannot determine the presence of a handoff compared to the current BS. To treat disturbances, we propose to reduce the likelihood of vertical flat off to a flat handoff opportunity that gives a direct impetus of the handoff potential. To this extent, the BS operating at the level $T_{K}$ is pressed into the level $\mathrm{T}_{\mathrm{J}}$, which means the BS J, which separates $\mathrm{r}$, $\mathrm{j}$ from the customer. The way to tell the reason behind Virtual BS is that the BSA does not meet the BS objectives of the BSJ level of service performance. The figure in [9] represents the zone focused on $L_{1}$ with $R_{J}$ is marked as ' $\mathrm{A}$ '. The level of service to the Level $\mathrm{T}_{\mathrm{K}}$ level indicates that all BSs of Level $\mathrm{T}_{\mathrm{J}}$ are outside A. Find the area $R_{J}^{\prime}=w_{j} R_{k}$ centred at $R_{2}$ on $L_{2}$, which is $\alpha_{j}$, which is a comparative division R. Since there is no B-level BS in 'B', BS will provide BS after development. If there is no vertical handoff between 1 and $T_{k}$ and $T_{j}$, then all the $B S$ zones of these two levels are outside the 'B' B' and except the $\mathrm{BS}_{\mathrm{k}}$, that is, the hidden area in the shape. $\mathrm{H}_{\mathrm{k}}$ means the level of opportunity, $j$ is from $T_{k}$ to level $T_{k}$. For $R_{K}$ and development guidance for a given partition, there is no handover between $T_{K}$ and $T_{J}$.

A client is related with level $T_{k}$ at area 11 and chooses whether changes to level $T_{j}$ when moving to area $1_{2}$. (Left) $T_{k}$ gives a bigger BRP to the ordinary client than $T_{j}$; (Right) $T_{k}$ gives a littler BRP to the ordinary client than $T_{j}$.

$$
P\left[H_{k, j}^{\prime} \mid r_{k}, \theta\right]=P\left[N\left(B^{\prime} \mid B^{\prime} I A^{\prime}\right)=0 \mid T_{k}=/ T_{j}\right]+P\left[N(B \backslash B \cap A)=0 \mid T_{k}=T_{j}\right.
$$

where $\mathrm{H}, \mathrm{J}, \mathrm{H}, \mathrm{J}$ and $\mathrm{N}$ are complementary (sp) speaks of the amount of BS in a given region. In (2), first the term refers to the vertical handoff and the next word is compared to the same handoff. When $T_{k}=T_{j}$, we have $r^{\prime}{ }_{j}=r_{k}$ and $R_{j}{ }_{j}=R_{k}$. Thus, the area $A^{\prime}$ must be indistinguishable from area $A, B^{\prime}=B$ when $T_{K}=T_{J}$. Using the illicit opportunity of PPP, we have

$$
P\left[H_{k, j} \mid r_{k}, \theta\right]=\exp \left(\Sigma \lambda_{i} \cdot\left|B^{\prime} \backslash B^{\prime} \cap A^{\prime}\right|\right)
$$

where $\left|B^{\prime}\right| B^{\prime} \cap A^{\prime}|=| B^{\prime}|-| B^{\prime} \cap A^{\prime} \mid$.

After certain controls, we acquire

$$
\left.P\left[H_{k, j} \mid r_{k}, \theta\right]=\exp \left\{\sum \lambda_{i}\left(\pi R_{i}^{2}-\mid r_{i}^{\prime}\left(\pi-\theta^{\prime}\right)+R_{i}^{\prime}\left[\theta^{\prime}-\sin ^{-1}\left(v \sin \theta^{\prime} / R_{i}^{\prime}\right)\right]-r_{i}^{\prime} v \sin \theta^{\prime}\right)\right]\right\}
$$

By averaging over $r_{k}$ and $\theta$, the likelihood of no handoff between levels $T_{k}$ and $T_{j}$ is

$$
\begin{aligned}
\mathrm{P}\left[H_{k, j}\right] & =E_{\theta}\left[E_{r k}\left[P\left[H_{k j} \mid r_{k}, \theta\right]\right]\right]=E_{\theta}\left[E_{r k}\left[P\left[H_{k j} \mid r_{k}, \theta\right]\right]\right] \frac{1}{\pi} \frac{1}{\pi} \int_{\theta=0}^{\pi} \int_{r=0}^{\infty} P[H k, j \mid r k \\
& =r, \theta] f r k(r) d r d \theta
\end{aligned}
$$


Where $\mathrm{f}_{\mathrm{rk}}(\mathrm{r})=\mathrm{e}^{-\lambda k \pi \mathrm{r}}{ }_{2} 2 \pi \lambda \mathrm{kr}$ [7]. Using (5), the handoff likelihood of the client related with level $T_{k}$ before handoff can be assessed as

$$
H_{k}={ }_{j=1}^{K} 1-\prod P[H k, \mathrm{j}]
$$

When $\{\alpha j\}=\alpha$, the handoff likelihood of level Tk is given by

$$
\begin{aligned}
& H_{k}=1-\left(2 \lambda_{k} / A_{k}\right) \int_{\theta=0}^{\pi} \int_{r=0}^{\infty} \exp \left\{-\sum_{j=1}^{K} \lambda j(P j B j) 2 / \alpha X\right. \\
& {\left[\begin{array}{l}
R 2\left(\pi-\theta^{\prime}+\sin -1\left(\frac{v \sin \theta^{\prime}}{R k(P j B j) 1 / \alpha} \text { QUOTE } \frac{\mathrm{v} \sin \theta^{\prime}}{\mathrm{Rk}(\mathrm{PjBj}) 1 / \pm} \frac{\mathrm{v} \sin \theta^{\prime}}{\mathrm{Rk}(\mathrm{PjBj}) 1 / \pm}\right)\right) \\
+r 2 \theta^{\prime}+r(P j B j)-1 / \alpha v \sin \theta^{\prime}
\end{array}\right] r r d r d \theta}
\end{aligned}
$$

At long last, the general handoff likelihood is acquired as

$$
H_{o}=\sum_{k=1}^{K} A_{k} H_{k}
$$

where the cell affiliation likelihood of the kth level $\mathrm{A}_{\mathrm{k}}$ is

$$
A_{k}=1 / \sum_{j=1}^{K} \lambda_{j}(P j B j)^{2 / \alpha j} \text { with } \lambda_{j}=\lambda_{\mathrm{j}} / \lambda_{k}
$$

There is a lot work related to vertical handoff that has been done and is mentioned in [10-15]. The effect of these methods can also be tested using the techniques mentioned in [16-23].

\section{Numerical results}

We initially accept creations determined by fertility by thinking of HCN with full-scale and pico levels under the accompanying structures: $\mathrm{L} 0=$ ions $38.54 \mathrm{~dB}, \mathrm{r} 0=1 \mathrm{~m},\{\lambda \mathrm{i}\}$ $2 \mathrm{i}=1=\{10,10\} \mathrm{km}-2,\{\mathrm{Pi}\} 2 \mathrm{i}=1=\{46,30\} \mathrm{dBm},\{2 \mathrm{i}\} 2 \mathrm{I}=1=0 \mathrm{~dB}$, and $\{\alpha \mathrm{i}\} 2 \mathrm{i}=1=4$ and $\{\mathrm{Bi}\} 2$ i. $=1=\{0,10\} \mathrm{dB}$. The guess work is featured on the outcome in [7], which implies that the subscriber velocity has a small logarithmic curve.

Figure 22.1 represents the per-level handoff certainty and the normal handoff certainty as elements of the speed scale. Nearby coordination between scientific results and reactions approves the accuracy of the investigation. This figure indicates that peacock customers are more likely to be in the hands of peacock customers than the inclusion of littler. For tests, scientific outcomes from [8] are considered. As it appears, their outcomes essentially ignore the handoff potential without including the vertical handoff. Besides, the full-scale 


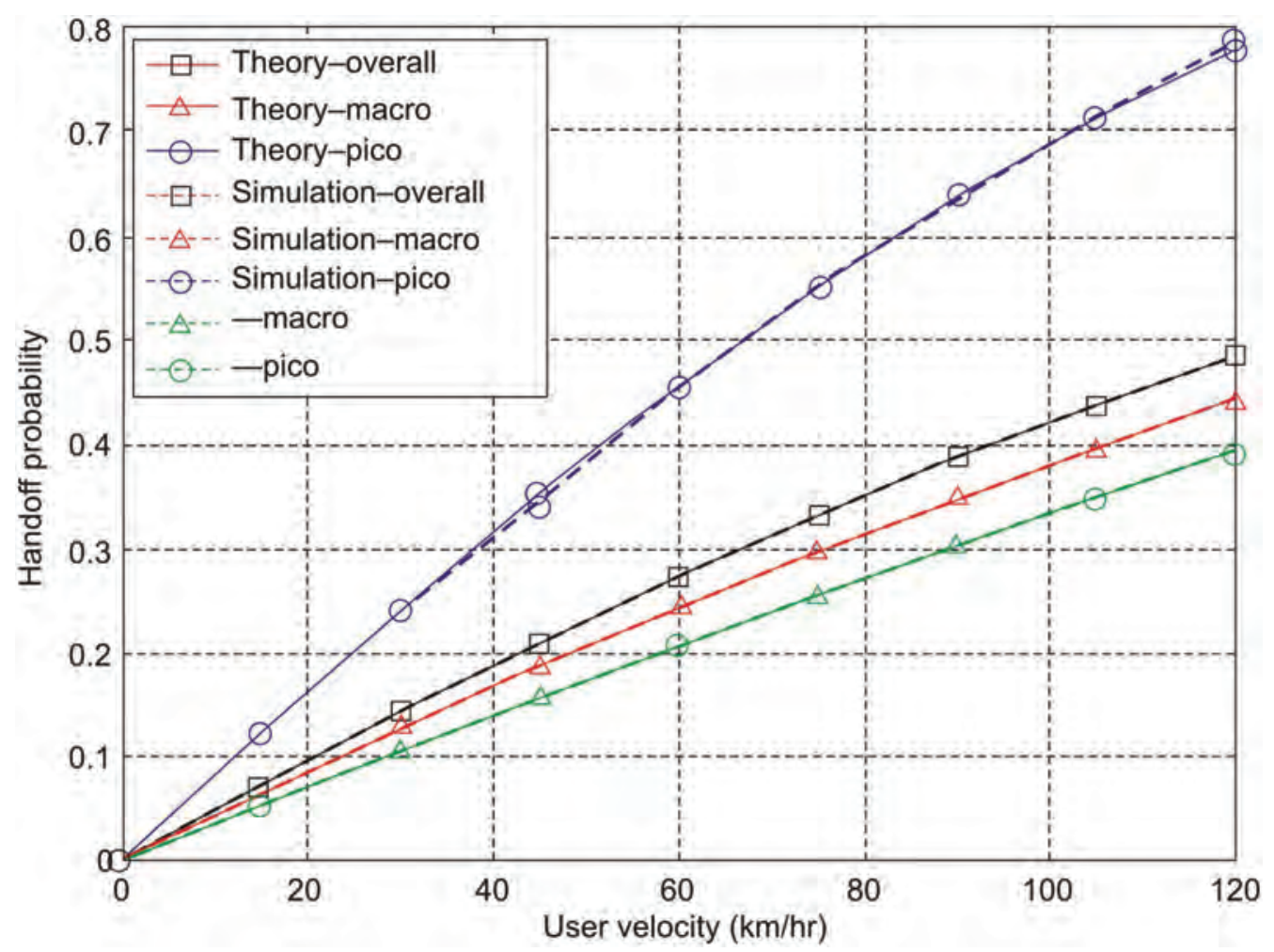

Figure 22.1 Handoff likelihood versus user speed.

and indirect probabilities of the pico levels cannot be distinguished from [8] when the two levels have the same BS thickness, which is not appropriate.

In Figure 22.1, we investigated the effect of BS thickness for handoff potentials under the altered thickness ratio $\lambda 2 /$ under 1 . The likelihood of the arms increases with the thickness ratio corrected to form. Expansion speeds gradually become clearer as the customer accelerates. The explanation is automatic because, with all the low-control picklock, including system density, the versatile client is likely to transform into a neighbouring $\mathrm{BS}$, giving it a higher ground connection quality than the present BS.

\section{Conclusion}

The research analysis described in this literature proposes a straightforward but correct and precise exposure technique to describe the execution of multi-level HCNs providing its best performance to a multifaceted user. Using random configuration and discussed similarity among vertical and flat handoffs, we determine the handover certainty and inclusion certainty, key execution markers to assess the portability method of HCN. Our outcomes can be utilized to decide significant structure parameters, for example, ideal REBs and rapidly evolving modern conditions, e.g., full load systems or non-uniform BS regimes. 


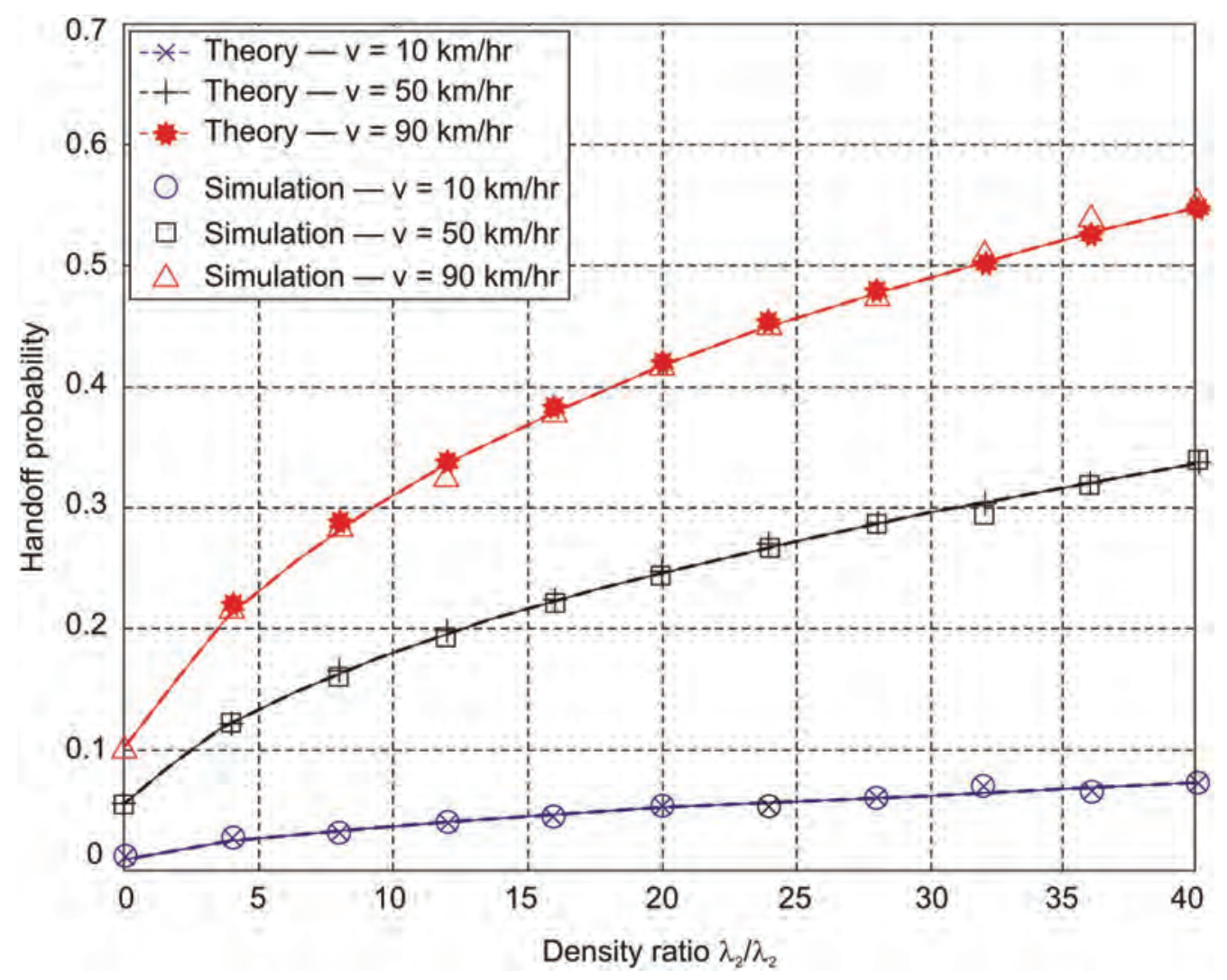

Figure 22.2 Result of density ratio to the overall handoff likelihood.

\section{References}

[1] N. Bhushan et al., "Network densification: the dominant theme for wireless evolution into 5G," IEEE Commun. Mag., vol. 52, no. 2, pp. 82-89, Feb. 2014.

[2] A. Imran, A. Zoha and A. Abu-Daiya, "Challenges in 5G: How to empower SON with big data for enabling 5G," IEEE Netw., vol. 28, no. 6, pp. 27-33, Nov. 2014.

[3] N. Zhang, N. Cheng, A. T. Gamage, K. Zhang, J. W. Mark and X. Shen, "Cloud assisted HetNets toward 5G wireless networks,” IEEE Commun. Mag., vol. 53, no. 6, pp. 59-65, June 2015.

[4] W. Bao and B. Liang, "Stochastic geometric analysis of user mobility in heterogeneous wireless networks," IEEE J. Sel. Areas Commun., vol. 33, no. 10, pp. 2212-2225, Oct. 2015.

[5] S. Sadr and R. S. Adve, "Handoff rate and coverage analysis in multitier heterogeneous networks," IEEE Trans. Wireless Commun., vol. 14, no. 5, pp. 2626-2638, May 2015.

[6] H.-S. Jo, Y. J. Sang, P. Xia and J. G. Andrews, "Heterogeneous cellular networks with flexible cell association: A comprehensive downlink SINR analysis," IEEE Trans. Wireless Commun., vol. 11, no. 10, pp. 3484-3495, Oct. 2012.

[7] J. G. Andrews, F. Baccelli and R. K. Ganti, "A tractable approach to coverage and rate in cellular networks,” IEEE Trans. Commun., vol. 59, no. 11, pp. 3122-3134, Nov. 2011.

[8] C. H. M. de Lima, M. Bennis, and M. Latva-Aho, "Modeling and analysis of handover failure probability in small cell networks," in Proc. IEEE INFOCOM, Toronto, ON, Canada, Apr./ May 2014, pp. 736-741.

[9] Shin-Ying Hsueh and Kuang-Hao Liu. "An equivalent analysis for handoff probability in heterogeneous cellular networks", IEEE Communications Letters, 2017. 
[10] Sunita Sharma and S.K.Bodhe "Design and performance evaluation of cost function based vertical handoff", International Journal of Engineering Research and Applications, vol. 2, no. 3, May-June 2012, pp. 257-262.

[11] E. Stevens-Navarro, V. Wong, and Y. Lin, "A vertical handoff decision algorithm for heterogeneous, wireless networks", Wireless Communications and Networking Conference March 2007, pp. 3199-3204.

[12] D. Niyato and E. Hossain, "A cooperative game framework for bandwidth allocation in 4g heterogeneous wireless networks", in: IEEE International Conference on Communications, ICC '06, vol. 9, June 2006, pp. 4357-4362.

[13] S. Bellovin, "A best-case network performance model" Tech. Rep., ATT Research, Feb. 1992.

[14] W. Zhang, "Handover decision using fuzzy madm in heterogeneous networks", in: IEEE, Wireless Communications and Networking Conference, vol. 2, March 2004, pp. 653-658 Vol. 2.

[15] S. Horrich, S.B. Jamaa and P. Godlewski, "Adaptive vertical mobility decision in heterogeneous networks", International Conference on Wireless and Mobile Communications, 2007, 44.

[16] S. R. Chopra, A. Gupta and H. Monga, "An LTE approach with MIMO by using suboptimal selection of antenna," 2018 International Conference on Intelligent Circuits and Systems (ICICS), Phagwara, pp. 167-172, 2018.

[17] R. Kausar, A. Gupta, I. B. Sofi and K. Arora, "Bit error rate based performance evaluation of LTE OFDMA system," 2018 International Conference on Intelligent Circuits and Systems (ICICS), Phagwara, pp. 161-166, 2018.

[18] A. Gupta, S. Dogra and I. B. Sofi, "Performance evaluation of spatial multiplexing using different modulation techniques in MIMO system for small and large scale fading channel", International Journal of Sensors Wireless Communications and Control, vol. 9, no. 2, pp. 188$202,2019$.

[19] S. R. Chopra, A. Gupta and H. Monga, "Performance analysis of space time trellis codes in Rayleigh fading channel", in Harmony Search and Nature Inspired Optimization Algorithms, Springer, Singapore, pp. 957-967, 2019.

[20] A. Gupta and S. R. Chopra, "Designing and testing of quadrifilar helix antenna for NOAA weather satellite," 2019 6th International Conference on Signal Processing and Integrated Networks (SPIN), Noida, India, pp. 785-790, 2019.

[21] R. Devi, R. K. Jha, A. Gupta, S. Jain and P. Kumar, "Implementation of an intrusion detection system using an adaptive neuro-fuzzy inference system for $5 \mathrm{G}$ wireless communication network", AEU-International Journal of Electronics and Communications, vol. 74, pp. 94$106,2017$.

[22] A. Gupta and R. K. Jha, "Security threats of wireless networks: A survey," International Conference on Computing, Communication \& Automation, Noida, pp. 389-395, 2015.

[23] A. Gupta and R.K. Jha, "Power optimization using massive MIMO and small cells approach in different deployment scenarios", Wireless Networks, vol. 23, no. 3, pp. 959-973, 2017. 


\title{
23 Secure communication channel for grid users using AES Encryption
}

\author{
Aditya Bakshi,*, Akhil Gupta ${ }^{2, *}$ \\ ${ }^{1}$ Inurture Education Solutions ltd., Karnataka, India \\ ${ }^{2}$ Lovely Professional University, Phagwara, India
}

\section{Introduction}

This is a public key cipher which uses modular exponentiation. First step of this algorithm is key generation then encryption and decryption takes place using the generated keys. It generates two keys which are public and private. Private keys are always kept secret. Data encrypted by one key is decrypted by the other key. Depending upon our requirement we decide which key should be used for encryption and which key should be used for decryption [1-4].

We need to achieve some security goals for server system as:

- Every user or computer must be identified before they can access to the resources. For this we need to collect user information and store them.

- User authentication must be done. We should ask for user name, password and other information related to the user. We can then allow users to access resources for which they are eligible.

- We should be able to identify and stop the modification of data while the data is passing through the communication channel.

- Users should not be able to deny the fact that they have committed something which they already have done.

\subsection{Microsoft Visual Studio}

It is an IDE which we use for developing software; it has a code editor, a source-level debugger and machine level debugger. It also has tools like form designer which we use for developing GUI applications, we use it for designing and developing websites, class and database schema also. It supports many programming languages. It has some built in languages and also supports some languages which can be installed by using language services [5-8]. Native codes are machine codes which as we know are executed by CPU directly and managed code (source code) both can be produced by it.

- There are many integrated development environments available in the software development environment. Visual Studio is one of the integrated development environments.

- The main functioning of the Visual Studio is to develop various types of the applications that may be windows or web console applications.

- Windows console applications are the applications that are used for the internal purpose of a single distributed system such as a network server connected to many systems.

*Emails: addybakshi@gmail.com, akhilgupta112001@gmail.com

DOI: 10.1201/9781003129103-23 
- Web console applications are the development of applications for a particular organization or any other that may acts as a customer for their own development of the application.

Key generation:

1. Choose two large prime numbers: $\mathrm{P}$ and $\mathrm{Q}$ of similar bit length, randomly

2. We calculate $\mathrm{N}=\mathrm{P} * \mathrm{Q}$.

3. We calculate $\Phi(\mathrm{N})=\Phi(\mathrm{P}) * \Phi(\mathrm{Q})=(\mathrm{P}-1) *(\mathrm{Q}-1)=\{\mathrm{N}-(\mathrm{P}+\mathrm{Q}+1)\}$

4. We choose an integer " $E$ " such that: $1<\mathrm{E}<\Phi(\mathrm{N})$ and GCD $(\mathrm{E}, \Phi(\mathrm{N}))=1$

5. Calculate $\mathrm{D}=\mathrm{E}$ inverse $(\bmod (\Phi(\mathrm{N}))$.

6. Public Key $=(\mathrm{N}, \mathrm{E})$.

7. Private $\mathrm{Key}=(\mathrm{N}, \mathrm{D})$.

8. P, Q, D and $\Phi(\mathrm{N})$ are kept secret.

Encryption: Plaintext $=\mathrm{M}<\mathrm{N}$.

Cipher text. $\mathrm{M}^{\wedge} \mathrm{E}(\bmod \mathrm{N})$.

Decryption: Cipher text $=\mathrm{C}$

Plaintext: $\mathrm{M}=\mathrm{C}^{\wedge} \mathrm{D}(\bmod \mathrm{N})$

The main features of Visual Studio:

- Code Editor is the important feature that is used for writing the code which has in built syntax and easy to access them at any time by just typing the name of the class.

- Debugger is available in Visual Studio that acts as both the machine and source level debugger. It also simply manages with the code and the core code which is written for the program of the application of any language the program may be written.

- Designer is the one use of designs for help in developing the applications. It contains various types of designers that are used for the development of the application.

Designs can be of various types and are selected according to the developer's issue. Various types of designers include

- Windows Form Designer

- Class Designer

- WPF Designer

- Mapping Designer

- Data Designer

- Web Designer or Development

Other tools include open tab browser, Team explorer, Data explorer, Server explorer, Properties editor, Object browser, Solution explorer, Dotfuscator software services community edition, ASP.NET website management tool, Text generation framework, Visual Studio tools for Office [9-12].

\subsection{Net framework}

- A frame of software which was developed by Microsoft that works on the platform that is developed by Microsoft. It consists of various types of classes and libraries of various functionalities that are available in other types of programming languages. 
- The main function of this framework is to get the access of the various functionalities of other ones and so that it is easy to use the functions that are used for the development of any application or the other console application.

- Two types of application are developed through this framework; they are Windows ApplicationS and Console or Web Application.

- There is a super class that contains various classes called as framework class library. FCL (framework class library) consists OF network procedure communication, algorithms of numeric, encryption of the code, data accessing activities like data abstraction, connectivity to the database and various functionalities.

- Programs can be written very easily in .NET framework as it consists of all types of classes and functions which are in built in the FCL so that it saveS more time and also in economical way.

- .NET framework architecture consists of the .NET core, assemblies, class library and common language infrastructure.

- Common language infrastructure (CLI) acts as a platform to obtain the needful functionalities for the execution and development of the application in a simple way that the other frameworks that are available.

- Class Library consists of all types of classes that are collected through the FCL classes for file handling, exception handling and many others. Standard libraries of classes are available so that they are ordered name space of the hierarchies.

- Base class library is a part of the class library and it also acts as a super class; it contains all the information about the classes that are required for developing the application. Various functions such as LNQ, WCF, WF, ASP.NET and ADO.NET are include in the base class collections.

- Framework class library is a small part of the class library and it contains .dll files which are available only in the framework class library that are only for the development of the application or the windows form

- $\quad$.NET core is one of the implementation prototypeS of .NET framework which is incomplete, which means cannot perform the total implementation of the .NET framework.

- Assemblies are used for the storage of the code that is present in the CLI. These are called simply CLI assemblies. These CLI assemblies are used for the .dll and .exe files that are obtained by developing the application using the FCL and CLI of the .NET framework.

- The development tool that is used by the .NET framework is Microsoft Visual Studio, operating system is Windows, server is Oracle Access and many other functions are used by the .NET framework.

\section{Literature review}

The paper in [1] discussed the side channel attacks. Firstly define a side channel attack, i.e. the context with respect of measurement that describe the leakage distribution of target device, then device input output control can be chosen adversary, lastly to check the complexity based upon data, time, memory and number of measurements. Implementation can be described mainly on two aspects. First, design type, in this how implementation criteria is taken into consideration, i.e. based on randomization and also with time randomization; second, leakage type functions provide features like linearity, noise distribution and variability. Last, the conclusion of this paper is to evaluate the attacks and the method that was adopted for the cryptanalysis [13-16]. 
The paper in [2] provides a summary about real world side channel analysis of encryption mechanism. They are using Xilinux FPGAs encryption mechanism. For analysis virtex 4, virtex 5 and Spartan 6 are used to show that encryption mechanism can be completely broken by applying some methods like correlation power analysis that is used to measure the power consumption of a device when the algorithm executes. Here results show the measurement of the algorithm based on the power consumption. For virtex 4 the power consumption measured is 50,000 and for virtex 5, 90,000. Analysis is done to find time instances of power traces with the help of known key where decryption happens. In today's world important topic is of IP theft and piracy of the product that results in insecurity. The attacks which happened beyond 8 bit hypothesis were taken into consideration for implementing a more secure system [17-19].

\section{Discussion}

Outcome of the proposed system, "Secure channel for grid users using AES encryption algorithm", provides secure communication for users in grid environment and in distributed environment. It will be difficult for anyone to see others' data. Scientific, research and even general applications and various user requirements in future will require more resources than we need now. So we will be in need of using grid computing services more often. Isolated grid systems will not be able to utilize the resources economically. We need commercialization of grid systems which means we are going to provide grid services on Internet infrastructure as well. We can charge accordingly for proving grid services. Client and providers will both get benefits by doing this. Providing grid services on Internet infrastructure is useful but then we will have to be cautious about its security aspect. The weakest link could be the security of communication channel, which is public.

Various protocols like TLS are there to ensure security but attack on them is possible if we do not strictly design our system to use strongest set of ciphers. Being strict on choosing strongest set of ciphers has advantage of secure system. But doing this will have disadvantages also, like users using old software for example old operating systems, old web browsers etc. will not be able to access the services. This problem of unavailability has a very simple solution, which is updating the software regularly.

\section{Conclusion}

Grids usefulness will be significant if it can be accessed by more and more users. Access to more users will utilize the resources provided by the grid. We can make it possible by using Internet infrastructure.

If we will apply constraints which will make a side-channel attack ineffective then we can apply AES to make our system secure. We also need to keep track of advances in cryptanalytic concepts and tools regularly.

\section{References}

[1] Imai, H., and Hirakawa, S. (1977). "A new multilevel coding method using error-correcting codes," IEEE Transactions on Information Theory, 23(3), 371-377.

[2] Ungerboeck, G. (1982). "Channel coding with multilevel/phase signals," IEEE transactions on Information Theory, 28(1), 55-67.

[3] Sklar, B. (2001). Digital Communications (Vol. 2). NJ: Prentice Hall. 
[4] Cheng, J. F., Chuang, C. H., and Lee, L. S. (1993, November). "Complexity-reduced multilevel coding with rate-compatible punctured convolutional codes," in Global Telecommunications Conference, 1993, including a Communications Theory Mini-Conference. Technical Program Conference Record, IEEE in Houston. GLOBECOM'93., IEEE (pp. 814-818). IEEE.

[5] Kofman, Y., Zehavi, E., and Shamai, S. (1994). "Performance analysis of a multilevel coded modulation system," IEEE Transactions on Communications, 42(234), 299-312.

[6] Morelos-Zaragoza, R. H., and Imai, H. (1998). "Binary multilevel convolutional codes with unequal error protection capabilities," IEEE Transactions on Communications, 46(7), $850-853$.

[7] Isaka, M., and Imai, H. (2001). "On the iterative decoding of multilevel codes," IEEE Journal on Selected Areas in Communications, 19(5), 935-943.

[8] Djordjevic, I. B., Vasic, B., and Neifeld, M. A. (2006). "Multilevel coding in free-space optical MIMO transmission with Q-ary PPM over the atmospheric turbulence channel," IEEE Photonics Technology Letters, 18(13/16), 1491.

[9] S. R. Chopra, A. Gupta and H. Monga, "An LTE approach with MIMO by using suboptimal selection of antenna," 2018 International Conference on Intelligent Circuits and Systems (ICICS), Phagwara, pp. 167-172, 2018.

[10] R. Kausar, A. Gupta, I. B. Sofi and K. Arora, "Bit error rate based performance evaluation of LTE OFDMA system," 2018 International Conference on Intelligent Circuits and Systems (ICICS), Phagwara, pp. 161-166, 2018.

[11] A. Gupta, S. Dogra and I. B. Sofi, "Performance evaluation of spatial multiplexing using different modulation techniques in MIMO system for small and large scale fading channel", International Journal of Sensors Wireless Communications and Control, 9(2), 188-202, 2019.

[12] S. R. Chopra, A. Gupta and H. Monga, "Performance analysis of space time trellis codes in Rayleigh fading channel", in Harmony Search and Nature Inspired Optimization Algorithms, Springer, Singapore, pp. 957-967, 2019.

[13] A. Gupta and S. R. Chopra, "Designing and testing of quadrifilar helix antenna for NOAA weather satellite," 2019 6th International Conference on Signal Processing and Integrated Networks (SPIN), Noida, India, pp. 785-790, 2019.

[14] R. Devi, R. K. Jha, A. Gupta, S. Jain and P. Kumar, "Implementation of an intrusion detection system using an adaptive neuro-fuzzy inference system for $5 \mathrm{G}$ wireless communication network", AEU-International Journal of Electronics and Communications, 74, 94-106, 2017.

[15] A. Gupta and R. K. Jha, "Security threats of wireless networks: A survey," International Conference on Computing, Communication \& Automation, Noida, pp. 389-395, 2015.

[16] A. Gupta and R.K. Jha, "Power optimization using massive MIMO and small cells approach in different deployment scenarios", Wireless Networks, 23(3), 959-973, 2017.

[17] A. Gupta, R. K. Jha, P. Gandotra and S. Jain, "Bandwidth spoofing and intrusion detection system for multistage $5 \mathrm{G}$ wireless communication network”, IEEE Trans. on Vehicular Technology, 67(1), 618-632, 2017.

[18] I. B. Sofi, A. Gupta and R. Kausar, "Performance evaluation of different channel estimation techniques in MIMO System for Hata channel model," 2018 International Conference on Intelligent Circuits and Systems (ICICS), Phagwara, pp. 155-160, 2018.

[19] M. Singh, K. Arora and A. Gupta, "Equalization in WIMAX system," 2018 International Conference on Intelligent Circuits and Systems (ICICS), Phagwara, pp. 330-333, 2018. 


\title{
24 SIC scheduling algorithm based MIMO technique for mobile ad-hoc networks
}

\author{
Krishan Kumar* \\ Professor, SEEE, LPU, Punjab, India
}

\section{Introduction}

Mobile ad-hoc networks have the ability to quickly respond to link fluctuations due to interference. Nowadays, the concept of directional antennas as well as smart antenna arrays and MIMO technology is adopted to improve the performance in ad-hoc networks. MIMO capability in a mobile ad-hoc network required a SIC for the diversity operation. This paper reviews the working of MIMO and adds the new concept of interference among different users. In MIMO-based ad-hoc networking need to face various interferences and synchronicity among users. Main sources of interference are co-interference due to more transmit and receive antennas, co-channel interference etc. MIMO is also affected by inter-carrier interference due to channel variation. In this adopted technique, SIC is highly effective to suppress co-channel interference. MIMO channels have better performance without channel interference [1]. SIC and MIMO concept are described to receive data streams. SIC is effective to boost the performance and increase SINR [3]. Network performance in MIMO with SIC is poor due to non-effective scheduling algorithm [4]. MANET is especially defenceless against security assaults due to its major nature. Specially appointed On-request Distance Vector is an exceptionally famous directing convention of MANETs; however, it has no safety efforts in-manufactured and is helpless against numerous kinds of assaults. Dark opening assault at system layer is the most considered assault in AODV steering convention. AODV is one of routing protocolss accessible for MANET. It is on interest directing convention which means it is receptive to the request of the system necessities. Mobile ad-hoc network became most popular due to various application like target detection, traffic monitoring etc. Energy consumption of nodes is main challenge because each node deployed in ad-hoc network has to operate without battery replacement for a long time in a harsh environment. Energy efficient technique is required to consume less transmission energy than single input single output (SISO) for the same bit error ratio (BER). The optimization of node energy consumption is a criterion to monitor network life time. Network protocols have been designed and implemented to control energy consumption in ad-hoc network. Wireless communication is an uncertain type channel and data transmission as well as energy efficiency are serious issues in the network.

\section{Related work}

Energy is a constraining variable in the effective sending of ad-hoc system since nodes are relied upon to have minimal potential for reviving their batteries. The vitality expenses

\footnotetext{
* Email: k_kraman@rediffmail.com
}

DOI: 10.1201/9781003129103-24 
of wireless network correspondence and talk about the components used to lessen these expenses for correspondence in ad-hoc network at that point centre around explicit MAC layer conventions can spare vitality by suspending the specialized gadget during transient inactive periods in correspondence that mean to diminish vitality utilization during both dynamic correspondence and idle periods in correspondence. Energy protection can be attained in one of two ways: saving vitality during active correspondence and saving vitality during idle occasions in the correspondence [5]. The primary focus is on the methods used to help correspondence in an ad-hoc system and commonly achieved by using vitality effective MAC and path protocol. The second spotlight is on decreasing the vitality devoured when the node is idle and not participating in communication by setting the node in a low-control state. Initially characterize the expenses related with correspondence in specially appointed systems and after that examine the utilization of correspondence time and idle time vitality protection [6]. Implementation of the new broadcasting technique to diminish the overhead of routing packets. This technique exploits the neighbourhood information utilizing rebroadcast deferral and furthermore coverage ratio of node. The network connectivity factor and the coverage ratio are utilized to calculate the rebroadcast probability [7]. The network connectivity factor is utilized to decide the quantity of node that is needed to get the route request bundle. Broadcasting is the essential mechanism utilized in reactive routing protocols. The significant issue is to lessen the quantity of rebroadcast bundles. The neighbour coverage area ratio and connectivity network are factors. This system progressively computes the rebroadcast delay, which finds the forward request and furthermore adequately discovers the neighbour inclusion information [8].

The consequence of the recreation demonstrates that the new system delivers less rebroadcast than the current convention. Because of less repetitive rebroadcast, the proposed instrument diminishes the system crash and dispute with the goal that it might likewise expand the parcel conveyance proportion and furthermore diminish the normal start to finish delay. This paper likewise proposes the strategy to decide the rebroadcast likelihood. The technique considers the revealed neighbours and availability metric and furthermore the nearby hub thickness to decide the rebroadcast likelihood. The rebroadcast likelihood comprises two sections. The rebroadcast delay isn't balanced in light of the fact that it is utilized to ascertain the request of dispersing neighbour inclusion data [9].

\section{Concept of SIC in MIMO models}

Recent advances in MIMO models attract the researchers to modify and design the technique to improve the performance in the network. In the joint MIMO model, SIC improves performance in the network. Based on the understanding of SIC, proposed model is used to cancel interference with SIC in the network. In this section, the MIMO model (Figure 24.1) is reviewed to add SIC for MIMO. Based on this background, the proposed concept is effective to cancel interference for MIMO in mobile ad-hoc networks (Figure 24.2).

\section{SIC scheduling algorithm}

A receive node is ready to catch signals from source node, including both signals from the unknown source node and interference from any unknown source node. In this SIC algorithm, receive node receives the signals from these unknown or known source nodes in five steps: 
$\mathrm{N}$-Transmitting Antennas

M-Recieving Antennas

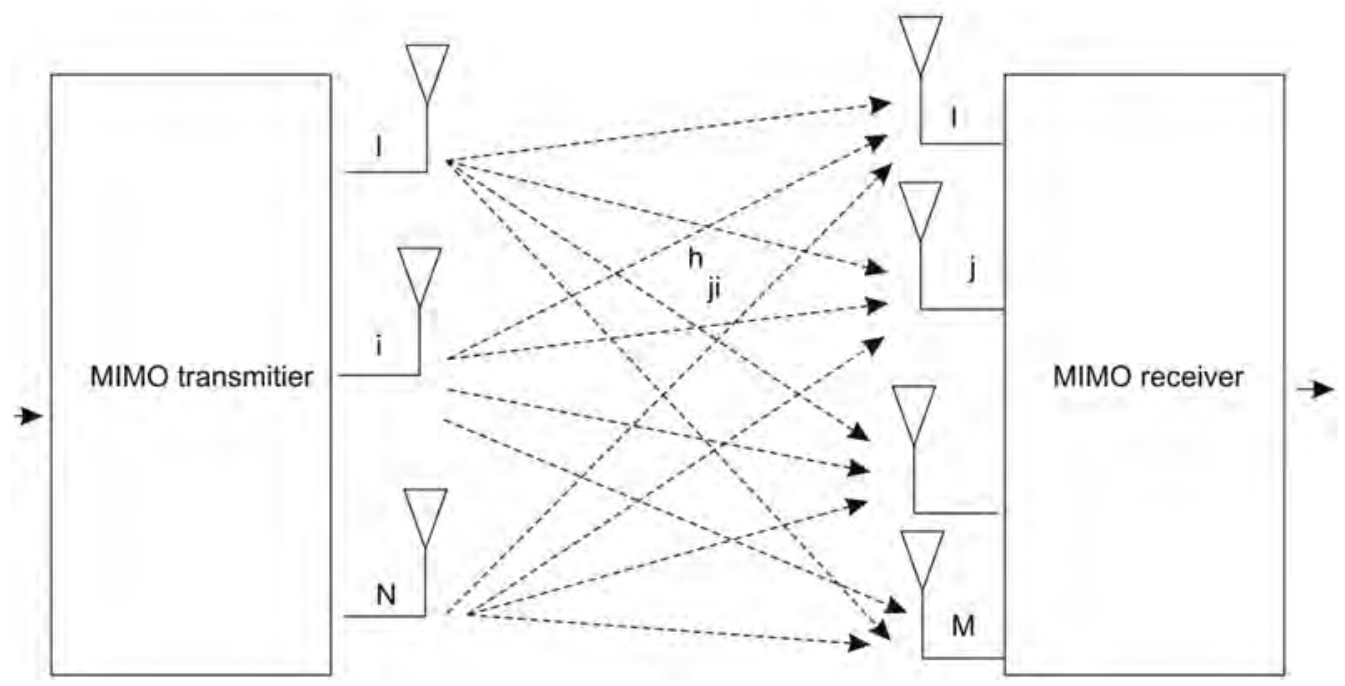

Figure 24.1 MIMO model.

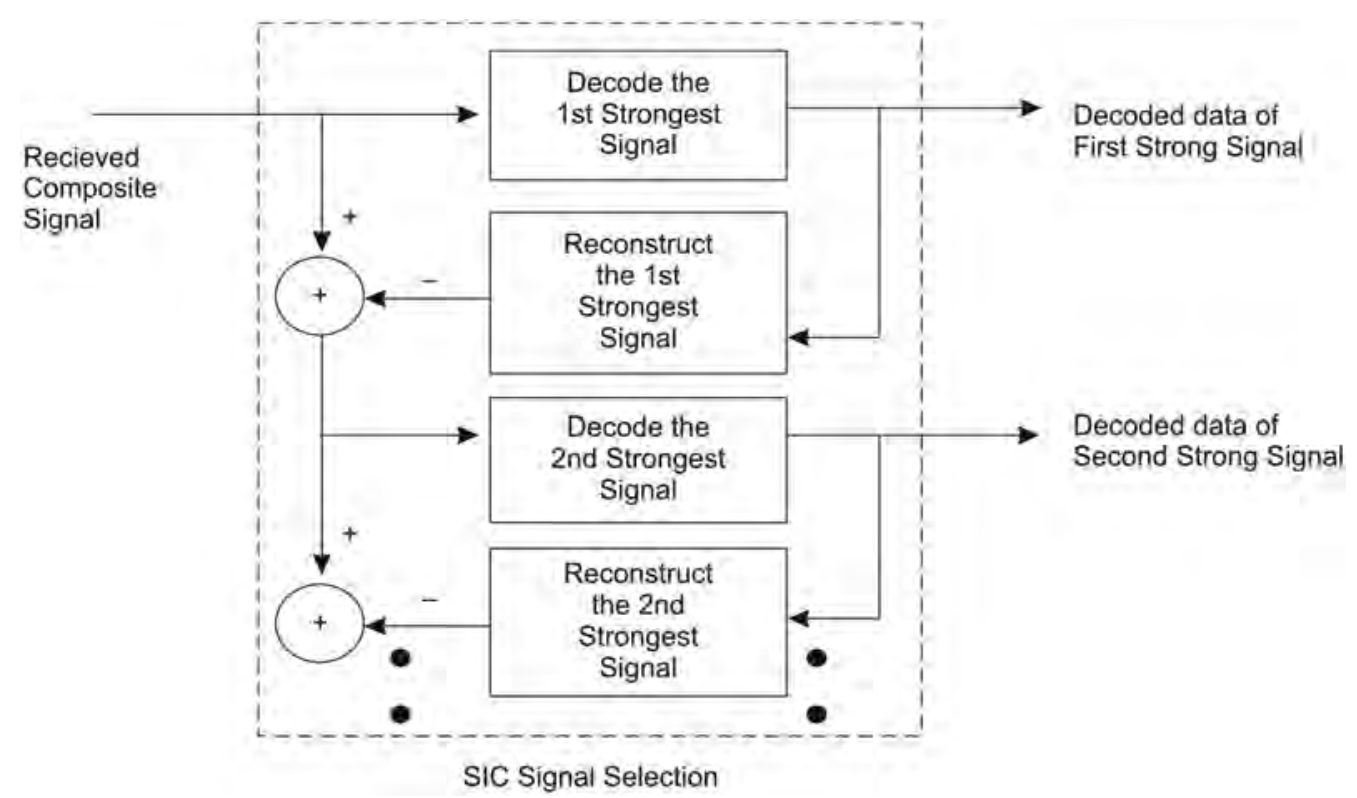

Figure 24.2 Data decoded by SIC. 
Step 1: Information from unexpected source nodes that are cancelled at the source nodes.

Step 2: Information from unexpected source nodes that are cancelled at receive node.

Step 3: Information from unknown source nodes that are received or decoded and subtracted from the composite signal, which is received by SIC at receive node before the known source node.

Step 4: Selection of signals from intended transmits or source nodes.

Step 5: Signal detected by SIC at receive node.

\section{Result analysis}

Performance is compared with MATLAB and NS2 simulation environment based on BER and throughput in new proposed SIC based MIMO technique and existing SIC based MIMO in mobile ad hoc networks.

\section{MATLAB simulation environment}

The performance of proposed SIC algorithm with MIMO was simulated using MATLAB2015. It is observed from simulation results that proposed technique has improvement as compared to existing SIC based MIMO. The simulation parameters are given in Table 24.1.

It is observed that from Figure 24.3 that Eb/No is better in proposed SIC scheduling algorithm with MIMO as compared to existing technique SIC with MIMO [1].

\section{NS2 simulation environment}

Simulation environment is framed with NS2 simulation software by using the network parameters to compare the network performance in mobile ad-hoc network. Simulation ad-hoc network parameters are shown in Table 24.2.

It is observed from the Figure 24.4 that more data transmitted successfully from one node to other node with respect to special period in the network. It is observed from the graph that throughput in proposed SIC algorithm based MIMO technique is better than the existing technique [1]. Throughput is a key parameter to increase the data delivery success in the network.

\section{Conclusion}

In this paper, the concept of SIC scheduling algorithm based MIMO technique is presented for mobile ad-hoc network (MANET). SIC scheduling algorithm is effective in interference cancellation to improve the performance in the network. The proposed technique is more effective and has better performance to improve the quality of services in the network.

Table 24.1 MATLAB parameters

\begin{tabular}{ll}
\hline Ad-hoc network parameters & Value \\
\hline Network size & $1000 \times 1000 \mathrm{~m}^{2}$ \\
Initial energy of nodes & $0.7 \mathrm{~J}$ \\
Packet size & $2000 \mathrm{bits}$ \\
Number of nodes & $60 \mathrm{nodes}$ \\
Transceiver energy consumption & $30 \mathrm{~nJ} / \mathrm{bit}$ \\
\hline
\end{tabular}




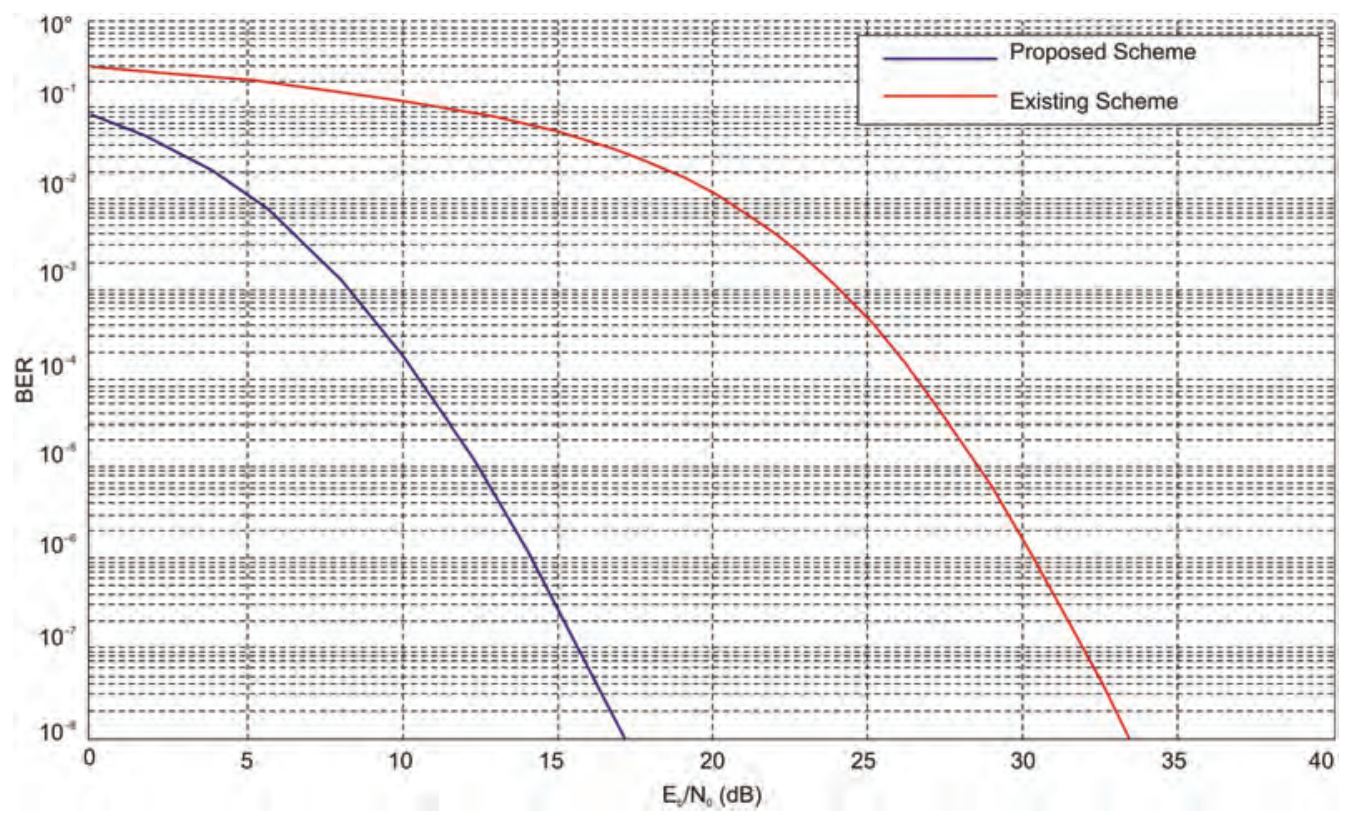

Figure 24.3 BER vs. Eb/no.

Table 24.2 NS2 simulation parameters

\begin{tabular}{ll}
\hline Mobile ad-hoc network parameters & Valueltype \\
\hline Channel & Wireless ad-hoc \\
Link model & Two ray ground \\
Medium access control & 802.11 \\
Antenna type & Directional \\
Network node & 60 nodes \\
Protocol & AODV \\
Initial energy value & $100 \mathrm{~J}$ \\
\hline
\end{tabular}

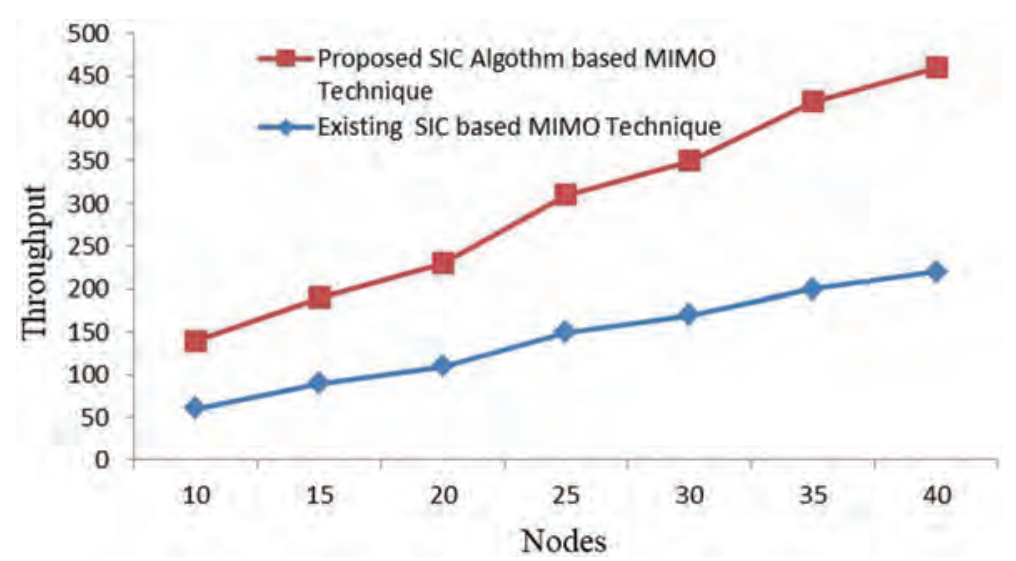

Figure 24.4 Throughput vs. time. 


\section{References}

[1] Brian A. Jalaian, Xu Yuan, Yi Shi, Y. Thomas Hou, Wenjing Lou, Scott F. Midkiff, Venkat Dasari. 2018. On the integration of SIC and MIMO DoF for interference cancellation in wireless networks. Wireless Networks 24:2357-2374.

[2] Taiwen Tang, Minyoung Park. 2004. A joint MIMO-OFDM transceiver and MAC design for mobile ad hoc networking. International Workshop on Wireless Ad-Hoc Networks.

[3] R.W. Heath, M. Scott, 2006. A joint MIMO-OFDM transceiver and MAC design for mobile ad hoc networking. EURASIP Journal on Wireless Communications and Networking 2:1-9.

[4] S. Xu, T. Saadawi. 2001. Does the IEEE 802.11 MAC protocol work well in multihop wireless ad hoc networks? IEEE Communication 39:7-13.

[5] K. Xu, M. Gerla, S. Bae. 2002. How effective is the IEEE 802:11 RTSKTS handshake in ad hoc networks. IEEE Glubecom, Taipei, Taiwan. 17-21.

[6] Stuber, G., Mclaughlin, S.W. 2004. Broadband MIMO-OFDM wireless communications. IEEE Conference 92:271-294.

[7] Thomas, T.A., Vook, E.W. 2003. Asynchronous interference suppression in broadband cyclic-prefix communications. IEEE Wireless Communications and Networking Conference, 568-572.

[8] Kumar Krishan, Singh, V.P. 2014. Power consumption based simulation model for mobile adhoc network. Wire. Pers. Commun. 77:1437-1448.

[9] Singh, V.P., Kumar Krishan. 2011. Survey on power control algorithms for mobile ad-hoc network. Wire. Pers. Commun. 60:679-685.

[10] Kumar Krishan, Khera Shelej. 2019. Optimization of transceiver energy with LEACH protocol for wireless sensor ad-hoc network. IEEE International Conference on Electrical, Computer and Communication Technologies, Combitore, India. 


\title{
25 Detection of nitrous oxide gas using silicene nanoribbons
}

\author{
Gurleen Kaur Walia ${ }^{1, *}$, Deep Kamal Kaur Randhawa ${ }^{2}$, \\ Kanwalpreet Singh ${ }^{3}$, Parulpreet Singh ${ }^{1}$, Harmandar Kaur ${ }^{2}$
}

\author{
${ }^{1}$ School of Electronics and Electrical Engineering, Lovely Professional University, \\ Phagwara, Punjab, India \\ ${ }^{2}$ Department of Electronics and Communication Engineering, Guru Nanak \\ Dev University, Regional Campus, Jalandhar, India \\ ${ }^{3}$ Department of Computer Science and Engineering, UIET, Panjab University, \\ Swami Sarvanand Giri Regional Centre, Hoshiarpur, Punjab, India
}

\section{Introduction}

With the rise in advances in technology and chemical activities, the environment as well as human health get affected badly due to discharge of toxic matter in the environment. To monitor the toxic matter, sensing of gas molecules is imperative to environment supervision, controlling the chemical processes, agricultural and medicinal applications [1-17]. For sensing mechanism, many gas sensors have been developed with high sensitivity and selectivity. Silicene, an analogue to graphene, is a 2-D allotrope of silicon that has great importance for sensing applications [18-24]. Many gas molecules have been sensed to date with regard to sensitivity and selectivity. Silicene is a buckled honeycomb structure mixed with $\mathrm{sp}^{2}$ and $\mathrm{sp}^{3}$ hybridization. Various experimental as well as theoretical studies have been done in order to cover many facets of silicene. In this paper, we mainly focus on adsorption behaviour of $\mathrm{N}_{2} \mathrm{O}$ (nitrous oxide) molecule on ASiNRs by using ab-initio method employing non-equilibrium's Green's function method (NEGF) and density functional theory (DFT). At present, there are $\mathrm{N}_{2} \mathrm{O}$ infrared gas sensors that measure this gas over the range $0-1 \%$ volume.

$\mathrm{N}_{2} \mathrm{O}$ is a greenhouse gas that affects the earth's climate and ozone layer. Earlier it was believed that soil microbes were solely responsible for its formation, but in 2018 an interdisciplinary research team from the University of Applied Sciences, Bingen and Heidelberg University, observed that plants too are the source of $\mathrm{N}_{2} \mathrm{O}$. The result of their study is that along with the human-induced global warming, the Earth's flora too emits detectable amounts of $\mathrm{N}_{2} \mathrm{O}$ contributing to the greenhouse effect. Long exposure to this gas may have some harmful effect such as dizziness, syncope, confusion, cardiac arrhythmias, seizures, leukopenia, megaloblastic anaemia, thrombocytopenia, myelopathy and peripheral neuropathy (especially posterior column findings) [25].

\footnotetext{
* Email: gurleenwalia.13@gmail.com
} 


\section{Computational methods}

DFT and NEGF combination is used for the calculation of transport and electronic attributes of P-ASiNRs. All calculations are performed using Atomistix Toolkit-Virtual NanoLab (ATK-VNL) issued by Quantumwise [26]. The exchange-correlation function of local density approximation (LDA) of Perdew-Zinger [PZ] is used. In this work, ASiNRs width is taken as 5 atoms and buckling height is $0.44 \AA$. Silicene can have three types of structure, namely high-buckling, low-buckling or planar structures, but the most stable structure is low-buckled. The mesh cut-off energy is taken as $150 \mathrm{Ry}$ and electron temperature is constant at $300 \mathrm{~K}$. The optimization of all geometries is carried out in a self-consistent way. The associated stress is kept lower than $0.1 \mathrm{GPa}$ with the HellmannFeynman force between the atoms less $0.05 \mathrm{eV} / \mathrm{A}$. K-point sampling of $1 \times 1 \times 99$ is used for electronic property calculations. A two-probe model is made, constituting of two electrodes and the central scattering region for the calculation of transport properties.

\section{Results and discussions}

First-principle investigations are utilized to understand and observe the behaviour of PASiNR before and after adsorption of $\mathrm{N}_{2} \mathrm{O}$ gas molecule. The paper mainly focuses on the investigation of the electronic and transport properties.

\subsection{Electronic properties}

The properties, namely, adsorption energy, band structure and DOS, have been studied.

Adsorption energy $\mathrm{E}_{\mathrm{ad}}$ is used to estimate how the foreign molecules react on the substrate. It also helps in estimating structural stability of adsorption of molecules on ASiNRs. It is calculated by relation:

$$
\mathrm{E}_{\mathrm{ad}}=\mathrm{E}_{\mathrm{ASiNR}+\text { Molecule }}-\mathrm{E}_{\mathrm{ASiNR}}-\mathrm{E}_{\text {Molecule }}
$$

where $\mathrm{E}_{\mathrm{ASiNR}+\text { Molecule }}$ symbolizes the entire optimized energy of the molecule-substrate complex. $\mathrm{E}_{\text {molecule }}$ and $\mathrm{E}_{\mathrm{ASiNR}}$ are the energies of single gas molecule and pristine ASiNR, respectively. In pristine ASiNR, $\mathrm{N}_{2} \mathrm{O}$ is $3.09 \AA$ away from ASiNR having adsorption energy $-0.02338 \mathrm{eV}$. The bond length between N-N is $1.127 \AA$ and N-O is $1.186 \AA$. On adsorption of the gas, the adsorption energy of the complex becomes $-0.54 \mathrm{eV}$, which shows an increase as compared with the substrate without the gas molecule.

3.1.1 Electronic band structures: It represents the energy range which the electrons may or may not occupy with regard to allowed energy bands and forbidden bands, respectively. It also helps in classifying the materials as metallic, semi-metallic or insulating, possessing indirect or direct band gaps. ASiNRs show off a varying range of characteristics in being a semi-metal, semiconductor or even an insulator. Figure 25.1 shows the results of band gap analysis. The band gap of pristine ASiNR is zero, displaying its semi-metallic behaviour, but after adsorption of $\mathrm{N}_{2} \mathrm{O}$ gas molecule the band gap rises to $0.28 \mathrm{eV}$, so the system becomes semiconductor.

3.1.2 Density of states: These characterize the total amount of electronic energetic states that are present per unit energy per unit volume. Higher values of DOS show there are more energy states available that can be occupied by electrons. DOS value is zero for no available state means. DOS is calculated as:

$$
\mathrm{D}(\mathrm{E})=\mathrm{dN}(\mathrm{E}) / \mathrm{dE}
$$



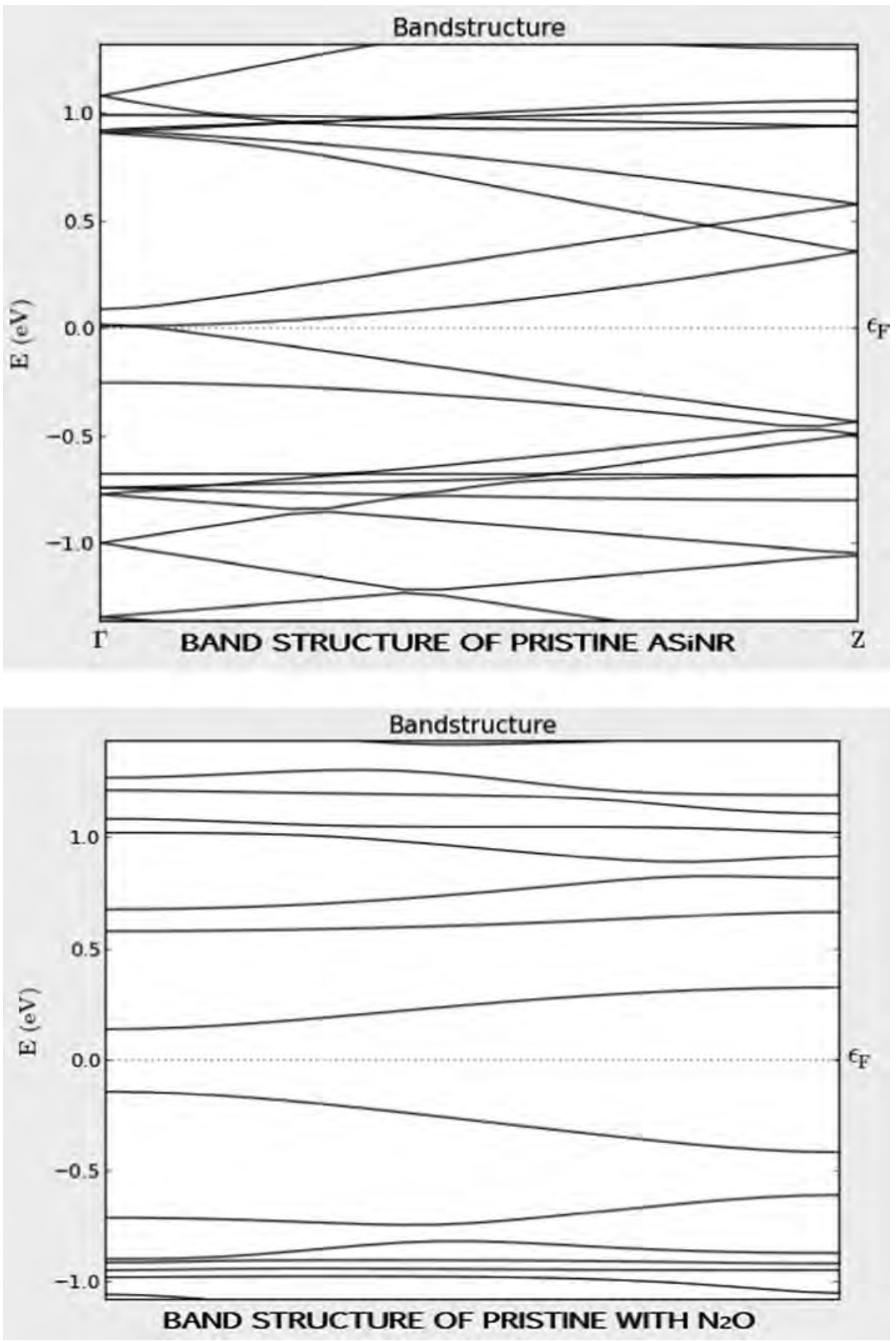

Figure 25.1 Band structures of ASiNR without and with $\mathrm{N}_{2} \mathrm{O}$. 


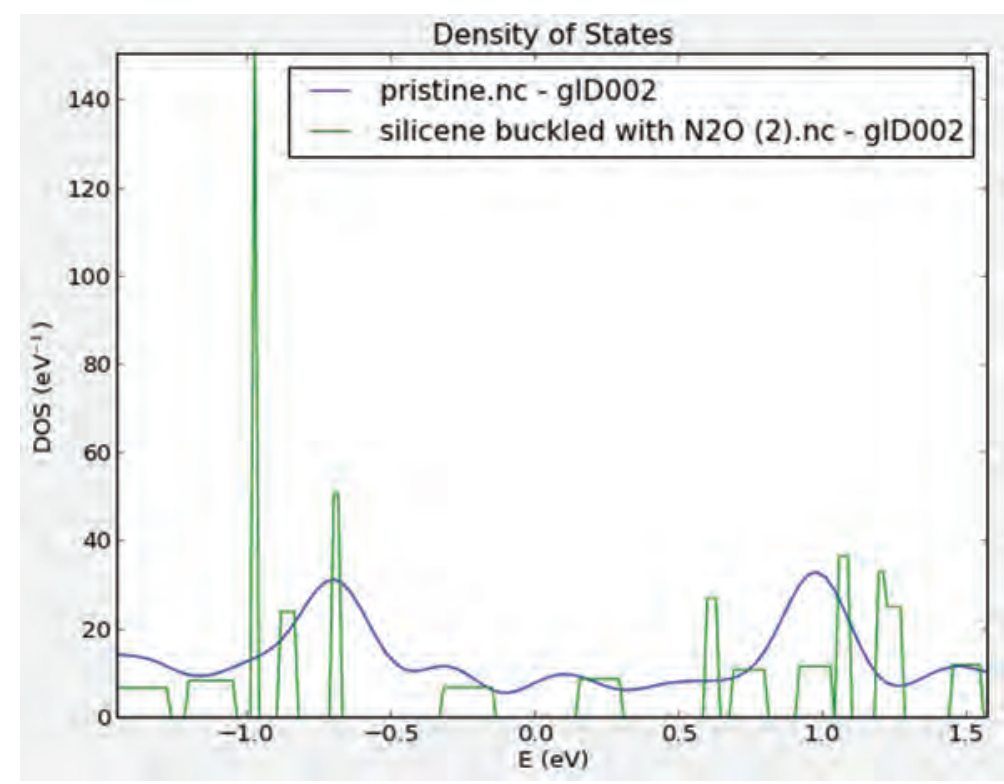

Figure 25.2 DOS comparison of ASiNR without and with $\mathrm{N}_{2} \mathrm{O}$.

where $\mathrm{N}$ symbolizes the number of electrons that possess energy $\mathrm{E}$ and having the energy range $\mathrm{dE}$. The total number of electrons is dependent on the energy. As per equation (2), DOS comes out to be inversely proportional to the energy slope, i.e. DOS values are lower for the steeper energy bands and vice versa. The results obtained from density of states analysis are shown in Figure 25.2. In this work, DOS peaks after adsorption possess much more magnitude as compared with the pristine nanoribbon, signifying the availability of a greater number of states.

\subsection{Transport properties}

The system is simulated by using a two-probe model for calculating the transport properties.

3.2.1 I-V curve: The current-voltage curve in Figure 25.3 shows that after the adsorption, there is increase in the conductivity, signifying the sensitivity.

\section{Conclusion}

In this paper, the interaction between $\mathrm{ASiNR}$ and $\mathrm{N}_{2} \mathrm{O}$ gas molecules is examined. Using DFT calculations, the properties of ASiNR are studied when the foresaid gas molecule is adsorbed on it. The results demonstrate that ASiNR is capable enough in sensing the $\mathrm{N}_{2} \mathrm{O}$ molecule even though it interacts with the substrate via weak van der Waals forces. The current-voltage characteristics also reveal better conductivity after $\mathrm{N}_{2} \mathrm{O}$ adsorption on ASiNR. Therefore, ASiNR is a suitable sensor for the $\mathrm{N}_{2} \mathrm{O}$ gas molecule. 


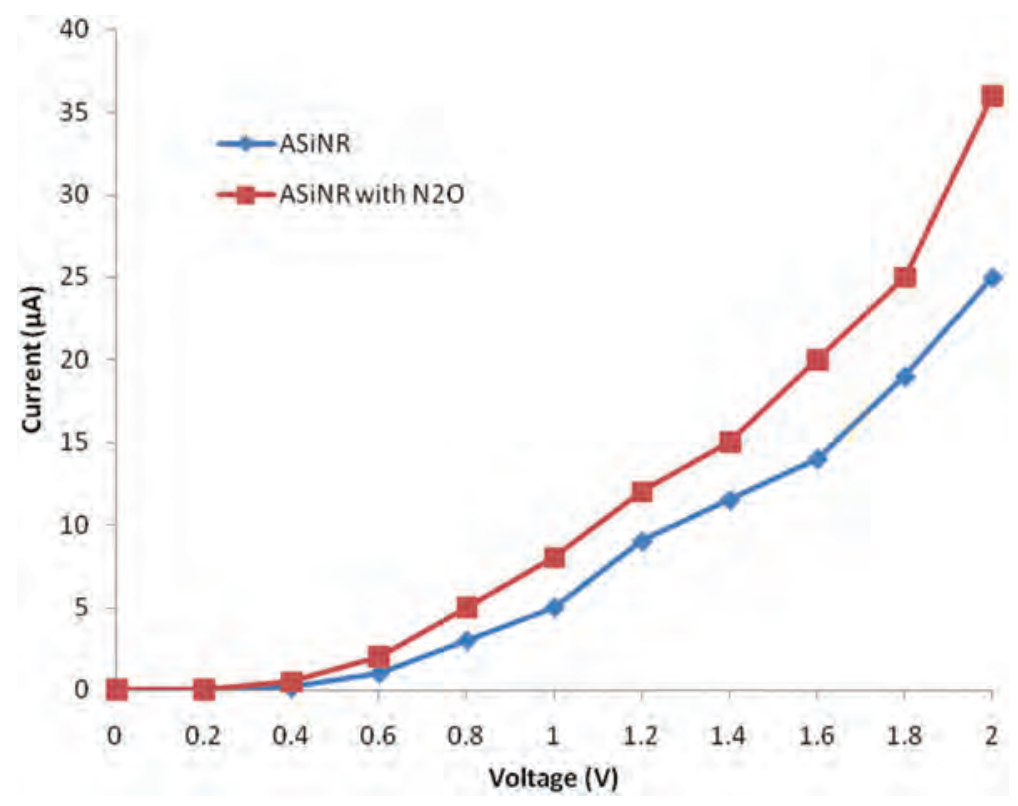

Figure 25.3 I-V curve.

\section{References}

[1] A.A. Peyghan and Z. Bagheri, "Electronic response of BC3 nanotube to $\mathrm{CS}_{2}$ molecules: DFT studies”, Comput. Theor. Chem. vol. 1008 (2012), pp. 1-7.

[2] A.A. Peyghan, M. Noei, and M.B. Tabar, "A large gap opening of graphene induced by the adsorption of Co on the Al-doped site", J. Mol. Model. vol. 19 (2013), pp. 3007-3014.

[3] J. Beheshtian, A. Ahmadi Peyghan, and Z. Bagheri, "Ab initio study of $\mathrm{NH}_{3}$ and $\mathrm{H}_{2} \mathrm{O}$ adsorption on pristine and Na-doped MgO nanotubes", Struct. Chem. vol. 24 (2013), pp. 165-170.

[4] G.K. Walia and D.K.K. Randhawa, "Density-functional study of hydrogen cyanide adsorption on silicene nanoribbons", J. Mol. Model. vol. 24 (2018), p. 242.

[5] G.-H. Fan, S. Zhu, X -K. Li, K. Ni, and H. Xu, "Ab initio investigation of pristine and doped single-walled boron nitride nanotubes as acetone sensor," Comput. Theor. Chem. vol. 1115 (2017), pp. 208-216.

[6] G.K. Walia and D.K.K. Randhawa, "Gas-sensing properties of armchair silicene nanoribbons towards carbon-based gases with single-molecule resolution," J. Struct Chem. vol. 29 (2018), pp.1893-1902.

[7] Y.-H. Zhang, Y.-B. Chen, K.-G. Zhou, C.-H. Liu, J.Zeng, H.-L. Zhang, and Y. Peng, "Improving gas sensing properties of graphene by introducing dopants and defects: a first-principles study", Nanotechnology. vol. 20 (2009), p. 185504.

[8] G.K. Walia and D.K.K. Randhawa, "First-principles investigation on defect-induced silicene nanoribbons - A superior media for sensing $\mathrm{NH}_{3}, \mathrm{NO}_{2}$ and $\mathrm{NO}$ gas molecules", Surf. Sci. vol. 670 (2018), pp. 33-43.

[9] A. Hosseinian, Z. Asadi, L. Edjlali, A. Bekhradnia, and E. Vessally, " $\mathrm{NO}_{2}$ sensing properties of a borazine doped nanographene: A DFT study”, Comput. Theor. Chem. vol. 1106 (2017), pp. 36-42.

[10] G.K. Walia and D.K.K. Randhawa, Adsorption and dissociation of sulfur-based toxic gas molecules on silicene nanoribbons: a quest for high-performance gas sensors and catalysts”, J. Mol. Model. vol. 24 (2018), pp. 94. 


\section{Intelligent Circuits and Systems}

[11] V. Nagarajan and R. Chandiramouli, " $\mathrm{TeO}_{2}$ nanostructures as a $\mathrm{NO}_{2}$ sensor: DFT investigation", Comput. Theor. Chem. vol. 1049 (2014), pp. 20-27.

[12] G.K. Walia and D.K.K. Randhawa, "Electronic and transport properties of silicene-based ammonia nanosensors: an ab initio study", J. Struc. Chem. vol. 29 (2018), pp. 257.

[13] B. Huang, Z. Li, Z. Liu, G. Zhou, S. Hao, J. Wu, B.-L. Gu, and W. Duan, "Adsorption of gas molecules on graphene nanoribbons and its implication for nanoscale molecule sensor", J. Phys. Chem. C. vol. 112 (2008) pp. 13442-13446.

[14] X.-L Wei, Y.-P Chen, W.-L Liu, and J.-X Zhong, "Enhanced gas sensor based on nitrogenvacancy graphene nanoribbons", Phys. Lett. A. vol. 376 (2012) pp. 559-562.

[15] L. Shao, G. Chen, H. Ye, H. Niu, Y. Wu, Y. Zhu, and B. Ding, "Sulfur dioxide molecule sensors based on zigzag graphene nanoribbons with and without Cr dopant", Phys. Lett. A. vol. 378 (2014) pp. 667-671.

[16] B. Huang, Z. Li, Z. Liu, G. Zhou, S. Hao, J. Wu, B.-L. Gu, and W. Duan, "Adsorption of gas molecules on graphene nanoribbons and its implication for nanoscale molecule sensor", J. Phys. Chem. C. vol. 112 (2008) pp. 13442.

[17] M.M. Monshi, S.M. Aghaei, and I. Calizo, "Doping and defect-induced germanene: A superior media for sensing $\mathrm{H}_{2} \mathrm{~S}, \mathrm{SO}_{2}$, and $\mathrm{CO}_{2}$ gas molecules", Surf. Sci. vol. 665 (2017) pp. 96.

[18] A. Kara, H. Enriquez, A.P. Seitsonen, L.C. Voon, S. Vizzini, B. Aufray, and H. Oughaddou, "A review on silicene - New candidate for electronics", Sci. Rep. vol. 67 (2012) pp. 1-18.

[19] P. Vogt, D.P. De Padova, C. Quaresima, J. Avila, E. Frantzeskakis, M.C. Asensio, A. Resta, B. Ealet, and G.L. Lay, "Silicene, compelling experimental evidence for graphene like twodimensional silicon", Phys. Rev. Lett. vol. 108 (2012) pp. 155501.

[20] J. Zhao, H. Liu, Z. Yu, R. Quhe, S. Zhou, Y. Wang, C.C. Liu, H. Zhong, N. Han, J. Lu, Y. Yao, and K. Wu, "Rise of silicene: A competitive 2D material", Progr. Mater. Sci. vol. 83 (2016) pp. 24-151.

[21] Y. Ding and J. Nia, "Electronic structures of silicon nanoribbons", Appl. Phys. Lett. vol. 95 (2009) pp. 083115.

[22] M.J.S. Spencer and T. Morishita. Silicene, Springer Series in Materials Science. 235 (2016). Switzerland.

[23] N.D. Drummond, V. Zólyomi, and V.I. Fal'ko, "Electrically tunable band gap in silicene", Phys. Rev. B Condens. Matter. vol. 85 (2012) p. 075423.

[24] R.G. Amorim and R.H. Scheicher, "Silicene as a new potential DNA sequencing device", Nanotechnology. vol. 26 (2015) pp. 154002.

[25] A. Schneir, "Nitrous oxide", Ch. 116 in Kent R. Olson (Ed.), Poisoning \& Drug Overdose, $6^{\text {th }}$ edn., McGraw-Hill, 2011.

[26] QuantumWise. Copenhagen, Denmark: Atomistix Toolkit version 2015.0. Available from: / www.quantumwise.com. 


\title{
26 Placement of solar DGs and capacitors in distribution network using combined approach
}

\author{
Shaik Aarif ${ }^{1,2 *}$, Suresh Kumar Sudabattula ${ }^{3}$
}

\author{
${ }^{1}$ Assistant Professor, Electrical and Electronics Engineering Department, \\ Prakasam Engineering College, Kandukur, Andhra Pradesh, India \\ ${ }^{2}$ Research Scholar, School of Electronics and Electrical Engineering, \\ Lovely Professional University, Phagwara, Punjab, India \\ ${ }^{3}$ Associate Professor, School of Electronics and Electrical Engineering, \\ Lovely Professional University, Phagwara, Punjab, India
}

\section{Introduction}

With the rapid escalation of power demands and pollution levels, it is viable to utilize renewable sources (RESs) in a suitable way. Further, placing these sources in DN is a challenging issue, because improper usage of these produces negative results. So, efficient utilization and connecting these sources in DN is a prominent research issue in today's scenario. Also, losses in DN is dominating as compared to transmission network [1]. Further, most feeders used in DN are of radial type which degrades the voltage profile of the farthest consumer. So, connecting compensating devices in suitable places is also an important issue. Finally, utilization of RESs and placement of compensating devices in $\mathrm{DN}$ is a most significant issue in active DN. So, in this article a suitable methodology is presented to solve this issue.

Further, in the literature authors solved DG placement problem (DGPP) and CPP and combination of both are solved and achieved good results. In [2], the authors used modified TLBO for solving DGPP in DN. Further, TLBO is compared with brute force method. In [3] the authors used QOTLBO method for solving DGPP in DN. Also, various test systems like 33, 69 and 118 bus are taken and the performance evaluated. In [4] the authors considered the BAT algorithm for solving solar DGPP in DN. Also, uncertainties of solar irradiance are taken into account. In [5], the FPA and LSF method is used to solve DGPP in DN. Further, efficacy of the method is tested with other approaches. In [6] the authors considered wind DGs in DN. Further, historical data are considered in the problem framework. Next, in [7], CPP is solved using TLBO. Further, cost and power loss are considered in the formulation. In [8], the ABC algorithm is applied for solving CPP in DN. Further, real and standard systems are considered for the testing. In [9], clustering-based OT is applied to solve CPP in DN. Further, the method is compared with various existing methods. In [10], the mine blast algorithm is presented to solve CPP in DN. Further, it is verified on 10 and 85 bus DN. Finally, DG and CPP are solved using different algorithms. In [11], the authors evaluate DG and CPP together using various OTs. Further, they compare this with other approaches in the literature. In [12], the dragonfly algorithm is used to solve DG and CPP in DN. Further, it is compared with other methods for validation.

\footnotetext{
* Corresponding author email: shaik.aarif202@gmail.com
} 


\section{Intelligent Circuits and Systems}

In this paper a combined method based on SA and DA is presented to solve solar DG and CPP in DN. Further, it is tested on a 69 bus system. Separate cases are considered for the evaluation and finally significant findings are observed from the results. The organization of the paper as follows: objective function (OF) and location of DGs and capacitor identification is given in Sections 2 and 3. Next, algorithmic steps to solve the problem are explained in Section 4. Finally, important observations and conclusions are given in Sections 5 and 6.

\section{Objective function}

The aim of this work is to improve power loss reduction of DN with allocation of solar DGs and capacitors in combination and the formulae for this given by Eq. (1)

$$
P_{\text {Tloss }}^{S D G / C a p}=\sum_{n=1}^{n b} P_{\text {loss }}^{S D G / C a p}
$$

Also, the OF satisfies various constraints of DN.

\subsection{Constraints}

\subsubsection{Solar DG}

$$
P_{S D G}^{\min } \leq P_{D G} \leq P_{S D G}^{\max }
$$

\subsubsection{Capacitor}

$$
\sum_{n=1}^{n_{c}} Q_{c} \leq 1.0 \sum_{n=1}^{n_{l}} Q_{L}
$$

\subsubsection{Voltage magnitude}

$$
V_{\min } \leq V \leq V_{\max }
$$

\section{Location of solar DGs and capacitors using SA}

Sensitivity analysis (SA) is a prominent approach to solve location identification of DGs and capacitors in DN [13]. Further, the SA formulas for finding out DGs and capacitors is given in Eq. (5) and Eq. (6).

$$
\begin{gathered}
\frac{\partial P_{\text {lineloss }}}{\partial P_{n+1, \text { eff }}}=\frac{2 P_{n+1, \text { eff }} R_{n \cdot n+1}}{\left|V_{n+1}\right|^{2}} \\
\frac{\partial P_{\text {lineloss }}}{\partial Q_{n+1, \text { eff }}}=\frac{2 Q_{n+1, \text { eff }} R_{n \cdot n+1}}{\left|V_{n+1}\right|^{2}}
\end{gathered}
$$


Calculate sensitivities and place both DGs and capacitors which have the highest sensitivities.

\section{Algorithmic steps to solving the sizing problem of solar DGs and capacitors}

DA is developed by Mirjalili and it is based on the swarming behaviour of dragonflies. The mathematical modelling of this algorithm is given in [14].

1. Read the data of the 69 bus system

2. Locations are found using SA

3. Given locations are input to the algorithm

4. Set the dimensions of search space $(d=3)$

5. Initialize the parameters [13]

6. Attraction towards the food sources represents the best sizes of solar DGs/capacitors given in Eq. (7)

$$
F^{k}=X^{+}-X
$$

7. Further, distraction towards enemy gives the sizes but those are not acceptable given that in Eq. (8)

$$
E^{k}=X^{-}+X
$$

8. Modify the step vector using Eq. (9)

$$
\Delta X_{k+1}=\left(s S_{k}+a A_{k}+c C_{k}+f F_{k}+e E_{k}\right)+w \Delta X_{k}
$$

9. Modify the position vector using Eq. (10)

$$
X_{k+1}=X_{k}+\Delta X_{k+1}
$$

End while

10. At the obtained sizes calculate the power loss: if it is less stop the procedure otherwise run the algorithm again until the desired output is achieved.

\section{Results and discussion}

MATLAB is used to implement the method and test system data are obtained from [15]. Further loss and voltage profiles of the system without solar DGs and capacitors are 225 $\mathrm{kW}$ and 0.9090 p.u. First locations of these sources are obtained using SA and those are 61, 49 and 12. After obtaining locations calculate the ratings of DGs and capacitors by DA. Finally, various cases are considered and results are presented in Table 26.1.

Base case

Case 1: 1 solar DG and 1 capacitor

Case 2: 2 solar DGs and 2 capacitors

Case 3: 3 solar DGs and 3 capacitors 


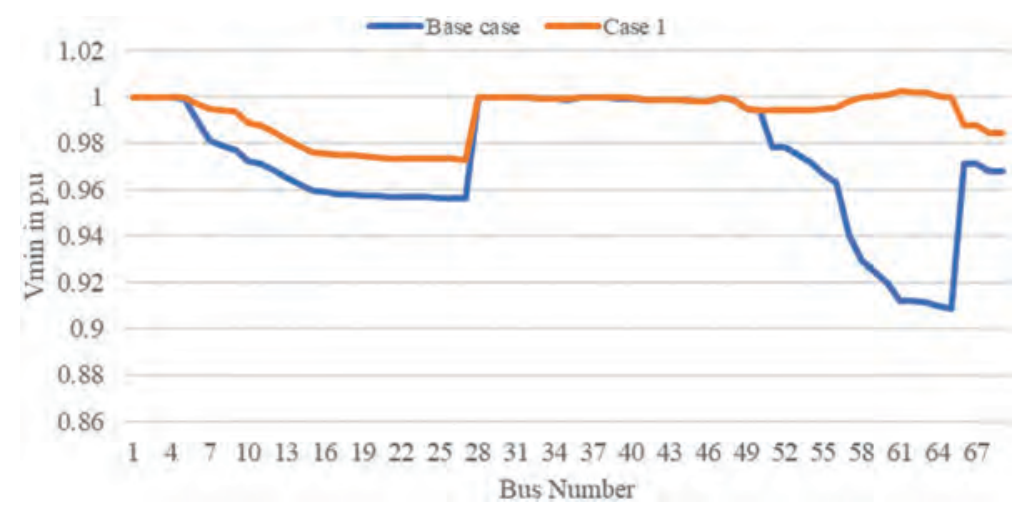

Figure $26.1 \mathrm{~V}_{\min }$ comparison for 69 bus system (base case and case 1).

Table 26.1 Optimal placement of DGs and capacitors (various cases)

\begin{tabular}{llllll}
\hline Particulars & $\begin{array}{l}\text { DG location } \\
\text { and sizes }(k W)\end{array}$ & $\begin{array}{l}\text { Capacitor } \\
\text { location and } \\
\text { sizes }(k V A r)\end{array}$ & $P_{\text {loss }}(k W)$ & $\begin{array}{l}\% P_{\text {loss }} \\
\text { reduction }\end{array}$ & $V_{\text {min }}(p . u)$. \\
\hline Base case & NA & NA & 225 & NA & 0.9090 \\
Case 1 & $61(1828.81)$ & $61(1303.95)$ & 23.17 & 89.70 & 0.9730 \\
Case 2 & $61(1833.38)$ & $61(1302.30)$ & 20.91 & 90.71 & 0.9733 \\
Case 3 & $49(810.40)$ & $49(448.32)$ & & & 0.9823 \\
& $61(1693.58)$ & $61(1159.22)$ & 6.431 & 97.41 & \\
& $49(977.50)$ & $49(708.48)$ & & & \\
\hline
\end{tabular}

In case 11 solar DG and 1 capacitor are placed at 61 bus, the power loss $\left(\mathrm{P}_{\text {loss }}\right)$ is reduced to $23.17 \mathrm{~kW}$ and the voltage profile is increased to $0.9730 \mathrm{p}$.u. As compared to base case the $\mathrm{P}_{\text {loss }}$ is reduced to $201.83 \mathrm{~kW}$ and the minimum voltage $\left(\mathrm{V}_{\min }\right)$ is improved in p.u to 0.064 . Further, in percentage comparison $\mathrm{P}_{\text {loss }}$ and $\mathrm{V}_{\min }$ is improved to $89.70 \%$ and $6.577 \%$. In case 2 , the $P_{\text {loss }}$ is minimized to $20.91 \mathrm{~kW}$ and $V_{\min }$ is enhanced to 0.9734 p.u. As compared to base case it is reduced to $204.1 \mathrm{~kW}$ and $\mathrm{V}_{\min }$ is enhanced to 0.0644 p.u. Further, in percentage improvement point view it is improved to $90.71 \%$ and $6.62 \%$. In comparison with case 1 it is reduced to $2.26 \mathrm{~kW}$ and from a percentage point of view it is enhanced up to $9.75 \%$. Finally, in case 3 the $\mathrm{P}_{\text {loss }}$ and $\mathrm{V}_{\min }$ after placing these are $6.43 \mathrm{~kW}$ and 0.9823 p.u.

In comparison with the base case it is reduced to $218.57 \mathrm{~kW}$ and voltage profile in p.u is improved to 0.0733 . Further, in percentage point of view power loss and voltage profile as compared to base case are improved to $97.14 \%$ and $7.46 \%$. Also, compared with case 1 it is improved to $72.21 \%$ and $0.946 \%$. Further, in comparison with case 2 it is improved up to $69.25 \%$ and $0.917 \%$. From the overall comparison maximum loss reduction and $\mathrm{V}_{\min }$ enhancement is achieved in case 3, which is the optimal number of DGs and capacitors in DN. Figures 26.1 and 26.2 show the comparison voltage profile for all cases. It is observed that voltage profiles at all buses increased to maximum values. 


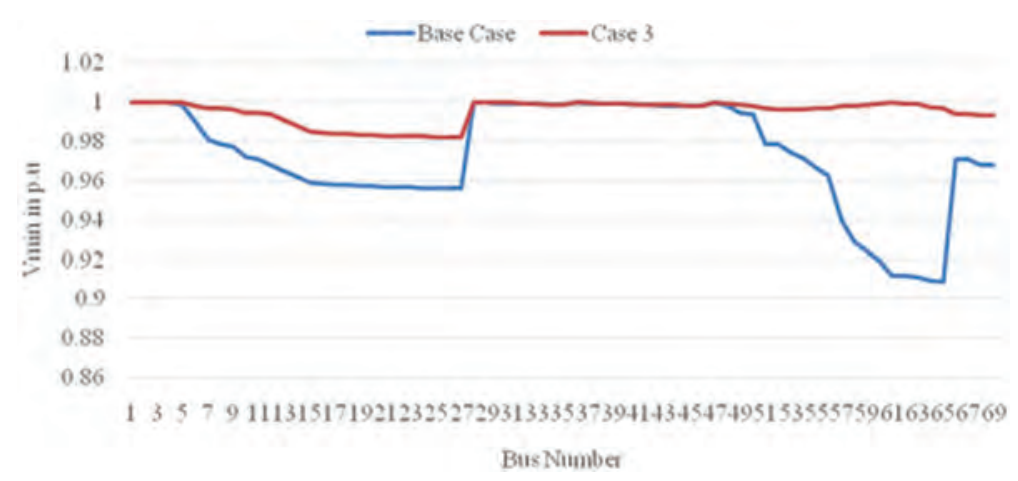

Figure $26.2 \mathrm{~V}_{\min }$ comparison for 69 bus system (base case and case 3).

\section{Conclusion}

A combined approach of DA and SA is applied to solve DG and CPP in DN. First, locations are found using SA and sizes tare obtained by applying DA. In the analysis various cases are taken and it is tested on a 69 bus system. It is verified that allocation of solar DGs along with capacitors reduces the losses to a suitable extent. Finally, minimum $\mathrm{P}_{\text {loss }}$ along with $\mathrm{V}_{\min }$ is obtained in case 3 (3 solar DGs and capacitors). So, it can be concluded that the optimal number of solar DGs along with capacitors minimizes the losses and improves the DN performance reasonably.

\section{References}

[1] G. Pepermans, J. Driesen, D. Haeseldonckx, R. Belmans and W. D’haeseleer, “Distributed generation: definition, benefits and issues”, Energy Policy vol. 33, no. 6 (2005) pp. 787-798.

[2] J.A.M. García and A.J.G. Mena, "Optimal distributed generation location and size using a modified teaching-learning based optimization algorithm”, Int. J. Electr. Power Energy Syst. vol. 50 (2013), pp. 65-75.

[3] S. Sultana and P.K. Roy, "Multi-objective quasi-oppositional teaching learning based optimization for optimal location of distributed generator in radial distribution systems", Int. J. Electr. Power Energy Syst. vol. 63 (2014), pp. 534-545.

[4] S.K. Sudabattula and M. Kowsalya., "Optimal allocation of solar based distributed generators in distribution system using bat algorithm”, Perspectives in Science. vol. 8 (2016), pp. 270-272.

[5] S.K. Sudabattula and M. Kowsalya, "Distributed energy resources allocation using flower pollination algorithm in radial distribution system”, Energy Procedia. vol. 103 (2016), pp. 76-81.

[6] A. Ouamm, H. Dagdougui and R. Sacile, "Optimal planning with technology selection for wind power plants in power distribution networks”, IEEE Syst. J. vol. 13, no. 3 (2019), pp. 3059-3069.

[7] S. Sultana and P.K. Roy, "Optimal capacitor placement in radial distribution systems using teaching learning based optimization”, Int. J. Electr. Power Energy Syst. vol. 54 (2014), pp. 387-398.

[8] A.A. El-Fergany and A.Y. Abdelaziz, "Capacitor placement for net saving maximization and system stability enhancement in distribution networks using artificial bee colony-based approach”, Int. J. Electr. Power Energy Syst. vol. 54 (2014), pp. 235-243. 


\section{Intelligent Circuits and Systems}

[9] J. Vuletić and M. Todorovski, "Optimal capacitor placement in radial distribution systems using clustering based optimization”, Int. J. Electr., Power Energy Syst. vol. 62 (2014), pp. 229-236.

[10] S.A. Elazim and E.S. Ali, "Optimal locations and sizing of capacitors in radial distribution systems using mine blast algorithm”, Electr. Eng. vol. 100, no. 1 (2018), pp. 1-9.

[11] N. Kanwar, N. Gupta, K.R. Niazi and A. Swarnkar, "Improved meta-heuristic techniques for simultaneous capacitor and DG allocation in radial distribution networks",. Int. J. Electr. Power Energy Syst. vol. 73 (2015), pp. 653-664.

[12] S.K. Sudabattula, M. Kowslaya and V. Suresh, "Simultaneous allocation of distributed generators and shunt capacitors in distribution system" ECTI Trans. Electr. Eng. Electron. Commun. vol. 17, no. 1 (2019), pp. 35-50.

[13] S. Kaur, G. Kumbhar and J. Sharma, "A MINLP technique for optimal placement of multiple DG units in distribution systems”, Int. J. Electr. Power Energy Syst. vol. 63 (2014), pp. 609-617.

[14] S. Mirjalili, "Dragonfly algorithm: a new meta-heuristic optimization technique for solving single-objective, discrete, and multi-objective problems", Neural Computing and Applications. vol. 27, no. 4 (2016), pp. 1053-1073.

[15] N.C. Sahoo and K. Prasad, "A fuzzy genetic approach for network reconfiguration to enhance voltage stability in radial distribution systems," Energy Convers. Manage. vol. 47 (2006), pp. 3288-3306. 


\title{
27 iPlanty
}

\section{A smart agriculture approach}

\author{
Anchit Sinha, $a^{1, *}$ Ayushman Trivedi ${ }^{1}$, Debaleena Das ${ }^{1}$, \\ Praveen Reddy, Sandeep Bansal, *
}

\author{
${ }^{1}$ Research Scholar, School of Electronics \& Electrical Engineering, \\ Lovely Professional University, India \\ ${ }^{2}$ Assistant Professor, School of Electronics \& Electrical Engineering, \\ Lovely Professional University, India
}

\section{Introduction}

As per the data of 2011 Census, India is the latest addition among the water-deficient nations list. Total urban population contributes almost 54\% of the global population in 2014. More than 100 countries are facing the problem of water scarcity [1]. As per the data gave by central water commission of India, it has been estimated that $85.3 \%$ of the total water is consumed by an agriculture sector only which has been predicted to be $83.3 \%$ by 2025 . There are several methods or techniques to control the wastage of water in agriculture while irrigating a field which is increasing at a very alarming rate. Any kind of climatic change such as an increase or decrease in average temperature, fluctuations in the rate of rainfall, change in weather conditions can disrupt the land and the crop associated with that land also. Concurrently, one of the major problems encountered by the farmer is of rodents like rats, rabbits etc. damaging their crops. According to the data, in 2009 only there is a huge rodent loss befallen in irrigated rice-crop system in Myanmar, Indonesia and the Philippines [2].

Almost $70 \%$ of the global use of water is for irrigation, which includes the water which came from rivers and pumped from the underground sources; the remaining $20 \%$ and $10 \%$ is used by the industries and residences respectively. By using the rule of thumb, it has been estimated that for the production of just one ton of grain it needs almost 1000 tons of water. Sometimes crops like fruits and vegetable processing a huge amount of water through the field simultaneously generate a huge volume of wastewater [3].

According to Union Agriculture Ministry statistics says that $48.6 \%$ of India's 140 million hectares of agriculture land are irrigated. There are some statistical data which clearly states that agriculture fields in Uttar Pradesh, Punjab and Haryana using canal's water for the irrigation with more copiousness use in the top northern areas which leads to scarcity of water in the plain areas. As a consequence, the part of Uttar Pradesh like Bundelkhand, Marathwada and other places in south India face a lot of water scarcity and eventually lead to the political war among the two states [4]. Similarly, some of the desert countries like Israel are performing at their best level in the agricultural sector of their respective economy. In the current scenario, $80 \%$ of Israel's wastewater is recycled for their agricultural uses.

Main features of this includes alerts to the intended user about their field at a particular point of time by providing all the data related to the field through a web application or

\footnotetext{
* Email: sandeep.15732@lpu.co.in
} 
any other mobile application interface. At the same time by analysing and construing the data given by the sensors used in the field with the help of machine learning in order to predict the best crop bestowing according to current climatic conditions and the real time data input from the sensors so that there will be no risk involved in the decision of farmer.

\section{Related work}

In 2005, the concept of IoT (Internet of Things) was introduced for the first time by ITU (International Telecommunication Union) [5]. Considering the fact that IoT is a very new technology, many works are going on for implementing IoT in agricultural sector in many innovative and efficient ways. Some of the innovations include the culture of soil, artificial photosynthesis technology, environment control technology (carbon dioxide, humidity, wind and pressure etc.) and also the intellectual irrigation system [6]. This work tells us about the method of usage of soil moisture sensor as mentioned in the paper and the calculation of the moisture is based on different types of units: they are base station unit (BSU), value unit (VU) and sensor unit (SU). They all work in a flowchart form. BSU as first the power of the get up send station unit address data to station unit address match later sensor unit (SU) its power-ups measures $\mathrm{V}$ and sends to the address to match with BSU and finally value unit (VU) it sends address to match with BSU then if yes change position of value [7].

It has been estimated that the world population in 2050 will be approximately around 9.7 billion and for the same reason there will be more urbanization and simultaneously agricultural land will decrease but the food supply will increase eventually so for that also IoT is blessing because it has been predicted that IoT device in agriculture sector will increase to 75 million in 2020 which is quite more than double which was 30 million in 2015 [8]. Some major application of IoT found in agriculture sector is like monitoring of farm field like to monitor the unwanted motion in between the fields by rodents and intruders. In this work, if there is any motion in between the field then it will get detected by the sensors like IR used for detection of unsolicited object and as a consequence buzzers get active as an alarm and the farm land owner will also get the notifications regarding the same in his/her mobile application interface. The combination of both the Blynk and Wi-Fi module is explained in this paper and also the parameters which are required to combine the software and hardware into a security system, as it was mentioned that the security mentioned in this report was for household purpose and this could be modified and can be used in the farms in more efficient and cost-effective way by introducing smart devices such as android and iOS mobiles with very small applications which could provide real-time data [9].

This paper is about the interfacing of temperature, rain sensor, soil sensor, water level sensor etc. These are integrated to collect the data and make the decisions accordingly in Arduino and display the output on the crystal display as monitor; basically the results are shown on serial monitor in the form of data and also in the graphical form [10]. This research deals about the selection of crop according to the suitable land and weather; basic idea behind this is to give a set of data which is in the puzzle form and then solving it by layer and layer and concluding the final crop by the help of machine learning and this is all about solving a puzzle and giving efficient output [11].

\section{Methodology}

In this work, drip irrigation system is set up with inlet and outlet pipes with controlled flow of water using pump. Sensors are used to monitor the field constraints using various 


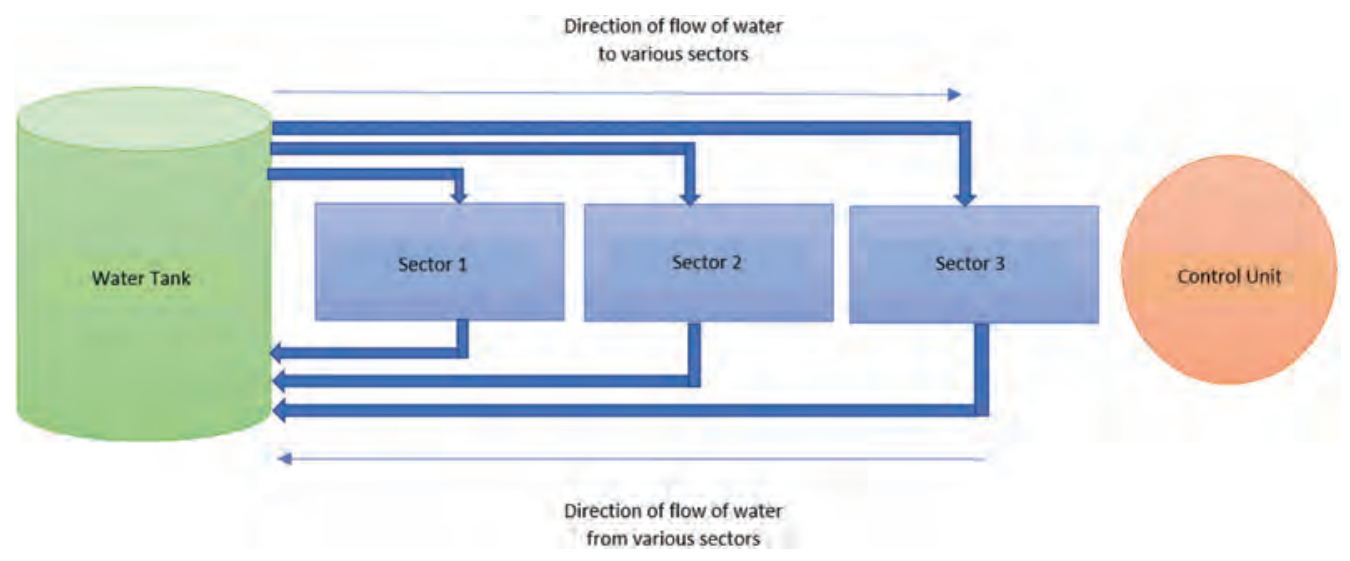

Figure 27.1 Block diagram of proposed system.

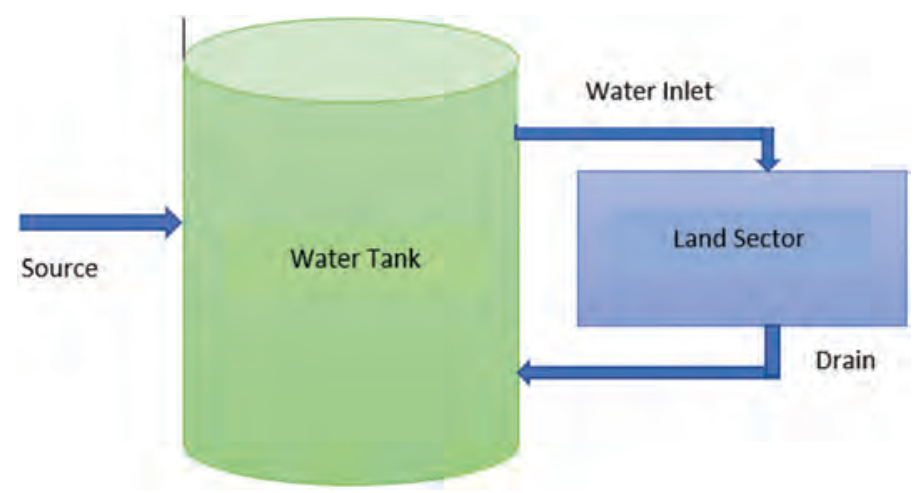

Figure 27.2 Control unit for flow of water.

sensors i.e. soil moisture sensor, IR sensor, LDR, raindrop and humidity and temperature sensor. Calibration is done using standard methods. The proposed system is quite simple yet efficient as shown in Figure 27.1. The whole system can be divided into four major parts i.e. water flow management, land sector division, sensor unit and control unit.

The water flow management consists of water pipes, pumps, water tanks, source and drain of water. The water is provided to the whole field using this system. The water enters through a water inlet which is connected to a water tank as shown in Figure 27.2. The water is pumped with the help of the electric pumps to the land sectors from the water tank whenever there is a deficiency of water in the field.

To avoid any wastage of water in field the leftover water from the sectors is collected in water tank and pumped back as shown in Figure 27.2 and it is again reprocessed whenever the need for water arises in the field. Rain also plays a vital role in irrigation. So, in order to make it a smart and efficient system, the rain drop sensor has been utilized so if there is no rainfall then only the whole irrigation system will be activated. Whole system will be automatically disabled in presence of rainfall. The whole field is divided into various sectors as shown in Figure 27.1. This is for the purpose of better water management, to 


\section{2}

avoid spreading of plant diseases, to detect and locate intruders and to grow multiple crops in a single field.

The control unit acts as the brain of the whole system. It contains various microcontrollers and will control all the functionality of the system. It is the decision-making unit of the system and it manages all the ongoing activities in the field and takes most suitable decisions in real-time data processing. The control unit is placed a bit far from the system as shown in the Figure 27.1 for ease of control. The sensor unit is used for real time measurement of various parameters from field. Some of the parameters that are measured by the sensor unit are Intruder Detection, Day or Night Detection, Rain Detection, Soil Moisture Detection, Temperature of Surrounding and Humidity of the Surrounding.

The measured values from real-time process are fed to control unit for processing and to take action of pump control, intruder detection and rainfall detection. Apart from this control system is directly involved in remote transmission of the real-time data to the user with help of mobile application and web interface.

\section{System design hardware/components}

In this work various sensors are used for field monitoring. These sensors are precise, reliable, cost-effective and easy to operate. Sensors used in this work are listed in Table 27.1.

\section{Rain-drop sensor}

A rain-drop sensor is used in the work to detect the presence of rainfall so as to make the system smarter and efficient. Figure 27.3 represents the sensor used to detect rainfall.

Table 27.1 Sensors used

\begin{tabular}{ll}
\hline Parameter & Sensors used \\
\hline Rainfall & YL-83 \\
Soil moisture & YL-69 \\
Intruder & RKI-3141 \\
Humidity & DHT-11 \\
Temperature & DHT-11 \\
Day and night & Light dependent resistor \\
\hline
\end{tabular}

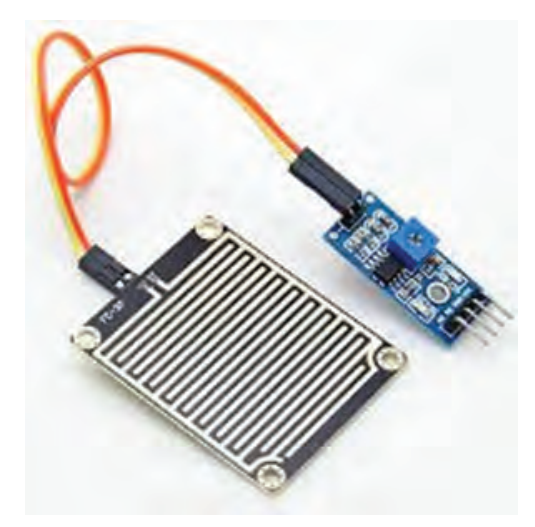

Figure 27.3 Rain-drop sensor. 


\section{Soil moisture sensor}

A soil moisture sensor is used to govern the amount of moisture present in the soil. It gives readings according to the variance in the level of moisture in the soil. Figure 27.4 portrays the soil moisture sensor. It operates on $5 \mathrm{~V} \mathrm{DC}$ with working temperature ranges from $10^{\circ} \mathrm{C}$ to $\sim 30^{\circ} \mathrm{C}$.

\section{Infrared sensor}

An infrared sensor is used to detect the light wavelengths which fall into the category of infrared sensors in light spectrum. In this sensor there is a LED known as IR emitter LED which emits wavelengths belonging to infrared region and a photodiode acts as the infrared receiver which detects the reflection of the wavelength transmitted by the IR emitter LED. Figure 27.5 illustrates IR sensor.

\section{DHT-11}

DHT-11 is a temperature and humidity sensor. It's a digital sensor giving the best and accurate reading. It uses a capacitive humidity sensor and a thermistor for measuring the condition and sends signal to data pin. The sensor gives value of temperature ranging from 0 to 50 degree Celsius and humidity from 20 to $90 \%$. Figure 27.6 illustrates DHT-11 temperature and humidity sensor.

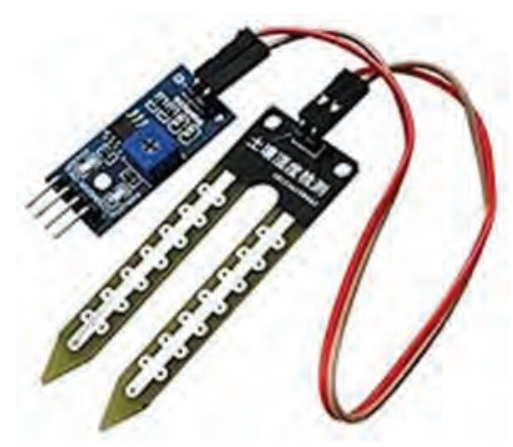

Figure 27.4 Soil moisture sensor.

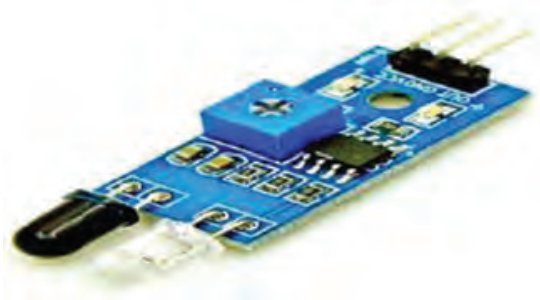

Figure 27.5 Infrared sensor. 


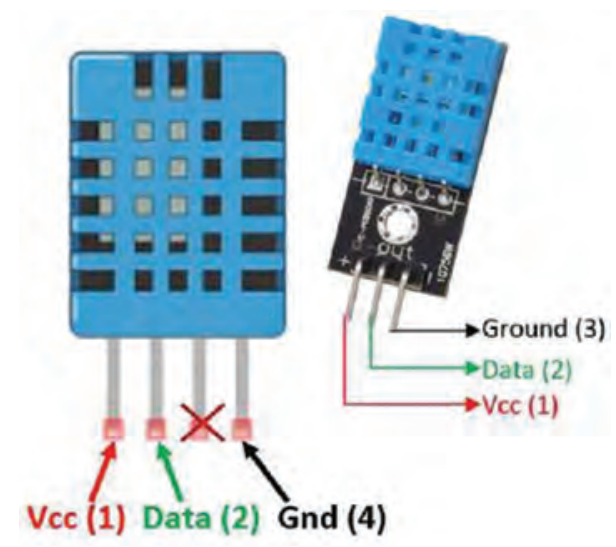

Figure 27.6 DHT-11.

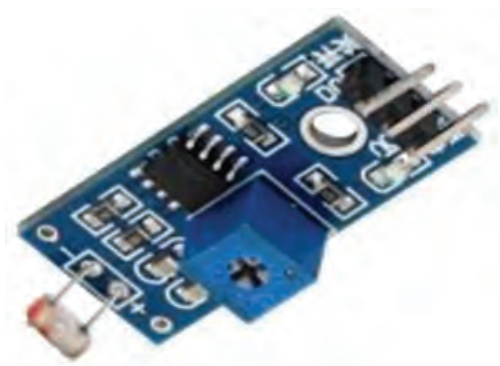

Figure 27.7 LDR sensor module.

\section{Light dependent resistor}

Light detecting resistance (LDR) is a type of variable resistance that increases or decreases the resistance value according to the intensity of light falling onto them. They are of two types: positive coefficient and negative coefficient.

The resistance decreases as the light intensity falling on LDR increases thereby making it more conducting with the increase in light intensity. Figure 27.7 illustrates an LDR sensor module.

\section{Results and discussion}

A smart irrigation system is developed in this work, which is capable of giving various real-time information with the help of IoT. This work will help in contributing to the protection of the crops by providing assistance and helps to make farming quite easy. The work also focuses on the protection of the field against various intruders. Thus, it also focuses on the safety and the security of the crops. Also, this irrigation technique saves water quite efficiently as it focuses the field as various small segments and irrigates the segments depending upon the need. Also, in this work water is not supplied to the fields if rainfall is present and thus again saves the water by a huge amount. Figure 27.8 shows the real time data being received at the user's end. 


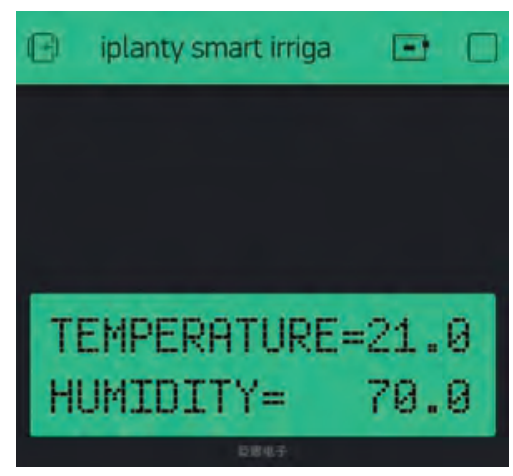

Figure 27.8 Real-time status of the field being acknowledged in mobile app.

In this work, the peripheral pumps are automatically controlled and the system is intended in such a way that if any specific place of the field is having less water, then the pumps will automatically switch on and irrigate the required sector. The entire arrangement is divided into three sectors and they are controlled autonomously by using several kinds of sensors like soil moisture sensors etc. Also, the system is intended in such a fashion that it will only identify the impostors after dusk. This system majorly focuses on being efficient and reduces the manpower. The real time data is received by the user as depicted in Figure 27.8.

\section{Conclusion}

The work mainly focuses on the construction of the smart irrigation system. The various sensors were used to make a wholly automated system which can work very precisely with very little human intervention. The execution of this work will help the farmers or any other client to take care of their field quite effectively and can monitor various parameters that are quite beneficial for the farmers. The various parameters include the presence of water in the various sectors of the field, the temperature in surrounding, humidity in the surrounding, rainfall status, etc. Apart from these lucrative features, there is another very beneficial feature included in this work, i.e. detection of the intruders. The system for detection of trespassers is planned in a very effective way and this procedure can send the messages if there are any interlopers present in the field and warn the agrarians.

This effectively provides safety and security to crops and the field. Also, this technique is only activated after dusk when the major mishaps took place and it also saves quite a lot of energy in this process. Water conservation is another major factor which is taken into contemplation while designing and implementing this work. Apart from drip irrigation this work also uses a proper drainage system that effectively reuses the water, i.e. left inside the pipes and reuses it. So, this work can easily be implemented in the places with less rainfall or the places which are facing the water shortage problem. Also, to increase the working precision of the sensors and to make the process of irrigation quite easy the whole field is divided into segments. So, the agrarians can easily monitor the whole field both physically and with the help of the application. These segments also allow the detection of the intruders quite effectively and easy as one can easily locate the presence of intruders in a particular sector with the help of the warning systems and the mobile application. 
So, it can be concluded that this work is based on how to save water and energy while helping the farmers to earn their share of profit quite easily and also the farmers can know their field in a much better way after implementing this technique. Also, this work focuses on the safety of the crops and the installed intruder detection system works quite effectively to serve for this purpose. This safety system allows the farmer or the owner of the land to take proper decision to save the field from any kind of damage.

\section{References}

1. Zhang, Yucui, and Shen, Yanjun. (2017). Wastewater irrigation: past, present, and future: Wastewater irrigation. WIREs Water. https://doi.org/10.1002/wat2.1234.

2. Wiley Interdisciplinary Reviews: Water. e1234. 10.1002/wat2.1234.

3. Singleton Grant R., Belmain Steven, Brown Peter R., Aplin Ken and Htwe Nyo Me (2010) Impacts of rodent outbreaks on food security in Asia. Wildlife Research 37, 355-359

4. Singh, R., and Phadke, V. (2006). Assessing soil loss by water erosion in Jamni River Basin, Bundelkhand region, India, adopting universal soil loss equation using GIS. Current Science, 90(10), 1431-1435. Retrieved January 23, 2020, from www.jstor.org/stable/24091996.

5. L. Li, H. Xiaoguang, C. Ke and H. Ketai, "The applications of WiFi-based Wireless Sensor Network in Internet of Things and Smart Grid," 2011 6th IEEE Conference on Industrial Electronics and Applications, Beijing, 2011, pp. 789-793.

6. Li Hong. "IOT and cloud computing: Advance strategic new industry" [M].Beijing, Posts \& Telecom Press, China, 2011.

7. Dursun, M., and Ozden, S. (2011). A wireless application of drip irrigation automation supported by soil moisture sensors. Scientific Research and Essays, 6(7), 1573-1582.

8. O. Elijah, T. A. Rahman, I. Orikumhi, C. Y. Leow and M. N. Hindia, "An overview of internet of Things (IoT) and data analytics in agriculture: benefits and challenges," in IEEE Internet of Things Journal, vol. 5, no. 5, pp. 3758-3773, Oct. 2018.

9. Yi, P., Iwayemi, A., and Zhou, C. (2010). Developing ZigBee deployment guideline under WiFi interference for smart grid applications. IEEE Transactions on Smart Grid, 2(1), 110-120

10. Anushree, M. K., and Krishna, R. (2018). A smart farming system using Arduino based technology. Ijariit Journal 4(4).

11. Harrington, P. (2012). Machine Learning in Action. Manning Publications Co. 


\title{
28 Electrostatically actuated graphene NEMS switch with different bridge structures
}

\author{
Rekha Chaudhary, Prasantha R. Mudimela* \\ School of Electronics and Electrical Engineering, Lovely Professional \\ University, Jalandhar, India
}

\section{Introduction}

In applications like remote control and communication systems, Microelectromechanical system (MEMS) technologies have een intensively developed and applied [1]. Radiofrequency (RF) MEMS switch is one of the devices of MEMS technology that has attracted the attention of researchers in recent years [2]. RF MEMS switches offer low actuation voltage, good isolation, low power consumption, and low insertion loss [35]. Metal-based RF MEMS switches suffer from the problem of stiction, self-actuation, and electromigration [6]. The success of the semiconductor industry depends upon the scalable manufacturing process of devices and their reliable performances. As the size of semiconductor devices is scaled down, the demands of such devices in applications have increased rapidly. The outstanding features like very small size, low power consumption, and low leakage current make a nanoelectromechanical (NEM) switch the best choice in many applications [7]. Also, NEM systems are suitable in harsh environments as these are insensitive to the external electric field [8], radiation [9], and temperature [10]. Graphene is a flat layer of carbon atoms connected in a honeycomb structure [11]. Graphene has remarkable electrical [12], mechanical [13] and thermal [14] properties. The outstanding mechanical properties have attracted the use of graphene as suspended in RF NEM switches as it could to lower the actuation voltage $[15,16]$. The fabrication of graphenebased NEM switches is much easier than carbon nano-tube based switches [17-20]. In the NEM switch, the actuation voltage can be reduced by various methods [20,21]. The reduction in spring constant is one of the methods that can be used as actuation voltage directly depends upon the spring constant of the suspended beam [22].

In this paper, graphene-based NEM switches with different bridge structures are simulated in COMSOL Multiphysics. The analysis of the actuation voltage of five different graphene bridge structures has been done. The electrostatic actuation mechanism has been used to actuate the switch. The total capacitance of the NEM switch is also simulated.

\section{Different bridge structures}

NEMS switch consists of a metal bridge structure suspended at some height over the transmission line. The central conductor of the transmission line is covered with a dielectric material. The central conductor at which bias voltage is applied is known as the actuation electrode. This structure in which the suspended beam is placed perpendicularly to the signal line (covered with the dielectric) is known as a shunt capacitive switch. In this work,

* Corresponding author email: prasantha.22708@lpu.co.in

DOI: $10.1201 / 9781003129103-28$ 

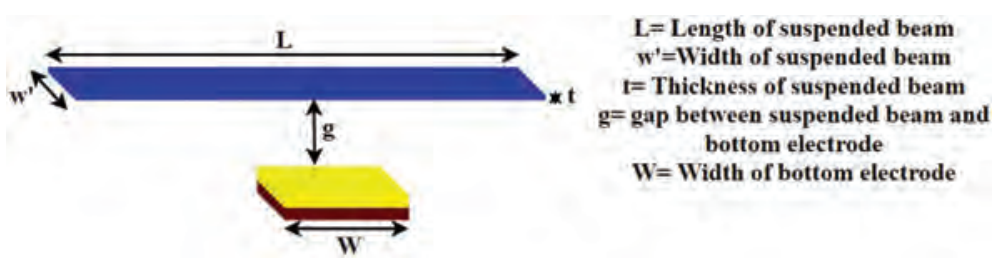

Figure 28.1 NEMS switch with intact beam structure.

Table 28.1 NEM switch device dimensions

\begin{tabular}{ll}
\hline Design parameters & Values \\
\hline Beam length $(\mathrm{L})$ & $10 \mu \mathrm{m}$ \\
Beam width $\left(\mathrm{w}_{\mathrm{b}}\right)$ & $5 \mu \mathrm{m}$ \\
Beam thickness $\left(\mathrm{t}_{\mathrm{b}}\right)$ & $9 \mathrm{~nm}$ \\
Gap $(\mathrm{g})$ & $250 \mathrm{~nm}$ \\
Electrode area & $2 \mu \mathrm{m} \times 5 \mu \mathrm{m}$ \\
\hline
\end{tabular}

graphene has been chosen as suspended beam material. The Young's modulus of graphene is taken as $860 \mathrm{GPa}$ and the Poisson's ratio is set as 0.17 [20]. Figure 28.1 shows the side view of the graphene NEM switch. For the actuation electrode, gold metal is taken and for dielectric material, $25 \mathrm{~nm}$ thick $\mathrm{HfO}_{2}\left(\varepsilon_{\mathrm{r}}=25\right)$ is taken [18]. The device dimensions for this switch structure are mentioned in Table 28.1.

As shown in Figure 28.2, the study of five different structures referred to as structures a, b, c, d, e, respectively, is done. The different bridge structures get actuated at a different voltage.

\section{A. Actuation mechanism}

The on-off switching of the NEMS switch is achieved by electrostatic actuation. The signal passes through the central electrode of the transmission line. The potential difference is applied between the actuation electrode and the graphene beam. When no voltage is applied to the electrode, the beam remains in its initial position and signal passes through the signal line without any interruption, the switch remains in on state. But when sufficient voltage is applied to pull the beam downward and the beam touches the bottom electrode, all the signal gets grounded through the suspended beam. In this state, the switch comes in off state. The voltage at which the beam comes in contact with the actuation electrode is known as pull-in/actuation voltage.

\section{B. Finite element modelling}

Finite element modelling (FEM) of five different bridge structures was done in COMSOL Multiphysics. For boundary conditions and electrostatic actuation mechanism, solid mechanics and electrostatics physics are used respectively. To save the computation time and reduce the meshing complexity, free tetrahedral meshing was used. All the simulations are performed in the air medium. The bias voltage is applied at the bottom actuation electrode while the suspended graphene beam is kept at ground potential. 
a

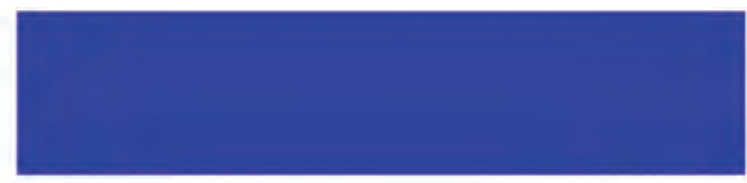

b

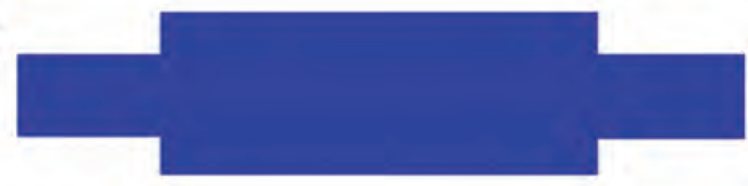

c

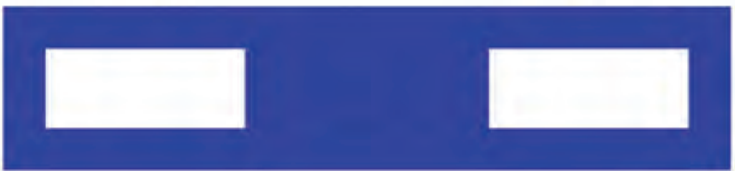

d

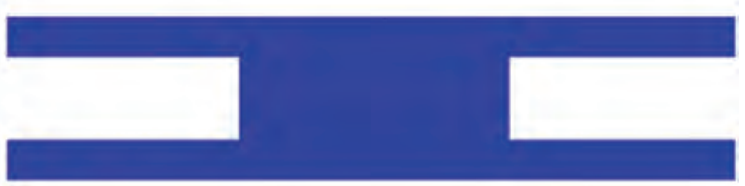

e

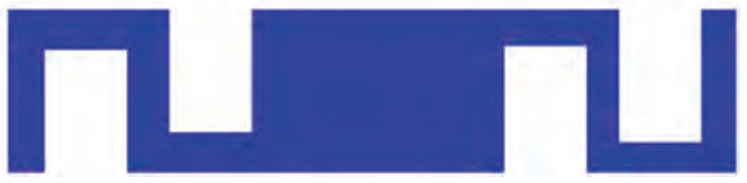

Figure 28.2 Different graphene bridge structures.

\section{Results and discussion}

The structural design and analysis of the NEM switch have been performed in COMSOL Multiphysics. Five different bridge structures are designed and simulated. Since bridge structure is considered, both the ends of the bridge are fixed. Graphene beam of $10 \mu \mathrm{m}$ length, $5 \mu \mathrm{m}$ width and $9 \mathrm{~nm}$ thick is considered as a suspended beam. The beam is suspended at a height of $250 \mathrm{~nm}$ from the dielectric material. The actuation voltage is applied at the bottom electrode. All the bridges structures get actuated at different actuation voltage. The total displacement of $250 \mathrm{~nm}$ of different graphene bridge structures is shown in Figure 28.3(a-e).

The results show that different voltages are required to snap down the bridge in different structures. As the bridge structures are different in all five cases, the spring constant of the bridge also changes, which in turn changes the actuation voltage.

When NEM switch is actuated between on-off states, two types of capacitance exist, one

is upstate capacitance $\left(\mathrm{C}_{\mathrm{up}}=\frac{\varepsilon_{o} w_{b} \mathrm{~W}}{g+\frac{t_{d}}{\varepsilon_{r}}}\right)$, the other is down state capacitance $\left(\mathrm{C}_{\mathrm{d}}=\frac{\varepsilon_{o} w_{b} \mathrm{~W}}{g}\right)$.

The down state capacitance is further divided into two parts: one is parallel plate capacitance $\left(C_{p}\right)$ while the other is tilted plate capacitance $\left(C_{t}\right)$. For accurate results, all three 

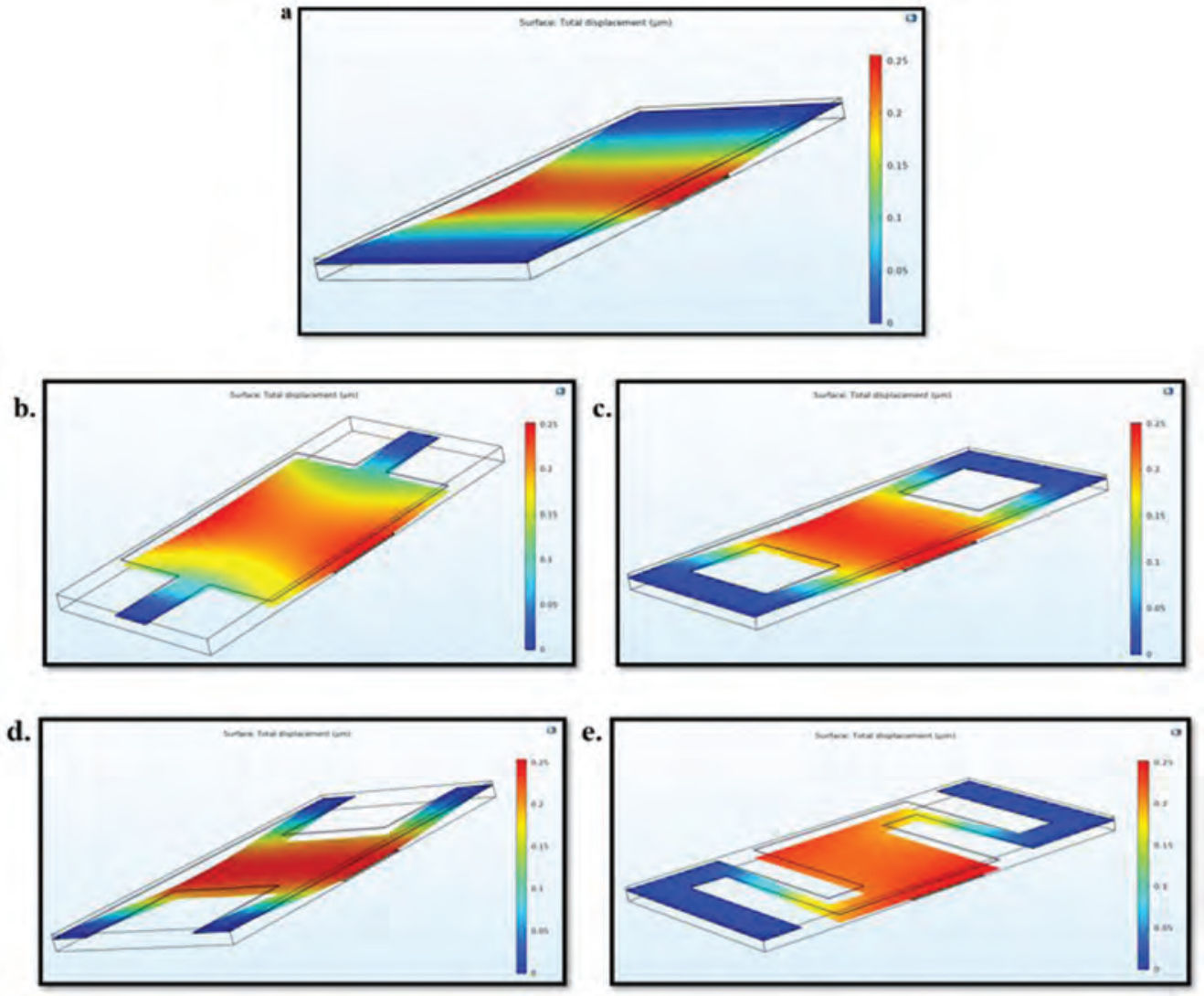

Figure 28.3 Total displacement of different structures: (a) structure-1, (b) structure-2, (c) structure3 , (d) structure-4, (e) structure-5.

Table 28.2 Actuation voltage and switching time for different bridge structures

\begin{tabular}{ll}
\hline Bridge structure & Pull-in voltage \\
\hline Structure-1 (intact) & $4.43 \mathrm{~V}$ \\
Structure-2 & $2.8 \mathrm{~V}$ \\
Structure-3 & $3.65 \mathrm{~V}$ \\
Structure-4 & $3.38 \mathrm{~V}$ \\
Structure-5 & $1.41 \mathrm{~V}$ \\
\hline
\end{tabular}

capacitances should be calculated. In the down state, the beam forms contact with the dielectric layer.

$$
\mathrm{C}_{\text {Total }}=\mathrm{C}_{\mathrm{up}}+\mathrm{C}_{\mathrm{d}} \text {, where } \mathrm{C}_{\mathrm{d}}=\mathrm{C}_{\mathrm{p}}+2 \mathrm{C}_{\mathrm{t}}
$$

For the simulated NEM switch, the obtained upstate, parallel plate and tilted plate capacitances are $0.3 \mathrm{fF}, 0.66 \mathrm{pF}$ and $0.85 \mathrm{fF}$, respectively. The area of the graphene beam 


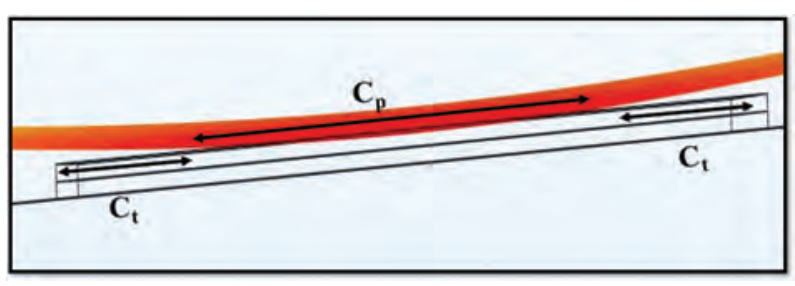

Figure 28.4 Down state capacitances.

that is in contact with the dielectric material is the same for all the cases. Hence the same value of capacitance is obtained in all the cases.

\section{Conclusions}

Graphene-based NEM switch with different bridge structures was designed and simulated in COMSOL Multiphysics. For different bridge structures, different actuation voltages are obtained. The intact structure requires the maximum actuation voltage $(4.43 \mathrm{~V})$ while the structure-5 requires minimum actuation voltage $(1.41 \mathrm{~V})$. The actuation voltage depends upon the bridge stiffness. The different bridge structures have different spring stiffness. The perforations reduce the mass of the suspended beam and make the beam less stiff which in turn changes the actuation voltage. Also, the total capacitance of $0.66 \mathrm{pF}$ was obtained for the simulated switch structure.

\section{References}

[1] Lai, Y. L., and L. H. Chang. 2008. Design of electrostatically actuated MEMS switches. Colloids and Surfaces A: Physicochemical and Engineering Aspects 313: 469-473.

[2] Yao, Z. J., S. Chen., S. Eshelman., D. Denniston., and C. Goldsmith. 1999. Micromachined low-loss microwave switches. Journal of Microelectromechanical Systems 8(2):129-134.

[3] Park, J. Y., G. H. Kim., K. W. Chung., and J. U. Bu., 2001. Monolithically integrated micromachined RF MEMS capacitive switches. Sensors and Actuators A: Physical 89(12): 88-94.

[4] Chaudhary, R., and P. R. Mudimela. 2020. Pull-in response and eigen frequency analysis of graphene oxide-based NEMS switch. Materials Today: Proceedings.

[5] Loh, O.Y., and H. D. Espinosa. 2012. Nanoelectromechanical contact switches. Nature Nanotechnology 7:283.

[6] Kim, J. H., Z. C. Chen., S. Kwon., and J. Xiang. 2014. Three-terminal nanoelectromechanical field effect transistor with abrupt subthreshold slope. Nano Letters 14(3):1687-1691.

[7] Li, Q., S. M. Koo., M. D. Edelstein., J. S. Suehle., and C. A. Richter. 2007. Silicon nanowire electromechanical switch for logic device application. MRS Online Proceedings Library Archive 1018.

[8] Jonsson, L. M., S. Axelsson., T. Nord., S. Viefers., and J. M. Kinaret. 2004. High frequency properties of a CNT-based nanorelay. Nanotechnology 15(11):1497.

[9] Lovellette, M. N., A. B. Campbell., H. L. Huges et al. 2004. IEEE Aerospace Conf. IEEE 2300-2305.

[10] Lee, T. H., S. Bhunia., and M. Mehregany. 2010. Electromechanical computing at $500 \mathrm{C}$ with silicon carbide. Science 329(5997):1316-1318.

[11] Novoselov, K. S., A. K. Geim., and S. V. Morozov et al. 2004. Electric field effect in atomically thin carbon films. Science, 306(5696):666-669. 


\section{Intelligent Circuits and Systems}

[12] Du, X., I. Skachko., A. Barker., and E. Y. Andrei. 2008. Approaching ballistic transport in suspended graphene. Nature Nanotechnology 3(8):491.

[13] Lee, C., X. Wei., J. W. Kysar., and J. Hone. 2008. Measurement of the elastic properties and intrinsic strength of monolayer graphene. Science 321(5887):385-388.

[14] Balandin, A. A., S. Ghosh., and W. Bao. et al. 2008. Superior thermal conductivity of singlelayer graphene. Nano Letters 8(3):902-907.

[15] Kim, S. M., E. B. Song., and S. Lee. et al. 2011. Suspended few-layer graphene beam electromechanical switch with abrupt on-off characteristics and minimal leakage current. Applied Physics Letters 99(2):023103.

[16] Chaudhary, R., and P. R. Mudimela. 2020. 3D modeling of graphene oxide based nanoelectromechanical capacitive switch. Microsystem Technologies 26(10).

[17] Acquaviva, D., A. Arun., and S. Esconjauregui. et al. 2010. Capacitive nanoelectromechanical switch based on suspended carbon nanotube array. Applied Physics Letters 97(23):233508.

[18] Ionescu, A. M., J. Dijon., and J. Robertson. 2011. Integration for all configurations. IEEE Microwave Magazine, 12(7):42-50.

[19] Sharma, P., J. Perruisseau-Carrier., C. Moldovan., and A. M. Ionescu. 2013. Electromagnetic performance of RF NEMS graphene capacitive switches. IEEE Transactions on Nanotechnology 13(1):70-79.

[20] Li, P., Z. You., G. Haugstad., and T. Cui. 2011. Graphene fixed-end beam arrays based on mechanical exfoliation. Applied Physics Letters 98(25):253105.

[21] Zulkefli, M., M. Mohamed., and K. Siow. et al. 2017. Three-dimensional finite element method simulation of perforated graphene nano-electro-mechanical (NEM) switches. Micromachines $8(8): 236$.

[22] Jaafar, H., K. S. Beh., N. A. M. Yunus., W. Z. W. Hasan., S. Shafie., and O. Sidek. 2014. A comprehensive study on RF MEMS switch. Microsystem Technologies 20(12): 2109-2121. 


\title{
29 Pre- and post-filter selection for image segmentation
}

\author{
Mohit Srivastava ${ }^{1 *}$, Manoj Kuri ${ }^{2}$ \\ ${ }^{1}$ Chandigarh Engineering College, Landran, Punjab, India \\ ${ }^{2}$ Government Engineering College, Bikaner, India
}

\section{Introduction}

The image processing is a procedure wherein the information from an image is digitized and different scientific activities are applied to the data utilizing a computer to generate an improved image [1]. Essentially, segmentation is the way toward sub-dividing an image into the constituent regions which pursues homogeneity and heterogeneity criteria inside the image. In this process homogeneous pixels/objects converge with one another to form a segment. The homogeneity of the formed segment and segment area are inversely proportional and hence segment homogeneity reduces as segment region grows.

Segmentation algorithms depend on either the dissimilarity or the similarity of intensity level values. Various image segmentation algorithms with expanded unpredictability have been created. Working of all these algorithms follows any of the three primary criteria: the inside segment homogeneity, detachment from neighbouring segments and shape homogeneity. Normally, threshold based [2,3], boundary/edge based [4,5] and region based [6] segmentation techniques are used.

\section{Region split and merge image segmentation}

The region split and merge based image segmentation is a part of the region based segmentation; every time a new seed pixel is to be chosen until all the pixels are assigned to some region or the other. The only drawback of this technique is that it doesn't adapt with image internal properties and hence generate region boundaries along with horizontal and vertical ones [6,7]. However, most of the existing region merging algorithms may provide solution based on local properties like coherence of brightness, colour, texture, motion, but can't guarantee a globally optimal solution of the merging result based on global properties like object symmetries [8], so the region merging remains local. This causes the merging output to be over-merged or under-merged or a combination of both [9], i.e. the output lacks behind in symmetric form.

Splitting is a recursive procedure, in which splitting of the image is done using the quad tree decomposition method. It splits the image into four equal quadrants. It is observed that boundaries of the image segments are not very sharp, therefore to sharpen and preserve the edge information pre-filtering is required. Laplacian, median and min spatial filters have been used as pre-filter to remove noise present in the image.

\footnotetext{
* Email: mohit.ece@cgc.edu.in
} 


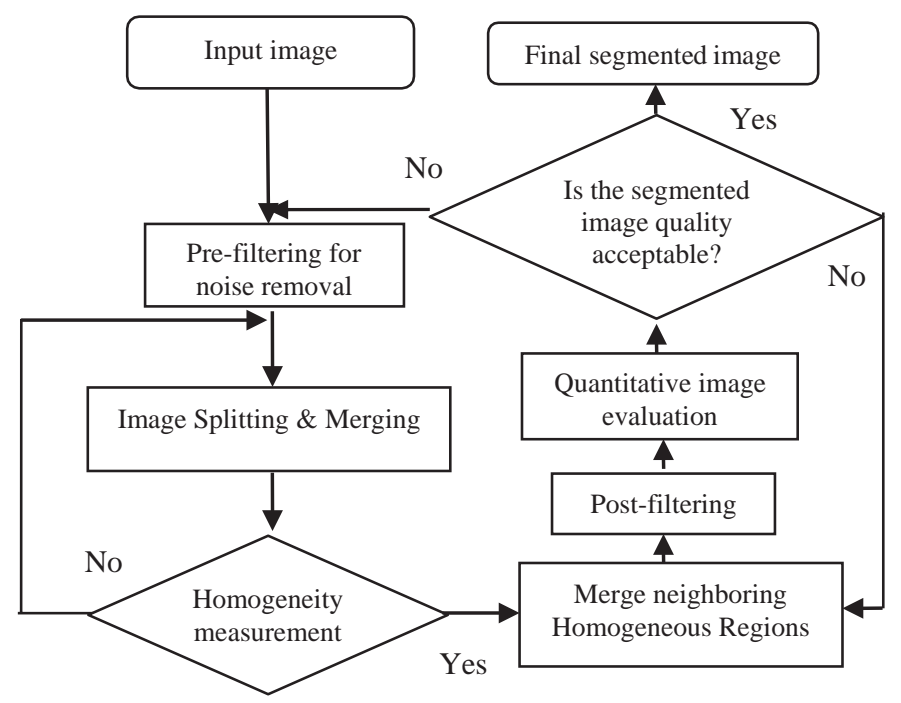

Figure 29.1 Flowchart of the methodology.

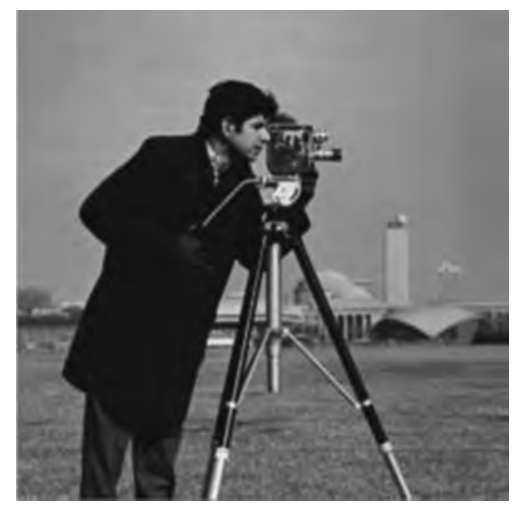

Figure 29.2 Grey image.

\section{Methodology adopted}

In this paper, region split and merge technique has been applied on pre-filtered images and then post-filtration of the segmented has been done to obtain perfect segments with closed boundaries. The process flowchart is shown in Figure 29.1.

\section{Experimental results and quality assessment}

\section{Pre- and post-filtration}

A standard "cameraman" image as shown in Figure 29.2 has been segmented using split and merge segmentation algorithm which is implemented using MATLAB. Initially, 
Laplacian, median, min, max, unsharp and LOG spatial filters have been used to do prefiltering of the image. Table 29.1 shows the variation in filter parameters for filtration process and selected filter types which give best response. Initially, first order min and max filter has been applied on image for removing the noise available in the image. Further, Laplacian spatial filter has been used as a pre-filter. Now, the image is passes through $3 \times 3$ Laplacian filter. We have varied the value of parameter alpha to find out the best filtered non-blurred image. Laplacian filter which is a derivative operator considers second order partial derivatives for filtration, and hence to generate grey level image the original image is added back every time to its derivatives [10]. Hence to obtain the grey level of image, the original image is again added back [11]. Laplacian-of-Gaussian (LOG) filter has been also used to filter the image. Mask size and standard deviation of the LOG filter is varied one by one and filtered images are generated. For this work image is filtered with a mask of $3 \times 3$ and $5 \times 5$, and standard deviation of values $0.3,0.5,0.6$. It is observed that a blurred image is generated when the value of standard deviation is chosen as 0.5 , and the image gets more blurred when its value less than 0.5 . The best filtered image with LOG filter is obtained at standard deviation of 0.6 with filter size of $3 \times 3$. The median spatial filter is also used to remove the noise present in the image. The median filter works on the basis of intensity value and has excellent noise reduction capabilities. Another spatial filter used in this work is unsharp filter, which enhances the edges of the image. A $3 \times 3$ size of unsharp filter with different alpha value has been used to do pre-filtration of the image. Selected filtered images through these spatial filters are further used for segmentation.

After doing pre-filtration of image, now image is segmented by making use of split and merge segmentation technique. From the filtered image, it is observed that clear boundary between object and background is obtained when Laplacian, min and median filtered image is used, which hence provides an optimal segmentation. Segmented image through LOG and unsharp filters are over-segmented. However, segmented image obtained through max filter causes under-segmentation. These segmented images are shown in Figure 29.3.

Table 29.1 Pre-filter information applied on grey image, parameters and values show selected parameters and values based on visual interpretation

\begin{tabular}{|c|c|c|c|c|}
\hline S.no & Filter type & $\begin{array}{l}\text { Mask size } \\
\text { (rows } \times \text { columns) }\end{array}$ & Parameters & \\
\hline \multirow[t]{2}{*}{1} & \multirow[t]{2}{*}{ Min } & $2 \times 3$ & \multirow[t]{2}{*}{ Order } & 1.0 \\
\hline & & $3 \times 3$ & & 1.0 \\
\hline \multirow[t]{4}{*}{2} & \multirow[t]{4}{*}{ Median } & $3 \times 3$ & \multirow[t]{4}{*}{ Padding } & Symmetric \\
\hline & & $3 \times 3$ & & Zeros \\
\hline & & $5 \times 5$ & & Symmetric \\
\hline & & $5 \times 5$ & & Zeros \\
\hline \multirow[t]{2}{*}{3} & \multirow[t]{2}{*}{ Unsharp } & $3 \times 3$ & \multirow[t]{2}{*}{ Alpha } & 0.20 \\
\hline & & $3 \times 3$ & & 0.85 \\
\hline \multirow[t]{2}{*}{4} & \multirow[t]{2}{*}{ Max } & $2 \times 2$ & \multirow[t]{2}{*}{ Order } & 1.0 \\
\hline & & $3 \times 3$ & & 1.0 \\
\hline \multirow[t]{3}{*}{5} & \multirow[t]{3}{*}{ Laplacian } & $3 \times 3$ & \multirow[t]{3}{*}{ Alpha } & 0.0 \\
\hline & & $3 \times 3$ & & 0.25 \\
\hline & & $3 \times 3$ & & 0.75 \\
\hline \multirow[t]{5}{*}{6} & \multirow[t]{5}{*}{ LOG (Laplacian-of-Gaussian) } & $3 \times 3$ & \multirow{5}{*}{$\begin{array}{l}\text { Standard } \\
\text { deviation }\end{array}$} & 0.30 \\
\hline & & $3 \times 3$ & & 0.50 \\
\hline & & $5 \times 5$ & & 0.50 \\
\hline & & $3 \times 3$ & & 0.60 \\
\hline & & $5 \times 5$ & & 0.60 \\
\hline
\end{tabular}



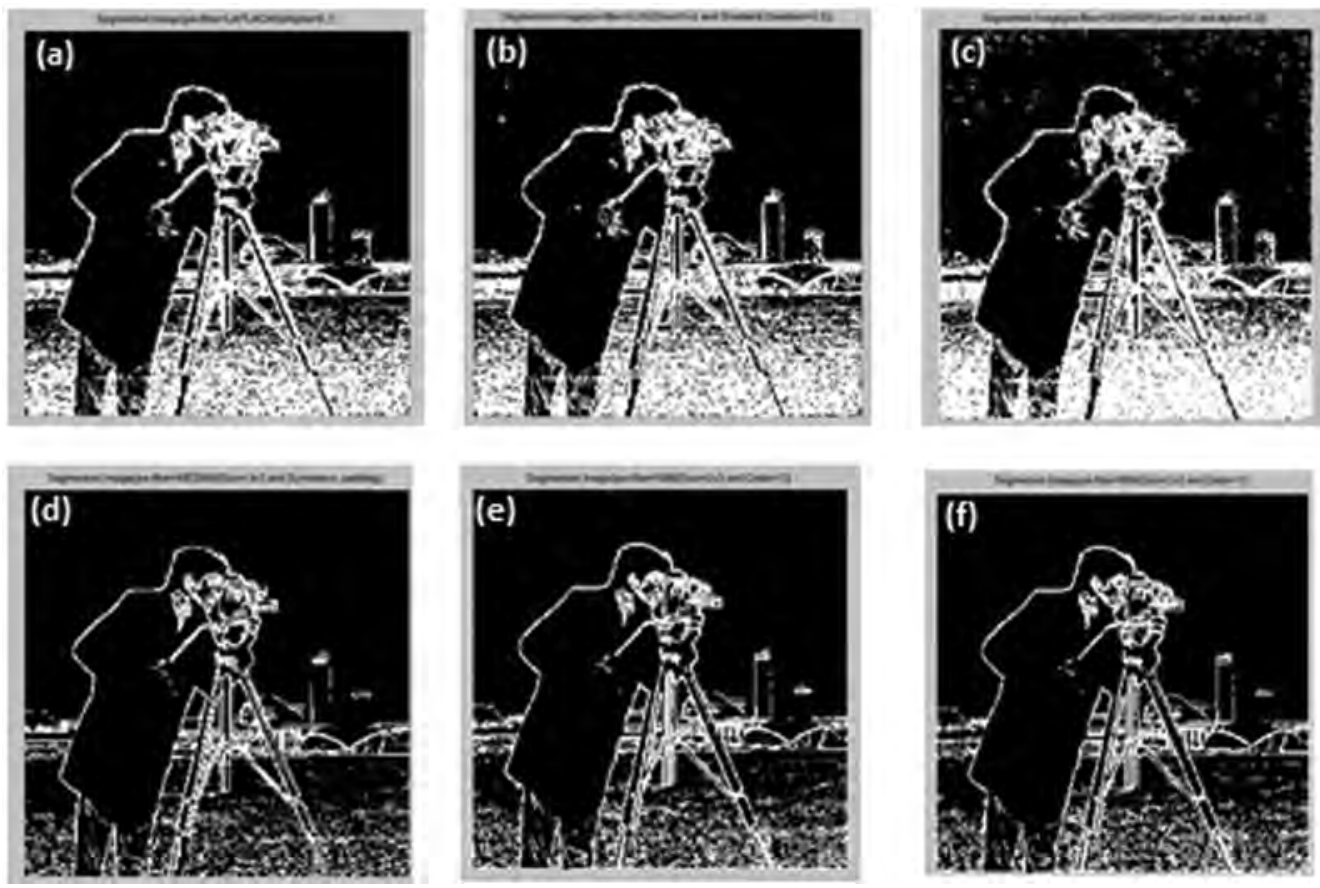

Figure 29.3 Output segmented image pre-filtered with (a) Laplacian filter, (b) LOG filter, (c) unsharp filter, (d) median filter, (e) min filter $($ size $=2 \times 3)$, (f) $\max$ filter $($ size $=3 \times 3)$.

In the segmented image it is observed that edges of the segments obtained from this filtered image are not continuous at some points. Therefore, it is required to apply post-filtering for smoothing the boundary. Laplacian, median and Sobel filters with varying parameters as listed in Table 29.2 are used for post-filtering operation.

\section{Quality evaluation}

Visual interpretation is best way to assess the segmentation quality, but it provides a qualitative assessment. Mean square error (MSE) is used to assess the quality of segmented and post filtered image quantitatively. The measured value of quantitative evaluation is shown in Table 29.2. Quantitative evaluation shows that quality of segmented image after applying the median filter as post filtering process appears highest with MSE $(\mathrm{dB})$ of 86.24, 85.89 and 85.72 applied over the Laplacian, min and median filtered image individually. It is because median filter has the advantage that it offers considerably less blurring with excellent noise reduction capabilities. Value of MSE obtained through a Sobel post filter shows that its filtration quality is comparatively poorer than median filter and the observed value of MSE $(\mathrm{dB})$ is $85.26,85.14$ and 84.89 when applied over Laplacian, median and min pre-filtered images, respectively. However, the quality of post Laplacian filtered image is lowest among all with MSE $(\mathrm{dB})$ value of $84.23,84.54$ and 85.25. Figure 29.4 to Figure 29.7 show the post-filtered images using the Laplacian, median, Sobel and LOG filters, respectively. From visual interpretation also it is observed that the final image generated by post-filtering using median and Sobel filters appears so accurate. 
Table 29.2 Quality assessment output images for finding effective pre and post-filtering techniques

\begin{tabular}{|c|c|c|c|c|c|c|}
\hline \multicolumn{3}{|l|}{ Pre-filter } & \multicolumn{3}{|l|}{ Post-filter } & \multirow{2}{*}{$\frac{\text { Evaluation }}{M S E(d B)}$} \\
\hline Name & Size & Parameter & Name & Size & Parameter & \\
\hline Laplacian & $3 \times 3$ & Alpha $=0.2$ & Median & $3 \times 3$ & Symmetric padding & 86.24 \\
\hline Median & $3 \times 3$ & Symmetric padding & & & & 85.54 \\
\hline Min & $2 \times 3$ & Order $=1$ & & & & 84.25 \\
\hline Laplacian & $3 \times 3$ & Alpha $=0.2$ & Laplacian & $3 \times 3$ & Alpha $=0.2$ & 84.23 \\
\hline Median & $3 \times 3$ & Symmetric padding & & & & 84. \\
\hline Min & $2 \times 3$ & Order $=1$ & & & & 85.89 \\
\hline Laplacian & $3 \times 3$ & Alpha $=0.2$ & Sobel & $3 \times 3$ & Search direction & 85.26 \\
\hline Median & $3 \times 3$ & Symmetric padding & & & (horizontal and vertical) & 85.14 \\
\hline Min & $2 \times 3$ & Order $=1$ & & & & 85.89 \\
\hline Laplacian & $3 \times 3$ & Alpha $=0.2$ & LOG & $3 \times 3$ & Standard deviation $=0.5$ & 76.41 \\
\hline Median & $3 \times 3$ & Symmetric padding & & & & 77.25 \\
\hline Min & $2 \times 3$ & Order $=1$ & & & & 74.32 \\
\hline
\end{tabular}
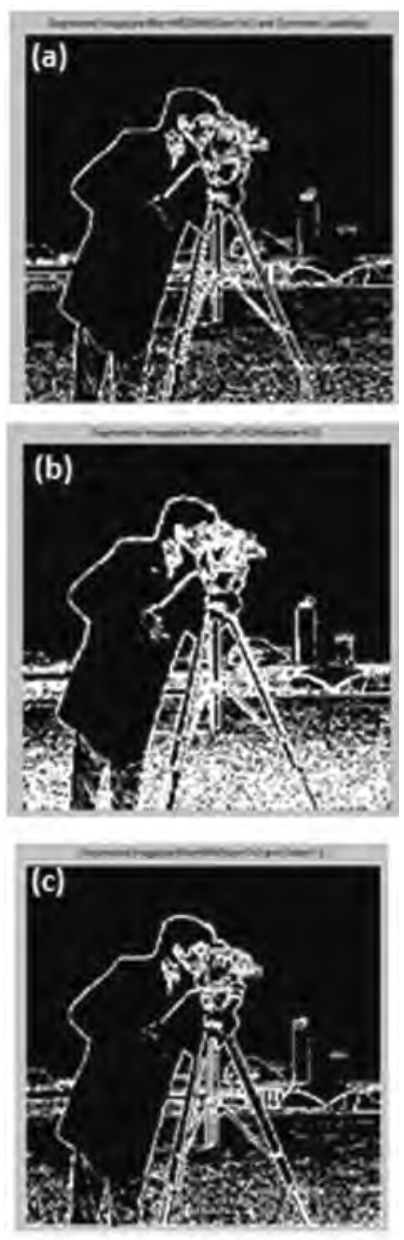

Figure 29.4 Output of Laplacian post-filtered segmented image applied on the image which is prefiltered using the (a) Laplacian filter, (b) median filter, (c) min filter. 

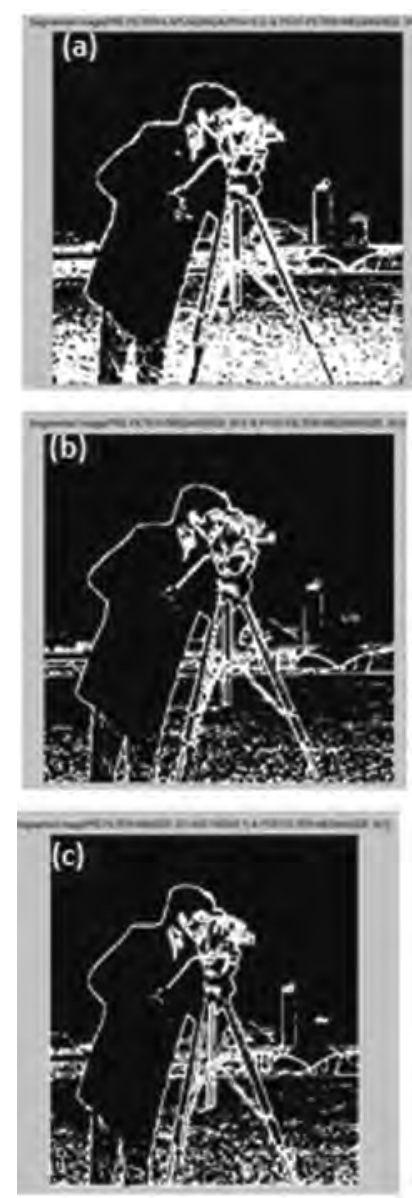

Figure 29.5 Output of Median post-filtered segmented image applied on the image which is pre-filtered using the (a) Laplacian filter, (b) median filter, (c) min filter.
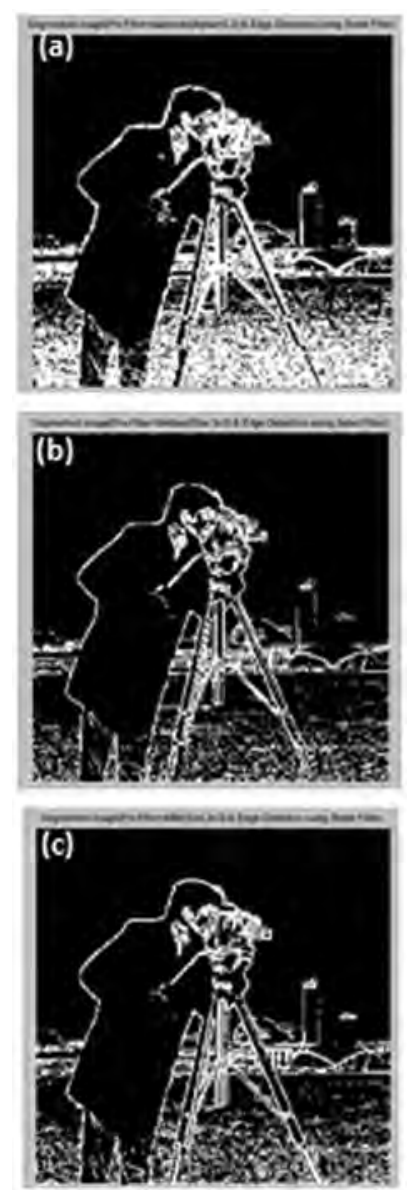

Figure 29.6 Output of Sobel post-filtered segmented image applied on the image which is pre-filtered using the (a) Laplacian filter, (b) median filter, (c) min filter.

\section{Conclusions}

This paper concludes with the assessment quality of pre and post-filters applied over an image. Image is first pre-filtered using min, median, max, Laplacian, unsharp and LOG filters. It is observed that pre-filter output of Laplacian, median and min is optimal, and hence they are considered for further processing. Post-filtering using Laplacian, median and Sobel filters has been applied to these pre-filtered and segmented images. Now the quantitative evaluation of these output images is done on the basis of the mean square error (MSE). The MSE value shows that Median filter acts proficiently as post-filter. Additionally, the combination of Laplacian and median filters as pre and post filter is most effective. 

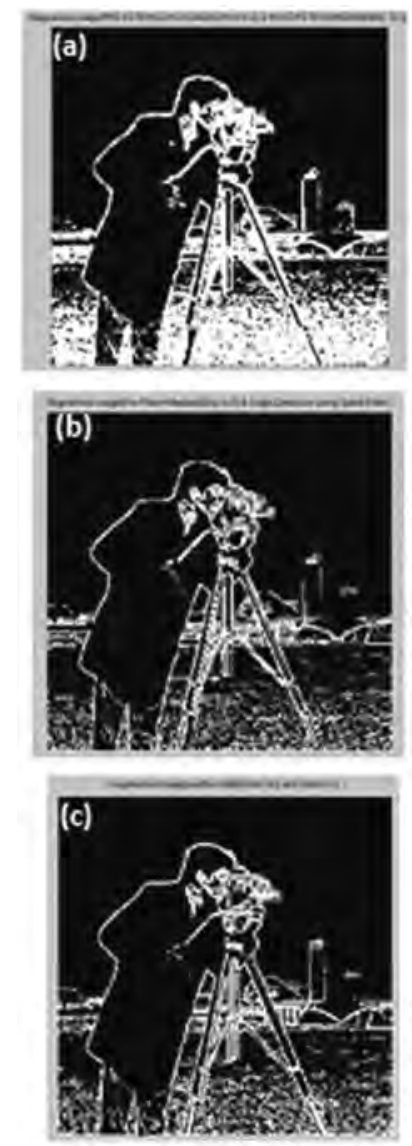

Figure 29.7 Output of LOG post-filtered segmented image applied on the image which is pre-filtered using the (a) Laplacian filter, (b) median filter, (c) min filter.

\section{References}

[1] Hoshelham, K., Li, Z., King, B. A split-and-merge technique for automated reconstruction of roof planes. Photogrammetric Engineering \& Remote Sensing Journal. 71, 855-862 (2005).

[2] Kandwal, R., Kumar, A., Bhargava, S. Review: existing image segmentation techniques. International Journal of Advanced Research in Computer Science and Software Engineering. 4, 153-156 (2014).

[3] Pun, C.M., An, N.Y., Cheng, M. A region-based image segmentation by watershed partition and DCT energy compaction. IEEE International Conference on Computer Graphics, pp. 131-135. China (2011).

[4] Thakare, P. A study of image segmentation and edge detection techniques. International Journal of Computer Science and Engineering. 3, 899-904 (2011).

[5] Palmer, P.L., Dabis, H., Kittler, J. A performance measure for boundary detection algorithms. Elsevier Computer Vision Image Understanding. 63, 476-494 (1996).

[6] Kelkar, D., Gupta, S. Improved quadtree method for split merge image segmentation. IEEE International Conference on Emerging Trends in Engineering and Technology, pp. 44-47. Nagpur, Maharashtra (2008). 


\section{Intelligent Circuits and Systems}

[7] Zhang, Y.J. A survey on evaluation methods for image segmentation. Elsevier Journal on Pattern Recognition. 29, 1335-1346 (1999).

[8] Wu, X. Adaptive split-and-merge segmentation based on piecewise least-square approximation. IEEE Transaction on Pattern Analysis and Machine Intelligence, 15, 808-815 (1993).

[9] Hunki, R., HaengSuk, L. A noble image segmentation using local area splitting and merging method based on intensity change. International Journal of Software Engineering and its Applications. 7, 99-112 (2013).

[10] Gonzalez, R.C., Woods, R.E. Digital Image Processing. Pearson Education, India (2009).

[11] Solomon, C.J., Breckon, T.P. Fundamentals of Digital Image Processing: A Practical Approach with Examples in MATLAB, 1st edn., Wiley-Blackwell (2010). 


\title{
30 Impact of temperature on $14 \mathrm{~nm}$ FINFET with high-K different oxide material
}

\author{
Shekhar Verma*, Suman Lata Tripathi* \\ School of Electronics \& Engineering, Lovely Professional University, \\ Punjab, India
}

\section{Introduction}

Designing of transistors in today's scenario plays a very crucial role as the scaling goes from $180 \mathrm{~nm}$ to $20 \mathrm{~nm}[1,2]$. As we scale down the transistor size, many factors come into picture that degrade the performance of the device on lower channel length. It's a very challenging task on the technology marketing, to overcome the effect on the performance of the device $[3,5]$. On below $20 \mathrm{~nm}$ technology leakage current and short channel effect are more prominent on the device performance. FinFETs [6,7] play an important and crucial role in this direction to overcome the scale issue in comparison to MOSFET devices. Efficient devices need to use minimum power to switch from on to off state or vice versa. In the case of FinFET, it uses the minimum bias voltage from 0.2 to 0.3 volt to change the working state of the device from on to off or vice versa [2]. In this research we analyse the effect of temperature on different oxide material of $14 \mathrm{~nm}$ FinFET that affects the mobility of the device or electron behaviour [4,6]. So, further analysis of the results in direction of electrical characteristics leads to enhancement in performance of the FinFET structure. In this paper, we used the SOI type structure for designing the $14 \mathrm{~nm}$ FinFET. Gate engineered and channel engineered multi-gate MOS structures are popular in low power digital and memory applications [8,9]. Gate engineering can be implemented by the use of high-K dielectric oxide material with metal gate of high work function [10-13]. In the proposed design, value of the fin height used is $21 \mathrm{~nm}$ with $10 \mathrm{~nm}$ width and oxide thickness is $1 \mathrm{~nm}$. In this design, the material for gate oxide is high- $\mathrm{K}$ material $\mathrm{HFO}_{2}$ (hafnium oxide) and low- $\mathrm{K}$ material $\mathrm{SiO}_{2}$ (silicon oxide). The contact material for source and drain terminal aluminum is used as shown in Figure 30.1. Temperature analysis is an important aspect to be checked for reliable device performance $[13,14]$. Therefore, the proposed structure has been analysed for temperature variation from 250 to $350 \mathrm{~K}$ in terms of subthreshold performance parameters.

\section{Device performance parameters}

\section{Saturation current}

For the performance of the device, we need to analyse all three states of cut off, linear and saturation region but in this paper we more focus on the saturation region to figure out the impact of temperature variation. For calculation of the drain current we used Equation 1.

$$
I_{D}=\varnothing+\mu C_{O X}\left(W_{g} \mid L_{g}\right)\left\langle\left(V_{g s}-V_{d s}\right) \mid 2 m\right\rangle
$$

\footnotetext{
*Emails: shekhar.14572@lpu.co.in, suman.21067@lpu.co.in
} 


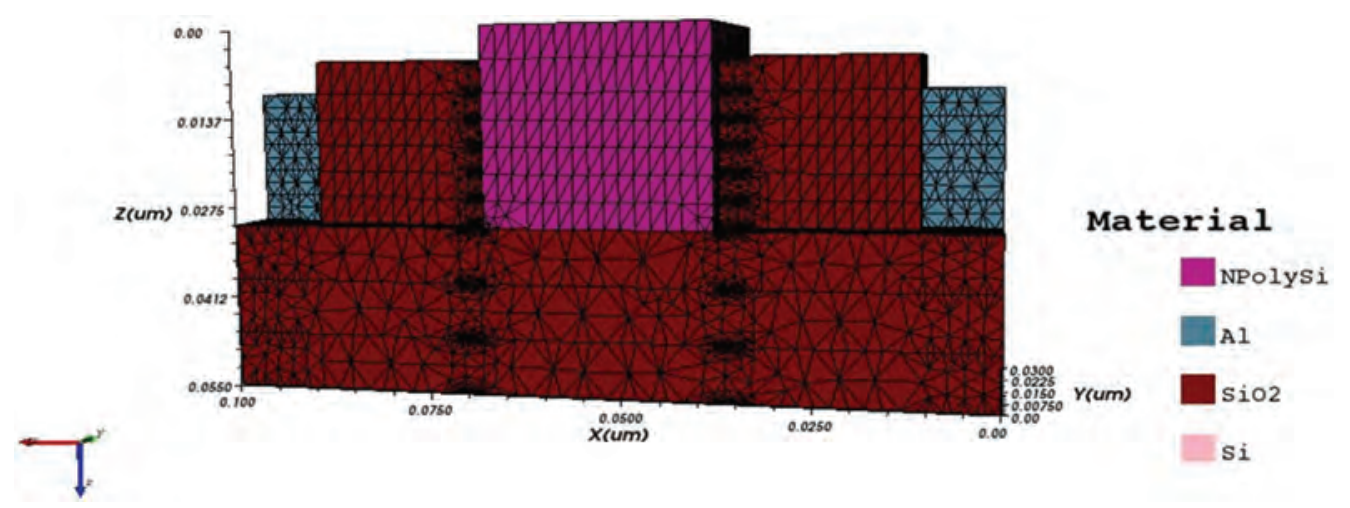

Figure 30.1 Structure of $14 \mathrm{~nm}$ SOI FinFET.

where $I_{D}$ is drain current at saturation region, $W_{g}$ is width of gate and $L_{g}$ is length of the gate and $V_{\mathrm{ds}}$ is drain to source voltage. $\varnothing$ is gate material work function, $\mu$ is mobility and $\mathrm{V}_{\mathrm{gs}}$ is gate to source voltage.

$$
m=1+\left(3 T_{O X} / T_{S I}\right)
$$

where $T_{\text {ox }}$ is gate oxide thickness and fin thickness is $T_{S I}$.

\section{Threshold voltage}

Minimum voltage is required to switch the device from off state to on state, which will be calculated as per Equation 3 below:

$$
V_{t h}=\varnothing+\left(\frac{K T}{q}\right) \ln \left(\frac{2 C_{O X} K T}{q^{2} n_{i} t_{S I}}\right)+\left(\frac{b^{2} \pi^{2}}{2 m_{d s} w_{s i}^{2}}\right)
$$

where $V_{\text {th }}$ is threshold voltage, $K$ is Boltzmann's constant, $q$ is charge of electron and $n_{i}$ is intrinsic silicon concentration, $\mathrm{h}$ is Planck's constant, fin thickness is $\mathrm{T}_{\mathrm{SI}}$, $\mathrm{T}$ is temperature $(\mathrm{K}), \mathrm{W}_{\mathrm{si}}$ is width of the fin.

\section{Electron moblility}

This factor on the FinFET device can be calculated as per Equation 4, where Lattice temperature is represented by $\mathrm{L}$ and $\mathrm{v}$ is $\mathrm{p}$ or $\mathrm{n}$ type of silicon.

$$
\mu_{v}^{L}=\mu_{\nu 300}^{L}\left(\frac{T_{L}}{300 K}\right)
$$

\section{Drain-induced barrier lowering (DIBL)}

This refers to the reduction of threshold voltage of the device at higher values of the drain voltage. This parameter in the proposed design will be calculated as per Equation 5. 
$D I B L=\frac{\Delta V_{t h}}{\Delta V_{d}}$

\section{Results and discussion}

In this paper, we have proposed the two designs of $14 \mathrm{~nm}$ FinFET by using the two different oxide material, high-K and low-K dielectric material, and the rest of the parameters are kept same for doing a fair simulation on both the devices.

\section{Transfer characteristics}

By changing the drain gate voltage from 0 to 1 volt with the step of 0.05 volt and keeping the constant value of 1 volt at drain source voltage, we oberved the performance of the device on both the oxide materials, high-K and low-K. As per Figure 30.2, drain current value is higher in comparison to the low-K oxide material. As the temperature increases from $200 \mathrm{~K}$ to $350 \mathrm{~K}$, the saturation current of the devices increases as per Figure $30.3(\mathrm{a}, \mathrm{b})$ due to lowering in the parasitic resistance. This resistance increases due to increasing in concentration of the minor charge as the temperatures rises from $200 \mathrm{~K}$ to $350 \mathrm{~K}$. In the proposed design $350 \mathrm{~K}$ gave the highest saturation current and $200 \mathrm{~K}$ the lowest current value.

\section{Output characteristics}

As per Figure 30.4, we observed the output characteristics of the $14 \mathrm{~nm}$ on different values of gate voltage. This shows the ideal nature of output characteristics with changing bias conditions.

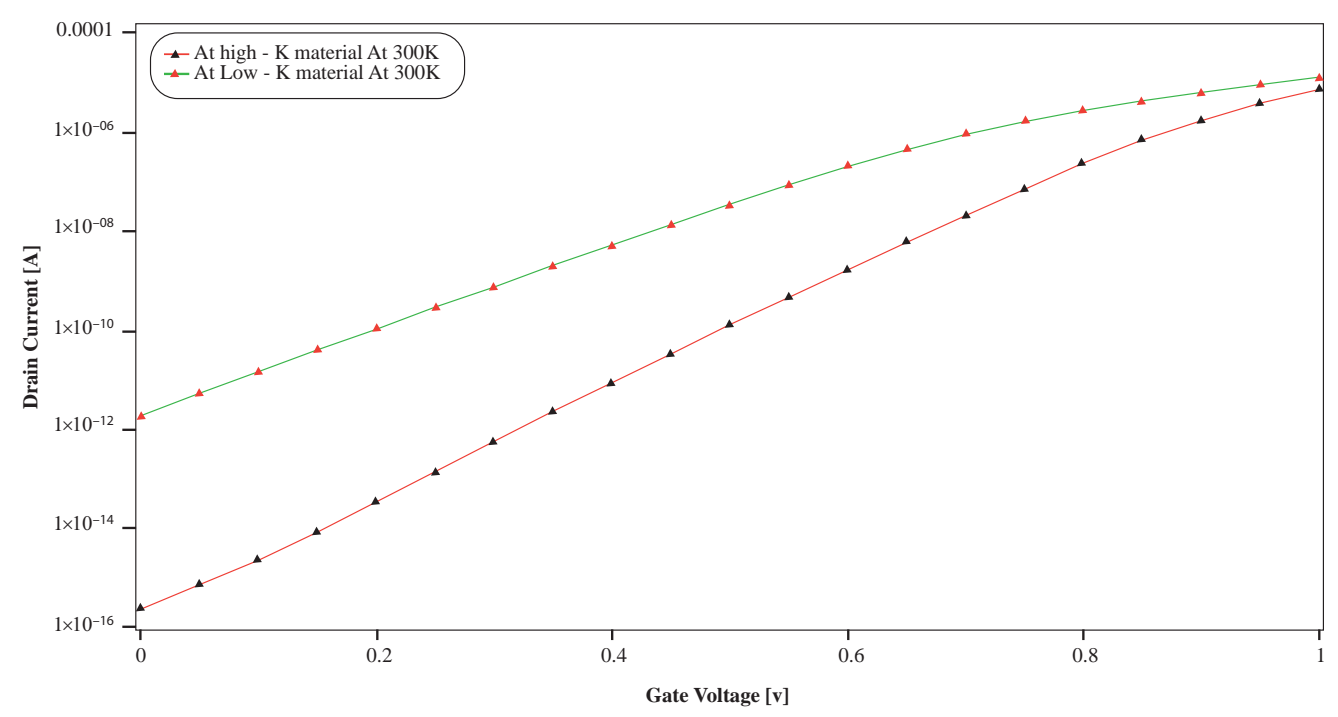

Figure 30.2 Gate voltage vs. drain current at $\mathrm{V}_{\mathrm{ds}}(1 \mathrm{~V})$. 


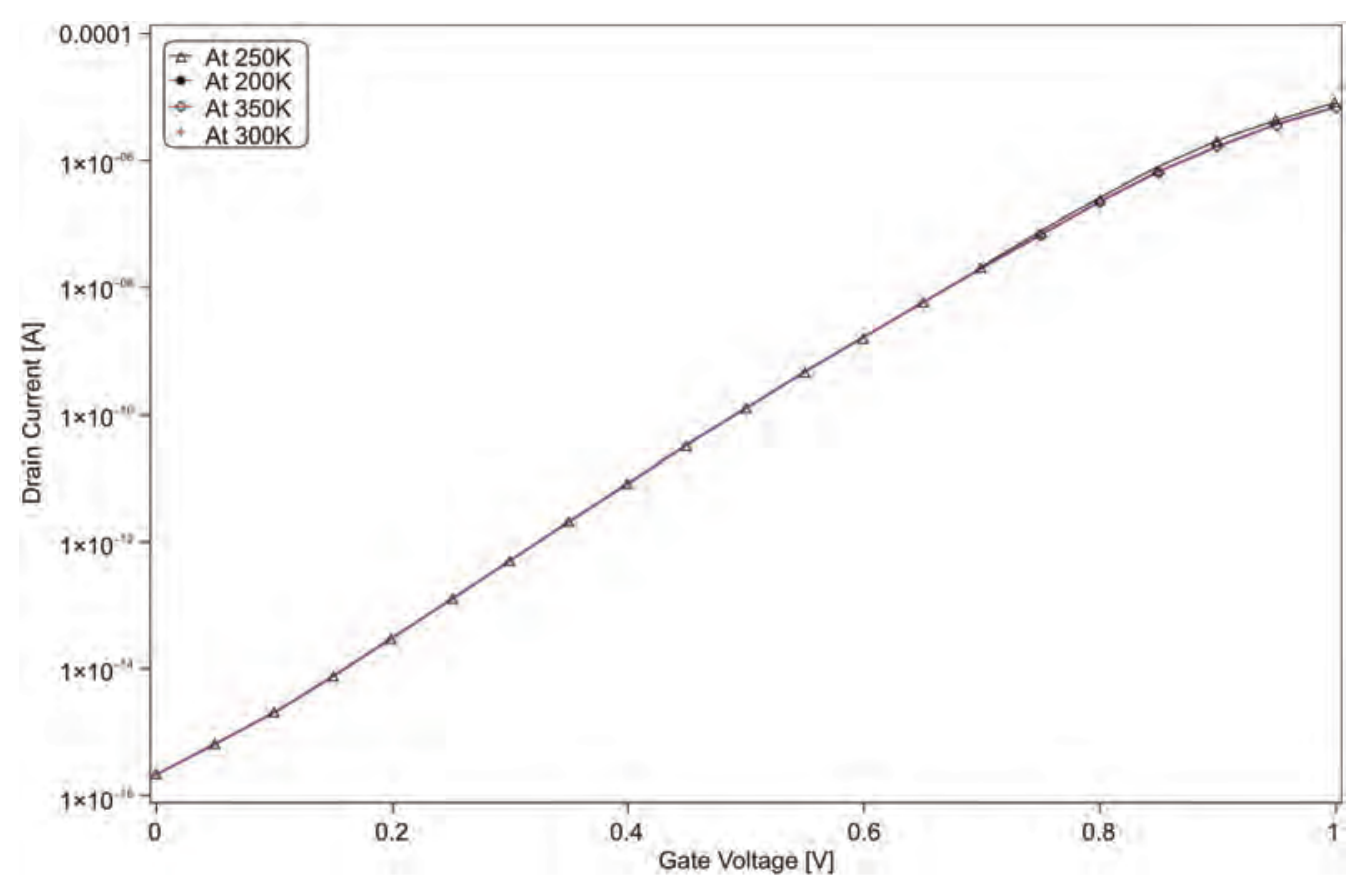

2.15E-16 2.15E-16 2.14E-16 2.14E-16

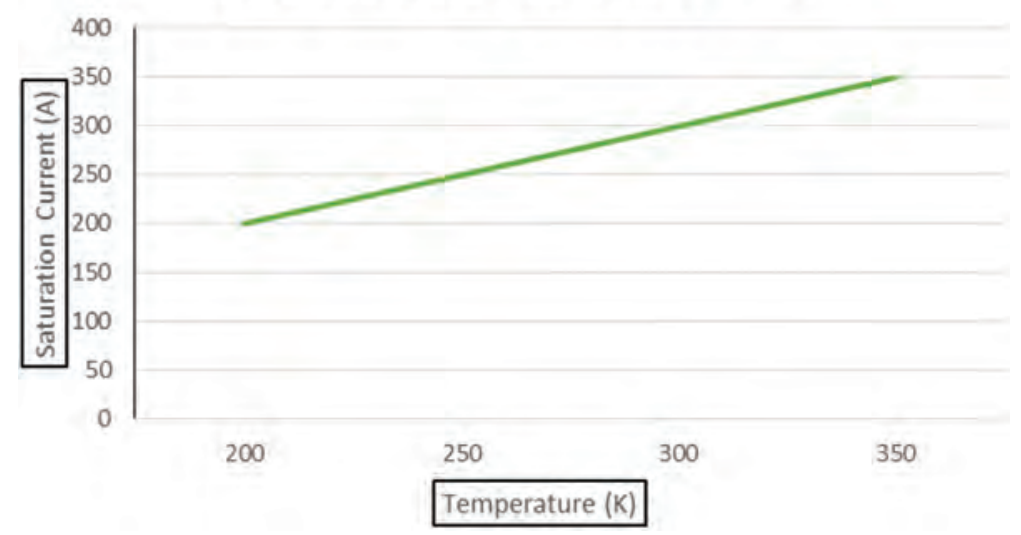

Figure 30.3 (a) Output characteristics. (b) Temperature vs. saturation current.

\section{Conclusion}

In this paper, we mainly focus on the impact of temperature on the performance of the $14 \mathrm{~nm}$ device. The results show that mobility decreases as the temperature rises due to the potential from biasing. The threshold voltage falls with increase in temperature, which increases saturation as well as off state current. Thus, the impact of temperature reduces the performance of FinFETs. 


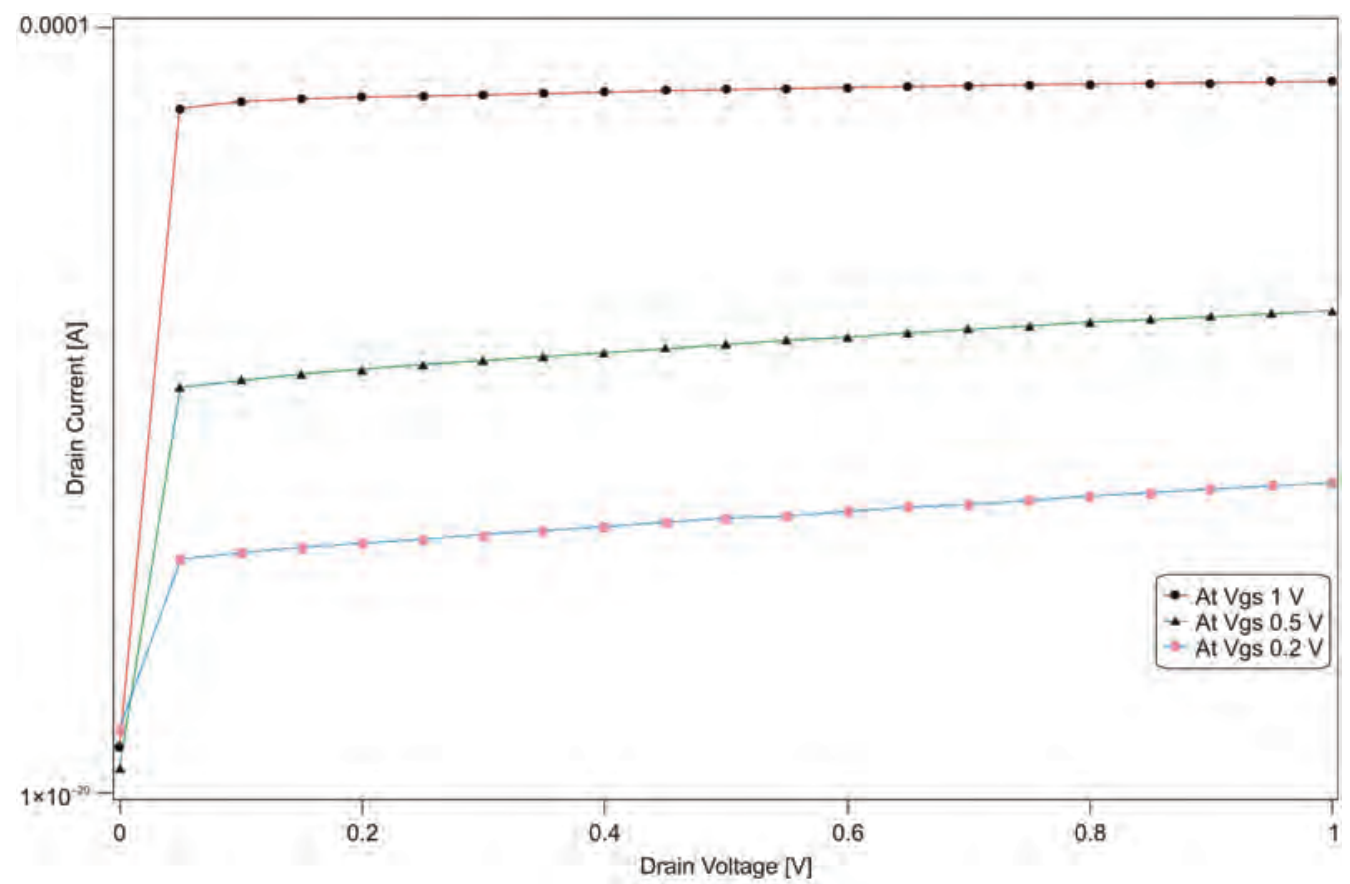

Figure 30.4 Drain voltage vs. drain current.

\section{References}

[1] A. Asenov, S. Kaya, and J.H. Davies, "Intrinsic threshold voltage fluctuations in decanano MOSFETs due to local oxide thickness variations", IEEE Trans. Electron Devices, vol. 49, no. 1, pp. 112-119, Jan. 2002. doi:10.1109/16.974757.

[2] S.E. Tyaginov, M.I. Vexler, A.F. Shulekin and I.V. Grekhov, "Statistical analysis of tunnel currents in scaled MOS structures with a non-uniform oxide thickness distribution", SolidState Electron., vol. 49, no. 7, pp. 1192-1197, 2005. doi:10.1016/j.sse.2005.04.007

[3] A.R. Brown, J.R. Watling, A. Asenov, G. Bersuker and P. Zeitzoff, "Intrinsic parameter fluctuations in MOSFETs due to structural nonuniformity of high- gate stack materials", in Proc. Int. Conf. Simulation Semiconductor Processes Devices, Sep. 2005, pp. 27-30. doi:10.1109/ SISPAD.2005.201464.

[4] Tripathi, S.L., and Patel, G.S., "Design of low power Si0.7Ge0.3 pocket junction-less tunnel FET using below $5 \mathrm{~nm}$ technology", Wireless Personal Communication,, pp.1-10, 2019. https:// doi.org/10.1007/s11277-019-06978-8.

[5] S.L. Tripathi, S. Verma and N. Dhanda, "Characterisation of ultra-small pocket Si0.7Ge0.3 junction-less tunnel FET with SOI”, Devices for Integrated Circuit (DevIC), pp. 79-83, March 23-24, 2019, Kalyani, India.

[6] S.L. Triapthi, "Low power high performance tunnel FET: analysis for IOT applications", IGI Global Publisher, pp. 47-57.

[7] G.S.Patel and S.L.Tripathi, "Performance enhnaced unsymmetrical FinFET and its applications", IEEE EDKCON, pp. 222-227, 2019.

[8] S.Verma, S.L.Tripathi and M.Bassi, "Performance analysis of FinFET device using qualitative approach for low-power applications", Devices for Integrated Circuit (DevIC), pp. 84-88, March 23-24, 2019, Kalyani, India. 


\section{Intelligent Circuits and Systems}

[9] S.L. Tripathi, R. Mishra and R.A. Mishra, "Characteristic comparison of connected DG FINFET, TG FINFET and independent gate FINFET on $32 \mathrm{~nm}$ technology", IEEE ICPCES, pp.1-7, December, 2012

[10] H. Nam and C. Shin, "Study of high-k/metal-gate work-function variation using Rayleigh distribution”, IEEE Electron Device Lett., vol. 34, no. 4, pp. 532-534, Apr. 2013. doi:10.1109/ LED.2013.2247376.

[11] Y.K. Verma, V. Mishra and S.K. Gupta, "A physics based analytical model for MgZnO/ZnO HEMT", Journal of Circuits, Systems, and Computers, vol. 29, no. 1, pp. 2050009-1, Jan. 2020; doi:http://doi.org/10.1142/S0218126620500097.

[12] Y.K. Verma, V. Mishra, P.K. Verma and S.K. Gupta, "Analytical modelling and electrical characterisation of ZnO based HEMTs", International Journal of Electronics, vol. 106, no. 5, pp. 707-720, Nov. 2019. doi:10.1080/00207217.2018.1545931.

[13] N. Patcharasardtra and W. Pengchan, "Influence of temperature to electron mobility on top of channel in 14 nm n-FinFET", IMECS 2017, March 2017, vol. 2, pp. 770-773.

[14] Tripathi, S.L., and Patel, G.S., "Design of low power $\mathrm{Si}_{0.7} \mathrm{Ge}_{0.3}$ pocket junction-less tunnel FET using below $5 \mathrm{~nm}$ technology”, Wireless Personal Communication (2019), pp.1-10. https://doi.org/10.1007/s11277-019-06978-8. 


\title{
31 Violation of independence assumption in ICA and its consequences
}

\author{
Bhaveshkumar C. Dharmani* \\ School of Electronics and Electrical Engineering, Lovely Professional University, \\ Jalandhar-Delhi, Punjab
}

\section{Introduction}

Independent Component Analysis (ICA) has been successfully used to solve both component analysis $[1,2]$ and blind source separation (BSS) $[3,4]$ problems. As a generative model, ICA assumes a latent (hidden) random vector $s$ linearly transformed to give observation random vector $x$, i.e.,

$$
\mathbf{x}=\mathbf{A} \mathbf{s}
$$

where $\boldsymbol{x}=\left(x_{1}, x_{2}, \ldots, x_{m}\right)^{T} ; \boldsymbol{s}=\left(s_{1}, s_{2}, \ldots, s_{n}\right)^{T} ; x_{i}, i=1: m, s_{i}, i=1: n$ are random variables with values in real number set $\mathfrak{R} ; m \geq n$ and $A$ is full rank. The ICA problem considers estimation of both the unknowns $A$ and $s$ together, with the only assumption of $s_{i}$ being mutually the "most independent possible" (m.i.p.). Let $y$ be the estimated source random vector given as

$$
\mathbf{y}=\mathbf{A}^{-1} \mathbf{x}=\mathbf{W} \mathbf{x}=\mathbf{W A S} \text {, }
$$

where $W$ is the estimated inverse of the mixing matrix. The optimization function, based on independence or dependence measures, used to give m.i.p. components on maximization are called either contrast functions or simply contrasts. Given $D$ a diagonal scaling matrix and $\boldsymbol{P}$ is a permutation matrix, $\mathbf{X}=\mathbf{A} \mathbf{S}=\mathbf{A}\left(\mathbf{D}^{-1}\left(\mathbf{P}^{-1} \mathbf{P}\right) \mathbf{D}\right) \mathbf{S}$. This implies that the estimated components $y$ could be any scaled and permuted version of $s$. Given the transformation is linear time invariant and the sources are independent and identically distributed $(i$ i.d.) with no more than one source being Gaussian, unique ICA solution can be obtained and the Darmois-Skitovitch Theorem ensures that the separated components are the same as the actual sources. The conditions for existence and uniqueness of ICA solution have been further detailed in $[1,5]$.

Various ICA algorithms use variety of contrasts derived through varying independence definitions and approximations. Most of the available contrasts may agree for the exact independence, but there are rare chances they will match for the "degree of independence". So, the components m.i.p. with respect to one of the independence measures do not necessarily ensure m.i.p. with respect to the other. In practice before an actual BSS application, it is rarely ensured whether the sources are the m.i.p. for the used independence measure or not. Overall, the scenario is: the ICA mathematics uses properties

*Email: Bhavesh.23409@1pu.co.in

DOI: $10.1201 / 9781003129103-31$ 
available through ideal independence, the ICA techniques allows the components to be m.i.p. and the real world ICA applications deal with the sources those are neither independent nor the m.i.p.. As a CA technique, ICA claims to inhibit the real nature of the data by finding the features with the least redundancy. So by definition, if the contrast changes, the ICs would change but still more or less be the least redundant directions for the same data. Compared to that, as a BSS techniques, ICA claims to obtain the components as the actual sources. Also, it is known that if the sources violate the ICA model assumption of being independent when there is a lack of samples then ICA results into overlearning $[6,7]$. Though wide applications, the overlearning phenomena has restricted the use of ICA.

The above facts motivate study on violations of independence assumption in ICA algorithms and their consequences on ICA solution, specifically for BSS applications. Accordingly, Section 2 describes a typical way ICA solution is obtained. Section 3 defines the types of violations by the components in ICA applications and their consequences. They are empirically verified through an experimental set-up using search for rotation based ICA (SRICA) in Section 4. Finally, Section 5 concludes the article with a discussion on considering ICA and BSS to be a different problems even in linear mixing conditions and suggests to consider "ICA as BSS" as a problem different than both ICA and BSS problems to accommodate large class of real world sources violating the ICA independence assumption.

\section{ICA solution}

$N$ sample extension of the ICA model brings $S$ as the source matrix, $X$ as the mixing matrix and $X=A S$. It is known that independence implies second order uncorrelatedness and zero mean uncorrelatedness implies orthogonality. So, ICs can be obtained through rotational orthogonal transformation of whiten components. Accordingly, the algorithm used is two steps. In the first step, whitened matrix $Z$ is obtained by transforming the zero mean $X$ through a whitening matrix $V$. So, $Z=V X=V A S$. In the next step, an optimal rotation of whiten matrix obtains ICs. The optimality is defined through a contrast giving maximization of independence. Let $R$ be the orthogonal matrix for rotation. Then, $U=R Z$ $=R V A S=W A S$, where $W=R V$ is the estimated unmixing matrix.

By definition, the random variables $y_{i}, i=1: n$, are mutually independent, if $p(y)=\prod_{i=1}^{n} y_{i}$. So, Kullback-Leibler divergence $(k l d)$ measuring mutual information between $p(y)$ and $\prod_{i=1}^{n} p_{i}\left(y_{i}\right)$ is the most canonical contrast for ICA [3]. As a contrast, it is defined as $\operatorname{mikld}\left(\boldsymbol{y}, y_{i}\right)=k l d\left(p(\boldsymbol{y}), \prod_{i=1}^{n} p_{i}\left(y_{i}\right)\right)=\int p(\boldsymbol{y}) \frac{p(\boldsymbol{y})}{\prod_{i=1}^{n} p_{i}\left(y_{i}\right)} d \boldsymbol{y}$, where $d \boldsymbol{y}=d y_{1} y_{2} \ldots y_{n}$.

The symmetrical distance measure Jensen-Shannon $\operatorname{KLD}\left(j s k l d\left(y, y_{i}\right)\right)$, maximization of Shannon entropy or minimization of the sum of marginal entropies minhyi $\left(\boldsymbol{y}, y_{i}\right)$ and normalize kurtosis $\left(\right.$ normk4 $\left.\left(\sum y_{i}\right)\right)$ are the other contrasts used for the experiments in Section 4 and are defined as: $\begin{aligned} & j s k l d\left(\mathbf{y}, y_{i}\right)=k l d(p(\mathbf{y}), \mathbf{M})+k l d\left(p\left(\prod_{i=1}^{n} y_{i}\right), \mathbf{M}\right), \operatorname{minhyi}\left(\mathbf{y}, y_{i}\right) \\ = & \sum_{i=1}^{n} \mathbf{H}\left(y_{i}\right), \operatorname{normk} 4\left(\sum y_{\mathbf{i}}\right)=\sum_{i=1}^{n}\left|k \operatorname{kurtosis}\left(y_{i}\right) 3\right|,\end{aligned}$ where $\mathbf{M}=\frac{1}{2}\left(p(\mathbf{y})+p\left(\prod_{i=1}^{n} y_{i}\right)\right), \boldsymbol{H}(\Theta)$ denotes entropy and $\mid q$ denotes absolute value. The contrast $\operatorname{radH}\left(\sum y_{i}\right)$ achieves minimization of sum of marginal entropies through spacing estimates of entropy. 


\section{Violation of independence assumption definitions and consequences}

There are two types of violations of the independence assumption: either based on the independence definition or the ICA independence assumption of being the m.i.p.. $\downarrow$ Weak Violation of Independence Definition $(w-V I D)$ : If the ICA sources are not statistically independent but are at least uncorrelated then there is said to exist $w$-VID. $\downarrow$ Strong Violation of Independence Definition $(s-V I D)$ : If the ICA sources are neither statistically independent nor even uncorrelated then there is said to exist $s$-VID. Weak Violation of ICA Independence Assumption (w-VIIA): If the ICA sources are not the m.i.p. but are at least uncorrelated then there is said to exist $w$-VIIA. Strong Violation of ICA Independence Assumption (s-VIIA): If the ICA sources are neither the m.i.p. with respect to the given contrast nor even uncorrelated then there is said to exist $s$-VIIA.

The strong violations always imply corresponding weak violations, i.e., $s$-VID implies $w$-VID and $s$-VIIA implies $w$-VIIA. Also, $s$-VIIA implies $s$-VID and $w$-VIIA implies $w$-VID. But $s$-VID implies neither $s$-VIIA nor $w$-VIIA as the sources may be correlated and still the m.i.p. It is known that even the laboratory generated test i.i.d. sources will not measure exact independence or uncorrelatedness. Accordingly, in the case of ICA for BSS of real world sources, there will be almost always $w$-VID and $s$-VID violations. Frequently the actual sources are the m.i.p. with respect to a particular contrast, but almost always it will not be the m.i.p. with respect to remaining contrasts in the literature. This brings almost always $w$-VIIA violation. The almost always violations of $s$-VID and $w$-VIIA will cause almost always violation of s-VIIA.

\subsection{Consequences}

The sources violating $w$-VID but not w-VIIA, i.e., those being m.i.p., can be obtained uniquely as a solution by an ICA algorithm. Thus, only a violation $w$-VID may not be affecting the ICA applications. The violation $w$-VIIA causes the m.i.p. components obtained as an ICA solution to be, though mathematically unique, different from the actual sources. It implies that there could be possible many solutions with the same or higher measure for the given sources using the given contrast. Also, based on the used contrast, estimation techniques and even the optimization techniques there are multiple solutions equally competent to be declared as the best solution. Thus, on $w$-VIIA violations, there is a problem of uniqueness of the solution, specifically when ICA is used for BSS as discussed in the introduction section. Most of the ICA algorithms assume orthogonal ICs either as a necessary condition to find the orthogonal ICA solution or to reduce the computation by reducing the possible search space. The $s$-VID or the $s$-VIIA violations cause the actual sources non-existing in the set of possible solutions for the ICA algorithms following orthogonal approach. As a solution to this, if the constraint of orthogonality is removed from the ICA algorithm then either the ICA algorithm may not work at all or may find a wrong solution due to misconvergence. Thus, on $s$-VIIA violations, there is a problem of existence of the solution, specifically when ICA is used for BSS.

\section{Empirical verifications and analysis}

The current article uses SRICA [8] algorithm based on genetic algorithm (GA) that follows the orthogonal approach, as discussed in Section 2, to find the optimal $n$-dimensional 
angle of rotation. As the estimated source matrix $Y$ is a permuted and scaled to univariant version of $S, Y=G S$ and $G=P W A D$, where $G$ is called gain matrix, $\boldsymbol{P}$ is a permutation matrix and $\boldsymbol{D}$ is a diagonal scaling matrix. Ideally $G$ being an identity matrix, Amari Performance Index (API) measures deviation of $G$ from diagonalization as a performance measure of ICA algorithms. Mathematically, $A P I(G)=\sum_{i=1}^{n}\left(\sum_{i=1}^{n} \frac{\mid G_{i j \mid}}{\max _{k}\left|G_{i k}\right|}-1\right)+\sum_{i=1}^{n}\left(\sum_{i=1}^{n} \frac{\mid G_{i j \mid}}{\max _{k}\left|G_{k j}\right|}-1\right)$. The nearer API is to zero, the better the performance of an ICA algorithm. The mean of the signal to interference ratio of the estimated sources (SIRS) with respect to the actual sources is also a performance measure defined as meanSIRS $=\frac{1}{n} \sum_{k=1}^{n} \operatorname{SIRS} S_{k}$, where $\operatorname{SIRS} S_{k}=\frac{\sum_{i=1}^{n} s_{k i}^{2}}{\sum_{i=1}^{n}\left(y_{k i}-s_{k i}\right)^{2}}$.

To verify the existence and uniqueness of the solution, the following experiment was done. Two i.i.d. sources were taken with the distributions randomly selected from the set of 21 densities with 18 densities as defined in [9] and 3 more densities, unimodal left-skewed, unimodal right skewed and Rayleigh, as in [8]. The added 3 densities are generated using power method. The sample size was kept to 1000 . The experiment was repeated for 50 simulation trials. The SRICA algorithm was used with 5 contrasts defined before. During experimentation, it was found that SRICA with a different contrast finds a different solution. To know whether the solution obtained is optimal or not, the independence measures for all the estimated sources were calculated. Also, GA allows use of the performance measures themselves as fitness or contrast function. The solutions available through them should be the best ideally. The comparison of the solutions answers the degree of approximations possible in the solution or whether the solution at all exists and is unique in the given solution space. For this verification, API and mean SIRS both were used as performance measures. To facilitate comparison with the existing ICA algorithms, results are also obtained for FastICA [10], EFICA [11], NPICA [12] and RADICAL [13] techniques, as they are the best considered so far $[9,13]$.

\subsection{Results and analysis}

The results have been tabularized in Table 31.1. The rows indicate the median obtained from 50 simulation trials through the contrast used with SRICA or the other ICA algorithm as shown in the first column, and the columns indicate corresponding independence and performance measures. The bold-face values represent the best obtained corresponding to that column, i.e., used performance measure. $A P I<0.1$ and $S I R S>10 \mathrm{db}$ imply good results.

\section{Observations}

All the algorithms have given satisfactorily approximated solutions with the following further observations:

- Though being m.i.p., mathematically, exact independence has been achieved neither by the estimated nor even by the laboratory generated i.i.d. sources. This confirms the obvious $w$-VID violation.

- An algorithm the best with respect to one of the performance measures does not imply that it is the best with respect to the others. There are multiple solutions qualifying 
Table 31.1 Problems of existence and uniqueness of the ICA solution for BSS demonstrated through performance measurement of SRICA using various contrasts, other state-of-the-art ICA algorithms, and performance measures used as contrasts with SRICA. The performances are measured using the conventional performance measures and contrast measures. Two i.i.d. sources had the distributions randomly selected from the set of 21 densities with sample size of 1000 . The entries show the median of 50 simulation trials. The boldface values denote the best per performance measure.

\begin{tabular}{|c|c|c|c|c|c|c|c|}
\hline \multirow{3}{*}{$\begin{array}{l}\text { SRICA (contrast) } \\
\text { and other ICA } \\
\text { algorithms used }\end{array}$} & \multicolumn{7}{|c|}{ Performance measures } \\
\hline & \multicolumn{5}{|c|}{ Independence measures of the estimated sources } & \multicolumn{2}{|c|}{$\begin{array}{l}\text { State-of-the-art } \\
\text { performance measures }\end{array}$} \\
\hline & $\begin{array}{l}\text { Jskld }(Y, y i) \\
\text { (bits) }\end{array}$ & $\begin{array}{l}\text { Mikld (Y,yi) } \\
\text { (bits) }\end{array}$ & $\begin{array}{l}\text { Minbyi } \\
\text { (Y,yi) (bits) }\end{array}$ & Normk4 $\left(\sum y_{i}\right)$ & $\begin{array}{l}\text { RadH } \\
\left(\sum y_{i}\right) 10^{-3}\end{array}$ & $A P I$ & $\begin{array}{l}\text { MeanSIRS } \\
\text { in }(d b)\end{array}$ \\
\hline Jskld(Y, yi) & 0.0035 & 0.0131 & 2.672 & 1.7274 & -4.3414 & 0.022 & 35.4952 \\
\hline Mikld(Y,yi) & 0.0035 & 0.0129 & 2.672 & 1.7305 & -4.3421 & 0.0227 & 35.0565 \\
\hline Minhyi(Y,yi) & 0.0035 & 0.0129 & 2.6718 & 1.7289 & -4.3423 & 0.019 & 36.2552 \\
\hline Normk4(y) & 0.0041 & 0.0152 & 2.6721 & 1.7504 & -4.333 & 0.0393 & 28.8385 \\
\hline $\operatorname{RadH}(y)$ & 0.0037 & 0.0138 & 2.6743 & 1.6662 & -4.3456 & 0.0232 & 33.9066 \\
\hline \multicolumn{8}{|c|}{ Other algorithms and source measures for comparison } \\
\hline FastICA & 0.0046 & 0.0169 & 2.6676 & - & -4.3316 & 0.0578 & - \\
\hline EFICA & 0.0042 & 0.0156 & 2.6754 & 1.7463 & -4.3295 & 0.0388 & 29.3483 \\
\hline NPICA & 0.0036 & 0.0136 & 2.6737 & 1.6769 & -4.3345 & 0.0191 & 35.6634 \\
\hline RADICAL & 0.0036 & 0.0133 & 2.6721 & 1.714 & -4.3443 & 0.0198 & 35.9338 \\
\hline Sources & 0.0015 & 0.0055 & 2.0335 & 1.7256 & -5.0161 & - & - \\
\hline \multicolumn{8}{|c|}{ Performance measures used as fitness measure, for Existence Problem } \\
\hline API & 0.0036 & 0.0133 & 2.6725 & 1.7318 & -4.3413 & 0.0077 & 56.1296 \\
\hline MeanSIRS & 0.0039 & 0.0146 & 2.6746 & 1.7317 & -4.3333 & 0.011 & 73.7894 \\
\hline
\end{tabular}


to be the m.i.p. based on the used contrast. This empirically validates the problem of uniqueness of the solution.

- Each SRICA algorithm with a particular contrast has found the best in terms of achieving the best contrast measure for the estimated sources but not necessarily with respect to other performance measures. This indicates that the optimization techniques have been successful in finding the best with respect to the used contrast but while doing so, they have not been able to follow the actual sources. There can be defined a property named "source following ability" of a contrast based on how much they ensure bringing the actual sources on maximization.

- It was expected that for the laboratory generated ideal sources without any added noise, at least the SRICA with performance measures themselves as contrasts should have been able to separate the actual sources and the mixing matrix. But none of the algorithms has been able to get the same sources or even sources with a nearby independence measure. This validates empirically the problem of existence of the solutions.

- The reason for the unmatched independence measures of the estimated source and actual sources is that the sources even violate $s$-VID being not exactly uncorrelated. This was separately also verified for the generated sources. All the algorithms in the test use the orthogonal approach. So, the actual sources do not exist at all in the solution space of those algorithms. This validates again the almost always happening $s-V I D$ and $s$-VIIA violations.

Overall, based on the above observations it can be concluded that the violation of independence assumptions are very common and almost always exist. These violations bring both existence and uniqueness problems, specifically when ICA is used for BSS applications. Current practice is to explain such results as an outcome of overlearning, where the phenomena is considered as the estimator's inability to follow the actual process that generated the samples and instead it follows the samples themselves; specifically a small number of samples result in over-assumption and over-reliance on independence. This article expands this understanding of overlearning in two ways. The widening paradigm says that there is almost always the violation of independence assumption and so almost always the overlearning. The sharpening paradigm says that the major reason for overlearning almost always existing is not necessarily the lack of insufficient samples as even with a sufficient number of samples there is a also violation of ICA model assumption. Though wide scope, the overlearning phenomena has restricted the use of ICA for BSS.

\section{Conclusion and discussion proposal on "ICA as BSS" as a new problem}

The article has defined violation of ICA independence conditions and empirically verified them. It is concluded that the sources almost always violate both the assumptions of being the m.i.p., as well, being uncorrelated. This has provided better understanding of the overlearning phenomenon through widening and sharpening paradigms. It has also exposed the problem of existence and uniqueness of the solution, specifically when ICA is used for BSS.

There are two possible queries to above conclusion. First, are the results not simply perturbation and allowed? More specifically, it is understood that the exact statistical behaviour would be when theoretically infinite number of samples and asymptotic behaviours are always acceptable. Also, this behaviour is already known to the research community and largely being studied as overlearning phenomenon. Is this understanding not 
acceptable to this article? This article is to denounce neither the asymptotic behaviour nor the current understanding in terms of overlearning, but it is to expose its dominance through the problem of existence and uniqueness of the solution almost always happening. To better understand this phenomena it has tried to understand the phenomena as a consequence of the violation of independence assumption.

The other query is whether the article wants to denounce the practice of using ICA for BSS by focusing on the problem of existence and uniqueness of the solution. In contrast to it, ICA has been used successfully for BSS since its origination, in fact, it is known that originally ICA was derived to solve the BSS problem [4]. The answer is, definitely not to denounce the existing practice. But the time has come to initiate a discussion on whether even in cases of linear mixing of stationary sources ICA can be considered equivalent to BSS or not. This article favours considering ICA and BSS both as a different problems in the linear mixing of stationary sources. At the same time, it wants the use of ICA for BSS to be continued. To signify a balancing act, it proposes to consider "ICA as BSS" to be a different problem than both ICA and BSS problems. All three being now different problems can have different mathematical requirements for existence and uniqueness of their solutions. Then, the basic model for all the three different problems - ICA, ICA as $B S S$ and BSS - remains same as in equation (1). The goal for the ICA as BSS problem is to separate the actual sources similar to that for BSS. But while BSS is open to use any "generic" assumption of independence, sparsity, second order uncorrelatedness or others, the assumption for "ICA as BSS" is independence but with more relaxed conditions. Specifically, the sources should be the m.i.p. with respect to a specific contrast, and allowed to be near the m.i.p. with respect to any other contrast. Also, they need be only near uncorrelated. This implies that the sources may have violations $s$-VID and $s$-VIIA. The relaxed assumptions on the sources would accommodate a large set of real world sources. But with a relaxed independence assumption, how to ensure the existence and uniqueness of the solution? To ensure the existence and uniqueness of the solution, some sort of regularization mechanism must be added with the basic assumption of independence. The regularization may be based on the second order statistic or bound on the independence measure of the sources or bound on the degree of uncorrelatedness or nearness of the separated sources or others. In general, the assumption should be "semi-generic" in nature, which is neither so widely applicable as if a "generic" nor as application specific as in semiBSS approach. Also, it should not be in the form of specific prior on the distributions of the sources or the mixing matrix as in Bayesian BSS approach. It is worth mention that current ICA definitions do not allow such regularization to achieve the actual sources. As stated before, the source following ability of a contrast may guide to decide the most suitable contrast for the given sources. Overall, the article opens up a discussion to consider ICA and BSS both as separate problems even in linear mixing conditions and the proposed solutions for more detailed study and further research to the research community.

\section{References}

[1] P. Comon. Independent component aalysis—a new concept? Signal Processing, vol. 36 (1994), pp. 287-314.

[2] Duda, Richard O., Hart, Peter E., and Stork, David G.. Pattern Classification, 2nd edn. WileyInterscience, 2000.

[3] J.-F. Cardoso. Blind signal separation: statistical principles. Proceedings of the IEEE, vol. 9, no. 10 (1998), pp. 2009-2025.

[4] P. Comon and C. Jutten, Handbook of Blind Source Separation: Independent Component Analysis and Applications, 1st edn. Academic Press, 2010. 


\section{Intelligent Circuits and Systems}

[5] J. Erikssonand and V. Koivunen. Identifiability, separability, and uniqueness of linear ICA models. Signal Processing Letters, IEEE, vol. 11, no. 7 (2004), pp. 601-604.

[6] J. Sarela and R. Vigario. Overlearning in marginal distribution-based ICA: analysis and solutions. The Journal of Machine Learning Research, vol. 4 (2003), pp. 1447-1469.

[7] J. Sarela and R. Vigario. A Bayesian approach to over- learning in ICA. Helsinki University of Technology, 2003.

[8] Dharmani Bhaveshkumar, C.. Proposal of a search for rotation based independent component analysis (SRICA) algorithm. In: Proceedings of the Fourth International Conference on Signal and Image Processing 2012 (ICSIP) (2012). Lecture Notes in Electrical Engineering, vol. 221, Springer India (2013), pp. 411-421.

[9] Bach, F., and Jordan, M.. Kernel independent component analysis. The Journal of Machine Learning Research, vol. 3 (2003), pp. 1-48.

[10] A. Hyvarinen and E. Oja. A fast fixed-point algorithm for independent component analysis. Neural Computation, vol. 9 , no. 7 (1997), pp. 1483-1492.

[11] Z. Koldovsky, P. Tichavsky and E. Oja. Efficient variant of algorithm fastica for independent component analysis attaining the cramer-rao lower bound. Neural Networks, IEEE Transactions on, vol. 17, no. 5 (2006), pp. 1265-1277.

[12] Boscolo, R., Pan, H., and Roychowdhury,V. Independent component analysis based on nonparametric density estimation. Neural Networks, IEEE Transactions on, vol. 15, no. 1 (2004), pp. 55-65.

[13] E.G. Learned-Miller and W.F. John III. ICA using spacings estimates of entropy. The Journal of Machine Learning Research, vol. 4 (2003), pp. 1271-1295. 


\title{
32 Architecture, key technologies and emerging applications of $5 \mathrm{G}$ cellular networks
}

\author{
Shailender Kumar Tomar ${ }^{1}$, Shelej Khera ${ }^{2}$ \\ ${ }^{1}$ Research Scholar (ECE), LPU Phagwara, Punjab, India \\ 2Professor \& HOD Communication Systems (SEEE), LPU Phagwara, \\ Punjab, India
}

\section{Introduction}

The speedy growth of mobile communication has resulted in an exponential increase in quantity of users and service providers. The network must therefore convene high system capacity, elevated data transmission rate, in addition to the communication must also make efficient use of the bandwidth resource to meet all emerging demands [1]. The fifthgeneration mobile network or just $5 \mathrm{G}$ is a major advance to today's 4G LTE networks. 5G is designed to meet the diverse increase of data and communication.

\section{Growth in wireless technology}

In Table 32.1 a comparative analysis of different generation attributes have been shown in detail.

\section{Requirements of fifth generation}

- Ultra low ranges of latency between $1 \mathrm{~ms}$ and $10 \mathrm{~ms}$.

- Provide sufficient capacity for infinite transfer of data.

- High resolution and high-speed Internet access.

- Higher reliability of the communication

- Supports virtual private network.

- System spectral efficiency is high

- Elevated increased peak bit rate

- Battery consumption is low

- Large number of supporting devices

- Elevated capability to permit additional system connectivity concomitantly and immediately [4]

IV. 5G technical requirements compared to $4 \mathrm{G}$

A set of eight requirements identified by industry are the following:

- Connections to field end points $1-10$ Gbps

- delay of the round-trip (latency): $1 \mathrm{~ms}$

- Bandwidth / unit area: 1000 times

- devices connected: 10-100times

DOI: $10.1201 / 9781003129103-32$ 
Table 32.1 Comparative analysis of different generation attributes [2,3]

\begin{tabular}{|c|c|c|c|c|c|}
\hline $\begin{array}{l}\text { Technology/ } \\
\text { feature }\end{array}$ & $1 G$ & $2 G$ & $3 G$ & $4 G$ & $5 G$ \\
\hline Deployment & 1980 & 1990 & 2001 & 2010 & 2020 onward \\
\hline $\begin{array}{l}\text { Frequency } \\
\text { band used }\end{array}$ & $800 \mathrm{MHz}$ & $900 \mathrm{MHz}$ & $2100 \mathrm{MHz}$ & $2600 \mathrm{MHz}$ & $3-90 \mathrm{GHz}$ \\
\hline Data rate & $2.4 \mathrm{kbps}$ & $64 \mathrm{kbps}$ & $2 \mathrm{Mbps}$ & 1 Gbps & 10 to 100 Gbps (expected) \\
\hline Technique & Analog & Digital & CDMA & Unified IP & WWWW and Unified IP \\
\hline Application & Only audio & Audio and data & $\begin{array}{l}\text { Audio, data and video } \\
\text { calling }\end{array}$ & $\begin{array}{l}\text { Dynamic information } \\
\text { access, wearable device } \\
\text { (online gaming, high } \\
\text { definition television) }\end{array}$ & $\begin{array}{l}\text { Dynamic information access, } \\
\text { wearable device with AI } \\
\text { capabilities (ultra high definition } \\
\text { video, virtual reality application) }\end{array}$ \\
\hline Multiplexing & FDMA & $\begin{array}{l}\text { TDMA, } \\
\text { CDMA }\end{array}$ & CDMA & OFDMA & $\begin{array}{l}\text { OFDM, filter-bank multicarrier, } \\
\text { non orthogonal, multiple access } \\
\text { (NOMA) }\end{array}$ \\
\hline Standards & $\begin{array}{l}\text { AMPS, } \\
\text { NMT, } \\
\text { TACS }\end{array}$ & $\begin{array}{l}2 \mathrm{G}-\mathrm{GSM} \\
2.5 \mathrm{G}-\mathrm{GPRS} \\
2.75 \mathrm{G}-\mathrm{EDGE}\end{array}$ & $\begin{array}{l}3 \text { G: UMTS, EDGE } \\
\text { CDMA2000/WCDMA } \\
3.5 \text { G- HSDPA } \\
\text { 3.75 G- HSUPA }\end{array}$ & $\begin{array}{l}\text { Long term evolution (LTE), } \\
\text { HSPA+VoLTE } \\
\text { Wi-max, Wi-Fi }\end{array}$ & Single unified standard WWWW \\
\hline Transfer of call & Inter network & Inter network & Inter network & Inter and intra network & Inter and intra network \\
\hline Connection & Circuit switching & $\begin{array}{l}\text { Circuit/packet } \\
\text { switching }\end{array}$ & All packet switching & Packet switching & 1 Packet switching \\
\hline
\end{tabular}


- Coverage: $100 \%$ (estimated)

- Availability: 99.999\% (estimated)

- Reduction energy usage in network: up to $90 \%$

- Battery life: up to 10 years [5]

\section{Promising technoligies for $5 \mathrm{G}$ wireless networks}

In this section, we discuss six novel techniques immerging as the basis of the fifth generation.

1. mmwave: A new radio called mmwave will be used in 5G. As we know, the radio frequency spectrum from $1 \mathrm{GHz}$ to $6 \mathrm{GHz}$ is very crowded (as shown in Figure 32.1) as many technologies use this range such as GSP, Wimax Wi-Fi, 4G, 3G, L-band, satellite, $\mathrm{S}$ band and C-band etc. Spectrum range from $30 \mathrm{GHz}$ to $300 \mathrm{GHz}$, known as millimetre wave, is utilized. It is a new territory. Thus the range from $24 \mathrm{GHz}$ to $100 \mathrm{GHz}$ is proposed for $5 \mathrm{G}[6]$.

2. Small cell: A mmwave has advantages but it has some disadvantages too; i.e. higher frequency signal will have more collisions with obstacles in the air, and thus they tend to lose energy more quickly. Therefore, mmwave signals cover shorter distances. They are also easily blocked by building and trees. The small cell concept will solve this problem as small cell stations will fill the coverage gap between a base station and user. A small area is covered by each cell station. The number of small cell stations and distance between them depend on the population at that location. In a highly populous district, for example, the distance between two small cells could vary from 10 to $100 \mathrm{~m}$. In near future we might see such small cells everywhere in our neighbourhood [8] as shown in Figure 32.2.

3. Massive Mimo: The relationship between the wave frequency and antenna size is inversely proportional (as shown in Figure 32.3) which means lower frequency signal needs a bigger antenna to transmit and receive while higher frequency signals can work well with smaller size antenna. The higher frequency wave we use, the smaller antenna we can get. Thus this mm wave helps to have many transmitters with receivers installed with minute cells, for example for older technology one cell can have 10 antennas, but for $5 \mathrm{G}$ this same cell may have 100 .

4. Beam-forming: Beam-forming makes the transmission more directional between user and base or cell station. Figure 3.4 shows the beam forming. This is termed as laser

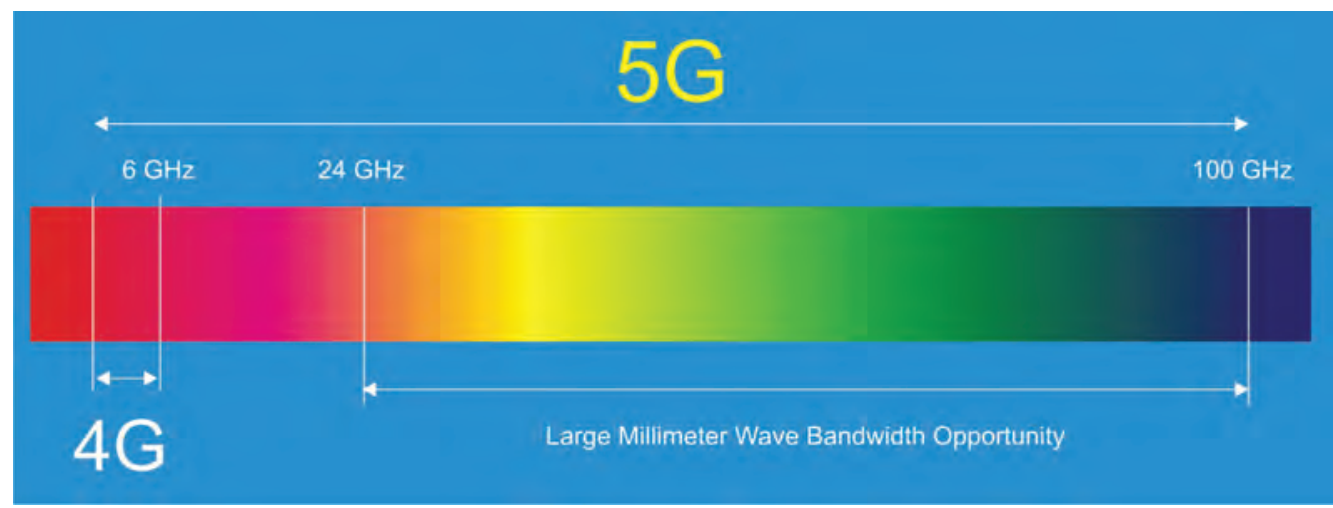

Figure 32.1 Millimetre wave. 


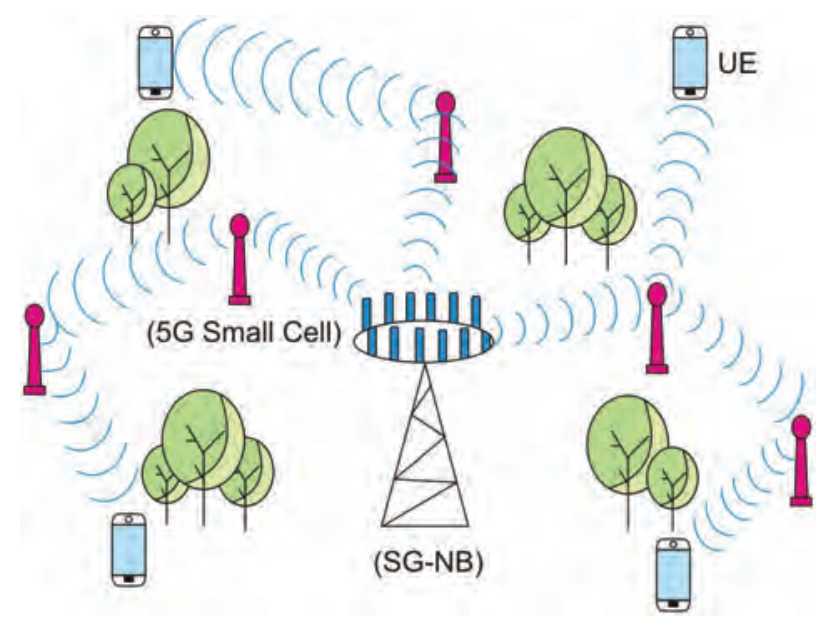

Figure 32.2 Small cells.

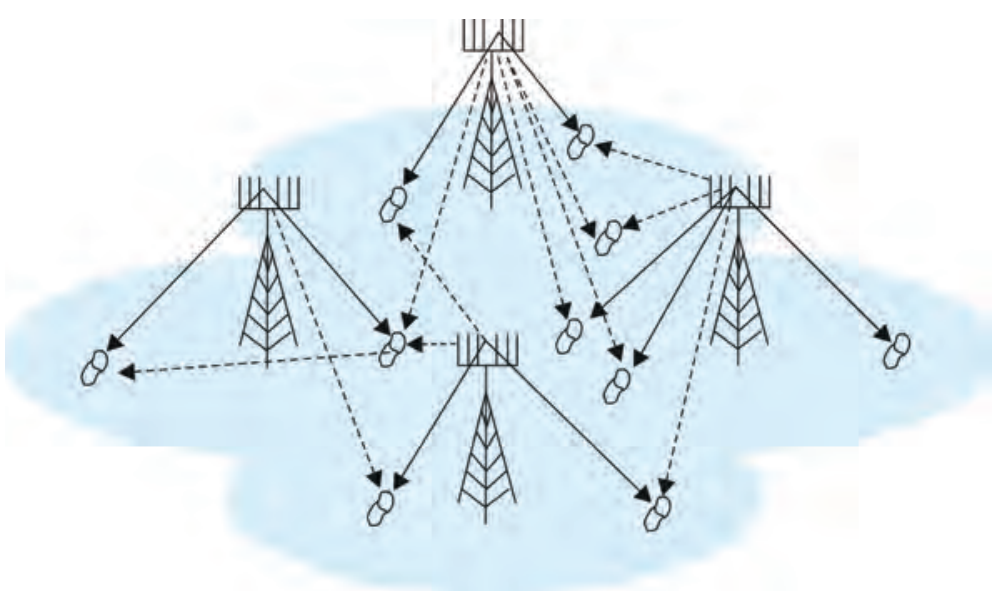

Figure 32.3 Illustration of massive MU-Mimo.

beam so that signals travel along an invisible cable and thus a faster data rate can be achieved [10].

5. NOMA (non-orthogonal multiple acess): In previous generations $1 \mathrm{G}$ through $3 \mathrm{G}$, multiple access methods are based on frequency, time and code. In 4G orthogonal multiple accesses are used. The idea of NOMA as shown in Figure 32.5 is to use the power level of the user device to access the base station. The new multiple access method would allow different signals to share the same channel simultaneously but NOMA provides a higher sum rate than the orthogonal method [11].

6. MEC (mobile edge computing): This technique as shown in Figure 32.6 utilizes cloud computing but brings cloud computing closer to user. Intensive and latency sensitive application like augmented reality, video conference, can be hosted at the edge of the network [11]. 


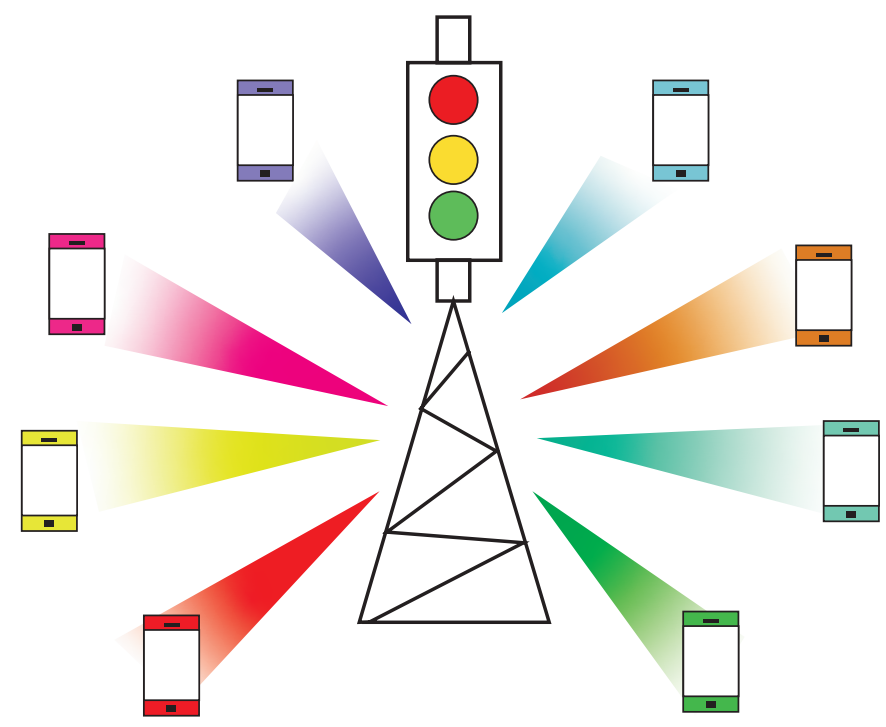

Figure 32.4 Beam-forming.

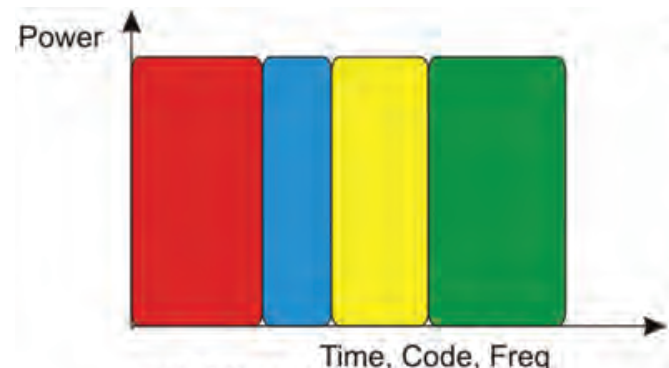

(a) Orthogonal Multiple Access

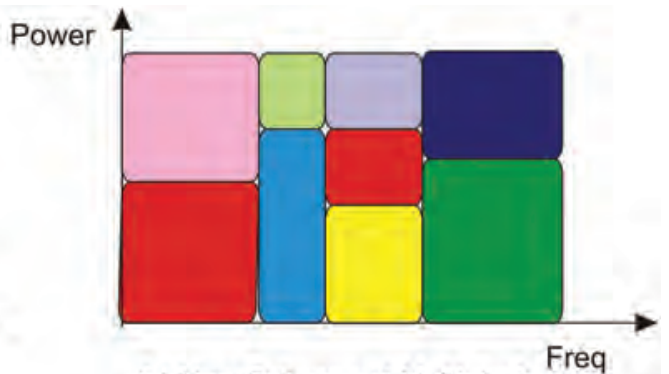

(b) Non-Orthogonal Multiple Access

Figure 32.5 NOMA.

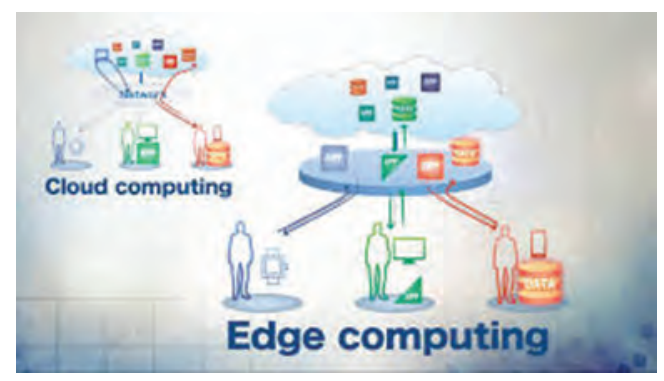

Figure 32.6 MEC. 


\section{Emerging applications of $5 \mathrm{G}$}

- Device2Device (D2D) communication

- Machine2Machine communication (M2M)

- Internet of Things (IoT): all things connected

- Artificial intelligence (AI) and machine learning

- Smart farming, smart home/buildings, smart cities

- Mission critical application, e.g. e-health

- Industry automation, augmented reality

- Self-driving cars

- Security and supervision[12]

\section{Conclusion}

$5 \mathrm{G}$ would be driven by a lot of new technologies .On the other hand, $5 \mathrm{G}$ would push other technologies to a new level, such as the Internet of Things (IOT), games, smart city, smartphone, self-driving cars, remote operations and machine learning. 5G is coming with a lot of potentials and possibilities. Some we know now, but there are more we don't know. But overall, the future looks bright.

\section{References}

[1] Huh H, Caire G, Papadopoulos C, et al. Achieving "massive mimo" spectral efficiency with a not-so-large number of antennas [J]. IEEE Transactions on Wireless Communications, 2011, 11(9); 3226-3239.

[2] Naser Al-Falahy and Omar Y. Alanig. Technologies for 5G networks: challenges and opportunities. IT Professional, Volume 19, Issue 1, Jan.-Feb. 2017, pp. 12-20. doi:10.1109/ MITP.2017.9.

[3] Akhil Gupta and Rakesh Kumar Jha. A survey of $5 \mathrm{~g}$ network: architecture and emerging technologies 2169-3536(c) 2015 IEEE.

[4] Siddhika Arunachalam, Shruti Kumar, Harsh Kshatriya, Mahendra Patil. Analyzing 5G: prospects of future technological advancements in mobile, IOSR Journal of Engineering (IOSRJEN) 2278-8719 volume 1, pp 06-s11.

[5] M. Sathiya, R. Gowthami, G. Karpagam, B. Saranya, U. Suganya. Cellular and network architecture for $5 \mathrm{~g}$ wireless communication networks in mobile technology, International Journal of Technical Research and Applications, Volume 3, Issue 2 (Mar-Apr 2015).

[6] Sandeep Singh and Mridul Chawla. A view on millimeter wave communication and effect on $5 \mathrm{G}$ system. Assistant professor of department of electronics \& communication, Chotu Ram University Science and Technology, ISSN No: 2393-8021.

[7] Theodore S. Rappaport and Shu Sun. Millimeter waves mobile communication for 5 G Cellular. Board of Governors of IEEE Vehicular Technology Society (VTS) (May 2013), INSPEC ISSN No: 2169-3536.

[8] Uzma Siddique and Hina Tabassum. Wireless backhauling of 5G small cells Department of Electrical and Computer Engineering, University of Manitoba (July 2015), ISSN No: $1536-1284$.

[9] Erik G. Larsson and Liesbet Van der Perre. Massive MIMO for 5G IEEE. 5 G Tech Focus vol. 1, no. 1 (March 2017) 7.

[10] Amy Nordum, Kristen Clark. 5G bytes: beamforming explained. IEEE spectrum staff (Jul 2017).

[11] 5G cellular networks: 6 new technologies. www.youtube.com/watch?v=hQvHNVRv_ms.

[12] Swati Yadav, Sugandha Singh. Review paper on development of mobile wireless technologies (1G to 5G), International Journal of Computer Science and Mobile Computing. 


\title{
33 Exploration of recent medium access control (MAC) protocols for efficient transmission of data in wireless body area network
}

\author{
Ms. Thota Sruthi*, Manwinder Singh* \\ School of Electronics and Electrical Engineering, Lovely Professional \\ University, Punjab
}

\section{Introduction}

The recent trends in the advances of the technology had changed the world into the smart world. In such era of technology the sensors are playing the major role in the home automation, defence, vehicular communication, monitoring the environmental parameters and majorly in the medical field to monitor the patient health condition. As a part of this, the people want to monitor their health parameters without disturbing their daily activities by frequent check-ups in the hospitals. Based on the conditions like mobility and real time health monitoring, an application had developed with the help of implanted sensors to monitor the parameters like heartbeat, temperature, blood pleasure, electrocardiogram (ECG), which can be collected and transferred by the personal digital assistant such as mobile through the wireless media to the doctors in the remote monitoring centres or primary health care units. This overall architecture of the network simply confined in Figure 33.1. This entire application was the new era in the wireless technology, i.e. wireless body area network (WBAN) [3] with best features like mobility and reliability.

The WBAN is widely used for the applications like elderly persons' health observations, telemedicine, emergency treatment. As the WBAN applications having the tiny sensors which are placed on the human body, these sensors are having very less battery capability and in some cases the patients need to have implanted biosensors even inside the body which are non-rechargeable, non-replaceable. Because of these reasons the WBAN main challenge is the energy consumption. For this the energy harvesting technique should be implemented by which the sensors will get the energy from various sources like body temperature, vibrations and external light sources [1] to increase the lifetime of the network. The data link layer's medium access control protocols are controlling the access of the nodes from the link through which the nodes are going to transfer the data. There should be efficient routing protocols in order to transmit the data without any interruption because of the energy limitation and also to maintain the better QoS [2]. This paper comprises the recent developments of the MAC protocols.

\section{Literature survey}

In [4], Chong-Qing et al. proposed an adaptive MAC protocol to serve few-transmit (FT) nodes. That is in WBAN application, there is no need to transmit the data of all the sensors continuously. So if those sensors are in sleep mode, then the energy can be saved greatly.

\footnotetext{
*Emails: thota.sruthi1993@gmail.com, manwinder.25231@lpu.co.in
} 

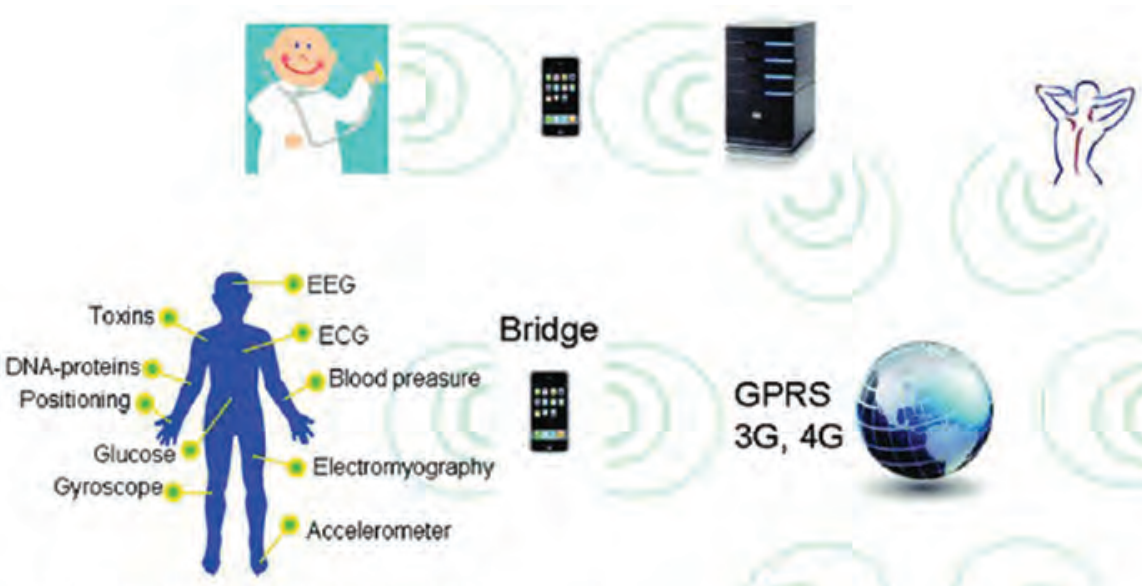

Bridge

Wireless Body Area Network

Bluetooth, UWB, etc
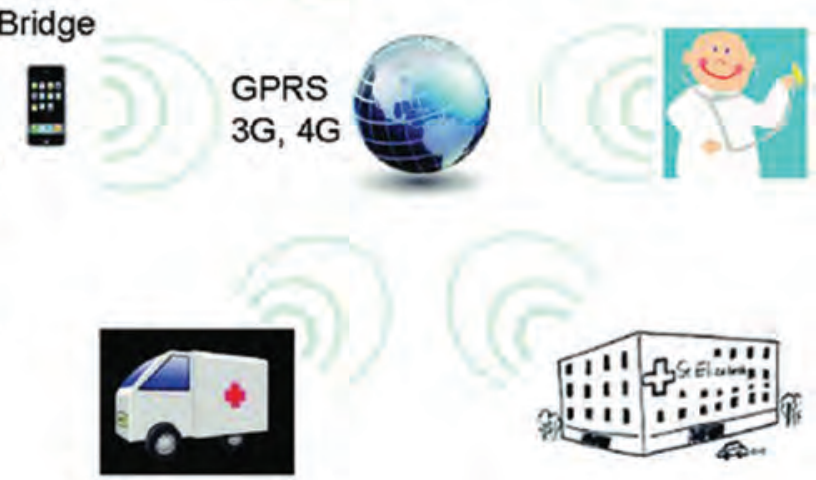

Figure 33.1 Overview of wireless body area network.

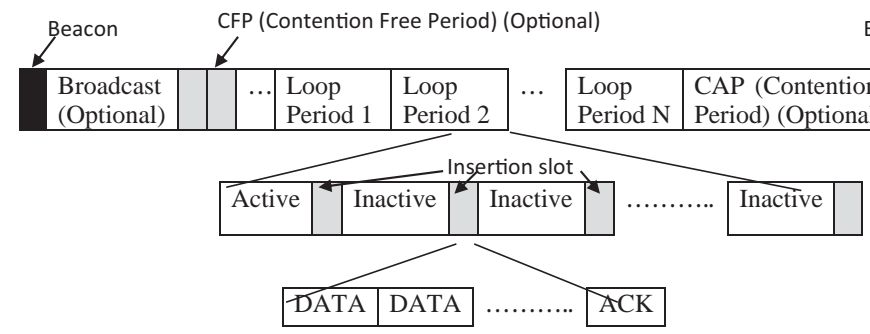

Figure 33.2 The super frame structure of FT-MAC.

But when the required time came to transmit the data those sensors must be activated. So, there should be an energy efficient protocol to manage this transmission. This paper had an FT-MAC protocol super frame structure and performance evolution of it. Figure 33.2 represents the FT-MAC protocol's super frame structure. It has the information regarding the time intervals.

CFP is having some optional guaranteed time slots (GTS) to communicate long frames between the coordinator and any single node. The loop period is starting with an active period for all-transmit mode, the inactive period is for few-transmit mode to save energy. The insertion slot can be used to make the communication between all nodes and coordinator to share data or network commands using slotted ALOHA technique. Then all the nodes retransmit the lost frames due to collisions in the transmission using carrier sense multiple access with collision avoidance mechanism (CSMA/CA) CAP.

The Data and ACK of insertion slot can be used to transfer the data or commands to/from nodes using download/upload insertion slots and to get the corresponding acknowledgements and also for special commands like SYNC, CAP, BREAK. The FT-MAC 


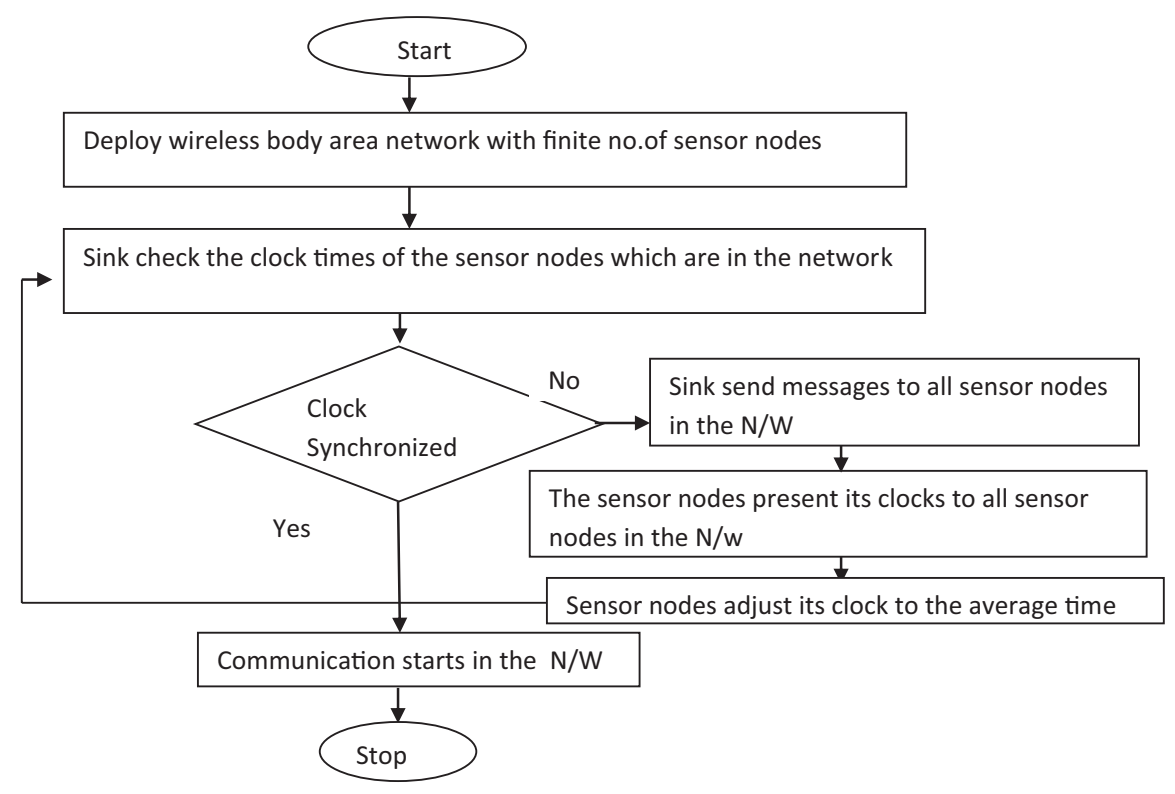

Figure 33.3 Flowchart for MAC protocol based on energy efficient TDMA.

adopts long super frames to reduce the energy consumption But, it is not applicable for heavy traffic applications of WBAN.

In [5] Bilandi et al. proposed a MAC protocol based on TDMA. This protocol avoids the performance degradation of the network due to packet collision because of the clock synchronized issues arising in the TDMA which lead to the packet loss. In this paper they had overcome this issue by setting the clock time in each sensor as the average time of all the sensors. After the clock synchronization the data can be transmitted efficiently. The flow of making nodes synchronized about the time is shown in the Figure 33.3.

In [6], Pallavi, Gupta, Bedi proposed an energy-efficient TDMA based MAC protocol in which there are three main phases. The Initial Phase is where Sink node broadcasts its location, which will be stored by all sensor nodes and they will send their ID, residual energy and location to the sink. Next stage is Selection of Forwarder Node, which involves in the calculation of Cost Function $(\mathrm{i})$ as distance ${ }_{(\mathrm{i})} /$ Residual Energy $_{(\mathrm{i})}$ to choose the forwarder node in each round. The last stage is Scheduling in which the forwarder node assigns TDMA schedule to children node to transfer their information in their allocated time slots. This method improves the throughput, decreases the path loss and saves the energy as the forwarder node was changed in each round. But, the nodes which are transmitting large amount of traffic will exhaust early.

In [7], Yuan et al. proposed an adaptive MAC protocol (A-MAC) based on IEEE 802.15.6. The assumptions of this protocol were

1. Priority allocation strategy: The three types of data in WBAN are emergency data, commands and control data having highest Priority $\mathrm{P}_{1}, \mathrm{P}_{2}$ for general periodical data; $\mathrm{P}_{3}$ as lowest priority for audio / video.

2. Superframe structure: The three access modes of IEEE 802.15.6 are beacon mode (superframe), non beacon mode with and without beacon period. The modification was done in beacon mode and that modified superframe structure is as in Figure 33.4. 


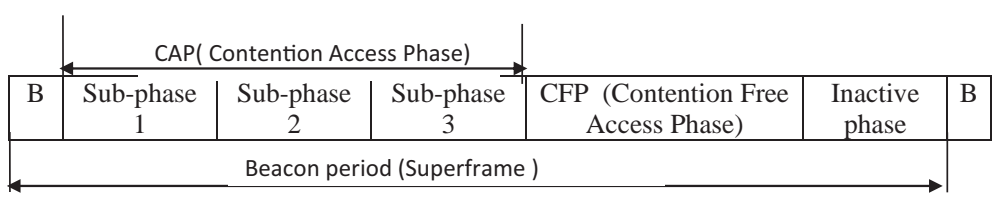

Figure 33.4 Superframe structure of beacon signal in A-MAC protocol.

CSMA/CA was used by the CAP nodes only. To reduce the probability of collision, there are three subphases in the CAP. Length of these subphases changes as $\mathrm{L}_{\mathrm{i}}=\mathrm{L}_{\mathrm{CAP}} *\left(\mathrm{~N}_{\mathrm{i}} / \mathrm{N}_{\mathrm{T}}\right)$ where, $\mathrm{L}_{\mathrm{i}}=$ length of subphase $(\mathrm{i}=1,2,3) ; \mathrm{L}_{\mathrm{CAP}}=\mathrm{L}_{\text {sum }}-\mathrm{L}_{\mathrm{CFP}} ; \mathrm{L}_{\mathrm{CFP}}=\mathrm{N}_{2}{ }^{*} \mathrm{~L}_{\text {sum }} /\left(\mathrm{N}_{1}+\mathrm{N}_{2}+\mathrm{N}_{3}\right)$.

$\mathrm{N}_{(1,2,3)}$ : Number of nodes for each priority; $\mathrm{N}_{\mathrm{T}}$ : Total number of nodes.

In order decrease the energy consumption and delay and to maximize the time slot's utilization all the subphase channels can be accessed by priority $\mathrm{P}_{1}$ service, the channels in the subphase 2, 3 can be accessed by $\mathrm{P}_{2}$ services and subphase 3 can be utilized by $\mathrm{P}_{3}$ services. The coordinator is responsible for time synchronization by sending the beacon signal. After that, random data from any node will be directed by the coordinator on confirmation of sending. For other type of data, a guaranteed time slot (GTS) will be assigned to the node by the coordinator and an ACK to the node after the successful transformation of the data, after that it enters into sleep until superframe ends. The IEEE 802.15.6 is only having two fixed access periods. The collision probability is very high as CSMA/CA is the only mechanism to access the channel which is also leading to network delays and time wastage. These issues were solved by this A-MAC protocol.

\section{Conclusion}

The implementation details of various MAC protocols for the applications of WBAN have been discussed here. Based on the literature survey, it is concluded that among various protocols, the adaptive MAC protocol which is based on priority of service and CSMA/ CA and GTS for the applications of WBAN should offer less delay, high reliability and should save the energy consumption for the longer life of the network. As in the example of elder people where they are more prone to abnormalities and should need some emergency medication and consultancy. Even for the patients who should have medical assistance immediately under serious conditions.

\section{Future scope}

Another major problem in the wireless technology is the signal fading under many dense structures even in homes. Therefore the delay occurs in the network as the signal gets lost by these obstacles. And also recharging of these sensors is a challenge. The energy harvesting techniques are still most reviewing topics.

\section{References}

[1] Akhtar, Rehmani, 2017, "Energy harvesting for self-sustainable for wireless body area networks", IEEE Computer Society (1520-9202).

[2] Ullah et al., 2017, "Medium Access Control (MAC) for Wireless Body Area Network (WBAN): Superframe structure, multiple access technique, taxonomy, and challenges". 
[3] Negra, Jemeli, Belghith, 2016, "Wireless Body Area Netwroks: Applications and technologies", The second International workshop on Recent Advances on Machine-to-Machine Communications, Procedia Computer Sciences 83 (1274-1281).

[4] Chong-Quing et al., 2017, “An energy-efficient MAC protocol for wireless body area networks”, ITM Web of Conferences 12, 03044.

[5] Bilandi, Verma, Dhir, 2018, "An energy-efficient TDMA based MAC protocol for wireless body area networks", First International Conference on Secure Cyber Computing and Communication (ICSCCC).

[6] Pallavi, Gupta, Bedi, 2018, "An improved energy-efficient TDMA based MAC protocol for WBAN", JCSSE International Journal of Computer Sciences and Engineering, Vol. 6, no. 3 (2347-2693).

[7] Yuan et al., 2019, "An adaptive MAC protocol based on IEEE802.15.6 for wireless body area networks", Hindawi Wireless Communication and Mobile Computing. 


\title{
34 A review paper on improving the network efficiency of IEEE 802.11e networks
}

\author{
Harpreet Bedi, Kamal Kumar, Gupta Raghav \\ School of Electronics and Electrical Engineering, LPU, Punjab, India
}

\section{Introduction}

A networking system is the backbone of any developing organization. In this paper different procedures were talked about to conquer the system. The network as per name defines has to collect and receive data from various types of applications and should provide more reliable enhanced features and updating in time to time. Thus to ensure all the must features QOS will be must for all users, as shown in Figure 34.1. To give QOS to such kind of usage, organization support is must.

\section{MAC layer}

This is the most important layer of data link layer which includes data transmission. It deals with and gives control to medium to access the data and transfer the data from sender side to receiver side. This system uses to access control mechanism and provide a full duplex arrangement in multipoint transmission. This channel or layer is a single or dual direction or can communicate with each other and other devices in the network.

\section{$D C F$}

DCF is the commonest method used in IEEE 802.11 which works on the CSMA. It operates on a transmission agenda with a first in first out algorithm. It reallocates and combines with MAC features and availability of the channel, if the channel is free or busy. If the channel is busy then MAC must ensure to wait for the time the channel is free and can then transmit the data. It keeps on checking the channel is free or not and if found busy, it sends an time interval DIFS. If the medium is still idle at the time of DIFS, then this layer converts the backoff counter, for every interval when the channel remains idle then the backoff counter range is decreased. If the service does not allow to use the medium in first phase, it will stop the backoff counter, look for the channel to free again for DIFS Frame and will enable to be repetitive. When the link changes the device will be accessible as shown in Figure 34.3. Each one must have to know their $\mathrm{CW}$ which is used to know the counter [12]. A signal will be sent by the receiver and will also be collecting the data. This message is sent over a SIFS which is smaller than DIFS. Immediately when SIFS is less than DIFS, the information is protected from other stations and is transmitted as shown in Figure 34.2. If the size of CW is less then the information will be lost. If the size will be double then backoff algorithm will be checked and analysed. All the features of the Mac will be checked and performed and depends upon bit frame and CW size. 


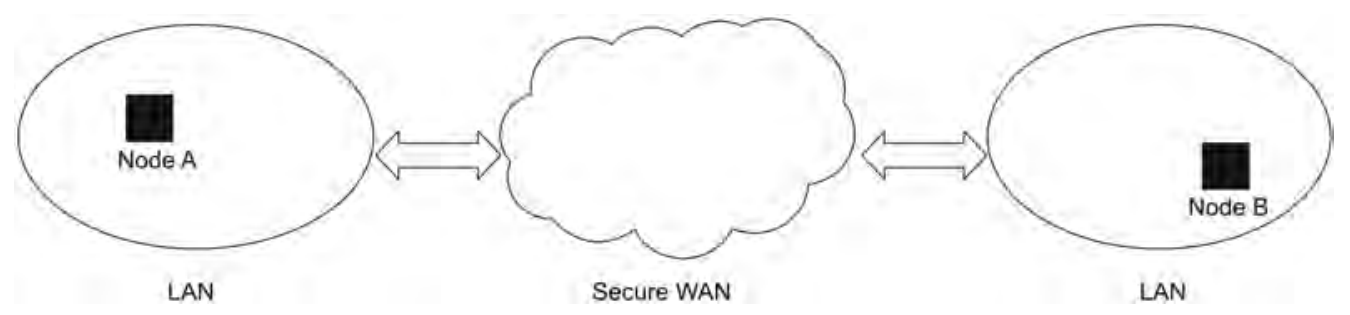

Figure 34.1 Partitioned network.

Immediate Access when medium is free >=

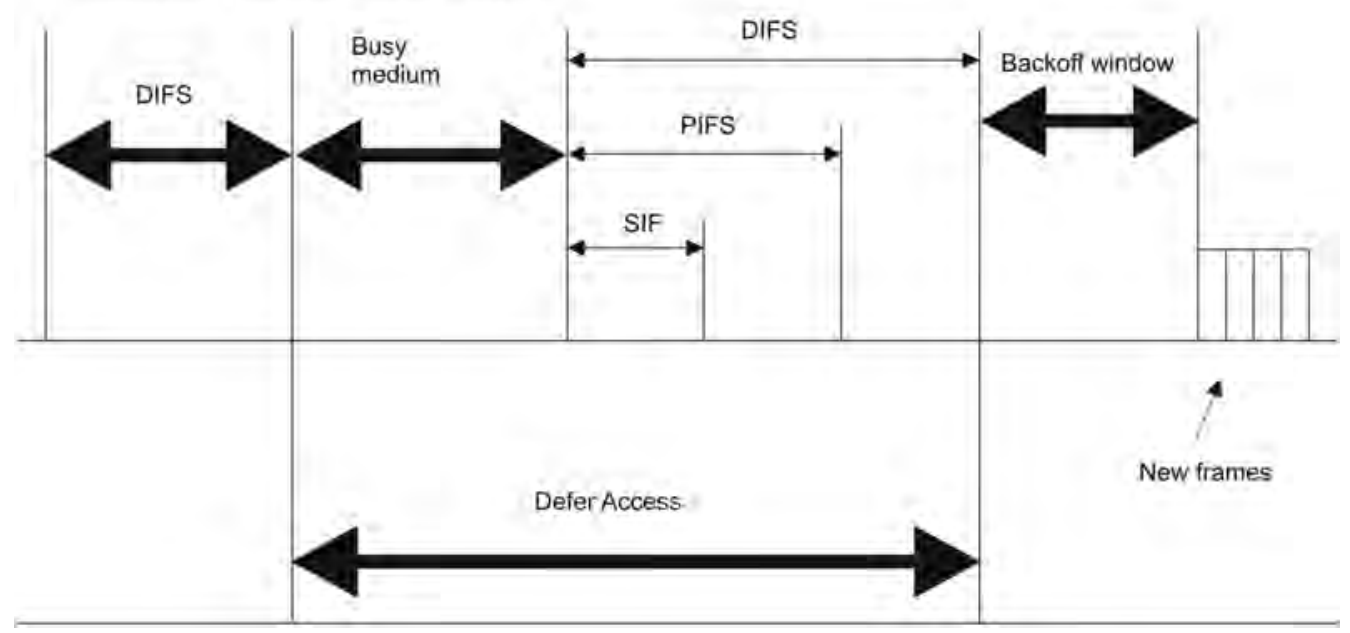

Figure 34.2 Timing relationship for DCF.

\section{EDCF}

EDCF is used to check and organized QOS with the help of increasing DCF. It helps in keeping separated and provide access to data transmission for QSTA which uses 8 stations to make changes in UP. Once it starts and reaches to MAC layer, each packet with a higher layer is provided with certain time and will be provided with a time range also for execution of the process. The system will use FIFO method to get access of access category that will help to send the information to the stations. This will be done from IEEE $802.1 \mathrm{~d}$ method. Various kinds of activities like video conferencing, on line mail and traffic will be transmitted into a respective Acs. For every AC, an improvised DCF will be sent which is EDCF. Diagram of EDCA is shown in Figure 34.3.

\section{Discussions and simulations}

In this article various simulator methods will be discussed and use the network simulator alongside Opnet and other analysis operating devices were used. The main purpose is to evaluate EDCA mechanism with the contention-based window whose size and variation 


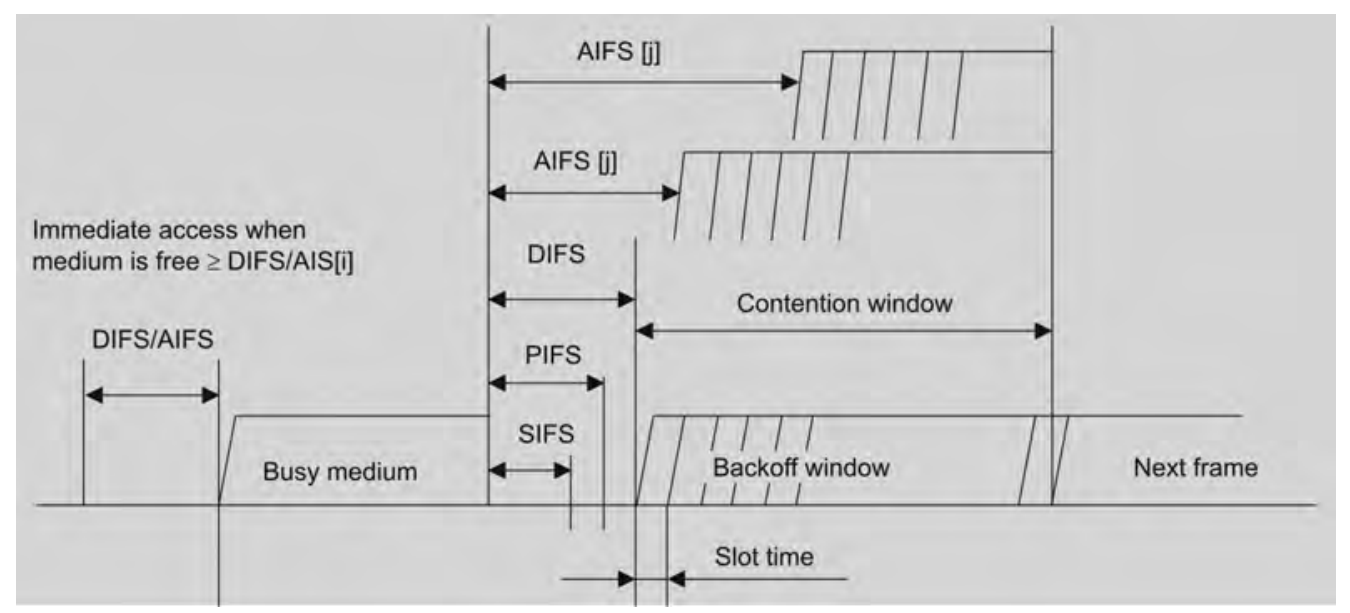

Figure 34.3 Timing relationship for EDCF.

Table 34.1 Simulation parameters

\begin{tabular}{lll}
\hline$A C$ & $C W \min$ & $C W \max$ \\
\hline 0 & 31 & 1023 \\
1 & 31 & 1023 \\
2 & $\left.\left(\mathrm{CW}_{\max }+\right) / 2-1\right)$ & $\mathrm{CW}_{\max }$ i.e. 15 \\
3 & Comes out to be $15^{\left.\left(\mathrm{CW}_{\max }+\right) / 4-1\right)}$ & $\left.\left(\mathrm{CW}_{\max }+\right) / 2-1\right)$ \\
& Comes out to be 7 & Comes out to be 15 \\
\hline
\end{tabular}

Table 34.2 Access category corresponding to an application

\begin{tabular}{lll}
\hline Access category & Applications configured & Destination \\
\hline AC $(0)$ & Videoconferencing & Excellent effort \\
AC $(1)$ & Online & Medium effort \\
AC $(2)$ & Back off & Poor \\
AC $(3)$ & Voip & Interactive media \\
\hline
\end{tabular}

varies with bit error rate and number of users in the channel or in medium. Table 34.1 shows various access categories with the size and variation of contention window.

\section{Analysis}

In the case of EDCF, the data has to travel into MAC layer from various layers which can be equivalent to different access categories, and will help to identify how to differentiate real time applications so that the access categories with highest priority have the capabilities to transmit and receive the data at a faster rate as compared to other access categories for various applications. Details are shown in Table 34.2. 
In one of the scenarios each station has different types of communications i.e. audio video and data to transfer to the same station. Bit rate for that application is $36 \mathrm{Mb} / \mathrm{s}$. The channel load was also discussed along with active station which was varying from 4 to 14. The EDCF can provide a comparison between the different access categories along with the different type of traffic i.e. high priority audio channel will be having highest throughput and less delay than video and data applications. If the channel and station are overloaded, there will be less delay with respect to audio channel as compared to other applications. The larger delays are only caused with respect to contention window size and variations for a high traffic network. This also demonstrates the various services provided by EDCF are effective and also explains that the quality of video and data applications will be degraded when the traffic is more and improved when the channel traffic is less as compared to other channels. The EDCA model explains the default values for contention window and its size varies for various applications for audio applications to video and data applications. The backoff algorithm and time for different applications were generated and cause fewer collisions between the video flows and lower data priority applications where we have many stations to transmit and receive the data. The graph shows the various analyses between the average packet delay and video throughput of the EDCF channel.

The graph explains the relation between throughput and bit error rate for different access category applications. This explains that both depend upon the data to be transmitted and are dependent on each other. BER is the important aspect to transmit the data and for wireless communications. Different 80.11e areas were discussed under different load channels which were critical to judge the performance of any wireless network.

\section{Conclusion}

In this article MAC layer and its most important protocols are discussed and a replica of EDCA is developed and covers all features of QOS [10]. From the analysis we find out that there is a stable throughput and that was compared with different existing models. The transmission of the data will be transmitted on the basis of prioritization of access categories of different media and then checked the overall system performance was much better than existing techniques.

\section{References}

1. T. Wark, P. Corke, P. Sikka, L. Klingbeil, Y. Guo, C. Rossman, P. Valencia, D. Swain, G. BishopHurley, Transforming agriculture through pervasive wireless sensor networks, IEEE Pervasive Computing 6 (2) (2007) 50-57, doi:10.1109/MPRV.2007.47.

2. D. Chen, P.K. Varshney, QoS support in wireless sensor networks: a survey, in: Proceedings of the 2004 International Conference on wireless Networks (ICWN 2004), Las Vegas, Nevada, USA, 2004, pp. 227-233.

3. K. Langendoen, G. Halkes, Energy-efficient medium access control, in R. Zurawski (Ed.), Embedded Systems Handbook, CRC Press, 2005 (Chapter 34).

4. O. Tsigkas, F.N. Pavudou, Providing QoS support at the distributed wireless MAC layer: a comprehensive study, IEEE Wireless Communications 15 (1) (2008) 22-31, doi:10.1109/ MWC.2008.4454701.

5. W. Pattara-Atikom, P. Krishnamurthy, S. Banerjee, Distributed mechanisms for quality of service in wireless LANs, IEEE Wireless Communications 10 (3) (2003) 26-34.

6. J.S. Vardakas, M.D. Logothetis, End-to-end delay analysis of the IEEE 802.11e with MMPP input-traffic, 2009 International Symposium on Autonomous Decentralized Systems, Athens, 2009, pp. 1-6. doi:10.1109/ISADS.2009.5207380. 


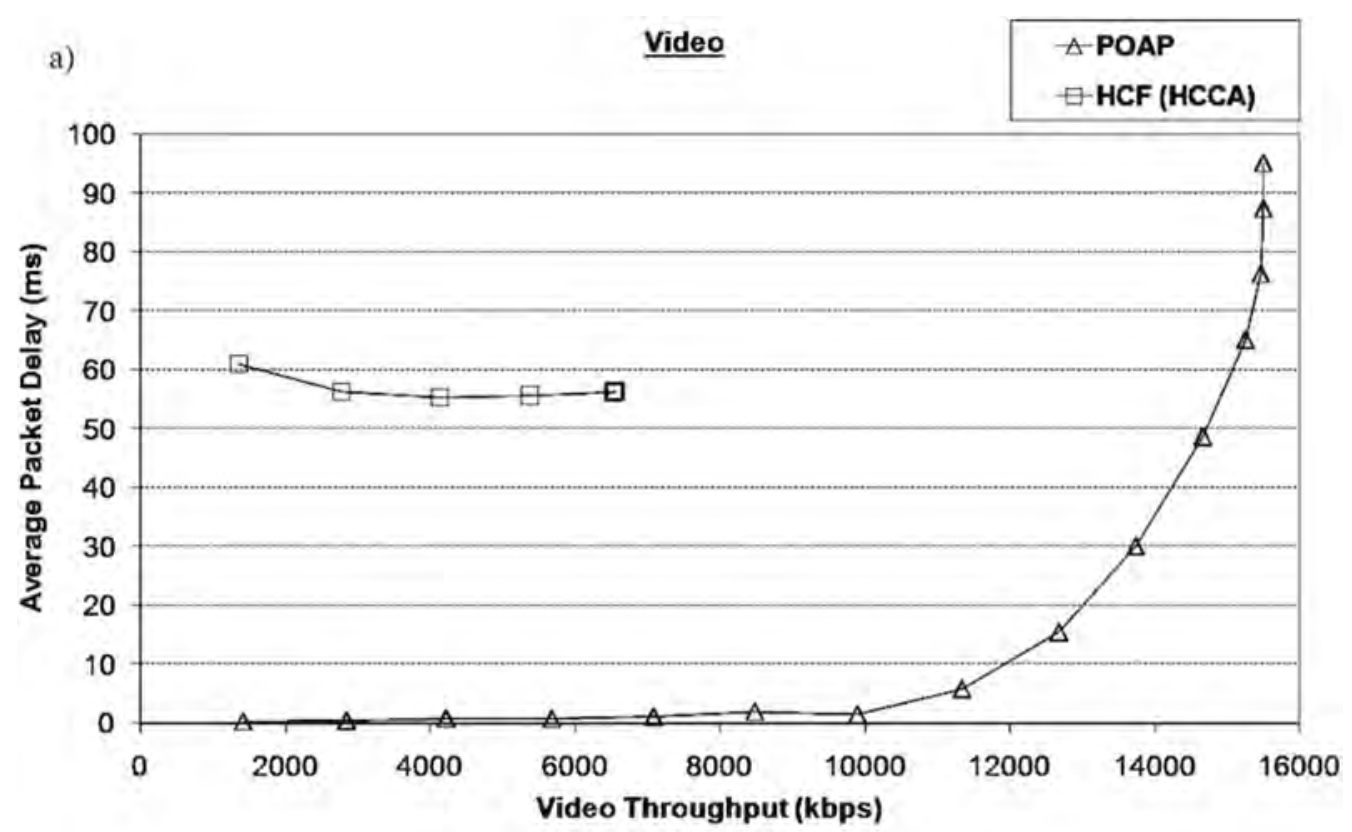
b)
$\underline{\text { Video }}$
$\triangle P O A P$
$\square$ HCF (HCCA)

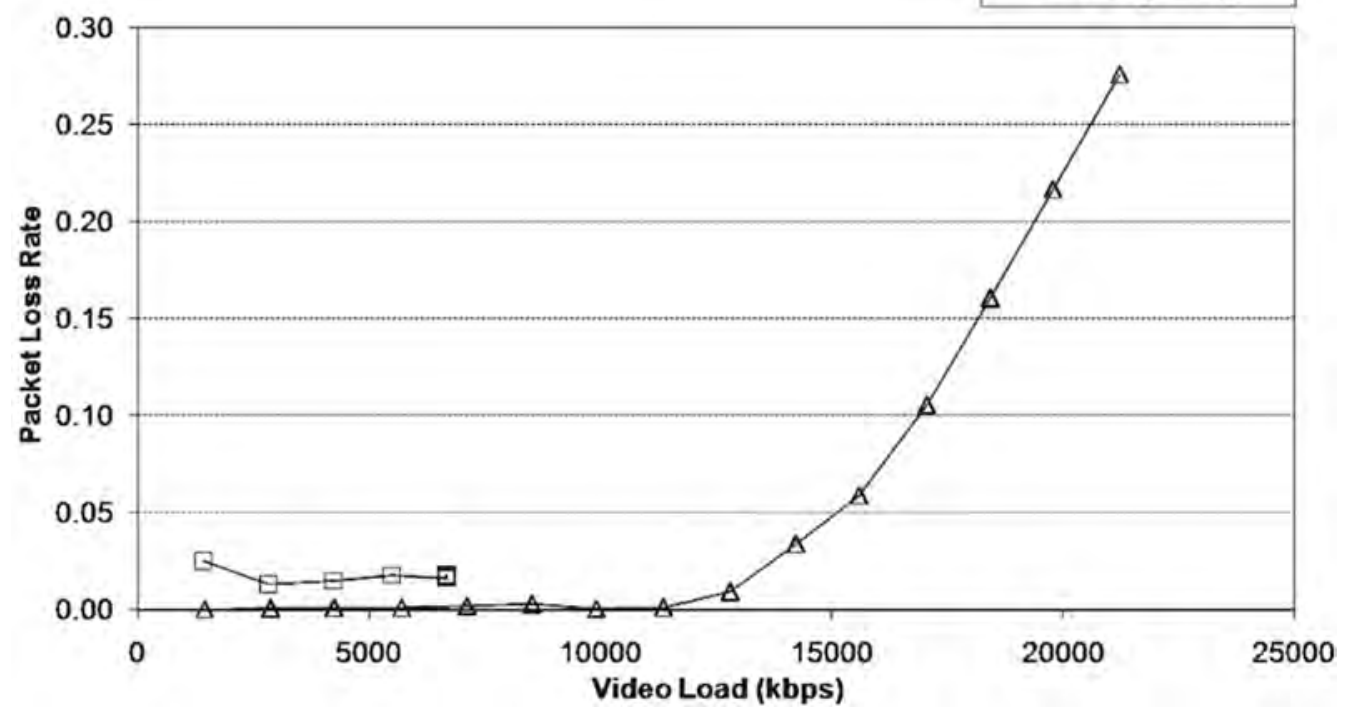

Graph 34.1 Relation between load and packets. 


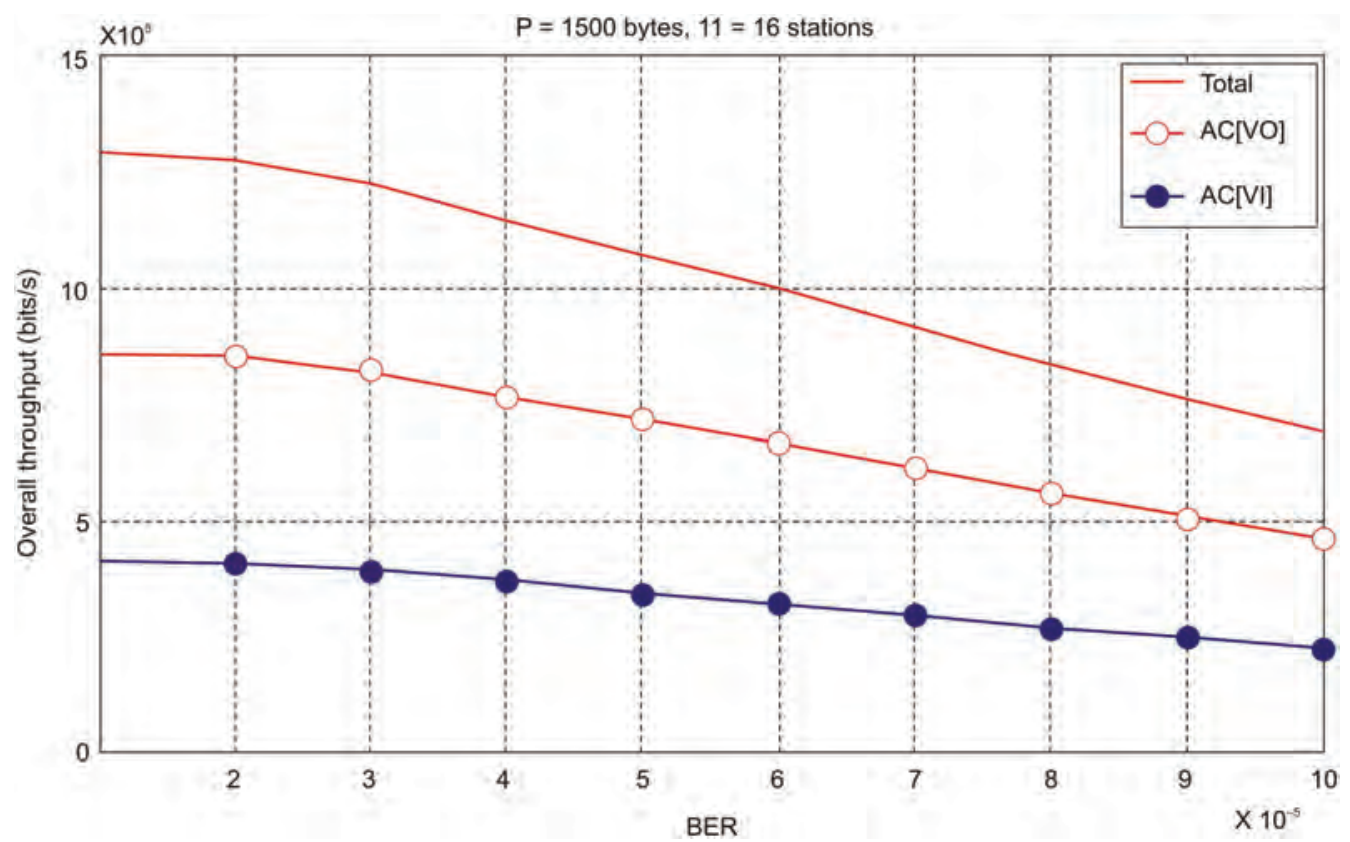

Graph 34.2 BER vs throughput.

7. Navdeep Singh Chauhan, Lehragaga Loveljeet Kaur, Implementation of QoS of different multimedia applications in WLAN, International Journal of Computer Applications 62 (8) (2013) 0975-8887.

8. Xin Liu, Wenjun Zeng, Throughput and delay analysis of the IEEE 802.15.3CSMAICA mechanism considering the suspending events in unsaturated traffic conditions. ieeexplore.ieee.org/ document $/ 5337030$.

9. Ikram Syed, Byeong-hee Roh, Delay analysis of IEEE 802.11e EDCA with enhanced QoS for delay sensitive applications (2016).

10. A. Jansang, A. Phonphoem, B. Paillassa, Analytical model for expected packet delay evaluation in IEEE 802.11e, 2009 WRI International Conference on Communications and Mobile Computing, Yunnan, 2009.

11. Shklyaeva A, Kubanek D, Analysis of IEEE 802.11e for delay sensitive traffic in wireless LANs in Proceedings of the Sixth International Conference on Networking (ICN'07).

12. M. Barry, A. T. Campbell, A. Veres, Distributed control algorithms for service differentiation in wireless packet networks, Proceedings IEEE INFOCOM 2001. Conference on Computer Communications. Twentieth Annual Joint Conference of the IEEE Computer and Communications Society (Cat. No.01CH37213), Anchorage, AK, USA, 2001.

13. A. Jiménez, J F. Botero, J P. Urrea, admission control implementation for QoS performance evaluation over SDWN, 2018 IEEE Colombian Conference on Communications and Computing (COLCOM), Medellin, 2018,

14. J. Y. Lee, H. S. Lee, A performance analysis model for IEEE 802.11e EDCA under saturation condition, in IEEE Transactions on Communications 57(1) (2009) 56-63.

15. Sunghyun, Del, Sai, Mangold S. IEEE 802.11e contention-based channel access (EDCF) performance evaluation, IEEE International Conference 2003.

16. Harpreet Bedi, A review paper on performance analysis of IEEE 802.11e, Proceedings of First International Conference on Computing, Communications, and Cyber Security (IC4S 2019)", Springer Science and Business Media LLC, 2020. 


\title{
35 Cluster head selection with dynamic clustering technique using mobility and stability parameters
}

\author{
Manoj Sindhwani*, Charanjeet Singh*
}

Department of Electronics and Electrical Engineering, Lovely Professional University, Phagwara, India

\section{Introduction}

Ad hoc is derived from a Latin word which signifies formed for. A network consisting of various independent devices sharing and exchanging information for communicating with each other resulting in formation of a multi hop radio network is termed an ad-hoc network. For communication, if the one party who is interested to communicate with the other party but they are far away from each other, needs an intermediary to exchange the information, for the purpose of which ad-hoc network came into existence. It implies that if the desired target node is distantly located and is unapproachable then the message is transmitted via other nodes.

An example of an ad-hoc network has been illustrated in Figure 35.1, where devices independent of each are considered among which communication is going to happen. The information cannot be directly sent to D from A, for the purpose of which $\mathrm{B}, \mathrm{C}$ are required through which $\mathrm{A}$ can communicate with $\mathrm{D}$. These nodes can be smart devices like PDAs, laptops, mobiles etc. On the bases of infrastructure, the two of the popular areas in ad-hoc networks are mobile ad-hoc network (MANETs) and vehicular ad-hoc networks (VANETs) [1,5].

In MANET the autonomous mobile users communicate over wireless links where nodes are mobile and the network topology is unpredictable. On the grounds of history, MANETs played a prominent role in battlefield communication but later the miniaturization offered in wireless communication led to its popular usage and made the way for major deployment of ad-hoc networks.

\subsection{Introduction to VANET}

Vehicular ad-hoc networks (VANET) is the sub-class of mobile ad-hoc networks (MANET). VANET can be considered as nodes on wheels. In this network the nodes have the capability to communicate with other nodes directly or indirectly. The main purpose behind the popularity of VANET $[2,4]$ is the safety and security of the vehicles and drivers, as the vehicle speed is increasing day by day with advanced technologies and smooth roads. VANET applications also include intelligent vehicles, reducing petrol consumption, entertainment etc.

\footnotetext{
*Emails: manoj.16133@lpu.co.in, charanjeet.21882@lpu.co.in
}

DOI: 10.1201/9781003129103-35 


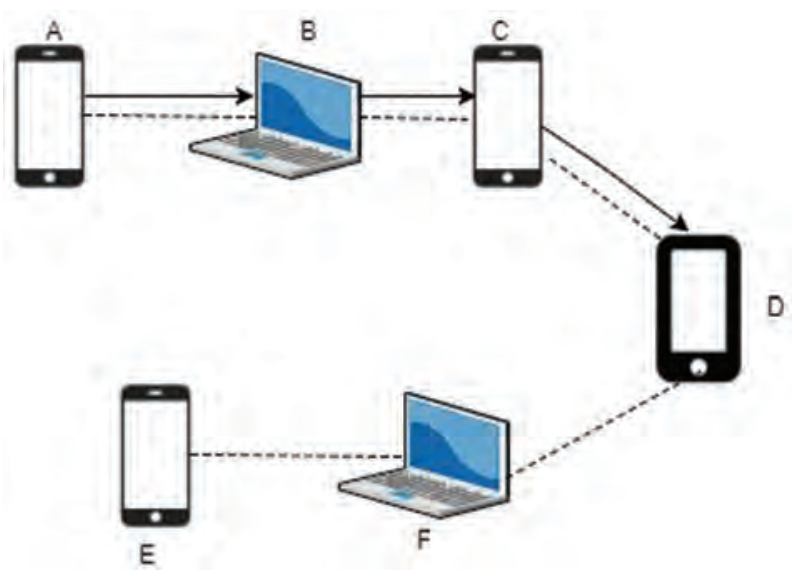

Figure 35.1 Ad-hoc network.

\subsection{Clustering in VANET}

Clustering is a control technique used in VANET to make frequent topology changes (because of more speed of vehicles) less dynamic. There are a number of clustering techniques [6,9] proposed by various researchers to ease the formation of clusters by applying different clustering algorithms

\section{System model}

In our system model, the clustering is on the basis of stability and mobility based clustering. We will be adopting the clustering algorithm shown in the figure for choosing a $\mathrm{CH}$ and the CM among the neighbouring nodes. It performs gateway node selection, cluster head selection and cluster member.

The number of hops to the cluster members from the cluster heads can be determined as single-hop or multi-hop where maximum of the clustering algorithms employ the singlehop clusters in which the distance between $\mathrm{CM}$ and $\mathrm{CH}$ is just one hop $[15,19]$ where it is easy to reduce or mitigate the issue of packet loss [12] where the vehicle is made aware of the one hop away vehicle information.

Neighbourhood discovery stands for the discovery of the nodes in a vehicular network to form the cluster. Referring to Figure 35.2, initially the willing node will enter the network and be a part of the vehicular network. Then the announcement of availability will be made by the broadcasting of periodic message being active or passive clustering. A database is prepared where the information regarding the neighbouring nodes is stored.

The general algorithm in VANET for clustering is shown in Figure 35.3. Cluster head selections chooses a leader among all. The fittest of all wins the race to be a leader termed as a cluster head. When all the data is gathered in the database then the $\mathrm{CH}$ is chosen on the basis of various parameters like mobility matrices, speed, velocity, relative distance etc. The cluster members are affiliated. Affiliation will be awarded to the $\mathrm{CM}$ and $\mathrm{CH}$. Announcement is done to know about the neighbours and affiliation process. Maintenance allows the maintenance process for the cluster heads $[13,17]$ as well as cluster members. 


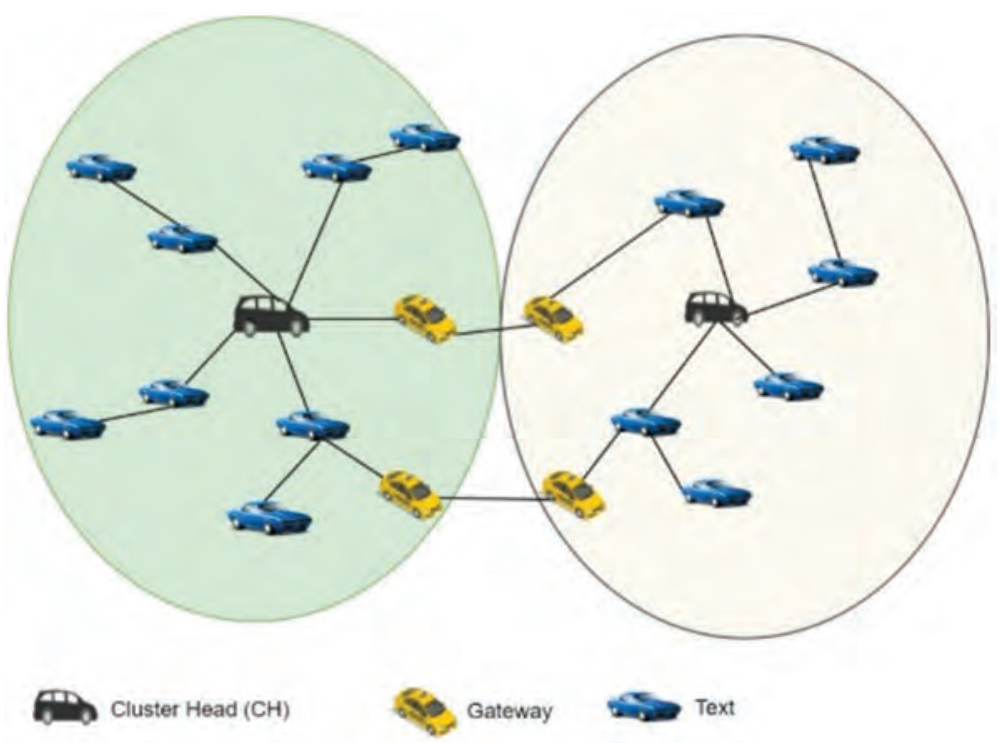

Figure 35.2 Clustering model.

It enables the cluster head to manage a cluster and merging of the cluster [14] and still maintains the network clusters. The nodes also periodically look for the links with the associated $\mathrm{CH}$. The strongest $\mathrm{CM}$ can be turned into a $\mathrm{CH}$ in case no $\mathrm{CH}$ is present. Some parameters have been considered prior to the implementation of the schemes which provides a platform for better outcome [20] and are necessary to be considered.

\section{Results and discussion}

As per the observations from vehicular ad-hoc networks, the less number of clusters are organized where the value of Dt is relatively kept high. In reference with the ETSC standards, the CAM messages are particularly broadcasted considering the frequency range between 1 and $10 \mathrm{nHz}$. The initial default value of beacon interval will be taken and set to $1.0 \mathrm{~s}$ and for future implementation and results it will be increased from a range of 0.5 to 2.0 or further in order to obtain ideal results in the simulation.

\section{Tools}

(i) SUMO, (ii) NS2, (iii) MATLAB

The simulation parameters are presented in Table 35.1.

The results showcased in Figure 35.4(a) represent the average cluster head duration with the total percentage of time. When the Dt increases, there can be observed a slight change in the cluster head duration and it maintains the stability.

The resulted showcased in Figure 35.4(b) represents the cluster member average duration where there is a significant increase in the size of the cluster. Also it is observable that Dt has a tiny impact on both the nodes whether it is cluster head or cluster member. Average CM duration is considered on the y-label, the cluster size on the x-axis, which specifies that the significant decrease is observed when the cluster member duration is 


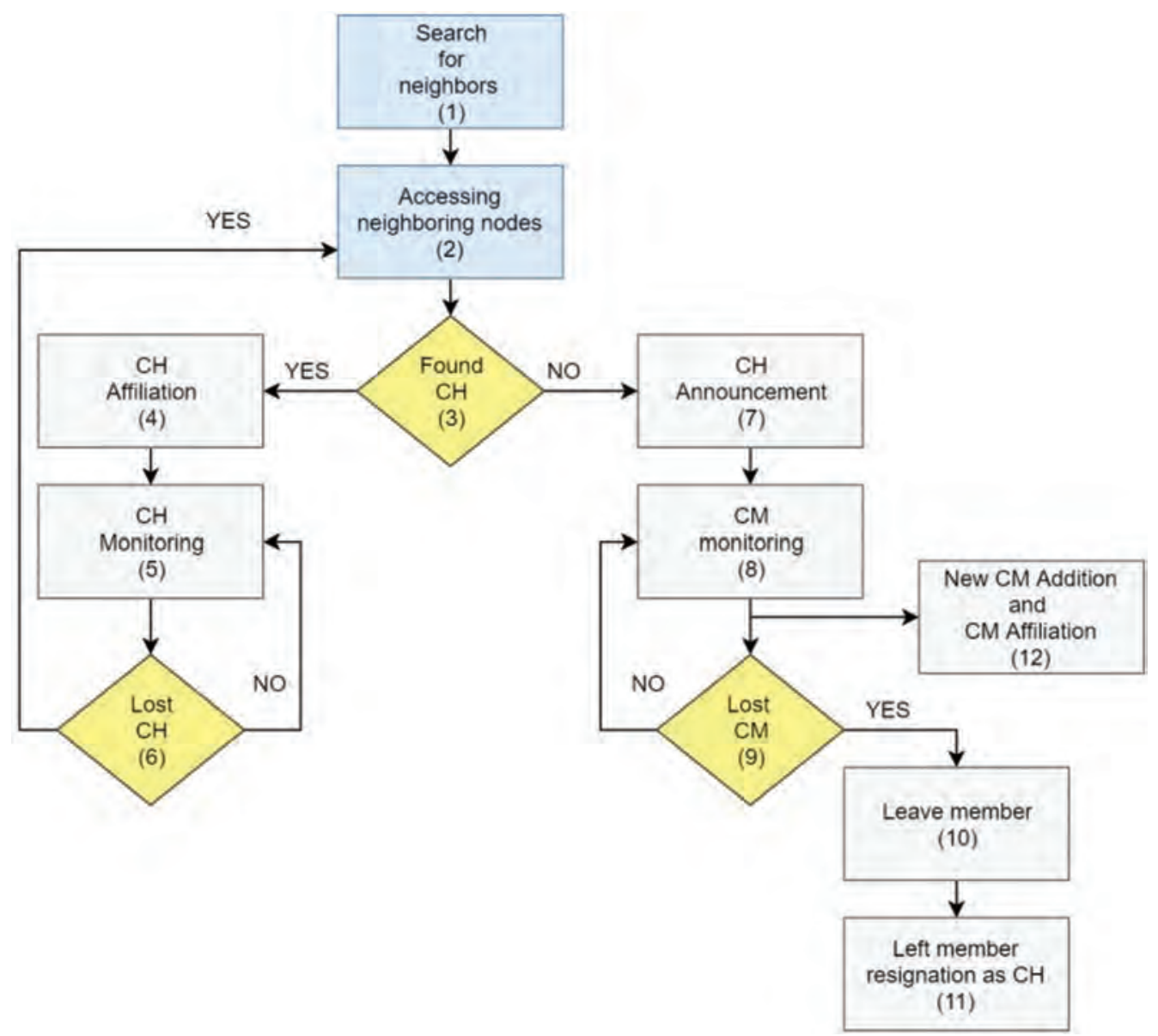

Figure 35.3 General algorithm in VANETS for clustering.

Table 35.1 Simulation parameters

\begin{tabular}{ll}
\hline Parameters & Values \\
\hline Simulation time & $300 \mathrm{~s}$ \\
MAC protocol & IEEE $802.11 \mathrm{p}$ \\
TR & $200 \mathrm{~m}$ \\
Number of vehicles & $100 \mathrm{~m}$ \\
Road length & $15 \mathrm{~km}$ \\
Car length & $5 \mathrm{~m}$ \\
Acceleration rate & $2.6 \mathrm{~m} / \mathrm{s}$ \\
Deceleration rate & $4.5 \mathrm{~m} / \mathrm{s}$ \\
Maximum lane speed (MLS) & $10-40 \mathrm{~m} / \mathrm{s}$ \\
Traffic flow rate & $1200 \mathrm{vehicles}$ per hour \\
$\mathrm{D}_{\mathrm{t}}$ & $100-200 \mathrm{~m}$ \\
$\mathrm{~B}_{\mathrm{I}}$ & $1.0 \mathrm{~s}$ \\
$\mathrm{M}_{\mathrm{I}}$ & $10.0 \mathrm{~s}$ \\
Tub & $5.0 \mathrm{~s}$ \\
Propagation model & Two-ray ground \\
Number of iterations & 10 \\
Mobility model & Car-following model \\
\hline
\end{tabular}



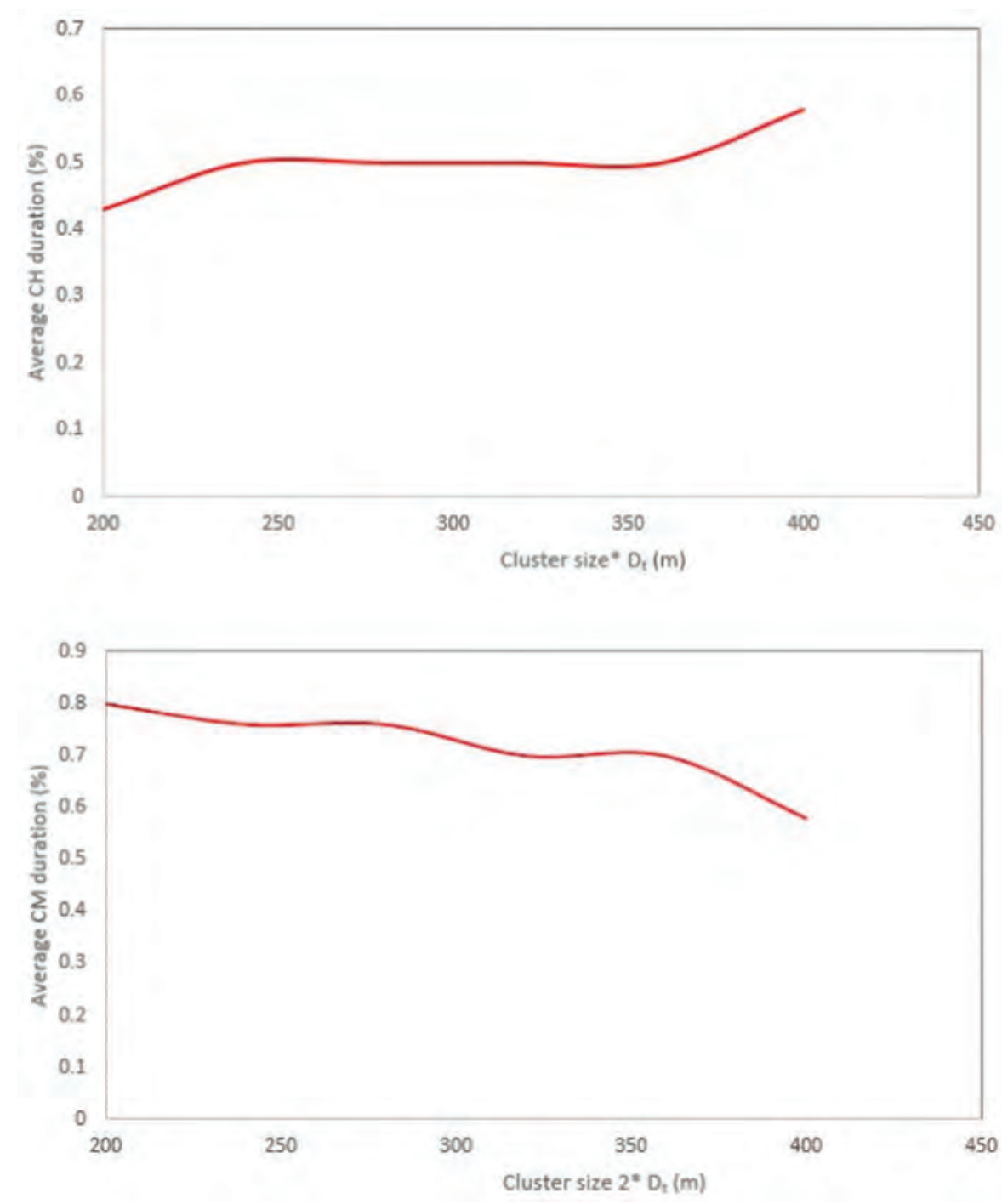

Figure 35.4 (a) Average $\mathrm{CH}$ duration, (b) average CM duration, (c) average number for UN for VMaSC.

increased. The number of clusters heads and the undecided nodes in comparison with the VMaSC are depicted in Figure 35.4(a) and (b). When the value of MLS is significantly made to rise up then the UN states of the cluster also change in VMaSC for only 1-hop between the CMs and the cluster head.

\section{Conclusion and future scope}

The work carried out to date has primarily focused on a technique formulated to implement dynamic clustering with $n$ number of hops while considering the two parameters of mobility and stability. The analysis describes the status of clustering algorithm. The 


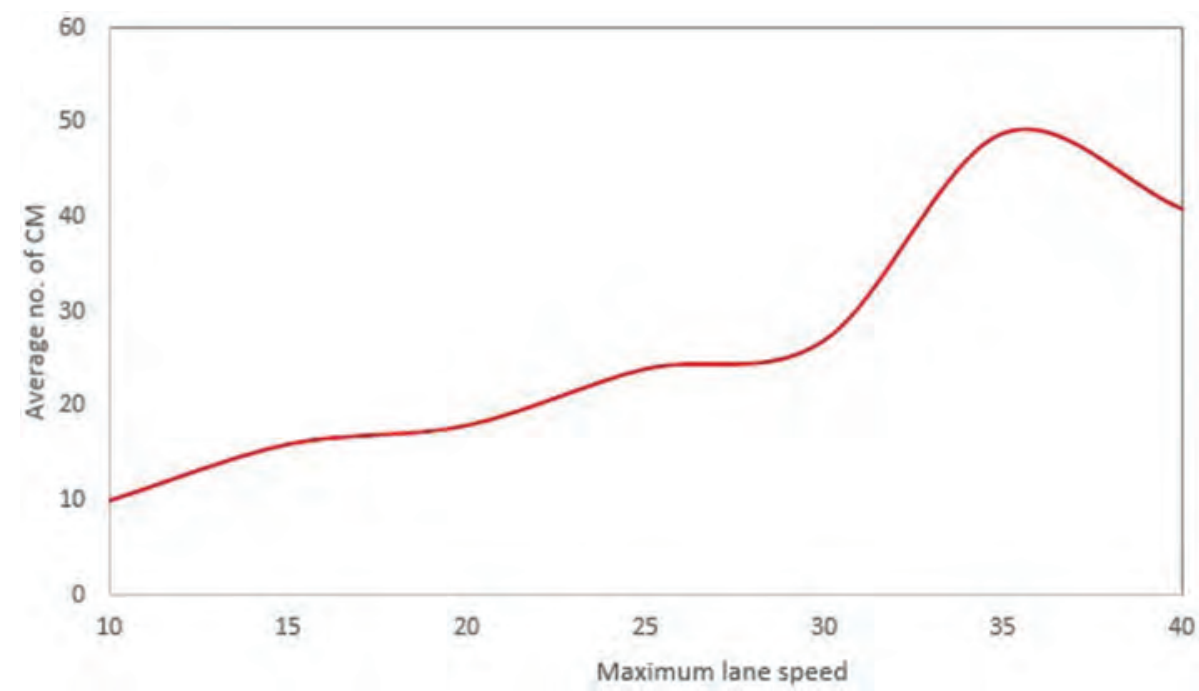

Figure 35.4 Continued

implemented clustering scheme has been compared with the VMaSC. The simulation tool is NS2 and the test scenario to be further considered is the highway scenario where traffic will also be generated with the help of SUMO (Simulation of Urban Mobility). The performance matrices calculated are average cluster head duration, average CM duration and the comparison of average number of Undecided nodes for VMaSC and 1-hop average number.

\section{Future scope}

(i) We can improve the stability of the cluster using dynamic clustering.

(ii) To use clustering for efficient $\mathrm{CH}$ selection and back-up cluster head.

(iii) To improve efficiency in network using increased hops.

\section{References}

[1] Rasmeet Singh Bali, Neeraj Kumar, Joel J. P. C Rodrigues, An intelligent clustering algorithm for VANETs. Connected Vehicles and Expo International Conference, October 2015.

[2] Weija Li, Xin Zhang, Zhongyi Shen, Meng Zhang, Dacheng Yang, Density based threshold algorithm in vehicular ad-hoc networks. IEEE International Conference on Communication Workshops (ICC), pp. 675-680, 2017.

[3] Weijia Li; Xin Zhang, Zhongyi Shen, Meng Zhang; Dacheng Yang, VANET: architectures, research issues, routing protocols, and its applications, International Conference on Computing, Communication and Automation, pp. 555-561, 2016.

[4] Saleha Mubarak AlMheiri, Hend Saeed AlQamzi, MANETs and VANETs clustering algorithms: a survey. Proceedings of the 8th IEEE GCC Conference and Exhibition, pp. 1-6, 2015.

[5] Meysam Azizian, Soumaya Cherkaoui, Abdel hakim Senhaji Hafi, A distributed D-hop cluster formation for VANET. IEEE Wireless Communications and Networking Conference, pp. 16, 2016.

[6] Jose Angel Leon Calvo, Rudolf Mathar, A two-level cooperative clustering scheme for vehicular communications. 6th International Conference on Information Communication and Management, pp. 205-201, 2016. 


\section{Intelligent Circuits and Systems}

[7] Rong Chai, XianleiGe, Qianbin Chen, Adaptive k-harmonic means clustering algorithm for VANETs, International Symposium on Communications and Information Technologies (ISCIT), pp. 233-237, 2014.

[8] Emna Daknou, Mariem Thaalbi, Nabil Tabbane, Clustering enhancement for VANETs in highway scenarios, pp. 1-5, 2015.

[9] Jyotsna Rao Dawande, Sanjay Silakari, Anjana Jayant Deen, Enhanced Distributed multi-hop clustering algorithm for VANETs based on neighborhood follow (EDMCNF) collaborated with Road Side Units. International Conference on Computational Intelligence and Communication Networks, pp. 106-113, 2015.

[10] Prabhjot Kaur Dhugga, Anshu Shanna, Meenakshi Sharma, An algorithm for static geographical clustering in VANET. International conference on MOOCs, pp. 420-426, 2015.

[11] Khalid Abdel Hafeez, Lian Zhao, Zaiyi Liao, Bobby Ngok-Wah Ma, A Fuzzy-logic-based cluster head selection algorithm in VANETs. International Conference on Communications, pp. 203-207, 2012.

[12] Pampapati Hubballi, A.V. Sutagundar, Ravikant Belagali, International Conference on Recent Trends in Electronics, Informational and Communication Technology, pp. 382-386, 2016.

[13] Chukwu Jeremiah, Agwu Joy Nneka, Issues and possibilities in vehicular ad-hoc networks (VANETs). International Conference on Computing Control, networking, Electronics, and Embedded System Engineering, pp. 254-259, 2015.

[14] Mengying Ren, Lyes Khoukhi, Houda Labiod, Jun Zhang, Veronique Veque, A new mobilitybased clustering algorithm for vehicular ad hoc networks (VANETs). Network Operations and Management Symposium, pp. 1203-1208, 2016.

[15] Giorgia V. Rossi, Zhong Fan, WoonHau Chin, Kin K. Leung, Stable clustering for ad-hoc vehicle networking. IEEE Wireless Communicationsand Networking Conference, pp. 16, 2017.

[16] Mickal Royer, Fabien Garcia, Alain Pirovano, An enhanced 1-hop clustering algorithm for publish / subscribe systems in AANETS. Digital Avionics Systems Conference, ENAC Toulouse, France, pp. 2D2-12D2-6, 2015.

[17] Ola Salman, Raghid Morcel, Obada Al Zoubi, Imad Elhajj, Ayman Kayssi, Ali Chehab, Analysis of topology based routing protocols for VANETs in different environments. International Multidisciplinary Conference On Engineering Technology, pp. 27-31, 2016.

[18] Hamayoun Shahwani, Toan Duc Bui, Jaehoon (Paul) Jeong, Jitae Shin, A stable clustering algorithm based on affinity propagation for VANETs. International Conference on Advanced Communication Technology, pp. 501-504, 2017.

[19] Peizhi Yang et al., Clustering algorithm in VANETs: a survey. International Conference on Anti-Counterfeiting And Security, pp. 166-170, 2015.

[20] Kai Zhang et al. Content aided clustering and cluster head selection algorithms in vehicular networks. Wireless Communication and networking Conference, pp. 1-6, 2017. 


\title{
36 Optimization of fire disaster management technique using fuzzy tools
}

\author{
Anil Kumar Rawat*, Kamal Kumar Sharma*, \\ Manwinder Singh*
}

Electronics and Communication Engineering, Lovely Professional

University, Phagwara, India

\section{Introduction}

Forest plays a significant role in maintaining the balance of nature as it includes diverse species of plants and animals. They have a major role in controlling the global warming. Apart from this, they have economic contribution to the country's wealth in terms of medicinal, food, wood and many more. From ancient times, the human race has relied on forests for food, shelter and clothes and therefore these forests were worshipped by the ancient people and hence taken due care of. Human negligence and ignorance have increased the incidences of forest fire apart from natural disaster. Therefore, there is an urgent need for a system which could keep an eye on the forest so as to effectively utilise its resources, take due care to maintain the ecosystem and eliminate or reduce the effect of natural or human triggered problems. They may include excessive mining, deforestation or fire. The global community is also concerned and has taken significant steps in protecting the environment.

To the best of our knowledge, utilizing the habitat including the creatures for fire/ smoke prediction is not done. Here the idea is that as soon as the danger of fire like smoke emerges, there is a rapid evacuation by the habitants of that ecosystem. As the aim is to protect the wild life including the habitat, the whole process is interlinked. Therefore, information of the location in terms of density and health of the habitat along with its environment are the key factors in monitoring the health of the ecosystem beyond fire.

WBAN has emerged as a possible solution to the problem of remote monitoring. It is a collection of intelligent body sensing nodes, placed in, on or around the target body thereby monitoring physiological parameters of individual as well its environment. The sensor nodes placed on the body are resource-constrained in terms of energy consumption rate, mobility, interference, and resource availability. Out of many applications like re-habitation, assisted living, sports, one such application of WBAN is health monitoring of the wild life ecosystem extending to wireless sensor networks wherein it is possible to remotely monitor the subject and its surrounding environment as well as for emergency situations like fire or other calamity. Figure 36.1 shows WBAN architecture.

The paper [1] discusses the evolutionary techniques in cognitive networks. Energy saving and efficient protocols are discussed in [2,3]. Role of big data, IoT and deep

\footnotetext{
* Emails: er.anilrawat1@gmail.com, kamalsharma111@gmail.com, singh.manwinder@gmail.com
} 


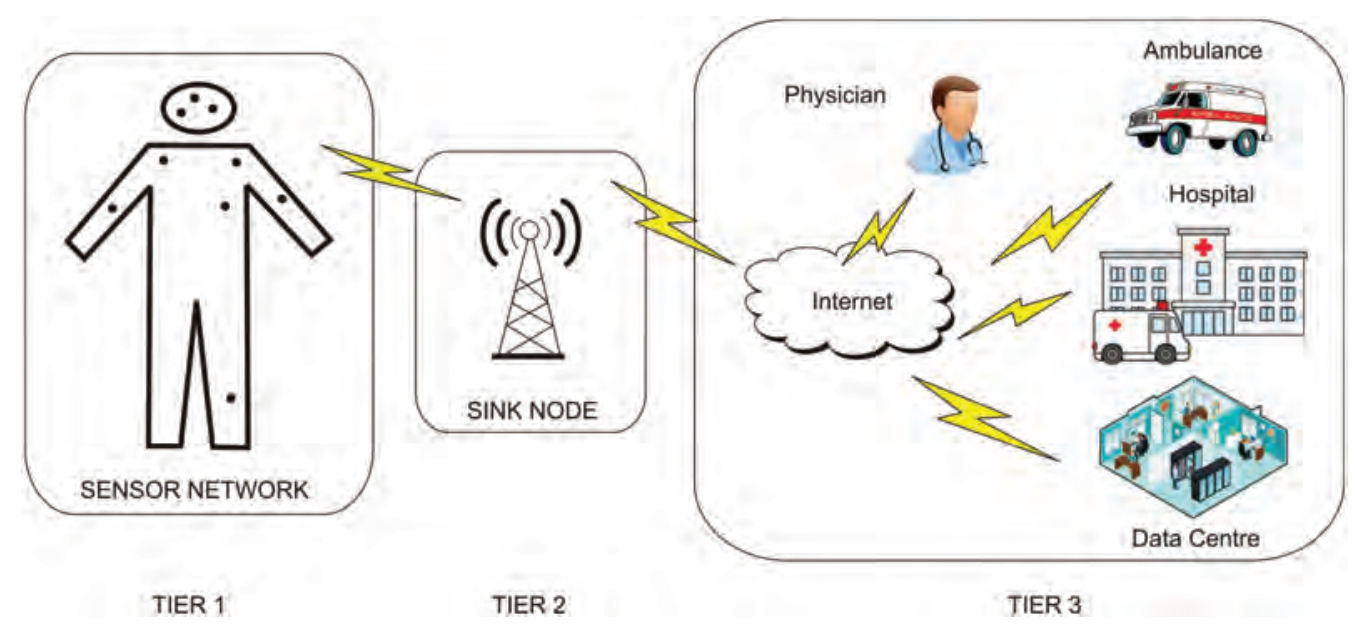

Figure 36.1 WBAN architecture.

learning in WBAN has considerable future. Privacy concerns related to WBAN technology are discussed in [4] and security concerns are thoroughly addressed in [5,6].

Health concerns related to in body implants were discussed in [7]. Switching and transmission concepts were discussed in [8,9]. Improving the life time of the network using multi-hop techniques is discussed in [10]. Thermal aware routing is discussed in [11]. Attacks in WBAN are discussed in [12,13]. WBAN authentication protocols used are elaborated and discussed in $[13,14]$.

A fire detection mechanism using only temperature as the parameter in WSN is discussed in [15]. To ensure whether the temperature rise is due to the natural process or a fire may not be distinguished. Another work related to fire identification in forest using artificial intelligence by testing and training is covered in [16]. A demonstration of the same is given highlighting promising results. A significant approach in a resource constraint or remote environment is discussed in [17], which includes the WBAN and a cloud network for providing the connectivity and decision-making possibility. It indicates post-fire measures to control the fire by studying its flow pattern.

In [18], a simulation based testing and training for the firefighters is discussed so as to effectively control the fire in risky areas. It is to ensure a co-ordination between the command centre through communication channels. Paper [19] presents an ethical view of the interaction of the human and the ecology and attempts at reducing the risk of damage unknowingly or through lack of awareness. A significant contribution in framing the prevention, detection and post-fire scenario is in [20]. A fuzzy based decision-making using pollutant gas and oxygen level has been discussed. A comparative analysis of the fire detection techniques in Canada and Korea is discussed in [21] addressing the accuracy, energy efficiency, hardware requirement and complexity. By studying the weather conditions effectively, a safe time can be calculated wherein restricted fire can be triggered to protect the place from uncontrolled and threatening conditions. As indicated in [26], an increase in $12.5 \%$ of wind speed can lead to $25 \%$ of potential fire burns in the region. Hence a prescribed burning is recommended in a safe manner in the suitable days. This can be predicted effectively by considering other factors like humidity, temperature, wind speed and the combustion fuel (forest wood and vegetation) 


\section{Proposed work}

The FWI system was developed in Canada, and consists of three moisture codes and three fire behaviour indices. The moisture codes capture moisture content of three generalized fuel classes and the behaviour indices reflect the spread rate, fuel consumption and intensity of a fire if it were to start [23,24],

Paper [25] considers only three parameters vis, temperature, humidity and time of the day. However, factors like wind speed have a key role in effectively coming to a better prediction mechanism. In this work, temperature, humidity and wind speed are used as the significant factors. The member function defined in the fuzzy logic will have three variables namely low $(\mathrm{L})$, medium $(\mathrm{M})$ and high $(\mathrm{H})$. The output parameter will be the chance of fire.

The possibilities of three parameters with three variables will be 27 . However, in the case of low temperature, the chances of fire are low irrespective of other factors. Hence those factors are not considered in fuzzy logic. The trends can be tabulated as in Table 36.1.

\section{Result analysis}

The input and output membership functions are defined in Figures 36.2, 36.3, 36.4 and 36.5 , respectively. Output effects due to the variation in input variables are shown

Table 36.1a Parameter range

\begin{tabular}{lll}
\hline Parameter & Range & Level \\
\hline Temperature & $27-1300^{\circ} \mathrm{C}$ & Low (L), Medium (M), High (H) \\
Humidity & $0-100 \%$ & Low (L), Medium (M), High (H) \\
Wind speed & $0-20 \mathrm{~km} / \mathrm{h}$ & Low (L), Medium (M), High (H) \\
\hline
\end{tabular}

Table $36.1 b$ Fire detection using fuzzy logic

\begin{tabular}{llll}
\hline Temp1 & Humidity & Wind speed & Fire alert \% \\
\hline High & High & High & Medium \\
High & Medium & Medium & High \\
High & Medium & High & Medium \\
High & Low & Low & High \\
High & High & Medium & Low \\
High & Low & High & High \\
High & High & Low & Medium \\
High & Low & Medium & High \\
High & Medium & Low & Medium \\
Medium & Low & Medium & Medium \\
Medium & Medium & Low & Low \\
Medium & Medium & Medium & Medium \\
Medium & High & High & Low \\
Medium & High & Medium & Medium \\
Medium & Medium & High & Low \\
Medium & Low & Low & Medium \\
Medium & High & Low & Low \\
Medium & Low & High & High \\
\hline
\end{tabular}




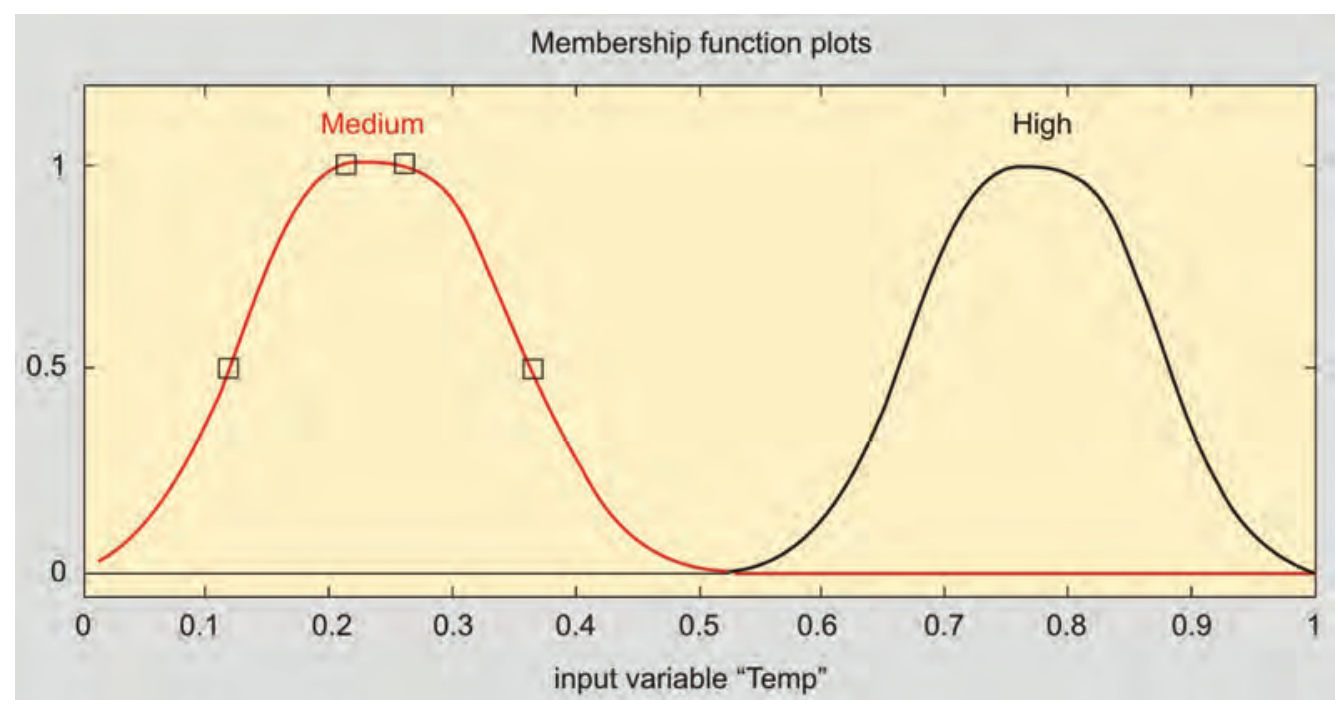

Figure 36.2 Membership function (temperature).

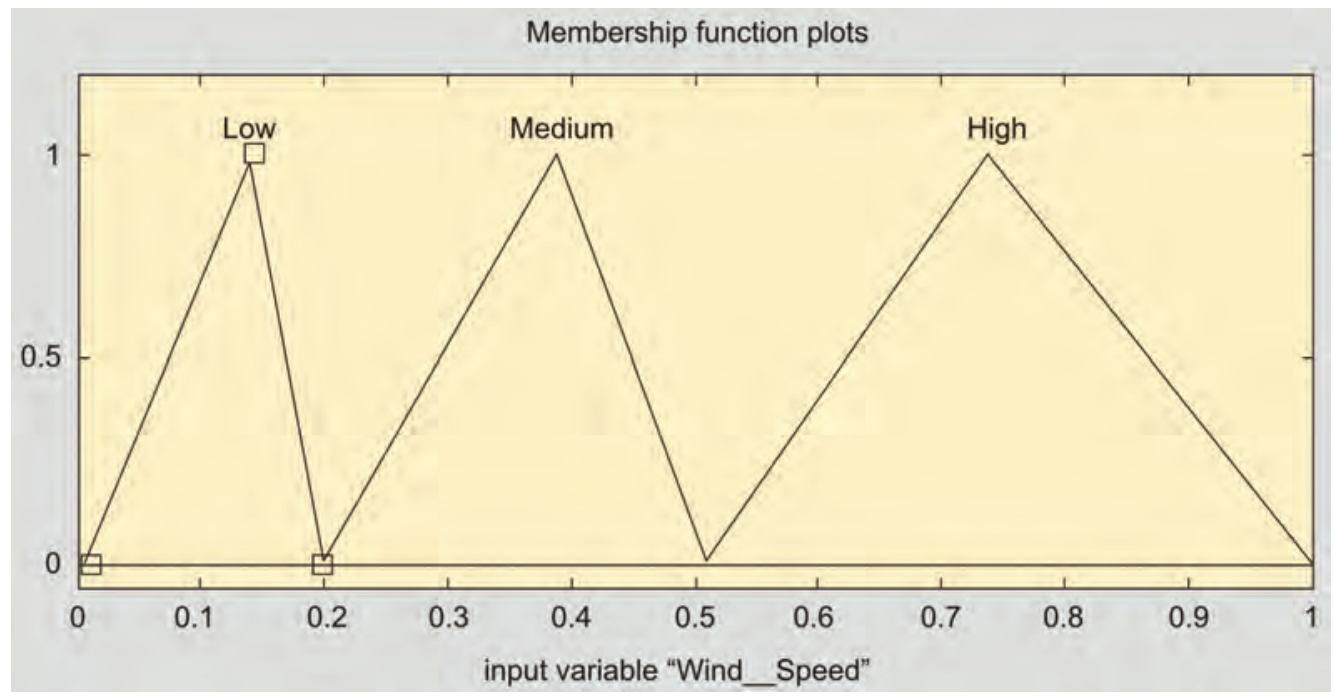

Figure 36.3 Membership function.

in Figure 36.6. The wind speed vs humidity graph (Figure 36.7) indicates that low humidity and high wind speed will result in significant fire alert indications. The wind speed and temperature graph (Figure 36.8) indicates high possibility of fire alert at high temperature and low wind speed. Its impact at high wind speed shows medium fire alert. Temperature controls the environment from being hostile resulting in fire as evident from Figure 36.9. Temperature vs humidity gives typically predictable graph indicating high temperate with low humidity to be a case of severe fire threat. High 


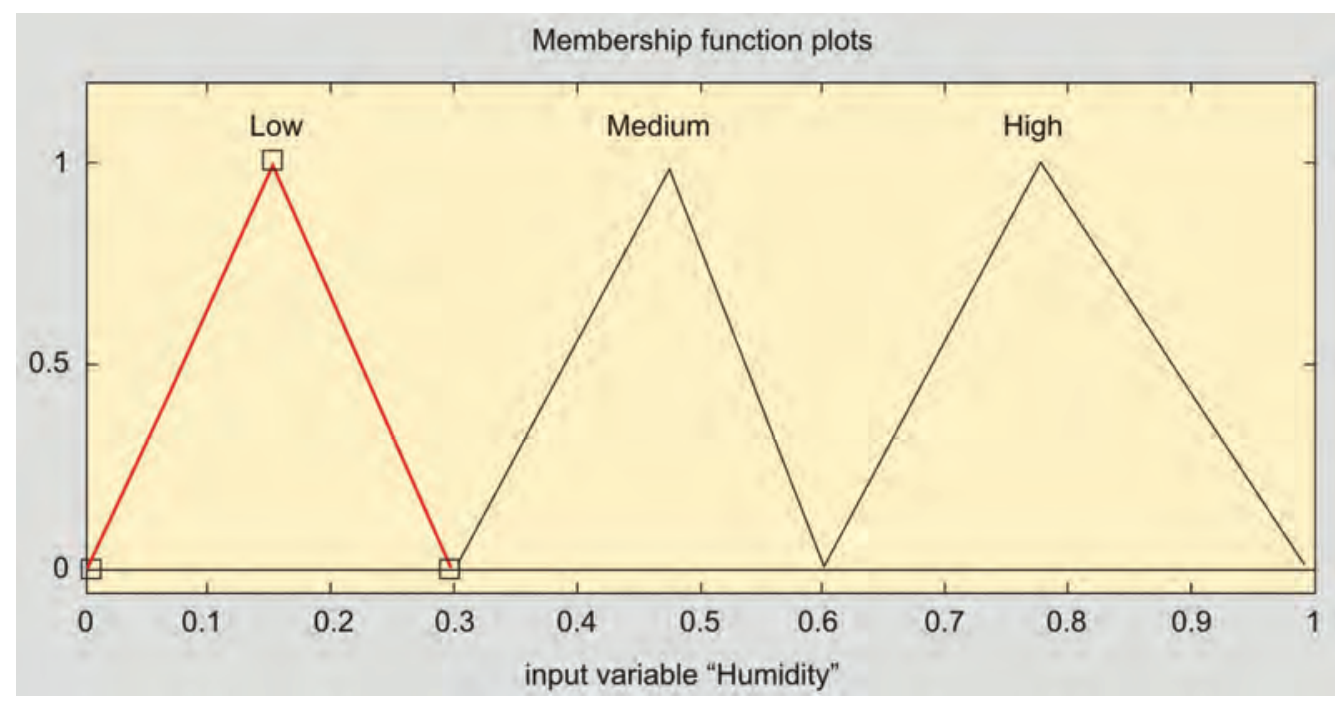

Figure 36.4 Membership function (wind speed).

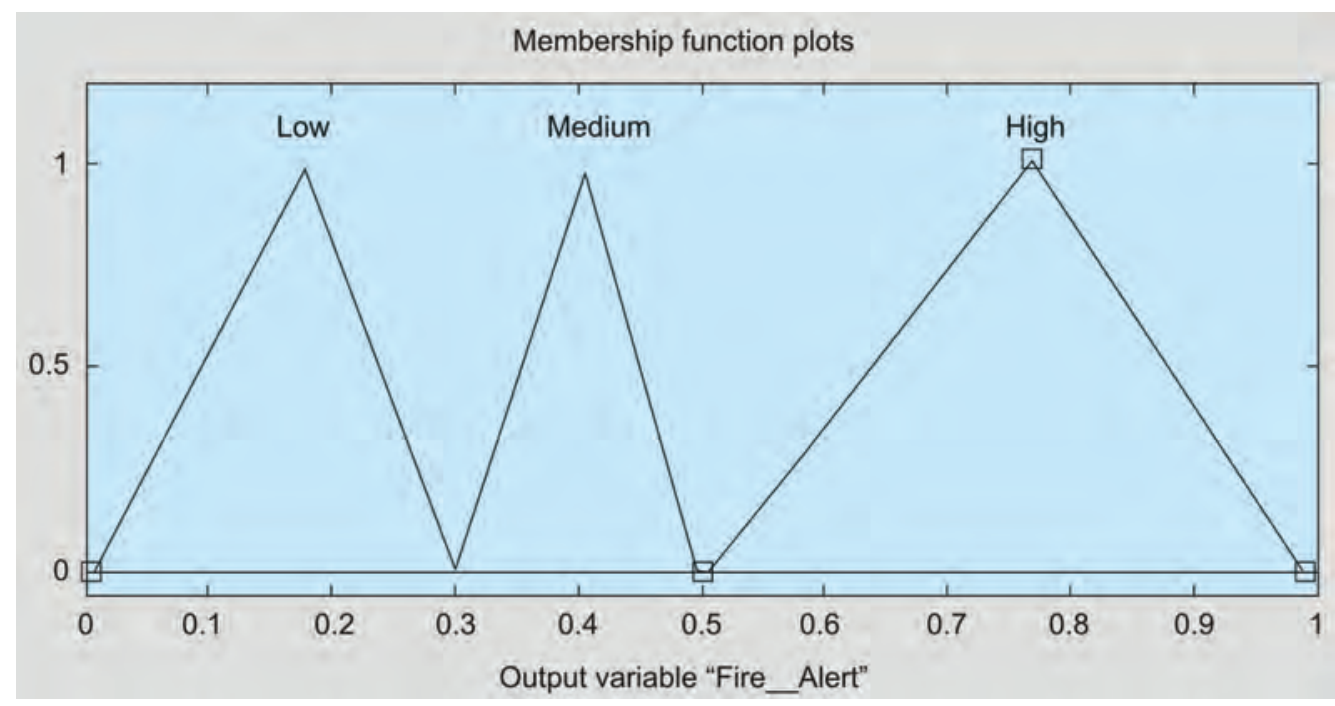

Figure 36.5 Membership function (fire alerts).

humidity will significantly reduce the impact of wind speed in fire scenario predictions (Figure 36.10). Combination of wind speed and temperature (Figure 36.11) and with the fire alert (Figure 36.12) indicates a certain combination of the two is required for the fire predictions. However, at medium humidity the fire alert is medium. This window can be used by the fire department for controlled fire scenarios for reducing the fuel and hence eliminating the chance of a fire. 

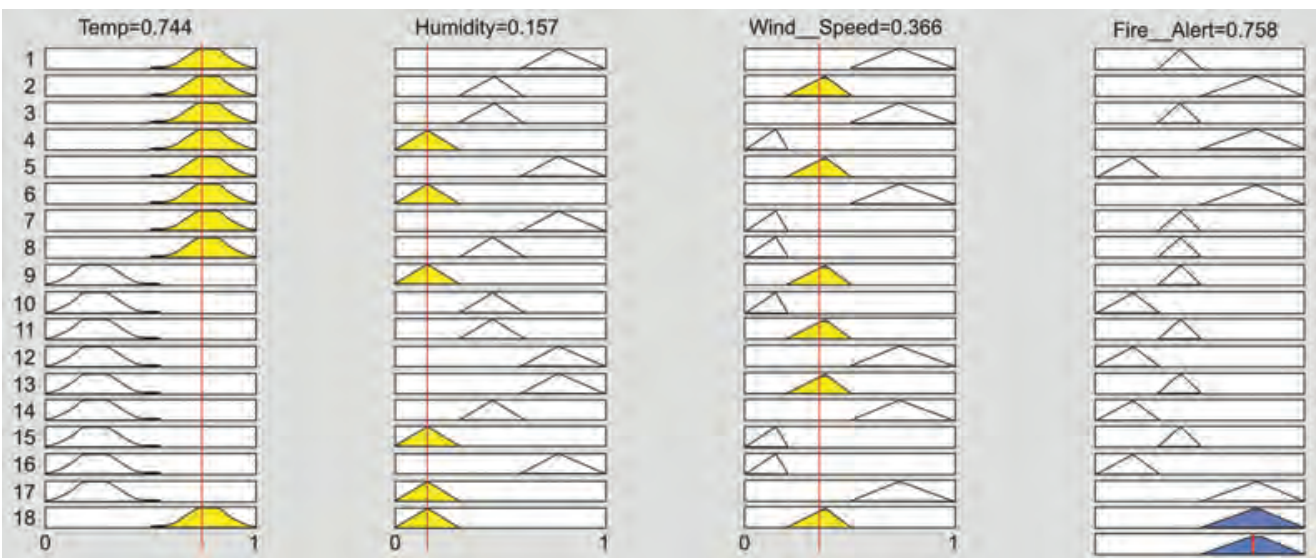

Figure 36.6 Output of corresponding inputs.

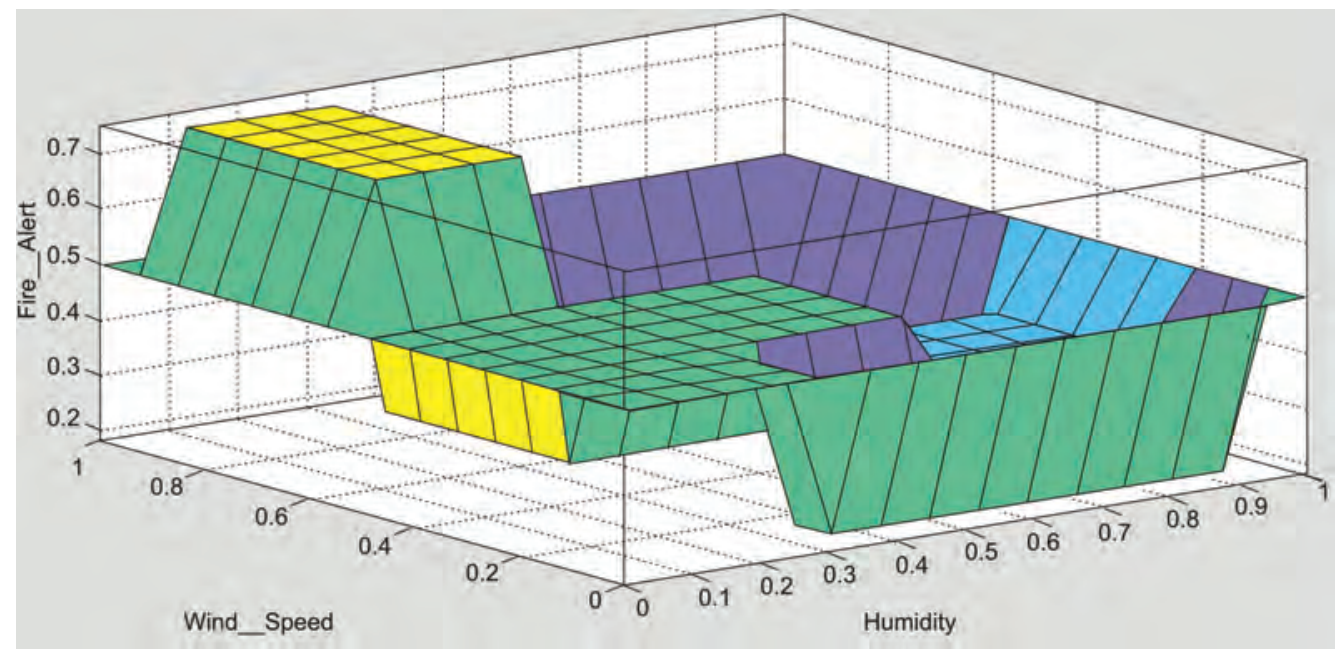

Figure 36.7 Wind speed vs humidity (fire alert).

\section{Challenges in WBAN and fire detection}

Security threats in WBAN exits at three levels, viz. data collection, transmission level and storage level [27]. Attacks at collection level include jamming attacks, data collision, data flooding, desynchronization, spoofing attack, selective forwarding and sybil attacks. At the transmission level, eavesdropping, man in the middle, data tampering, scrambling, signalling, unfair allocation, message modification, hello flood, data interception and wormhole attack. Finally, at the storage level, data inference, unauthorized access, malware or social engineering attacks.

Sensors are connected to the different parts of the body signifying varied information ranging from EEG, ECG, temperature, blood pressure etc. Each sensor belongs to different data types with varied data storage, format, different rates resulting in diverse 


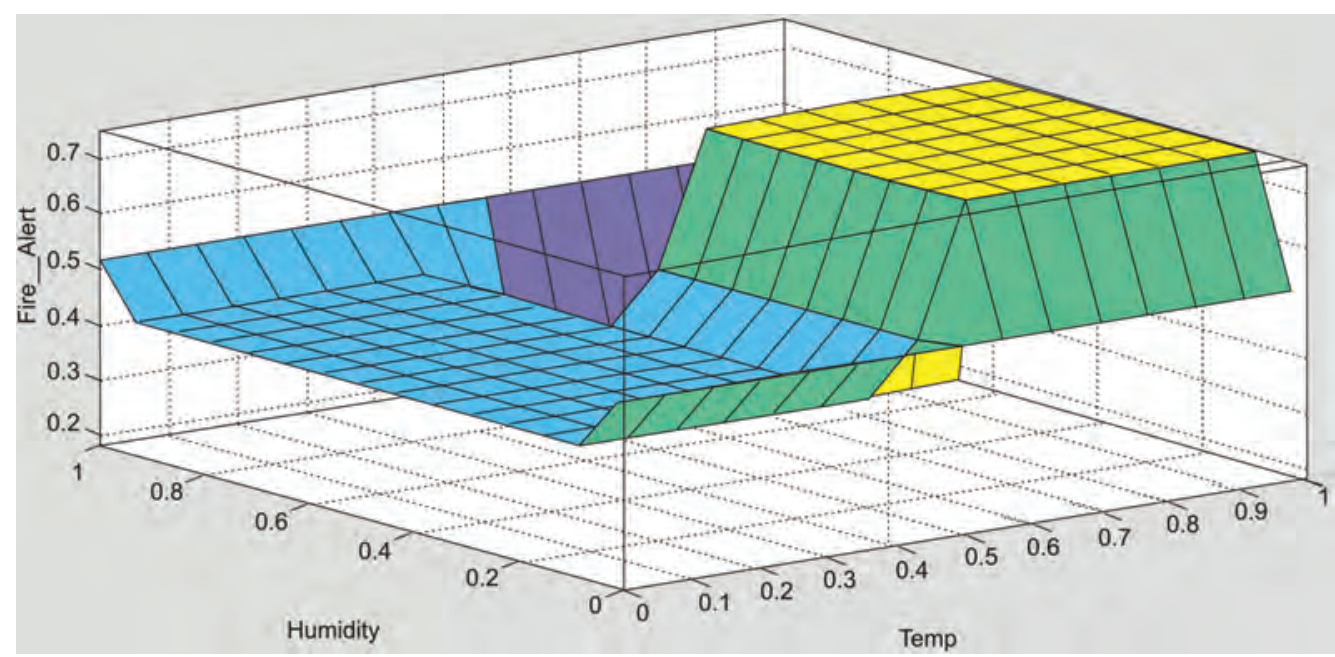

Figure 36.8 Temperature vs fire alert.

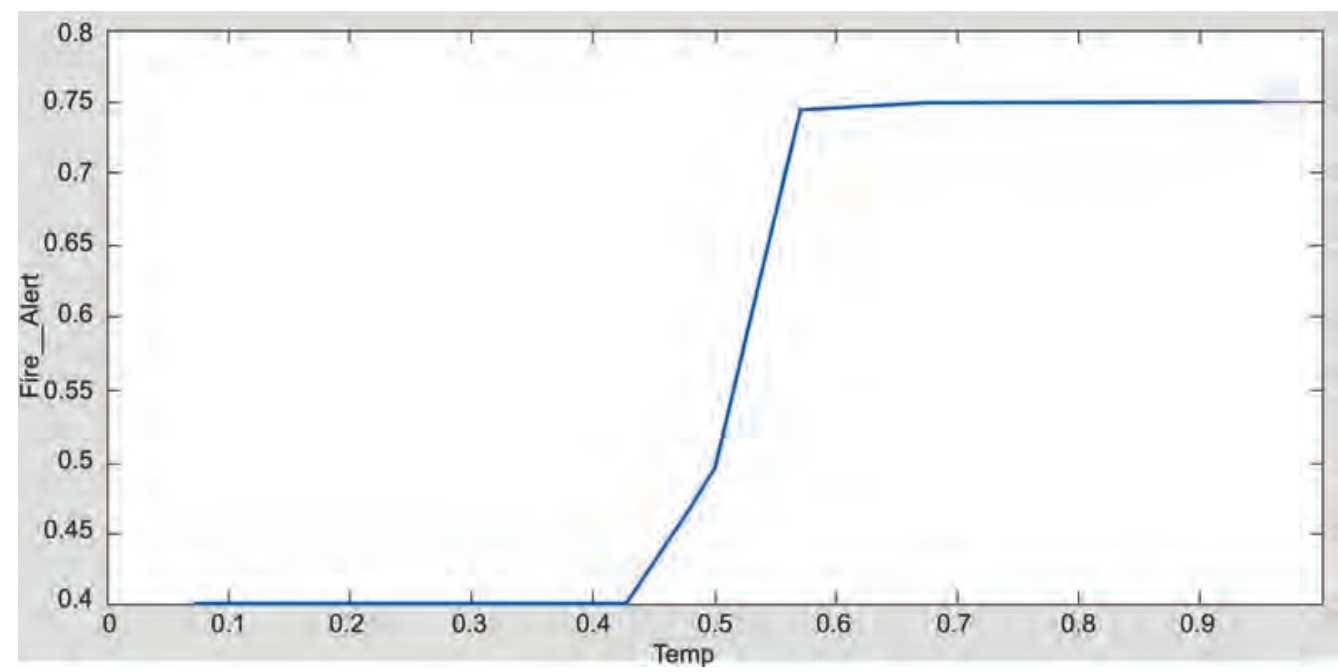

Figure 36.9 Wind speed vs temperature (fire alert).

QoS constraints and designing models with multiple data delivery [28]. Simultaneous requirement of multiple sensor data for monitoring.

Real time data collection and transmission is vital for emergency conditions, posing a challenge for remote healthcare monitoring. Delay of transmission has to be kept within the stipulated time to handle emergency scenarios.

Energy consumption poses a major constraint in WBANs. For example, in implantable sensing devices like pace-makers, frequently replacing the power source is not possible. Replacement of batteries frequently because of high energy consumption may pose a threat to the patient and adds to the inconvenience, limiting its usage. The QoS routing should be energy-aware and consume energy efficiently to meet delivery requirements. 


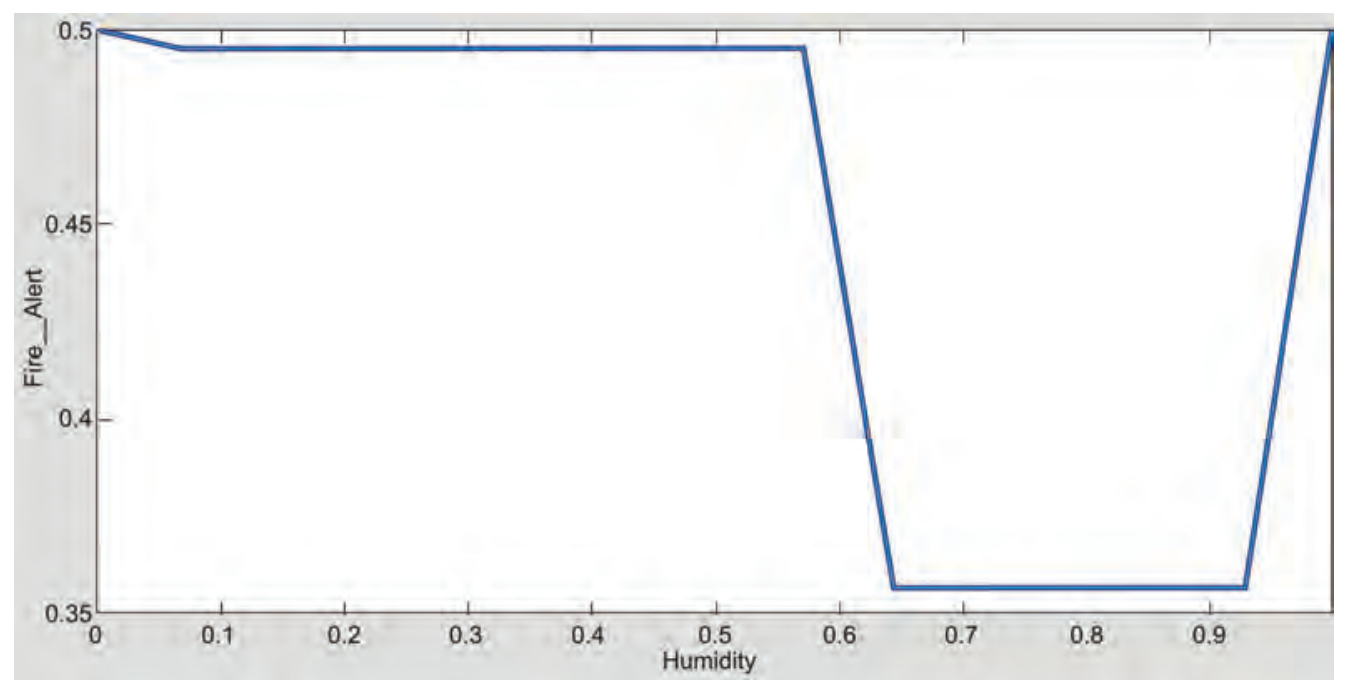

Figure 36.10 Humidity vs temperature (fire alert).

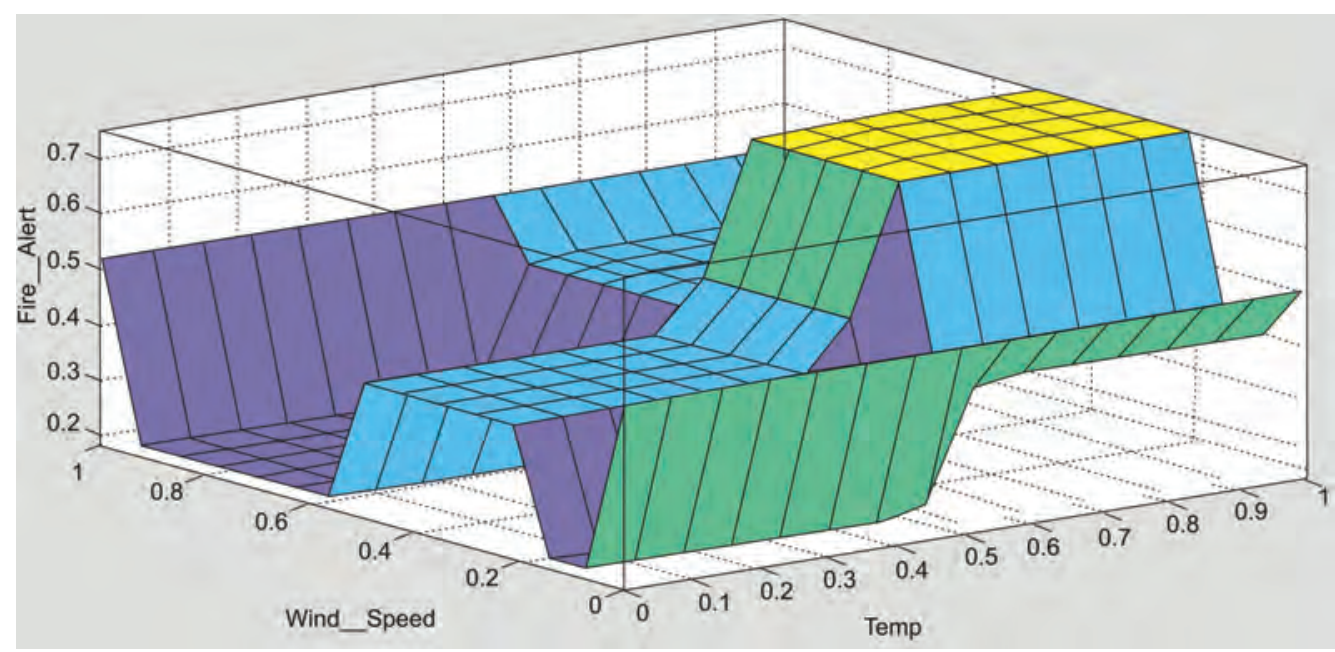

Figure 36.11 Humidity vs fire alert.

It is the transmitted power with respect to the received signals. Intra-WBAN interference caused due to neighbouring sensors and inter-WBAN interference as a result of multiple WBAN networks in the nearby vicinity due to the common communication channel bandwidth lead to path loss.

Temperature and humidity parameters change during a fire. By governing significant variation in the same, fire and its extent can be known. In [29], a combined effect of temperature increase and a decrease in humidity is utilized with insignificant maintenance.

Early detection of flames could drastically reduce the damage but poses a challenge due to the time constraints. A new local binary pattern method shows a much better result than the SVM or k-nearest classifier [30]. 


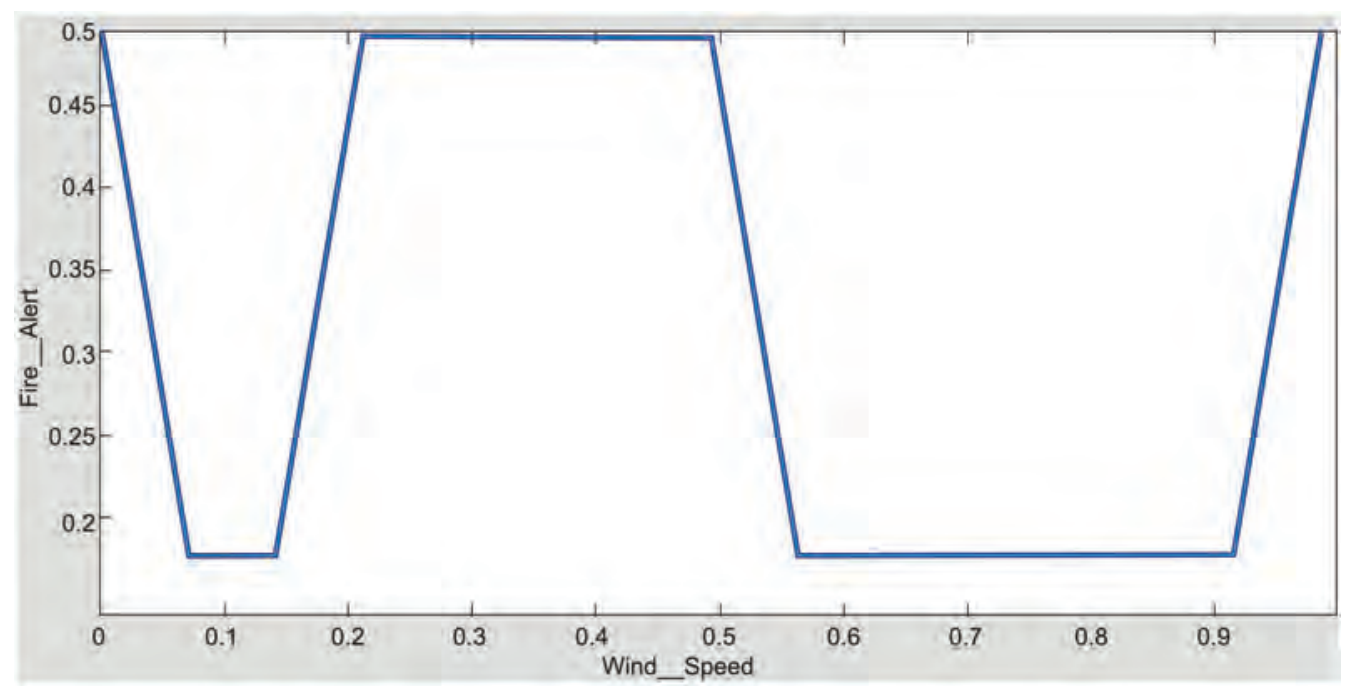

Figure 36.12 Wind speed vs fire alert.

\section{Future directions}

Energy is the major concern in a WBAN which restricts the efficient utility of other parameters. Instead of being dependent on replacing or external recharging which at times is not feasible, a self-energizing module in desired for the future device. There is a need for efficiently harnessing the renewable sources of energy like solar, wind, vibration, thermal, motion, electromagnetic etc. [31-32].

The IEEE 802.15.6 is a great initiative to bring improvements to channel assignment strategies in order to avoid collision, increase reliability and at the same time ensure network efficiency. The problem lies with the dynamic and varied types of data traffic. This modified standard provides flexibility to define its own superframe [33,34].

Routing algorithms ensure the data are efficiently and timely sent to the sink. It is desired to have an efficient routing protocol as all the sensor nodes and the sink nodes drain energy thereby reducing the network lifetime. There are various types of routing protocols like temperature based, cluster based, cross-layer based and many more. Finding the shortest path considering factors like interference, link quality, residual energy, distance is a challenging task [35]. Deep learning algorithms can provide a suitable solution to the given problem.

UWB helps to reduce the reflection, multipath fading, overlapping signals thereby a potential viable future spectrum for WBAN networks. UWB can solve the problem of limited energy constraints [35].

The future advances will depend on inter-dependence on multi-technological merging which includes big data, IoT, deep learning, 5G, nano-technology, energy harvesting. The need of the hour is researchers to be aware of and receptive towards the trends and to be able to collaborate to be a part of the innovation race [36].

\section{Conclusion}

This paper highlighted the issue of disaster management for wildlife. In this, the major challenge in early detection is presented so that the damage can be prevented or minimized. 


\section{Intelligent Circuits and Systems}

Fuzzy based sensor networks including WSN and WBANs are utilized as a potential solution. Simulation results shows that challenges in sensor networks are presented; the major one is energy constraints along with false detection issues. Three-stage disaster management is proposed including prevention, pro-activeness and post-evacuation for damage control. Fast response and co-ordination between firefighters can also be improved with WBANs for better and effective control measure. Hence a three-fold is a very viable solution in a confined environment, specifically a wildlife sanctuary. A smart system within the forest is suggested which has a wider role to play in nurturing, monitoring and disaster management. Finally, a request to the research community to collaborate with multidimensional expertise so as to preserve the ecosystem.

\section{References}

[1] H.Anandakumar, R. Arulmurugan, and C. C. Onn, Computational Intelligence and Sustainable Systems, pp. 61-70, Springer, 2019.

[2] Hisham Alshaheen, Energy saving and reliability of wireless body area networks for health applications. Submitted in partial fulfilment of the requirements of the Degree of Doctor of Philosophy, 2018.

[3] A. Tauqir, N. Javaid, S. Akram, A. Rao, and S. N. Mohammad, "Distance aware relaying energy-efficient: DARE to monitor patients in multi-hop body area sensor networks," pp. 1-8.

[4] M. Khan, B. Jan, and H. Farman, Deep Learning: Convergence to Big Data Analytics. Springer Singapore, 2019.

[5] C. Chen, A. Knoll, H. Wichmann, and A. Horsch, "A review of three-layer wireless body sensor network systems in healthcare for continuous monitoring," August 2013, 2014.

[6] D. Das, S. Maity, B. Chatterjee, and S. Sen, "Enabling covert body area network using electroquasistatic human body communication," Sci. Rep., vol. 9, no. 1, pp. 1-14, 2019.

[7] A. Nadeem, M. A. Hussain, O. Owais, A. Salam, and S. Iqbal, "Application specific study, analysis and classification of body area wireless sensor network applications," Comput. Networks, 2015.

[8] J. Zhu, G. Zhang, Z. Zhu, and K. Yang, "Joint time switching and transmission scheduling for wireless-powered body area networks," vol. 2019, 2019.

[9] W. Zang, F. Miao, R. Gravina, F. Sun, G. Fortino, and Y. Li, "CMDP-based intelligent transmission for wireless body area network in remote health monitoring," Neural Comput. Appl., vol. $0123456789,2019$.

[10] M. M. Yaqoob, K. Fatima, S. Shamshirband, and A. Mosavi, "AMHRP: adaptive multi-hop routing protocol to improve network lifetime for multi-hop wireless body area network keywords," May, 2019.

[11] F. Jamil, M. Iqbal, R. Amin, and D. Kim, "Adaptive thermal-aware routing protocol for wireless body area network," Electronics, vol. 8, no. 1, p. 47, 2019.

[12] S. Macwan, N. Gondaliya, and N. Raja, "Survey on wireless body area network," vol. 5, no. 2, pp. 107-110, 2016.

[13] R. Dautov and G. Tsouri, "On the negatively correlated eavesdropper in indoor wireless body area networks," 2019 16th IEEE Annu. Consum. Commun. Netw. Conf., pp. 1-2, 2019.

[14] J. Shen, S. Chang, J. Shen, Q. Liu, and X. Sun, "A lightweight multi-layer authentication protocol for wireless body area networks," Futur. Gener. Comput. Syst., 2016.

[15] A. A. A. Alkhatib, "Index Terms-Wireless Sensor Network, WSN, efficient system for forest fire detection, efficient detection method for forest fire systems, Wireless Sensor Network for Forest Fire Detection and Decision Making,” pp. 299-309.

[16] M. Montebello, Ambient Intelligence, vol. 840. 2019.

[17] A. Bagula, N. Boudriga, and S. Berrahal, "Reacting to fire incidents in infrastructure less zones. A WBAN cloud based approach,” July 2018, pp. 38-43, 2016.

[18] A. Molina-Pico, D. Cuesta-Frau, A. Araujo, J. Alejandre, and A. Rozas, "Forest monitoring and wildland early fire detection by a hierarchical wireless sensor network," J. Sensors, vol. 2016, 2016. 
[19] J.-F. Dwyer, “The human dimensions of urban forest ecosystem management," Proc. 7th Natl. Urban For. Conf., pp. 236-245, 1995.

[20] J. Toledo-Castro et al., "Forest fire prevention, detection, and fighting based on fuzzy logic and wireless sensor networks," Complexity, vol. 2018, 2018.

[21] K. Bouabdellaha, H. Noureddine, and S. Larbi, "Using wireless sensor networks for reliable forest fires detection," Procedia Comput. Sci., vol. 19, pp. 794-801, 2013.

[22] P. Charbonneau, "Forest fires," Natural Complexity, pp. 130-153, 2017.

[23] C. Vitolo, F. Di Giuseppe, B. Krzeminski, and J. San-Miguel-Ayanz, "Data descriptor: A 19802018 global fire danger re-analysis dataset for the Canadian fire weather indices," Sci. Data, vol. 6, pp. 1-10, 2019.

[24] R. D. Field et al., "Development of a global fire weather database," Nat. Hazards Earth Syst. Sci., vol. 15, no. 6, pp. 1407-1423, 2015.

[25] R. Srivastava and M. Hasan, "Forest fire prediction modelling using fuzzy logic," pp. 668-676.

[26] A. M. Chiodi, N. K. Larkin, J. M. Varner, and J. K. Hiers, "Sensitivity of prescribed burn weather windows to atmospheric dispersion parameters over southeastern USA," Int. J. Wildl. Fire, vol. 28, no. 8, pp. 589-600, 2019.

[27] R. Nidhya and S. Karthik, Body Area Network Challenges and Solutions. 2019.

[28] N. Yessad, M. Omar, A. Tari, and A. Bouabdallah, "QoS-based routing in wireless body area networks: a survey and taxonomy," Computing, vol. 100, no. 3, pp. 245-275, 2018.

[29] V. Chowdary, M. Kumar Gupta, and R. Singh, "A review on forest fire detection techniques: a decadal perspective,” Int. J. Eng. Technol., vol. 7, no. 3.12, p. 1312, 2018.

[30] O. Maksymiv, T. Rak, and D. Peleshko, "Video-based flame detection using LBP-based descriptor: influences of classifiers variety on detection efficiency," Int. J. Intell. Syst. Appl., vol. 9, no. 2, pp. 42-48, 2017.

[31] A. Tony and L. Hiryanto, "A review on energy harvesting and storage for rechargeable wireless sensor networks," IOP Conf. Ser. Mater. Sci. Eng., vol. 508, no. 1, 2019.

[32] N. Zhao, F. R. Yu, H. M. Wang, T. Q. Duong, and Z. Chang, "IEEE Access special section editorial: exploiting the benefits of interference in wireless networks: energy harvesting and security," IEEE Access, vol. 6, pp. 30612-30616, 2018.

[33] D. Yuan, G. Zheng, H. Ma, J. Shang, and J. Li, "An adaptive MAC protocol based on IEEE802.15.6 for wireless body area networks," Wirel. Commun. Mob. Comput., vol. 2019, pp. 1-9, 2019.

[34] J. Ghosh and Z. Jako, "MAC protocol operation in energy harvesting for 5 G networks," ICST Trans. Mob. Commun. Appl., vol. 3, no. 9, p. 153554, 2018.

[35] Y. Qu, G. Zheng, H. Ma, X. Wang, B. Ji, and H. Wu, "A survey of routing protocols in WBAN for healthcare applications," Sensors (Switzerland), vol. 19, no. 7, 2019.

[36] B.-S. Kim, K. Kim, B. Shah, F. Chow, and K. Kim, "Wireless Sensor Networks for Big Data Systems," Sensors, vol. 19, no. 7, p. 1565, 2019. 


\title{
37 Plant species recognition using CNN
}

\author{
Rosepreet Kaur Bhogal*, Ajmer Singh*
}

\author{
School of Electronics and Electrical Engineering, Lovely Professional University, \\ Phagwara, Punjab, India
}

\section{Introduction}

We humans can clearly recognize and differentiate different features of artefacts when it comes to the recognition of pictures. This is because our minds were unwittingly equipped with the same set of images that culminated in capability growth to differentiate items effortlessly. As we perceive the real world, we are hardly sure of it. Meeting and identifying with easily different entities of the visual world is no threat to us. The subconscious mind executes all the operations without any difficulty. In comparison to human brains, computers interpret graphics as a collection of numerical values and look for patterns in the digital image to recognize and differentiate key features of the image, whether it is photo, video, illustration, or even live. The way a picture is perceived by a machine is entirely different from humans. Computer vision utilizes software for image processing to interpret and recognize images from a single image or image series. An illustration of computer vision is the precise detection of pedestrians and vehicles on the road, categorization and screening of millions of useruploaded photographs. Image recognition is a program or software's ability to identify object objects, persons, places, and behaviour. To recognize photos through a camera system, it combines machine vision technology including artificial intelligence and qualified algorithms.

Image-based approaches for species recognition are deemed a successful solution. A customer can use the built-in camera of a mobile device to capture a photograph of the field plant and examine it with an activated identification program to classify the species or receive at least a set of nearby species list when there is no particular match [2]. Nonprofessionals could also use a computer-aided plant species identification system to participate in this operation. Plants provide us with a breathable atmosphere, heat, shelter, medication, and electricity to sustain the Earth's ecological balance. Photo analysis and pattern recognition technologies have come to existence for developing the catalogues of plant species to protect and restore the living plant species through the systematic use of digital images of plant parts such as stem, base, leaves, fruit and flower. Leaves of a plant are the most part of it, because it has smooth, 2D surfaces with distinctive form, texture and colour, which makes a leaf very useful for the detection or identification of different species of plants. The big drawback to using leaves for identification is that they are delicate and vulnerable to being quickly destroyed by different kinds of biological and environmental factors [1]. As its signature form, colour and texture may be changed, a weakened leaf lacks its ability to provide distinguishing signals. Consequently, a scheme of acknowledgement centred on such properties may yield inaccurate and unstable outcomes. The present research focused on addressing the situation in order to build a robust system of identification of plants that can clearly accommodate blurred photographs of the root. As a fragmented leaf has the ability to provide fewer details, the study also tried to extract

\footnotetext{
* Emails: rosepreetkaur12@gmail.com, ajmersinghbhinder@gmail.com
}

DOI: $10.1201 / 9781003129103-37$ 


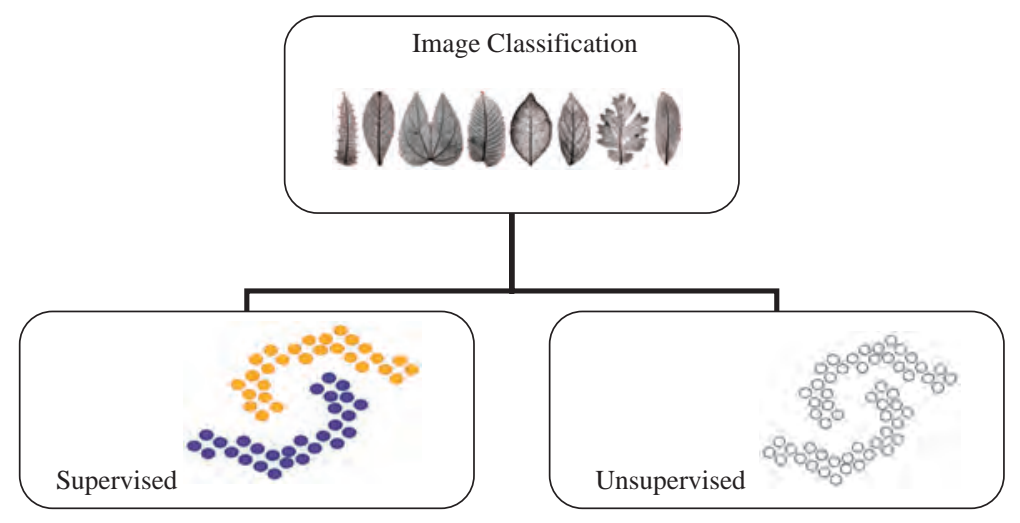

Figure 37.1 Image classification.

a threshold of fragment size; apart from that outcomes are uncertain and whether these thresholds are uniform or vary with respect to other variables [3].

Classification of images relates to the process of removing groups of knowledge from a multiband raster image. There are two forms of classification, based on the relationship between the analyst and the machine during diagnosis: controlled and unsupervised. Using the spectral signatures of training samples, supervised classification is able to classify a test image. To represent the groups with which to collect, it can easily create training samples. Unmonitored classification identifies spectral classes (or clusters) without the involvement of the analyst in a multiband picture. The unattended classification is by providing access to cluster formation, the ability to analyze cluster output as shown in Figure 37.1.

Architecture of the neural network was used as an effective solution to derive highlevel data functionality with the creation of neural networks. With simplified details, CNN architectures may extract highly abstract properties accurately while retaining the most upto-date raw data properties. For grouping or estimation, this is helpful. CNN has recently emerged as an important tool for the definition of image processing capabilities and identities. $\mathrm{CNN}$ will automatically learn basic filters and hierarchically merge them to explain the fundamental principles for pattern recognition. $\mathrm{CNN}$ does not have to measure technological functions that take time and effort. The method's generalization makes it a functional and flexible solution to the various classification and identification implementation issues [2].

\section{Literature review}

In the literature, there are several scientific studies that use the properties of the leaves such as colour and texture to distinguish plants. Different techniques in data processing have been used to classify the leaf species. It is difficult to guess the number of clusters (K-value) during the K-means clustering process [4]. The specifics of the initial seed and the artefacts have a significant impact on the final studies. K-means clustering is very prone to scale. The rescaling of the data would totally change the results. Such function does not extend to the identification of pieces of plants. Sparse classification based on representation [4] is unreliable using occluded data and therefore not ideal for detecting fragments of grain. Gabor filters [5] resolve one wavelet transform restriction and are able to handle the directed edges, but lose the input picture spectral detail. Most of the previous studies for the identification of plant species were based mainly on characteristics of form, 
texture, and colour. LBP was one of the best methods of extraction of functionality until about 10 years ago. Several reports of research can be included in the literature focused on enhanced LBP (local binary pattern) process and plant identification system LBP. Ahmed et al. [6] used the SVM classification and the simple LBP system to characterize the broadleaf and grass weed photos with high accuracy and computational efficiency. Ren et al. [7] used multi-scale LBP to suggest a framework for constructing leaf picture descriptors. A multi-scale pyramid is originally used to maximize the use of leaf data and each training picture is separated into several overlapping blocks to collect LBP histograms in each scale. However, a PCA eliminates the LBP flexibility factor. The authors found that a compact and discriminative description of the leaf can be given by the derived multi-scale overlapping block LBP descriptor.

In recent years, some reports have proposed the use of CNN in the classification of the crop. Jassmann et al. [8] established a leaf-based method for plant classification. The system uses a CNN in a mobile app to categorize the nature of the herb, which is fitted with the ImageCLEF data set. The planned design comprises a circular sheet, accompanied by a composite layer and two fully connected layers added to the input picture of $60 \times$ 80 pixels [9]. A simpler edition of AlexNet was introduced for the identification of the root. Instead of ReLU, they used parametric linear units (PReLU). Identification of plant disease requires manipulating leaf identification. Sladojevic et al. [10] were interested in a new way of creating a picture classification model for disease-identification using CNN. Thirteen healthy plant leaf diseases were recognized by the evolutionary model with the ability to discriminate plants from the surrounding environment.

\section{Dataset}

The collection of the Swedish leaf has pictures of 15 leaf species, with 75 photographs per plant [11]. The accompanying Figure 37.2 shows a few comparison images. For the untrained eye, certain leaves would not be distinguishable. To train the proposed design, the random selection of 50 pictures from each leaf class for training and the remaining 25 photos for research were used. This dataset is commonly used for evaluating strategies for shape-based matching [12].

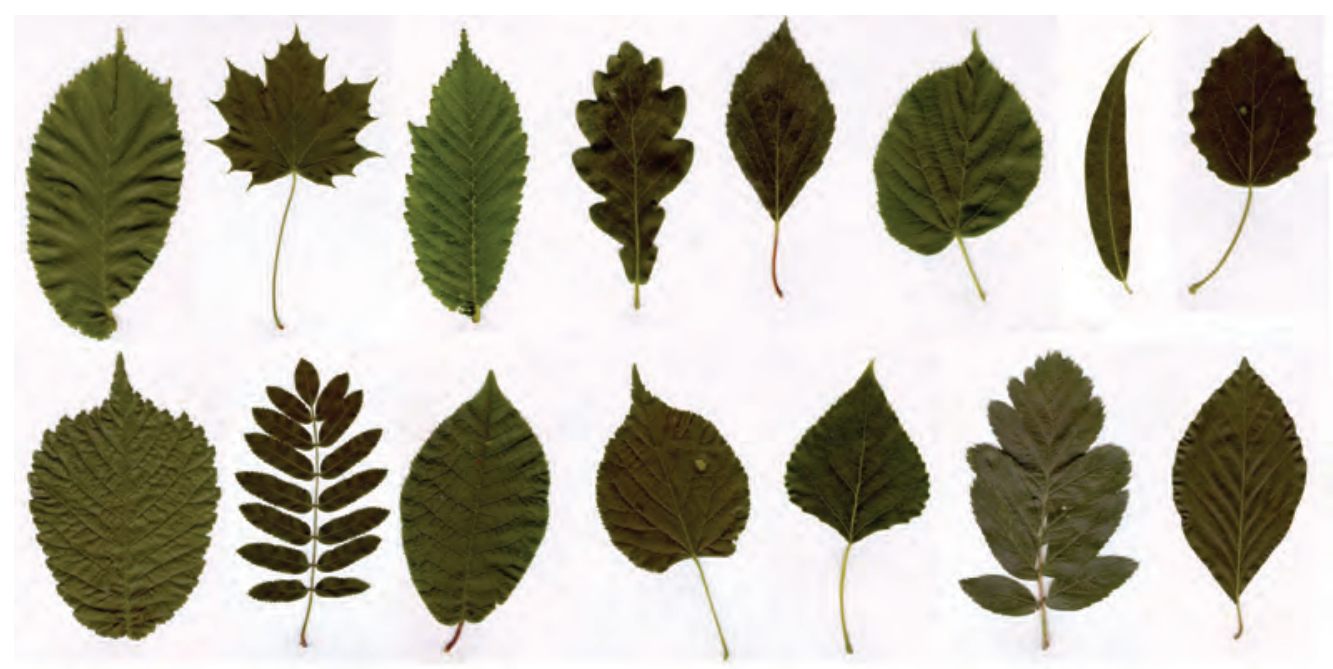

Figure 37.2 Some sample images from Swedish dataset. 
This dataset has several apparent features. First, the picture leaves are well placed manually and only with very little rotation. Third, it catches only the same single side of the leaves. But in reality, it is usually different on both sides of the leaves. Eventually, without significant partial damage, the leaves have good quality. Because the dataset has three characteristics described above, this dataset has a clear spatial structure prior to it. The good spatial knowledge could be used to improve the accuracy of the classification. Such a precedent, though, does not occur in reality.

\section{Methodology}

$\mathrm{CNN}$ is a special type of neural network that operates like a normal NN except that at the beginning it has a convolution layer. The image is broken up into a series of tiles; instead of being fed the whole picture as a collection of numbers, the computer instead attempts to guess what each tile is. The machine is actually trying to predict what's in the image based on reading all the tiles. This lets the computer organize the processes and identify the target regardless of where it is in the image.

The Swedish dataset consists of 1125 images with variable sized pixels. The collection contains 15 classes that are mutually exclusive (do not overlap) with 75 items in each class. The photos are low, clearly labelled and have no noise, rendering the dataset perfect with much less pre-processing for this mission.

\section{Step 1: Pre-processing}

First, it is necessary to add a bit of variance to the data as the data set images are very organized and contain little noise. However, the size of all the images per class is not the same. In the step, all images were made a size of $128 \times 128$ in RGB format.

\section{Step 2: Splitting of dataset}

This takes a long time to measure the model's gradient using a dataset as a whole. Use a small batch of photos during each optimizer iteration. The dataset is then split into 750 image training set and 375 image evaluation set.

\section{Step 3: Building of network}

A CNN incorporates trained input data attributes and utilizes convolutional 2D layers. It implies that this network type is appropriate for $2 \mathrm{D}$ image processing. In reality, CNNs use very little pre-processing relative to other algorithms for image classification. This ensures they will acquire the filters that other algorithms need to have hand-made [13]. CNNs can be used in lots of applications including image and video recognition, image description and recommendation applications for natural language processing and diagnostic image analysis. CNNs have an origin layer, an output layer, and layers that are covered. Typically, the secret layers consist of convolution layers, layers of ReLU, pooling layers, and layers that are completely linked. Convolutional layers add an action of convolution to the data. It moves the knowledge to the next layer. In the next step, pooling blends the contributions of neuron clusters into a single neuron. Fully connected layers, as shown in Figure 37.3, link each neuron in one layer to the next layer of each neuron.

\section{Experimental results}

An uncertainty matrix, also known as an error matrix in the area of machine learning, is primarily for the problem of statistical classification. A matrix of uncertainty is a table 


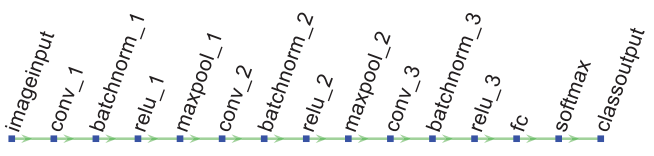

Figure 37.3 Network flow diagram of proposed architecture.

Confusion Matrix

\begin{tabular}{|c|c|c|c|c|c|c|c|c|c|c|c|c|c|c|c|c|}
\hline leat & $\begin{array}{c}23 \\
6.1 \%\end{array}$ & $\begin{array}{c}\mathbf{0} \\
0.0 \%\end{array}$ & $\begin{array}{c}0 \\
0.0 \%\end{array}$ & $\begin{array}{c}0 \\
0.0 \%\end{array}$ & $\begin{array}{c}0 \\
0.0 \%\end{array}$ & $\begin{array}{c}0 \\
0.0 \%\end{array}$ & $\begin{array}{c}\mathbf{0} \\
0.0 \%\end{array}$ & $\begin{array}{c}\mathbf{0} \\
0.0 \%\end{array}$ & $\begin{array}{c}1 \\
0.3 \%\end{array}$ & $\begin{array}{c}\mathbf{0} \\
0.0 \%\end{array}$ & $\begin{array}{c}\mathbf{0} \\
0.0 \%\end{array}$ & $\begin{array}{c}0 \\
0.0 \%\end{array}$ & $\begin{array}{c}0 \\
0.0 \%\end{array}$ & $\begin{array}{c}0 \\
0.0 \%\end{array}$ & $\begin{array}{c}0 \\
0.0 \%\end{array}$ & $\begin{array}{c}95.8 \% \\
4.2 \% \\
\end{array}$ \\
\hline leaf10 & $\begin{array}{c}0 \\
0.0 \% \\
\end{array}$ & $\begin{array}{c}25 \\
6.7 \% \\
\end{array}$ & $\begin{array}{c}0 \\
0.0 \% \\
\end{array}$ & $\begin{array}{c}0 \\
0.0 \%\end{array}$ & $\begin{array}{c}0 \\
0.0 \%\end{array}$ & $\begin{array}{c}0 \\
0.0 \%\end{array}$ & $\begin{array}{c}0 \\
0.0 \%\end{array}$ & $\begin{array}{c}\mathbf{0} \\
0.0 \%\end{array}$ & $\begin{array}{c}0 \\
0.0 \%\end{array}$ & $\begin{array}{c}\mathbf{0} \\
0.0 \% \\
\end{array}$ & $\begin{array}{c}\mathbf{0} \\
0.0 \%\end{array}$ & $\begin{array}{c}0 \\
0.0 \%\end{array}$ & $\begin{array}{c}0 \\
0.0 \%\end{array}$ & $\begin{array}{c}0 \\
0.0 \% \\
\end{array}$ & $\begin{array}{c}0 \\
0.0 \% \\
\end{array}$ & $0.0 \%$ \\
\hline leaf11 & $\begin{array}{c}0 \\
0.0 \%\end{array}$ & $\begin{array}{c}0 \\
0.0 \%\end{array}$ & $\begin{array}{c}25 \\
6.7 \%\end{array}$ & $\begin{array}{c}0 \\
0.0 \%\end{array}$ & $\begin{array}{c}\mathbf{0} \\
0.0 \%\end{array}$ & $\begin{array}{c}\mathbf{0} \\
0.0 \%\end{array}$ & $\begin{array}{c}\mathbf{0} \\
0.0 \%\end{array}$ & $\begin{array}{c}0 \\
0.0 \%\end{array}$ & $\begin{array}{c}\mathbf{0} \\
0.0 \%\end{array}$ & $\begin{array}{c}\mathbf{0} \\
0.0 \%\end{array}$ & $\begin{array}{c}\mathbf{0} \\
0.0 \%\end{array}$ & $\begin{array}{c}0 \\
0.0 \%\end{array}$ & $\begin{array}{c}0 \\
0.0 \%\end{array}$ & $\begin{array}{c}0 \\
0.0 \%\end{array}$ & $\begin{array}{c}0 \\
0.0 \%\end{array}$ & $\begin{array}{l}100 \% \\
0.0 \%\end{array}$ \\
\hline leaf12 & $\begin{array}{c}0 \\
0.0 \%\end{array}$ & $\begin{array}{c}\mathbf{0} \\
0.0 \%\end{array}$ & $\begin{array}{c}0 \\
0.0 \%\end{array}$ & $\begin{array}{c}\mathbf{2 5} \\
6.7 \%\end{array}$ & $\begin{array}{c}0 \\
0.0 \%\end{array}$ & $\begin{array}{c}\mathbf{0} \\
0.0 \%\end{array}$ & $\begin{array}{c}\mathbf{0} \\
0.0 \%\end{array}$ & $\begin{array}{c}\mathbf{0} \\
0.0 \%\end{array}$ & $\begin{array}{c}0 \\
0.0 \%\end{array}$ & $\begin{array}{c}\mathbf{0} \\
0.0 \%\end{array}$ & $\begin{array}{c}0 \\
0.0 \%\end{array}$ & $\begin{array}{c}0 \\
0.0 \%\end{array}$ & $\begin{array}{c}0 \\
0.0 \%\end{array}$ & $\begin{array}{c}0 \\
0.0 \%\end{array}$ & $\begin{array}{c}0 \\
0.0 \%\end{array}$ & $\begin{array}{l}0 \% \\
0 \%\end{array}$ \\
\hline leaf13 & $\begin{array}{c}0 \\
0.0 \% \\
\end{array}$ & $\begin{array}{c}0 \\
0.0 \% \\
\end{array}$ & $\begin{array}{c}\mathbf{0} \\
0.0 \% \\
\end{array}$ & $\begin{array}{c}0 \\
0.0 \% \\
\end{array}$ & \begin{tabular}{|c|}
25 \\
$6.7 \%$ \\
\end{tabular} & $\begin{array}{c}0 \\
0.0 \%\end{array}$ & $\begin{array}{c}0 \\
0.0 \%\end{array}$ & \begin{tabular}{|c|}
$\mathbf{0}$ \\
$0.0 \%$ \\
\end{tabular} & $\begin{array}{c}0 \\
0.0 \%\end{array}$ & $\begin{array}{c}\mathbf{0} \\
0.0 \%\end{array}$ & $\begin{array}{c}1 \\
0.3 \% \\
\end{array}$ & \begin{tabular}{|c|}
$\mathbf{0}$ \\
$0.0 \%$
\end{tabular} & \begin{tabular}{|c|}
0 \\
$0.0 \%$ \\
\end{tabular} & \begin{tabular}{|c|}
0 \\
$0.0 \%$ \\
\end{tabular} & \begin{tabular}{|c|}
0 \\
$0.0 \%$
\end{tabular} & $\begin{array}{l}96.2 \% \\
3.8 \%\end{array}$ \\
\hline leaf14 & \begin{tabular}{|c|c}
0 \\
$0.0 \%$ \\
\end{tabular} & $\begin{array}{c}0 \\
0.0 \% \\
\end{array}$ & \begin{tabular}{|c|}
$\mathbf{0}$ \\
$0.0 \%$ \\
\end{tabular} & $\begin{array}{c}0 \\
0.0 \% \\
\end{array}$ & \begin{tabular}{|c|}
0 \\
$0.0 \%$ \\
\end{tabular} & \begin{tabular}{|c|}
$\mathbf{2 5}$ \\
$6.7 \%$ \\
\end{tabular} & $\begin{array}{c}0 \\
0.0 \% \\
\end{array}$ & \begin{tabular}{|c|}
$\mathbf{0}$ \\
$0.0 \%$ \\
\end{tabular} & $\begin{array}{c}0 \\
0.0 \% \\
\end{array}$ & $\begin{array}{c}\mathbf{0} \\
0.0 \% \\
\end{array}$ & $\begin{array}{c}0 \\
0.0 \% \\
\end{array}$ & \begin{tabular}{|c|}
$\mathbf{0}$ \\
$0.0 \%$ \\
\end{tabular} & \begin{tabular}{|c|}
0 \\
$0.0 \%$ \\
\end{tabular} & \begin{tabular}{|c|}
$\mathbf{0}$ \\
$0.0 \%$ \\
\end{tabular} & \begin{tabular}{|c|}
$\mathbf{0}$ \\
$0.0 \%$ \\
\end{tabular} & $\begin{array}{l}100 \% \\
0.0 \% \\
\end{array}$ \\
\hline leaf1 & $\begin{array}{c}0 \\
0.0 \% \\
\end{array}$ & $\begin{array}{c}0 \\
0.0 \% \\
\end{array}$ & \begin{tabular}{|c|}
0 \\
$0.0 \%$ \\
\end{tabular} & $\begin{array}{c}0 \\
0.0 \% \\
\end{array}$ & $\begin{array}{c}0 \\
0.0 \% \\
\end{array}$ & $\begin{array}{c}0 \\
0.0 \% \\
\end{array}$ & $\begin{array}{c}25 \\
6.7 \% \\
\end{array}$ & \begin{tabular}{|c|}
$\mathbf{0}$ \\
$0.0 \%$ \\
\end{tabular} & $\begin{array}{c}0 \\
0.0 \% \\
\end{array}$ & $\begin{array}{c}\mathbf{0} \\
0.0 \% \\
\end{array}$ & $\begin{array}{c}0 \\
0.0 \% \\
\end{array}$ & \begin{tabular}{|c|}
$\mathbf{0}$ \\
$0.0 \%$ \\
\end{tabular} & \begin{tabular}{|c}
$\mathbf{0}$ \\
$0.0 \%$ \\
\end{tabular} & \begin{tabular}{|c|}
$\mathbf{0}$ \\
$0.0 \%$ \\
\end{tabular} & \begin{tabular}{|c|}
$\mathbf{0}$ \\
$0.0 \%$ \\
\end{tabular} & $\begin{array}{l}0 \% \\
0 \% \\
\end{array}$ \\
\hline leaf2 & \begin{tabular}{|c|}
0 \\
$0.0 \%$ \\
\end{tabular} & $\begin{array}{c}0 \\
0.0 \% \\
\end{array}$ & $\begin{array}{c}\mathbf{0} \\
0.0 \% \\
\end{array}$ & $\begin{array}{c}0 \\
0.0 \% \\
\end{array}$ & $\begin{array}{c}0 \\
0.0 \% \\
\end{array}$ & $\begin{array}{c}0 \\
0.0 \% \\
\end{array}$ & $\begin{array}{c}0 \\
0.0 \% \\
\end{array}$ & \begin{tabular}{|c|}
25 \\
$6.7 \%$ \\
\end{tabular} & $\begin{array}{c}0 \\
0.0 \% \\
\end{array}$ & $\begin{array}{c}\mathbf{0} \\
0.0 \% \\
\end{array}$ & \begin{tabular}{|c|}
0 \\
$0.0 \%$ \\
\end{tabular} & $\begin{array}{c}\mathbf{0} \\
0.0 \% \\
\end{array}$ & \begin{tabular}{|c|}
$\mathbf{0}$ \\
$0.0 \%$ \\
\end{tabular} & \begin{tabular}{|c|}
$\mathbf{0}$ \\
$0.0 \%$ \\
\end{tabular} & $\begin{array}{c}0 \\
0.0 \% \\
\end{array}$ & $\begin{array}{l}100 \% \\
0.0 \% \\
\end{array}$ \\
\hline leaf3 & $\begin{array}{c}2 \\
0.5 \% \\
\end{array}$ & $\begin{array}{c}0 \\
0.0 \% \\
\end{array}$ & $\begin{array}{c}0 \\
0.0 \% \\
\end{array}$ & $\begin{array}{c}0 \\
0.0 \% \\
\end{array}$ & $\begin{array}{c}0 \\
0.0 \% \\
\end{array}$ & $\begin{array}{c}\mathbf{0} \\
0.0 \% \\
\end{array}$ & $\begin{array}{c}0 \\
0.0 \% \\
\end{array}$ & $\begin{array}{c}0 \\
0.0 \% \\
\end{array}$ & $\begin{array}{c}24 \\
6.4 \% \\
\end{array}$ & $\begin{array}{c}\mathbf{0} \\
0.0 \% \\
\end{array}$ & $\begin{array}{c}0 \\
0.0 \% \\
\end{array}$ & $\begin{array}{c}\mathbf{0} \\
0.0 \% \\
\end{array}$ & $\begin{array}{c}0 \\
0.0 \% \\
\end{array}$ & $\begin{array}{c}0 \\
0.0 \% \\
\end{array}$ & $\begin{array}{c}0 \\
0.0 \% \\
\end{array}$ & $\begin{array}{l}.3 \% \\
7 \% \\
\end{array}$ \\
\hline leaf4 & $\begin{array}{c}0 \\
0.0 \%\end{array}$ & $\begin{array}{c}0 \\
0.0 \%\end{array}$ & $\begin{array}{c}0 \\
0.0 \%\end{array}$ & $\begin{array}{c}0 \\
0.0 \%\end{array}$ & $\begin{array}{c}0 \\
0.0 \%\end{array}$ & $\begin{array}{c}0 \\
0.0 \%\end{array}$ & $\begin{array}{c}\mathbf{0} \\
0.0 \%\end{array}$ & $\begin{array}{c}\mathbf{0} \\
0.0 \%\end{array}$ & $\begin{array}{c}\mathbf{0} \\
0.0 \%\end{array}$ & $\begin{array}{c}25 \\
6.7 \%\end{array}$ & $\begin{array}{c}\mathbf{0} \\
0.0 \%\end{array}$ & $\begin{array}{c}0 \\
0.0 \%\end{array}$ & $\begin{array}{c}0 \\
0.0 \%\end{array}$ & $\begin{array}{c}0 \\
0.0 \%\end{array}$ & $\begin{array}{c}0 \\
0.0 \%\end{array}$ & $\begin{array}{l}100 \% \\
0.0 \%\end{array}$ \\
\hline leaf5 & $\begin{array}{c}0 \\
0.0 \%\end{array}$ & $\begin{array}{c}0 \\
0.0 \%\end{array}$ & $\begin{array}{c}0 \\
0.0 \%\end{array}$ & $\begin{array}{c}\mathbf{0} \\
0.0 \%\end{array}$ & $\begin{array}{c}0 \\
0.0 \%\end{array}$ & $\begin{array}{c}0 \\
0.0 \%\end{array}$ & $\begin{array}{c}0 \\
0.0 \%\end{array}$ & $\begin{array}{c}0 \\
0.0 \%\end{array}$ & $\begin{array}{c}\mathbf{0} \\
0.0 \%\end{array}$ & $\begin{array}{c}\mathbf{0} \\
0.0 \%\end{array}$ & $\begin{array}{c}24 \\
6.4 \%\end{array}$ & $\begin{array}{c}0 \\
0.0 \%\end{array}$ & $\begin{array}{c}0 \\
0.0 \%\end{array}$ & $\begin{array}{c}0 \\
0.0 \%\end{array}$ & $\begin{array}{c}0 \\
0.0 \%\end{array}$ & $\begin{array}{l}100 \% \\
0.0 \%\end{array}$ \\
\hline leaf6 & $\begin{array}{c}0 \\
0.0 \%\end{array}$ & $\begin{array}{c}0 \\
0.0 \%\end{array}$ & $\begin{array}{c}0 \\
0.0 \%\end{array}$ & $\begin{array}{c}0 \\
0.0 \%\end{array}$ & $\begin{array}{c}0 \\
0.0 \%\end{array}$ & $\begin{array}{c}0 \\
0.0 \%\end{array}$ & $\begin{array}{c}\mathbf{0} \\
0.0 \%\end{array}$ & $\begin{array}{c}0 \\
0.0 \%\end{array}$ & $\begin{array}{c}\mathbf{0} \\
0.0 \%\end{array}$ & $\begin{array}{c}\mathbf{0} \\
0.0 \%\end{array}$ & $\begin{array}{c}\mathbf{0} \\
0.0 \%\end{array}$ & $\begin{array}{c}25 \\
6.7 \%\end{array}$ & $\begin{array}{c}0 \\
0.0 \%\end{array}$ & $\begin{array}{c}1 \\
0.3 \%\end{array}$ & $\begin{array}{c}\mathbf{0} \\
0.0 \%\end{array}$ & $\begin{array}{l}96.2 \% \\
3.8 \%\end{array}$ \\
\hline leaf7 & $\begin{array}{c}0 \\
0.0 \%\end{array}$ & $\begin{array}{c}0 \\
0.0 \%\end{array}$ & $\begin{array}{c}0 \\
0.0 \%\end{array}$ & $\begin{array}{c}0 \\
0.0 \%\end{array}$ & $\begin{array}{c}0 \\
0.0 \%\end{array}$ & $\begin{array}{c}\mathbf{0} \\
0.0 \%\end{array}$ & $\begin{array}{c}0 \\
0.0 \%\end{array}$ & $\begin{array}{c}\mathbf{0} \\
0.0 \%\end{array}$ & $\begin{array}{c}0 \\
0.0 \%\end{array}$ & $\begin{array}{c}0 \\
0.0 \%\end{array}$ & $\begin{array}{c}\mathbf{0} \\
0.0 \%\end{array}$ & $\begin{array}{c}0 \\
0.0 \%\end{array}$ & $\begin{array}{c}25 \\
6.7 \%\end{array}$ & $\begin{array}{c}0 \\
0.0 \%\end{array}$ & $\begin{array}{c}0 \\
0.0 \%\end{array}$ & $\begin{array}{l}100 \% \\
0.0 \% \\
\end{array}$ \\
\hline leaf8 & $\begin{array}{c}0 \\
0.0 \% \\
\end{array}$ & $\begin{array}{c}\mathbf{0} \\
0.0 \%\end{array}$ & $\begin{array}{c}0 \\
0.0 \% \\
\end{array}$ & $\begin{array}{c}0 \\
0.0 \%\end{array}$ & $\begin{array}{c}0 \\
0.0 \%\end{array}$ & $\begin{array}{c}0 \\
0.0 \%\end{array}$ & $\begin{array}{c}\mathbf{0} \\
0.0 \%\end{array}$ & $\begin{array}{c}\mathbf{0} \\
0.0 \%\end{array}$ & $\begin{array}{c}0 \\
0.0 \%\end{array}$ & $\begin{array}{c}\mathbf{0} \\
0.0 \% \\
\end{array}$ & $\begin{array}{c}\mathbf{0} \\
0.0 \%\end{array}$ & $\begin{array}{c}0 \\
0.0 \%\end{array}$ & $\begin{array}{c}0 \\
0.0 \%\end{array}$ & $\begin{array}{c}24 \\
6.4 \% \\
\end{array}$ & $\begin{array}{c}0 \\
0.0 \% \\
\end{array}$ & $\begin{array}{l}100 \% \\
0.0 \% \\
\end{array}$ \\
\hline \multirow[t]{2}{*}{ leaf9 } & $\begin{array}{c}0 \\
0.0 \%\end{array}$ & $\begin{array}{c}0 \\
0.0 \%\end{array}$ & $\begin{array}{c}0 \\
0.0 \% \\
\end{array}$ & $\begin{array}{c}0 \\
0.0 \%\end{array}$ & $\begin{array}{c}0 \\
0.0 \%\end{array}$ & $\begin{array}{c}0 \\
0.0 \%\end{array}$ & $\begin{array}{c}0 \\
0.0 \%\end{array}$ & $\begin{array}{c}\mathbf{0} \\
0.0 \% \\
\end{array}$ & $\begin{array}{c}0 \\
0.0 \%\end{array}$ & $\begin{array}{c}\mathbf{0} \\
0.0 \% \\
\end{array}$ & $\begin{array}{c}0 \\
0.0 \%\end{array}$ & $\begin{array}{c}0 \\
0.0 \%\end{array}$ & $\begin{array}{c}0 \\
0.0 \% \\
\end{array}$ & $\begin{array}{c}0 \\
0.0 \% \\
\end{array}$ & $\begin{array}{c}25 \\
6.7 \% \\
\end{array}$ & $\begin{array}{l}100 \% \\
0.0 \%\end{array}$ \\
\hline & $\begin{array}{l}92.0 \% \\
8.0 \%\end{array}$ & $\begin{array}{l}100 \% \\
0.0 \%\end{array}$ & $\begin{array}{l}100 \% \\
0.0 \%\end{array}$ & $\begin{array}{l}100 \% \\
0.0 \%\end{array}$ & $\begin{array}{l}100 \% \\
0.0 \% \\
\end{array}$ & $\begin{array}{l}100 \% \\
0.0 \%\end{array}$ & $\begin{array}{l}100 \% \\
0.0 \%\end{array}$ & $\begin{array}{l}100 \% \\
0.0 \%\end{array}$ & $\begin{array}{l}96.0 \% \\
4.0 \%\end{array}$ & $\begin{array}{l}100 \% \\
0.0 \%\end{array}$ & $\begin{array}{c}96.0 \% \\
4.0 \%\end{array}$ & $\begin{array}{l}100 \% \\
0.0 \%\end{array}$ & $\begin{array}{l}100 \% \\
0.0 \%\end{array}$ & $\begin{array}{l}96.0 \% \\
4.0 \%\end{array}$ & $\begin{array}{l}100 \% \\
0.0 \%\end{array}$ & $\begin{array}{c}98.7 \% \\
1.3 \%\end{array}$ \\
\hline
\end{tabular}

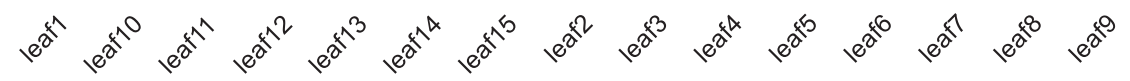

Target Class

Figure 37.4 Uncertainty matrix of statistical classification.

often used to describe the performance (or "classifier") of a classification algorithm on a set of test data specified for the true values. It is fairly easy to understand the ambiguity equation itself, but the associated jargon can be challenging. A matrix of uncertainty is a list of effects of estimation on a question of classification. The amount of correct and incorrect assumptions is summed and broken down by each class with count values. This is the secret to the structure of the uncertainty. The uncertainty matrix reveals whether, when making predictions, the classification process becomes confused. The confusion matrix based on classification of plant leaf species has been given in Figure 37.4. 
Table 37.1 Class mapping with the leaf species

\begin{tabular}{llllll}
\hline $\begin{array}{l}\text { Class } \\
\text { name }\end{array}$ & Leaf species name & $\begin{array}{l}\text { Class } \\
\text { name }\end{array}$ & Leaf species name & $\begin{array}{l}\text { Class } \\
\text { name }\end{array}$ & Leaf species name \\
\hline leaf1 & Ulmus carpinifolia & leaf6 & Betula pubescens & leaf11 & Salix sinerea \\
leaf2 & Acer & leaf7 & Salix alba'Sericea' & leaf12 & Populus \\
leaf3 & Salix aurita & leaf8 & Populus tremula & leaf13 & Tilia \\
leaf4 & Quercus & leaf9 & Ulmus glabra & leaf14 & Sorbus intermedia \\
leaf5 & Alnus incana & leaf10 & Sorbus aucuparia & leaf15 & Fagus silvatica \\
\hline
\end{tabular}

Table 37.2 Comparison with other methods

\begin{tabular}{lll}
\hline Year & Method & Accuracy $(\%)$ \\
\hline- & Proposed method & 98.7 \\
2012 & {$[14]$} & 91.33 \\
2014 & {$[15]$} & 97 \\
2018 & {$[16]$} & 98.03 \\
\hline
\end{tabular}

There are 15 output classes versus target classes of leaf species in form of matrix as shown in Figure 37.4. The class name with respect to leaf species name is given in Table 37.1, which has been used in confusion matrix. The total number of images in dataset are 1125 where 75 images belong to each class. From each class 50 images have been used for training and 25 images for testing. For calculation of the correct rate of the confusion matrix, in the first column 23 leaf species have been classifying as Ulmus carpinifolia but the other 2 of the 25 have been classified as Salix Aurita. Similarly, the other columns have their own number of identification of leaf species. The system has been tested for all leaf species images. The number of random leaf species has been used to test this proposed method. After testing the overall system by all classes the correct classification rate is $97.7 \%$. This proposed method is good in comparison with other methods which are used for leaf species identification. The comparison with other methods is given in Table 37.2.

\section{Conclusion}

In this paper, it is proved that an architecture of the CNN can distinguish the plant type from the sequences of images. In comparison, in this study, $\mathrm{CNN}$ is considered to be a superior form of selection of features rather than a system of classification. The leaf identification using CNN is overall a good way to examine the species with accuracy of $98.7 \%$. The comparison with other method has been given in Table 37.2. It may need to include more types of tropical plants, particularly those with dense leaves, as a work in the future of this science and include additional taxonomic characteristics to further enhance the identification process for plant species that can be used by botanists and the public.

\section{References}

[1] T.Q. Bao, N.T.T. Kiet, T.Q. Dinh, and H.X. Hiep, "Plant species identification from leaf patterns using histogram of oriented gradients feature space and convolution neural networks," Journal of Information and Telecommunication, pp. 1-11, 2019. 


\section{Intelligent Circuits and Systems}

[2] J. Chaki, N. Dey, L. Moraru, and F. Shi, "Fragmented plant leaf recognition: Bag-of-features, fuzzy-color and edge-texture histogram descriptors with multi-layer perceptron," Optik, vol. 181, pp. 639-650, 2019.

[3] M. Turkoglu and D. Hanbay, "Leaf-based plant species recognition based on improved local binary pattern and extreme learning machine," Physica A: Statistical Mechanics and its Applications, vol. 527, p. 121297, 2019.

[4] S. Zhang, H. Wang, and W. Huang, "Two-stage plant species recognition by local mean clustering and Weighted sparse representation classification," Cluster Computing, vol. 20, no. 2, pp. 1517-1525, Nov. 2017.

[5] J. Chaki, R. Parekh, and S. Bhattacharya, "Plant leaf recognition using texture and shape features with neural classifiers," Pattern Recognition Letters, vol. 58, pp. 61-68, 2015.

[6] F. Ahmed, A.H. Bari, A. Shihavuddin, H.A. Al-Mamun, and P. Kwan, "A study on local binary pattern for automated weed classification using template matching and support vector machine," 2011 IEEE 12th International Symposium on Computational Intelligence and Informatics (CINTI), 2011.

[7] X.-M. Ren, X.-F. Wang, and Y.Zhao, "An efficient multi-scale overlapped block LBP approach for leaf image recognition," Lecture Notes in Computer Science Intelligent Computing Theories and Applications, pp. 237-243, 2012.

[8] T. J. Jassmann, R. Tashakkori, and R.M. Parry, "Leaf classification utilizing a convolutional neural network," SoutheastCon 2015, 2015.

[9] Y.-H. Wu, L. Shang, Z.-K. Huang, G. Wang, and X.-P. Zhang, "Convolutional neural network application on leaf classification," Intelligent Computing Theories and Application Lecture Notes in Computer Science, pp. 12-17, 2016.

[10] S. Sladojevic, M. Arsenovic, A. Anderla, D. Culibrk, and D. Stefanovic, "Deep neural networks based recognition of plant diseases by leaf image classification," Computational Intelligence and Neuroscience, vol. 2016, pp. 1-11, 2016.

[11] O. Söderkvist, Computer vision classification of leaves from Swedish trees (Master's thesis), Department of Electrical Engineering, Linköping University, Linköping, Sweden, 2001.

[12] P.F. Felzenszwalb and J.D. Schwartz, "Hierarchical matching of deformable shapes," 2007 IEEE Conference on Computer Vision and Pattern Recognition, 2007.

[13] K. Lee and D.-C. Park, "Image classification using fast learning convolutional neural networks," 2015.

[14] Hu, R.X., Jia, W., Ling, H., et al., "Multiscale distance matrix for fast plant leaf recognition," IEEE Trans. Image Process., 2012, 21, (11), pp. 4667-4672.

[15] Wang, X., Feng, B., Bai, X., et al., "Bag of contour fragments for robust shape classification," Pattern Recognit., 2014, 47, (6), pp. 2116-2125.

[16] Tan, J.W., Chang, S.W., Kareem, S.B.A., et al., "Deep learning for plant species classification using leaf vein morphometric," IEEE/ACM Trans. Comput. Biol. Bioinf., 2018, p. 1. 


\title{
38 Multilevel coding scheme with trellis codes as component code in $5 \mathrm{G}$ scenario
}

\author{
Shakti Raj Chopra*, Akhil Gupta* \\ School of Electronics \& Electrical Engineering, Lovely Professional University, \\ Phagwara, Punjab, India
}

\section{Introduction}

Fading of multi-paths is one of the main causes for incorrect reception of wireless signal transmission. Research community has studied several error-correcting codes to prevent the adverse effects of fading multi-paths. New coding technique was introduced, called multilevel coding by Imai and Hirakawa that uses multiple level for the correction of errors in signal transmission codes [1]. The multilevel coding scheme is designed to provide robust data transfer speeds and enhanced throughput and spectral efficiency. It operates as follows. Initially, the input stream data is divided into several block of data streams then, parallel processing of these blocks via a collection of encoders for generating error-fixing codes, known as part codes. Each part code of an expanded constellation of signals is mapped to the symbols. The mapping comprises fragmenting the extended constellation of signals into subsets successively until a single constellation point is in the subset. Ungerboeck developed this special technique of partitioning the signal spectrum, and it is called set partitioning [2]. To create convoluted codes the mapped symbols, called multilevel codes, are modulated and combined. The multilevel codes are eventually communicated across the wireless transmission device. Convolutionary codes are a form of error correction codes which are commonly used to ensure reliable data transmission in wireless communication [3]. We use the convolutionary codes in this paper as component codes for data encoding and transfer using multilevel schemes. The split level coding system can provide the required data transmission rate, encoding gain, and error output with convolution codes as component codes [4-8]. The paper is laid out in the following way. Section II discusses the device model of multilevel coding schemes with convolution codes as part codes and the performance of the model is shown in Section III.

\section{A. Background study}

In wireless communications, fading is one of the main causes of false signal reception. The research community has studied numerous error correction codes to limit the harmful impact of fading multi-paths. The paper developed a new coding method, called multilevel coding, which uses several codes for signal transmission error correction. The key objective of the MLC scheme is to provide a flexible information transfer rate, thereby improving the spectral efficiency. It functions as follows. First, there are several secondary data streams which divide the input data stream. These sub-data sources are managed by

\footnotetext{
* Emails: shaktirchopra2006@gmail.co.in, akhilgupta112001@gmail.com
} 
a set of encoders in parallel to generate error correction codes known as component codes, which are assigned to symbols from the large constellation of signals [2].

To explain constellation mapping: mapping involves dividing a large constellation of signals into subsections, respectively, till the section includes only one point of constellation. This particular technique has been introduced by partitioning the constellation of signals, and is called a set partition. Proposed complicated codes are constructed by combining the modulated mapped symbols, and these complicated codes are called multilevel codes that are then transmitted through a wireless channel [5].

Convolutional codes are a form of code to correct an error, widely used in wireless communications to achieve reliable data transmission. In the convolutional encoder, a convolution of the impulse response of the encoder with the input data stream is performed. They use multilevel coding to achieve better quality and higher throughput.

Explaining the diversity and orthogonal code in October 1998, Alamouti presented a paper which utilizes the transmit diversity scheme and allows low effective bit error rate (BER). It is extremely difficult to reduce the consequence of the multipath fading channel. In the AWGN channel, it requires 1 or $2 \mathrm{~dB}$ change in SNR to reduce the BER from $10^{-2}$ to $10^{-3}$. However, it may require about $10 \mathrm{~dB}$ improvements in SNR in multipath fading. The improvement in the value of SNR cannot be achieved by increasing the power of transmission or increasing bandwidth because it is contrary to the next-generation requirements. The paper shows that this reduction in bit error rate can be achieved using diversity. This is one of the popular and simple techniques for MIMO technology. The encoding technique of symbols has the orthogonality property to a low complexity maximum likelihood decoding of the transmitted signals. This transmission scheme was presented for two transmit antenna and one receive antenna, which achieves full rate and the full diversity of 2 over complex constellation symbols. It is also shown that it has dual diversity property, i.e., it can achieve the same full diversity of 2 with 2 receive antenna and 1 transmit antenna using maximal-ratio receiver combining (MRRC). This transmission technique can be extended for 2 transmit antenna and $M$ antennas at receiving end, which again provides a full diversity of order of $2 \mathrm{M}$. So by this scheme, neither feedback from the receiver to the transmitter is required nor is expansion in bandwidth needed. This is the only scheme that achieves the full rate over complex constellation symbols [6].

\section{System model of multileval coding scheme with convolutional codes}

In the multilevel environment first information is divided into different blocks and each block is parallel encoded by a different number of encoders.

Figure 38.1 shows the block diagram of multilevel coding scheme.

By the use of a serial to parallel converter, the input information $u$ is partitioned into L data-streams.

$$
\begin{gathered}
u \rightarrow\left\{\begin{array}{llll}
u_{1} & u_{2} & \ldots & u_{L}
\end{array}\right\} \\
u_{i} \in\{0,1\}^{k} ; i=1,2, \ldots, L
\end{gathered}
$$

$k$ is denoted as the data-stream length. 


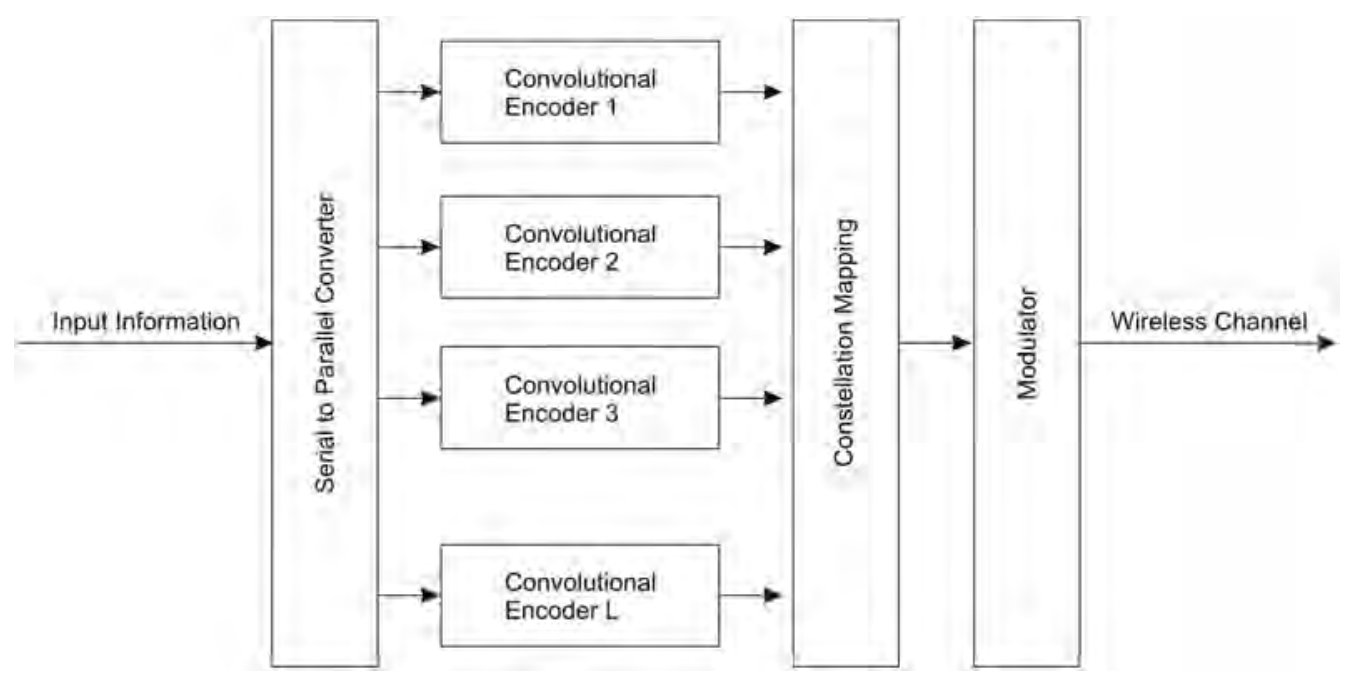

Figure 38.1 Multilevel coding scheme.

There are $L$ levels and the data streams of information are passed through an encoder at their respective levels to produce error-correcting codes which are the codes of the components. The encoders which are used at different levels are the same. The code of component $c_{i}$ is of the form. The component code is of the form

$$
c_{i} \in\{0,1\}^{n} ; i=1,2, \ldots, L
$$

where $n$ is the length of code-word.

The generated codes are mapped to constellation M-QAM using Gray mapping. For $M$-QAM constellation, the number of levels required is $\log _{2} M$. The constellation set of signal points, $Q$, can be represented as

$$
Q=\left\{M\left(c_{1}^{1} c_{2}^{1} \ldots c_{L}^{1}\right), M\left(c_{1}^{2} c_{2}^{2} \ldots c_{L}^{2}\right), \ldots, M\left(c_{1}^{n} c_{2}^{n} \ldots c_{L}^{n}\right)\right\}
$$

where $M($.$) is the mapping function.$

The symbols mapped are modulated and communicated through the wireless interface. The authors use additive white Gaussian noise (AWGN) channel for simplicity purposes.

A noisy multilevel coded signal is received by the receiver which is demodulated and then decoded. The signal received at time $t$ may be represented as

$$
Y_{t}=Q_{t}+n_{t}
$$

The receiver extracts the information using parallel independent decoding [8]. Figure 38.2 shows the decoding procedure at the receiver. 


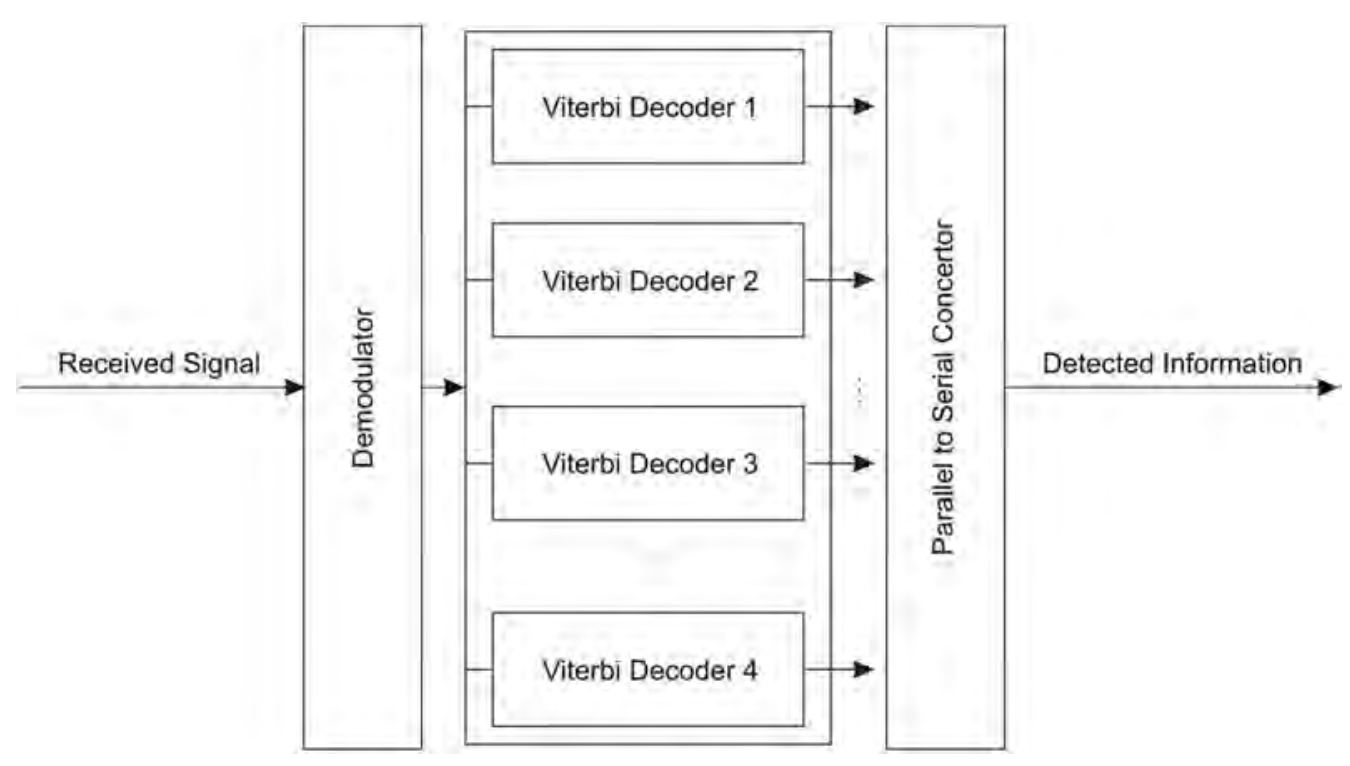

Figure 38.2 Decoding procedure at receiver.

A Viterbi decoder is used for the reliable information extraction at each stage. To determine the corresponding code-word, the Viterbi decoder performs a search to optimize the probability function. The probability function may be defined as

$$
L\left(c_{i}\right)=P\left(Y_{t} \mid c_{i}\right)=\log \left(\sum_{\substack{c_{j} \\ j=1,2, \ldots, L \\ j \neq i}} \exp \left(\frac{\left|Y_{t}-Q_{t}\right|^{2}}{2 \sigma_{n}^{2}}\right) ; i=1,2, \ldots, L\right.
$$

where $\sigma_{n}^{2}$ is the noise variance.

The information sub-streams obtained at each stage are transmitted through a serial converter to obtain the actual information.

$$
\left\{\begin{array}{llll}
\widehat{u_{1}} & \widehat{u_{2}} & \ldots & \widehat{u_{L}}
\end{array}\right\} \rightarrow \hat{u}
$$

\section{Error performance}

Figures 38.3 and 38.4 display the performance analysis of multilevel coding schemes with the codes of convolution as component codes used in Section II. The output is measured for a system which constructs data input through 4 stages. That implies there really are 4 parallel encoders. The encoders used at the different levels are encoded the codes of 


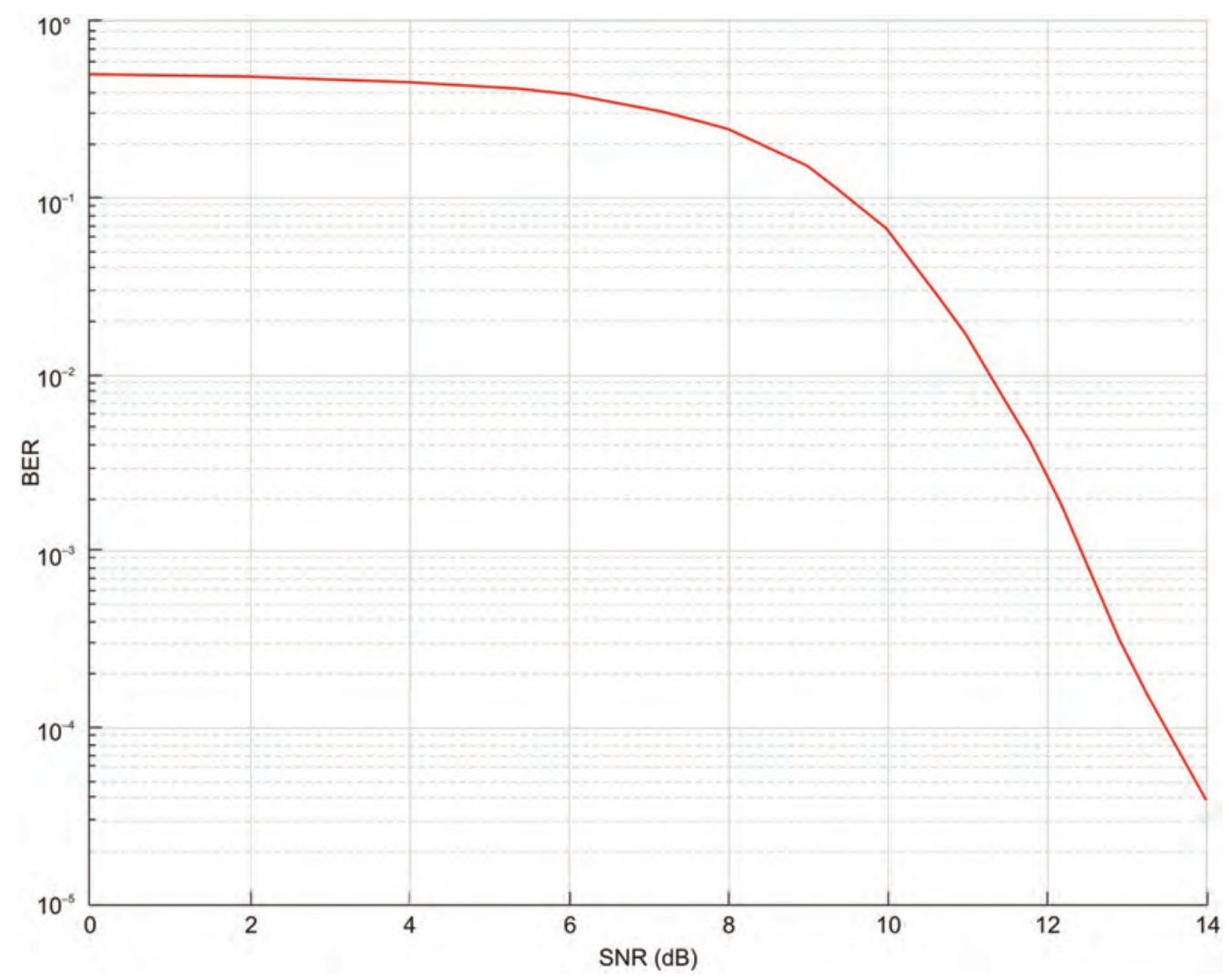

Figure 38.3 Bit error rate (BER) vs signal to noise ratio (SNR) curves for multilevel coding scheme with trellis codes as component codes.

rate $2 / 3$. For the production of part codes, the sub-information streams are recycled at their respective level via a convolutionary encoder. Using Gray mapping, codes generated for components are mapped into 16-QAM constellations. The symbols being mapped are modulated and forwarded to the receiver. The receiver demodulates the signal, and at each point the information is detected using the Viterbi decoder with parallel independent decoding. This device requires the energy of $\sim 13.5 \mathrm{~dB}$ for achieving $10^{-4}$ bit error rate (BER) and $\sim 13 \mathrm{~dB}$ for achieving symbol error rate (SER) of $\sim 10^{-3}$

\section{Conclusion}

This paper addresses a multi-level scheme for coding with convolution codes as part codes to minimize the detrimental multi-path fading effects. This scheme improves wireless communication performance by delivering increased gain in coding, spectral efficiency, data transfer rate and low complexity of decoding. The performance can further be improved if the generated codes are used in a massive MIMO (multiple input multiple output) environment. 


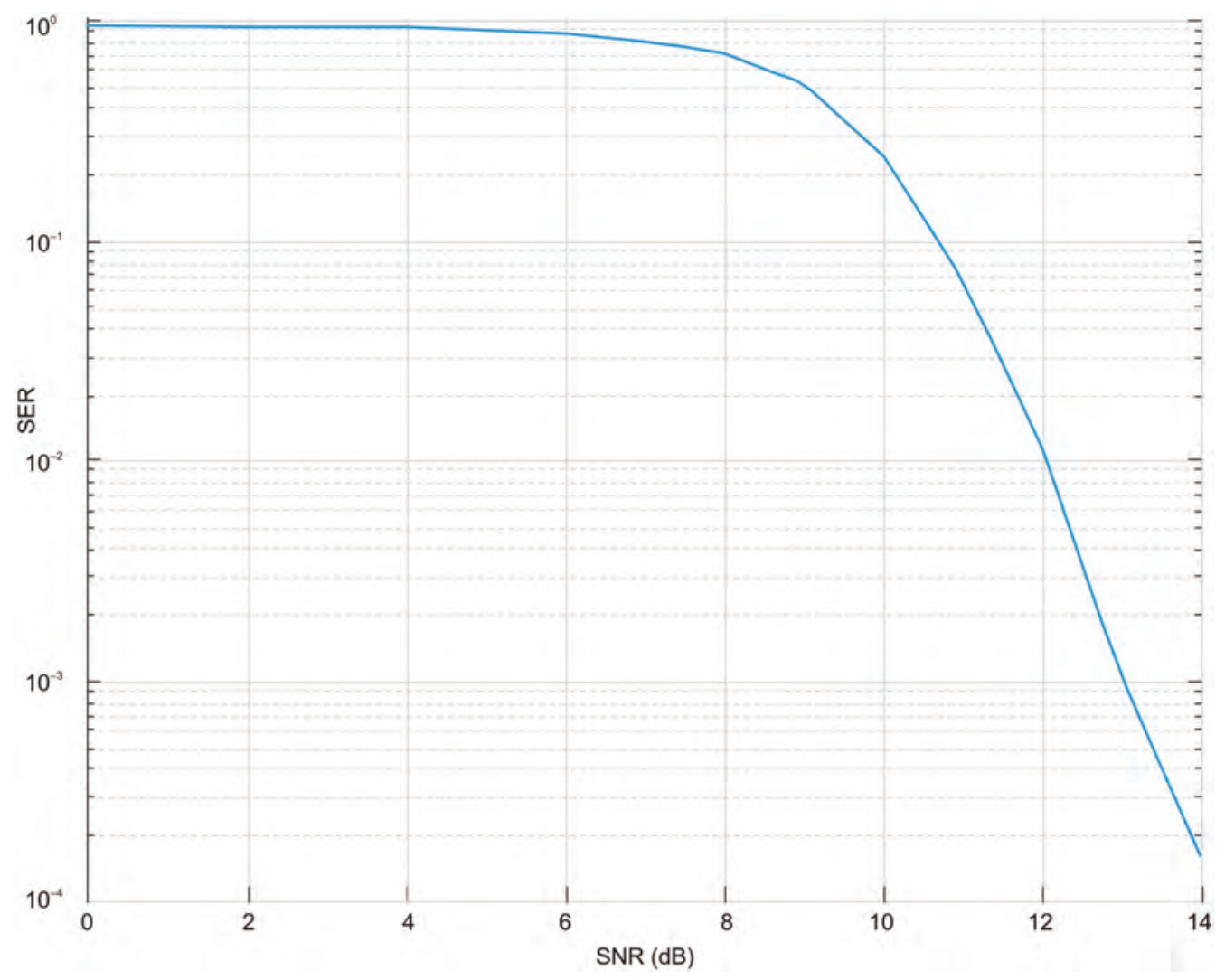

Figure 38.4 Symbol error rate (SER) vs signal to noise ratio (SNR) curves for multilevel coding scheme with trellis codes as component codes.

\section{References}

[1] Imai, H., and Hirakawa, S. (1977). A new multilevel coding method using error-correcting codes. IEEE Transactions on Information Theory, 23(3), 371-377.

[2] Ungerboeck, G. (1982). Channel coding with multilevel/phase signals. IEEE transactions on Information Theory, 28(1), 55-67.

[3] Sklar, B. (2001). Digital Communications (Vol. 2). NJ: Prentice Hall.

[4] Cheng, J.F., Chuang, C.H., and Lee, L.S. (1993, November). Complexity-reduced multilevel coding with rate-compatible punctured convolutional codes. In Global Telecommunications Conference, 1993, including a Communications Theory Mini-Conference. Technical Program Conference Record, IEEE in Houston. GLOBECOM'93, IEEE (pp. 814-18). IEEE.

[5] Kofman, Y., Zehavi, E., and Shamai, S. (1994). Performance analysis of a multilevel coded modulation system. IEEE Transactions on Communications, 42(234), 299-312.

[6] S.M. Alamouti, "A simple transmitter diversity scheme for wireless communications", IEEE Journal on Selected Areas in Communications, vol. 16, pp. 1451-1458, 1998.

[7] Isaka, M., and Imai, H. (2001). On the iterative decoding of multilevel codes.IEEE Journal on Selected Areas in Communications, 19(5), 935-943.

[8] Djordjevic, I.B., Vasic, B., and Neifeld, M.A. (2006). Multilevel coding in free-space optical MIMO transmission with Q-ary PPM over the atmospheric turbulence channel. IEEE Photonics Technology Letters, 18(13/16), 1491. 
[9] S R. Chopra, A. Gupta and H. Monga, "An LTE approach with MIMO by using suboptimal selection of antenna," 2018 International Conference on Intelligent Circuits and Systems (ICICS), Phagwara, pp. 167-172, 2018.

[10] R. Kausar, A. Gupta, I.B. Sofi and K. Arora, "Bit error rate based performance evaluation of LTE OFDMA system," 2018 International Conference on Intelligent Circuits and Systems (ICICS), Phagwara, pp. 161-166, 2018.

[11] A. Gupta, S. Dogra and I.B. Sofi, "Performance evaluation of spatial multiplexing using different modulation techniques in MIMO system for small and large scale fading channel", International Journal of Sensors Wireless Communications and Control, vol. 9(2), pp. 188202, 2019.

[12] S.R. Chopra, A. Gupta and H. Monga, "Performance Analysis of space time trellis codes in Rayleigh fading channel", in Harmony Search and Nature Inspired Optimization Algorithms, Springer, Singapore, pp. 957-967, 2019.

[13] A. Gupta and S.R. Chopra, "Designing and testing of quadrifilar helix antenna for NOAA weather satellite," 2019 6th International Conference on Signal Processing and Integrated Networks (SPIN), Noida, India, pp. 785-790, 2019.

[14] R. Devi, R.K. Jha, A. Gupta, S. Jain and P. Kumar, "Implementation of an intrusion detection system using an adaptive neuro-fuzzy inference system for $5 \mathrm{G}$ wireless communication network", AEU-International Journal of Electronics and Communications, 74, 94-106, 2017.

[15] A. Gupta and R.K. Jha, "Security threats of wireless networks: A survey," International Conference on Computing, Communication \& Automation, Noida, pp. 389-395, 2015.

[16] A. Gupta and R.K. Jha, "Power optimization using massive MIMO and small cells approach in different deployment scenarios", Wireless Networks, vol. 23(3), pp. 959-973, 2017.

[17] A. Gupta, R.K. Jha, P. Gandotra and S. Jain, "Bandwidth spoofing and intrusion detection system for multistage $5 \mathrm{G}$ wireless communication network", IEEE Trans. on Vehicular Technology, vol. 67(1), 618-632, 2017.

[18] I.B. Sofi, A. Gupta and R. Kausar, "Performance evaluation of different channel estimation techniques in MIMO system for Hata channel model," 2018 International Conference on Intelligent Circuits and Systems (ICICS), Phagwara, pp. 155-160, 2018. 


\title{
39 Optimal control of renewable energy system integrated with electric vehicle
}

\author{
Muzafar Ahmad Shah" *, Mohammad Imran Mir ${ }^{1}$, \\ Javed Dhillon ${ }^{2, *}$ \\ ${ }^{1}$ Research Scholar, School of Electronics and Electrical Engineering, \\ Lovely Professional University, Punjab, India \\ ${ }^{2}$ Assistant Professor, School of Electronics and Electrical Engineering, \\ Lovely Professional University, Punjab, India
}

\section{Introduction}

Power systems are a complex and wide electrical network which usually consist of power generator, transmission lines and distribution networks together with loads that are spread over a wide geographic area throughout the network [1]. The machine load in the power system always keeps on altering from period to period, depending on the consumer's needs. In order to uphold the trustworthiness of the power system and to ensure its stable operation, properly designed controllers are necessary to regulate system variations. The industry's rapid growth has further contributed to the power system's increased complexity. Frequency is extremely reliant on active power, and reactive energy is highly hooked on voltage. Thus, the power system governor complexity can be divided into two sections. One has to do with regulating the effective power sideways with frequency, while the other has to do with the reactive power via voltage regulation [2]. The Automatic Load Frequency Control (ALFC) is commonly known as the real power control and the other as the frequency control. The automatic LFC (ALFC) essentially contracts through the control of the generator's actual power output yield and its frequency and/or speed. The prime loop is moderately fast in one to numerous seconds where changes occur. The prime control will respond through or via the speed administrator to frequency changes and the flow of steam or hydro is controlled.

The secondary/subordinate loop is faster than the main loop. The secondary/subordinate loop ensures excellent frequency control and also maintains sufficient real exchange of energy between the majority of the pool participants. This loop is impervious to rapid fluxes in load and also in the frequency, even though it focuses on rapid changes that occur over minute periods. Load uproar due to persistent and regular load variability with lower values often creates problems for ALFC. Owing to the revolution in the real power claim load in a zone, tie line power drifts from the nearby interrelated system and changes in system speed, making the system deranged. So, we are in need of the ALFC to primarily uphold the firmness when load deviations occur. This is completed by minimalizing, apart from the power exchange, transient frequency deviations and also by keeping and making the effort to minimize steady state frequency error to very nearly zero [3]. Inequality surrounding demand generation induces variations in frequency. Now if the frequency

\footnotetext{
* Emails: shahaatiif@gmail.com, javeddah@gmail.com
}

DOI: 10.1201/9781003129103-39 
deviates and is not upheld within the prescribed values, so it can lead to lines tripping, and system collapse, and the blackouts [4].

\section{Micro-grid isolated mode}

\section{A. Micro-grid}

MGs are the systems with the distributed loads, distributed low voltage energy resources like microturbines, WTs, pvs [5]. DMS administers the power grid, and the MGDS manages the operation of the MG. Now to calculate real-time information/data of the isolators/ disconnectors, distributed generation (DG) resources, EV(s), PMUs and also loads are built on the MG [7].

\section{B. Electric-vehicle model}

Modelling EVs can be carried out with equivalent EVs with different inverter capacity, since the number of EVs in each EV station is different. Figure 39.1 illustrates the load frequency control equivalent model that may be used. As noted, the LFC signal response can be reduced by the number and convenience of the controllable EVs.

$$
E_{\text {control }}^{\min } \leq E_{\text {control }} \leq E_{\text {control }}^{\max }
$$

where $\mathrm{E}_{\text {control }}$ is the controllable EV's total energy whereas the lower energy and the higher energy limits are the $\mathrm{E}_{\min }$ controls and the $\mathrm{E}_{\max }$ controls. These limits are measured in the $[5,6]$ energy capability that depends on SOC defined.

\section{Diesel generator model}

Diesel generator (DG) has the characteristics of fast starting speed, long service [9] life and high efficiency as a small electricity generator. Within short intervals, DG can track variations in demand via power control mechanisms [10]. The DG will change its output through fuel control when demand fluctuates. The DG model for LFC is shown in Figure 39.2. The time transfer function represents the correlation between LFC and DG's output-power. This model is composed of governor and generator, which are referred to respectively by the first-order system plants [11].

DG is a limited power generating system with structures of fast opening speed, toughness and high effectiveness. In short intervals of time [12], DG may obey changes in load demand through mechanisms for power control. The DG adjusts performance via the fuel control when the demand for energy fluctuates.

The figure shows the DG template for LFC's continuous time transfer feature. The figure shows the association [13] amongst the LFC signal and the DG output yield power. While the DG stands for the increase in output power, i.e. $\Delta \mathrm{P}_{\mathrm{DG}}=0$ is that condition/ state for which the generation of the DG is pointed to a value that only balances [14] generation-generation and grid load [15]. The frequency deviation is as such zero under such conditions, i.e. all $\Delta \mathrm{f}=0$. Eventually, the power balance of grid decides this certain power level [16-20]. The reference to DG indicates that the power generated by DG is greater than needed, and the opposite indicates that the power generated by DG is lower than the actual power required [7]. 


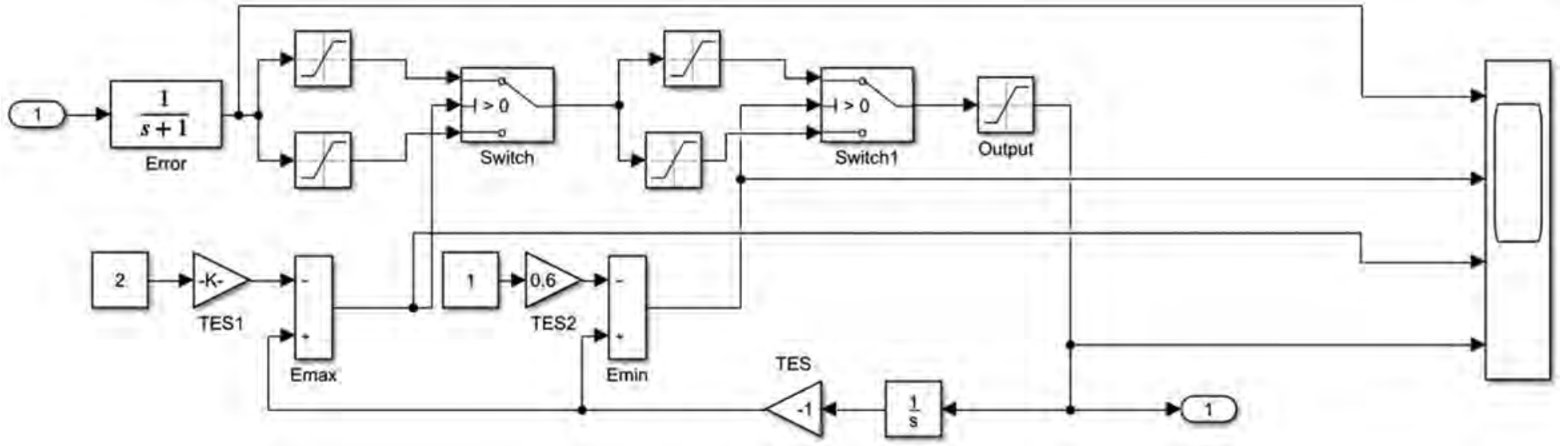

Figure $39.1 \mathrm{EV}$ model for LFC. 


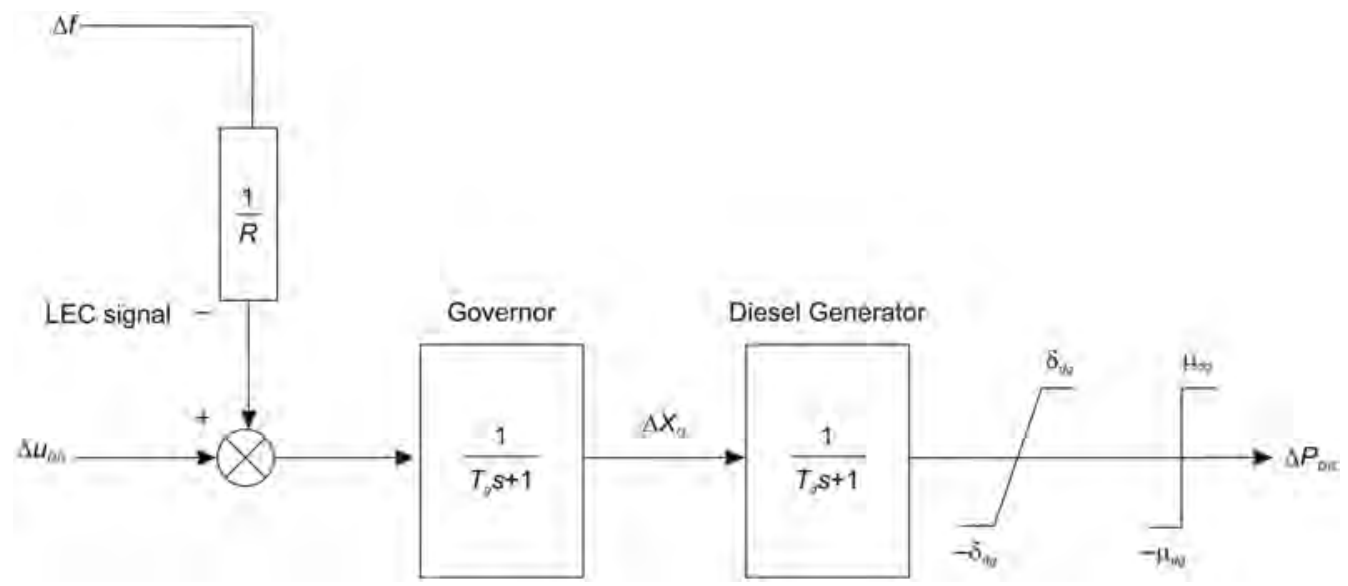

Figure 39.2 DG transfer function model.

\section{Wind turbine model}

A large part of renewable energy is produced by wind power and is steadily increasing as the generation cost is attractive. Wind energy costs can be lower than most other available technologies for electricity generation. Nevertheless, unfortunately, wind generators do not support the system. There will therefore be stability issues if more and more wind generators are added to the national grid.

Wind turbines extract kinematic wind energy and turn it into useful mechanical energy. These are designed to capture as much wind energy as possible for the generation of power by maximizing the number and speed of the turbine rotor blades.

Kinetic energy is the source of power found in wind and can be expressed by the following equation.

$$
P_{w}=0.5 \rho A V^{3}
$$

where $\rho, \mathrm{A}$ and $\mathrm{V}$ are the density of air, normal area to the speed of wind and velocity of wind expressed in $\mathrm{kg} / \mathrm{m}^{3}, \mathrm{~m}^{2}$ and $\mathrm{m} / \mathrm{s}$, respectively.

The wind data here is taken from the hourly wind data for Kutch, India. The respective wind power output for each hour is being given to the grid through lookup table as shown in Figure 39.3.

\section{E. Micro-grid scheme}

In Figure 39.4 the LFC and the isolated MG proposed, consisting of a generic DEG, WTG and EV, are illustrated. Power electronic interfaces for synchronization of DEG and WTG sources, and for reversing dc voltage, PV panels, FCs, and energy-storage systems, connects the DGs to this MG $[5,11]$. Each micro source has a circuit breaker that is designed for the disconnection of the network and for main purposes avoids the effects of severe disturbances through the MG. The DEG produces a certain amount of compulsory power for the secondary frequency control that is considered to be the spin reservoir [20]. 


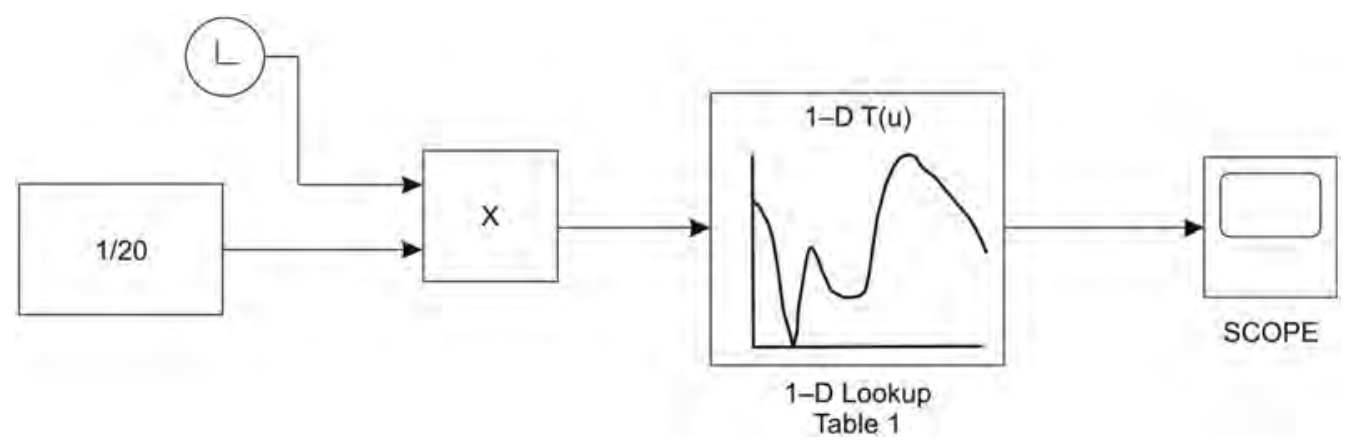

Figure 39.3 Wind data MATLAB model.

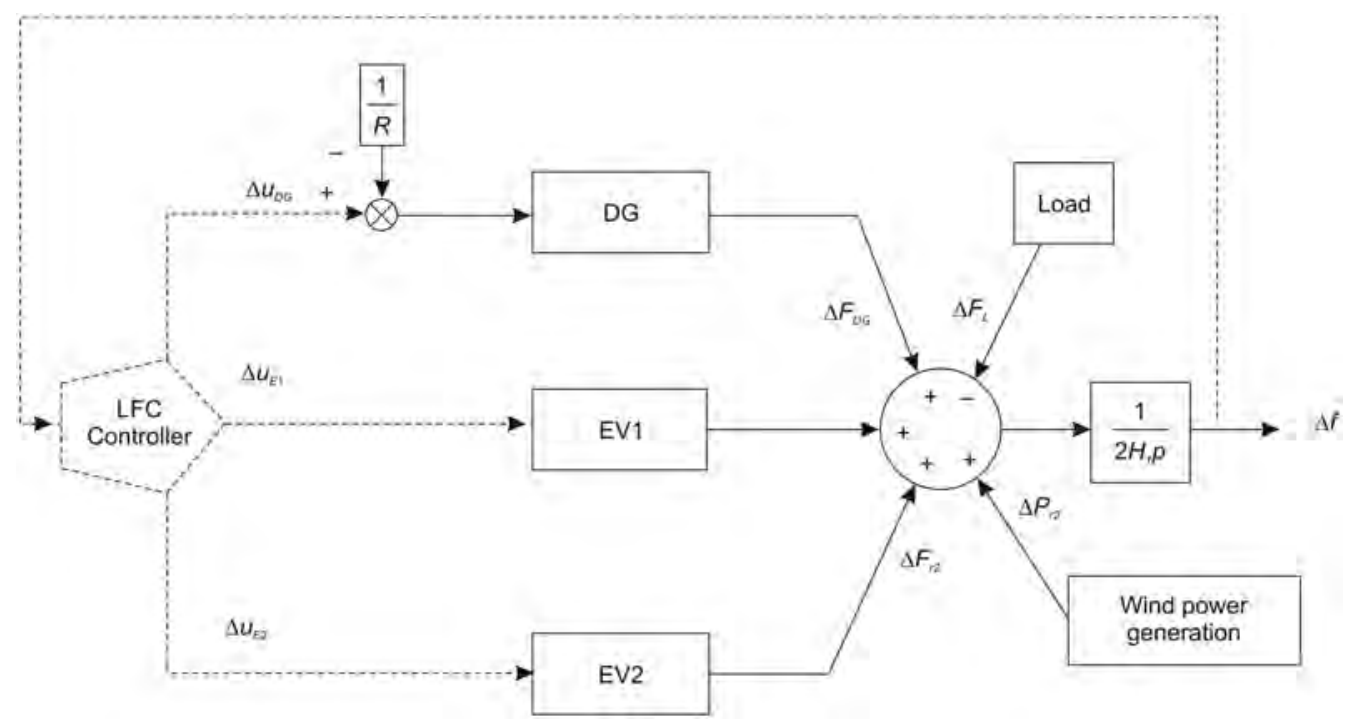

Figure 39.4 LFC with control model of micro-grid.

\section{Optimization technique used}

The controllers should be regularly replaced so as to achieve the required performance. In order to achieve better performance, many new fine-tuning approaches were proposed. A robust approach based on particle swarm optimization (PSO) is used to avoid the retuning of the PID controller. PSO is using information from swarms. The approach was inspired by the social interaction, animal behaviours seen among birds and fish.

The swarm intelligence (SI) is based on the PSO algorithm. The approach was inspired by studying social interaction, animal behaviours seen between birds, fish, etc. PSO follows the process found in fish, where by competing and cooperating with each other they seek food. The swarm has so-called elements in which each unit represents different possible collections of unknown constraints that should be optimized. Typically, a "swarm" is prepared by a random solution population. Particles travel in a randomly 
multi-dimensional search area in this process. It continues to change its location to its own understanding and also to understand its neighbouring particle's experience. The particle's aim is to look for a solution to accomplish this very effectively. The elements swarm and move to the finest function called fittingfunction. Then it congregates to a solution of one min or so. A function is used to evaluate swarm performance, so we can find out if the best solution has been achieved as shown in Figure 39.5.

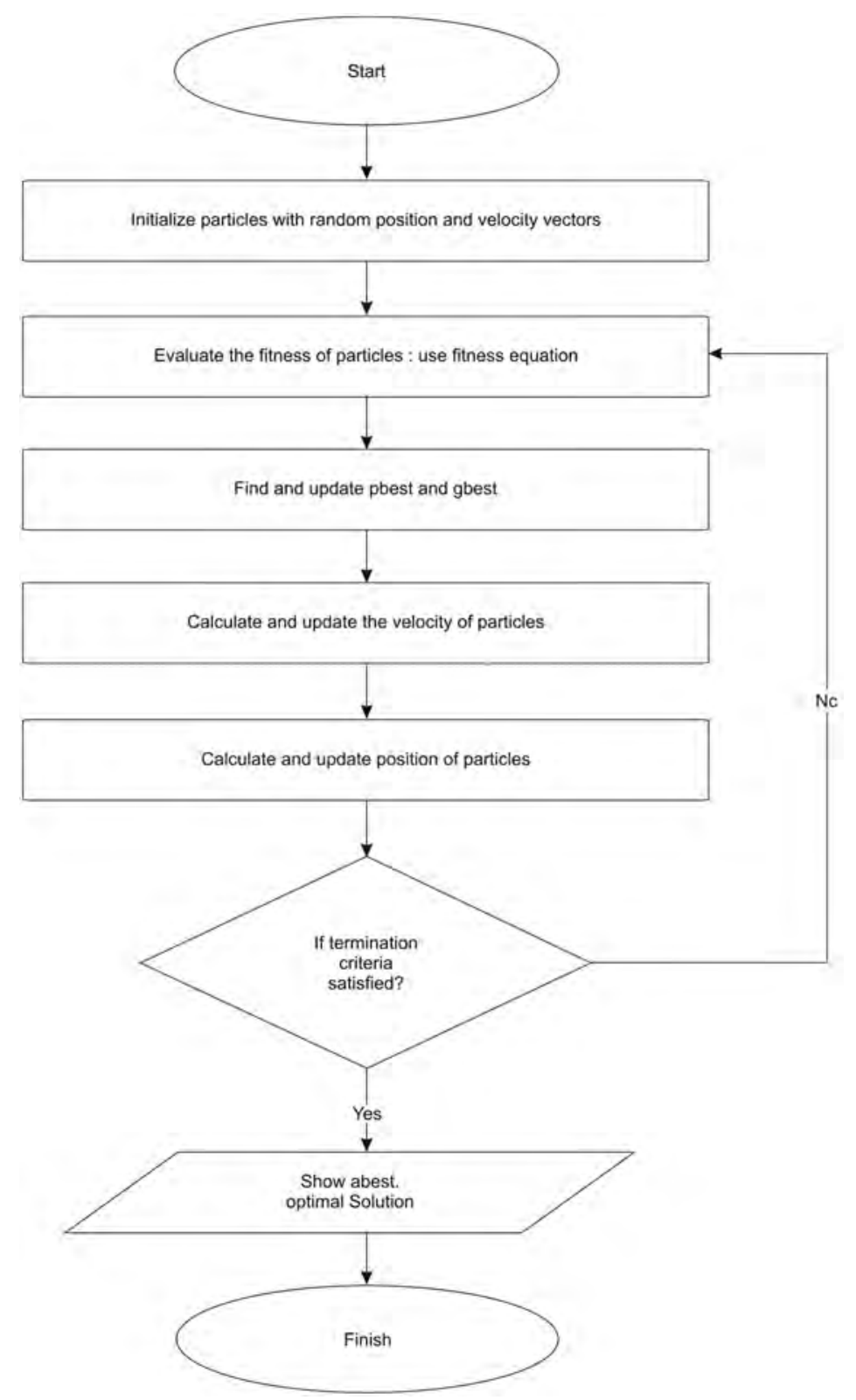

Figure 39.5 Flowchart of PSO algorithm. 


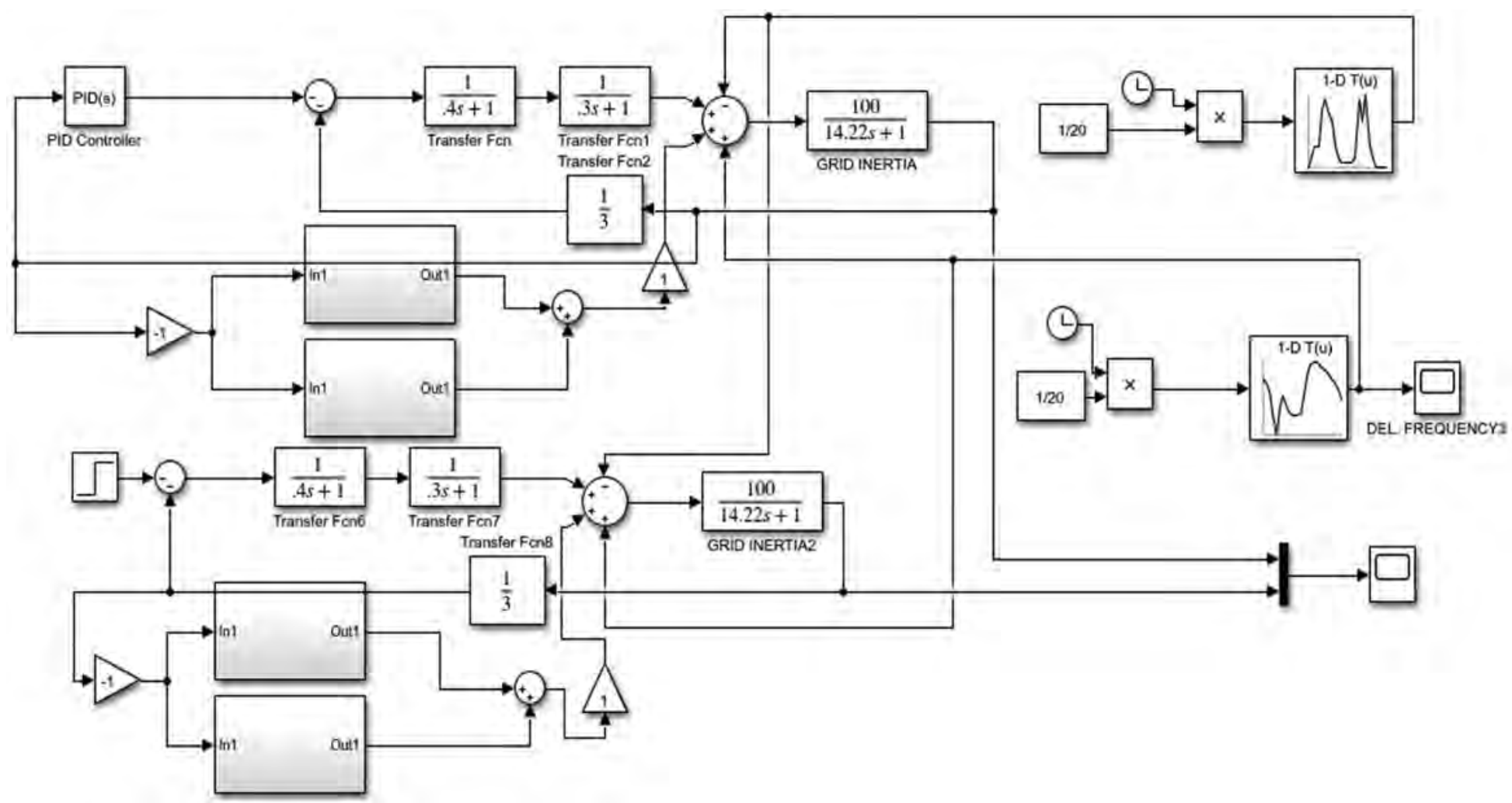

Figure 39.6 MATLAB Simulink model. 


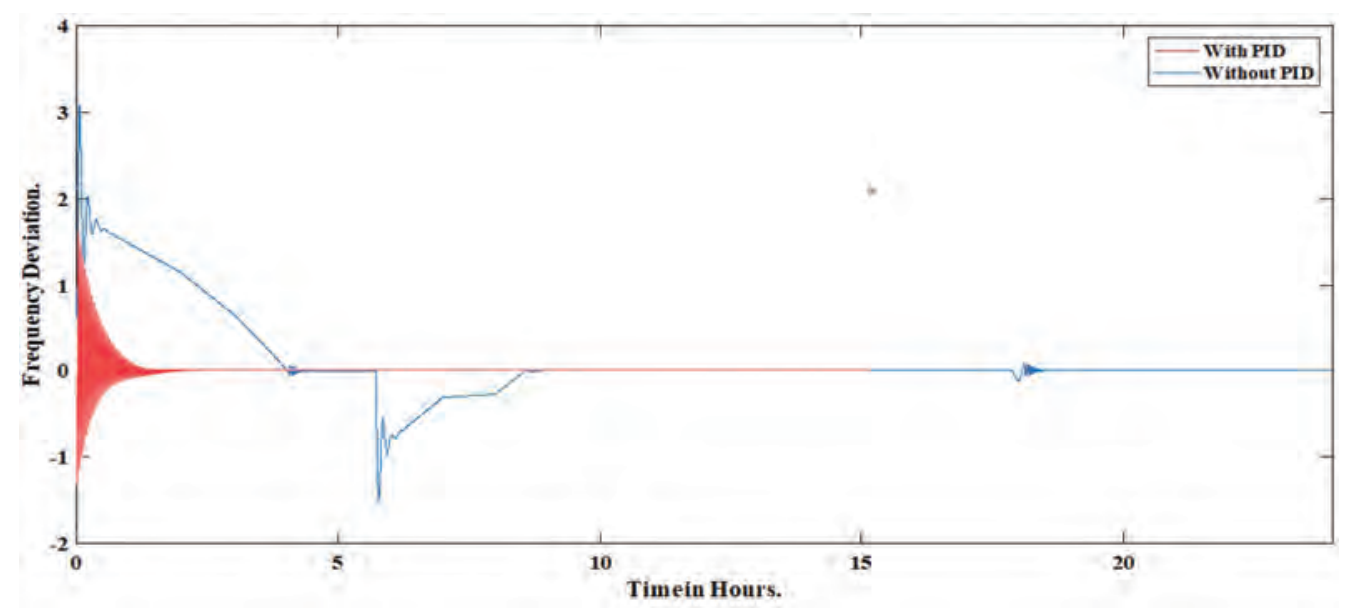

Figure 39.7 Frequency deviation with and without PID.

\section{Results and disucssion}

In order to inspect the presentation a well-tuned PID controller/supervisor has been premeditated in the simulation software. The classical system constraints are provided and the parameters of controllers are presented.

The MATLAB Simulink model for the proposed work is shown in Figure 39.6, where an electric vehicle is integrated with an existing system. The variable load disturbance is applied via lookup table. The results for the change in the frequency are analysed for different cases as shown in Figures 39.7 and 39.8. The results clearly show the difference in the frequency deviations for the systems with and without PID controller. The frequency deviations are supressed quite efficiently by the use of the proposed controller. The same can be seen from the results shown in Figure 39.7.

The MG unit in this chapter is simulated to monitor the performance and the operation of implemented controller in the MATLAB Simulink environment. Compared to optimal PID controllers, the performance was obtained. In order to represent a fair comparison, altogether parameters of these control systems are optimized using the PSO optimization technique.

\section{Conclusion}

LFC is one of the important and leading aspects when it comes to the hybrid grid model. The existing grid incorporated with the wind power is integrated with electric vehicles which will act as the alternative sources of energy. A PID controller is being used for the minimization of the power and frequency deviations. The proposed methodology which integrates electric vehicles with the existing grid system was quite efficient in LFC control. The same can be verified from the above results which clearly show that the frequency deviations are efficiently suppressed with the use of controller. Wind power variations w.r.t. speed of wind can be seen from the graph. The results were taken for a period of 24 hours. 


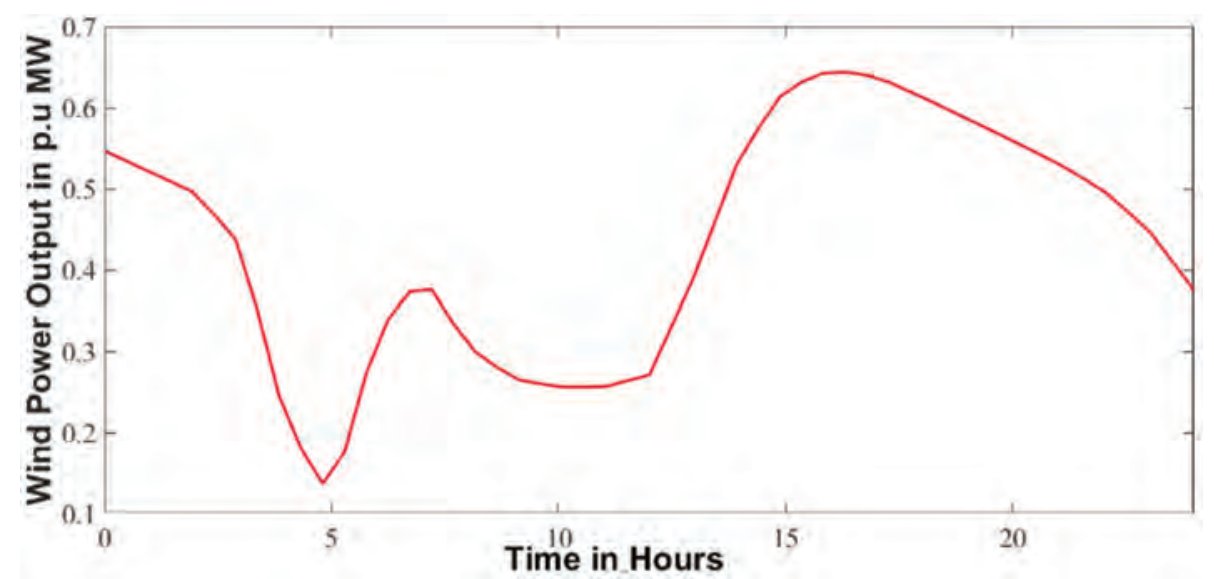

Figure 39.8 Wind power output.

\section{References}

[1] Yao Zang, "Load frequency control of multiple-area power systems”, Tsinghua University, July 2007. Master of science in Electrical Engineering.

[2] I. J. Nagrath and D. P. Kothari, Modern power system analysis-TMH 1993.

[3] Elgerd Ol. Electric Energy Systems Theory - An Introduction, 2nd edn. Tata McGrawHill, 2000.

[4] Greg Turner, Kelley Jay P, Storm Caroline L, Wetz DA, Lee W-J. Design and active control of a microgrid testbed. IEEE Trans Smart Grid 2015;6(1):73-81.

[5] Khalghani, Reza Mohammad, Khooban Mohammad Hassan, Mahboubi- Moghaddam Esmaeil, Vafamand Navid, Goodarzi Mohammad. A self-tuning load frequency control strategy for microgrids: human brain emotional learning. Int J Electr Power Energy Syst 2016;75:311-9.

[6] Mojica-Nava, Eduardo, Macana Carlos Andrés, Quijano Nicanor. Dynamic population games for optimal dispatch on hierarchical microgrid control. Syst Man Cybern: Syst IEEE Trans 2014;44(3):306-17.

[7] Jun Yang, Zeng Zhili, Tang Yufei, Yan Jun, He Haibo, Wu Yunliang. Load frequency control in isolated micro-grids with electrical vehicles based on multivariable generalized predictive theory. Energies 2015;8(3):2145-64.

[8] Zheng S, Tang X, Song B, Lu S, Ye B. Stable adaptive PI control for permanent magnet synchronous motor drive based on improved JITL technique. ISA Trans 2013;52(4):539-49.

[9] Bevrani H, Habibi F, Babahajyani P, Watanabe M, Mitani Y. Intelligent fre quency control in an ac microgrid: online PSO-based fuzzy tuning approach. IEEE Trans Smart Grid 2012;3(4):1935-44.

[10] Shiqi Zheng, Tang Xiaoqi, Song Bao, Lu Shaowu, Ye Bosheng. Stable adaptive PI control for permanent magnet synchronous motor drive based on improved JITL technique. ISA Trans 2013;52(4):539-49.

[11] Yesil Engin. Interval type-2 fuzzy PID load frequency controller using Big Bang-Big Crunch optimization. Appl Soft Comput 2014;15:100-12.

[12] Pahasa Jonglak, Ngamroo Issarachai. Coordinated control of wind turbine blade pitch angle and PHEVs using MPCs for load frequency control of microgrid. IEEE Syst J 2014;99:1-9.

[13] Mahto Tarkeshwar, Mukherjee Vivekananda. Quasi-oppositional harmony search algorithm and fuzzy logic controller for load frequency stabilisation of an isolated hybrid power system. IET Gener Transm Distrib 2015;9(5):427-44. 
[14] Singh Vijay P, Mohanty Soumya R, NandKishor, Ray Prakash K. Robust H- infinity load frequency control in hybrid distributed generation system. Int J Electr Power Energy Syst 2013;46:294-305.

[15] Lee Dong-Jing, Li Wang. Small-signal stability analysis of an autonomous hybrid renewable energy power generation/energy storage system part I: time-domain simulations. IEEE Trans Energy Convers 2008;23(1):311-20.

[16] S. Dhundhara and Y. P. Verma, Capacitive energy storage with optimized controller for frequency regulation in realistic multisource deregulated power system, Energy 2018.

[17] Ghaemi Mostafa, Hosseini-Sani Seyyed Kamal, Khooban Mohammad Hassan. Direct adaptive general type-2 fuzzy control for a class of uncertain non-linear systems. IET Sci Meas Technol 2014;8(6):518-27.

[18] Khooban Mohammad Hassan, Niknam Taher, Sha-Sadeghi Mokhtar. A time- varying general type-II fuzzy sliding mode controller for a class of nonlinear power systems. J Intell Fuzzy Syst 2015:1-11.

[19] Niknam Taher, Khooban Mohammad Hassan, Kavousifard Abdollah, Soltan- pour Mohammad Reza. An optimal type II fuzzy sliding mode control design for a class of nonlinear systems. Nonlinear Dyn 2014;75(1-2):73-83.

[20] Khooban Mohammad Hassan, Alfi Alireza, Abadi Davood Nazari Maryam. Control of a class of non-linear uncertain chaotic systems via an optimal Type- 2 fuzzy proportional integral derivative controller. IET Sci Meas Technol 2013;7(1):50-8.

[21] R. K. Selvaraju and G. Somaskandan, Acs algorithm tuned anfis-based controller for lfc in deregulated environment, J Appl Res Technol 2017;15(2):152-166. 


\title{
40 Energy efficient concurrent MAC transmission based UWASN for tsunami warning
}

\author{
Vikram Nayyar ${ }^{1 * *}$, Krishan Kumar², Monika Agrawal ${ }^{3}$
}

\author{
${ }^{1}$ Research Scholar, SEEE, LPU, Punjab, India \\ ${ }^{2}$ Professor, SEEE, LPU, Punjab, India \\ ${ }^{3}$ Professor, CARE, IIT, Delhi, India
}

\section{Introduction}

Tsunami is among the deadliest disasters that claim several thousands of lives. Geological event monitoring is the key to predict tsunami. Currently the tsunami prediction methods utilize terrestrial or cabled underwater systems for the parameter monitoring. Some of the parameters can also be collected from the different layers in the atmosphere. As the occurrence of tsunami is usually an underwater event terrestrial monitoring is not capable of detecting all the detection parameters. Moreover the oceans are vast and hostile. Therefore underwater cables become unreliable, complicated and expensive. There is a need for improved method for monitoring of tsunami events. The interplate joints, landslide prone areas and volcanoes are the major causes of tsunami. These sources mostly prevail in seafloor of the oceans (Nayak and Kumar 2008; Barrick 1979; Chatfield and Brajawidagda 2013; Joseph 2011; Penteado et al. 2010).

For real-time monitoring of tsunami UWASN is essential. It is the most capable wireless method to collect different types of data from the oceans. Among various waves for communication acoustic waves have the least absorption in underwater conditions. Thus acoustic wave is preferred medium for wireless communication. The SNs can be deployed randomly or fixed with tripods or anchors (Xiao 2010; Petroccia et al. 2018; Han et al. 2013; Alfouzan et al. 2019; Sandeep and Kumar 2017).

\section{Related work}

A random deployment of UWASN is considered to consist of a number of depth based layers. The depth of a layer is contained within the node transmission range. As the propagation delay of the acoustic waves is large this allows simultaneous transmission of the nodes separated by considerable layers. Similarly the nodes separated by certain hops within the same layer transmit simultaneously. This considerably improves the throughput and network delay. The network lifetime is reduced as the upper layers are required to receive considerable data from the lower layers (Alfouzan et al. 2019).

The grid division of the network is proposed for efficient routing. The nodes compute the coordinates on the basis of a localization scheme. The nodes within the grid compete to become CHs. The $\mathrm{CH}$ s forward to the $\mathrm{CH}$ coordinates that are closer to the sink. Depending on the mobility of the nodes the localization and clustering is periodically

\footnotetext{
*Email: vikramnayyar@live.com
}

DOI: 10.1201/9781003129103-40 
required. As the sink is required to be capable of transmitting to every node the topology cannot have wider seafloor coverage (Wang et al. 2016).

Seismic monitoring of the seabed can be accomplished with 1-hop and 2-hop sensor network. On the basis of the propagation delay of the sensor nodes the sink node allots time slots for data accumulation. The sensor nodes transmit on command of sink node. The 1-hop network is simple and demands lower computational capability of the sensor nodes. The simpler computation in sensor nodes reduces the network cost and energy consumption. The 2-hop network increases the seabed coverage of the network. The 1-hop nodes accumulate the data from 2-hop nodes. For data collection the data accumulating nodes use the similar scheme as sink node. This causes hotspot problems at the data accumulating nodes to reduce the network lifetime. With increased number of nodes the overall delay in the monitoring also increases (Morozs et al. 2017; 2019).

Inherently UWASNs possess long propagation delays and small bandwidth. Moreover the energy of the SNs is hard to replenish (Zhang et al. 2018). Therefore we propose energy efficient concurrent transmission UWASN for real-time monitoring of underwater tsunami parameters. Unlike the methods proposed for seafloor monitoring the transmission range within the cluster is significantly smaller. This conserves the energy of the cluster. The following tsunami parameters can be measured with underwater sensors:
a. Electromagnetic currents
b. Surface waves
c. Temperature and pressure
d. Water current

Monitoring these parameters requires the deployment of underwater sensors. With the existence of marine life and deep water currents the accuracy of monitoring can degrade (Batsi et al. 2019). Therefore a number of distributed sensors are proposed to be deployed in large expanse of the underwater environment. The larger number of sensors also increases the monitoring area to improve tsunami analysis. On alarming of significant number of sensors the alarm is raised.

\section{Network topology}

As shown in Figure 40.1 a two-hop network consisting of a sink node, CHs and SNs is considered. The sink node can be suspended from a ship or a buoy at a depth of about 5 $\mathrm{m}$. CH and SNs are placed on the seabed using anchors. This fixes node positions to eliminate periodic localization and clustering requirement. $\mathrm{CH}$ is at one-hop distance from the sink node. The depth of the seabed is about $5 \mathrm{~km}$. The distance between the SNs and $\mathrm{CH}$ is within $500 \mathrm{~m}$. This gave good results in a one-hop separation. On this basis the circular coverage on the seabed can have a diameter of about $2 \mathrm{~km}$. Our method has following considerations:

a. The position of the sink node is maintained at about the centre of the seabed coverage. The anchored $\mathrm{CH}$ s and SNs have fixed positions.

b. As the energy consumption of $\mathrm{CH}$ is significantly higher it is allotted additional energy resource. The network has dedicated $\mathrm{CHs}$ that perform data accumulation only.

c. On the basis of propagation distance the SNs adjust their transmit power. This conserves the energy and reduces interference.

d. CHs have a transmission of about $6 \mathrm{~km}$. Thus they can broadcast to all the SNs in the network. 


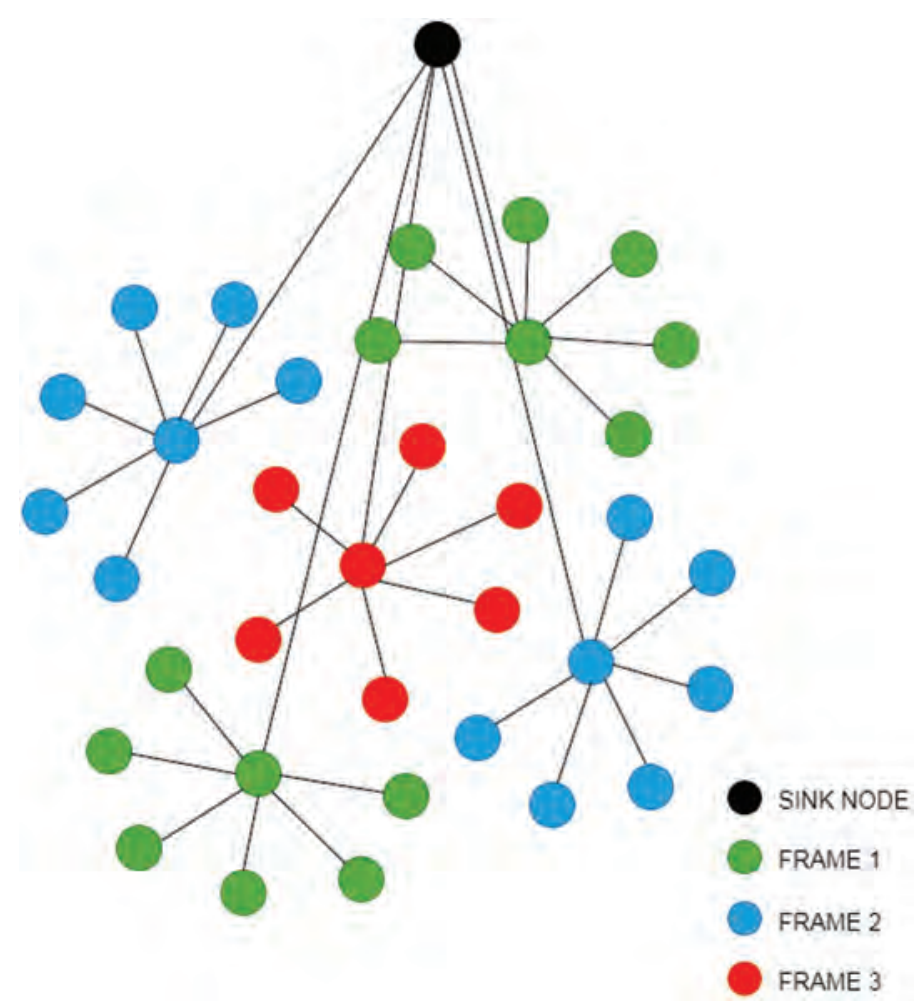

Figure 40.1 Proposed network topology.

\section{Frame allotment}

Five CHs collect the data from the SNs. This is shown in Figure 40.2. CH using frame 3 is at the centre of the circular coverage. Thus it forms a circular cluster. It has the shortest distance to the sink. Frame 1 and frame $2 \mathrm{CHs}$ are placed at the edge of the cluster to form minimum angle to the sink. This improves the connectivity to the sink with uniform circular coverage.

To reduce the network delay we propose frame sharing among the opposite clusters. First two frames are allotted to two pairs of opposite clusters. Third frame is allotted to the central cluster. As the central cluster has the largest number of nodes it is allotted the last frame. This improves the network fairness. With more nodes frame 3 is larger in comparison to other frames.

Dynamic time frame for $\mathrm{CHs}$ is computed according to the average packet length. Average packet length $l_{\text {avg }}$ of a $\mathrm{CH}$ is given as

$$
l_{\text {avg }}=\frac{l_{1}+l_{2}+\ldots \ldots \ldots+l_{n}}{n}
$$

$n$ is the number of cycles taken for average and $l_{1}, l_{2}, l_{3}, \ldots, l_{n}$ are the individual packet lengths for cycles $1,2,3, \ldots \ldots, n$, respectively. The duration of $\mathrm{CH}$ frame is calculated as 


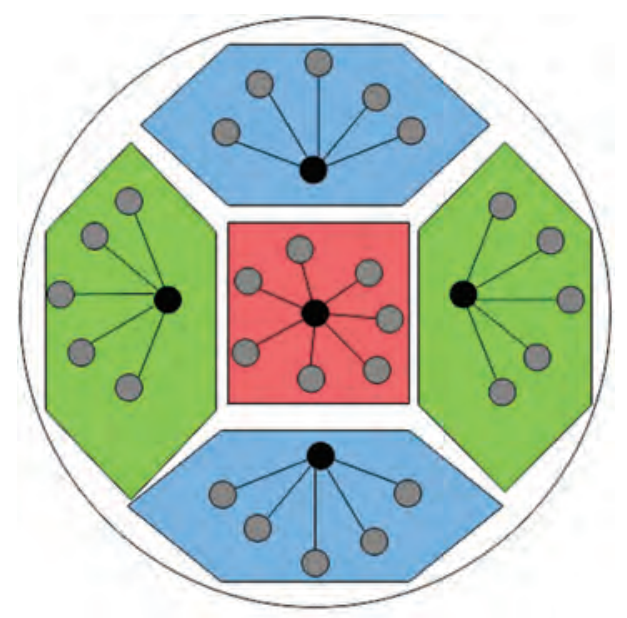

Figure 40.2 Top view of network topology.

$$
t_{s}=l_{\text {avg }}+2 l_{b}+\Phi
$$

$l_{b}$ is the length of stored buffer data of $\mathrm{CH}$. $\Phi$ is the time slot allowance to prevent buffer overloading. This is obtained as

$$
\Phi=\frac{3\left(l_{\max }-l_{\text {avg }}\right)}{2}
$$

$l_{\max }$ is the maximum packet length in the duration of $n$ packet cycles. $\Phi$ and $\mathrm{l}_{\mathrm{b}}$ ensure the contention of $\mathrm{CH}$.

The duration of the dynamic slot for $\mathrm{CH}$ pair is equal to the slot duration of $\mathrm{CH}$ with greater average traffic. Therefore the dynamic slot for $\mathrm{CH}$ pair is determined as

$$
t_{\text {pair }}=\max \left(t_{s 1}, t_{s 2}\right)
$$

$t_{s 1}$ and $t_{s 2}$ are the respective data slot durations of individual clusters in the pair.

$\mathrm{CHs}$ broadcast $t_{\text {pair }}$ at the beginning of the data frame. This enables the adjoining clusters to set their sleep cycle. The SN joining request is also mentioned using this broadcast message. SNs in the adjoining cluster are requested to join on the basis of cluster pair balance. The cluster pair balance is the difference of time slot durations of the $\mathrm{CH}$ pair. This is computed as

$$
\Delta t_{s}=\left|t_{s 1}-t_{s 2}\right|
$$

The difference is compared with the threshold to determine the condition

$$
\Delta t_{s}>t_{t h}
$$




\section{8}

$t_{t h}$ is the threshold of the difference in $\mathrm{CH}$ time slots. If the condition holds then the $\mathrm{CH}$ with smaller time slot requests the SNs in the adjoining $\mathrm{CH}$ pair to join cluster. The SNs receiving the message determine the distance to the $\mathrm{CH}$ and $\Delta t_{s}$ of their existing pair. SNs acknowledge before the data frame end of requesting $\mathrm{CH}$. Due to reduced traffic $\mathrm{CH}$ is idle at this duration. The requesting $\mathrm{CH}$ receives the acknowledgements and selects the closest $\mathrm{SN}$ to be part of the cluster. It can accept multiple SNs based on the pair balance. $\mathrm{CH}$ responds with assigning data slot to the SNs in the next cycle. Thus the unused frame portion is optimally utilized.

\section{Data transmission}

SNs are allotted transmission slots in round robin. $\mathrm{CH}$ performs the allotment of data slots. The SNs with different propagation delays and at opposite ends of $\mathrm{CH}$ use the same data slot. This is shown in Figure 40.3. As node 2 and node 4 have different propagation delays they concurrently transmit to the $\mathrm{CH}$. Similarly, node 1 and node 3 transmit concurrently. The difference in the propagation delays of the nodes in the pair is

$$
\tau_{d}=\left|\tau_{1}-\tau_{2}\right|
$$

$\tau_{1}$ and $\tau_{2}$ are the propagation delays of respective $\mathrm{SNs}$ to $\mathrm{CH}$. The pair is determined on the basis of $\tau_{d}$. The transmitter adjusts the transmit power on the basis of the propagation distance. This reduces the interference and allows concurrent transmission within the cluster.

\section{Conclusion}

We described the monitoring arrangement for principal tsunami regions. Due to remote environment and immenseness of the oceans such regions are not being monitored in real time. The transmission requirement of the sensors is significantly less than $\mathrm{CH}$. Thus the topology conserves the energy and allows the network to be dense at the seabed. The proposed network is also capable to stretch to deep seafloor. The wireless communication enables the data to be monitored in real time.

The data collection can aid to reveal more information and improve tsunami predictions. There is a significant improvement in delay as the $\mathrm{CH}$ pair accumulates the data concurrently. Moreover concurrent transmission within the cluster reduces the network delay and improves the energy consumption. The larger propagation delay allows a number of nodes to transmit concurrently. So the warning is raised immediately.

Dedicated CHs eliminate the need of cluster formation and updating. This reduces the computational requirement of the nodes. The cost of the nodes and energy consumption is reduced.

\section{References}

1. Alfouzan, Faisal Abdulaziz, Alireza Shahrabi, Seyed Mohammad Ghoreyshi, and Tuleen Boutaleb. 2019. "An energy-conserving collision-free Mac protocol for underwater sensor networks.” IEEE Access 7: 27155-71. https://doi.org/10.1109/ACCESS.2019.2901646.

2. Barrick, Donald E. 1979. "A coastal radar system for tsunami warning." Remote Sensing of Environment 8 (4): 353-58. https://doi.org/10.1016/0034-4257(79)90034-8. 


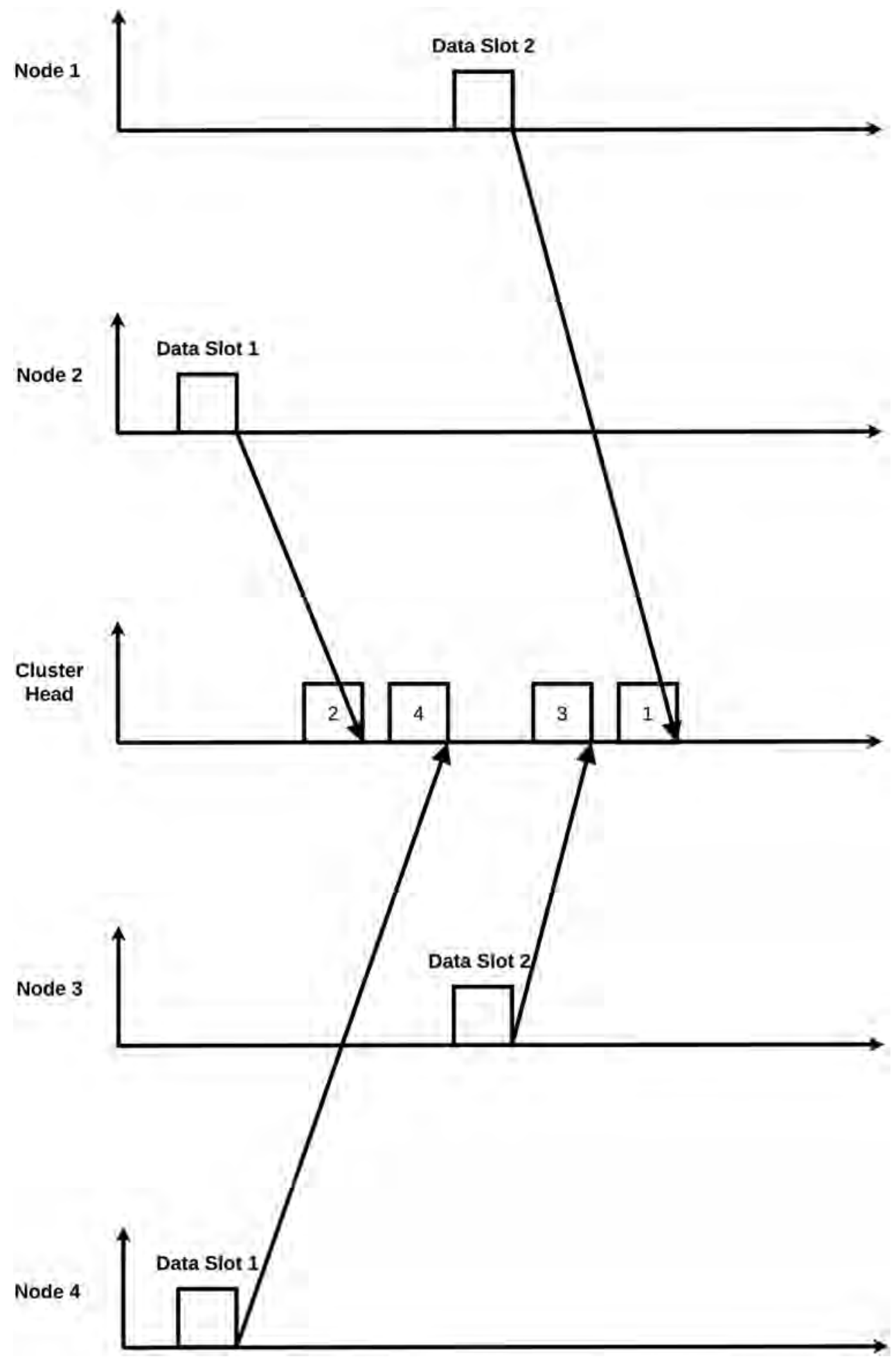

Figure 40.3 Concurrent transmission of nodes to $\mathrm{CH}$. 
3. Batsi, Evangelia, Eve Tsang-Hin-Sun, Frauke Klingelhoefer, Gaye Bayrakci, Emmy TY Chang, Jing-Yi Lin, David Dellong, Clément Monteil, and Louis Géli. 2019. "Nonseismic signals in the ocean: indicators of deep sea and seafloor processes on ocean-bottom seismometer data." Geochemistry, Geophysics, Geosystems 20 (8): 3882-3900. https://doi.org/10.1029/ 2019 GC008349.

4. Chatfield, Akemi Takeoka, and Uuf Brajawidagda. 2013. "Twitter early tsunami warning system: a case study in Indonesia's natural disaster management." Proceedings of the Annual Hawaii International Conference on System Sciences, 2050-60. https://oi.org/10.1109/ HICSS.2013.579.

5. Han, Guangjie, Chenyu Zhang, Lei Shu, Ning Sun, and Qingwu Li. 2013. "A survey on deployment algorithms in underwater acoustic sensor networks." International Journal of Distributed Sensor Networks 9 (12): 314049. https://doi.org/10.1155/2013/314049.

6. Joseph, Antony. 2011. "Tsunamis: detection, monitoring, and early-warning technologies." Choice Reviews Online 49 (04): 49-2078-49-2078.

7. Morozs, Nils, Paul D. Mitchell, and Yuriy Zakharov. 2019. "Dual-hop TDA-MAC and routing for underwater acoustic sensor networks." IEEE Journal of Oceanic Engineering 44 (4): 865 80. https://doi.org/10.1109/JOE.2019.2933130.

8. Morozs, Nils, Paul Mitchell, and Yuriy V. Zakharov. 2017. "TDA-MAC: TDMA without clock synchronization in underwater acoustic networks." IEEE Access 6: 1091-1108. https:// doi.org/10.1109/ACCESS.2017.2777899.

9. Nayak, Shailesh, and T. Srinivasa Kumar. 2008. "Indian tsunami warning system." The International Archives of the Photogrammetry, Remote Sensing and Spatial Information Sciences, Beijing 37 (1): 1501-6.

10. Penteado, D., L.H.M.K. Costa, and A.C.P. Pedroza. 2010. "Deep-ocean data acquisition using underwater sensor networks." In The Twentieth International Offshore and Polar Engineering Conference. International Society of Offshore and Polar Engineers.

11. Petroccia, Roberto, Jan Sliwka, Alberto Grati, Vittorio Grandi, Piero Guerrini, Andrea Munafò, Marin Stipanov, João Alves, and Robert Been. 2018. "Deployment of a persistent underwater acoustic sensor network: the CommsNet17 experience.” In OCEANS-MTS/IEEE Kobe Techno-Oceans (OTO), 1-9. IEEE. https://doi.org/10.1109/OCEANSKOBE.2018.8559262.

12. Sandeep, D.N., and Vinay Kumar. 2017. "Review on clustering, coverage and connectivity in underwater wireless sensor networks: a communication techniques perspective." IEEE Access 5: 11176-99. https://doi.org/10.1109/ACCESS.2017.2713640.

13. Wang, Kun, Hui Gao, Xiaoling Xu, Jinfang Jiang, and Dong Yue. 2016. “An energy-efficient reliable data transmission scheme for complex environmental monitoring in underwater acoustic sensor networks." IEEE Sensors Journal 16 (11): 4051-62. https://doi.org/10.1109/ JSEN.2015.2428712.

14. Xiao, Yang, ed. 2010. Underwater Acoustic Sensor Networks. CRC Press. https://doi.org/ $10.1201 / 9781420067125$.

15. Zhang, Rongqing, Xilin Cheng, Xiang Cheng, and Liuqing Yang. 2018. "Interference-free graph based TDMA protocol for underwater acoustic sensor networks.” IEEE Transactions on Vehicular Technology 67 (5): 4008-19. https://doi.org/10.1109/TVT.2017.2778752. 


\title{
41 Comparative analysis of congestion pricing using application of PTDF method and Bialek's method in Indian Utility 146-Bus system
}

\author{
Tanuj Mishra, Amit Kumar Singh, \\ Vikram Kumar Kamboj
}

SEEE, Lovely Professional University, Jalandhar, Punjab, India

\section{Introduction}

In the electrical energy market, the introduction of deregulation has triggered big competition among the suppliers and consumers, resulting in the congestion of transmission lines. The major issue in terms of security of the power system is congestion management. Congestion of transmission lines is a condition under which economic dispatch can never be maintained and the generators with high operating costs have to take over in order to maintain stability in the transmission system operation. The main reason for the occurrence of congestion in the network is the violation of some of the constraints of the power system like active and reactive power of the generator, voltage limits and the thermal limits of the transmission lines $[5,10]$. The effect of congestion is that the generation cost can be increased due to dispatch of more costly generators and that cost will be reflected in the transmission pricing and also in congestion costs that will in turn decrease the overall efficiency of the system [6]. So, it is very important to relieve congestion and charge the network consumers who are responsible for such a condition in the system. Independent system operators (ISOs) are formed in the deregulated electrical market environment in order to control and operate a transmission network in an open and transparent manner for every participant of the wholesale electrical market [7]. The cost of congestion should be properly reflected in the pricing schemes for using the transmission network otherwise in a few locations the demand is more than supply and, in some cases, there is surplus of supply [2]. The cost of congestion can be evaluated from the condition of those who were unable to use the network due to congestion. The present transmission system demands that sufficient capacity is constructed for transmission where congestion rarely occurs. And for the same a justified method is a requirement that can evaluate the cost of congestion.

\section{Methods for pricing congestion}

The congestion cost is calculated using three processes: re-dispatch of generators, cost allocated for congestion constraints and contribution of load. Initially, ISOs check for any violation of constraints and the cause for congestion in the system. If the congestion is found to be due to constraint violation then the re-dispatch of generation is carried out to relieve the congestion. The total congestion cost (TCC) is estimated as the difference

DOI: $10.1201 / 9781003129103-41$ 
between the production cost before and after re-dispatch [1]. Thereafter, the contribution of each load for line flow is estimated using the proposed PTDF method application and Bialek's tracing method and the congestion cost for each load is calculated. They are then assigned and collected from each load and paid to generator companies for the re-dispatch operation undertaken to eliminate congestion.

\subsection{Re-dispatch of generators}

Under this method an OPF is utilized for re-dispatching of generators considering the constraints and the congestion is removed by regulating the generator output (MW). OPF considers both equality and inequality constraints. Equality constraints include power balance equations, voltage limits etc. and the inequality constraints include generator's output, transmission line MVA (mega-volt ampere) flow etc. [3]. All the equality constraints and some of the inequality constraints like maximum active power flow have to be satisfied. The primary aim of OPF is to minimize the production cost or to adjust the generators' output considering both equality and inequality constraints for a congestionfree transmission system.

The function is:

$$
\begin{aligned}
& \min \sum_{i=1}^{n} C_{i}\left(P_{G i}\right)=\sum_{i=1}^{n}\left(a_{i} * P_{G i}^{2}+b_{i} * P_{G i}+c_{i}\right) \text { (Generation cost) } \\
& {\left[P_{G}\right]-\left[P_{D}\right]=[B][\theta] \text { (Equation for power balance) }} \\
& P_{i j} \leq P_{i j}^{\max } \text { where } P_{i j}=\frac{1}{x_{i j}}\left(\theta_{i}-\theta_{j}\right) \text { (Limits for line flow) } \\
& P_{G \min } \leq P_{G} \leq P_{G \max } \text { (Active power limits for generators) }
\end{aligned}
$$

where $a_{i}, b_{i}, c_{i}$ are cost coefficients for generator at bus ' $\mathrm{i}$ ', $C_{i}\left(P_{G i}\right)$ is the generator cost, $\left(P_{G i}\right)$ is the generated power at bus 'i', $P_{D}$ is the power demand, $\theta_{i}$ is the voltage angle at bus ' $i$ ', $x_{i j}$ is the $\mathrm{i}-\mathrm{j}$ line reactance and $P_{i j}$ is the power flow at line $\mathrm{i}-\mathrm{j}$.

The susceptance matrix ' $\mathrm{B}$ ' is estimated from the following criteria:

$$
[B]=\left\{\begin{array}{lr}
B_{i j}=\sum_{j} \frac{1}{x_{i j}} & \text { for } i \neq r e f \\
B_{i j}=0.0 & \text { for } i=r e f \\
B_{i j}=\frac{-1}{x_{i j}} & \text { for } i \neq r e f ; j \neq r e f \\
B_{i j}=0.0 & \text { for } i=\text { ref } ; \text { for } j=\text { ref }
\end{array}\right.
$$

During the re-dispatch process the active power flow constraint is considered due to which the production cost of generators tends to increase. This increase in generator's production cost is called the total congestion cost (TCC) [4]. 


\subsection{Cost allocated for congestion constraints}

It is difficult to allocate the TCC to the congestion caused by multiple lines in the network and it is also not fair to charge all the congested lines equally as some lines are more responsible for congestion compared to others. So a method has been developed to charge congested lines according to their contribution [8]. In this method the effect of any line $i-j$ is calculated by removing it from the system and this impact is known as the relief cost. This method considers the following: total cost of production with all lines is calculated. Relief cost for all the lines $\mathrm{i}-\mathrm{j}$ are calculated.

The constrained allocation cost is calculated based on proportional sharing principle using TCC.

$$
T C_{i j}=\frac{R C_{i j} *\left(P_{i j}-P_{\max }\right)}{\sum_{i j} R C_{i j} *\left(P_{i j}-P_{\max }\right)} * T C C
$$

where $\left(P_{i j}-P_{\max }\right)$ is the minimum MW relief needed in order to relieve congestion.

\subsection{Contribution of load}

Contribution of load for congestion is charged using load distribution factors. These factors $D_{i j, k}^{n}$ are estimated for each load and line flow and the power contribution of each load for congestion in the line is calculated as $D_{i j, k}^{n} * P_{L k}$. Using this method the distance between load and the congested lines can be calculated as:

Distance $=1 / \mathrm{D}$-factor

Application method for congestion pricing: an unconstrained load flow is run for base case. Generators are re-dispatched for any constraints violation. OPF is run to eliminate congestion by re-dispatching generators [9].

The production costs of generators are calculated for constrained and unconstrained cases.

$$
\begin{aligned}
& P C_{k}=a * P_{G k}^{2}+b * P_{G k}+c \text { (Unconstrained) } \\
& P C_{k}^{\prime}=a * P_{G k}^{\prime 2}+b * P_{G k}^{\prime}+c \text { (Constrained) } \\
& a, b, \text { care cost coefficients for generator }
\end{aligned}
$$

TCC is calculated taking the difference between constrained and unconstrained cost of production:

$$
T C C=P C_{k}^{\prime}-P C_{k}
$$

Then a constrained allocation cost (TC) is calculated for each congested line. Distribution factors are estimated for a line flow after re-dispatching the generators. 
Power load contribution to each congested line is evaluated using:

$$
D_{i j, k}^{n} * P_{L k}
$$

The congestion cost due to each load is calculated as:

$$
C C_{k}=\sum \frac{D_{i j, k}^{n} * P_{L k}}{\sum_{k}\left(D_{i j, k}^{n} * P_{L k}\right)} *\left(T C_{i j}\right)
$$

Incremental congestion cost (ICC), which is the amount of change in congestion cost for any change in load (MW), is calculated as:

$$
I C C_{k}=\frac{C C_{k}}{P_{L k}}
$$

This method charges the user according to their actual contribution to congestion in the congested lines. In this method ICC gives the load impact for its congestion cost. ICC is proportional to the distance between congested lines and load, i.e. ICC will be less for the distant load location.

\section{Results}

An Indian Utility 146-Bus system consists of 24 generators and 100 loads. The system has 169 transmission lines. Some of the buses have both generators and loads connected. Using line data, generator data, load data and line limits, load flow analysis is obtained. After running Newton-Raphson AC Load Flow two transmission lines, line 28-27 and line 28-34, are found to be congested. The generator and load contributions to power flow are determined for both unconstrained and constrained cases.

The line flow of transmission line 28-27 in the constrained case has changed from $-343.15 \mathrm{MW}$ to $-201.53 \mathrm{MW}$ and the line flow for line 28-34 in the constrained case has changed from -974.68 MW to $-897.65 \mathrm{MW}$ after re-dispatch of generators and they are now under the line limit of $300 \mathrm{MW}$ and $900 \mathrm{MW}$, respectively.

The total generation cost is determined for constrained and unconstrained cases and the constraint is taken as the line limits. It can be seen using Bialek's method that generation at buses $28,29,34,36,37,58,61,62$ and 88 is reduced and generation at buses 1, 38, $49,63,75,79$ and 83 has increased during re-dispatch in order to relieve the congestion on the transmission lines. The increase in generation cost, which is also termed the total congestion cost (TCC), is determined. The TCC for the Indian Utility 146-Bus system is found to be -2098.43 ( $\$ / \mathrm{h})$. The PTDF has been calculated using power flow by programming in MATLAB; from the obtained PTDF, other distribution factors like GGDF and GLDF can also be determined. Using the distribution factors obtained from PTDF, load and generation contribution on line flow have been determined. Also, the generator and load contributions to line flow and each other are also determined using Bialek's method.

The calculated relief cost for the congested lines is as follows: $\mathrm{RC}_{27-28}=-9.5(\$ / \mathrm{h})$, $\mathrm{RC}_{28-34}=-8.8(\$ / \mathrm{h})$. So, the calculated constrained allocation cost which is the distribution 


\section{TRANSMISSION CHARGES FOR GENERATOR CONTRIBUTION PER YEAR}

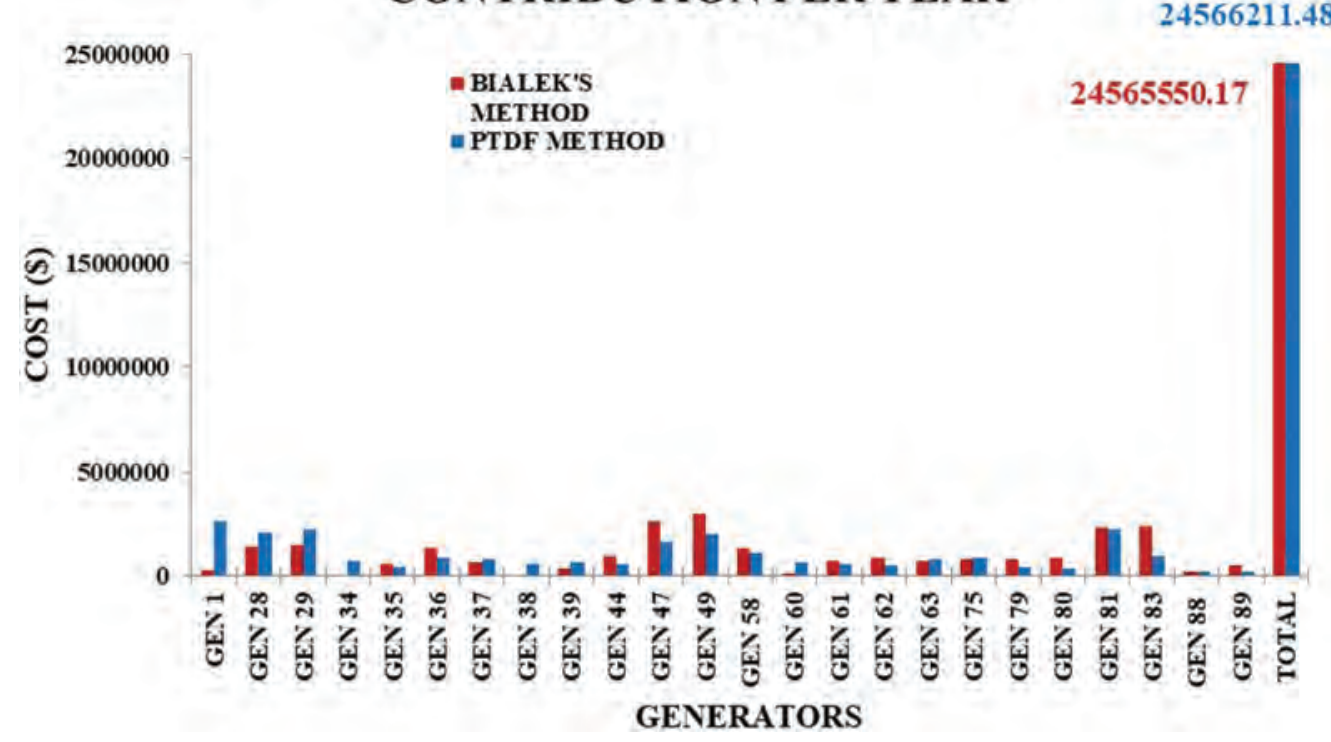

Figure 41.1 Comparison of transmission charges for generator's contribution per year of Indian Utility 146-Bus system using BIALEK'S method and application of PTDF method.

of TCC among each line is: $\mathrm{TC}_{27-28}=-1875.46(\$ / \mathrm{h}), \mathrm{TC}_{28-34}=-541.96(\$ / \mathrm{h})$. These constraint costs are divided among all the loads responsible for congestion using the proposed application of PTDF method by calculating the load contribution on line flow.

By comparing the results obtained using the proposed application of PTDF method and Bialek's method it is found that both methods result in a similar cost for congestion, i.e. according to Bialek's method the congestion cost is $-2098.43(\$ / \mathrm{h})$ and from the proposed application of PTDF method it is $-2417.61(\$ / \mathrm{h})$. The congestion cost calculated has a negative value because after re-dispatching the generators the total cost of generation is reduced and hence this is reflected in the congestion cost. The transmission cost for generator contribution per year can also be estimated using the proposed application of PTDF method and this has also been verified by comparing the results with Bialek's method shown in Figure 41.1. As seen from the bar chart in Figure 41.1, there is some variations of transmission charges calculated by each method. This is because proposed application of PTDF method takes into account all the positive and negative contributions of generator but Bialek's method considers only positive contribution and other contributions are neglected. The proposed application of PTDF method also evaluates the negative flow of power in the lines.

\section{Conclusion}

The purpose of performing congestion studies is to estimate the congestion cost for Indian Utility 146-Bus, using the proposed application of PTDF method and Bialek's method then to compare the results and check their accuracy. The evaluated congestion cost for Indian utility 146-Bus system using Bialek's method is $-2098.43 \$ / \mathrm{h}$ and using the proposed 
PTDF method it is $-2417.61 \$ / \mathrm{h}$. Here it can be seen that congestion cost using proposed PTDF method is slightly greater than Bialek's method. The values of congestion cost for IEEE 30-Bus system and Indian Utility 146-Bus system are negative because after applying the line limits and re-dispatching the generators the cost of generation is less than the unconstrained case. So, the congestion cost is actually a smaller cost for generation of the same energy. That is why the congestion cost values are negative.

To determine the congestion cost a proposed PTDF method is used which uses other distribution factors to calculate the generator and load contribution on line flow; similarly, Bialek's method is used to determine the same. The evaluated load and generators' contribution using both the methods are used to further determine the transmission costing. The transmission cost using proposed PTDF method is 2804.36 \$ h and using Bialek's method is $2804.29 \$ / \mathrm{h}$, which are almost the same. By this it can be concluded that proposed PTDF method can be used for determining the congestion cost and also for transmission costing.

\section{References}

1. Acha, E., Fuerte-Esquivel, C. R. and Ambriz-Perez, H. 1997. Topological generation and load distribution factors for supplement charge allocation in transmission open access: discussion. IEEE Transactions on Power Systems 12(3):1191-1193.

2. Baldick, R. 2011. Optimal charging arrangements for energy transmission: Final Report U.K. OFGEM. Accessed 21-11-2019.

3. Bialek, J. 1996. Tracing the flow of electricity. IEE Proceedings-Generation Transmission and Distribution 143(4):313-320.

4. Bialek, J. 1997. Elimination of merchandise surplus due to spot pricing of electricity. IEE Proceedings-Generation Transmission and Distribution 144(5):399-405.

5. Christie, R. D., Wollenberg, B. F., and Wangensteen, I. 2000. Transmission management in the deregulated environment. Proceedings of the IEEE 88(2):170-195.

6. Kirschen, D., Allan, R., and Strbac, G. 1997. Contributions of individual generators to loads and flows. IEEE Transactions on Power Systems 12(1):52-60.

7. Liu, M., and Gross, G. 2004. Role of distribution factors in congestion revenue rights applications. Power Systems, IEEE Transactions on 19(2):802-810.

8. Perez-Arriaga, I. J. 1995. Marginal pricing of transmission services: an analysis of cost recovery. Power Systems, IEEE Transactions on 10(1):546-553.

9. Rau, N. S. 2000. Transmission loss and congestion cost allocation-an approach based on responsibility. Power Systems, IEEE Transactions on 15(4):1401-1409.

10. Rudnick, H., Palma, R. and Fernandez, J. E. 1995. Marginal Pricing and supplement cost allocation in transmission open access. IEEE Transactions on Power Systems 10(2): 1125-1132. 


\title{
42 Motion detection and tracking of surveillance videos under distorted environments
}

\author{
Chinthakindi Kiran Kumar', Gaurav Sethi², \\ Kirti Rawal 3 *
}

${ }^{1} \mathrm{PhD}$ Scholar, School of Electronics \& Electrical Engineering, Lovely Professional University, Phagwara, Punjab, India; Assistant Professor, Malla Reddy College of Engineering \& Technology, Doolapally, Hyderabad, India

${ }^{2}$ Professor, School of Electronics \& Electrical Engineering, Lovely Professional University, Phagwara, Punjab, India

${ }^{3}$ Associate Professor, School of Electronics \& Electrical Engineering, Lovely Professional University, Phagwara, Punjab, India

\section{Introduction}

The quality of videos that are acquired with the surveillance cameras usually depends on several factors; one of such important factor is illumination. There are some other factors like dirt and water on the lens of the camera which also deteriorate the quality of the videos. Among these, lighting conditions are one of the crucial factors in identifying the object in surveillance videos.

In recent days, most of the crimes are solved with the help of CCTV surveillance video recordings. So, the demand for these surveillance videos has grown to the saturation to protect the public and their properties [1].

Until now, several algorithms were designed to identify the objects and track the objects or humans in the surveillance videos but most of them are experimental or considered a constant illumination environment [2] but in reality it is not and it can be observed that this illumination varies very often [3]. When this change of illumination occurs the concept of object detection becomes more complex and it becomes difficult to detect the object accurately. The application of object detection becomes more difficult when the videos are distorted with noise and illumination changes [4]. The problem of illumination change can be defined as change in the visibility or appearance of the object with varying illumination [5]. This paper presents a review on illumination correction algorithms for surveillance videos and also suggests an approach that is adaptive and efficient in mitigating the problem of illumination variation.

\section{Literature survey}

To correct the changes in varying illumination of surveillance videos, several authors have proposed different methods and approaches that are strong at some aspects and weak at others. So, the detailed study of these algorithms makes the researchers find the research gaps and further frame new objectives that could meet those limitations. 
Chen et al. [6] introduced a single stage approach of semi-supervised deep neural network (SDNN). This network aims to detect the objects in multiple successive frames in continuous video sequences. This approach fuses the context information which is present in different frames and later combines all the forecasts from multi-scale features which are present in different layers. All the features in the frame are fused together in order to complete the entire process. The SDNN has achieved an average precision rate of 0.64 which is 0.05 greater than conventional R-CNN network model.

Dorai et al. [7] proposed tracklet algorithm for object tracking in the surveillance video that processes the sequence of frames with respective detection. Later, an association is employed for analysing objects in the video sequences. It is often observed that object tracking algorithms are based on appearance models for forecasting the moments in objects in each frame. In most of the scenarios the models are taught with ample amount of training samples and these are used for the estimation of the object tracking.

Zhang et al. [8] proposed a multi-task oriented visual tracking algorithm with sparse learning. This algorithm could handle the particles or the objects independently. In this work, they employed particle filter concept to estimate and track the position of the object and these elements are represented with updated version of dictionary template. This approach is more robust against some of the noisy elements with the help of proximity optimization concept. The algorithm provides a high accuracy in tracking the objects with high computational complexity.

Sreedevi et al. [9] proposed an architecture to detect whether the moving object is human or not. In this approach they have employed background separation and SAD algorithm to detect the movement and store those frames in which the movement is observed. In this work, they have considered videos that are properly and constantly illuminated.

Phadke and Velmurgan [10] have proposed an illumination correction algorithm with $\log$ discrete cosine transforms and the object is tracked with mean shift filtering. It was observed that this method could effectively handle the problem of illumination variation for several video recordings. In this paper, they have considered web cam based videos where the illumination is varied synthetically. However, when surveillance videos are being considered, the illumination variation is quite different.

The main motive of this work [10] is to modify the DC coefficients obtained after DCT transformation for low illumination video frames. In this work every video frame that is captured is converted from RGB to YUV colour space and then a logarithmic transformation is applied on the Y-plane of YUV from which the Y-plane (luminance) is separated from reflectance planes which is then followed by DCT transformation. When observed, the DCT matrix does contain DC component at its first position which is directly proportional to the illumination level of that respective frame so higher DC value means a high illumination. So in order to attain a constant illumination video sequence the first $\mathrm{K}$ consecutive frames are taken into consideration. The DC components of these K consecutive frames are modified by increasing the illumination of darker frames. The correction is achieved with correction factor convolved with DC components; later a means shift algorithm is employed for tracking the objects. The main limitation of this approach is that it could not handle the problem of scaling and orientation of the target region, hence it can be said that it cannot sustain the sudden changes in the illumination.

Muthuswamy and Rajan [11] proposed a particle filter approach with Monte Carlo which aims to approximate the posterior distribution recursively with finite weight sample information.

The main concept behind the particle is straight with weight set of samples $\left.x_{k-1}^{(i)}, w_{k-1}^{(i)}\right\} N_{i=1}$ which are estimated using the distribution function $p\left(x_{k-1} \mid Z_{k-1}\right)$; later the new samples are estimated based on the distribution that relies on previous state and the 
updated measurements $x_{k}^{i}=q_{p}\left(x_{k} \mid x_{k-1}^{i}, z_{k}\right)$. So to maintain consistent new samples with respect to the weights then the new particles are distributed according to $p\left(x_{k} \mid Z_{k}\right)$. The overall performance of the particles relies on the quality of the proposed distribution and the state model $\mathrm{p}\left(\mathrm{x}_{\mathrm{k}} \mid \mathrm{x}_{\mathrm{k}-1}\right)$ and this new update of weights becomes the particle likelihoods.

Mahmoud [12] proposed a method for colour constancy using deep networks. In this work he used deep networks for estimating the illuminant colour changes and gamma correction for the extracted semantic regions. From the results it was observed that this approach yielded an improvement of $40 \%$ when compared with traditional retinex algorithms. On the other hand Wang and Shang [13] have deployed the concept of deep network for estimating illumination correction for which they have designed 11 convolutional layer structure and include global averaging pooling concept. It was observed that this approach has reduced $60 \%$ of the average angular error.

Ma et al. [14] tried to propose accurate scale estimation of tracking objects. The approach is termed as scale invariant tracking with average peak to correlation energy (SITUP), which aims to mitigate the problem of fixed template size for tracking the objects. The proposed approach could manage the scale changes effectively and was able to discriminate the correlation filter based trackers and when tested on benchmark datasets it was observed that this method meets the requirement of scale variation challenge by proving more accurate results. Further in another article [15] the authors have proposed a FAST (fast and accurate scale estimation for tracking). In this work they have incorporated a scale searching scheme that obtains a robust and accurate scale estimation which is used for accurate tracking with peak to correlation energy and multiresolution filter framework.

\section{Conclusions}

This paper presents a brief review on the methods and approaches that were used to handle the problem of illumination variations in the surveillance videos. From the review, it has been observed that many of the object detection tracking and identifications algorithms were proposed in the literature. But, these algorithms have been implemented by just considering the constant illumination. However, in real life situations when the illumination is drastically changing, the identification of the objects becomes very difficult. It is concluded from the literature survey that there is a need to propose a method that can handle the abrupt changes in the illumination. This can be possible only with deep neural networks where the high efficiency outputs with accurate illumination correction can be sustained and retained.

\section{References}

[1] N. Gaba, N. Barak and S. Aggarwa. 2016. "Motion detection, tracking and classification for automated video surveillance," IEEE 1st International Conference on Power Electronics, Intelligent Control and Energy Systems (ICPEICES), Delhi, pp. 1-5.

[2] Kalpesh R. Ranipa and Kiritkumar Bhatt. 2014. "Illumination condition effect on object tracking: a review", Global Journal of Computer Science and Technology: Graphics \& Vision, vol. 14, no. 5 Version 1.0.

[3] W. Hu, T. Tan, L. Wang and S. Maybank. 2004. "A survey on visual surveillance of object motion and behaviors," IEEE Trans. Syst., Man, Cybern. C: Appl. Rev., vol. 34, no. 3, pp. 334-350.

[4] A. Yilmaz, O. Javed and M. Shah. 2006. "Object tracking: a survey," ACM Comput. Surv, vol. 38 . 
[5] B. Wu and R. Nevatia. 2007. "Detection and tracking of multiple, partially occluded human by Bayesian combination of edgelet based part detectors," International Journal of Computer Vision, vol. 75 , no. 2, pp. 247-266.

[6] Chen, J., et al. 2019. "Semi-supervised deep neural networks for object detection in video surveillance systems." In Lin, Z., et al. (Eds.), Pattern Recognition and Computer Vision. PRCV 2019, Lecture Notes in Computer Science, vol. 11857. Springer, Cham.

[7] Y. Dorai, F. Chausse, S. Gazzah and N.E.B. Amara. 2017. "Multi target tracking by linking tracklets with a convolutional neural network," VISIGRAPP (6: VISAPP), pp. 492-498.

[8] T.Zhang, B. Ghanem and S. Liu. 2012. "Robust visual tracking via multi-task sparse learning," in EEE Conference on CVPR, pp. 16-20.

[9] Sreedevi, M., Yaswanth Kumar Avulapati, Anjan Babu and Sendhil Kumar. 2012. "Real time movement detection for human recognition," in Procedure of the World Congress on Engineering and Computer Science, vol. 1.

[10] G. Phadke and R. Velmurgan. 2013. "Illumination invariant mean-shift tracking," IEEE Workshop on Applications of Computer Vision (WACV), Tampa, FL, pp. 407-412.

[11] K. Muthuswamy and D. Rajan. 2015. "Particle filter framework for salient object detection in videos," IET Computer Vision, vol. 9, no. 3, pp. 428-438.

[12] Mahmoud Afifi. 2018. Semantic White Balance: Semantic Color Constancy Using Convolutional Neural Network, Lassonde School of Engineering, York University, Canada.

[13] Minquan Wang and Zhaowei Shang. 2018. Deep Separable Convolution Neural Network for Illumination Estimation, Chongqing University, China.

[14] H. Ma, S. T. Acton and Z. Lin. 2020. "SITUP: scale invariant tracking using Average peak-tocorrelation energy," IEEE Transactions on Image Processing, vol. 29, pp. 3546-3557.

[15] H. Ma, Z. Lin and S. T. Acton. 2020. "FAST: fast and accurate scale estimation for tracking," IEEE Signal Processing Letters, vol. 27, pp. 161-165. 


\title{
43 An effective strategy for denoising of MRI images to detect brain tumour
}

\author{
Bandana Bali', Brij Mohan Singh ${ }^{2}$, \\ Kamal Kumar Sharma ${ }^{3, *}$
}

\begin{abstract}
${ }^{1} \mathrm{PhD}$ Research Scholar, Department of Computer Science and Engineering, Uttarakhand Technical University, Dehradun, India

${ }^{2}$ Professor, CS and IT Department, College of Engineering, Roorkee, India

${ }^{3}$ Professor, Lovely Professional University, Punjab, India
\end{abstract}

\section{Introduction}

Digital image processing always remains a challenging area of programming for numerous motives. Radiology medical practice is all about detection, monitoring and segmentation of medical images for diagnosis. Research of AI has been running pervasive in the area of radiology from last decade. AI technology in medical imaging pillars like CT, MRI, and PET scan is advancing in terms of validity, sensitivity and resolutions. Deep learning with convolutional neural network $(\mathrm{CNN})$ is recently gaining more attention due to its high performance in recognizing medical images for predictions. AI has also covered other major circles of radiology like thoracic imaging, colonoscopy, mammography, brain imaging, radiation oncology, DNA and RNA sequencing. There are two approaches used in radiology known as machine learning and deep learning. In machine learning we provide predefined feature, but this approach is having demerits to adopt variation in imaging data available from CT, MRI and PET scans. Another method is known as deep learning method, which automatically learn features from available data, hence deep learning is robust in case of undesired variations in image data. Recent research shows that deep learning approach is superior in terms of accuracy, sensitivity and specificity, perfect in its interpretation with lesser errors and utilizes more time on patient's treatments $[1,2]$. Fast evaluations in AI and its applications into routine clinical imaging will cause a major change to the practice of radiology. Machine learning in form of image processing, computer vision and natural language processing of AI technology enhances support to the new form of radiology in future. For precise development of this area, there are still untangled challenges glimpsing the adoption of artificial intelligence like how to over fit the problem of machine learning due to unavailability of larger reviewed recent trades of AI in radiology. It can be further divided into analogue image processing and digital image processing (DIP). DIP permits the utilization of highly complex and complicated algorithms, and therefore can overture both more disenchanted performance about easy, simple tasks assigned, and the implementation or employment of techniques that are decreed to be impassable by the analogue methods. Specifically, DIP technology is applied for classification, extraction of features, recognizing pattern, and many more. Figure 43.1 depicts the digital image processing architecture.

\footnotetext{
* Email: bandanasharma1@gmail.com
} 


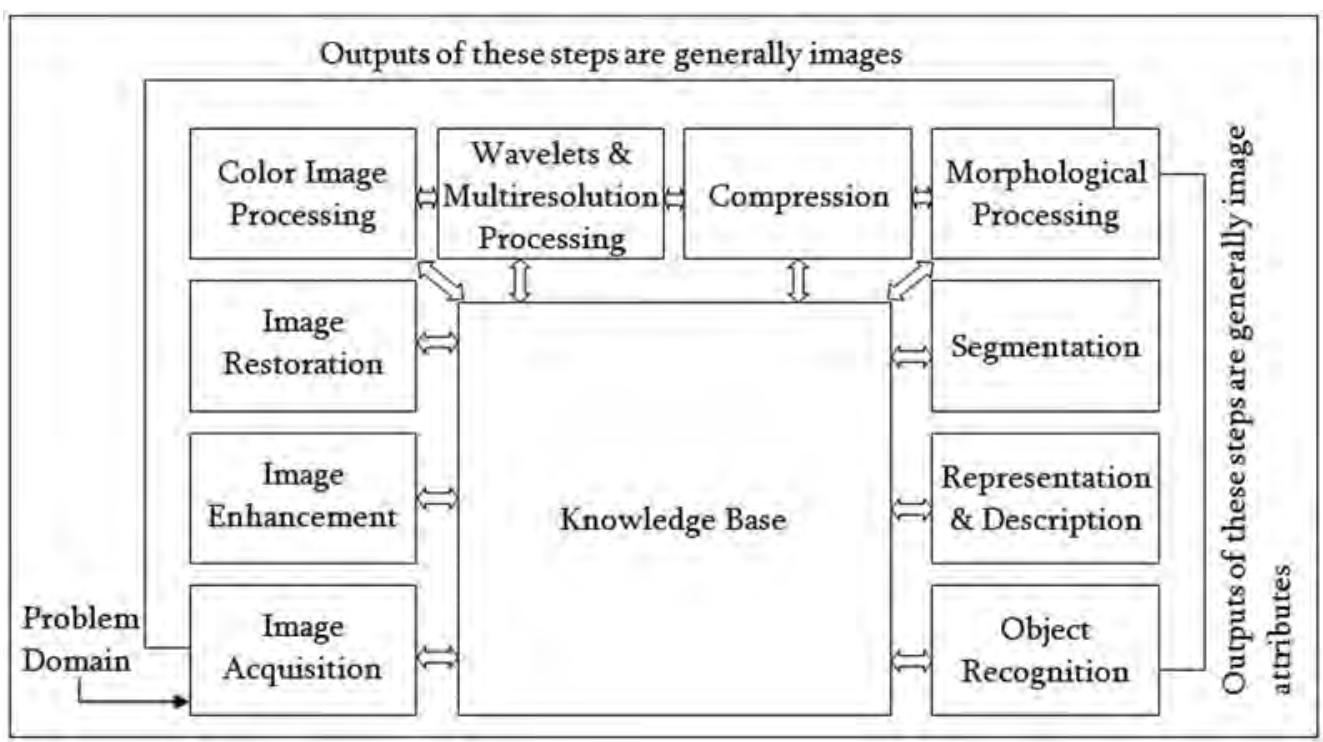

Figure 43.1 Digital image processing.

\section{Medical image processing}

The method and course of devising the perceptible portrayal of a human body interior for medical arbitration and clinical purposes (diagnosis or examination of diseases) is known as medical imaging. It attempts to disclose the human body's internal structures covered by the bones and skin, likewise to determine and prescribe or operate on a disease. It also creates a database of common, normal physiology anatomy for making it attainable for identifying the abnormalities or deformity.

Even though there is requirement of imaging of detached tissues and organs achieved for medical analysis, such measures are not generally cited as medical imaging. In far reaching sense, this is an important component of life concerning imaging and assimilates radiology, paramount medicine, analytical radiological sciences and endoscopy, medical drawing and microscopy for example personal medicinal and other pathological examination. The methods of measurement and storing that are not generally devised to generate images, as magnetoencephalography (MEG), electroencephalography (EEG), electrocardiography (EKG or ECG) and many others, but that generates data easily and are liable to be depicted and expressed as maps, may be perceive as types of medical imaging. In the United States, in year 2006, radiation disclosure arising out of medical imaging comprises about 50 percent of complete ionizing radiation exposure. In the clinical framework, imperceptible light medical imaging is commonly associated with radiology and medical experts or professionals are responsible and accountable at intervals for achieving the images and for interpreting, i.e. translating the images. Perceptible light medical imaging includes static pictures or digital videos that are able to be perceived without specialized equipment. The two approaches that make use of the perceptible light imaging are dermatology and wound care. The diagnostic radiography entitles the technical facets relating to medical imaging, in specific medical image acquirement. 
There are different types of medical images:

- X-ray: These are electromagnetic high energy and very short wavelength rays which are able to pass over many tissues impervious to light.

- Computed tomography (CT): Computed tomography (CT) scans, also known as CAT (computed axial tomography) scans, produce multiple cross-sectional images of the body by using special X-rays and computer enhancements.

- Echocardiography: An echocardiogram is an image attained when ultrasound is performed for imaging the heart. ECG can also identify heart muscles where pumping has weakened because of constrained blood supply or heart attack damage. A unique type of echo can evaluate the flow of blood through the heart. It also indicates the persistence of heart to brain to blood flow shortage involving low cardiac output or low ejection and carotid artery can promote cognitive injury and such injury may lead to vascular dementia.

- Magnetic resonance imaging (MRI): MRI delivers extremely full images of human tissue organs as well as bones without using X-rays.

Gaussian noise: This noise is analytical noise which has the PDF (probability density function) comparable and identical to that of the normal distribution, i.e. Gaussian distribution. Additionally, the values which noise can endure on are Gaussian distributed. There is an exclusive and unique case is WGN (white Gaussian noise), in which the values at any instant are analytically self-reliant and not correlated. Gaussian noise is frequently used as additive white noise for yielding additive WGN. If the sequence of white noise is a Gaussian sequence, then it is referred as white Gaussian noise sequence. This kind of noise enhances a random value of Gaussian distributed noise, the unique pixel value. The probability distribution function $(\mathrm{PDF})$ is given by:

$$
F(g)=\frac{1}{\sqrt{2 \pi \alpha}} e^{-(g-m)^{2} / 2 \sigma^{2}}
$$

Here, $g$ represents the grey level and $m$ is the mean of the function. Graphically, it is as shown in Figure 43.2.

Step wise analysis of noise reduction using SVM, iterative bilateral filter and LMRD in different type of medical images:

Step 1: In this step, MATLAB is used to execute the results.

Step 2: In this step, the project has been opened to start the programme to de-noise the image.

Step 3: In this step, we select the load input image to de-noise the image.

Step 4: In this step, the image has been loaded for de-noising the medical image.

Step 5: Here, we have selected the original medical image and noisy image has been generated, indicated in the right side of the image.

Step 6: After selecting the noisy image, we apply filter to de-noise the medical image. That is showing on the left side in de-noise image block.

Step 7: Here we have operated the second technique, i.e. LMRD, to de-noise the medical image. The noise has been reduced while applying this method.

Step 8: Now the third technique, SVM, has been operated for image de-noising. Here more noise has been reduced after applying SVM to de-noise the medical image. 


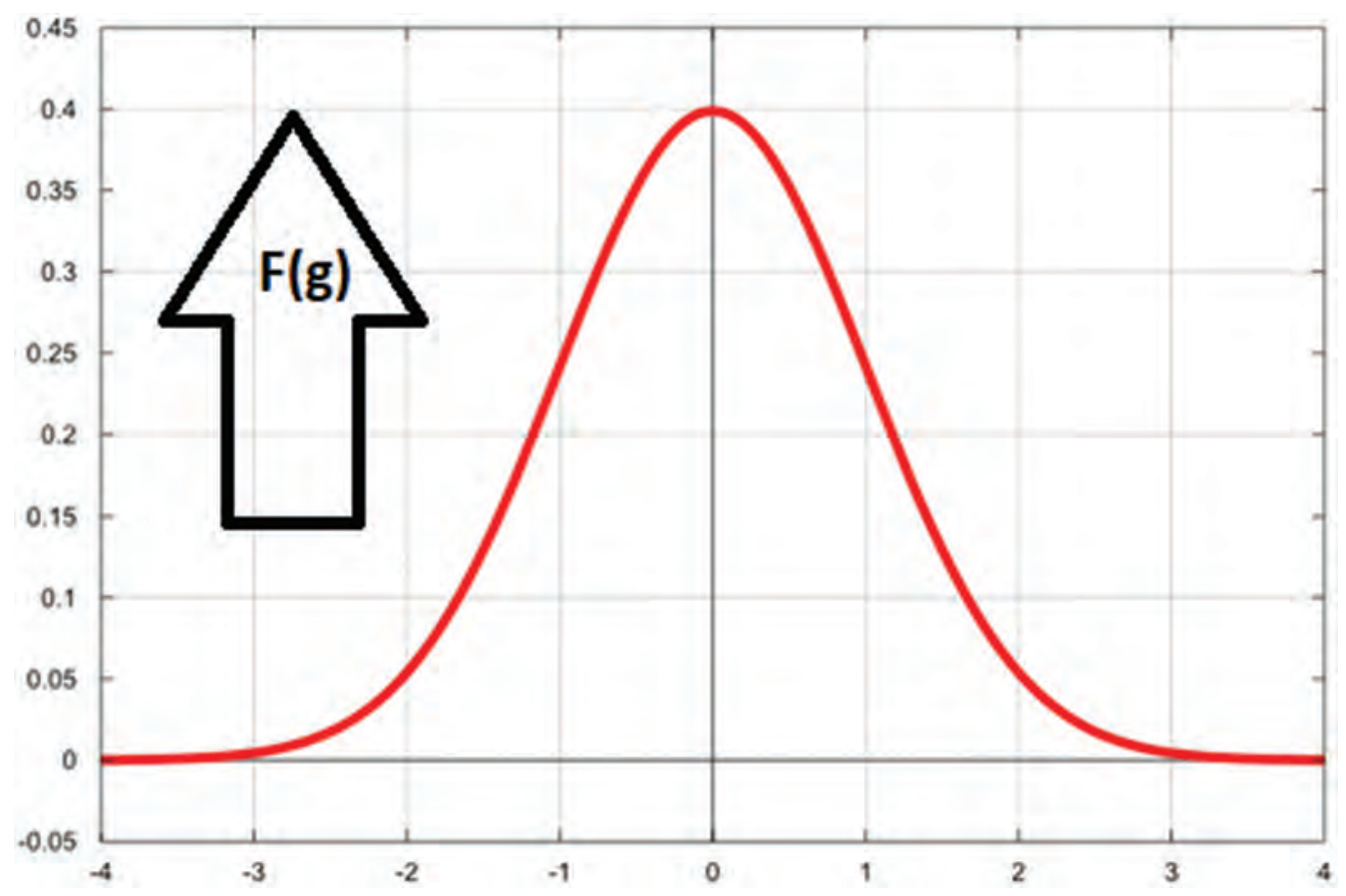

Figure 43.2 Gaussian noise distribution.

Step 9: In this step, we have operated the de-noised image step for further result. The noise has been removed in this step, indicated on the left side of the image.

Step 10: In this step, after applying the three techniques the PSNR (peak signal noise ratio) parameter has been calculated and the graphical comparison of PSNR value between previous and our algorithm is indicated in Figure 43.3. The result indicates that the PSNR value is higher in our algorithm as compared to previous work.

Step 11: In this step, MSE (mean square error) parameter has been calculated and the graphical comparison of MSE value between previous and our algorithm is indicated in the image. The result indicates that the MSE value is lesser of our algorithm as compare to previous work.

Step 12: In this step, the result has been calculated while using different methods to conclude SSIM (structural similarity index matrix) parameter. The result indicates that the SSIM value of our proposed work is on higher side as compared to previous concluded works.

\section{Results and discussion}

We have defined a novel de-noising technique for parallel MRI by exploiting the self-similarity between multi-channel coil images and inside themselves. The method that has been proposed removes noise and the aliasing artefacts together by influencing sparse and low rank matrix factorization as shown in Figures 43.4 and 43.5. Trial results establish that our projected algorithm may give benefit for both visual diagnostic and quantitative methodologies. The formalism is accessible and validates that the LRMD and SVM methods 


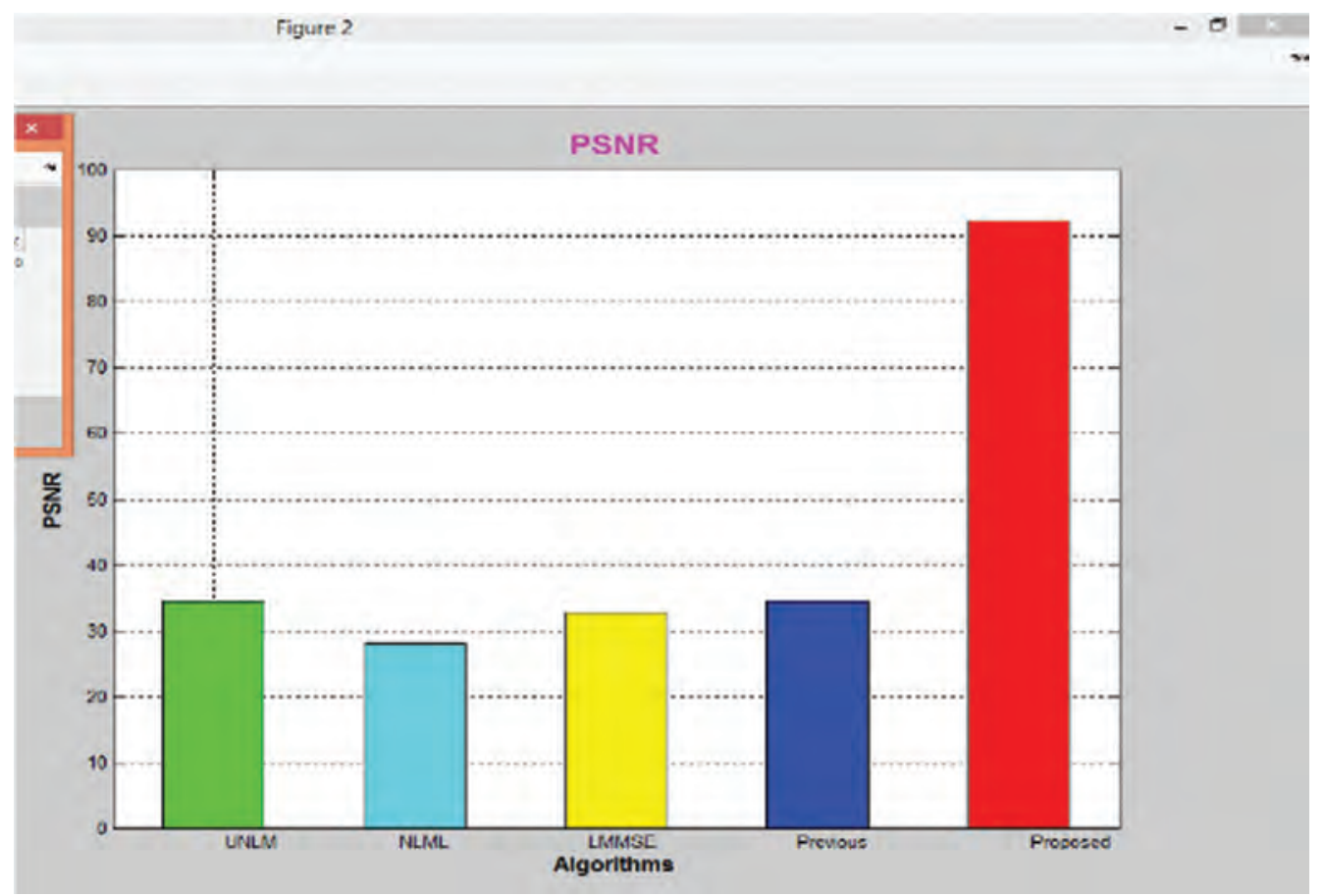

Figure 43.3 Comparison of PSNR between previous techniques and proposed work.

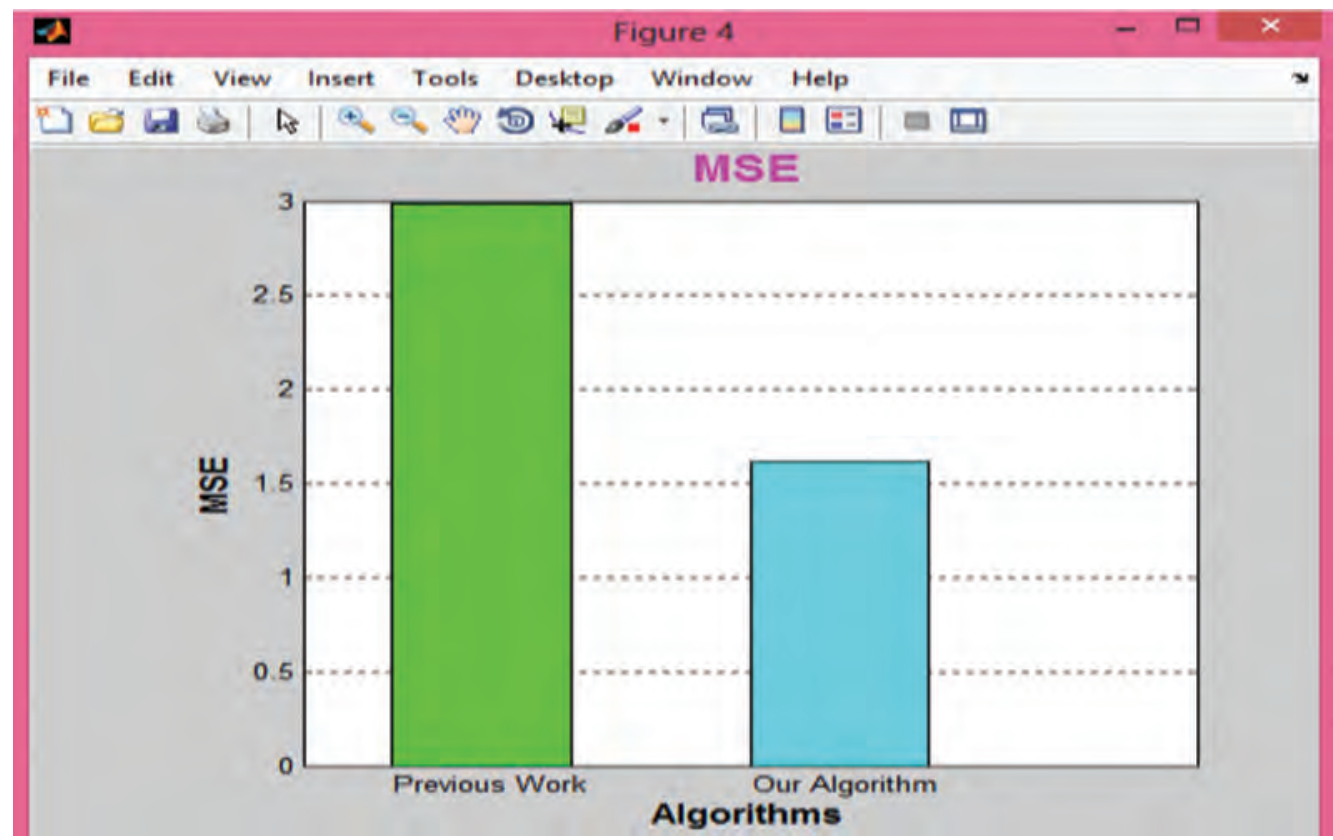

Figure 43.4 Comparison of MSE between previous and proposed work. 


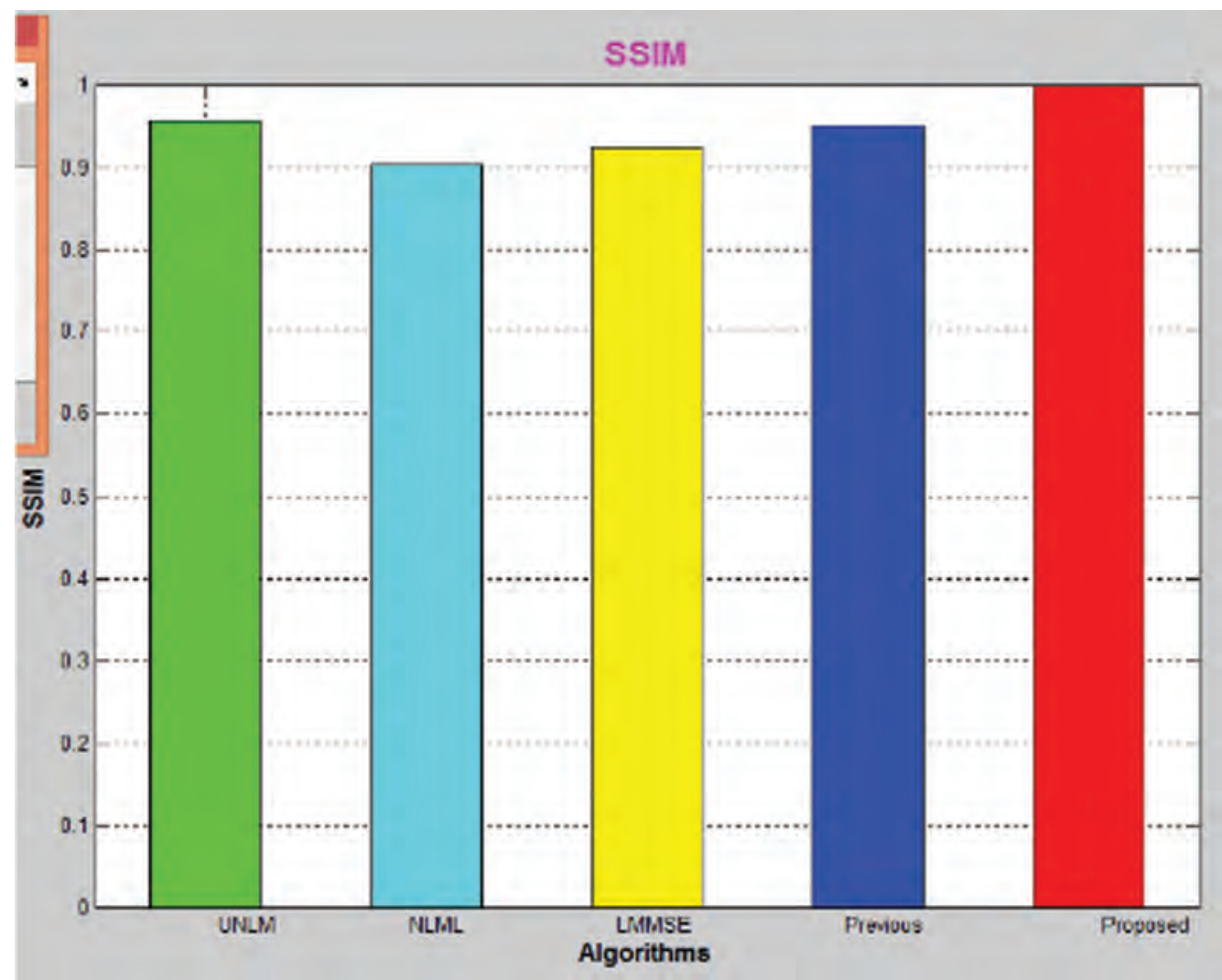

Figure 43.5 Comparison of SSIM between previous techniques and proposed work.

can jointly suggest a new system to further decrease the noise in MR images. The projected method drastically progresses the excellence of parallel MRI scanning in specific medical images. The proposed approach may also be an effective method to de-noise the medical images used in digital image processing techniques.

\section{References}

[1] S.R. Telrandhe, A. Pimpalkar, A. Kendhe, "Brain tumor detection using object labeling algorithm \& SVM", International Engineering Journal For Research \& Development, vol. 2, pp. 28, Nov. 2015.

[2] SobriMuda, M. Mokji, "Brain lesion segmentation from diffusion-weighted MRI based on adaptive thresholding and gray level co-occurrence matrix", J. Telecommunication Electronic and Computer Engineering, vol. 3, 2011.

[3] S. Cha et al., "Review article: update on brain tumor imaging: from anatomy to physiology", Journal of Neuroradiology, vol. 27, pp. 475-487, 2006.

[4] R. Adams, L. Bischof, "Seeded region growing”, IEEE Trans. On Pattern Analysis and Machine Intelligence, vol. 16, no. 6, pp. 641-647, 1994.

[5] J. Selvakumar, A. Lakshmi, T. Arivoli, "Brain tumor segmentation and its area calculation in brain MR images using k-mean clustering and fuzzy c-mean algorithm", Proceedings in IEEEInternational Conference On Advances In Engineering Science And Management, pp. 186$190,2012$. 
[6] R. Muthukrishnan, M. Radha, "Edge detection techniques for image segmentation", International Journal of Computer Science \& Information Technology, vol. 3, no. 6, Dec. 2011.

[7] M. Saritha, K. Paul Joseph, "Classification of MRI brain images using combined wavelet entropy based spider web plot and probabilistic neural network", Elsevier Pattern Recognition Letters, vol. 34, pp. 2151-2159, 2013.

[8] P.N. Gopal, R. Sukanesh, "Wavelet statistical feature based segmentation and classification of brain computed tomography images”, lET Image Process, vol. 7, pp. 25-32, 2013.

[9] Laxmi, P. Samata, "Optimization of visual presentation of MRI image for accurate detection of tumor in human brain using virtual instrument", The Biomedical Engineering International Conference, 2012.

[10] G. Hamerly, C. Elkan, "Learning the k in K-means", Proceedings 7th Annual Conference on Neural Information Processing Systems (NIPS), pp. 281-288, 2003. 


\title{
44 Wideband MIMO antenna with improved isolation
}

\author{
Pankaj Kumar Keshri*, Richa Chandel
}

School of Electronics and Electrical Engineering, Lovely Professional

University, Phagwara, Punjab, India

\section{Introduction}

In present day communication requires high data rate, wide bandwidth, and high capacity along with very low power consumption which is fulfilled by ultra wideband (UWB) system. The frequency spectrum which is officially decided by FCC for UWB is 3.1-10.6 $\mathrm{GHz}$ in 2002 [1]. The UWB technology is suitable only for short distance communication because of low power spectral density and high throughput. However, in personal wireless devices applications, multipath fading and reliability are the major issues [2]. In order to improve these issues MIMO technology is combined with UWB system in [3-6]. In MIMO system, multiple antennas are present which require high isolation among these antenna elements. There are various decoupling structure like defected ground structure [7], meta material [8,9], a tree-like structure [10] presented in the literature. Further a mushroom type electromagnetic band gap (EBG) structure [11], meander line resonator [12], neutralization line [13] are presented in literature for isolation improvement. A tapered micro strip fed in polygon shaped radiator with two L-shaped slit are presented for isolation improvement in [14]. In [15], for isolation improvement CSRR and stubs are used.

In the present paper, compact and novel wideband MIMO antenna is designed and this antenna is working with frequency range of $4-15.2 \mathrm{GHz}$. This frequency range is suitable for various wireless hand-held device applications. The proposed antenna consists of two modified rectangular symmetric element and these are placed orthogonally on top surface. To obtain wideband we modify the ground and truncate the rectangular patch. The isolation is improved by placing two elements orthogonally on the top surface. The proposed antenna is design on HFSS simulation software and simulated result has wider impedance bandwidth $(11.2 \mathrm{GHz})$, stable gain, good return loss, quasi-omnidirectional radiation patterns and very good (less than $-19 \mathrm{~dB}$ ) isolation.

\section{Structure of proposed antenna}

Top view of structure of proposed antenna with optimized dimension is presented in Figure 44.1. In this proposed antenna, $50 \mathrm{ohm}$ micro strip fed line is used and the size of antenna is $39 \mathrm{~mm} \times 39 \mathrm{~mm}$. This antenna has $1.6 \mathrm{~mm}$ thickness and made with low cost FR4 substrate. The designed antenna is simulated on HFSS simulation software and optimized parameter of novel designed antenna are described in Table 44.1.

Table 44.2 describes the antenna performance of previous literature with proposed designed antenna. The performance is measured in terms of size, isolation coefficient and

*Email: pankaj.16384@lpu.co.in

DOI: $10.1201 / 9781003129103-44$ 


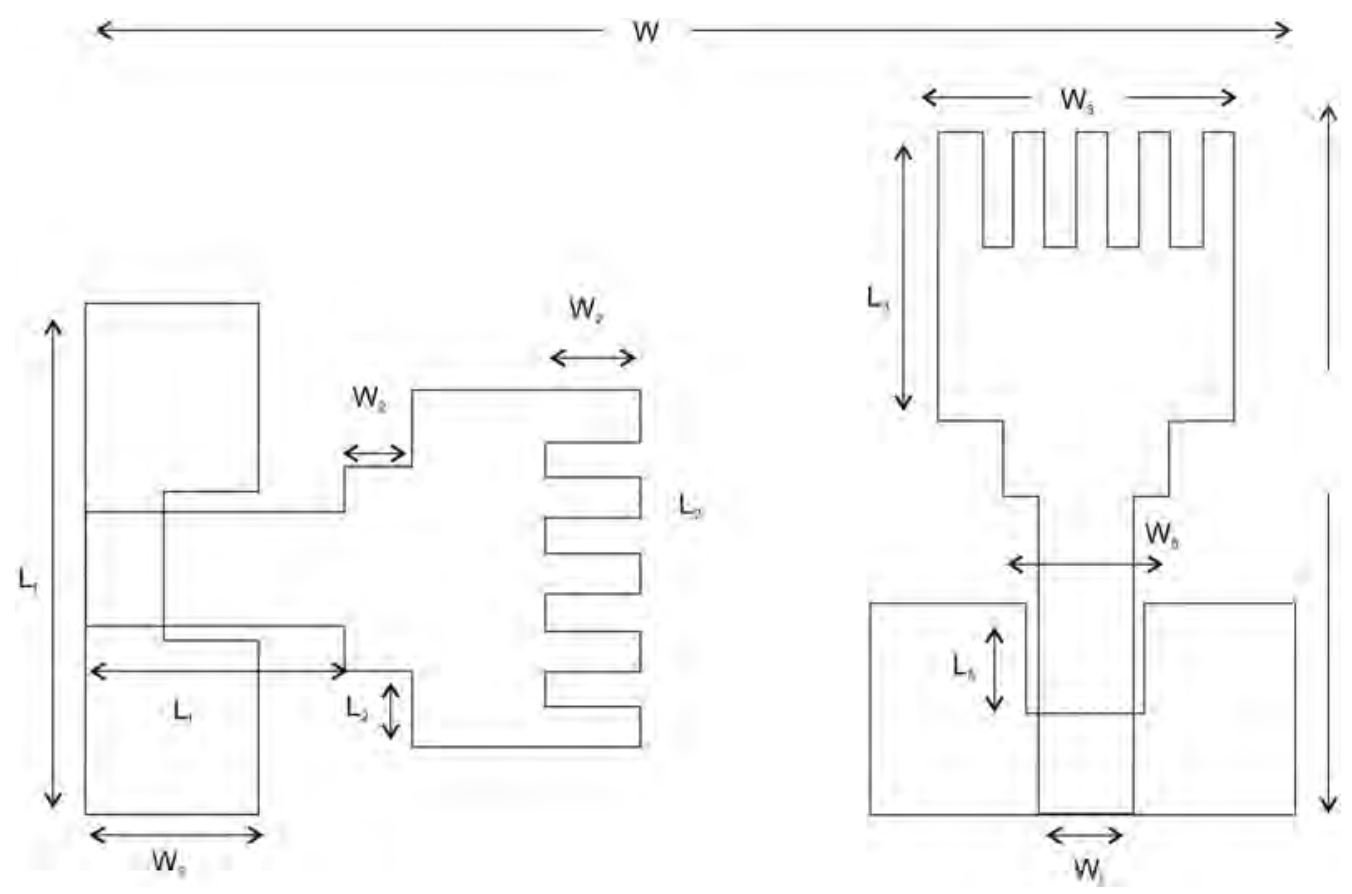

Figure 44.1 Top view of antenna with parameters.

Table 44.1 Optimized parameters with dimensions

\begin{tabular}{llllllll}
\hline Parameters & $W$ & $W_{g}$ & $W_{f}$ & $W_{s}$ & $W_{1}$ & $W_{2}$ & $W_{3}$ \\
\hline Dimensions $(\mathrm{mm})$ & 39 & 5.8 & 3 & 4 & 2 & 7.5 & 9.5 \\
Parameters & $\mathrm{L}$ & $\mathrm{L}_{\mathrm{g}}$ & $\mathrm{L}_{\mathrm{f}}$ & $\mathrm{L}_{\mathrm{s}}$ & $\mathrm{L}_{1}$ & $\mathrm{~L}_{2}$ & $\mathrm{~L}_{3}$ \\
Dimensions $(\mathrm{mm})$ & 19 & 13.6 & 8.5 & 2 & 2 & 1 & 7.5 \\
\hline
\end{tabular}

Table 44.2 Performance of previous designs with proposed antenna

\begin{tabular}{llll}
\hline Published literature & Size $(\mathrm{mm} \times \mathrm{mm})$ & Bandwidth $(\mathrm{GHz})$ & Isolation $(\mathrm{dB})$ \\
\hline$[16]$ & $33 \times 45.5$ & 7.5 & $<-15$ \\
{$[17]$} & $30 \times 40$ & 7.6 & $<-16$ \\
{$[18]$} & $31 \times 20$ & 4 & $<-15$ \\
Proposed Antenna & $39 \times 19$ & 11.2 & $<-20$ \\
\hline
\end{tabular}

bandwidth. The proposed antenna is wide band, with better isolation and compact size with respect to other published antenna.

\section{Results and discussion}

Figure 44.2 presents simulated S-parameters of designed compact novel wideband MIMO antenna which covers wideband from $4 \mathrm{GHz}$ to $15.2 \mathrm{GHz}$ with good reflection coefficient 


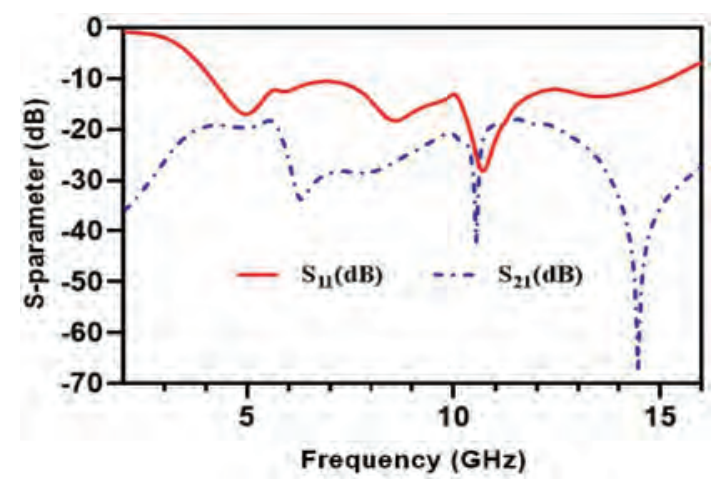

Figure $44.2 S_{11}$ and $S_{21}$ parameters of designed antenna.

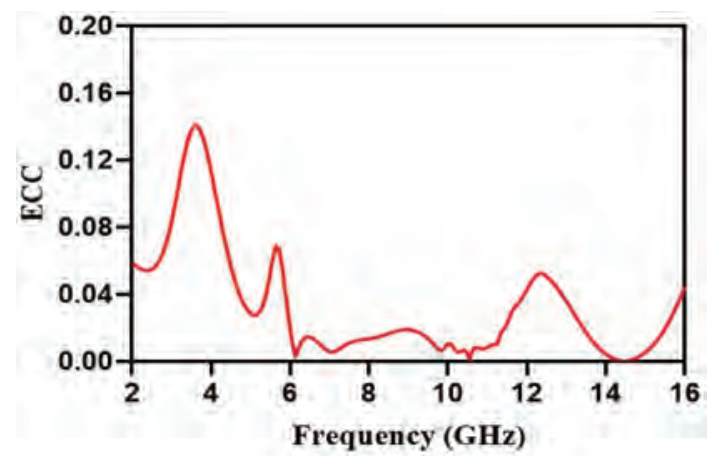

Figure 44.3 Simulated ECC.

less than $-10 \mathrm{~dB}$ for both port S11 and S22. The isolation coefficient S12 and S21 is less than $-20 \mathrm{~dB}$ in whole operating range. The proposed designed antenna is suitable for various wireless hand-held devices. The performance of MIMO antenna is evaluated in terms of envelope correlation coefficient (ECC). The practical acceptable value of ECC should be lower than 0.5 . Figure 44.3 shows the simulated value of ECC which is less than 0.1 throughout the frequency band.

The simulated surface current distribution is presented in Figure 44.4, and is uniformly distributed to all patch surface and more at near to feed point when second port is excited. The port 2 is excited at $4.8 \mathrm{GHz}, 8.05 \mathrm{GHz}$ and $10.8 \mathrm{GHz}$. Figure 44.5 presents the simulated gain of designed antenna which varirs from $2 \mathrm{dBi}$ to $7 \mathrm{dBi}$ at frequency band from 4 to $15.2 \mathrm{GHz}$.

Figure 44.5 presents radiation pattern at $4.8 \mathrm{GHz}, 8 \mathrm{GHz}$ and $10.8 \mathrm{GHz}$ for E-plane and $\mathrm{H}$-plane which is quasi-omnidirectional for $\mathrm{H}$-plane and for E-plane is directional pattern.

\section{Conclusion}

A novel and compact multiple input multiple output antenna is presented in this literature. The high isolation and compact size are achieved by placing antenna element orthogonal 


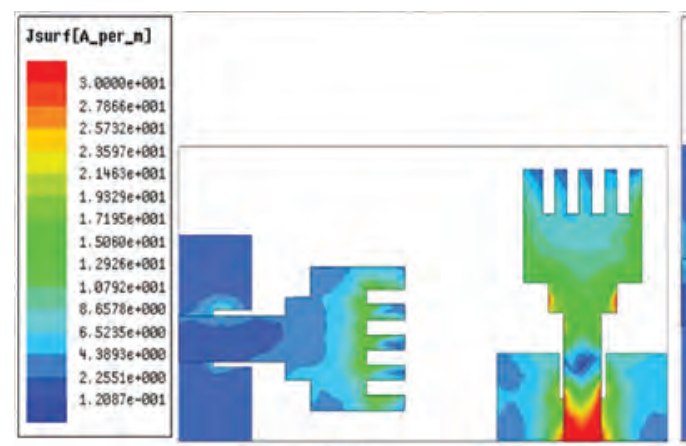

(a)

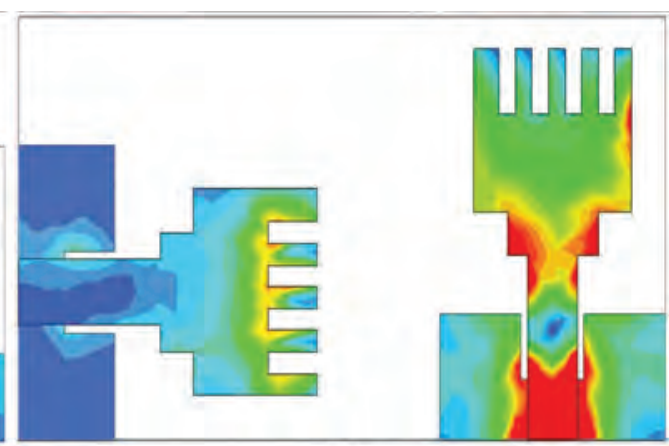

(b)

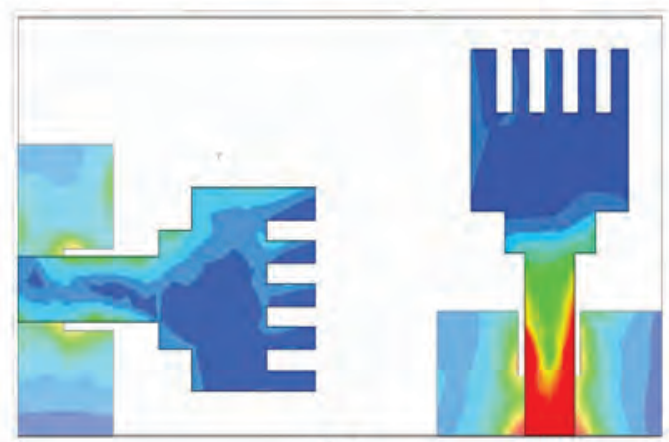

(c)

Figure 44.4 Simulated current distribution at (a) $4.8 \mathrm{GHz}$, (b) $8.05 \mathrm{GHz}$, (c) $10.8 \mathrm{GHz}$.

and modifying ground structure. Moreover the major parameters like S-parameter, radiation pattern, isolation and gain are investigated by simulation which is carried out on HFSS simulation software. The above result shows the good return loss, wider bandwidth, high isolation, and quasi-omnidirectional radiation pattern. Therefore, the proposed designed antenna is suitable for various wireless hand-held devices.

\section{References}

[1] "Revision of part 15 of the commissions rules regarding ultra-wideband transmission systems first report and order," Federal Commun. Commission, Washington, DC, USA, Tech. Rep. FCC 02.V48, 2002.

[2] L. Liu, S.W. Cheung, and T. I.Yuk, "Compact MIMO antenna for portable devices in UWB applications," IEEE Trans. Antennas Propag., vol. 61, no. 8, pp. 4257-4264, Aug. 2013.

[3] S. Tripathi, A. Mohan, and S. Yadav, "A compact Koch fractal UWB MIMO antenna with WLAN band-rejection,” IEEE Antennas Wireless Propag. Lett., vol. 14, pp. 1565-1568, 2015.

[4] C. M. Luo, J. S. Hong, and L. L. Zhong, "Isolation Enhancement of a very compact UWBMIMO slot antenna with two defected ground structures," IEEE Antennas Wireless Propag. Lett., vol. 14, pp. 1766-1769, 2015.

[5] L. Kang, H. Li, X. H. Wang, and X. W. Shi, "Compact offset microstrip fedMIMO antenna for band-notched UWB applications," IEEE Antennas Wireless Propag. Lett., vol. 14, pp. 17541757,2015

[6] C. X. Mao and Q. X. Chu, "Compact co-radiator UWB-MIMO antenna with dual polarization,” IEEE Trans. Antennas Propag., vol. 62, no. 9, pp. 4474-4480, Sep. 2014. 


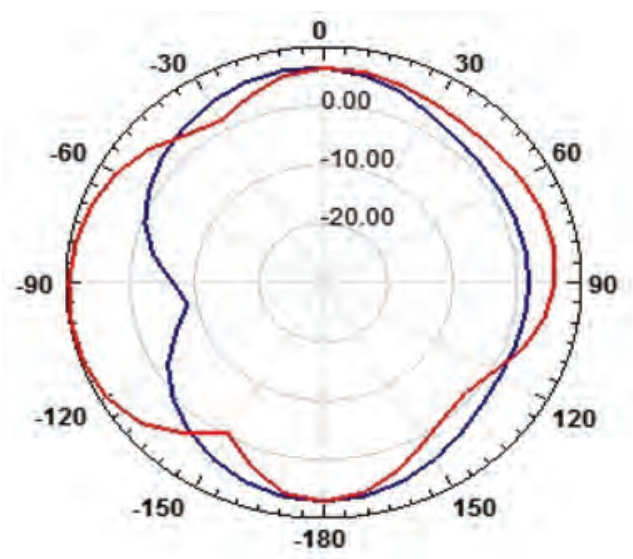

(a)

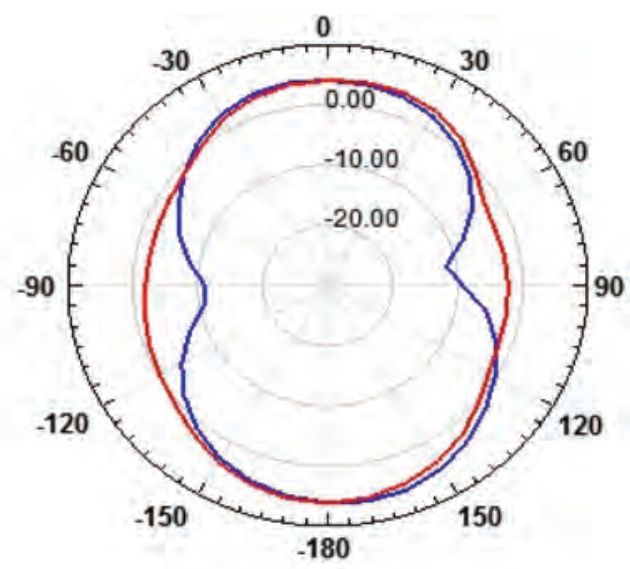

(b)

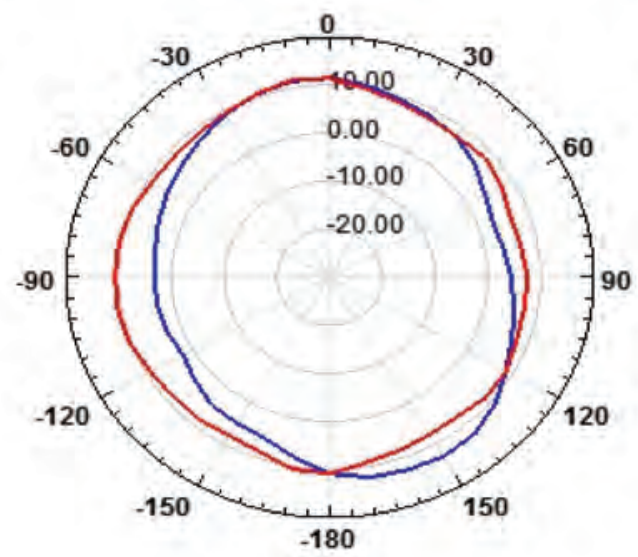

(c)

Figure 44.5 Simulated radiation pattern at (a) $6.51 \mathrm{GHz}$, (b) $8.03 \mathrm{GHz}$, (c) $11 \mathrm{GHz}$.

[7] J. Zhu, B. Feng, B. Peng, L. Deng, and S. Li, "A dual notched band MIMO slot antenna system with Y-shaped defected ground structure for UWB applications," Microw. Opt. Technol. Lett., vol. 58, no. 3, pp. 626-630, Mar. 2016.

[8] D. A. Ketzaki and T. V. Yioultsis, "Metamaterial-based design of planar compact MIMO monopoles," IEEE Trans. Antennas Propag., vol. 61, no. 5, pp. 2758-2766, May 2013.

[9] J. Zhao and J. Wang, "Correlation reduction in antennas with metamaterial based on newly designed SRRs," in Proc. Asia-Pacific Int. Symp. Electromagn. Compat, 2010, pp. 981-984.

[10] S. Zhang, Z. Ying, J. Xiong, and S. He, "Ultrawideband MIMO/Diversity antennas with a tree-like structure to enhance wideband isolation," IEEE Antenna Wireless Propag. Lett., vol. 8, pp. 1279-1282, 2009.

[11] J. Kumar, “Compact MIMO antenna,” Microw. Opt. Technol. Lett., vol. 58, no. 6, pp. 1294$1298,2016$.

[12] J. Ghosh, S. Ghosal, D. Mitra, and S. R. B. Chaudhuri, "Mutual coupling reduction between closely placed microstrip patch antenna using meander line resonator," Prog. Electromagn. Res. Lett., vol. 59, pp. 115122, Apr. 2016.

[13] S. Zhang and G. F. Pedersen, "Mutual coupling reduction for UWBMIMO antennas with a wideband neutralization line," IEEE Antennas Wireless Propag. Lett., vol. 15, pp. $166-$ $169,2016$. 
[14] R. Chandel, A. K. Gautam, and K. Rambabu, "Tapered fed compact UWB MIMO-diversity antenna with dual band-notched Characteristics," IEEE Trans. on Antennas and Propag., vol. 66, no. 4, pp. 1677-1684, 2018.

[15] M. S. Khan, A.D. Capobianco, S. M. Asif, D. E. Anagnostou, R. M. Shubair, and B. D. Braaten, "A compact CSRR-enabled UWBdiver- sity antenna," IEEE Antennas Wireless Propag. Lett., vol. 16, pp. 808-812, 2017.

[16] M. S. Khan et al., "Compact ultra-wideband diversity antenna with a floating parasitic digitated decoupling structure," Microw., Antenna Propag., vol. 8, pp. 747-753, Mar. 2014.

[17] J.-Y. Deng, L.-X. Guo, and X.-L. Liu, "An ultrawideband MIMO Antenna with a high isolation," IEEE Antenna Wireless Propag. Lett., vol. 15, pp. 182-185, 2016

[18] J. Kumar, "Compact MIMO antenna," Microw. Opt. Technol. Lett., vol. 58, no. 6, pp. 12941298, 2016. 


\title{
45 BBO based location optimization of target nodes using single mobile anchor node in WSNs
}

\author{
Parulpreet Singh*, Gurleen Kaur Walia, \\ Gagandeep Singh Walia
}

\author{
Lovely Professional University, Phagwara, India
}

\section{Introduction}

WSNs are the basic development drawing in huge interest. Various researches have resulted in different sensors of very small size and less cost which can communicate very effectively and with great efficiency. With the help of these ultra small sensors which are capable of communicating very effectively, it has led to ways to control many applications with great ease. These can cover a wide variety of applications related to defence range, medical health care, sensing climatic conditions etc. One of the most important aspect of WSN network is to find the location of the nodes, which is referred to as localization. If we follow the configuration in a manual way to find out the information of the location, it makes the process very complex. Secondly if we take help of GPS system, that also adds to the cost factor and moreover it is not feasible to find locations for areas like underwater and indoors [1,2].

\section{Applications of CI based BBO algorithm for WSN localization}

Biogeography is referred to as studying distribution of plants and animals in the geographical manner [3]. It relies upon the possibility of biogeography, which deals with the transport of species that depend upon different parts like precipitation, contrasting characteristics of geographical highlights, hotness and land territory. In biogeography based advancement wellness work is named as HSI (habitat suitability index) [4]. High HSI indicates a territory is where the organisms would love to live, and low HSI indicates a territory where organisms would not like to stay. The portrayal of the structures of the territory is named as SIV (suitability index variables). The migration (immigration) and resettlement (emigration) rates rely on the quantity of species in the territories. Low habitat suitability index habitat with high migration $\lambda$ is found in territories which are having less HSI and territories with high habitat suitability index has high resettlement $\mu$ [5]. The estimations of migration and resettlement degrees are given by conditions (1) and (2)

$$
\lambda=I\left[I-\frac{k}{n}\right]
$$

\footnotetext{
* Email: parulpreet.23367@lpu.co.in
}

DOI: 10.1201/9781003129103-45 


$$
\mu=\frac{E}{n}
$$

where I represents maximum migration rates and $\mathrm{E}$ represents maximum resettlement rates. Values of $\mathrm{I}$ and $\mathrm{E}$ may not be the same. $\mathrm{k}$ represents sum of species of the $\mathrm{k}^{\text {th }}$ individual whereas $n$ represents species number. $S_{\max }$ represents total species in a territory (habitat).

\section{Hilbert curve order with respect to size of the network}

In 1891, David Hilbert proposed a space-filling curve which on his name is referred to as the Hilbert curve [6]. While the curve was being planned it was made sure that it must visit all the spots in the whole target area. The most important step is to make sure that the right order of the curve should be selected so that the full possibility of the network is ensured. The least order should always be selected to cover the entire network. Assume considering $\mathrm{N}$ sub areas we consider the whole network and all the sub areas are subsquares of size 1 . The sub-square size is given by Equation (3).

$$
l=\frac{L}{2^{k}}
$$

where L represents network grid size and $\mathrm{k}$ represents curve order. Equation 4 calculates the total number of sub-squares

$$
N=4^{k}
$$

\section{Node localization optimization using single anchor node}

Here the position of the target nodes is found only with the help of a single anchor node which is mobile and will cover all the areas following the Hilbert trajectory curve. All the nodes referred to as target nodes whose location is to be found are distributed in the whole field in a random manner. We divide the whole field into various grids and grid interspacing should be less than anchor node range. Anchor nodes while moving through each grid will be sending a beacon signal which will make the target nodes localize individually. All the nodes which comes in the range of mobile anchor nodes receive beacon signal for a certain period of time and hence collect anchor node received signal strength information. Now, the Euclidean distance between the anchor and the target nodes is found out with the help of RSS information. After finding the distance, we deploy six ANs virtually at the same distance (corresponding to Euclidian distance) all at 60 degrees. Out of these six virtual ANs only two will be selected to find out the position of the TN which is coming under the range of the AN. The transmitting range of each node is kept same and each node hardware configuration is also kept same $[2,7,8]$.

\section{Simulation results and discussion}

The simulations using 50 fixed target nodes and single dynamic anchor nodes (following Hilbert trajectory [second order]) have been conducted in MATLAB and BBO based soft computing algorithm has been implemented in these simulations. In $15 \times 15$ length units 
field, these target nodes are deployed. The reference node is moving with the fixed speed following the above-mentioned trajectory. Whenever any TN comes into the range of reference node, using RSS measures, distance is calculated between that target and reference node. Further, with same space and an angle change of 60 degrees, six VA nodes are projected in the whole 360 degrees. Furthermore, using angle information, two VA nodes are selected to find out the centroid and BBO based optimization algorithm is implemented on that centroid.

In the proposed work, each TN localized by only dynamic AN runs BBO algorithms distinctly to localize itself. Population size of 20 with probability of alteration of particle weight is considered as 0.05 . The rate of emigration as well as immigration is considered as 1 . In the simulations, 100 iterations have been considered.

In mobility based scenario, BBO based optimization scheme is investigated. The fitness function is considered as the average localization error. Due to stochastic behaviour of the algorithm, there is no indistinguishable solution for the similar deployment. The simulation has been conducted in MATLAB software and the altitudinal results of suggested localizing algorithm using solicitation of BBO algorithm is given in Figure 45.1.

Figure 45.1 gives the results of the localized node at the first position of the $\mathrm{AN}$ at $(0$, $0)$. It can be clearly seen that 4 TNs are coming in the range of anchor node and are being localized. Similarly at various positions of the AN, the results of localized TN are given in Table 45.1. The actual difference between estimated and localized target node is termed the localization error. It is clearly depicted in Figure 45.1 that the localization algorithm will run for 16 different counts with fixed intervals of time. The performance parameters considered in the table are MiLE, M $\times$ LE, ALE and TIR, which can be abbreviated as

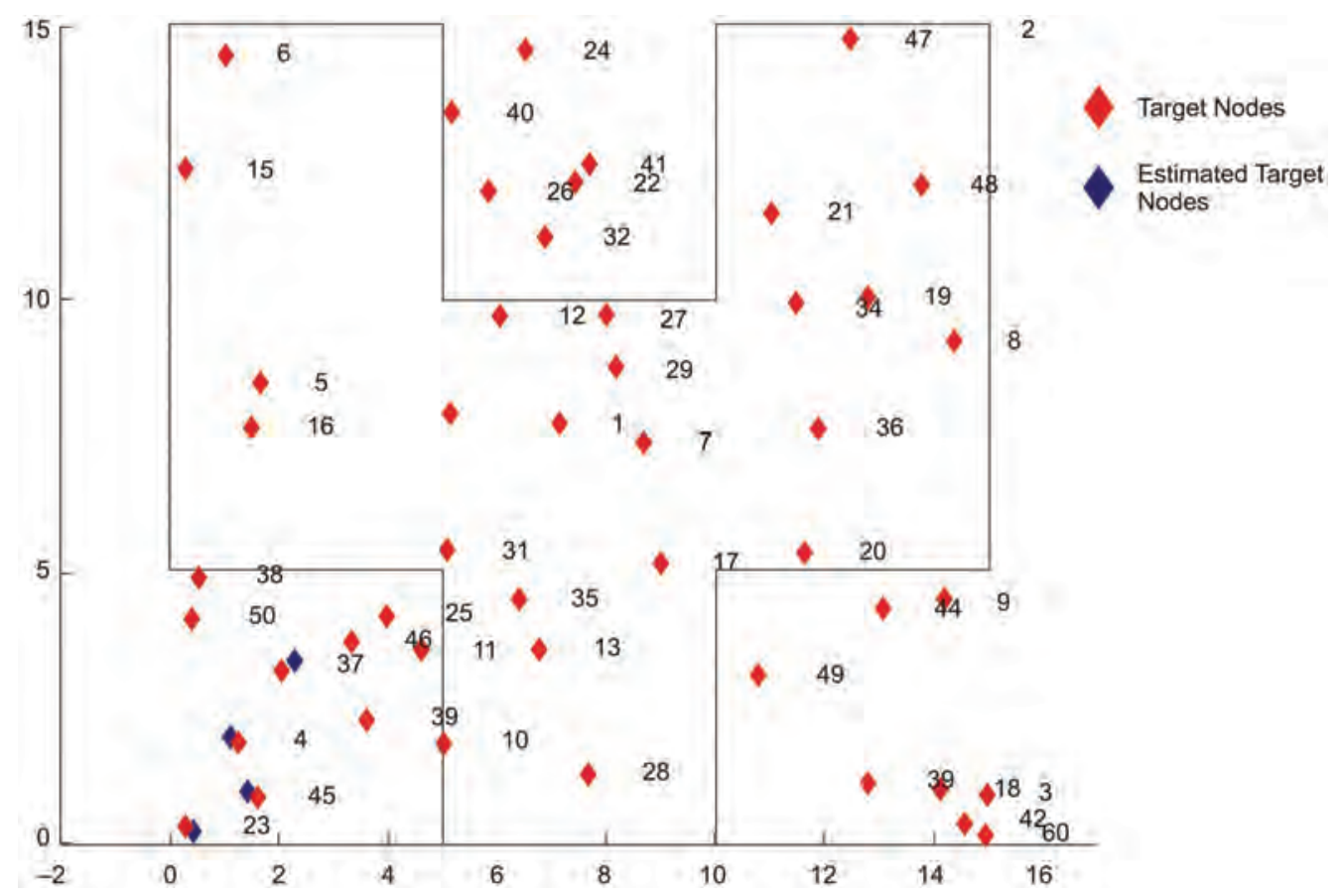

Figure 45.1 Target nodes localized at various positions of the moving anchor node using application of $\mathrm{BBO}$. 
Table 45.1 Summary of simulation results for BBO algorithm for all 16 positions of anchor node

\begin{tabular}{lcrlllr}
\hline $\begin{array}{l}\text { Anchor } \\
\text { position }\end{array}$ & $\begin{array}{l}\text { Anchorposition } \\
\text { coordinates }\end{array}$ & & $\begin{array}{l}\text { M×LE } \\
\text { (L units) }\end{array}$ & $\begin{array}{l}\text { MiLE } \\
\text { (L units) }\end{array}$ & $\begin{array}{l}\text { ALE } \\
\text { (L units) }\end{array}$ & $\begin{array}{l}\text { Number } \\
\text { of TIR }\end{array}$ \\
\cline { 2 - 3 } & $x$ & $y$ & & & & \\
\hline 1 & 0 & 0 & 0.58061 & 0.2281 & 0.3980 & 4 \\
2 & 5 & 0 & 1.1784 & 0.1702 & 0.6003 & 5 \\
3 & 5 & 5 & 1.4205 & 0.2374 & 0.6162 & 12 \\
4 & 0 & 5 & 0.9831 & 0.0459 & 0.5579 & 6 \\
5 & 0 & 10 & 1.1463 & 0.3997 & 0.6735 & 3 \\
6 & 0 & 15 & 0.5821 & 0.3713 & 0.4767 & 2 \\
7 & 5 & 15 & 0.6846 & 0.5196 & 0.6078 & 3 \\
8 & 5 & 10 & 1.3225 & 0.2014 & 0.5784 & 12 \\
9 & 10 & 10 & 0.8132 & 0.1056 & 0.4402 & 9 \\
10 & 10 & 15 & 0.9334 & 0.2110 & 0.4633 & 5 \\
\hline
\end{tabular}

minimum, maximum and average error of localization by bearing in mind all localized nodes. For mobility based scenarios, there is a trade-off that the period of the convergence should be less, which means that the convergence should be very fast.

The algorithm proposed in this this work has less convergence time. The average time of convergence for BBO at any position of the AN is 0.2077 seconds. The algorithm proposed in this work has very few convergence periods as well as providing good localization accuracy. Table 45.1 gives the localization error with all positions of the AN, following Hilbert trajectory.

\section{Discussion and future aspects}

In this work, node location finding procedure employing the BBO algorithm for isolated and isotropic WSNs is anticipated by employing only dynamic AN following Hilbert trajectory. The reference node is moving with the fixed speed following the above-mentioned trajectory. Whenever any TN comes into the range of reference node, using RSS measures, distance is calculated between that TN and reference node. Further, with same space and an angle change of 60 degrees, six VA nodes are projected in the whole 360 degrees. Furthermore, using angle information, two VA nodes are selected to find out the centroid and the BBO based optimization algorithm is implemented on that centroid. The proposed algorithm can be utilized for the various applications in logistics, military and patient health monitoring in medical applications.

\section{References}

1. H. Karl, A. Willig, Protocols and Architectures for Wireless Sensor Networks. John Wiley \& Sons, 2007.

2. P. Singh, A. Khosla, A. Kumar, M. Khosla, " $3 \mathrm{~d}$ localization of moving target nodes usingsingle anchor node in anisotropic wireless sensor networks", AEU-International Journal of Electronics and Communications 82, 2018.

3. D. Simon, "Biogeography-based optimization", IEEE Transactions on Evolutionary Computation 12 (6) (2008) 702-713.

4. Z. Wang, P. Liu, M. Ren, Y. Yang, X. Tian, "Improved biogeography-based optimization based on affinity propagation”, ISPRS International Journal of Geo-Information 5 (8) (2016) 129. 


\section{Intelligent Circuits and Systems}

5. A. Kumar, A. Khosla, J. S. Saini, S. Singh, "Meta-heuristic range based node localization algorithm for wireless sensor networks", in: IEEE International Conference on Localization and GNSS, IEEE, 2012, pp. 1-7.

6. H. Sagan, "Hilbert space filling curve", in Space Filling Curves. Springer, 1994, pp. 9-30.

7. P. Singh, A. Khosla, A. Kumar, M. Khosla, "Computational intelligence based localization of moving target nodes using single anchor node in wireless sensor networks", Telecommunication Systems 69 (3) (2018) 397-411.

8. P. Singh, A. Khosla, A. Kumar, M. Khosla, "Optimized localization of target nodes using single mobile anchor node in wireless sensor network", AEU-International Journal of Electronics and Communications 91 (2018) 55-65. 


\title{
46 Smart queue management system for public transportation
}

\author{
Rohith Sai Veedula, Praneeth Atagara Meti, \\ Sri Gayathri Pabbareddy, Towfeeq Fayaz, \\ Gurjot Singh Gaba*, Lavish Kansal
}

\author{
School of Electronics and Electrical Engineering, Lovely Professional \\ University, Phagwara, Punjab, India
}

\section{Introduction}

Since the first commercial flight in 1914, air travel has changed a lot. Then air travel was an experience reserved for the most privileged class of the society and the cabins in the aero plane were spacious and passengers experienced a luxury in-flight [1]. But the categorization of air travel tickets into different classes has allowed people having different financial status have an airline travel.

The growth of countless new routes and development of hundreds of new airports and thousands of new planes and pilots in the past decades created a new market, making different airline companies provide tickets at competitive low prices. According to the International Air Transport Association (IATA) in 2016 there were a staggering 3.8 billion air travellers and it is predicted to rise to 7.2 billion passengers by 2035 [2]. This increase in the number of passengers has led to the processes of increasing the number of seats on a flight, which technically involves reduction in leg space, number of lavatories on board etc. This reduction in the number of lavatories would possess a difficulty for the passengers in making of use of them, mainly in the case of international air travel (long journeys). The reduction in the size of the lavatory to expand the seating arena can be seen in Figure 46.1.

Many recommendations and patents were filed for facilitating the passengers with limited lavatories, but most of them remained on paper and have not been implemented on real grounds. We have designed our lavatory management system in a way easy to implement, having all possible functions integrated on a single system.

\section{Literature review}

This section elaborates the various research works carried out to solve the aforementioned issue.

A queue management system providing a token number to the passenger making a reservation through their system had been proposed by the inventors [4]. This system works on the basic principle of serial queue token allocation and cancellation and does not discuss other advanced features like urgency request and passenger authentication.

A system for providing reservation of lavatory in public transport system such as airplanes comprising in-screen seat display, higher priority to passengers with urgency,

* Corresponding author email: gurjot.17023@lpu.co.in 

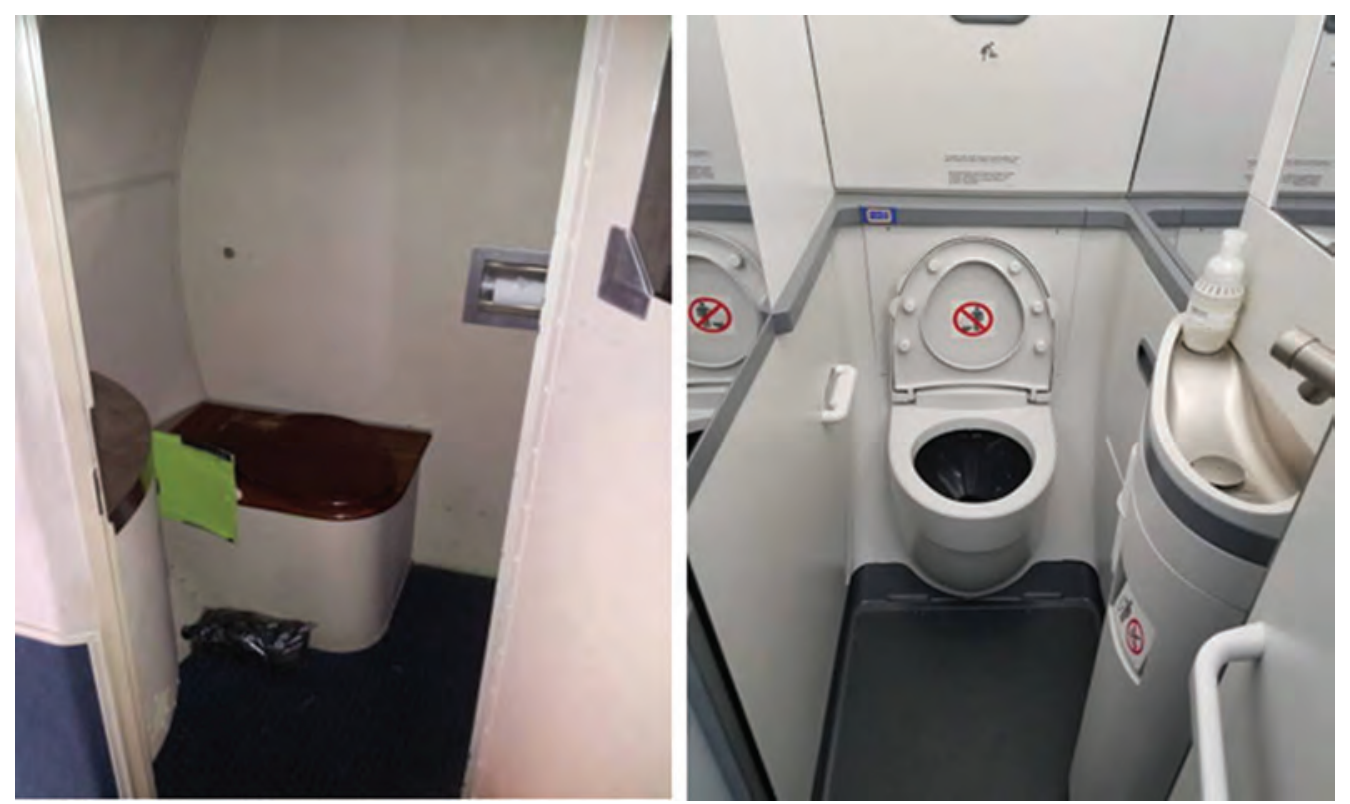

Figure 46.1 Washrooms in the past and present generations [3].

access code for authentication of passengers at the lavatory door has been mentioned by the inventor [5]. However, the proposed system fails to display a waiting time to the passengers in the queue.

Another system providing a mechanism for automatic reservation of lavatory facility to the passengers through mobile and display the waiting time for occupying the lavatory to the passengers has been proposed. A facility for emergency reservation and user authentication for availing various services were also mentioned in the proposed work [6]. However, the proposed system fails to present the facility for the passenger in the lavatory to extend the time in case of emergency.

In the above reviewed proposed systems, most of them are serving the purpose of reserving a lavatory and standing in the queue virtually avoiding a physical strain and discomfiture to the other passengers. But, those systems lack the ability to arrange passengers depending on their requirement of lavatory usage, such as some of the passengers might have reserved lavatory for the purpose of facewash or for sanitizing their hands; these activities would not take much time and allotment of equal time span to all would make the movement of passengers slow. A more efficient algorithm based on pre-arrangement of passengers based on their specificities would allow much optimized use of the available lavatories.

The traditional solutions also fail to prevent misuse of the lavatory, who would be reserving the lavatory too often such as making reservation immediately just after making use of the lavatory thereby avoiding legitimate passengers making use of the reservation system. A complete system consisting of the collective features of reservation with user authentication using in-screen displays or mobile phones, emergency requests for reservation and time extension, lavatory proximity, lavatory misuse avoidance feature would be ideal and serve the complete purpose. 


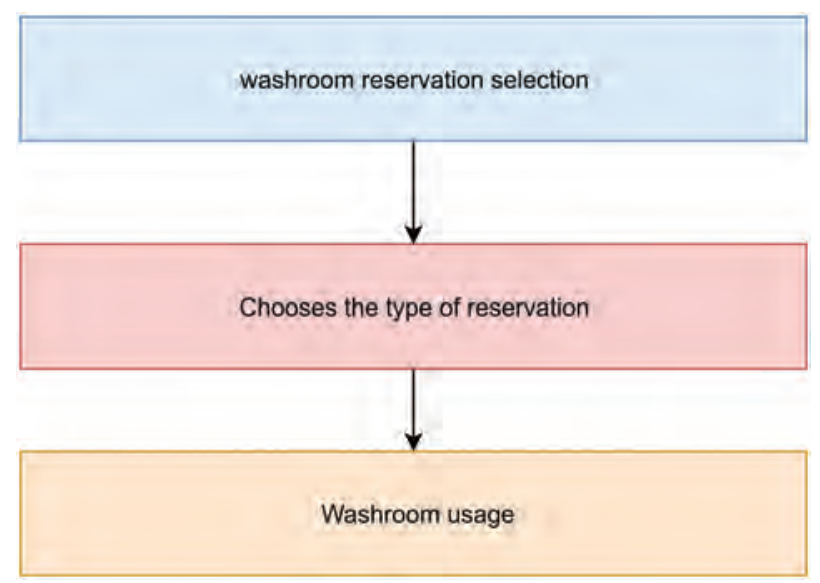

Figure 46.2 Working of lavatory management system.

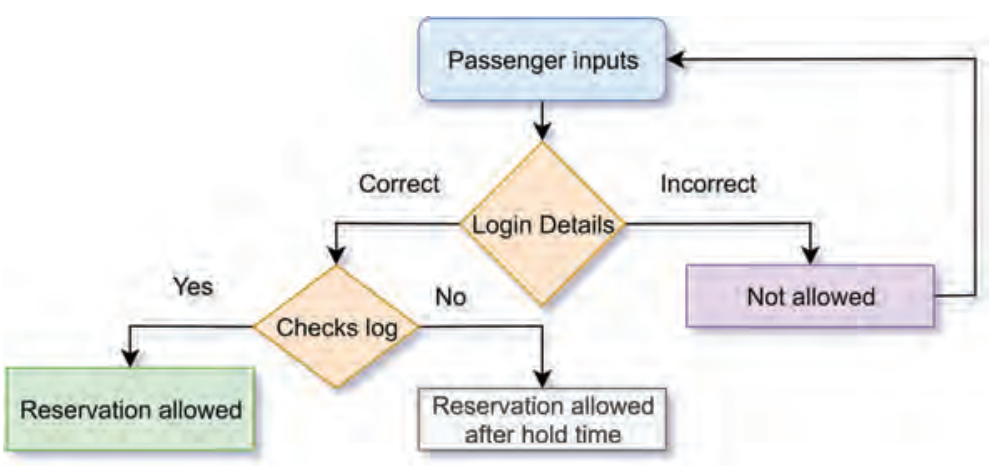

Figure 46.3 Lavatory reservation process.

\section{Proposed system}

Figure 46.2 illustrates the three stage working of the automatic lavatory management system.

In stage 1 of lavatory reservation selection, passengers select lavatory reservation through their mobiles or using the in-screen seat display where they are prompted to login with their respective credentials. If the provided login information is correct, the passenger is allowed to make a reservation, if passenger had not made any reservation within past 20 minutes. This buffer time allows the prevention of the misuse of the reservation system by people who make multiple reservation requests creating ambiguity in the queue. Figure 46.3 depicts the complete process.

During stage 2, passenger chooses the type of reservation followed by defining the sensitivity of request i.e., urgency or normal. Figure 46.4 depicts the user interface for booking the lavatory. The normal request enables the passenger to reserve the lavatory for facewash etc. This allows the system to shuffle the passengers in the queue with respect to the usage time of lavatory. 


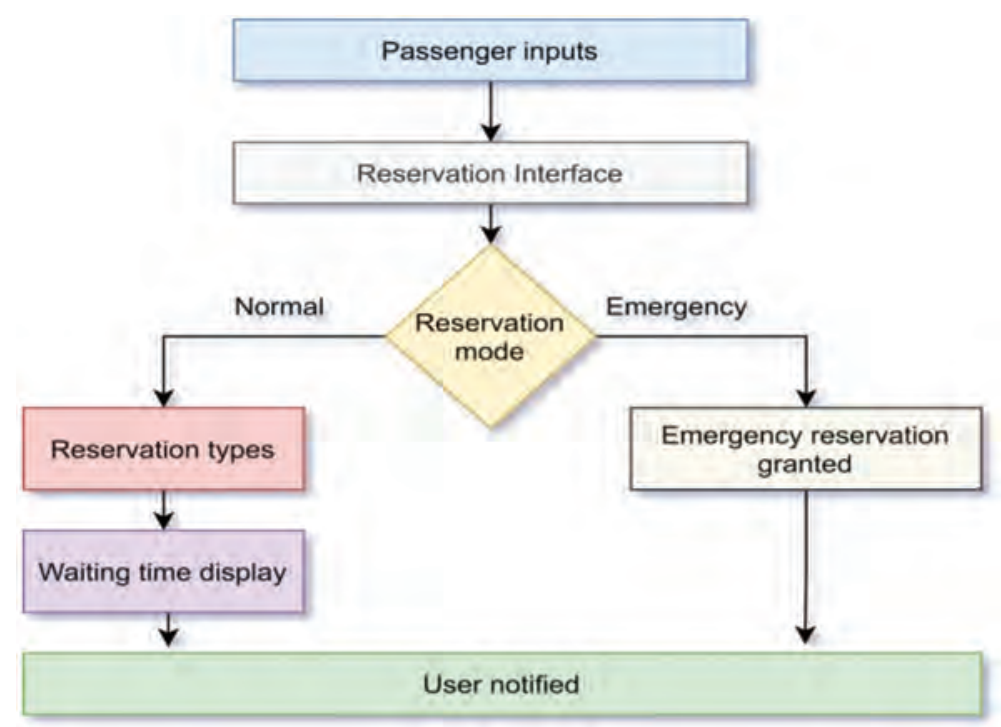

Figure 46.4 Sensitivity of reservation.

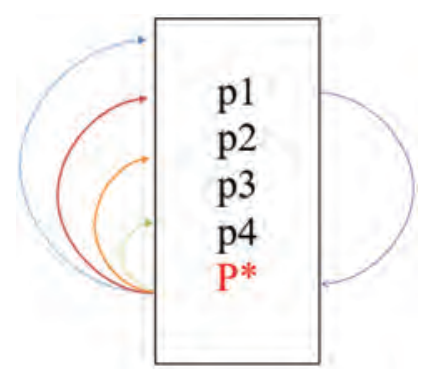

Figure 46.5 Passenger request for emergency.

\section{A. Emergency request working}

In the case of emergency reservation by a passenger, other passengers in the queue are requested for a swap in their position backwards by a place, so that the user with emergency can make use of the lavatory in less time. The swapping process can be observed in Figure 46.5.

\section{B. Lavatory allocation methodology}

Passengers are allocated to the nearest washroom from the location of their seat for convenient movement in the path between the seats, as food trolleys, movement of other passengers and vendors in the aisle might create any form of inconvenience to the users in reaching the lavatory. Therefore, nearest available washrooms if available would be given priority initially and further forms of queue optimizations are followed.

Further, lavatories are allocated to the passengers dynamically by checking the availability in other sections of the transport system (such as other cabin classes in aero planes 


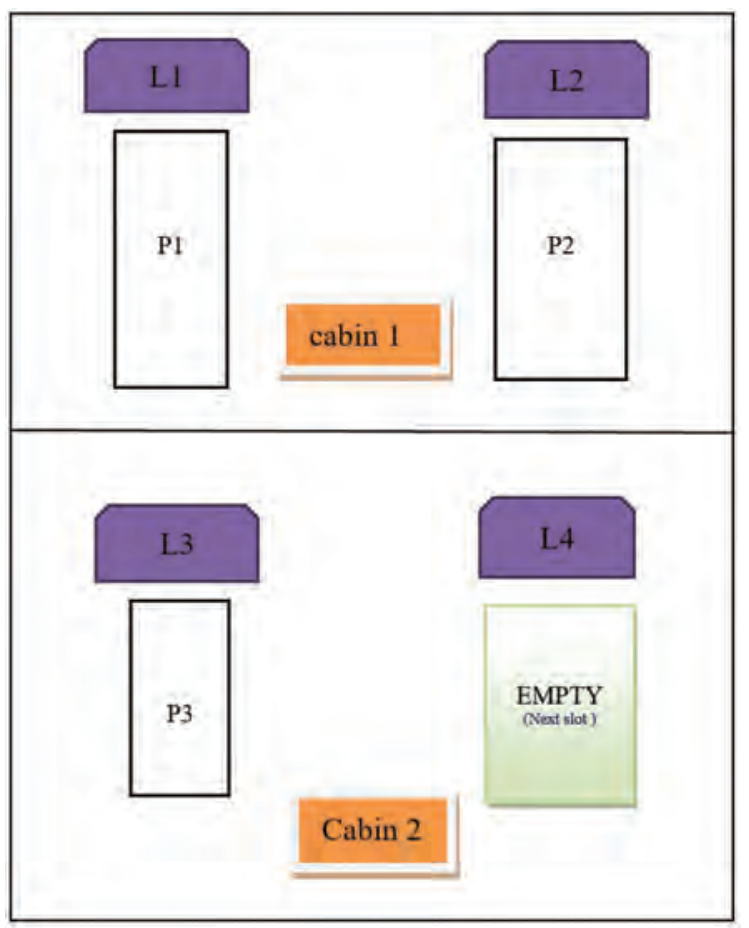

Figure 46.6 Dynamic washroom allocation.

or compartments in railways) lavatories on board and allocating them if any of them are free and if not free then the passenger is added to the queue with least waiting list. This allows the maximum usage of resources. Figure 46.6 depicts the process of dynamic allocation of washrooms.

The usage type selection such as facewash, lavatory, other would allow the system to assign the passenger reservation based on time and arrange the queue based on this time allocation.

The queue at a lavatory having total waiting time higher than the queue at another lavatory would indicate the system to push next new reservation to the queue with lesser overall time, else if no new reservation is made then rearrange the existing queues in an optimal way. Figure 46.7 justifies the process of optimization.

If a queue at one lavatory is clearing quickly than other lavatories passengers from other longer and long waiting queues will be swapped to the quicker ones to provide early turns. Arrangement of passenger queue with respect to the time of usage would allow the algorithm to function in a more efficient manner, as the queue having "facewash reservation" requests would clear faster when compared to the other options.

A waiting time for next lavatory availability is displayed to the passengers and they are notified when their turn comes.

Stage 3 demonstrates the process when passenger visits the lavatory. The passenger has to enter the same authentication pattern which was earlier used by him during the reservation process. This allows only legitimate passengers to make use of the lavatory. Though usage time is allocated to passengers, to encounter uncertainty an extension push button is provided inside the lavatory. The same would be notified in the waiting time interfaces of the passengers in the queue. Figure 46.8 shows the stepwise execution of stage 3. 


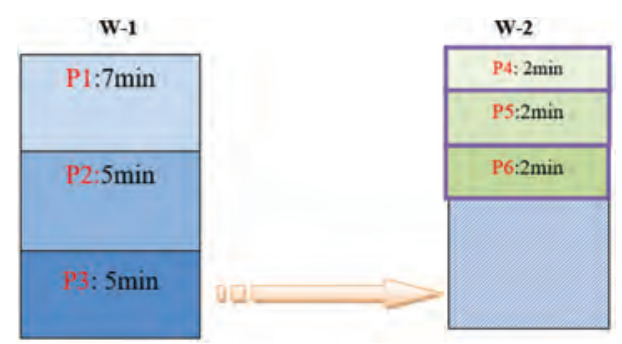

Figure 46.7 Queue arrangement based on usage time.

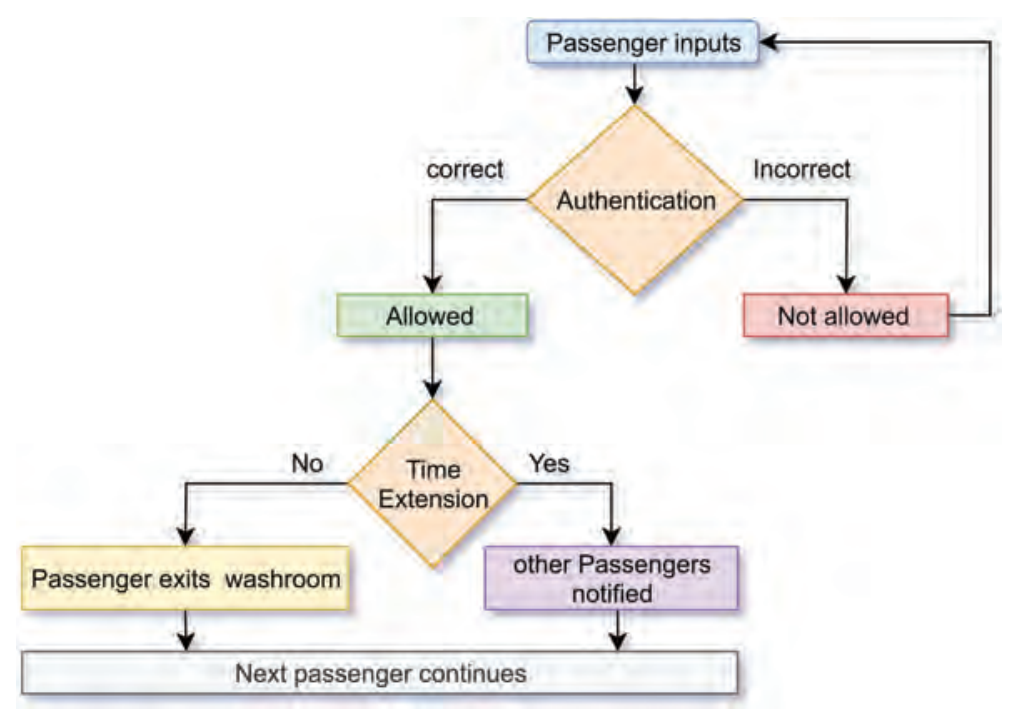

Figure 46.8 Smart time extension feature in lavatory.

The components used for the design and development of the lavatory management system include a microcontroller unit which is embedded with a Wi-Fi on it (node MCU) or an external connection to the local network [7]. Passengers enter the input through either their mobile, laptop or the screens available on board. The serial ID associated with the passenger while a reservation is made is displayed on a seven segment display board in front of the lavatory. Figure 46.9 shows the integrated model of smart queue management system.

The electronic system has been simulated and tested on hardware for reliability and performance analysis. An authentication ID in the form of pass code or pattern is entered through a numerical pad or LCD touch display to allow only legitimate users to access the lavatory. In addition, extra time requirement could be requested through a push button made available in the lavatory.

\section{Results and discussion}

The passenger is advised to connect with the local Wi-Fi network if personal device is used instead of screen panels. The user can access the URL of the lavatory management system 


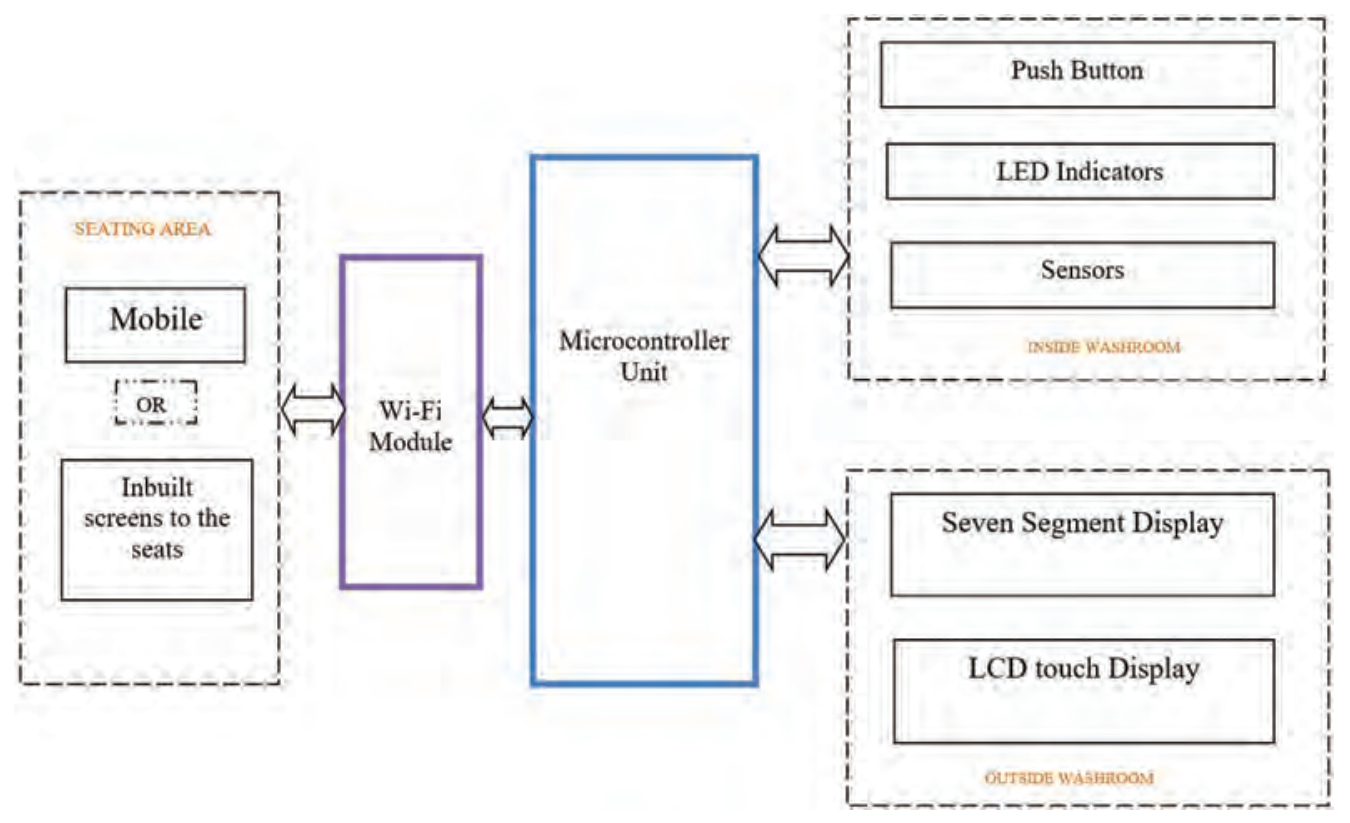

Figure 46.9 Block diagram of smart queue management system.

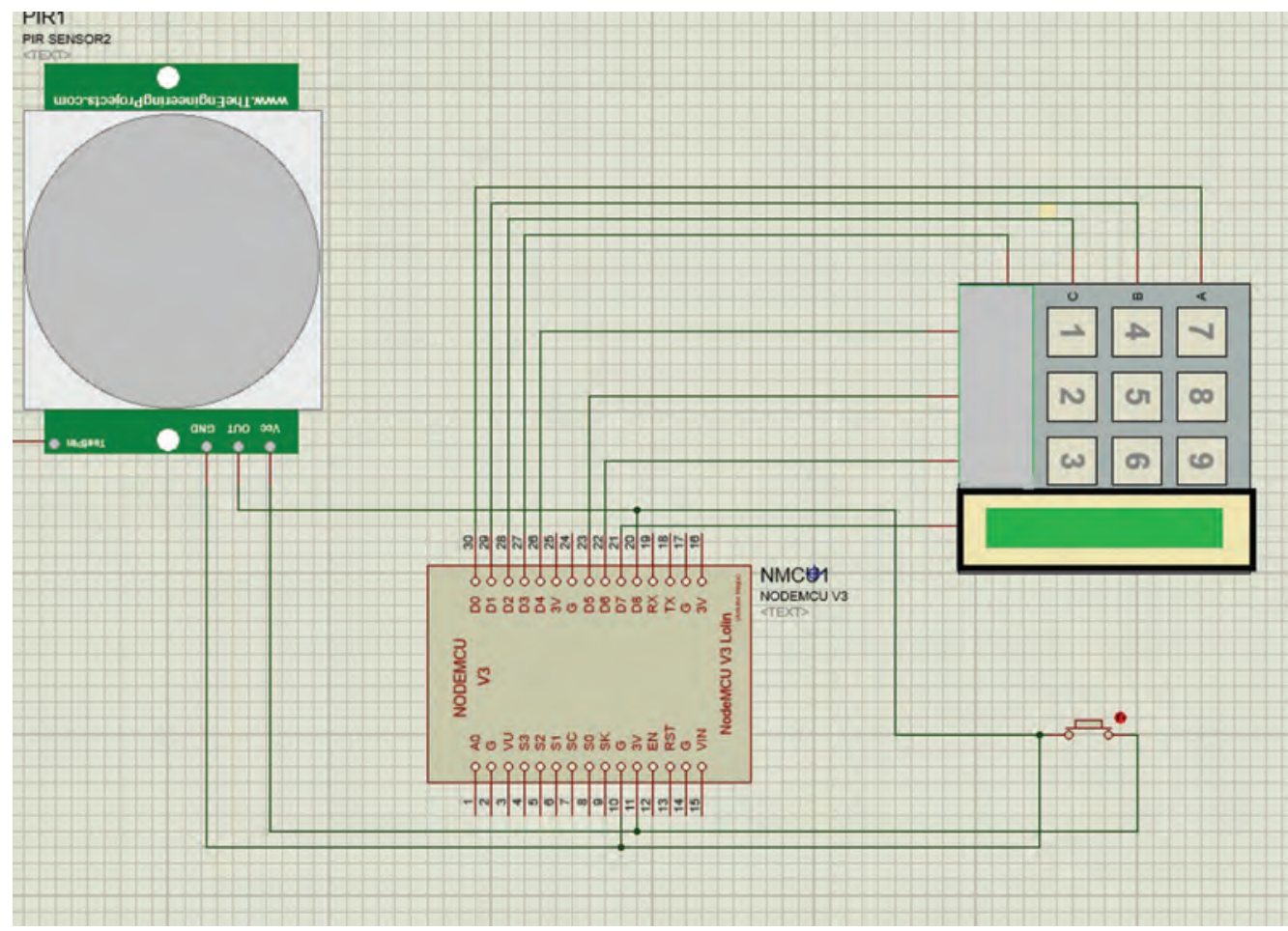

Figure 46.10 Simulation model of queue management system. 


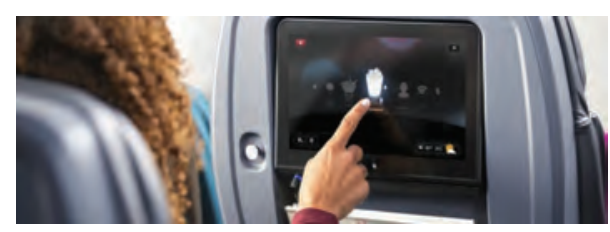

Figure 46.11 Passenger hardware for interface selection [8].

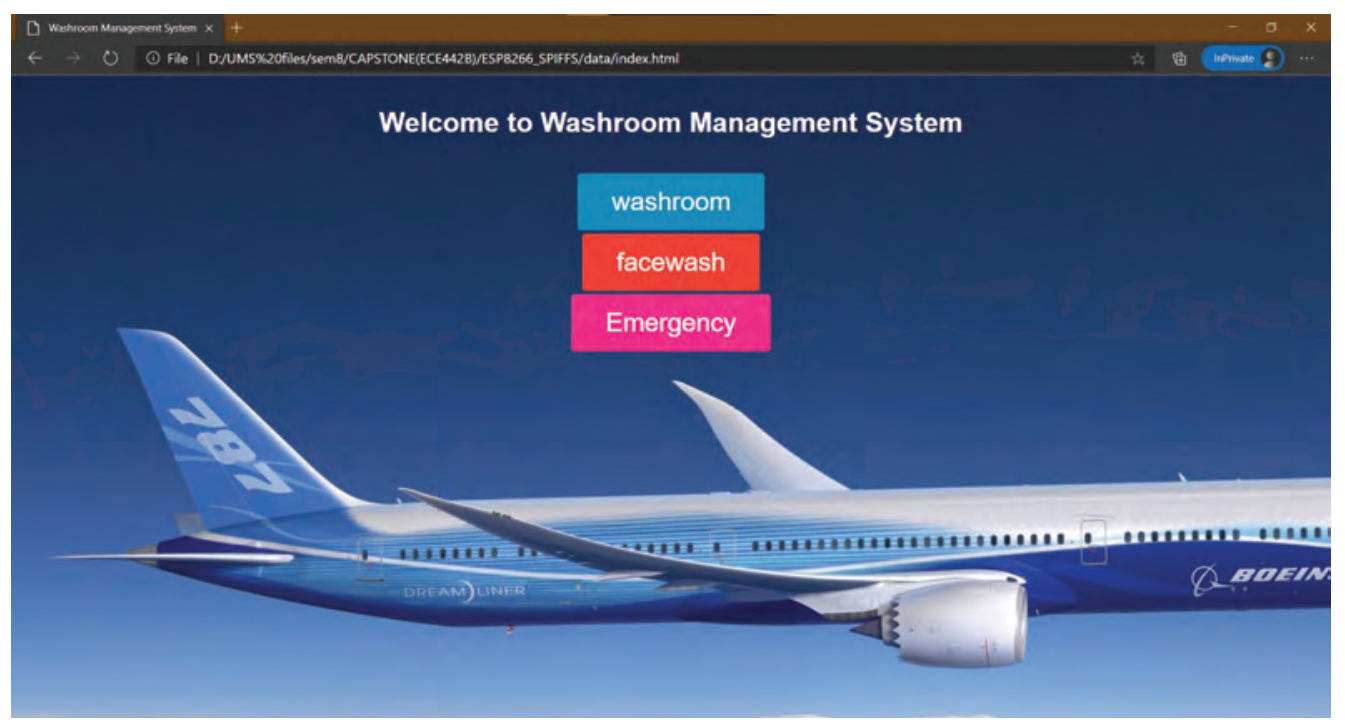

Figure 46.12 Lavatory slot booking interface.

as and when required to book the slot. Once the passenger visits the web interface of the system, they are advised to log in through their details such as their ticket number.

Figure 46.11 depicts the hardware reference to be used for a successful login. The passenger can book the restroom for a normal reservation process for the type of usage (facewash, toilet, others) of the lavatory as displayed in Figure 46.12. The passenger can even request the emergency use of the lavatory.

Figure 46.13 depicts a sorted queue arrangement after the passenger "p2" in the queue accepted the request of $\mathrm{P} *$ for making an emergency use of the washroom.

A rearranged queue of lavatory is depicted in Figure 46.14, in which the new user reservation is pushed to the queue of lavatory with less total waiting time.

\section{Conclusion and future scope}

This system of lavatory management provides passengers with a comfortable experience during their travel and helps the organizations in serving the passengers in the best possible manner. As travel in the modern world is taking the terminology of not just travelling to various places on earth but exploring various planets and developing a habitat on possible ecosystem in the universe the discussion on the basic amenity of the humans sound as 


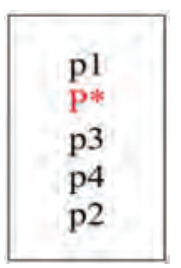

Figure 46.13 Passenger P* emergency request accepted.

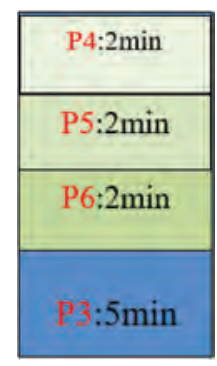

Figure 46.14 Updated queue after calculating users' total waiting time.

an area to find enough answers for everyone's comfiture. An effective and simple interface would help in achieving in reaching any amateur and add on to the advantage of society. The process of working of this system of "queue management" could not only be limited for its usage at the locations of lavatories in transport systems, but also can be applied at different locations of public usage like shopping malls, stores etc.

\section{References}

[1] "Aircraft lavatory", En.wikipedia.org, 2020. [Online]. Available at: https://en.wikipedia.org/ wiki/Aircraft_lavatory. Accessed: 2 Feb. 2020.

[2] "As billions more fly, here's how aviation could evolve", Nationalgeographic.com, 2017. [Online]. Available at: www.nationalgeographic.com/environment/urban-expeditions/transportation/air-travel-fuel-emissions-environment/. Accessed: 2 Feb. 2020.

[3] "Aircraft toilets / toilets of the world", Toilets of the World, 2020. [Online]. Available at: https:// toilet-guru.com/aircraft.php. accessed: 2 feb. 2020.

[3] "Two problems with American Airlines lavatories: size and... sexism? - View from the wing", View from the Wing, 2020. [Online]. Available at: https://viewfromthewing.com/two-problemswith-american-airlines-lavatories-size-and-sexism/. Accessed: 2 Feb. 2020.

[4] International Business Machines Corp, "System and method for providing reservations for restroom use", US6329919B1.

[5] “Airplane Lavatory Reservation System”, US 7535367B2.

[6] Good2Go, Inc., San Francisco, CA (US), “Facility And Resource Access System”, US $1018592 B 1$.

[7] M. Schwartz, Internet of Things with ESP8266. Packt Publishing, 2016.

[8] “American Airlines - entertainment online", Entertainment.aa.com, 2020. [Online]. Available at: https://entertainment.aa.com/en/seatback/. Accessed: 2 Feb. 2020. 


\title{
47 Proposal of new performance measures for ICA
}

\author{
Bhaveshkumar C. Dharmani* \\ School of Electronics \& Electrical Engineering, Lovely Professional \\ University, Punjab, India
}

\section{Introduction}

Independent Component Analysis (ICA) has been successfully applied for dimensionality reduction, feature extraction, source separation and others [1-4]. The ICA model assumes that the available observed data matrix $X$ with dimension $n \times N$ was generated due to linear transformation of hidden component matrix $S$ with dimension $n \times N$, i.e.,

$$
\mathbf{X}=\mathbf{A S},
$$

where $A$ with dimension $n \times n$ is the linear time invariant mixing transformation. More specifically, $\boldsymbol{x}=\left(x_{1}, x_{2}, \ldots, x_{m}\right)^{T}$ is an observation random vector $(r . v e c.) ; x_{i}, i=1: n$ are observation random variables $(r$ vars. $), s=\left(s_{1}, s_{2}, \ldots, s_{n}\right)^{T}$ is a source $r . v e c . ; s_{i}, i=1: n$ are $r$. vars. with values in real number set $\Re ; m \geq n N$ is the number of samples and $A$ is full rank.

As a component analysis tool, it aims finding component vectors in the directions of maximum information. The well-known Principal Component Analysis (PCA) assumes variance as the measure of information and finds the components that are orthogonal and in the directions of maximum statistical variance. Compared to this, ICA assumes statistical independence as the measure of information and finds the components that are "most independent possible" (m.i.p.). The ICA problem is to find $A$ and $S$ together from $X$ with the only assumption of statistical independence among the components of $S$.

Historically, ICA was developed as a solution to blind source separation (BSS) problem in linear time-invariant mixing. When ICA is used for BSS, the components are called sources. Given the sources are independent and identically distributed (i.i.d.) with no more than one source being Gaussian and $A$ is full rank, a unique ICA solution is obtained and it matches the actual sources as ensured by the Darmois-Skitovitch theorem $[1,4,6]$. Let $W$ be the estimated inverse of the mixing matrix $A$, then

$$
\mathbf{Y}=\mathbf{A}^{-1} \mathbf{X}=\mathbf{W} \mathbf{X}=\mathbf{W} \mathbf{A S} \text {. }
$$

As an optimization problem ICA can be expressed as

$$
\mathbf{Y}=\max _{\mathbf{w}} \varnothing(\mathbf{W} \mathbf{x})
$$

\footnotetext{
*Email: Bhavesh.23409@lpu.co.in
}

DOI: $10.1201 / 9781003129103-47$ 
where $\varnothing(\bullet)$ is a contrast function or simply contrast, which measures independence or dependence and ensures optimal $W$ on maximization giving components or sources among $y$ to be the most independent possible.

The performance of the ICA algorithms is measured in terms of their ability to obtain back the actual test sources. All the state-of-the-art performance measures are based on the time sequence matching of the estimated sources $y_{i}, i=1: n$, and actual sources $s_{i}, i=1: n$. As the model assumes the sources to be $r$. var., this article proposes to use statistical matching among the estimated and actual sources as a criterion for performance measurement. Empirical validations are also provided with two such performance measures. The second proposed criterion uses existing independence measures of the estimated sources as the performance measure. The third one uses performance measure of the estimated sources, when the same performance measure is used as the independence measure, as the reference for comparison with the outcomes through other algorithms.

Section 2 discusses the conventional contrast and performance measures for ICA. Section 3 proposes the novel performance criteria and measures. Section 4 provides empirical verification of the proposals made in Section 3. Finally, Section 5 provides discussion on consequences and conclusion.

\section{ICA contrasts and performance measure}

ICA needs independent components $\left(y_{i} s\right)$ from the correlated mixtures $\left(x_{i} s\right)$ of them. Independence implies uncorrelatedness (the opposite is true only for Gaussian random variable). There are techniques available to get zero mean uncorrelated components of the data matrix. So, if we think of a transformation, from the former to the latter, then it must be an orthogonal transformation. Overall, the algorithm is two steps. First, let a zero mean observed mixture data matrix $X$ be linearly transformed through a whitening matrix $V$ to give a zero mean univariant whiten data matrix $Z$, i.e., $\mathbf{Z}=\mathbf{V X}=\mathbf{V A S}$. As a next step, let $\boldsymbol{R}$ be the optimal orthogonal matrix for rotation giving m.i.p. components with respect to a given contrast. Then, $\mathbf{Y}=\mathbf{R Z}=\mathbf{R V A S}=\mathbf{W} \mathbf{A S}$, where $\mathbf{W}=\mathbf{R V}$ is the estimated unmixing matrix. The first step is obtained through standard techniques for PCA through eigen value decomposition (EVD) or singular value decomposition (SVD). This article achieves the optimal rotation matrix $R$ through the Search for Rotation based ICA (SRICA) algorithm [7], which uses the genetic algorithm (GA) as a global search based optimization technique.

\subsection{Contrasts}

By definition, the random variables $y_{i}, i=1: n$, are mutually independent, if $p(\boldsymbol{y})=\prod_{i=1}^{n} p\left(y_{i}\right)$. So, based on the distance measures between both the joint $p d f$ and the product of the marginal $p d f$ s of a random vector following ICA contrasts are popular.

Kullback-Leibler divergence $(k l d)$, measuring mutual information between the true dis-

tribution $p(y)$ and the approximated distribution $\prod_{i=1}^{n} p\left(y_{i}\right)$, is the most canonical contrast for ICA [3] and is denoted here as $\operatorname{mikld}\left(\boldsymbol{y}, y_{i}\right)=k l d\left(p(\boldsymbol{y}), p\left(\prod_{i=1}^{n} y_{i}\right)\right)=\int p(\boldsymbol{y}) \frac{p(\boldsymbol{y})}{\prod_{i=1}^{n} y_{i}} d \boldsymbol{y}$, where $d \boldsymbol{y}=d y_{1} y_{2} \ldots y_{n}$. The symmetrical version of $k l d$, also known as the Jensen-Shannon $k l d$ divergence, is defined as $j \operatorname{skld}\left(\mathbf{y}, y_{i}\right)=k l d(p(\mathbf{y}), \mathbf{M})+\operatorname{mikld}\left(p\left(\prod_{i=1}^{n} y_{i}\right), \mathbf{M}\right)$, where 
$\mathbf{M}=\frac{1}{2}\left(p(\mathbf{y})+p\left(\prod_{i=1}^{n} y_{i}\right)\right)$. As $\boldsymbol{R}$ is orthogonal, $\mathbf{H}(\mathbf{y})=1 / \log (\mathbf{R}) \cdot \mathbf{H}(\mathbf{z})$ is constant. So, $\operatorname{minhyi}\left(\mathbf{y}, y_{i}\right)=\mathbf{H}(\mathbf{y})-\sum_{i=1}^{n} \mathbf{H}\left(y_{i}\right)=\sum_{i=1}^{n} \mathbf{H}\left(y_{i}\right)$, where $\boldsymbol{H}(\cdot)$ denotes entropy, defines maximization of the sum of marginal entropy, through Shannon, as the ICA contrast. There are multiple ways possible to estimate the marginal entropies. For example, the article [12] has used $m$-spacing based entropy estimates to estimate this contrast. In Section 4, this contrast has been denoted as $\operatorname{radH}\left(\sum y_{i}\right)$. Another popular contrast is based on the definition that independence implies farthest possible from Gaussian and accordingly ICA tries to find the maximally non-Gaussian components. For example, normalized kurtosis,

defined as $\operatorname{normk} 4\left(\sum y_{i}\right)=\sum_{i=1}^{n}\left|\operatorname{kurtosis}\left(y_{i}\right)-3\right|$, is such a contrast, where $|\cdot|$ denotes absolute value.

\subsection{Performance measures}

As $\mathbf{X}=\mathbf{A S}$, scaling to any source $s_{i}$ can be compensated by dividing corresponding column $a_{i}$ of $A$. So, both being unknown, the scaling or the variances and the signs of the ICs cannot be estimated. Similarly, if $\boldsymbol{P}$ is the permutation matrix and $\boldsymbol{L}$ is a scaling matrix then $\mathbf{X}=\mathbf{A S}=\mathbf{A}\left(\mathbf{L}^{-1}\left(\mathbf{P}^{-1} \mathbf{P}\right) \mathbf{L}\right) \mathbf{S}$. This implies that the estimated components $y_{i}$ can be obtained as a scaled and permuted version of actual distributions $s_{i}$. To achieve a unique solution, all the mixtures are scaled to univariance. So, let $\mathbf{Y}=\mathbf{G U}$, where $\boldsymbol{u}$ is the scaled to univariate version of $S$ and $G$ is the Gain Matrix. This achieves $\mathbf{G}=$ PWAD, where $D$ is a diagonal scaling matrix. Ideally, $G$ should be an identity matrix. So, the Amari Performance Index (API) measures deviation of $G$ from diagonalization. The better the performance, the nearer $A P I$ is to zero.

$$
A P I(G)=\sum_{i=1}^{n}\left(\sum_{i=1}^{n} \frac{\mid G_{i j \mid}}{\max _{k}\left|G_{i k}\right|}-1\right)+\sum_{j=1}^{n}\left(\sum_{i=1}^{n} \frac{\mid G_{i j \mid}}{\max _{k}\left|G_{k j}\right|}-1\right)
$$

The presence of other than the diagonal components indicates interference to the actual source being estimated. Accordingly, the SIRG for kth source and summarized form is defined as,

$$
\text { meanSIRG }=\frac{1}{n} \sum_{k=1}^{n} \operatorname{SIRG}_{k}, \text { where } \operatorname{SIRG}_{k}=\frac{G_{k k}^{2}}{\sum_{i=1, i \neq k}^{n} G_{k i}^{2}} .
$$

Signal to interference ratio from the actual and estimated sources (SIRS), i.e., the deviation in the time sequence of the actual and the estimated source, is measured as a performance index for that source. And, for overall performance of the separation, the mean of the SIRS of all the sources, i.e.,

$$
\text { meanSIRS }=\frac{1}{n} \sum_{k=1}^{n} \operatorname{SIRS}_{k} \text {, where } \operatorname{SIRS} S_{k}=\frac{\sum_{i=1}^{n} s_{k i}^{2}}{\sum_{i=1}^{n}\left(y_{k i}-s_{k i}\right)^{2}} .
$$




\section{Novel performance measures}

Ideally, when the estimated components are same as the actual sources, i.e., $\mathbf{Y}=\mathbf{U}$, then $\mathbf{G}=\mathbf{I}$ (identity matrix). So, all the three previously defined performance measures for ICA are based on the same criteria of source time sequence matching, whereas, by definition, both the estimated and actual sources are random variables. Also, the ICA algorithms are expected to find the "most independent components" and while trying to search for them, should not be considered responsible for not being able to follow and find the same latent sources. So, the time sequence matching criterion and the performance measures based on it are too ideal for ICA, though the criterion is useful for performance measurements when ICA is used for BSS application.

A few more observations can be noted about the conventional performance measures. As these measures are based on the same criterion of source time sequence matching, it is expected that an ICA algorithm which is the best with respect to one is also the best with respect to the other. But, simple mathematics and the observations show that this may not be always true. Also among these measures, SIRG and SIRS give performances for individual sources and then summarize them taking mean or median to characterize the overall performance. They do not simultaneously consider the overall structure of the source vector. Compared to this, a measure depending upon the overall structure is more preferable.

As a natural outcome of the above discussion, three different performance criteria and performance measures based on them for ICA can be suggested.

\subsection{Random source matching as a performance criterion}

Statistical matching or nearness of the estimated and the actual sources can serve as a new performance criterion. Accordingly, an asymmetric distance measure mikld $(\mathbf{s} \mathbf{y})$ ) and a symmetric distance measure jskld $(\mathbf{S}, \mathbf{y})$ have been tested as performance measures for ICA algorithms and verified in the next section. As these measures are depending upon the joint pdf, and not on the aggregation of individual performances, they can be considered to give a summarized or holistic performance.

$$
\begin{aligned}
& \operatorname{mikld}(\mathbf{s}, \mathbf{y})=\sum_{n b i n}^{n \max } P\left(s_{n b i n}\right) \log \left(\frac{P\left(s_{n b i n}\right)}{P\left(y_{n b i n}\right)}\right), \\
& j \operatorname{skld}(\mathbf{s}, \mathbf{y})=\operatorname{kld}(p(\mathbf{y}), \mathbf{M})+\operatorname{mikld}(p(\mathbf{s}), \mathbf{M}),
\end{aligned}
$$

where $\mathbf{M}=\frac{1}{2}(p(\mathbf{y})+p(\mathbf{s}))$. The lower the value of these performance measures, the better are the performances. Many such statistical distance measures can be defined to be used as performance measures for ICA algorithms. This criterion can be used for ICA as CA, as well, for BSS.

\subsection{Independence measures as the performance measures}

The ideal ICA algorithm should be considered responsible to find the m.i.p. components and not necessarily the sources. So, ICA algorithm performance can be measured based on how much the estimated components are independent and any independence measure can 
be used as a performance measure. This can be considered a good performance measure for ICA. In the absence of any knowledge about the hidden sources for matching, independence measure can be considered suitable performance measure for ICA as BSS. Previously defined all the optimization functions or contrasts are used as performance measures in the next section.

\subsection{Performance with respect to the best possible}

SRICA facilitates the use of the performance measures themselves as the contrasts or the independence measures. Assuming this gives the highest performance with respect to the given performance measure, relative performance of any ICA algorithm can be measured with respect to it.

\section{Empirical verification}

To validate the proposed performance measures, the following experiment was done. Two i.i.d sources were taken with the distributions randomly selected from the set of 21 densities. The set contains 18 densities as defined in [8] and 3 more densities added are the unimodal left-skewed, unimodal right-skewed and Gaussian as in [7]. The added 3 densities are generated using power method. The sample size was kept 1000 . The experiments were done using 50 trials. GA allows use of any optimization function, whether continuous, discrete, with or without multiple local optima, as a fitness function. So, the SRICA algorithm was used with all the 5 contrasts defined before. To facilitate comparison with the existing ICA algorithms, results are also obtained for FastICA [9], EFICA [10], NPICA [11] and RADICAL [12] techniques, as they are the best considered so far $[8,12]$. These ICA algorithms were compared for their performances using the state-of-the-art source time sequence matching criterion based performance measures API, SIRS and SIRG, the proposed statistical source matching criterion based performance measures mikld (s, y) and $j \operatorname{skld}(\mathbf{S}, \mathbf{y})$, as well, the used contrasts or independence measures were also used as performance measures. To further allow the comparison in a better way, SRICA was also used with the performance measures themselves as fitness or contrast functions.

The results are tabulated in Table 47.1. The rows indicate the performance measures for the outcome obtained through the used contrast with SRICA or the other ICA algorithm as denoted in the first column. The columns indicate performance measures for the algorithms in the first column. Each entry is a median of 50 trials. Thus, each entry in the table denotes the median value of the performance measure shown in the first row of the corresponding column and obtained for the solution due to ICA algorithm in the first column of the corresponding row. The bold-face values represent the best obtained corresponding to that column, i.e., used performance measure. $A P I<0.1$ and SIRG, SIRS $>10 \mathrm{db}$ imply good results. Accordingly, all the algorithms have given satisfactorily approximated solutions.

\section{Observations and discussion}

The following observations can be made.

- The best obtained through one of the performance measures do not ensure the best corresponding to the other.

- The statistical source matching criteria for performance measures have worked equally good. This validates their use as a performance measure for ICA algorithms. 
Table 47.1 Performances of varying independence measures and performance measures as fitness measures in SRICA; comparison with other ICA algorithms; the performances are measured using the conventional and suggested performance measures. Number of sources $=2$, selected randomly from all 21 types of source distributions; GA parameters: float version; population size $=10$; generations $=15$; kernel density estimation method with Gaussian kernel; Each entry is a median of the 50 trials.

\begin{tabular}{|c|c|c|c|c|c|c|c|c|c|c|}
\hline \multirow[t]{3}{*}{ SRICA (Contrast) and algo. used } & \multicolumn{10}{|c|}{ Performance measures Contrasct } \\
\hline & \multicolumn{5}{|c|}{ Contrasts as performance measures } & \multicolumn{3}{|c|}{ Conventional } & \multicolumn{2}{|c|}{ Stat matching } \\
\hline & $\begin{array}{l}\text { jskld } \\
\text { (Y, yi) in } \\
\text { (bits) }\end{array}$ & $\begin{array}{l}\text { mikld } \\
\text { (Y, yi) in } \\
\text { (bits) }\end{array}$ & $\begin{array}{l}\text { minhyi } \\
(Y, y i) \\
\text { in (bits) }\end{array}$ & $\begin{array}{l}\text { normk4 } \\
\left(\sum y_{i}\right)\end{array}$ & $\begin{array}{l}\mathrm{radH} \\
\left(\sum y_{i}\right)^{*} \\
1 e-03\end{array}$ & $A P I$ & $\begin{array}{l}\text { mean } \\
\text { SIRG } \\
(d b)\end{array}$ & $\begin{array}{l}\text { mean } \\
\text { SIRS } \\
(d b)\end{array}$ & $\begin{array}{l}\text { mikld } \\
(s, y) \\
\text { in bits }\end{array}$ & $\begin{array}{l}\text { jskld } \\
(s, y) \\
\text { in bits }\end{array}$ \\
\hline jskld(Y, yi) & 0.0035 & 0.0131 & 2.6720 & 1.7274 & -4.341 & 0.0220 & 36.1895 & 35.4952 & 0.1988 & 0.0385 \\
\hline $\operatorname{mikld}(\mathrm{Y}, \mathrm{yi})$ & 0.0035 & 0.0129 & 2.6720 & 1.7305 & -4.342 & 0.0227 & 36.1331 & 35.0565 & 0.2016 & 0.0388 \\
\hline $\operatorname{minhyi}(\mathbf{Y}, \mathrm{yi})$ & 0.0035 & 0.0129 & 2.6718 & 1.7289 & -4.342 & 0.0190 & 37.0690 & 36.2552 & 0.1653 & 0.0353 \\
\hline $\operatorname{normk} 4\left(\sum \gamma_{i}\right)$ & 0.0041 & 0.0152 & 2.6721 & 1.7504 & -4.333 & 0.0393 & 28.9497 & 28.8385 & 0.2436 & 0.0520 \\
\hline $\operatorname{radH}()$ & 0.0037 & 0.0138 & 2.6743 & 1.6662 & -4.346 & 0.0232 & 34.1744 & 33.9066 & 0.1785 & 0.0364 \\
\hline \multicolumn{11}{|c|}{ Other algo. and source measures for comparison } \\
\hline FastICA & 0.0046 & 0.0169 & 2.6676 & - & -4.332 & 0.0578 & 28.3762 & - & 0.2935 & 0.0585 \\
\hline EFICA & 0.0042 & 0.0156 & 2.6754 & 1.7463 & -4.330 & 0.0388 & 29.3615 & 29 & 0.1700 & 0.0397 \\
\hline NPICA & 0.0036 & 0.0136 & 2.6737 & 1.6769 & -4.335 & 0.0191 & 35.6442 & 35.6634 & 0.1611 & 0.0355 \\
\hline RADICAL & 0.0036 & 0.0133 & 2.6721 & 1.7140 & -4.344 & 0.0198 & 36.1129 & 35.9338 & 0.1765 & 0.0386 \\
\hline Sources & 0.0015 & 0.0055 & 2.0335 & 1.7256 & -5.016 & - & - & - & - & - \\
\hline \multicolumn{11}{|c|}{ Performance measures used as fitness measure for better comparis } \\
\hline API & 0.0036 & 0.0133 & 2.6725 & 1.7318 & -4.341 & 0.0077 & 61.6951 & 56.1296 & 0.1700 & 0.0401 \\
\hline mea & 0.0038 & 0.0143 & 2.6737 & 1.7037 & -4.337 & 0.0124 & 59.0524 & 55.3 & 0.1454 & 0.0292 \\
\hline meanSIRS & 0.0039 & 0.0146 & 2.6746 & 1.7317 & -4.333 & 0.0110 & 72.8616 & 73.7894 & 0.1474 & 0.0328 \\
\hline & 0.0038 & 0.0138 & 2.6721 & 1.7067 & -4.340 & 0.0170 & 41.7181 & 42.3173 & 0.1386 & 0.0284 \\
\hline $\operatorname{jskld}(s, y)$ & 0.0038 & 0.0138 & 2.6719 & 1.7070 & -4.341 & 0.0165 & 43.9772 & 42.9636 & 0.1354 & 0.0251 \\
\hline
\end{tabular}




\section{Intelligent Circuits and Systems}

- All the contrasts with SRICA have sufficiently good performance with respect to the other contrasts used as performance measures, though not the best. This justifies use of any contrast or independence measure as a performance measure.

- Each SRICA algorithm has found the best with respect to the independence measure it used, both as a contrast and as a performance measure.

- The best estimated through each contrast is different. Similarly, the best corresponding to each performance measure is also different. This implies there are multiple solutions and not a unique solution qualifying to be the best.

- When a performance measure itself used as a contrast, it achieved the best with respect to itself. This justifies such performance to be used as a reference to compare the performance of other ICA algorithms. Thus, the comparison with the best achieved by using it as a contrast would be a better performance measure specifically when ICA is used as for CA application or for a real world BSS application where the sources are not available for matching.

\section{Conclusion}

The article has successfully proposed three novel sets of performance criteria for ICA algorithms: statistical source matching, independence measures or the ICA contrasts and the relative performance with the performance measures used as the contrast. Section 4 has provided the empirical validation of these proposed performance measures. The latter two criteria based performance measures could be a potential choice when ICA is used for CA applications, as well, for real world BSS applications where sources not available for matching.

\section{References}

[1] P. Comon. Independent component analysis-a new concept? Signal Processing vol. 36, (1994), pp. 287-314.

[2] Duda, Richard O., Hart, Peter E., and Stork, David G. Pattern Classification (2nd edition). 2: Wiley-Interscience (2000).

[3] A. Hyvarinen, J. Karhunen and E. Oja. Independent Component Analysis. John Wiley, New York (2001).

[4] J.-F. Cardoso. Blind signal separation: statistical principles. Proceedings of the IEEE vol. 9, no. 10 (1998), pp. 2009-2025.

[5] P. Common and C. Jutten. Handbook of Blind Source Separation: Independent Component Analysis and Applications, 1st edn. Academic Press (2010).

[6] J. Eriksson and V. Koivunen. Identifiability, separability, and uniqueness of linear ICA models", Signal Processing Letters, IEEE vol. 11, no. 7 (2004), pp. 601-604.

[7] Dharmani Bhaveshkumar, C. Proposal of a search for rotation based independent component analysis (SRICA) algorithm. Proceedings of the Fourth International Conference on Signal and Image Processing, Coimbtore, India (2012), Lecture Notes in Electrical Engineering, vol. 221, Springer India (2013), pp. 411-421.

[8] Bach, F., and Jordan, M. Kernel independent component analysis. The Journal of Machine Learning Research 3 (2003), pp. 1-48.

[9] A. Hyvarinen and E. Oja. A fast fixed-point algorithm for independent component analysis. Neural Computation 9(7) (1997), pp. 1483-1492.

[10] Z. Koldovsky, P. Tichavsky, and E. Oja. Efficient variant of algorithm FastICA for independent component analysis attaining the Cramer-Rao lower bound. IEEE Trans. Neural Netw. 17(5) (2006), pp. 1265-1277. 
[11] Boscolo, R., Pan, H., and Roychowdhury,V. Independent component analysis based on nonparametric density estimation. Neural Networks, IEEE Transactions on 15(1) (2004), pp. 55-65.

[12] E.G. Learned-Miller and W.F. John III. ICA using spacings estimates of entropy. The Journal of Machine Learning Research 4 (2003), pp. 1271-1295. 


\title{
48 WSN sensor deployment and sampling interval \\ Role of dynamics
}

\author{
Aarti Kochhar,*, Naresh Kumar ${ }^{2}$ \\ ${ }^{1}$ UIET, Panjab University, Chandigarh, India; Lovely Professional University, \\ Phagwara, India \\ ${ }^{2}$ UIET, Panjab University, Chandigarh, India
}

\section{Introduction}

WSN is an emerging technology which supports IoT (Internet of Things). Sensors are considered to be eyes and ears of IoT technology. Figure 48.1 shows the basic architecture of a sensor node. Networks of sensors sense the data and after processing send it to a remote station using transceiver. Data received at remote station is used for making decisions or building decision support systems. In agriculture, crops or plants are grown over a large area. Data collection from these large fields is necessary to make important decisions but it is a tedious task. WSN can help in data collection from these large agricultural fields.

Over the years, many researchers have widely studied the application of WSN in the field of agriculture. While few tried to focus on a particular crop or a particular disease related to a crop, others focused on general scenarios like irrigation. While rice, tomato etc. are the crops for which application and utility of WSN or IoT is extensively studied, tea is one of the least worked upon crops in the field of IoT. Out of it, most of the available literature is from tea boards in collaboration with other government institutions like C-DAC. Although use of electronics for tasting and grading of tea has substantial research available, the application of WSN in tea garden areas does not have much significant work. Upon searching with different keywords on the IEEE digital library, the authors found less than 10 relevant articles for application of WSN in tea plantations.

Tea is not only a widely consumed beverage in India but also an important export item. So it contributes a significant sum in economic growth of India. According to the 63rd annual report of the Tea Board of India, in year 2016-17 a total of 227.63 million $\mathrm{kg}$ of tea was exported out of India at value of Rs. 4632.5 crores [1]. According to Chapter 5 of the same report, the fund allocation to tea research institutions for R \& D was Rs. 9 crore for year 2016-17. Two major varieties of tea plant are Camellia sinensis (Chinese variety) and Camellia assamica (Indian variety). The rest of the paper is organized as follows. Section III presents the problem and methodology. In Section IV, results are discussed. Section V concludes the paper.

\section{Related work}

Sun et al. [2,3] implemented a set up of 25 nodes in the Tea Research Institution, Guangdong Academy of Agricultural Sciences. Out of 25, 10 nodes were deployed for soil

* Email: aarti.kochhar92@gmail.com

DOI: $10.1201 / 9781003129103-48$ 


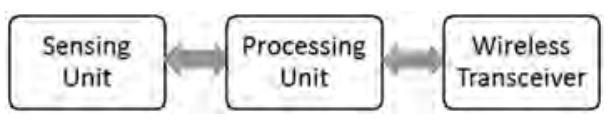

Figure 48.1 Architecture of a sensor node.

moisture content, 1 for sensing of temperature and humidity, 13 data relay nodes and 1 for connection to server room. Nodes acquire data every second and send it to base station. Quality of service (QoS) was analysed for different network scales. ATmega128L was used for processing and CC2520 was used as transceiver. Nodes were deployed randomly and in a diamond-shaped network. Packet loss ratio (PLR) was less for diamond-shaped deployment.

Saikia et al. [4] proposed the use of sensor networks during fermentation and drying of tea leaves. After plucking of tea leaves, it has to go through a process of withering, rolling, cutting, fermentation and drying. Duration, temperature and relative humidity of fermentation process decides the quality of tea. A total of 6 sensor nodes were deployed. These sensors were calibrated according to the environment before deployment.

Wang et al. [5] proposed to plant the tea in WSN monitored greenhouse to make it sprout early. Hong and Shuhui [6] proposed a real time system based on Z-BEE WSN to prevent frosting on tea leaves. Two sensor nodes were deployed, one at $6 \mathrm{~m}$ height from ground and other at the height of the plant's canopy. Along with two sensors, one data gathering device SZ06-2K-9-S was used. SZ02-ZBEE was used at data processing and transport layer. Difference in the temperature captured by two sensors was used to make decision for switching on the fan to prevent frosting. Dasgupta [7] used water-level and temperature sensor in collaboration with National Instruments' LabVIEW to check water accumulation in roots of tea plants.

Rupanjali et al. [8] used sensors and camera to monitor the tea plantation area. Two WSN nodes were placed at distance of $10 \mathrm{~m}$ from each other and both the nodes send the data to a gateway placed $50 \mathrm{~m}$ away. Gateway was connected to server PC using Ethernet cable. Data is sent to gateway every 15 minutes. Gupta et al. [9] proposed a setup for larger geographical area and used integration of WSN and GIS (geographic information system). Thirteen field stations were set up over the area of 205 hectares. The paper focused on building a spatial map of information using interpolation. Inverse distance weighted (IDW) was used as interpolation algorithm to predict the value of soil $\mathrm{pH}$, soil moisture and soil temperature at unsampled locations of large field. Thus the use of WSN in tea gardens can help in improving overall productivity.

\section{Problem and proposed solution}

Since Camellia assamica is mostly grown on slopes of mountains in large areas stretched over hundreds of hectares, so there are huge variations across the field. Soil moisture level at the end of slope is always different from the tip of the slope. Areas in upper mountains have more frequent variations because of intermittent cloud and sunshine. Shady areas are less prone to climatic changes than open areas. Also parts of tea estates touching Meleng River and Western Ghats have more frequently changing environment than areas far from river banks. Other than these, micro-climatic zones are also present in a tea plantation area. Most of the present literature assumes these large areas to be uniform.

Authors propose to consider the dynamics of a field of Camellia assamica to increase network lifetime. As stated in the literature [10], most of the energy consumption in the 


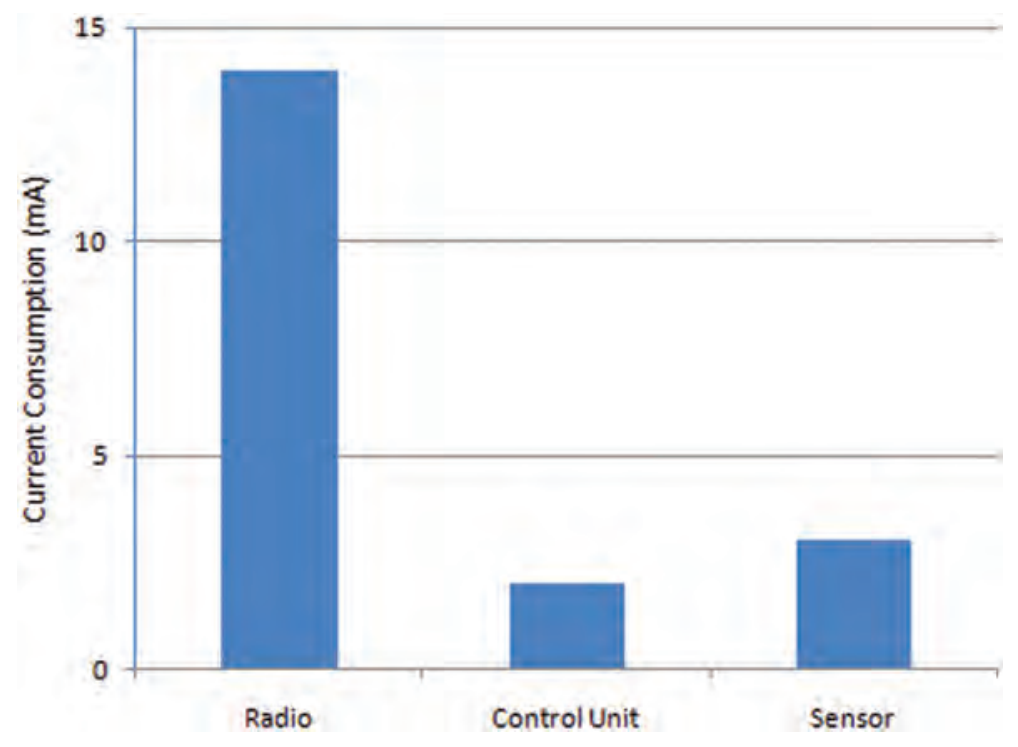

Figure 48.2 Current consumption in a WSN node [10].

setup of a WSN is consumed by transceiver or radio. As shown in Figure 48.2, radio consumes maximum energy in a wireless network. So optimizing a transceiver can help in reducing overall energy consumption of network and hence prolonging the network lifetime.

\section{Results and discussion}

The proposed work was compared with two setups [8,9] implemented by the Tea Association of India in collaboration with C-DAC, West Bengal at Tocklai Experimental Station, Jorhat, Assam.

In the first setup [8], two WSN nodes were placed at distance of $10 \mathrm{~m}$ from each other and transmission interval is 15 minutes. Since in a tea plantation parameters don't change much over a distance of $10 \mathrm{~m}$, one node is assumed to send data every 15 minutes while the other node sends data on an hourly basis. CC2520 mote of Zigbee is used as transceiver. Considering modelling of current for Zigbee/CC2520 mote [12], earlier setup consumes 0.207507 Joule of energy over the time of one day. However, the proposed setup consumes 0.129688 Joule of energy/day. Hence the proposed setup saves almost $37.5 \%$ of energy/day. The operating voltage of nodes is $12 \mathrm{~V}$. For comparison, data sent was assumed to be one byte. The percentage of energy saved will vary with number of bytes sent and duration of experiment.

In the second setup [9], 13 field stations were set up over the area of 205 hectares. The authors propose to divide the area into two regions representing separate dynamics. Region-1 is assumed to have more frequent variations than region-2. Authors propose to operate 8 nodes on region-1 at transmission or sampling interval of 15 minutes while the other 5 nodes operate in region- 2 at transmission interval of one hour. Operating voltage of nodes is $12 \mathrm{~V}$. Earlier setup which assumes uniform field area consumes 1.3 Joule/ day while proposed setup with two separate regions consumes 0.95 Joule/day. Hence the proposed scenario saves $26.17 \%$ of energy/ day. Data sent was assumed to be one byte. 
CC2520 mote of Zigbee is used as transceiver. Further, following the same concept, area can be divided into more than two regions and the least dynamic region can operate with one sensor node also. Thus it can save more energy and network will operate for longer duration.

\section{Conclusion}

The proposed work optimizes the number of sensor nodes and rate of data transmission to reduce energy consumption and prolong network lifetime of WSN in a tea garden area. Authors suggest not assuming environment of large plantation areas to be uniform. Exploring the dynamic nature of parameters both spatially and temporally can help in saving a precious resource of WSN, i.e. energy. In future work, packet length can also be optimized to prolong lifetime of nodes.

\section{References}

[1] 63rd annual report of Tea Board of India for 2016-17. www.teaboard.gov.in/pdf/Annual_ Report_Combined_2016_17_Total_Book_27_11_2017_Final_Curve_pdf4622.pdf.

[2] Sun, D., Jiang, S., Wang, W., and Tang, J. 2010. WSN design and implementation in a tea plantation for drought monitoring. In Proceedings - 2010 International Conference on CyberEnabled Distributed Computing and Knowledge Discovery, CyberC 2010, 156-159. http:// doi.org/10.1109/CyberC.2010.36.

[3] Sun, D., Wang, W., Lu, J., and Lin, Z. 2010. Design of WSN nodes and network performance analysis in a tea plantation. Wireless Sensor Network, 2010. IET-WSN. IET International Conference on, 144-147. http://doi.org/10.1049/cp.2010.1043.

[4] Saikia, D., Boruah, P. K., and Sarma, U. 2015. A sensor network to monitor process parameters of fermentation and drying in black tea production. Mapan - Journal of Metrology Society of India, 30(3), 211-219. http://doi.org/10.1007/s12647-015-0142-4

[5] Wang, F., Zheng, J., Mei, L., Ding, Z., Feng, W., and Wang, L. 2014. Design and development of intelligent monitoring system for plastic tea greenhouse. In IFIP Advances in Information and Communication Technology book series (vol. 419, pp. 443-449). Retrieved from https:// hal.inria.fr/hal-01220947; https://hal.inria.fr/hal-01220947/document.

[6] Hong, C., and Shuhui, Z. 2012. A temperature auto-monitoring and frost prevention real time control system based on a Z-BEE networks for the tea farm. In 2012 IEEE Symposium on Electrical Electronics Engineering (EEESYM), 644-647.

[7] Dasgupta, Y. 2014. Application of wireless sensor network in remote monitoring: waterlevel sensing and temperature sensing, and their application in agriculture. In 2014 First International Conference on Automation, Control, Energy and Systems (ACES), 28-31.

[8] Rupanjali, D.B., Bhagat, R. M., Saikia, M., Saikia, A., Pathak, L. B., Gupta, N., and Bhattacharyya, N. 2013. Using wireless sensor network for monitoring growing environment of tea in North-East India. Journal of Environmental Research And Development, 8(2).

[9] Gupta, N., Gupta, P. P., Pramanik, P., Saikia, A., Sengupta, L., Bhagat, R. M., and Bhattacharya, N. 2014. Integration of geoinformatics and wireless sensors for smart agriculture in tea. In Second International Conference on Remote Sensing and Geoinformation of the Environment (RSCy2014), 9229,92290W. http://doi.org/10.1117/12.2066366.

[10] Didioui, A. 2014 Energy-aware transceiver for energy harvesting wireless sensor network. Signal and Image Processing.

[11] Sampaio, H., and Motoyama, S. 2017. Sensor nodes estimation for a greenhouse monitoring system using hierarchical wireless network. 2017 25th International Conference on Software, Telecommunications and Computer Networks (SoftCOM).

[12] Casilari, E., Cano-García, J. M., and Campos-Garrido, G. 2010. Modeling of current consumption in 802.15. 4/ZigBee sensor motes. Sensors, 10, 5443-5468. http://doi.org/10.3390/ s10060. 


\title{
49 Recognition and categorization of plant from leaf image using visual traits
}

\author{
Parul Mittal* \\ Department of Electrical and Electronics Engineering, Lovely Professional \\ University, Punjab, India
}

\section{Introduction}

Plants are imperative for prevalence of life on earth [1] being a radix of diverse as natural assets. The plants at galore exist on earth with similar appearance which makes it arduous for mankind to differentiate these based on their physical view. Conversely an appropriate recognition of plants is vital to circumvent any mishap due to venomous or perilous plants. As an elixir to this problem the digital system is required to identify plants from their digital image, since their error rate is infinitesimal when compared to manual recognition by humans.

In such systems, features are automatically drawn out from input image and based on these attributes classification is done by the classifier. There are copious traits, such as leaves, bark, flowers, stem and fruits on the basis of which these can be distinguished. As flowers and fruits are season dependent and may wither, leaves being independent of such constraints form the optimal parameter for recognition [2-4].

The organization pattern of the paper follows proposed methodology in Section II, the process of image pre-processing in Section III, detailed methods of obtaining attributes in Section IV, categorization as well as recognition using diverse classifiers in Section V, assessment variables in Section VI, verdict of experiment in Section VII, and Section VIII incorporates conclusion and future enhancement scope of the paper.

\section{Proposed system}

The coloured leaf image in RGB colour space is input to the system. The image preprocessing is done which includes obtaining binary image, boundary image, $R, G, B$ cropped image, grey scale cropped image and grey image. Various features like shape, colour, texture and vein features are extracted using pre-processed images. The drawn out features are utilized for classification using classifiers.

\section{Image pre-processing}

Data set used is Flavia dataset [5] with all leaves on white background. Coloured digital image of the leaf is input to the system. Coloured image is in three dimensional space. Three dimensional space coloured image is converted into two dimensional space by transforming it into grey image. Image is resized to $256 * 256$ for optimal handling. Since an input image is in a RGB colour space, for segmentation R, G, B elements are obtained

\footnotetext{
*Email: parulmittal641@gmail.com
}

DOI: 10.1201/9781003129103-49 


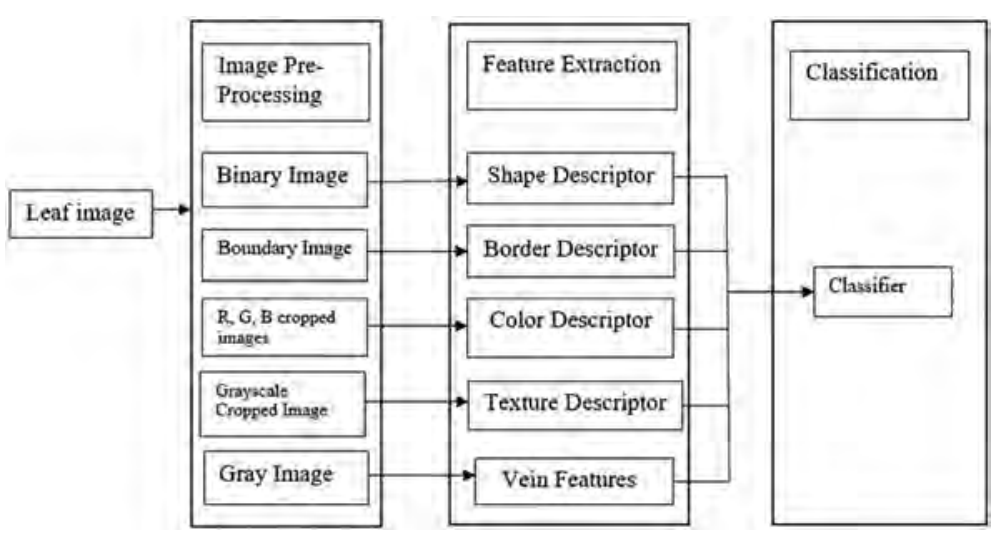

Figure 49.1 Proposed methodology flow diagram.

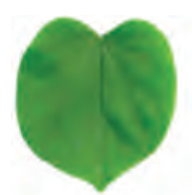

(a)

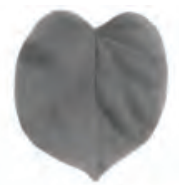

(b)

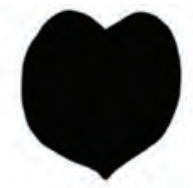

(c)

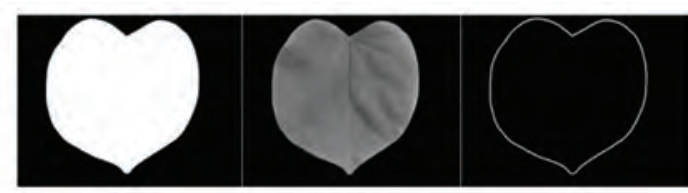

(d) (e)

(f)

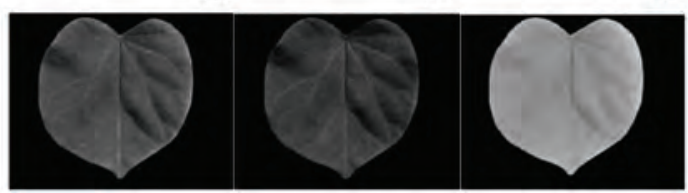

(g)

(h)

(i)

Figure 49.2 Leaf image pre-processing: (a) input image, (b) grey image, (c) binary image, (d) complement of binary image, (e) cropped image, (f) boundary image, (g) R component cropped image, (h) G component cropped image, (i) B component cropped image.

from an image. The process of OR is carried on obtained R, G, B elements to attain segmented image with content in black colour on white colour background.

White image on black background is obtained for further processes by complementing the resultant of the OR operation. The resultant image obtained is not clean image, due to the presence of white holes on black background and black holes on white area, thus morphological hole filling operation is done to obtain clean image [6]. Leaf boundary extraction image is acquired by keeping only those pixels which construct the outline of an image. The subtraction is done of dilated image from real image to acquire an image of the leaf boundary. Grey scale cropped and R, G, B component cropped images are obtained for extracting texture features and colour features respectively.

\section{Extraction of features}

The extraction of visible low feature attributes such as, texture, colour and shape is illustrated by this paper. 


\section{A. Shape features}

This work demonstrates the extraction of diverse shape descriptors [7]. The 28 rudimentary and morphological attributes have been extracted from binary image which included various boundary [8] and regional [12] descriptors as well as Euler number. The vivid plant species can be distinguished based on these as these have the potential to discriminate the diverse shapes.

The dispersion highlights an irregularity of the leaf shape [10].

\section{B. Vein features}

Vein attributes from leaf image are obtained by carrying out morphological opening operations on greyscale image with structuring elements the shape of which are flat disk and radius one, two, three and four respectively [11]. The subtraction is performed on aftermath images and greyscale image where the former is subtracted from the latter. As a result, in total there are 5 vein features V1 = Area1/Area, V2 = Area2/Area, V3 = Area3/ Area, V4 = Area4/Area, V5 = Area4/Area1. Area1, Area2, Area3, Area4 correspond to image parts which are retained after performing subtraction. Area corresponds to the area of the binary input image.

The algorithm can be explained as:

1) Input RGB scale image is converted into greyscale image (g) and binary image (b).

2) Four flat disk shaped structuring elements S1, S2, S3, S4 are considered with radius of $1,2,3,4$ respectively.

3) Opening operation is performed on greyscale image with S1, S2, S3, S4; thus at output $\mathrm{i} 1, \mathrm{i} 2, \mathrm{i} 3, \mathrm{i} 4$ are obtained respectively.

4) Output images are subtracted from greyscale image, thus as a resultant there are o1, o2, o3, o4 images representing veins of image (Figure 49.3).

5) Area of images b, o1, o2, o3, o4 are calculated as A, A1, A2, A3, A4 respectively for calculating vein features V1, V2, V3, V4, V5.

$$
\begin{aligned}
& v 1=\frac{A 1}{A} \\
& v 2=\frac{A 2}{A}
\end{aligned}
$$

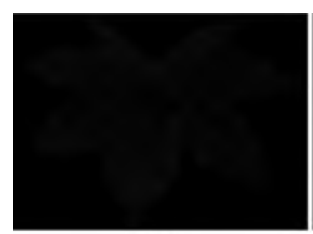

(a)

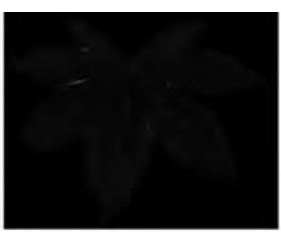

(b)

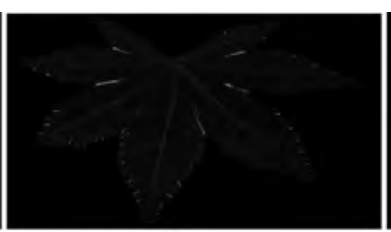

(c)

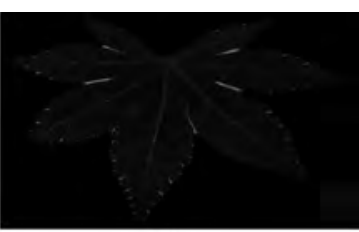

(d)

Figure 49.3 Images of leaf vein processed with structuring disk one, two, three, four, respectively. 


$$
\begin{aligned}
& v 3=\frac{A 3}{A} \\
& v 4=\frac{A 4}{A} \\
& v 5=\frac{A 4}{A 1}
\end{aligned}
$$

\section{Colour features}

Colour moments $[12,13]$ have been proposed for colour features extraction. As colour of a leaf is uniform probability distribution is followed by colour in a leaf, which makes utilization of colour moments optimum.

As sum of pixels in an image are $\mathrm{M}$, calculation of colour moments at i-th colour channel at $\mathrm{j}$-th image pixel has been done for all pixels. Consequently, for each colour channel 7 colour moments have been obtained and 21 in total.

The algorithm is explained as:

1) The R, G, B channels are extracted from the image.

2) Mean is calculated for all three channels using expression

$$
\mu=\frac{\sum P i j}{M}
$$

3) Standard deviation is calculated for all three channels using expression

$$
\sigma=\sqrt{ }\left(\frac{\left(\sum P i j-\mu\right)^{2}}{M}\right)
$$

4) Skewness is calculated for all three channels using expression

$$
\theta=\sqrt{ }\left(\frac{\left(\sum P i j-\mu\right)^{3}}{M^{*} \sigma^{3}}\right)
$$

5) Kurtosis is calculated for all three channels using expression

$$
\gamma=\sqrt{ }\left(\frac{\left(\sum P i j-\mu\right)^{4}}{M * \sigma^{4}}\right)
$$

6) Smoothness is calculated for all three channels.

7) Entropy is calculated for all three channels using expression

$$
E=-\sqrt{ }\left(\sum M * \log _{2} M\right)
$$




\section{4}

Intelligent Circuits and Systems

8) Uniformity is calculated for all three channels using expression

$$
U=\sum(P i j)^{2}
$$

9) Thus, at output for each channel $7 * 1$ matrix has been obtained.

10) Steps $1-8$ are repeated for each image in the database.

\section{Texture features}

The texture traits has been attained by a technique of grey-level co-occurrence matrix (GLCM) $[15,16]$ which counts grey value pixels appearance at particular distance. Twenty traits, such as correlation, entropy, homogeneity, variance [17] etc. have been computed at distance of 1 for diverse angles, such as $0^{\circ}, 45^{\circ}, 90^{\circ}$ and $135^{\circ}$; as a result, aggregately 81 attributes have been obtained.

\section{Recognition and classification}

The classification is done by classifiers. It classifies the input images in one of the leaf class by comparing the input image features with feature vectors dataset prepared as the output of the training. In this research paper, for the purpose of classification and identification probabilistic neural network (PNN) [5,18], support vector machine (SVM) [19-21], artificial neural network with training algorithm gradient descent (GD) and scalar conjugate gradient (SCG) have been implemented. Recognition is identification of the object in the input image. Recognition has been performed in this research work with the use of the SVM and PNN classifiers. The testing image is the input given to the trained classifier and the obtained output is the plant name.

\section{Evaluation parameters}

The performance of plant recognition and classification system based on disparate attribute extraction methods and classifiers can be gauge based on parameters, such as, accuracy, precession, recall, confusion matrix etc.

\section{Result analysis}

The proposed algorithm has been implemented with the help of different classification techniques as mentioned above. Following results are obtained after applying evaluation parameters on the obtained outputs of different classifiers. Moreover those have been compared on the basis of parameters, such as classes and aggregate of input leaf images. It has been experimentally found that the results of the above used described methods are improved as compared to the existing methodologies.

Table 49.1 shows the results obtained.

In the above, performance parameters show an enhanced result of the used research methodology, where on analysis it has been analysed that $100 \%$ accuracy has been attained with extracted features from plant leaf image and classifiers like SVM and PNN.

The confusion matrix can be generated for different classifiers with different number of the inputs and the classes of the leaf images to visualize performance accuracy of classifier. One of the matrixes is shown in Figure 49.4, which depicts the performance accuracy of scalar conjugate gradient with 301 leaf images as input of 5 different classes from Flavia dataset. 
Table 49.1 Result analysis

\begin{tabular}{|c|c|c|c|c|c|}
\hline \multirow[t]{2}{*}{ Classifier } & \multicolumn{2}{|l|}{ Input parameters } & \multicolumn{3}{|c|}{ Evaluation parameters } \\
\hline & $\begin{array}{l}\text { Number of input } \\
\text { leaf images }\end{array}$ & $\begin{array}{l}\text { Number of } \\
\text { input classes }\end{array}$ & Accuracy & Precision & Recall \\
\hline PNN & \multirow[t]{4}{*}{320} & \multirow[t]{4}{*}{32} & $100 \%$ & 1 & 1 \\
\hline SVM & & & $100 \%$ & 1 & 1 \\
\hline SCG & & & $97.5 \%$ & - & - \\
\hline GD & & & $88 \%$ & - & - \\
\hline SVM & \multirow[t]{4}{*}{640} & \multirow{4}{*}{32} & $100 \%$ & 1 & 1 \\
\hline PNN & & & $100 \%$ & 1 & 1 \\
\hline SCG & & & $96 \%$ & - & - \\
\hline GD & & & $92 \%$ & - & - \\
\hline SVM & \multirow[t]{4}{*}{1280} & \multirow[t]{4}{*}{32} & $100 \%$ & 1 & 1 \\
\hline PNN & & & $100 \%$ & 1 & 1 \\
\hline SCG & & & $98.5 \%$ & - & - \\
\hline GD & & & $98 \%$ & - & - \\
\hline SVM & \multirow[t]{4}{*}{1536} & \multirow[t]{4}{*}{32} & $99.3651 \%$ & 0.9938 & 0.9948 \\
\hline PNN & & & $100 \%$ & 1 & 1 \\
\hline SCG & & & $98.5 \%$ & - & - \\
\hline GD & & & $98 \%$ & - & - \\
\hline SVM & \multirow[t]{4}{*}{1850} & \multirow[t]{4}{*}{32} & $100 \%$ & 1 & 1 \\
\hline PNN & & & $100 \%$ & 1 & 1 \\
\hline SCG & & & $99 \%$ & - & - \\
\hline GD & & & $98.5 \%$ & - & - \\
\hline SVM & \multirow[t]{4}{*}{1162} & \multirow[t]{4}{*}{20} & $100 \%$ & 1 & 1 \\
\hline PNN & & & $100 \%$ & 1 & 1 \\
\hline SCG & & & $96.8 \%$ & - & - \\
\hline GD & & & $96.2 \%$ & - & - \\
\hline SVM & \multirow[t]{4}{*}{611} & \multirow[t]{4}{*}{10} & $100 \%$ & 1 & 1 \\
\hline PNN & & & $100 \%$ & 1 & 1 \\
\hline SCG & & & $99.8 \%$ & - & - \\
\hline GD & & & $99.2 \%$ & - & - \\
\hline SVM & \multirow[t]{4}{*}{301} & \multirow[t]{4}{*}{5} & $100 \%$ & 1 & 1 \\
\hline PNN & & & $100 \%$ & 1 & 1 \\
\hline SCG & & & $100 \%$ & - & - \\
\hline GD & & & $98.3 \%$ & - & - \\
\hline
\end{tabular}

\section{Conclusion and future scope}

The paper exhibits an intensified performance of methods utilized for recognition and classification of leaves images. The research method indicates an implementation of extraction of attributes, such as shape, colour etc., where aggregately 140 attributes have been obtained on which operation of recognition and classification has been implemented utilizing diverse classifiers, such as SVM, PNN etc.

The results depicted manifest that for various classes and integer of input images for operation of classification, accuracy differs, where $100 \%$ accuracy has been attained with extracted features and classifiers like SVM, PNN. The results obtained are thus compared as shown above, where it proves in comparison to existing methods accuracy has been reinforced. 

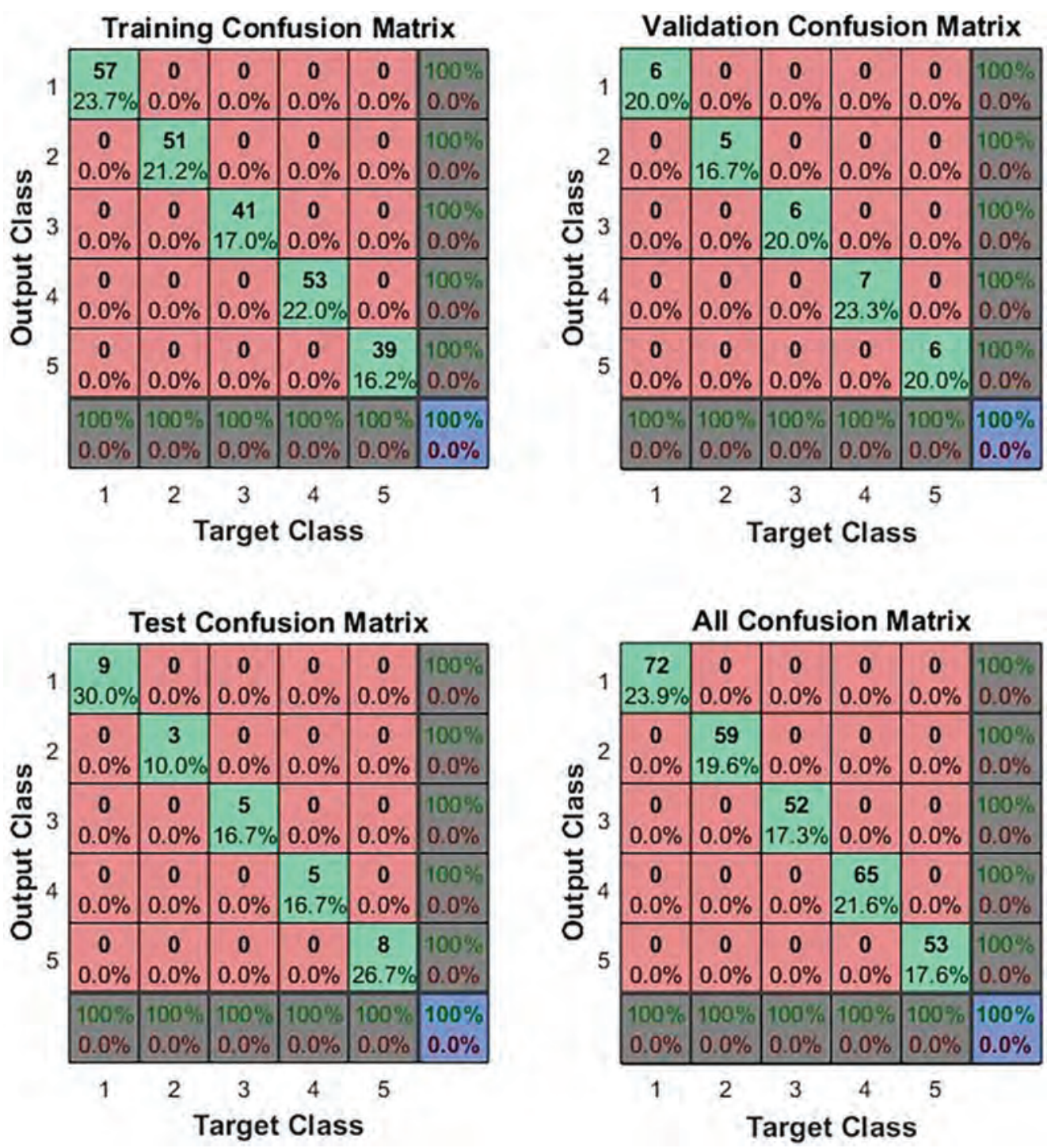

Figure 49.4 Output SGD confusion matrix 301 leaves 5 classes.

Furthermore improvement in algorithm can be attained to uphold the accuracy by reducing the complexity of the algorithm through fewer extracted features followed by combined classifiers.

\section{References}

[1] Sapna Sharma and Dr. Chitvan Gupta, "A review of plant recognition methods and algorithms", IJIRAE, issue 6, vol. 2, June 2015.

[2] Sachin D. Chothe and V.R.Ratnaparakhe, "Plant leaf identification system", International Journal on innovative Research in Science, Engineering and Technology vol.4, special issue 6, May 2016. 
[3] Nisar Ahmed, Usman Ghani Khan and Shahzad Asif, "An automatic leaf based plant identification system", 5th International. Multidisciplinary Conference, 29-31 Oct.

[4] Andrade, M., Mayo, S. J., Kirkup, D., and Van Den Berg, C., "Comparative morphology of population of Monstera Adans. (Araceae) from natural forest fragments in northeast Brazil using elliptic Fourier analysis of leaf outlines”, Kew Bulletin, 63, 193-211, 2008.

[5] Stephen Gang Wu, Forrest Sheng Bao, Eric You Xu, Yu-Xuan Wang, Yi-Fan Chang and Qiao-Liang Xiang, "A leaf recognition algorithm for plant classification using probabilistic neural network", IEEE 7th International Symposium on Signal processing and Information Technology.

[6] R. Gonzales, R. E. Woods, Digital Image Processing, 2nd edn. New Jersey, Prentice Hall, 2002.

[7] JX Du, XF Wang and GJ Zhang, "Leaf shape based plant species recognition", Applied Mathematics and Computation, volume 185, issue 2, pp. 883-893, 15 February 2007.

[8] Mingqiang Yang, Kidiyo Kpalma and Joseph Ronsin, "A survey of shape feature extraction techniques”, Pattern Recognition, IN-TECH, pp.43-90, 2008.

[9] Dengsheng Zhang and Guojun Lu, "Evaluation of MPEG-7 shape descriptors against other shape descriptors", Multimedia Systems 9: 15-30 (2003).

[10] Nixon, M.S., and Aguado, A.S. Feature Extraction and Image Processing. Woburn, Newness, 2002.

[11] A. Kadir Gadjah, L. E. Nugroho Gadjah, A. Susanto Gadjah Mada and P.I. Santosa, "Neural network application on foliage plant identification", International Journal of Computer Applications, volume 29, no. 9, September 2011.

[12] Noah Keen and Dr. Bob Fisher "Color Moments”, https://pdfs.semanticscholar.org/bccc/f2b2 142f62a217c035c1e8003b0363c43d2a.pdf.

[13] Martinez, W., and Martinez, A. 2002. Computational Statistics Handbook With MATLAB. Bocca Raton, CRC Press LLC.

[14] Abdul Kadir, Lukito Edi Nugroho, Adhi Susanto and Paulus Insap Santosa, "Leaf classification using shape, color, and texture features", International Journal of Computer Trends and Technology, July to Aug issue 2011.

[15] Acharya, T., and Ray, A. 2005. Image Processing Principles and Applications. New Jersey: John Wiley \& Sons.

[16] Abdul Kadir, "A model of plant identification system using GLCM, lacunarity and Shen features”, https://pdfs.semanticscholar.org/a70a/c3a6ffc33055a4ae9e40d68f8bb70096efe4. pdf.

[17] R. Haralick, "Statistical and structural approaches to texture", Proc. IEEE, 67, 5, 786804, 1979.

[18] V. Cheung and K. Cannons, "An introduction to probabilistic neuralnetworks", www.ijirae. com/volumes/Vol2/iss6/15.JNAE10092.pdf.

[19] Cortes, C., and Vapnik, V.N., "Support vector networks", Machine Learning 20, 273297, 1995.

[20] Pradeep Nijalingappa and V. J. Madhumathi, "Plant identification system using its leaf features", IEEE 2015 International Conference on Applied and Theoretical Computing and Communication Technology (iCATccT), 29-31 Oct. 2015.

[21] P. Mittal, M. Kansal and H. kaur Jhajj, "Combined classifier for plant classification and identification from leaf image based on visual attributes," 2018 International Conference on Intelligent Circuits and Systems (ICICS), Phagwara, 2018, pp. 184-187. 


\title{
50 Fast method of face recognition in real time using Raspberry-Pi and Intel Movidius NCS
}

\author{
Navjot Rathour*, Rajesh Singh, Anita Geblot
}

Lovely Professional University, Phagwara, Punjab, India

\section{Introduction}

Deep learning has played a significant role in the field of computer vision. The level of accuracy that has been achieved with various deep learning algorithms is unbeatable in terms of accuracy in the area of computer vision. Such accuracy demands some cost to provide that unbeatable accuracy. That price is well known in the embedded community as computational resources because embedded devices are mainly lacking in these resources. When it comes to working with devices like Raspberry-Pi, which is resourceconstrained, a CPU is always required, as the smallest model takes multiple orders when being trained on CPU only. When the devices like Raspberry-Pi are considered, nobody can even think of training a deep learning model because of its low computational capability and being so underpowered. The next vital parameters that need to be taken into consideration are memory and computational capability. Talking about RAM, RaspberryPi can have 1-4 GB of RAM. This RAM needs to take care of the deep learning model but also the internal operations of Raspberry-Pi. In addition to that computation capability of embedded devices also plays an important role. Most of the embedded devices don't consume much power. Drawing less power also points towards a less powerful machine. To work with embedded devices like Raspberry-Pi, thinking of training deep learning is entirely incorrect. Instead of that, one need to follow the steps shown in Figure 50.1.

\section{Literature review}

Face recognition is not confined to one discipline, and instead it is multi-disciplinary. There are various face detection and facial expression detection techniques, but this was first introduced in 1978 [2]. Face recognition plays an essential role in the field of security applications, i.e., identification and verification of people via images and video frames [3]. It has been observed from the last couple of years that face recognition technology has replaced various biometric security systems [4-6]. The primary reason is the capability of recording and even performing the interaction. The human face is more reliable in comparison with other biometric techniques such as iris and fingerprint [7]. On the other hand, there are specific techniques like fingerprint scanning, which is more accurate in comparison with face recognition. There are several face recognition techniques in the literature [8]. Deep networks have proven to be efficient for face recognition. A nine-layer network, which has trained on $\sim 4$ million images with a primary classifier, has achieved an accuracy of $97.35 \%$ with deep learning [9]. Various algorithms are available to detect a

* Email: er.rathour@gmail.com

DOI: $10.1201 / 9781003129103-50$ 


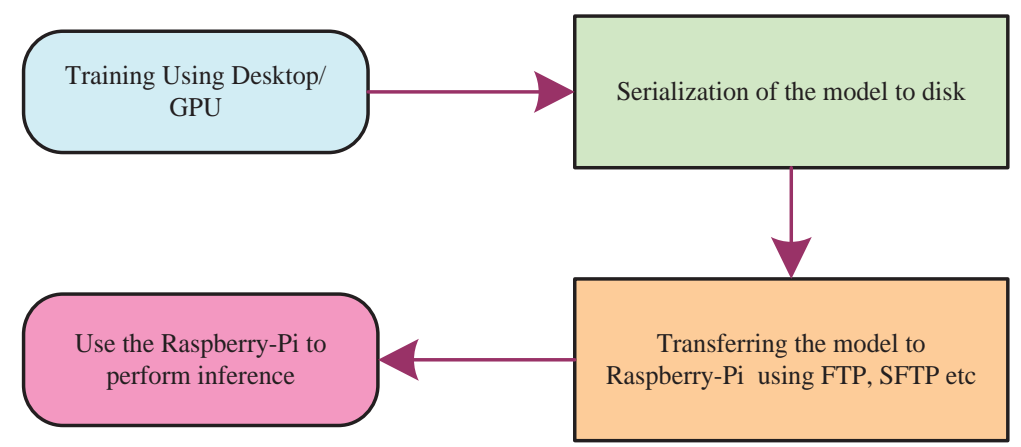

Figure 50.1 Steps to implement deep learning model on Raspberry-Pi.

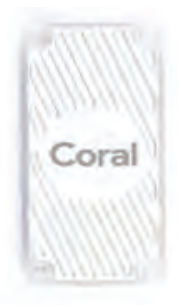

a)

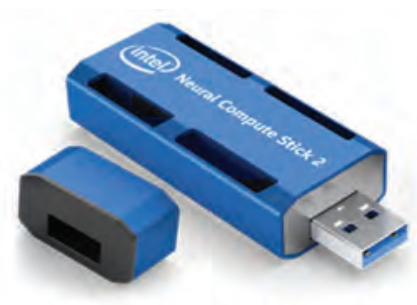

b)

Figure 50.2 Coprocessors for Raspberry-Pi. (a) Google coral TPU USB Accelerator. (b) Neural compute stick2.

face in real-time, out of which the Viola-Jones algorithm is the most popular one $[10,11]$. Hough transform is also a new technique used for face recognition [12]. Raspberry-Pi has achieved quite the right amount of popularity over the last couple of years, and various applications have been proposed like home automation that helps the house owner to get the alerts, and the control can be done via the Internet [13].

\section{Use of coprocessor}

There are situations when deep learning are performed on Raspberry-Pi. In such a situation coprocessors need to be utilized. The most popular coprocessors that can be used with the Raspberry-Pi are Google coral TPU USB Accelerator, and the other one is Intel's Neural Compute stick. The Neural Compute stick can be used by simply plugging in and accessing it via OpenVINO Toolkit or NCS2 SDK. This powerful device can run between 80 and 150 GFLOPs and, too, with a minimal power requirement of $1 \mathrm{~W}$ [14].

The second coprocessor can be Google coral TPU USB Accelerator as shown in Figure 50.2(a). This coprocessor also works similarly as discussed for Intel's NCS, but Google has already reported that their Coral series products are $10 \times$ faster than Intel's NCS shown in Figure 50.2(b). But the practical implementation and comparative analysis say that to achieve a speed of $10 \times$ faster than NCS, one needs to have USB 3. Working with Raspberry-Pi $3 / 3 \mathrm{~B}+$ gives a similar speed. To get the $10 \times$ speed, one needs to use Raspberry-Pi 4, which has USB 3. The iteration rate is quite comparable between Coral 


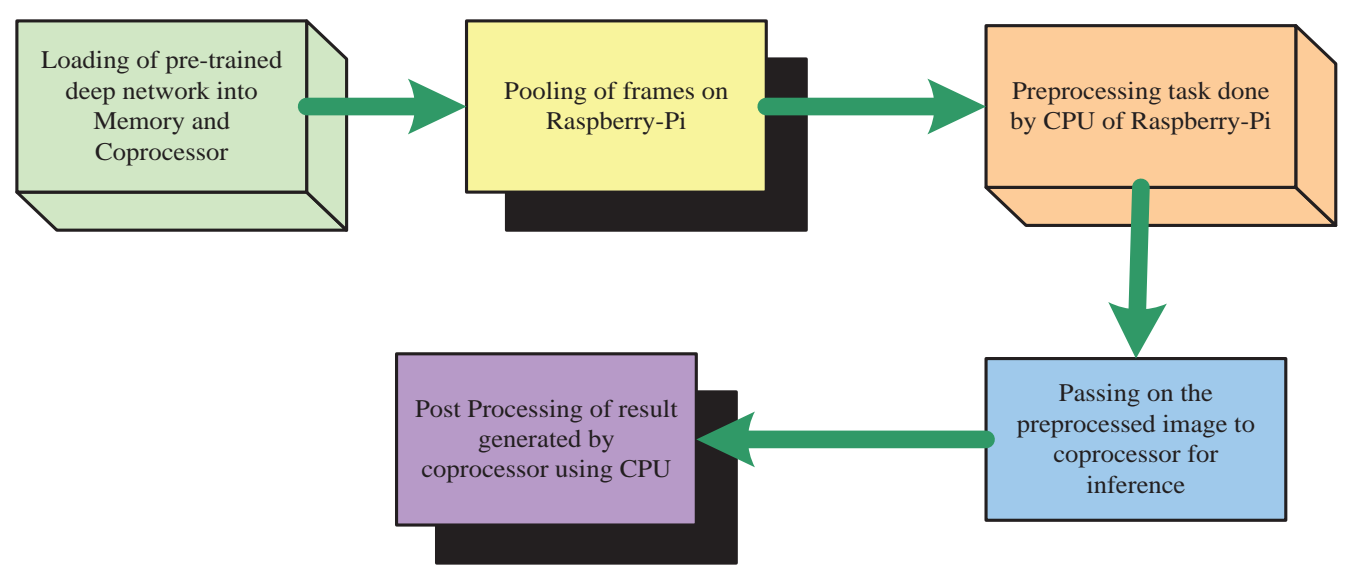

Figure 50.3 Pipeline with coprocessors.

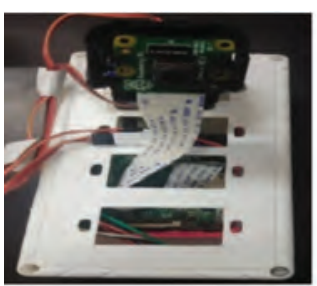

a).

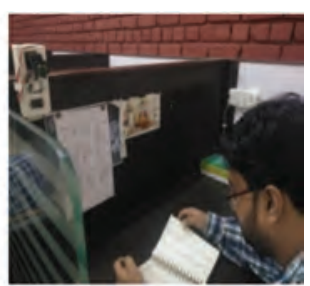

b).

Figure 50.4 (a) Hardware setup using Raspberry-Pi and Pi Cam. (b) Dataset creation.

and NCS when it comes to USB 3 with Coral. Figure 50.3 shows the pipeline process with coprocessor using Raspberry-Pi.

\section{Methodology}

This section explains the complete methodology used for fast recognition of face using Intel's Neural Compute Stick 2 and Raspberry-Pi 4B Model. The entire process starts with the creation of a facial dataset.

\subsection{Dataset creation}

The dataset has been created automatically by using OpenCV and webcam. A dataset of 20 people working in a private organization has been created under various lighting conditions and with different emotional states and facial expressions at 6 different time slots of the day. In compilation, a total of 6 images of each person has been added in the facial image dataset of 120 images, as shown in Figure 50.4(a).

Hardware setup having Raspberry-Pi and Pi-Camera has been used, and the dataset of facial images is captured as shown in Figure 50.4(b). These images are captured using OpenCV, and a few images of the dataset are shown in Figure 50.5. 


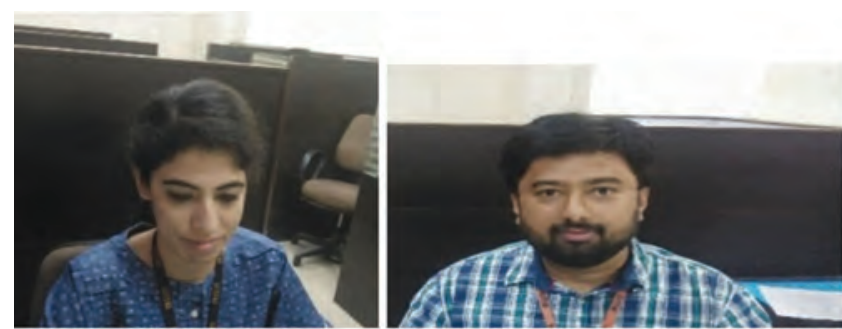

a) b)

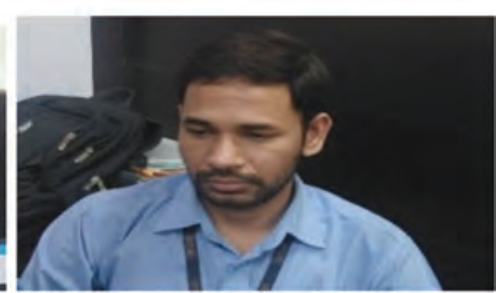

c)

Figure 50.5 Dataset images. (a) Himani. (b) Swanil. (c) Ravi.

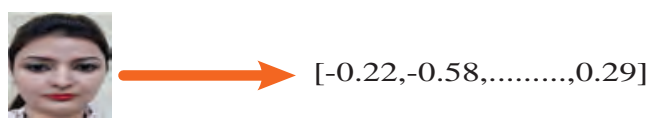

Figure 50.6 Generation of the 128-d real-valued number feature vector of each training image in the dataset.

\subsection{Face detection and facial embeddings extraction using Movidius NCS}

The primary step after the creation of the dataset is to quantify it and to measure the faces in the training dataset. The deep network has not trained here using Raspberry$\mathrm{Pi}$; instead has been used to extract the facial embeddings from the dataset using a pre-trained deep network. The network that has been used is already pre-trained with $\sim 3$ million images at Carnegie Melon University as a part of the OpenFace project [15]. The network architecture that has been used is based on ResNet-34, as mentioned in a paper for Deep Residual Learning for Face Recognition [16] with some reductions in the number of layers and filters. This network has been trained by Davis King [17] on public dataset Labeled Faces in the Wild (LFW). This network achieves around $99.38 \%$ accuracy when compared with other state-of-the-art methods. The famous Adam Gietgey and Davis King, who are the authors of the face recognition module [d] and creator of dlib, have explained the details about how the network has been trained how the network produces 128-d to quantify the faces as shown in Figure 50.6. These 128 dimensions are a unique measurement of each face and can act as a base for the classifier to recognize the face. To achieve this step with Movidius NCS, a shell script has been used to set up the environment for NCS using OpenVINO. The detector used to detect the face using NCS is a Caffe-based deep learning face detector that helps to localize the faces in the images, and the embedding model that has been used is OpenCV deep learning Torch, the embedding model.

The time taken to process 120 images of the dataset was 57 seconds with Intel's NCS and USB 3 of the Raspberry-Pi 4B model. Figure 50.7 shows the completion of embeddings computations.

\subsection{Training}

The next important step is training a standard machine learning model so that the trained model could be able to identify an actual person via those extracted 128 -d embeddings that are unique for each face. Plenty of options are available, like Random Forest, SVM, 


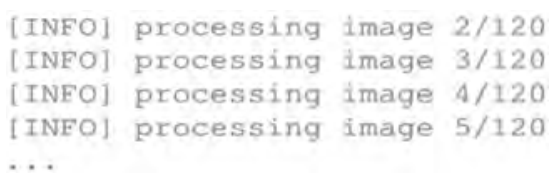

Figure 50.7 Computation of facial embeddings with OpenCV and Movidius.

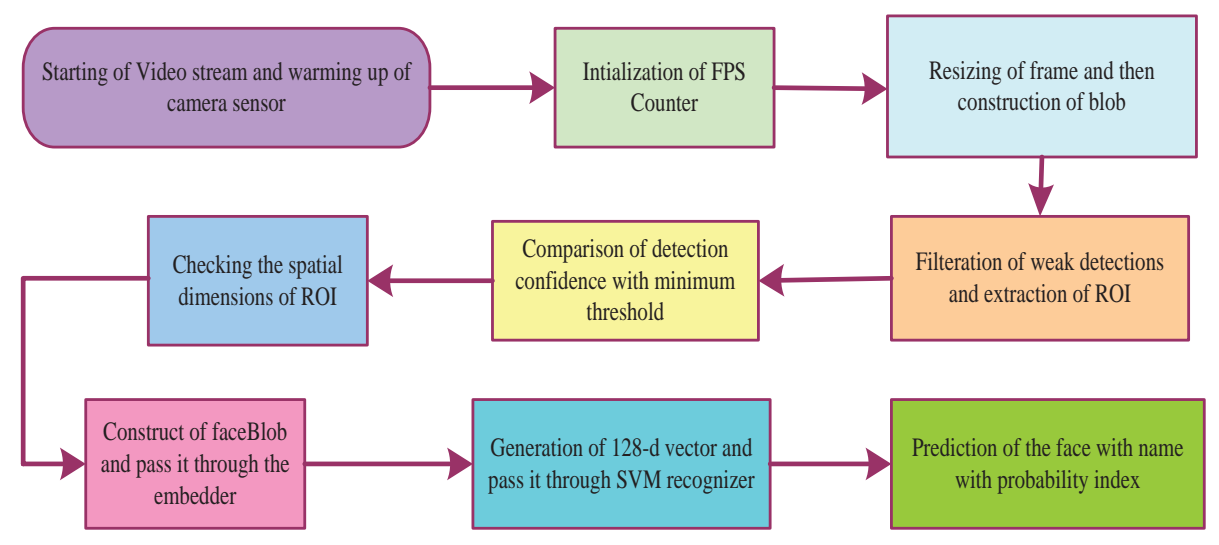

Figure 50.8 Process flow of face recognition in real time using Movidius NCS.

and k-NN. When it comes to training a smaller dataset, k-nearest neighbour is useful via the face_recognition [18] library and dlib [17]. In this work, a more robust classifier known as support vector machine (SVM) is used. It has been achieved with the help of Scikit-learn. The kernel that has been used in this process is the Radial Basis kernel [19]. The kernel is quite tricky to tune when compared with another linear kernel. So, to use this kernel, a process known as 'grid searching' has been used. This process is helping to find the optimal parameters during machine learning for a particular model.

\subsection{Real-time face recognition}

Once the network has been trained the next step is to recognize the face in real time, the detailed process of face recognition in video streams using Movidius NCS shown in following Figure 50.8; this part of the methodology is a pre-trained model or deep learning models like previous two stages of detector and embedder. Instead, this is an SVM based machine learning model for face recognition. In this stage, the CPU of the Raspberry-Pi has been utilized to recognize the faces in real time. Figure 50.8 shows the complete process of face recognition in real time using Movidius NCS. The entire process starts with the warming up of the camera sensor and the beginning of the video stream. A counter for counting frames per second has been initialized to benchmark. After capturing the frame from video, it has been resized, and to detect the face in that frame, a blob is also constructed. After the formation of blobs, the face is detected, but before that, weak detection needs to filter out and then extract the ROI. The pointed ROI is used to detect spatial dimensions to ensure correct recognition. After ensuring that the spatial dimensions are large enough and are more than minimum probability, then finally, the face blobs are constructed and passed through the embedder to generate a $128-\mathrm{d}$ vector. The SVM embedder is then finally predicted with the name and probability index. 


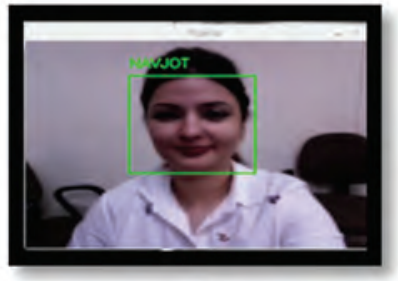

a)

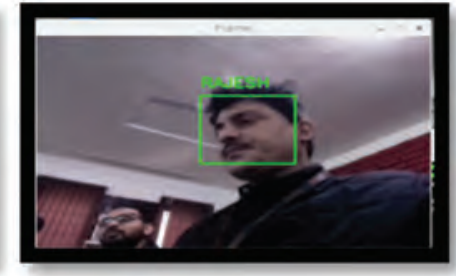

b)

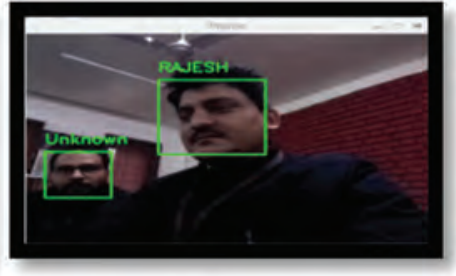

c)

Figure 50.9 Face recognition results. (a) Face recognized as Navjot. (b) Face recognized as Rajesh. (c) Unknown face detected.

\section{Results and discussion}

The results of face detection are shown in Figure 50.9. The faces in the video frames are correctly identified. With this setup of Raspberry-Pi 4B using Intel Movidius NCS, an efficiency of 6.29 FPS has been achieved, which is almost $243 \%$ more than the efficiency achieved with Raspberry-Pi's CPU only, which was only 2.59 FPS.

\section{References}

[1] Samuel Albanie. convnet-burden - Memory consumption and FLOP count estimates for convnets.https://github.com/albanie/convnet-burden. 2018.

[2] M. SOWN, A preliminary note on pattern recognition of facial emotional expression. In The 4th International Joint Conferences on Pattern Recognition, 1978.

[3] Nguyen, A., J. Yosinski and J. Clune. Deep neural networks are easily fooled: High confidence predictions for unrecognizable images. In Proceedings of the IEEE Conference on Computer Vision and Pattern Recognition. 2015.

[4] Mandal, B., et al. A wearable face recognition system on google glass for assisting social interactions. In Asian Conference on Computer Vision. 2014. Springer.

[5] Wang, X., et al. Computerized-eyewear based face recognition system for improving social lives of prosopagnosics. In Pervasive Computing Technologies for Healthcare (Pervasive Health), 2013 7th International Conference on. 2013. IEEE.

[6] Chen, S., A. Pande and P. Mohapatra. Sensor-assisted facial recognition: an enhanced biometric authentication system for smartphones. In Proceedings of the 12th Annual International Conference on Mobile Systems, Applications, and Services. 2014. ACM.

[7] Houmb, S.H., et al.An integrated security verification and security solution design tradeoff analysis approach. Integrating Security and Software Engineering: Advances and Future Visions/Mouratidis, Haralambos, 2007: 190-219.

[8] Yang, J., et al. Two-dimensional PCA: a new approach to appearance-based face representation and recognition. IEEE Transactions on Pattern Analysis and Machine Intelligence, 2004, 26(1): 131-137.

[9] Taigman, Y., et al. Deepface: Closing the gap to human-level performance in face verification. In Proceedings of the IEEE Conference on Computer Vision and Pattern Recognition. 2014.

[10] Viola, P., and M. Jones. Rapid object detection using a boosted cascade of simple features. In Computer Vision and Pattern Recognition, 2001. Proceedings of the 2001 IEEE Computer Society Conference. 2001. IEEE.

[11] Jones, P., P. Viola and M. Jones. Rapid object detection using a boosted cascade of simple features. University of Rochester. Charles Rich. 2001. Citeseer. 


\section{Intelligent Circuits and Systems}

[12] Varun, R., et al., Face recognition using Hough transform based feature extraction. Procedia Computer Science, 2015, 46: 1491-1500.

[13] Xu, Z., et al., Raspberry Pi based intelligent wireless sensor node for localized torrential rain monitoring. Journal of Sensors, 2016.

[14] Wikipedia Contributors. Movidius - Products. https://en.wikipedia.org/wiki/ Movidius\# Products. 2019.

[15] CarnegieMellonUniversity-SatyaLab.OpenFace. https://cmusatyalab.github. io/openface/. 2016 (cited on pages 299, 317).

[16] Kaiming Heetal. "DeepResidualLearningforImageRecognition”.In: CoRR abs/1512.03385 (2015). http://arxiv.org/abs/1512.03385 (cited on pages 18, 79).

[17] Davis E. King. "Dlib-ml: a machine learning toolkit". J. Mach. Learn. Res. 10 2009, 10: 17551758. http://dl.acm.org/citation. cfm?id=1577069.1755843 (cited on pages 79, 306).

[18] Adam Geitgey. face_recognition - The world's simplest facial recognition api for Python and the command line. https://github.com/ageitgey/face_recognition. 2016 (cited on pages $79,306)$.

[19] Wikipedia Contributors. Radial basis function kernel. https://en.wikipedia.org/ wiki/Radial_ basis_function_kernel. 2019 (cited on page 308). 


\title{
51 Design of an automatic on-load tap changing distribution transformer
}

\author{
Krishan Arora ${ }^{1 *}$, Tarun Dhandhel \\ ${ }^{1}$ Assistant Professor and Head, School of Electronics and Electrical Engineering, \\ Lovely Professional University, Punjab, India \\ ${ }^{2} \mathrm{M}$-Tech Scholar, School of Electronics and Electrical Engineering, Lovely \\ Professional University, Punjab, India
}

\section{Introduction}

Nowadays all equipment running in home or in industry or in office or any place you can imagine needs electricity. Only electricity is not enough to run any equipment. Electricity with proper voltage, required current and in required form is given to any equipment, then only equipment can run. As all know alternate current can be transferred to desirable place with proper voltage and can be changed in required form to operate it. But when equipment is made to run on frequently changing voltage, then equipment can get damaged or may catch fire which can do a huge loss to a person. To avoid it, consumers have to use a small stabilizer in homes but for each equipment one stabilizer is required which can be a tough call for being pocket friendly. Also, industries too have the same concept for their equipment in their industry. Industries get their supply in 3-phase form. Nowadays all the world is shifting towards the use of electricity rather than use of fossil fuels. For different voltages to supply authors would require different transformer. So, to avoid it a country specifies a standard for a country. In India $220 \mathrm{~V}, 50 \mathrm{~Hz}$ is specified. But still we require different voltages for use in some industries as equipment made in another country or with different standards are made and cannot work in another country. So, to break this barrier we are making phase changing and on-load tap changing transformer.

\section{Review of literature}

In discrete steps, various turns ratios can be selected in transformer with tap changer strategy. With this type of arrangement in transformer, variation can be obtained with turn ratio with the connection of access points termed as taps connected on either primary or secondary winding. Transformers are essential for the transmission, distribution and utilization of alternating current electrical energy. Technically, power transformer is a combination of two or more windings and, through electromagnetic induction, transmits the voltage and current to another system to serve the purpose of transmitting power. Refashioning in transformation ratio helps in regulation of voltage at tap windings, so revising in number of turns. The process of altering the ratio of transformation by tapping the windings is termed as tap changing. Tapping may be changed mainly in two different ways: when the transformer is disconnected from the supply, called as, off-load tap changing; when the transformer is operating on load (without de-energized), known as on-load

* Email: krishan.12252@lpu.co.in

DOI: 10.1201/9781003129103-51 


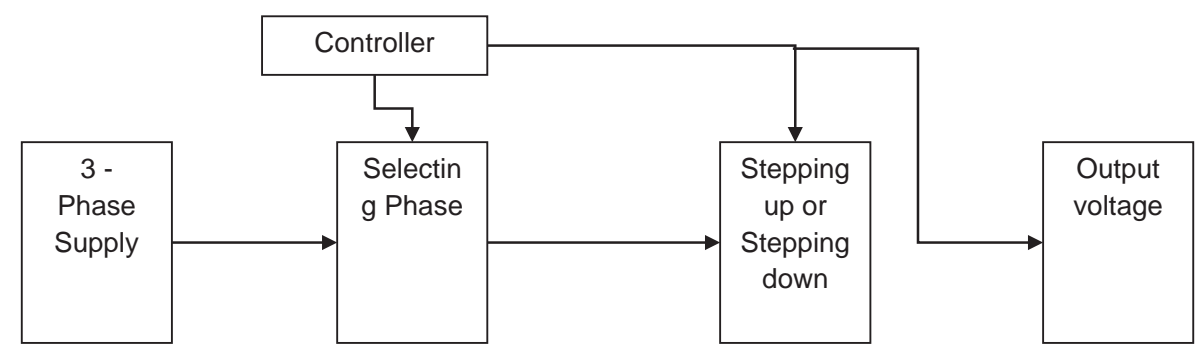

Figure 51.1 Block diagram phase changing and tap changing.

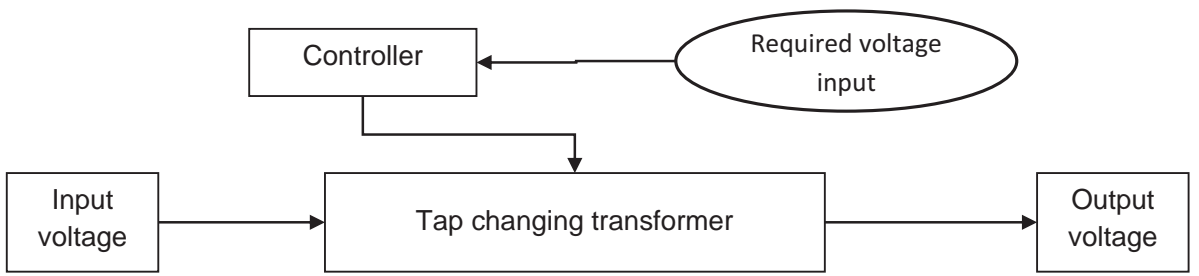

Figure 51.2 Design solution for tap changing transformer.

tap changing. During off-load tap changing, the transformer is completely de-energized, in order to avoid arcing at the point of breath. The method of off-load tap changing is not suitable for large power supply systems. This system call for power transformer with a voltage regulating winding, the tapping of which is changed over under loaded condition by on-load tap changer. Schemes employed for on-load tap changer involve the use of more complicated and expensive tap changing equipment.

\section{Block diagram}

In this block diagram, the basic circuit operation is represented. As shown in Figure 51.1 authors have used 3-phase supply which is given to system and then phase selector selects one phase as per requirement of output than stepping up or stepping down is done if required and then output is given. All this operation is controlled by controller.

\section{Design solutions}

In the early stages of the research work, various design solutions were employed. The initial design solution can be seen in Figure 51.2.

The tap changing transformer can do step up or step down if they have tapping in secondary windings. In the design shown firstly it is asked what output voltage is required than tap is changed of the transformer by controller keeping in mind what voltage is given in input. Limitation of this design is that it can only make variation in output voltage as per the tapings made in the transformer which are in secondary windings. In this case output voltage is not independent of input voltage. So the author may require more number of voltages in input side. Next design was varied to suit these needs, as is shown in Figure 51.3.

In this design tap changing transformer system is added with phase changing transformer to have variations in input voltage which will help it have more precise and 


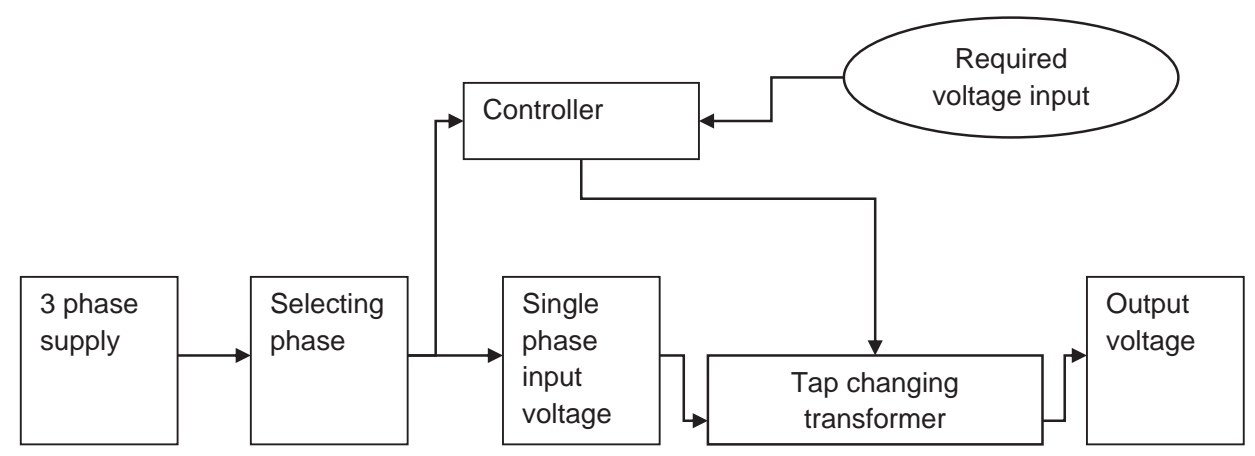

Figure 51.3 Design solution for tap changing transformer with phase selector system.

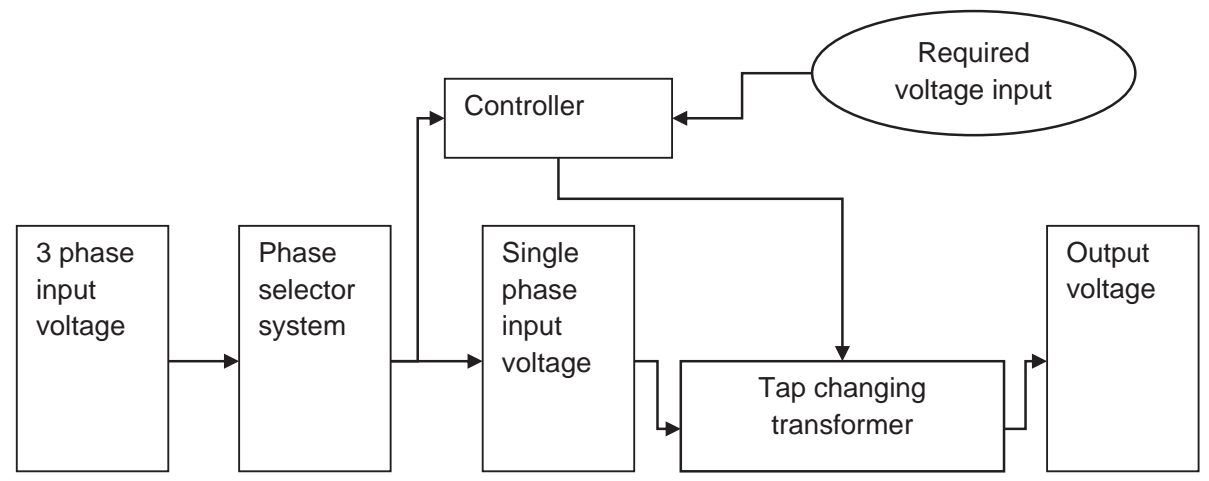

Figure 51.4 Design solution for tap changing autotransformer with phase selector system.

accurate output. This system too has a limitation after having phase selector system that 1-phase input voltage can step up or step down depending on which transformer is used. If the author uses step up transformer but as per output required the author needs to do step down then this system will fail. So to tackle this limitation the next design was made, as shown in Figure 51.4.

In the design shown in Figure 51.4, the author can step up and step down during onload conditions depending on the voltage required for the output but precision of output voltage would be compromised. If the author needs output of 220 volts but all the voltages in 3-phase system are 60 volts, 150 volts and 250 volts, in this case author can step down 250 volts to 220 volts but it would require a lot of tapings in secondary windings which could create a problem to control it through a microcontroller. So to overcome this problem the author has replaced the combination of step up and step down transformer with an autotransformer which can act as both step up and step down by changing the direction of input and output.

This final design uses a servo motor which rotates the autotransformer to desirable output voltage. Also combination of relays are used to change the input and the output side of the autotransformer to make it step up autotransformer or step down autotransformer as per required of the system. 


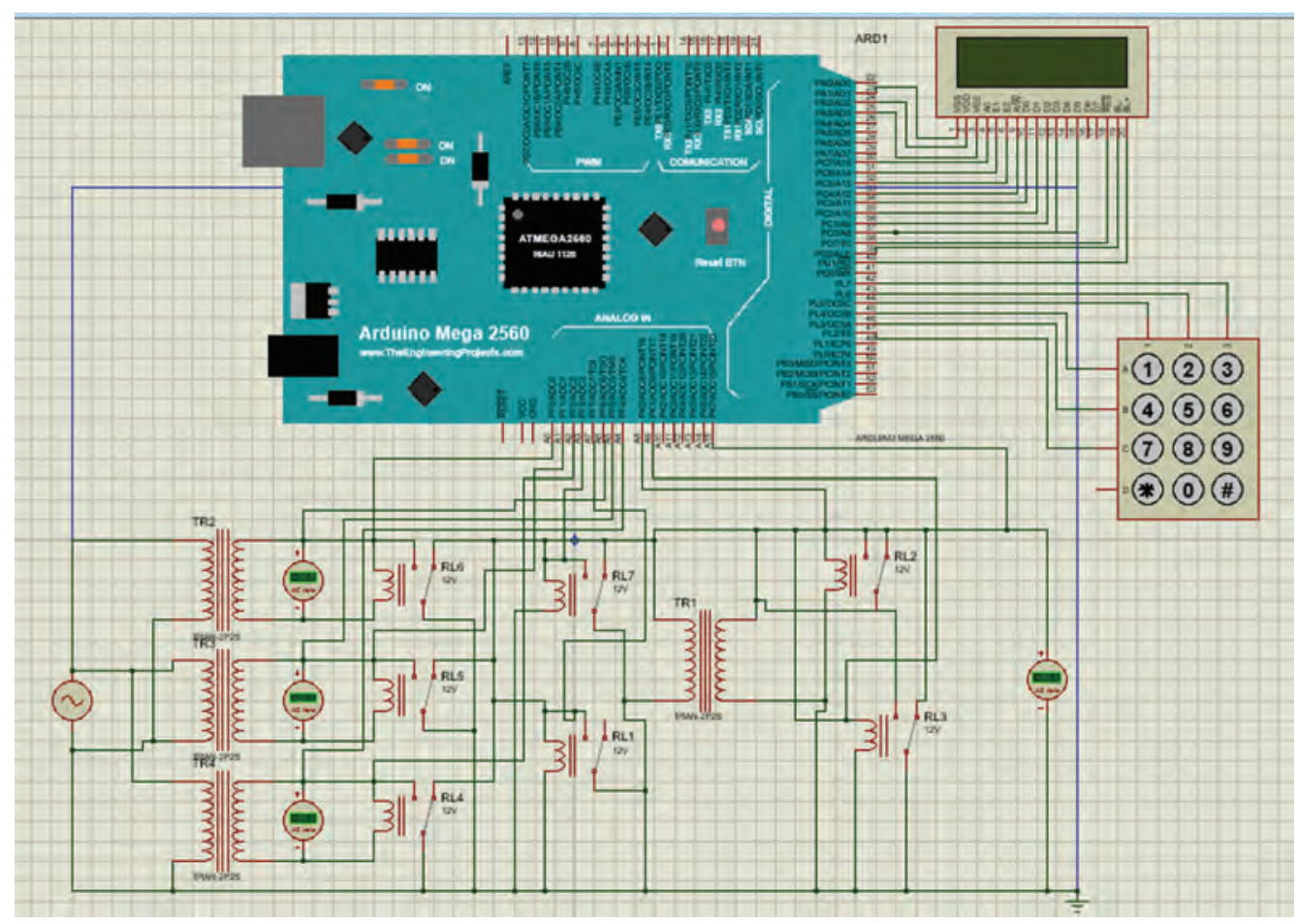

Figure 51.5 Circuit representation of on-load tap changer.

\section{Working}

This research work focuses on the planning and construction of phase changing and automatic on-load tap changing distribution transformer as shown in Figure 51.5. This ensures continuous offer of power to the load as per the input provided until it's asked to prevent it. Thus this method first asks for input voltage that is required to control the instrumentation then the system compares the required voltage with all the three voltages in the 3-phase system and whichever voltage in the 3-phase system is nearest to required voltages that is forwarded to next step. In the next step the needed voltage is compared with incoming voltage then Arduino mega decides how the connected autotransformer would work, i.e., it ought to work as step up transformer or step down transformer. Arduino mega decides how the transformer would work and switch the relays on and off as per demand. In this circuit, three different autotransformer acts as three phases which are received by the industry for industrial purposes. Also each autotransformer is attached with AC voltage sensor in parallel. Also, each AC supply coming from the autotransformer is connected with the relay module which acts as switch and for our phase selector circuit. All the three relays are controlled by the Arduino mega which acts as its brain and responds as per required voltage. In the next part of this circuit where the author gets a single phase supply, it can step up or step down the voltage of the single phase supply as per the requirement of the output voltage. In this circuit, an error sensing feedback control called servo is used to correct system performance. 


\section{Conclusion}

This research focuses on the design and construction of a phase changing and automatic on-load tap changing distribution transformer. This ensures continuous supply of power to the load as per the input provided till it is asked to stop it. The supply comes to industry in the form of 3-phase in the industry. So this system firstly ask for input voltage which is required to operate the equipment; after the required voltage is input then the system compares the required voltage with all the three voltages in the 3-phase system and whichever voltage in 3-phase system is nearest to required voltages that is forwarded to next step. In next step required voltage is compared with incoming voltage then Arduino mega decides how the connected autotransformer would work, i.e., it should work as step up transformer or step down transformer.

\section{References}

[1] Gautham Ram Chandra Mouli, Pavol Bauer, Thiwanka Wijekoon, Ara Panosyan, "Design of a Triac OLTC for grid voltage regulation.” 2013 IEEE (2277-7970) vol. 2, no. 4, 2012.

[2] S.V.M. Bhuvanaika Rao, B. Subramanyeswar, "Fine voltage control using OLTC by static tap change mechanism". International Journal of Advanced Computer Research, vol. 2, Dec. 2002.

[3] Vigneshwaran. S, Yuvaraja. T "Voltage regulation by solid state tap change mechanism for distributing transformer.” International Journal of Engineering Research \& Technology (IJERT), vol. 4, issue 02, February 2015.

[4] Vivek Thomas Chacko1, Mohamed Noufal, Nidhin Mohan, Sriram Vinod, Eldhose K, "Solid state on load tap changer for transformer using Arduino." International Research Journal of Engineering and Technology (IRJET), vol. 04, Apr. 2017.

[5] S.M. Bashi, "Microcontroller-based fast on-load semiconductor tap changer for small power transformer." Journal of Applied Sciences vol. 5, no. 6, 999-1003, 2005. 


\title{
52 An advanced driver assistance system for autonomous vehicles
}

\author{
Design and test in virtual environment
}

\author{
Rahul Kumar Mahato, Avinash Kumar, Jyoti Prasad \\ Kurmi, Sanaboina Snigdha, Gurjot Singh Gaba*, \\ Rajan Miglani
}

\author{
School of Electronics and Electrical Engineering, Lovely Professional \\ University, Punjab, India
}

\section{Introduction}

For drivers throughout the globe poor visibility and bad weather conditions are major reasons for concern. It is for the same reason that even autonomous vehicles find their credibility challenged in foggy conditions. Fog is basically the cloud on the ground and the particles of fog range within 1-100 $\mu \mathrm{m}$ in diameter, with most particles in the 10-15 $\mu \mathrm{m}$ region, while rain drops are 10-100 times bigger than fog particles and range from 0.5 to $5 \mathrm{~mm}$ in diameter. The size of these tiny particles is very crucial because their size decides the reflection of vehicle light waves back onto the eye of the driver.

It is very clear that driving in fog can be very challenging. Fog reduces the visibility and this reduced visibility increases the chance of fatalities. Low visibility can also be caused due to heavy rainfall and snow. Reduced visibility may result in driver missing seeing potholes, sign board, pedestrians, traffic lights, exit lanes etc. or misjudging the distance, thus leading to possible road accidents. There are many cases reported when many vehicles collides into one another due to poor visibility. Nearly 50 people were injured when more than 60 vehicles crashed into eachother on 22 December 2019, in eastern Virginia due to heavy fog [1]. In Rewari district, on the Delhi-Jaipur highway, a pile up due to fog was reported in which two people lost their lives and at least 12 got injured [2]. Wikipedia shares some major pile-ups in [3] and fog is one of the causes of almost every accident. According to "Times of India", in 2016, as many as 9,317 people died in fog-related road crashes, and the number increased to 11, 090 in 2017. According to "Ministry of Road Transport \& Highways", India, there were 44,011 and 28,026 road accidents due to heavy rain and fog, respectively in the year 2018 [4].

With the advances in automobiles technology, there have been many techniques developed such as: advanced driver assistance systems (ADAS), adaptive cruise control (ACC), driver drowsiness detection system, lane monitoring, advanced parking system (APS), traction control and many more [5]. However, these techniques are integrated in premium cars with limited functionality.

In this paper, our focus is to propose a smart electronic system to assist drivers in poor visibility which also lowers down speed automatically to prevent accidents. The

* Corresponding author email: gurjot.17023@lpu.co.in

DOI: $10.1201 / 9781003129103-52$ 
remaining paper is structured as follows: Section II discusses the related work, and Section III describes our proposed system. Section IV presents the results followed by conclusions in Section V.

\section{Related work}

Researchers at MIT, USA have developed a new imaging system that can calculate the distance of objects obscured by fog so thick that humans can't see through it. They have developed an algorithm that divides the raw data into two parts: light reflected form the object; light reflected from the fog. Further, light reflected from the object is used for distance measurement. They have used the concept of optical depth, which describes the amount of light that penetrates the fog and this is independent of the distance of the objects [6]. This system is expected to be used in autonomous vehicles for knowing the distance of obstacles behind the thick fog through which we can't see and may meet with a severe accident. Although, todays' automation in automobiles is mainly at "level 0 " or "level 1", implication of this system can help manufactures to reach higher level of automation and the accuracy sensing will increase exponentially for low visibility conditions.

Amir Mukhtar et al. in have introduced a collision avoidance system (CAS). They have listed the causes of accidents; one of them is fog and heavy rainfall. Their paper provides a review on a huge range of sensors along with some techniques to track the CAS for autonomous vehicles. Driver assistance system (DAS) and CAS are sometimes used interchangeably; however, DAS is a subset of CAS. Companies such as Volvo, Ford, Honda, Toyota, Nissan, Subaru and Mercedes-Benz have developed their CAS and promote it as a premium feature to the customers [7]. Nissan, for CAS, uses high quality radar to detect the obstacle and the relative speed of the obstacle [8]. Automobile company BMW also provides various safety features and explains the advantages of those techniques in context of security as well in riding comfort [9].

\section{A. Use of camera, LiDAR and radar}

The camera is a very popular device to get the on-field video for various purposes. They are popular because of their availability, diversity, cost and multi-functionality. LiDAR provides precise depth perception and correct distance of up to a few centimetres, making it a better option over cameras. But majority of literature has contributions where cameras have been used for detection of fog or analysing road environment. Sahana V. et al. in their paper [10] have discussed about some of the techniques used to detect the dense fog using in-vehicle cameras for the evaluation of road condition. Using image acquisition, image layer decomposition, layer enhancement, feature extraction, backscattered veil detection they were able to find the fog levels and the intensity of the objects.

Light detection and ranging sensor (LiDAR) is another popular device for fog detection and ranging. It continuously sends a laser pulse in the direction of obstacle and depending upon the time pulse propagation and reception, the distance of the obstacle is calculated. LiDAR is used for more accurate precision. But, in contrast, they also suffer from environmental factors like fog and smog. Car manufacturers worldwide have successfully incorporated radar and LiDAR in a few vehicles as a part of testing [11,12].

\section{Proposed scheme}

In our system, we intend to use two ultrasonic sensors to detect the obstacles. The two ultrasonic sensors are placed at the middle of the each half of the length of front bumper 


\section{2}

Intelligent Circuits and Systems

of vehicle. The purpose of this setup is to provide higher azimuthal angle for the reliable performance. As observed from Figure 52.1, the azimuthal angle of each sensor is $15^{\circ}$.

Also, use of these two sensors would help us to predict if the obstacle is present on left or right. Figure 52.2 shows the setup of sensors and three different levels of the system's functionality. When the obstacle is far enough to witness any collision, the obstacle's distance is known to the system and alerts as "obstacle detected". When the obstacle enters a range that has the probability of collision if no deceleration is performed, in this case driver is alerted to apply brakes. If the distance decreases further such that immediate braking is required then the system autonomously applies the brake to avoid chances of collision.

\section{A. Experimental setup}

As discussed earlier, there are two major conditions that make low visibility: rain and fog. The experimentation has been performed in a chamber where fog and rain conditions were artificially created to evaluate the proposed system performance. Figure 52.3 illustrates the experimental setup created to evaluate the behaviour and performance of the proposed

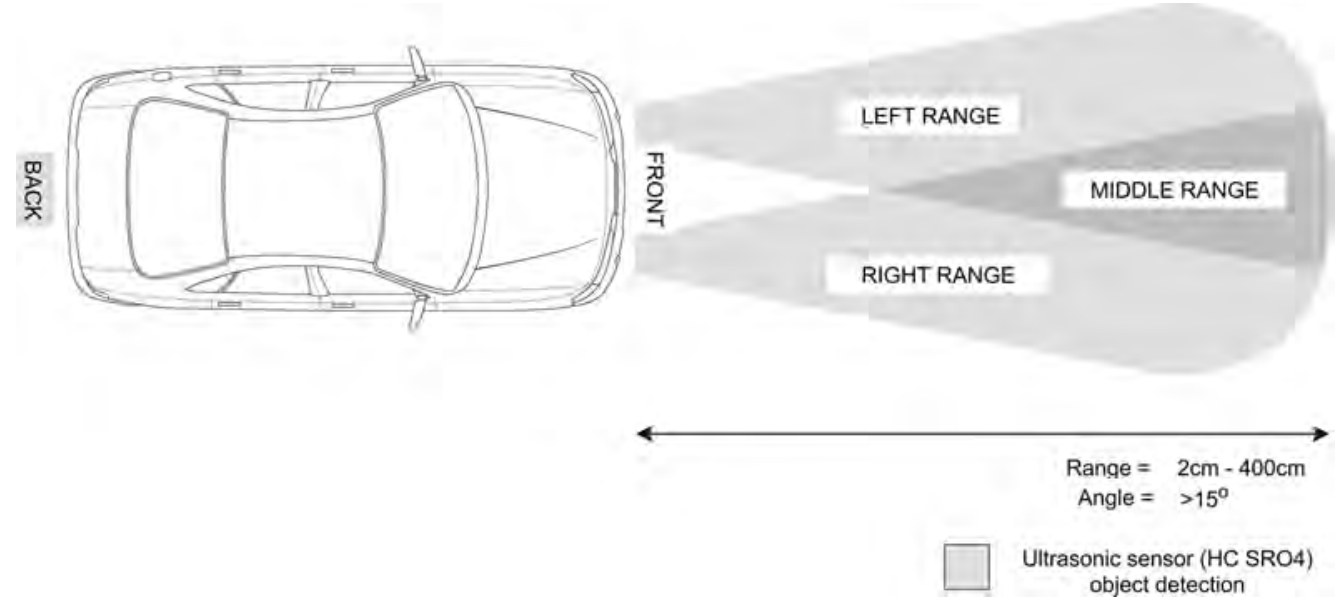

Figure 52.1 Placement of sensors in vehicle with its operational characteristics.
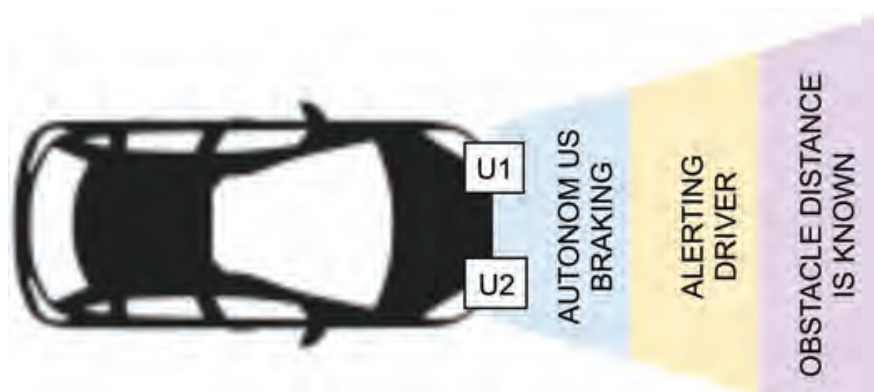

U: Ultrasonic sensor

Figure 52.2 Alerts and action model of proposed system. 


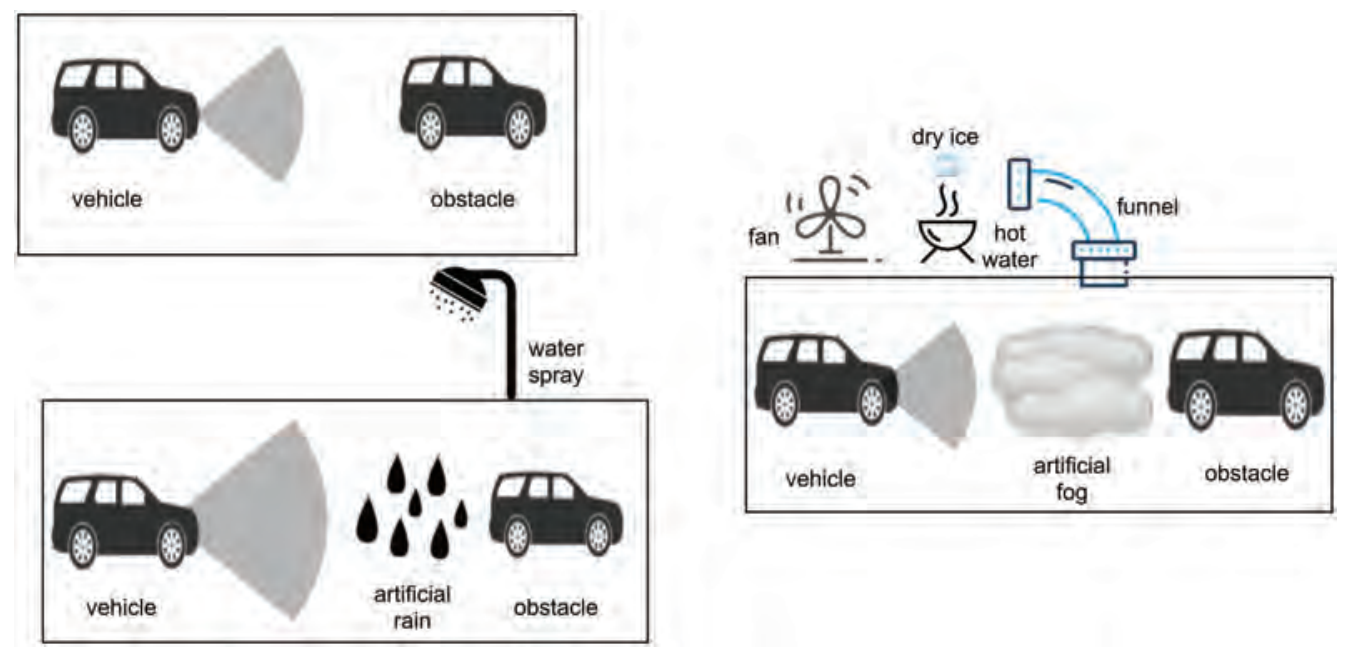

Figure 52.3 Experimental setup of chamber tested under fog and rain conditions.

system in different weather conditions. We've sprayed fine water droplets and made the fog artificially using dry ice and hot water and pushed them continuously in between our system and the obstacle to provide a real test case. One may find other ways to artificially produce fog.

Figure 52.4 portrays the relationship of various units of the proposed embedded system. Input unit represents the two ultrasonic sensors. These two sensors are the input module for our system; they're the on-field ranging and sensing component. The outputs of both the sensors are fed to the Processing unit; this block contains Arduino-uno (a micro controller) and a motor driver (L293D). This block processes the data using the algorithm to find the distance of the obstacle from the vehicle under consideration. Once the distance is known, the algorithm keeps its track and performs the necessary calculation for deceleration if required. Motors are hereby representing the tyres of the car. Motor driver helps to run the motor simultaneously after it receives command from the Arduino; for a need to perform deceleration, the rpm is varied according to the distance between object and the vehicle (refer Figure 52.5).

The flowchart in Figure 52.6 defines the system work: how the process is carried out step by step to achieve the required outcomes of the system. Through Figure 52.6, we can infer the background process of our system. The process object detection programs the system to continuously detect the obstacle. Once the system detects any obstacle from any of the sensors, at that instant, the signal is flagged accordingly. Through this work, we can predict the side at which the obstacle is present and then alert the driver accordingly.

Until this, the system only acts as a guide to the driver but, if the distance reduces to less than predefined threshold (i.e., $100 \mathrm{~cm}$ in our test case), a decision has to be made by the system for autonomously braking to prevent collisions. The system will gradually decrease the speed and then releases the control back to the driver once it comes out of danger of collision. Figure 52.7 provides the pseudo code of the algorithm which is helpful in understanding the overall working behaviour of the proposed system. Table 52.1 lists the various components used in the research and experimentation work along with their specifications. 


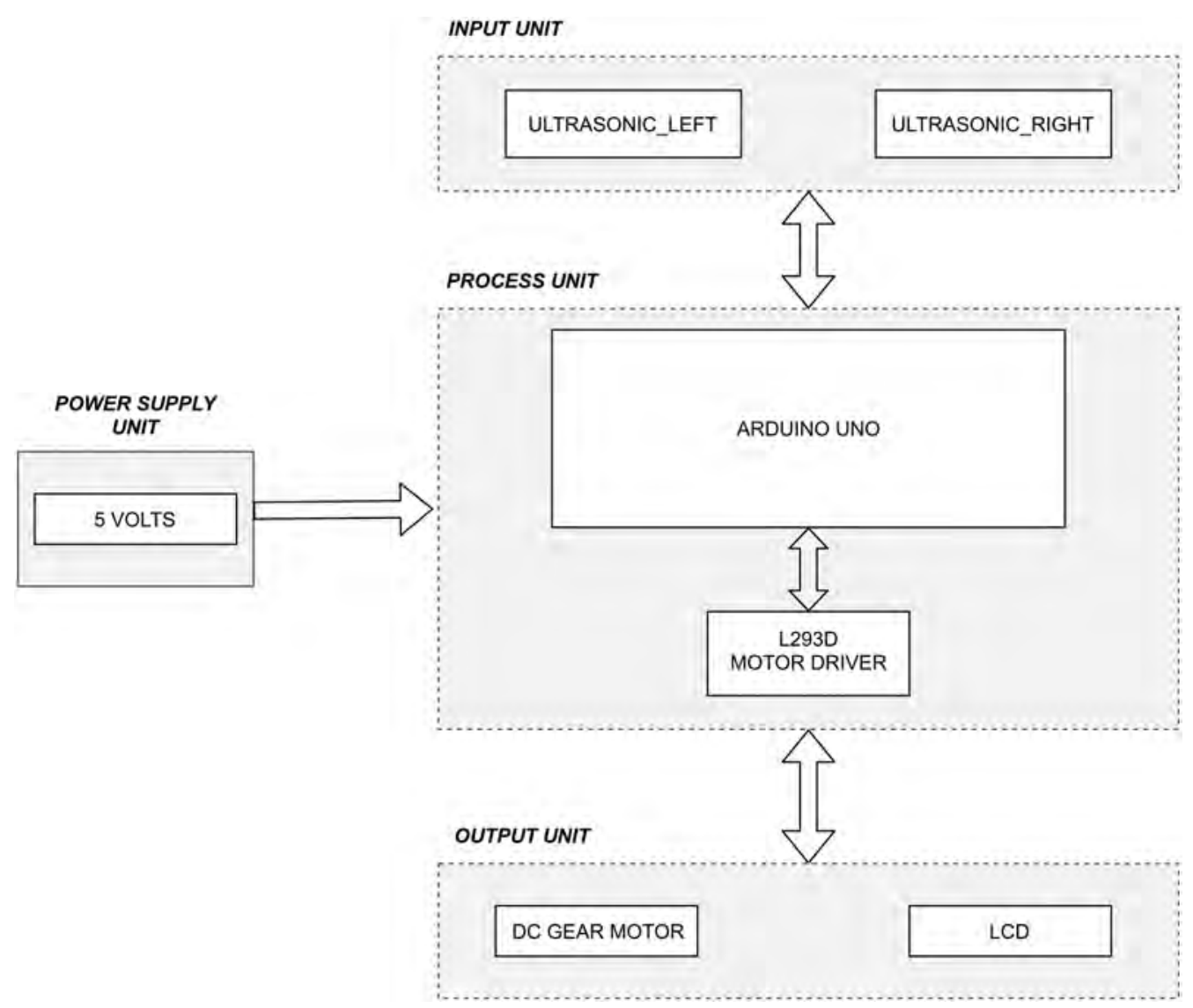

Figure 52.4 Relationship of various units of embedded system.

\section{Results and discussion}

The results shared in this section have been carried out using our experimental setup. We have performed the experiment in a few environmental conditions such as rainy, heavy fog and sunny conditions. Figure 52.8 shows object distance at frequent time intervals for heavy fog conditions, compared with no fog condition. We manually placed an object at a distance $125 \mathrm{~cm}$ in fog condition and observed the response of the system. Similarly, the same setup has been used for analysing the system in rainy conditions. Figure 52.9 shows a comparative graph (with no-rain condition) of the obstacle when placed at a distance of $132 \mathrm{~cm}$. Figure 52.10 shows us the functionality of the system: how the system reacts when the object is too close to meet with an accident. We see that the system doesn't perform any operation while the distance of the object is greater than $100 \mathrm{~cm}$ (can be different according to the experimental setup and the use); as soon as the object distance reaches below $100 \mathrm{~cm}$, the system autonomously applies the brakes to prevent the accident. Refer to the example demonstrated in Figure 52.10: suppose a car (equipped with our system) is travelling with a constant speed of $50 \mathrm{kmph}$ in foggy weather. A vehicle (travelling with $<50 \mathrm{kmph}$ ) was detected in front of him as an obstacle by the system. Table 52.2 provides the accuracy and error of the proposed system in diverse weather conditions. 


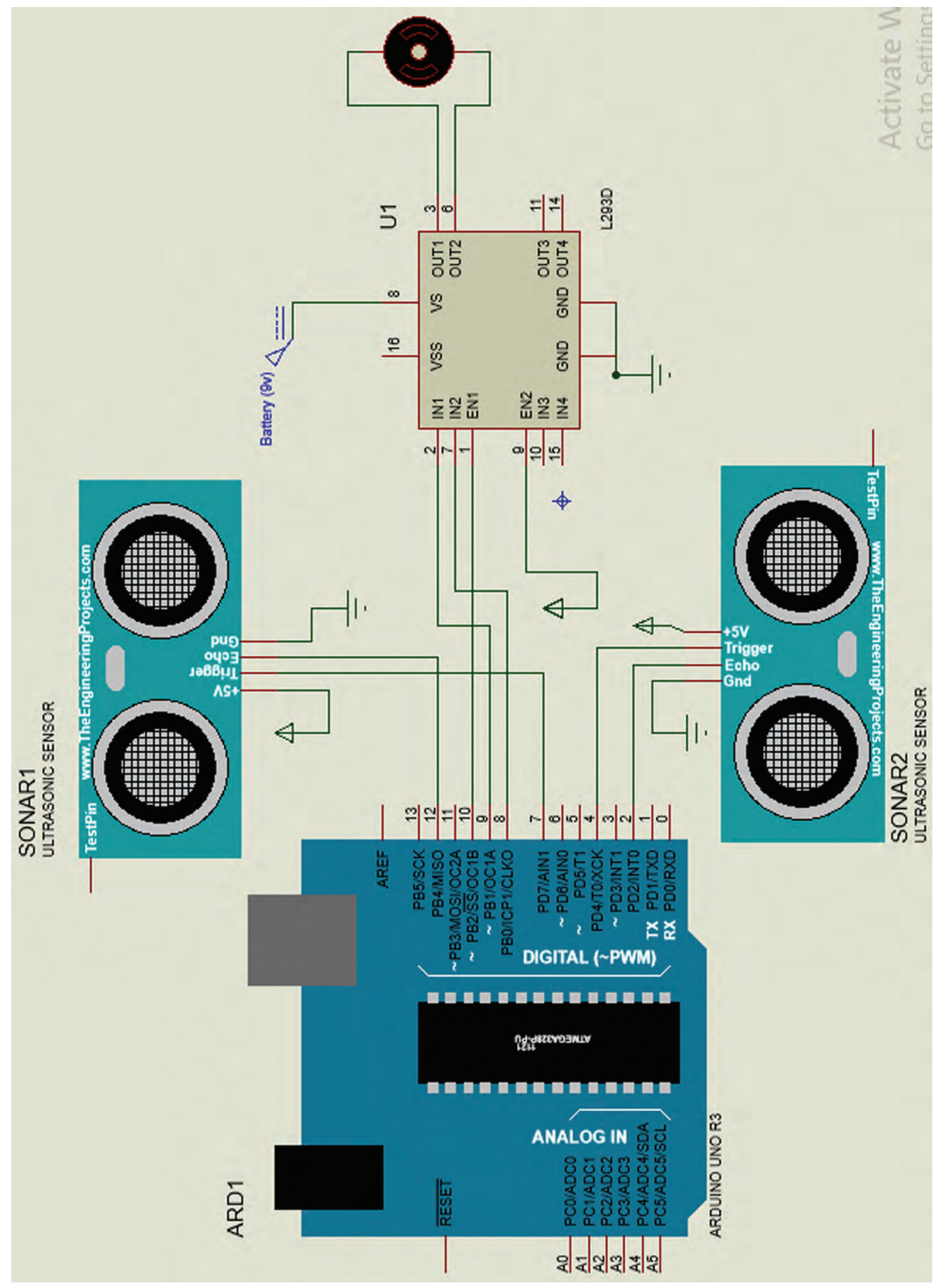




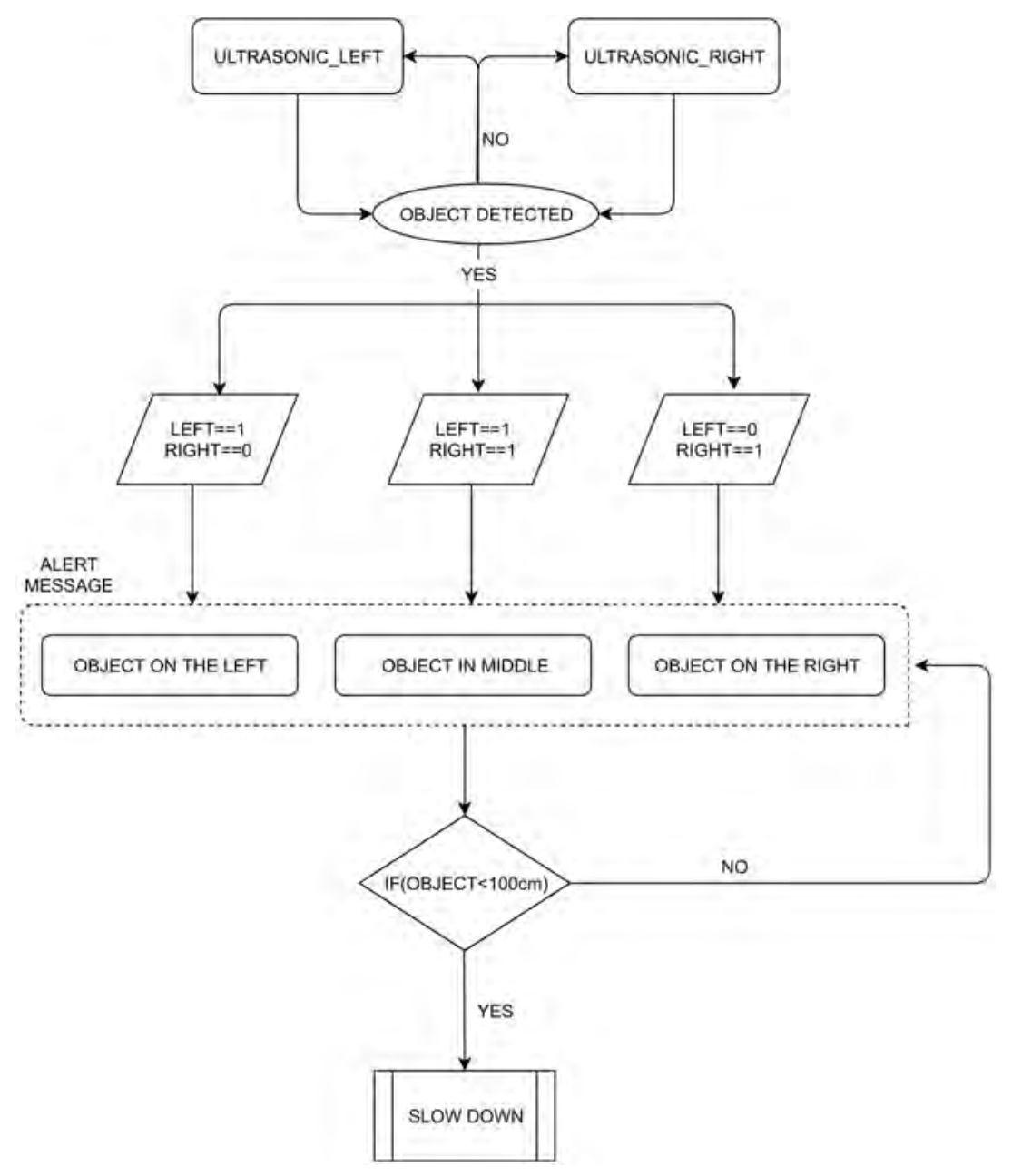

Figure 52.6 Stages of execution.

The system alerts the driver about the obstacle. Somehow if the driver ignores the alert and keeps travelling at the same speed, then the system takes over the control from the driver and applies the brakes automatically to prevent accident. The system made the vehicle to stop because the obstacle remained in the path as the driver did not change the lane and nor the obstacle. If the driver had changed the lane, then the system would have released the control immediately if there's no vehicle (under $100 \mathrm{~cm}$ distance) in that lane.

\section{Conclusions}

With the passage of time the automobile industry has also become smarter; vehicles, nowadays use sensors to help the driver in many ways. Even after implementing technologies in the vehicles, there are thousands of people who die in road accidents. Some of these accidents are caused due to low visibility, when the driver couldn't locate the 


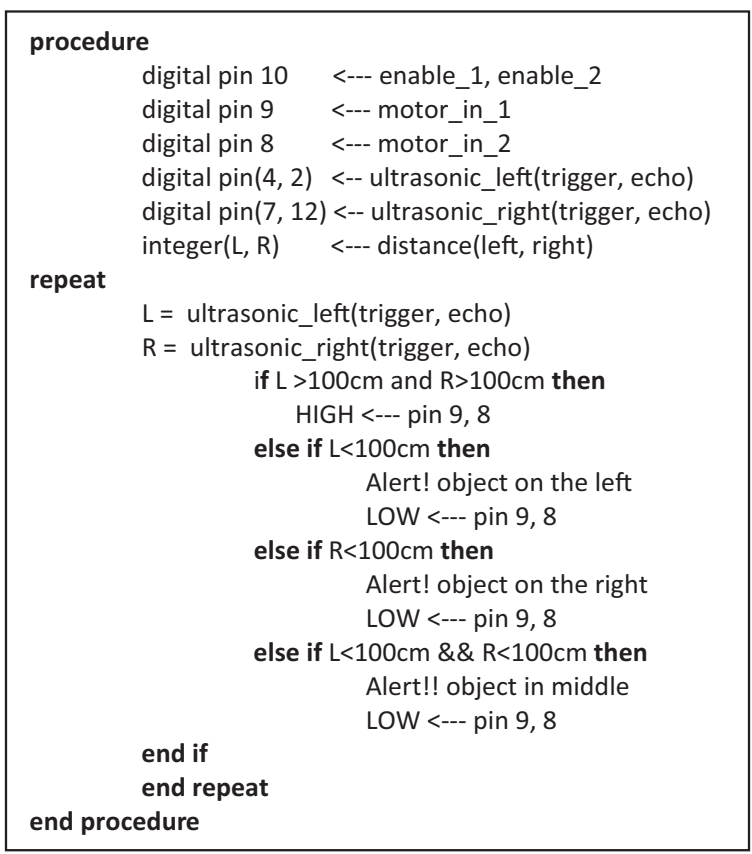

Figure 52.7 Pseudo code of algorithm used in the proposed system.

Table 52.1 Components and specifications

\begin{tabular}{ll}
\hline Components & Specifications \\
\hline Arduino UNO & ATMEGA328P \\
Ultrasonic sensor & HC-SR04 \\
LCD & $16 * 2$ alphanumeric display \\
Motor & $5 \mathrm{~V}$ DC \\
Battery & $9 \mathrm{~V}$ Alkaline \\
\hline
\end{tabular}

obstacle in time. In this paper, we have discussed the use of ultrasonic sensors to prevent the accident due to poor visibility conditions. We have categorized the gap (between vehicle and obstacle) into three different parts; each part has some or no control over the vehicle depending upon the gap. We experimented with the system in fog, rainy and sunny conditions. Each of these conditions is analysed for their accuracy and error percentage based on data obtained from the ultrasonic sensors. Moreover, the system has the capability to work on its own in critical conditions and the same has been successfully performed in our experimental setup. This paper might help you to get an idea about a safety system in foggy weather. 


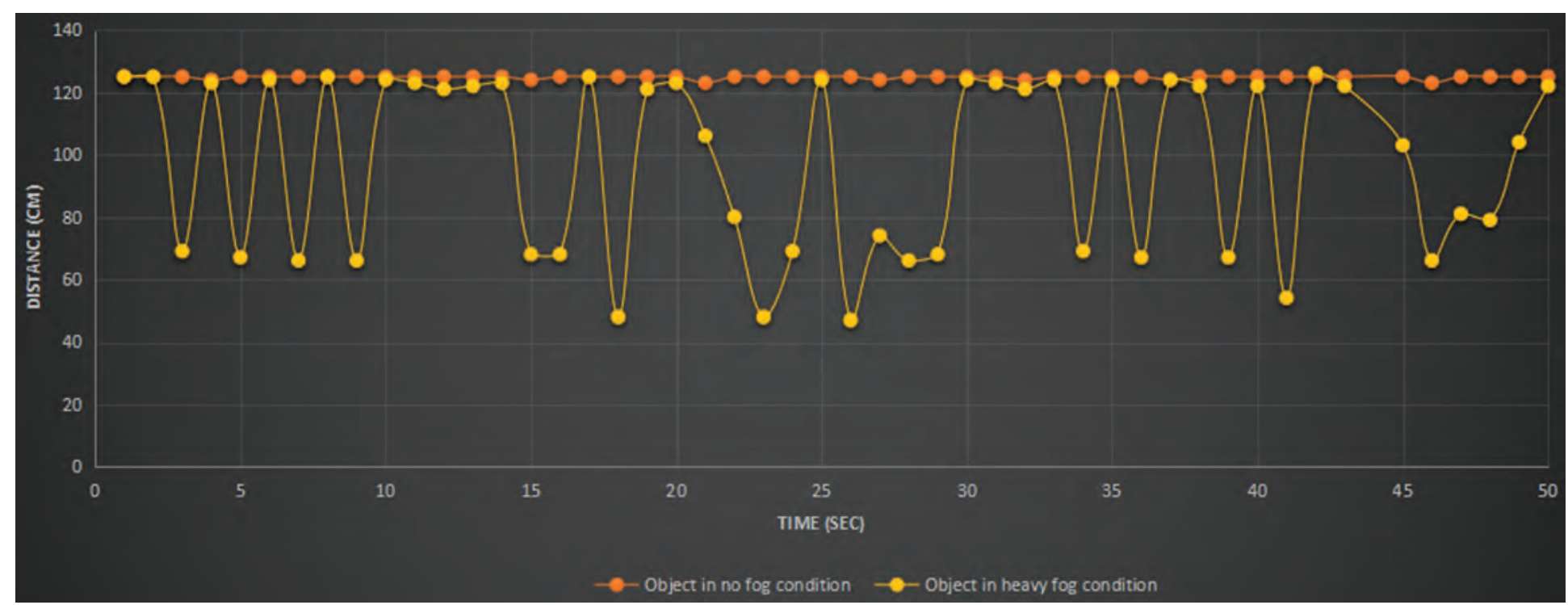

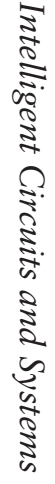

Figure 52.8 Detection of obstacle in foggy conditions. 


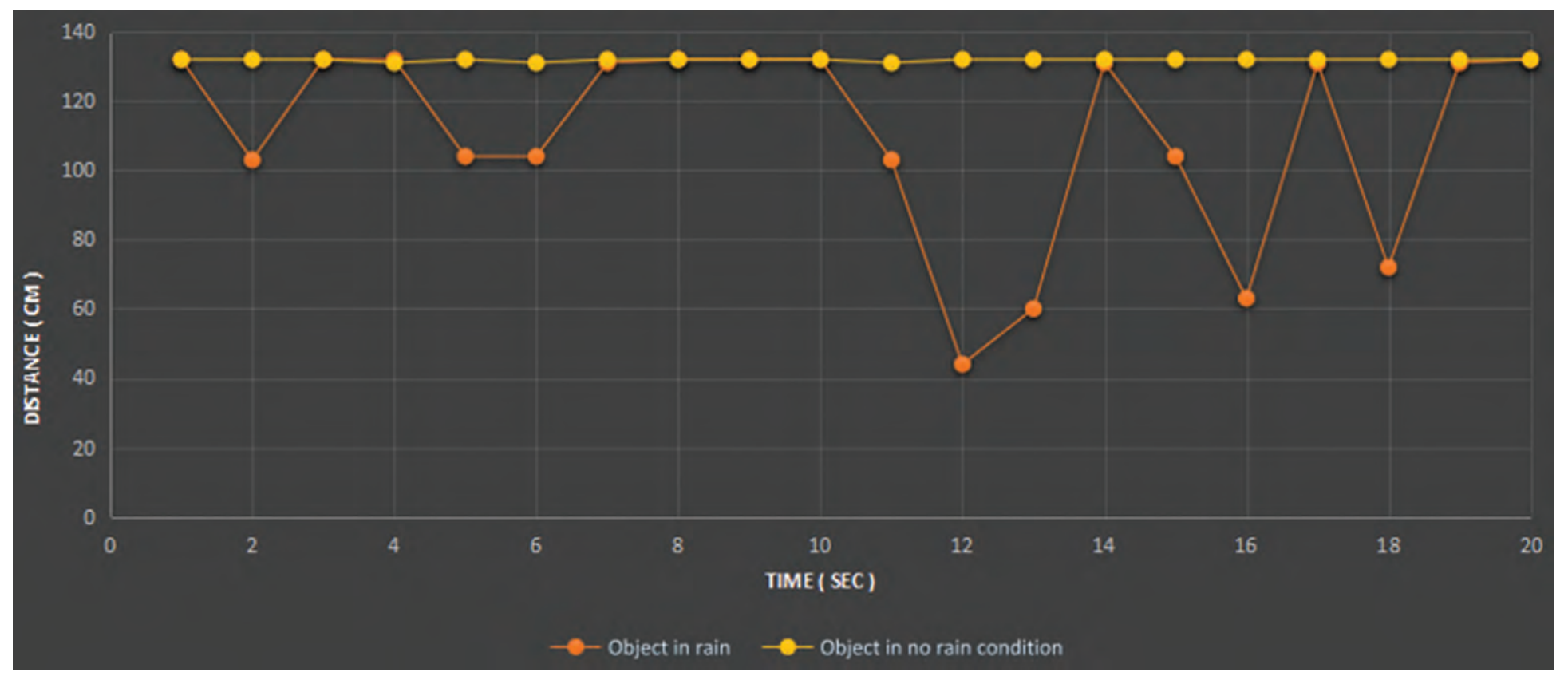

Figure 52.9 Detection of obstacle in rainy conditions. 
TIME

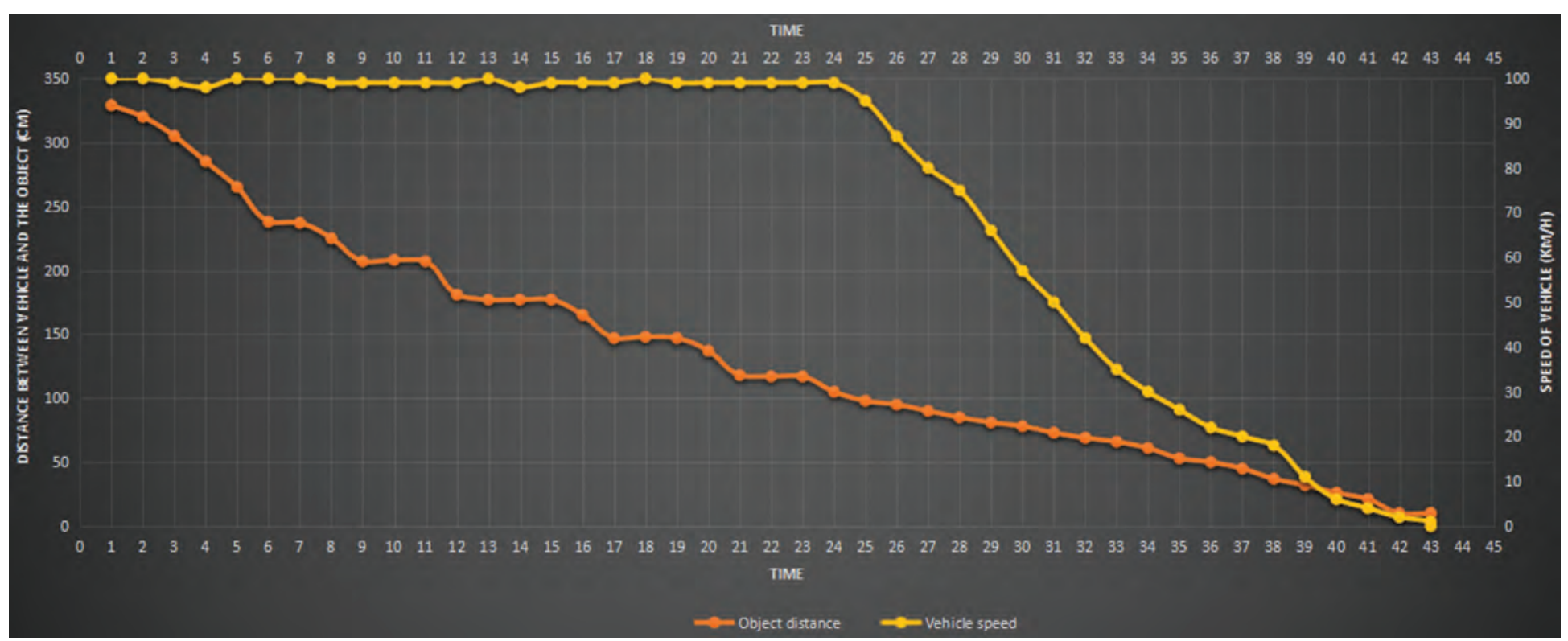

Figure 52.10 Autonomous braking to prevent collisions. 
Table 52.2 Accuracy and error in diverse weather conditions

\begin{tabular}{llrr}
\hline & Weather conditions & & \\
\cline { 2 - 4 } & Sunny day & Fog & Rain \\
\hline Distance between vehicle and obstacle $(\mathrm{cm})$ & Variable & 125 & 132 \\
Error $(\%)$ & 5 & 48 & 45 \\
Accuracy $(\%)$ & 95 & 52 & 55 \\
\hline
\end{tabular}

\section{References}

[1] "Virginia pile-up: More than 50 hurt in US motorway crash", BBC News, 2019. [Online]. Available: www.bbc.com/news/world-us-canada-50888357. [Accessed: 23-Feb-2020].

[2] "2 killed, 12 injured in vehicle pile-up due to fog in Rewari”, Hindustan Times, 2019. [Online]. Available: https://www.hindustantimes.com/cities/2-dead-12-injured-in-12-vehicle-pileup-infog-near-rewari/story-HOJAxJP1CVDP4tNJfuGx3N.html. [Accessed: 23-Feb-2020].

[3] “Multiple-vehicle collision", Wikipedia, 2020. [Online]. Available: https://en.wikipedia.org/ wiki/Multiple-vehicle_collision\#Major_pileups. [Accessed: 23-Feb-2020].

[4] Ministry of Road Transport and Highways, India, "Road Accidents in India - 2018”, 2019.

[5] "Advanced driver-assistance systems", Wikipedia, 2020. [Online]. Available: https:// en.wikipedia.org/wiki/Advanced_driver-assistance_systems. [Accessed: 23-Feb-2020].

[6] D. Weaver, "MIT researchers are tackling a major challenge for self-driving cars - fog", CNBC, 2018. [Online]. Available: https://www.cnbc.com/2018/03/31/mit-developed-imagingtechnology-that-can-see-through-fog.html. [Accessed: 23-Feb-2020].

[7] A. Mukhtar, L. Xia, and T. B. Tang, "Vehicle detection techniques for collision avoidance systems: a review", in IEEE Transactions on Intelligent Transportation Systems, vol. 16, no. 5, pp. 1-21 October 2015.

[8] "Foreward collision avoidance assist concept", Nissan Technological Development Activities, 2020. [Online]. Available: https://www.nissan-global.com/EN/TECHNOLOGY/OVERVIEW/ fcaac.html. [Accessed: 23-Feb-2020].

[9] M. Hartwig, "Overview of the main driver assistance systems I BMW.com", BMW.com, 2020. [Online]. Available: /www.bmw.com/en/innovation/the-main-driver-assistance-systems.html. [Accessed: 23-Feb-2020].

[10] Patil, Usha , Thouheed Ahmed, Syed, and Sahana. "Evaluation of dense fog to assess the road condition using backscattered veil approach", First Annual International Conference on Computers Communication and Applied Sciences, At ThinkSoft Research and Information Technologies, Bangalore, 2016.

[11] R. H. Rasshofer and K. Gresser, "Automotive radar and Lidar systems for next generation driver assistance," in Advances in Radio Science. BMW Group Research and Technology, Munich, Germany, 3 May 2005.

[12] C. Baduea, R. Guidolinia, R. V. Carneiroa, P. Azevedoa, V. B. Cardosoa, A. Forechib, L. Jesusa, R. Berriela, T. Paixao, F. Mutzc, L. Veronesea, T. Oliveira-Santosa and A. F. D. Souzaa, "Selfdriving cars: a survey," in Robotics, pp. 1-34, 2019. [Online]. Available: https://arxiv.org/pdf/ 1901.04407.pdf. [Accessed: 23-Feb-2020]. 


\title{
53 Evaluation of additive manufacturing using Marlin firmware
}

\author{
Jonnada Sai Rohit*, Nitin Kumar* \\ School of Electronics and Electrical Engineering, Lovely Professional University, \\ Jalandhar, India
}

\section{Introduction}

Additive manufacturing [2], which is also known as 3d printing [1] or rapid prototyping, is the process in which different types of objects can be made by the technique of combining materials based upon $3 \mathrm{~d}$ CAD models. They are printed in the form of layers, one upon the other. This technique has been used for prototyping purposes for many years. But still there are certain issues which need to be resolved and need to be improved. Initially the prototypes which were printed using these techniques did not offer certain demands for regular use. But today additive manufacturing is one of the most important technologies which is being considered vastly in almost every field. There are different processes in additive manufacturing which have their own and unique advantages in materials and their applications. But nowadays additive manufacturing [4] is being considered widely for the manufacture of end products with more applications. Hence due to this use in manufacturing of end products, additive manufacturing [3] has been termed rapid manufacture and rapid tooling technique.

\section{System description}

We have used Slic3r which is a $3 \mathrm{D}$ slicing engine software for 3D printing which uses rebuilding of G-code from 3D CAD files. After completing, the required G-code file is sent to the printer for the production of the 3D modelled part for the manufacturing of a physical object. About half of the 3D printers tested are considered to be supported by slic3r [5].

\section{Features:}

- Faster G-code generation.

- It uses brim for the best adhesion.

- It has micro layering feature which saves time, increase accuracy.

- Slic3r can manage multiple printers, filaments and build styles.

- It has user friendly interface and are also available from command line.

- It can also print using multiple extruders.

Figure 53.1 shows the 3D slicing software which is used in this work.

\footnotetext{
* Emails: jonnada1969@gmail.com,nitin.14652@lpu.co.in
}

DOI: $10.1201 / 9781003129103-53$ 


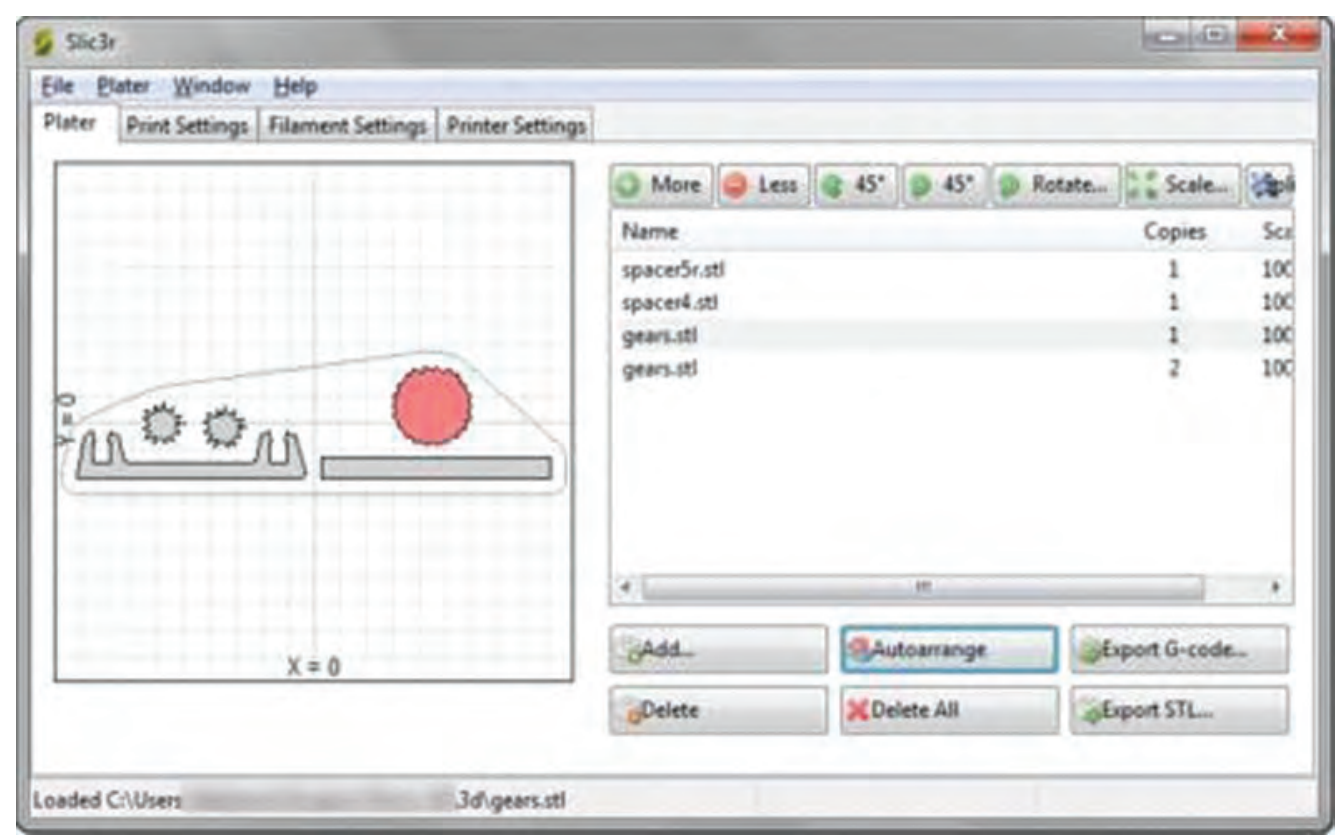

Figure 53.1 3D slicing software.

\subsection{Pronterface}

Pronterface is a graphical interface based firmware supplied with the printer. Once connected pronterface is used to control the all the three $\mathrm{X}, \mathrm{Y}, \mathrm{Z}$ axes and the extruder manually using this tool. Pronterface is also used to monitor the temperature of the nozzle and bed where the 'watch' option is selected and then the graph of real time temperature value is shown in it.

\section{Features:}

- It has a fully featured GUI host.

- It allows one to interactively control the machine.

- Pronterface can be used to slice the object directly from the host as shown in Figure 53.2.

- It also allows one to print objects from the host.

\subsection{Repetier-Host}

The Repetier-Host is a host software which helps settling position of the STL files on the simulated print bed and slice them all together as shown in Figure 53.3. The slicer is usually used or else we use the well-known Skein forge.

\section{Features:}

- The GUI of the tool is easy to use.

- It also communicates in classic ASCII mode or by using binary repetier protocol. 


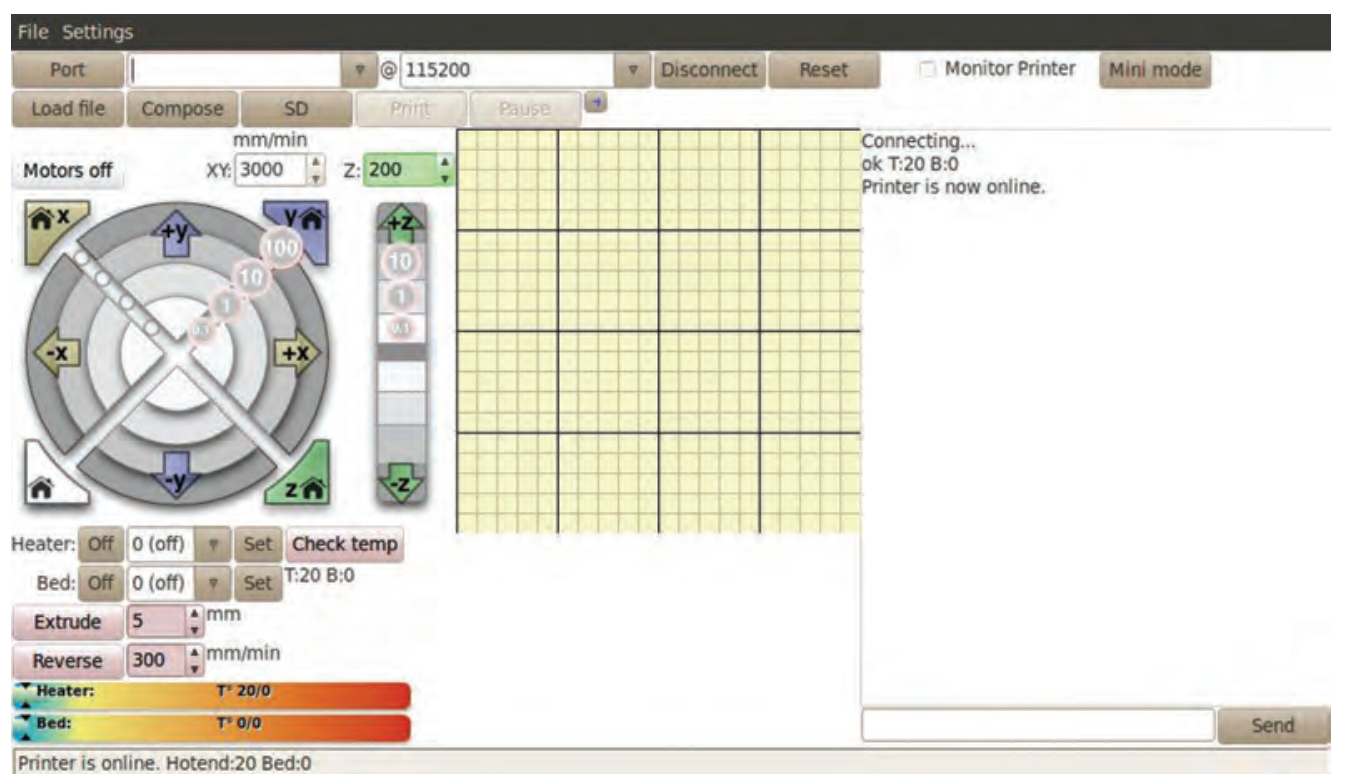

Figure 53.2 Pronterface interface.

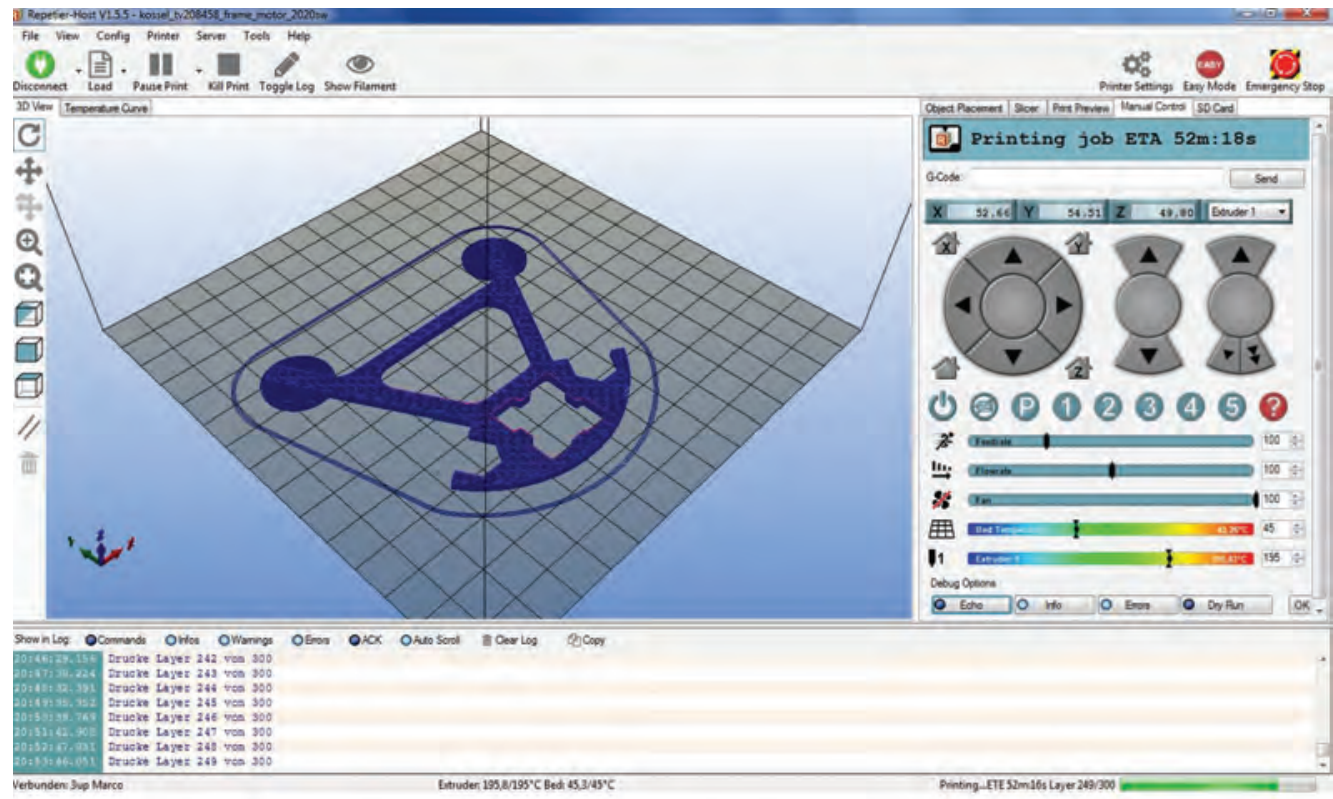

Figure 53.3 Repetier-Host software. 


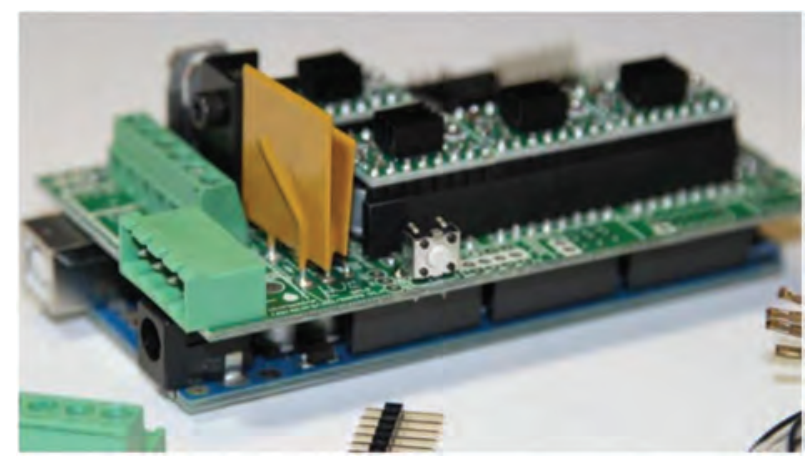

Figure 53.4 Connection with Raspberry Pi.

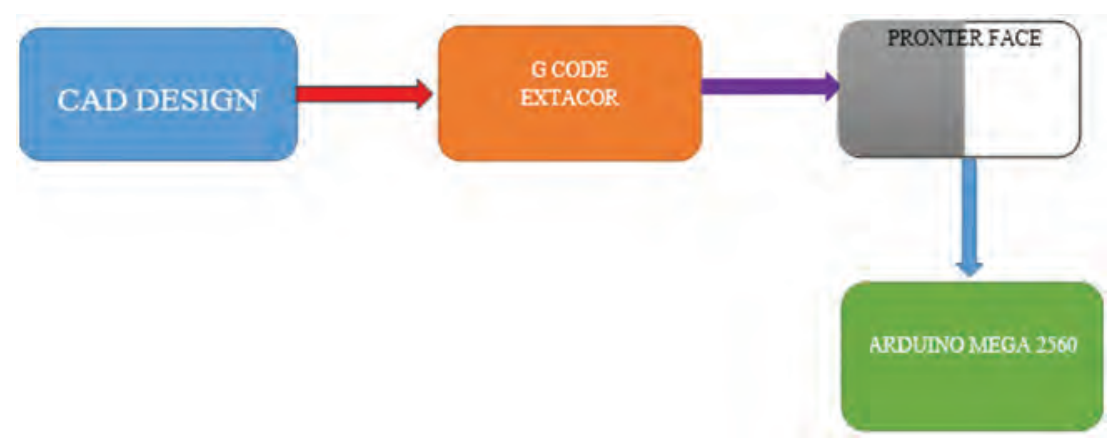

Figure 53.5 Block diagram.

- We can visually preview the G-code and can even change the code, to see the difference.

- It is used to visualize the G-code.

\subsection{Wiring and working}

The jumpers control the motor movement with precision. Figure 53.4 shows the connection with Raspberry Pi. Figure 53.5 shows the block diagram showing the working of ramps.

\section{CAD design}

For additive manufacturing we basically need a design of a model. The design can be done in many softwares like BS Solid Works, Auto CAD, Maya etc. The format of the design file should be in $.3 \mathrm{mf}$ or .stl or .obj.

\subsection{Conversion of CAD to G-code}

A software known as slic3r is used in our project that is used to extract the G-code from the CAD models which we have. 


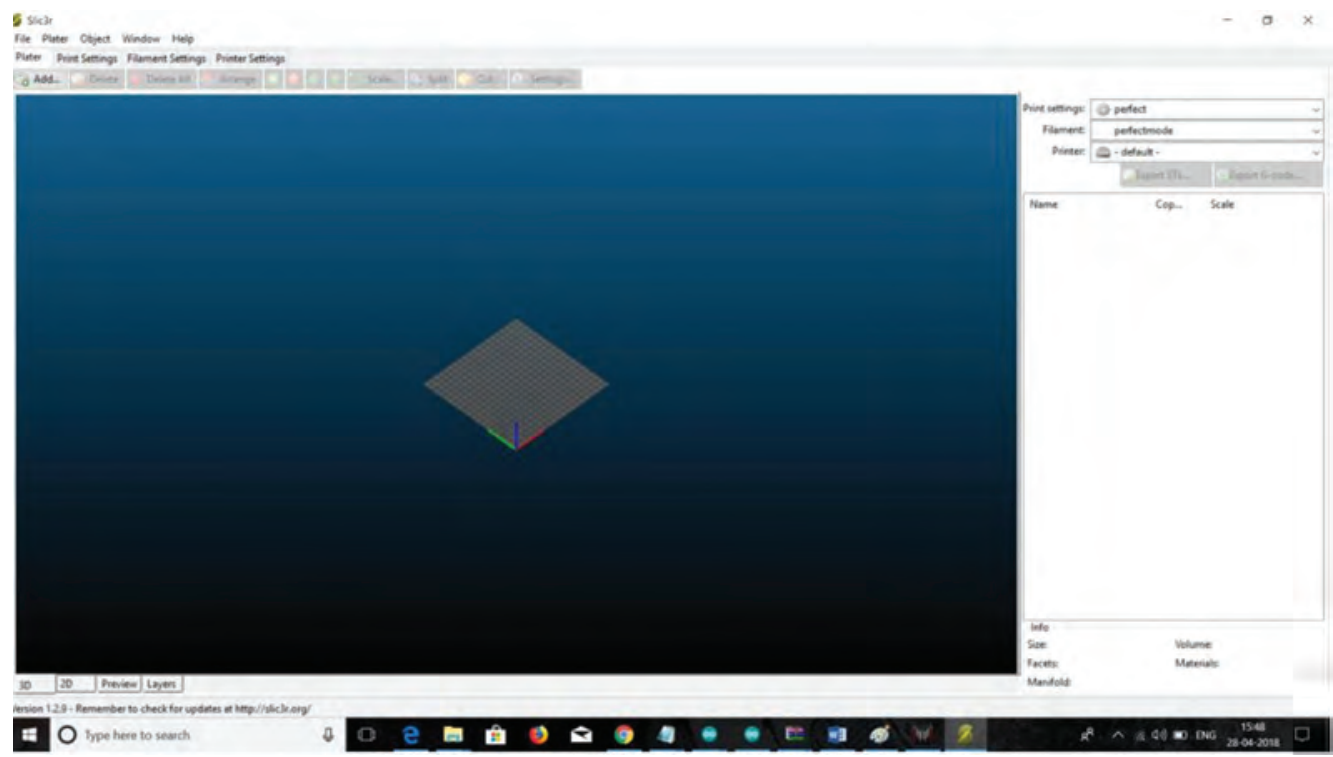

Figure 53.6 Slic3r interface (1).

From Figure 53.6, we can see the slic3r interface. To add our design file just press the Add option, which is shown in the figure, and the image directly comes onto the screen. The rotation of the CAD model can be changed by pressing the arrow marks in the interface.

From the Figures 53.7 and 53.8, we can see the interface of slic $3 r$ interface. To add our design file just press Add option which is shown in the above figure the image directly comes on to the screen. The rotation of the cad model can be changes by pressing the arrow marks in the interface.

In the print settings menu we need to set the settings as we are using $0.4 \mathrm{~mm}$ nozzle; the minimum layer height should be $0.2 \mathrm{~mm}$; the solid layers defines the completely filled layers on top and the bottom, as we can see from the figure.

Fill density is used to define the percentage required to be filled completely and the strength of the object also. They are many patterns available in slic3r.

\section{Connecting Arduino to pronterface}

Pronterface is the software which sends the G-code to Arduino. We need to load the Gcode file by pressing the Load file button from Figure 53.9. After loading the G-code file it shows the design on the screen.

By pressing the connect button it connects to Arduino; later on we need to just press the print button; it takes $7-8$ min to start printing; meanwhile it heats up the heat bed and hot end. 


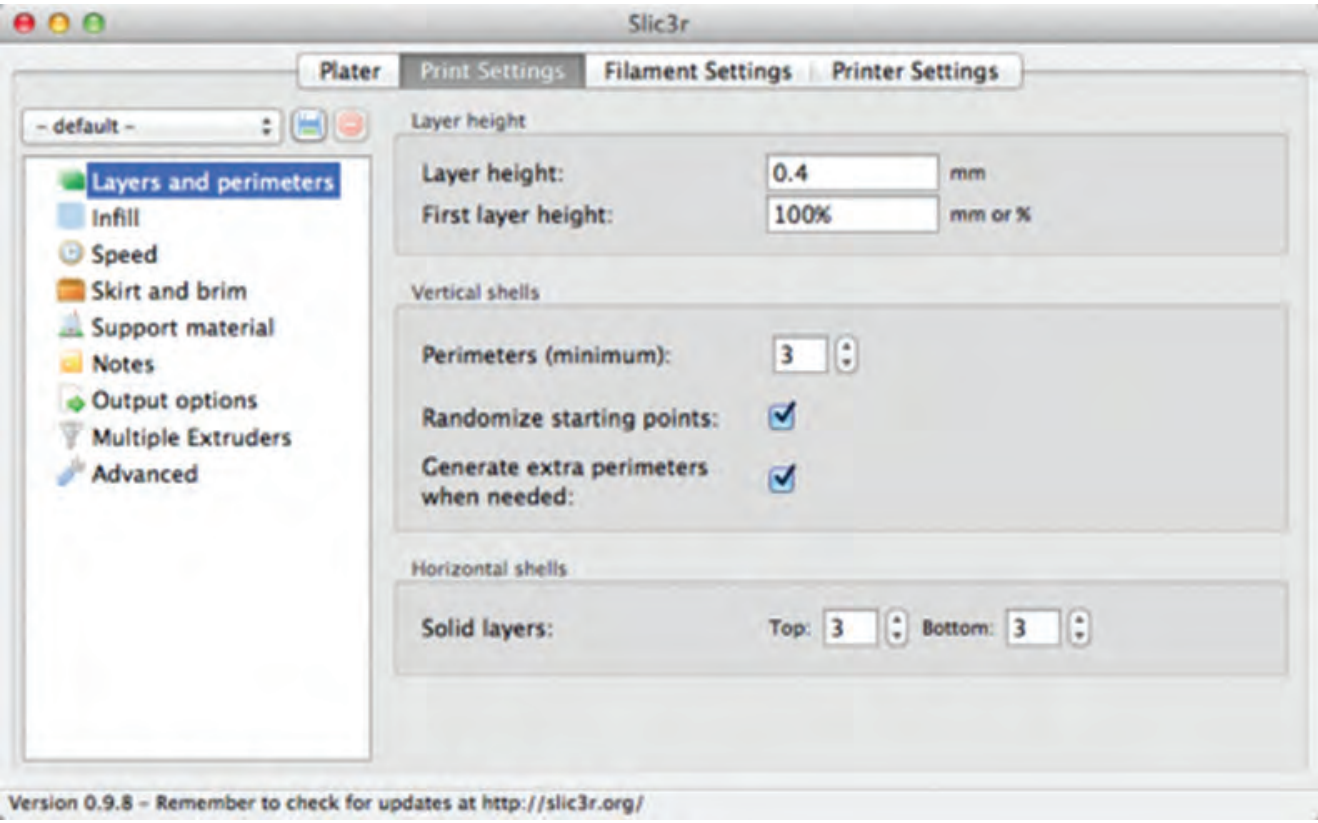

Figure 53.7 Slic3r interface (2)

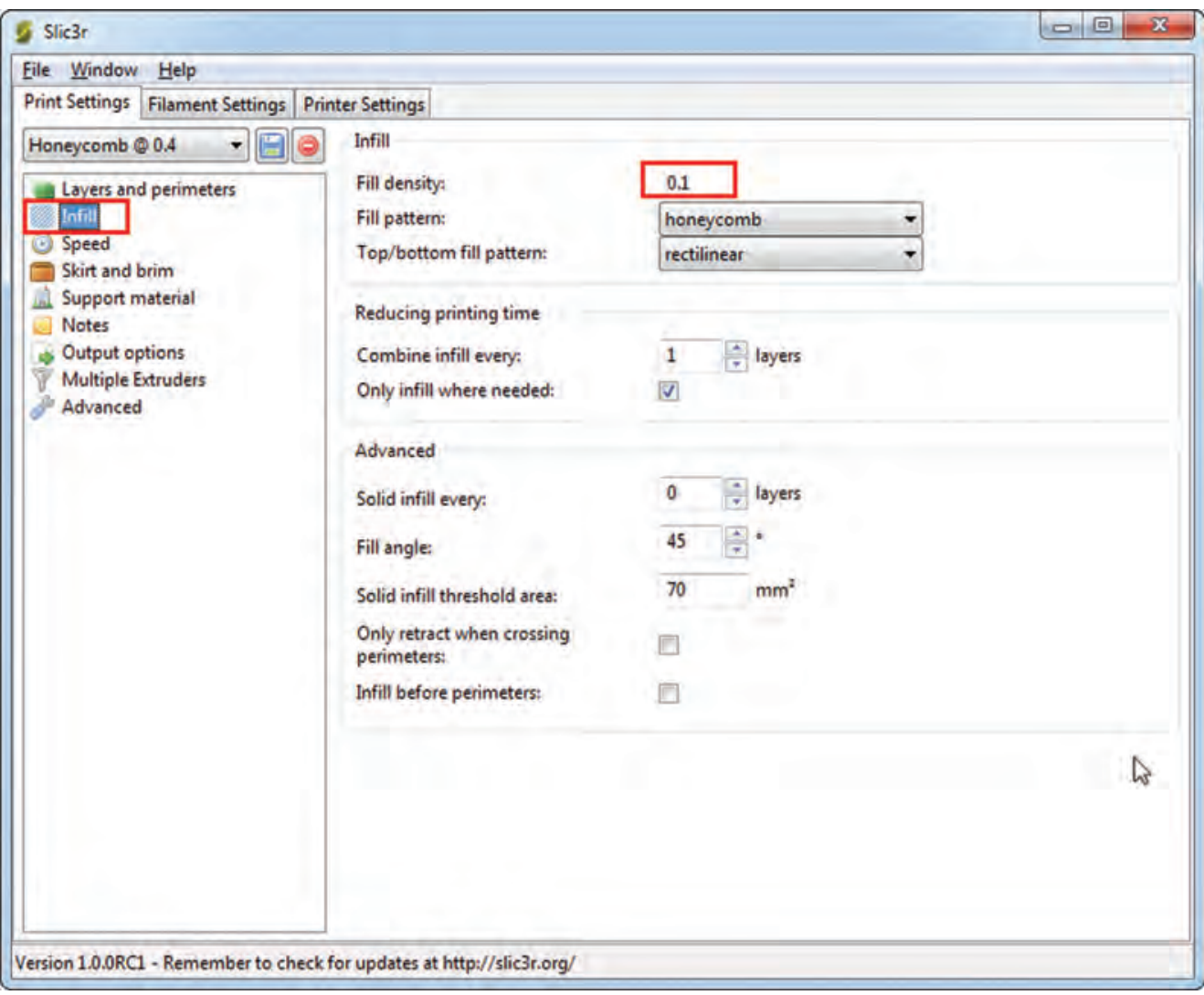

Figure 53.8 Slic3r interface (3) 


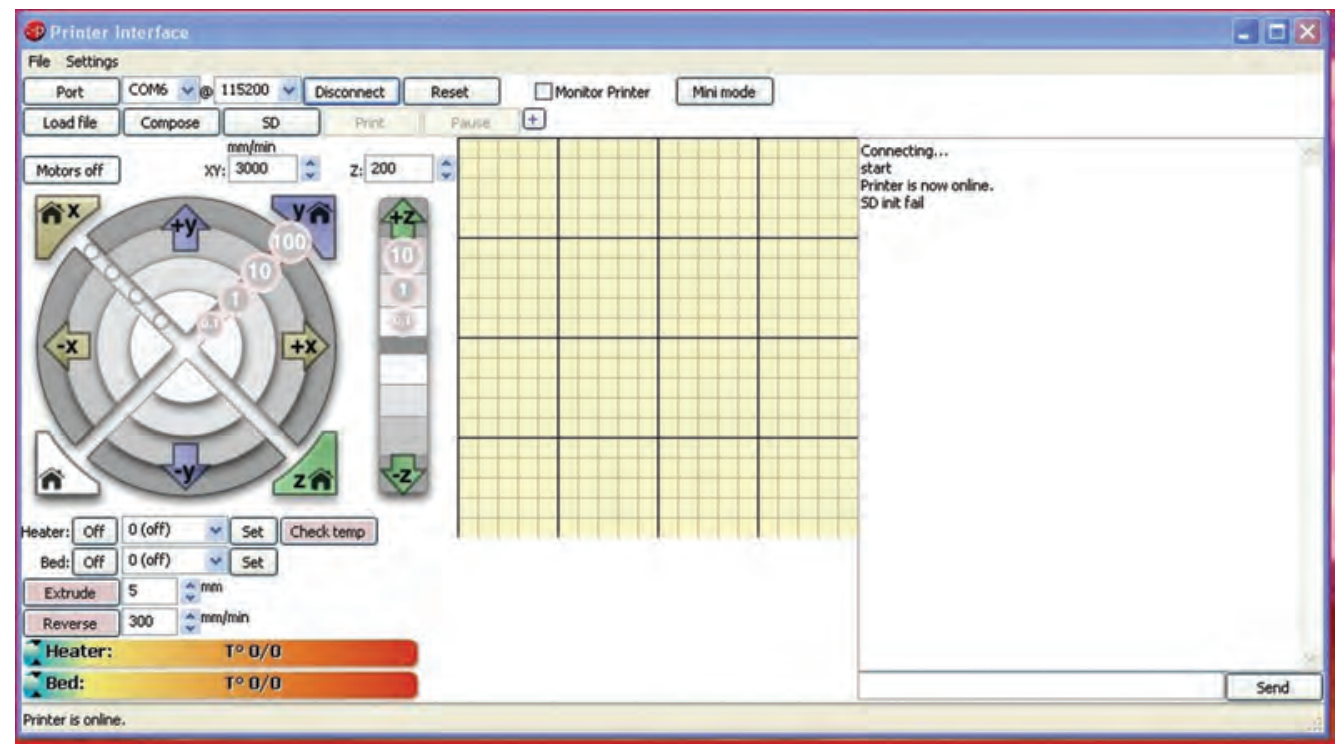

Figure 53.9 Printer interface.

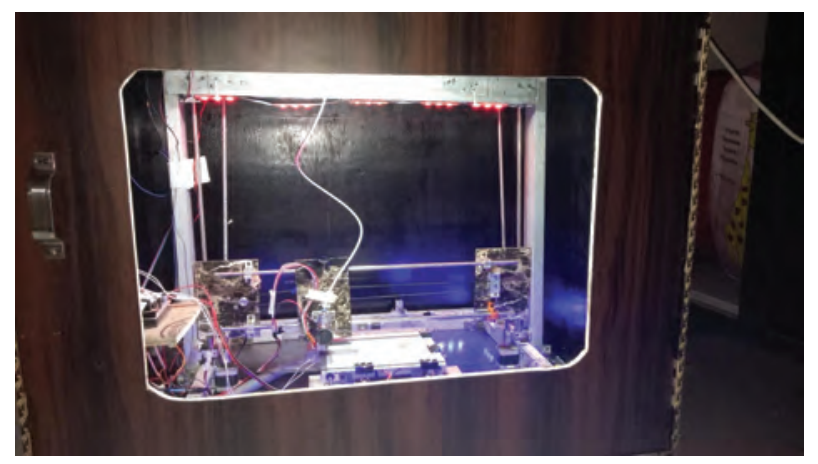

Figure 53.10 Working mode of 3D printer.

\section{RESULTS AND OUTCOMES}

\section{Project working}

Mentioned below are the few pictures of our project working and the final view of our 3D printer. Figure 53.10 shows the working model of 3D printer of the proposed work.

\section{Outputs of $3 \mathrm{D}$ printer}

Mentioned below are the few pictures of outcomes which were printed using our 3D printer. 


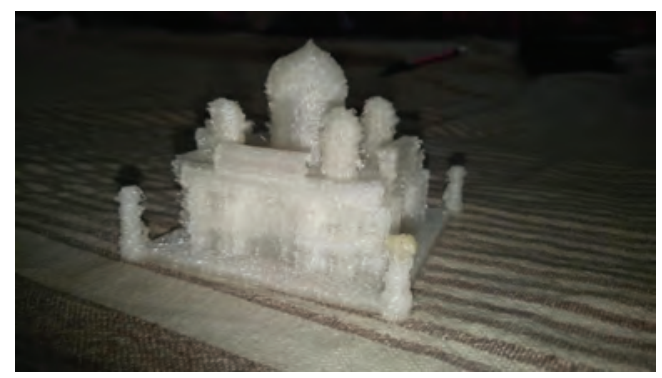

Figure 53.11 3D Taj Mahal.

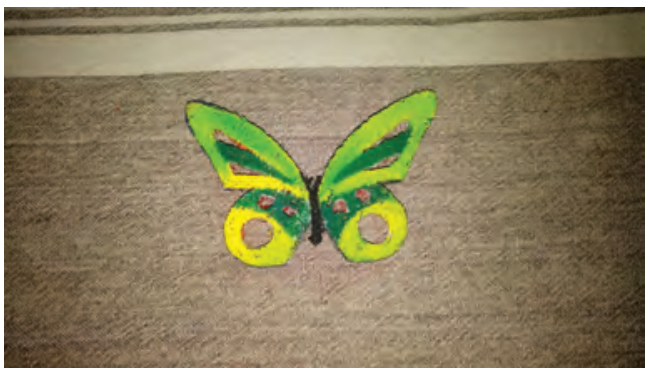

Figure 53.12 3D Butterfly.

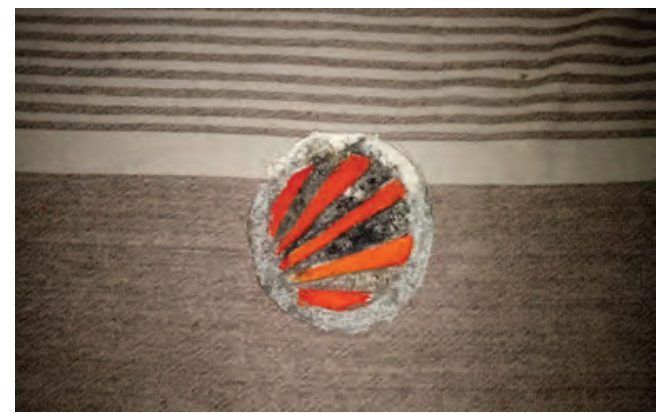

Figure 53.13 3D LPU logo.

I. 3D model of Taj Mahal printed using our 3D printer (Figure 53.11).

II. 3D model of Butterfly printed using our 3D printer (Figure 53.12).

III. 3D model of LPU logo printed using our 3D printer (Figure 53.13).

\section{Conclusion}

This system proposes an economical, easy accessible, easily maintainable 3D object printing. It uses additive manufacturing to enhance rapid manufacture by using rapid tooling technique. 


\section{References}

1. C. Bailey, S. Stoyanov, T. Tilford and G. Tourloukis, "3D-printing and electronic packaging," 2016 Pan Pacific Microelectronics Symposium (Pan Pacific), Big Island, HI, 2016, pp. 1-7.

2. K. A. Nate, J. Hester, M. Isakov, R. Bahr and M. M. Tentzeris, "A fully printed multilayer aperture-coupled patch antenna using hybrid 3D / inkjet additive manufacturing technique," 2015 European Microwave Conference (EuMC), Paris, 2015, pp. 610-613.

3. L. Hao, D. Raymond, G. Strano and S. Dadbakhsh, "Enhancing the sustainability of additive manufacturing," 5th International Conference on Responsive Manufacturing - Green Manufacturing (ICRM 2010), Ningbo, 2010, pp. 390-395.

4. S. Lüngen, T. Tiedje, K. Meier, K. Nieweglowski and K. Bock, "Reliability of 3D additive manufactured packages," 2018 7th Electronic System-Integration Technology Conference (ESTC), Dresden, 2018, pp. 1-5.

5. D. Ahlers, F. Wasserfall, N. Hendrich and J. Zhang, "3D Printing of Nonplanar Layers for Smooth Surface Generation," 2019 IEEE 15th International Conference on Automation Science and Engineering (CASE), Vancouver, BC, Canada, 2019, pp. 1737-1743. 


\title{
54 Analysis of 5G channel parameters using mmWave channel simulator NYUSIM
}

\author{
Akbil Gupta*, Meghana Tripathi*, Proddatur Lok Pavan \\ Kumar*, Alamuru Venkata Harsha Vardhan*
}

School of Electronics and Electrical Engineering, Lovely Professional University Phagwara, India

\section{Introduction}

The NYUSIM simulator utilized in the investigation performs Monte Carlo reproductions [1] that engender categorical numbers of samples of the channel impulse replications at explicit TR disseverment of separations, where the quantity of tests and scope of transmitter disseverment are inspected and determined by clients, as elucidated in the accompanying section [1]. It takes around 22 minutes to engender and spare $100 \mathrm{CIR}$ values and yield the documents. The following sections give a clear view of how parameters are undertaken in NYUSIM. The measurements of $5 \mathrm{G}$ and channel models are developed from $28 \mathrm{GHz}$ to $73 \mathrm{GHz}$.

\section{A. Information structures}

There are of 28 info parameters in the test system out of which we have used recurrence, T-R disunion, Scenario, Foliage loss, RX locations, and antenna spacing as the editable parameters. Also, we have kept some parameters fixed like RF bandwidth, TX azimuth elevation, RX azimuth elevation and TX array type which is ULA (uniform linear array).

1. Frequency: The carrier frequency in $\mathrm{GHz}$ is taken as $28 \mathrm{Ghz}, 38 \mathrm{Ghz}$ and $60 \mathrm{Ghz}$ for simulation purposes. It is an editable parameter where we can analyse TR separation vs. received power as a function of frequency. This simulator can handle a wide range of carrier frequencies up to $100 \mathrm{GHz}$ and radio frequency (RF) bandwidth up to $800 \mathrm{MHz}$. Millimetre wave spectrum is generally considered to be a spectrum band between $6 \mathrm{GHz}$ and $100 \mathrm{GHz}$, with $5 \mathrm{G}$ trials being conducted in a variety of bands within this range.

2. RF bandwidth: The bandwidth is kept fixed by default as $800 \mathrm{Mhz}$ for different scenarios: an editable parameter which may range from 0 to $800 \mathrm{MHz}$ [1]. 5G wireless technology utilizes UWB (ultra wide band) systems with a higher data transfer capacity at a low vitality level. Band width is $4000 \mathrm{Mbps}, 400$ times faster than today's wireless networks. Uses a smart antenna and a CDMA.

\footnotetext{
* Emails: akhilgupta112001@gmail.com, meghanatripathi19@gmail.com, pavankumar1324@gmail.com, harshaalamuru997@gmail.com
}

DOI: 10.1201/9781003129103-54 
Table 54.1 Type of cell used

\begin{tabular}{llc}
\hline Type of cell & Lower bound & Upper bound \\
\hline Small cell & $10 \mathrm{~m}$ & $40 \mathrm{~m}$ \\
Urban cell & $50 \mathrm{~m}$ & $450 \mathrm{~m}$ \\
\hline
\end{tabular}

Table 54.2 Tx power with changing frequency

\begin{tabular}{lll}
\hline Frequency & Indoor & Outdoor \\
\hline $28 \mathrm{GHz}$ & $23.9 \mathrm{dBm}$ & $30.1 \mathrm{dBm}$ \\
$38 \mathrm{GHz}$ & $19.1 \mathrm{dBm}$ & $21.2 \mathrm{dBm}$ \\
$60 \mathrm{GHz}$ & $19.1 \mathrm{dBm}$ & $19.1 \mathrm{dBm}$ \\
\hline
\end{tabular}

Table 54.3 Number of users considered

\begin{tabular}{lc}
\hline Type of the cell & No. of Rx locations \\
\hline Small cell outdoor & 20 \\
Small cell indoor & 20 \\
Urban cell outdoor & 100 \\
Urban cell indoor & 20 \\
\hline
\end{tabular}

3. Scenario: It is a selectable parameter taken as UMa (urban macrocell) and Umi (urban microcell) for the analysis purpose mentioned in Table 54.1.

4. Environment: A parameter that is selectable and it denotes the environment, either LOS or NLOS. The default setting is LOS.

5. Lower and upper bound of TR separation: It means the biggest separation among Tx and $\mathrm{Rx}$ in metres. In the given analysis, small cells and urban cells are considered.

Trransmitted power $(\mathrm{dBm})$ : It is an editable parameter mentioned in Table 54.2, denoting the transmission power in decibels per milliwatt; $30(\mathrm{dBm})$ is the default value and it can be set to any value ranging from 0 to $30(\mathrm{dBm})$. Table 2 shows the values depending on the indoor and outdoor scenarios as a function of the frequency.

1. No. of Rx locations: It denotes the number of users, which can be any positive integer from 1 to 10,000 as mentioned in Table 54.3.

2. Rain rate: This is the editable parameter which denotes the rain rate in millimetre/ hour used in the assessing spread of way misfortune instigated by the downpour rate. The default value is $0(\mathrm{~mm} / \mathrm{h})$, and the typical range is 0 to $150(\mathrm{~mm} / \mathrm{h})$ [2].

3. No. of transmitter and receiver antenna elements: The editable antenna elements which range from 1 to 128 .

4. Transmitter antenna and receiving antenna spacing: The editable parameter mentioned in Table 54.4, which denotes the distance between the antennas at the transmitter in a number of carrier wavelengths. The default value is 0.5 and can be set to any positive number with up to one decimal place from 0.1 to 100 . The antenna spacing varies according to the frequency. 
Table 54.4 Tx and Rx antenna spacing

\begin{tabular}{ll}
\hline Frequency & Antenna spacing \\
\hline $28 \mathrm{GHz}$ & $5.35 \mathrm{~mm}$ \\
$38 \mathrm{GHz}$ & $3.94 \mathrm{~mm}$ \\
$60 \mathrm{GHz}$ & $2.5 \mathrm{~mm}$ \\
\hline
\end{tabular}

\section{B. Output files}

\section{Received power vs. absolute propagation time}

Test repetition, position, T-R division, RMS postponement, omnidirectional strength, omnidirectional method of abuse, in which the unfortunate type (PLE) [2,4] is shown in the PDP plot. The red strong line on the PDP is the clammer edge (that is, the base of the intensity of each solvable multipath segment received), which is controlled by the transmit power, the dynamic scope $(180 \mathrm{~dB})$ of our estimation framework, and the 10 decibel signal for the noise ration. That is, 170 decibels increase the transmit power on the edge logarithmic scale [4].

\section{3-D AOA power spectrum}

Receiving antenna azimuthal angle and height HPBWs are set to 10.90 and 8.60 on both transmitter and receiver, approximately $28 \mathrm{GHz}$ to coordinate the antenna HPBWs used in $[2,3]$.

\section{Omnidirectional power delay profile}

The omnidirectional antenna pattern and the resulting omnidirectional receiving power must then be connected to several novel pointing angles, where the transmitted and received antennas are routed across a wide range of azimuth and elevation planes [5].

\section{Simulation environment}

There are two types of the environment considered, that is LOS and NLOS. The massive MIMO technique and beamforming property of mm waves ensure that strict LOS isn't a requirement. So, most of the simulation results are analysed under the NLOS and various environments include urban macrocells, urban microcells and the rural macrocells. The simulation results show a network of three-dimensional features and different power delay profiles for different mmWave bands, while considering the time-space lobbying procedure for different bands of mmWave. The latest networks are ready to use mmWave bands to amaze capital. Unused parts of the spectrum. However, due to the loss of high latency at mmWave frequencies, mmWave signal coverage can be minimized, especially for non-line-of-visual umph, where mmWave signals cross obstacles. But it looks tight. In this work, simulations and results are mostly carried out for the NLOS environment [6]. 


\section{Intelligent Circuits and Systems}

Table 54.5 Fixed input parameters

\begin{tabular}{ll}
\hline Fixed parameter & Values \\
\hline Carrier frequency & $28 \mathrm{GHz}$ \\
Tx antenna spacing & $5.35 \mathrm{~mm}$ \\
Rx antenna spacing & $5.35 \mathrm{~mm}$ \\
Scenario & $\mathrm{UMa}(\mathrm{urban}$ macrocell) \\
RF bandwidth & $800 \mathrm{MHz}$ \\
Base station height & $35 \mathrm{~m}$ \\
Environment & $\mathrm{NLOS}$ \\
Rain rate & $20 \mathrm{~mm} / \mathrm{h}$ \\
Foliage attenuation & $0.4 \mathrm{~dB} / \mathrm{m}$ \\
\hline
\end{tabular}

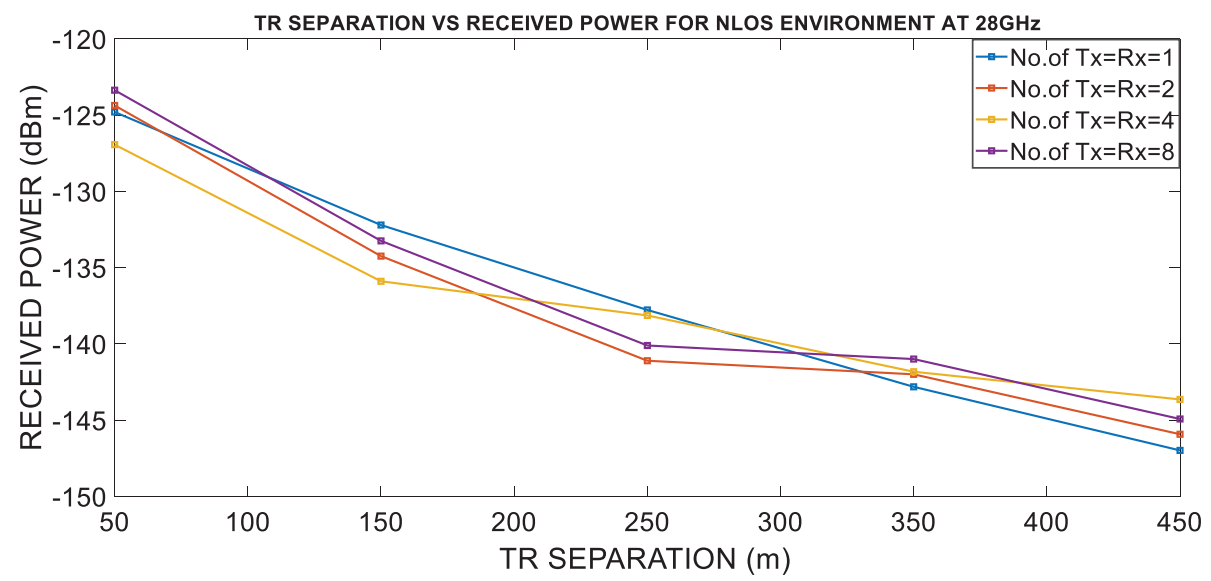

Figure 54.1 TR separation vs. received power as number of antenna elements.

Table 54.6 Output values after the multiple runs

\begin{tabular}{llll}
\hline $\begin{array}{l}\text { TR separation in } \\
\text { metres }(m)\end{array}$ & $\begin{array}{l}\text { Received power } \\
(d B m)\end{array}$ & Path loss $(d B)$ & RMS delay $(n s)$ \\
\hline 50 & -126.93 & 135.13 & 38.67 \\
150 & -135.89 & 148.87 & 45.07 \\
250 & -138.13 & 151.54 & 33.35 \\
350 & -141.82 & 155.51 & 35.15 \\
450 & -143.637 & 157.48 & 38.26 \\
\hline
\end{tabular}

\section{A. Urban cell indoor scenario}

The graphical analysis is done under the input parameter mentioned in Table 54.5, as TR separation.

The results mentioned in Figure 54.1 and Figure 54.2 analyse the effect on the received power and path loss by increasing the TR separation. The results are also mentioned in Table 54.6. 


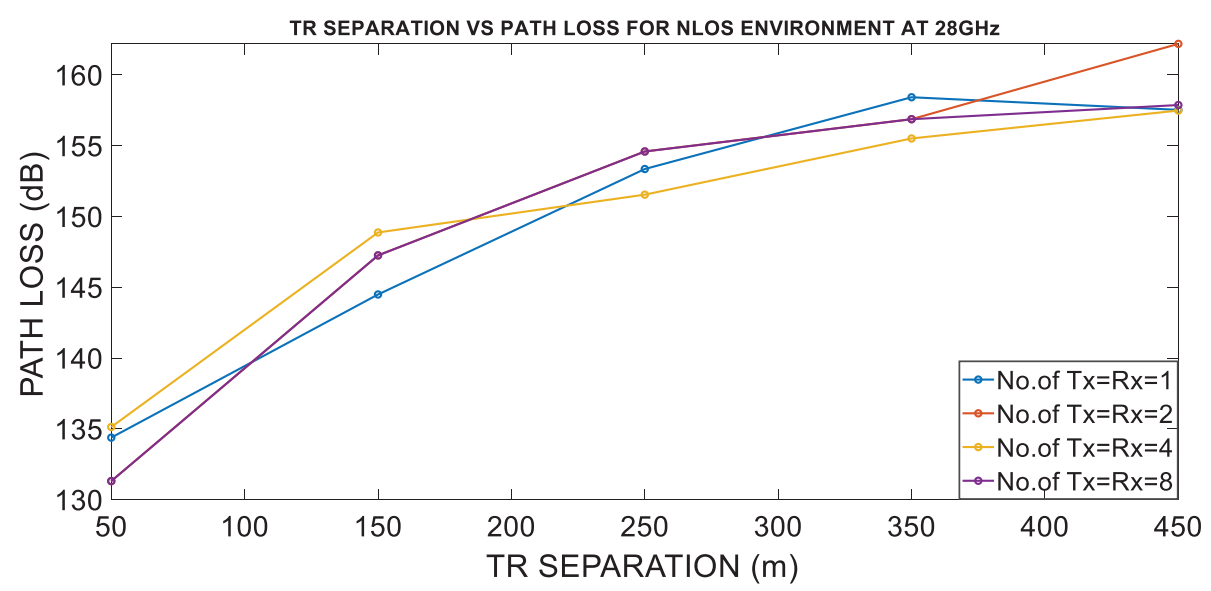

Figure 54.2 TR separation vs. path loss as a function of the number of antenna elements.

\section{Channel model supported by NYUSIM}

The separation of free space connection and path loss model around your anchor point, with additional attenuation of the term due to various weather factors [7], is utilized in NYUSIM, which is communicated as $[3,8,9]$ :

$$
P L^{C I}(f, d)[d B]=F S P L(f, 1 m)[d B]+10 n \log _{10}(d)+A T[d B]+\chi_{\sigma}^{C I} \text { where } d \geq 1 m
$$

If $\mathrm{f}$ is a carrier frequency in $\mathrm{GHz}, \mathrm{d}$ is a three-dimensional transmitter separator, $\mathrm{n}$ is a path loss exponent (PLE), AT is a climate-induced attenuation term, and CI is a Jeromemon Gaussian random variable with standard deviation in $\mathrm{dB}$, and FSPL (F; $1 \mathrm{~m}$ ) is the free space path loss at $1 \mathrm{~m}$ drop at the carrier frequency in the TR partition [9].

$$
\operatorname{FSPL}(f, 1 m)[d B]=20 \log _{10}\left(\frac{4 \pi f \times 10^{9}}{c}\right)=32.1[d B]+20 \log _{10}(f)
$$

where $\mathrm{c}$ is the speed of light, and $\mathrm{f}$ is in GHz. The term AT is classified by [7]:

$$
A T[d B]=\alpha\left[\frac{d B}{m}\right] \times d[m]
$$

Attenuation factor $\mathrm{d}$ in $\mathrm{dBm}$ for the oscillation frequency range of $1 \mathrm{GHz}$ to $100 \mathrm{GHz}$, which has the total attenuation effect of dry air, dihydrogen monoxide vapour, rain and fog [7], is the 3DT-R decomposition distance.

The analysis is done in different weather conditions for the understanding of the changing parameters. 


\section{The new version: human blockage and spatial consistency}

Spatial consistency: It is the realistic and continuous channel evolution implemented using NYUSIM version 2.01. In the local area, the implementation is done along the user terminal (UT) trajectory. Large-scale parameters with spatial correlation are generated, such as shadow attenuation, (LOS) / (NLOS) state, to achieve spatial stability.

Human blockage: Human blockage can be simulated as the shadow loss effect due to a person close to the mobile phone and shadowing loss about several hundreds of milliseconds.

\section{Conclusion}

From the use of the software, it was found that NYU wireless was used to run simulations from 2012 to 2017 and collected 1 terabyte of data [1]. The number of wireless links between deployed sensors is rapidly increasing and provides traditional features to deal with. The main objectives of $5 \mathrm{G}$ research and development are to provide faster Internet speeds at a lower cost, reduce battery drainage, lower latency and improve security and connectivity. For the larger community, a proper data set is required to elaborate on the wireless connections and channels in a different environment. The speed it is said to serve is hard to get and we need a good level of infrastructure for 5G technology deployment. We aim to use our analysis in further optimizing the parameters of the wireless channel models. The actual rendering of channel response in different scenarios has resulted in visualizing the effects in urban microcell as well as urban macrocell scenarios. Through this analysis, a broad student community aims towards learning of the support of realistic channel modelling [12]. The technology of 5G is said to be a dramatic change. Longer bandwidth and low dormant times will allow for the development of new services and the improvement of existing ones.

\section{References}

[1] Version 1.5 Copyright 2017 New York University and NYU WIRELESS User Manual.

[2] T. S. Rappaport, S. Sun, R. Mayzus, H. Zhao, Y. Azar, K. Wang, G. N. Wong, J. K. Schulz, M. Samimi, and F. Gutierrez, "Millimeter-wave mobile communications for $5 \mathrm{G}$ cellular: It will work!”, IEEE Access, vol. 1, pp. 335-349, 2013.

[3] S. Sun, G. R. MacCartney and T. S. Rappaport, "A novel millimeter-wave channel simulator and applications for 5G wireless communications," 2017 IEEE International Conference on Communications (ICC), Paris, 2017, pp. 1-7.

[4] T. S. Rappaport, G. R. MacCartney, Jr., M. K. Samimi, and S. Sun, "Wideband millimeter-wave propagation measurements and channel models for future wireless communication system design (Invited Paper)", IEEE Transactions on Communications, vol. 63, no. 9, pp. 30293056, 2015.

[5] S. Sun, G. R. MacCartney, M. K. Samimi and T. S. Rappaport, "Synthesizing omni directional antenna patterns, received power and path loss from directional antennas for 5G millimeterwave communications," 2015 IEEE Global Communications Conference (GLOBECOM), San Diego, CA, 2015, pp. 1-7

[6] Khawaja, W., Ozdemir, O., Yapici, Y., Erden, F., Ezuma, M., and Guvenc, I. (2019). Coverage enhancement for NLOS mmWave links using passive reflectors. arXiv preprint arXiv:1905.04794.

[7] H. J. Liebe, G. A. Hufford and M. G. Cotton, "Propagation modelling of moist air and suspended water/ice particles at frequencies below $1000 \mathrm{GHz}$ ”, AGARD Conference Proceedings, $542,1993$. 
[8] S. Sun et al., "Investigation of prediction accuracy, sensitivity, and parameter stability of largescale propagation path loss models for $5 \mathrm{G}$ wireless communications", IEEE Transactions on Vehicular Technology, vol. 65, no. 5, pp. 2843-2860, 2016.

[9] 3GPP, "Study on channel model for frequency spectrum above $6 \mathrm{GHz}$ ", 3rd Generation Partnership Project (3GPP), TR 38.900 V14.2.0, 2016.

[10] S. Ju, O. Kanhere, Y. Xing and T. S. Rappaport, "A millimeter-wave channel simulator NYUSIM with spatial consistency and human blockage," 2019 IEEE Global Communications Conference (GLOBECOM), Hawaii, USA, Dec. 2019, pp. 1-6.

[11] S. R. Chopra, A. Gupta and H. Monga, "An LTE approach with MIMO by using suboptimal selection of antenna," 2018 International Conference on Intelligent Circuits and Systems (ICICS), Phagwara, pp. 167-172, 2018.

[12] R. Kausar, A. Gupta, I. B. Sofi and K. Arora, "Bit error rate based performance evaluation of LTE OFDMA system," 2018 International Conference on Intelligent Circuits and Systems (ICICS), Phagwara, pp. 161-166, 2018.

[13] A. Gupta, S. Dogra and I. B. Sofi, "Performance evaluation of spatial multiplexing using different modulation techniques in MIMO system for small and large scale fading channel", International Journal of Sensors Wireless Communications and Control, vol. 9(2), pp. 188$202,2019$.

[14] S. R. Chopra, A. Gupta and H. Monga, "Performance analysis of space time trellis codes in Rayleigh fading channel”, In Harmony Search and Nature Inspired Optimization Algorithms, Springer, Singapore, pp. 957-967, 2019.

[15] A. Gupta and S. R. Chopra, "Designing and testing of quadrifilar helix antenna for NOAA weather satellite," 2019 6th International Conference on Signal Processing and Integrated Networks (SPIN), Noida, India, pp. 785-790, 2019.

[16] R. Devi, R. K. Jha, A. Gupta, S. Jain and P. Kumar, "Implementation of an intrusion detection system using an adaptive neuro-fuzzy inference system for $5 \mathrm{G}$ wireless communication network", AEU-International Journal of Electronics and Communications, vol. 74, pp. 94$106,2017$.

[17] A. Gupta and R. K. Jha, "Security threats of wireless networks: A survey," International Conference on Computing, Communication \& Automation, Noida, pp. 389-395, 2015.

[18] A. Gupta and R.K. Jha, "Power optimization using massive MIMO and small cells approach in different deployment scenarios”, Wireless Networks, vol. 23(3), pp. 959-973, 2017. 


\title{
55 Raspberry Pi-based smart waste management system using Internet of Things
}

\author{
Shaik Vaseem Akram*, Rajesh Singh*, Anita Geblot* \\ Lovely Professional University, Phagwara, Punjab, India
}

\section{Introduction}

Waste management is one of the most primary problems that the world is facing the irrespective of the case of developing or developed countries. The primary problem in waste management is that waste bins are full in public places before the next cleaning process begins. Every time labourers must visit the garbage bins in the city area to check whether they are filled or not. If garbage bins are not filled, then the human resources invested goes in vain. To overcome and enhance the waste management system many researches proposed different kind of mechanism and framework. C.L. Popa et al. (2017) developed a distinct kind of module for managing the different type of garbage automatically and developed azure IoT reference model for waste management [1]. Rybnytska et al. (2018) implemented the decision support system for optimal routing path for the trucks for garbage collection and reducing the emission of $\mathrm{CO}_{2}$ while transporting waste from garbage bins to the incinerator. JSPRIT open source Java platform was implemented for the vehicle routing problem [2]. Misra et al. (2018) proposed a system that is used for checking the level of the waste in bin and pungent gas too. Things speak server, Arduino based micro controller, ultrasonic sensor (HC SR04) and gas sensors are used in this study [3]. Maksimovic's (2018) green Internet of Things (IoT) platform proposes waste management for reducing the impact of waste and reduce the emission of greenhouse gas from waste [4]. Chu et al. (2018) proposed a multilayer deep learning system (MDLS) model which is used to classify the waste disposed in the waste bin in public area by using Alex net convolutional neural network (CNN) algorithm. When compared to the CNN model the accuracy rate of MDLS in both tests is more than 90\% [5]. Memon et al. (2019) Internet of Things (IoT) based waste management suggests for real-time monitoring of bins with the assistance of ultrasonic sensors and WeMos [6]. In Chaudhary et al. (2019), to enhance the efficiency of waste collection and transportation, a GIS (geographic information system) based waste management model is proposed [7]. Yerraboina et al. (2018) designed a smart garbage bin for real-time monitoring of garbage bins with the assistance of the Internet of Things (IoT) [8]. Bevish Jinila et al. (2019), to reduce the problem of the overflow of garbage from the bins, proposed a smart container with cloud server capability [9]. Using the advantage of a Raspberry Pi controller and moistures sensor we are proposing an architecture for a waste segregation system by leveraging the cloud server and Raspberry Pi controller. In this study we are proposing a system for segregating the waste at the initial point without human intervention.

\footnotetext{
*Emails: vaseemakram5491@gmail.com, srajssssece@gmail.com, eranita5@gmail.com
}

DOI: 10.1201/9781003129103-55 


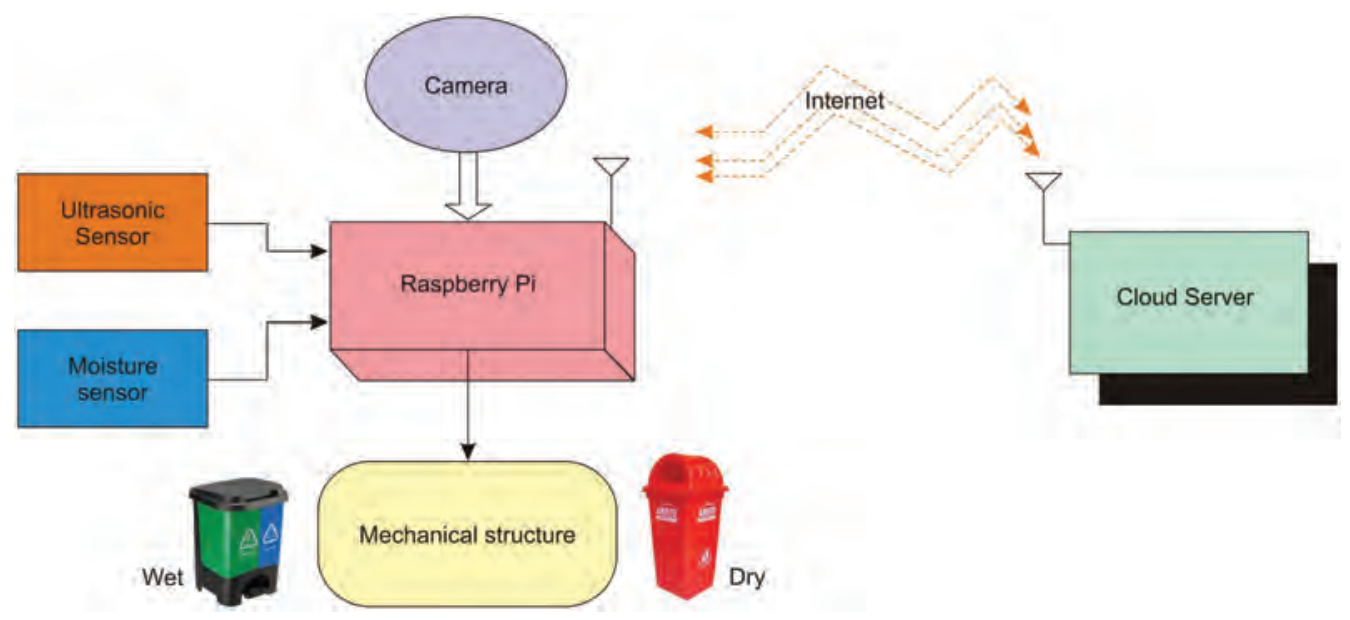

Figure 55.1 Architecture of waste segregation system.

\section{Proposed architecture}

Waste segregation is a primary activity for segregating the waste for reducing the extra burden for municipal authority for performing the activity. In this study, an automatic segregation system is proposed for enhancing the existing segregation system is shown in Figure 55.1. The system consists of a Raspberry Pi controller, Arduino uno, moisture sensor, pi camera, ultrasonic sensor and cloud server. In this analog output moisture sensor is connected to A1 of Arduino board and ultrasonic sensor pins trigger and echo pins are connected to 20 and 21 pins of Raspberry Pi. Camera is connected to Pi to take the picture of dust bins when Pi gives command to it and it is connected to the USB port of Pi as shown in Figure 55.2. When waste is brought to dispose of in the bins, the moisture sensor senses the moisture and confirms whether the waste is dry or wet. If the waste is a wet one, then the bin which is allocated for wet waste opens the lid for disposal of the waste. Simultaneously, the Pi camera captures the visuals of the disposal waste so that it adds an additional feature for real time monitoring. As Raspberry Pi controller is integrated with inbuilt Wi-Fi adapter, which enables it to transmit the data like visuals and sensory data of the bin to cloud server via Internet. As data logs into the cloud server, it enhances the user for real time monitoring of the bins through Internet from any location.

\section{Hardware implementation}

\section{Raspberry Pi 3 controller}

Raspberry Pi 3 controller is a system on chip (SoC) device which comes with a Broadcom BCM2837 64bit quad core processor and it is built in with 40 pins. Of 40 pins, 26 GPIO (general purpose input/output) pins are for controlling the physical devices using physical computing as shown in the Figure 55.2. This controller has in built 802.11n Wi-Fi capability for transmitting the data through Internet and controller is powered through USB port with a voltage of $5 \mathrm{~V}$. With the assistance of HDMI port, the Raspberry Pi interfaced to the computer and the USB ports are usefully connecting the camera. 


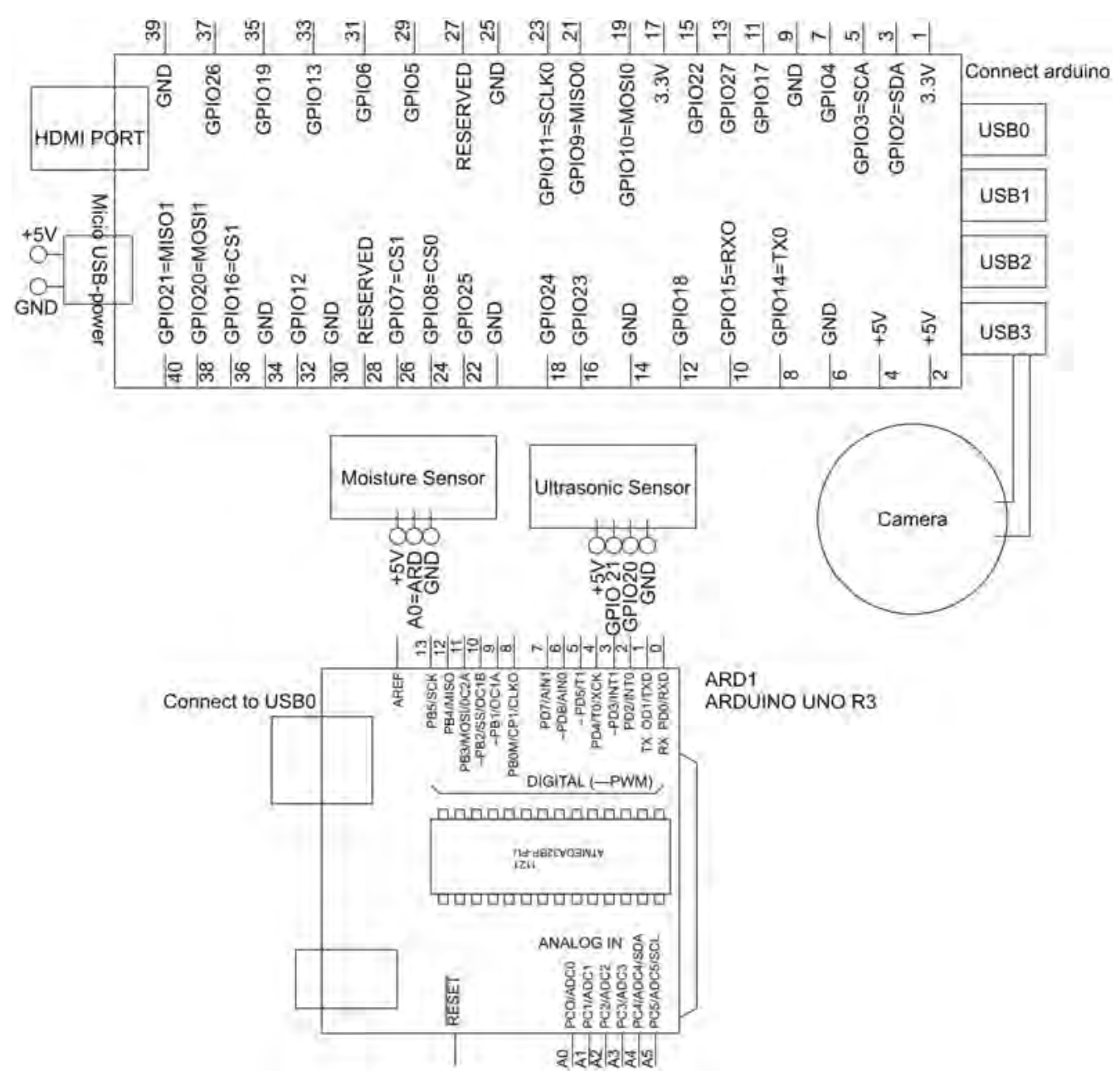

Figure 55.2 Circuit diagram of waste segregation system.

\section{Ultrasonic sensor}

Ultrasonic sensor works based on ultrasonic waves. Generally, this sensor is used to measure the distance to an object. This sensor estimates the distance to an object by measuring the time taken between transmitted and received ultrasonic pulses. Ultrasonic sensor is capable of measuring the distance range of $2 \mathrm{~cm}$ to $400 \mathrm{~cm}$.

\section{Moisture sensor}

Moisture sensor is useful measuring the volumetric content of water in the soil. It uses the capacitance concept to calculate surrounding medium dielectric permittivity. In this case the dielectric permittivity is a function of the water content. The sensor generates a voltage that is proportional to the dielectric permittivity, and hence the soil water content. Compared with dry waste, the water content of a wet waste material will be more. Moisture sensor is three pin module and they are analog pin (A0), Vcc and GND. 


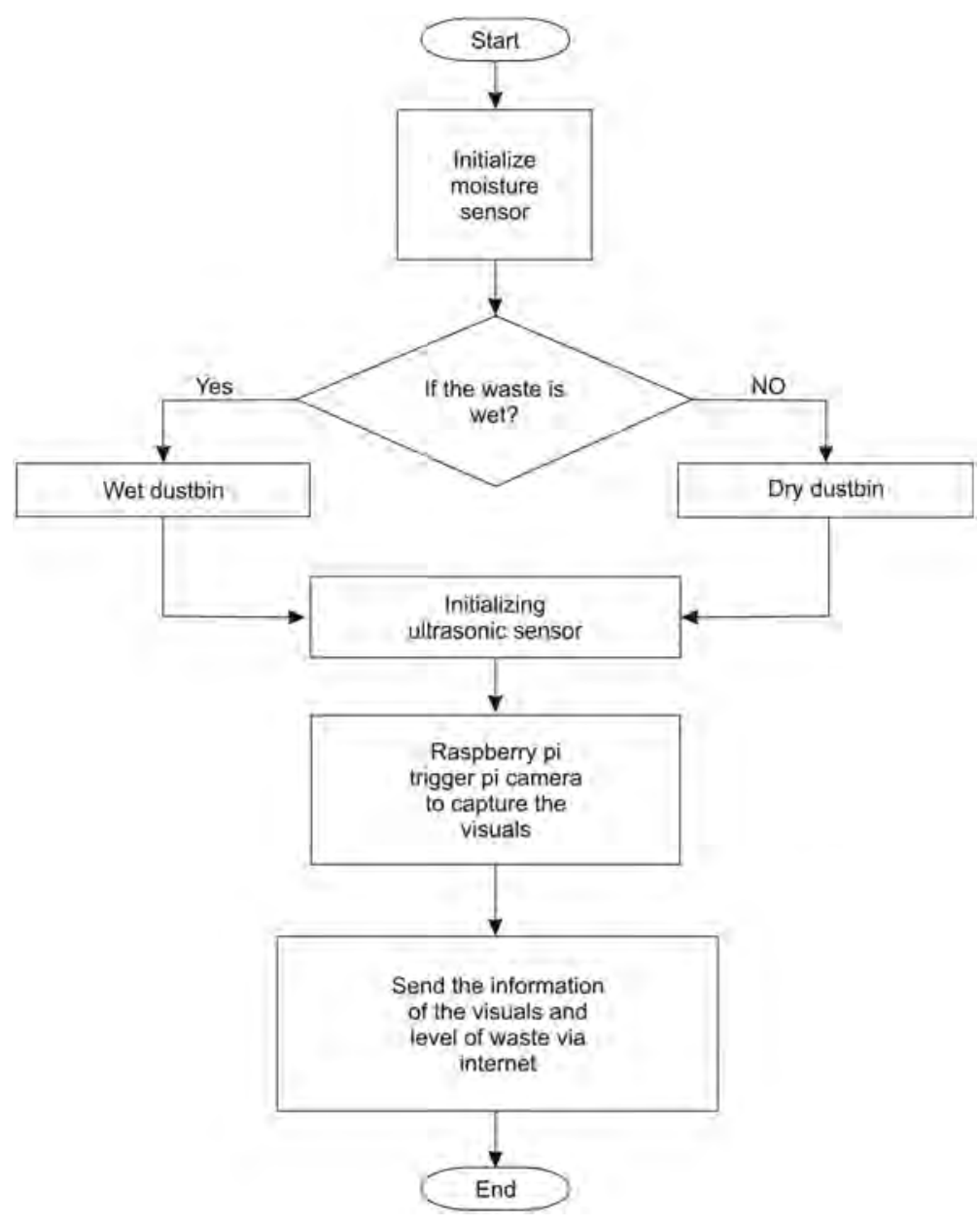

Figure 55.3 Flowchart of proposed architecture.

\section{Flowchart}

Figure 55.3 shows the flowchart of the waste management system. When the waste is placed on the sheet moisture sensor sense, whether it is wet or dry, it will send to the Arduino, which is connected to the Raspberry Pi. If it is wet, waste sends to the wet bin, and if it is dry, it sends it to another dry bin. Amount of space left in the bins is given by the ultrasonic sensor. As Raspberry Pi controller is integrated with inbuilt Wi-Fi adapter, it enables it to transmit the data regarding the bin to cloud server via the Internet.

\section{Conclusion}

Waste segregation system is the challenging area in the waste management. Due to the rise in the population, the amount of waste generation is also increasing with the same rate. To enhance the waste management system, the segregation of waste needs to process at the disposal point. To make it possible we are proposing a Raspberry Pi-based waste 


\section{2}

Intelligent Circuits and Systems

segregation system with the assistance of cloud server. The moisture sensor triggers the bin through controller to open lid by analysing which kind of the waste they are disposing of and the camera which is integrated with the controller captures the image during the disposal and communicates to the cloud server for real-time monitoring.

\section{References}

1. Popa, Cicerone Laurentiu, George Carutasu, Costel Emil Cotet, Nicoleta Luminita Carutasu, and Tiberiu Dobrescu. "Smart city platform development for an automated waste collection system." Sustainability 9, no. 11 (2017): 2064.

2. Rybnytska, Olga, Frada Burstein, Andrei V. Rybin, and Arkady Zaslavsky. "Decision support for optimizing waste management.” Journal of Decision Systems 27, no. sup1 (2018): 68-78.

3. Maksimovic, Mirjana. "Leveraging internet of things to revolutionize waste management." International Journal of Agricultural and Environmental Information Systems (IJAEIS) 9, no. 4 (2018): 1-13.

4. Chu, Yinghao, Chen Huang, Xiaodan Xie, Bohai Tan, Shyam Kamal, and Xiaogang Xiong. "Multilayer hybrid deep-learning method for waste classification and recycling." Computational Intelligence and Neuroscience 2018 (2018).

5. Misra, Debajyoti, Gautam Das, Triankur Chakrabortty, and Debaprasad Das. "An IoTbased waste management system monitored by cloud." Journal of Material Cycles and Waste Management 20, no. 3 (2018): 1574-1582.

6. Memon, Saadia Kulsoom, Faisal Karim Shaikh, Naeem Ahmed Mahoto, and Abdul Aziz Memon. "IoT based smart garbage monitoring \& collection system using WeMos \& ultrasonic sensors." In 2019 2nd International Conference on Computing, Mathematics and Engineering Technologies (iCoMET), pp. 1-6. IEEE, 2019.

7. Chaudhary, Shailendra, Chaitanya Nidhi, and Nek Ram Rawal. "GIS-Based model for optimal collection and transportation system for solid waste in Allahabad City." In Emerging Technologies in Data Mining and Information Security, pp. 45-65. Springer, Singapore, 2019.

8. Yerraboina, Sirisha, Nallapaneni Manoj Kumar, K. S. Parimala, and N. Aruna Jyothi. "Monitoring the smart garbage bin filling status: an IoT application towards waste management.” Int J Civ Eng Technol 9, no. 6 (2018): 373-381.

9. Jinila, Y. Bevish, Shahzad Alam, and Prabhu Dayal Singh. "Cloud-based scheme for household garbage collection in urban areas." In Advances in Big Data and Cloud Computing, pp. 539546. Springer, Singapore, 2019. 


\title{
56 Comparative performance analysis of service discovery protocol and interference management schemes for device to device communication in $5 \mathrm{G}$ network
}

\author{
Koushik Barman*, Ajay Roy*, Narbada Prasad Gupta*
}

Lovely Professional University, Punjab, India

\section{Introduction}

D2D is essential for LTE-A and beyond LTE-A or 5G standards. It becomes a challenge for the researches to re-investigate possible architectures, protocols and resource allocation and interference cancellation algorithms to ensure measurable QoS for underlying cellular networks [1]. It has been found from literature survey that in LTE-A D2D communication exists in licensed band spectrum which is more secure than hotspot or Wi-Fi where ISM band is used for D2D communication [2]. Allocation of available resource blocks (RBs) depends on the operating mode of the cellular network. The operating modes are broadly classified into three categories e.g. reuse mode, dedicated mode and cellular mode. In the case of reuse mode device to device users utilize similar spectrum as traditional cellular user therefore it causes interference among themselves [3]. In this case the base station coordinates the transmit power for both the links. In dedicated mode D2D users get $50 \%$ of the total available resources of cellular user. As the resources are assigned prior to the communication and both link may utilize different resources, it provides no interference between cellular and D2D communication. To achieve maximum throughput, transmitter node need to transmit maximum power. In case of cellular mode, the device to device users communicate with each other via the base station and the corresponding BS acts like a relay node. All nodes use orthogonal resources and communication between UEs to BS uses $25 \%$ of the resources whereas BS to UEs uses $25 \%$ for D2D transmission. Rest $50 \%$ remain reserved for cellular communication [4]. Out of these three modes reuse mode has highest spectrum efficiency but it requires advanced interference cancellation schemes. In dedicated mode of operation no interference occurs between cellular user and D2D user but it has poor spectral efficiency because resources are divided between D2D user and cellular user. Dedicated mode is preferred in overlaying two-way cellular networks. In the case of cellular mode BS acts as a relay node [5]. Interference cancellation schemes are required for reuse and cellular mode of operation. Another important research challenge is to develop service discovery protocols [6]. A UE can itself search for a neighbour UE to establish a D2D link. An eNodeB or base station can broadcast messages to UEs for D2D connection. A UE can request $\mathrm{eNodeB}$ for $\mathrm{D} 2 \mathrm{D}$ connection without getting any message from eNodeB. Therefore, it is a real challenge for the researcher to make a proper service

\footnotetext{
*Emails: koushik.15737@lpu.co.in, ajoy.22652@lpu.co.in, narbada.24806@lpu.co.in
}

DOI: $10.1201 / 9781003129103-56$ 


\section{4}

discovery protocols which can work under the above said conditions and can provide the optimal solution for D2D service request based on their location in the cell. Proximity services using licensed band spectrum have been introduced in a technical report (3GPP TR22.803) of 3GPP in June 2013. The report was named "Feasibility Study for Proximity Services (Prose)" [7]. This report describes technical feasibility requirements of mobile UEs under LTE-A for commercial (e.g. discovery/social networks), network offloading, and public safety applications [7]. In this report, 13 general use cases and 13 public safety use cases were recognized in $\mathrm{D} 2 \mathrm{D}$ communication scenario.

\section{Types of service discovery protocols}

Proximity service discovery is a process to discover UEs in close proximity for D2D communication. It includes both device discovery and service discovery. There are two types of service discovery protocols, e.g. reactive protocol and proactive protocol. Reactive protocol uses following steps to initiate D2D request. Consider UE-E is Enabler UE and $\mathrm{UE}-\mathrm{R}$ is receiver $\mathrm{UE}$.

a) UE-E sends request to UE-R. This initiation request signal consist of service type, location address of UE-E and other related fields.

b) UE-R calculates various parameters like distance, probability of time delay, channel state information and optimal route. UE-R forwards a signal to base station which contains all this information. Base station provides permission to UE-R to establish connection to UE-E.

c) Base station also checks profile and authenticity of UE-E. If it is authorized, then base station checks resource blocks are available or not. If RBs are available then it replies to the UE-R, otherwise it sends negative response to UE-R.

d) UE-R relays to UE-E response of base station.

e) UE-E transmits "D2D request" to contact corresponding services.

In proactive protocol, base station will send notifications to all authenticated UEs (provided proximity area or geographical location) using multicast message for D2D communication. The process of D2D connection can be establish as per following:

a) Base station periodically transmits "Service advertisement message" to all user equipment available under its coverage.

b) UE-R transmits "Multicast D2D message" to group of devices. This message contains service type information and location information.

c) UE-E sends reply "D2D response" message to UE-R.

d) UE-R asks base station for D2D request permission by sending information about UE-E.

e) Base station initiates to provide resource block for establishment of D2D link.

Figure 56.1 shows non-roaming reference architecture mentioned in 3GPP release 12 . [7] According to this architecture direct discovery is possible if the UEs are under radio coverage of E-UTRAN. Direct discovery is the process in which a UE discovers a nearby UE for D2D communication. All UEs must have D2D proximity service discovery application through which they will communicate with D2D proximity application server which is connected to proximity service function block. Table 56.1 shows comparison of various service discovery protocols. 


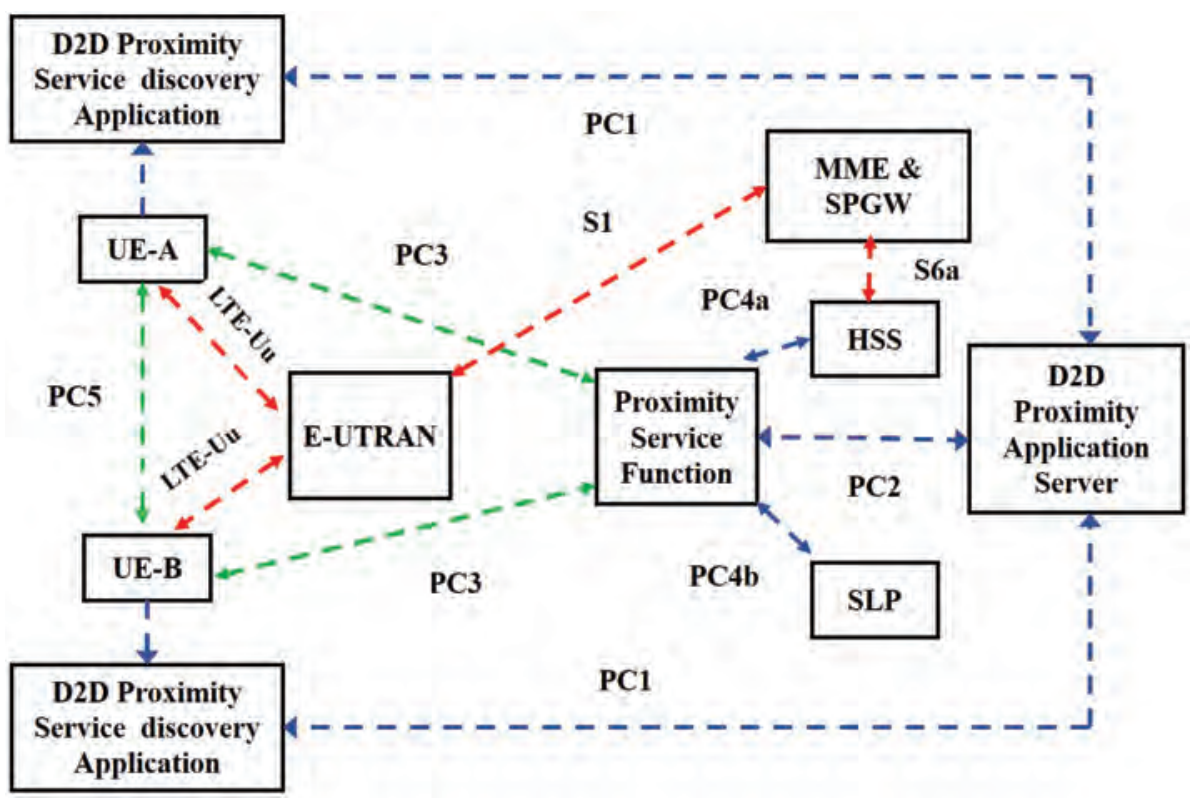

Figure 56.1 LTE-A non-roaming architecture for supporting service discovery D2D communication.

Table 56.1 Existing service discovery protocols

\begin{tabular}{|c|c|c|}
\hline Network types & $\begin{array}{l}\text { Distributed networks } \\
\text { (MANETs/ WLAN/ } \\
\text { WiFi-direct) }\end{array}$ & Centralized networks (LTE-A) \\
\hline $\begin{array}{l}\text { Device discovery } \\
\text { mechanism }\end{array}$ & Proactive and reactive & $\begin{array}{l}\text { Direct discovery } \\
\text { EPC assist discovery }\end{array}$ \\
\hline Operating frequency band & Unlicensed ISM band & Licensed band \\
\hline $\begin{array}{l}\text { Service discovery protocol } \\
\text { types }\end{array}$ & Carrier sense multiple access & $\begin{array}{l}\text { Direct discovery: push mechanism } \\
\text { based protocol } \\
\text { EPC level discovery: network } \\
\text { assistance based protocol (Ref:ITU } \\
\text { 3GPP release 12) }\end{array}$ \\
\hline Limitations & $\begin{array}{l}\text { 1. Security challenges } \\
\text { 2. Resource allocation and } \\
\text { management challenges } \\
\text { 3. Protocol Overhead } \\
\text { problems }\end{array}$ & $\begin{array}{l}\text { Protocol design challenges in the case } \\
\text { of out of coverage area of eNodeB }\end{array}$ \\
\hline
\end{tabular}

Let us consider there are total $\mathrm{N}$ numbers of UEs which generates $\mathrm{M}$ numbers of $\mathrm{D} 2 \mathrm{D}$ request per time slot. Hence pdf of generated D2D request can be expressed as,

$$
\rho=\frac{1}{\sigma \sqrt{ } 2 \pi} \exp \left(-\frac{(M-\mu)^{2}}{2 \sigma^{2}}\right)
$$


Here $\mu$ is the mean of device to device request, $\sigma$ is the standard deviation of a Gaussian random process. D2D request generation process has been considered as a Gaussian random process.

Protocol overhead for proactive is given by

$$
(\mathrm{PO})_{\mathrm{P}}=(2 *(\mathrm{~T}-\mathrm{K})+\mathrm{K} *(2+(14 * \mathrm{M}))) / \mathrm{T}
$$

Protocol overhead for reactive is given by

$$
(\mathrm{PO})_{\mathrm{R}}=\left(\mathrm{K}^{*} 15 * \mathrm{M}\right) / \mathrm{T}
$$

where $\mathrm{K}$ is the time slot where device to device request generated and $\mathrm{T}$ is the total number of time slots. Figure 56.1 shows comparison curve of control overhead for proactive and reactive protocols. Result shows that proactive protocol is better for more D2D pair requests whereas reactive protocol is better for fewer D2D requests.

\section{Interference management schemes}

The objectives of interference management are minimization of cellular interference on nearby cellular link, minimization of cellular interference on D2D link, minimization of D2D interference on cellular link and minimization of D2D interference on nearby D2D link. Interference management schemes are classified in three different categories. They are power control schemes, retransmission schemes and resource management schemes.

Figure 56.3 shows a plot between signal to noise plus interference and normalized base station power for different values of D2D link power. This graph provides a comparative analysis of D2D link power in the presence of cellular link. It illustrates that, for a range of -4 to $10 \mathrm{dBm}$, SINR at D2D receiver normalized base power varies to control D2D link power without compromising cellular link to degrade.

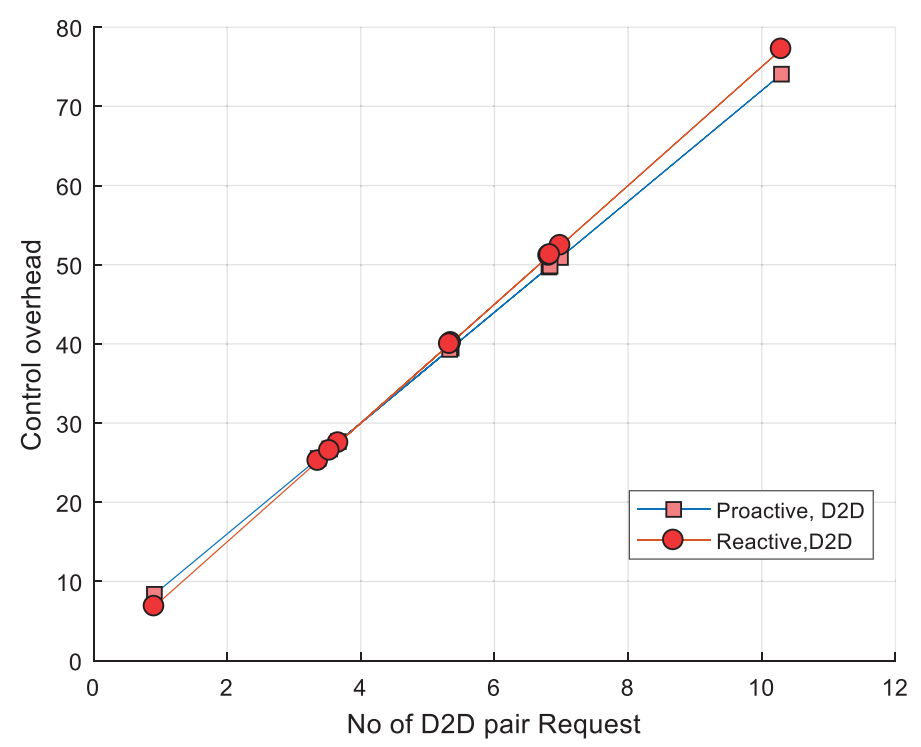

Figure 56.2 D2D request vs control overheads plot of proactive and reactive protocols. 


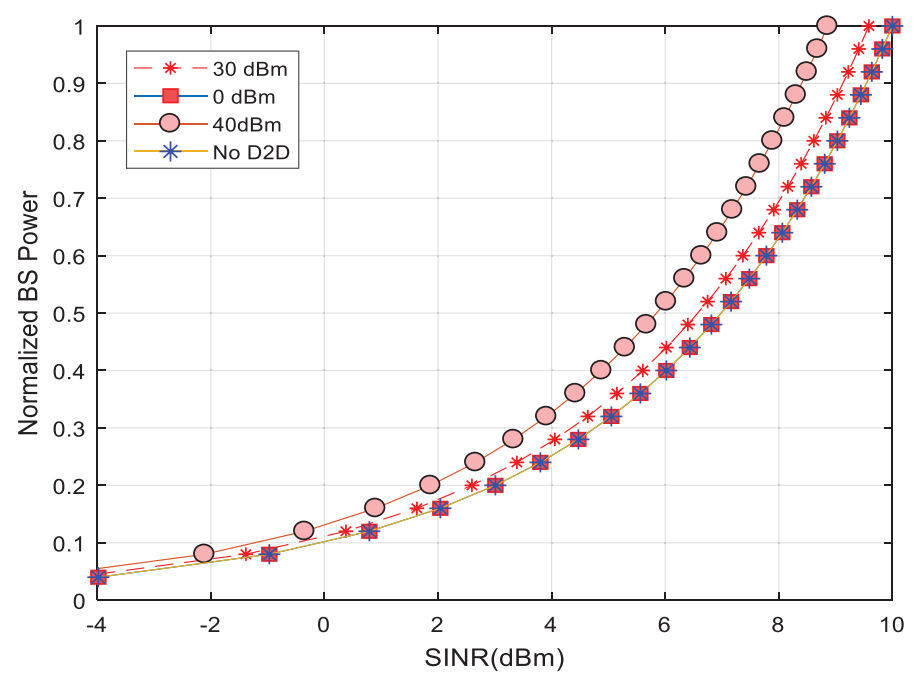

Figure 56.3 SINR vs normalized BS power.

\section{Conclusion}

This paper presents a study of various service discovery protocols. A comparative analysis has been reported for proactive, reactive protocols, distributed resource access protocols and direct discovery protocols. We have presented various proximity based services of 3 GPPP releases. Control overhead has been calculated for proactive and reactive protocols. It has been shown that for increasing numbers of D2D link requests proactive protocols perform better than reactive protocols. Also interference management technique based on power control has been discussed.

\section{References}

[1] Faustin Ahishakiye and Frank Y. Li, "Service discovery protocols in D2D-enabled cellular networks: reactive versus proactive," in Proceeding of IEEE Globecom Workshop, pp. 833838, Jan. 2014.

[2] M.N. Tehrani, M. Uysal and H. Yanikomeroglu, "Device-to-device communication in 5G cellular networks: challenges, solutions, and future directions, "IEEE Commun. Mag. vol. 52, pp. 86-9252, May 2014.

[3] A. Gupta and R. K. Jha, "A survey of 5 G network: architecture and emerging technologies," IEEE Access, vol. 3, pp. 1206-1232, July 2015.

[4] Brett Kaufman and Behnaam Aazhang, "Cellular networks with an overlaid device to device network" in IEEE Asilomar Conference on Signals, Systems and Computers, Pacific Grove, USA, Oct. 2008.

[5] Gjendemsjo, D. Gesbert, G. E. Oien and S. G. Kiani, "Binary power control for sum rate maximization over multiple interfering links," IEEE Trans. Wirel. Commun. vol. 7, no. 8, pp. 31643173, Aug. 2008.

[6] S.-Y. Lien, C.-C. Chien, F.-M. Tseng and T.-C. Ho, "3GPP device-to-device communications for beyond 4G cellular networks”, IEEE Commun. Mag., vol. 54, pp. 29-35, Aug. 2016.

[7] 3GPP, “TS 23.303 version 12.2.0 Release 12", Available online: www.etsi.org/deliver/etsi_ts/ 123300_123399/123303/12.02.00_60/ts_123303v120200p.pdf(Accessed 18 November 2019). 


\title{
57 Raspberry-Pi and Intel Movidius Assisted Framework for Fruit Quality Monitoring
}

\author{
Navjot Rathour*, Pranab Kumar Mishra, Kartik Gaur, \\ Abhishek Maddheshiya
}

\author{
School of Electronics and Electrical Engineering, Lovely Professional University, \\ Phagwara, Punjab, India
}

\section{Introduction}

Agriculture collectively concerns the overall economic development in India. Being an integral part of economic boost, agriculture marks the livelihood for two-thirds of the population in India. The interruptions of technology and new innovations have witnessed the growth in production and maintenance of products. Still, innovations in the development of agriculture are in a way a bit sluggish [1].To make fruits reach a large part of the population it needs a quality production. Fruits contain vitamins A, C, E and other minerals that help our metabolism go well [2]. Hence increase in quality production of fruits has become an important concern nowadays. Moreover, manual inspection reduces the quality checking of fruits because of inconsistency and inaccurate decisions. Thus, quality monitoring of fruits and their maturity check-up at the time of their growth has become utmost important [3-5]. Diseases being the greatest hurdle in the growth of a healthy fruit, they need to get checked periodically and treated. Fruit quality monitoring depends on various factors such as size, texture, colour, degree of ripeness and defect free $[6,7]$. Diseases on the fruits are monitored and images are captured and compared to high accuracy and decisions are taken to treat them well [8]. The autonomous robot embedded with Raspberry Pi and camera setup thus just moves around the orchard to capture the best real-time images for further processing. A computer vision based autonomous system makes it more efficient and accurate and reduces computational complexity to its highest extent $[4,5]$. In the commercial products the use of machine perception can be increased by using OpenCV-Python. It gives a common platform for computer vision applications. OpenCV-Python basically gives preference to real-time image processing [5] [9]. Image processing further provides different clusters of image properties to analyse and classify between images thus making it easier to detect the maturity and disease growth in the fruits [10]. Raspberry Pi being a powerful computing system contributes to the most accurate and efficient decision making. Raspberry Pi4 B-Model as shown in Figure 57.1 is a single-board computer which uses the 2GB LPDDR4 RAM, 64-bit quad-core CortexA72 processor, Broadcom 2711, 2 USB 3.0 super speed ports, True Gigabit Ethernet port, Bluetooth 5.0,802.11b/g/n/ac wireless LAN $(2.5 \mathrm{GHz}$ and $5 \mathrm{GHz}), 4 \mathrm{~K}$ UHD video support, Dual micro-HDMI ports. Raspberry $\mathrm{Pi}$ is using an operating system named Raspbian, a version of GNU Linux [12]. Intel Neural Compute stick is used on low power applications for real-time interference to fine tune, develop and deploy CNN. It has dimensions of

* Corresponding author email: er.rathour@gmail.com

DOI: 10.1201/9781003129103-57 


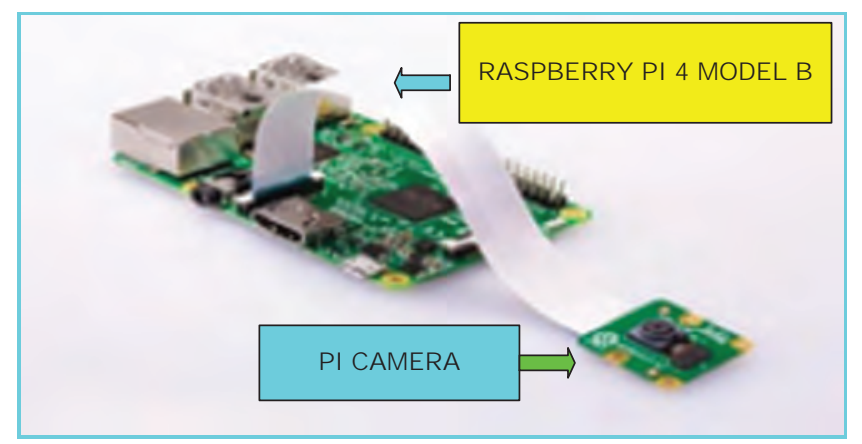

Figure 57.1 Raspberry Pi 4 Model B with Pi camera module.

1.06 in $\times 2.85$ in $\times 0.55$ in $(27 \mathrm{~mm} \times 72.5 \mathrm{~mm} \times 14 \mathrm{~mm})$ and the operating temperature ranges from $0^{\circ}$ Celsius to $40^{\circ}$ Celsius and moreover it can be connected or attached to the 3.0 USB port. Raspberry Pi provides the hardware support [13].

\section{Methedology}

\subsection{Dataset selection}

The given name to the dataset is Fruit-360 and it has been taken from the addresses pointed to in the literature as shown in Figure 57.2. In this work only apple images are included. In this dataset a total of 1,919 images have been taken. Later the dataset is categorized into four categories named as:

\begin{tabular}{ll}
\hline Category & Number of images \\
\hline Good apples & 658 \\
Healthy apples & 624 \\
Average apples & 473 \\
Bad/rotten apples & 164 \\
\hline
\end{tabular}

This dataset is completely high quality to get accurate result. In datasets if the fruit image is noisy or background changes that can lead to the less effective model results. So, dataset plays a huge role in getting accurate results from the model. The dataset has been trained on an external system. For training process AlexNet or GoogLeNet can be used.

\subsection{Platform selection}

The Raspberry Pi has a small size like a credit card and it is a single board powerful computing system. The Raspberry Pi Foundation from the UK introduced the basic embedded system. Compared to its previous version named Raspberry pi 3 Model B+, it has high quality multimedia performance, high memory, a good processing speed and connectivity, while retaining the power consumption amount and the compatibility. For the user, Raspberry Pi4 B-Model gives a performance matched to entry-level x86PC systems. This also has attributes such as 2.4 or $5.0 \mathrm{GHz}$ dual-band wireless LAN, a 64-bit high 


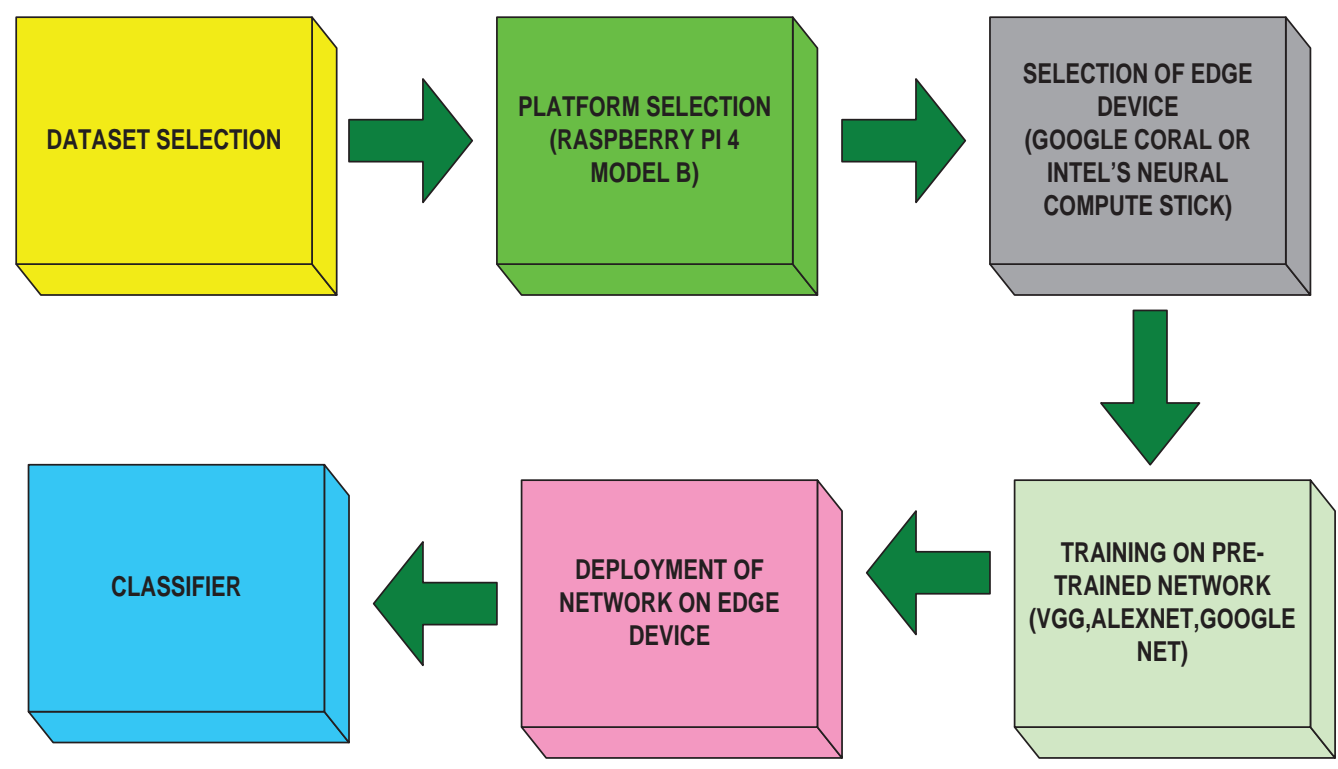

(a)

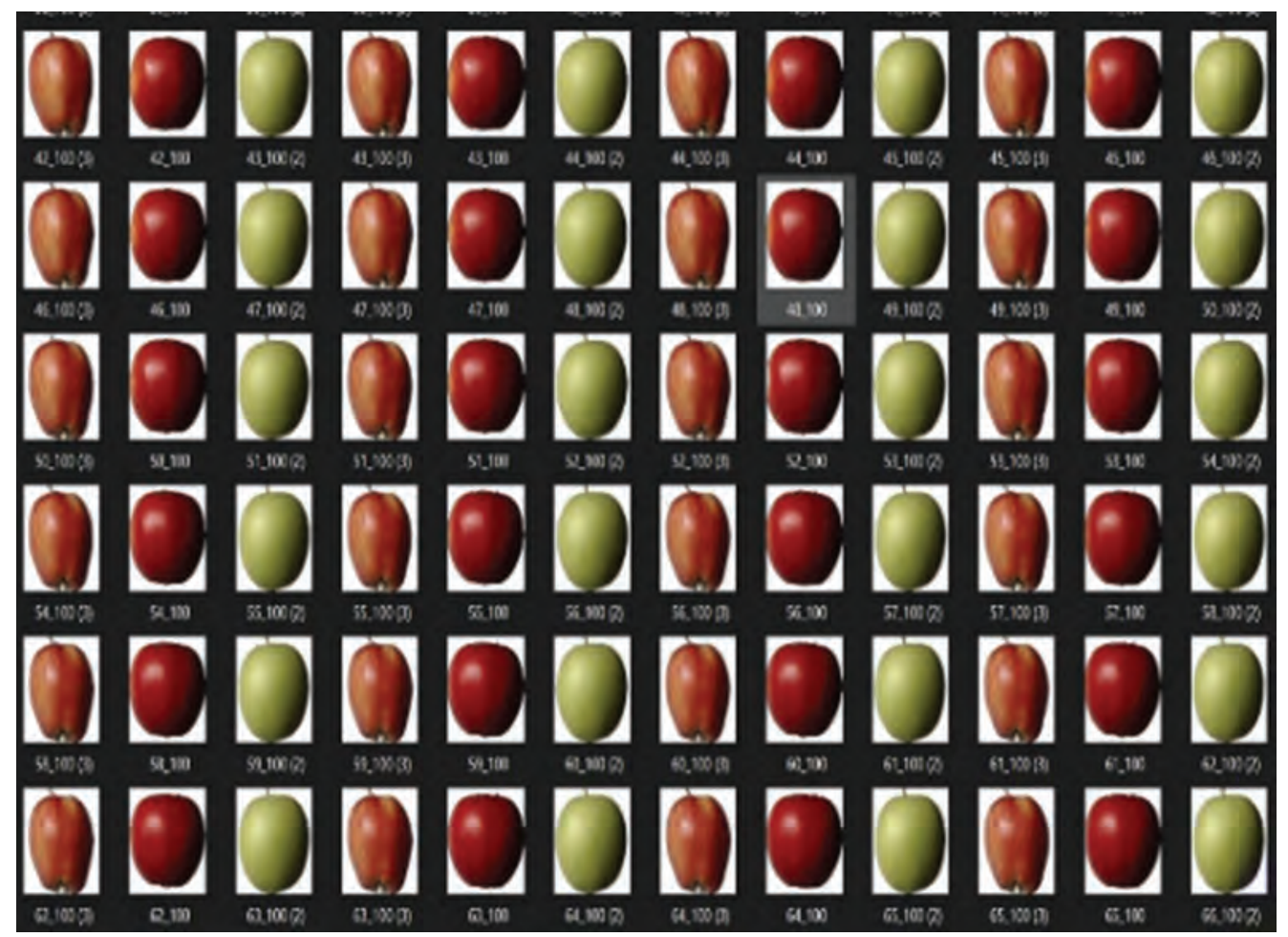

(b)

Figure 57.2 (a) Methodology of work flow and (b) sample apple dataset. 
performance quad-core processor, up to 4 GB of RAM, Bluetooth 5.0, a support of dual display via two micro-HDMI ports having resolutions to $4 \mathrm{~K}$, gigabit Ethernet, USB 3.0 [14-16].

Raspberry Pi specifications include:

- Processor of Broadcom BCM2711, quad-core Cortex-A72 (ARM v8) 64-bit SoC at $1.5 \mathrm{GHz}$.

- Memory space of 2 GB/4 GB LPDDR4 having 40-pin standard GPIO header.

- Operating temperature ranging from 0 to $50^{\circ} \mathrm{C}$.

- Connectivity of $2.4 \mathrm{GHz}, 5.0 \mathrm{GHz}$ IEEE $802.11 \mathrm{~b} / \mathrm{g} / \mathrm{n} / \mathrm{ac}$ wireless LAN, Bluetooth 5.0, two 2.0 USB ports, two 3.0 USB ports, BLE Gigabit Ethernet.

\subsection{Selection of edge device}

The Google Coral TPU USB accelerator co-processor works in a very similar way, but Google has reportedly suggested that Coral series products are 10 times faster than Intel's NCS2. But NCS2 is cost effective and practically it provides the same speed when connected to USB 3.0. In order to achieve the 10 times faster speed the use of Raspberry $\mathrm{Pi} 4 \mathrm{~B}-$ Model having 3.0 USB is more viable. A CNN (convolutional neural network) that has been trained on a related wide-ranging problem such as AlexNet and GoogLeNet is used in the task of visual recognition without training the early layers. For feature detection, those layers are fixed. The upper layers are finely tuned to match the conventional problems. This process is called transfer learning since a $\mathrm{CNN}$ is being used by the system that has been trained on a distinct but correlated scenario. This is normally done to reduce the need for enormous training datasets and to accelerate the learning process. So the CNN will adapt smoothly as long as the transfer learning process takes place in related scenarios. This is for the reason that the signals in visual recognition or other natural stimuli are compositional. The first few layers of any CNN will learn pretty much the same basic features such as colour, edge or gradient features for visual recognition tasks $[17,18]$.

\subsection{Training on pre-trained network}

The main work of CNN is to identify the attributes or characteristics of images. For that purpose, the CNN needs to be built and trained separately. Or, we can use a predefined model that has been trained already as shown in Figure 57.3. In this scenario the transfer learning can be more feasible.

For the formation of neural networks, Keras is a good library, having a simple API.

The transfer learning shown in Figure 57.4, is just to train a model with large amount of data and then transfer all of its info to a smaller dataset. This is the basic understanding of the transfer learning.

For image processing with CNN, starting convolutional layers need to be freeze and only last few layers need to train which makes the prediction accurate as well as useful. The main concept is that convolutional layers extract general, low-level attributes or characteristics that are in the images or dataset - such as colour, texture, gradients, curves, shapes, etc. [18]. Thus, we can transfer the learning of the network trained on pre-trained datasets. Image pre-processing and data augmentation go hand in hand by providing transformations on trained images. 


\begin{tabular}{|l|c|c|c|c}
\hline MODEL & LAYERS & PARAMETERS & MACCs & $\begin{array}{l}\text { ERROR- } \\
\mathbf{5} \%\end{array}$ \\
\hline AlexNet & 8 & 60 & 650 & 19.7 \\
\hline ZefNet & 8 & 60 & 650 & 11.2 \\
\hline VGG16 & 16 & 138 & 7800 & 10.4 \\
\hline SqueezeNet & 18 & 1.2 & 860 & 19.7 \\
\hline GoogleNet & 22 & 5 & 750 & 6.7 \\
\hline Inception-v3 & 48 & 23.6 & 5700 & 5.6 \\
\hline Inception-v4 & 70 & 35 & 6250 & 5 \\
\hline ResNet-101 & 101 & 40 & 3800 & 6.8 \\
\hline ResNet-152 & 152 & 55 & 5650 & 6.7 \\
\hline ResNet-200 & 200 & 65 & 6850 & 5.8 \\
\hline ResNeXt & 101 & 68 & 4000 & 5.3 \\
\hline DenseNet-201 & 201 & 16.5 & 1500 & 6.3 \\
\hline SENet-154 & 154 & 100 & 10,500 & 4.5 \\
\hline MobileNet-v1 & 28 & 4.2 & 569 & 10 \\
\hline MobileNet-v2 & 28 & 3.5 & 300 & 9 \\
\hline ShuffleNet & 11 & 5.3 & 260 & 10 \\
\hline
\end{tabular}

Figure 57.3 Complexity and accuracy of known convolutional neural network (CNN) networks.

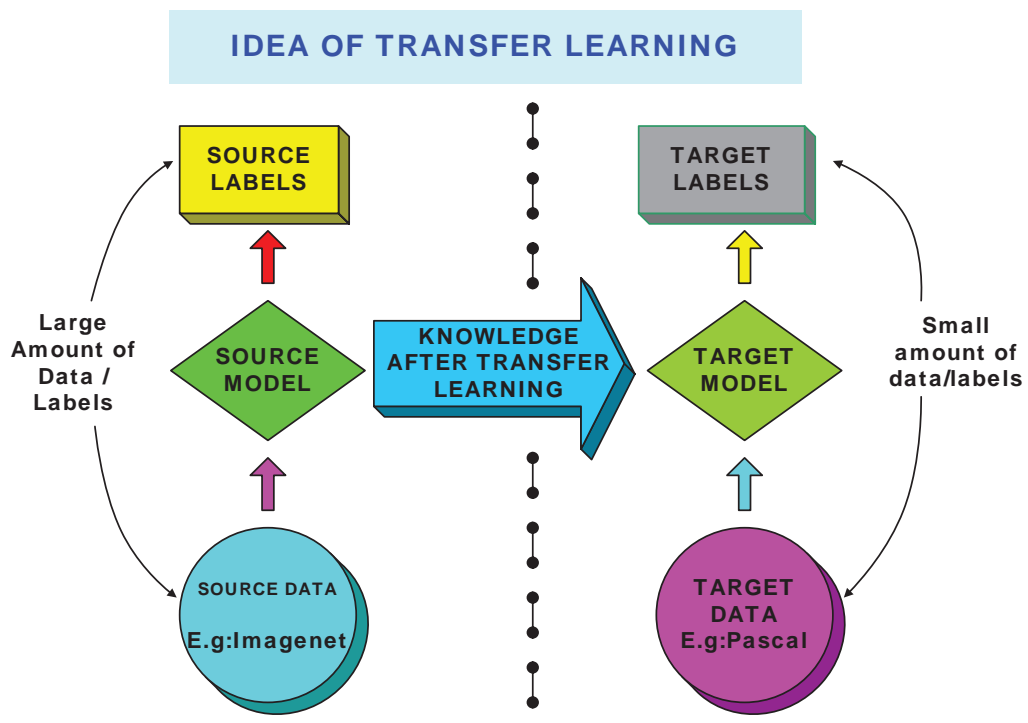

Figure 57.4 Transfer learning.

\section{Conclusion and future scope}

Fruit quality monitoring using a resource constrained device like Raspberry Pi along with the Intel Movidius Neural Compute stick is an efficient and cost-effective solution to the existing and heavy and bulky fruit grading systems. Current work presented in the paper has been tested for apples only but can be extended for various classes of fruits. 


\section{References}

[1] Mhaski, R. R., Chopade, P. B., and Dale, M. P. (2015, November). Determination of ripeness and grading of tomato using image analysis on Raspberry Pi. In 2015 Communication, Control and Intelligent Systems (CCIS) (pp. 214-220). IEEE.

[2] www.betterhealth.vic.gov.au/health/healthyliving/fruit-and-vegetables.

[3] P. Yimyam and S. Jaitrong, "Mango maturity classification by using physical properties q1," in 12th Annual Conference of Thai Society of Agricultural Engineering, 2011.

[4] Halil Durmus, Ece Olcay Gunes, K. Murvet and Burak Berk Ustundag, "The design of general purpose autonomous agricultural mobile robot: "Agrobot", IEEE Fourth International conference on Agro-Geoinformatics, pp. 49-53, July 2015.

[5] C. S. Nandi, B. Tudu and C. Koley, "An automated machine vision based system for fruit sorting and grading," in Proceedings of the International Conference on Sensing Technology, ICST, 2012.

[6] Mustaffa, I. B., and Khairul, S. F. B. M. (2017, November). Identification of fruit size and maturity through fruit images using opencv-python and rasberry pi. In 2017 International Conference on Robotics, Automation and Sciences (ICORAS) (pp. 1-3). IEEE.

[7] Patil, B., Panchal, M. H., Yadav, M. S., Singh, M. A., and Patil, M. D. (2017). Plant monitoring using image processing, raspberry Pi \& Iot. International Research Journal of Engineering and Technology (IRJET), 1337-1342.

[8] Patil. J.K., and Raj Kumar, "Feature extraction of diseased leaf images", Journal of Signal and Image Processing, 2012.

[9] M. P. Arakeri and Lakshmana, "Computer vision based fruit grading system for quality evaluation of tomato in agriculture industry," Procedia Comput. Sci., vol. 79, pp. 426-433, 2016.

[10] Tichkule, S. K., and Gawali, D. H. (2016, November). Plant diseases detection using image processing techniques. In 2016 Online International Conference on Green Engineering and Technologies (IC-GET) (pp. 1-6). IEEE.

[11] Gawande, G., and Chinchamalatpure, M. Smart fruit grading system using Raspberry Pi.

[12] E. Element14, “Element14.com,” Premier Farnell plc., 2016.

[13] https://software.intel.com/en-us/neural-compute-stick.

[14] Shilpashree, K. S., Lokesha, H., and Shivkumar, H. (2015). Implementation of image processing on Raspberry Pi. International Journal of Advanced Research in Computer and Communication Engineering, 4(5), 199-202.

[15] https://projects.raspberrypi.org/en/projects/getting-started-with-picamera.

[16] https://static.raspberrypi.org/files/product-briefs/Raspberry-Pi-4-Product-Brief.pdf.

[17] /www.datacamp.com/community/tutorials/convolutional-neural-networks-python.

[18] https://towardsdatascience.com/transfer-learning-with-convolutional-neural-networks-inpytorch-dd09190245ce. 


\title{
58 Control scheme of doubly-fed induction generators applied in wind energy conversion systems
}

\author{
Bhagwan Shree Ram*, Narbada Prasad Gupta
}

SEEE, Lovely Professional University, Phagwara, Punjab, India

\section{Introduction}

During the transient period, flux power angle magnitude is increased with respect to sudden rise in stator current. So the differential value of real and reactive powers of DFIG is changed and also controls the rotor side control (RSC) of DFIG. Simultaneously, the magnitude of $d q$ frame of grid current is increased. This incremental current changes the real and reactive powers of grid and it controls the GSC. The exponential value of slip angular frequency value of the direct torque control depends upon $d q+$ frame rotor voltage and current. During the transient period, the short circuit current of stator is increased. Simultaneously, 3-phase rotor current is also increased. This increases the magnitude of $d q+$ frame of rotor current and this current controls the error signals which are given to the PI controller of RSC.

In the direct power control (DPC) technique, the control techniques are implemented in both rotor and grid side converters. Leakage factor, stator angular frequency, magnitude of reference value of rotor flux and flux power angle control the change in real and reactive powers of DFIG [1-3]. Rotor side control (RSC) and GSC are controlled by actual value of voltage and current of DFIG and grid, respectively, by DPC technique. Separate control scheme is implemented to control the GSC in DPC, that is, differential value of grid voltage $d V_{\text {GRID }}$ and current $d i_{\text {GRID }}$ effectively control the GSC.

\section{Characteristics of DFIG with wind speed variations}

The behavior of DFIG is analysed at transient and post-transient conditions and the test system is shown in the Figure 58.1. The time at end of the 2-second 3-phase short circuit fault is applied across the terminal and the length of the fault is extended up to 0.15 second. During the transient period the speed of the wind turbine connected to the DFIG is oscillated and it is shown in Figure 58.1.

The performance of DFIG is analysed with the $40 \%$ of rise of speed from its rated value and the test system at the time of $2 \mathrm{~s}$, the speed is stepped up from 1 to 1.4 p.u., as shown in Figure 58.2.

In the DTC control technique, during the stepping up of the wind speed, the pulsation rating of DFIG parameters is controlled by controlling $T_{e}$. But in DPC, the stator current and voltage, gird voltage and current control the converters of DFIG. Hence, the

* Corresponding author email: bhagwan.24828@lpu.co.in

DOI: 10.1201/9781003129103-58 


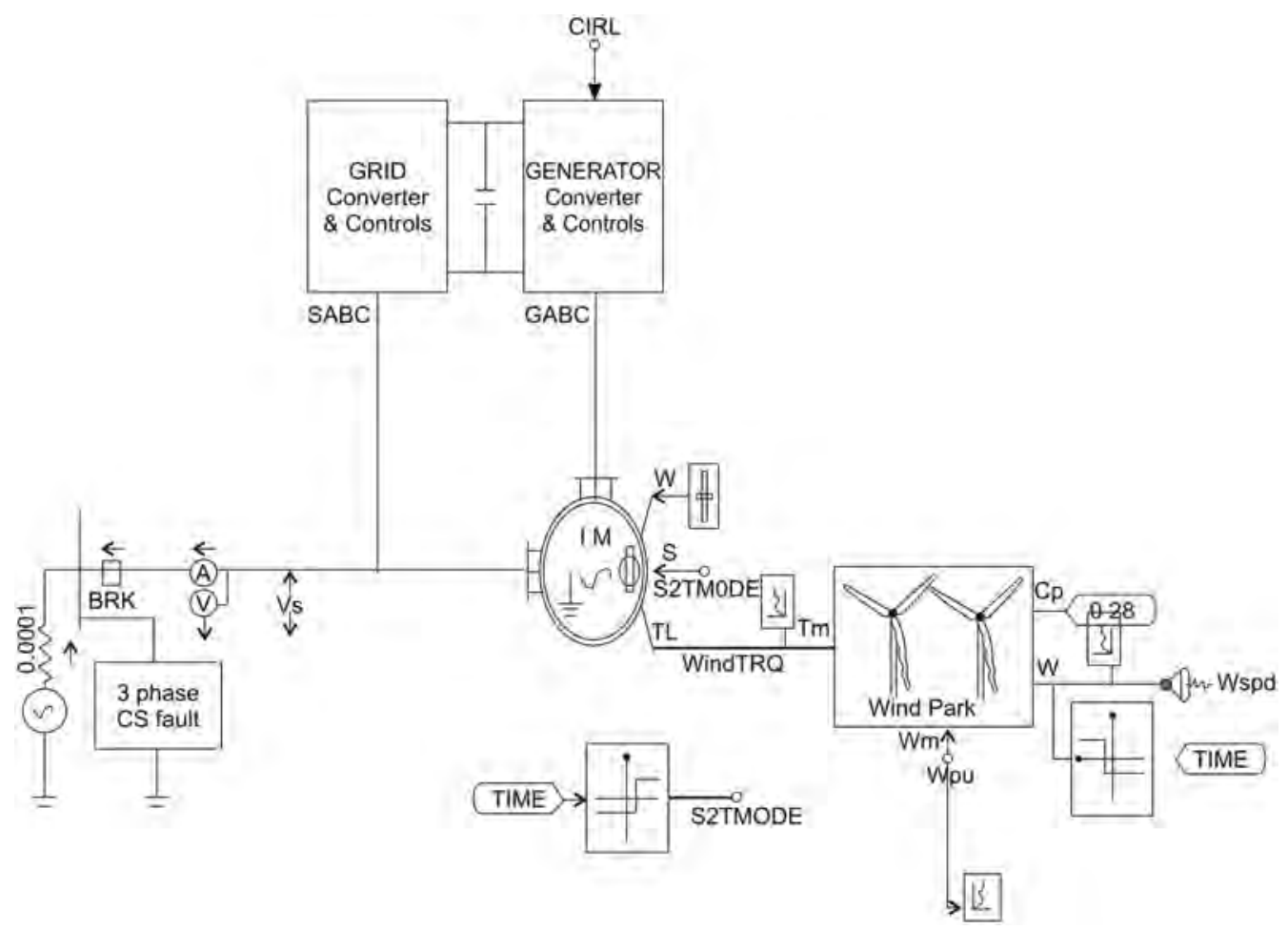

Figure 58.1 DFIG with 3-phase short circuit fault.

magnitude of pulsation values are $0.89 \%, 1.79 \%, 5 \%, 13.04 \%$ of $V_{s}, T_{e}, P_{D F I G}, Q_{D F I G}$ less than DTC technique, respectively.

\section{Pulsation of DFIG parameters with the DTC and DPC techniques}

The simulation studies of DFIG parameters are carried out at unbalanced load which is connected across the DFIG and the system is similar to as shown in Figure 58.1 and in Table 58.1.

\section{Analysis of the result of the DFIG parameters with the DTC and DPC}

DTC: With the unbalanced condition, pulsation of real and reactive powers and THD value of $i_{s}$ and $i_{r}$ are controlled by electromagnetic torque. The oscillation of $T_{e}$ can be controlled by $d q+$ and $d q$ - frames of rotor current in RSC and this controlling procedure is already discussed in case 1 and 2 . But in the resonant controller technique, the rise time and steady state error are reduced by $K_{p}$ and $K_{i}$ and overshoot problem is minimized by $C_{d}$ and $C_{q}$. Also, the fundamental components of PI and harmonic component of resonant controllers control the RSC. Hence, the pulsation of DFIG parameters and total harmonic distortion of stator and rotor currents are less than resonant controller. With the steppedup speed, $V_{s}$ is magnified from its rated value. But, controlling of RSC is achieved by rotor current in $d q+$ and $d q$-frames through $e^{j s l}$ and $e^{j s}$. So, the pulsation of torque $T_{e}$ is $2.54 \%$ less than the resonant controller based on sine and cosine components of $T_{e}$. With the 


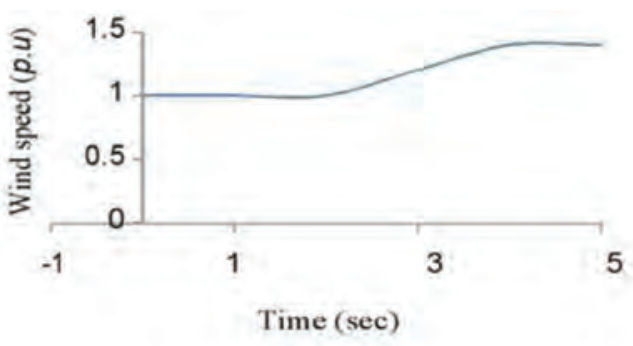

(a)

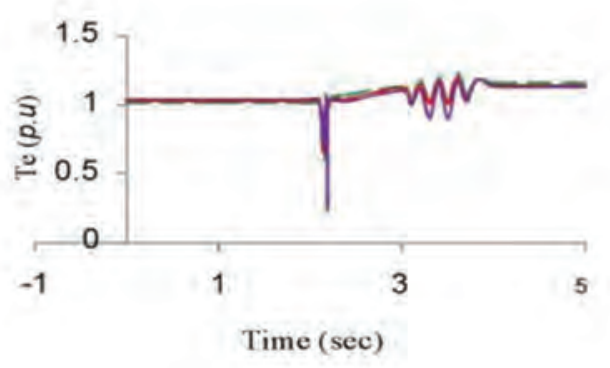

(c)

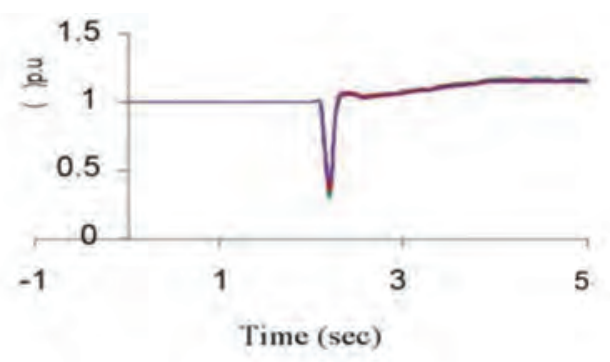

(b)

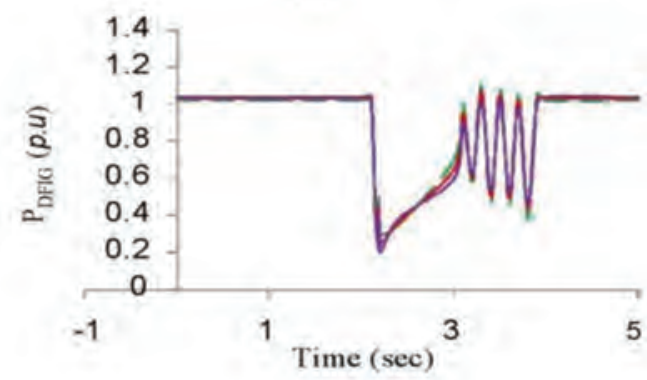

(d)

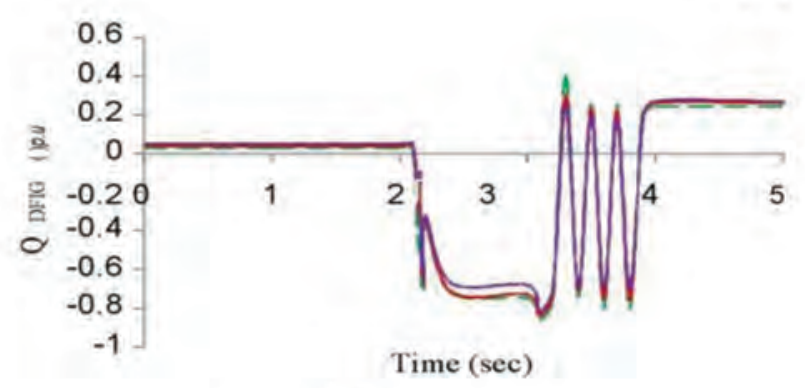

(e)

--Resonant -DTC -DPC

Figure 58.2 Characteristics of DFIG with DTC and DPC at variable wind speed.

Table 58.1 Pulsation of DFIG parameters with DTC and DPC techniques

\begin{tabular}{llllll}
\hline $\begin{array}{l}\text { DFIG } \\
\text { controllers }\end{array}$ & $\begin{array}{l}T_{e} \\
\text { pulsation } \\
(\%)\end{array}$ & $\begin{array}{l}P_{\text {DFIG }} \\
\text { pulsation } \\
(\%)\end{array}$ & $\begin{array}{l}Q_{\text {DFIG }} \\
\text { pulsation } \\
(\%)\end{array}$ & $\begin{array}{l}\text { THD } \\
\text { value of } i_{s} \\
(\%)\end{array}$ & $\begin{array}{l}\text { THD } \\
\text { value of } i_{r} \\
(\%)\end{array}$ \\
\hline DTC & \pm 6.56 & \pm 4.18 & \pm 7.24 & 0.85 & 6.84 \\
DPC & \pm 4.82 & \pm 3.26 & \pm 6.56 & 0.04 & 5.27 \\
\hline
\end{tabular}


controlling of electromagnetic torque of DFIG, oscillation of $V_{s}, P_{D F I G}, Q_{D F I G}$ are $0.93 \%$, $7.14 \%$ and $8 \%$ less than the resonant controller respectively. At the post-transient period, flux power angle of RSC and grid current in $d q$ frame are within the normal range and the system is maintained at the stable region shown in Figure 58.2(a-e). In this control technique, grid voltage and current control the GSC. But the hysteresis band only controls the GSC in torque controller.

The pulsation magnitudes of $V_{s}, T_{e}, P_{\text {DFIG }}, Q_{\text {DFIG }}$ are $3.15 \%, 3.92 \%, 14.29 \%$ and $16.67 \%$ less than the DTC controller, respectively.

DPC: RSC and GSC are controlled by the actual value of voltage and current of DFIG and grid respectively by the DPC technique. A separate control scheme is implemented to control the GSC in DPC, that is, differential value of grid voltage $d V_{\text {GRID }}$ and current $d i_{G R I D}$ effectively control the GSC [4,5]. Slip $s$ and the differential value of real and reactive powers such as $d P_{D F I G}$ and $d Q_{D F I G}$ are controlling the RSC [6]. But ${ }_{s}$ is not considered in DTC. Hence pulsation of $T_{e}, P_{D F I G}, Q_{D F I G}$, THD of $i_{s}, V_{G R I D}$ are less than DTC technique shown in Table 58.1.

\section{Conclusion}

This paper has presented the torque equation of DTC based on sine and cosine components and mathematical expressions of the stator power equation of DPC. Also we discussed the designing of DTC and DPC control techniques of DFIG and analysed its performance in the grid system. From the simulation results, the following points are observed. In the DTC technique, electromagnetic torque $T_{e}$ only controls the pulsation of DFIG parameters and the hysteresis band technique is adopted in GSC. The separate control schemes are implemented in converters of DFIG in DPC control technique. Actual value of real and reactive powers of DFIG and grid controls the RSC and GSC respectively. So the pulsation magnitude of $V_{s}, T_{e}, P_{D F I G}$ and $Q_{D F I G}$ are minimized compared with DTC. Based on the above discussions, the performance of DFIG is improved with the DPC technique compared with DTC in the grid.

\section{References}

1. M. Liserre, R. Teodorescu, and F. Blaabjerg, "Multiple harmonics control for three-phase grid converter systems with the use of PI-RES current controller in a rotating frame," IEEE Trans. Power Electron., vol. 21, no. 3, pp. 836-841, May 2006.

2. R. Teodorescu, F. Blaabjerg, M. Liserre, and P. C. Loh, "Proportional resonant controllers and filters for grid-connected voltage-source converters," IEE Proc. Electr. Power Appl., vol. 153, no. 5, pp. 750-762, Sep. 2006.

3. J. Hu, Y. He, L. Xu, and B. W. Williams, "Improved control of DFIG systems during network unbalance using PI-R current regulators," IEEE Trans. Ind. Electron., vol. 56, no. 2, pp. 439451, Feb. 2009.

4. I. Etxeberria-Otadui, U. Viscarret, M. Caballero, A. Rufer, and S. Bacha, "New optimized PWM VSC control structures and strategies under unbalanced voltage transients," IEEE Trans. Ind. Electron., vol. 54, no. 5, pp. 2902-2914, Oct. 2007.

5. J. Hu, H. Nian, B. Hu, Y. He, and Z. Q. Zhu, "Direct active and reactive power regulation of DFIG using sliding-mode control approach," IEEE Trans. Energy Convers., vol. 25, no. 4, pp. 1028-1039, Dec. 2010.

6. R. Pena, R. Cardenas, J. Proboste, G. M. Asher, and J.C.Clare, "Sensorless control of doubly-fed induction generators using a rotor-current-based MRAS observer," IEEE Trans. Ind. Electron., vol. 55, no. 1, pp. 330-339, Jan. 2008. 


\title{
59 Stability assessment of single-area thermal automatic generation control system using eigenvalue analysis
}

\author{
Megha Khatri*, S. Hareesh Reddy, Himanshu Aggarwal
}

SEEE, Lovely Professional University, Phagwara, Punjab, India

\section{Introduction}

The assessment of instability of a power system can be carried out from the location of poles near the imaginary axis. A single area mid-sized thermal power automatic generation control (AGC) system is considered for analysis at various operating points, which are obtained with the help of sequential computation of eigenvalues. Generally it is not essential or feasible to calculate all the poles of a power system model [1-5]. Therefore those poles which are allied with electromechanical modes that are closer to the imaginary axis or poorly damped and negatively damped with stable, critically stable and unstable modes are considered.

It is very common that due to poorly adjusted controller gains the real poles of a system may reach an unstable region [6,7].

The changes in controller parameters may be required due to the extensive usage of phasor measurement units, the contact component between the power plant, controller and the grid, i.e., communication units. The state space representation of the thermal system is used to identify the eigenvalues and the location of roots using stability locus [8].

It is a matter of fact that the eigenvalues which are poorly damped hamper the convergence of the system stability and lead to the critical analysis of the system. Despite several investigations and advances in the stability analysis techniques the time domain based method is straightforward and systematic to find out the stability criteria of the system [9-12].

The controller parameters space $\mathrm{K}_{\mathrm{i}}-\mathrm{K}_{\mathrm{p}}$ recognizes the stability region of single area thermal power plant. When the imaginary and real values of the system characteristic equation $t$ are equal to zero, then the stability boundary locus can be found. This leads to the main contribution of the work, which is visualization of the system stability in two-dimensional plane. It is a simple and fast way to find out the controller parameters [13-16].

The proposed work is outlined as follows: Section II presents mathematical modelling of single area PI controlled thermal AGC system and the eigenvalues are determined to analyse the location of poles and Section III is about the simulations and discussions followed by the conclusions.

* Corresponding author: megha.25035@lpu.co.in

DOI: $10.1201 / 9781003129103-59$ 


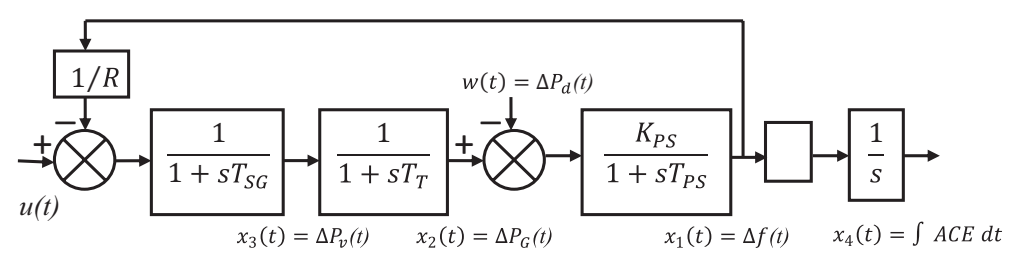

Figure 59.1 Block diagram of single-area thermal power system.

\section{System modelling with proposed method}

The diagram of a single area thermal power system is elaborated in Figure 59.1. The mathematical modelling of the respective system is formulated using a set of elements/ coefficients of differential equations presented with state-space method in Equation (5). Sequentially to find out the eigenvalues of the system, the input state space variable $u(t)$ is exhibited with the controller parameters [16-20]. This results in dense state matrices as shown in Equation (6).

A dynamic model of the system with speed governor, turbine and the power system under investigation has $\mathrm{R}$ as the governor speed parameter in p.u.; $\Delta \mathrm{P}_{\mathrm{v}}$ as the adjustable in valve positions of governor in p.u.; $\mathrm{K}_{\mathrm{PS}}$ is the power system gains; $\mathrm{T}_{\mathrm{SG}}$ is the speed governor time constant in s; the turbine time $\mathrm{T}_{\mathrm{T}}$ constant (in seconds); the power system time $\mathrm{T}_{\mathrm{PS}}$ constants (in seconds); the synchronizing coefficient $\beta$. The nominal parameters taken for the simulations are $\mathrm{f}=60 \mathrm{~Hz}, \beta=1 ; \mathrm{R}=2.4 \mathrm{~Hz} /$ p.u.; $\mathrm{T}_{\mathrm{T}}=0.3 \mathrm{~s}, \mathrm{~T}_{\mathrm{PS}}=20 \mathrm{~s} ; \mathrm{K}_{\mathrm{PS}}=120, \mathrm{~T}_{\mathrm{SG}}=0.08 \mathrm{~s}$.

The transfer functions of each component of the power system represented in frequency domain are given below.

Transfer function of turbine:

$$
G_{T}(s)=\frac{\Delta P_{G}(s)}{\Delta P_{v}(s)}=\frac{1}{1+s T_{T}}
$$

Transfer function of governor:

$$
G_{G}(s)=\frac{\Delta P_{v}(s)}{\Delta P_{\mathrm{e}}(s)}=\frac{1}{1+s T_{S G}}
$$

The speed governing system has one direct input $\Delta P_{\text {ref }}$, feedback $\Delta f$ with single output $\Delta P_{e}(s)$ given as

$$
\Delta P_{e}(s)=\Delta P_{r e f}(s)-\frac{1}{R} \Delta f(s)
$$

The plant transfer function

$$
G_{P}(s)=\frac{K_{P} s}{1+s T_{P s}}
$$


Table 59.1 Eigenvalue locations for stable, marginally stable and unstable regions

\begin{tabular}{lll}
\hline Stable & Critically stable & Unstable \\
\hline $\mathrm{K}_{\mathrm{p}}=0.0, \mathrm{~K}_{\mathrm{i}}=0.20$ & $\mathrm{~K}_{\mathrm{p}}=0.0, \mathrm{~K}_{\mathrm{i}}=0.9571$ & $\mathrm{~K}_{\mathrm{p}}=0.0, \mathrm{~K}_{\mathrm{i}}=1.10$ \\
-13.264 & -13.166 & -13.147 \\
$-1.021+2.346 \mathrm{i}$ & $0.0000+2.5860 \mathrm{i}$ & $0.1042+2.663 \mathrm{i}$ \\
$-1.021-2.346 \mathrm{i}$ & $0.0000-2.5860 \mathrm{i}$ & $0.1042-2.663 \mathrm{i}$ \\
-0.575 & -2.716 & -2.944 \\
\hline
\end{tabular}

Using state space illustration

$$
\dot{x}(t)=A x(t)+B u(t)+F w(t)
$$

where $x(t)=\left[\Delta f \Delta P_{G} \Delta P_{v} \int A C E\right]^{T}$

$$
A=\left[\begin{array}{cccc}
\frac{-1}{T_{P S}} & \frac{K_{P S}}{T_{P S}} & 0 & 0 \\
0 & \frac{-1}{T_{T}} & \frac{1}{T_{T}} & 0 \\
\frac{-1}{R T_{S G}} & 0 & \frac{-1}{T_{S G}} & 0
\end{array}\right], B=\left[\begin{array}{llll}
0 & 0 & \frac{1}{T_{S G}} & 0
\end{array}\right]^{T} \text { and } F=\left[\begin{array}{cccc}
\frac{-K_{P S}}{T_{P S}} & 0 & 0 & 0
\end{array}\right]^{T}
$$

With PI controller control is taken as follows

$$
u(t)=\left(K_{p}+\frac{K_{i}}{s}\right) x(t)
$$

Now, Equation (5) can be rewritten as

$$
\dot{x}(t)=(A+B K C) x(t)+F w(t))
$$

where $K=\left[\begin{array}{ll}K_{p} & K_{i}\end{array}\right], C=-\left[\begin{array}{llll}1 & 0 & 0 & 0 \\ 0 & 0 & 0 & 1\end{array}\right]$

\section{Simulations and discussion}

In this section, the stability margins are obtained by simulating the considered single area thermal system fitted with the PI controller. Then eigenvalues are obtained for different controller gains to find the stability regions presented in Table 59.1.

Figure 59.2 shows the range of controller gain $K_{i}$ for different values of proportional gain $\mathrm{K}_{\mathrm{p}}$ for which the system remains stable (blue shaded area). Moreover, one can choose any value of gains from this stability region, such that the automatic generation system remains stable. Furthermore, if the gains are chosen on the stability boundary the system 


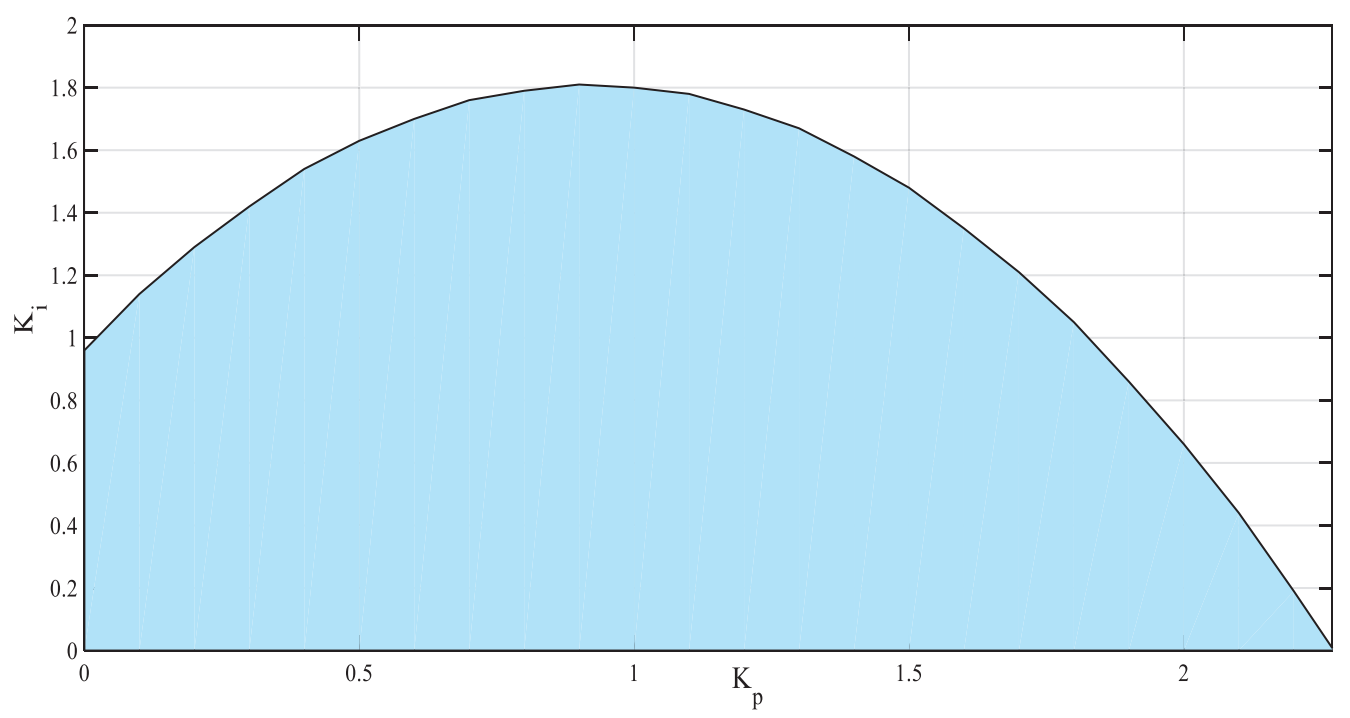

Figure $59.2 \mathrm{~K}_{\mathrm{i}}-\mathrm{K}_{\mathrm{p}}$ stability margin region.

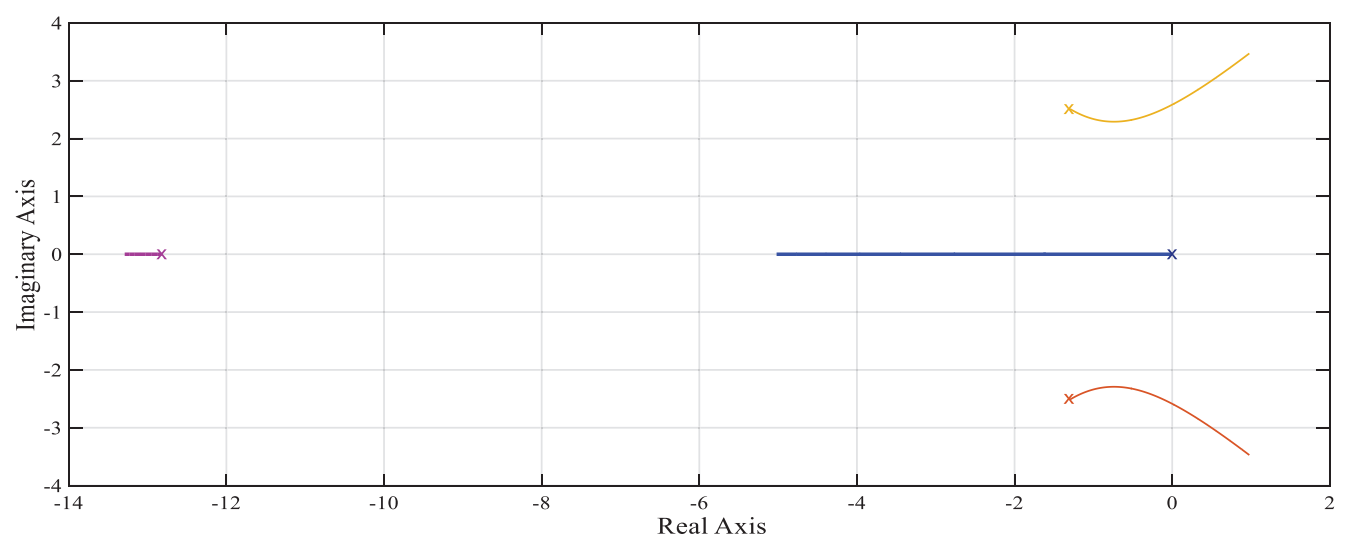

Figure 59.3 Closed-loop system poles position with $\mathrm{K}_{\mathrm{p}}=0$ and varying $\mathrm{K}_{\mathrm{i}}$.

is marginally stable, and if gains are chosen outside this boundary, the system becomes unstable.

The eigenvalues calculated by taking controller gain $\mathrm{K}_{\mathrm{p}}=0$ and different values of gains $K_{i}$ are shown in Figure 59.3. The closed loop poles' movement can be seen with the increase of $K_{i}$. The figure shows open loop poles with ' $\mathrm{x}$ ' mark and as $\mathrm{K}_{\mathrm{i}}$ reaches 0.9571 the imaginary poles cross the imaginary axis, which gives the stability region as with $\mathrm{K}_{\mathrm{p}}=0, \mathrm{~K}_{\mathrm{i}}$ can takes values up to 0.9571 . To show the effectiveness of the presented approach, the considered automatic generation control system is simulated with $1 \%$ step load perturbation at $\mathrm{t}=0$, with $\mathrm{K}_{\mathrm{p}}=0$, while taking three values of $\mathrm{K}_{\mathrm{i}}$ in different stability regions found using the proposed technique. The time domain dynamic responses for the 


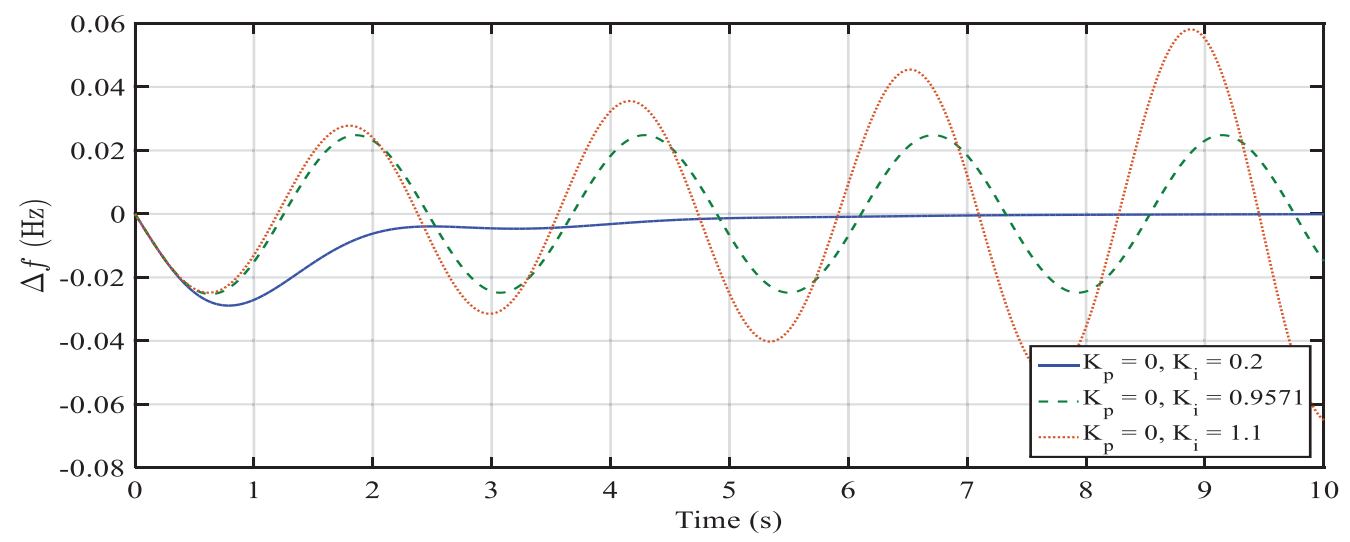

Figure 59.4 Dynamic response with $1 \%$ step load disturbance and controller gain in stable, critically stable and unstable regions.

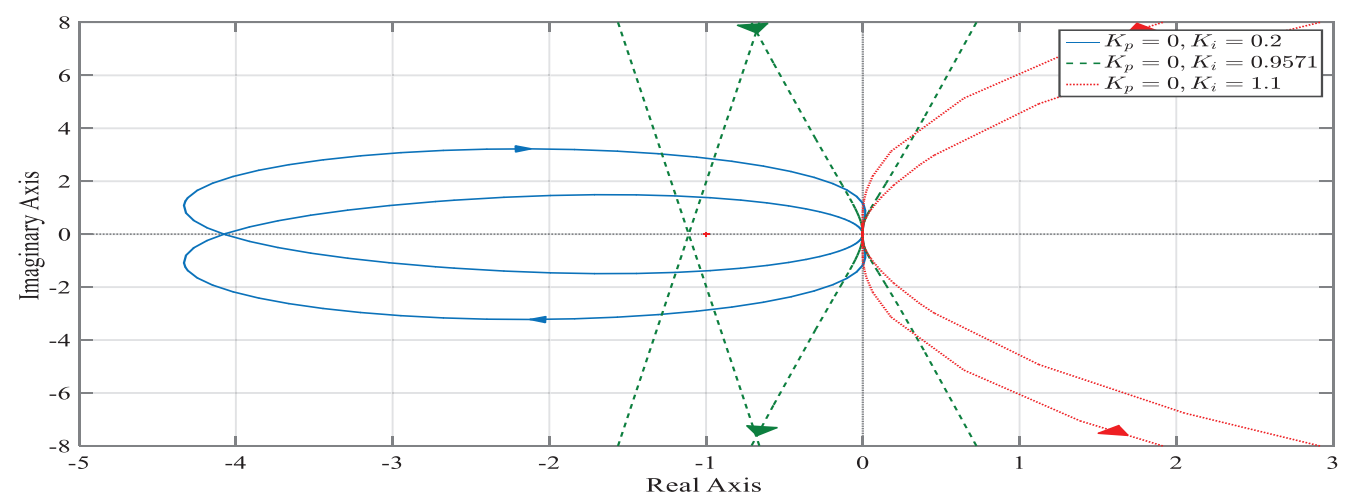

Figure 59.5 Nyquist plot of the system with chosen PI gains.

applied load disturbance are shown in Figure 59.4. The results show that the controller gain taken in the stable region (i.e. $\mathrm{K}_{\mathrm{p}}=0, \mathrm{~K}_{\mathrm{i}}=0.2$ ) stabilizes the frequency, gains taken on the stability boundary (i.e. $K_{p}=0, K_{i}=0.9571$ ) give undamped frequency and gains taken outside the boundary (i.e. $\mathrm{K}_{\mathrm{p}}=0, \mathrm{~K}_{\mathrm{i}}=1.1$ ) give unstable response. Figure 59.5 depicts the locus of stability with the chosen PI controller gains.

\section{Conclusions}

The stability margins for the single-area thermal automatic generation system fitted with the PI controller are investigated. The stability margins in PI controller gains space are found by calculating the location of closed loop poles using the state-space technique. The proposed scheme gives the stability regions on the graph, so that the controller gains can be chosen effectively. The efficacy of the proposed scheme is the evaluated using the time domain simulations. 


\section{References}

[1] P. Kundur, J. Paserba, V. Ajjarapu, G. Andersson, A. Bose, C. Canizares, N. Hatziargyriou, D. Hill, A. Stankovic, C. Taylor et al., "Definition and classification of power system stability ieee/cigre joint task force on stability terms and definitions," IEEE Transactions on Power Systems, vol. 19, no. 3, pp. 1387-1401, 2004.

[2] R. A. Horn and C. R. Johnson, Topics in Matrix Analysis. Cambridge University Press, 1991.

[3] L. Jiang, W. Yao, Q. H. Wu, J. Y. Wen and S. J. Cheng, "Delay dependent stability for load frequency control with constant anytime-varying delays," IEEE Trans. Power Syst., vol. 27, no. 2, pp. 932-941, May 2012.

[4] K. Meerbergen, A. Spence and D. Roose, "Shift-invert and Cayley transforms for detection of rightmost eigenvalues of nonsymmetric matrices," BIT, vol. 34, no. 3, pp. 409-423, Sep. 1994.

[5] S. Sonmez, S. Ayasun and U. Eminoglu, "Computation of timedelay margins for stability of a single-area load frequency control system with communication delays," WSEAS Trans. Power Syst., vol. 9, pp. 67-76, 2014.

[6] A. Semlyen and L. Wang, "Sequential computation of the complete eigen system for the study zone in small signal stability analysis of large power systems," IEEE Trans. Power Syst., vol. 3, no. 2, pp. 715-725, May 1988.

[7] N. Tan, I. Kaya, C. Yeroglu and D. P. Atherton, "Computation of stabilizing PI and PID controllers using the stability boundary locus," Energy Convers. Manage., vol.47, pp.30453058, 2006.

[8] F. Milano and M. Anghel, "Impact of time delays on power system stability," IEEE Trans. Circuit Syst.-I, vol. 59, no. 4, pp. 889-900, Apr. 2012.

[9] J. Ma, Z. Y. Dong and P. Zhang, "Comparison of BR and QR eigen value algorithms for power system small signal stability analysis," IEEE Trans. Power Syst., vol. 21, no. 4, pp. 1848-1855, Nov. 2006.

[10] T. Smed, "Feasible eigenvalue sensitivity for large power systems," IEEE Trans. Power Syst., vol. 8, no. 2, pp. 555-563, May 1993.

[11] H. Ye, Y. Liu and P. Zhang, "Efficient eigen-analysis for large delayed cyber-physical power system using explicit infinitesimal generator discretization," IEEE Trans. Power Syst., vol. 31, no. 3, pp. 2361-2370, May 2016.

[12] G. Angelidis and A. Semlyen, "Improved methodologies for the calculation of critical eigen values in small signal stability analysis," IEEE Trans. Power Syst., vol. 11, no. 3, pp. 12091217, Aug. 1996.

[13] S. Gomes, Jr., N. Martins and C. Portela, "Computing small-signal stability boundaries for large-scale power systems,” IEEE Trans. Power Syst., vol. 18, no. 2, pp. 747-752, May 2003.

[14] F. Milano and M. Anghel, "Impact of time delays on power system stability," IEEE Trans. Circuit Syst.-I, vol. 59, no. 4, pp. 889-900, Apr. 2012.

[15] Z. Du, W. Liu and W. Fang, "Calculation of rightmost eigen values in power systems using the Jacobi-Davidson method," IEEE Trans. Power Syst., vol. 21, no. 1, pp. 234-239, Feb. 2006.

[16] H. Ye, W. Gao, Q. Mou and Y. Liu, "Iterative infinitesimal generator discretization-based method for eigen-analysis of large delayed cyberphysical power system," Elec. Power Syst. Res., vol. 143, pp. 389-399, Feb. 2017.

[17] C. Li, G. Li, C. Wang and Z. Du, "Eigenvalue sensitivity and eigenvalue tracing of power systems with inclusion of time delays," IEEE Trans. Power Syst., vol. 33, no. 4, pp. 37113719, Jul. 2018.

[18] H. Ye, Q. Mou and Y. Liu, "Enabling highly efficient spectral discretization-based eigenanalysis methods by Kronecker product," IEEE Trans. Power Syst., vol. 32, no. 5, pp. 4148 4150, Sep. 2017.

[19] J. Rommes and N. Martins, "Computing large-scale system eigen values most sensitive to parameter changes, with applications to power system small-signal stability," IEEE Trans. Power Syst., vol. 23, no. 2, pp. 434-442, May 2008.

[20] F. Milano, "Small-signal stability analysis of large power systems with inclusion of multiple delays,” IEEE Trans Power Syst., vol. 31, no. 4, pp. 3257-3266, Jul. 2016. 


\title{
60 Design and analysis of a shared charged dynamic latch comparator
}

\author{
Tejender Singh ${ }^{1,2}$, Suman Lata Tripathi ${ }^{3}$
}

\author{
${ }^{1}$ Research Scholar, School of Electronics and Electrical Engineering, Lovely \\ Professional University, Punjab, India \\ ${ }^{2}$ Assistant Professor, CMR Institute of Technology, Hyderabad, Telangana, India \\ ${ }^{3}$ School of Electronics and Electrical Engineering, Lovely Professional University, \\ Punjab, India
}

\section{Introduction}

An electronic component which compares two input voltages is called a comparator. It is generally driven by two input voltages, and then it compares and produces a differential output voltage which can be either a high level signal or a low level signal. Comparators are related closely to Op-Amps. But the designed structure is different as it has positive feedback and its output is saturated at one power rail or the other power rail [1-3].

In Figure $60.1 V_{S_{-}}$and $V_{S_{+}}$are negative and positive supply voltages respectively. The above comparator is used for sensing random varying input signal which reaches either a defined threshold level or a reference level. Due to the advances in technology a comparator which offers high speed and operates on low power supply voltages by producing less leakage current is more challenging. A new proposed CMOS based dynamic latch comparator is designed which has significant improvement in power delay, power consumption, power delay product and offset voltage [4].

\section{CMOS-based dynamic latch comparator}

CMOS technology has been mostly adopted in IC technology due to its high levels of scalability and higher levels of integration. In this section, a detailed discussion is carried out on the characteristics and operation of the existing dynamic comparators and a proposed dynamic comparator is been defined.

\section{A. Single tail current dynamic latch comparator (STDLC)}

A single tail current based dynamic latch comparator is most used ADCs whose input impedance is high; consumption of static power is zero and it also has negligible output swing. Its operation is classified into dual phase: they are reset phase and regeneration phase. Here clock is kept low in reset phase and Mtail transistor is maintained in OFF state. A logical level $\mathrm{V}_{\mathrm{dd}}$ is obtained by pulling both the output terminals Outn and Outp by M7 and M8 transistors and it is called the starting state of the regeneration phase. When CLK switches to $\mathrm{V}_{\mathrm{dd}}$, the Mtail transistor will turn ON while M7 and M8 will go to OFF state by switching back the circuit in regeneration phase. Here Outp and Outn terminals will get discharged, which were pre-charged at the first stage due to supply as

DOI: $10.1201 / 9781003129103-60$ 


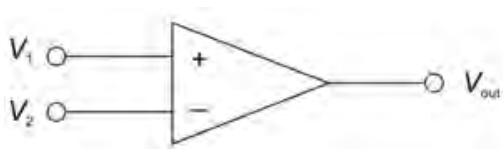

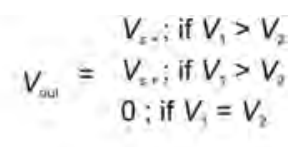

Figure 60.1 Comparator.

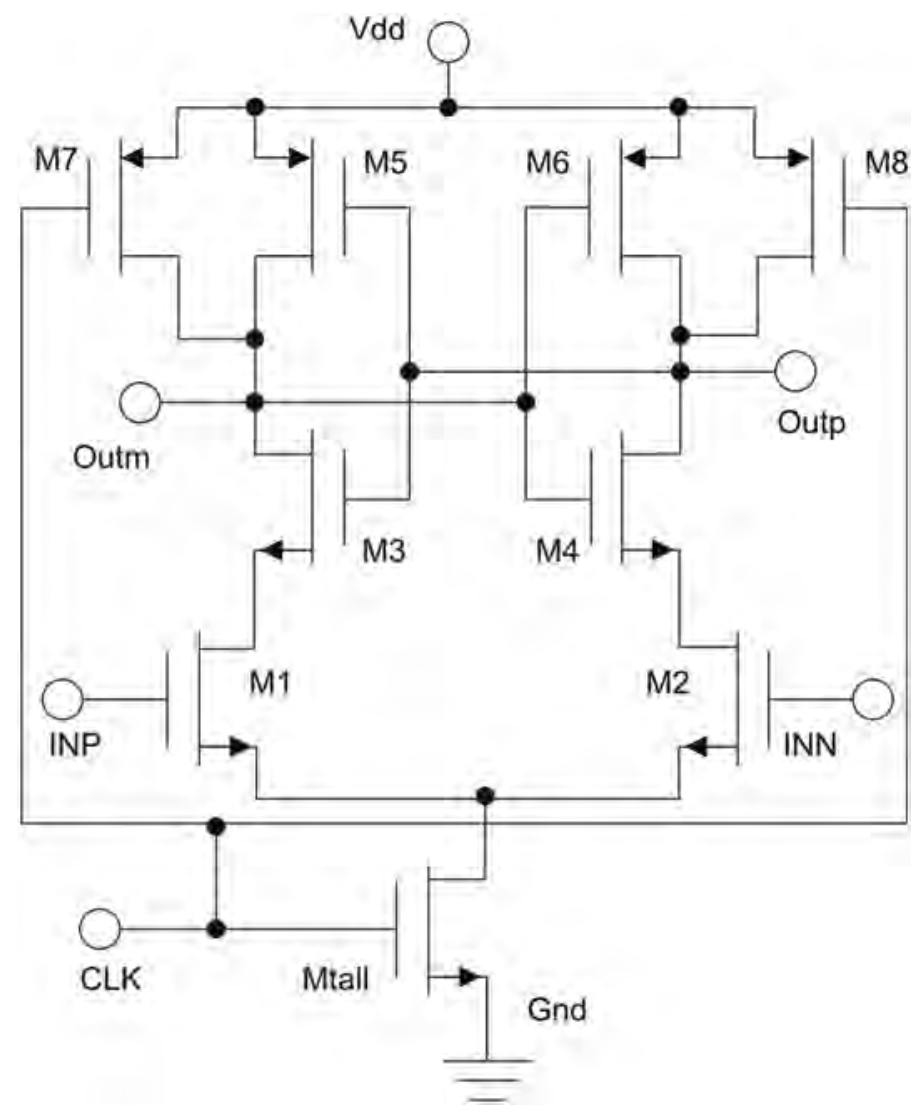

Figure 60.2 Single tail current DLC.

shown in Figure 60.2. Voltage applied at input terminals i.e. $I_{N P}$ and $I_{N N}$ determines the discharge rate. Assuming that $\left(\mathrm{V}_{\mathrm{INP}}>\mathrm{V}_{\mathrm{INN}}\right)$ such that Outn gets discharged quicker than Outp and falls down to $\mathrm{V}_{\mathrm{dd}}-\mathrm{V}_{\mathrm{tp}}$. such that (PMOS-M6) transistor turns ON and starts the second stage, i.e. regeneration process produced by cross coupled inverter by making the output terminal Outn to completely discharge to GND and to charge the output terminal Outp to supply voltage $\mathrm{V}_{\mathrm{dd}}$. [5-9].

It has advantages like rail to rail output swing, zero static power, and higher input impedance. The disadvantages of the circuit are high supply voltage due to stacking and it has only one current path. 
B. Double tail current dynamic latch comparator (DTDLC)

This DLC circuit is made of fewer transistors and has two stages of different tail currents. In stage one it produces low offset by handling a small current, whereas in stage two it provides short delay time by handling a large current and the circuit is made up of latch as shown in Figure 60.3. For optimization at differential stage a tail current is required

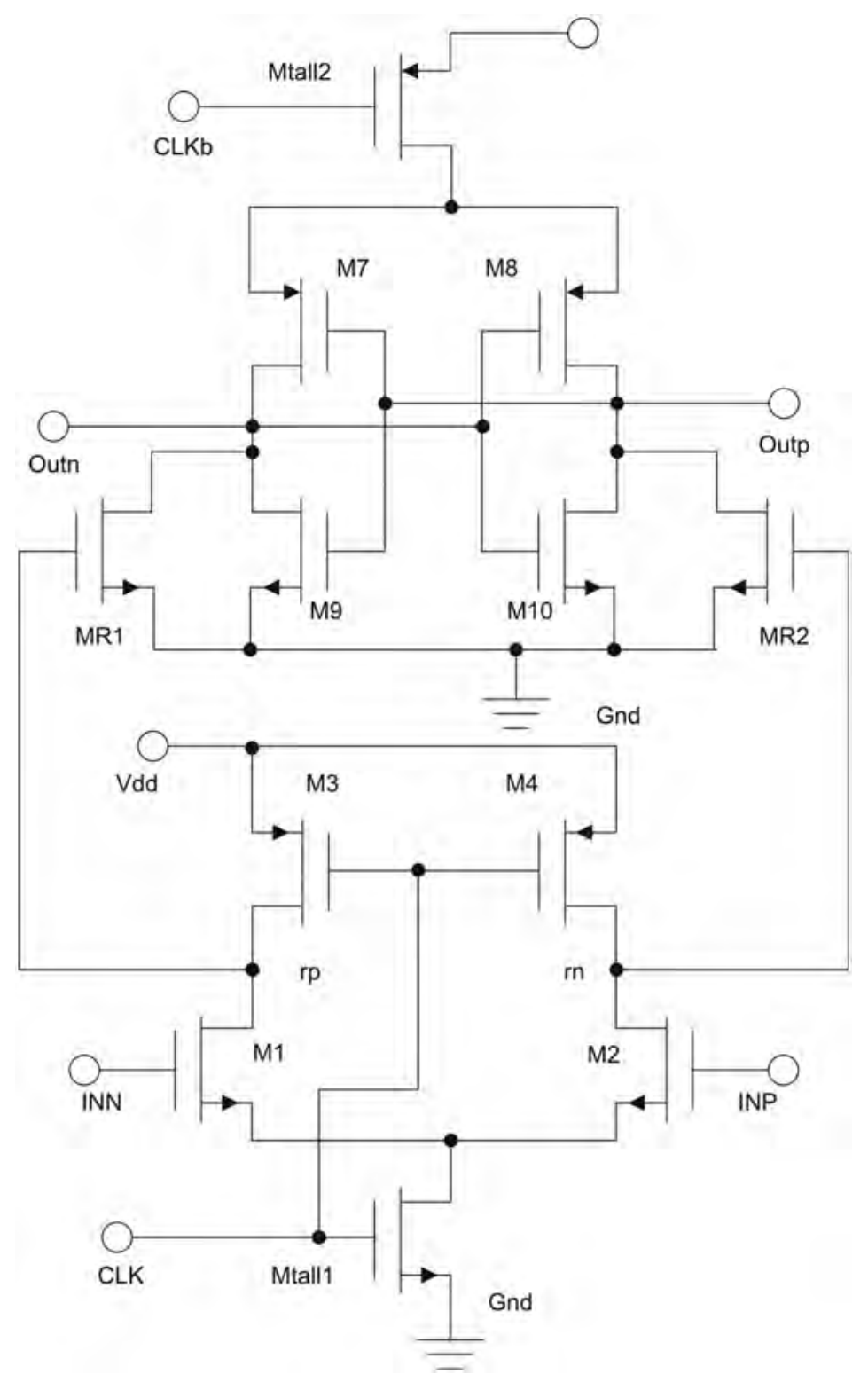

Figure 60.3 Conventional double tail current DLC. 
for achieving an integration time and for fast latching. It works in two phases similar to STDLC, i.e. reset phase and regeneration phase.

In the first stage, i.e. the reset stage, the clock is maintained low, current sources Mtail1, Mtail2 are switched OFF and this results in zero static power [6]. The ON transistors M3, M4 pulls the terminal fp\&fn to $V_{\text {dd }}$. Similarly MR1 and MR2 transistors are switched ON and pull Outp and Outn towards GND. In second stage Mtail1, Mtail2 are turned ON by making transistor M3, M4 to switch OFF at high CLK. Therefore $\mathrm{fn}$ and fp get discharged with different speeds and when any one of the output of reset stage reaches below $V_{\text {th }}$ of MR1 and MR2 transistors turn OFF. In a latch there are two outputs due to the positive feedback: one reaches $V_{\mathrm{dd}}$ and other reaches to ground. The amplified differential input voltage is driven to cross coupled inverters by the middle stage and reduces kickback noise [10-12]. It has the advantages of reduced overall delay and low operating voltage, whereas higher supply voltage, higher power consumption are the disadvantages.

\section{Proposed comparator (PSC-DTDLC)}

The architecture of a proposed designed comparator, i.e. the proposed shared charge double tail current dynamic latch comparator (PSC-DTDLC), is represented in Figure 60.4. At stage one i.e. charge sharing stage the clock signal is set to ground (CLOCK $=0$ ) and both Mtail1 and Mtail2N transistors are turned OFF which prevents consumption of static power, whereas transistors $\mathrm{M} 3, \mathrm{M} 4$ pull down $\mathrm{fn}$, fp terminals to $\mathrm{V}_{\mathrm{dd}}$ by turning the transistors MR1 and MR2 to ON state. Here PMOS transistor shorts the Outp and Outn output terminals and makes it a shared charge transistor i.e. a charge is shared between Outp and Outn terminals and it will be approximately at $0.7 \mathrm{~V}$ i.e. half of that of the supply voltage $\mathrm{V}_{\mathrm{dd}}$. The evaluation stage is the second stage which is also called the decision-making stage where the clock signal is set at high supply voltage $\left(C L O C K=V_{d d}\right)$, NMOS transistors Mtail1 and Mtail2N will be ON, PMOS transistors M3, M4 will move to OFF state. Therefore between $\mathrm{fn}$ and $\mathrm{fp}$ node an input differential voltage is formed which passes to cross coupled latch and provides a faster decision. Now, through inputs M1 and M2 transistors fn and fp terminals start discharging at different rate at the specified input voltages.

Assuming that $\mathrm{V}_{\text {INP }}<\mathrm{V}_{\text {INN }}$ at that time the Outp i.e. output terminal and its voltage at fp terminal discharges faster than that of the Outn i.e. output terminal and its voltage at fn terminal and vice versa. By making M7 transistor to ON and Outn terminal charges to $\mathrm{V}_{\mathrm{dd}}$ whereas M8 transistor remains in OFF state. On other hand when Outp is discharged to GND, M9 transistor will go in to a cut-off state and draws zero current from $\mathrm{V}_{\mathrm{dd}}$ after a short time. Therefore at the end of the regeneration phase Outn $=V_{d d}$ and Outp $=$ GND. The proposed method has the advantages such as lower input offset voltage over a common mode voltage, less kickback noise, less transistor stacking and a better optimization is achieved in terms of power and delay as it has two separate tail current transistors. The performance of the proposed comparator can be further enhanced by including other techniques at circuit [13] and transistor level [14] as per their compatibility with the existing circuit.

\section{Simulation and results}

In this section, we have done the implementation of the proposed comparator with the other two discussed comparators and the proposed comparator is simulated using Cadence virtuoso schematic editor tool in $90 \mathrm{~nm}$ technology at $1 \mathrm{GHz}$ clock frequency, common 


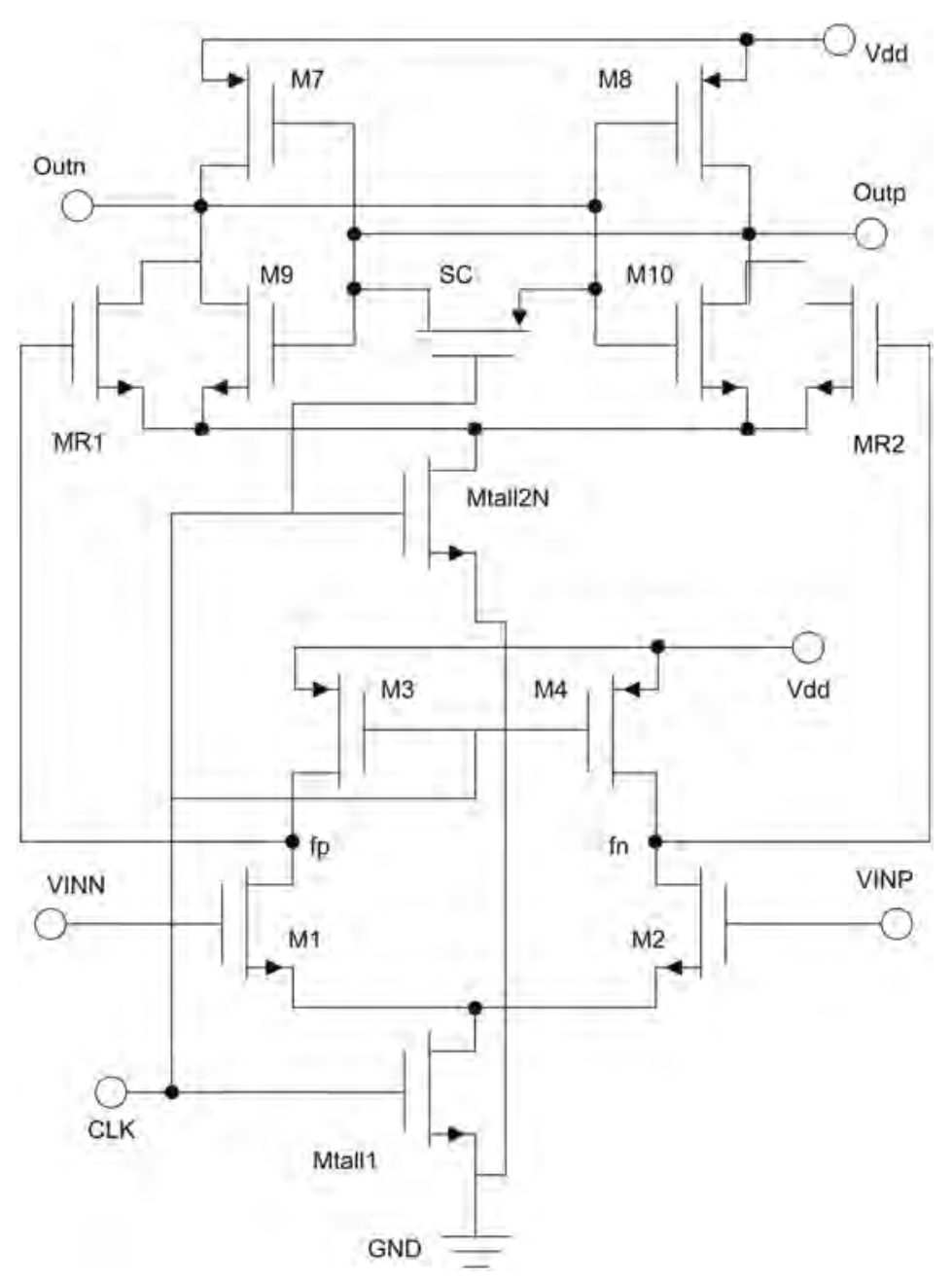

Figure 60.4 Proposed shared charge double tail current DLC.

Table 60.1 Comparisons of different comparator architectures

\begin{tabular}{llll}
\hline Comparator architecture & Delay $($ ps) & Power $(\mu w)$ & Power delay product $(f J)$ \\
\hline STDLC & 63.43 & 32.51 & 2.06 \\
DTDLC & 53.41 & 21.23 & 1.13 \\
PSC-DTDLC & 41.27 & 24.23 & 0.99 \\
\hline
\end{tabular}

mode voltage at $0.7 \mathrm{~V}$, load capacitance at $7 \mathrm{pf}, \mathrm{V}_{\mathrm{dd}}=1 \mathrm{~V}$, and temperature at $27^{\circ} \mathrm{C}$ and the results are tabulated and plotted. Comparison of different comparator architectures based on delay (ps), power delay product $(\mathrm{fJ})$ and power $(\mu \mathrm{w})$ is given in Table 60.1 and analysis of various comparators are shown in Figure 60.5. 


\section{Comparator Analysis}

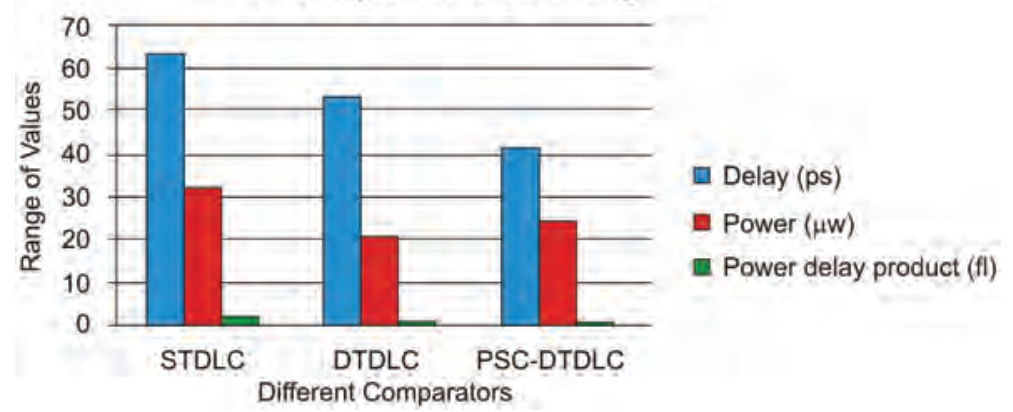

Figure 60.5 Comparisons of different comparator.

\section{Conclusion}

Here a novel latch comparator known as "Proposed Shared Charge Double Tail dynamic latch Comparator" (PSC-DTDLC) has been designed and analysed using CMOS technology at $90 \mathrm{~nm}$. Its circuit is simulated by the Cadence virtuoso schematic editor tool and results are obtained based on power delay, offset voltage, power consumption and power delay product and found to be promising compared to the other comparators that were designed using the same tool.

\section{References}

[1] P. Nandhini, P. Prasana, K. Jaishri, G. Muthumeenakshi, "Design of FinFET based low power dynamic comparator," SSRG International Journal of Electronics and Communication Engineering (SSRG - IJECE), Mar 2019,.

[2] Samaneh Babayan-Mashhadi, Reza Lotfi, "Analysis and design of a low voltage low-power double-tail comparator," IEEE Trans. VLSI Systems Feb 2014.

[3] B. Goll and H. Zimmermann, "A comparator with reduced delay time in 65-nm CMOS for supply voltages down to 0.65," IEEE Trans. Circuits System II, Exp. Briefs, vol. 56, no. 11, pp. 810-814, Nov. 2009.

[4] Mir Muntasir Hossain, Satyendra N. Biswas, "Analysis and design of a 32nm FinFET dynamic latch comparator," IEEE, 2019.

[5] Allen, P. E. and Douglas R. Holberg, "CMOS Analog Circuit Design", Oxford University Press, 2002.

[6] Uthaichana, Patheera and Ekachai Leelarasmee, "Low power CMOS dynamic latch comparators," Conference on Convergent Technologies for the Asia-Pacific Region, TENCON, IEEE Proceeding. 2003. 605-608.

[7] Priyesh P. Gandhi, N. M. Devashrayee, "A novel low offset low power CMOS dynamic comparator", Analog Integrated Circuits and Signal Processing (2018) 96:147-158.

[8] Schinkel, D., et al., "A low-offset double-tail latch-type voltage sense amplifier," 18th Annual Workshop on Circuits, Systems and Signal Processing, Pro RISC. Utrecht: Technology Foundation STW, 2007, 89-94.

[9] D. Xu, S. Xu and G. Chen, "High-speed low-power and low power supply voltage dynamic comparator," IEEE Electron. Lett., vol. 51, no. 23, pp. 1914-1916, Nov. 2015.

[10] Srinivasa Rao Vemu, P.S.S.N. Mowlika, S. Adinarayana, “An energy efficient and high speed double tail comparator using cadence EDA tools”, 2017, IEEE. 
[11] Vijay Savani, N. M. Devashrayee, "Analysis and design of low-voltage low-power highspeed double tail current dynamic latch comparator," Analog Integrated Circuits and Signal Processing (2017) 93:287-298.

[12] N. Bala, B. Abdul and B. Nagendra, "Domino logic based high speed dynamic comparator," IEEE Int. Innovations in Information Embedded and Comm. Systems, 2015.

[13] Sanjeet Kumar Sinha, Suman Lata Tripathi "BDD based Logic synthesis and optimization for low power comparator circuit," IEEE ICICS, pp. 37-41, 2018.

[14] Suman Lata Tripathi, Sobhit Saxena, "Asymmetric gated Ge-Si ${ }_{0.7} \mathrm{Ge}_{0.3}$ nHTFET and pHTFET for steep subthreshold characteristics," International Journal of Microstructure and Materials Properties, vol. 14 (6), pp. 497-509, 2019. 


\title{
61 Load flow studies of LV microgrid using ETAP
}

\author{
Chandan Kamble ${ }^{1}$, Javed Dhillon ${ }^{2}$ \\ ${ }^{1}$ PhD Scholar, LPU, Phagwara, Punjab, India \\ ${ }^{2}$ Assistant Professor, LPU, Phagwara, Punjab, India
}

\section{Introduction}

The microgrid (MG) is a scaled-down version of the centralized power system. It has the capability of generating, distributing and controlling the power in a small locality. Microgrid comprises micro generation, energy storage elements such as flywheels, supercapacitors, batteries, along with controllable loads to ensure efficient control credentials to perform local network operation, power electronic units like converters as shown in Figure 61.1. An advanced control system ensures those components operate in a coordinated and optimized manner.

In order to make power balance between the supply and demand, the microgrid central control (MGCC) should forecast the local generation capacity and consumer demand for the next period. If the generation exceeds the consumption, the output generation of an under control DGs should be relaxed, and during this transient condition, the surplus power is dissipated through dummy resistor loads if the overvoltage exists. On the other hand if generation is less than consumption, two ways out are possible: one can reduce/curtail the consumption or increase the generation. The particular DGs will either make ready to supply the deficit power or the part of loads will be disconnected from the microgrid as per requirement.

Due to technological development such as computer based software it is possible to study and analyse complex electrical system problems with a possible solution. The study mainly includes power flow, transient analysis, voltage profile, reactive power control and monitoring, etc. This research work focuses on the load flow study in a microgrid system consisting of solar and wind source using ETAP software.

The rest of the paper is organised as follows: Section II includes the concept of the microgrid and its ETAP. Section III is the One Line view of the study system and description; this diagram is considered under real time data used in ETAP for simulation purposes. Section IV contains load modelling and simulation of microgrid in software and finally the conclusive remarks and observations of this research work.

\section{Concept of microgrid and ETAP}

The microgrid is connected to the main utility grid and can be connected or disconnected/ islanded from the traditional grid in the case of fault condition. An energy storage system is installed to store the energy from PV and wind during availability and fed to the critical load whenever the main grid is disconnected from the microgrid to meet the demand. Microgrids may be classified in three ways as AC, DC and AC-DC microgrids. 

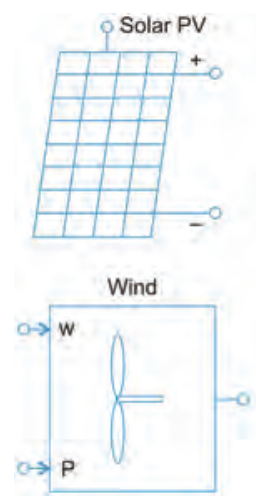
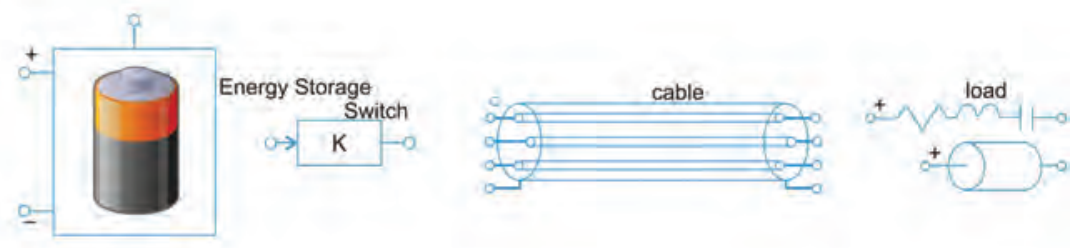

Figure 61.1 Components of microgrid [1].

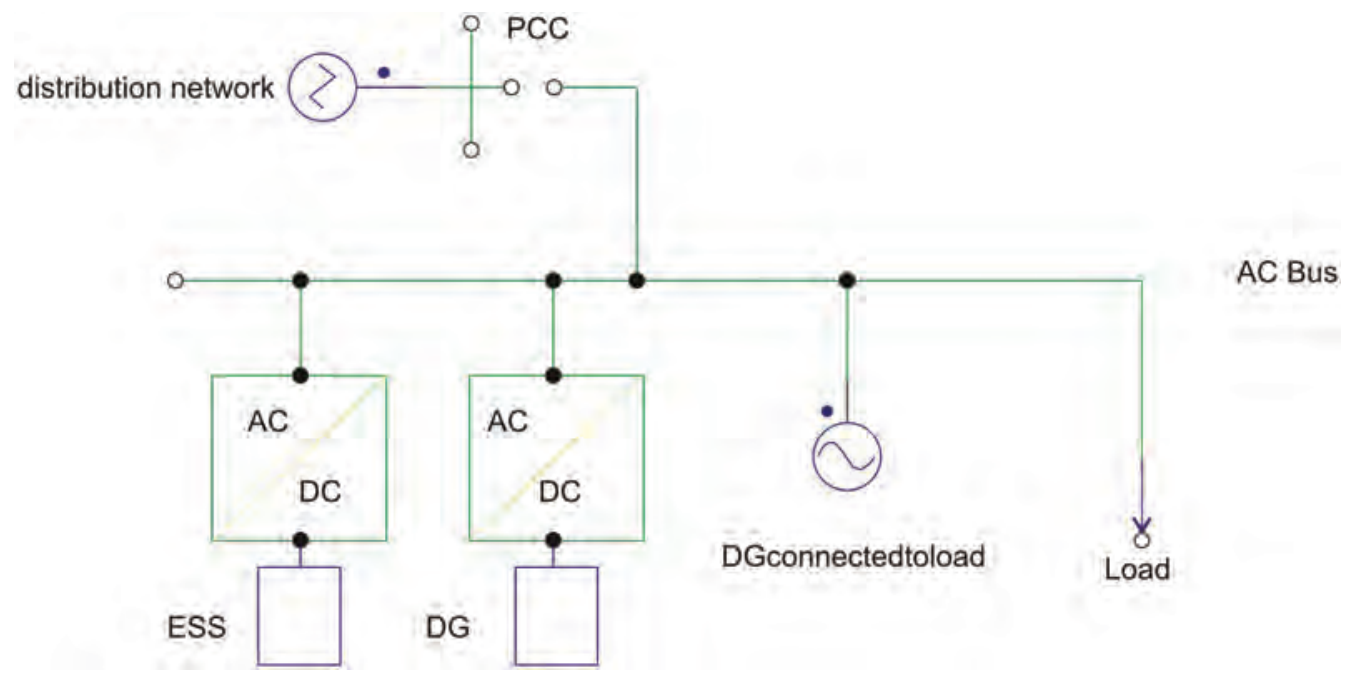

Figure 61.2 AC microgrid.

In a DC microgrid energy storage, distributed generators, DC load are connected to DC bus through converter whereas in $\mathrm{AC}$ microgrid distribution system is connected to $\mathrm{AC}$ bus through circuit breaker switches and DG, energy storage is connected to the AC bus through inverter as shown in Figure 61.2.

About ETAP: One of the application of this software is to analyse the load flow and voltage drip with more accuracy and reliability in calculation. It has salient features such as automatic device alerts, warning and evaluation of summary sheet, load flow, transient study analyser and evolved graphics that make this software the most promising load flow analysis tool nowadays. The load flow software module can also help to validate system models and obtain accurate and reliable results. Due to DG (distributed generation) coordination and performance of the microgrid is becoming very important as uncertain and stochastic behavior of solar and wind microgrid experiences voltage and power fluctuations due to which the consumer suffers from poor quality of supply. So analysing the microgrid containing the monitoring of various parameter, their loading 
modeling, simulation and parameter optimization of distribution system

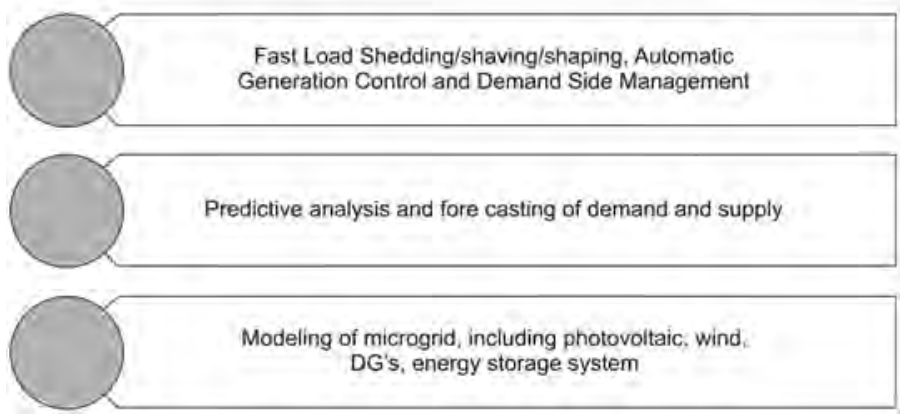

Figure 61.3 ETAP microgrid key functionality.

capacity and usage needs to be done in detail. The ETAP microgrid solution platform provides this opportunity by combining DG technologies with this software to both monitor, forecast, regulate and optimize energy supply and demand for the smart grid applications.

ETAP software allows one to design, model, analyse, optimize and control the microgrid. Using the ETAP microgrid solution it is possible to design a model and validate it with real time data.

\section{System description}

The main focus of the study is to check the effectiveness of the microgrid when operated under islanded mode. For that a real time example has been taken where an education premises is supplied by the main grid to supply the demand but when due to outage continuity is maintained through solar PV array installed on the terrace of the building described in Figures 61.4 and 61.5. The two cases are intentional islanded mode and grid connected mode. For both cases bus loading and real and reactive power have been examined. One power transformer of $250 \mathrm{kVA}$ is available to supply the demand. The cables of around $950 \mathrm{~m}$ long are going to three wings. Based on the survey an ETAP model is designed and load flow is performed.

\section{Results and conclusion}

The aim of study is to see the effectiveness of a PV operated microgrid system. In Figure 61.6 bus 3 loading is $95.41 \%$ loaded when the power is available from the main grid but when the power is fed from solar bus 2 is $96.83 \%$ and bus 3 is $93.04 \%$ loaded as shown in Figure 61.7. This means that load bus is expected to have $0.4 \mathrm{kV}$ but actually it is 0.374 . This condition is called the undervoltage condition (range of bus undervoltage is set to $95 \%$ ).

A further load flow report is obtained in both the cases, viz. grid connected and isolated grid connected mode, as shown in Table 61.2. 


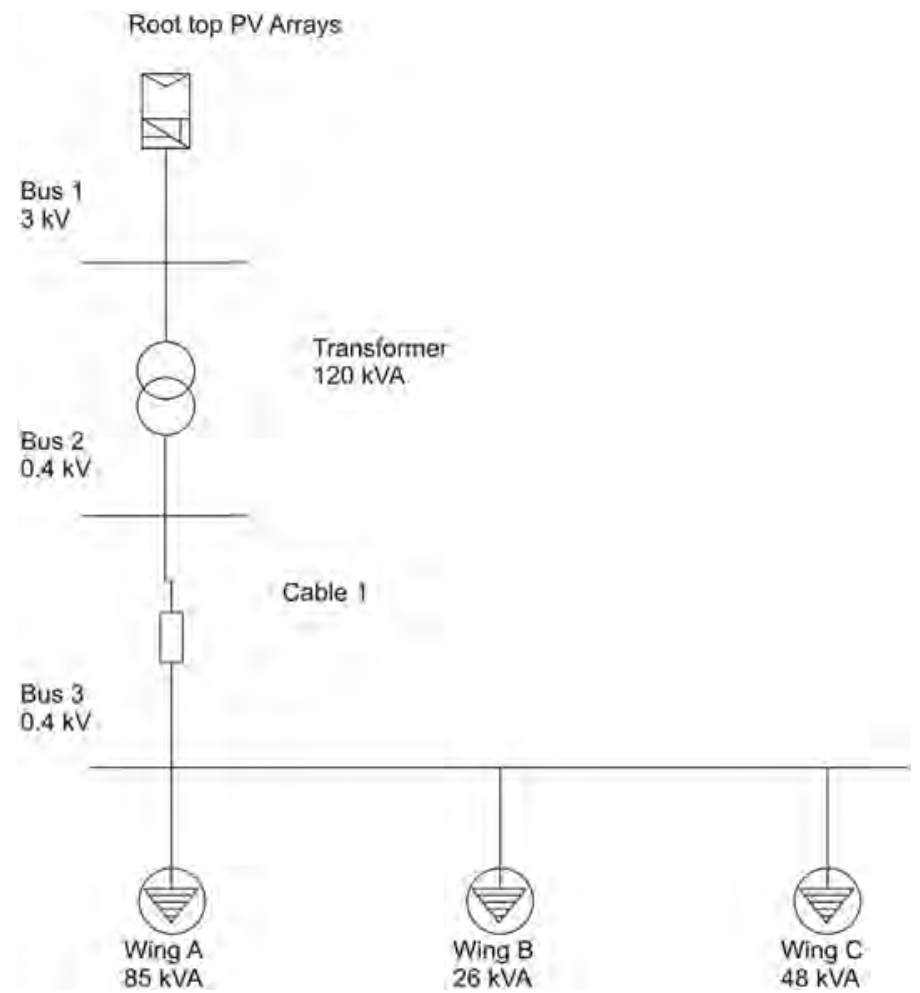

Figure 61.4 Study system.

Table 61.1 Survey of connected load of wing B

\begin{tabular}{|c|c|c|c|c|c|c|c|}
\hline Sr. no. & Room no./lab & Light & Fan & Others & $\begin{array}{l}\text { Computer } \\
\text { load }\end{array}$ & $A C$ & Total \\
\hline 1. & Classroom & $2800 \mathrm{~W}$ & 2450W & & & & $5250 \mathrm{~W}$ \\
\hline \multirow[t]{4}{*}{2.} & Corridor ground floor & $320 \mathrm{~W}$ & & 1000W & & & 2440W \\
\hline & First floor & $280 \mathrm{~W}$ & & & & & \\
\hline & Second floor & $360 \mathrm{~W}$ & & & & & \\
\hline & Third floor & $480 \mathrm{~W}$ & & & & & \\
\hline 3. & Computer lab & $360 \mathrm{~W}$ & $50 \mathrm{~W}$ & & 9860W & $17412 \mathrm{~W}$ & $27682 \mathrm{~W}$ \\
\hline 4. & Chemistry lab & $1040 \mathrm{~W}$ & $1470 \mathrm{~W}$ & 1000W & & & $3510 \mathrm{~W}$ \\
\hline 5. & Dept. of chemistry & $280 \mathrm{~W}$ & $250 \mathrm{~W}$ & $1000 \mathrm{~W}$ & & & $1530 \mathrm{~W}$ \\
\hline 6. & HOD office & $320 \mathrm{~W}$ & 200W & 1000W & & & $1520 \mathrm{~W}$ \\
\hline 7. & NA lab & $480 \mathrm{~W}$ & $180 \mathrm{~W}$ & $1000 \mathrm{~W}$ & & & $1660 \mathrm{~W}$ \\
\hline 8. & Dept. of physics lab & $760 \mathrm{~W}$ & $1280 \mathrm{~W}$ & & & & 2040W \\
\hline 9. & Knowledge centre & $240 \mathrm{~W}$ & 200W & & & & $440 \mathrm{~W}$ \\
\hline 10. & Power electronic & $720 \mathrm{~W}$ & $800 \mathrm{~W}$ & & & & $1520 \mathrm{~W}$ \\
\hline
\end{tabular}



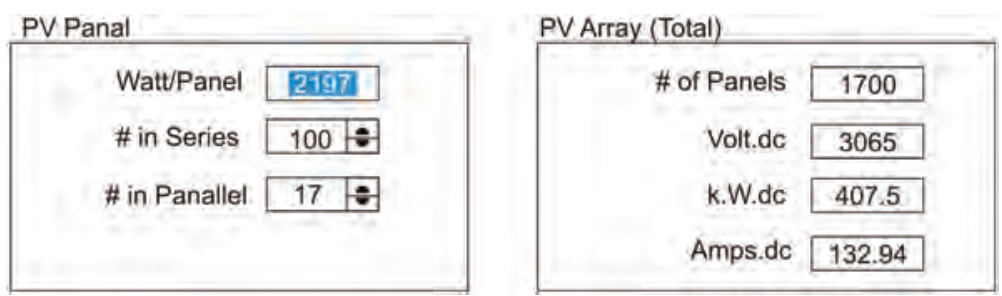

Figure 61.5 PV array rating.

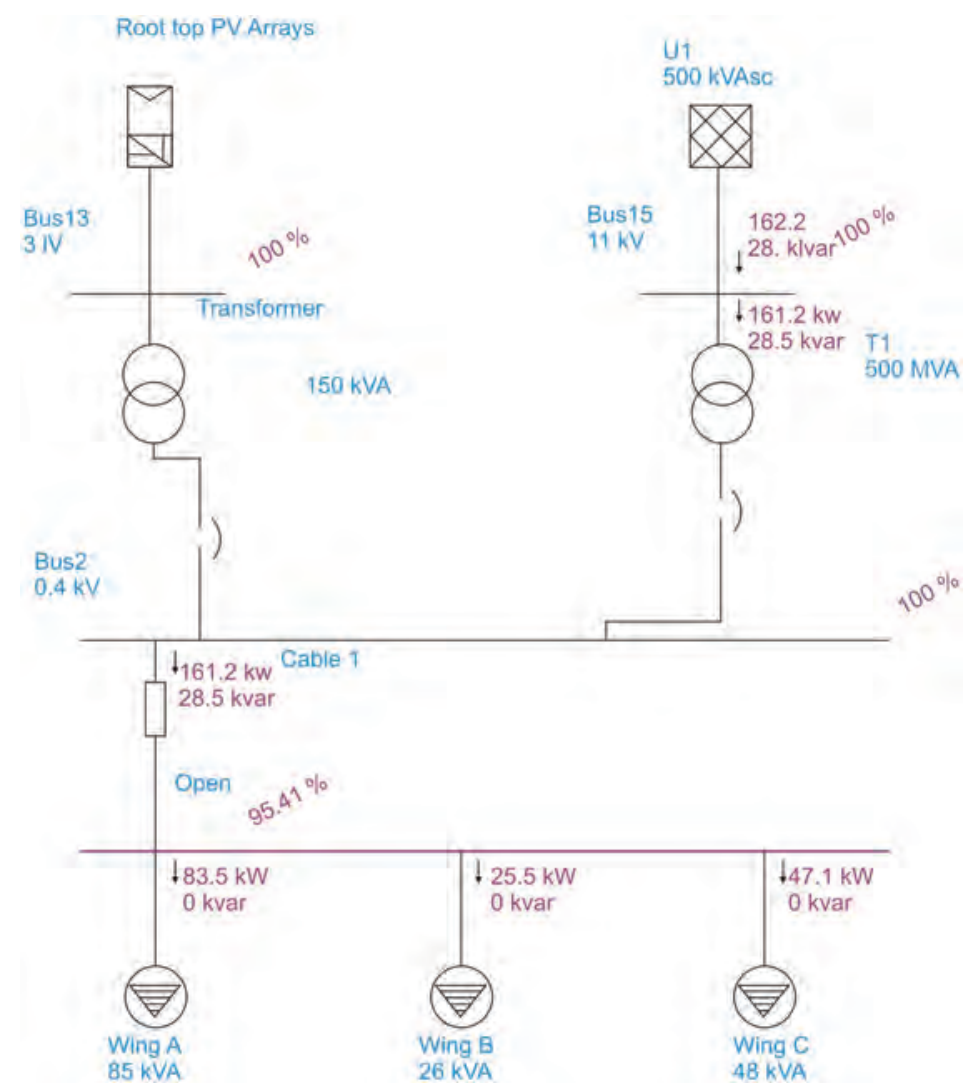

Figure 61.6 Simulation of load flow during grid connected mode.

\section{Conclusion}

From the analysis it is found that when the microgrid is operated in off-grid connected mode voltage stability and power instability happens. It is also observed that voltage drop is not only the line impedance but also the type of load connected. With the help of load flow analysis it is possible to analyse every element connected to the microgrid and its operating condition. The ETAP Load Flow Analysis module helps in calculating the bus voltages, branch power factors, currents, and power flows under steady state and 


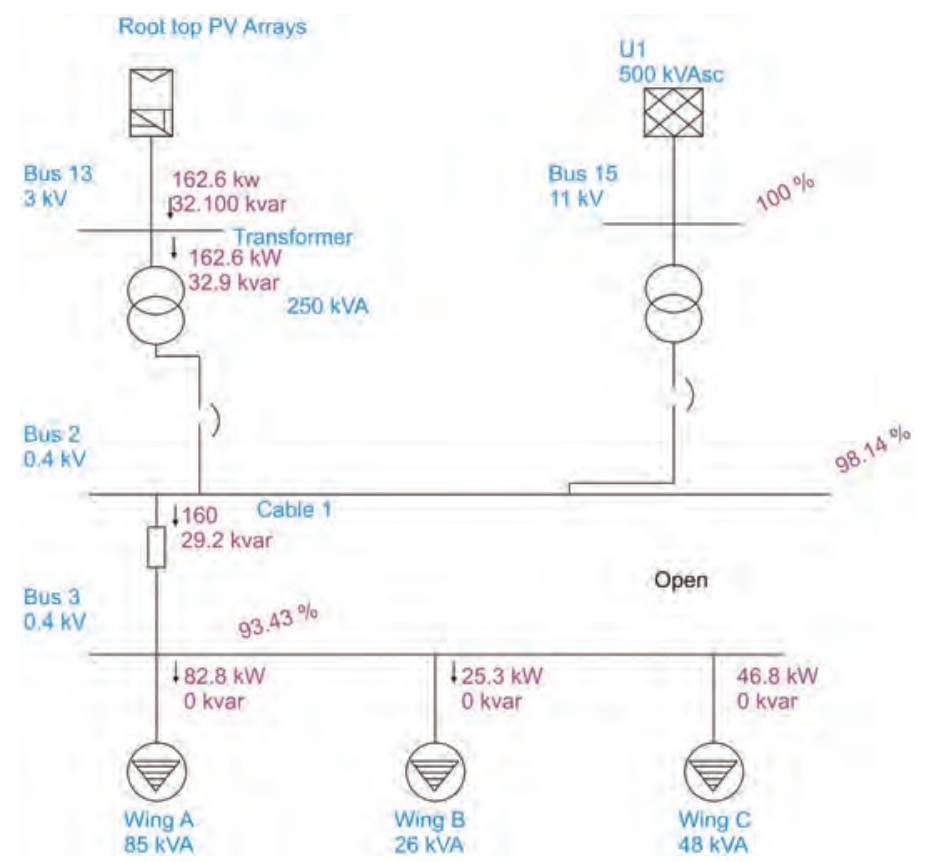

Figure 61.7 Simulation of load flow during off-grid connected mode.

Table 61.2 Load flow report

\begin{tabular}{lllc}
\hline Bus & Bus voltage $(k V)$ & $\begin{array}{l}\text { Bus voltage loading in } \% \\
\text { (grid connected mode) }\end{array}$ & $\begin{array}{l}\text { Bus voltage loading \% } \\
\text { (off-grid connected mode) }\end{array}$ \\
\hline BUS 2 & 0.4 & $100 \%$ & $98 \%$ \\
BUS 3 & 0.4 & $95 \%$ & $93 \%$ \\
BUS 13 & 3 & $100 \%$ & $100 \%$ \\
\hline
\end{tabular}

transient conditions applied to the electrical system. ETAP allows you to select from several different methods in order to achieve the best calculation efficiency. Further scope of work is to study the microgrid system with uncertainty of PVs, voltage and power imbalance and its solution.

\section{References}

[1] Bevrani H, Francois B, Ise T, Microgrid Dynamics and Control, 1st edition. NJ: Wiley.

[2] J. Arockiya Xavier Prabhu, Kaustubh S. Nande, Smriti Shukla, Chirag N. Ade, "Design of electrical system based on short circuit study using ETAP For IEC projects", Proc. IEEE International Conf. 6th Power Systems (ICPS), pp. 1-6, Oct. 2016.

[3] Muhammad Aman Ullah, Arslan Qaiser, Qamar Saeed, Abdul Rehman Abbasi, Iftikhar Ahmed, Abdul Qadir Soomro, "Load flow voltage stability and short circuit analyses and remedies for a $1240 \mathrm{MW}$ combined cycle power plant using ETAP”, Proc. IEEE International Conf. ICIEECT., pp. 1-6, May 2017. 
[4] N. Nisar, M. B. Khan, S. Gondal and M. Naveed, "Analysis and optimization of 132KV grid using ETAP," 2015 Power Generation System and Renewable Energy Technologies (PGSRET), pp. 1-6Islamabad, 2015.

[5] Kamble CS and Rewatkar Rajni, "Load-flow analysis of distribution systems using ETAP" International Journal of Advanced Engineering, Management and Science, 2017. 


\title{
62 A universal memory that never runs out of steam
}

\author{
Urvashi Sharma ${ }^{1,2, *}$, Sachin Mishra ${ }^{2, *}$, Gulshan \\ Kumar $^{1,3, *}$, Reji Thomas ${ }^{1,4, *}$
}

\author{
${ }^{1}$ Division of Research and Development, Lovely Professional University, \\ Punjab, India \\ ${ }^{2}$ School of Electronics and Electrical Engineering, Lovely Professional University, \\ Punjab, India \\ ${ }^{3}$ School of Computer Science and Engineering, Lovely Professional University, \\ Punjab, India \\ ${ }^{4}$ School of Chemical Engineering and Physical Sciences, Lovely Professional \\ University, Punjab, India
}

\section{Introduction}

Digital semiconductor memories are inevitable in computers and use electronic assembly for information storage and processing. The semiconductor memories are categorized as: (1) volatile memories (loss of data with the shutdown of power); (2) non-volatile memories (NVMs) (no loss of information with the shutdown of power). random-access memory (RAM) is volatile memories that support read and write of data multiple times; they can be further classified into Static random-access memory (SRAM) and dynamic random-access memory (DRAM) [1-3], respectively, used as cache memory and primary memory. The "cache memory" in the central processing unit (CPU), configured with six transistors (i.e. 2cross coupled transistors and 4-pass transistors) is an SRAM having less power required for retention/write/re-write and excellent read-out [4]. The cross-coupled transistors are used to store memory bits in inverter pairs of complementary metal-oxide semiconductor (CMOS). The p-MOS configuration is being replaced with a 4-pass transistor structure of high impedance resistors. This reduces the storage of information with an increase in power dissipation. Synchronous DRAM (SDRAM) and mobile DRAM (MDRAM) come into the group of high-density volatile memories. In DRAM, the arrangement of 1-capacitor (used for storing) and 1-transistor form a cell used for storing one bit of information. The size of this single cell is $6 \mathrm{~F}^{2}$ (" $\mathrm{F}$ " is the smallest feature size) having read/write time $\sim 10 \mathrm{~ns} / 10 \mathrm{~ns}$. The DRAM is generally termed as "primary memory" [4]. The capacitor is at logic " 1 " considered as charged while discharged state with logic " 0 ". These logic " 1 " and " 0 " designate the data storage on the capacitor. The inconsistent power requirement in refreshing the bits after every few milliseconds (ms) raises the complication in processors. This is due to the constant requirement of power for refreshing the state of the cell after every few ms. When battery life is limited, continuous consumption of power is a concern for prolonged operations.

The Flash and read-only memory (ROM) are examples of non-volatile memories. Erasable-programmable read-only memory (EPROM), electrically erasable programmable

\footnotetext{
*Emails: aurvashi.11814489@lpu.in, bsachin.20444@lpu.co.in, cgulshan3971@gmail.com, dreji.22648@1pu.co.in
}

DOI: $10.1201 / 9781003129103-62$ 


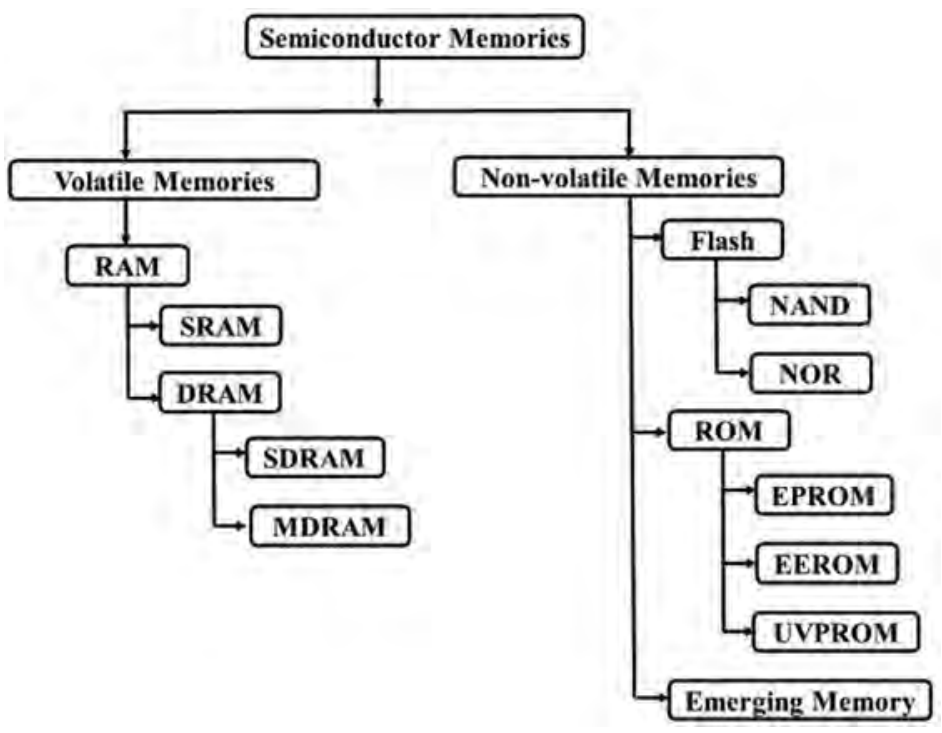

Figure 62.1 The arrangement of memories regarding their functional traits.

read-only memory (EEPROM) and ultraviolet erasable read-only memory (UVEPROM) are the subclasses of ROM. Flash memory based on floating-gate transistors (FGT) is considered as secondary or external memory with write/erase time $\sim 1 \mathrm{~ms} / 10 \mathrm{~ms}$ [4]. It is similar to the metal-oxide-semiconductor field-effect transistor (MOSFET) along with additional gate (termed as the floating gate (FG)) in between control gate and body. The NOR and NAND [5] are the classifications of Flash. As compared to page-write and cell size of NAND, NOR consists of large cell size with direct-write properties.

For over 30 years, since the evolution of solid-state technologies, DRAM, SRAM and Flash dominated the semiconductor industry for memory. However, features such as low energy consumption, non-volatility, density and fastness are prerequisites of the future semiconductor memory. The emerging memories mentioned in Figure 62.1 have the potential to replace any of the existing memory technology for information processing and storage. Such an accomplishment in memory may result in "universal memory" that can replace conventional memories. In short, this "universal memory" will be the combination of the high speed of SRAM, NV characteristics of FLASH, great endurance of DRAM and SRAM and large scalability of DRAM and Flash [6] (see Table 62.1). This communication is about a memory that can be considered as a memory of universal acceptance.

\section{Emerging memories}

The aforementioned memories have been scaled down successfully to achieve higher speed and large density at a lower production cost from one technology generation to the next by following Moore's law. However, the integrated circuits are approaching their physical limits (where the quantum limitation comes to define further miniaturization) in the near future and we need more from Moore's law that takes us to 'more than Moore's' world right now. The emerging memories [7] resistive random-access memory (RRAM) [8], magneto-resistive random-access memory (MRAM) [6], phase-change random-access memory (PCRAM) [7] and ferroelectric memory [5,9] (Figure 62.2) are considered for the replacement of current technologies in that direction and are discussed briefly here in this 
Table 62.1 Characteristics required for universal memory

\begin{tabular}{lllll}
\hline Memory hierarchy & $\begin{array}{l}\text { Speed (writelerase } \\
\text { time) }\end{array}$ & $\begin{array}{l}\text { Scalability (cell } \\
\text { size) }\end{array}$ & $\begin{array}{l}\text { Endurance } \\
\text { (cycles) }\end{array}$ & $\begin{array}{l}\text { Energy } \\
\text { efficiency }\end{array}$ \\
\hline SRAM & High $(0.3 \mathrm{~ns} / 0.3 \mathrm{~ns})$ & Low $\left(140 \mathrm{~F}^{2}\right)$ & High $\left(10^{6}\right)$ & Volatile \\
DRAM & High $(10 \mathrm{~ns} / 10 \mathrm{~ns})$ & High $\left(6 \mathrm{~F}^{2}\right)$ & High $\left(10^{6}\right)$ & Volatile \\
FLASH & Low $(1 \mathrm{~ms} / 10 \mathrm{~ms})$ & High $\left(10 \mathrm{~F}^{2}\right)$ & Low $\left(10^{5}\right)$ & Non-volatile \\
Universal memory & High & High & High & Non-volatile \\
\hline
\end{tabular}

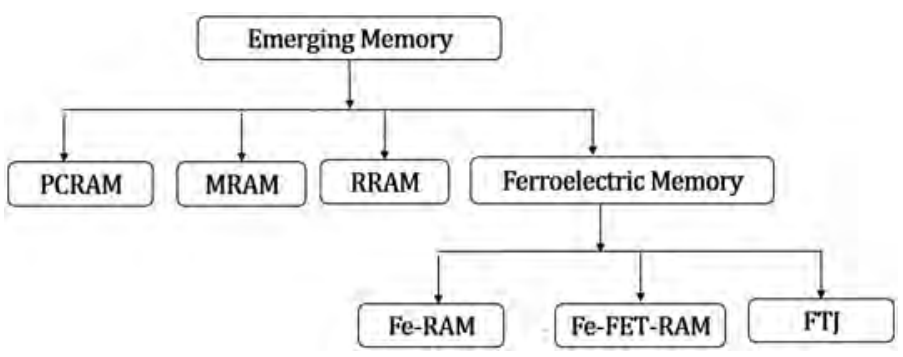

Figure 62.2 The classification of emerging memories.

session. Systematic introduction of oxides and nitrides (e.g. $\mathrm{SiO}_{2} / \mathrm{SiON} / \mathrm{HfO}_{2} / \mathrm{ZrO}_{2} / \mathrm{Ta}_{2} \mathrm{O}_{5}$ ) from one node to another prolonged the ultra-large-scale integration, be it a process or memory so far. However, the industry is in a situation where more and more oxides need to be introduced to move further as the currently used oxides may not stand the extreme downscaling.

An RRAM [10,11] is a type of resistor having switchable electrical resistance and is considered for the next generation NVM devices. These memories store information in the form of a resistance state in the MIM (metal-insulator-metal) [5,8] configuration. The magnitude of resistance depends upon the voltage applied. These memories possess low power consumption, modest structure, high speed, and scalability. The RRAM has been realized with binary and complex metal oxides, chalcogenides, organic materials and solid electrolytes. Initially, it was investigated with single layer tantalum oxide but recently multilayer structures perform better in terms of scaling potential and cycling. RRAM and its mechanism can be categorized into (1) anion-type oxygen ions (negative charge), (2) cation-type resistive random-access memory (CRRAM), (3) carbon-based resistive random-access memory (CBRRAM) and (4) oxide-based-electrode resistive randomaccess memory (OERRAM) [10]. The anion-type and cation-type RRAM are subjected to a negative charge and redox reaction/migration of positive charge respectively. The carbon-based properties and its composition are included in CBRAM while switching mechanisms and electrode based on oxide materials are subjected to oxide-based electrode RRAM [12]. Among these, anion-type RRAM with its excellent reliability is much well-suited for CMOS fabrication technology. It is a metal/silicon dioxide-based RRAM. CBRAM involves the migration of doped ionizing cations along with redox reactions. It is capable of multi-bit storage, large dynamic margin and small operation voltage. These suffer from poor reliability and compatibility for integrated circuits (IC) fabrication. CBRRAM or $\alpha-\mathrm{C}: \mathrm{H}$ centred RRAM is simple composition memory having a low cost, excellent endurance, great retention of data, multi-bit storage and self-protection for forming process. These involve addition reaction and C-C bond lengths. Lastly, oxidebased electrode RRAM is the most well-matched for the optoelectronics industry and less 
operating voltage with good consistency for IC technology. These are more compatible with conventional semiconductor fabrication process of semiconductor.

The PCRAM is one of the family members of resistive memory, different phases of the same material show different resistance states. Generally, a thin layer of chalcogenide, e.g., $\mathrm{Ge}_{2} \mathrm{Sb}_{2} \mathrm{Te}_{5}$, sandwiched between two electrodes is used for this type of memory. With the application of heat or energy, chalcogenide materials undergo switching from amorphous state to crystalline state $[5,13]$. These memories perform well during the course of random access with reasonable write throughput, density and durability as NAND Flash. However, questions like optimizing write latency, energy-saving and endurance slowed down its manufacturing. Another emerging technology, MRAM [5,6,13,14], uses the properties of magnetic materials and has high endurance and fast read/write operations. Siemens, Motorola, Hitachi, Hewlett Packard and many more examined a lot on MRAM for several years. MRAM comprises combining the feature of high read/writes speeds with unlimited endurance. The storage of data is realized using magnetic tunnel junction (MTJ), i.e. two ferromagnetic layers are separated using an insulating oxide layer. Spin-torque-transfer RAM (STT-RAM) and spin-orbit-torque RAM (SOTRAM) are two promising technologies in this category of MRAM. IBM and Samsung invented the spintransfer memory and Flexible foundries memory respectively and collaborated for the manufacturing of STT-MRAM $[15,16]$. The merits like low power consumption, nonvolatility (flash, tape storage and hard drives) and high speed (faster than SRAM) make this technology very interesting. Another variant, magneto-electric memory, manufactured by Cornell University consumes less energy (10-time less than STT RAM manufactured by IBM and Samsung) [15]. Even though a promising memory, switching current distribution in MRAM is a challenge as it restricts large scale manufacturing. With this, thermal instability, failure and degradation of the junction are also some problematic aspects with MRAM. Compared with PCRAM [17] and MRAM [14], RRAM offers lower energy consumption and greater density respectively. The RRAM also offers better performance than flash and good stability with $10 \mathrm{~nm}$ technology [18].

Some of the dielectric materials, ferroelectric materials are also used in non-volatile memories. The perovskite materials, $\mathrm{PbTi}_{\mathrm{X}} \mathrm{Zr}_{1} \times \mathrm{O}_{3}(\mathrm{PZT})$ and layered strontium-bismuthtantalite alloy (SBT) are the ferroelectric choice for this so far [5]. This non-volatile memory is termed as ferroelectric RAM (Fe-RAM). The cell construction is the same as DRAM (1T-1C) with a non-linear ferroelectric capacitor instead of a linear dielectric capacitor [9]. Fe-RAMs are based on remnant polarization at zero applied electric field in ferroelectric crystal. These have the ability to switch polarization $( \pm \mathrm{Pr})$ with an external electric field [5]. It has the non-volatility of Flash, but having demerits of lower density (<DRAM) and high manufacturing cost ( SRAM). Fe-RAM is used in local area network (LAN) bypass, advanced metering, automotive shift-by-wire, process control in industries, navigation, solid-state drive (SSD), gaming, motion control etc. The widely investigated ferroelectricmemories possess low voltage to $0.9 \mathrm{~V}$, endurance more than $10^{13}$ write/read cycles, write speed of $100 \mathrm{~ns}$ at low voltage with retention of about 10 years [19]. However, the low density is the hindrance in replacing Flash, EEPROM, DRAM and SRAM to realize a "Universal Memory" with ferroelectric and this aspect is discussed in the following section.

\section{Universal memory}

The SRAM, Flash and DRAM bear complementary properties suited for the cache, data storage, and active memory respectively. But as mentioned above, each of these memories has demerits when high-speed operation with huge data handling capability is required. Such issues of these memories can be overcome with alternatives or so-called emerging memories. Among those emerging memories, ferroelectric memories are of great 
interest due to the fastness in operation and its radiation resistance. The Fe-RAM started with boundless potential. But scaling the lateral size of the ferroelectric capacitor to less than $100 \mathrm{~nm}$ resulted in underachieving the high density. In fact, the polarization in the nano-sized ferroelectric capacitor might not be enough to provide a suitable signal for detection. Thus, 3-D capacitors were proposed to increase the area vertically to achieve large signal strength for error-free detection. However, it is very difficult to develop 3D ultra-thin ferroelectric films with conformity (i.e., uniform film thickness around the high-aspect-ratio of the 3-D structure). Henceforth, Fe-RAM 3-D capacitor technology for high-density is not considered rigorously. The ferroelectric field-effect transistor ( $\mathrm{Fe}-$ FET) [20] and ferroelectric tunnel junctions (FTJ) [21] are the other two types of "ferroelectric memory" considered for the high density. Configuration of 1-transistor instead of $1 \mathrm{~T}-1 \mathrm{C}$ with the gate dielectrics is replaced with the ferroelectric layer as the first option to increase the density of the memory. This memory (Fe-FET) has the potential to replace DRAM. But inter-diffusion with semiconductor deteriorates the junction and retention of the data is a concern and hence Fe-FET with ferroelectric directly on semiconductor has not been considered rigorously these days.

Fe-RAM and Fe-FET memories have destructive readout; information is lost while reading and requires re-writing after each read operation. So a new paradigm with nondestructive readout has been proposed using FTJ with high density [5,21]. It is a twoterminal device with an ultra-thin ferroelectric layer between two metal electrodes. The applied field modulates the height of the barrier that further alters the tunnelling current across the stack. This change in tunnelling current due to the polarization reversal results in two resistance states. This tunnelling electro-resistance (TER) of FTJ and its bi-stability are exploited in non-volatile memories. Compared to other ferroelectric memories, FTJ has long retention times and superior endurance.

\section{Conclusion}

The article deliberates the possibility of a "Universal Memory" that has the combined features of Flash memory's non-volatility, SRAM's fastness, DRAM's high scalability and DRAM/SRAM's endurability. Hence, the prospects of emerging memories viz., resistive RAM, magnetic RAM, phase-change RAM and ferroelectric RAM are discussed. Among the ferroelectric RAM, Fe-RAM, Fe-FET and FTJ are discussed in terms of density, retention and speed. FTJ, which is not rigorously considered so far, can be used for realizing the universal memory as it can be manufactured in high density, operated with non-destructive read-out and high speed. FTJ has much potential if a CMOS-compatible ferroelectric material is discovered for the practical application. However, the fabrication, design, conception and modelling of non-volatile memories is still a subject of intense research.

\section{Acknowledgements}

Financial support from the Science and Engineering Research Board (SERB), Department of Science and Technology (DST), Govt. of India for the project "CMOS compatible Ferroelectric Tunnel Junction for Universal Memory" under the Core Research Grant CRG/2018/003539 is gratefully acknowledged.

\section{References}

[1] J. F. Scott and C.A.P. D. Araujo, Ferroelectric memories, Science, vol. 246, pp. 1400-1405, 1989.

[2] C. A. P. D. Araujo, L. D. Mcmillan, B. M. Melnick, J. D. Cuchiaro and J. F. Scott, Ferroelectric memories, Ferroelectrics, vol. 104, pp. 241-256, 1990. 
[3] M. Melanotte, R. Bez and G. Crisenza, Nonvolatile memories - status and emerging trends, Microelectronics Engineering, vol. 15, pp. 603-612, 1991.

[4] D. S. Jeong, R. Thomas, R. S. Katiyar, J. F. Scott, H. Kohlstedt, A. Petraru and C. S. Hwang, Emerging memories: resistive switching mechanism and current status, Reports on Progress in Physics, vol. 75, pp. 076502-1-31, 2012.

[5] R. Bez and A. Pirovano, Non-volatile memory technologies: emerging concepts and new materials, Materials Science in Semiconductor Processing, vol. 7, pp. 349-355, 2004.

[6] R. Cowburn, The future of universal memory, Materials Today, vol. 6, pp. 32-38, 2003.

[7] M. Asadinia and H. S. Azad, Chapter One: Introduction to non-volatile memory technologies, Advances in Computers, vol. 118, pp. 1-13, 2020.

[8] M. J. Rozenberg, I. H. Inoue and M. J. Sánchez, A model for non-volatile electronic memory devices with strongly correlated materials, Thin Solid Films, vol. 486, pp. 24-27, 2005

[9] H. Kohlstedt, Y. Mustafa, A. Gerber, A. Petraru, M. Fitsilis, R. Meyer, U. Bötterger and R. Waser, Current status and challenges of ferroelectric memory devices, Microelectronic Engineering, vol. 80, pp. 296-304, 2005.

[10] A. Makarov, V. Sverdlov and S. Selberherr, Emerging memory technologies: Trends, challenges, and modeling methods, Microelectronics Reliability, vol. 52, pp. 628-634, 2012.

[11] Y. Wang, K. Kang, M. Kim, H. LEE, R. Waser, D. Wouters, R. Dittmann, J. J Yang and H. Park, Mott-transition-based RRAM, Materials Today, vol. 28, pp. 63-80, 2019.

[12] T. C. Chang, K. C. Chang, T. M. Tsai, T. J. Chu and S. M. Sze, Resistance random access memory, Materials Today, vol. 19, pp. 254-264, 2016.

[13] K. Kim and S. Y. Lee, Future emerging new memory technologies, Integrated Ferroelectrics: An International Journal, vol. 64, pp. 3-14, 2004.

[14] J. Åkerman, Towards a universal memory, Applied Physics, vol. 743, pp. 2003-2006, 2005.

[15] A. I. Kingon, J. P. Maria and S. K. Streiffer, Alternative dielectrics to silicon dioxide for memory and logic devices, Nature, vol. 406, pp. 1032-1038, 2000.

[16] C.S. Hwang, Prospective of semiconductor memory devices: from memory system to materials, Advanced Electronic Materials, vol. 1, pp. 1400056-1-30, 2015.

[17] B. C. Lee, P. Zhou, J. Yang, Y. Zhang, B. Zhao, E. Ipek, O. Mutlu and D. Burger, Phase-change technology and the future of main memory, vol. 30, pp. 131-141, 2010.

[18] J. Boukhobza and P. Olivier, 10 emerging non-volatile memories, Flash Memory Integration, pp. 203-224, 2017.

[19] P. Zurcher, R. E. Jones, P. Y. Chu, D. J. Taylor, B. E. White, S. Zafar, B. Jiang, Y. J. T. Lii and S. J. Gillespie, Ferroelectric nonvolatile memory technology: applications and integration challenges, IEEE Transactions on Components, Packaging, and Manufacturing Technology, vol. 20, pp. 175-181, 1997.

[20] M. Si, A. K. Saha, S. Gao, G. Qiu, J. Qin, Y. Duan, J. Jian, C. Niu, H. Wang, W. Wu, S. K. Gupta and P. D. Ye, A ferroelectric semiconductor field-effect transistor, Nature Electronics, vol. 2, pp. 580-586, 2019.

[21] S. Fujiii and M. Saitoh, Chapter 10.3 - Ferroelectric tunnel junction, Ferroelectricity in Doped Hafnium Oxide: Materials, Properties and Devices, pp. 437-449, Woodhead Publishing, 2019. 


\title{
63 Fault simulation algorithm for transistor single stuck short faults
}

\author{
Durgesh Addala, P. Teja, Sobhit Saxena \\ School of Electronics and Electrical Engineering, Lovely Professional \\ University, Phagwara, Punjab, India
}

\section{Introduction}

CMOS technology includes pull-up and pull-down structures in which pull-up structures consist of one or more PMOS transistors and pull-down structures consist of the same number of NMOS transistors as PMOS. In 2-input NAND gate at circuit level the pull-up network is arranged in parallel manner and the pull-down network is arranged in series [1]. Transistor faults are two types: struck short and short open faults; in struck short we have one main condition which is quiescent current (IDDQ), the current path from vdd to gnd. We generally face fault in the form of IDDQ so we want do a test by comparing the fault free and faulty circuits test vectors identified in the IDDQ case [2].

\section{Two input CMOS NAND gate}

\section{A. Fault free circuit (NAND gate)}

A two input CMOS NAND gate is represented in Figure 63.1. The circuit consists of two PMOS transistors and two NMOS transistors. Digital input signals are two types high input signal and low input signal which are represented as ' 1 ' and ' 0 '. The working of the circuit is expressed in Table 63.1.

\section{B. Single stuck short faults}

The faulty circuit consists of a defect due to stuck short defect generally caused in transistor [3]. In this circuit consisting of four transistors the maximum possible faults is sixteen. Formula for getting possible faults is $2^{\text {n }}$; here $n$ is number of transistors. But here we are considering one fault at a time as shown in Figure 63.2, so only four faults are considered. Fault can be identified in the IDDQ case.

In Table 63.2, we can find the test vector in the form of IDDQ. When PMOS-1 or PMOS-2 stuck-short we get the IDDQ case at $\mathrm{a}=1$ and $\mathrm{b}=1$ then the test vector is $(1,1)$, NMOS-1 stuck-short the IDDQ case is found at $(0,1)$. When NMOS-2 stuck-short the IDDQ case is found at $(1,0)[4,5]$.

\section{Verilog program for testing CMOS NAND gate}

In Verilog PMOS and NMOS the switching logicis represented as pmos (out, data, control), nmos (out, data, control). We have to develop the Verilog program for fault free and faulty circuits. Figure 63.3a shows symbols used for NMOS and PMOS.

DOI: 10.1201/9781003129103-63 


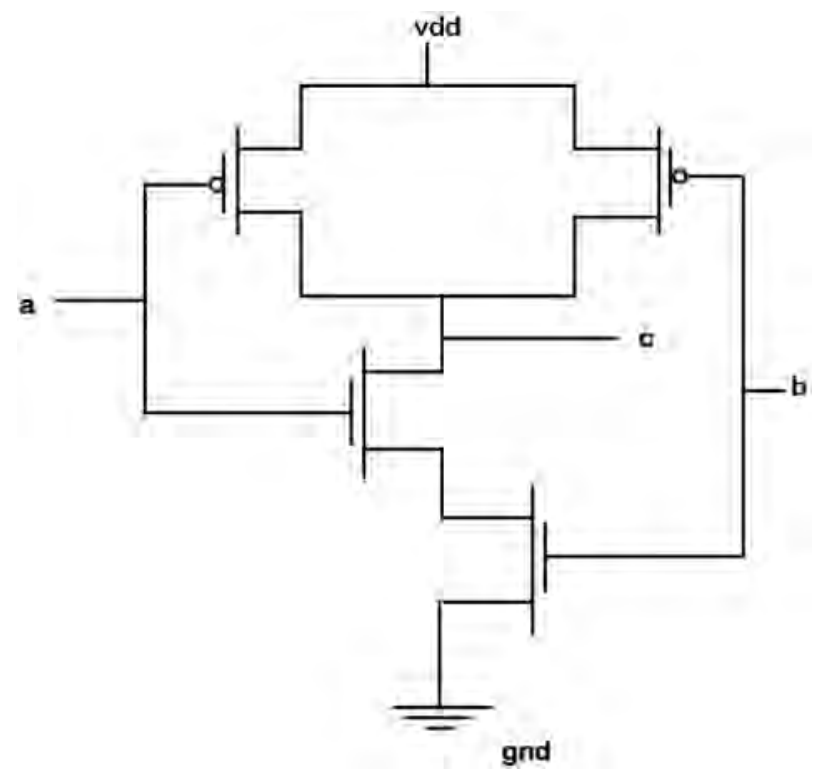

Figure 63.1 CMOS 2-input NAND gate.

Table 1 Truth table of 2-input NAND gate

\begin{tabular}{llll}
\hline s. no. & \multicolumn{2}{l}{ Truth table } & \\
\cline { 2 - 4 } & Input-a & Input- $b$ & Output-c \\
\hline 1. & 0 & 0 & 1 \\
2. & 0 & 1 & 1 \\
3. & 1 & 0 & 1 \\
4. & 1 & 1 & 0 \\
\hline
\end{tabular}

A. Verilog program for fault free circuit (NAND gate)

module nandgate $(\mathrm{a}, \mathrm{b}, \mathrm{c}) ;$ // declaration of module and ports input a,b; // input declaration output c;// output declaration wire w; // internal wire declaration supply0 gnd; // supply1 vdd; // pmos pmos1(c,vdd,a); // pmos switch operation pmos pmos2 (c,vdd,b); // pmos switch operation nmos nmos1 $(\mathrm{c}, \mathrm{w}, \mathrm{a})$; //nmos switch operation nmos nmos2(w,gnd,b); // nmos switch operation endmodule // 

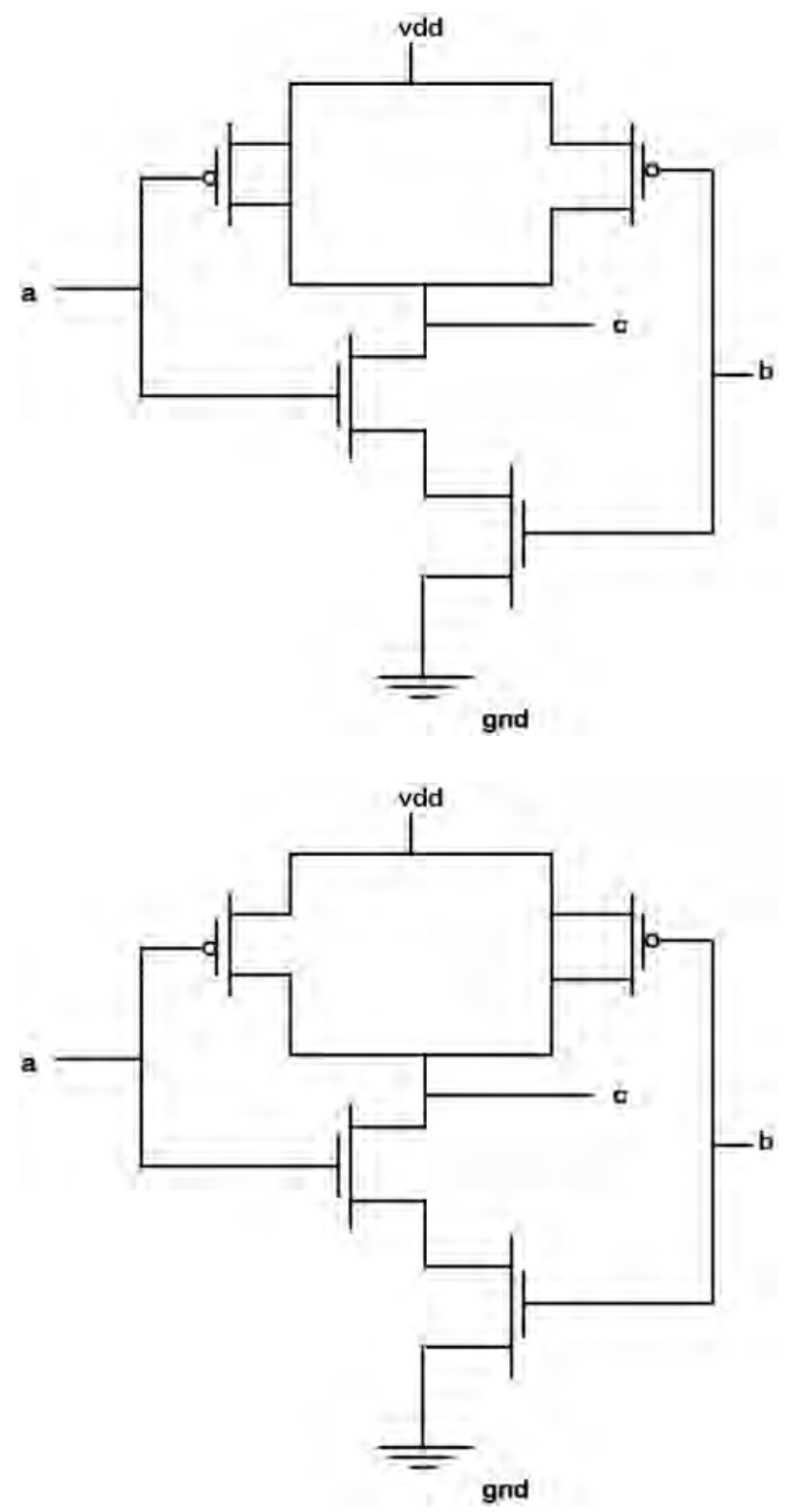

Figure 63.2 (a) PMOS-1 stuck-short. (b) PMOS-2 stuck-short. (c) NMOS-1 stuck-short. (d) NMOS-2 stuck-short. 

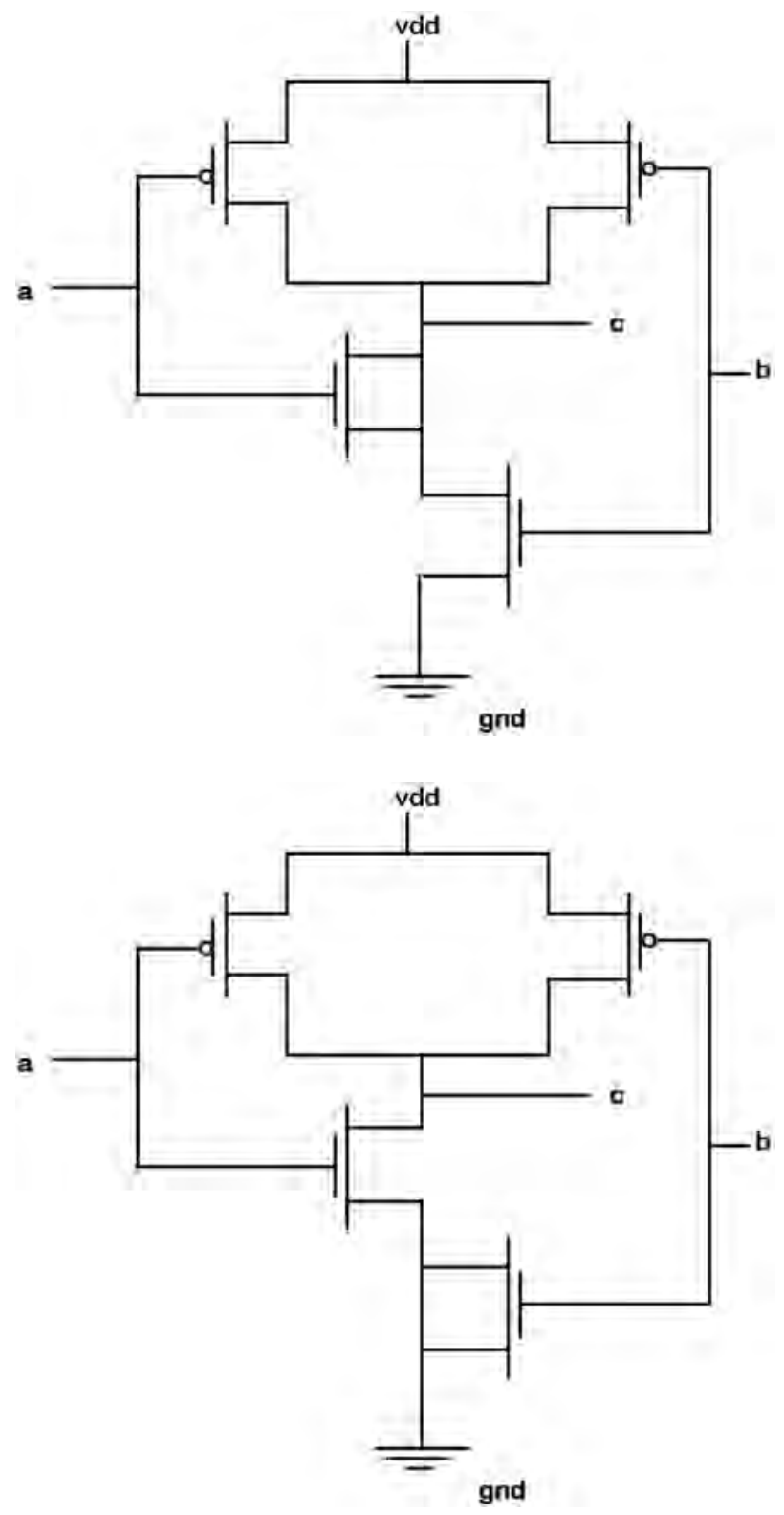

Figure 63.2 (Continued)

Table 63.2 Truth table for faulty free and faulty circuits

\begin{tabular}{lllll}
\hline$a b$ & 00 & 01 & 10 & 11 \\
\hline $\mathrm{c}$ & 1 & 1 & 1 & 0 \\
PMOS-1 stuck-short(n) & 1 & 1 & 1 & IDDQ \\
PMOS-2 stuck-short(o) & 1 & 1 & 1 & IDDQ \\
NMOS-1 stuck-short(p) & 1 & IDDQ & 1 & 0 \\
NMOS-2 stuck-short(q) & 1 & 1 & IDDQ & 0 \\
\hline
\end{tabular}



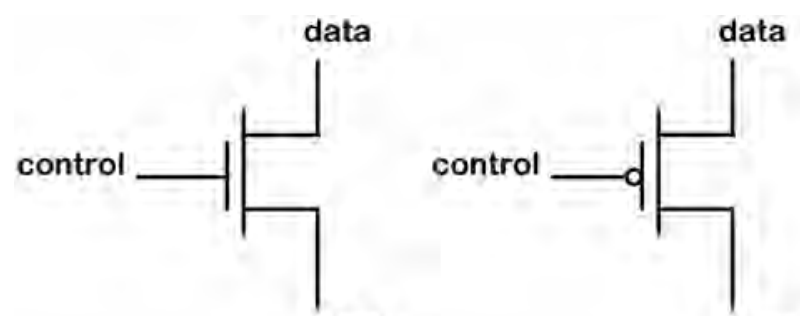

out

out

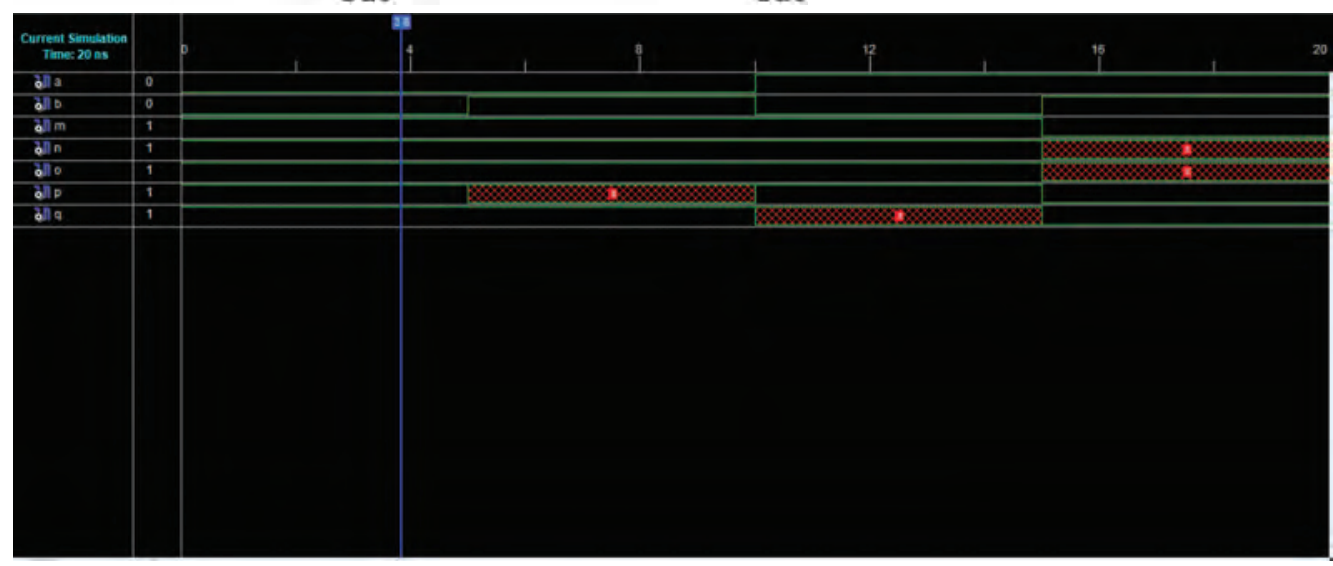

Figure 63.3 (a) NMOS and PMOS switching logic. (b) Simulated waveforms for finding test vectors.

\section{B. Verilog program for faulty circuits (NAND gate)}

- For faulty circuit just remove the mos switch and short the data to output. In this manner we can write Verilog program for all possible faults.

For example, pmos- 1 stuck-short

module nandgatep $1(\mathrm{a}, \mathrm{b}, \mathrm{c}) ; / /$ declaration of module and ports

input a,b; // input declaration

output c; // output declaration

wire $\mathrm{w}$;// internal wire declaration

supply0 gnd; //

supply1 vdd; //

assign $\mathrm{c}=\mathrm{vdd}$; // injecting fault

nmos nmos1(c,w,a); // nmos switch operation

nmos nmos2(w,gnd,b);// nmos switch operation

endmodule //

- For pmos-2 stuck-short

module nandgatep $2(a, b, c) ; / /$ declaration of module and ports

input $\mathrm{a}, \mathrm{b} ; / /$ input declaration

output c;// output declaration

wire w; // internal wire declaration

supply0 gnd; //

supply1 vdd; // 
assign $\mathrm{c}=\mathrm{vdd}$; // injecting fault

nmos nmos1(c,w,a); // nmos switch operation

nmos nmos 2 (w,gnd,b); // nmos switch operation

endmodule //

- Similarly, for nmos-1 stuck-short

module nandgaten $1(\mathrm{a}, \mathrm{b}, \mathrm{c}) ; / /$ declaration of module and ports

input $\mathrm{a}, \mathrm{b} ; / /$ input declaration

output c;// output declaration

wire $\mathrm{w}$; // internal wire declaration

supply0 gnd; //

supply1 vdd; //

pmos pmos1(c,vdd,a); // pmos switch operation

pmos pmos2(c,vdd,b); // pmos switch operation

nmos nmos2(c,gnd,b); // nmos switch operation

endmodule //

- For nmos-2 stuck-short

module nandgaten $2(\mathrm{a}, \mathrm{b}, \mathrm{c})$; // declaration of module and ports

input $\mathrm{a}, \mathrm{b} ; / /$ input declaration

output c; // output declaration

wire $\mathrm{w}$; // internal wire declaration

supply0 gnd; //

supply1 vdd; //

pmos pmos1(c,vdd,a); // pmos switch operation

pmos pmos2(c,vdd,b); // pmos switch operation

nmos nmos1(c,gnd,a); // nmos switch operation

endmodule //

- In final module we have all modules for finding test vectors

module testvector(m,n,o,p,q,a,b);

input $a, b$;

output m,n,o,p,q;

nandgate $\mathrm{x} 1(\mathrm{a}, \mathrm{b}, \mathrm{m}) ; / /$ fault free circuit

nandgatep1 x2(a,b,n); // pmos-1 stuck-short

nandgatep2 x3(a,b,o); // pmos-2 stuck-short

nandgaten $1 \times 4(\mathrm{a}, \mathrm{b}, \mathrm{p}) ; / /$ nmos-1 stuck-short

nandgaten $2 \times 5(\mathrm{a}, \mathrm{b}, \mathrm{q}) ; / /$ nmos-2 stuck-short

endmodule

- Testbench

module durgesh_tb;

reg a,b;

wire m,n,o,p,q;

testvector $\mathrm{u}(\mathrm{m}, \mathrm{n}, \mathrm{o}, \mathrm{p}, \mathrm{q}, \mathrm{a}, \mathrm{b})$;

initial begin

$a=0 ; b=0$;

\#5 a=0; b=1;

\#5 $\mathrm{a}=1 ; \mathrm{b}=0$;

\#5 $\mathrm{a}=1 ; \mathrm{b}=1$;

\#5 \$stop;

end

endmodule 


\section{Simulation result}

Finally, by comparing fault free and faulty circuits we can find the test vectors in IDDQ condition. The don't care conditions obtained in the waveforms indicate the test vectors as shown in Figure 63.3b.

\section{Conclusion}

The testing of CMOS-based NAND gate circuits is successfully implemented using Verilog. The ease of finding the test vectors make this Verilog code useful to implement in other transistor level testing.

\section{References}

[1] Wadsack, R. L. "Fault modeling and logic simulation of CMOS and MOS integrated circuits." Bell System Technical Journal 57.5 (1978): 1449-1474.

[2] Min, Y., and Li, Z. "IDDT testing versus IDDQ testing." J. Electron. Testing: Theory Appl. 13.1 (1998): 15-55.

[3] Maxwell, Peter, et al. "Current ratios: A self-scaling technique for production IDDQ testing." Proceedings International Test Conference 2000 (IEEE Cat. No. 00CH37159). IEEE, 2000.

[4] Bryant, Randal E., and Michael D. Schuster. "Fault simulation of MOS digital circuits." VLSI Design 4.6 (1983): 24-30.

[5] Konuk, Haluk. "Fault simulation of interconnect opens in digital CMOS circuits." International Conference on Computer Aided Design: Proceedings of the 1997 IEEE/ACM international conference on Computer-aided design. Vol. 9, no. 13. 1997. 


\title{
64 Qualitative performance analysis of greyscale image denoising techniques
}

\author{
Amandeep Singh,*, Gaurav Sethi ${ }^{1}$, G.S. Kalra ${ }^{2}$ \\ ${ }^{1}$ School of Electronics and Electrical Engineering, Lovely Professional University, \\ Jalandhar, Punjab, India \\ ${ }^{2}$ Electronics and Communication, CT Group of Institutions, Jalandhar, \\ Punjab, India
}

\section{Introduction}

Images captured by a digital camera can have various sources of noise, i.e. compression, environment effect on transmission, dust on camera lenses and damaged memory locations. This dilutes the details and information present in the image. So, image denoising plays an important role to maintain the image quality and details of the image. Image denoising is required to remove the noise without affecting the details of image like texture, edges [1-4]. In certain images, it is very difficult to identify the difference between objects and their boundaries. It is important to understand that the solution of every noise cannot be the same as denoising is the inverse problem. There are different types of noises playing roles in image distortion. But impulse noise plays a major role in image distortion. So, it becomes important to detect and remove impulse noise. Impulse noise is also known as salt and pepper noise, which affects the image pixel with minimum or maximum value of intensity possible in the image i.e., 0 or 255. This noise can affect the image at any location, which makes it hard to identify the noise location and it is further difficult to remove the impulse noise or restore the original value [5]. In the field of greyscale image denoising, various algorithms are available for denoising but it is hard to find a desirable one. In this paper, we will compare three existing denoising algorithms on the basis of their performance on different noise levels. The performance evaluation is made in the form of peak signal to noise ratio (PSNR) [6]. These three denoising algorithms are median filter (MF), progressive switching median filter (PSMF) and centred weighted median filter (CWMF). We have no intention to give you a detailed review of these methods. More focus in this paper is on the qualitative result-based comparison. This manuscript is systematized as follows. In Section 2, the process of comparative analysis is presented. Section 3 is dedicated to results and discussion (denoising noise of greyscale images). The concluding remarks are drawn in Section 4.

\section{Comparative analysis procedure}

The comparative analysis is achieved between three algorithms of denoising, i.e., MF, PSMF and CWMF. Centred weighted median filter (CWMF) denoises the given image by providing more weight to its centre values and hence can provide decent denoising while avoiding blurring of image [7]. Progressive switching median filter (PSMF) uses a recursive

\footnotetext{
* Corresponding author: amansandhu6788@gmail.com
} 


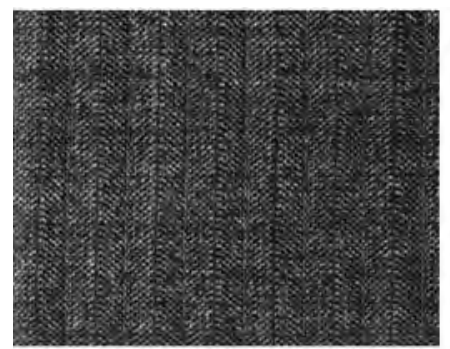

(a)

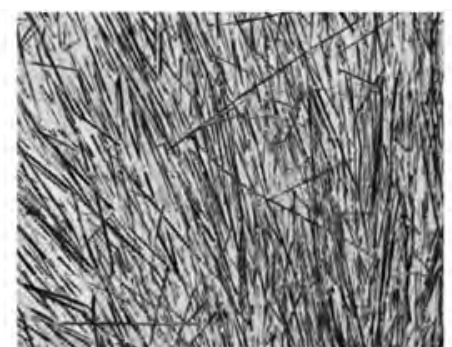

(b)

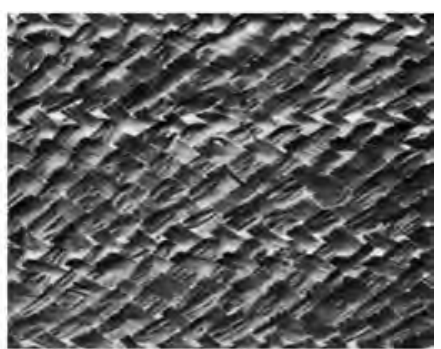

(c)

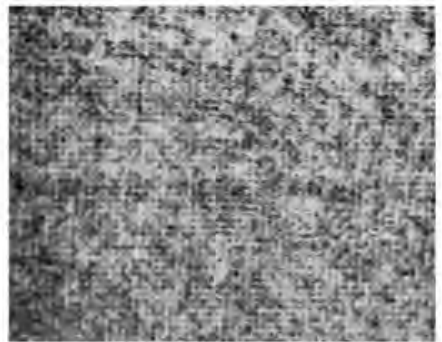

(d)

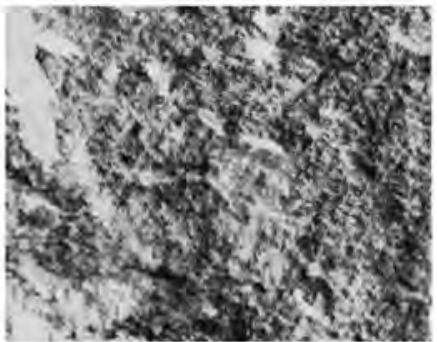

(e)

Figure 64.1 Original texture image data set. (a) Texture-A image, (b) texture-B image, (c) texture-C image, (d) texture-D image, (e) texture-E image.

mechanism in two stages for image denoising which gives it an advantage over the median filter when noise is high but it has high computational cos $t[8]$. Median filter (MF) is one of the classic filters: it denoises the image but at high noise levels it introduces a blurring effect. The advantage of this filter is its low computational complexity [9]. In this paper, the first data set of noisy images will be applied to denoising methods one by one and then calculated PSNR will be compared with each other to know how well these algorithms perform under the different ranges of noise levels. The five original images of standard texture data set are used in this analysis as shown in Figure 64.1 [10].

\section{Results and discussion}

The denoising stage requires to remove the noise from the image with minimum countereffects to the image. In this paper, the algorithms are tested on the data set with a wide range of noise levels to ensure their robustness. In these results noisy images are produced from original images shown in Figure 64.1(a) by adding Salt and pepper noise of increasing order having a range of 10 to 50 percent. Samples of denoising results from a noisy image are shown in Figure 64.2(b-d) to Figure 64.6(b-d) for side by side comparison. In Figure 64.2 image (b) contains the image denoised by median filter, image (c) contains the image denoised by progressive switching median filter and the image (d) represents the resultant image denoised by centred weighted median filter.

This same pattern results of a denoised image from a noisy image is shown in Figure 64.3 to Figure 64.6 and, similarly, results of greyscale texture images i.e. Figure 64.1 (b) to (d) can be calculated. The visual results show that all algorithms have reduced performance when noise is increased in the image. The performance of these algorithms is measured with the parameter PSNR, which is shown in Figure 64.7 to Figure 64.11. These parameter results 


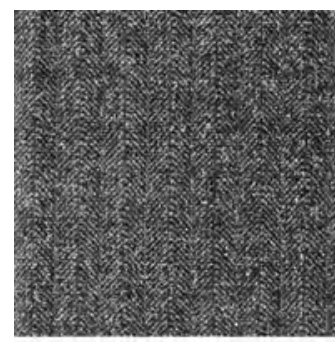

(a)

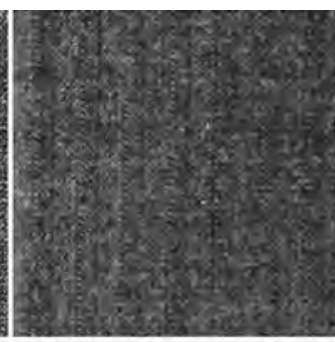

(b)

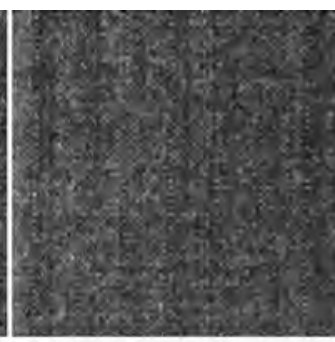

(c)

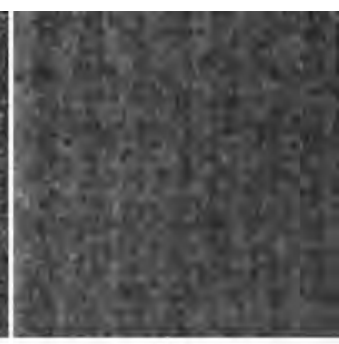

(d)

Figure 64.2 (a) Image affected by 10\% noise. (b) MF denoising, (c) PSMF denoising, (d) CWMF denoising.

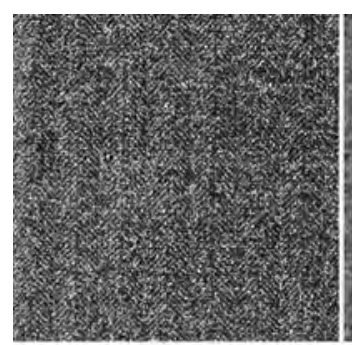

(a)

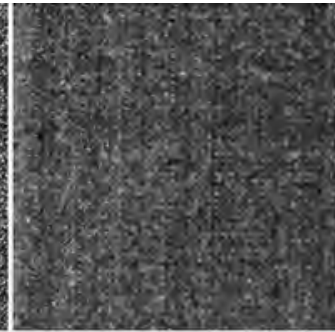

(b)

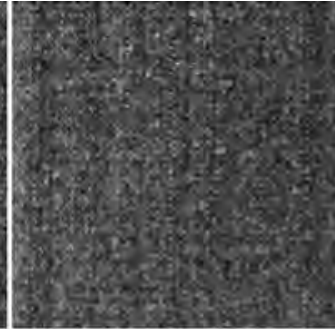

(c)

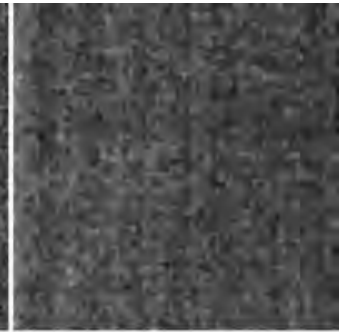

(d)

Figure 64.3 (a) Image affected by 20\% noise. (b) MF denoising, (c) PSMF denoising, (d) CWMF denoising.

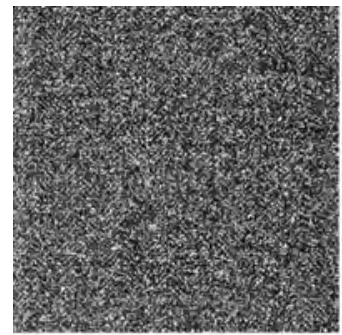

(a)

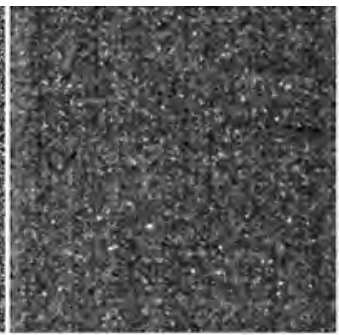

(b)

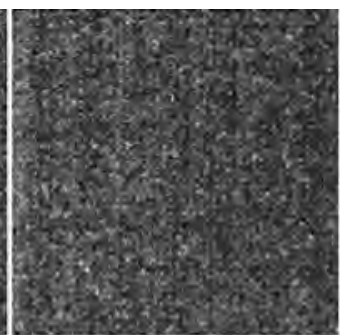

(c)

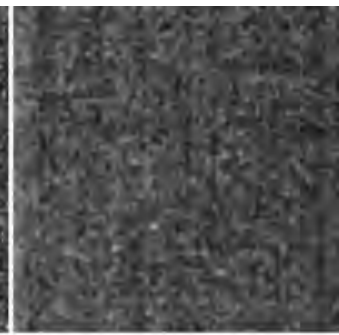

(d)

Figure 64.4 (a) Image affected by 30\% noise. (b) MF denoising, (c) PSMF denoising, (d) CWMF denoising

unfold the fact that the algorithms which perform better at low noise may not yield a similar performance at high noise levels. Let's consider the case of MF, which has a PSNR value of 16.96 at noise level of $10 \%$ for the image shown in Figure 64.2(a), whereas PSMF and CWMF values of PSNR are 16.89 and 15.65 for same case (shown in Figure 64.7). But this scenario of results changes when we analyse the results for denoising of image with 50\% noise (as shown in Figure 64.7): the algorithm CWMF obtained a PSNR value 14.43 in comparison to smaller values of MF (PSNR = 12.20) and PSMF (PSNR = 13.09). 


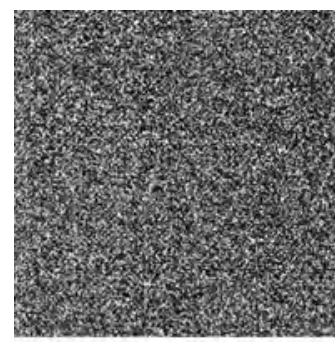

(a)

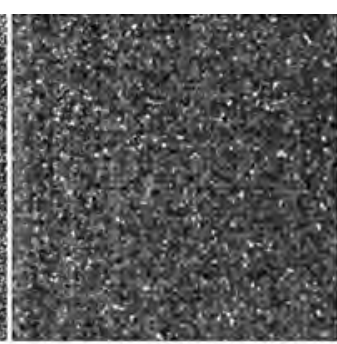

(b)

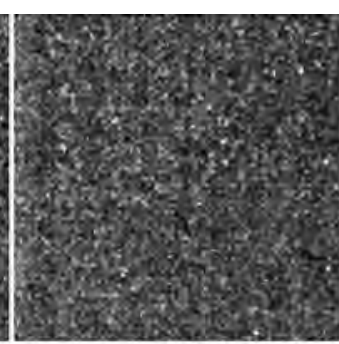

(c)

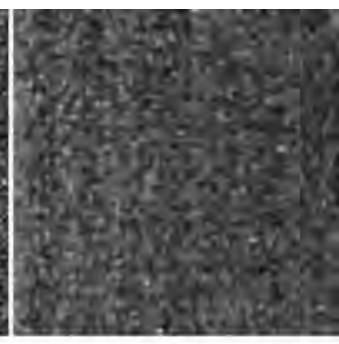

(d)

Figure 64.5 (a) Image affected by 40\% noise. (b) MF denoising, (c) PSMF denoising, (d) CWMF denoising.

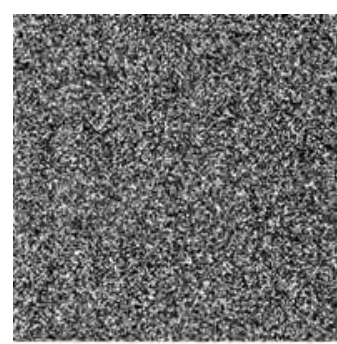

(a)

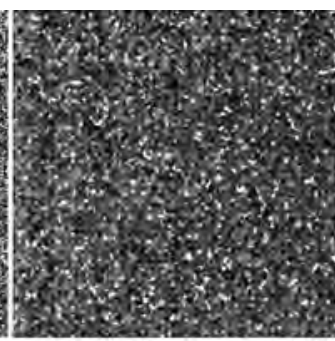

(b)

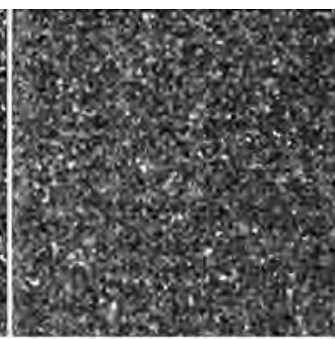

(c)

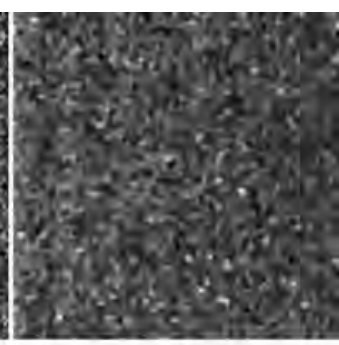

(d)

Figure 64.6 (a) Image affected by 50\% noise. (b) MF denoising, (c) PSMF denoising, (d) CWMF denoising.

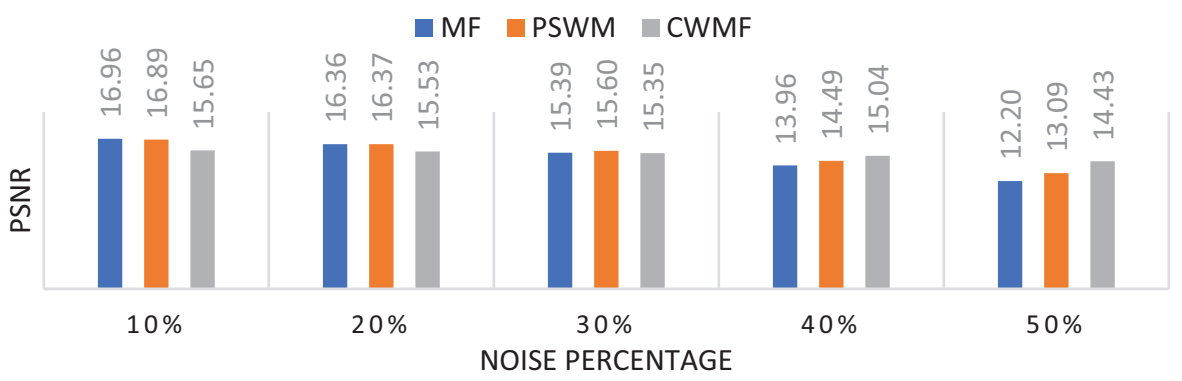

Figure 64.7 PSNR results for texture-A image denoised by MF, PSMF and CWMF.

Table 64.1 is presented to provide the overall behaviour of these algorithms. The MF filter performs better for the low noise levels ranging from $10 \%$ to $20 \%$ and CWMF performs better for the high noise level of $40 \%$. In this table, each value contains the mean of PSNR values, which is calculated from five denoised images of a noise level.

\section{Conclusion}

In this work analysis of three algorithms, MF, PSMF and CWMF, is presented for the greyscale texture dataset. The denoising is performed for salt and pepper noise and the 


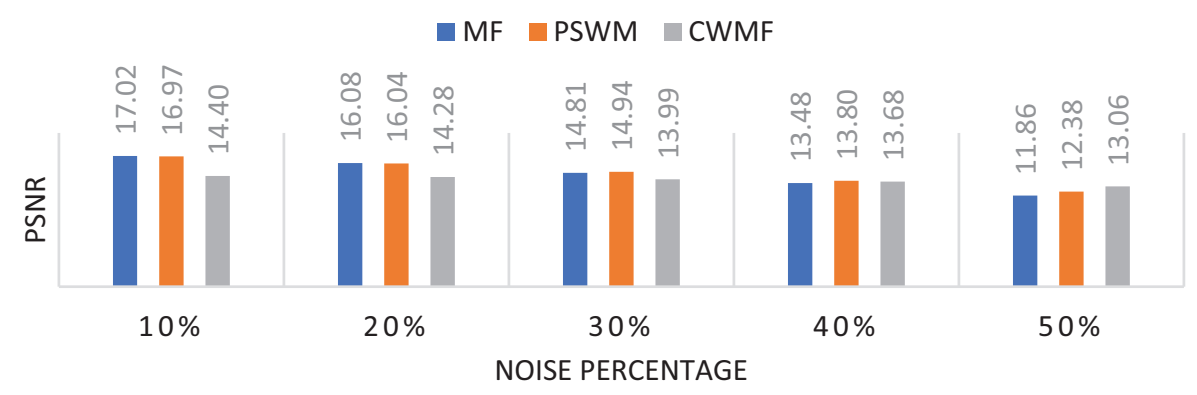

Figure 64.8 PSNR results for texture-B image denoised by MF, PSMF and CWMF.

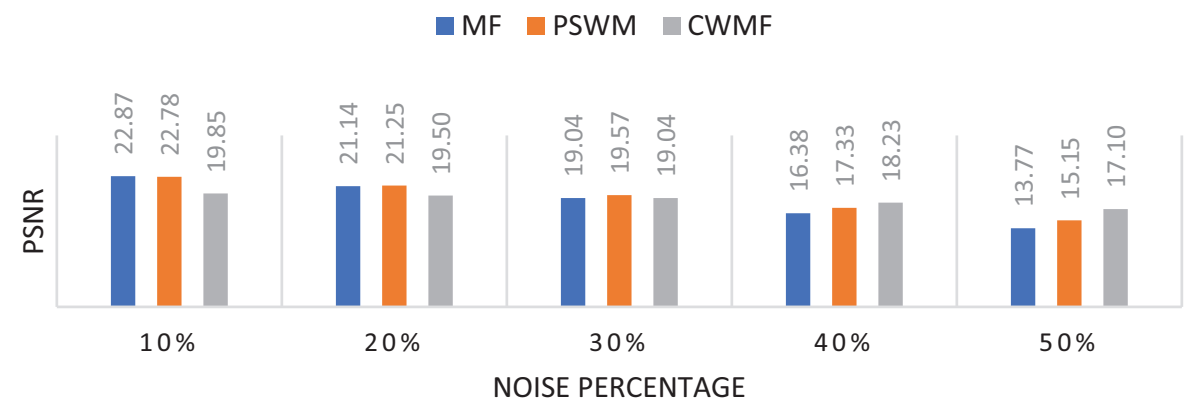

Figure 64.9 PSNR results for texture-C image denoised by MF, PSMF and CWMF.

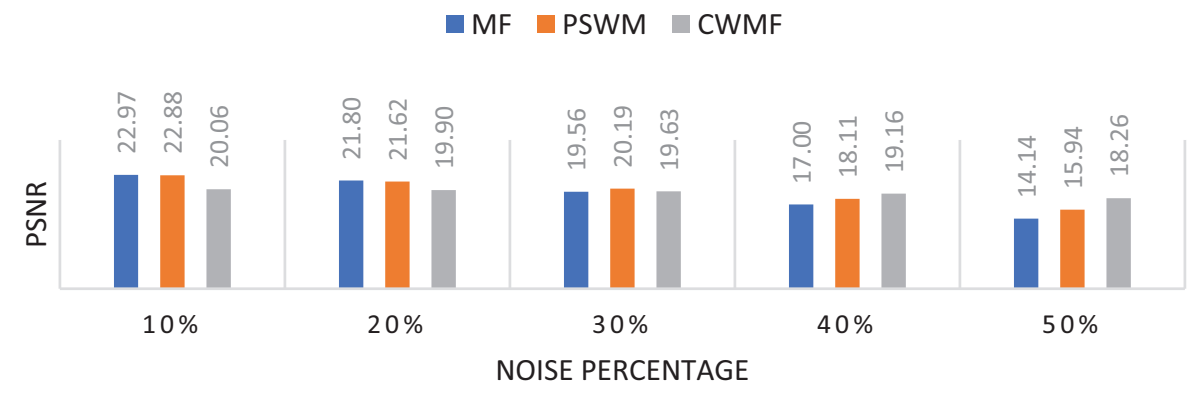

Figure 64.10 PSNR results for texture-D image denoised by MF, PSMF and CWMF.

Table 64.1 Comparative analysis of mean PSNR values of algorithms

\begin{tabular}{llllll}
\hline Noise level & $10 \%$ & $20 \%$ & $30 \%$ & $40 \%$ & $50 \%$ \\
\hline MF & 20.34 & 19.19 & 17.47 & 15.37 & 13.11 \\
PSMF & 20.27 & 19.16 & 17.86 & 16.19 & 14.31 \\
CWMF & 17.87 & 17.68 & 17.35 & 16.90 & 16.01 \\
\hline
\end{tabular}




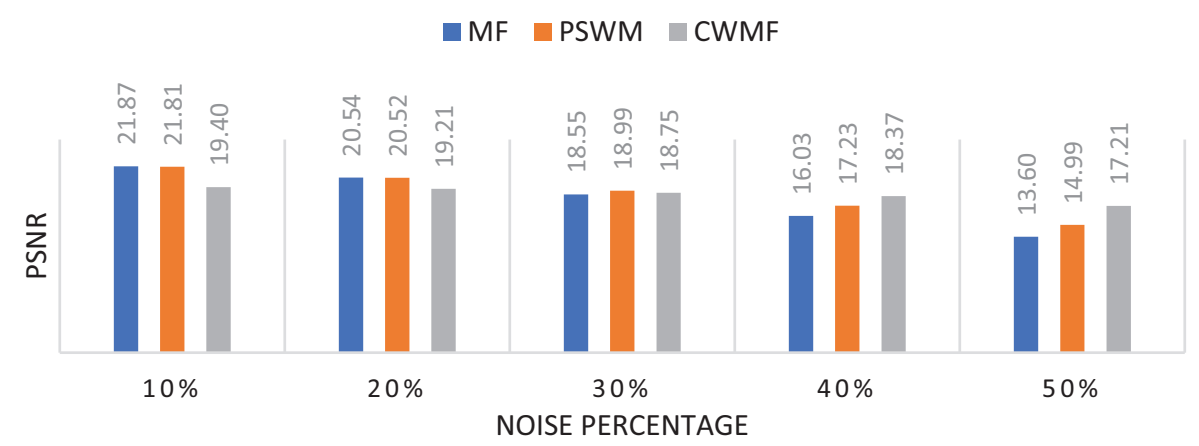

Figure 64.11 PSNR results for texture-E image denoised by MF, PSMF and CWMF.

PSNR parameter is obtained to evaluate the performance. Noise in the image is added in steps ranging from $10 \%$ to $50 \%$ with a step size of $10 \%$ to evaluate the robustness of the algorithms. As these algorithms have shown different characteristics at low and high noise levels, it is very difficult to choose one algorithm for a wide range of noise-affected images. A hybrid algorithm can be constructed by combining the MF algorithm and the CWMF algorithm to make an algorithm which can operate well on both low and high levels of noises.

\section{References}

[1] A. Buades, B. Coll, and J. M. Morel, "A review of image denoising algorithms, with a new one," Multiscale Modeling and Simulation. 2005.

[2] R. Chandel and G. Gupta, "Image filtering algorithms and techniques: a review," Int. J. Adv. Res. Comput. Sci. Softw. Eng., vol. 3, no. 10, pp. 2277-128, 2013.

[3] Y. Li, Q. Pan, S. Wang, H. Peng, T. Yang, and E. Cambria, "Disentangled variational autoencoder for semi-supervised learning," Inf. Sci. (Ny)., 2019.

[4] Z. Sun, B. Han, J. Li, J. Zhang, and X. Gao, "Weighted guided image filtering with steering kernel,” IEEE Trans. Image Process., vol. 29, pp. 500-508, 2020.

[5] P. Karthikeyan and S. Vasuki, "Efficient decision based algorithm for the removal of high density salt and pepper noise in images," J. Commun. Technol. Electron., vol. 61, no. 8, pp. 963-970, 2016.

[6] V. Gupta, D. K. Gandhi, and P. Yadav, "Removal of fixed value impulse noise using improved mean filter for image enhancement," in 2013 Nirma University International Conference on Engineering (NUiCONE), 2013, pp. 1-5.

[7] T. Sun, M. Gabbouj, and Y. Neuvo, "Center weighted median filters: Some properties and their applications in image processing," Signal Processing, vol. 35, no. 3, pp. 213-229, 1994.

[8] G. M. Daiyan and M. A. Mottalib, "Removal of high density salt \& pepper noise through a modified decision based median filter," 2012 Int. Conf. Informatics, Electron. Vision, ICIEV 2012, vol. 18, no. 5, pp. 565-570, 2012.

[9] I. Aizenberg and C. Butakoff, "Effective impulse detector based on rank-order criteria," IEEE Signal Process. Lett., vol. 11, no. 3, pp. 363-366, 2004.

[10] "Brodatz Textures." [Online]. Available at: www.ux.uis.no/ tranden/brodatz.html [Accessed: 16-Jun-2017]. 


\title{
65 A novel hybrid GWO-PS based solution approach for combinatorial unit commitment problem
}

\author{
Meenakshi Gupta1, Vikram Kumar Kamboj², \\ R.K. Sharma ${ }^{2}$
}

${ }^{1}$ Ph.D Research Scholar, School of Electronics and Electrical Engineering, Lovely Professional University, Punjab, India

${ }^{2}$ School of Electronics and Electrical Engineering, Lovely Professional

University, Punjab, India

\section{Introduction}

The size of the power system is growing exponentially due to heavy demand for electricity in all the sectors. Nowadays, in the power sector, there are different kinds of electric power generating stations like nuclear, thermal and hydro power plants etc. During a day, the demand for electric power is changing continuously and achieves various peak values. In this way, it is essential to choose which power generation units have to turn on and in which hour it is required in the power system framework to arrange and, furthermore, for what time period the generating unit should keep in shut down condition to maintain the cost viability of turning on and closing down of particular unit. The whole procedure of making and computing on these choices is recognized as unit commitment (UC). The power generating unit that is planned for association with the network of electrical power sector is known as the committed unit [1]. In power systems, the unit commitment problem refers such kind of problem which are associated with deciding the off/on conditions of producing units to limit the working expense for a specified time period. Power generators can't be directly turned on to fulfil up load demand of the electric power. So it is necessitated that the arranging of producing units is to be done in such a way that there is sufficient amount of power generation accessible to satisfy the demand of the electrical load along with preventing malfunctions and failures under adverse conditions. Unit responsibility handles the power generation plan for the electrical system to minimize fuel and operational costs while fulfilling system constraints, for example, demand of electrical load and system save necessities over a set of time spans. UCP is fundamentally about discovering the most appropriate timetable to turn off or turn on the power units to fulfil the electrical power need for a while and simultaneously keep the expense of power generation as low as could be expected. UCP contains a large-scale, nonlinear and mixed integer constrained optimization which has a place with combinatory improvement issues. Here are numerous constraints included with UCP and consequently it is a tedious and complex process for computation and finding out the optimum solution for UCP [2]. The UCP is to decide a small amount of price turn-off and turn-on arrangement for a set of power generation units to fulfil an electricity need while fulfilling system physical and operational requirements connected with different power generation units. The price for start-up, shutdown, no load cost and fuel are included in the cost for

DOI: $10.1201 / 9781003129103-65$ 


\section{8}

production. The operational constraints which must be thought about include: the total amount of electric power generation should achieve the electricity demand along with losses to that generating system; there should be sufficient spinning reserve to withstand any shortfall in electricity generation; the generation limit of each generator unit must be within the limit of its maximum and minimum capacity, and minimum down time and minimum up time of each and every power generating unit should be pragmatic. The unit commitment is intended to figure out with an appropriate generator commitment schedule for electric power sector over a time horizon of one day to a week. The fundamental objective of SCUCP is to minimize the total generation cost and satisfy some physical and system constraints forced on the system, for example, operating constraint, load-power generation balance, spinning reserve, minimum down time horizon and minimum up time horizon and initial status of such generating unit and so on [3]. A few ordinary techniques are accessible to take care of the UCP. The premier target of UCP is to locate the optimum scheduling for working of the accessible generating units to regulate the total cost of power generation and along with total cost for operation of that power generating unit. The complete cost of electric power generation incorporates the cost of shut down of the unit, cost of fuel and the cost of start-up of the unit. The costs of fuel depend on the price of the fuel, the qualities information of power generating units, for example, heat pace of producing units, turn-on and off schedule and initial status of the power generation unit. So, the main objective of security constrained unit commitment problem is to minimize the total operating cost while satisfying those constraints [4-6].

\section{Problem formulation}

The generating power is distributed along with utilities of generator scheduling which will meet the time varying load demand for a specific time period, which is known as the unit commitment problem (UCP). The actual objective of UCP is minimization of overall cost for production considering different system constraints. The overall costs of production including sum of shutdown cost and start-up cost, cost of fuel are given below:

$$
\min (T C)=\sum_{b=1}^{H} \sum_{i=1}^{N G}\left\{F_{\text {cosh } i}\left(P_{b i}\right)+S U C_{b i}+S D C_{b i}\right\}
$$

The total cost of fuel over the scheduled time span h,

$$
\begin{aligned}
& T C=\sum_{b=1}^{H} \sum_{i=1}^{N G}\left[F_{\text {cosh } i} \times U_{b i}+S U C_{b i}\left(1-U_{i,(b-1)}\right) \times \mathrm{U}_{b i}\right] \\
& T C=\sum_{b=1}^{H} \sum_{i=1}^{N G}\left[\left(A_{i} P_{i}^{2}+B_{i} P_{i}+C_{i}\right) \times U_{i, h}+S U C_{i, h}\left(1-U_{i,(b-1)}\right) \times \mathrm{U}_{i, h}\right]
\end{aligned}
$$

Here, cost for fuel $F_{\text {cosib }}\left(P_{i b}\right)$ is stated as a quadratic design that is mostly used by researchers, also named as equation of convex function.

The cost of fuel of $(n)$ unit at $(\mathrm{t})$ hour can be mathematically represented as such an equation which is given below:

$$
F_{\text {cosih }}\left(P_{i}\right)=A_{i} P_{i}^{2}+B_{i} P_{i}+C_{i}
$$

where $A_{i} B_{i}$ and $C_{i}$ are represented as coefficients of cost that may expressed as $\$ / \mathrm{h}, \$ . \mathrm{MWh}$ and $\$ / \mathrm{MWh}^{2}$, correspondingly. 
Start-up cost can be mathematically represented by a step function which is given below:

$$
S T C_{i b}==\left\{\begin{array}{l}
H S U_{i} ; \text { for } T_{i}^{D W} \leq T_{i}^{U P} \leq\left(T_{i}^{D W}+T_{i}^{C O L D}\right) \\
C S U_{i} ; \text { for } T_{i}^{U P}>\left(T_{i}^{D W}+T_{i}^{C O L D}\right)
\end{array}\right.
$$

the usual value of the shutdown cost for standard system is denoted as zero and this can be established as a fixed cost followed by the eqn. (5).

$$
\mathrm{SDC}_{i b}=\mathrm{KP}_{i b}
$$

where $\mathrm{K}$ is represented as incremental cost for shut-down.

This is is subjected through some constraints followed by: (1) system constraints and (2) unit constraints

\section{System constraints}

System constraints are interrelated with all generating units existing in the systems. The systems constraints are characterised into two types: power balance or load balance constraints.

In the power system the constraint including power balance or load balance is more important parameter requiring that the summation of whole committed generating unit at $\mathrm{t}^{\text {th }}$ time span must be larger than or equivalent to the power demand for the particular time span $t$.

$$
\sum_{i=1}^{N U} P_{i, h} \times U_{i, h}=P D_{i}
$$

\section{Spinning reserve (SR) constraints}

Reliability of the system can be considered as facility of extra capability of power generation that is more important to happen instantly when failure occurrs due to sudden change in load demand for such power generating unit which is already running. The extra capability of power generation is recognized as Spinning reserve, which is exactly represented as:

$$
\sum_{i=1}^{N U} P_{i, b}^{M A X} \times U_{i, h} \geq P D_{b}+S R_{b} .
$$

Constraints for power generating unit: The specific constraints related to a particular power generating unit exist in the systems are called generating unit constraints.

Thermal unit constraints: Thermal power units are controlled manually. This type of unit needs to undertake the change of temperature gradually. So it takes a certain time span to make the generating unit accessible. So some crew members are essential to execute the maintenance and procedure of some thermal power generating units.

Minimum up time: This constraint is defined as there will be a minimum period of time before the unit can be start again when the unit has already been shut down, which is mathematically defined as:

$$
T_{i, h}^{\mathrm{ON}} \geq T_{i}^{U P}
$$




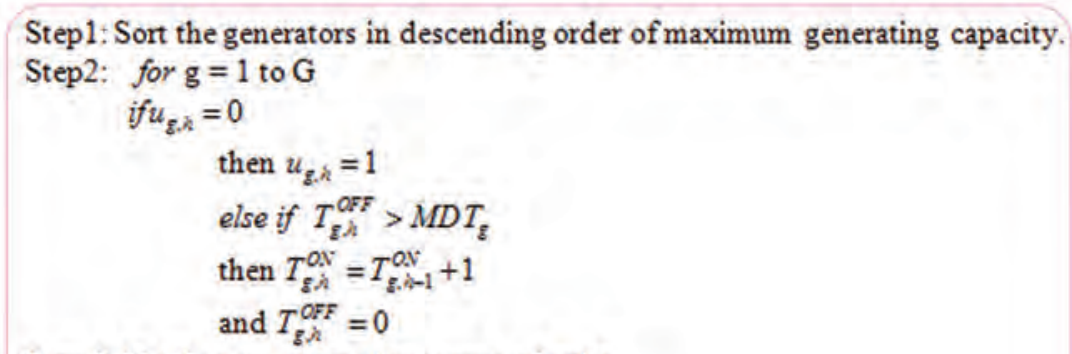

Step-3: Verify new generating power of units.

Step-4: if $\sum_{j=1}^{N G} P_{\mathrm{j} z a x} u_{j \lambda} \geq D_{h}+R_{h}$ then stop the algorithm, else go to step-2.

Step-5: if $T_{O F F}^{g, h}<M D T_{g}$ then do $l=h-T_{g, h}^{O F F}+1$ and set $u_{g, h}=1$

Step-6: Calculate $T_{g}^{i}=T_{g j-1}^{O N}+1$ and $T_{g t}^{O F F}=0$

Step-7:if $1>\mathrm{h}$, Verify generator output power for $\sum_{j=1}^{N} P_{j=\max } u_{j, h} \geq P D_{i}+S R_{i}$, else increment 1 by 1 and go to step- 5

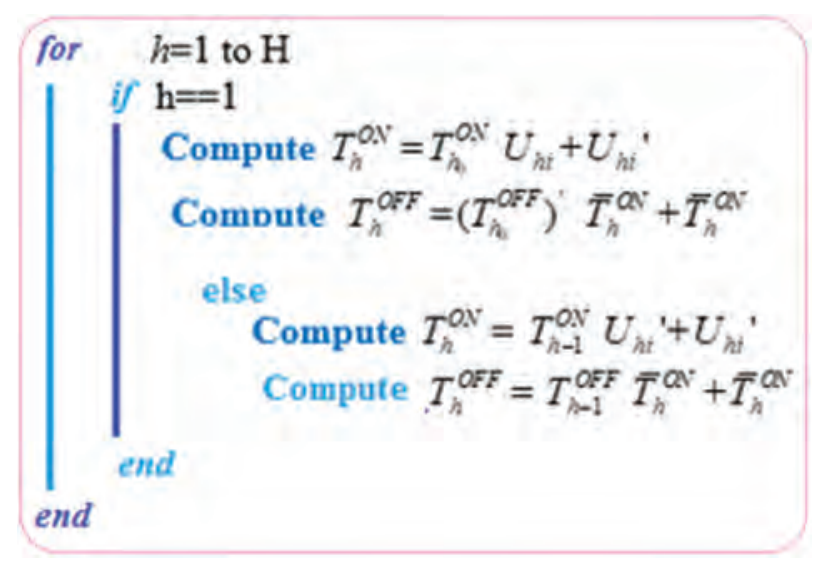

Figure 65.1 (a) Pseudo code of SR repairing. (b) Pseudo code for MUD/MUT constraints.

where $T_{i, h}^{O N}$ is defined as interval through which the generating unit i is constantly ON (in hours) and $T_{i}^{U P}$ is defined as minimum up time (in hours) for the generating unit $\mathrm{n}$.

Minimum down time: When the power generating units will be de-committed, there is required a least period of time for recommitting the unit, which is mathematically given as:

$$
T_{i, b}^{O F F} \geq T_{i}^{D W}
$$

where $T_{i, b}^{O F F}$ is time period for which generating unit $\mathrm{n}$ is constantly OFF (in hrs) and $T_{i}^{D W}$ is denoted as minimum down time (in hours) for the unit.

For an adequate minimum downtime and up time repair by heuristic mechanism, those stages are stated as in Figure 65.1(b). 
Max and min electric power generating limits: All electricity generating units have their individual max/min electric power generating limit, below and outside which it cannot produce and this is known as maximum and minimum power limits, which is mathematically written as:

$$
P_{i}^{M I N} \leq P_{i, b} \leq P_{i}^{M A X}
$$

Initial status for operation of electrical units: Every unit haas an initial operating position that must proceed as the day's earlier generation schedules are taken into consideration, thus each and all generating units can fulfil its lowest down/up time.

Unit accessibility constraint: The constraint shows accessibility of power generating unit surrounded by any of the resulting various circumstances:

- Accessible or non-accessible

- Must outage or out

- Must running condition

Initial status of electricity generation unit: It signifies value of initial grade of power generating unit. Its favourable rate signifies the position of current generating unit which already in up condition, which mean about numeral time periods of the generating units are previously up, and for its negative value which is an index of the integer of hour then power generating unit has been previously in down condition. For the position of generating unit + /- earlier the 1 st hour through the schedule which is an essential feature to define where its newest situation interrupts the constraint of $T_{i}^{U P}$ and $T_{i}^{D W}$. LOLH is properly taken into consideration while solving the SCUC problem.

Crew constraint: When any power plant consists of added units and they couldn't turn on int the same time period. So here need more than one crew member to attend such units in the same time while starting up.

\section{Hybrid GWO-PS algorithm}

In the proposed research, the recently developed grey wolf optimizer [7] has been hybridized with pattern search algorithm to improve the exploitation phase of the existing GWO algorithm. The mathematical formulation for the hybrid GWO-PS algorithm can be found in [8] and pseudo code for the proposed optimizer is been shown in Figure 65.2.

\section{Results and discussion}

The recently developed GWO-PS algorithm has been applied to solve security constrained unit commitment problem of electric power system. In order to validate the results using GWO-PS algorithm, a standard IEEE Test system with 4 and 10 generating units has been taken into consideration [9] and the generation schedule of committed units for the 4-unit system is shown in Table 65.1 and committed status of the 10-unit system is shown in Figure 65.3 and its corresponding dispatch schedule is shown in Figure 65.4. The 40 search agents are taken into consideration for effective research study. 


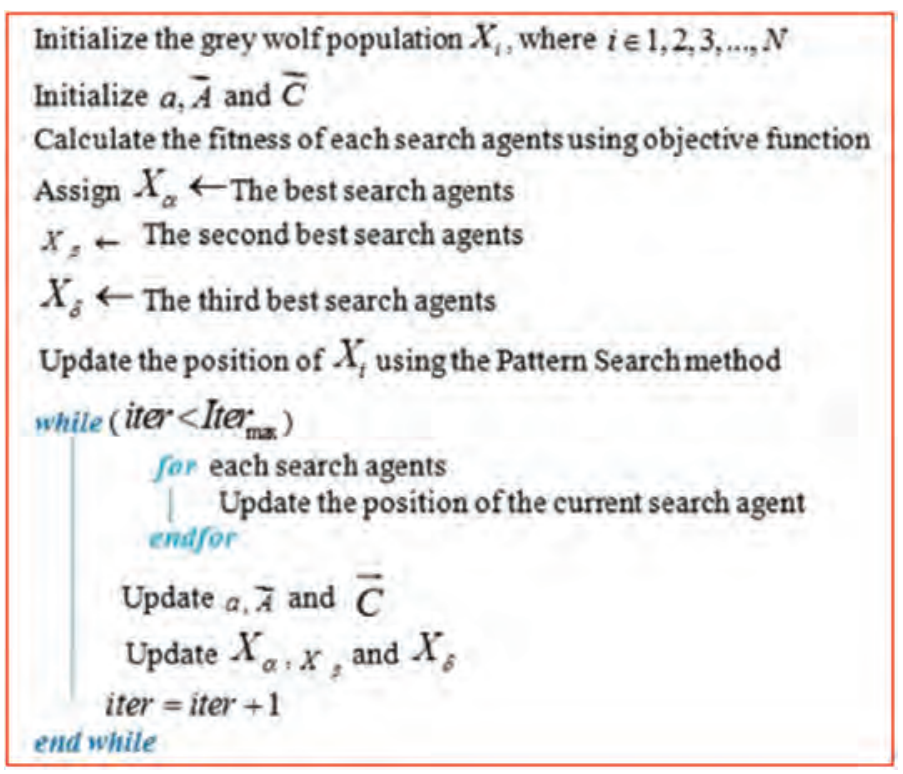

Figure 65.2 Pseudo code for proposed GWO-PS algorithm.

Table 65.1 Test results for 4-generating-unit system

\begin{tabular}{|c|c|c|c|c|c|c|c|c|}
\hline \multirow[t]{2}{*}{ Hour } & \multicolumn{4}{|c|}{ Commitment status } & \multicolumn{4}{|c|}{ Generation schedule } \\
\hline & U1 & $U 2$ & U3 & U4 & $U 1$ & $U 2$ & $U 3$ & U4 \\
\hline 1 & 1 & 1 & 0 & 0 & 300 & 150 & 0 & 0 \\
\hline 2 & 1 & 1 & 1 & 0 & 300 & 205 & 25 & 0 \\
\hline 3 & 1 & 1 & 1 & 1 & 300 & 250 & 30 & 20 \\
\hline 4 & 1 & 1 & 1 & 0 & 300 & 215 & 25 & 0 \\
\hline 5 & 1 & 0 & 1 & 1 & 300 & 0 & 80 & 20 \\
\hline 6 & 1 & 0 & 1 & 0 & 255 & 0 & 25 & 0 \\
\hline 7 & 1 & 0 & 1 & 0 & 265 & 0 & 25 & 0 \\
\hline \multirow[t]{3}{*}{8} & 1 & 1 & 0 & 0 & 300 & 200 & 0 & 0 \\
\hline & & & & & \multicolumn{2}{|c|}{ Total cost $(\$)$} & \multirow{2}{*}{\multicolumn{2}{|c|}{$\begin{array}{r}74476.00 \\
20.853569\end{array}$}} \\
\hline & & & & & & ne (s) & & \\
\hline
\end{tabular}

\section{Conclusions}

The proposed GWO-PS has been successful applied to the unit commitment problem and its effectiveness has been verified for standard IEEE test systems consisting of 4 and 10 generating units and experimentally it has been found that the commitment results of GWO-PS are better than PSO and classical GWO algorithm and similar kind of algorithm can be applied to solve the security constraint unit commitment problem and the profitbased unit commitment problem as future research. 


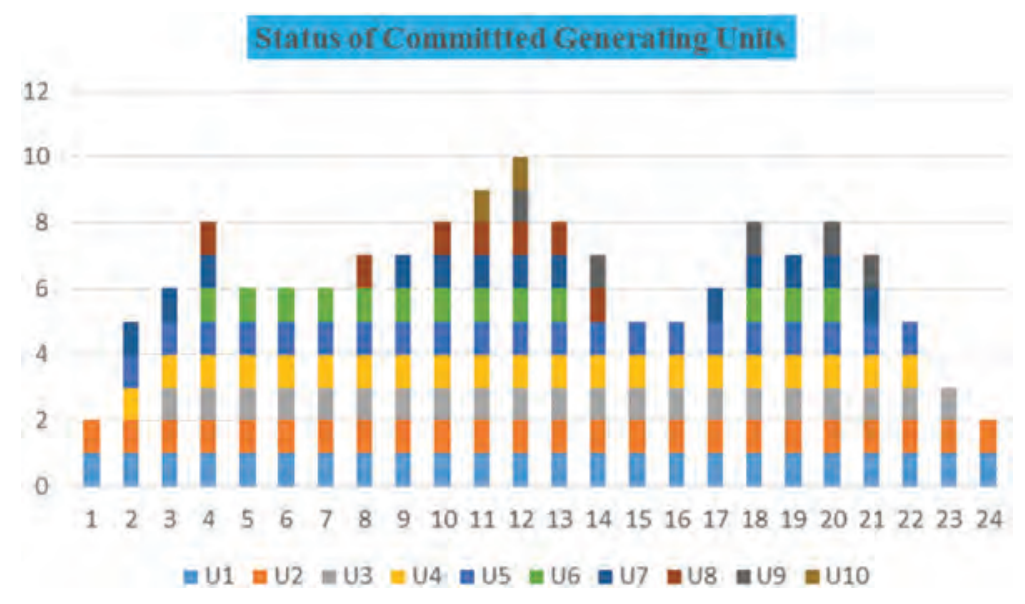

Figure 65.3 Committed units for 10 -unit system at SR $=10 \%$.

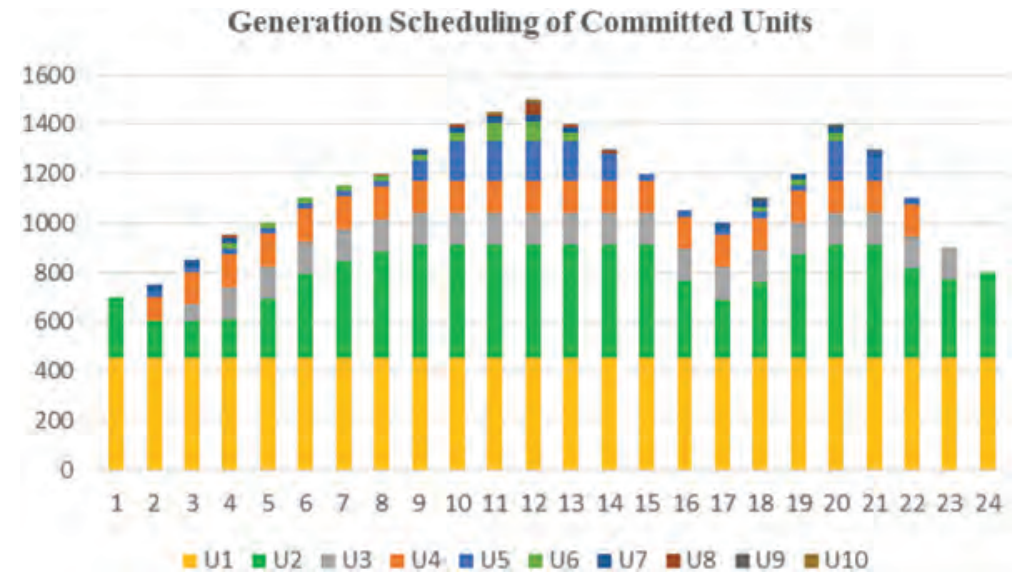

Figure 65.4 Hourly dispatch of committed units for 10 -unit system at SR $=10 \%$.

\section{References}

[1] V. Kumar, S. K. Bath, and J. S. Dhillon, "Electrical power and energy systems implementation of hybrid harmony search / random search algorithm for single area unit commitment problem," Int. J. Electr. Power Energy Syst., vol. 77, pp. 228-249, 2016.

[2] M. Reza Norouzi, A. Ahmadi, A. Esmaeel Nezhad, and A. Ghaedi, "Mixed integer programming of multi-objective security-constrained hydro/thermal unit commitment," Renew. Sustain. Energy Rev., vol. 29, pp. 911-923, 2014.

[3] E. Nasrolahpour and H. Ghasemi, "A stochastic security constrained unit commitment model for reconfigurable networks with high wind power penetration," Electr. Power Syst. Res., 2014.

[4] V. K. Kamboj, S. K. Bath, and J. S. Dhillon, "Solution of non-convex economic load dispatch problem using Grey Wolf Optimizer,” Neural Comput. Appl., 1934.

[5] V. K. Kamboj, S. K. Bath, and J. S. Dhillon, "Multiobjective multiarea unit commitment using hybrid differential evolution algorithm considering import/export and tie-line constraints," Neural Comput. Appl., vol. 28, no. 11, pp. 3521-3536, 2017. 


\section{Intelligent Circuits and Systems}

[6] V. K. Kamboj, S. K. Bath, and J. S. Dhillon, "A novel hybrid DE-random search approach for unit commitment problem," Neural Comput. Appl., vol. 28, pp. 1559-1581, 2017.

[7] S. Mirjalili, S. M. Mirjalili, and A. Lewis, "Grey Wolf optimizer," Adv. Eng. Softw., vol. 69, p. 46, 2014.

[8] V. K. Kamboj, A. Bhadoria, and N. Gupta, "A novel hybrid GWO-PS algorithm for standard benchmark optimization problems," Ina. Lett., vol. 3, no. 4, pp. 217-241, 2018.

[9] V. K. Kamboj, "A novel hybrid PSO-GWO approach for unit commitment problem," Neural Comput. Appl., vol. 27, pp. 1643-1655, 2015. 


\title{
66 Power quality based comparative analysis of resonant inverters
}

\author{
Syed Iqra ${ }^{1, *}$, Himanshu Sharma ${ }^{2, *}$ \\ ${ }^{1}$ Research Scholar, Lovely Professional University, Punjab, India \\ ${ }^{2}$ Assistant Professor, Lovely Professional University, Punjab, India
}

\section{Introduction}

Inverters are DC to AC converters which find their uses in solar power grids, UPSs (uninterruptible power supply) in computers, basic block of SMPS (switch mode power supply), industrial and home equipment like pumps, centrifugal fans, mixers, conveyor belts, etc. These are also used as autonomous power supplies (e.g. in aircraft). The various types of inverters are categorized based on source of load (CSI and VSI), output waveform (sine wave, square wave, modified sine wave), PWM modulation (single PWM, multiple PWM, sinusoidal PWM, modified SPWM), type of load (single phase load and three phase load) and the number of levels at the output (two level and multilevel inverter). Multilevel inverters have a further classification as diode-clamped multilevel inverter, flying-capacitor multilevel inverter and cascaded multilevel inverter. A considerable improvement in the various types of multilevel inverters has been made over the years which provides not only minimized harmonic distortion in the output voltage and current waveforms on the load side, but also a reduced voltage stress on the switches used. These are widely used for medium-voltage and high power applications [1]. The problem encountered in these inverters is that the switches operate in hard switching mode. As a result the switching losses increase and hence contribute to the power losses. Also the rate of change of voltage and current across the switch is high, resulting in switching stress [2] and high radiated electromagnetic interference [3]. To mitigate this problem, a quasi-resonant inverter, which is shown in Figure 66.1, has been developed that helps in providing a zero voltage switching at the time of switching $\mathrm{ON}$ and zero current switching at the time of switching OFF of the switch. The goal of ZCS and ZVS is achieved by connecting a resonant inductor in series with IGBT switches and resonant capacitor in parallel with neutral point switches [4].

Another category of inverters called Z-source (shown in Figure 66.2) has found wide applications in the power electronics field. It was first introduced by Peng in 2002. Over the years the Z-source topologies have been improved and applied in various power electronic converters as in choppers, rectifiers, inverters and cycloconverters. They find their industrial applications in photovoltaic systems, energy storage systems, motor drives, power factor correction and fuel cells [5]. Due to its configuration, it operates as a buck-boost converter without making use of conventional DC-DC converters. Also it overcomes the problem encountered in voltage source inverters (VSI) and current source inverter (CSI) [6]. In the conventional VSI and CSI the two switches in the same arm can't be triggered as it will short-circuit the arm and can destroy the converter. But in the case of the developed

* Emails: syediqraa55@gmail.com, himanshu.23441@1pu.co.in

DOI: $10.1201 / 9781003129103-66$ 


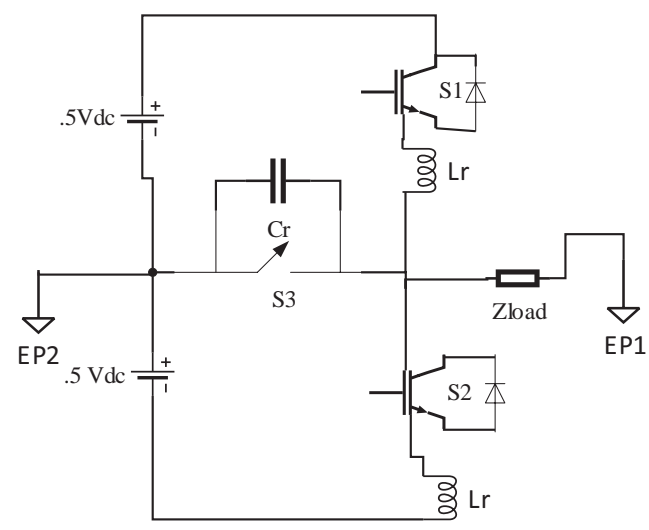

Figure 66.1 Quasi-resonant three level inverter.

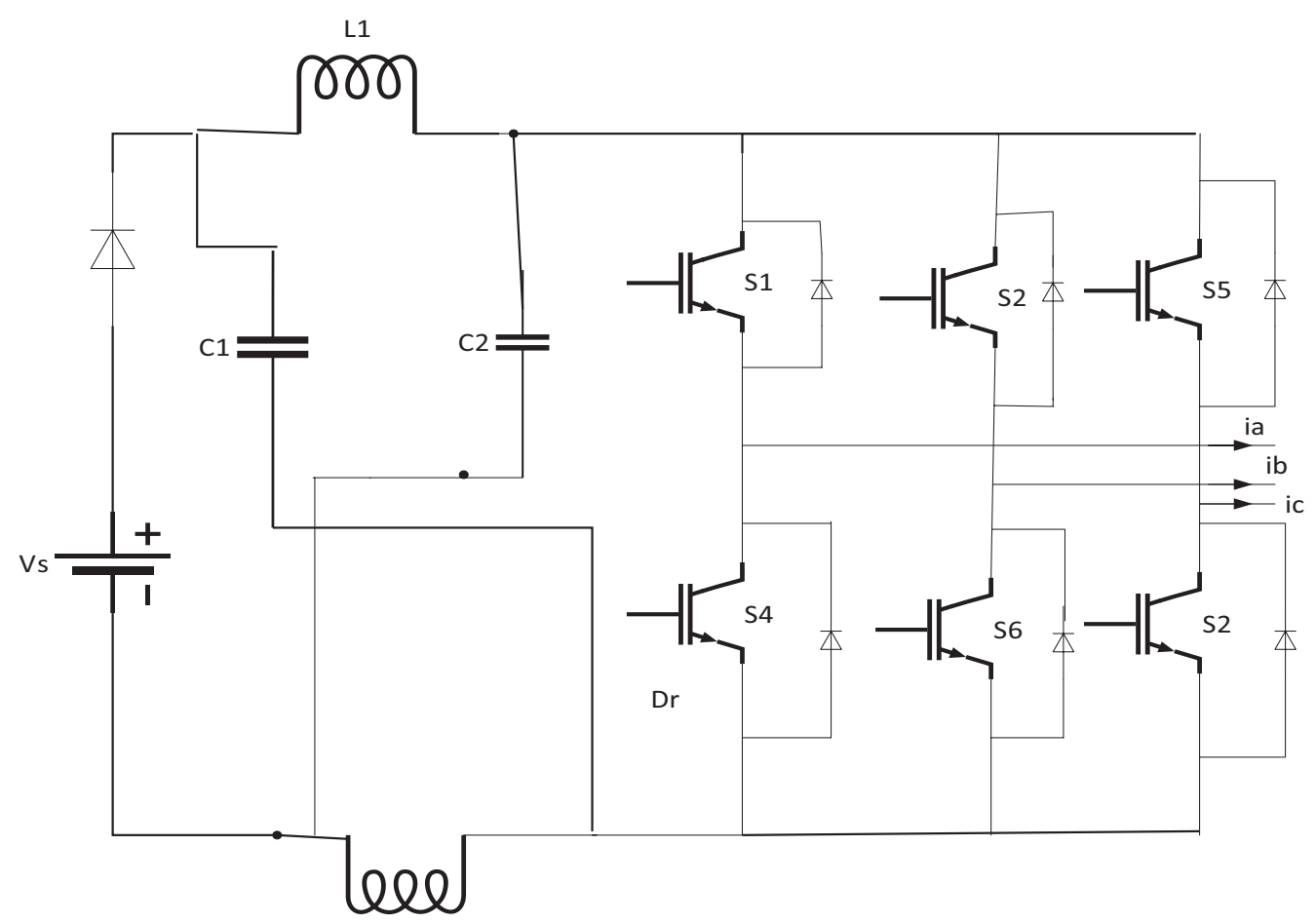

L2

Figure 66.2 Structure of a Z-source inverter.

Z-source inverter the switches of the same leg are triggered and shooting of the current results in greater DC voltage and gives a higher output of desired magnitude [7]. In a PWM control of Z-source inverter, along with the six active switches, the two zero switching states can be replaced partially or fully by the shoot through zero state depending on how much boosting is required on DC side while keeping active states unchanged to give a desired voltage waveform [8]. This inverter is more reliable, compact and cost efficient. 
Table 66.1 Switching state and circuit behaviour

\begin{tabular}{|c|c|c|}
\hline & Positive half-cycle & Negative half-cycle \\
\hline & $S 3 S 1$ & $S 3 S 2$ \\
\hline Switching state & ON OFF & ON OFF \\
\hline Circuit behaviour & $\mathrm{S} 3$ is freewheeling & $\mathrm{S} 3$ is freewheeling \\
\hline Switching state & OFF ON & OFF ON \\
\hline Circuit behaviour & Quasi-resonant mode & Quasi-resonant mode \\
\hline Switching state & OFF OFF & OFF OFF \\
\hline Circuit behaviour & D1 freewheeling & D2 freewheeling \\
\hline
\end{tabular}

\section{Operating principles of the two inverters}

A. Quasi-resonant three level inverter: A Simulink model of the inverter is shown in Figure 66.3. Switches s1 and s2 are connected in series with Lr1 and Lr2 respectively in the same leg of the three phase inverter and with the proper configuration of the converter a zero current turn OFF of the same switches is obtained. Also S3 is a bidirectional switch and is connected across a resonant capacitor which provides a zero voltage turn $\mathrm{ON}$ for the switch. For simplicity, the switching states and the circuit behaviour of a single phase quasi-resonant inverter are shown in Table 66.1.

B. Z-source inverter: The characteristic feature of this inverter is the introduction of an impedance tank which comprises two same-valued inductors and capacitors connected in cross connecting the tank with the input source. The simulink model of Z-source inverter is shown in the Figure 66.4. During the condition of high input voltage, the shoot-through zero state is not activated and it acts as a normal buck converter. At the time of low input voltage, the shoot-through zero state of the inverter is activated and the desired output is achieved while acting as a buck-boost converter.

\section{Simulation results and comparisons}

The simulation is observed for the voltage across the resonant capacitor which is connected along the switch and the current is measured across the resonant inductor. The progress of these two parameters is observed while varying the value of inductor and capacitor. The simulink results are shown in Figure 66.5.

\section{Case 1}

Here the capacitors are kept at a value of $0.33 \times 10^{-12} \mathrm{~F}$ and inductors are kept at a value of $17.6 \times 10^{-12} \mathrm{H}$.

\section{Case 2}

Figure 66.6 shows the output results of voltage across $C$ and current across $L$ for an input voltage of 150 volts.

The values are decreased for inductors and increased for capacitors and a change in current is observed.

Here $\mathrm{Cr}=0.33 \times 10^{-10} \mathrm{~F}$ and $\mathrm{Lr}=17.6 \times 10^{-5}$. 


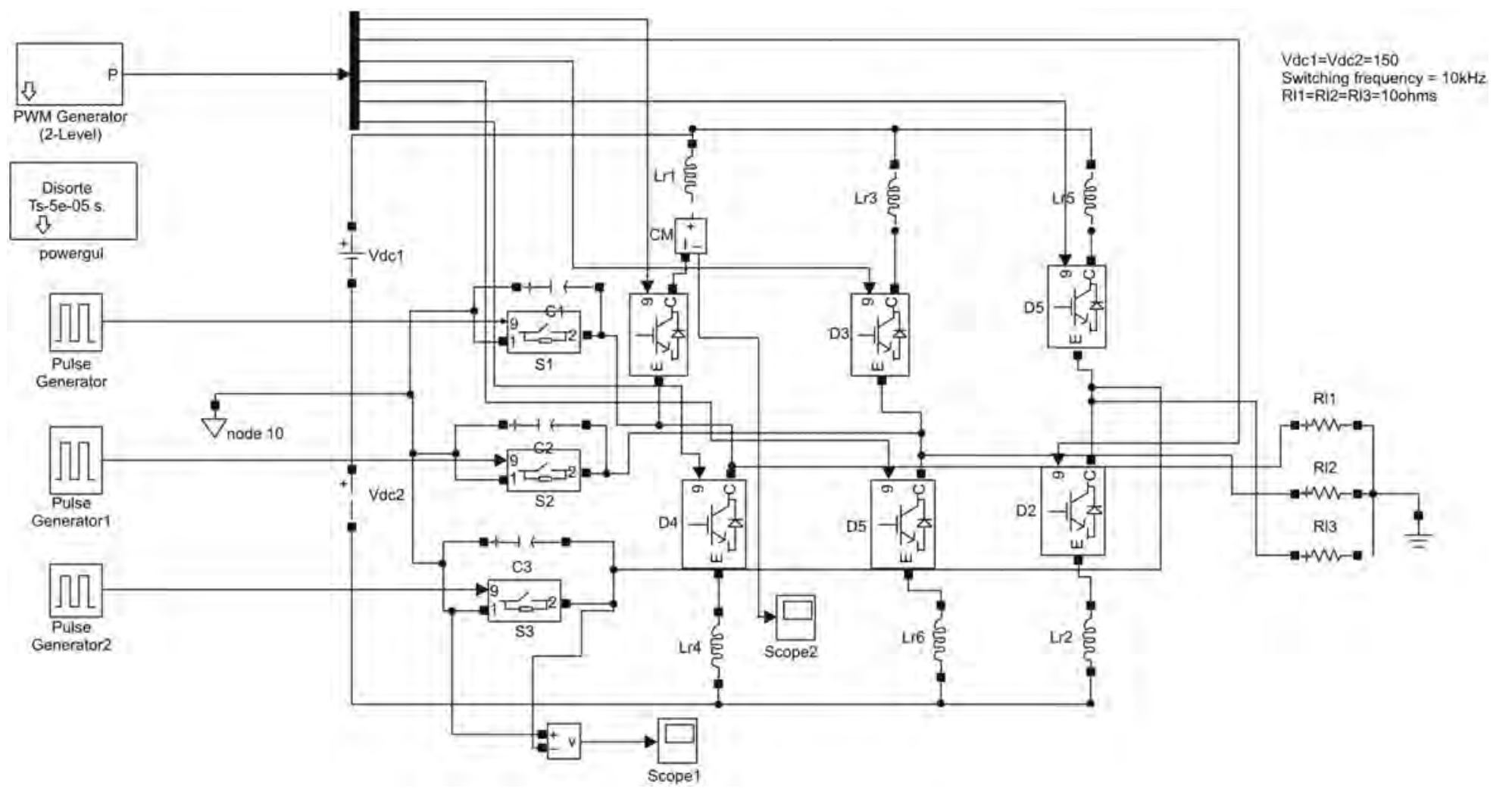

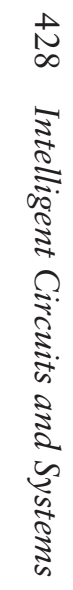

Figure 66.3 Simulink model of a three phase quasi-resonant inverter. 


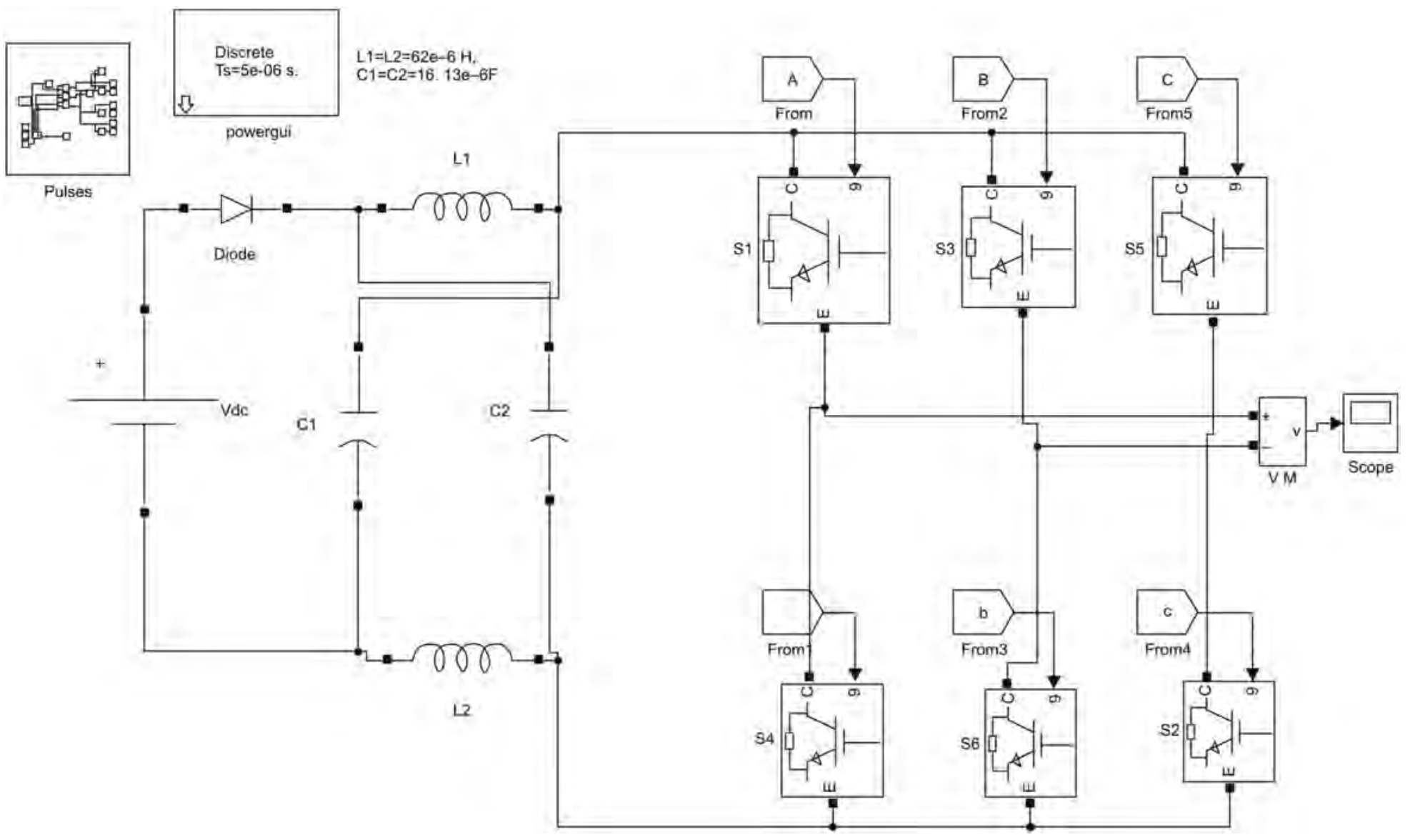


Table 66.2 Comparison between the quasi-resonant inverter and Z-source inverter

\begin{tabular}{|c|c|}
\hline Quasi-resonant inverter & Z-source inverter \\
\hline $\begin{array}{l}\text { It helps in soft switching turn ON and turn } \\
\text { OFF, preventing switching losses and } \\
\text { reduction in EMI. }\end{array}$ & $\begin{array}{l}\text { It activates the shoot-through zero state when the } \\
\text { input voltage is lower and hence gives desired } \\
\text { output. }\end{array}$ \\
\hline $\begin{array}{l}\mathrm{dv} / \mathrm{dt} \text { and } \mathrm{di} / \mathrm{dt} \text { are decreased and hence the } \\
\text { switching stress is reduced. }\end{array}$ & $\begin{array}{l}\text { It acts as buck-boost converter without the need } \\
\text { of incorporating DC-DC converters. }\end{array}$ \\
\hline $\begin{array}{l}\text { These were first used in DC-DC converters } \\
\text { but were later modified and applied in DC- } \\
\text { AC inverters. }\end{array}$ & $\begin{array}{l}\text { Developed in 2002, these were implemented } \\
\text { in choppers, rectifiers, inverters and } \\
\text { cycloconverters. }\end{array}$ \\
\hline $\begin{array}{l}\text { The values of } \mathrm{Lr} \text { and } \mathrm{Cr} \text { need to be optimized } \\
\text { so that area of intersection is less and hence } \\
\text { less power loss. }\end{array}$ & $\begin{array}{l}\text { The modulation index can be adjusted to obtain } \\
\text { the boosted voltage without stressing the } \\
\text { switches. }\end{array}$ \\
\hline $\begin{array}{l}\text { These have applications in industries and in } \\
\text { home appliances. }\end{array}$ & $\begin{array}{l}\text { These are applied in renewable energy sources, } \\
\text { motor drives and electric vehicles. }\end{array}$ \\
\hline
\end{tabular}

\section{Case 3}

In this case output voltage is equal to 150 volts and the current is shooting very high as the value of the inductor is reduced to $17.6 \times 10^{-7} \mathrm{H}$ and the value of the capacitor is increased to $0.33 \times 10^{-8} \mathrm{~F}$. The output voltage across capacitor and current across inductor is shown in Figure 66.7.

It can be observed how the changing values of the resonant capacitor and the resonant inductor vary the output voltage waveform and current waveform.

The three phase quasi-resonant inverter where the three phases differ by an angle of 120 and incorporated with capacitor and inductor in order to provide a soft switching turn $\mathrm{ON}$ and turn OFF and gives the no load phase voltage shown in Figure 66.8. 


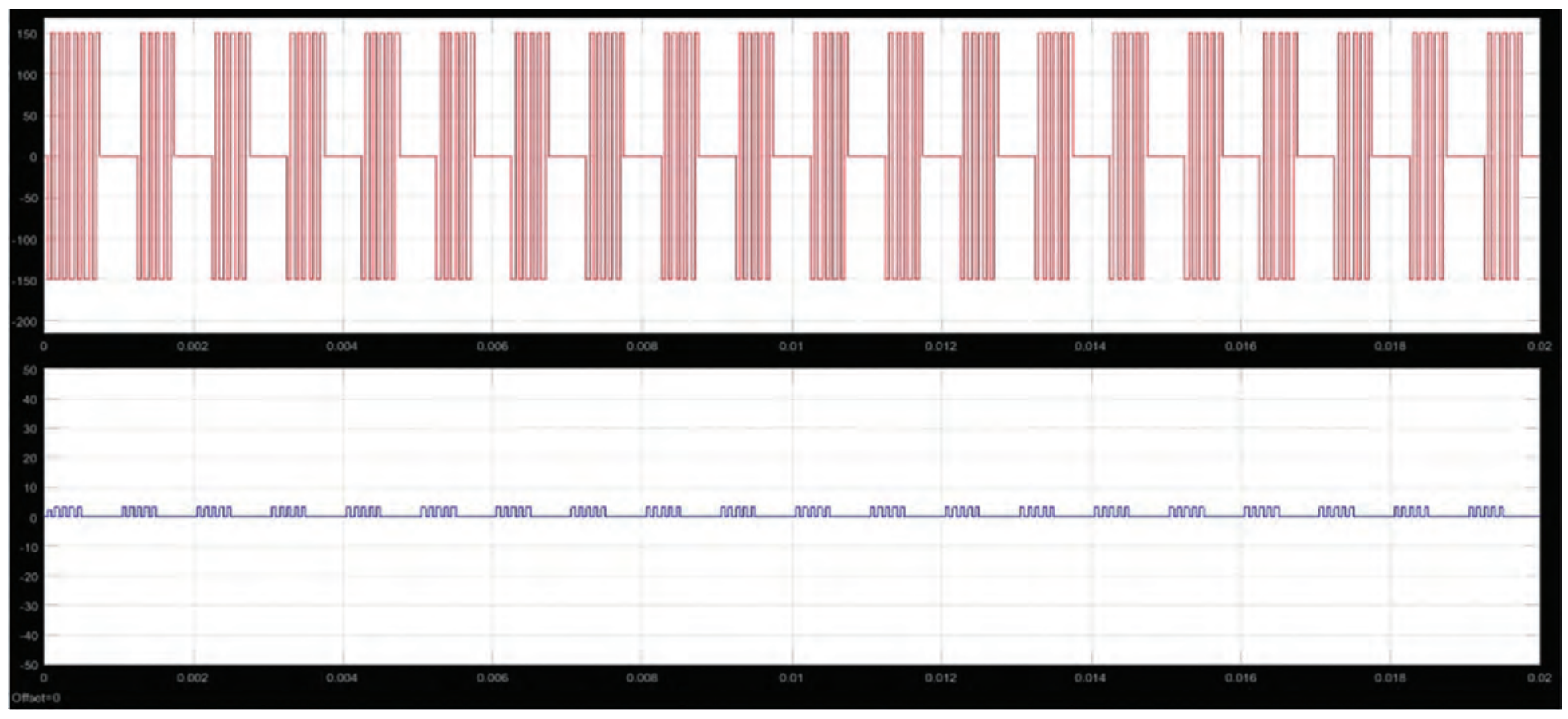

Figure 66.5 Output results of voltage across C and current across L for an input voltage of 150 volts. 


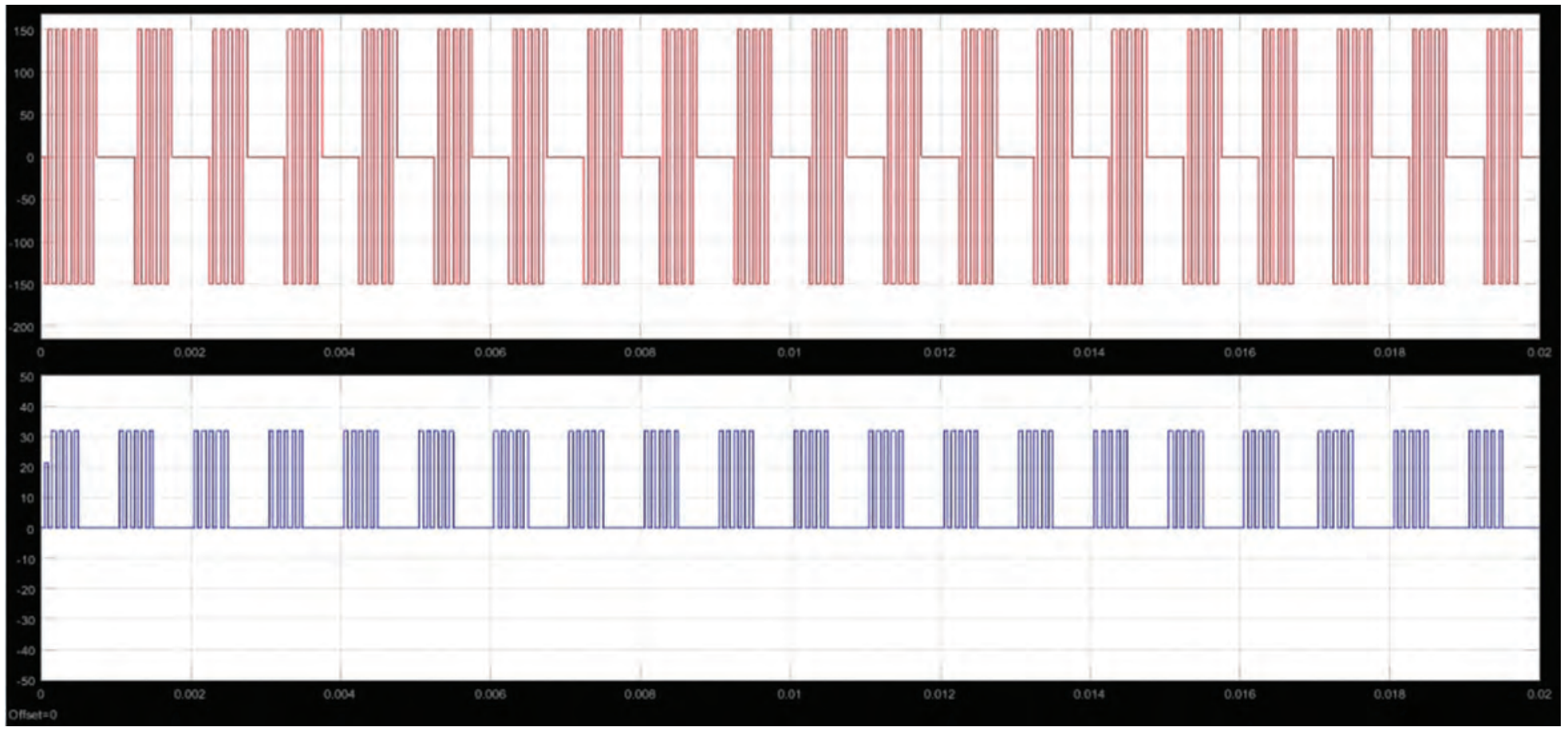

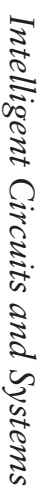

Figure 66.6 Output results of voltage across C and current across L for an input voltage of 150 volts. 


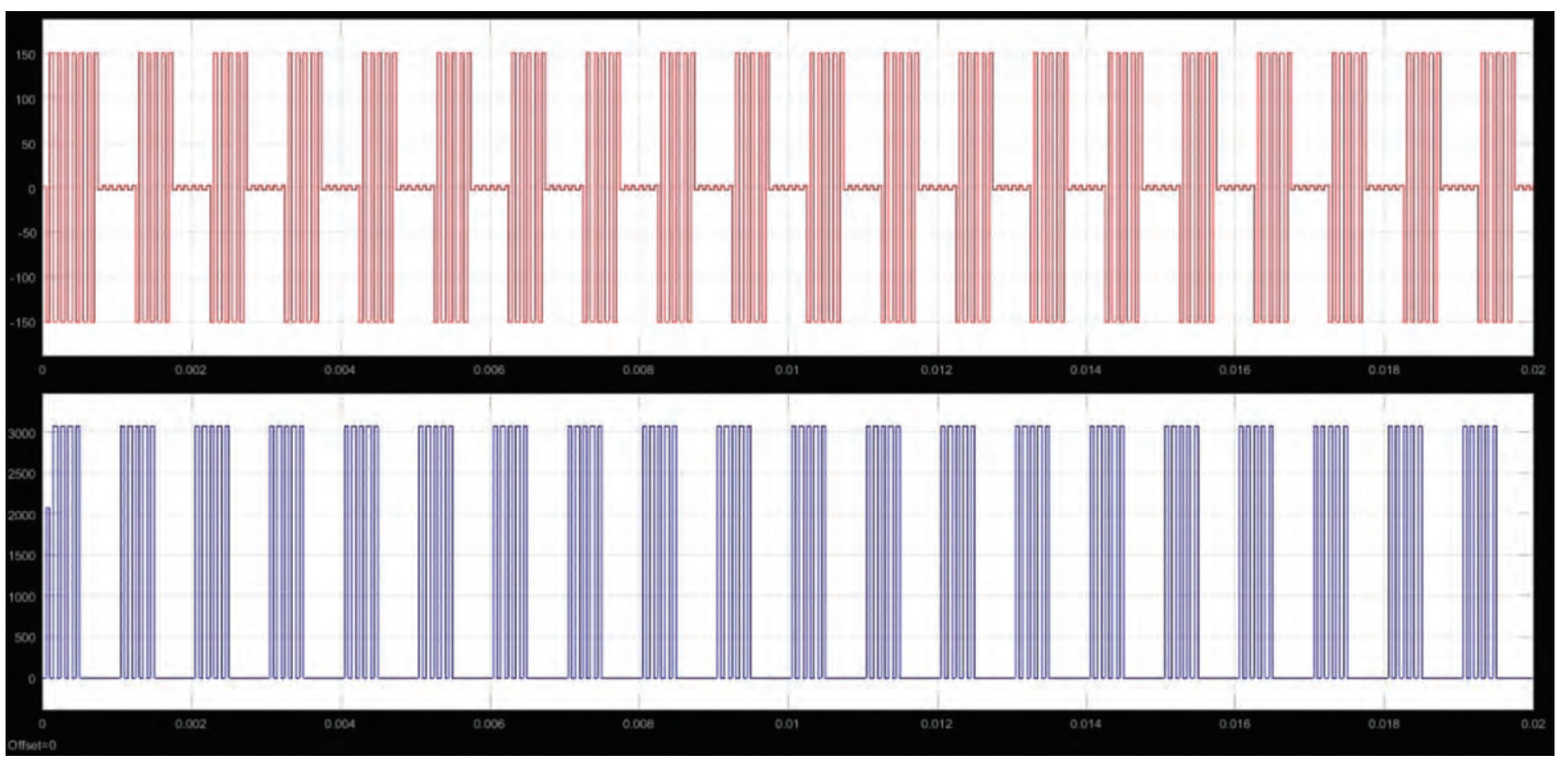

Figure 66.7 Output results of voltage across C and current across L for an input voltage of 150 volts. 


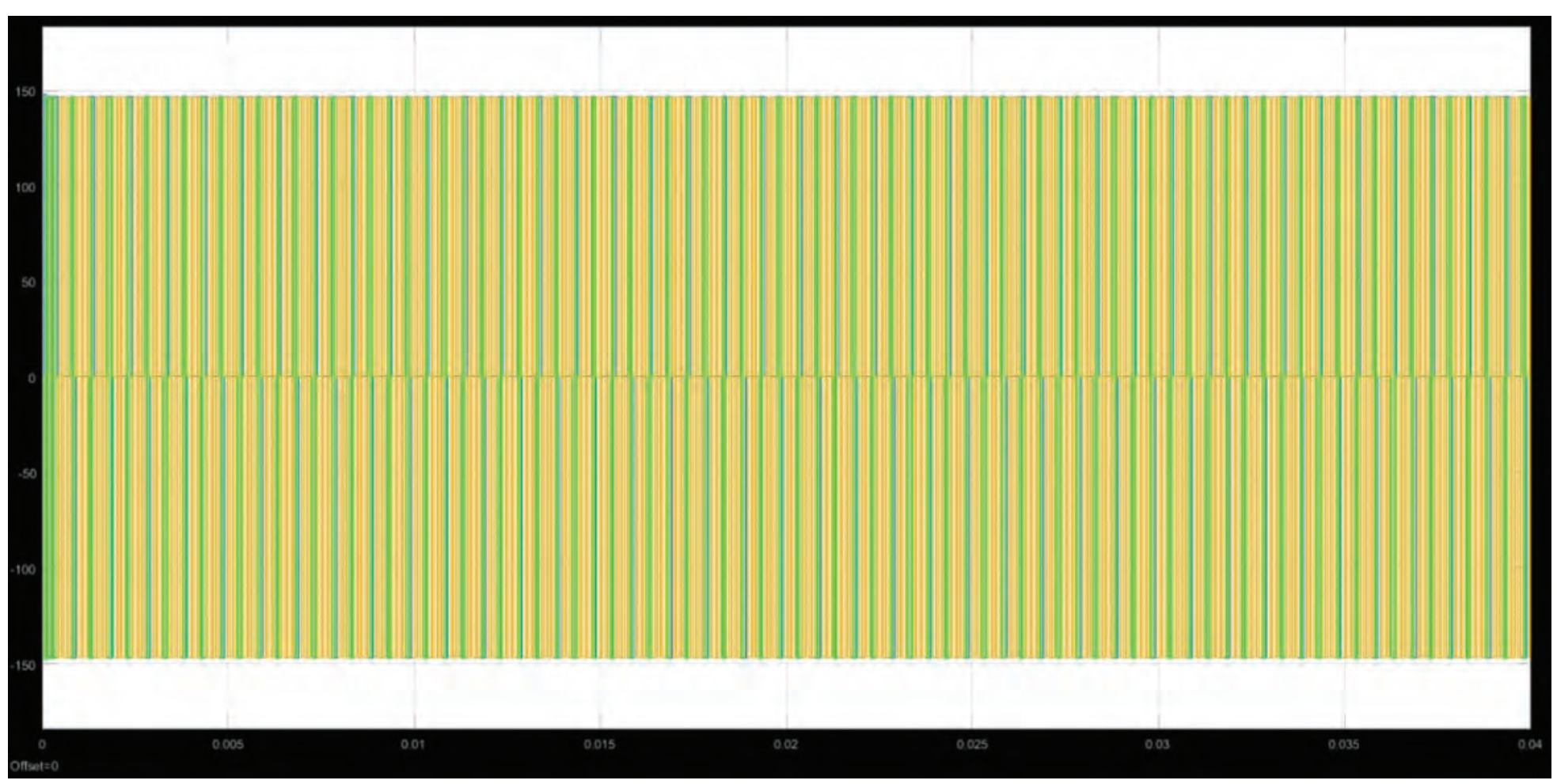

Figure 66.8 Output no-load voltage waveform of the converter. 


\section{References}

[1] B. Wu, "High-power converters and ac drives," High-Power Convert. ac Drives, pp. 1-332, 2005.

[2] T. Chen and M. Narimani, "Soft-switching T-type multilevel inverter," Journal of Power Electronics, vol. 19, no. 5, pp. 1182-1192, 2019.

[3] H. Zhu, J. S. Lai, A. R. Hefner, Y. Tang, and C. Chen, "Modeling-based examination of conducted EMI emissions from hard- and soft-switching PWM inverters," IEEE Trans. Ind. Appl., vol. 37, no. 5, pp. 1383-1393, 2001.

[4] T. Chen and M. Narimani, "A new ZVZCS three-level inverter," Proc. IECON 2017-43rd Annu. Conf. IEEE Ind. Electron. Soc., pp. 601-606, 2017.

[5] IEEE Transactions on Industrial Electronics, "Z-source converters: topologies, modulation techniques, and application - Part I,” vol. 65, no. 6, pp. 5092-5095, 2018.

[6] J. G. Webster and F. Z. Peng, "Z-source inverters," Wiley Encycl. Electr. Electron. Eng., no. i, pp. 1-11, 2017.

[7] F. Z. Peng, M. Shen, S. Member, Z. Qian, and S. Member, "Maximum boost control of the Z-source inverter," IEEE Transactions on Power Electronics, vol. 20, no. 4, pp. 833$838,2005$.

[8] M. Shen et al., "Constant boost control of the $\mathrm{Z}$-source inverter to minimize current ripple and voltage stress," IEEE Transactions on Industry Applications, vol. 42, no. 3, pp. 770-778, 2006. 


\title{
67 A comprehensive analysis of biosensor and performance parameters
}

\author{
Pawandeep Kaur ${ }^{1}$, Avtar Singh Buttar ${ }^{2}$, \\ Ashutosh Sharma ${ }^{3}$, G.P. Singh ${ }^{4}$ \\ ${ }^{1}$ PhD Scholar at I.K. Gujral Punjab Technical University, Kapurthala, \\ Punjab, India \\ ${ }^{1,3,4}$ Lovely Professional University, Jalandhar, Punjab, India \\ ${ }^{2}$ Associate Professor and Head, I.K. Gujral Punjab Technical University, \\ Kapurthala, Punjab, India
}

\section{Introduction}

Biosensor breaks into the words as bio relates to biology (nature) and sensor termed as the device which is capable of recognizing the participle present in nature as favourable form or non-favourable form. Thus biosensor is a powerful device that is a composite of a bio recognition component and analyses the throughput in the measurable form to detect the pathogens, toxins, pesticides, medical illness, environmental contaminants, food nutrients, increase the productivity and addition of the healthy nutrients during the growth of plants, the formation of the healthy seeds, controlling the additives as fermentation and monitor their lifecycle. According to the International Union of Pure and Applied Chemistry (IUPAC) [1] a biosensor is an independently analytic device which is a composite of biological or its derived recognition component and the transduction. A larger amount of charge moving with the same speed means a higher value of the measured current. The doping elements, for example affected by biological elements, are able to change the amount of potential difference across the sensor-sensitive material to control the current between source and drain of transistor junctions. Fundamental progress in semiconductor materials led to the development of such biosensors to achieve high sensitivity in biological sensors [2]. Further advances CMOS techniques are introduced with the scaling feature adapted the sensing arrays in parallel and integrated with circuits and electronic systems. With this solid state biosensors have the following characteristics

\subsection{Biosensor components}

(1) Bioreceptor as a recognition device, (2) a transducer as a physical component and (3) a signal processing system as conditioners.

In Figure 67.1 are the various components representing the principle of a biosensor. To understand the physical quantity, chemical reactions and corresponding electrical signals only when the bio components interact with the receptor and transducer interface [3]. These analyse terms as macromolecules like antigen, cells, antibodies, nucleic acids, enzymes or complex systems such as eukaryotic cells, bacterial cells, plant tissues or slices 


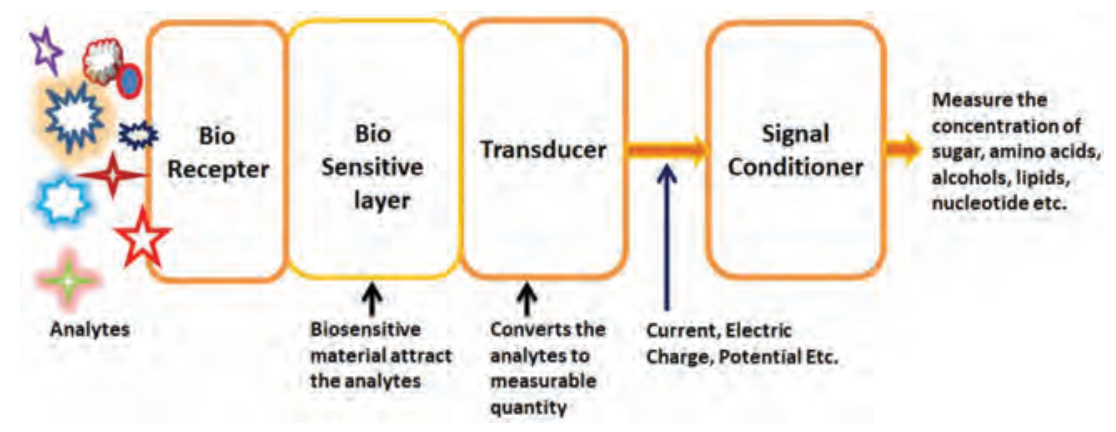

Figure 67.1 Structure represents principle of biosensors.

of mammalian. The study reflects that the immobilization of the enzymes, tissues or cells is used a number of times for an appreciable time on the interface of the transducer with a receptor. After this, the bio component as analyte binds with the receptor to make a bond, which results in the output in the measurable form. The transducer sensitive materials are current, voltage, conductance, impedance, optical, fluorescence, colour oriented or surface plasma resonating in order to quantify the concentration of the amino acids, sugar (glucose), lipids, and nucleotide which can be identified by biosensors.

\subsection{Performance characteristics of biosensors}

For any sensing device design, there are performance parameters that make the device commercial and easy to use. For biosensors the immobilization of the bio components is the foremost part, thus the performance factors of the biosensor are based on the structure [5] of the biosensor for various applications.

1. Sensitivity: this is the response time of the sensing material when the reactions are taking place at the interface of the receptor and the transducer area. Now the sensitivity is measured in parts per billion (ppb).

2. Detection limit: this is the parameter that indicates the minimum amount of the concentration of the target used for identification.

3. Selectivity: this termed as the crucial parameter as to how effectively it avoids any environmental disturbance or interference for quantifying the desired species out of others species with a similar structure.

4. Reversibility: play a vital role in the design of sensors as there is a tendency of the analytes that, after interacting with the interface which goes under the kinetic reactions, because of catalytic nature they return to their original form. The literature reviews show that some bio molecules such as antibodies and antigens are an example of non-reversibility which restricts the design of the immune biosensors.

5. Stability and the life: the life of the biosensor degrades with the passage of time and effects the accuracy, response time and reusability of the sensor. The stability of the biosensor depends upon the techniques used for immobilization of the analytes.

The main performance factor in a biosensor is the online observation or monitoring, correct, fast recognition of the analyte concentration. 
Table 67.1 Comparison on channel and structure of MOSFET based biosensors

\section{Semiconductor (MOSFET)}

Junctioned [17]

leakage current and complex fabrication process.

Inversion channel mobility in metal oxide semiconductor transistor is reduced by this electric field.

In inversion mode transistor reduces mobility of electron due to surface roughness scattering.

Current drive of IM MuGFETs decreases with temperature (mobility decreases with increasing temperature) more than IM DG FET.

IM DG FET lower bipolar gain compared to JL DG FET.

IM MOSFET high degradation of Gm compared to junctionless MOSFET.

Maximum S/D series resistance compare to JL device.
Junctionless MOSFET based biosensor

Dual Gate [18] Cylindrical [19]

Junctionless

Leakage current (Ioff) increases

extremely low leakage current and simple fabrication processes [20].

Electric field perpendicular to the current flow is found to be significantly lower in junctionless transistor.

Bulk conduction of junctionless transistor is credited for reduced surface roughness scattering

Current drive of JL MuGFETs increases with temperature (mobility remains constant with temperature) [21].

JL DG FET characterized by a higher bipolar gain.

Junctionless MOSFET slower degradation of $\mathrm{Gm}$ for high gate voltage.

Lower S/D series resistance is also found in JL device. as we move the body further away from the control of the gate.

Gate control over channel, thus reduce the leakage currents as gate is covered from all sides.

Cylindrical structure enhances the carrier transport and $I_{\text {on }} / I_{\text {off }}$ ratio.

to more leakage.

Sensitivity is less in the case of dual gate as per structure limitation.

Challenge with dual gate beyond $7 \mathrm{~nm}$ without compromising silicon body thickness (tsi) and oxide thickness (tox).

Package density is less with dual gate than cylindrical due to short channel.

Corner effect dominant in dual gate, weakens the gate controllability.

Dual gate alignment issue in DGDM biosensor
Increasing sensitivity of sensors more surface to volume is required, which is possible with cylindrical structure.

The channel length is reduced as per ITRS [22] by 2023 to $5 \mathrm{~nm}$ which is achievable with cylindrical structure without compromising the silicon body thickness $(t s i)$ and oxide thickness (tox).

Short devices increase the packing density of the device on chip and further reduce the cost per chip $[23,24]$.

Corner effect is eliminated in cylindrical gate MOSFETs [21]

In the case of cylindrical no issue on alignment at the time of fabrication. 


\section{Types of biosensors}

The classification of the biosensor is based on the components as biological components, transduction based along with the semiconductor as an emerging technology for sensing the bio species. In the following section, biosensors based on electronics are explored.

\subsection{Transduction based}

2.1.1 Electrochemical biosensors: These sensors' numerous advantages are easy and simple construction, low detection limit, easy operation, and particularity. These biosensors are available in a portable form as single chip devices for in vivo observation, because of miniaturization in devices as per advances in electronic instruments [4]. Classifications of the electrochemical biosensors are as:

(a) Amperometric biosensors: the output produced during oxidation or chemical reactions of electro active product is a current having units ampere, so named as an amperometric biosensor. The analytes like pollution contaminates are detected through screen printed sensor, and are explored with nanowires [5].

(b) Conductometric biosensors: in this biosensor the output produced after reactions is captured as a change in conductance. The main advantages of impedimetric methods is their ability to report charge transfer resistance during hybridization process between probe DNA and target DNAs or between antigen and antibodies affinity interactions [6]. Units are in mho.

(c) Potentiometric biosensors: this is basically a type of ISFET, in which reference electrodes are used for measuring potential with respect to bio sensing electrode [7]. The potential has units in volts and the measured volt is small in amount further signalling boosting system is required.

2.1.2 Optical biosensor: These sensors based on the principle that the output is measured after biochemical reactions at receptor end in the form of light absorbed or emitted. Based on their absorption or emitting techniques biosensors of different types are absorption, fluorescence, luminescence, surface plasmon resonance (SPR) [8], colorimetric, fibre optic based. The measurement is the construct of the refractive index when electromagnetic waves are propagating after illumination, at the surface of the metal film, termed as surface plasmons. Biomolecule kinetics changes the plasmon mode at the surface, due to surface plasmons are profoundly reliant on the orientation of the plasmonic structure and the ecological parameters [9]. The colorimetric biosensor is based on the change in colour density when analytes are interacting with receptor and transduction area. The essential application is in quantifying the pathogens in food and water.

2.1.3 Thermal biosensors: When there is a change in thermal with respect to the interception of bio species onto the interface of bio receptor and transducer. The chemical reactions composite of the change in heat and enthalpy which further can be recorded by thermistor of high sensitivity [10].

2.1.4 Piezoelectric biosensor: These utilize precious crystals to make an electrical potential by versatile disfigurements. The piezoelectric biosensors include estimation of mass change happening because of bimolecular communication [11]. Piezoelectric crystal is to utilize gauge to change the mass by corresponding with the adjustment of swaying occurrence of piezo-crystal.

2.1.5 Chemiluminescence biosensor: Sensor relies on the emission of light or termed luminescence after the chemical reaction results in energy production, which emits light. 
In [12], this happens due to reactions the iota and particles changes their states from energized to relax (ground) level as results by-products of light are released because of kinetic reactions. The basic principle of chemiluminescence is an interaction between the bio species and the transduction area, after immobilized bio species, results in the generation of light, thus this emitted light is quantified by the photomultiplier tube (PMT) .Various literature reviewers depict that the chemiluminescence biosensors have high sensitivity and fast response, with ease of handling of instrumentation along with broad calibration range.

2.1.6 Barometric biosensor: These are used in observing the respiration of bacteria by measuring the pressure and respiration hindrance as a sign of toxicity. The major application areas are in waste water detection of harmful pathogens, using ambient light sensor (ALS) of a smart phone rather than computational imaging based phone cameras .This allows light weight, portable, and low cost. [13].

\section{Semiconductor field effect transistor based biosensor}

\subsection{Limitations of ISFET}

There are numerous drawbacks associated with ISFET based biosensors. Foremost is electric potential influenced by the ion concentration of the analytes in solution [14]. Secondly, analytes have different layers (bulk solution, diffusion layer and stern layer) with different ion concentration thus may affect the electrical signal [15]. Third problem is that maintaining and controlling the samples like urine, saliva, blood serum as real human samples is challenging [16]. Poor sensitivity in the case of neutral biomolecules.

\section{Conclusion}

Research in the zone of biosensors and gadgets brings every one of the controls like engineering, material science, applied science, and therapeutic (medical) science on a typical stage where every one of them has their own significance. Semiconducting innovation has thought of a lead job and discovers fast development in the zone of scaled down biosensors. Advances in the zone of biosensors and gadgets with electronic detecting innovations will lift up the fate of diagnostics and social insurance that involves health medication, bedside clinical diagnostics, poisonous virus, drug disclosure and microorganism. In field of semiconductors biosensors help to detect the charged and neutral analytes.

\section{References}

[1] S. E. Braslavsky, "Glossary of terms used in photochemistry, (IUPAC Recommendations 2006)," Pure and Applied Chemistry, vol. 79, pp. 293-465, 2007.

[2] L. C. Clark Jr and C. Lyons, "Electrode systems for continuous monitoring in cardiovascular surgery," Annals of the New York Academy of Sciences, vol. 102, pp. 29-45, 1962.

[3] H. Im, X.-J. Huang, B. Gu, and Y.-K. Choi, "A dielectric-modulated field-effect transistor for biosensing," Nature Nanotechnology, vol. 2, p. 430, 2007.

[4] J. Hopkins, K. Fidanovski, A. Lauto, and D. Mawad, "All-organic semiconductors for electrochemical biosensors: An overview of recent progress in material design," Frontiers in Bioengineering and Biotechnology, vol. 7, p. 237, 2019.

[5] G. Hernandez-Vargas, J. E. Sosa-Hernández, S. Saldarriaga-Hernandez, A. M. VillalbaRodríguez, R. Parra-Saldivar, and H. Iqbal, "Electrochemical biosensors: A solution to pollution detection with reference to environmental contaminants," Biosensors, vol. 8, p. 29, 2018. 
[6] R. Eivazzadeh-Keihan, P. Pashazadeh-Panahi, T. Mahmoudi, K. K. Chenab, B. Baradaran, M. Hashemzaei, et al., "Dengue virus: a review on advances in detection and trends-from conventional methods to novel biosensors," Microchimica Acta, vol. 186, p. 329, 2019.

[7] J. Ding and W. Qin, "Recent advances in potentiometric biosensors," TrAC Trends in Analytical Chemistry, p. 115803, 2020.

[8] M. Bocková, J. Slabý, T. Špringer, and J. Homola, "Advances in surface plasmon resonance imaging and microscopy and their biological applications," Annual Review of Analytical Chemistry, vol. 12, pp. 151-176, 2019.

[9] Y. Zhao, R.-j. Tong, F. Xia, and Y. Peng, "Current status of optical fiber biosensor based on surface plasmon resonance," Biosensors and Bioelectronics, p. 111505, 2019.

[10] C. Pundir and A. Malik, "Bio-sensing of organophosphorus pesticides: A review," Biosensors and Bioelectronics, p. 111348, 2019.

[11] M. Pohanka, "Overview of piezoelectric biosensors, immunosensors and DNA sensors and their applications," Materials, vol. 11, p. 448, 2018.

[12] M. J. Molaei, "A review on nanostructured carbon quantum dots and their applications in biotechnology, sensors, and chemiluminescence," Talanta, vol. 196, pp. 456-478, 2019.

[13] S. Dutta, "Point of care sensing and biosensing using ambient light sensor of smartphone: Critical review," TrAC Trends in Analytical Chemistry, vol. 110, pp. 393-400, 2019.

[14] W. M. Siu and R. S. Cobbold, "Basic properties of the electrolyte-SiO 2-Si system: physical and theoretical aspects," IEEE Transactions on Electron Devices, vol. 26, pp. 1805$1815,1979$.

[15] F. Puppo, M.-A. Doucey, T. S. Moh, G. Pandraud, P. M. Sarro, G. De Micheli, et al., "Femtomolar sensitive field effect transistor biosensors based on silicon nanowires and antibodies," in SENSORS, 2013 IEEE, 2013, pp. 1-4.

[16] J.-Y. Pyo and W.-J. Cho, "High-performance SEGISFET pH sensor using the structure of double-gate a-IGZO TFTs with engineered gate oxides," Semiconductor Science and Technology, vol. 32, p. 035015, 2017.

[17] B. Buvaneswari, "A survey on multi gate MOSFETS," International Journal of Innovative Research in Science, Engineering and Technology, vol. 3, pp. 783-788, 2014.

[18] R. Narang, M. Saxena, and M. Gupta, "Investigation of dielectric modulated (DM) double gate (DG) junctionless MOSFETs for application as a biosensors," Superlattices and Microstructures, vol. 85, pp. 557-572, 2015.

[19] A. Chakraborty and A. Sarkar, "Analytical modeling and sensitivity analysis of dielectricmodulated junctionless gate stack surrounding gate MOSFET (JLGSSRG) for application as biosensor," Journal of Computational Electronics, vol. 16, pp. 556-567, 2017.

[20] E. Rahman, A. Shadman, and Q. D. Khosru, "Effect of biomolecule position and fill in factor on sensitivity of a dielectric modulated double gate junctionless MOSFET biosensor," Sensing and Bio-Sensing Research, vol. 13, pp. 49-54, 2017.

[21] D.-Y. Jeon, S. J. Park, M. Mouis, S. Barraud, G.-T. Kim, and G. Ghibaudo, "Low-temperature electrical characterization of junctionless transistors," Solid-State Electronics, vol. 80, pp. 135-141, 2013.

[22] N. M. Ravindra, "International technology roadmap for semiconductors (ITRS). Symposium," Journal of electronic materials, vol. 30, pp. 1478-1627, 2001.

[23] A. Ortiz-Conde and F. J. G. Sanchez, "Unification of asymmetric DG, symmetric DG and bulk undoped-body MOSFET drain current," Solid-state Electronics, vol. 50, pp. 1796-1800, 2006.

[24] N. Mendiratta, S. L. Tripathi, S. Padmanaban, and E. Hossain, "Design and analysis of heavily doped $\mathrm{n}+$ pocket asymmetrical junction-less double gate MOSFET for biomedical applications," Applied Sciences, vol. 10, p. 2499, 2020. 


\title{
68 A review: Building fire detection and emergency evacuation system
}

\author{
G.S. Birajdar ${ }^{1, *}$, Rajesh Singh ${ }^{2, *}$, Anita Geblot ${ }^{3, *}$ \\ ${ }^{1}$ Research Scholar, SEEE, LPU Punjab, India \\ ${ }^{2}$ Professor, SEEE, LPU Punjab, India \\ ${ }^{3}$ Associate Professor, SEEE, LPU, Punjab, India
}

\section{Introduction}

With the rapid growth in population, buildings are also growing vertically due to the shortage of occupancies. Due to this there is a rise of problems regarding safe evacuation of people during an emergency situation like a fire. Similarly, a threat to the life of building and human is increasing due to excessive use of insulating material in building which can catch fire easily. Many people are killed every year and more are hospitalized due to fire in India. Many of these casualties can be avoided if we detect a fire early and guide people to a safe location.

\section{Development in fire detection and emergency evacuation}

\subsection{Hardware platforms}

After detecting a fire event it needs to be processed for further course of actions, for which an effective hardware platform plays a key role.

\subsubsection{Arduino board}

Arduino boards are equipped with many digital inputs and output pins that can be interfaced with different expansion boards or shields and other required circuits. These boards feature a USB for loading programs from personal computers.

\subsubsection{Raspberry Pi}

The Raspberry Pi is a cheaper and small sized computer that plugs into a computer monitor or TV, and uses a standard keyboard and mouse. It can be connected to the Internet easily using Ethernet port and USB Wi-Fi dongles. Multiprocessing is possible using this board. Programming languages like Python, C, C++ and Ruby can be used.

\subsection{Fire sensing sensors and devices}

These involve multi-sensor modules for sensing parameters like heat, smoke, flame, gas etc. (Figure 68.1), signal conditioning, amplifier circuit, processing unit, alarm system and safe evacuation path display.

\footnotetext{
*Emails: gajananbirajdar20@gmail.com, rajesh.23402@lpu.co.in, anita.23401@1pu.co.in
}

DOI: 10.1201/9781003129103-68 


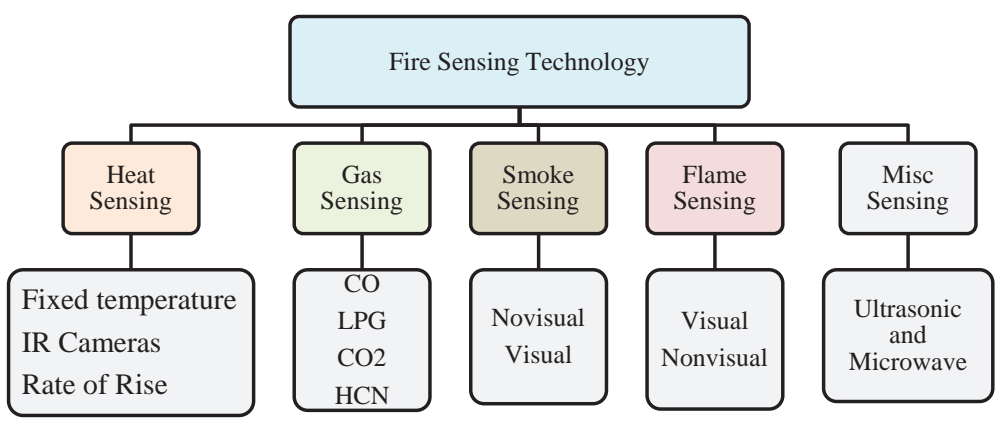

Figure 68.1 Fire-sensing technologies.

\subsection{Simulation tools}

\subsubsection{BIM}

Building information modelling (BIM) enables us to present a facility layout in threedimensions (3D) and include the disaster-prevention objects. Specifically, it is used in the design of fire sprinklers and alarm system. e.g. BIM 360, AutoCAD, Revit, etc. [3].

\subsubsection{FDS}

Fire dynamics simulator (FDS) is a computational fluid dynamics model of fire-driven fluid flow. It is developed by the Building Fire Research Laboratory of NIST. FDS can analyse three-dimensional fires very quickly. It enables the visualization of smoke and heat transport from fire; besides, FDS includes an analysis of fire detectors and water sprinklers.

\subsubsection{MATLAB}

MATLAB based image processing can be used to detect fire accidents. The input image for the system can be taken from real-time video from any CCTV camera installed in the premises of the building. Fire growth is estimated by comparing two consecutive fire images. If fire growth continues over certain frames, the emergency alarm is actuated.

\section{Suggestions for a future fire detection and evacuation system}

\subsection{Long-range communication technology}

Connected devices have so far run on traditional protocols like Bluetooth, WiFi and cellular network etc. But embedded sensors and other IoT devices have different network requirements like low power, long range etc. LoRa and LPWAN can fulfil these requirements.

\subsection{Hardware selection and customization}

Selection of hardware is a key parameter while designing fire detection and evacuation systems. The parameters like accuracy, quick response, cost, size, robustness, and reliablity need to be considered while selecting hardware. Instead of considering ready boards for 
Table 68.1 Comparison of hardware, fire sensing, simulation, communication method and UI

\begin{tabular}{|c|c|c|c|c|c|}
\hline References & Hardware / technology & Fire-sensing devices & Simulation software & $\begin{array}{l}\text { Communication } \\
\text { technology }\end{array}$ & User interface \\
\hline Jiang Huixian et al. & $\begin{array}{l}\text { Mobile terminal indoor } \\
\text { location technology } \\
\text { and Bluetooth }\end{array}$ & Temperature sensor & GIS & $\begin{array}{l}\text { Bluetooth } \\
\text { technology }\end{array}$ & $\begin{array}{l}\text { Sending alarm message } \\
\text { to user mobile with fire } \\
\text { location }\end{array}$ \\
\hline Faisal Saeed et al. & Raspberry Pi & $\begin{array}{l}\text { LM 35, MQ9 } \\
\text { Smoke sensor }\end{array}$ & FDS & GSM & No work on evacuation \\
\hline Selahattin et al. & Arduino & $\begin{array}{l}\text { RFM23B RFID, LM35, HC- } \\
\text { 05, PIR }\end{array}$ & BIM & $\begin{array}{l}\text { Bluetooth } \\
\text { technology }\end{array}$ & Audio signal, text message \\
\hline Majumder et al. & $\begin{array}{l}\text { nRF24L01 } \\
\text { Laptop with Arduino }\end{array}$ & IR flame sensor & $\begin{array}{l}\text { MATLAB's robotics } \\
\text { package }\end{array}$ & $\mathrm{RF}$ transceivers & HTML/CSS based website \\
\hline Angel et al. & FPGA, microcontroller & Temperature sensor & TimeNET & $\begin{array}{l}\text { Wireless } \\
\text { transreceiver }\end{array}$ & $\begin{array}{l}\text { Direct alarm system is } \\
\text { provided at each floor }\end{array}$ \\
\hline Kun-Ming Yu et al. & IP based cameras & $\begin{array}{l}\text { Temp., humidity, illumination } \\
\text { and CO levels }\end{array}$ & GCM & $\begin{array}{l}\text { ZigBee\& } \\
\text { RFID }\end{array}$ & $\begin{array}{l}\text { Smartphone } \\
\text { Digital signage }\end{array}$ \\
\hline Huan-Po Hsu et al. & Zigbee, RFID & $\begin{array}{l}\text { Temperature, humidity and } \\
\text { light }\end{array}$ & mySQL databese & WSN technology & LCD display \\
\hline Poy et al. & Microcontroller & Smoke & BAS & Cloud & App on smartphone \\
\hline
\end{tabular}


designing system it is recommended to develop customized hardware which assists in reducing cost and size.

\subsection{Intelligent digital display}

During fire accidents people caught inside buildings need to be evacuated through a safe exit path. A static fire exit path may lead to the wrong destination as fire spreads in any direction. Hence, an intelligent digital display is to be adopted to guide people based on the real-time situation.

\subsection{Density of people}

Safe evacuation of people from the building during fire emergency is of utmost important. People density is a key parameter in rescue operations during fire events. We need to install an effective system which can measure the number of persons stuck in a fire.

\section{Conclusion}

Fire accident is one of the dangerous events which need to be addressed effectively with the help of smart technology. In view of this, we have surveyed the available methods and systems for fire detection and evacuation systems. Efforts have been taken to review the important literature to give insights into techniques used for fire detection and safe evacuation. We have identified some areas where still improvement is possible. Suggestions are also made for the future development of fire detection and evacuation systems.

\section{References}

1. Jiang Huixian and Zhang Shaoping. 2018. Design of intelligent fire-fighting system of a big public building emergency evacuation navigation system for mobile terminal. IEEE.

2. Faisal Saeed, Anand Paul, Abdul Rehman, Won Hwa Hong and Hyuncheol Seo. 2017. IoTbased intelligent modeling of smart home environment for fire prevention and safety. $m d p i$, Sensor Jornal 7(1)

3. Selahattin Gokceli, Nikolay Zhmurov, Kurt Gunes Karabulut, and Berna Ors. 2017. IoT in Action Design and Implementation of a Building Evacuation Service. Hindawi, Journal of Computer Networks and Communications: 02.

4. Majumder, Swarnadeep, Sean O’Neil, and Ryan Kennedy. 2017. Smart apparatus for fire evacuation - an iot based fire emergency monitoring and evacuation system. IEEE.

5. A Sheeba Angel and R. Jayaparvathy. 2017. Design and implementation of an intelligent emergency evacuation system. Computation of Power, Energy Information and Communication (IEEE): 013-017.

6. Kun-Ming Yu and Shao-Tsai Cheng. 2015.Intelligent evacuation system integrated with image recognition technology. IEEE (doi:10.1109/UMEDIA.2015.7297422).

7. Huan-Po Hsu, Kun-Ming Yu, and Shao-Tsai Cheng. 2014. Emergency evacuation base on intelligent digital signage systems. IEEE (doi:10.1109/U-MEDIA.2014.31).

8. Poy, Hector Moner, and Brian Duffy. 2014. A cloud-enabled building and fire emergency evacuation application. IEEE Cloud Computing 1.4: 40-49. 


\title{
69 Statistical evaluation of colour image denoising techniques
}

\author{
Amandeep Singh,*, Gaurav Sethi², G.S. Kalra ${ }^{3}$ \\ 1,2School of Electronics and Electrical Engineering, Lovely Professional \\ University, Jalandhar, Punjab, India \\ ${ }^{3}$ Electronics and Communication, CT Group of Institutions, \\ Jalandhar, Punjab, India
}

\section{Introduction}

Images nowadays are considered as a vital source of information, so these are transmitted, stored and processed in high numbers. Any loss in image information can affect the overall performance of the application containing the image processing stage. This demands more technological advances to maintain image quality [1-4]. Due to these reasons, image denoising is still a valid challenge for the researchers. The image denoising process is required to achieve maximum denoising while keeping the prime details of images like high-frequency components and object boundaries. The image loses some important information in the denoising stage as there is a very less intensity difference between the object and its boundaries. It is worth taking a note here that image denoising is an inverse problem, so the solution may vary from problem to problem. Salt and pepper is the noise which has a major role is image distortion. This noise can reduce the image details, further it is important to detect and remove the noise before providing the image to the image processing stage. Salt and pepper noise can have two possible values, i.e., 0 or 255 [5]. The occurrence of impulse noise is random in images and can produce any pattern, which makes it is even more difficult to detect the location of noise and to predict the original value of the noisy pixel. This problem is considered as a classic problem in digital image processing but it is still attracting the attention of various researchers as the enhanced image visual clarity is always in demand. To achieve this various algorithms are available, hence it will be a challenging task to find a suitable method for colour images. Various methods were designed earlier for the greyscale image but in comparison less work is performed with respect to colour image denoising. In this paper, a comparative analysis will be achieved on the basis of peak signal to noise ratio (PSNR) [6]. These three denoising algorithms are used for the comparative analysis, i.e. centred weighted median filter (CWMF) [7], progressive switching median filter (PSMF) [8] and median filter (MF) [9]. As these algorithms do not support the colour image as such so the image is first divided into three parts and the algorithm is applied sequentially to make the algorithms compatible with colour images. This manuscript is systematized as given: in Section 2, the process of comparative analysis is presented. Section 3 is dedicated to results and discussion (denoising of colour images). The concluding remarks are in Section 4.

* Corresponding author email: amansandhu6788@gmail.com

DOI: 10.1201/9781003129103-69 


\section{Comparative analysis procedure}

In this paper, noise is added to the image in the range of different levels to form a noisy image dataset. This range of noise levels is typically from $10 \%$ to $50 \%$ with a step size of $10 \%$. These noise-affected images are provided as input to algorithms for denoising and PSNR is calculated from denoised images. The comparative analysis is performed between three algorithms of denoising, i.e. MF, PSMF and CWMF. Median filter (MF) is one of the renowned filters due to its low complexity and computational cost but its filtration efficiency is good for low noise level only; its performance decrease as noise levels rise. Progressive switching median filter (PSMF) uses a repetitive mechanism of two stages to perform image denoising, which gives its performance a boost over the median filter but it has a high computational cost. Centred weighted median filter (CWMF) achieves denoising by inclining the weight distribution towards the centre and hence can provide decent denoising while avoiding damage to the edges of an image. Colour images are formed by a combination of three individual matrices of colour, red, green and blue, which can in combination represent any colour. So algorithms which are designed to denoise greyscale images can be provided with these individual matrixes one by one to denoise a colour image and then again combine the three individual denoised matrixes to form a denoised colour image.

\section{Results and discussion}

To ensure the appropriate selection of the denoising algorithm for the salt and pepper noise-affected images, it is required to evaluate the popular denoising algorithms on the different range of salt and pepper noise. This is done to identify the versatility and reallife utilization capabilities of these algorithms. For this process, five colour images of the standard data set [10] [presented in Figure 69.1(a) to Figure 69.1(e)] are affected by salt and pepper noise to create noise-affected images. These images are provided as input to the denoising algorithms (MF, PSMF, CWMF) to obtain a denoised image for the comparative analysis. The salt and pepper noise is added in the image in the steps of $10 \%$ starting from the initial noise level of $10 \%$ and maximum up to $50 \%$. These various levels of noise-affected colour images are considered as the noisy colour image data set for this comparison. The noisy colour image data is applied to the MF, PSMF, CWMF algorithms for this comparison and results are compared with the statistical parameter PSNR. Samples of noisy image data are presented in images shown in Figure 69.2(a) to Figure 69.6(a) and denoised images from the noisy image are shown in Figure 69.2(b)(d) to Figure 69.6(b)-(d). Denoising results produced by the MF algorithm are shown in Figure 69.2(b) to Figure 69.6(b) whereas results produced by the PSWN algorithm are shown in Figure 69.2(c) to Figure 69.6(c) along with denoising results of the CWMF algorithm, which are presented in Figure 69.2(d) to Figure 69.6(d). Resulting images are presented to provide a clear depiction of image denoising provided by these algorithms to the human eye.

On a similar basis results of other images of the colour image data set (as shown in Figure 69.1(b) to Figure 69.1(e)) can be calculated. The results of the statistical parameter PSNR are provided in Table 69.1 to Table 69.5. The results are shown in the form of the PSNR parameter for the corresponding denoised image. From these results, a pattern can be noticed that as the noise level in the images increases the performance of the denoising algorithms reduces. 


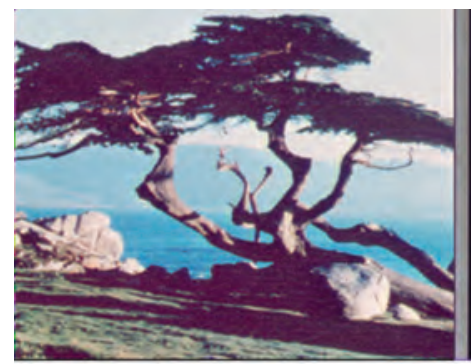

(a)

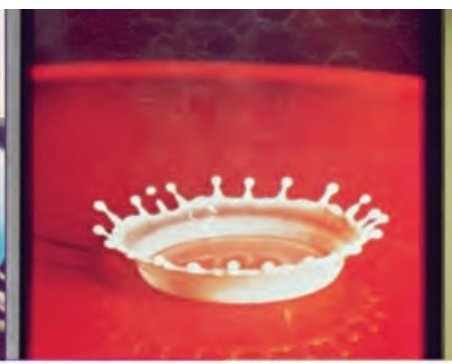

(b)

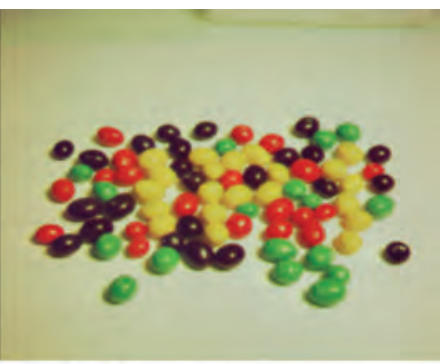

(c)

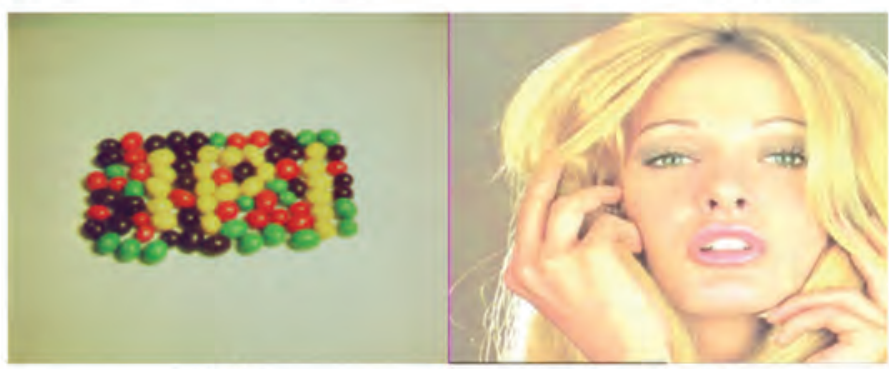

(d)

Figure 69.1 Original colour image data set. (a) Tree image, (b) drop image, (c) jelly beans-A image, (d) jelly beans-B image, (e) tiffany image.

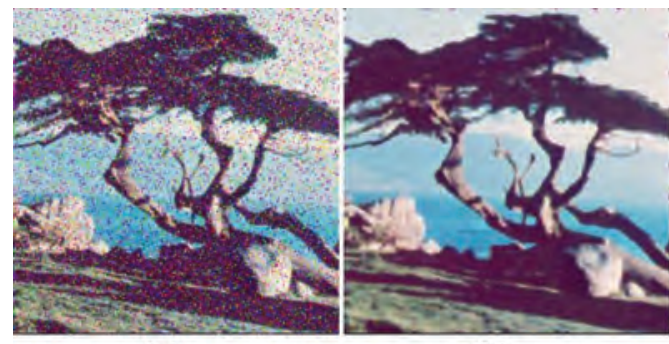

(a)

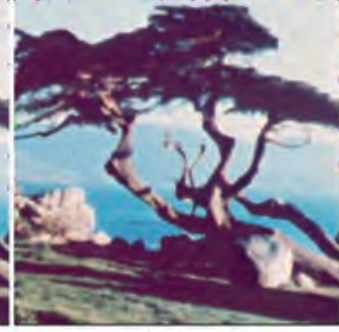

(c)

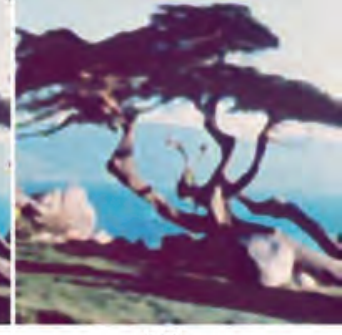

(d)

Figure 69.2 (a) Tree image affected by 10\% noise. (b) MF denoising, (c) PSMF denoising, (d) CWMF denoising.

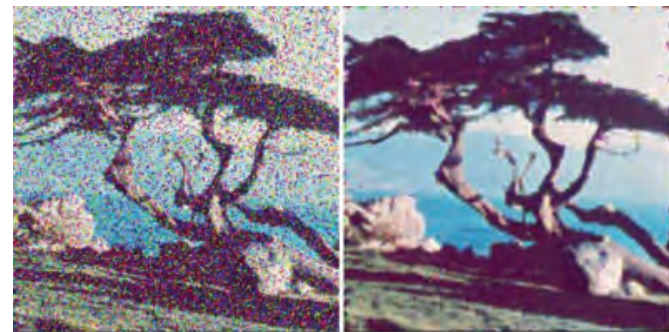

(a) (b)

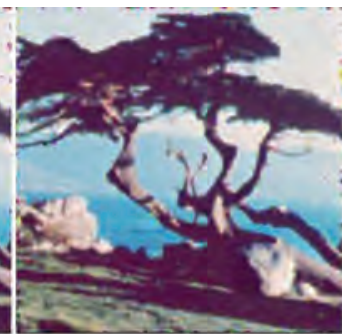

(c)

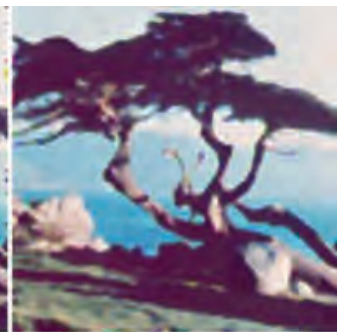

(d)

Figure 69.3 (a) Tree image affected by 20\% noise. (b) MF denoising, (c) PSMF denoising, (d) CWMF denoising. 


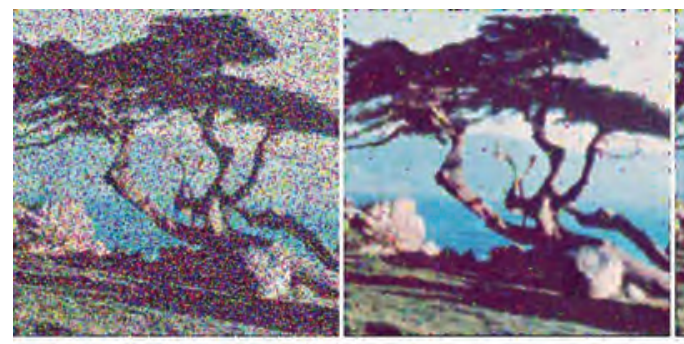

(a)

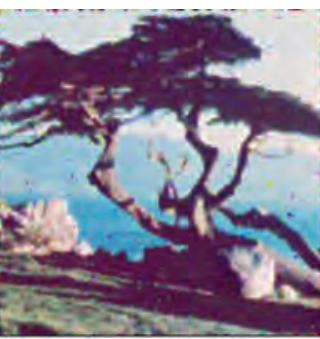

(c)

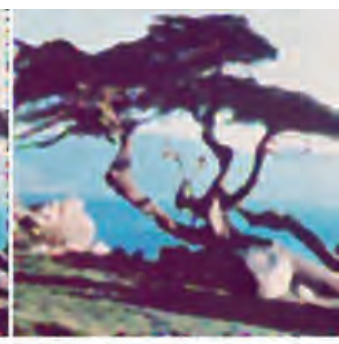

(d)

Figure 69.4 (a) Tree image affected by 30\% noise. (b) MF denoising, (c) PSMF denoising, (d) CWMF denoising.

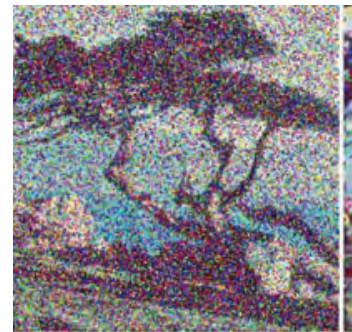

(a)

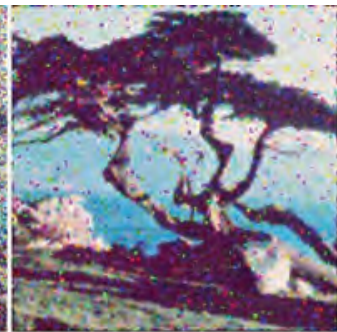

(b)

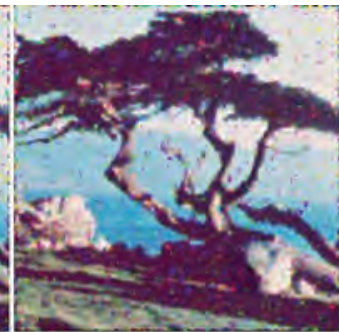

(c)

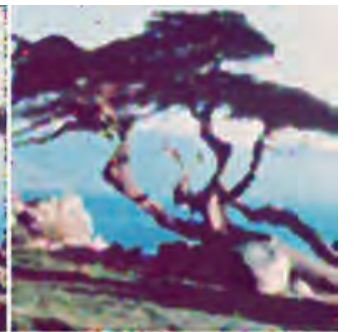

(d)

Figure 69.5 (a) Tree image affected by 40\% noise. (b) MF denoising, (c) PSMF denoising, (d) CWMF denoising.

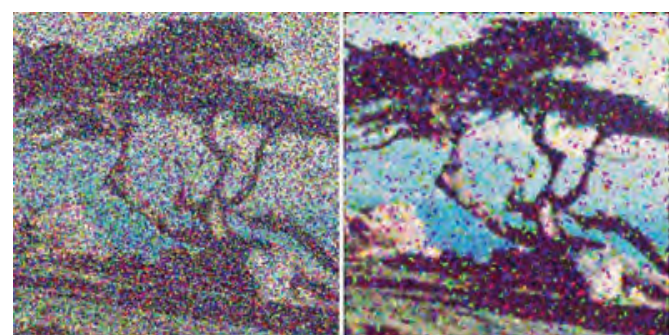

(a) (b)

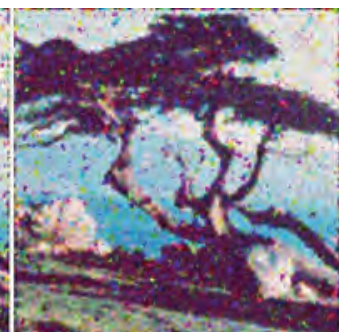

(c)

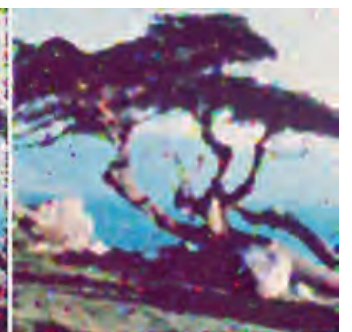

(d)

Figure 69.6 (a) Tree image affected by 50\% noise. (b) MF denoising, (c) PSMF denoising, (d) CWMF denoising.

Every algorithm has a different range for its best performance, i.e. MF provides better performance when an image is affected by low level of noises, typically $10 \%$, where its performance is generally high; similarly the PSMF algorithm also provides the desired performance when images are affected in the noise level range of $10 \%$ and $20 \%$. But the CWMF algorithm is unable to perform better than the MF algorithm 
Table 69.1 PSNR results for tree image denoised by MF, PSMF and CWMF

\begin{tabular}{llllll}
\hline Noise level & $10 \%$ & $20 \%$ & $30 \%$ & $40 \%$ & $50 \%$ \\
\hline MF & 26.43 & 23.91 & 20.92 & 17.46 & 14.21 \\
PSMF & 26.39 & 24.13 & 21.69 & 19.00 & 16.06 \\
CWMF & 23.51 & 23.19 & 22.70 & 21.83 & 20.23 \\
\hline
\end{tabular}

Table 69.2 PSNR results for drop image denoised by MF, PSMF and CWMF

\begin{tabular}{llllll}
\hline Noise level & $10 \%$ & $20 \%$ & $30 \%$ & $40 \%$ & $50 \%$ \\
\hline MF & 33.23 & 27.48 & 22.42 & 18.35 & 14.74 \\
PSMF & 32.62 & 28.41 & 24.32 & 20.40 & 17.19 \\
CWMF & 32.99 & 32.42 & 31.34 & 29.35 & 25.14 \\
\hline
\end{tabular}

Table 69.3 PSNR results for jelly beans-A image denoised by MF, PSMF and CWMF

\begin{tabular}{llllll}
\hline Noise level & $10 \%$ & $20 \%$ & $30 \%$ & $40 \%$ & $50 \%$ \\
\hline MF & 32.36 & 27.50 & 22.37 & 18.08 & 14.78 \\
PSMF & 32.41 & 27.92 & 24.12 & 20.49 & 17.04 \\
CWMF & 31.40 & 30.42 & 29.58 & 27.59 & 23.93 \\
\hline
\end{tabular}

Table 69.4 PSNR results for jelly beans-B image denoised by MF, PSMF and CWMF

\begin{tabular}{llllll}
\hline Noise level & $10 \%$ & $20 \%$ & $30 \%$ & $40 \%$ & $50 \%$ \\
\hline MF & 32.18 & 27.33 & 22.62 & 18.25 & 14.48 \\
PSMF & 31.82 & 28.55 & 24.69 & 20.76 & 17.02 \\
CWMF & 29.52 & 28.63 & 28.00 & 26.26 & 23.19 \\
\hline
\end{tabular}

Table 69.5 PSNR results for tiffany image denoised by MF, PSMF and CWMF

\begin{tabular}{llllll}
\hline Noise level & $10 \%$ & $20 \%$ & $30 \%$ & $40 \%$ & $50 \%$ \\
\hline MF & 31.01 & 26.92 & 21.80 & 17.25 & 13.92 \\
PSMF & 30.62 & 26.89 & 23.23 & 19.66 & 16.27 \\
CWMF & 28.49 & 28.34 & 28.05 & 26.70 & 23.39 \\
\hline
\end{tabular}

when an image is affected with $10 \%$ of salt and pepper noise. CWMF shines in the performance as noise increases from low level to high, typically when images are affected with $30 \%$ of noise level to $50 \%$. To get better performance on a wide range of noiseaffected images, it is required to design a new denoising algorithm which can have the advantages of MF (to have high denoising capabilities when an image is affected by low level of noises) and CWMF (to have high denoising performance for images affected with high noise levels). 


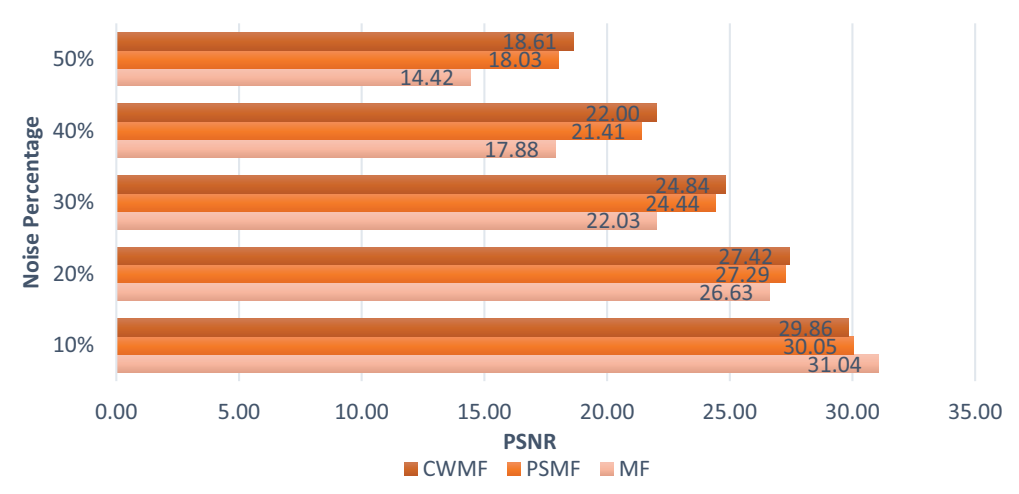

Figure 69.7 Comparative analysis of mean PSNR values of the algorithms.

To obtain the overall performance pattern of the considered algorithms, the mean values of all the images are calculated for the respective noise level and presented in Figure 69.7. For $10 \%$ noise level affected colour images the MF filter performs better than other algorithms and for the images affected by the noise level of $20 \%$ to $30 \%$ the CWMF algorithm achieves the higher mean PSNR.

\section{Conclusion}

We evaluate the performance of different image denoising algorithms for a wide range of noise levels, typically for the range of $10 \%$ to $50 \%$. For this process, noisy images are produced by adding salt and pepper noise with increasing levels. All the algorithms considered for this evaluation have a delay in the performance whenthe noise level increases. The median filter performs comparatively higher than the other two algorithms (PSMF and CWMF) for a 10\% noise level. But the drawback of median filter is that its performance reduces when the noise level exceeds the $10 \%$ mark. On the other hand, the CWMF algorithm has a mean performance better than the other two (MF and PSMF) algorithms for the noise range of $20 \%$ to $50 \%$. It is suggested that a hybrid algorithm can be made by combining the qualities of both MF and CWMF algorithms to achieve a desirable denoising performance for the wide range of noise-affected images.

\section{References}

[1] O. S. Faragallah and H. M. Ibrahem, "Adaptive switching weighted median filter framework for suppressing salt-and-pepper noise," AEU-Int. J. Electron. Commun., vol. 70, no. 8, pp. 1034-1040, 2016.

[2] Z. Sun, B. Han, J. Li, J. Zhang, and X. Gao, "Weighted guided image filtering with steering kernel,” IEEE Trans. Image Process., vol. 29, pp. 500-508, 2020.

[3] M. Li and Y. Xu, "Improved non-local means algorithm for image denoising," in 2019 IEEE 11th International Conference on Communication Software and Networks, ICCSN 2019, 2019.

[4] Zhengguo Li, Jinghong Zheng, Zijian Zhu, Wei Yao, and Shiqian Wu, "Weighted guided image filtering," IEEE Trans. Image Process., vol. 24, no. 1, pp. 120-129, Jan. 2015.

[5] Kamarujjaman, M. Mukherjee, and M. Maitra, "A new decision-based adaptive filter for removal of high density impulse noise from digital images," in 2014 International Conference on Devices, Circuits and Communications, ICDCCom 2014-Proceedings, 2014. 


\section{Intelligent Circuits and Systems}

[6] M. S. Nair and G. Raju, "A new fuzzy-based decision algorithm for high-density impulse noise removal," Signal, Image Video Process., vol. 6, no. 4, pp. 579-595, 2012.

[7] T. Sun, M. Gabbouj, and Y. Neuvo, "Center weighted median filters: Some properties and their applications in image processing," Signal Processing, vol. 35, no. 3, pp. 213-229, 1994.

[8] G. M. Daiyan and M. A. Mottalib, "Removal of high density salt \& pepper noise through a modified decision based median filter," 2012 Int. Conf. Informatics, Electron. Vision, ICIEV 2012, vol. 18, no. 5, pp. 565-570, 2012.

[9] I. Aizenberg and C. Butakoff, "Effective impulse detector based on rank-order criteria," IEEE Signal Process. Lett., vol. 11, no. 3, pp. 363-366, 2004.

[10] Volume 3: Miscellaneous-USC Viterbi I Ming Hsieh. Department of Electrical and Computer Engineering. [Online]. Available at: https://minghsiehece.usc.edu/volume-3-miscellaneous-2/ [Accessed: 25-Jan-2020]. 


\title{
70 Smart water quality analysis system using datapine
}

\author{
Vikas Tiwari, K.P. Sharma, Nonita Sharma, \\ Prashant Kumar
}

\author{
Department of Computer Science \& Engineering, Dr. B.R. Ambedkar \\ National Institute of Technology, Jalandhar, India
}

\section{Introduction}

Nearly $70 \%$ of Earth's surface is covered with water, out of which only $2.5 \%$ of water is available as freshwater. This fresh water is present in rivers, lakes, ponds and in the form of glaciers. Whereas less than $0.3 \%$ of water is present in streams, lakes and rivers. But as development in industrialization is taking place and also due to other human actions, the quality of water is degrading day by day. According to a report presented by the Central Ground Water Board in 2017, the most important reasons for water pollution are excessive use of natural resources [1]. Fast growth in industrialization and release from industries and run-off from the agricultural fields are also the major concern for water pollution. A very small percentage of water is available as fresh water which is fit for human consumption. And that also is being affected by human actions, which makes water quality monitoring an important topic of concern. The term water quality monitoring refers to monitoring the fluctuations in various quality parameters of water such as $\mathrm{pH}$, turbidity, conductivity, total dissolved solids (tds), temperature, dissolved oxygen etc. But the monitoring of water needs to be done using smart ways [2].The smart solution for water quality monitoring deploys sensors for measuring various parameters of water quality, transmits the measured values to the cloud storage and when the value exceeds some predefined threshold, the end users are notified [4,5].

\section{Materials and tools}

The data for river Satluj and Beas are collected from the government website: www.data. gov.in [6,7]. The analysis of the data is done using the data analysis tool called datapine [8]. The different water quality indicators namely $\mathrm{pH}, \mathrm{BOD}$, conductivity, temperature, nitrate, total coliform, fecal coliform are compared for both the rivers in Himachal Pradesh and Punjab states. The analysis is done in order to draw a conclusion to decide the type of sensors required for proper analysis of water quality. The prototype for analysing water quality is inferred from the review presented by Geetha and Gouthami [9], where they discussed the previous work done in this field and proposed model using TI CC3200 MCU kit (Figure 70.1) [9].

\section{Analysis of various parameters comparing both data streams}

The following comparisons are drawn from the in-depth analysis of the data.

DOI: $10.1201 / 9781003129103-70$ 


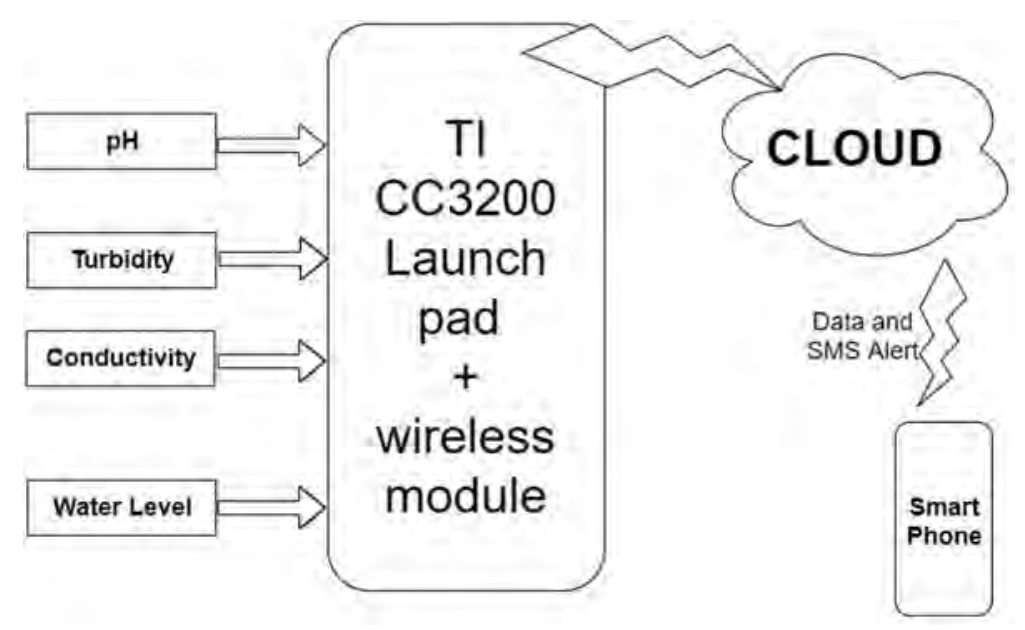

Figure 70.1 Block diagram of proposed system [9].

\subsection{Biochemical oxygen demand}

Biochemical oxygen demand or BOD is a chemical method for finding the measure of dissolved oxygen required by aerobic microorganisms to decompose organic substances existing in a given water, tested at a specific temperature for a particular time frame.

The greater the BOD measure, the more is food available for microorganisms, and the more polluted is the water source. A water source having a BOD measure of 3-5 ppm is viewed as moderately clean, whereas the water having a BOD measure $6-9$ ppm is considered a little bit polluted as some organic materials are present there breaking this waste. The BOD comparison for the rivers Satluj and Beas for Punjab and Himachal Pradesh can be viewed in Figure 70.2.

\subsection{Conductivity}

Conductivity and salinity are measures of water's capability to carry current. It provides an estimation of the salts dissociated in water. The greater conductivity value demonstrates that more synthetic substances are dissociated in the water. The conductivity of distilled water is in the range 0.5 to $3 \mu \mathrm{S} / \mathrm{cm}$, whereas most streams stretch from 50 to $1500 \mu \mathrm{S} / \mathrm{cm}$. Freshwater streams in a perfect world ought to have conductivity values lying in 150 to $500 \mathrm{HS} / \mathrm{cm}$ to help grouped maritime life. The comparisons between both the river streams is depicted in Figure 70.3.

\subsection{Temperature}

The chemical and physical properties of water also depend on the temperature of the water, as it governs the rate at which the biological and chemical reactions take place and also controls the maximum amount of dissolved oxygen in the water. Temperature oversees the sorts and kinds of amphibian life in water. In normal water sources, the temperature might not exceed 89 degrees (Fahrenheit), whereas temperature in cold water sources should not exceed 68 degrees (Fahrenheit). The analysis of both the river streams is shown in Figure 70.4. 


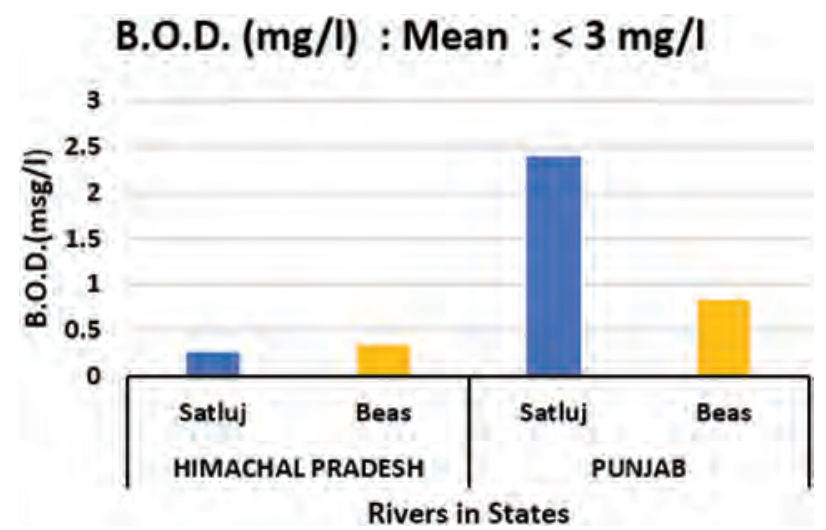

Figure 70.2 Comparison of BOD of Satluj and Beas in HP and Punjab.

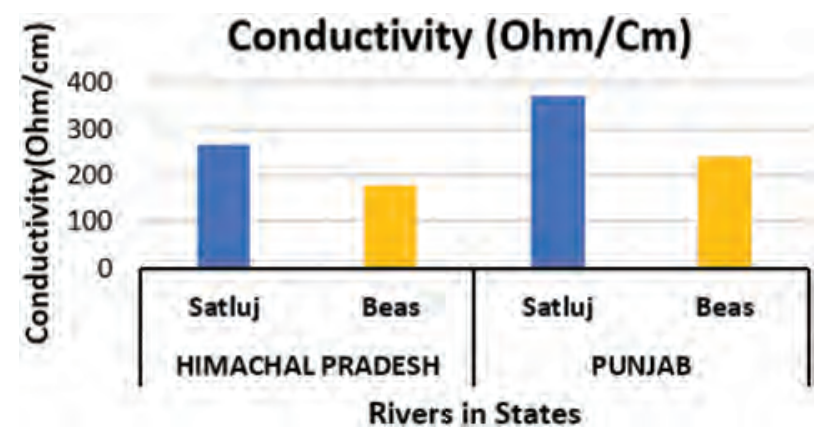

Figure 70.3 Comparison of conductivities of Satluj and Beas in HP and Punjab.

\section{Temperature}

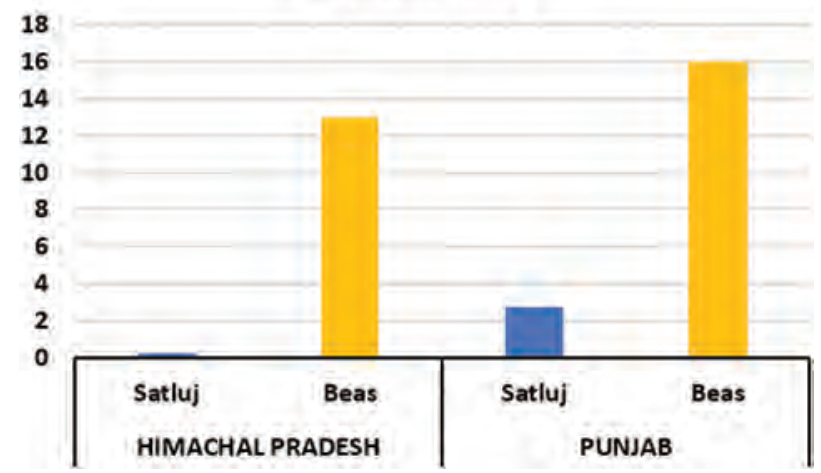

Figure 70.4 Comparison of water temperature (in degree Celsius) of Satluj and Beas in HP and Punjab. 


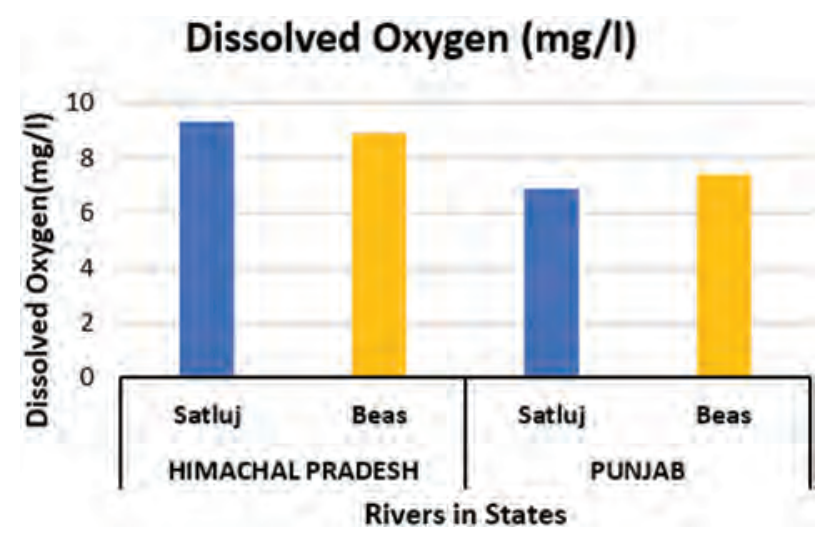

Figure 70.5 Comparison of dissolved oxygen in water for Satluj and Beas in HP and Punjab.

\subsection{Dissolved oxygen}

In water bodies, the parameter dissolved oxygen or DO is measured in terms of concentration. The DO in a water source is generally measured in milligram per litres of water. Oxygen present in the atmosphere generally diffuses into the water source and is further used by fish and other water-going creatures to breathe. In IOT, the DO is generally measured using a specially calibrated oxygen water sensor, which usually measures the DO level as a percentage. Figure 70.5 demonstrates the analysis of DO in the two river streams. The concentration level of dissolved oxygen should be above $6.5-8 \mathrm{mg} / \mathrm{L}$ and between about $80-120 \%$.

\section{$3.5 \mathrm{pH}$}

$\mathrm{pH}$ is used to determine the acidic or basic nature of water. $\mathrm{pH}$ generally is hydrogen ion concentration in ion. On the off chance that the water of the stream is excessively acidic or basic, the hydrogen $\left(\mathrm{H}^{+}\right)$ion or hydroxyl $(\mathrm{OH}-)$ ion vitality might disrupt aquatic creatures' biochemical responses by either hurting or killing the creatures. Safe water sources usually have $\mathrm{pH}$ between 6 and 9. For safe drinking water $\mathrm{pH}$ ranges from 6.5 to 8.5, whereas for oceanic life it goes from 6.5 to 9. Analysis of $\mathrm{pH}$ is done in Figure 70.6.

\section{Correlation indicator table among the water quality parameters}

\subsection{Correlation indicators for Satluj River}

The correlation indicators (Table 70.1) for the Satluj River shows strong negative correlation between nitrate and $\mathrm{DO}$, which means as nitrate concentration increases the dissolved oxygen concentration decreases. This is due to fact that nitrate facilitates survival of oxygen-consuming microorganisms and vegetation in water [10]. $\mathrm{pH}$ is strongly negatively correlated with nitrate and temperature. This is because the presence of nitrate in water makes it acidic, because of formation of its conjugate acid nitric acid (i.e. $\mathrm{HNO}_{3}$ ) [11]. Fecal coliform is strongly positively correlated with BOD, as the more there is fecal coliform, the more there are oxygen-consuming microorganisms and vegetation in water, and the greater is the BOD value. Strong positive correlation between conductivity and $\mathrm{BOD}$, and conductivity and nitrate is also observed. 


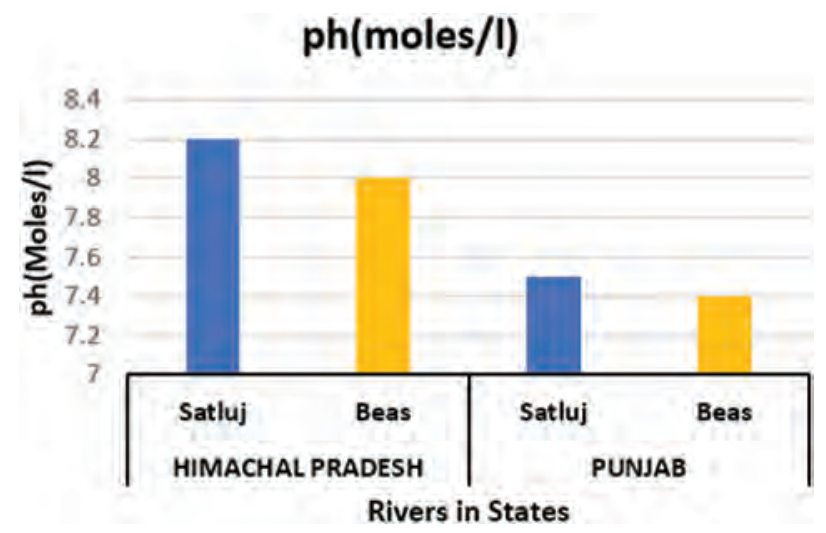

Figure 70.6 Comparison of water pH values of Satluj and Beas in HP and Punjab.

Table 70.1 Correlation indicators for Satluj River

\begin{tabular}{|c|c|c|c|c|c|c|c|c|}
\hline & $B O D$ & D.O & $p H$ & Nitrate & $\begin{array}{l}\text { Fecal } \\
\text { coliform }\end{array}$ & Conductivity & $\begin{array}{l}\text { Total } \\
\text { coliform }\end{array}$ & Temperature \\
\hline $\mathrm{BOD}$ & - & -0.86 & -0.71 & 0.80 & 0.89 & 0.93 & 0.92 & - \\
\hline DO & -0.86 & - & 0.80 & -0.96 & - & -0.84 & - & -0.48 \\
\hline $\mathrm{pH}$ & -0.71 & 0.80 & - & -0.83 & - & -0.68 & - & -0.76 \\
\hline Nitrate & 0.80 & -0.96 & -0.83 & - & & 0.85 & & \\
\hline $\begin{array}{l}\text { Fecal } \\
\text { coliform }\end{array}$ & 0.89 & & & & & 0.71 & & \\
\hline Conductivity & 0.93 & -0.84 & -0.68 & 0.85 & 0.71 & & 0.76 & \\
\hline $\begin{array}{l}\text { Total } \\
\text { coliform } \\
\text { Temperature }\end{array}$ & 0.92 & & & & & 0.76 & & \\
\hline
\end{tabular}

\subsection{Correlation indicators for Beas River}

On the basis of the correlation table for the Beas River (Table 70.2), the table shows strong negative correlation between DO and nitrate. This clearly indicates that as the nitrate in the water increases, the dissolved oxygen and $\mathrm{pH}$ decreases in correlation with that. This means the greater the nitrate concentration in water the more acidic is the water. Also dissolved oxygen and conductivity are strongly negatively correlated. The correlation matrix [12] also indicates strong negative correlation between fecal coliform and dissolved oxygen. This is due to the fact that the greater the fecal coliform value, the more there are organisms and vegetation matter consuming oxygen present [13]. Moreover, a strong negative correlation between conductivity and dissolved oxygen and a strong positive correlation between conductivity and temperature are also depicted.

\section{Conclusion}

On the basis of the above analysis of water quality data collected for rivers Satluj and Beas flowing in the region Himachal Pradesh and Punjab, it can be concluded that water quality 
Table 70.2 Correlation indicators for the Beas River

\begin{tabular}{|c|c|c|c|c|c|c|c|c|}
\hline & $B O D$ & D.O & $p H$ & Nitrate & $\begin{array}{l}\text { Fecal } \\
\text { coliform }\end{array}$ & Conductivity & $\begin{array}{l}\text { Total } \\
\text { coliform }\end{array}$ & Temperature \\
\hline BOD & - & -0.86 & & 0.69 & 0.85 & 0.76 & 0.77 & - \\
\hline D.O & -0.86 & - & & -0.77 & -0.76 & -0.85 & - & -0.70 \\
\hline $\mathrm{pH}$ & & & - & -0.81 & -0.70 & & -0.64 & \\
\hline Nitrate & 0.69 & -0.77 & -0.81 & - & 0.85 & & 0.73 & \\
\hline $\begin{array}{l}\text { Fecal } \\
\text { coliform }\end{array}$ & 0.85 & -0.76 & -0.70 & 0.85 & & 0.71 & & \\
\hline Conductivity & 0.76 & -0.85 & & & & & 0.64 & 0.85 \\
\hline $\begin{array}{l}\text { Total } \\
\quad \text { coliform }\end{array}$ & 0.77 & & -0.64 & 0.73 & - & 0.64 & & \\
\hline Temperature & & -0.70 & & & & 0.85 & & \\
\hline
\end{tabular}

is lower in Punjab when contrasted with water quality in Himachal Pradesh. The water quality of Satluj River is poorer when compared with the water quality of river Beas in both the states. Also correlation insights generated from the simulation indicate that most the prominent indicators are $\mathrm{BOD}$, temperature, $\mathrm{pH}$ and conductivity. All other indicators can be evaluated from these parameters.

\section{References}

[1] Central Ground Water Board (2017) Ministry of Water Resources, River Development and Ganga Rejuvenation, Government of India. http://cgwb.gov.in/. Accessed 24 July 2017.

[2] Tuna, Gurkan, Orhan Arkoc, and Kayhan Gulez. "Continuous monitoring of water quality using portable and low-cost approaches." International Journal of Distributed Sensor Networks 9, no. 6 (2013): 249598.

[3] Lambrou, Theofanis P., Christos C. Anastasiou, Christos G. Panayiotou, and Marios M. Polycarpou. "A low-cost sensor network for real-time monitoring and contamination de- tection in drinking water distribution systems." IEEE Sensors Journal 14, no. 8 (2014): 2765-2772.

[4] Chen, Yiheng, and Dawei Han. "Water quality monitoring in smart city: A pilot project." Automation in Construction 89 (2018): 307-316.

[5] Sharma, Nonita, and Ajay K. Sharma. "Cost analysis of hybrid adaptive routing protocol for heterogeneous wireless sensor network." S'adhanª 41, no. 3 (2016): 283-288.

[6] https://data.gov.in/resources/water-quality-river-satluj-2013.

[7] https://data.gov.in/resources/water-quality-river-beas-2013.

[8] www.datapine.com/documentation/G.

[9] Geetha, S., and S. Gouthami. "Internet of things enabled real time water quality monitoring system.” Smart Water 2, no. 1 (2016): 1.

[10] Srivastava, Satyam, Saikrishna Vaddadi, and Shashikant Sadistap. "Smartphone-based system for water quality analysis.” Applied Water Science 8, no. 5 (2018): 130.

[11] Jiang, Peng, Hongbo Xia, Zhiye He, and Zheming Wang. "Design of a water environment monitoring system based on wireless sensor networks." Sensors 9, no. 8 (2009): 6411-6434.

[12] Sharma, Nonita, and Akanksha Juneja. "Extreme gradient boosting with squared logistic loss function.” In Machine Intelligence and Signal Analysis, Springer, Singapore (2019): 313-322.

[13] Srivastava, S., Vaddadi, S., and Sadistap, S. "Smartphone-based system for water quality analysis.” Applied Water Science 8, no. 5 (2018): 130.

[14] Ubidots (2017) IoT platform - Internet of Things. https://ubidots.com/. Accessed 22 June 2017. 
[15] Bhatt, Jayti, and Jignesh Patoliya. "IoT based water quality monitoring system." IRFIC (2016).

[16] Charef, Azedine, Antoine Ghauch, Patrick Baussand, and Michel Martin-Bouyer. "Water quality monitoring using a smart sensing system." Measurement 28, no. 3 (2000): 219-224.

[17] Cloete, Niel Andre, Reza Malekian, and Lakshmi Nair. "Design of smart sensors for real- time water quality monitoring." IEEE Access 4 (2016): 3975-3990. 


\title{
71 Design of MPPT based controller for BLDC motor drive
}

\author{
Aaryan Raj, Aman Ganesh \\ School of Electronics and Electrical Engineering, Lovely Professional \\ University, Phagwara, India
}

\section{Introduction}

The technological advances and developments related to solar PV cells and arrays are to achieve higher conversion efficiency. But once a type of solar panel/array is selected the next big challenge is to utilize them for maximum electrical efficiency. The power obtained or supplied by a PV solar cell depends on both physical and mechanical parameters such as irradiance, temperature, mounting or tilt angle, sun tracking etc. The power obtained is the function of both voltage and current or in other words the impedance of the system. So many electronic circuits and algorithms in the literature can be found to track the maximum available DC power [1]. The basic power electronics based DC-DC converter is the heart of the MPPT controller, where the single LC (inductor-capacitor) component guarantees a wide input operating voltage range over varying insolation and load parameters. Similarly for maximizing the output from the PV array there are many algorithms such as voltage or current based peak power point tracking. Fractional open circuit voltage or current, perturb and observe (P\&O), incremental conductance (INC), intelligent algorithm (fuzzy/neural/neuro-fuzzy) based peak power point tracking, load voltage or current have been proposed [2-6].

At present the trend is toward the use of battery-operated vehicles. The vehicles work under varying load conditions and hence draw significant current from the battery thus exhausting them at a faster rate. So a potential solution is to use a PV array and MPPT based driver circuit. The benefit of using a vehiclemounted PV array is that PV energy can directly drive the load and can also charge the battery. The MPPT based driver circuit will help to draw maximum power from the PV source, thus indirectly increasing battery life and reducing operating cost. The objective of this paper to select the best MPPT algorithm among P\&O, FOPC and IC implemented for a DC-DC converter based driving circuit for a PV array mounted BLDC motor based e-vehicle.

The paper is organized in four sections. The first section gives the introduction, the second section gives the basics of the PV solar cell, different MPPT algorithms, the BLDC motor control scheme, and the third section gives insight about the DC-DC driver circuit and BLDC motor. Simulation results are given in the fourth section, followed by the conclusion in the last section. 


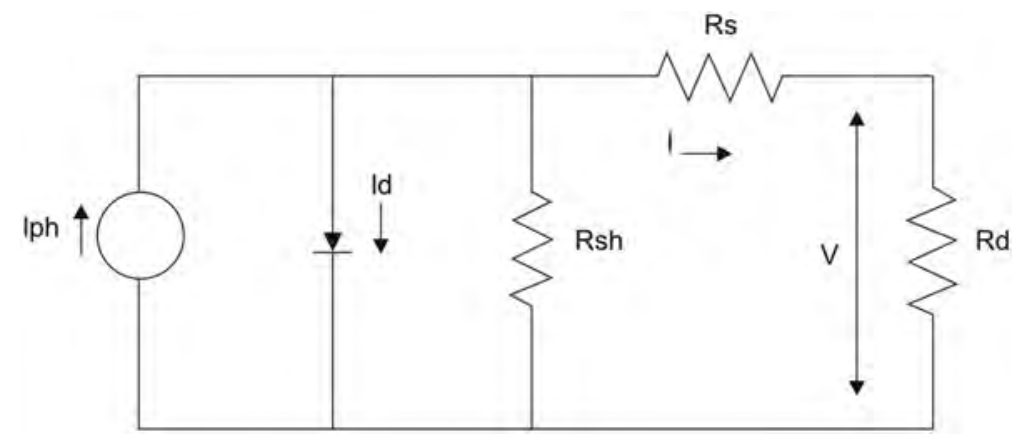

Figure 71.1 Equivalent electrical model of PV solar cell.
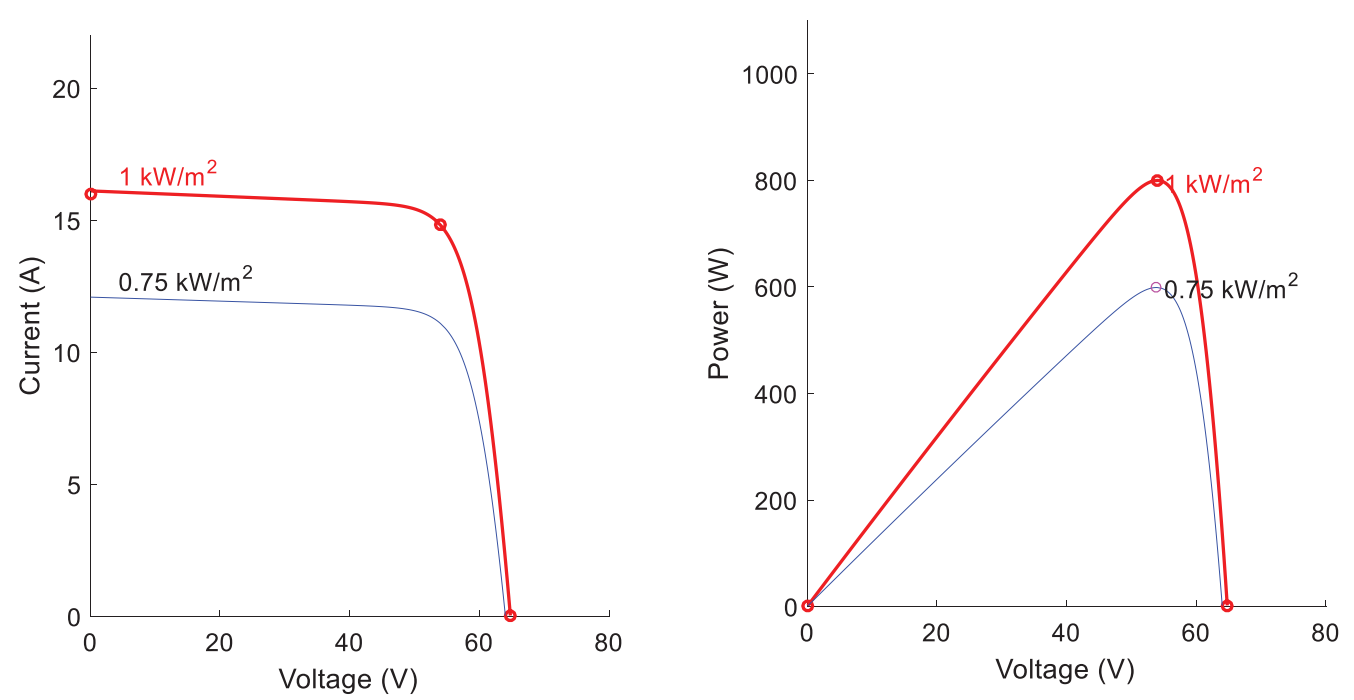

Figure 71.2 Performance characteristics of PV cell.

\section{Methodology}

\subsection{Solar cell equivalent circuit and its characteristics}

The PV solar cell governing equations can be found in [7] for ready reference. Figure 71.1 shows the electrical equivalent of the PV solar cell.

The photocurrent is represented as Iph, reverse saturation current is $I_{d}$, the shunt and series resistance is represented by $\mathrm{R}_{\text {sh }}$ and Rs respectively. The performance characteristics of the selected PV array $(2 * 2)$ with Isc of $8 \mathrm{~A}$, Voc of $32.4 \mathrm{~V}$ and peak power $(\mathrm{Mpp}=200 \mathrm{~W})$ at peak voltage of $27 \mathrm{~V}$ is shown in Figure 71.2 under the varying irradiance levels of 1 $\mathrm{kW} / \mathrm{m}^{2}$ and $0.75 \mathrm{~kW} / \mathrm{m}^{2}$. 


\subsection{MPPT control algorithm}

In the current study, three algorithms are selected. A brief description of each is presented in the following sections.

\subsubsection{Hill climbing / perturb and observe (P\&O) MPPT}

This is a conventionally and majorly used algorithm works by measuring the output voltage level and then varying (perturbing) it in small steps in both directions (positive and negative) for obtaining maximum power. It is a continuous process, so the variations are done in small steps so as to achieve maximum power. The higher the step size the lower will be the tracking. This puts a limitation on the electronic circuit realization of the algorithm $[2,3]$. The algorithm is best explained with the flowchart shown in Figure 71.3(A).

\subsubsection{Incremental conductance MPPT}

The I-V curve slope gives the information about the conductance, hence the point of the maximum power has a defined/fixed maximum power point. In the incremental conductance method use of both voltage and current sensor is there. So the conductance is evaluated at each sampling instant and is compared against the reference conductance [4]. This control algorithm is depicted in Figure 71.3(B) with the help of flowchart. The incremental conductance change $(\mathrm{dI} / \mathrm{dV})$ is calculated with respect to power change $(\mathrm{dP} / \mathrm{dV})$

\subsubsection{Fractional open circuit voltage MPPT}

The algorithm is relatively easy to understand and implement. It explores the somewhat linear relationship between $\mathrm{V}_{\text {MPP }}$ and Voc. For each PV module it is defined and has to be pre-determined experimentally. So mathematically $\mathrm{V}_{\mathrm{MPP}}=\mathrm{K} \mathrm{V}_{\mathrm{OC}}$. The algorithm is implemented by measuring the module voltage at a given sampling rate and then modulating the duty cycle of the driver circuit for maximizing the PV module output. Simple and relatively easy to implement electronic circuitry is the advantage of this algorithm. But as the $\mathrm{V}_{\mathrm{MPP}}$ changes with the change in temperature and insolation this algorithm limits itself while considering $\mathrm{V}_{\mathrm{MPP}}$ to be a fixed voltage only. But still in the literature it can be found that FOCV comparatively gives better results [5,6]. Figure 71.3(c) gives a pictorial representation of the FOCV algorithm.

\subsection{BLDC motor control scheme}

In the current work the battery has not been included so the BLDC motor will extract power from the installed PV array. The control mechanism layout is shown in Figure 71.4.

The level shifted MPPT driver output voltage is fed to the DC-AC converter circuit which generates rectangular pulses for driving the BLDC motor. Since the current emphasis is on extracting maximum power from the panel, the DC level shifter and BLDC basic model details are discussed in the following section.

\subsubsection{DC-DC converter design}

The basic DC-DC driver circuit used in the current study is a boost converter. The circuital elements of an boost converter are: inductor, a switching device (MOSFET in 


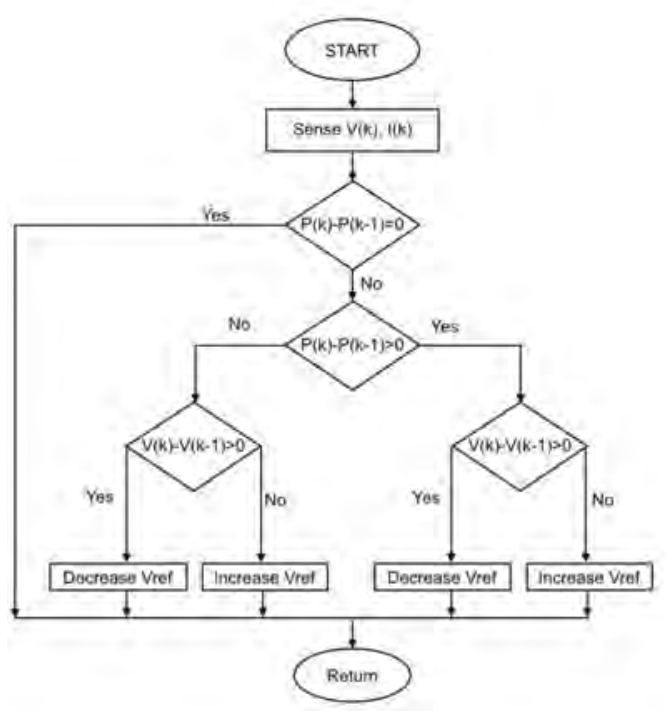

(a)

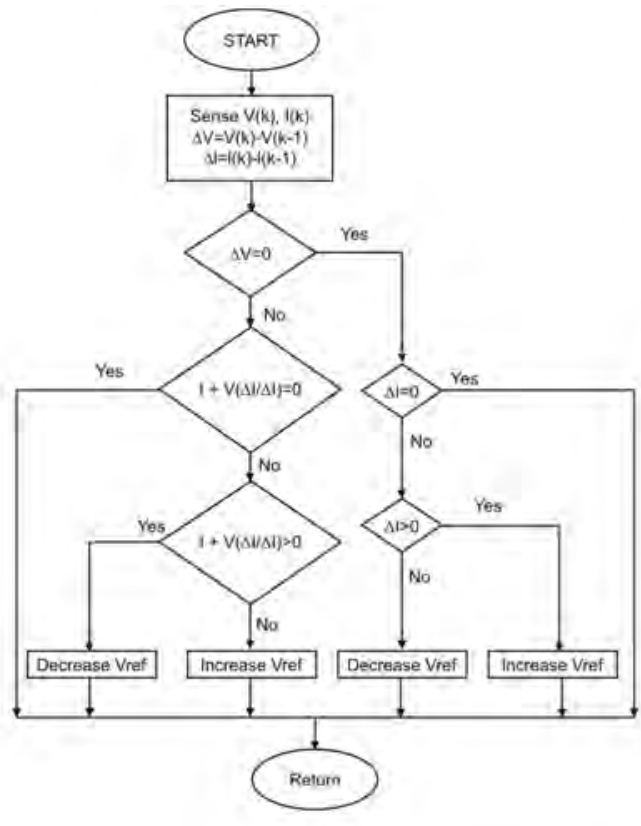

(b)

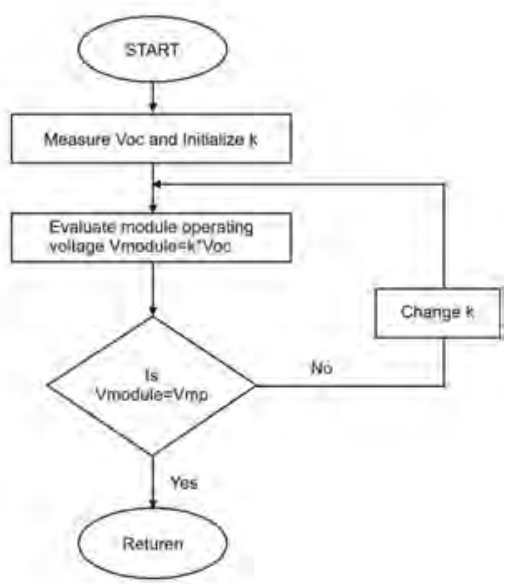

(c)

Figure 71.3 Algorithm flowchart: (a) P\&O, (b) INC and (c) FOCV. 


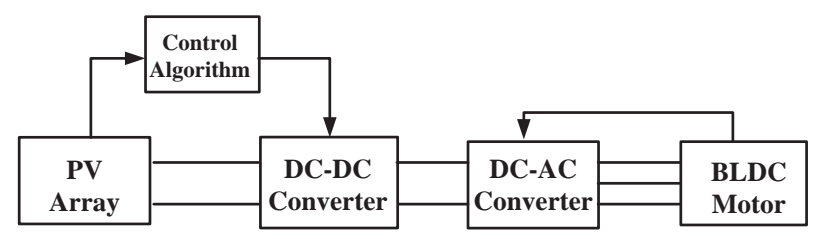

Figure 71.4 Vehicle control layout.

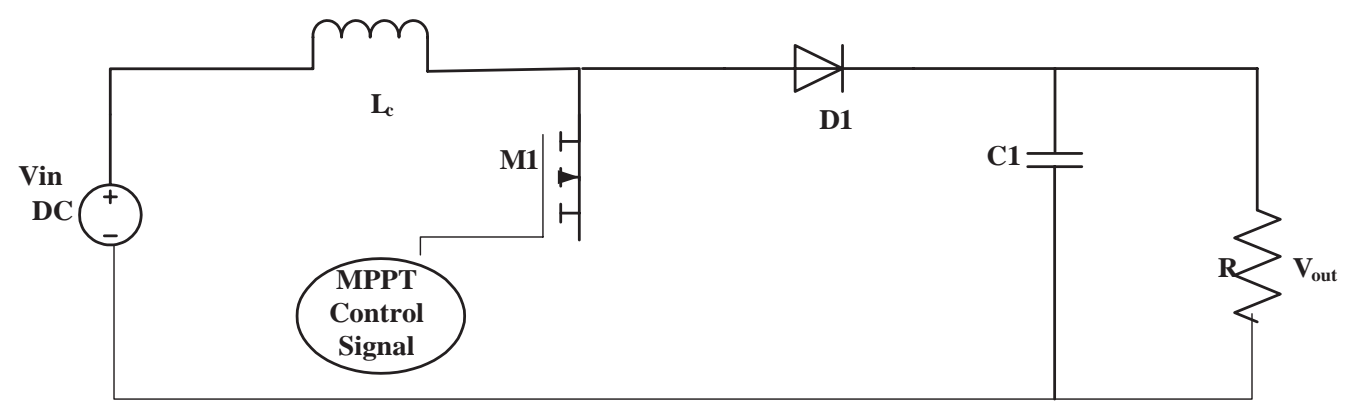

Figure 71.5 DC-DC driver circuit.

the current case), gate driver circuit (for controlling the switching of MOSFET), a diode, a capacitor and a load (represented by a resistance). The circuit diagram is shown in Figure 71.5.

For the ideal boost converter the expression for the obtained output voltage is

$$
\mathrm{V}_{\text {out }}=\frac{\mathrm{V}_{\text {in }}}{1-\mathrm{K}}
$$

For continuous conduction mode, there will be continuous flow of current through the inductor, i.e. the inductor current will never fall to zero . For this, the ripple current and ripple voltage are expressed as (2), while the critical value of the inductor and the conductor are given as (3).

$$
\begin{aligned}
\Delta \mathrm{I} & =\frac{\mathrm{KV}_{\mathrm{S}}}{\mathrm{fL}} \text { and } \Delta \mathrm{V}=\frac{\mathrm{KI}_{\mathrm{a}}}{\mathrm{fC}} \\
\mathrm{L}_{\mathrm{C}} & =\frac{\mathrm{K}(1-\mathrm{K}) \mathrm{R}}{2 \mathrm{f}} \quad \text { and } \quad \mathrm{C}_{\mathrm{c}}=\frac{\mathrm{K}}{2 \mathrm{fR}}
\end{aligned}
$$

\subsubsection{Dynamic model of BLDC motor}

The electrical model of the BLDC motor is shown in Figure 71.6. The phase current is represented as $i_{a b c}, e_{a b c}$ is the phase back emf, $R_{a b c}$, and $L_{a b c}$ are the phase resistance and inductance. 


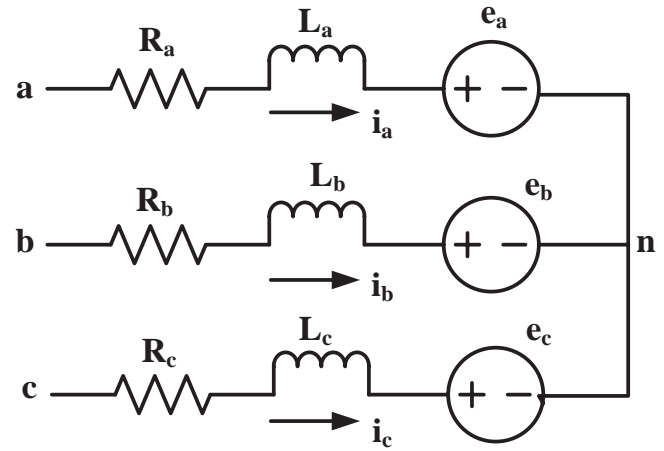

(a)

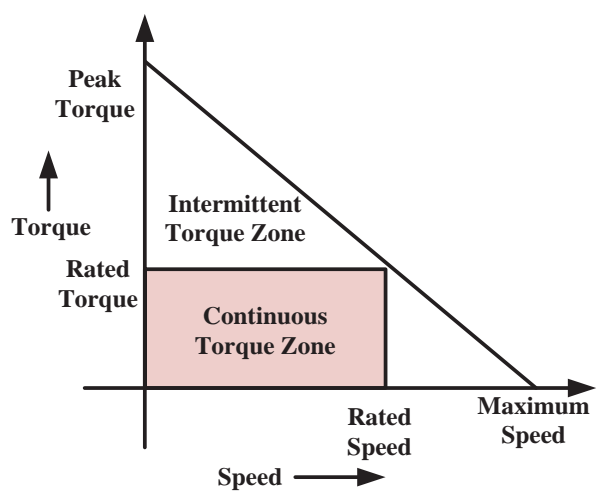

(b)

Figure 71.6 (a) Electrical equivalent of BLDC motor, (b) speed-torque characteristics.

The BLDC transfer function is expressed as

$$
\frac{\omega(s)}{V_{a n}(s)}=\frac{\frac{k_{t}}{J_{m} L_{a}}}{s^{2}+\left(\frac{J_{m} R_{a}+B L_{a}}{J_{m} L_{a}}\right) s+\left(\frac{B R a+k_{t} K_{e}}{J_{m} L_{a}}\right)}
$$

where $\omega$ is the motor speed, $V_{a n}$ is the phase a stator voltage, $k_{t}$ is the electromagnetic torque constant, $\mathrm{J}_{\mathrm{m}}$ is the rotor moment of inertia, $\mathrm{k}_{\mathrm{e}}$ is the back emf constant.

The torque equation is

$$
T_{e m}(s)=k_{t} \frac{V_{a n}(s)-k_{e} \omega(s)}{R_{a}+s L_{a}}
$$

The desired BLDC motor characteristics [8] can be obtained using (4) and (5). The speed torque characteristics are also shown in Figure 71.6. From the characteristics it is clear that the BLDC motor is capable of providing rated torque at rated speed. The speed of the BLDC motor is basically voltage controlled and it uses a basic inverter circuit for generating AC pulses from the DC voltage source. The PWM voltage control technique is used for controlling the supply voltage to the motor.

\section{Results and discussion}

The comparative performance of the three control algorithms viz. P\&O, INC and FOCV for a defined DC-DC converter circuit connected to a solar PV panel is done to determine the best control algorithm for tracking the maximum power under the test conditions of variation in both irradiance and load. The simulations are performed under the condition that the speed is controlled by the supply voltage. So as per the characteristics shown in Figure $71.6(\mathrm{~b})$ as the voltage drops, the speed drops, which results in the increase in load 


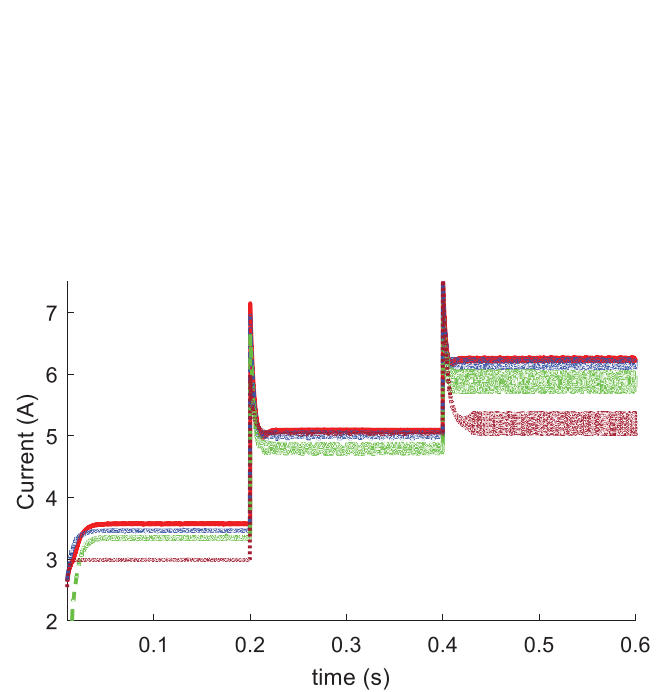

Effect of load variation under fixed irradiance
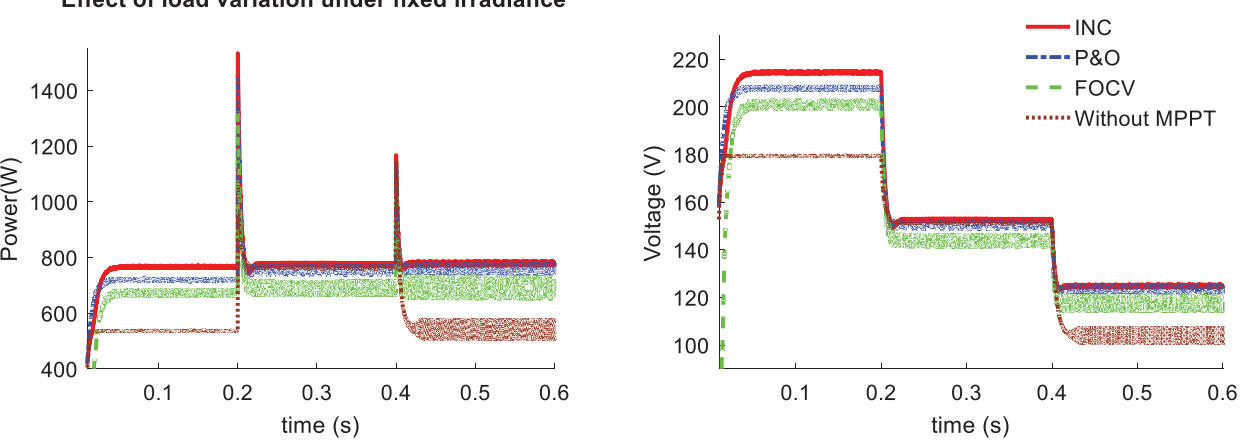

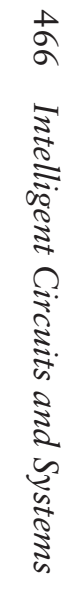
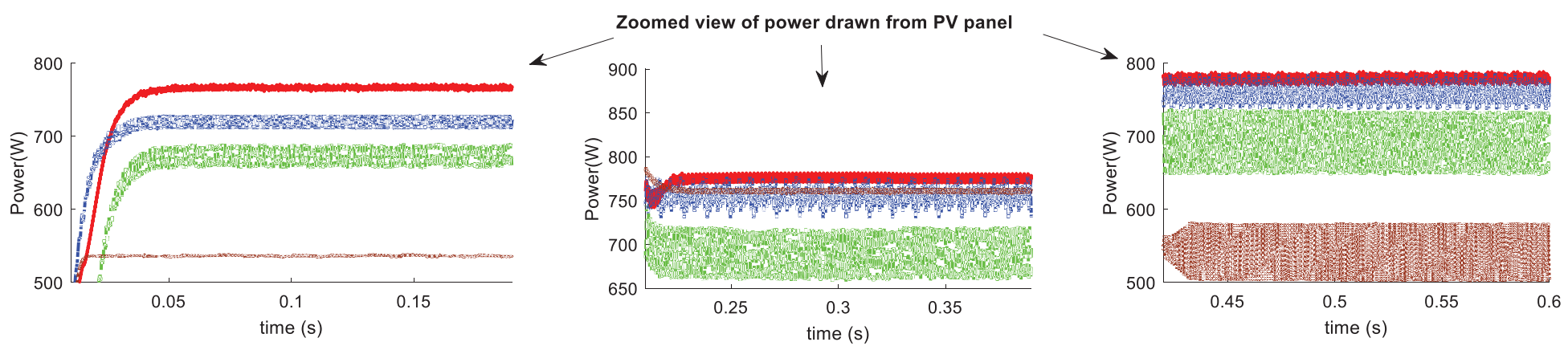

Figure 71.7 Maximum power tracking under fixed irradiance and variable load conditions. 


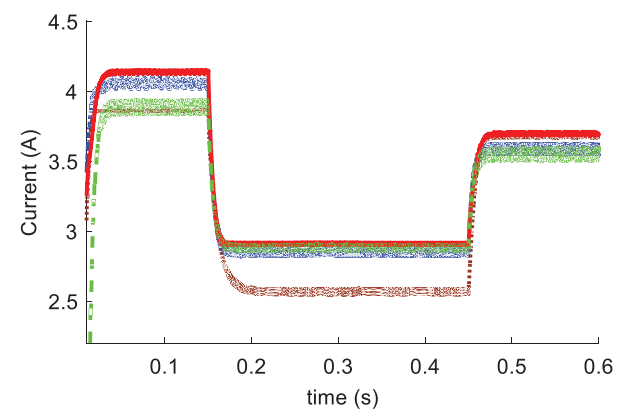

Effect of irradiance variation under fixed load
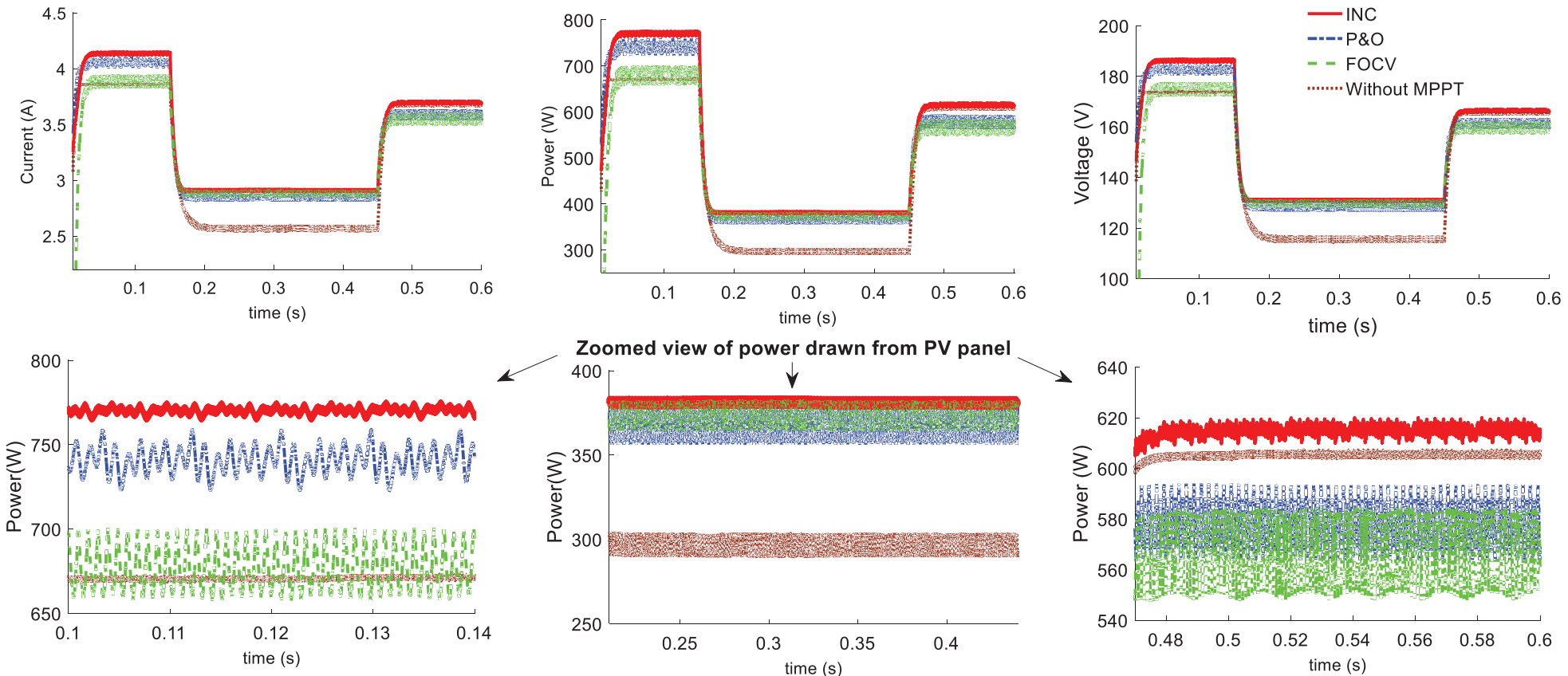

Zoomed view of power drawn from PV panel
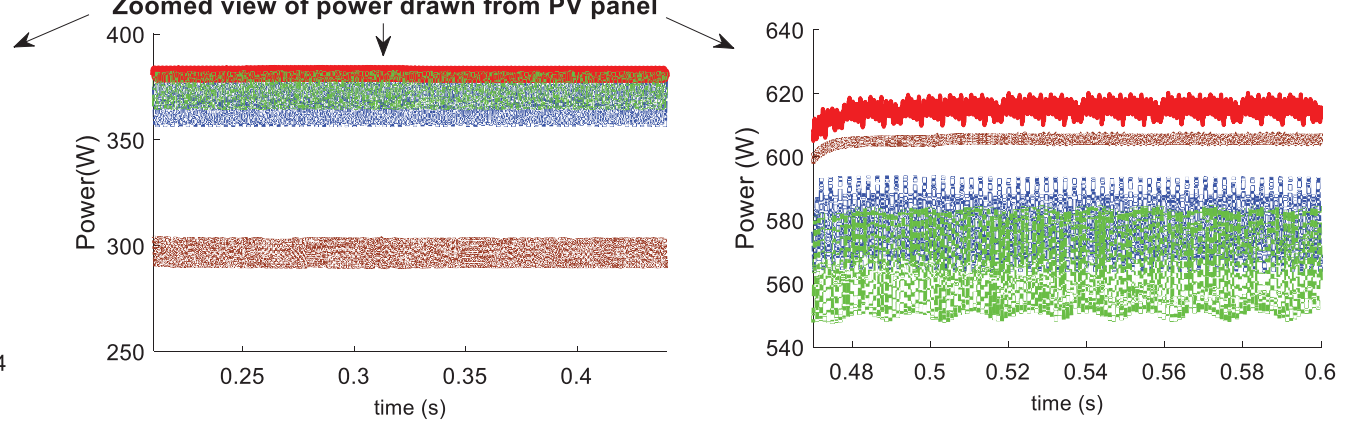

Figure 71.8 Maximum power tracking under fixed load and variable irradiance conditions. 
torque, and this drop is proportional to the phase resistance. The same concept is used where the characteristics of the BLDC motor are varied under the differential resistance settings of $9 \Omega, 6 \Omega$ and $3 \Omega$ set at the start of the simulation and then varied with a step size of 0.2 second. The irradiance level of $1000 \mathrm{~W} / \mathrm{m}^{2}$ is maintained for the entire simulation time. The corresponding simulations are shown in Figure 71.7.

From the simulation results it is clear that the incremental conductance based control algorithm (shown in red) provides the maximum power tracking under variable load conditions while tracking the power of $770 \mathrm{~W}$. The power tracked is nearly the same in the three conditions, whereas the second best tracking is provided by the P\&O MPPT represented by the blue curve.

The second set of simulations, Figure 71.8, is under the condition of varying irradiance level under fixed load conditions. The selected irradiance levels are $1000 \mathrm{~W} / \mathrm{m}^{2}$ at the start of the simulation, $500 \mathrm{~W} / \mathrm{m}^{2}$ from $1.5 \mathrm{~s}$ to $4.5 \mathrm{~s}$ and final transition to $750 \mathrm{~W} / \mathrm{m}^{2}$ is done at $4.5 \mathrm{~s}$ till the end of the simulation time. All this is done under fixed load represented by $\mathrm{r}=45 \Omega$.

From the results it is evident that the INC based controller circuit provides better tracking in comparison to the other two selected algorithms, i.e. P\&O and FOCV. The results are far superior in comparison to a DC-DC converter based driver circuit without MPPT control.

\section{Conclusion}

The paper has presented a driver circuit for a BLDC based e-vehicle. A comparative performance analysis of three different control algorithms was presented under the varying conditions of load and irradiance. It can be concluded from the simulation results that the incremental conductance based controller is able to extract maximum power from the installed PV assembly in comparison to the P\&O and FOCV based controller under the different test conditions. The INC based controller not only showed superior results but it was able to draw same power, thus exhibiting that the controller does not allow the load characteristics of the motor to be impinged upon by the characteristics of the installed PV panel.

\section{References}

[1] G.K. Singh. 2013. Solar power generation by PV (photovoltaic) technology: A review. Energy, 53: $1-13$.

[2] D. Verma, S. Nema and R. K. Nema. 2017. Implementation of perturb and observe method of maximum power point tracking in SIMSCAPE/MATLAB. International Conference on Intelligent Sustainable Systems (ICISS), Palladam, 148-152.

[3] N. Kumar, I. Hussain, B. Singh and B. K. Panigrahi. 2018. Framework of maximum power extraction from solar PV panel using self predictive perturb and observe algorithm. IEEE Transactions on Sustainable Energy, 9(2): 895-903.

[4] M.H. Anowar and P. Roy. 2019. A modified incremental conductance based photovoltaic MPPT charge controller. International Conference on Electrical, Computer and Communication Engineering (ECCE), Cox's Bazar, Bangladesh, 1-5.

[5] S. Negi, A. Maity, A. Patra and M. Sharad. 2019. Adaptive fractional open circuit voltage method for maximum power point tracking in a photovoltaic panel. 32nd International Conference on VLSI Design and 2019 18th International Conference on Embedded Systems (VLSID), India, 482-487. 
[6] Y. Huang. 2014. A rapid maximum power measurement system for high-concentration photovoltaic modules using the fractional open-circuit voltage technique and controllable electronic load. IEEE Journal of Photovoltaics, 4 (6): 1610-1617.

[7] Nguyen, X.H., and Nguyen, M.P. 2015. Mathematical modeling of photovoltaic cell/module/ arrays with tags in MATLAB/Simulink. Environ. Syst.Res. 4 (24): 1-13.

[8] P. Sarala, S. F. Kodad and B. Sarvesh,. 2016.Analysis of closed loop current controlled BLDC motor drive. International Conference on Electrical, Electronics, and Optimization Techniques (ICEEOT), Chennai, 1464-1468. 


\title{
72 Hybrid channel estimation via fuzzy method over Nakagami-m fading in MIMO-OFDM system
}

\author{
Harmandar Kaur, *, Mamta Khosla ${ }^{2}$, R.K. Sarin ${ }^{2}$, \\ Gurleen Kaur Walia ${ }^{3}$
}

\author{
${ }^{1}$ Department of Electronics and Communication Engineering, Guru Nanak \\ Dev University, Regional Campus, Jalandhar, India \\ ${ }^{2}$ Department of Electronics and Communication Engineering, Dr. B. R. \\ Ambedkar National Institute of Technology, Jalandhar, India \\ ${ }^{3}$ School of Electronics and Electrical Engineering, Lovely Professional \\ University, Phagwara, India
}

\section{Introduction}

The channel estimation method highly impacts the overall functioning of the communication system. The effectiveness of channel state estimation method becomes greatly crucial for highly mobile nodes, which have associated Doppler offset that accounts for spreading of the received signal, deteriorating the system performance of the underlying communication system. For simplification, it is assumed in the literature by numerous techniques of channel estimation that the receiver already has the knowledge of Doppler offset. For example, it is assumed that the mobile terminal has a known velocity and the Monte Carlo technique is applied to solve the CSI and frequency offset estimation issue $[1,2]$. By using the expectation maximization (EM) algorithm for a high mobility scenario, carrier offset and channel estimate values are jointly calculated [3]. Assuming that the nodes are immobile, the Kalman filter technique is utilized to solve the estimation of channel coefficients problem [4] for the MIMO system [5] and the MIMO-OFDM systems [6-8]. The conventional Kalman filter tracking is not suitable for estimating the non-linear estimation issue [10]. A type-1 Takagi-Sugeno (TS) fuzzy is capable of performing interpolation by weighted fuzzy bases along with the Kalman filter technique [11-14]. So, in the proposed channel estimation approach, we use the advantages of Kalman filtering scheme and fuzzy aided interpolation method over Nakagami- $m$ fading for obtaining the channel estimates. The Nakagami fading channel can efficiently fit a wider range of fading channel variations and it also has an added advantage that it easily fits to the empirical data pertaining to the channel [15-18]. The manuscript is organised as follows. Section 2 presents a general description to the MIMO-OFDM system. The proposed TS fuzzy Kalman filter for the channel state coefficients estimation is presented in Section 3. The outcomes of simulation and discussion are contained in Section 4. Section 5 summarizes the key findings.

* Corresponding author email: harmandar.ecejal@gndu.ac.in

DOI: $10.1201 / 9781003129103-72$ 


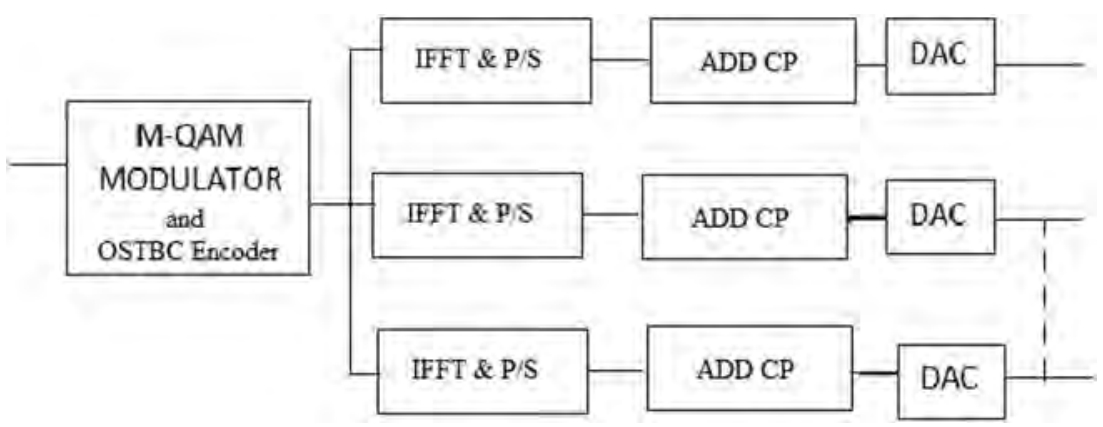

Figure 72.1 MIMO-OFDM transmitter system

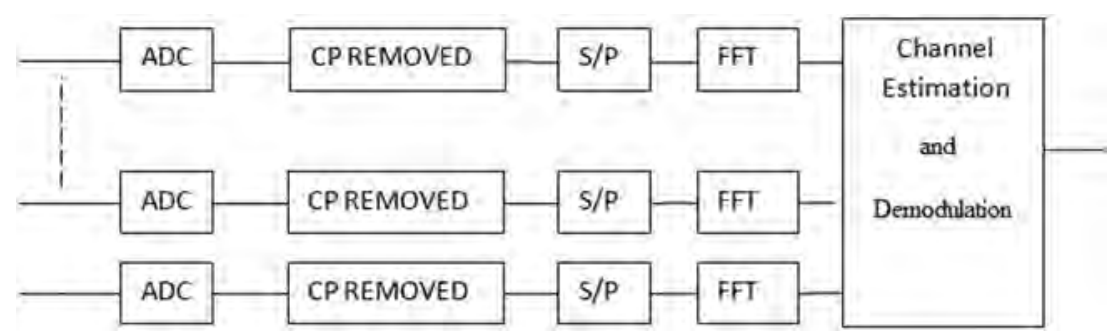

Figure 72.2 MIMO-OFDM receiver system

\section{System description}

The MIMO-OFDM system considered comprises 2 antennas each for transmission $\left(\mathrm{N}_{\mathrm{t}}\right)$ and reception $\left(\mathrm{N}_{\mathrm{r}}\right)$ of the signal, and the input information bit stream undergoes quadrature amplitude modulation (QAM). Orthogonal space-time block coder (OSTBC) is adopted to ensure full diversity gain. The data undergoes multicarrier modulation including inverse Fourier transform, followed by parallel-to-serial conversion and incorporation of cyclic prefix as shown in Figure 72.1.

In the receiver section shown in Figure 72.2, the fast Fourier transform (FFT) operation is applied after cyclic prefix removal and lastly serial-to- parallel conversion (S/P). The processed signal is then used to extract the CSI by the estimation technique employed and is demodulated.

The OSTBC encoded signal for the $t^{\text {th }}$ sub channel and the $n^{\text {th }}$ symbol is:

$$
R_{n}(t)=S_{n}(t) H_{n}(t)+V_{n}(t)
$$

where $R_{n}(t)$ is the received signal, $S_{n}(t)$ is the generated symbol, $S_{n}(t)$ is the OSTB coded transmitted symbol, $H_{n}(t)$ is the frequency response of the channel, $a_{1, n-1}$ is $e_{j 2 \pi f d t}$ where $f_{d}$ is the associated Doppler offset frequency [19-24], $V_{n}(t)$ is the additive white Gaussian noise with covariance $Q_{v}$ and $W_{n}(t)$ is the zero-mean white Gaussian process with covariance $Q_{w}$. 


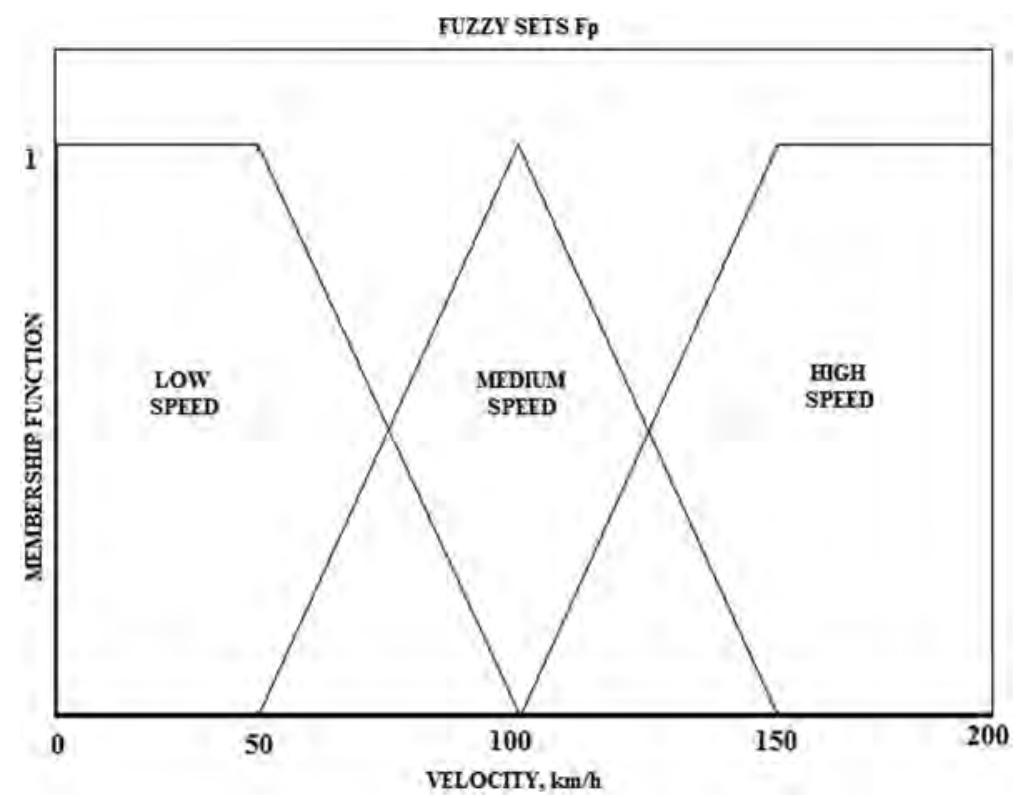

Figure 72.3 Membership functions for velocity.

\subsection{TS fuzzy Kalman filter aided channel coefficient estimation}

The purpose of using fuzzy interpolation together with the Kalman filtering is to get an approximate piecewise linear solution for the non-linear channel state estimation through its MFs. The TS fuzzy uses type- 1 membership functions (MFs) for representing three different mobile node velocity ranges, i.e. low $(0-50 \mathrm{~km} / \mathrm{h})$, medium $(50-150 \mathrm{~km} / \mathrm{h})$ and high $(100-200 \mathrm{~km} / \mathrm{h})$ as depicted in Figure 72.3.

The state space model uses the weighted fuzzy bases $\mu_{i}$ required to perform interpolation via P linear systems as follows [25]:

$$
\mathbf{X}_{\mathbf{n}}=\frac{\sum_{\mathbf{i}=1}^{\mathbf{P}} \mathbf{F}_{\mathbf{i}}\left(\hat{\mathbf{v}}_{\mathbf{n}-1}\right)\left(\mathbf{a}_{\mathbf{i}, \mathbf{n}-1}\left(\mathbf{H}_{\mathbf{n}-1}\right)+\mathbf{W}_{\mathbf{n}}\right)}{\sum_{\mathbf{i}=1}^{\mathbf{P}} \mathbf{F}_{\mathbf{i}}\left(\hat{\mathbf{V}}_{\mathbf{n}-1}\right)}
$$

Equation (2) creates P linear models by utilizing the weighted fuzzy bases $\mu_{i}$ by P mobile velocities given as:

$$
\begin{aligned}
& \text { If } \hat{v}_{n-1 \mid n-1} \text { is } F_{i} \\
& \text { then } X_{n \mid n}=G\left(X_{n-1 \mid n-1}\right)+K_{i, n} e_{n} \\
& \mathrm{H}_{\mathrm{n}}=\sum_{\mathrm{i}=1}^{\mathrm{P}} \mu_{\mathrm{i}}\left(\hat{\mathrm{v}}_{\mathrm{n}-1}\right)\left(\mathrm{a}_{\mathrm{i}, \mathrm{n}-1}\left(\mathrm{H}_{\mathrm{n}-1}\right)+\mathrm{K}_{\mathrm{i}, \mathrm{n}} \mathrm{e}_{\mathrm{n}}\right)
\end{aligned}
$$

where $e_{n}$ is the error, $K_{n}$ is the Kalman gain 

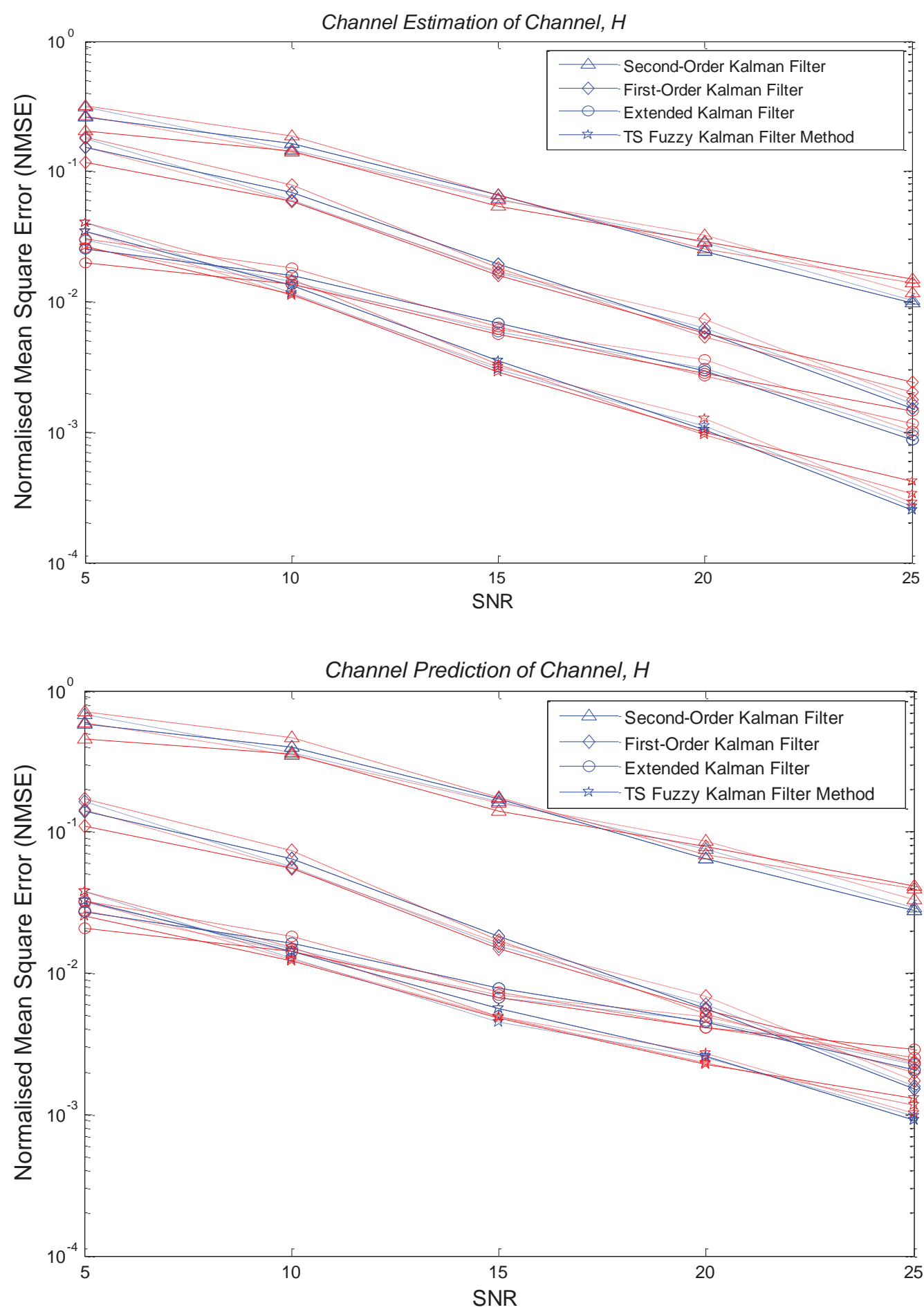

Figure 72.4 NMSE of $2 \times 2$ MIMO-OFDM system with proposed channel estimation and prediction over Nakagami- $m$ fading for different values of fade severity $(m=1.5,2,3,4,5$ and 5.5$)$. 


\section{Simulation results}

The simulation has been carried out extensively and the results obtained support the superior performance of our proposed TS fuzzy Kalman filter aided channel estimation scheme for a wide range of fade severity over Nakagami- $m$ channels. The simulation is run for 1000 frames in the MIMO-OFDM system each equipped with 2 transmitter and 2 receiver antennas, for $2 \mathrm{GHz}$ central frequency. The mobile velocities vary between $5 \mathrm{~km} / \mathrm{h}$ and $200 \mathrm{~km} / \mathrm{h}$ and are defined in terms of MFs of the fuzzy system. The system supports 128 cyclic prefixes and 512 sub-channels. We have considered a fair comparison based on SER and NMSE values among the first-order Kalman filter [26], extended Kalman filter [27], second-order Kalman filter [28-31] and the proposed TS fuzzy Kalman filter based channel coefficient estimation for mobile node scenario over least severe fade condition and most severe fade condition.

Figure 72.4 depicts the proposed TS fuzzy-Kalman filtering for channel state estimation which gives superior outcomes for time varying multipath fading channel having dynamic Doppler offset for both least $(m=5.5)$ and worst $(m=1.5)$ fading conditions. The SER variation with varying SNR is presented in Figure 72.5 and it is observed that the proposed TS fuzzy-Kalman filtering outperforms the considered Kalman Filter variants for different fade severity values.

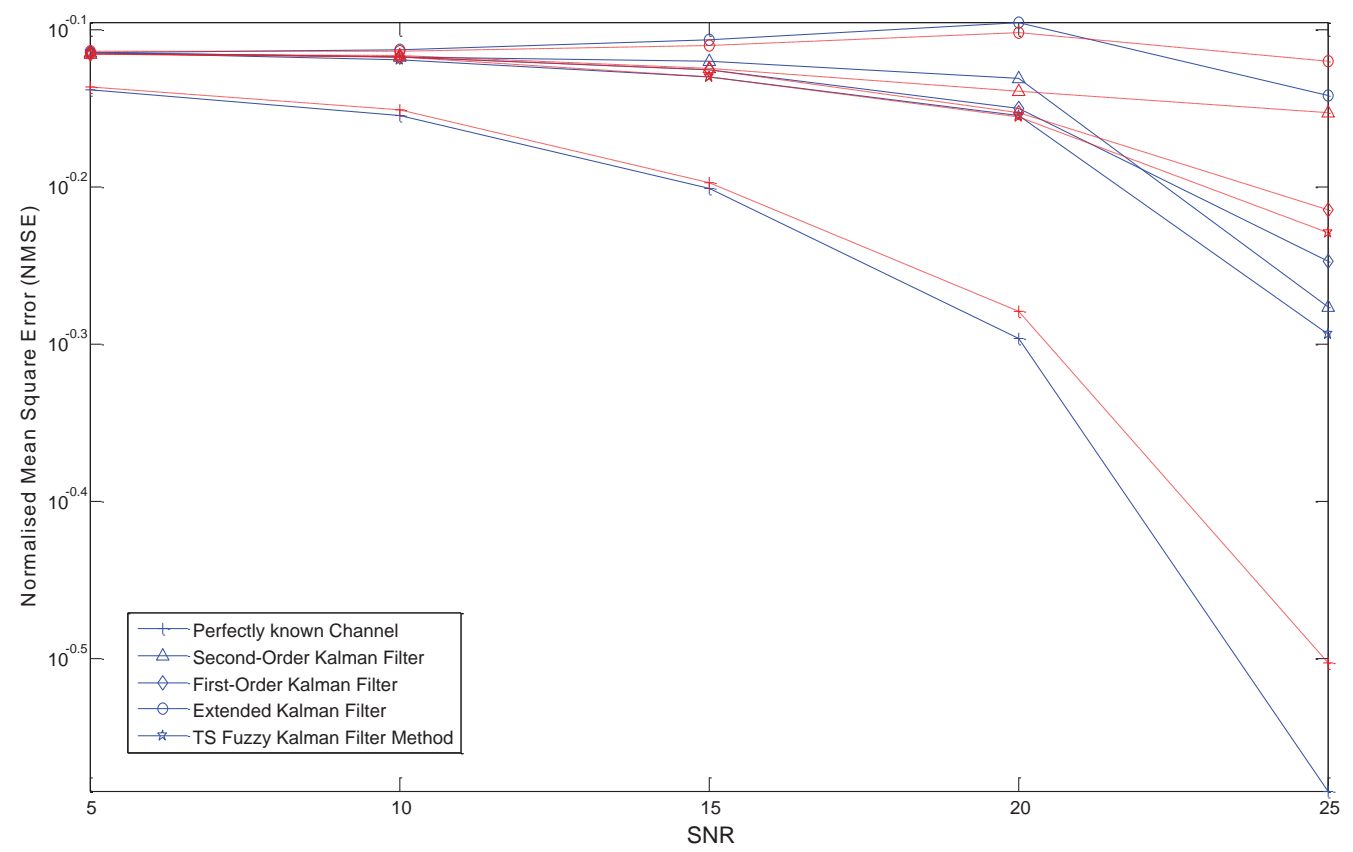

Figure 72.5 SER of $2 \times 2$ MIMO-OFDM system over Nakagami- $m$ fading for different values of fade severity $(m=1.5,5.5)$. 


\section{Conclusion}

The channel estimation scheme must be created to improve the system performance in the practical scenario. A hybrid channel estimation approach employing TS fuzzy based Kalman filter method is utilized for estimating of the channel with non-linear characteristics. The proposed channel estimation approach has been used at varied levels of fade severity and is shown to outperform the other approaches considered for all fade intensities considered. The proposed hybrid channel estimation is based on TS fuzzy bases with the Kalman filter to interpolate through varied velocity range. Simulation results based on SER and NMSE clearly corroborate that the proposed TS fuzzy aided Kalman filter for estimating the channel coefficient performs efficiently for mobile communicating nodes in a MIMO-OFDM system under Nakagami- $m$ fading channel conditions.

\section{References}

1. Smith, T.F., and Waterman, M.S. (1981) Identification of common molecular subsequences. J. Mol. Biol. 147, 195-197.

2. May, P., Ehrlich, H.C., and Steinke, T. (2006) ZIB structure prediction pipeline: composing a complex biological workflow through web services. In: Nagel, W.E., Walter, W.V., and Lehner, W. (eds.) Euro-Par 2006. LNCS, vol. 4128, pp. 1148-1158. Springer, Heidelberg.

3. Foster, I., and Kesselman, C. (1999) The grid: blueprint for a new computing infrastructure. Morgan Kaufmann, San Francisco.

4. Czajkowski, K., Fitzgerald, S., Foster, I., and Kesselman, C. (2001) Grid information services for distributed resource sharing. In: 10th IEEE International Symposium on High Performance Distributed Computing, pp. 181-184. IEEE Press, New York.

5. Foster, I., Kesselman, C., Nick, J., Tuecke, S. (2002) The physiology of the grid: an open grid services architecture for distributed systems integration. Technical report, Global Grid Forum.

6. Abouraddy, A.F., and Elnoubi, S.M. (2000) Statistical modelling of the indoor radio channel at $10 \mathrm{GHz}$ through propagation measurements - Part 1: Narrow-band measurements and modelling. IEEE Transactions on Vehicular Technology, Vol. 49 No.5, pp.1491-1507.

7. Baranyi, P., K'oczy, L. and Gedeon, T. (2004) A generalized concept for fuzzy rule interpolation. IEEE Transactions on Fuzzy Systems, Vol. 12 No. 6, pp. 820-837.

8. Beaulieu, N. C. and Cheng, C. (2001) An efficient procedure for Nakagami-m fading simulation. In Globecom 2001: IEEE Proceedings of Globecom 2001, Vol. 6, pp. 3336-3342.

9. Chang, Y., Chen, S., and Liau, C. (2008) Fuzzy interpolative reasoning for sparse fuzzy-rulebased systems based on the areas of fuzzy sets., IEEE Transactions on Fuzzy Systems, Vol. 16 No. 5, pp.1285-1301.

10. Chen, S., and Ko, Y. (2008) Fuzzy interpolative reasoning for sparse fuzzy rule-based systems based on $\alpha$-cuts and transformations techniques, IEEE Transactions on Fuzzy Systems, Vol. 16 No. 6, pp.1626-1648.

11. Haykin, S. (2002) Adaptive Filter Theory, 4th edn. Englewood Cliffs, NJ: Prentice-Hall.

12. Huang, Z., and Shen, Q. (2006) Fuzzy interpolative reasoning via scale and move transformations, IEEE Transactions on Fuzzy Systems, Vol. 14 No. 2, pp.340-359.

13. K'oczy, L., and Hirota, K. (1993) Approximate reasoning by linear rule interpolation and general approximation., International Journal of Approximate Reasoning, Vol. 9 No. 3, pp.197-225.

14. Kalofonos, D. N., Stojanovic, M., and Proakis, J. G. (2003) Performance of adaptive MCCDMA detectors in rapidly fading rayleigh channels, IEEE Transactions on Wireless Communications, Vol .2 No. 2, pp.1375-1387.

15. Kim, K., Pun, M., and Iltis, R. (2010) Joint carrier frequency offset and channel estimation for uplink MIMO-OFDMA systems using parallel Schmidt Rao-Blackwellized particle filters., IEEE Transactions on Communications, Vol. 58 No. 9, pp. 2697-2708. 
16. Komninakis, C., Fragouli, C., Sayed, A. H., and Wesel, R. D. (2002) Multi-input multi-output fading channel tracking and equalization using Kalman estimation., IEEE Transactions on Signal Processing, Vol. 50 No. 5, pp. 1065-1076.

17. Lindbom, L. (1993) Simplified Kalman estimation of fading mobile radio channels: High performance at LMS computational load, in ICASSP1993: Proceedings of International Conference on Acoustics, Speech, and Signal Processing, pp. 352-355.

18. Liu, Z., Ma, X., and Giannakis, G. B. (2002) Space-time coding and Kalman filtering for timeselective fading channel, IEEE Transactions on Communications, Vol. 50 No. 2, pp. 183-186.

19. Mahfouz, S., Chehade, F.M., Honeine, P., Farah, J., and Snoussi, H. (2014) Target tracking using machine learning and Kalman filter in wireless sensor networks, IEEE Sensors Journal, Vol. 14 No. 10, 2014.

20. Min, C., Chang, N., Cha, J., and Kang, J. (2007) MIMO-OFDM downlink channel prediction for IEEE802.16e systems using Kalman filter. In WCNC 2007: Proceedings of IEEE WCNC, Kowloon, Hongkong, pp. 942-946.

21. Nakagami, M. (1960) The m-Distribution, a general formula of intensity of rapid fading. In William C. Hoffman (ed.), Statistical Methods in Radio Wave Propagation: Proceedings of a Symposium, Oxford, England, pp. 3-36.

22. Pajala, E., Isotalo, T., Lakhzouri, A., and Lohan, E. S. (2006) An improved simulation model for Nakagami-m fading channels for satellite positioning applications, In WPNC 2006: Proceedings of the 3rd Workshop on Positioning, Navigation and Communication (WPNC06), Hannover, March 2006, pp. 81-90.

23. Patel, H. A., and Thakore, D. G. (2013) Moving object tracking using Kalman filter, International Journal of Computer Science and Mobile Computing, Vol. 2 No. 4, pp. 326-332.

24. Salmond, D. (2001) Target tracking: introduction and Kalman tracking filters, Target Tracking: Algorithms and Applications, Enschede, Netherlands, pp.1-16.

25. Schafhuber, D., Matz, G., and Hlawatsch, F. (2003) Kalman tracking of time varying channels in wireless MIMO-OFDM systems. In Asilomar 2003: Proceedings of 36th Asilomar Conference on Signals, Systems and Computers, pp. 1261-1265.

26. Shantaiya, S., Verma, K., and Mehta, K. (2015) Multiple object tracking using Kalman filter and optical flow, European Journal of Advances in Engineering and Technology, Vol. 2 No. 2, pp.34-39.

27. Shen, Q., and Yang, L. (2011) Generalisation of scale and move transformation-based fuzzy interpolation, Journal of Advanced Computational Intelligence and Intelligent Informatics, Vol. 15 No. 3, pp. 288-298.

28. Simon, D. (2003) Kalman filtering for fuzzy discrete time dynamic systems, Applied Soft Computing, Vol. 3 No. 3, pp.191-207.

29. Kaur, H., Khosla, M., and Sarin, R.K. (2018) Interval type-2 fuzzy Kalman filter aided individual channel estimation in MIMO relay system, International Journal of Communication Systems, Vol. 31 No. 17, 31:e3792.

30. Kaur, H., Khosla, M., and Sarin, R.K. (2017) Hybrid channel estimation for MIMO relay systems with Doppler offset influences, International Journal of Communication Systems, Vol. 31 No. 3, 31:e3469.

31. Kaur, H., Khosla, M., and Sarin, R.K. (2018) Channel estimation in a MIMO relay system: Challenges \& approaches, Second International Conference on Inventive Systems and Control (ICISC), Comboitre, 2018. 


\title{
73 Analysis of IoT sensors for monitoring the oil pipeline parameters
}

\author{
Chavala Lakshmi Narayana*, Rajesh Singh*, \\ Anita Gehlot*
}

Lovely Professional University, Phagwara, India

\section{Introduction}

The oil industry is one of the biggest industries in this world. Oil pipelines are considered as an economical and safe mode to transport petroleum products over long distances. However, they are subject to hazardous damage like fire, explosions, casualties, and environmental damage that leads to huge economic losses that have severe consequences on human lives and infrastructure [1]. Statistical failure results like PHMSA in the United States [3], EGIG in Europe [4], UKOPA in the United States, and PNGPC in China various countrie's pipeline issues and frequency of failures were analysed by comparison. Oil pipeline failure frequencies in the United States from the year 2004 to 2015 are shown in Figure 73.1.

Based on the statistical results, the pipeline failures for oil transportation were 432, all of which are happening as significant incidents from the year 2010 to 2015 in the database; the main oil pipeline failure causes are failure of pipe welding material, equipment failure and corrosion, as shown in Figure 73.2.

This paper shows different sorts of sensors in IoT like liquid temperature, humidity, vibration, pressure, flow rate, and smoke and an acoustic sensor. Various sorts of sensors are utilized for different parameters of the oil pipeline to make an IoT empowered keen condition. Also, this paper breaks down the sort of sensors suitable for measuring parameters. Sensors have a significant impact on the mechanization of any implementation by estimating and preparing the gathered information for distinguishing modifications in physical things. At whatever point there is an adjustment in the sensor physical condition, it delivers a quantifiable reaction.

\section{Types of sensors used to monitor oil pipelines}

\subsection{Pressure and flow rate sensors}

These sensors measure the power and convert it into electrical signals. This kind of sensor can be utilized in gauge pressure variations of an oil pipeline and moreover measures the flow rate of oil by flow rate sensors [5].

\footnotetext{
*Emails: Laxminarayana0706@gmail.com, srajssssece@gmail.com,dranitagehlot@gmail.com
} 


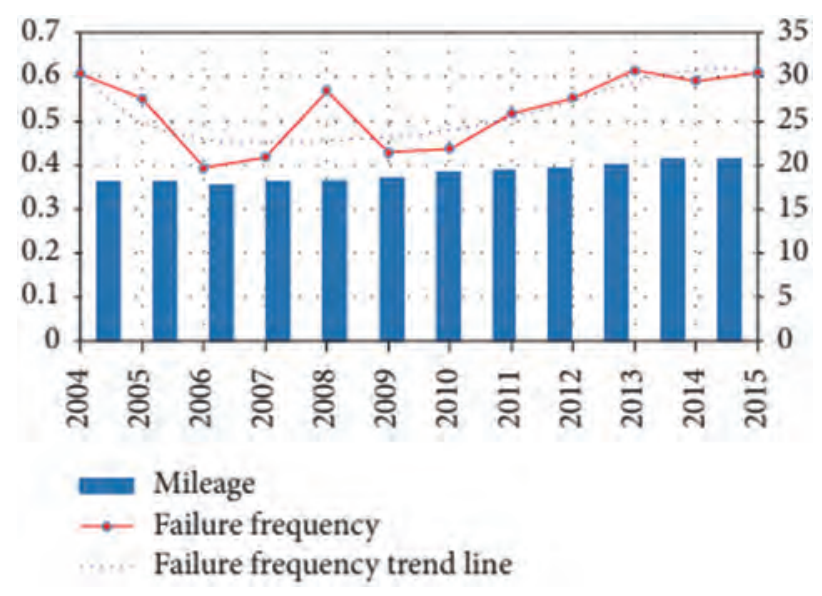

Figure 73.1 Failure and mileage frequencies for oil pipelines [2].

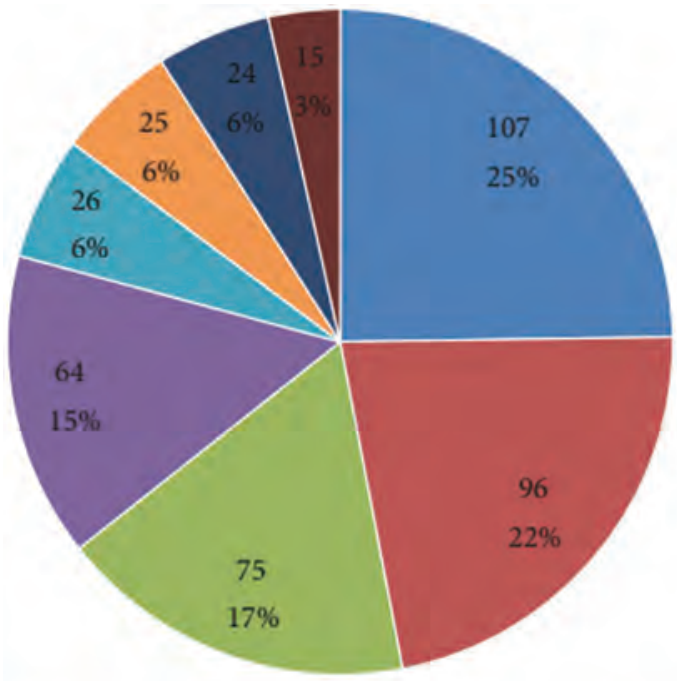

Corrosion

Pipe/weld material failure

Equipment failure

Excavation damage
Other outside force damage

Incorrect operation

Others

natural force damage

Figure 73.2 Causes of oil pipeline damage [2]. 


\subsection{Temperature sensor}

The authors in [6] utilized temperature sensors for the visualizing of biological conditions of nature. By utilizing Wi-Fi, the information gathered is sent to the cloud for investigating. A comparable sort of sensor is additionally utilized by the authors in [7] for agribusiness and empower ranchers to expand their general yield and quality by getting continuous data from their domain. In this, we are going to measure the temperature of oil in the pipeline. The characteristics of the sensor are low power and high sensitivity that should not catch fire.

\subsection{Humidity sensor}

This sensor gauges temperature of air similarly as dampness and measures the moistness in nature [7]. Utilized humidity sensors for keen farming also, empower ranchers to build their general yield and item quality by getting ongoing live information on their land. The humidity sensor is useful in pipeline monitoring to check the moisture level in the pipeline before transporting oil.

\subsection{Vibration sensor}

The vibration of the pipeline can be detected by accelerometer sensors [8]. This sensor is tied on the pipeline and vibration data can be collected. This is aimed to detect third-party damages and leak of the pipeline.

\subsection{Fire detection sensor}

Smoke sensors are utilized to distinguish smoke when the pipeline is harmed and spills oil which bursts into flames. Yu et al. [9] proposed deployment of a sensor network in the forest for fire detection. Different IoT sensors are used for powerful ecological condition tracking [10]. LPG gas leak detection using IoT sensors is given in [11].

\subsection{Acoustic sensor}

The significant classes of acoustic sensors are specifically aquaphones and geophones. Aquaphones require direct contact with hydrants as well as valves, while geophones tune in to spills superficially straightforwardly over the pipeline. Simultaneously, steel poles can likewise be embedded into the covered channel to pass signals to sensors mounted on the bars [12].

\section{Conclusion}

This world is changing by IoT by making a savvy circumstance between us. In savvy application using IoT, sensors expect a key activity in the computerization of utilization by making it increasingly splendid to respond with no human intervention. This paper presents different sorts of sensors for monitoring the oil pipeline in IoT empowered conditions. IoT incorporates different kinds of sensors being able to talk with each other remotely and brilliantly. This paper breaks down various IoT sensors and clarifies which sensors are utilized in various parameter measurements of the pipeline. Later on, this assessment will help us with building up an IoT application based on various sensors for monitoring oil in long-distance oil pipelines. 
Table 73.1 Various IoT sensors for the oil pipeline monitoring

\begin{tabular}{|c|c|c|c|c|}
\hline Sensor & Sensor model & $\begin{array}{l}\text { Deployment } \\
\text { of sensor }\end{array}$ & $\begin{array}{l}\text { Parameters to } \\
\text { be monitored }\end{array}$ & Specifications \\
\hline \multirow[t]{6}{*}{$\begin{array}{l}\text { Pressure } \\
\text { sensor }\end{array}$} & & $\begin{array}{l}\text { Inside the } \\
\text { pipeline }\end{array}$ & $\begin{array}{l}\text { Gauge } \\
\text { pressure of } \\
\text { pipeline }\end{array}$ & $\begin{array}{l}\text { Power supply: } 0-5 \text { V DC } \\
\text { Accuracy: }-0.5 \% \text { FS } \\
\text { Measuring range: } 0-5 \mathrm{kpa}\end{array}$ \\
\hline & & & & \\
\hline & $\begin{array}{l}\text { Diaphragm } \\
\text { pressure sensor }\end{array}$ & & & \\
\hline & & $\begin{array}{l}\text { Inside the } \\
\text { pipeline }\end{array}$ & $\begin{array}{l}\text { Gauge } \\
\text { pressure of } \\
\text { pipeline }\end{array}$ & $\begin{array}{l}\text { Measuring range: } 0-1000 \\
\text { bar }\end{array}$ \\
\hline & $\begin{array}{l}\text { Bourdon tube } \\
\text { sensor }\end{array}$ & & & \\
\hline & $\begin{array}{l}\text { Digital pressure } \\
\text { sensor (GPT204) }\end{array}$ & $\begin{array}{l}\text { Inside the } \\
\text { pipeline }\end{array}$ & $\begin{array}{l}\text { Gauge } \\
\text { pressure of } \\
\text { pipeline }\end{array}$ & $\begin{array}{l}\text { Power supply: } 10-30 \mathrm{~V} \text { DC } \\
\text { Accuracy: }-0.5 \% \text { FS } \\
\text { Measuring range: } 0-100 \\
\text { Mpa }\end{array}$ \\
\hline \multirow[t]{2}{*}{$\begin{array}{c}\text { Flow rate } \\
\text { sensor }\end{array}$} & Z41WC & $\begin{array}{l}\text { Inside the } \\
\text { pipeline }\end{array}$ & $\begin{array}{l}\text { Flow rate of } \\
\text { oil }\end{array}$ & $\begin{array}{l}\text { Power supply: } 5 \text { to } 18 \mathrm{~V} \\
\text { Measuring range: } 3 \text { litres/ } \\
\text { min }\end{array}$ \\
\hline & YF-S201 & $\begin{array}{l}\text { Inside the } \\
\text { pipeline }\end{array}$ & $\begin{array}{l}\text { Flow rate of } \\
\text { oil }\end{array}$ & $\begin{array}{l}\text { Power supply: } 5-24 \mathrm{~V} \text { DC } \\
\text { Measuring range: } 1-30 \\
\text { litres/min }\end{array}$ \\
\hline \multirow[t]{2}{*}{$\begin{array}{l}\text { Temperature } \\
\text { sensor }\end{array}$} & T100 RTD & $\begin{array}{l}\text { Inside the } \\
\text { pipeline }\end{array}$ & $\begin{array}{l}\text { Temperature } \\
\text { of the oil }\end{array}$ & $\begin{array}{l}\text { Power supply: } 0-5 \mathrm{~V} \text { DC } \\
\text { Nominal resistance } \\
100 \mathrm{ohm} \\
\text { Measuring range: }-20^{\circ} \mathrm{C} \\
\text { to } 150^{\circ} \mathrm{C} \text {. }\end{array}$ \\
\hline & PT 1 & $\begin{array}{l}\text { Inside the } \\
\text { pipeline }\end{array}$ & $\begin{array}{l}\text { Temperature } \\
\text { of the oil }\end{array}$ & $\begin{array}{l}\text { Power supply: } 0-5 \mathrm{~V} \mathrm{DC} \\
\text { Nominal } \\
\text { resistance: } 1000 \mathrm{ohm} \\
\text { Temperature range }-50^{\circ} \mathrm{C} \\
\text { to } 90^{\circ} \mathrm{C}\end{array}$ \\
\hline \multirow[t]{4}{*}{$\begin{array}{l}\text { Vibration } \\
\text { sensor }\end{array}$} & & $\begin{array}{l}\text { On the } \\
\text { pipeline }\end{array}$ & $\begin{array}{l}\text { Vibrations } \\
\text { of the } \\
\text { pipeline }\end{array}$ & $\begin{array}{l}\text { Power supply: } 0-5 \mathrm{~V} \text { DC } \\
\text { Acceleration ranges from } \\
\pm 2 \text { to } \pm 16 g\end{array}$ \\
\hline & MPU6050 & & & \\
\hline & ADXL335 & $\begin{array}{l}\text { On the } \\
\text { pipeline }\end{array}$ & $\begin{array}{l}\text { Vibrations } \\
\text { of the } \\
\text { pipeline }\end{array}$ & $\begin{array}{l}\text { Power supply: } 1.8-5 \mathrm{~V} \mathrm{DC} \\
\text { Acceleration ranges from } \\
\text { up to } \pm 3 g\end{array}$ \\
\hline & $\begin{array}{l}\text { Piezoelectric } \\
\text { accelerometers }\end{array}$ & $\begin{array}{l}\text { On the } \\
\text { pipeline }\end{array}$ & $\begin{array}{l}\text { Vibrations } \\
\text { of the } \\
\text { pipeline }\end{array}$ & $\begin{array}{l}\text { Power supply: } 0-5 \text { V DC } \\
\text { Acceleration ranges from } \\
\quad \pm 4 \text { to } \pm 6000 g\end{array}$ \\
\hline $\begin{array}{r}\text { Acoustic } \\
\text { sensor }\end{array}$ & 点 & $\begin{array}{l}\text { On the } \\
\text { pipeline }\end{array}$ & $\begin{array}{l}\text { Leaks of the } \\
\text { pipeline }\end{array}$ & $\begin{array}{l}\text { Working voltage: } \\
\text { DC } 3.3-5 \mathrm{~V} \\
\text { Induction range: } 0.5 \mathrm{M}\end{array}$ \\
\hline
\end{tabular}


Table 73.1 Cont.

\begin{tabular}{|c|c|c|c|c|}
\hline Sensor & Sensor model & $\begin{array}{l}\text { Deployment } \\
\text { of sensor }\end{array}$ & $\begin{array}{l}\text { Parameters to } \\
\text { be monitored }\end{array}$ & Specifications \\
\hline \multirow[t]{2}{*}{$\begin{array}{l}\text { Smoke } \\
\text { detection } \\
\text { sensor }\end{array}$} & MQ-4 & $\begin{array}{l}\text { On the } \\
\text { pipeline }\end{array}$ & $\begin{array}{l}\text { Detect smoke } \\
\text { caused due } \\
\text { to fire }\end{array}$ & $\begin{array}{l}\text { Operating voltage: } 4.5 \\
\text { to } 5 \text { V DC } \\
\text { Detection concentration: } \\
200-10000 \text { ppm }\end{array}$ \\
\hline & MQ-214 & $\begin{array}{l}\text { On the } \\
\text { pipeline }\end{array}$ & $\begin{array}{l}\text { Detect smoke } \\
\text { caused due } \\
\text { to fire }\end{array}$ & $\begin{array}{l}\text { Operating voltage: } 4.5 \\
\text { to } 5 \text { V DC } \\
\text { Detection concentration: } \\
500-10000 \text { ppm } \\
\text { Sensitivity to methane gas }\end{array}$ \\
\hline \multirow[t]{2}{*}{$\begin{array}{l}\text { Humidity } \\
\text { sensor }\end{array}$} & DHT11 & $\begin{array}{l}\text { Inside the } \\
\text { pipeline }\end{array}$ & $\begin{array}{l}\text { Humidity } \\
\text { inside the } \\
\text { pipeline }\end{array}$ & $\begin{array}{l}\text { Humidity range: } \\
20-80 \% \mathrm{RH} \\
\text { Power supply voltage } 3.3 \\
\text { to } 5 \mathrm{~V} \mathrm{DC}\end{array}$ \\
\hline & & $\begin{array}{l}\text { Inside the } \\
\text { pipeline }\end{array}$ & $\begin{array}{l}\text { Humidity } \\
\text { inside the } \\
\text { pipeline }\end{array}$ & $\begin{array}{l}\text { Humidity range: } \\
0-100 \% \mathrm{RH} \\
\text { Power supply voltage } 3.3 \\
\text { to } 5 \mathrm{~V} \mathrm{DC}\end{array}$ \\
\hline
\end{tabular}

\section{References}

[1] Wang, T., W. Xuan, X. Wang et al. Overview of oil and gas pipeline failure database, in Proceedings of International Conference on Pipelines and Trenchless Technology, ICPTT, 2013, pp.1161-1167, October 2013.

[2] Lianshuang Dai, Dongpo Wang, Ting Wang, Qingshan Feng and Xinqi Yang. Analysis and comparison of long-distance pipeline failures, Journal of Petroleum Engineering, 2017, Article ID 3174636.

[3] PHMSA, http://primis.phmsa.dot.gov/comm/reports/safety/PSI.html, 2015.

[4] EGIG, 9th report of the gas pipeline incidents of European gas pipeline incident data group, 2015.

[5] Wang, B.-Y., Hsieh, F.-C., Lin, C.-Y., Chen, S.-E., Chen, F.-Z., and Wu, C.-C. Development of a piezoelectric vacuum sensing component for a wide pressure range. Sensors 2014, 14, 22099-22112.

[6] S. Zafar, G. Miraj, R. Baloch, D. Murtaza and K. Arshad. An IoT based real-time environmental monitoring system using Arduino and cloud service, Engineering, Technology \& Applied science Research, 8(4), 2018, pp. 3238-3242.

[7] N. Nayyar and V. Puri. Smart farming: IoT based smart sensors agriculture stick for live temperature and moisture monitoring using Arduino, cloud computing \& solar technology, In: Proc. of The International Conference on Communication and Computing Systems (ICCCS-2016), 2016.

[8] Ismail, M.I.M., Dziyauddin, R.A., Ahmad Salleh, N.A., Muhammad-Sukki, F, Bani, N.A., Izhar, M.A.M., And Latiff, L.A. 2019. A review of vibration detection methods using accelerometer sensors for water pipeline leakage. IEEE access 7, pp. 5165-51981.

[9] Yu, Liyang, Neng Wang and Xiaoqiao Meng. Real-time forest fire detection with wireless sensor networks, in Proceedings of International Conference on Wireless Communications, Networking and Mobile Computing, Vol. 2, 2005.

[10] D. Punniamoorthy, V. S. Kamadal, B. Srujana Yadav and V. S. Reddy. Wireless sensor networks for effective environmental tracking system using IoT and sensors, 2018 2nd International Conference on I-SMAC (IoT in Social, Mobile, Analytics and Cloud). 
[11] V. Suma, R. R. Shekar and K. A. Akshay. Gas leakage detection based on IOT, 3rd International conference on Electronics, Communication and Aerospace Technology (ICECA), Coimbatore, India, 2019, pp. 1312-1315.

[12] Mutiu Adesina Adegboye, Wai-Keung Fung and Aditya Karnik. Recent advances in pipeline monitoring and oil leakage detection technologies: principles and approaches, Sensors, 19(11), 2019, p. 2548. 


\title{
74 Crop survival analysis using machine learning and Internet of Things
}

\author{
Ch Sai Dinesh Reddy, *, Swapnil Bagwari ${ }^{2}$
}

\author{
${ }^{1}$ Student, Lovely Professional University, Phagwara, Punjab, India \\ ${ }^{2}$ Assistant Professor, Department of Electronics and Communication \\ Engineering, Lovely Professional University, Phagwara, Punjab, India
}

\section{Introduction}

Most information is shared via the Internet. The concept of linking objects and devices using the Internet to share or store the data among devices is known as IoT. IoT supports different types of technologies like Lora, ZigBee, RFID, NFC, LTE and M2M [1]. Lora technology is used for long-range communication and can cover in rural areas. Medar Ramesh et al. [2] defined how remote sensing data and machine learning techniques have focused on the prediction of the crop yield. This method is cost-effective when compared to ground data. Charoen-Ung and Mittrapiyanuruk [3] contributed to this work by developing a machine learning-based prediction algorithms like gradient boosting and random forest models for yield prediction which can predict more accurately than human experts. Ghadge et al. [4] explained how soil plays an important role in the field of agriculture. The system used two algorithms and the best will be chosen based on the output, thereby helping the farmers by reducing the difficulties. Priya et al. [5] proposed that farmers can attain a good accuracy for the prediction of crop yield using the random forest classifier. They also explained how data mining plays a key role in the field of agriculture. Vanitha et al. [6] explained how to organize the dataset acquired from the field accurately. This also explains how to select the best features like rainfall in the dataset which will show a great impact on the results and how to build a model using it and alerting the farmer when needed. Reshma et al. [7] explained how IoT and machine learning help the farmers in increasing productivity by using the available land without wastage and how to control the pests.

The dataset used for processing is obtained from an IoT setup that connected with sensors like soil moisture, light sensor, DHT 11 and rainfall sensor module. The data is collected and sent to the Thingspeak cloud platform. The data is extracted into a CSV file. The dataset is provided to various machine learning models like SVM, k-NN and random forest. Python is used for analysing the agricultural data set. Spyder is the software/platform that supports the Python language which is used to predict the accuracy of crop survival. Arduino IDE is the software used to program NodeMCU.

The temperature conditions required for the growth of sugarcane are explained in Table 74.1.

Depending on the variety of sugarcane, the cultivation of crops requires 12-18 months. There are several stages involved in the cultivation like germination, stem elongation, growth phase, ripening phase. The growth phase requires a huge amount of sunlight.

\footnotetext{
* Email: chsdr24@gmail.com
}

DOI: $10.1201 / 9781003129103-74$ 
Table 74.1 Temperature and humidity values of sugarcane crops

\begin{tabular}{llllll}
\hline S.no. & Sugarcane crop stages & $\begin{array}{l}\text { Growth stage } \\
\text { duration }\end{array}$ & Temp & Humidity & $\begin{array}{c}\text { Sunshine } \\
\text { in hours }\end{array}$ \\
\cline { 3 - 4 } & & max-min & max-min & \\
\hline 1 & Germination period & $\begin{array}{c}\text { 15 to 30 days } \\
\text { after planting }\end{array}$ & $30-15$ & $70-50$ & 10 \\
2 & Stem elongation stage & $\begin{array}{l}31 \text { to } 120 \\
121 \text { to } 210\end{array}$ & $30-15$ & $70-50$ & 10 \\
3 & Growth phase & 211 to 365 & $15-12$ & $65-80$ & 11 \\
4 & Ripening phase & & & & 10 \\
\hline
\end{tabular}

Parameters like soil moisture and rainfall are given as input to the machine learning models to compare and predict the survival of the sugarcane crop.

\section{Data and methods}

\subsection{Proposed methodology}

Figure 74.1 represents how data is collected from the field with the help of sensors and sent to the cloud. Firstly, NodeMCU is initialized and connected to a network. Sensors like DHT 11, soil moisture, rainfall and LDR module are connected and the data is sent to the cloud platform. Thingspeak is an example of a cloud platform that is used to store and visualize the data to the user. Machine learning models such as SVM, k-NN and random forest are implemented with the help of a programming language called Python.

\subsection{Methods to approach}

The methodology involved in the development of this system is to use low-cost and lowpower sensors, a controller that is used to store, upload and visualize the data in the cloud. The block diagram of the model is shown in Figure 74.2.

The block diagram consists of DHT 11, rainfall sensor, light sensor, soil moisture sensors which are connected to the NodeMCU (an IoT device) which is capable of sensing and uploading the data in the cloud. The Thingspeak cloud contains the visualizations for different sensors to monitor. Figures 74.1 and 74.2 were designed using an online editor called draw.io which can be accessed through this link: www.draw.io.

\subsection{Experimental setup}

In Figure 74.3 a prototype was designed to explain the task of the system. The setup plays a good role in the field of precision agriculture by collecting information related to temperature, humidity, rainfall, soil moisture, light, and sorting the data using machine learning models.

\section{Result and analysis}

In Figure $74.4 \mathrm{a}$ the graph represents the train and test accuracy for the dataset. It shows that the bias gives good results by avoiding overfitting or leakage of data under any circumstances. In Figure 74.4b the numbers of 0's and 1's are plotted based on two 


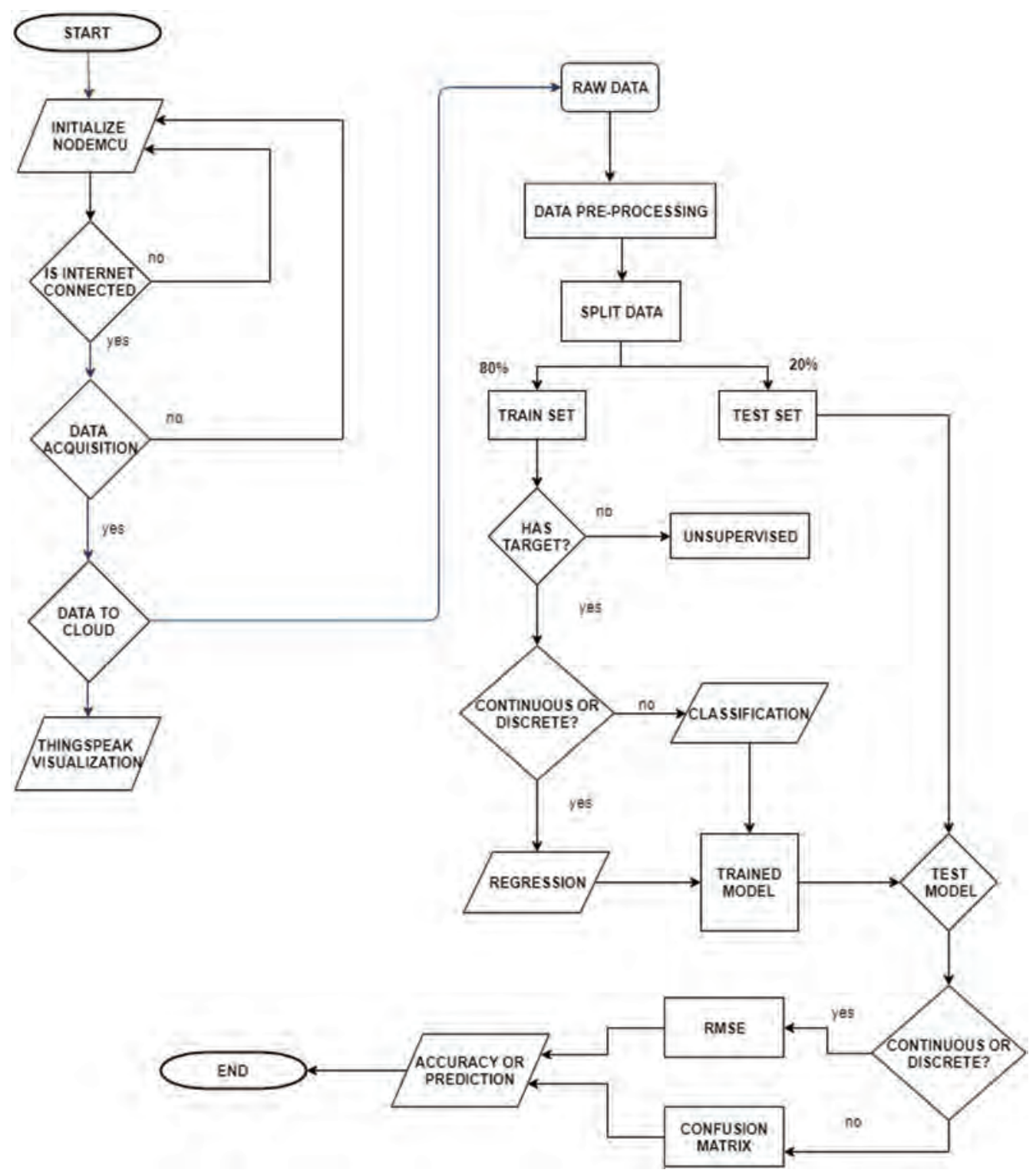

Figure 74.1 Proposed system architecture.

parameters, i.e., temp and soil Moisture. Green dots are 1's and red dots are 0's. The line that divides the green and red dots is known as prediction line. One part contains the dots of other parts and the accuracy depends on how the prediction line divides the points exactly. In Figure 74.4c the prediction line will divide the two categories mostly in two equal parts. This determines that the model is running fine and the prediction results are accurate.

Table 74.2 compares the results obtained between k-NN, SVM and random forest. It shows that the prediction accuracy is more for $\mathrm{k}-\mathrm{NN}$. The area under curve (AUC/ROC 


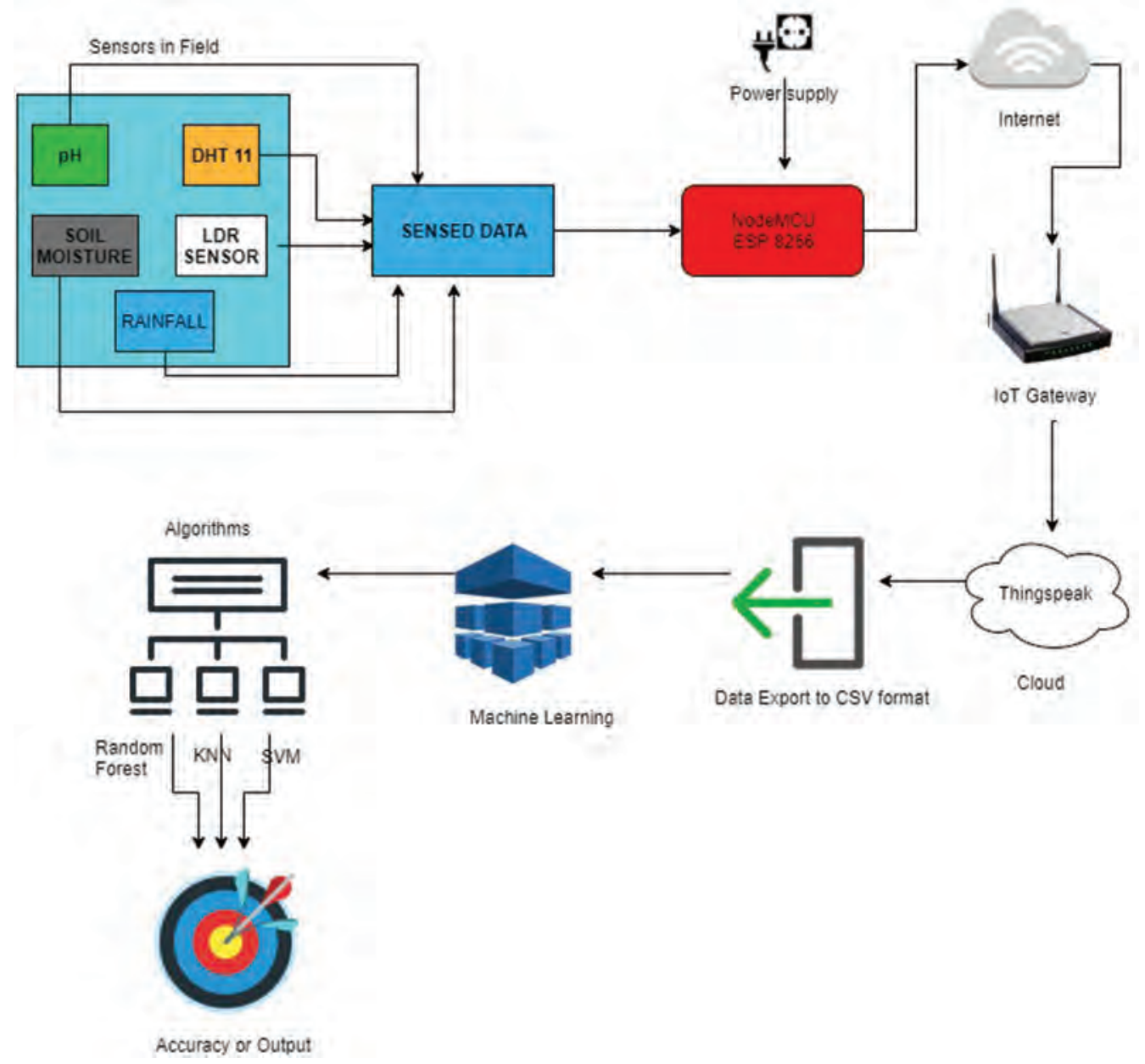

Figure 74.2 Block diagram of the proposed system.

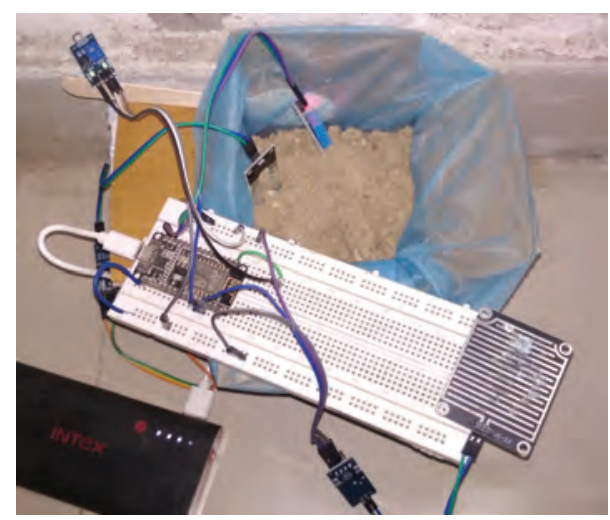

Figure 74.3 Experimental setup. 


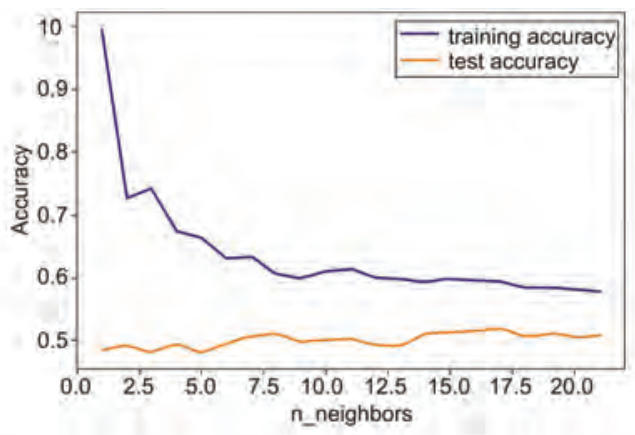

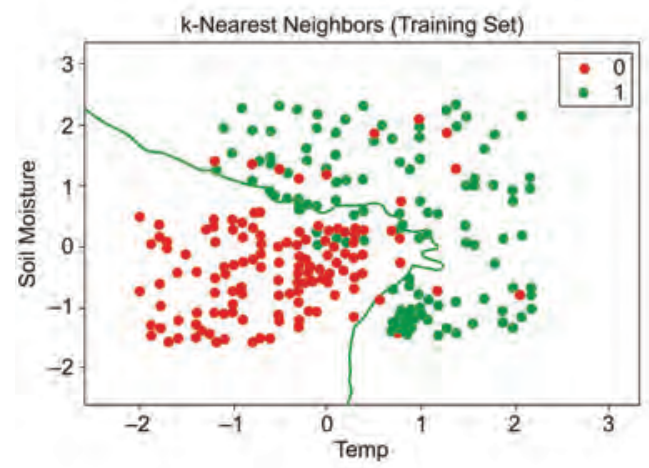

B

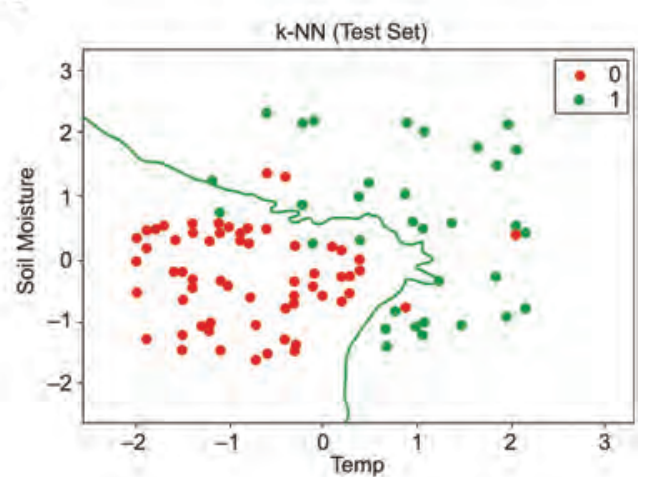

c

Figure 74.4 (a) Train v/s test accuracy. (b) Prediction on training set for k-NN. (c) Prediction on test set for k-NN.

Table 74.2 Results of the proposed method

\begin{tabular}{|c|c|c|c|c|}
\hline \multirow[t]{2}{*}{ S. no. } & & \multicolumn{3}{|c|}{ Machine learning models } \\
\hline & & $k-N N$ & $S V M$ & Random forest \\
\hline 1 & Training accuracy & $89 \%$ & $85 \%$ & $86 \%$ \\
\hline 2 & Testing accuracy & $87 \%$ & $84 \%$ & $83 \%$ \\
\hline 3 & Prediction accuracy & $88 \%$ & $84 \%$ & $85 \%$ \\
\hline 4 & Error $(\%)$ & $12 \%$ & $16 \%$ & $15 \%$ \\
\hline 5 & Area under curve & $90 \%$ & $87 \%$ & $89 \%$ \\
\hline 6 & Specificity $=\operatorname{Tn} /(\operatorname{Tn}+F p)$ & $88 \%$ & $83 \%$ & $87 \%$ \\
\hline 7 & Precision $(P)=T p /(T p+F p)$ & $92 \%$ & $88 \%$ & $84 \%$ \\
\hline 8 & Sensitivity (or) recall $[R]=T p /(T p+F n)$ & $89 \%$ & $85 \%$ & $86 \%$ \\
\hline 9 & F1 score $2(\mathrm{P} . \mathrm{R}) /(\mathrm{P}+\mathrm{R})$ & $90 \%$ & $87 \%$ & $85 \%$ \\
\hline
\end{tabular}

curve) is a parameter that tells us how much data is useful. The error tells that how much data is unused by the model. Specificity, precision and F1 score tell us how our model is performing. If those values are good then our model performance is good. Specificity avoids false alarms and false positives. Precision is to be chosen if our measurement meets the true value. If false positives ( $\mathrm{Fp}$ ) are better than false negatives (Fn) then Recall will be chosen to analyse the model. Out of all the three model performances, $\mathrm{k}-\mathrm{NN}$ provides 
better results in terms of accuracy and fewer error results. The results can be improved by using different methods in the models like parameter tuning or by building our custom models which give better accuracy.

\section{Conclusion and future scope}

The proposed architecture was implemented and evaluated. By this, we can conclude that this paper describes an architecture of how IoT and machine learning play an important role in the field of agriculture. The application of this system in the field will advance the harvest and production of the crop. By implementing IoT in agriculture there will be less need for manpower and the cost of labour is also reduced. The whole system is efficient and consumes less power. The performance of each model is identified and compared with other models. The highest accuracy is found to be $90 \%$ in a loamy type of soil. By this, we can see that k-NN shows good accuracy among the other two classifiers. In the future, a weed detection technique using ESP-32 cam and fertilizer prediction required for the soil is our concern. Adding LoRa (long-range) technology to this system will help the user to get the results that are some kilometres far away.

\section{References}

[1] Khanna, Abhishek, and Sanmeet Kaur. "Evolution of Internet of Things (IoT) and its significant impact in the field of precision agriculture." Computers and Electronics in Agriculture 157 (2019): 218-231.

[2] Medar, Ramesh A., Vijay S. Rajpurohit and Anand M. Ambekar. "Sugarcane crop yield forecasting model using supervised machine learning." International Journal of Intelligent Systems and Applications 11.8 (2019): 11.

[3] Charoen-Ung, Phusanisa, and Pradit Mittrapiyanuruk. "Sugarcane yield grade prediction using random forest and gradient boosting tree techniques." 2018 15th International Joint Conference on Computer Science and Software Engineering (JCSSE). IEEE, 2018.

[4] Ghadge, Rushika, et al. "Prediction of crop yield using machine learning." International Research Journal of Engineering and Technology (IRJET) 5 (2018).

[5] Priya, P., U. Muthaiah and M. Balamurugan. "Predicting yield of the crop using machine learning algorithm.” International Journal of Engineering Sciences \& Research Technology 7.1 (2018): $1-7$.

[6] Vanitha, C. N., N. Archana and R. Sowmiya. "Agriculture analysis using data mining and machine learning techniques.” 2019 5th International Conference on Advanced Computing \& Communication Systems (ICACCS). IEEE, 2019.

[7] Reshma, SR Juhi, and Anitha S. Pillai. "Impact of machine learning and Internet of Things in agriculture: state of the art." International Conference on Soft Computing and Pattern Recognition. Springer, Cham, 2016. 


\title{
75 A review on video forensics system using soft computing techniques
}

\author{
Sunpreet Kaur Nanda ${ }^{1}$, Deepika Ghai, * \\ ${ }^{1}$ Research Scholar, School of Electronics and Electrical Engineering, \\ Lovely Professional University, Punjab; Assistant Professor, Electronics and \\ Communication Engineering Department, P.R. Pote College of Engineering and \\ Management, Amravati, India \\ ${ }^{2}$ Assistant Professor, School of Electronics and Electrical Engineering, \\ Lovely Professional University, Punjab, India
}

\section{Introduction}

Person identification is becoming a growing need day by day in almost all sectors. Video forensics would prove to be fruitful for identification purpose in legal matters, banks, etc. This method not only identifies a person but lets us know whether the data presented is authentic or not [1]. Video forensics is addressing the global level problems as identification and authentication of that report for persons or criminals. It is not only required in a specific region but it is a global level problem which needs to be addressed. Anomalous behaviour of any human being if identified at an early stage would help to solve several problems in or before time. As advanced crime increments exponentially, the requirement for video forensics criminological aptitude in law authorization develops with it [2]. The Indian forensics organization is also taking efforts to improve the efficiency of the available video forensics methods. Documentation of proof is significant in numerous parts of a case, from essentially recording the state of proof to upgrading subtleties that may not be observable or obvious to the natural eye. Thus, there is a great deal of extension for development in video legal sciences [3]. With every single positive edge these gadgets have additionally become the observers to practically all occasions identified with its clients (common and criminal), so these are to be researched to extricate the privileged and exact data that is connected and required [4].

Nowadays, there is a drastic change and advance in information technology. Due to this growth, the technology is easily accessible to the majority of the human beings $[5,6]$. The human reliance on this innovation has expanded a ton. Practically all the information is being put away electronically now. Subsequently, these gadgets have become an integral part of our everyday life [7]. These gadgets have become observers to all the occasions identified with its clients, with every single positive plot for common and criminal and are utilized to examine and remove the privileged and precise data that is required. Computerized gadgets are explored in all cases nowadays in urban regions. Laptops, personal computers, personal digital assistants (PDAs), compact discs (CDs), pen drives, secure digital (SD) \& microSD cards, etc. are the generally used digital devices [8].

* Corresponding author email: deepika.21507@lpu.co.in 
The remaining part of the paper is structured as follows: Section 2 describes the detailed literature review about the work done in video forensics. The discussion about video forensics is described in Section 3. The conclusion and future scope of video forensic techniques are concluded in Section 4.

\section{Related work}

Numerous approaches have been proposed for video forensic techniques. This article classifies approaches according to the different types of image and video forensics and discusses the performance evaluation metrics also. Various techniques such as deep learning-based anomaly detection approach (DeepAnt), convolutional auto-encoders (ConvAE), convolutional long short-term memory (ConvLSTM), etc. have been used for video forensics [1-3]. Table 75.1 shows the comparison of various existing video forensic techniques on the basis of the performance evaluation metrics and on various public datasets.

\section{Discussion}

We will extensively study and try to explore deep learning architectures that are meant for early anomaly detection in videos. From the literature review, it is observed that very good performance has been shown by both the inception and residual networks and that too with relatively low computational cost. Embedded processing capabilities and the potential of deep features are motivated by the recent improvements in them; we will investigate deep CNNs or deep learning in Python for video forensics that improve detection accuracy and also minimize the false warning rate. It combines both the architectures to further boost the performance with the good classification result. The problem is posed as a classification problem whereas the overall goal is to identify normal and abnormal human behaviour patterns in the public domain. It is necessary to build a training and testing framework for normal, abnormal and detection of suspicious activity from the video using deep learning techniques. At the same time, it is equally important to design and develop a proposed system for person identification from the abnormal and suspicious activity video. We also need to validate the performance of the proposed system in terms of accuracy, computation time, with other state-of-art methods on the various benchmark datasets.

\section{Conclusion and future scope}

Very little work has been done in the field related to video forensics till the current date. Hence, video forensics has become a very popular topic these days. There are several software applications such as Cognitech, Elecard StreamEye, Ocean Systems dTective, etc. available for video analysis. Despite this, several techniques such as correlation matching, line segment matching, motion segmentation, shape matching, median and average frames, total variation denoise, etc. have been advanced from picture crime scene investigation; video signals present new difficulties in the video measurable applications world $[20,21]$. This is a result of the multifaceted nature and the measure of information to be prepared and furthermore because of the wide work of pressure strategies, which may bring about changing or deleting impressions left by past sign adjustments. The examination movement on video legal sciences is as yet a progressing procedure since the unpredictability of video altering prospects requires extra exploration endeavours to make these strategies increasingly strong. There are many applications of video forensics such as civil 
Table 75.1 Comparison of various existing video forensic techniques on the basis of the performance evaluation metrics

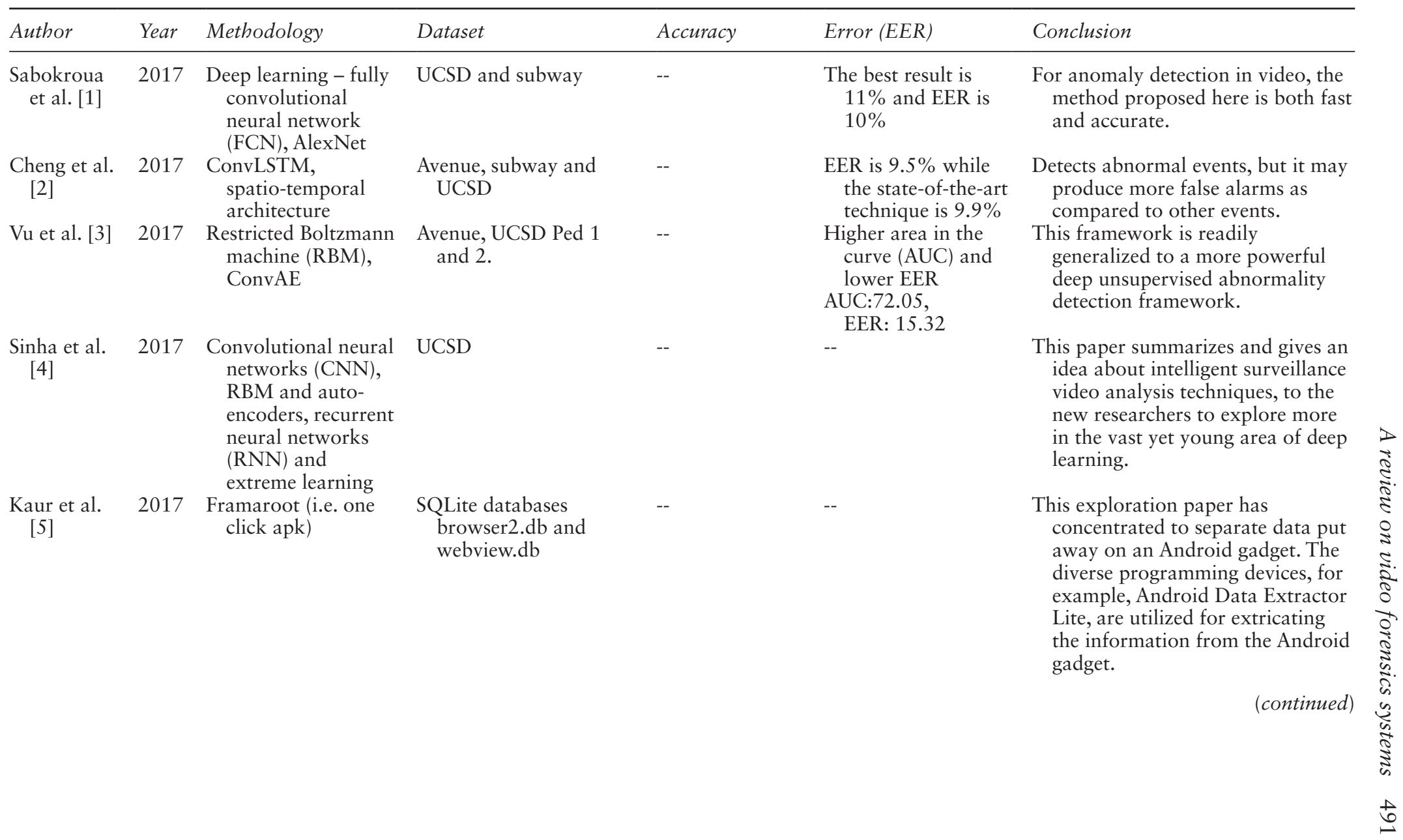




\begin{tabular}{|c|c|c|c|c|c|c|}
\hline Author & Year & Methodology & Dataset & Accuracy & Error (EER) & Conclusion \\
\hline $\begin{array}{l}\text { Muhammad } \\
\text { et al. [6] }\end{array}$ & 2018 & $\mathrm{CNN}$ & $\begin{array}{l}\text { Own datasets: } \\
\text { Dataset 1: } 17 \text { fire videos } \\
\text { and } 14 \text { normal videos } \\
\text { without fire } \\
\text { Dataset } 2: 107 \text { images } \\
\text { belong to fire class } \\
\text { and } 119 \text { images } \\
\text { belong to non-fire } \\
\text { class from } 226 \text { images }\end{array}$ & $\begin{array}{l}\text { For Dataset } \\
\text { 1: accuracy of } \\
93.55 \% \text { with } \\
11.67 \% \text { false } \\
\text { alarms. } \\
\text { For Dataset } \\
\text { 2: better than } \\
\text { RNN }\end{array}$ & -- & $\begin{array}{l}\text { This paper improved the false } \\
\text { detection accuracy, but the } \\
\text { number of false alarms is high } \\
\text { and more research has to be done. }\end{array}$ \\
\hline $\begin{array}{l}\text { Bajestani } \\
\text { et al. [7] }\end{array}$ & 2018 & $\begin{array}{l}\text { Faster region } \\
\text { convolutional } \\
\text { neural network } \\
\text { (R-CNN) }\end{array}$ & $\begin{array}{l}\text { PASCAL VOC, UMN } \\
\text { and USDC }\end{array}$ & $\begin{array}{l}\text { True positive } \\
(56 \%), \text { false } \\
\text { positive } \\
(36 \%)\end{array}$ & -- & $\begin{array}{l}\text { This method improves the true } \\
\text { positive with the tradeoff of trivial } \\
\text { false positive. }\end{array}$ \\
\hline $\begin{array}{l}\text { Kaushala } \\
\text { et al. [8] }\end{array}$ & 2018 & $\begin{array}{l}\text { Neural network, } \\
\text { fuzzy logic, hybrid } \\
\text { of neuro-fuzzy }\end{array}$ & VOT, ALOV, BMC & -- & -- & $\begin{array}{l}\text { Different delicate processing based } \\
\text { methodologies for moving } \\
\text { article discovery and following } \\
\text { in recordings. Article provides } \\
\text { various techniques along with } \\
\text { scope, pros, cons and the } \\
\text { limitation associated with each } \\
\text { of them. R-CNN proves to be the } \\
\text { best. }\end{array}$ \\
\hline $\begin{array}{l}\text { Nasir et al. } \\
\text { [9] }\end{array}$ & 2018 & Fog computing & -- & -- & -- & $\begin{array}{l}\text { This low force PC can supplant an } \\
\text { exorbitant cloud arrangement, } \\
\text { yet in addition holds potential } \\
\text { for versatility for empowered } \\
\text { applications without including } \\
\text { any critical expense. }\end{array}$ \\
\hline $\begin{array}{l}\text { Muhammad } \\
\text { et al.[10] }\end{array}$ & 2018 & $\begin{array}{l}\text { Probabilistic and } \\
\text { lightweight } \\
\text { algorithm }\end{array}$ & UNSW NB15 & -- & -- & $\begin{array}{l}\text { Trial results check the effectiveness, } \\
\text { security, and heartiness of } \\
\text { proposed calculation contrasted } \\
\text { and data encryption standard } \\
\text { (DES). }\end{array}$ \\
\hline
\end{tabular}




\begin{tabular}{|c|c|c|c|c|c|c|}
\hline $\begin{array}{l}\text { Munir et al. } \\
\text { [11] }\end{array}$ & 2018 & DeepAnT & $\begin{array}{l}\text { Genuine just as } \\
\text { manufactured datasets } \\
\text { from various areas } \\
\text { including street } \\
\text { traffic, organize use, } \\
\text { on-line commercial, } \\
\text { web traffic, space } \\
\text { transport, and } \\
\text { wellbeing }\end{array}$ & $\begin{array}{l}\text { Accuracy } \\
95.23 \% \text { in } \\
\text { road traffic } \\
\text { dataset }\end{array}$ & -- & $\begin{array}{l}\text { Evaluation of DeepAnT on } 10 \\
\text { different datasets comprising } 433 \\
\text { time series in all and provide a } \\
\text { total comparison with } 15 \text { state- } \\
\text { of-the-art anomaly detection } \\
\text { methods. Support vector machine } \\
\text { (SVM) classifier proves to be } \\
\text { better. }\end{array}$ \\
\hline $\begin{array}{l}\text { Singh et al. } \\
\text { [12] }\end{array}$ & & $\begin{array}{l}\text { Passive-blind } \\
\text { technique, inter- } \\
\text { frame forgery } \\
\text { detection }\end{array}$ & $\begin{array}{l}\text { Detection evaluation } \\
\text { dataset }\end{array}$ & $\begin{array}{l}\text { Detection } \\
\text { accuracy is } \\
97 \%, 96.1 \%, \\
\text { and } 93.3 \% \\
\text { at } 9,6 \text { and } \\
3 \text { Mbps, } \\
\text { for MPEG } \\
\text { compression }\end{array}$ & -- & $\begin{array}{l}\text { Presents a store of data with respect } \\
\text { to the sorts of alteration assaults a } \\
\text { video can experience the ill effects } \\
\text { of and a far reaching wellspring of } \\
\text { references for the inactive visually } \\
\text { impaired methods proposed for } \\
\text { identifying assaults. }\end{array}$ \\
\hline $\begin{array}{l}\text { Sultani et al. } \\
\text { [13] }\end{array}$ & 2018 & $\begin{array}{l}\text { Deep multiple } \\
\text { instance learning } \\
\text { (MIL) ranking } \\
\text { model, binary } \\
\text { SVM classifier }\end{array}$ & $\begin{array}{l}\text { UMN, UCSD, Avenue, } \\
\text { CAVIAR, MoSIFT }\end{array}$ & $\begin{array}{l}\text { False } \\
\quad \text { alarm: } 1.9 \%\end{array}$ & $\begin{array}{l}\text { Area under the curve } \\
\text { (AUC): } 75.41\end{array}$ & $\begin{array}{l}\text { Another huge scope peculiarity } \\
\text { dataset comprising an assortment } \\
\text { of genuine oddities is presented. }\end{array}$ \\
\hline $\begin{array}{l}\text { Castillo } \\
\text { et al. [14] }\end{array}$ & 2018 & $\begin{array}{l}\text { Darkening and } \\
\text { contrast at } \\
\text { learning and } \\
\text { test (DaCoLT), } \\
\text { brightness guided } \\
\text { preprocessing } \\
\text { approach }\end{array}$ & COCO Dataset & $\begin{array}{l}\text { Precision of } \\
\quad \text { around } 73 \%\end{array}$ & -- & $\begin{array}{l}\text { This paper proposed brightness } \\
\text { guided preprocessing approach. } \\
\text { DaCoLT model shows very high } \\
\text { potential even in low quality } \\
\text { recordings and gives acceptable } \\
\text { outcomes as a programmed alert } \\
\text { framework. }\end{array}$ \\
\hline $\begin{array}{r}\text { Bouindour } \\
\text { et al. [15 }\end{array}$ & 2019 & CNN & UCF101 & -- & $\begin{array}{l}\text { Equal error rate } \\
\text { of frame level } \\
\text { (EERFL): } 6.25 \%, \\
\text { the equal error } \\
\text { rate of pixel level } \\
\text { (EERPL): } 9.82 \%\end{array}$ & $\begin{array}{l}\text { This strategy is hearty, considers } \\
\text { uncommon typical occasions } \\
\text { present in the preparation stage. } \\
\text { Furthermore, it very well may be } \\
\text { joined in online CCTV. }\end{array}$ \\
\hline
\end{tabular}

(continued) 


\begin{tabular}{|c|c|c|c|c|c|c|}
\hline Author & Year & Methodology & Dataset & Accuracy & Error (EER) & Conclusion \\
\hline $\begin{array}{l}\text { Camerona } \\
\text { et al. [16] }\end{array}$ & 2019 & $\mathrm{CNN}$ & $\begin{array}{l}\text { PASCAL VOC 2012, } \\
\text { VOC } 2007\end{array}$ & $\begin{array}{l}\text { Pre CNN } \\
\text { approach } \\
89 \% \text { on } \\
\text { their custom } \\
\text { dataset. } \\
\text { Faster R-CNN- } \\
\text { Very high } \\
\text { YOLO- Low } \\
\text { SSD- Medium } \\
\text { ResNet- High }\end{array}$ & -- & $\begin{array}{l}\text { This work has featured a portion } \\
\text { of the useful difficulties of } \\
\text { planning a handling framework } \\
\text { for a CNN-based computerized } \\
\text { observation framework utilizing } \\
\text { off-the-rack equipment and open- } \\
\text { source calculations }\end{array}$ \\
\hline $\begin{array}{l}\text { Xiao et al. } \\
\text { [17] }\end{array}$ & 2019 & DeepAnT & -- & -- & -- & $\begin{array}{l}\text { Proposed a structure for video } \\
\text { based advanced criminology } \\
\text { examination, valuable for hostile } \\
\text { to wrongdoing or quick reaction } \\
\text { when wrongdoing exercises or } \\
\text { practices are distinguished. }\end{array}$ \\
\hline $\begin{array}{l}\text { Saleem et al. } \\
\text { [18] }\end{array}$ & 2019 & Deep CNNs & Trecvid 2016 UET & -- & $\begin{array}{l}\text { EER: } 35 \% \text { for } \\
\text { Trecvid dataset. } \\
\text { EER: } 52 \% \text { for UET } \\
\text { dataset. }\end{array}$ & $\begin{array}{l}\text { It is a structure for creating multi- } \\
\text { line printed depictions for video } \\
\text { subtitling. Accomplishments } \\
\text { rich model stretches out element } \\
\text { lattice to visual (2-D and 3-D) } \\
\text { and facial highlights. Spatio- } \\
\text { transient qualities are enveloped } \\
\text { by utilizing profound neural } \\
\text { systems. }\end{array}$ \\
\hline $\begin{array}{l}\text { Sreenu et al. } \\
\text { [19] }\end{array}$ & 2019 & Big data computing & $\begin{array}{l}\text { ImageNet } 2012 \\
\text { PASCAL VOC } \\
\text { MediaEval } 2013 \text { dataset } \\
\text { UCSD pedestrian, } \\
\text { Violent-flows, CUHK, } \\
\text { UCF50, Rodriguez's, } \\
\text { the mall and finally } \\
\text { the worldExpo's } \\
\text { dataset }\end{array}$ & -- & -- & $\begin{array}{l}\text { Methods analysing behaviour of } \\
\text { crowd are discussed. }\end{array}$ \\
\hline
\end{tabular}


and criminal attorneys, law enforcement corporations, insurance investigation, private investigation, domestic disputes, bank theft and robbery, etc. Numerous methods have been proposed in the past for anomaly detection in videos. These approaches considered the different attributes related to videos such as deblur filters, demultiplexing, denoise filters, detection filters, enhancement, histogram editor, segmentation, tracking, zoom, photogrammetry etc. [22,23]. This paper gives an expansive grouping of the different procedures and calculations proposed before. This paper likewise uncovered a presentation examination table of various procedures that was proposed before for oddity discovery in recordings. Each approach has its own advantages and limitations. Despite the fact that there are numerous quantities of calculations, there is no single brought together methodology that fits for all the applications. Future examination has still to explore increasingly complex preparing chains where every procedure on the sign might be iterated on various occasions. These situations end up being progressively reasonable since the chance of transmitting and disseminating video content over the web favours the dispersion of duplicates of a similar interactive media content which has been altered on numerous occasions. This paper tells us that many tools are already readily available in the market for video tampering detection but almost all of them are good for basic functionality. No tool is yet able to perform higher-level functions. The need is to develop tools which are able to detect hidden data in a video as well as tampering done to it. It should be able to present the evidence in a proper way so that the user can use it as evidence for law enforcement. Video forensics will surely be the most handy and reliable method for finding originality of evidence.

\section{References}

[1] M. Sabokroua, M. Fayyazb, M. Fathyc, Z. Moayedd, R. Kletted, "Deep-Anomaly: fully convolutional neural network for fast anomaly detection in crowded scenes," Journal of Computer Vision and Image Understanding, pp. 1-30, 2017.

[2] C.B. Jin, S. Li, H. Kim, "Real-time action detection in video surveillance using sub-action descriptor with multi-CNN," Computer Vision and Pattern Recognition, pp. 1-29, 2017.

[3] H.T. Vu, T.D. Nguyen, D. Phung at "Energy-based localized anomaly detection in video surveillance," in: Proceedings of Advances in Knowledge and Data Mining, Jeju, South Korea, pp. 641-653, 2017.

[4] R.K. Sinha, R. Pandey, R. Pattnaik, "Deep learning for computer vision tasks: a review," in: International Conference on Intelligent Computing and Control, pp. 1-5, 2017.

[5] H. Kaur, K.R. Choudhary, "Digital forensics: implementation and analysis for Google Android framework," Information Fusion for Cyber-Security Analytics, Springer, pp. 307-331, 2017.

[6] K. Muhammad, J. Ahmad, I. Mehmood, S. Rho, S.W. Baik, "Convolutional neural networks based fire detection in surveillance videos," IEEE Access, vol. 6, pp. 18174-18183, 2018.

[7] M.F. Bajestani, S.S.H.R. Abadi, S.M.D. Fard, R. Khodadadeh, "AAD: adaptive anomaly detection through traffic surveillance videos”, Computer Vision and Pattern Recognition, 2018. https://arxiv.org/abs/1808.10044.

[8] M. Kaushala, B.S. Khehrab, A. Sharma, "Soft computing based object detection and tracking approaches: state-of-the-art survey,” Applied Soft Computing, vol. 70, pp. 423-464, 2018.

[9] M. Nasir, K. Muhammad, J. Lloret, A.K. Sangaiah, M. Sajjad, "Fog computing enabled costeffective distributed summarization of surveillance videos for smart cities," Journal of Parallel and Distributed Computing, vol. 126, pp. 161-170, 2019.

[10] K. Muhammad, R. Hamza, J. Ahmad, J. Lloret, H.H.G. Wang, S.W. Baik, "Secure surveillance framework for IoT Systems using probabilistic image encryption," IEEE Transactions on Industrial Informatics, vol. 14, pp. 3679-3689, 2018.

[11] M. Munir, S.A. Siddiqui, A. Dengel, S. Ahmed, "DeepAnT: a deep learning approach for unsupervised anomaly detection in time series” IEEE Access, vol. 7, pp. 1991-2005, 2019. 
[12] R.D. Singh, N. Aggarwal, "Video content authentication techniques: a comprehensive survey," Multimedia Systems, vol. 24, pp. 211-240, 2018.

[13] A. Sultani, C. Chen, M. Shah, "Real-world anomaly detection in surveillance videos," Computer Vision and Pattern Recognition, 2018 <https://arxiv.org/abs/1801.04264>.

[14] A. Castillo, S. Tabik, F. Perez, R. Olmos, F. Herrera, "Brightness guided preprocessing for automatic cold steel weapon detection in surveillance videos with deep learning," Neurocomputing, vol. 330, pp. 151-161, 2019.

[15] S. Bouindour, H. Snoussi, M.M. Hittawe, N. Tazi, T. Wang, "An on-line and adaptive method for detecting abnormal events in videos using spatio-temporal ConvNet," Applied Sciences, vol. 9, pp. 1-17, 2019.

[16] J.A.D. Cameron, P. Savoie, M.E. Kaye, E.J. Scheme, "Design considerations for the processing system of a CNN-based automated surveillance system," Expert Systems with Applications, vol. 136, pp. 105-114, 2019.

[17] J. Xiao, S. Li, Q. Xu, "Video-based evidence analysis and extraction in digital forensic investigation," Special Section on Deep Learning: Security and Forensics Research Advances and Challenges, IEEE Access, vo. 7, pp. 55432-55442, 2019.

[18] S. Saleem, A. Dilawari, U.G. Khan, R. Iqbal, S. Wan, T. Umer, "Stateful human-centered visual captioning system to aid video surveillance," Computers and Electrical Engineering, vo. 78, pp. 108-119, 2019.

[19] G. Sreenu, M.A. Saleem Durai, "Intelligent video surveillance: a review through deep learning techniques for crowd analysis", Journal of Big Data, vol. 48, pp. 1-27, 2018.

[20] A.K. Kushwaha, A. Wadhe, "Design and implementation of forensic framework for video forensics," International Journal of Current Engineering and Technology, vol.5, pp. 1015$1018,2015$.

[21] J. Kamenicky, M. Bartos, J. Flusser, B. Mahdian, J. Kotera, A. Novozamsky, S. Saic, F. Sroubek, M. Sorel, A. Zita, B. Zitova, Z. Sima, P. Svarc, J. Horinek, "PIZZARO: forensic analysis and restoration of image and video data," Forensic Science International, vol. 264, pp. 153166, 2016.

[22] M. Jerian, S. Paolino, F. Cervelli, S. Carrato, A. Mattei, L. Garofano, "A forensic image processing environment for investigation of surveillance video," Forensic Science International, vol. 167, pp. 207-212, 2007.

[23] S. Milani, M. Fontani, P. Bestagini, M. Barni, A. Piva, M. Tagliasacchi, S. Tubaro, "An overview on video forensics," APSIPA Transactions on Signal and Information Processing, vol. 1, pp. 1-18, 2012. 


\title{
76 A new method for calculation of permittivity of spacer in gas insulated busduct
}

\author{
Janaki Pakalapati ${ }^{1}$, N. Karthick ${ }^{1}$, G. V. Nagesh Kumar ${ }^{2}$ \\ ${ }^{1}$ Lovely Professional University, Jalandhar, Punjab, India \\ ${ }^{2} J N T U A C E P$, Pulivendula, A.P. India
}

\section{Introduction}

Nowadays, the gas insulated busduct is gaining popularity due to its high transforming capacity of power to the industry and the entire world due to its compactness, ideal use in restricted areas. The high demand for electrical power and energy efficiency in urban areas made it necessary for power consumers to boost the voltage network [1,2]. Cong Liu et al. have identified that the breakdown of solid insulators is mainly based on the electrical insulation performance of the filler materials [3]. Okabe et al. [4] have reported that solid insulators in GIB are usually made of epoxy resin material and lose their accumulated charge under several operating conditions, leading to heavy flashovers, and Kokkinaki. et al. [5] have observed that the insulation capacity of the composites deteriorates gradually for in-service insulators due to environmental, electrical and mechanical stresses. Nakanishi et al. [6] proposed a method by fitting the insulator with shielding for reducing the electric field along the insulator for reducing the charge accumulation. Several researchers $[7,8]$ have identified a new method in order to differentiate from the complex methods, where the structures of the insulators are to be modified by using several composite materials along with epoxy materials by using nanoparticles like $\mathrm{SiO}_{2}$. Zhang et al. [9] mixed $\mathrm{TiO}_{2}$ nanoparticles into epoxy with various mass fractions, reaching a conclusion that as the concentration of $\mathrm{TiO}_{2}$ increases, the amount of surface charge accumulated on the composite material increases at first but then decreases. A rapid rise in the power density of electrical equipment and electronic equipment highlights the need for thermally conductive but electrically insulating products.

Polymer materials have been widely used as dielectrics to provide electrical insulation in power equipment and electrical devices because of their low cost and easy processing. However, most of the polymer materials have low thermal conductivity, which makes the thermal management of high-power-density equipment and devices become a large challenge [10-12]. The surges or any event of flashovers will damage the spacer and hence a spacer material has to be chosen to get rid of these situations in regular testing in plant or onsite. Qi et al. [13] observed the situation of the insulator for its smooth operation by using epoxy resin along with $\mathrm{TiO}_{2}$, which is a nanoparticle, and mixed in nanofiller composition to develop composite materials. Yang et al. [14] have manganese oxide composites prepared using the bonded-exfoliated method.

\section{Governing equation of the Lichtenecker Rother rule}

From the literature, it was observed that there are several methods to mix the components of dielectric materials for obtaining the effective dielectric permittivity. The Lichtenecker

DOI: $10.1201 / 9781003129103-76$ 
Table 76.1 Concentration of carbides with epoxy material

\begin{tabular}{llll}
\hline Filler concentration & Epoxy concentration & $\varepsilon_{\text {eff }} B_{4} C$ & eff $S i C$ \\
\hline 0 & 1 & 3.49 & 3.49 \\
0.2 & 0.8 & 3.71 & 3.94 \\
0.4 & 0.6 & 3.96 & 4.47 \\
0.6 & 0.4 & 4.22 & 5.07 \\
0.8 & 0.2 & 4.5 & 5.75 \\
1 & 0 & 4.8 & 6.5 \\
\hline
\end{tabular}

Rother rule is identified as the effective dielectric function method to meet the requirement. It was applied in a wide range of mixtures including biological materials, geophysical applications. This mixture formula can be derived from Maxwell's equation when conservation of charge is applied to the mixture and its components. Lichtenecker's logarithmic mixture formula for dielectrics has proven to be a useful practical formulation for determining the permittivity of homogenized dielectric mixtures. The other variants of the Maxwell Garnett formula and the Bruggeman formula can be derived directly from the Lichtenecker logarithmic mixture formula. The permittivity of two-phase dielectric satisfying the Lichtenecker Rother mixing rule can be written as

$\log \varepsilon_{\mathrm{c}}=\mathrm{x} \log \varepsilon_{1}+\mathrm{y} \log \varepsilon_{2}$

where $\quad \varepsilon_{\mathrm{c}}=$ resultant composite permittivity

$\mathrm{x}, \mathrm{y}=$ concentrations of filler and polymer

$\varepsilon_{1}, \varepsilon_{2}=$ permittivity of filler and epoxy

\section{Preparation of polymer materials}

A composition of nanofillers and nanomaterials is used for the preparation of the insulating material for the insulators in the GIB. In this paper several combinations of carbides, nitrides, oxides are considered along with epoxy material to improve the permittivity of the insulating material, which can further be used in the design of solid insulator. A few types of carbide like boron carbide $\left(\mathrm{B}_{4} \mathrm{C}\right)$, silicon carbide $(\mathrm{SiC})$ are used along with the composition of epoxy material in different filler ratios to get the new material for insulation. The permittivity of epoxy material is $3.49, \mathrm{~B}_{4} \mathrm{C}$ is 4.8 and that of $\mathrm{SiC}$ is 6.5 . These two filler materials are mixed with epoxy material at different compositions from the above formula and the effective permittivity is calculated as shown in Table 76.1. From Table 76.1, it is clear that with only a little mixture of the epoxy and the carbides the effective permittivity is increased. Figure 76.1 and Figure 76.2 represent the variation of permittivity ranges of the mixture of boron carbide and silicon carbide along with epoxy material. Figure 76.3 represents the comparison of both the carbides and it is observed that the permittivity of silicon carbide is more than that of boron carbide.

Nitrides are the other filler composite materials used in the design of polymeric spacers. The different types of nitrides used here are boron nitride $(\mathrm{BN})$, aluminium nitride (AlN) and silicon nitride (Si3N4) and they are used along with the composition of epoxy material in different filler ratios to get the new material for the solid insulator. The permittivity of epoxy material is $3.49, \mathrm{BN}$ is 4 , AlN is 9 and that of $\mathrm{Si}_{3} \mathrm{~N}_{4}$ is 7.5 . These filler materials are mixed with epoxy material at different compositions and the effective permittivity is calculated from the above formula and tabulated as in Table 76.2. From Figures 76.4, 


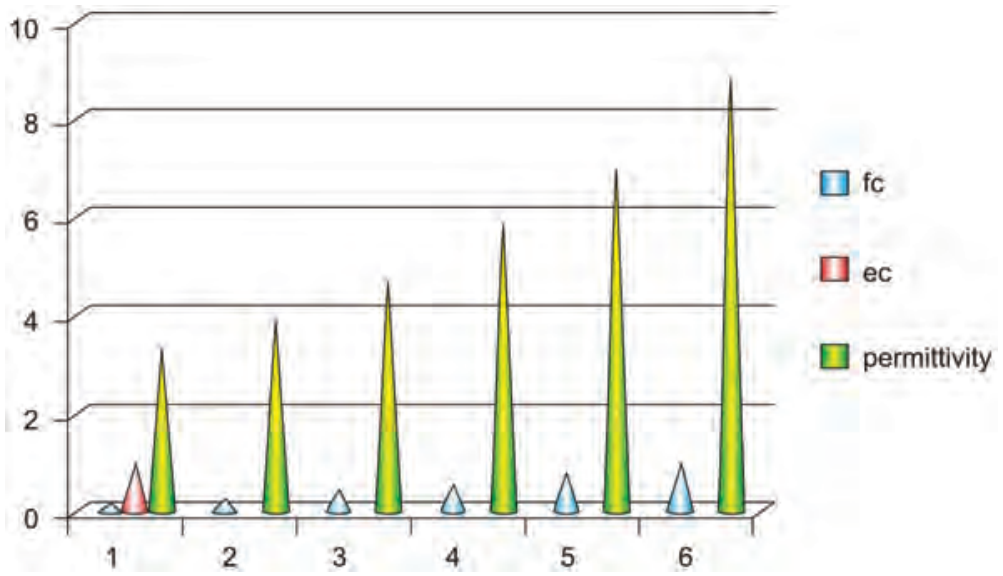

Figure 76.1 Filler concentration of $\mathrm{B}_{4} \mathrm{C}$.

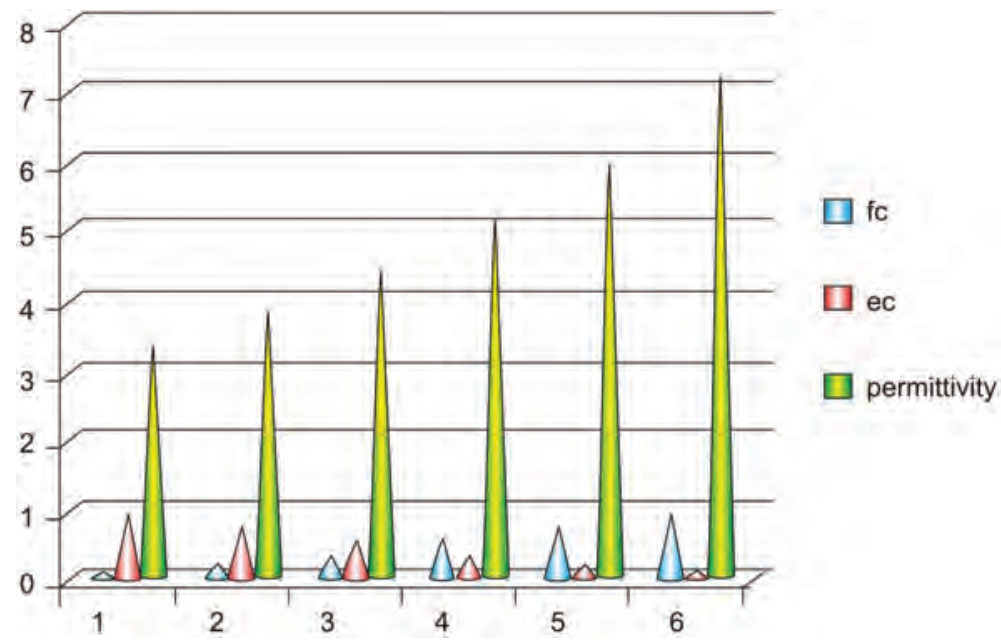

Figure 76.2 Filler concentration of SiC.

Figure 76.5 and Figure 76.6 it is clear that with only some quantity of the mixture of epoxy and nitrides the effective permittivity increases. Figure 76.7 represents the comparison of all the nitrides and it was observed that aluminium nitride has more permittivity than other nitrides.

Oxides are the other filler composite materials used in the design of polymeric spacers. The different types of oxides used here are titanium oxide $\left(\mathrm{TiO}_{2}\right)$, Aluminum oxide $\left(\mathrm{Al}_{2} \mathrm{O}_{3}\right)$, silicon dioxide $\left(\mathrm{SiO}_{2}\right)$, magnesium oxide $(\mathrm{MgO})$ and they are used along with the composition of epoxy material in different filler ratios to get the new material for the solid insulator. The permittivity of epoxy material is $3.49, \mathrm{TiO}_{2}$ is $86, \mathrm{Al}_{2} \mathrm{O}_{3}$ is $9.8, \mathrm{MgO}$ is 3.2 , $\mathrm{SiO}_{2}$ is 3.9. These filler materials are mixed with epoxy material at different compositions and the effective permittivity is calculated from the above formula and tabulated as in Table 76.3. From Figures 76.8, Figure 9, Figure 76.10 and Figure 76.11 it is observed that 


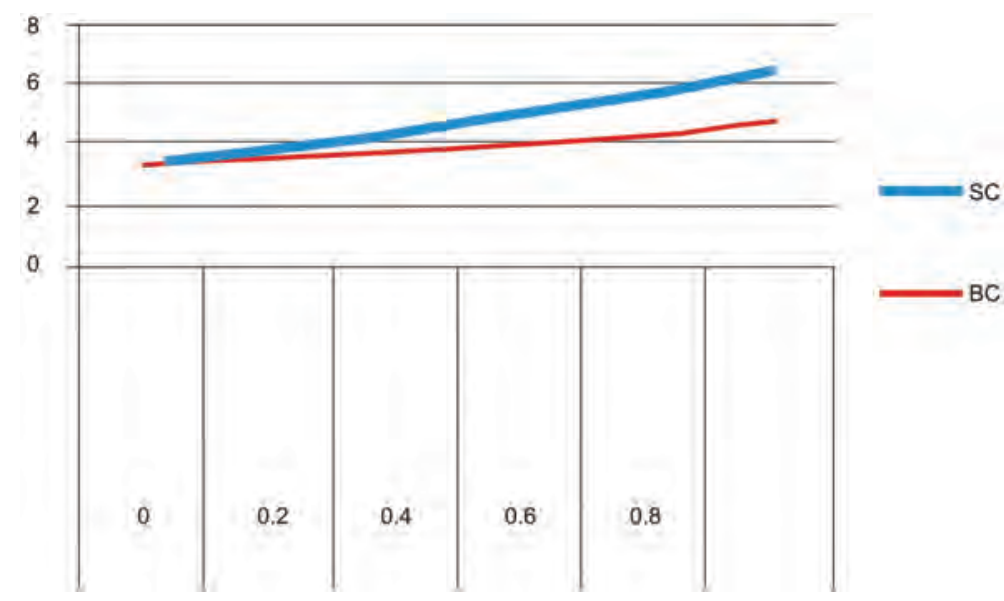

Figure 76.3 Comparison of carbides.

Table 76.2 Concentration of nitrides with epoxy material

\begin{tabular}{lllll}
\hline $\begin{array}{l}\text { Filler } \\
\text { concentration }\end{array}$ & $\begin{array}{l}\text { Epoxy } \\
\text { concentration }\end{array}$ & $\varepsilon_{\text {eff }} B N$ & eff AlN & $\varepsilon_{\text {eff }} S i_{3} N_{4}$ \\
\hline 0 & 1 & 3.49 & 3.49 & 3.49 \\
0.2 & 0.8 & 3.54 & 4.16 & 3.89 \\
0.4 & 0.6 & 3.63 & 5.37 & 4.67 \\
0.6 & 0.4 & 3.71 & 6.02 & 5.49 \\
0.8 & 0.2 & 3.89 & 7.41 & 6.3 \\
1 & 0 & 4.6 & 9 & 7.5 \\
\hline
\end{tabular}

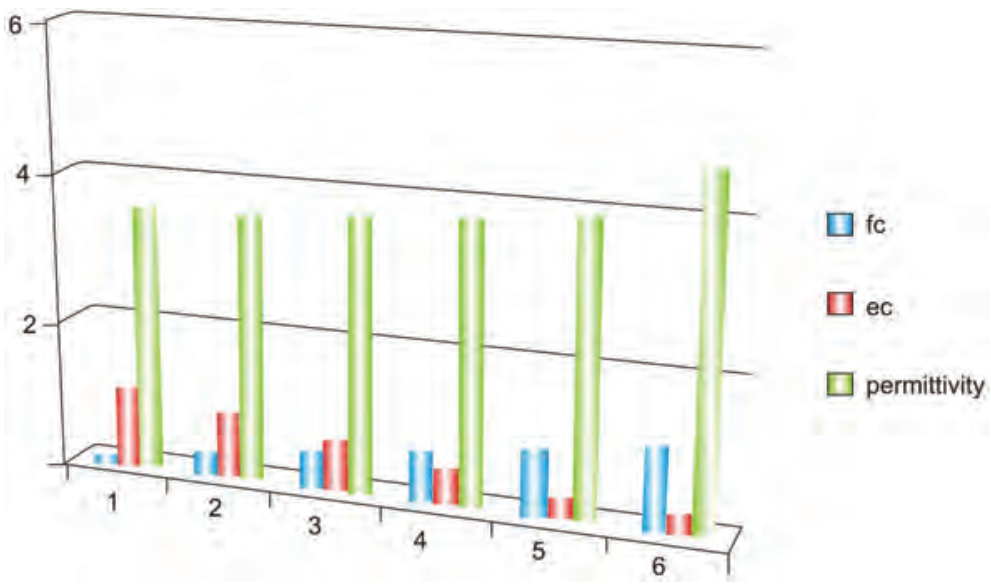

Figure 76.4 Filler concentration of epoxy with BN. 


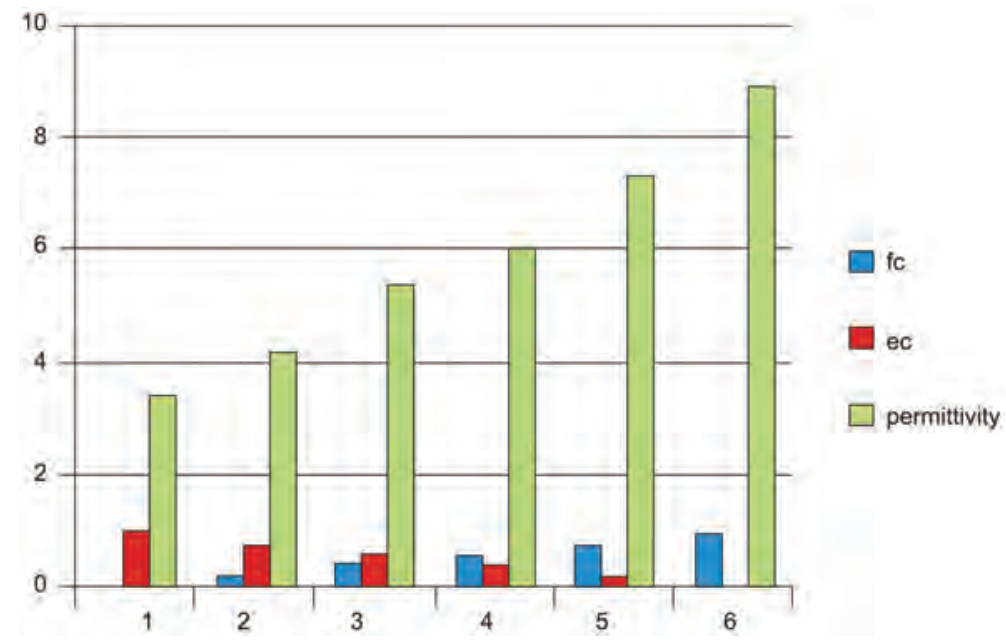

Figure 76.5 Filler concentration of epoxy with AlN.

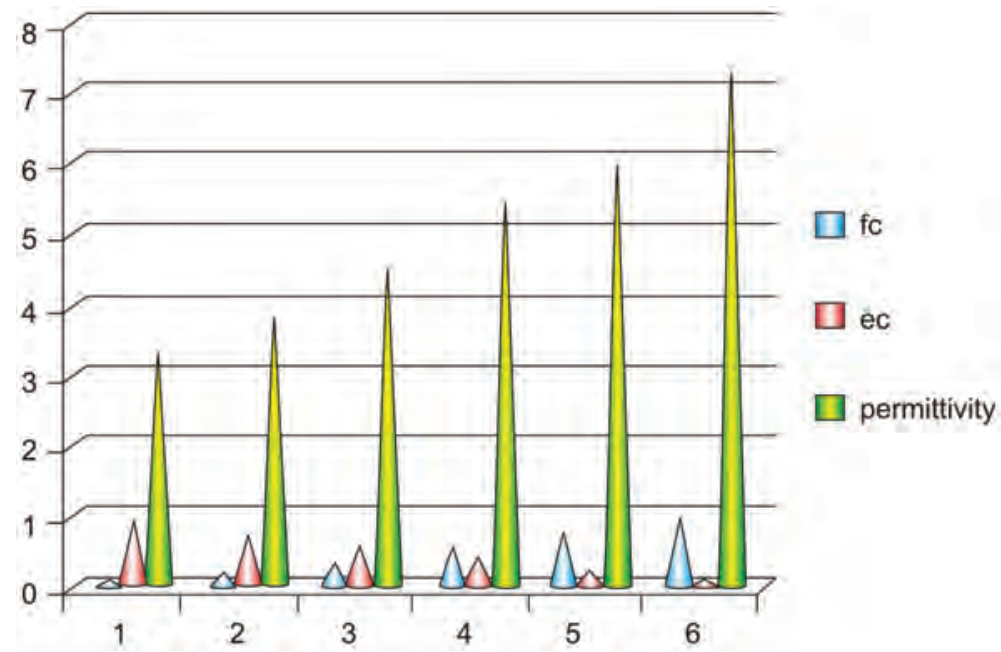

Figure 76.6 Filler concentration of epoxy with $\mathrm{Si}_{3} \mathrm{~N}_{4}$.

with only a small quantity of different oxides along with the epoxy material the effective permittivity increases.

\section{Conclusion}

In this paper, the different concentrations of polymeric materials are combined with epoxy material using the Lichtenecker Rother mixing formula to improve the dielectric strength of the insulators in GIB. The obtained results show that the permittivity of the filler concentrations has been improved drastically with a small quantity of polymers like carbides, nitrides and oxides along with epoxy material. The permittivity of silicon carbide is more than that of boron carbide. Similarly, boron nitride has resulted in high 


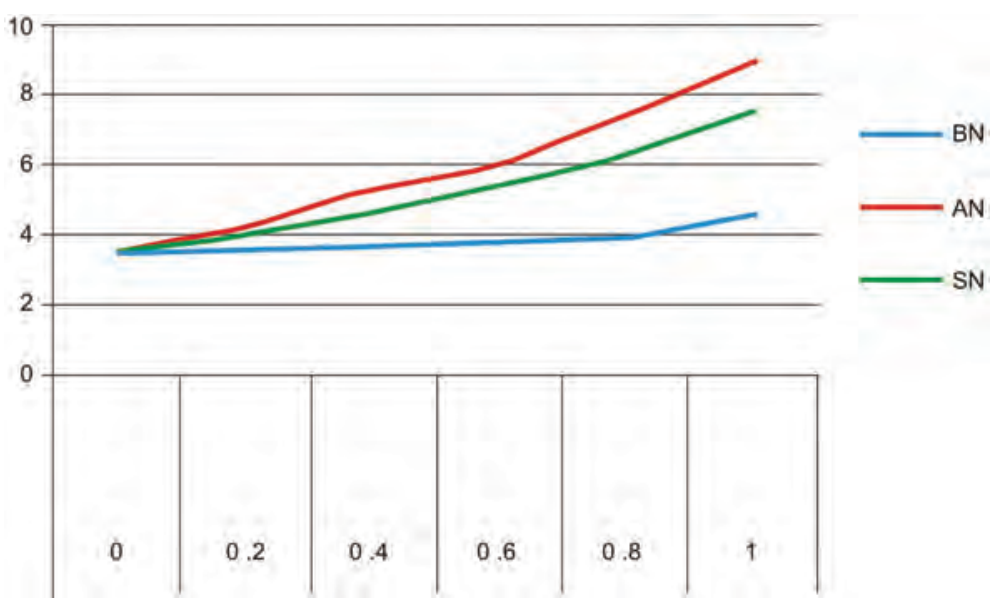

Figure 76.7 Comparison of permittivity of all the nitrides.

Table 76.3 Concentration of oxides with epoxy material

\begin{tabular}{llclll}
\hline $\begin{array}{l}\text { Filler } \\
\text { concentration }\end{array}$ & $\begin{array}{l}\text { Epoxy } \\
\text { concentration }\end{array}$ & $\varepsilon_{\text {eff }} \mathrm{TiO}_{2}$ & eff $\mathrm{Al}_{2} \mathrm{O}_{3}$ & $\varepsilon_{\text {eff }} \mathrm{MgO}$ & ${ }_{\text {eff }} \mathrm{SiO}_{2}$ \\
\hline 0 & 1 & 3.49 & 3.49 & 3.49 & 3.49 \\
0.2 & 0.8 & 6.60 & 4.16 & 3.38 & 3.54 \\
0.4 & 0.6 & 12.3 & 5.01 & 3.31 & 3.63 \\
0.6 & 0.4 & 23.44 & 6.16 & 3.31 & 3.71 \\
0.8 & 0.2 & 44.66 & 7.41 & 3.23 & 3.80 \\
1 & 0 & 86 & 9.1 & 3.2 & 3.9 \\
\hline
\end{tabular}

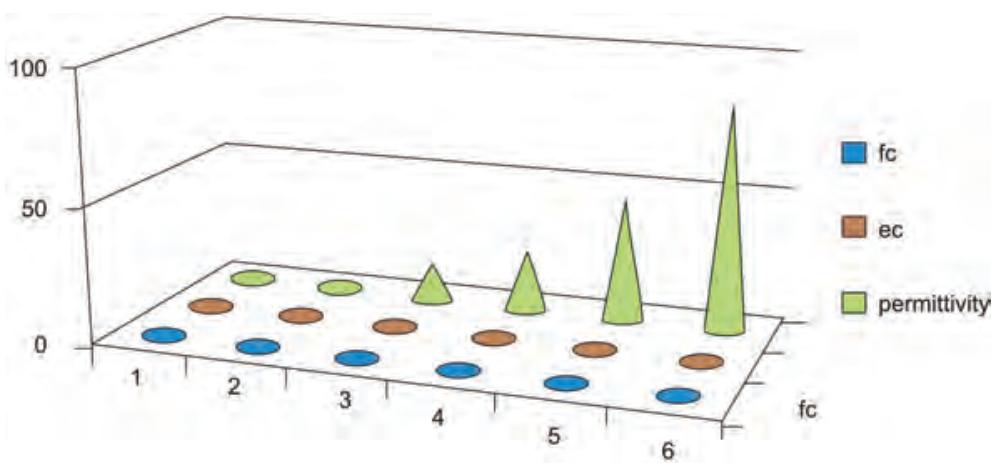

Figure 76.8 Filler concentration of epoxy with $\mathrm{TiO}_{2}$.

permittivity when compared to aluminium nitride and silicon nitride. Similarly, titanium oxide has resulted in high permittivity than aluminium oxide, silicon dioxide and magnesium. So all these compositions of polymers can be used to reduce the magnitude of the electrical field in the solid insulator for the smooth operation of gas insulated busducts. 


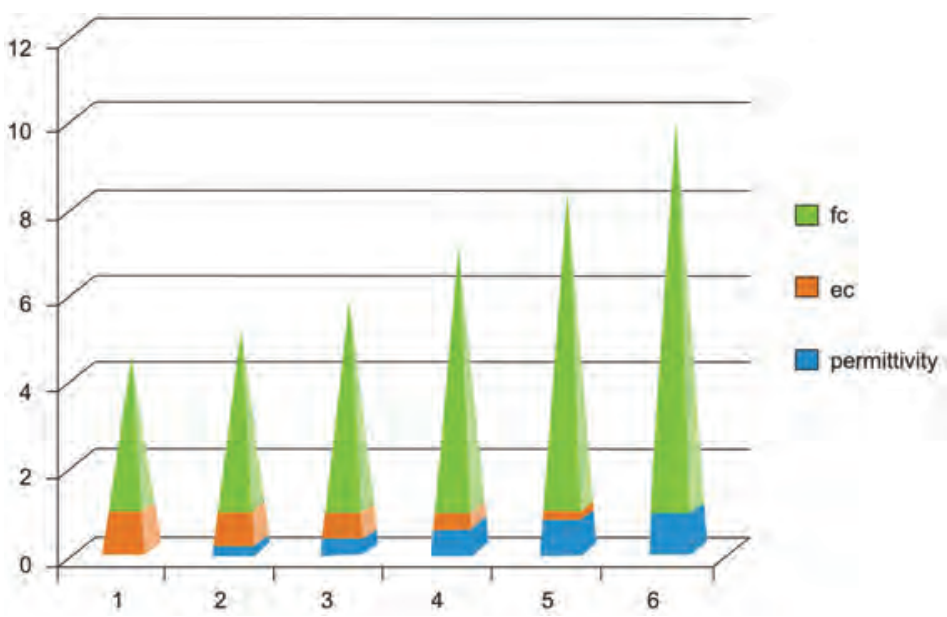

Figure 76.9 Filler concentration of epoxy with $\mathrm{Al}_{2} \mathrm{O}_{3}$.

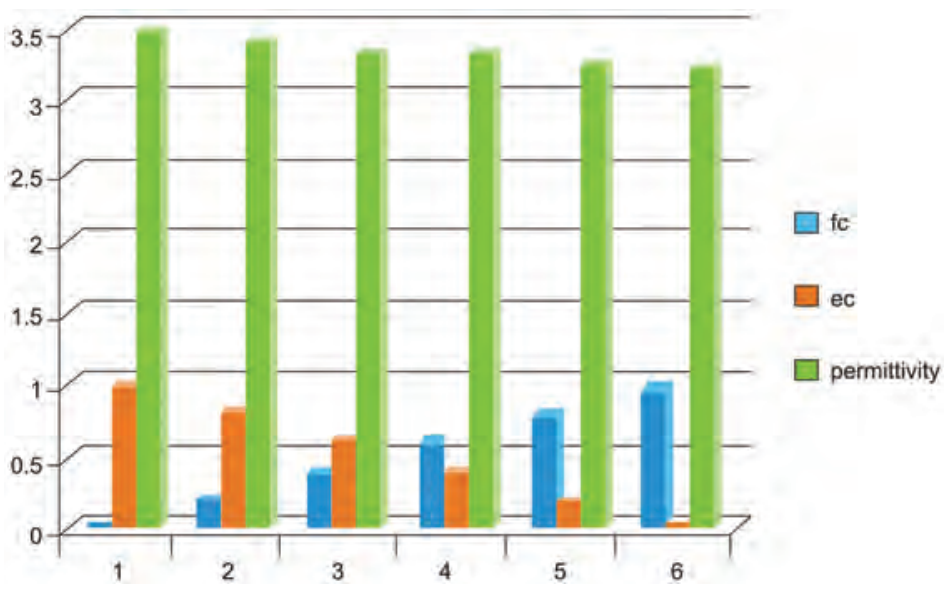

Figure 76.10 Filler concentration of epoxy with $\mathrm{MgO}$.

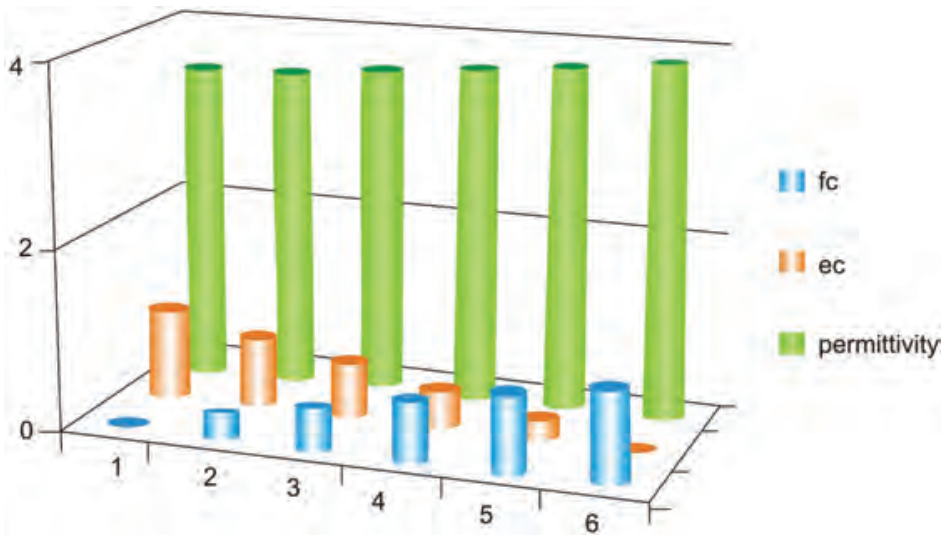

Figure 76.11 Filler concentration of epoxy with $\mathrm{SiO}_{2}$. 


\section{References}

1. O.Dwyer, J.J. "Breakdown in solid dielectrics", IEEE Trans. Electr. Insul., vol. 17(6), pp. 484-487,1982.

2. Helgee, B., Bjellheim, P. "Electric breakdown strength of aromatic polymers: dependence on film thickness and chemical structure", IEEE Trans. Electr. Insul., vol. 26(6), pp. 11471152, 1991.

3. Cong Liu, Yu Chen, Kai Yao, Yi Hao, Shuang Wang, Zengbin Wang, Ruilei Gong, Chaoyun Du. "Combined electrical and thermal aging of alumina filled epoxy solid insulators for GIS", IEEE, 2019.

4. Okabe, S. Koto, M., Endo, F., Kobayashi, K. "Insulation characteristics of GIS spacer for very fast transient overvoltage", IEEE Transactions on Power Delivery, vol. 11(1), pp. 210$218,2002$.

5. Kokkinaki, O., Klini, A., Polychronaki, M., Mavrikakis, N.C., Siderakis, K., G., Koudoumas, E., Pylarinos, D., Thalassinakis, E., Kalpouzos, K., Anglos, D. "Assessing the type and quality of high voltage composite outdoor insulators by remote laser-induced breakdown spectroscopy analysis: A feasibility study", Spectrochimica Acta - Part B Atomic Spectroscopy, vol. 165 , art. no. $105768,2020$.

6. Nakanishi, K., Yoshioka, A., Arahata, Y., Shibuya, Y. "Surface charging on epoxy spacer at DC stress in compressed SF6 gas", IEEE Transactions on Power Apparatus and Systems, vol. 102(12), pp. 3919-3927, 1983.

7. Tanaka, T. "Dielectric nanocomposites with insulating properties", IEEE Trans. Dielectr. Electr. Insul., vol. 12(5), pp. 914-928,2005.

8. Lau, K.Y., Vaughan, A.S., Chen, G. "Nanodielectrics: opportunities and challenges", IEEE Electr. Insul., vol. 31(4), pp.45-54, 2015.

9. Zhang, L., Zhou, Y.X., Tian, J.H., Sha, Y.C., Zhang, Y.X., Wu, H.Z., Wang, Y.S. “Experiment and simulation of space charge suppression in LDPE/MgO nanocomposite under external DC electric field", Journal of Electrostatics, vol. 72, pp. 252-260, 2014.

10. Lau, K.Y., Vaughan, A.S., Chen, G. "Nanodielectrics: opportunities and challenges", IEEE Electr. Insul. Mag., vol. 31(4), pp. 45-54, 2015.

11. Tanaka, T., Montanari, G.C., Mulhaupt, R. "Polymer nanocomposites as dielectrics and electrical insulation-perspectives for processing technologies, material characterization and future applications”, IEEE Trans. Dielectr. Electr. Insul., vol. 11(5), pp. 763-784, 2004.

12. Diaham, S., Zelmat, S., Locatelli, M. "Dielectric breakdown of polyimide films: area, thickness and temperature dependence”, IEEE Trans. Dielectr. Electr. Insul., vol. 17(1), pp.18-27, 2010.

13. Bo Qi, Chunjia Gao, Shun Liu, Linjie Zhao, Chengrong Li. "Surface charge distribution on GIS insulator under DC/AC voltage", IEEE Trans. Dielectr. Electr. Insul., vol. 24(5), pp. 31733181, 2017.

14. Yang, X.S., Zhao, T., Liu, Q.Y., Zhao, K., Wei, Z.T., Zhao, Y. “Transport properties for Bi2Se3/ La0.7Sr0.3MnO3 composites”, Ceramics International, vol. 46(4), pp. 4748-4753, 2020. 


\title{
77 A performance study and PAPR assessment of OFDM system for the diverse modulation schemes
}

\author{
Prabal Gupta ${ }^{1, *}$, H. Pal Thethi $i^{2, *}$ \\ ${ }^{1}$ Department of ECE, SCSE, Lovely Professional University, Punjab, India \\ ${ }^{2}$ Department of ECE, SEEE, Lovely Professional University, Punjab, India
}

\section{Introduction}

OFDM is well known for the high data rates of information in wireless communication system. It is considered as highly spectrum efficient because it provides the orthogonality among the subcarriers; in addition to this, it also has many advantages like diminishing the effect of inter symbol interferences (ISI) and providing a very easy estimation of channel [1]. But it also has the key disadvantage of very high PAPR. OFDM system is the most significant wireless communication system which has been utilized in the long term evolution (LTE), worldwide interoperability for microwave access (WiMAX) (IEEE 802.16) etc. [2]. Numerous methods have been projected to reduce the issue of PAPR; in [3] a modified sequence discrete Hartley transform based system for PAPR reduction and preserving out of band interference $(\mathrm{OBI})$ is proposed.

In [4], the authors focus on the scaled particle swarm optimization which is incorporated in the partial transmitted sequence (PTS).

In [5], a novel PTS technique has been proposed which offers good performance for both PAPR diminishing and bit error rate (BER).

In [6], the authors suggested a technique which is based upon PTS and Bose Chaudhuri Hocquenghem $(\mathrm{BCH})$ codes and it achieves remarkable performance.

In [7], a review of the PTS technique is given. In this study, the PAPR performance and computational complexity level are deliberated.

The organization of the work is that Section 1 is the introduction. Section 2 describes OFDM. The mathematical equations related to OFDM are described in Section 3, followed by simulation results and discussion in Section 4 and finally the conclusion is demonstrated in Section 5.

\section{OFDM}

The simulations of MATLAB are used in order to represent the performance study and PAPR assessment of OFDM system. Figure 77.1 shows the block diagram representation of OFDM system. The transmitter and receiver of OFDM has the following procedures:

1. Information bits of source are modulated by digital modulation scheme (Mod.) like $\mathrm{M}$-ary quadrature amplitude modulation and M-ary phase-shift keying (M-PSK). In

\footnotetext{
* Emails: prabal.gupta9@gmail.com, harpalnitr@gmail.com
} 


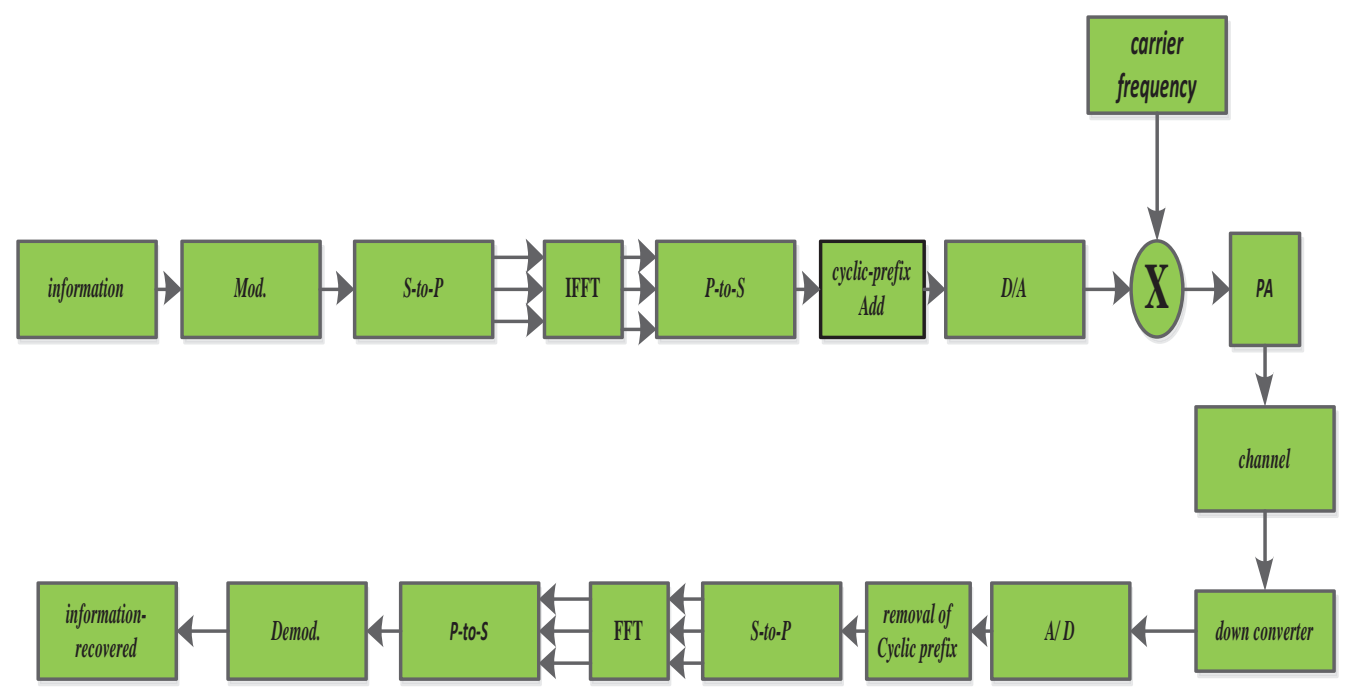

Figure 77.1 Block diagram representation of the OFDM [2].

OFDM modulation is utilized like BPSK,16-QAM, QPSK, 64-QAM and 8-PSK etc. Generally, it has been observed that higher order modulation leads to the improvement in the speed of data rates.

2. After performing the mapping of symbols, the data is converted into parallel with the assistance of serial-to-parallel (S/P).

3. Now, several orthogonal sub-carriers are generated with the help of inverse fast Fourier transform (IFFT), hence symbols can be transmitted easily. There are many sizes of IFFT depicted in the literature like 64, 128, 256, 512, 1024 and 2048. IFFT [8] diminishes the computational complexity in contrast to IDFT (inverse discrete Fourier transform); several DSP (digital signal processing) chips with implementation of both IFFT and FFT are available. Precisely, OFDM actually converts frequency selective-fading channel into numerous flat-fading sub-channels, hence allowing the utilization of very easy frequency domain equalizers, again converting from parallel to serial.

4. Both the inter-symbol interferences (ISI) and inter-carrier interferences (ICI) [8] can be diminished significantly, with the assistance of the guard intervals in between several symbols of OFDM. This particular interval is the cyclic extension of signal and concatenated at the start of symbols of OFDM, known as cyclic prefix (CP).

5. Then a digital to analog conversion (D/A) is applied and multiplied by carrier frequency, passed through a power amplifier (PA) and, finally, through the channel.

At the receiver, all the above- mentioned procedures which are performed at the transmitter will be reversed like the down conversion, including conversion from analog to digital converter (A/D), then cyclic-prefix removal is applied, then serial-to parallel (S/ $\mathrm{P})$, fast Fourier transform (FFT), parallel-to-serial (P/S) and, finally, bits will be obtained from the symbols after applying demapping of the symbols known as digital demodulation (demod.). 


\section{Mathematical equations}

The information $\mathrm{h}[\mathrm{n}]$ after digital modulation passes through IFFT is represented by [6],

$$
\mathbf{h}[\mathbf{n}]=\frac{1}{\sqrt{\mathrm{N}}} \sum_{\mathrm{k}=0}^{\mathrm{k}=(\mathrm{N}-1)} \mathcal{H}[\mathrm{k}] \mathrm{e}^{\frac{(2 . \mathrm{k} . \mathbf{n} . \pi)}{(\mathrm{N})}} \quad 0 \leq \mathbf{n} \leq \mathbf{N}-1
$$

where subcarriers are equated to $\mathrm{N}$ whereas time domain representation is through $\mathrm{n}$ and frequency domain is depicted with the help of $\mathrm{k}$.

\subsection{PAPR}

It is well-demonstrated by the highest power in time-domain to the average-power [6],

$$
\operatorname{PAPR}(\mathbf{h}[\mathbf{n}])=\frac{\max \left(|\mathbf{h}[\mathbf{n}]|^{2}\right)_{0 \leq \mathrm{n} \leq \mathrm{N}-1}}{\mathrm{E}\left(\left.\mathbf{h}[\mathbf{n}]\right|^{2}\right)}
$$

where operator of expectation which is used for average power is E[.].

\subsection{Complementary-cumulative-distribution functions (CCDF)}

It is well-explained by the possibility that PAPR is obtained to be higher in comparison to a particular specific threshold $[1,6]$,

$$
\operatorname{CCDF}\left(\text { PAPR_ }_{-}(\mathbf{h}[\mathbf{n}])\right)=\operatorname{Prb}\left(\text { PAPR_ }_{-}(\mathbf{h}[\mathbf{n}])>\text { Specific threshold }\right)
$$

Specific threshold can also be represented by PAPR0.

\section{Simulation results and discussion}

The modern-day requirement is more information so new wireless applications have been created for the current technologies which ultimately provide high speed data rate transmission along with better utilization of the spectrum. Henceforth, OFDM is the excellent solution for the achievement of this goal. It provides the clear choice for the upcoming high data rate system. Precisely, in the OFDM method, numerous data bits are simultaneously modulated with the assistance of numerous subcarriers. This method divides the entire band of frequency into many smaller sub-bands. OFDM [8] enhances the spectrum efficiency because the sub-bands are orthogonal to each other. This implies that each of the individual sub-bands is independent of the others. It has been determined tha, the PAPR of a discrete time signal which has undergone oversampling provides an accurate approximation [7] of the continuous time signal of OFDM only if the oversampling factor is found to be at least $4(\mathrm{~L} \geq 4)$. In this projected work, we have chosen an oversampling value $\mathrm{L}$ as 4,8 and $\mathrm{N}=32,64,128$, OFDM blocks = 3200, modulation = 8-PSK and 16-PSK. Figure 77.2 to Figure 77.5 depict the comparison of CCDF performance for OFDM under numerous subcarriers, oversampling and modulations. The analysis shows that OFDM at $\mathrm{N}=32$ achieves lowest PAPR and outperforms the OFDM for $\mathrm{N}=64$ and 128. The performance 


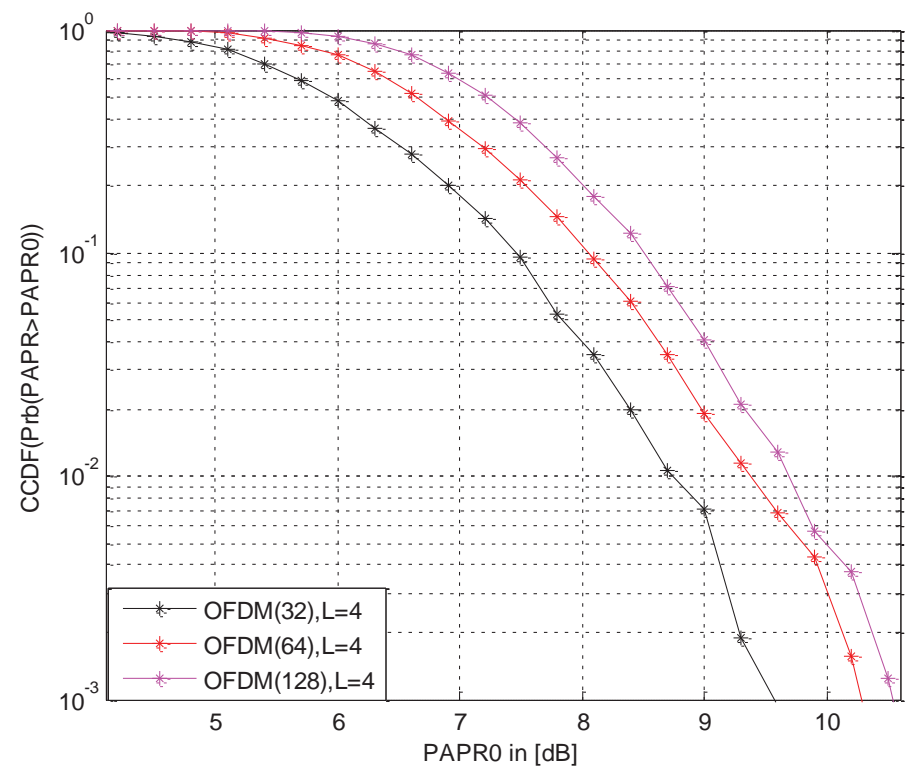

Figure 77.2 CCDF for $\mathrm{N}=32,64,128$ under $\mathrm{L}=4$ and 8-PSK modulation.

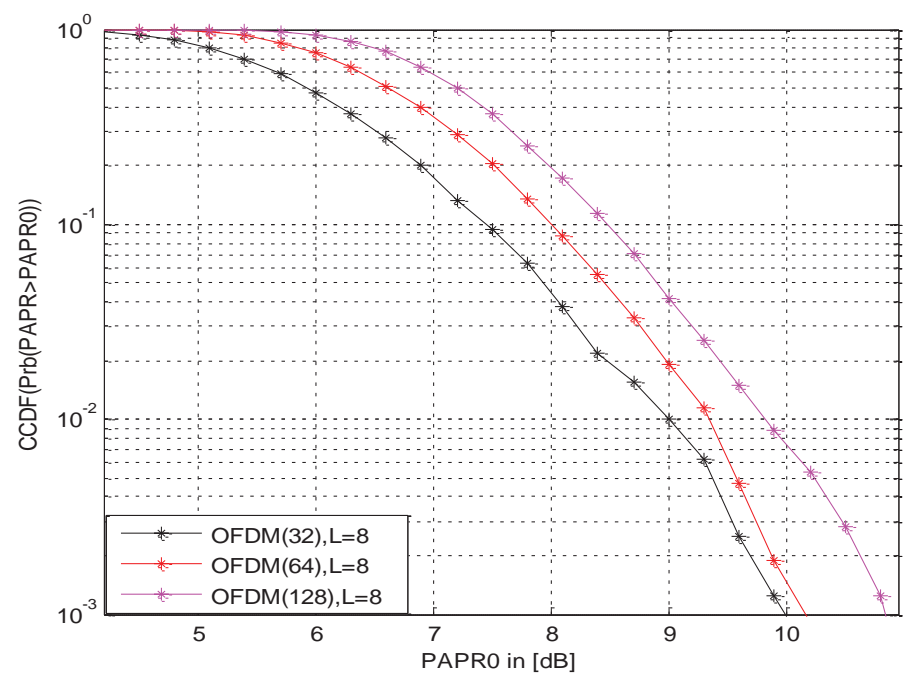

Figure $77.3 \mathrm{CCDF}$ for $\mathrm{N}=32,64,128$ under $\mathrm{L}=8$ and 8-PSK modulation.

enhancement arises from the condition that as the number of subcarriers are increasing so the corresponding increment in PAPR issue is observed. It is obvious from Figure 77.2 that in order to obtain a CCDF $=0.1 \%$, for 8 -PSK and $\mathrm{L}=4$ based OFDM system over $\mathrm{N}=128$ requires the PAPR of $10.50 \mathrm{~dB}$, but this condition falls to $10.30 \mathrm{~dB}$ for an OFDM system over $\mathrm{N}=64$; for an OFDM system over $\mathrm{N}=32$ the PAPR value further falls to 9.6 


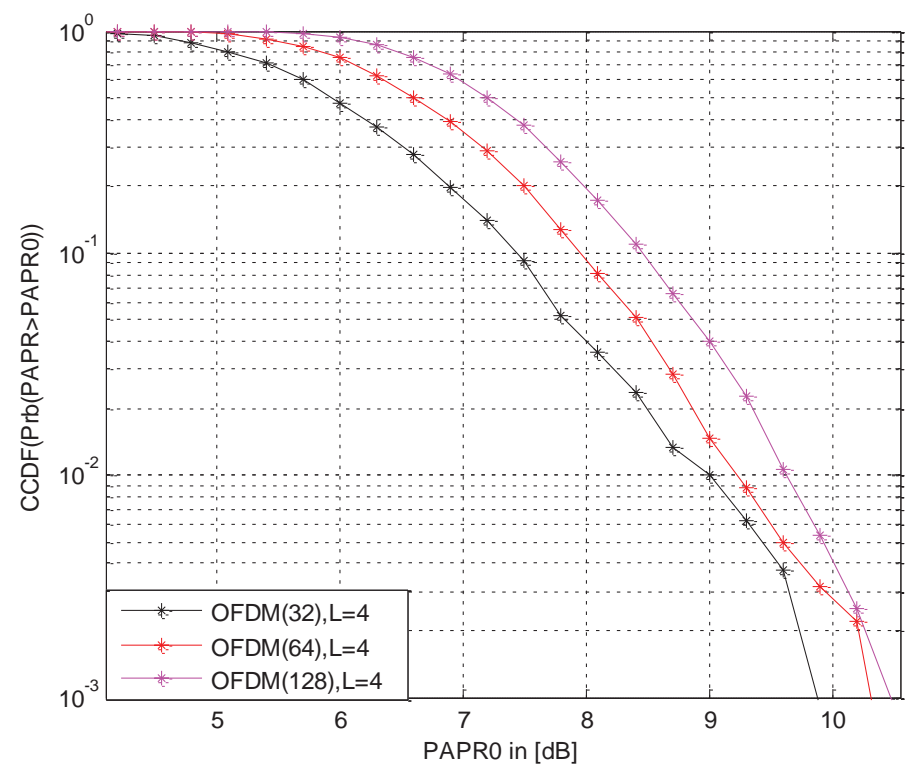

Figure 77.4 CCDF for $\mathrm{N}=32,64,128$ under $\mathrm{L}=4$ and 16-PSK modulation.

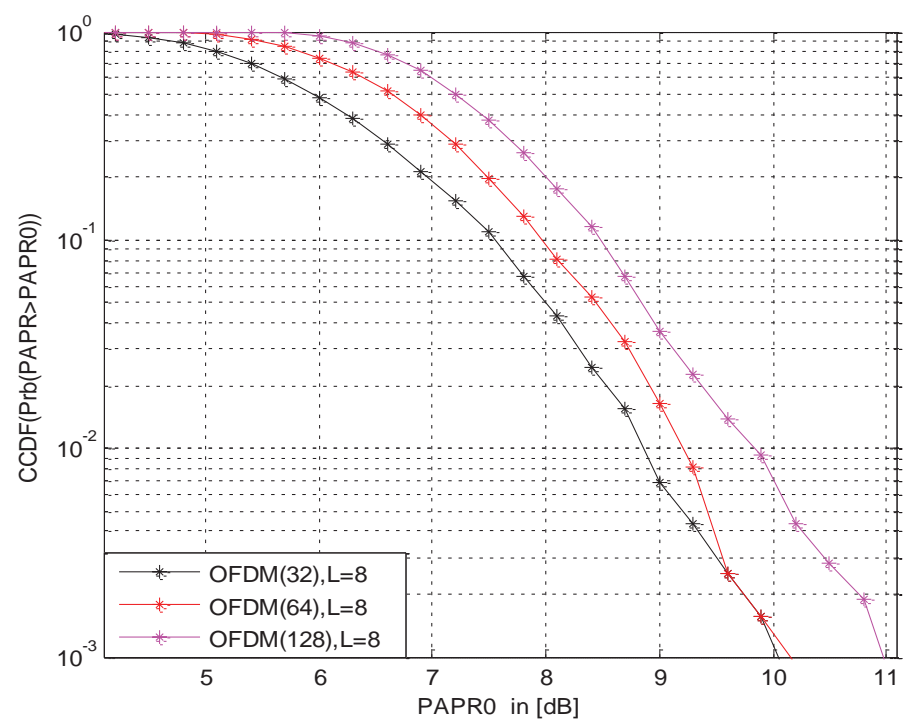

Figure 77.5 CCDF for $\mathrm{N}=32,64,128$ under $\mathrm{L}=8$ and 16-PSK modulation.

dB. Similar observations also have been obtained from Figure 77.3 to Figure 77.5. The values of PAPR $(\mathrm{dB})$ necessary to attain a CCDF $=0.1 \%$ for OFDM system over $\mathrm{N}=128$, 64 and 32 considering diverse numbers of modulation method and oversampling values are depicted in Table 77.1. 
Table 77.1 CCDF performance analysis of OFDM system for diverse modulation methods, subcarriers and oversampling values

$\operatorname{PAPR}(d B)$ to attain $C C D F=0.1 \%$

OFDM system

\begin{tabular}{lllr}
\hline Modulation and oversampling & $N=128$ & $N=64$ & $N=32$ \\
\hline $\mathrm{L}=4$ and 8-PSK & 10.50 & 10.30 & 9.60 \\
$\mathrm{~L}=8$ and 8-PSK & 10.90 & 10.10 & 10.00 \\
$\mathrm{~L}=4$ and 16-PSK & 10.40 & 10.30 & 9.90 \\
$\mathrm{~L}=8$ and 16-PSK & 10.98 & 10.10 & 10.01 \\
\hline
\end{tabular}

\section{Conclusion}

This paper studied and analysed the PAPR for diverse modulation procedures like 8-PSK, 16-PSK, oversampling factor for values 4 and 8 under subcarriers with values like 32, 64 and 128. Performance of the OFDM system at $\mathrm{N}=32$ is better in all the cases because it achieves minimum PAPR in comparison with other subcarriers like 64 and 128 and also the best performance is achieved by OFDM system at $\mathrm{N}=32$ under $\mathrm{L}=4$ and 8 -PSK modulation with the lowest PAPR of $9.60 \mathrm{~dB}$. The most important conclusion which can be drawn from here is that as the $\mathrm{N}$ is increased then a corresponding increment in PAPR is also observed. This can be easily observed from all the figures and Table 77.1.

\section{References}

[1] Gupta, P., R.K. Singh, H.P. Thethi, B. Singh, and S.K. Nanda. 2019. Discrete cosine transform matrix based SLM algorithm for OFDM with diminished PAPR for M-PSK over different subcarriers. Journal of Computer Networks and Communications 2019. https://doi.org/ $10.1155 / 2019 / 2893207$.

[2] Sandoval, Francisco, Gwenael Poitau, and Francois Gagnon. 2017. Hybrid Peak-to-average power ratio reduction techniques: review and performance comparison. IEEE Access 5: $27145-$ 61. https://doi.org/10.1109/ACCESS.2017.2775859.

[3] Satyavathi Kakara and B. Rama Rao 2019. Modified phase sequence in hybrid PTS scheme for PAPR reduction in OFDM systems. Innovations in Electronics and Communication Engineering. Vol. 33. Springer, Singapore. https://doi.org/10.1007/978-981-10-8204-7.

[4] Prasad, Sanjana, and Ramesh Jayabalan. 2019. PAPR reduction in OFDM using scaled particle swarm optimisation based partial transmit sequence technique. The Journal of Engineering 2019(5): 3460-68. https://doi.org/10.1049/joe.2018.5340.

[5] Merah, Hocine, Mokhtaria Mesri and Larbi Talbi. 2019. Complexity reduction of Pts technique to reduce Papr of OFDM signal used in a wireless communication system. IET Communications 13(7): 939-46. https://doi.org/10.1049/iet-com.2018.5705.

[6] Gupta, Prabal, B. Arun Kumar and Sandeep Kumar Jain. 2015. Peak to average power ratio reduction in OFDM using higher order partitioned PTS sequence and Bose Chaudhuri Hocquenghem codes. In International Conference on Signal Processing and Communication Engineering Systems - Proceedings of SPACES 2015, in Association with IEEE, 443-47. https://doi.org/10.1109/SPACES.2015.7058303.

[7] Jawhar, Yasir Amer, Lukman Audah, Montadar Abas Taher, Khairun Nidzam Ramli, Nor Shahida Mohd Shah, Mustafa Musa and Mustafa Sami Ahmed. 2019. A review of partial transmit sequence for PAPR reduction in the OFDM systems. IEEE Access 7: 18021-41. https://doi.org/10.1109/ACCESS.2019.2894527.

[8] Rahmatallah, Yasir, and Seshadri Mohan. 2013. Peak-to-average power ratio reduction in Ofdm systems: a survey and taxonomy. IEEE Communications Surveys and Tutorials 15(4): 1567-92. https://doi.org/10.1109/SURV.2013.021313.00164. 


\title{
78 Power system security of distributed network using contingency analysis
}

\author{
Mohammad Imran Mir ${ }^{1, *}$, Amit Kumar Singh ${ }^{2, *}$, \\ Muzafar Ahmad Shah,*
}

\author{
${ }^{1}$ Research Scholar, Department of Electrical and Electronics Engineering, \\ Lovely Professional University, Jalandhar, Punjab, India \\ ${ }^{2}$ Associate Professor, Department of Electrical and Electronics Engineering, \\ Lovely Professional University, Jalandhar, Punjab, India \\ ${ }^{3}$ Research Scholar, Department of Electrical and Electronics Engineering, \\ Lovely Professional University, Jalandhar, Punjab, India
}

\section{Introduction}

Effective (active) and reactive energy flows from the power plant via different network buses and divisions in all three-phase ac power systems. The effective and reactive energy flow is defined as load flow or power flow. Energy flow evaluation is used to assess a power system's continuous working state. During the design and maintenance of the distribution system, power flow modelling is commonly used by power distribution practitioners. [1]. Power flow evaluation is used to assess a power system's stable operation. In preparation and operation of the power distribution network, the power flow evaluation is commonly used by power distribution specialists. In order to deal with uncertainty, load flow analyses can be split into deterministic load flow and uncertain load flow. The deterministic load flow study would not consider the uncertainty sparked by both power and load comportments. There are many strategies to take into account the uncertainties, like probabilistic, possibilities, decision theories of information gaps, solid optimization and interval testing. In the case of power system engineers, a load flow analysis describes the conditions of the power system at different operating periods. This seeks to minimize the disparity between the quantities measured $[2,3]$.

$$
\begin{aligned}
& P i=P G i-P L i \\
& \Delta P i=P i-P i, \text { calc } \\
& \Delta Q i=Q i-Q i, \text { calc }
\end{aligned}
$$

\section{Power system security}

The security of the network system ensures that the electrical system can withstand unexpected disturbances, such as electrical short circuits or accidental loss of system elements of the power system. The most significant concern is the economic operation of each

\footnotetext{
*Emails: mirimraniitk@gmail.com, amit.20267@lpu.co.in, shahaatiif@gmail.com
}

DOI: $10.1201 / 9781003129103-78$ 


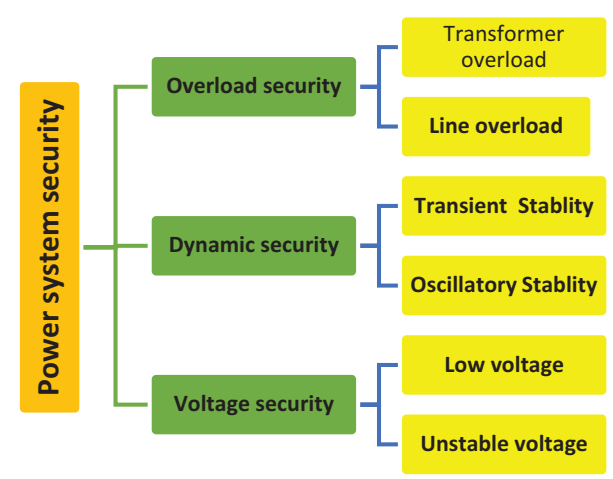

Figure 78.1 Types of power system security.

power system. Security systems require strategies designed to keep the network running if components collapse. One with a small chance of failure or infrastructure loss is a strategically secure power network. If the system failure pattern continues, it can crash entirely or its major components. The ultimate economic objective is therefore the safe function of the utility. The network is to be run at minimal cost, with assured emergency condition reduction, by the energy management system (EMS). Contingencies are responsible for most breaches. A certain system has been designed to be protected only for one or even more definite cases of contingency, and a certain set of amounts assessed for system breaches.

The power system security evaluation involves main three steps.

(a) Monitoring of the system.

(b) Analysis of contingency.

(c) Counteractive action analysis.

\section{(a) Monitoring of the system}

This provides information to the power system operators or dispatches up-to-date information in real time on the conditions of the power system as load and generation shifts, including data monitoring and transmission in all substations in the transmission network. Telemetric devices calculate, track and transmit information, voltage, currents, and circuit breaker status. In addition, other vital and significant data, for example frequency, output of generators and position of taps in transformers, can be remotely assessed.

\section{(b) Analysis of contingency}

The assessment of contingency is mainly a tool for "preview" analysis. The outcomes of problems that may happen in the near future in the power system are simulated and evaluated. Contingency analysis is used as an off-line analysis tool for contingency incidents and as an on-line tool to illustrate the consequences of potential outages to operators. This makes it possible for operators to respond more strongly to failures using previously designed retrieval scenarios. The contingency programs are housed in current operating 
computers. These anticipate potential system problems before they take place. They investigate failures and alert operators to possible overloads or severe voltage infringements. This means an emergency identification analysis and "what if" simulations can be carried out $[3,5,6]$.

\section{(c) Counteractive action analysis}

It allows the controller to alter procedure if, in the event of a certain breakdown, a contingency analysis program anticipates a serious problem. This therefore provides precautionary and post-emergency control

\section{Contingency selection}

To mitigate the above problem, the contingency testing or contingency selection process is used. Practically, any overload or overvoltage is caused in all possible failures on other power system equipment. The selection of contingencies is called the process for determining contingencies which lead to a violation of the operating limits. The standard power flow algorithms for individual contingencies will measure these indices in offline mode [4-6].

\subsection{Concept and algorithm of contingency analysis}

The current approach arranges the buses and network branches in homogeneous groups, depending on true power and reactive flow in the branches from a resolved power flow or state approximation estimate, according to some of the following principles. When this organization has been accomplished, questions such as: "How far is this unit's power? Or "which generators provide this load?" A line parameterized chart can also display the state of the network. Finding some answers to questions such as "How much has the generator used this line?" or "What's the proportion of losses in a system generated? "Well into the past there were several frequent outages in the integrated power system. Thus, power systems ought to be run more cost-effectively so that power is distributed securely. Reliable service ensures that sufficient power is generated and can be transmitted to users efficiently. The majority of power plants are built to handle all key failure incidents with plenty of redundancy. Two key loss incidents explore potential consequences and solutions in this regard: line blackouts and device malfunction generation. The line or system failure that causes line flows or voltages to exceed the limit is specifically established [7-9]. There were several frequent outages in the past in the integrated electricity system. Thus, it must be made sure that power systems are operated more economically to guarantee a reliable delivery of power. A reliable unit means a sufficient generation of power and it can efficiently be transferred to the loads. Many power systems have been developed with sufficient redundancy to support all main fail activities. The potential consequences and corrective action of two key failure incidents have been examined here: line interruptions and failure of generation. It should be noted that $\mathrm{N}-1$ contingency analysis has been done on a 12-bus system [10-12].

The single line diagram of the 12-bus system with data given at each bus is shown in Figure 78.2. 
Table 78.1 Shows power system generator data for contingency analysis

\begin{tabular}{lllll}
\hline Gen no. & Bus type & Voltage $(k V)$ & $\begin{array}{l}\text { Apparent power } \\
\text { (MVA) }\end{array}$ & $\begin{array}{l}\text { Real power } \\
\text { p(MW) }\end{array}$ \\
\hline 1 & P, $\delta$ & 66 & 470.60 & 400 \\
2 & PQ & 66 & 353 & 300 \\
3 & PQ & 66 & 294.12 & 250 \\
\hline
\end{tabular}

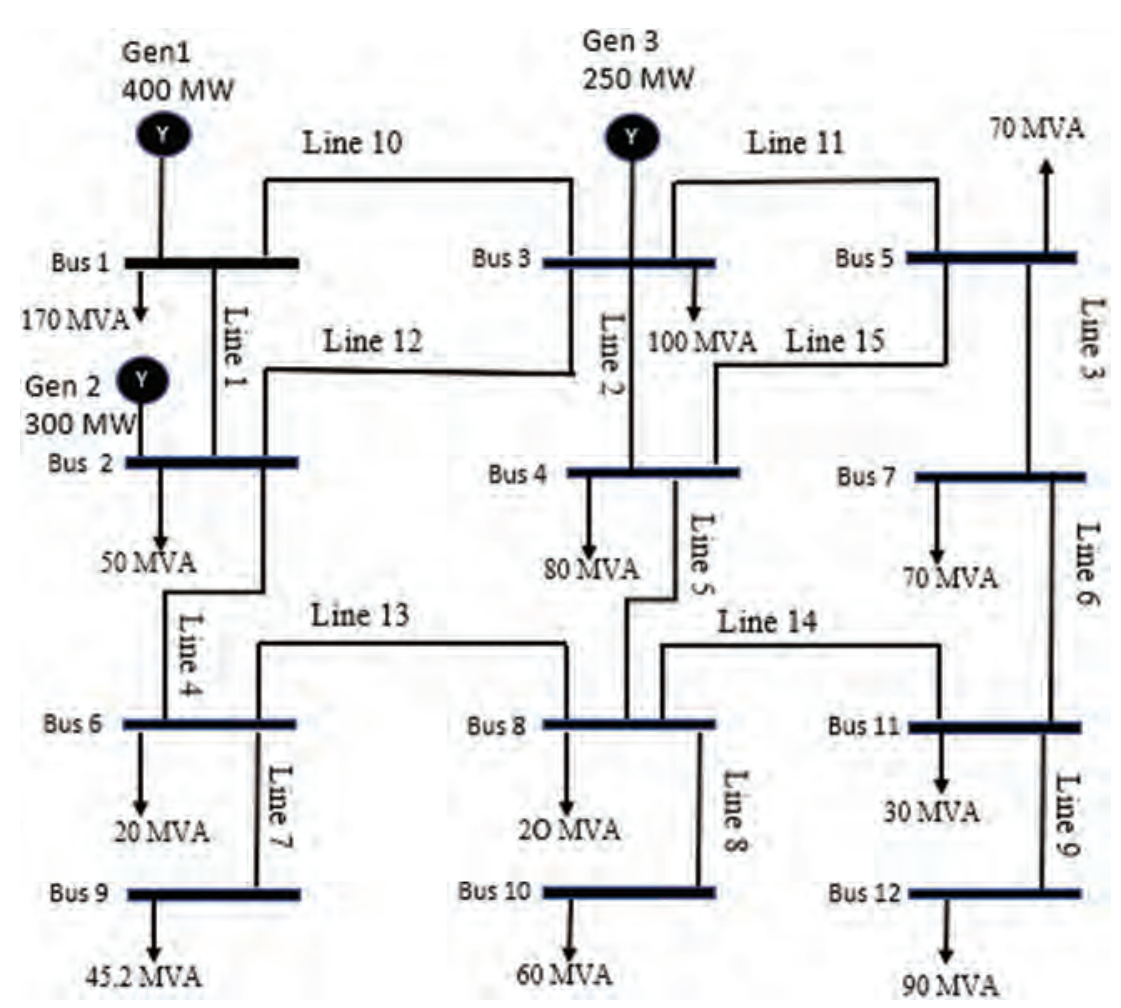

Figure 78.2 Shows the single line diagram of the 12-bus system.

\section{Results}

The results obtained from the evaluation of contingency analysis are given in Figure 78.3 and Table 78.3.

The graph shown in Figure 78.3 clearly illustrates the effect of outages on each line. When the outage takes place some of the lines are violating the limits and therefore necessary action should be taken against such violations otherwise it could lead to the system insecurity. The analysis has been done on the basis of MW violation, e.g. the orange line in the graph shown in Figure 78.3 depicts that when the line 10 which is between bus1 and bus 3 goes out of the system, it results in the increase in the power flow at other buses which cause the limit violation on some of the lines. Similarly, the effect of each outage on the overall system has been observed and results are shown in the graph of Figure 78.3 as well as in Table 78.3. 
Table 78.2 Shows distribution system 12-bus branch data for $66 \mathrm{kV}$

\begin{tabular}{ccllll}
\hline From bus & To bus & $X_{P U}$ & $R_{P U}$ & $X^{1}$ & $B / 2$ \\
\hline 1 & 2 & 0.1126 & 0.01840 & 1 & 0 \\
1 & 3 & 0.1876 & 0.0314 & 1 & 0 \\
2 & 3 & 0.16 & 0.026 & 1 & 0 \\
2 & 6 & 0.0376 & 0.0064 & 1 & 0 \\
3 & 4 & 0.076 & 0.0 .126 & 1 & 0 \\
3 & 5 & 0.1126 & 0.0188 & 1 & 0 \\
4 & 5 & 0.16 & 0.026 & 1 & 0 \\
4 & 8 & 0.1126 & 0.0188 & 1 & 0 \\
5 & 7 & 0.1126 & 0.0188 & 1 & 0 \\
6 & 8 & 0.076 & 0.0126 & 1 & 0 \\
6 & 9 & 0.0376 & 0.0064 & 1 & 0 \\
7 & 11 & 0.076 & 0.0126 & 1 & 0 \\
8 & 10 & 0.0376 & 0.0064 & 1 & 0 \\
8 & 11 & 0.1126 & 0.0188 & 1 & 0 \\
11 & 12 & 0.076 & 0.0126 & 1 & 0 \\
\hline
\end{tabular}

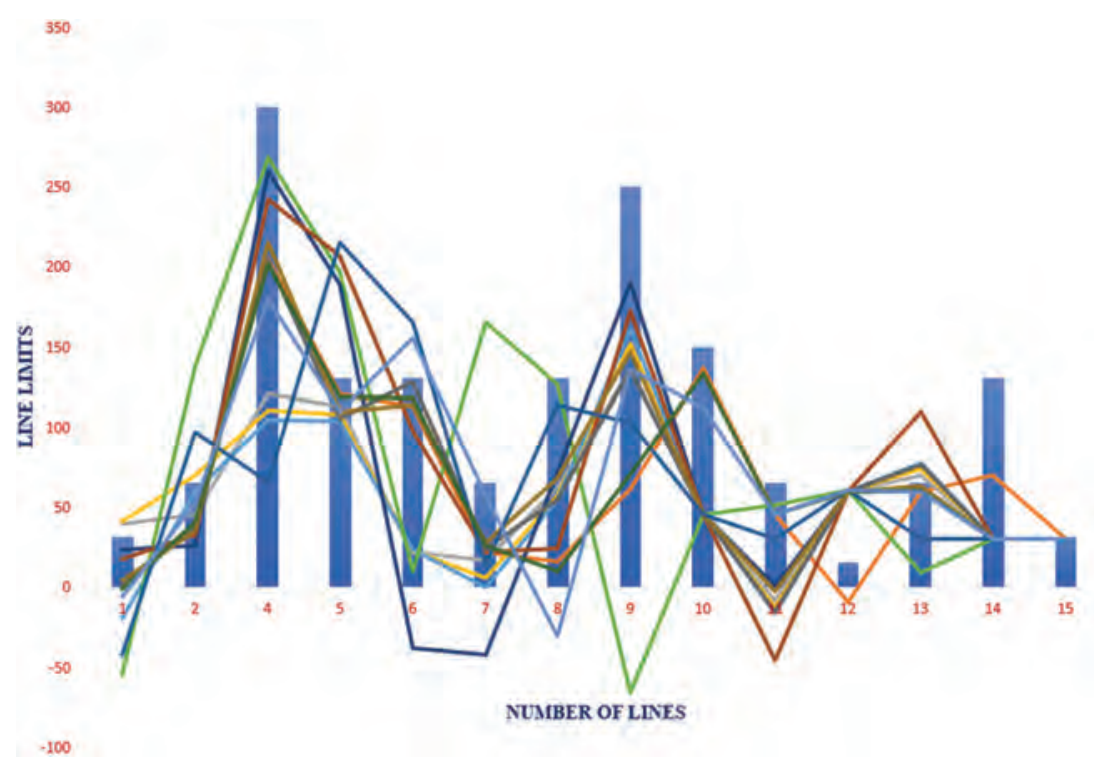

Figure 78.3 Security constraint contingency analysis.

\section{Conclusion}

The N-1 contingency analysis has been carried out on a 12-bus system. It has been observed that while performing the $\mathrm{N}-1$ contingency analysis in which the effect of each outage is observed one by one, the lines 2, 5, 6, 12 and 13 are breaching the limits. These lines are triggering the network imbalance. A further aspect has always been that lines 12 and 13 are the most sensitive lines in the system, creating considerable chaos for all buses. Safeguarding of lines 12 and 13 is therefore the most influential factor compared with others. 
Table 78.3 Outage-based contingency analysis

\begin{tabular}{|c|c|c|c|c|c|c|c|c|c|c|c|c|c|c|}
\cline { 2 - 12 } \multicolumn{1}{c|}{} & 1 & 2 & 4 & 5 & 6 & 7 & 8 & 9 & 10 & 11 & 12 & 13 & 14 & 15 \\
\hline - limits & 32 & 65 & 300 & 130 & 130 & 65 & 130 & 250 & 150 & 65 & 16 & 65 & 130 & 32 \\
\hline - No outage & 0.11 & 39.24 & 205.11 & 120.36 & 112.71 & 21.93 & 16.40 & 61.82 & 136.86 & 45.34 & -9.03 & 60.27 & 70.27 & 30.14 \\
\hline - Outage 1-2 & 39.61 & 45.98 & 121.03 & 113.37 & 21.93 & 17.02 & 62.42 & 135.71 & 45.35 & -8.46 & 60.28 & 69.71 & 30.14 & 30.14 \\
\hline - Outage 1-3 & 41.24 & 69.79 & 110.74 & 107.67 & 22.84 & 6.08 & 57.79 & 152.10 & 45.35 & -12.99 & 60.29 & 74.46 & 30.15 & 30.14 \\
\hline - Outage 2-3 & -19.31 & 60.31 & 104.78 & 104.19 & 23.32 & -0.14 & 54.96 & 161.47 & 45.35 & -15.74 & 60.28 & 77.28 & 30.14 & 30.14 \\
\hline - Outage 2-6 & -55.03 & 137.14 & 268.16 & 196.73 & 10.16 & 165.00 & 126.51 & -65.46 & 45.46 & 51.51 & 60.44 & 9.36 & 30.22 & 30.14 \\
\hline - Outage 3-4 & 23.81 & 26.21 & 260.24 & 188.71 & -37.76 & -42.24 & 71.96 & 189.53 & 45.36 & 0.60 & 60.33 & 60.54 & 30.17 & 30.14 \\
\hline - Outage 3-5 & 17.56 & 32.62 & 242.43 & 206.73 & 97.83 & 22.17 & 24.39 & 172.58 & 45.36 & -45.77 & 60.31 & 109.48 & 30.17 & 30.14 \\
\hline - Outage 4-5 & -0.10 & 39.80 & 204.11 & 105.78 & 128.59 & 24.22 & 55.09 & 135.89 & 45.34 & -15.59 & 60.27 & 77.09 & 30.14 & 30.14 \\
\hline - Outage 4-8 & 3.84 & 35.78 & 215.36 & 109.56 & 113.74 & 27.89 & 68.67 & 146.79 & 45.35 & -2.38 & 60.28 & 63.42 & 30.14 & 30.14 \\
\hline - Outage 6-8 & -41.81 & 97.39 & 65.61 & 215.21 & 165.76 & 14.07 & 113.83 & 103.26 & 45.33 & 30.47 & 60.34 & 30.13 & 30.17 & 30.14 \\
\hline -Outage 7-11 & -1.16 & 40.59 & 201.41 & 118.80 & 117.93 & 26.34 & 10.48 & 71.13 & 133.27 & 45.34 & 60.27 & 60.96 & 30.14 & 30.14 \\
\hline - Outage 8-11 & -5.77 & 52.71 & 179.88 & 109.46 & 155.25 & 57.66 & -29.99 & 135.93 & 112.29 & 45.34 & 60.92 & 60.27 & 30.18 & 30.14 \\
\hline
\end{tabular}

\section{References}

[1] C.L. Wadhwa, Load flows, Chapter 18. In Electrical Power Systems. New Academic Science, 2017.

[2] Idoniboyeobu and Udoha, D.C., "A comparative power flow analysis of Dumez $11 \mathrm{kv}$ distribution network in Nigeria," American Journal of Engineering Research (AJER), vol. 6, no. 12, pp. 9, 2017.

[3] ] R. Bulbul, C.-W. Ten and A. Ginter, "Cyber-contingency evaluation for multiple hypothesized substation outages," in Proc. IEEE PES Innovative Smart Grid Technologies, Feb. 1922, 2014.

[4] ] N. M. Mahdi, "Power flow analysis of Rafah Governorate distribution network using ETAP software," International Journal of Physical Sciences, vol. 1, p. 8, 2013.

[5] B. M. Weedy, Electric Power Systems. 5th edn. Chichester,: John Wiley \& Sons, 2012.

[6] K. Radha Rani, J. Amarnath and S. Kamakshaiah, "Contingency analysis under deregulated power systems”, ICGST-ACSE Journal, vol. 11, no. 2, 2011.

[7] T. Guler, G. Gross, M. Liu, "Generalized line outage distribution factors", Power Systems, EEE Trans., vol. 22, no. 2, 2007, pp. 879-881.

[8] I.J. Nagrath and D. P. Kothari, Power system security, Chapter 13. In Modern Power system analysis. Tata McGraw-Hill Education, 2003.

[9] Acharya, N., Mahat, P., and Mlthulananthan, N., "An analytical approach for DG allocation in primary distribution network", International Journal of Electrical Power and Energy Systems, vol. 28, no. 10, pp. 669-678, 2006.

[10] K. Bhattacharya, M. Bollen and J. Daalder, Operation of Restructured Power Systems. Kluwer Academic Publishers, 2001.

[11] R. Bacher, "Graphical interaction and visualization for the analysis and interpretation of contingency analysis results", Proceedings of IEEE Power Industry Computer Application Conference, 1995, pp. 128-134, 1995.

[12] M.K. Enns, J.J. Quada and B. Sackett, "Fast linear contingency analysis", IEEE Trans. On PAS, vol. PAS101, No. 4, pp. 783-791, 1982. 


\title{
79 4K HDMI USB3 capture card reference design
}

\author{
Yashwant Reddy Keesara ${ }^{1, *}$, Rashi Vatsa, *, \\ Someet Singh $h^{3, *}$
}

${ }^{1}$ Department of Electronics and Communication Engineering, Lovely

Professional University Jalandhar, India

${ }^{2}$ Electronics and Communication, Institute of Technology, Nirma University, Abmedabad, India

${ }^{3}$ Department of Electronics and Communication Engineering, Lovely

Professional University, Jalandhar, India

\section{Introduction}

When using most high-end modern cameras, having HDMI/MHL OUT ports, to record high resolution videos, there is a constant need to swap the SD/memory cards in them and this makes it quite hard to use them as sources for real-time streaming or displaying the videos over a host computer or PC because of the unavailability of HDMI input ports on the PC. These cameras can be used to stream the videos in real-time over to an HDMI enabled display but that will only enable a person to visualize the video over a display with no room for editing or processing the video stream on the fly. This gives the need to develop a design that can act as a bridge between the HDMI OUT port of the camera (source) and the host controller (PC).

IHS Markit is an organization which conducts different surveys. One of the surveys about wired connectivity is carried out by the organization which reports as shown in Figure 79.1 [1].

The following results were concluded from the Markit survey:

- HDMI, Display Port and MHL are most popular wired video and audio interface technologies.

- HDMI has biggest base of all wired video interface technologies. It is the most popular interface in digital and smart televisions, HD and UHD set-top boxes, Blu-ray players/ recorders.

- Display Port has become a more popular solution across the PC and the mobile phone segment. The release of alternate mode (Alt Mode) specification has changed the scenario.

- MHL is the only wired interface supported by mobile phones but with Type-C USB dynamics have changed.

Cypress Ez-USB FX3 [2] is a superspeed peripheral controller which is a USB3.1 Gen1 compliant device and can be used to add USB 3.0 functionality to a device. It also provides a powerful, integrated and fully configurable GPIF II interface for connecting devices like ASIC, FPGA seamlessly with configurable data lanes.

\footnotetext{
* Emails: yashwantreddy143@gmail.com,18mece17@nirmauni.ac.in, someet.17380@lpu.co.in
}

DOI: 10.1201/9781003129103-79 


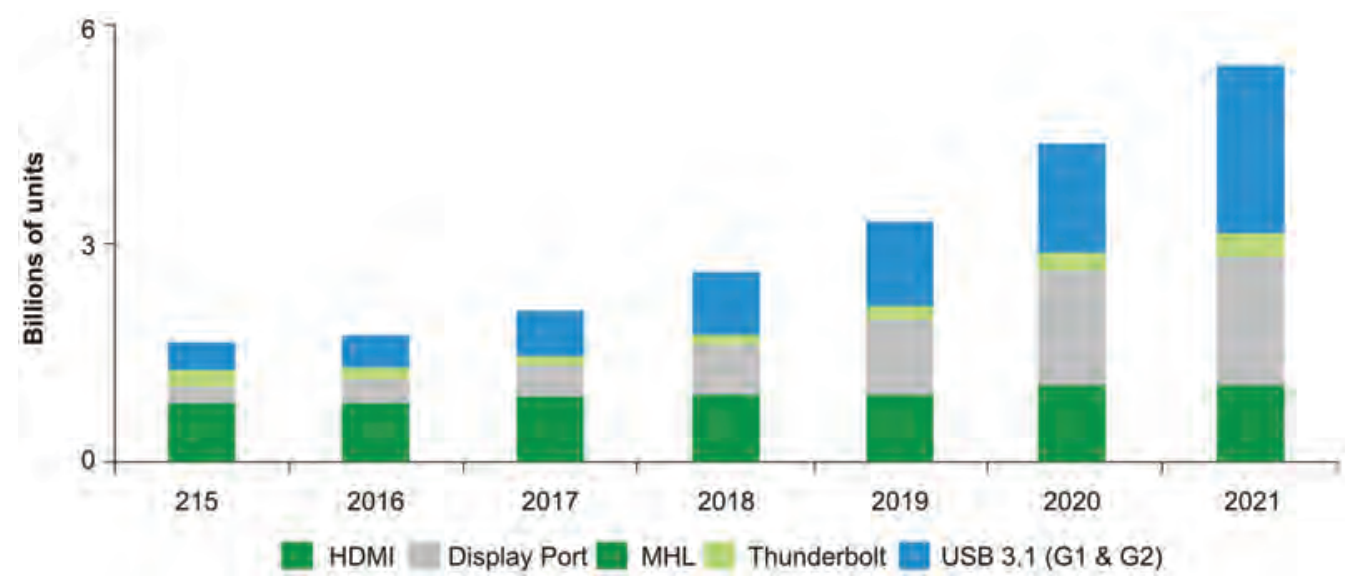

Figure 79.1 Wired Markit survey [1].

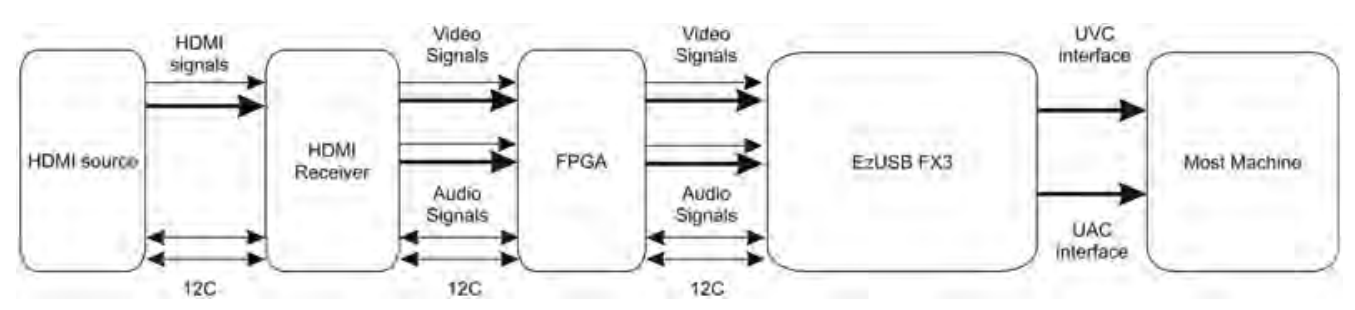

Figure 79.2 System level block diagram.

The mentioned USB 3.0's application is compatible with HDMI 1.4 and MHL 2.1 sources which enable streaming high data rate audio and video signals to a USB host machine. HDCP (high-bandwidth digital content protection) [3] protected HDMI signals are received by the HDMI receiver and are then modified to achieve maximum bandwidth of GPIF II interface and this data is transferred over DMA channels internal of FX3 to the USB host. Since the application is a composite USB device with UAC (universal audio class) [4] and UVC (universal video class) [5] device class interface, it reduces the device development time as OS platforms have support for native UAC and UVC drivers. The system level block diagram of the design is shown in Figure 79.2.

\section{Methodology}

The reference design acts as a base for an industrial camera and USB3 Vision applications using Cypress EZ-USB FX3 SuperSpeed USB Controller and ITE IT6801FN: single port MHL2.1/HDMI1.4 receiver with 3D support and Lattice ECP5 FPGA with HelionVision ISP IP.

This paper aims to provide the architectural, functional and design details of the reference design for 4K HDMI USB3 vision camera systems. The reference design aims to demonstrate a combined implementation of using FX3 and Lattice ECP5 FPGA to interface an HDMI camera along with an HDMI receiver module to a USB host system. The architectural block diagram of the reference design is shown in Figure 79.3. 


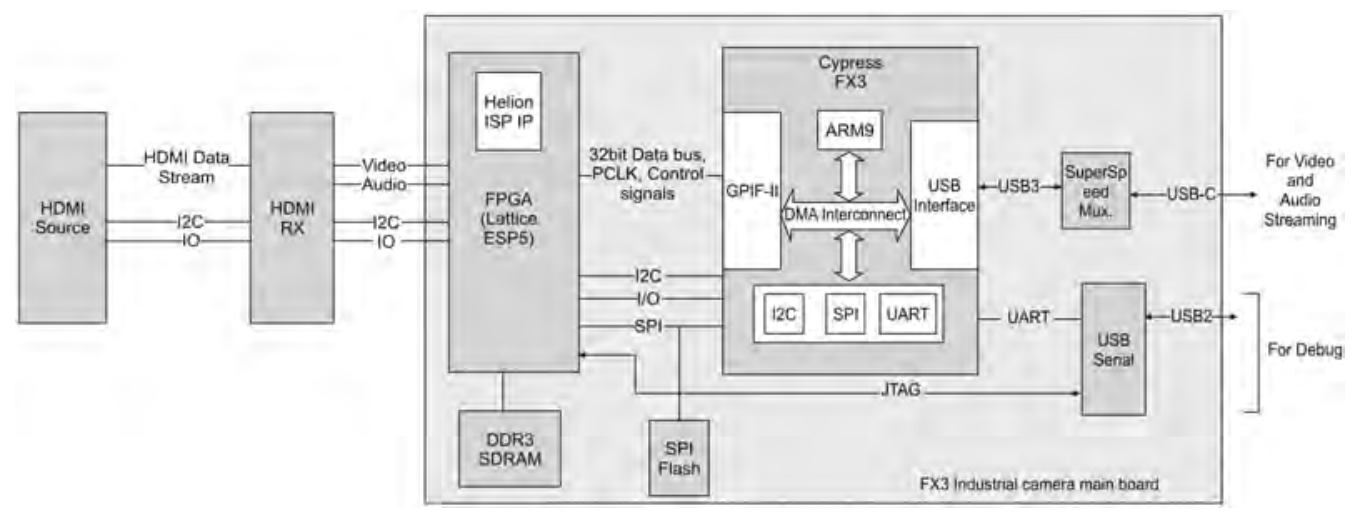

Figure 79.3 Architectural block diagram.

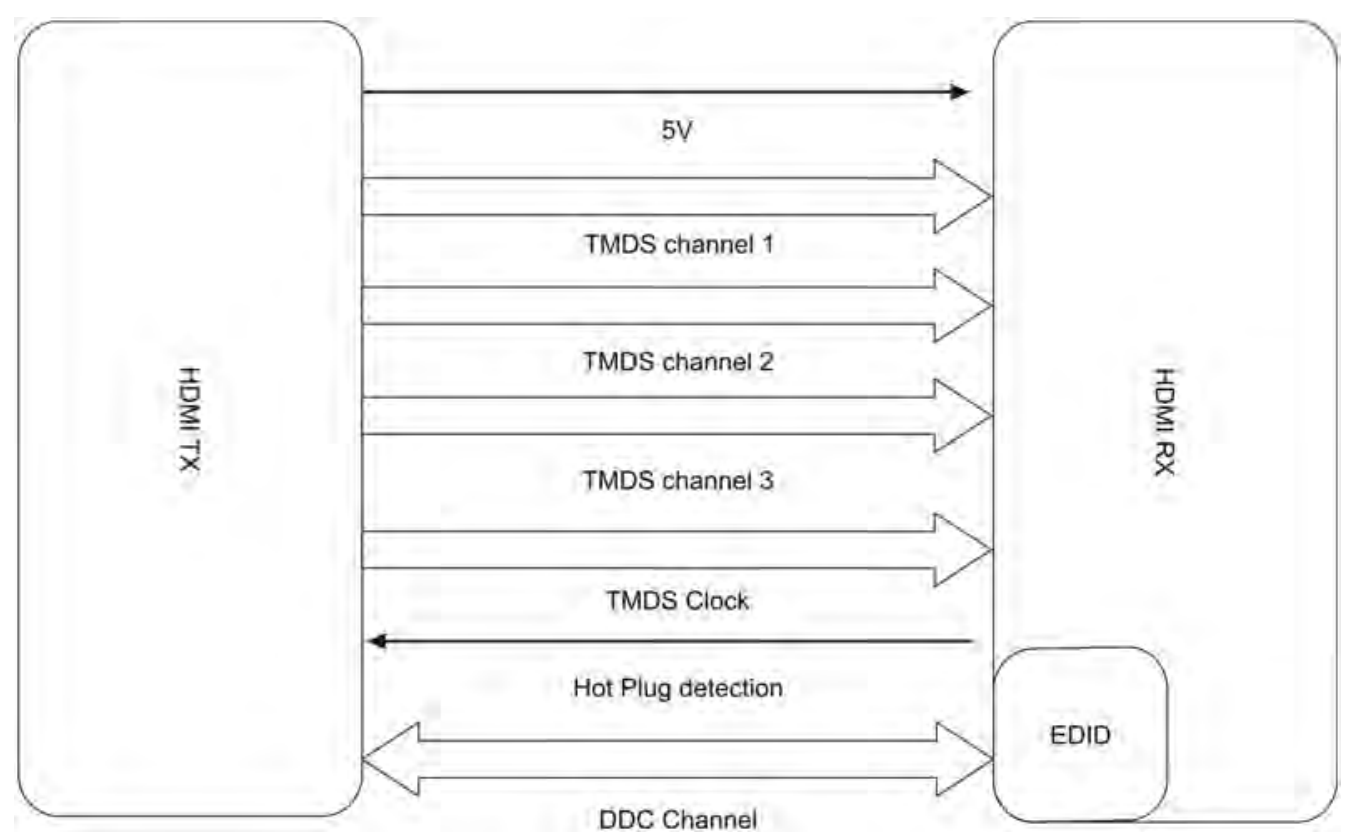

Figure 79.4 HDMI transmitter and receiver connections.

An image sensor with an LVDS (low-voltage differential signalling) or a parallel interface acts as a hardware source for both image and audio data. These are transported in the form of the differential voltage pulses over a 16-bit parallel interface between a defined voltage range from the image sensor to the HDMI receiver. The communication [3] between an HDMI Tx (transmitter) and an HDMI Rx (receiver) is as shown in Figure 79.4.

The HDMI receiver takes in the parallel data stream containing audio and video signals in one stream and separates them into individual audio and video differential signals and acts as a bridge between the video and audio signals from the image sensor module and the ISP (image signal processor). 


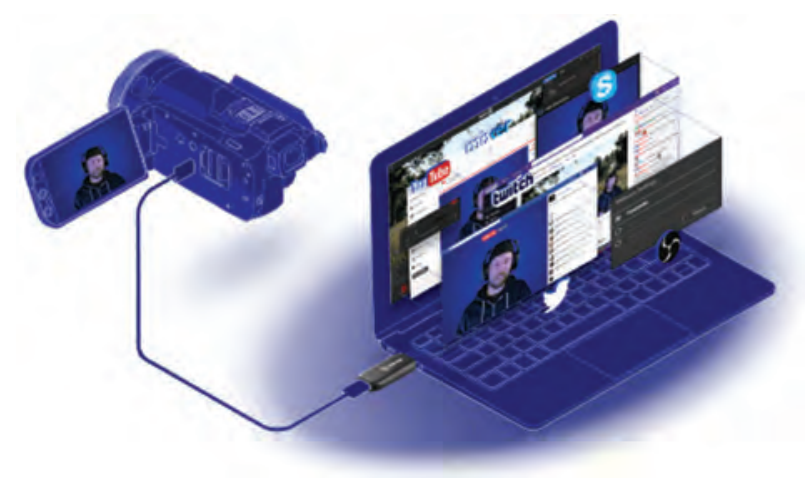

Figure 79.5 Use case [8].

The HelionVision ISP IP is used to process the image data and stream it over the GPIF-II (general programmable interface, generation II) and is implemented on the Lattice ECP5 FPGA to be able to carry out the image processing and to enable the interfacing of LVDS or parallel interface image sensors to the host system. The Lattice ECP5 FPGA is responsible for taking the individual data streams of audio and video from the HDMI receiver and then processing them to attain the maximum bandwidth supported by the GPIF-II interface of FX3 by padding the video data and sending the audio data over the GPIF interface when there is a blanking time of the image data; i.e., when the image data is not being sent, audio data is sent by the receiver over the same interface which can be recognized by the GPIF-II state machine that runs on FX3 and sends the audio data over a separate channel to the host. The FPGA also has a DDR3 controller which is used for colour conversion in the case of ultra-HD resolutions. One of the use cases of such a design is shown in Figure 79.5.

\section{Implementation}

The FX3 firmware is developed in such a way that all the blocks, FPGA, HDMI Rx and the HDMI image sensor, are configured over I2C before the device enumerates as a composite UVC and UAC device. The configuration register files are compiled with the firmware of FX3 and are sent one after the other configuring each block and on completion, passing on to the next block. Once all the blocks are configured, FX3 sends the USB descriptors to the host, which makes it enumerate as a composite device with UAC and UVC interfaces to be able to stream audio and video to host applications.

The interface between FX3 and FPGA is that of a SlaveFIFO [6] wherein the FPGA acts as master and provides clock and data to FX3 and this data is then sent to the host via DMA channels inside the FX3 through the USB interface. FX3 has an internal state machine that configures it to act as a slave based on the application note and accept the data that the master provides and pass it to the host, and the same happens over FPGA where FPGA has a master state machine based on the AN87216 [7] by going on with a 32-bit data bus and clock and some control signals that are used to control the data acquisition.

The steps done by each block are as follows:

1. Audio and video data stream acquisition from HDMI image sensor to HDMI Rx. 
The HDMI image source (Tx) follows the HDMI/MHL specification and sends the image data and audio data in a single stream over the TMDS (transition minimized differential signalling) [3], channel which improves the signal integrity by reducing the number of transitions that the signals need to go through in the cable.

The TMDS on HDMI mixes the video, audio and auxiliary data using three packet types:

- Video data period: the pixels of video line are transmitted.

- Data island period: the audio and auxiliary data is sent during the horizontal and vertical blanking intervals.

- Control period: this occurs between the video and data island periods.

2. Processing done in HDMI Rx and passing on data to FPGA.

HDMI Rx is responsible for the following functions:

- Separating combined audio and video data received from the HDMI image sensor $(\mathrm{Tx})$ to individual video and audio data streams.

- Passing on the individual video and audio data streams along with a PCLK for synchronous transfer by generating HSYNC, VSYNC which acts as the horizontal lines and vertical columns for the pixel placement in video data and sending as a frame whereas audio is sent over its I2S (Inter-IC Sound) transmitter interface to the FPGA.

The interface diagram between HDMI Rx and FPGA is shown in Figure 79.6.

3. Processing done on FPGA and the interface between FPGA and FX3's GPIF-II interface.

The main function of FPGA is checking the video streaming resolution from internal registers and based on the value, it does the following:

- If resolution is 1080p and lower, two 16-bit frames of data are packed and sent over GPIF as a single packet of 32-bit to the FX3 in order to utilize the maximum bandwidth supported by the GPIF-II interface of FX3 as shown in Figure 79.7.

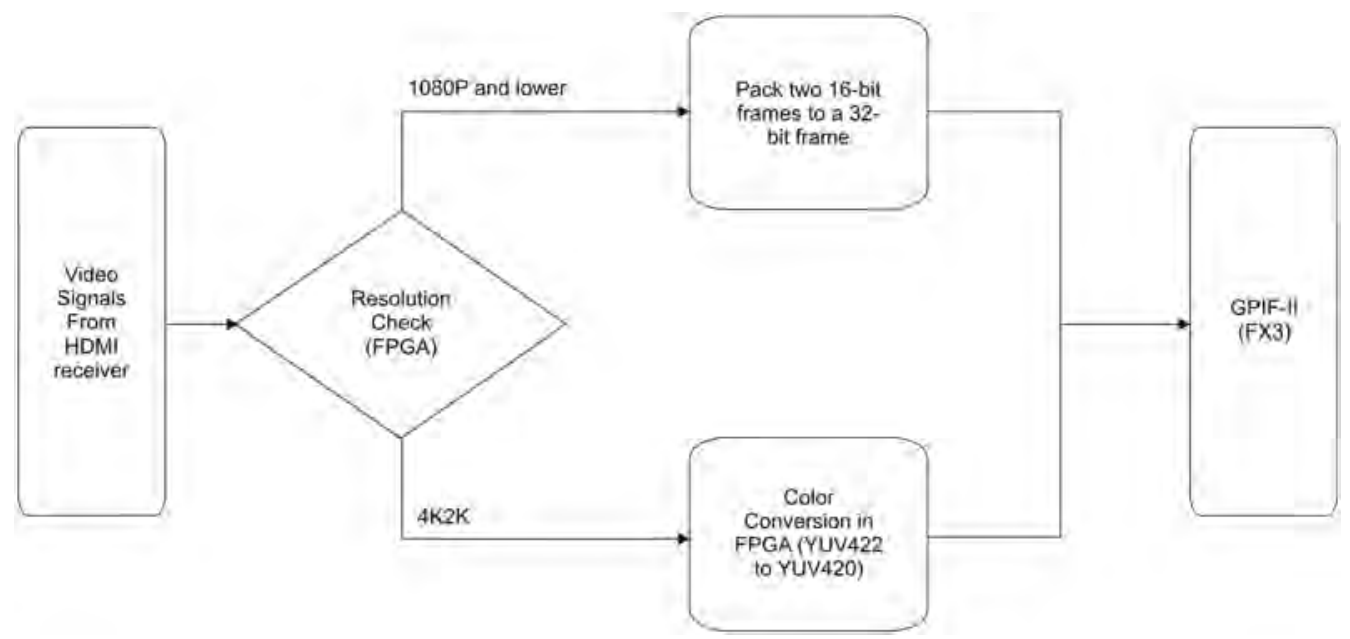

Figure 79.6 HDMI Rx and ECP5 connections. 


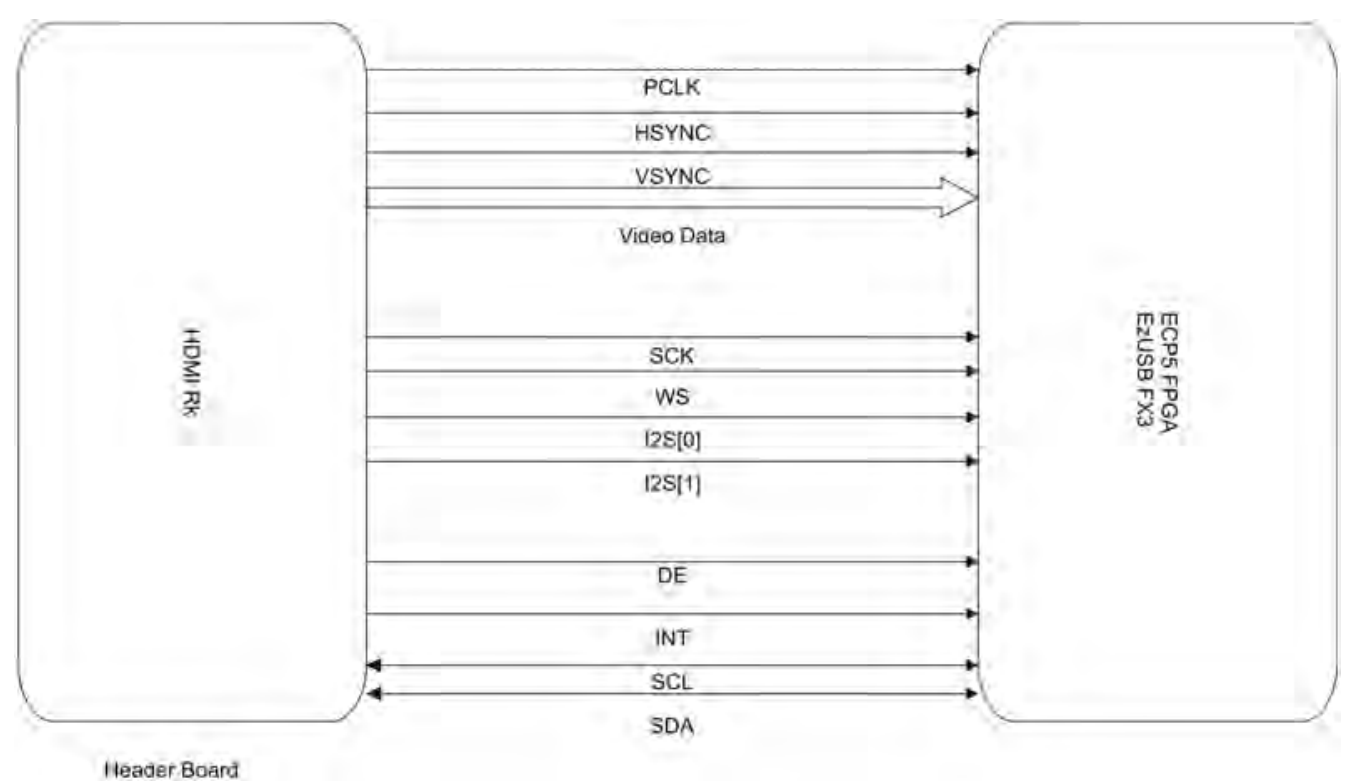

Figure 79.7 Flow of instructions in FPGA.

- If resolution is $4 \mathrm{~K} 2 \mathrm{~K}$, there is an additional step that needs to be performed by the FPGA where the FPGA needs to convert the native 16-bit YUV422 colour format to 12-bit YUV420 because of the bandwidth limitations of the GPIF-II interface. The colour conversion is performed in the DDR3 RAM of the FPGA which acts as a buffer for incoming frames, takes off the ' $\mathrm{V}$ ' pixels from the frames and then packs two and half such frames to make it a 32-bit packet and sends it to FX3 over GPIF-II interface as shown in Figure 79.7.

FPGA also adds the UVC headers to the frames before sending them to FX3 so that the data frames sent from FX3 are recognized by native UVC compliant host applications and video players.

The FPGA acts as master and follows the timing diagrams as mentioned in the application note AN87216 by Cypress and passes the video data over to the GPIF-II interface and implements an algorithm where it sends the audio data between the video data packets to the FX3.

4. FX3 usage case in the design

FX3 acts as a USB SuperSpeed controller which takes up the data from the FPGA and commits it to the host PC over the UVC interface for video data and over the UAC interface for audio data using DMA channels internally without CPU intervention at superspeed data rates of up to 5 Gbps.

\section{Outcomes}

The first phase of the design and the following work is completed: 


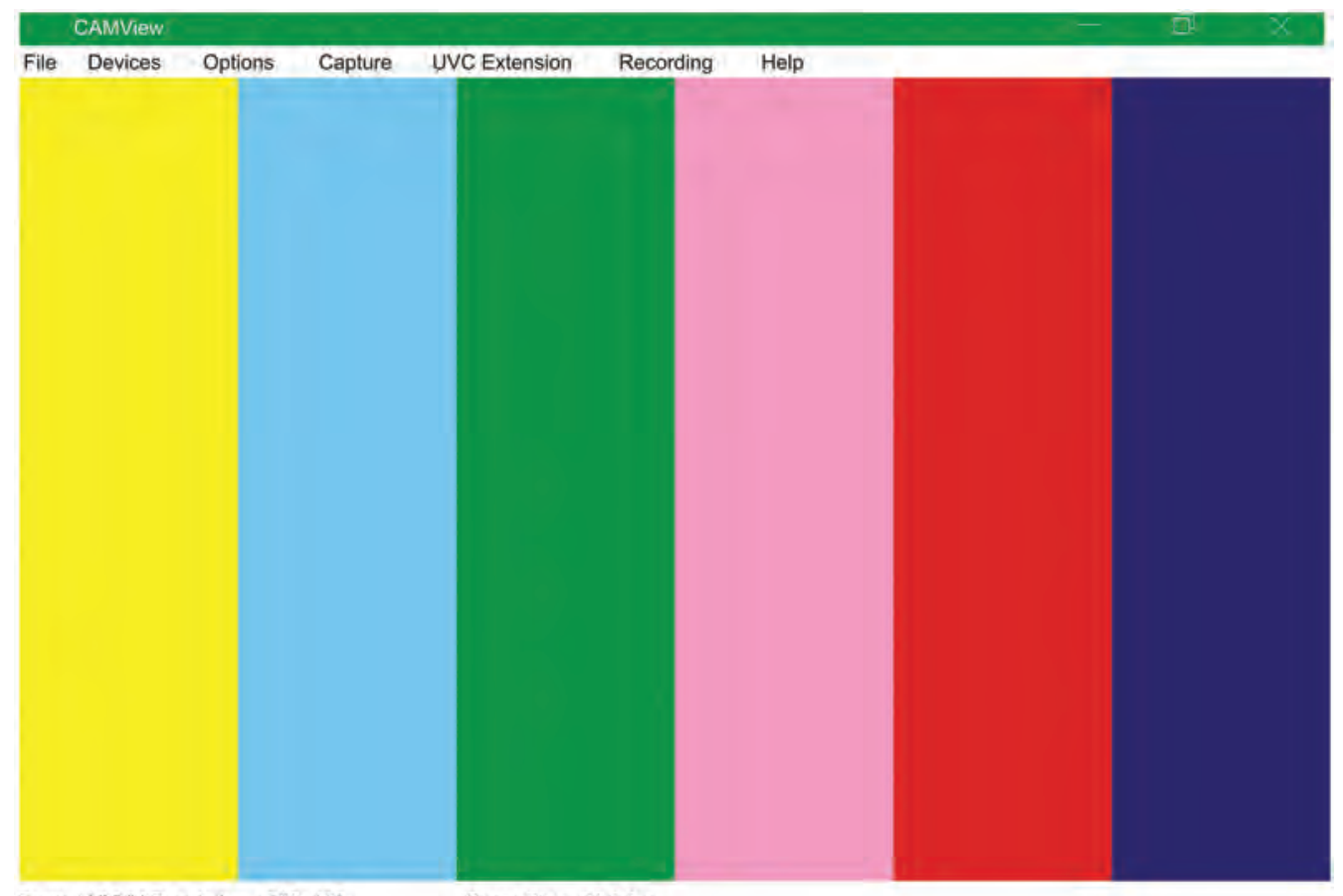

Figure 79.8 Colour bar project result.

- The schematics design of the header board containing the HDMI Rx and the HDMI connector for the HDMI camera, which is to be compatible with the pre-existing base board containing the ECP5 along with FX3.

- Modified the FX3 firmware to add the UAC interface descriptors, creation of DMA channels for the audio interface and testing it using a hardcoded audio file from the SPI flash to check the correct functioning of the audio channel in FX3 and the HDMI $\mathrm{RX}$ configuration registers settings have been added and sent over I2C to configure the RX and was successfully configured.

- Completed the understanding and modification in the colour bar project by adding the I2C slave interface for communication with the HDMI receiver and FX3. This I2c slave would read/write from/to appropriate registers as shown in Figure 79.8.

- Understood the DDR3 controller demo test example to implement ping pong buffer implementation for colour conversion.

\section{Future scope}

The DDR3 controller ping pong buffer implementation needs to be added to the firmware and make it work with the FPGA for colour conversion for ultra-HD 4K2K resolutions.

Testing of the prototype board needs to be performed and tweak it to the state of making it commercially available as a product to the industrial and consumer electronics market. 


\section{Conclusion}

This paper presents a reference design for 4K HDMI USB3 Capture Card that can be used to bridge the gap between HDMI camera sources and host computers by providing USB 3.0 as the interface to mediate the data. The testing and results of the design need to be validated against some of the market competitors to enhance it and go beyond what is being currently made available in the market.

\section{References}

[1] D. Kithany, S. P. Analyst, and S. H. Appliances, "Interface battleground 2017,” vol. 1. 2021.

[2] USB Implementers Forum, "SuperSpeed USB," USB 3.1 Specif., 2013.

[3] P. C. Electronics, B. V International, S. Image, S. Corporation, and T. Corporation, "HighDefinition Multimedia Interface," 2009.

[4] USB Implementers Forum, "Universal Serial Bus Device Class Definition for Video Devices $1.1, " 2005$.

[5] U. I. Forum, "Universal Serial Bus Device Class Definition for Video Devices,” p. 97, 2001, doi: 10.1109/WESCON.1997.632372.

[6] A. Rama and S. Krishna, "Designing with the EZ-USB FX3 Slave FIFO interface more information," no. 001, pp. 1-70.

[7] A. Sai and K. Vakkantula, "Designing a GPIF TM II master interface USB host U Cypress host more information," no. 001, pp. 1-31.

[8] "Cam Link elgato.com", elgato.com, 2020. [Online]. Available: www.elgato.com/en/gaming/ cam-link-4k [Accessed: 05- Feb- 2020]. 


\title{
80 A comparative review of different TMOs for HDR images
}

\author{
N. Neelima1, Y. Ravi Kumar ${ }^{2}$ \\ ${ }^{1}$ Associate Professor, CMR Institute of Technology, Hyderabad, Telangana, India \\ ${ }^{2}$ Scientist-G, SINT (E) Division, DLRL, Hyderabad, India
}

\section{Introduction}

An HDR image first stores the pixel values which span the total range of real world scenes. For each particular scene a dynamic range will define the ratio between the bright and dark part of that particular scene. The dynamic range of real world scenes is quite high, i.e., 100000:1, and for low dynamic range images of JPEG format it won't exceed 255:1, whereas for human eyes it can be near 100000:1, which is greater than the CRT, whose dynamic range won't exceed 100:1.

The different luminance values for some lighting environments are as shown in Table 80.1.

Formats for LDR images are JPEG, PNG, TIFF and so on. LDR images can be displayed on conventional picture tubes such as CRT, which is designed for accommodating the capabilities of display devices. This paper is structured as follows: in Section 2 the advances and different types of TMOs are presented. The different assessment parameters are characterized in Section 3. In Section 4, results of tone mapped images with different quality assessment parameters and their profiles are discussed. Finally it ends with conclusions in Section 5.

\section{HDR imaging implementation and tone mapping}

In this section, the implementation of the HDR image and tone mapping are discussed. The block diagram, to perform the operation of real world scene with tone mapping is shown Figure 80.1. As shown in Figure 80.1 in two environments, the real world image is seen. Viewer 2 directly views the image in one environment and viewer 2 in another environment observes the scene after applying the tone mapping method. To have similarity between viewer 1 and 2, how the scene is viewed is the main objective [1].

\subsection{Tone mapping operators}

In this paper four prominent TMOs, gamma, Benterle, Chiu and Raman, are described below.

\section{(i) Gamma TMO}

The nonlinear relationship between the input voltage and output light in CRT given by power law as 
Table 80.1 Different luminance values for lighting environments

\begin{tabular}{ll}
\hline Condition & Luminance $\left(\mathrm{cd} / \mathrm{m}^{2}\right)$ \\
\hline Star light & $10^{-3}$ \\
Moon light & $10^{-1}$ \\
Indoor lighting & $10^{2}$ \\
Sun light & $10^{5}$ \\
Maximum intensity of CRT monitors & $10^{2}$ \\
\hline
\end{tabular}

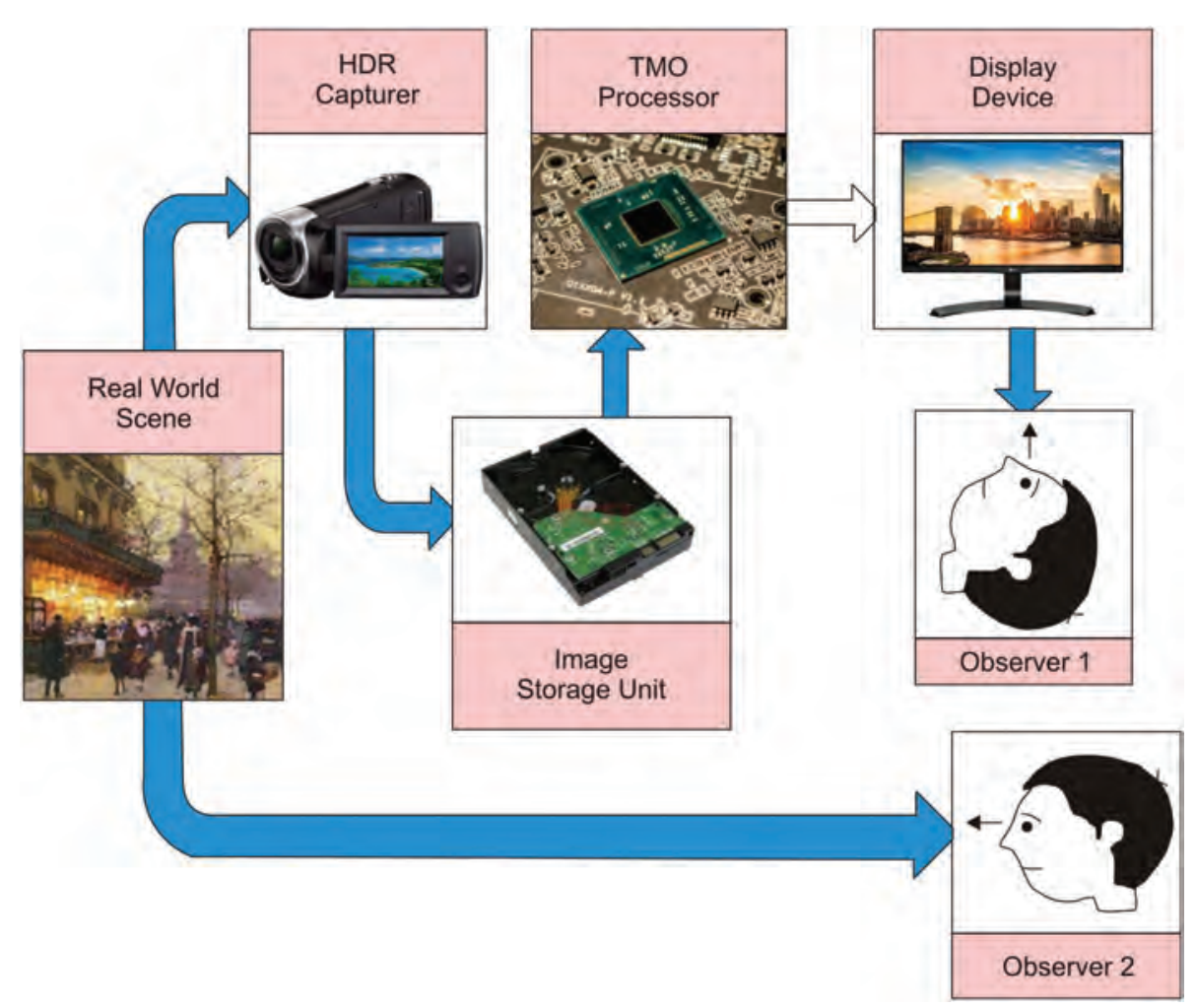

Figure 80.1 Block diagram of real world scenes and tone mapped.

$$
\begin{aligned}
& L \propto V^{\gamma} \\
& L=K V^{\gamma}
\end{aligned}
$$

where $\mathrm{K}$ is constant and $\gamma$ is the nonlinearity obtained by a particular operation of the CRT; it varies for various monitors. These nonlinearities can be given by the look up tables which convert values into voltages. The amount of contrast can be maximized using the contrast control.

Once the gamma value is known, images can be sent to the display, which should contain normalised values between 0 and 1 given by 


$$
\begin{aligned}
& R^{\prime}=R^{1 / \gamma} \\
& G^{\prime}=G^{1 / \gamma} \\
& B^{\prime}=B^{1 / \gamma}
\end{aligned}
$$

\section{(ii) Benterle TMO}

The Benterle TMO was tested with values varying from $0.015 \mathrm{~cd} / \mathrm{m}^{2}$ to $3000 \mathrm{~cd} / \mathrm{m}^{2}$. The input source image is segmented into different zones of dynamic range. This TMO is a hybrid operator. In this, the first is to linearize the input image. The range of the image can be obtained by inverting a TMO. The range expansion can be controlled and is given by

$$
L_{W}(x)=\frac{1}{2} L_{W, M a x} L_{W h i t e}\left(L_{d}(x)-1+\sqrt{\left(1-L_{d}(x)\right)^{2}+\frac{4}{L_{W h i t e}^{2}} L_{d}(x)}\right.
$$

where the maximum output luminance in $\mathrm{cd} / \mathrm{m}^{2}$ is $L_{W, \text { Max }} \cdot L_{W h i t e} €(1,+\infty)$ gives the shape of the expansion curve. While limiting artefacts due to expansion $L_{W \text { bite }} \cong L_{W, \text { Max }}$ contrast can be increased [7].

\section{(iii) С̆іи TMO}

Chiu proposed the first operator that preserves the local contrast. This TMO scales the scene luminance by multiplying each pixel by a factor $\mathrm{S}(\mathrm{x}, \mathrm{y})$ by averaging the neighbouring pixels given as shown in equation (7)

$$
L_{d}(x, y)=S(x, y) L_{w}(x, y)
$$

where $\mathrm{S}(\mathrm{x}, \mathrm{y})$ represents the scaling function which is used to compute the local average of neighbouring pixels. Equation (8) is used to compress the HDR images by taking the reciprocal of these pixels.

$$
L_{d}(x, y)=\frac{1}{k L_{w}^{b l u r}(x, y)} L_{w}(x, y)
$$

where $\mathrm{K}$ is the proportionality constant.

\section{(iv) Raman TMO}

This approach to Tone mapping HDR images follows in four steps:

1. Extraction of luminance channel: the luminance is obtained by the equation given below [8]:

$$
\mathrm{L}=0.2126 \mathrm{I}_{\mathrm{R}}+0.7152 \mathrm{I}_{\mathrm{G}}+0.0722 \mathrm{I}_{\mathrm{B}}
$$




\section{Intelligent Circuits and Systems}

2. Computation of contrast measure is done using two approaches: (i) contrast by means and (ii) constant by variance, given by equations (9) and (10)

$$
\operatorname{var}=\frac{1}{p} \sum_{p=0}^{p-1}\left(\mathrm{f}_{\mathrm{p}}-\mu\right)^{2}
$$

$$
\text { where } \mu=\frac{1}{p} \sum_{p=0}^{p-1} \mathrm{f}_{\mathrm{p}}
$$

(i) Histogram adjustment:

The contrast value is scaled to a maximum value of 1 . Contrast threshold value $\mathrm{C}$ is scaled to the range $0-1$.

(ii) Design of kernel:

A Gaussian kernel in the logarithmic domain is applied on the luminance channel to achieve tone mapping. It is given as shown in equation (11):

$$
\varnothing(c)=\frac{\alpha}{\sigma \sqrt{2 \pi}} e^{\left(\frac{\alpha^{2}}{\sigma^{2}}\right)}
$$

where $\alpha$ is the scaling factor, $\sigma$ is the local contrast in the tone mapped image, where $\alpha, \sigma$ are taken as 0.5 .

(iii) Generation of tone mapped image: the following equations (12), (13), (14) and (15) are used to find new luminance and restore the colour information.

$$
\begin{aligned}
& L_{\text {new }}=e^{\varnothing(c) \ln (L)} \\
& I_{\text {Rnew }}=\left(\frac{L_{\text {new }}}{L}\right) I_{R} \\
& I_{\text {Bnew }}=\left(\frac{L_{\text {new }}}{L}\right) I_{B} \\
& I_{\text {Gnew }}=\left(\frac{L_{\text {new }}}{L}\right) I_{G}
\end{aligned}
$$

\section{Quality assessment parameters}

Assessment parameters are defined as follows. 


\subsection{Luminance}

Luminance is obtained by taking the logarithmic average of an image. For colour images, the luminance value is obtained by finding sum and is given as shown in equation (16):

$$
\text { Lum }=0.213 \text { RED + 0.715 GREEN + 0.072 BLUE }
$$

\subsection{Mean square error (MSE)}

The mean square errors for images $\mathrm{A}$ and $\mathrm{B}$ are as shown in equation (17):

$$
\operatorname{MSE}(A, B)=\frac{1}{M} \sum_{i=1}^{M}\left(a_{i}-b_{i}\right)^{2}
$$

\section{3 mPSNR}

Modified peak signal to noise ratio is shown in equation (18):

$$
m P S N R=10 \log _{10} \frac{(c o l * 255)^{2}}{M S E}
$$

\subsection{Tone mapped quality index (TMQI)}

The TMQI is given by

$$
\operatorname{TMQI}(H, L)=A_{R I}\left[s_{f}(H, L)\right]^{\alpha}+\left(1-A_{R I}\right)\left[s_{n}(L)\right]^{\beta}
$$

where $s_{n}$ represents statistical naturalness, $\alpha$ and $\beta$ represent two factor sensitivities, $A_{R I}\left(0 \leq A_{R I} \leq 1\right)$ adjusts their relative value, $s_{f}$ represents the structural fidelity, LDR and HDR images as L,H $[10,11]$.

\subsection{Feature similarity index tone mapped (FSITM)}

The feature similarity index for tone mapped (FSITM) is as shown in equation (20):

$$
\operatorname{FSITM}_{\mathrm{CH}}=\varphi F_{C H}(H, L)+(1-\varphi) F_{C H}(\log H, L)
$$

$c \in\{R, G, B\}$ is RGB of images, where $\varphi(0 \leq \varphi \leq 1)$ is the channel $C H$, and FSITM $_{C H}$ which represents the feature similarity index.

\section{Results and discussion}

The HDR source image is as shown in Figure 80.2(a). Different tone mapping operators discussed in 2.1 are applied for the HDR image study table to change to low dynamic 


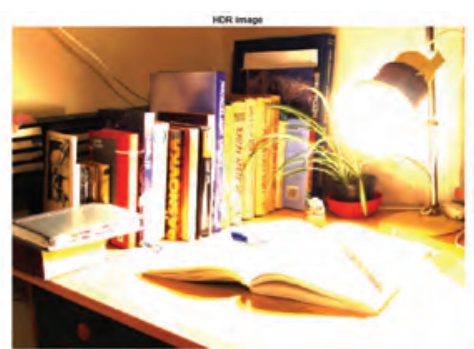

(a)

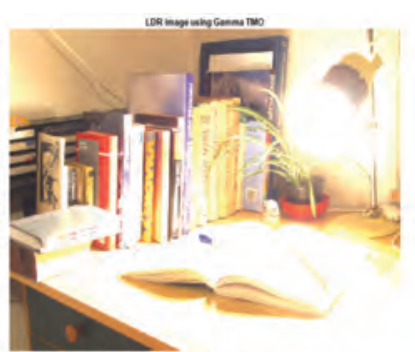

(b)

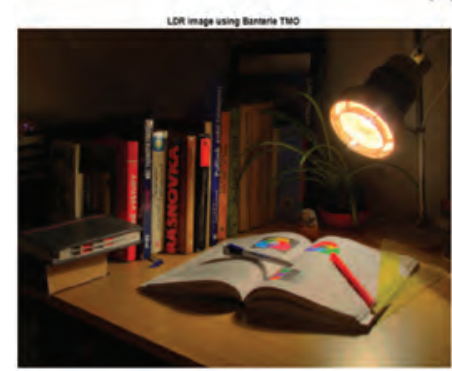

(c)

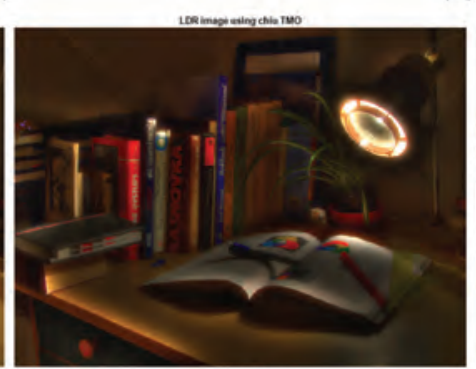

(d)

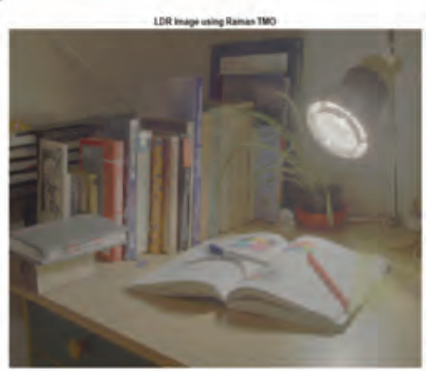

(e)

Figure 80.2 (a) HDR image of size $535 \times 713$ of study table. (b) Low dynamic image after gamma tone mapping. (c) Low dynamic image after Banterle TMO. (d) Low dynamic image after Chiu TMO. (e) Low dynamic range image after Raman TMO.

Table 80.2 Quality assessment parameters for study table HDR image

\begin{tabular}{lllrll}
\hline Type of TMO & Luminance & TMQI & mPSNR & MSE & FSITM \\
\hline Gamma TMO & 0.7924 & 0.2037 & 13.4572 & 0.0293 & 0.8103 \\
Benterle TMO & 0.2086 & 0.2199 & 9.9760 & 0.2825 & 0.8847 \\
Chiu TMO & 0.1637 & 0.2133 & 9.2270 & 0.3581 & 0.8030 \\
Raman TMO & 0.4138 & 0.2097 & 12.0628 & 0.1287 & 0.9014 \\
\hline
\end{tabular}

range image (LDR). The gamma tone mapping operator is applied on the source image study table for gamma $=2.2$ and the corresponding LDR image is shown in Figure 80.2(b).

The profiles of mPSNR, luminance, MSE are shown in Figure 80.3(a) and 3(b). From the profiles it is clear that gamma TMO has $\mathrm{mPSNR}=13.4572$ greater than other TMOs. The luminance and MSE of gamma TMO are 0.7924 and 0.0293 better than other TMOs.

The profile of TMQI and FSITM is as shown in Figure 80.4, which indicates that the Raman TMO has better tone mapped quality index compared to the other TMOs.

\section{Conclusion}

To display HDR images in conventional manner using LDR displays tone mapping operators play a prominent role. To display HDR images more realistically and accurately 


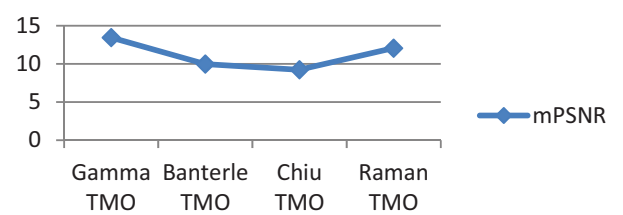

(a)

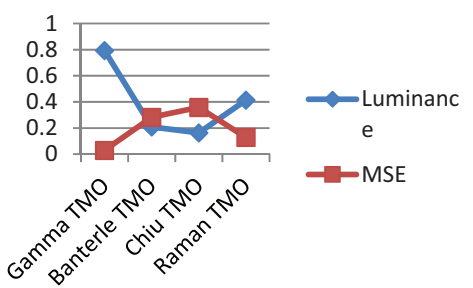

(b)

Figure 80.3 Profiles of mPSNR, luminance and MSE of different TMOs.

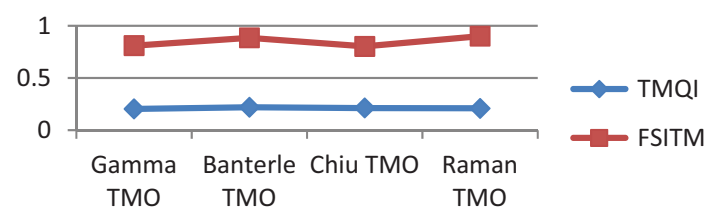

Figure 80.4 Profile of TMQI and FSITM of different TMOs.

there is a need for efficient and effective TMOs though already existing TMOs such as Benterle, gamma correction, Raman are Chiu are developed and put to use. Luminance, mPSNR, MSE, TMQI and FSITM, which are considered to be the performance, are recorded while calculating for existing tone mapping operators. Compared to other tone mapping operators, promising results can be achieved using gamma TMO.

\section{References}

[1] N. Neelima and Y. Ravi Kumar, Performance assessment of TMOs, Procedia Computer Science, 89, 700-709 (2016).

[2] Hojatollah Yeganeh and Zhou Wang, Objective quality assessment of tone-mapped images, IEEE Transactions on Image Processing 22(2), 657-667 (2013).

[3] F. Drago, W. L. Martens, K. Myszkowski and H. P. Seidal, Perceptual evaluation of tone mapping operators, In Proceedings of SIGGGRAPH Conference Sketches Applications (2003).

[4] Francesco Banterle, Alessandro Artusi, Kurt Debattista and Alan Chalmers, Advanced High Dynamic Range Imaging: Theory and Practice. A K Peters Ltd. (2011).

[5] E. Reinhard, M. Stark, P. Shirley and J. Ferwerda, Photographic tone reproduction for digital images, Proceedings of the 29th Annual Conference on Computer Graphics and Interactive Technology 21, 267-276 (2002).

[6] F. Drago, K. Myszkowski, T. Annen and N. Chiba, Adaptive logarithmic mapping for displaying high contrast scenes, Computer Graphics Forum 22(3), 419-426 (2003).

[7] Shanmuganathan Raman and Subhasis Chaudhuri, Bilateral filter based compositing for variable exposure photography, Eurographics 2009. Image Fusion, IEEE Transactions on Image Processing. 24(11) (2015).

[8] Keda Ma, Kai Zeng and Zhou Wang, Objective quality assessment for color-gray image conversion, IEEE Transactions on Image Processing 24(12) (2015).

[9] Lukas Krasula, Manish Narwaria and Karel Fliegel, Influence of HDR reference on observers preference in tone-mapped images evaluation, QoMEX IEEE, (2015). 


\section{Intelligent Circuits and Systems}

[10] Jino Lee and Rae-Hong Park, Image quality assessment of tone mapped images, IJCGA 5(2) (2015).

[11] Keda Ma, Hojatollah Yeganeh, Kai Zeng and Zhou Wang, High dynamic range image compression by optimizing tone mapped image quality index, IEEE Transactions on Image Processing 24(10) (2015). 


\title{
81 Classification of P2P file-sharing traffic using heuristic based and statistical based techniques
}

\author{
Max Bhatia*, Vikrant Sharma
}

Department of Computer Science Engineering, Lovely Professional University, Punjab, India

\section{Introduction}

P2P technology has grown rapidly over the past decade and has been one of the largest contributors of the Internet traffic [1]. The traffic such as WWW, FTP, Email, etc. use the client-server approach where the client requests data from the server and the server provides the data back to the client, which leads to an asymmetric kind of traffic and does not consume large bandwidth. However, with the advent and popularity of various P2P applications, the peers involved in communication act as client and server simultaneously due to which transfer of data takes place in both directions in huge amounts, which leads to a symmetric kind of network traffic and consumes a lot of network bandwidth. Therefore, non-P2P application protocols such WWW, FTP, Email, HTTPS, etc. are unable to get their fair share of network bandwidth and suffer with poor quality of service.

File-sharing applications such as BitTorrent are the largest contributor of P2P traffic which accounts for more than $44 \%$ of the Internet traffic in EMEA (Europe, the Middle East and Africa) [2]. As of late, the action taken against the utilization of P2P applications has constrained them to discover alternative ways (such as port disguising, traffic tunnelling, traffic encryption and so forth) to stow away their traffic on the Internet. While the authors in [3] argue that P2P traffic is diminishing, it still represents a major portion of the Internet traffic, and is seen as a burden on the network resources [4]. Due to the massive adoption of P2P applications by the users in the past few years, it has attracted great attention of the network administrators and the Internet service providers (ISPs) as it poses various commercial problems such as network security, congestion, etc.

The oldest and the simplest network traffic classification is port-based classification, which classifies the traffic by analysing the TCP/UDP port number of the packet header. This is due to that fact that there are well-known port numbers ranging between 0 and 023 that are assigned by IANA [5] to various protocols such as HTTP, SMTP, DNS, FTP, etc. This technique is fast, easy to implement and hence is most often used to build certain rules in the firewalls and access control lists [6]. However, the port-based technique cannot be used to classify all kinds of traffic flows since there exists some protocols such as peerto-peer (P2P) and FTP (operating in passive mode) which mostly use random or ephemeral port numbers for communication and hence they do not usually function on their default port numbers. In addition to this, many P2P applications masquerade the traffic to avoid

\footnotetext{
* Corresponding author: max.16870@lpu.co.in
} 
their detection. The authors in [7] and [8] found that only 30-70\% of the traffic could be detected by using port-based classification technique.

The payload-based classification technique overcomes the limitation of the port-based technique. It classifies the traffic by examining the packet-payload of each packet to search for the application signature and map it with the database containing the signatures of previously stored application protocols. For example, eDonkey P2P traffic contains the string ' $\mathrm{xe} 3 \mathrm{x} 38$ '. This technique is also known as deep packet inspection (DPI) and is the most accurate technique in classifying traffic. There also exist various open-source and commercial tools based on the DPI technique which can classify the traffic such as nDPI [9], Libprotoident [10,11], L7-filter [12], Cisco's NBAR [13], etc. However, this technique suffers from various limitations, for example, it is infeasible in high-speed networks, involves a lot of processing load and complexity [14], is unable to deal with encrypted traffic, application protocol-signatures need to be found every time as new applications emerge, direct analysis of packet payload may breach the privacy policy of some organizations, etc.

Due to these limitations, a modern technique known as classification in the dark is used for traffic classification, which classifies the traffic flow by analysing its statistical properties or by observing its behavioural patterns on the basis of a pre-defined set of heuristics rules. The statistical-based technique makes use of packet-level or flow-level properties of the traffic such as inter-arrival time of packet, packet length, duration of flow, etc. [15] in order to classify the traffic. On the other hand, the heuristic-based classification uses a pre-defined set of rules such as observing the number of connections made by a host, host acting both as client as well as server, number of port used by the host, etc.

The main objective of this research work is to classify P2P file-sharing (P2P-fs) traffic. We specifically focus on P2P file-sharing traffic since it is the largest contributor of P2P Internet traffic as a whole. For this purpose, a 2-step classification approach has been employed which is categorized into packet-level and flow-level classification modules. In packet-level classification, the P2P-port based technique has been utilized and in the flowlevel classification, a combination of heuristic-based and statistical-based technique (using C4.5 decision tree) has been utilized for classifying the traffic. The experimental results show that the proposed technique achieves high accuracy (above 98.5\%) in classifying P2P-fs traffic. In addition to that, the proposed technique (a) works on both TCP and UDP protocols, (b) uses minimum heuristics for classification (and hence has less overhead) and (c) works with encrypted traffic and can be used for real-time classification as well. The remaining paper is organized as follows. In Section 2, related work is discussed. the Proposed traffic classification methodology is discussed in Section 3. In Section 4, traffic datasets, validation and experimental results are discussed. Finally, Section 5 concludes the research work.

\section{Related work}

Park et al. in [16] identify the peers that use the popular BitTorrent client program known as uTorrent. The authors proposed a methodology to identify copyrighted file sharers by analysing request-response packets of HTTP and UDP protocols as well as the handshake process of the BitTorrent client program. But their methodology focuses only on identifying peers using a single file sharing program (i.e. uTorrent) and did not address the traffic encryption issue as well. Reddy and Hota in [17] proposed a methodology to classify P2P traffic based on host behaviour. They identified a set of heuristics which analyses the host behaviour from the headers of the transport layer and found an average detection rate of $99 \%$. Ye and Cho in [1] classified P2P traffic using a hybrid technique 
which is the combination of packet-level and flow-level classification processes. At packetlevel, the traffic is classified with the combination of signature-based and heuristic-based techniques. At flow-level, a combination of statistical-based and pattern heuristic-based technique is used to classify remaining unknown traffic. The authors achieved a classification accuracy of $98 \%$ but their technique does not classify P2P traffic which uses the UDP protocol for communication. Jamil et al. in [18] proposed a technique that utilizes the combination of SNORT and ML based techniques to classify P2P traffic. The technique used chi-square and fuzzy as feature selection algorithms along with ML algorithms, SVM, C4.5 decision tree and ANN, and achieved classification accuracy of 99.5\%. Abdalla et al. in [19] proposed an approach based on feature selection and analytical methods (scatter and ANOVA) for detecting the optimal set of flow features which could be used online for $\mathrm{P} 2 \mathrm{P}$ traffic classification. Their methodology used a four-stage process in order to narrow down and identify the optimal features for traffic classification. The ML algorithms used with the selected features were J48 and naïve Bayes, for which they achieved classification accuracy of $99.5 \%$. Sajeev and Nair in [20] proposed a hybrid technique which makes use of header and payload information to classify P2P traffic. In the first step, a communication module is created by analysing header information and then the LASER, i.e. Longest Common Subsequence (LCS)-based Application Signature ExtRaction, algorithm is used for extracting signatures from the payload. Both the header and payload information are fed into a statistical-based classifier that uses a C4.5 decision tree to classify P2P traffic. The technique was able to achieve a detection rate of $95 \%$ but since it also makes use of a signature-based technique, it may not be able to achieve good classification accuracy where the whole traffic is encrypted.

Most of the studies discussed above generally classify traffic as either P2P or non-P2P. The technique proposed in this paper focuses on classifying P2P file-sharing traffic specifically, as it is one of the largest contributors of P2P Internet traffic as a whole. For this purpose, a combination of heuristic-based and statistical-based classification approaches (using C4.5 decision tree) has been utilized to classify whether a flow belongs to P2P filesharing traffic or not.

\section{P2P-fs traffic classification methodology}

A 2-step classification approach has been employed to classify P2P-fs traffic as shown in Figure 81.1. The classification process has been categorized into two levels, namely packet-level classification and flow-level classification. In the packet-level classification process, the $\mathrm{P} 2 \mathrm{P}$-port based technique has been utilized, and in the flow-level classification process, a combination of heuristic-based and statistical based technique (using C4.5 decision tree) has been utilized in order to classify the traffic as either P2P-fs or non-P2P-fs.

In the traffic classification process, a flow is generally defined as a combination of 5tuples (i.e. source-IP, source port, destination-IP, destination port, protocol). In order to identify packets belonging to the same flow, packet-hash is calculated by concatenating 5 -tuple flow information as shown in Figure 81.2. In this way, packets of a flow which are travelling in either direction will have the same packet-hash. It is used to identify those packets whose flow has already been classified as P2P-fs. In this research work, a P2P flow table is used to store both the flow details and packet-hash of the flows which have been classified as P2P-fs.

In the packet-level classification process, initially a P2P port-based technique is employed by extracting the port number from the packet header and mapped with wellknown P2P port numbers used by various P2P-fs applications [21] as shown in Table 81.1. If a match is found, then the packets belonging to a particular flow are labelled as P2P-fs 
Table 81.1 Default port numbers used by various P2P-fs applications

\begin{tabular}{ll}
\hline P2P-fs applications & Default ports used \\
\hline eMule & $4662,4672,4711$ \\
uTorrent & $771,6881-6889,6890,6891-6900,6901,6902-6968,6969,6970-6999$, \\
& 7000,9091 \\
Transmission & 9091 \\
qBitTorrent & 9000 \\
\hline
\end{tabular}

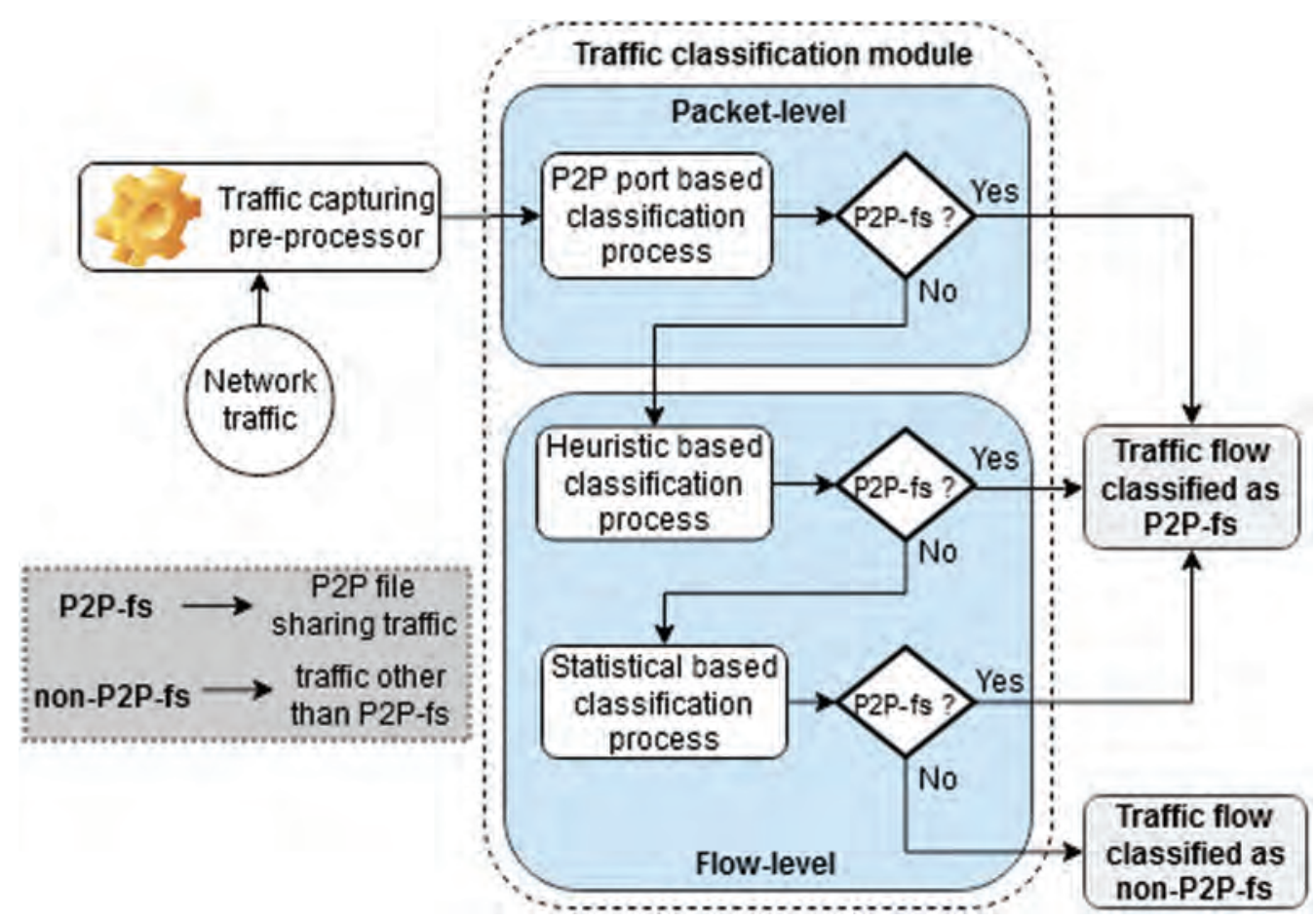

Figure 81.1 P2P (file-sharing) traffic classification technique.

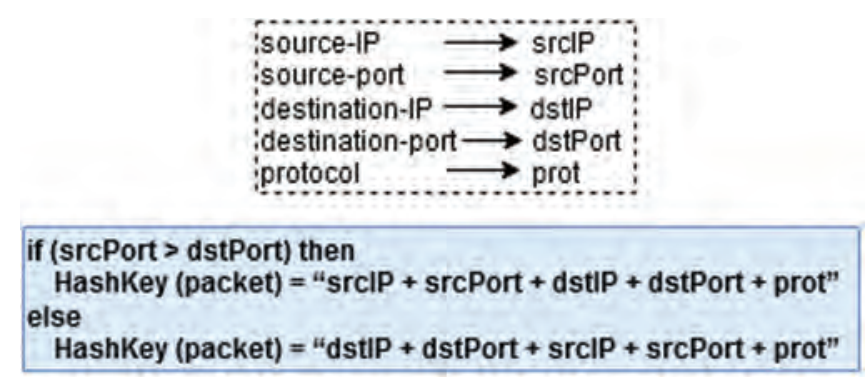

Figure 81.2 Calculation of hash-key of packet. 
and the flow details are added to the P2P flow table. Although the port-based technique is inefficient in classifying the traffic (since many P2P applications use random port numbers or masquerade port numbers to avoid detection), yet it has been utilized here to perform an early classification if any of the P2P-fs applications are still using well-known P2P port numbers for communication. The traffic which remains un-classified as P2P-fs is further fed to the flow-level classification process where the heuristic-based technique is employed on it. The heuristics that are used in the proposed work are described below:

(a) Usage of TCP and UDP (heuristic_1): If both source and destination peers use TCP and UDP protocols simultaneously, then such kind of behaviour is shown by P2P applications (such as Skype, BitTorrent, etc.) where TCP could be used for establishing the connection with other peers and UDP could be used for transferring the data amongst those peers (or vice versa). Hence, such kind of traffic is considered as P2P traffic, but it may not necessarily be P2P-fs traffic.

(b) TCP segment-length or UDP datagram-length ratio (heuristic_2): This heuristic is based on the observation that P2P-fs traffic has a unique packet size distribution in which the TCP segment-length (or UDP datagram-length) for most of the packets in a flow has a range either between 0 and 100 bytes or $>1000$ bytes. The reason behind such behaviour could be that smaller segments/datagrams (i.e. $0-100$ bytes in size) are used for data-request and larger segments/datagrams (i.e. > 1000 bytes in size) are used for data-response. Hence, we define packet-size-distribution ratio (psd_ratio) of a flow as follows:

$$
p s d_{-} \text {ratio }=\frac{\text { total_TCP_segment_length }(\text { or total_UDP_datagram_length })}{\text { total_number_of } \_ \text {packets }}
$$

Here, total_TCP_segment_length (or total_UDP_datagram_length) refers to all those packets of a flow which have segment-length (or datagram-length) in the range 0-100 bytes or $>1000$ bytes. Hence, the TCP/UDP flows which have the psd_ratio > thresholdvalue are classified as $\mathrm{P} 2 \mathrm{P}$-fs traffic. For experimental purposes, the threshold-value taken here is 0.70 .

(c) Usage of ephemeral port numbers (heuristic_3): This heuristic is based on the fact that if both source and destination peers of a flow use ephemeral port numbers (i.e. above 1023) for communication, then such a flow is considered as belonging to P2P traffic; but it may not necessarily belong to P2P-fs traffic, since similar behaviour could be found in non-file-sharing P2P traffic as well (e.g. VoIP traffic).

(d) Data transfer between peers (heuristic_4): This heuristic is based on the fact that both source and destination peers involved in P2P file-sharing transfer a large number of bytes to each other, since the source peer downloads the required data possessed by the destination peer and uploads the data requested by the destination peer. Such kind of traffic differs from FTP traffic in a way that P2P-fs traffic involves transfer of data in both directions, but FTP traffic involves transfer of data in a single direction only. Hence, in a flow, if the number of bytes transferred in both directions is greater than a threshold value, then the flow is classified as P2P-fs traffic. For experimental purposes, the threshold value taken here is $3 \mathrm{MB}$.

The heuristic-based classification technique is mentioned in Algorithm 1, where the proposed heuristic rules have been used to classify the traffic either as P2P-fs or nonP2P-fs. Here, we analyse that if source and destination IPs are involved in TCP and UDP 


\section{8}

communication with each other (i.e. heuristic_1) and their psd_ratio $>0.70$ (i.e. heuristic_ 2), then the flow is labelled as P2P-fs traffic. Otherwise, if source and destination use either the ephemeral ports for communication with each other (i.e. heuristic_3) or transfer data $>3 \mathrm{MB}$ to each other (i.e. heuristic_4) and their psd_ratio > 0.70 (i.e. heuristic_2), then such flow is also labelled as P2P-fs traffic. It is to be noted that the heuristics, namely heuristic_1, heuristic_3 and heuristic_4, alone may not be sufficient to verify whether a flow belongs to P2P-fs traffic or not, since such behaviour could be shown by non-file-sharing P2P traffic as well (e.g. VoIP traffic).

\begin{tabular}{|c|c|}
\hline Legends & Algorithm 1 \\
\hline $\begin{array}{l}\text { src } \rightarrow \text { source peer } \\
\text { dst } \rightarrow \text { destination peer } \\
\text { psd_ratio } \rightarrow \text { ratio of all packets with segment } \\
\text { length ranging between } 0-100 \text { and }>1000 \text { bytes } \\
\text { to total number of packets of a flow } \\
\text { data(src_to_dst }) \rightarrow \text { data transferred from source } \\
\text { to destination } \\
\text { data(dst_to_src) } \rightarrow \text { data transferred from } \\
\text { destination to source } \\
\text { data(threshold) } \rightarrow 3 \mathrm{MB} \\
\text { heuristic_1 } \rightarrow \text { both src and dst uses TCP and UDP } \\
\text { simultaneously } \\
\text { heuristic_2 } \rightarrow \text { psd_ratio }>=0.7 \\
\text { heuristic_3 } \rightarrow \text { both src and dst use } \\
\text { emphemeral ports } \\
\text { heuristic_4 } \rightarrow \text { data(src_to_dst) and data(dst_to__ } \\
\text { src) }>\text { data(threshold) }\end{array}$ & $\begin{array}{l}\text { Begin } \\
\text { while(trafficFlows_not_finished) }\{ \\
\text { flow = fetch_next_trafficFlow( }) \\
\text { if(flow.heuristic_1() } \rightarrow \text { satisfied) }\{ \\
\text { if(flow.heuristic_2() } \rightarrow \text { satisfied })\{ \\
\text { write: flow } \rightarrow \text { P2P_flowTable } \\
\} \\
\} \\
\text { else if }(\text { (flow.heuristic_3() } \rightarrow \text { satisfied) or } \\
\text { (flow.heuristic_4() } \rightarrow \text { satisfied })\{ \\
\text { if(flow.heuristic_2() } \rightarrow \text { satisfied) }\{ \\
\text { write: flow } \rightarrow \text { P2P_flowTable } \\
\} \\
\} \\
\} \\
\text { End }\end{array}$ \\
\hline
\end{tabular}

The flows which still remain un-classified as belonging to P2P-fs traffic are fed to the statistical-based classification process where the C4.5 decision tree is utilized on the statistical properties of the traffic flow in order to verify whether the remaining traffic contains any trace of P2P-fs traffic or not.

\section{Datasets, validation and experimental results}

The proposed technique classifies the traffic as either P2P-fs traffic or non-P2P-fs traffic (i.e. any traffic other than P2P file-sharing traffic). It is implemented in Java using jNetPcap library [22] and weka [23]. The metrics which is used to evaluate the proposed methodology are accuracy, false positive and false negative rates, where accuracy is defined as follows:

$$
\text { Accuracy }=\frac{\mathrm{TP}+\mathrm{TN}}{\mathrm{TP}+\mathrm{TN}+\mathrm{FP}+\mathrm{FN}}
$$

Here, TP, TN, FP and FN refer to true positive, true negative, false positive and false negative, respectively. Accuracy measures the capability of the classifier in identifying positive and negative cases. The experiment using the proposed technique has been conducted on offline traffic traces, where two datasets have been utilized consisting of both P2P and non-P2P traffic flows, whose details are shown in Table 81.2. 
Table 81.2 The number of flows in Dataset-1 and Dataset-2

\begin{tabular}{llll}
\hline Dataset & P2P_fs flows & non-P2P_fs flows & Total \\
\hline Dataset-1 & 7599 & 71399 & 78998 \\
Dataset-2 & 20821 & 10967 & 31788 \\
\hline
\end{tabular}

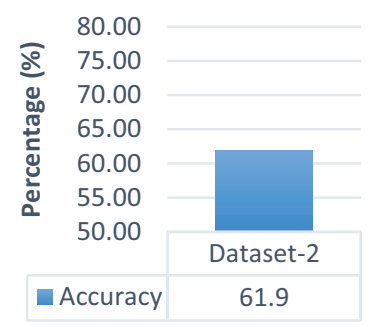

Figure 81.3 Accuracy of packet-level classification process.

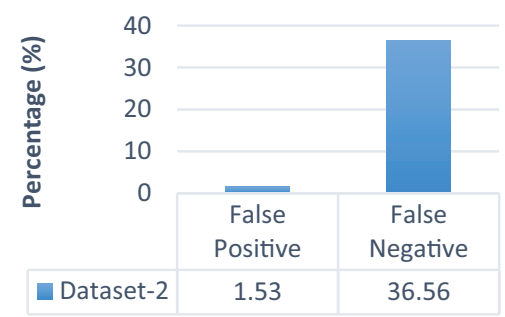

Figure 81.4 FP and FN rates of packet-level classification process.

The Dataset-1 is UNIBS traffic traces which belongs to the University of Brescia and is available publicly [24,25]. The Dataset- 2 consists of real-traffic traces which have been captured in a campus area network in a controlled environment using the Wireshark [26] tool, due to which it is known in advance regarding the flows that belong specifically to P2P-fs traffic and hence such traffic flows are labelled with actual applications accordingly for ground-truth verification. It consists of P2P traffic traces of popular filesharing applications, namely uTorrent, eMule, Transmission and qBitTorrent for analysis purposes. Both datasets (i.e. Dataset-1 and Dataset-2) consist of a mixture of various P2P applications (including P2P file-sharing applications) and non-P2P applications such as Skype, BitTorrent, Transmission, FTP, HTTP, SSL, DNS, etc.

The experiment conducted on Dataset- 2 shows that the packet-level classification process is able to achieve a classification accuracy of $61.90 \%$ only (as shown in Figure 81.3). In addition to that, it has $\mathrm{FP}$ and $\mathrm{FN}$ rates of $1.53 \%$ and $36.56 \%$, respectively (as shown in Figure 81.4). The packets related to connection establishment (i.e. initial communication) among the peers have also been captured in Dataset-2. Here, we observe that some of the peers used well-known P2P port numbers to establish the connection, after which they use random port numbers for communication. Therefore, some of the traffic gets identified at an early stage. However, if this initial communication is missing (or not captured), then the performance of the packet-level classification process would be much poorer. 


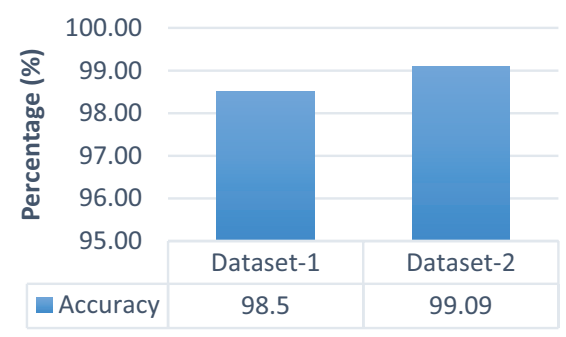

Figure 81.5 Overall classification accuracy of P2P-fs classification technique.

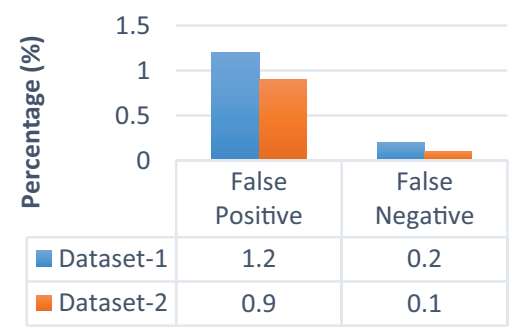

Figure 81.6 FP and FN rates of P2P-fs classification technique.

The combination of packet-level and flow-level classification processes achieved overall accuracy ranging between $98.5 \%$ and $99.05 \%$ (as shown in Figure 81.5 ). In addition to that, it has FP and FN rates ranging between 0.9 and $1.2 \%$ and 0.1 and $0.2 \%$, respectively (as shown in Figure 81.6). The proposed classification technique not only achieves high classification results, but also has low overhead (as minimum heuristics are used), classifies both TCP and UDP flows and works with encrypted traffic as well.

\section{Conclusion}

With the rapid development of the Internet, various P2P applications and services have emerged and are being adopted by large numbers of users. P2P applications consume a lot of network bandwidth and are one of the largest contributors of Internet traffic. Due to this fact, it poses various problems to the ISPs and network administrators such as dealing with network congestion, security, maintaining quality of service for other application, etc. Traditional traffic classification techniques such as port-based and payload-based are inefficient in classifying P2P traffic as various limitations are associated with them. In this research work, a 2-step P2P traffic classification technique has been proposed which specifically classifies P2P file-sharing traffic. We specifically focus on P2P file-sharing traffic since it is the largest contributor of P2P Internet traffic as a whole. The experimental results show that proposed technique achieves high accuracy over $98.5 \%$ in classifying P2P filesharing traffic. In addition to that, the proposed technique (a) works on both TCP and UDP protocols, (b) uses minimum heuristics for classification (and hence has less overhead) and (c) works with encrypted traffic and can be used for real-time classification as well.

However, the proposed technique has been made to work on offline traffic datasets and classifies P2P file-sharing traffic only. Therefore, our future work will focus on extending our technique to fine-grained P2P traffic classification, i.e. identifying other kinds of P2P 
traffic as well (in addition to file-sharing traffic) which contributes to P2P Internet traffic as a whole.

\section{References}

[1] W. Ye and K. Cho, "Hybrid P2P traffic classification with heuristic rules and machine learning," Soft Computing, vol. 18, no. 9, pp. 1815-1827, 2014.

[2] "Global Internet Phenomena," Sandvine, 2019.

[3] C. Labovitz, S. Iekel-Johnson, D. McPherson, J. Oberheide and F. Jahanian, "Internet interdomain traffic," ACM SIGCOMM Computer Communication Review, vol. 40, no. 4, pp. 75-86, 2010.

[4] J. Seibert, R. Torres, M. Mellia, M. M. Munafo, C. Nita-Rotaru and S. Rao, "The internetwide impact of $\mathrm{p} 2 \mathrm{p}$ traffic localization on isp profitability," IEEE/ACM Transactions on Networking, vol. 20, no. 6, pp. 1910-1923, 2012.

[5] "Service Name and Transport Protocol Port Number Registry" [Online]. Available: www. iana.org/assignments/service-names-port-numbers/service-names-port-numbers.xhtml.

[6] Y. Qi, L. Xu, B. Yang, Y. Xue and J. Li, "Packet classification algorithms: From theory to practice," in IEEE INFOCOM 2009, IEEE, 2009, pp. 648-656.

[7] A. W. Moore and K. Papagiannaki, "Toward the accurate identification of network applications," in International Workshop on Passive and Active Network Measurement, Springer, 2005, pp. 41-54.

[8] A. Madhukar and C. Williamson, "A longitudinal study of P2P traffic classification," in 14th IEEE International Symposium on Modeling, Analysis, and Simulation, 2006.

[9] “nDPI" [Online]. Available: www.ntop.org/products/deep-packet-inspection/ndpi [Accessed 2019].

[10] "WAND Network Research Group" [Online]. Available: http://research.wand.net.nz/software/libprotoident.php [Accessed 2019].

[11] S. Alcock and R. Nelson, "Libprotoident: traffic classification using lightweight packet inspection,” WAND Network Research Group, Tech. Rep., 2012.

[12] "Application Layer Packet Classifier for Linux" [Online]. Available: http://17-filter.sourceforge. net/.

[13] “Network Based Application Recognition (NBAR)," Cisco [Online]. Available: www.cisco. $\mathrm{com} / \mathrm{c} / \mathrm{en} / \mathrm{us} / \mathrm{products} / \mathrm{collateral} /$ ios-nx-os-software/network-based-application-recognitionnbar/prod_case_study09186a00800ad0ca.html.

[14] M. Bhatia and M. K. Rai, "Identifying P2P traffic: a survey," Peer-to-Peer Networking and Applications, vol. 10, no. 5, pp. 1182-1203, 2017.

[15] T. T. Nguyen and G. J. Armitage, "A survey of techniques for internet traffic classification using machine learning," IEEE Communications Surveys and Tutorials, vol. 10, no. 1-4, pp. 56-76, 2008.

[16] S. Park, H. Chung, C. Lee, S. Lee and K. Lee, "Methodology and implementation for tracking the file sharers using BitTorrent," Multimedia Tools and Applications, vol. 74, no. 1, pp. 271286, 2015.

[17] J. M. Reddy and C. Hota, "Heuristic-based real-time P2P traffic identification," in 2015 International Conference on Emerging Information Technology and Engineering Solutions, 2015.

[18] H. A. Jamil, B. M. Ali, M. Hamdan and A. E. Osman, "Online P2P Internet traffic classification and mitigation based on Snort and ML," European Journal of Engineering Research and Science, vol. 4, no. 10, pp. 131-137, 2019.

[19] B. M. A. Abdalla, H. A. Jamil, M. Hamdan, J. S. Bassi, I. Ismail and M. N. Marsono, "Multistage feature selection for on-line flow peer-to-peer traffic identification," in Asian Simulation Conference, 2017.

[20] G. Sajeev and L. M. Nair, "LASER: A novel hybrid peer to peer network traffic classification technique," in 2016 International Conference on Advances in Computing, Communications and Informatics (ICACCI), 2016. 


\section{Intelligent Circuits and Systems}

[21] "List of TCP and UDP port numbers" [Online]. Available: www.wikiwand.com/en/List_of_ TCP_and_UDP_port_numbers [Accessed 2019].

[22] “jNetPcap" [Online]. Available: https://sourceforge.net/projects/jnetpcap/ [Accessed 2019].

[23] "Weka" [Online]. Available: www.cs.waikato.ac.nz/ml/weka [Accessed 2019].

[24] F. Gringoli, L. Salgarelli, M. Dusi, N. Cascarano, F. Risso and others, "Gt: picking up the truth from the ground for internet traffic," ACM SIGCOMM Computer Communication Review, vol. 39, no. 5, pp. 12-18, 2009.

[25] M. Dusi, F. Gringoli and L. Salgarelli, "Quantifying the accuracy of the ground truth associated with Internet traffic traces,” Computer Networks, vol. 55, no. 5, pp. 1158-1167, 2011.

[26] "Wireshark" [Online]. Available: www.wireshark.org [Accessed 2019]. 


\title{
82 A review of crop disease and pest forewarning using machine learning
}

\author{
Sachit Dubey, *, Raju Barskar ${ }^{2, *}$, Anjna Jayant Deen ${ }^{2, *}$ \\ ${ }^{1}$ Reseach Scholar and ${ }^{2}$ Assistant Professor, Department of Computer Science and \\ Engineering, University Institute of Technology, RGPV, Bhopal (M.P.), India
}

\section{Introduction}

Agriculture is the backbone of the Indian economy as it accounts for nearly $13.9 \%$ of India's gross domestic product (GDP) and employs $54.6 \%$ of its workforce. Although the loss due to pest and disease has declined after the green revolution they still pose a significant threat. Weather plays an important role in the development of crop diseases as well as in pest attacks. Favourable weather conditions often greatly affect the pathogenic life cycle; some pathogens lie dormant in normal weather conditions but may become active in the case of extreme weather conditions. On the basis of weather and pest interaction, various weather-based prediction rules were developed by various eminent research scholars and scientists. Pest surveillance and forecasting of pest and disease are key protection strategies under the climatic change scenario. Forecasting generally refers to the estimation of the forthcoming outbreak of crops and disease. For the purpose of the development and use of this model, we require both metrological and biological data. Advances in the field of artificial intelligence and machine learning have completely revolutionized the area of predictive modelling. The machine learning technique provides more accuracy and fewer errors than traditional regression techniques and is being used in various fields for performing classification tasks. Through this paper, we aim to look at various developments that have taken place in the field of disease and pest management using machine learning and find the future scope of improvement. For understanding crop disease and pest management, one has to study their classification as well as their relationship with various weather parameters in great detail.

\section{Literature survey}

P.B. Jawade et al. (2019) [1] developed a disease prediction module to predict the thrips outbreak in mango using machine learning and IoT (Internet Of Things). The authors used both past as well as live weather data using in-field sensors to forecast the attack in real time. The experiment was performed near Devgad Taluka. The authors made use of a random forest algorithm for the development of the prediction model.

Rajni Jain et al. (2007) [2] performed a case study on the performance of various machine learning algorithms (logistic regression, decision tree, C 4.5, rough set theory, rough set based decision tree (RDT)) for predicting the outbreak of powdery mildew of mango (PWM) caused by Oidium mangiferae Bertehet. The authors used the metrological

\footnotetext{
*Emails: sachit30@gmail.com, rajubaraskar@rgtu.net, anjnadeen@yahoo.co.in
}

DOI: 10.1201/9781003129103-82 
Table 82.1 A detailed overview of various research articles that have used the machine learning approach for predicting crop diseases

\begin{tabular}{|c|c|c|c|c|c|}
\hline Crop & Disease/pest & Algorithm & Data set & Result & Author \\
\hline Mango & Thrips & Random forest & $\begin{array}{l}\text { Temperature and } \\
\text { humidity }\end{array}$ & Gives low error estimates & $\begin{array}{l}\text { P.B. Jawade et al. } \\
\text { (2019) [1] }\end{array}$ \\
\hline Mango & Powdery mildew & $\begin{array}{l}\text { Rough sets, linear } \\
\text { regression, rough sets } \\
\text { based decision, decision } \\
\text { tree }\end{array}$ & $\begin{array}{l}\text { Temperature and } \\
\text { humidity }\end{array}$ & Average accuracy of $75 \%$ & $\begin{array}{l}\text { Rajni Jain et al. } \\
\text { (2007) [2] }\end{array}$ \\
\hline Rice & Blast & $\begin{array}{l}\text { Long-term memory } \\
\text { network (LSTM) }\end{array}$ & $\begin{array}{l}\text { Temperature and } \\
\text { humidity }\end{array}$ & Maximum accuracy of $79.4 \%$ & $\begin{array}{l}\text { Yongseon Kim et al. } \\
\text { (2017) [3] }\end{array}$ \\
\hline Coffee & Rust & Fuzzy & $\begin{array}{l}\text { Temperature and } \\
\text { humidity }\end{array}$ & $\begin{array}{l}\text { Fuzzy was able to present } \\
\text { competitive error rate as } \\
\text { compared to the classical } \\
\text { model }\end{array}$ & $\begin{array}{l}\text { Marcos E. Cinta } \\
\text { et al. (2011) [4] }\end{array}$ \\
\hline Cherry & $\begin{array}{l}\text { A general disease of } \\
\text { cherry }\end{array}$ & Discriminant Analysis & $\begin{array}{l}\text { Temperature, } \\
\text { humidity, rainfall } \\
\text { and wind speed }\end{array}$ & $\begin{array}{l}\text { maximum prediction accuracy of } \\
93.6 \% .\end{array}$ & Ilic et al. (2018) [5] \\
\hline Wheat & $\begin{array}{l}\text { Yellow rust, aphids, } \\
\text { and powdery mildew }\end{array}$ & $\begin{array}{l}\text { Linear discriminant } \\
\text { analysis (LDA) }\end{array}$ & Spectral features & Maximum accuracy of $87 \%$ & $\begin{array}{l}\text { Zhang et al. (2017) } \\
{[6]}\end{array}$ \\
\hline Rice & Blast & $\begin{array}{l}\text { Artificial neural network } \\
\text { (ANN) }\end{array}$ & $\begin{array}{l}\text { Temperature and } \\
\text { humidity }\end{array}$ & The average accuracy of $72 \%$. & $\begin{array}{l}\text { Jia-you Hsieh et al. } \\
\text { (2019) [7] }\end{array}$ \\
\hline Pomegranate & $\begin{array}{l}\text { The general diseases of } \\
\text { pomegranate }\end{array}$ & Hidden Markov chain & $\begin{array}{l}\text { Temperature, } \\
\text { humidity and } \\
\text { wind speed }\end{array}$ & Accuracy of $80.7 \%$ & $\begin{array}{l}\text { Dnyanesh Nawale } \\
\text { et al. (2018) [8] }\end{array}$ \\
\hline
\end{tabular}


parameters temperature and humidity for forewarning the spread of an outbreak. The results showed that JCP and RJP had better prediction accuracy than other models.

Yongseon Kim et al. (2017) [3] developed a model for predicting the outbreak of rice blast using a long-term memory network (LSTM). The dataset consisted of historical data on rice blast occurrence in three regions of Cheol won, Iheon and Milyong in Korea as well as climatic data associated with the regions for the duration of 2003-2006. The result provided a maximum accuracy of $79.4 \%$ with LSTM.

Marcos E. Cinta et al. (2011) [4] developed a model for forewarning the outbreak of coffee rust using a metrological dataset. The authors compared the performance of traditional as well as the fuzzy decision tree algorithm. The result showed that a fuzzy decision tree (fuzzy) was able to present a competitive error rate as compared to the classical model.

Ilic et al. (2018) [5] used a data mining method to detect disease in cherry plants using discriminant classification analysis. The data set consisted of metrological parameters such as temperature, humidity, rainfall and wind speed. The authors performed a comparison of various algorithms that come under Discriminant analysis namely linear discriminant analysis, quadratic discriminant analysis, pseudo linear discriminant analysis, and compact classification tree. The result showed that the compact classification tree provided maximum prediction accuracy of $93.6 \%$.

Leonardo G. Cordova et al. [6] developed a system for forecasting anthracnose and botrytis fruit rots in strawberry. The model used leaf wetness and temperature to predict the outbreak of disease. The authors developed an online advisory system to predict the outbreak of these diseases using the Florida agriculture weather network.

Jia-you Hsieh et al. [7] developed a model for predicting rice blast disease based on machine learning and neural network. The dataset used in the experiment used weather data collected from different zones of Taiwan from 2014 to 2018. The authors mainly used two parameters, namely temperature and humidity. The classifier provided an accuracy of $72 \%$.

Dnyanesh Nawale et al. (2018) [8] used both machine learning and IoT for detecting Pomegranate disease. Pomegranate is a high profile fruit from Asia. But due to continuous changing climatic factors, the losses caused by pest and disease have been increasing in recent years. In order to minimize the loss due to climatic factors, the authors developed a model for predicting the disease outbreak using metrological parameters such as temperature, humidity, moisture and wind speed. The model was developed using hidden Markov chain, providing an accuracy of $80.7 \%$. Table 1 shows a detailed overview of various research articles that have used Machine learning approach for predicting crop diseases.

Table 82.2 Various metrological parameters required in a dataset

\begin{tabular}{llll}
\hline S. no. & Parameter & Description & Units \\
\hline 1 & Maximum temperature & Highest air temperature recorded for the day & ${ }^{\circ} \mathrm{C}$ \\
2 & Minimum temperature & Lowest air temperature recorded that day & ${ }^{\circ} \mathrm{C}$ \\
3 & Average temperature & Average temperature recorded for particular location & ${ }^{\circ} \mathrm{C}$ \\
4 & Relative humidity & It is a measure of the actual amount of water vapour & $\%$ \\
& & in the air compared to the total amount of vapour & that can exist in the air at its current temperature \\
5 & Precipitation & Condensation of rain and water vapour from & $\mathrm{mm}$ \\
& Evapotranspiration & atmosphere & $\mathrm{mm}$ \\
\hline
\end{tabular}




Algorithm Concept
$\begin{aligned} & \text { (I) Supervised learning } \\ & \text { k-Nearest }\end{aligned}$ The k-nearest neighbour algorithm is a supervised learning algorithm that
neighbour
makes a decision on the basis of "feature similarity" between unlabelled
and labelled values present in the training data set. It is based on the
philosophy that similar things exist in close proximity to one another
just like "birds of a feather flock together". k-NN captures the idea of
similarity in proximity in mathematical terms as defined as a distance
between two points within a dimensional space. This distance can be
Euclidian, Manhattan or Hamilton. Generally, the Euclidian distance
between two points is used to calculate the proximity between two data
points.

$$
d(\mathbf{p}, \mathbf{q})=\sqrt{\sum_{i=1}^{N}\left(p_{i}-q_{i}\right)^{2}}
$$

Advantage

Disadvantage

Equation 1: Euclidian distance between two points $p$ and $q$

The k-NN has the following steps:

- Step 1: load the data set.

- Step 2: initialize value of $\mathrm{k}$ on the basis of the chosen number of neighbours.

- Step 3: for each unlabelled data value the following steps are performed:

(I) Calculate proximity between the unlabelled data value and each labelled value present in the training dataset.

(II) Arrange the unlabelled data value in ascending order of their proximity with the data values present in the training dataset.

(III) Choose the value which is closest to the unlabelled value.

(IV) Put the unlabelled data value in the same class as the chosen value in the previous step.

(i) No training period, i.e. it learns only at the time of making prediction

(ii) New data can be added easily without disturbing the accuracy

(iii) It is easy to implement as it requires only two parameters (i) Not good for classification of large dataset

(ii) Struggles with high dimensional data

(iii) Feature scaling is done before applying k-NN in order to avoid wrong prediction

(iv) Has high sensitive towards noisy data

- Step 4: END. 
Decision tree

Decision tree is a supervised learning algorithm which makes use of decision rules inferred from data values inferred from values to predict a target parameter. The decision tree is a structure that includes a root node, branch node and leaf node. Each internal node represents a test on an attribute and each branch represents the outcome of a test and leaf nodes hold class labels. For predicting weather data the decision tree splits the attributes repeatedly into subgroups using recursive portioning. Recursive portioning is a statistical method used for multivariable analysis. Recursive portioning generates a decision tree in which data members are classified using independent variables. The following steps are involved in each

recursive phase of the algorithm.

- Step 1: select the right attribute to perform the split. Various criteria can be used to select the right attribute for splitting.

- Step 2: the training dataset is split into various subgroups.

- Step 3: generate a subtree for each branch until no further splitting is possible.

Random

forest

Random forest is a supervised learning algorithm that creates a forest with the various decision trees in which each tree outputs a class and the class with most votes is chosen for production. It is based on the philosophy that the performance of several models operating as a committee will outperform any of the individual models. There are mainly two phases in the random forest procedure:

First Phase: creating the random forest

- Randomly select k-features from a given set of $\mathrm{M}$ features such that $\mathrm{k}<\mathrm{M}$.

- Find the node with best split point among $\mathrm{k}$-features.

- Divide the selected node into various daughter nodes.

- Perform steps 1 to 3 until a determined outcome is reached.

- Perform steps 1 to 4 till $\mathrm{N}$ decision trees are created.

Second Phase: prediction from random forest

- Calculate the value provided by every diction tree.

- Enumerate vote for every predicted value.

- Choose value with the highest vote.

(i) Requires less effort in pre-processing data

(ii) Dataset need not be normalized before use

(iii) Does not require feature scaling

(iv) Presence of noise does not affect process of creating decision tree

(v) Easy to explain technical terms to stakeholder

(i) Can be used to solve both classification and regression problems

(ii) Works well for both categorical and continuous value

(iii) Handling of nonlinear parameters is efficient

(iv) Introduction of new data point does not affect stability of the system (i) Small changes in dataset can alter the structure of the decision tree, leading to instability

(ii) Calculations can become more complex compared to other algorithms

(iii) It requires a long training period

(iv) It proves inadequate for regression and continuous values

(i) May lead to high complexity as it takes into consideration output from various trees

(ii) Has a longer training period 
Table 82.3 Cont.

\begin{tabular}{|c|c|c|c|}
\hline Algorithm & Concept & Advantage & Disadvantage \\
\hline $\begin{array}{l}\text { Support } \\
\text { vector } \\
\text { machine } \\
\text { (SVM) }\end{array}$ & $\begin{array}{l}\text { SVM or supervised learning is an algorithm which is used for both } \\
\text { classification and regression practices. The SVM looks at the extreme of } \\
\text { data sets and draws a boundary known as a hyperplane and segregates the } \\
\text { two classes. Data points that are close to each other's class are known as } \\
\text { support vectors. The algorithm looks only at the extreme ends of a class } \\
\text { for classification rather than looking at every value of the dataset. It can be } \\
\text { used for both linear and nonlinear datasets. For transforming a nonlinear } \\
\text { dataset into a linear data set SVM uses kernel functions which export the } \\
\text { data set into multidimensional space. } \\
\text { There are various steps involved in SVM prediction: } \\
\text { - Step 1: check whether the data set is linear or not. If data set is nonlinear } \\
\text { than export the data set to multidimensional space by mapping input } \\
\text { features to higher dimensional space using kernel. } \\
\text { - Step } 2: \text { search for the optimal hyperplane for which margin of separation } \\
d \text { is maximum. Consider a hyperplane } w^{T} x+b=0 \text {, where w is the weight } \\
\text { vector, x is input vector and b is bias. To find the maximum separation } \\
d \text { we need to minimize }\|w\| \text { and no data point should be found between } \\
\text { margin, that is }\end{array}$ & $\begin{array}{l}\text { (i) More effective for high } \\
\text { dimensional space } \\
\text { (ii) Works well if number } \\
\text { of samples is less than } \\
\text { number of dimensions } \\
\text { (iii) Is memory efficient }\end{array}$ & $\begin{array}{l}\text { (i) Not suited for large } \\
\text { dataset } \\
\text { (ii) Does not perform } \\
\text { well for noisy } \\
\text { data sets } \\
\text { (iii) It under-performs } \\
\text { when number of } \\
\text { features for each } \\
\text { data point exceeds } \\
\text { the number of } \\
\text { training data } \\
\text { samples }\end{array}$ \\
\hline
\end{tabular}

minimizing $\|w\|$ such that discrimination boundary is obeyed. Langrenger for SVM is given as

$\min \mathrm{L}_{\mathrm{p}}=\frac{1}{2}\|w\|^{2}-\sum_{i=1}^{l} a_{i} y_{i}\left(x_{i} \cdot w+b\right)+\sum_{i=1}^{1} a_{i}, \forall i, a_{i} \geq 0$

- Step 3: on differentiating $L p$ w.r.t $w$ and $b$ and equating it to zero we get

$w=\sum_{i=1}^{l} a_{i} y_{i} x_{i} \quad \& \sum_{i=1}^{l} a_{i} y_{i}=0$ 
Artificial

neural

network

$(\mathrm{ANN})$

An artificial neural network is an interconnected multi-layer network of special nodes known as neurons. These neurons are special nodes which trigger a response only when the input reaches a particular threshold value which is checked using a special function known as an activation function. The general system of the neural network consists of the input layer, the hidden layer and the output layer. The output of one layer is treated as an input for the other layer. The neurons are connected to one another using a link known as a synapsis; each of these links has a value associated with it known as weight.

The prediction mechanism of the neural network consists of two phases.

Phase 1: Propagation

- Generate output by feeding values to the network

$$
y=\varnothing\left(\sum_{j=0}^{n} w_{k j} * x_{j}\right)
$$

where $w$ is weight and $x$ is input.

- Calculate the cost and determine the error term.

- Estimate the difference between the actual value and predicted value.

Phase 2:

- Change the weight of the neurons accordingly such that the difference between the actual and predicted value is minimized.

\section{(II) Unsupervised learning}

k-Means clustering
$\mathrm{k}$-Means clustering is an unsupervised learning algorithm that partitions the dataset into predefined non-overlapping clusters where each data value belongs to a single cluster. It tries to make members of a cluster as similar as possible. It assigns an unknown data point to a particular cluster on the basis of proximity of the given data point from the centroid of the cluster, i.e. the distance between the data point and the centroid. The mechanism of k-means clustering consists of the following phases.

Phase 1 initialize clusters

- Step 1: specify the number of clusters.

- Step 2: initialize centroid by shuffling the dataset and randomly select $\mathrm{k}$ data point for centroid.

- Step 3: perform steps 1 and 2 till there is no change to the centroid. (i) Can work with incomplete information

(ii) High fault tolerance

(iii) Supports parallel processing capability (i) Has high hardware dependency

(ii) There is no specific rule to determine the structure of a neural network (i) Its implementation is simple

(ii) Works well with large dataset

(iii) Can easily adapt for new examples

(iv) Provides convergence (i) It depends highly on initial value

Generates cluster with varying size and density (iii) Problems of scaling for higher dimensional data 


\begin{tabular}{|c|c|c|c|}
\hline Algorithm & Concept & Advantage & Disadvantage \\
\hline $\begin{array}{l}\text { Fuzzy } \\
\text { clustering }\end{array}$ & $\begin{array}{l}\text { Fuzzy clustering is an unsupervised learning algorithm in which each data } \\
\text { point is assigned a probability of belonging with respect to each predefined } \\
\text { cluster. Unlike the k-means clustering algorithm in which the probability of } \\
\text { belonging for a particular cluster can be either } 0 \text { or } 1 \text { the fuzzy values can } \\
\text { be anything between } 0 \text { and } 1 \text {. In the fuzzy clustering centroid of data, the } \\
\text { point is calculated first followed by a distance of each point from a given } \\
\text { centroid. This process is repeated until clusters become constant. } \\
\text { The algorithm involves the following steps } \\
\text { - Step 1: initialize the data points into the desired number of clusters } \\
\text { randomly. }\end{array}$ & $\begin{array}{l}\text { (i) Performance briefly } \\
\text { for overlapping } \\
\text { dataset } \\
\text { (ii) Data points belong } \\
\text { to all the clusters to } \\
\text { some degree }\end{array}$ & $\begin{array}{ll}\text { (i) A priori specification } \\
\text { for number of clusters } \\
\text { (ii) } & \text { Lower value of } \\
& \text { terminal citation give } \\
& \text { better result but more } \\
& \text { iteration }\end{array}$ \\
\hline
\end{tabular}

$\gamma$ is fuzzy membership value, $m$ is the fuzziness parameter and $x_{k}$ is data point.

- Step 3: find the distance of data point from centroid.

- Step 4: update membership value.

$$
\gamma=\left[\left\{\sum_{1}^{n}\left(d_{k i}^{2} / d_{k j}^{2}\right)\right\}^{1 / m-1}\right]^{-1}
$$

- Step 5: perform steps 2 and 4 until the clusters become constant. 


\section{Parameters used for disease forecasting}

All past research work used past metrological data associated with the region where a given crop is grown such as temperature, humidity, moisture, wind speed, etc. for prediction. They also used information about incidents of disease outbreak and metrological data associated with the region to devloped a correlational model for forecasting. Table 2 shows various metrological parameters required in a dataset.

\section{Machine learning algorithms used for forecasting}

There are various machine learning algorithms that operate in the domain of predictive analysis. Each of these algorithms performs differently for different types of datasets; some may perform better with statistical data sets while others may perform better for a dataset consisting of a multimedia dataset. There are mainly two broad categories of machine learning algorithms, namely supervised and unsupervised. Table 3 shows various Machine learning algorithm's used for disease forecasting.

\section{Conclusion}

Pest and disease pose a major threat to agricultural production across the world. On the basis of weather and pest interaction, we can develop prediction rules and find conical weather conditions which support outbreaks of pest and disease. The weather-based forewarning and modelling of pest disease is an important component of crop production strategies. In this paper, we survey various machine learning techniques that are being used in crop disease modelling. We also studied the interaction and interrelation between various climatic factors and crop disease. In the end, we discussed and prosed a future scope in the field of weather-based crop disease modelling.

\section{References}

[1] Jawade, P. B., Chaugule, D., Patil, D., and Shinde, H. (2019, March). Disease prediction of mango crop using machine learning and IoT. In International Conference on E-Business and Telecommunications (pp. 254-260). Springer, Cham.

[2] Jain, R., Minz, S., and Ramasubramanian, V. (2009). Machine learning for forewarning crop diseases. J Ind Soc Agril Stat, 63(1), 97-107.

[3] Kim, Y., Roh, J. H., and Kim, H. Y. (2018). Early forecasting of rice blast disease using long short-term memory recurrent neural networks. Sustainability, 10(1), 34.

[4] Cintra, M. E., Meira, C. A., Monard, M. C., Camargo, H. A., and Rodrigues, L. H. (2011, November). The use of fuzzy decision trees for coffee rust warning in Brazilian crops. In 2011 11th International Conference on Intelligent Systems Design and Applications (pp. 13471352). IEEE.

[5] Ilic, M., Ilic, S., Jovic, S., and Panic, S. (2018). Early cherry fruit pathogen disease detection based on data mining prediction. Computers and Electronics in Agriculture, 150, 418-425.

[6] Cordova, L. G., Madden, L. V., Amiri, A., Schnabel, G., and Peres, N. A. (2017). A meta-analysis of a web-based disease forecast system for control of anthracnose and Botrytis fruit rots of strawberry in the Southeastern United States. Plant Disease, 101(11), 1910-1917.

[7] Hsieh, J. Y., Huang, W., Yang, H. T., Lin, C. C., Fan, Y. C., and Chen, H. (2019). Building the Rice Blast Disease Prediction Model based on Machine Learning and Neural Networks (No. 1197). EasyChair.

[8] Pawara, S., Nawale, D., Patil, K., and Mahajan, R. (2018, April). Early detection of pomegranate disease using machine learning and the Internet of Things. In $20183 \mathrm{rd}$ International Conference for Convergence in Technology (I2CT) (pp. 1-4). IEEE. 


\title{
83 Modelling of inverters using $\mathrm{MoS}_{2}$ based transistors
}

\author{
Murali Krishna M. ${ }^{1,2, *}$, Prasantha R. Mudimela ${ }^{1, *}$ \\ ${ }^{1}$ School of Electronics \& Electrical Engineering (SEEE), Lovely Professional \\ University, Phagwara, Punjab, India \\ ${ }^{2}$ Department of Electronics and Communication Engineering, \\ Anurag Group of Institutions, Hyderabad, India
}

\section{Introduction}

The scaling of conventional silicon transistors has now become complex due to the significant degradation of mobility of carriers and increase in wire resistance, so the researchers now have started to look to various unique materials in accordance with meeting ITRS goals. Two dimensional (2D) structures like CNTs, graphene, GNRs and TMDs with chemical $\mathrm{MX}_{2}$ forms (e.g. $\mathrm{MoS}_{2}$ ) with its smooth and stable honeycomb structure and excellent properties have received significant attention [1-3]. The researchers are interested in 2D materials such as CNTs, graphene and TMDs. [4]. In particular, until recently there have been missing experimental studies on atomic 2D crystals due to the complexity in identifying them [4,5]. Graphene is semi-metallic in its pure 2D structure and has zero band gap, while a band gap can be opened by separating graphene into graphene nanoribbons (GNRs) of less than $10 \mathrm{~nm}$. Problems such as process variability, loss of mobility and roughness of the line edge significantly deteriorate its efficiency [5,6]. TMDs, in particular, have a $1-2 \mathrm{eV}$ band gap by default without being scaled down to the thinner nanometre scale, significantly reducing manufacturing difficulties and also minimizing unintended process variance results. So TMD transistors with field effect transistors (FETs) were deemed as a useful design for transistors in the post CMOS era [7, 8]. The 2D TMDs with chemical compounds $\mathrm{MX}_{2}$, where $\mathrm{M}$ is of a transition metal and $\mathrm{x}$ is for $\mathrm{S}$, Se, or Te, are members of the same class proposed for the potential of this layered material family for wearable electronics, which could be a major part of wearable devices and sensors [9]. The TMDs are chosen as the channel material whereas the substrate, the oxide of the gate and the transistor electrodes are made of different materials. The simulation of electrical properties is essential in order to improve $2 \mathrm{D}$ material-based transistor design, as is device creation optimization, output prediction and low-power switching circuit [10-13]. Here we propose a $\mathrm{MoS}_{2}$ transistor model and study the voltage-current (I-V) properties of single layer $\mathrm{MoS}_{2}$ FETs. In this work, the compact $\mathrm{MoS}_{2}$ FET model is introduced and is further simplified by obtaining more precisely formed approximations which reduce computational difficulty to become compatible with SPICE. The model is implemented in SPICE and simulations are performed to calculate circuit levels in order to determine the efficiency of $\mathrm{MoS}_{2}$ FET-based circuits. It enables the exploration of space and the measurement of process transitions at the circuit level. A monolayer $\mathrm{MoS}_{2}$ has low leakage current with a high-effective mass value and a significantly larger band gap than a 10 -nm tube.

\footnotetext{
*Emails: murali.41800082@lpu.in, prasantha.22708@1pu.co.in
}

DOI: $10.1201 / 9781003129103-83$ 
In this article, we analysed a single layered $\mathrm{MoS}_{2}$ transistor system in accordance with the ITRS specifications and tested and verified the results against the various channel dimension values and technical requirements. The remaining part of this paper is presented as: Section II gives more information on $\mathrm{MoS}_{2}$ transistor modelling and introduces the proposed versatility supporting a SPICE-compatible platform. The experimental results are discussed in Section III. The conclusions are drawn in Section IV.

\section{II. $\mathrm{MoS}_{2}$ transistor modelling}

The physical effective thickness, gate length of the oxide and the power supply of the 2026 LOP devices, predicted by ITRS, are $0.5 \mathrm{~nm}, 5.8 \mathrm{~nm}$, and $0.43 \mathrm{~V}$ respectively [14]. The channel is undoped. Strongly doped drain and source contacts are believed to be $10^{20} \mathrm{~cm}^{-3}$ doping performance for transistor $\mathrm{MoS}_{2}$ and $10^{19} \mathrm{~cm}^{-3}$ for silicon transistor ultra-thin body. Figure 83.1 shows the transistor spice model in which drain $(D)$, gate $(G)$, source $(S)$, and bulk (B) and $I_{d s}$ are the terminal electrodes and $I_{d s}$ is drain current. Capacitors formed from the neighbouring electrodes are $C_{g s}, C_{g d}, C_{b d}$, and $C_{b s}$. Such capacitors are viewed as parallel plate capacitors and we can calculate with $C_{b s}=C_{b d}=$ W.L.C. $C_{b}$ and $C_{g s}=C_{g d}=$ W.L.C. $C_{t}$ and $E_{0}$ which is the zeroth sub-band energy, is useful in calculation of $V_{c}$. $E_{0}$ is determined from the band gap $E_{g}$, which depends on the material. The effective channel length $L \_e f f=L+\left(\left|V_{-} d s\right|\right) / v_{-} s a t$ where $v_{\text {sat }}$ is the velocity of the carrier in saturation. Such doping volumes cause the source lead bands to be aligned with the Fermi source of the various devices. The drain and source electrodes contact with the single layer $\mathrm{MoS}_{2}$ and are considered as ohmic. The source terminal is grounded, and the reference potential is taken into consideration. The electrostatics of this unit are easily understandable through the capacitive equivalent circuit depicted in Figure 83.1. The $C_{q}$ represents the $2 \mathrm{D}$ sheet's quantum capacitance; the top and bottom oxide capacitances are $C_{t}$ and $C_{b}$. Through integrating the expression for overall energies, the charge density per unit area is measured and expressed as $Q_{c}=Q_{p}+Q_{n}=-q \int_{0}^{\infty} D(E) f\left(E-E_{F}\right) d E$; here $E$ represents energy, $Q_{p}$ is hole charge contribution, $Q_{n}$ is electron charge contributions. $f(E)$ represents the Fermi-Dirac function, $E_{F}=q V_{c}$ represents the Fermi level, the semiconductor mid-gap is considered as the reference point. The $V_{c}$ is the channel voltage which gives the voltage drop at the $C_{q}$ or surface potentials. The quantum capacitance is given by $C_{-} q=\left(d Q_{-} c\right) /\left(d V_{-} c\right)$. The total charge $Q_{c}$ as a function of $V_{c}$ is linear for $q\left|V_{c}\right| \geq\left|E_{0}\right|$. For the measurement of hole and electron concentrations $V_{p}(x)$ and $V_{n}(x)$ respectively two different levels of

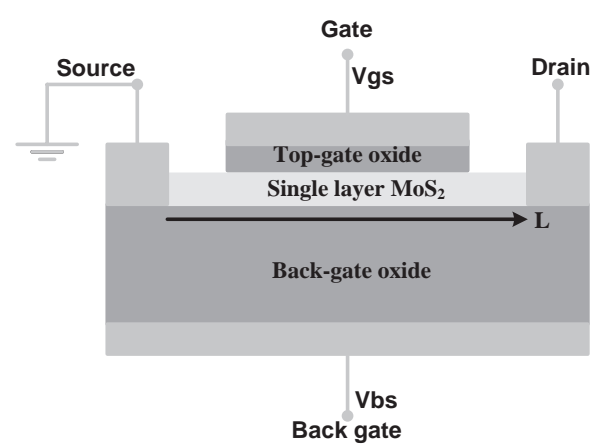

(a)

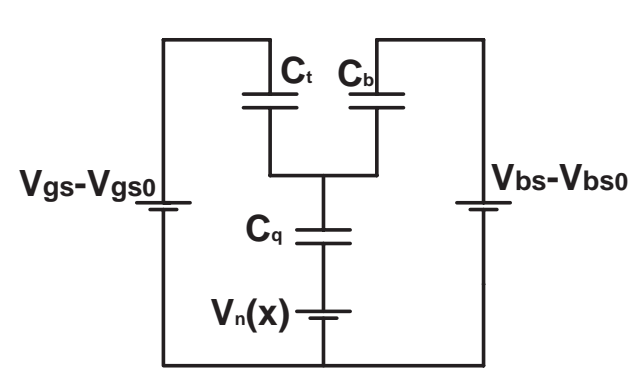

(b)

Figure 83.1 (a) Cross section of single layer $\mathrm{MoS}_{2}$ transistor. (b) Transistor equivalent capacitive circuit. 


\section{4}

Intelligent Circuits and Systems

quasi-Fermi should be taken into consideration. The $x$ shows the direction of transport of the charges. We considered the modelling of unipolar n-type $\mathrm{MoS}_{2}$ FETs. The same can be extended to p- type $\mathrm{MoS}_{2}$ FETs. By using the basic circuit law for the capacitive circuit, we get the $V_{c}$ channel voltage relation as shown:

$$
V_{c}(x)=\frac{Q_{n}\left(V_{c}\right)}{C_{b}+C_{t}}+\left(V_{g s}-V_{g s 0}-V_{n}(x)\right) \frac{C_{t}}{C_{b}+C_{t}}+\left(V_{b s}-V_{b s 0}-V_{n}(x)\right) \frac{C_{b}}{C_{b}+C_{t}}
$$

$V_{n}(x)$ is the given voltage at channel position $x . V_{g s}$ is the top gate to source voltage and $V_{g s}$ is the voltage of back gate to source. The top gate capacitance $C_{t}$ is calculated by using $C_{-} t=\varepsilon_{-}$topgate $/ T_{-}($ox,topgate $) \quad$ and $\quad C_{-} b=\varepsilon_{-}$bottomgate $/ T_{-}$(ox, bottomgate $)$, $V_{n}(x=0)=V_{s}=0, V_{n}(x=L)=V_{d s}$, the drain to source voltage. $V_{b s}-\bar{V}_{b s 0}$ and $V_{g s}-V_{g s 0}$ are the back gate and top gate source voltage overdrive, respectively. When $\left|q V_{c}\right| \geq\left|E_{0}\right|$, the $Q_{c}\left(V_{c}\right)$ is linear and exponential when $\left|q V_{c}\right| \leq\left|E_{0}\right|$. For modelling of the drain current of a single layer n-type $\mathrm{MoS}_{2}$ FET a drift diffusion transportation is assumed which takes the form of $I_{d s}=-W Q_{n}(x) V(x)$, here $W$ is the width of the gate, and $V(x)$ is the drift velocity of an electron and is given by $V=\mu E$, where $E$ is the electric field, $\mu$ is the effective electron mobility which is considered to be not dependent on the carrier's density, field, or the temperature. This proposed model pertains as long as the length of the channel is much above the electron mean free path. The electric field $E$ is given by $E=\left(d V_{-} n(x)\right) / d x \cong\left(d V_{-} n\right) / L$. The $I_{-} d s=\mu W Q_{-} n(x)\left(d V_{-} n\right) / L$. Integration of the corresponding expression over the channel length of the device is used to express the explicit drain current in expression $I_{d s}=\mu \frac{W}{L} \int_{0}^{V_{d s}} Q_{n} \frac{d V_{n}}{d V_{c}} d V_{c}$.
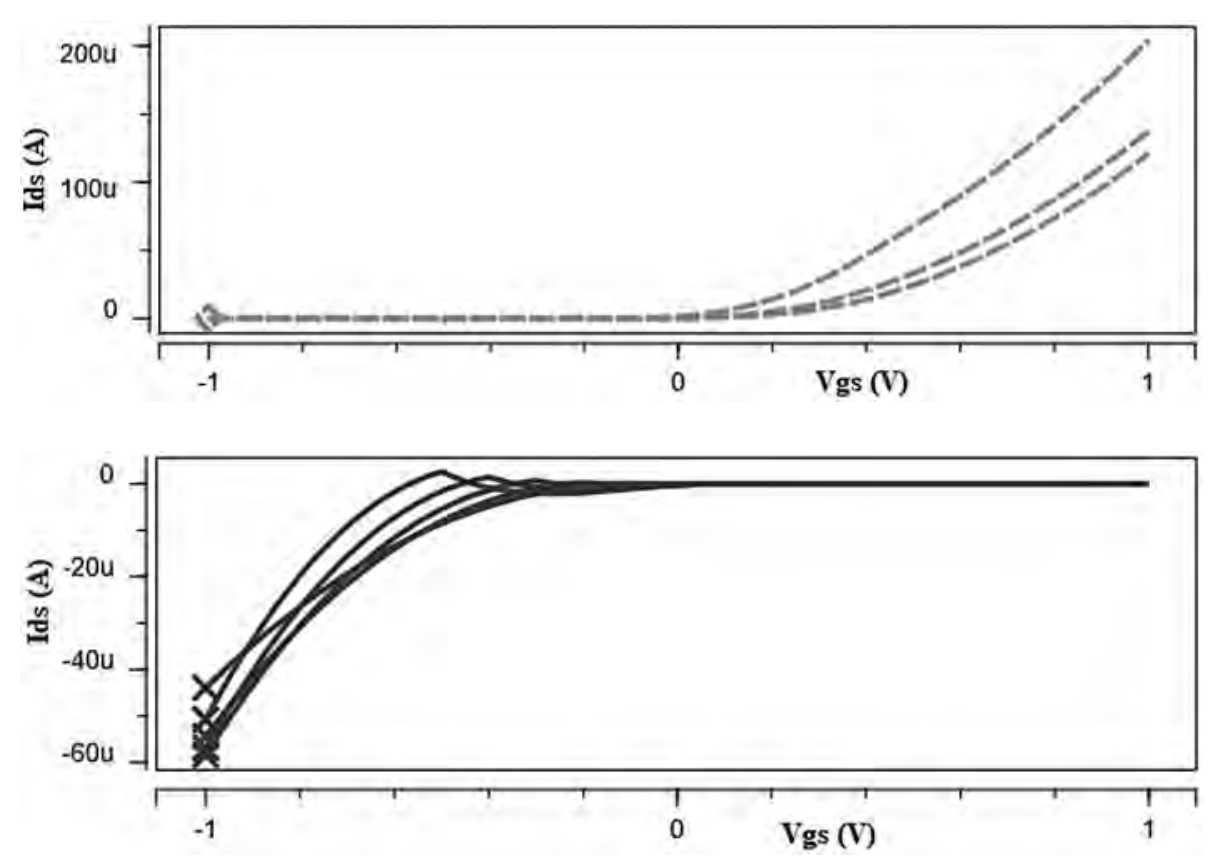

Figure 83.2 (a) n-Type $\mathrm{MoS}_{2}$ based transistor $I_{d s}$ vs. $V_{g s}$. (b) p-Type $\mathrm{MoS}_{2}$ based transistor $I_{d s}$ vs. $V_{g s}$. (c) Inverter based on $\mathrm{MoS}_{2}$ transistor. $V_{\mathrm{dd}}, V_{\text {in }}, V_{\text {out }}$, power. 

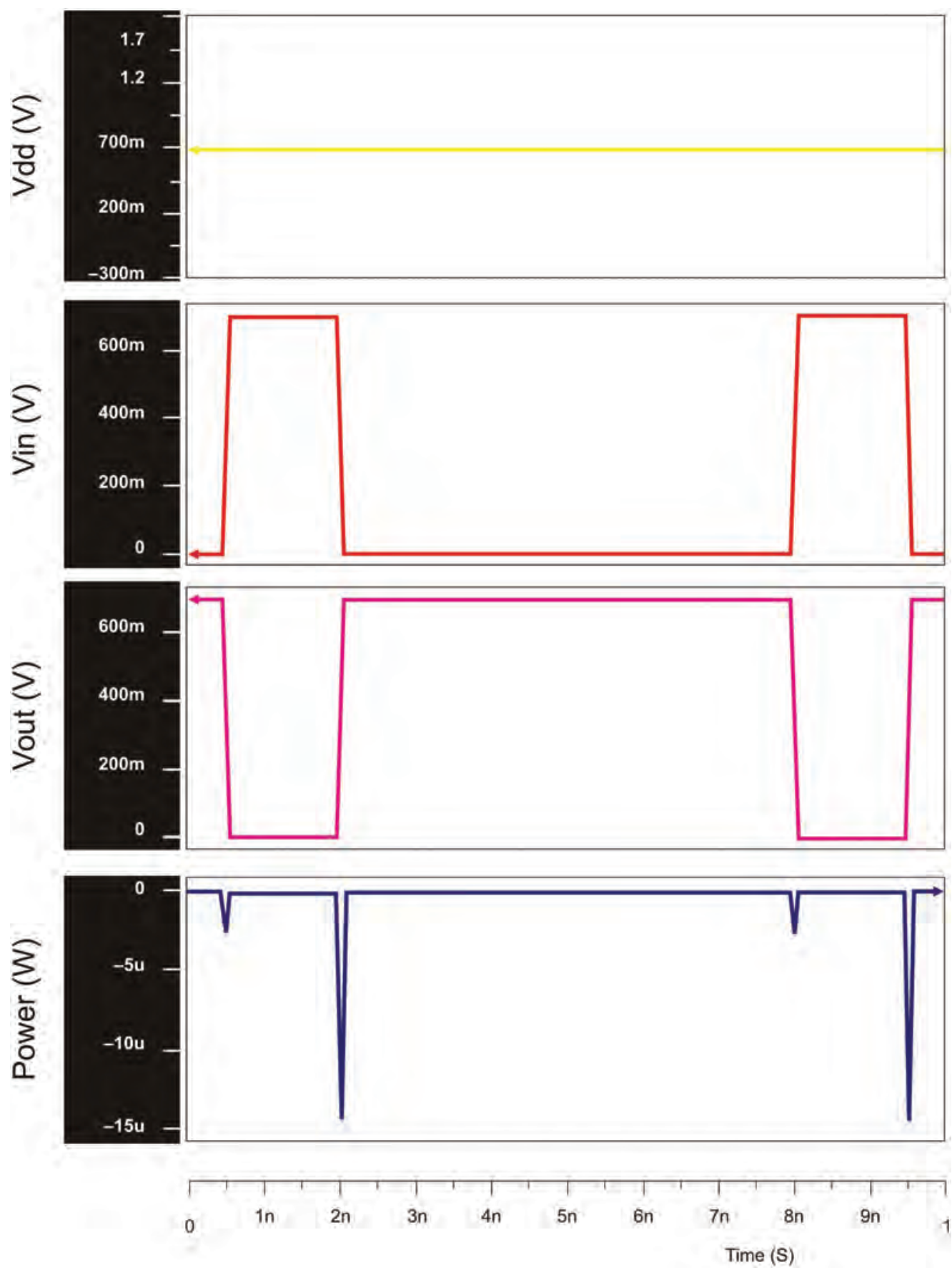

Figure 83.2 (Continued) 
Table 83.1 Technology parameters used in the model

\begin{tabular}{lll}
\hline Technology node & $V_{D D}(V)$ & $V_{t h}(V)$ \\
\hline $32 \mathrm{~nm}$ & 0.9 & 0.30 \\
$16 \mathrm{~nm}$ & 0.7 & 0.29 \\
$10 \mathrm{~nm}$ & 0.6 & 0.25 \\
\hline
\end{tabular}

\section{Simulation results of $\mathrm{MoS}_{2}$ transistor-based inverter}

We simulated the n-type and p-type $\mathrm{MoS}_{2}$ FET by considering the channel lengths of 0.032 $\mu \mathrm{m}, 0.016 \mu \mathrm{m}$ and $0.010 \mu \mathrm{m}$. SPICE simulations are carried out by using the SPICE model designed in Section II and tested. In an attempt to have an accurate simulation describing the $\mathrm{MoS}_{2}$ FET, we have used parameter sets from Table 83.1. In our simulations, p-type and n-type $\mathrm{MoS}_{2}$ FET characteristics are verified from channel lengths $0.032 \mu \mathrm{m}, 0.016 \mu \mathrm{m}$ and $0.010 \mu \mathrm{m}$. The models are simulated for $V_{\mathrm{DD}}$ values from $0.6 \mathrm{~V}$ to $1 \mathrm{~V}$ to check the results of threshold voltages. The voltages are tabulated in Table 83.1.

\section{Conclusion}

The single layer $\mathrm{MoS}_{2}$ transistor designed with $10 \mathrm{~nm}, 16 \mathrm{~nm}, 32 \mathrm{~nm}$ technology node for n-type and p-type $\mathrm{MoS}_{2}$ FETs are modelled and analysed for their suitability for circuit levels by considering an inverter. The designed single layer $\mathrm{MoS}_{2}$ transistor and the systematic simulations for versatile n-type and p-type $\mathrm{MoS}_{2} \mathrm{FET}$ and an inverter were carried out and verified. From the observations the $\mathrm{MoS}_{2}$ FET utilizes much less power and outperforms Si based CMOS designs.

\section{References}

1. Morasae Samadi, Navid Sarikhani, Mohammad Zirak, Hua Zhang, Hao-Li Zhang and Alireza Z. Moshfegh, "Group 6 transition metal dichalcogenide nanomaterials: synthesis, applications and future perspectives," Nanoscale Horizons 3, 90-204, 2018.

2. Devin Verreck, Goutham Arutchelvan, Cesar J. Lockhart de la Rosa, Alessandra Leonhardt, Daniele Chiappe, Anh Khoa Augustin Lu, Geoffrey Pourtois, Philippe Matagne, Marc M. Heyns, Stefan De Gendt, Anda Mocuta and Iuliana P. Radu, "The role of nonidealities in the scaling of MoS2 FETs", IEEE Transactions on Electron Devices 65(10), 4635-4640, 2018.

3. K. Alam and R. Lake, "Monolayer $\mathrm{MoS}_{2}$ transistors beyond the technology road map", IEEE Trans. on Electron Devices, 2012.

4. X. Song, J. Hu and H. Zeng, "Two-dimensional semiconductors: recent progress and future perspectives", J. Mater. Chem. C, 2013.

5. K. S. Novoselov, D. Jiang, F. Schedin, T. J. Booth, V. V. Khotkevich, S. V. Morozov and A. K. Geim, "Two-dimensional atomic crystals”, Proc. Natl. Acad. Sci. 102(30), 10451-10453, 2005.

6. M. Gholipour, Y.-Y. Chen and A. Sangai, "Analytical SPICE-compatible model of Schottkybarrier-type GNRFETs with performance analysis”, IEEE Tran. on Very Large Scale Integration Systems, 2015.

7. Jiahao Kang, Deblina Sarkar, Wei Liu, Debdeep Jena and Kaustav Banerjee, "A computational study of metal-contacts to beyond-graphene $2 \mathrm{~d}$ semiconductor materials", in IEEE International Electron Devices Meeting, 2012.

8. Y. Yoon, K. Ganapathi and S. Salahuddin, "How good can monolayer MoS2 transistors be?", Nano Letters, 2011. 
9. V. Podzorov, M. E. Gershenson, C. Kloc, R. Zeis and E. Bucher, "High-mobility field-effect transistors based on transition metal dichalcogenides", Appl. Phys. Lett. 84, 3301, 2004.

10. David Jimenez, "Explicit drain current, charge and capacitance model of graphene field-effect transistors", IEEE Transactions on Electron Devices 58(12), 4377-4383, 2011.

11. Available: www.itrs.net/.

12. Han Liu, Mengwei Si, Sina Najmaei, Adam T. Neal, Yuchen Du, Pulickel M. Ajayan, Jun Lou and Peide D. Ye, "Statistical study of deep submicron dual-gated field-effect transistors on monolayer chemical vapor deposition molybdenum disulfide films", Nano Letters, 2013.

13. K. S. Novoselov, D. Jiang, F. Schedin, T. J. Booth, V. V. Khotkevich, S. V. Morozov and A. K. Geim, "Two-dimensional atomic crystals”, Proc. Natl. Acad.Sci.102(30), 10451-10453, 2005.

14. Mihir R. Choudhury, Youngki Yoon, Jing Guo, Kartik Mohanram, K. F. Mak, C. Lee, J. Hone and J. Shan, "Graphene nanoribbon FETs: technology exploration for performance and reliability”, IEEE Transactions on Nanotechnology 10(4) 2011. 


\title{
84 Hardware based design of smart warehouse monitoring system
}

\author{
Mohit Charan Mullapudi*, Manasij Chakraborti*, \\ Akhil Kumar Panda*, Garv Sehgal, S.K. Sinha* \\ Department of Electrical \& Electronics Engineering, Amity University, \\ Uttar Pradesh, Noida, India
}

\section{Introduction}

As the world is moving towards a future of online purchase of goods, it has become essential for online retailers to provide proper warehouse monitoring systems for various types of goods they are going to store in their warehouses. These goods, depending on their nature, need to be monitored dynamically. Temperature, for example, is an important aspect of a monitoring system as each good has a specified temperature range it can be stored at otherwise the good may perish or become unusable. If the temperature is not in the specified range, the temperature sensor of the monitoring system senses the abnormality and raises an alarm so that the hardware can adjust the HVAC system of the warehouse.

Fire-fighting systems are provided for the safety of goods as well as the personnel working in the warehouse. Thus, this hardware design also aims to provide a smoke detection solution for extra redundancy of the system. Smoke sensors are installed in the monitoring system so that in the case of fire, the notification will be sent to the entry/exit system which would enable the employees of the warehouse to be aware of any fire and reach safety as soon as possible. The system would also send a notification of high priority to all the concerned authorities for immediate action.

Without any power supply, the whole warehouse system with its devices would be rendered useless, thus a power supply monitoring hardware is also installed in this system. This will ensure that there are no interruptions in working which may result in loss of time and money for the client. Notification will be sent to the system in the case of abnormality in the power supply and if the delay is long, the system sends the command to the generator system for any further actions to take place [1].

A warehouse is a vast system which involves numerous small systems bound together in order to provide for a system we know as a "warehouse". Monitoring of each and every system would be next to impossible from regular checks, thus this hardware design for monitoring a warehouse would be using various sensors in order for proper monitoring of the warehouse and notify the operators in the case of any abnormalities [2].

\footnotetext{
* Emails: mohitcharan2012@gmail.com, manasijchakraborti@gmail.com, akhilpanda333@gmail.com, garv.sehgal1970@gmail.com,sksinha6@amity.edu
}

DOI: $10.1201 / 9781003129103-84$ 


\section{System description}

The components used in this work are:

Temperature sensor (DHT11) - a temperature sensor has an important role in any kind of electronic related application. For example, maintaining a specific temperature in a cold storage is extremely important otherwise the products may get damaged. This piece of equipment thus helps in sensing and sending the information to the cooler to keep the temperature in check.

In this work, a digital type temperature sensor has been used as a preventative reliability device. It is used to monitor the surrounding of the warehouse and provide realtime data in any temperature changes it observes. This helps provide a safe environment for goods and people as well in the warehouse.

DHT11 is a digital temperature sensor which can be easily interfaced with Arduino with DHT11 library in Arduino IDE. DHT11 is multipurpose and can be also used as a humidity sensor. It can write temperature and humidity values at the same time. Thus being able to sense and report the temperature and humidity of the entire warehouse would prove to be a huge asset for a monitoring system as a whole. This can be especially useful for food storage units where the temperature and humidity levels of the entire warehouse affect the quality of food.

Smoke detectors - the monitoring system hardware also includes a smoke detector system, which works alongside the fire detection system, thus providing a better allround protection system. Smoke detectors, as the name would suggest, are used for detecting smoke caused by even the smallest of fires (including cigarettes). This would be especially beneficial as the operator would be notified before any fire is started; it would imply that personnel working there would have more time at hand to evacuate in case of any major emergency. This has been included keeping the safety in mind. In case of fire, smoke detectors will give an indication about any sort of fire that may prove fatal if kept unchecked. These are generally stationary detectors. This way the detectors should cover a larger area for detection of any sort of smoke, if placed strategically. Another thing that this kind of digital smoke detector can do is detect any kind of harmful gases that may be dangerous for work and provide a notification to the operator as well as an alarm. It is especially important for warehouses that deal with such dangerous chemicals or toxic matter, for example an LPG storage unit or a chemical storage unit.

NodeMCU - NodeMCU is the key component of the prototype hardware; it can be labelled as the "brains" of the entire monitoring system. It is a low cost/economical option for an IoT based platform for operation. It is based on the Espressif ESP8266-12E WiFi system-on-chip, loaded with an open source, LUA based firmware. It is perfect for IoT applications and other situations where wireless communication is required. Thus it is highly used by developers who want to explore the IoT platform. Since NodeMCU is an open platform, it means that developers can easily modify/build. It is a Wi-Fi enabled chip that can use the TCP/IP protocol. It also supports serial communication such as USB, USART, SPI, I2c etc. Using a serial protocol is especially beneficial for the hardware prototype as one can add various other components to it; one can connect LCD display units, SD cards, touch screens, GPS modules etc. which would be helpful in complete and smart monitoring of the warehouse. It would also be advantageous as one would have scope for further development in case a need arises [3]. 
As discussed earlier, NodeMCU is based on Lua. Lua is a scripting language that is easy to learn and thus easy to develop.

Arduino could have also been used but NodeMCU has numerous advantages over Arduino in the development process for the hardware as well as software based design of the warehouse monitoring system [4].

The reasons for using NodeMCU over Arduino are listed below:-

- Its cost is low compared to Arduino.

- It has a CPU core, faster WiFi, more input/output pins and also supports Bluetooth.

- It also comes with touch sensitive pins.

- It has full TCP/IP stack and microcontroller capability.

Buzzer - the buzzer is used for alarming the operator about any fault that takes place in the circuit. A buzzer circuit includes a single transistor, a ferrite inductor and a piezo transducer. The transistor and the inductor are the main components of the buzzer and can also be called the "heart" of the component. When a specified voltage, let's say $5 \mathrm{~V}$, is introduced in the circuit, the transistor starts to conduct. When it is conducting, the piezo electric element starts to operate; however, this leads to grounding of the base of the transistor through the centre tap element of the piezo which leads to switching off of the transistor and piezo. This cycle repeats itself, generating an oscillation which we are able to hear as buzzing. In case of a fault, the voltage will vary and the buzzer will give out a beeping sound which will alarm the operator.

Indicator lights - indicator lights have also been used to indicate faults in the circuit as well as how the circuit is functioning. A green light would indicate that there is no fault in the circuit whereas a red light indicates that there is a fault. This is useful for personnel/ operator to easily pinpoint the faults and necessary actions can be taken so as to correct the fault. The indicating lights thus are used solely for indicative purposes.

Printed circuit board (PCB) - this is a board which mechanically supports and electrically connects electrical or electronic components using conductive pads and tracks. It can be said that a PCB is the simplest electronic product of all. PCBs can be single sided or multi-sided depending upon the needs. Copper layers are used for the conduction purposes.

Fault detection system - as suggested before, a fault detection system is of extreme importance for a warehouse monitoring system so as to guarantee user safety as well as safety of goods and equipment of the warehouse. This fault detection system controller includes short-circuit fault, open circuit, over-current and over-voltage fault detection for the power supply of the warehouse. During operation, the controller is able to pick up faults and transmit them to the user interface module as soon as possible. Open circuit faults are detected by the means of reading the voltage fed into the controller and the voltage coming out; if there is any difference of voltage at the two ends, the fault is detected and an alarm is sent. For faults related to voltage such as over-voltage or undervoltage, MCU MC9S08DZ128 is used as the main control chip. The main part of this circuit is the voltage comparator. The output of comparator serves as the control signal to a smart switch. When the control signal is high, the switch is open and MCU is connected or else the MCU won't work as it would not have any power [5-7].

Fire-fighting system - a fire-fighting system is of utmost importance for a warehouse and its corresponding monitoring system. From numerous surveys that have been conducted, we concluded that around $40 \%$ damage to property and life in warehouses is caused due to fire. The system that is being developed thus includes a digital fire detector to prevent 
any such mishaps from happening. It is a precautionary measure that one needs to adopt in order to provide safety and security of goods as well as the people working in the warehouses. The fire detector detects the amount of carbon monoxide in the atmosphere of the warehouse; if the level of carbon monoxide surpasses the minimum set value, it gives out an alarm.

A fire-fighting system is installed in most warehouses but it is not integrated with the monitoring system. Integration with the warehouse monitoring system will help the owners of warehouse, inventory owners and fire-fighting authorities to get information in case of the fire. The monitoring device is connected to a server via Internet. The data is sent to the concerned authorities via the server.

\section{Experimental prototype}

This work aims at a simple and economical monitoring system that can be easily put together and function at a level that would be comparable to some of the major prototypes out there. Thus the client would be able to gain more for a price far less than his competitors. The hardware design would consist of parts that are easily available and won't cost much, hence the overall cost of this work would be less. One of the benefits of using low cost hardware is that it is easy replaceable and also has further scope for improvement.

In the monitoring device all sensors are connected to NodeMCU, which is a WiFi enabled microprocessor which is connected to the server via Internet. All the data fetched by the microprocessor is processed by the microprocessor and accordingly the output devices are activated. The microprocessor converts the analog values to digital values via analog-digital converters. All the essential values and alarms are sent to the server $[8,9]$.

Another thing that this kind of digital smoke detector can do is detect any kind of harmful gases that may be dangerous for work and provide a notification to the operator as well as an alarm. It is especially important for warehouses that deal with such dangerous chemicals or toxic matter, for example an LPG storage unit.

The smoke sensor, temperature sensor, buzzer and LED indicators will be connected to the NodeMCU as shown in Figure 84.1 and the NodeMCU will be programmed accordingly. If the temperature or the smoke level in the room crosses the threshold

\section{Monitoring device}

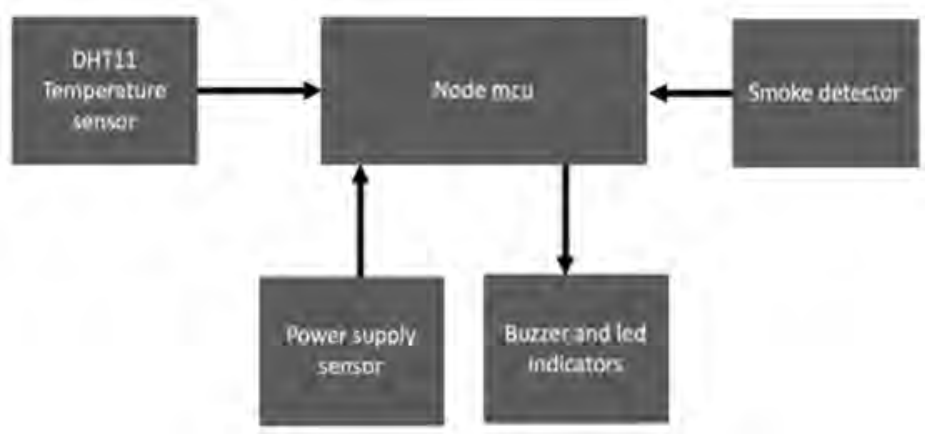

Figure 84.1 Block diagram. 
level, the buzzer and LED indicators will give signals to the user, thereby preventing any hazard [10].

\section{Conclusion}

This work gives details for the design and hardware required for a warehouse monitoring system. Using this circuit, the physical parameters of a warehouse such as temperature, humidity or any faults that may occur in the warehouse can be monitored. NodeMCU is the primary component that is used in this work. It is the controlling device that is used to bind all the various sensors (input/output) such as the temperature sensor, smoke detector and buzzer alarm. The temperature sensor DHT11 doubles as a temperature and humidity sensor. Node MCU is a Wi-Fi enabled controller that can transmit data wirelessly which can be viewed by the operator on a PC. This system is especially useful for warehouses that have special requirements such as a cold storage unit where all the physical parameters are to be monitored dynamically so that the goods may not get damaged.

With the growth of online retailers, there is a constant strife to provide best-in-class storage facilities at a lower cost. Thus, this work once implemented can bring about massive changes in the online retailing and storage sector and will be especially beneficial for warehouses that have specific needs as well as providing a safe environment for the personnel working in the warehouse [12].

\section{Results}

The warehouse monitoring system is a part of the warehouse management system. In a warehouse monitoring system, goods as well as personnel are monitored for security, protection, temperature etc. In this system, the master-controller fetches data from all the sensors and processes them according to the set conditions. If any parameter is not in the set range then the corresponding alarm will give notification and the error will be shown in the serial monitor in the corresponding PC. Smoke detectors are used for fire sensing, temperature sensors are used to measure ambient temperature of the warehouse so that proper temperature is maintained. For warehouses, electric power supply is especially important; if there is a power cut, the warning system sends a notification to generator system. As per the requirement, the monitoring system can be programmed and other parameters can be monitored [13-15].

From Figure 84.2 (experimental prototype) one can see that the NodeMCU is the core of the monitoring system; it is serially connected to the computer of the operator via USB. Various other sensors such as temperature + humidity sensor, buzzer, indicative lights etc. are also connected to their respective pins on the controller.

The sensors are able to monitor the surrounding perfectly of a given room and display the data on the monitor of the controller as shown in Figure 84.3. From the serial output data, one is able to find that the temperature of the room was 28 degree Celsius as well the humidity that was around $51 \%$. In the case of a fire, such as a burning matchstick nearby, the prototype was able to detect a fire, sound an alarm and flash warning lights; an emergency notification was also sent to the operator [16]. 


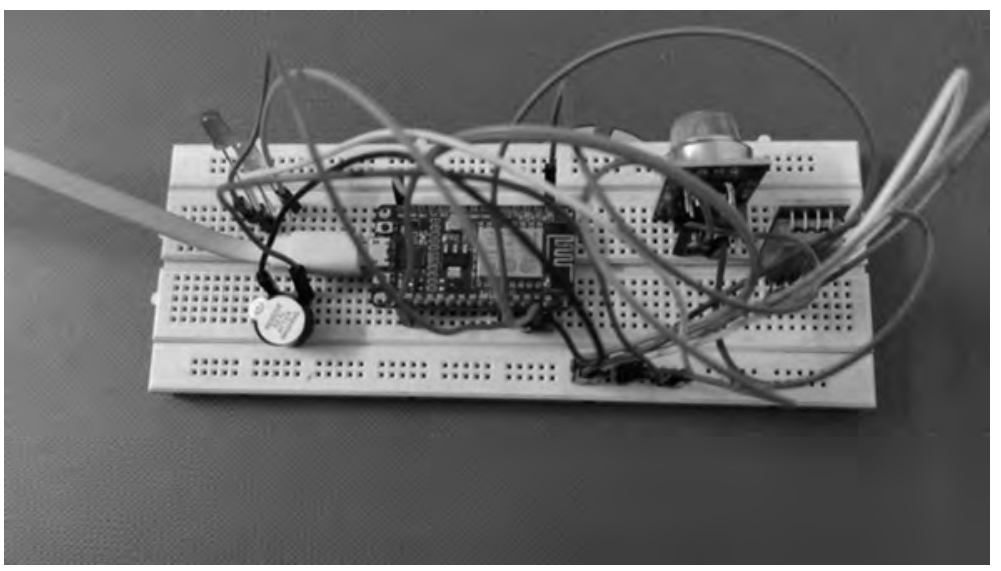

Figure 84.2 Experimental prototype.

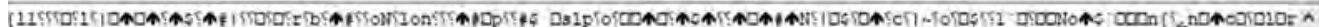
$18: 43: 48.037 \rightarrow$ Alrte 1202

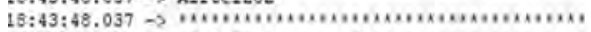

L8:43:51.335 $\rightarrow$ WHiFi connected 4 H

Iemperatute: $26.10 \mathrm{deg}$ C. Humidity: 51.009. Connecting to Thingspeak.

$16: 43: 51.662 \rightarrow$ Sending....

lenperature: 28.10 deg C. Huridicy: 51.00 . Connecting to Thingspeak.

L: $: 44: 02.248 \rightarrow$ Sending....

Temperature: 28.40 deg C. Hunidicy: 51 .ovit. Connecting to Thingspeak.

$18: 44: 12.994 \rightarrow$ Sending....

Temperature: $28.10 \mathrm{deg} C$. Humidicy: $51.06 \mathrm{~s}$. Connecting to Iningspeale,

18:44:22.949 $\rightarrow$ Sending...

Temperature: $2 e .20$ deg C. Humidicy; 51.004 . Connecting to Thingspeal.

18:44:33.271 $\rightarrow$ Sending....

$<$

ĐAutoscoll ఐShow timestanp Newine $\sim 115200$ boud $\approx$ dear output

Figure 84.3 Serial output.

\section{References}

[1] A. M. Atieh et al., "Performance improvement of inventory management system processes by an automated warehouse management system," in Procedia CIRP, 2016.

[2] R. Pulungan, S. P. Nugroho, N. El Maidah, T. B. Atmojo, P. D. Hardo, and P. Pawenang, "Design of an intelligent warehouse management system," in Information Systems International Conference (ISICO), 2013.

[3] B. Yan, C. Yiyun, and M. Xiaosheng, "RFID technology applied in warehouse management system," in Proceedings - ISECS International Colloquium on Computing, Communication, Control, and Management, CCCM 2008, 2008.

[4] C. K. M. Lee, Y. Lv, K. K. H. Ng, W. Ho, and K. L. Choy, "Design and application of Internet of things-based warehouse management system for smart logistics," International Journal of Production Research. 2017.

[5] A. Giuseppe, E. Mario, and M. Cinzia, "Analysis of a warehouse management system by means of simulation experiments," in 10th International Conference on Modeling and Applied Simulation, MAS 2011, Held at the International Mediterranean and Latin American Modeling Multiconference, I3M 2011, 2011. 


\section{Intelligent Circuits and Systems}

[6] F. Petropoulos, S. Makridakis, V. Assimakopoulos, and K. Nikolopoulos, “'Horses for courses' in demand forecasting," Eur. J. Oper. Res., 2014.

[7] E. Zunic, H. Hasic, K. Hodzic, S. Delalic, and A. Besirevic, "Predictive analysis based approach for optimal warehouse product positioning," 41st International Convention on Information and Communication Technology, Electronics and Microelectronics (MIPRO), 2018.

[8] E. Zunic, A. Besirevic, R. Skrobo, H. Hasic, K. Hodzic, and A. Djedovic, "Design of optimization system for warehouse order picking in real environment," in ICAT 2017-26th International Conference on Information, Communication and Automation Technologies, Proceedings, 2017.

[9] E. Zunic, K. Hodzic, H. Hasic, R. Skrobo, A. Besirevic, and D. Donko, "Application of advanced analysis and predictive algorithm for warehouse picking zone capacity and content prediction," in ICAT 2017-26th International Conference on Information, Communication and Automation Technologies, Proceedings, 2017.

[10] E. Zunic, A. Besirevic, S. Delalic, K. Hodzic, and H. Hasic, "A generic approach for order picking optimization process in different warehouse layouts," 41st International Convention on Information and Communication Technology, Electronics and Microelectronics (MIPRO), 2018.

[11] O.Vallis, J.Hochenbaum, and A. Kejariwal, “A novel technique for long-term anomaly detection in the cloud," Proceedings 6th USENIX Work. Hot Top. Cloud Comput. (USENIX'14), 2014.

[12] Dan Ding and R. A. Cooper, "Electric-power wheelchairs," IEEE Control System Magazine, 2005, 25(2):22-43

[13] R. A. Cooper. Wheelchair Selection and Configuration. New York: Demos Medical, 1998.

[14] Ma Xiao-zhen and Wan Shun-yun. "Fault detection of electric-powered wheelchair kinetic control system," China Micromotors, 2010, 43(6):88-89.

[15] INA139 Data sheet. High-side measurement current shunt monitor. Texas Instruments, 2005.

[16] MC9S08DZ128 Data sheet Rev.1. Freescale, 2008. 


\title{
85 Design of battery management and monitoring system for electric vehicles
}

\author{
B. Manish Reddy*, Anoop Kumar Verma*, Siddhant \\ Treasure*, Vijay Kumar Tayal*
}

Department of Electrical \& Electronics Engineering, Amity University, Noida, India

\section{Introduction}

As energy and environmental problems are becoming extremely serious, the electric vehicle has become, in recent years, a new vehicle that is developing rapidly. As the basis of the development of electric vehicles, batteries play an increasingly important role. A Li-ion battery was the preferred choice of packaging because of its energy performance advantages [2]. However, electric vehicle power Li-ion batteries have high capacity, which, combined with security, uniformity, durability and cost related issues, place restrictions on its widespread use. For long lifetime and proper functioning of a lithium ion battery in an electric vehicle it is necessary to maintain its state of health $(\mathrm{SOH})$ and to monitor its performance continuously. For this purpose, our BMS (battery management system) came into existence for maintaining each cell condition, balance charging and various other parameters. But till today also monitoring of each cell is difficult because it requires many probes.

In this paper, the different characteristic performance of a battery under load, its temperature fault effect and prevention of cell going under deep-discharge state using MATLAB-Simulink have been discussed. Further, a prototype design of BMMS (battery management and monitoring system) to perform both managing and monitoring functions using proteus is presented. In order to account for the results of a battery, every simulation of the battery is performed either using a prismatic cell having characteristic features of a lithium cell or by directly using a lithium cell in case of temperature faults. The concept of every simulation is based on state of health $(\mathrm{SOH})$. It is defined as the current condition of the battery in comparison to its ideal condition (at the time of manufacturing). Though it is not possible to directly measure the state of health thus, the focus is on measuring the state of charge, equal balancing of each cell and temperature effects. This will altogether give the battery state of health.

In this paper, the effect of a prismatic cell under RL load is studied. Further, to be more specific constant load is required when a vehicle is operating at the same speed. Secondly, if a lithium cell undergoes temperature fault, how its characteristics differs from a normal Li cell is checked. The battery along with motor load and relay to prevent it from going under deep discharge state is simulated. On the basis of the following results obtained from simulations the prototype of BMMS (battery management and monitoring system) for electric vehicles is to be designed. This paper is organized as follows: Section 2 represents the literature review, system description is discussed in detail in Section 3, simulation results are discussed in Section 4 and the conclusion is presented in Section 5.

\footnotetext{
*Emails: manishbaddam@gmail.com, anoopkumar20061998@gma il.com, tsiddhant30@gmail.com, vktayal@amity.edu
} 


\section{Literature review}

Menghua and Bing developed an Android microcontroller system for lithium ion battery monitoring in electric vehicles [1]. The state of charge and state of health estimation of lithium ion battery has been proposed by Huang et al. [2]. Feng et al. [3] have worked on parameter identification and dynamic simulation of Li-ion power battery based on DP model. A battery monitoring system using LoRa technology has been developed by Tsyani et al. [4]. Electric vehicle technologies' future has been discussed by Yong et al. [5]. Watrin et al. [6] presented an estimation of SoC and SoH for lithium batteries. GPRS based monitoring of batteries in an online mode has been developed by Luo et al. [7]. Lead acid battery monitoring in real time utilizing IoT technology has been presented by Rauniyar et al. [8]. The future trends regarding developments of batteries have been discussed by Yonghua et al. [9]. Lu et al. reviewed the management of lithium ion batteries [10]. Sharma and Tayal [11] proposed the advanced design of a micro-controller based PV panel control for maximum power tracking. Singh et al. [12] discussed design improvements for an efficient and automatic battery charging of a car. The major reasons for the requirement of a monitoring system are as follows.

a. Provide low cost maintenance and operation: During the design and development of any system, capital plays an important role. It is required at each and every step of the complete process. By this simulation we will be able to reduce the extra cost as we have already done the required simulations.

b. Long life of battery: The system is designed in order to monitor the electrical parameters of the battery. When the parameters are appropriately monitored and studied, along with its temperature and other fault effects, so a particular and appropriate system can be designed which will help in increasing the battery life.

c. Easy detection of any abnormal activity or fault: Sensors and relay used along with the system will provide us a way to analyse so that faults can be detected very easily and the fault will not affect the performance of the battery.

\section{System description}

A prismatic cell under RL load as shown in Figure 85.1 is used to define the Li-ion cell working under constant load condition. This block diagram is used to explain that when an electric vehicle is driving at constant speed then how the current will be drawn and the state of charge will vary with the usual passage of time. The blocks used in this simulation are Prismatic Cell, Bus Selector, Scope, RL-Load, Voltage Measurement, Power GUI.

\section{Simulation results}

The output in Figure 85.2 shows that while operating under constant load conditions the voltage of the battery is $96-100 \mathrm{~V}$, which is the maximum voltage the motor will require, and also the continuous load current is $162.5 \mathrm{~A}$.

\section{A. Lithium cell under temperature fault conditions}

This block model is simulated in order to determine or depict the behaviour of the two kind of cells: one working under fault condition and the other working under non-fault condition. This will help us to determine how to differentiate the faulty cell from rest of the battery pack system which will help us in equal balancing of the cells. Also it will help 


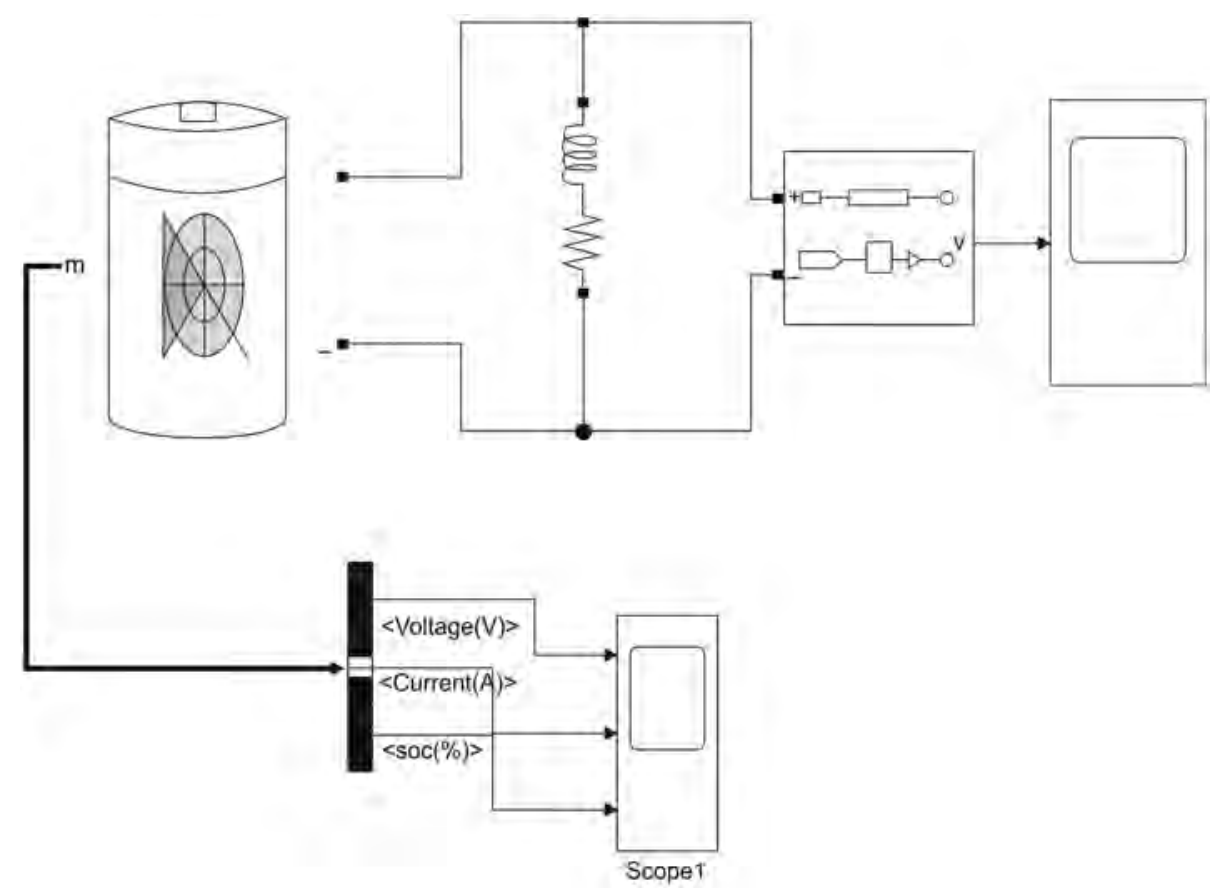

Figure 85.1 Battery modelling at continuous load.

us to isolate the faulty cell from the rest of the cells of the battery pack so that it might not affect the performance of the other cells during operation. The blocks used in this simulation, as shown in Figure 85.3, are Lithium Cell, MUX, Scope, Conduction Heat Source, Convection Heat Source, Temperature Block.

The output in Figure 85.4 shows the temperature difference between the faulty cell and normal cell. The figure clearly shows that the faulty cell will go under uneven heating and should be managed as soon as possible.

\section{B. Thermal analysis}

The block model in Figure 85.5 is used to compare the two prismatic cells' behaviour, one with a thermal stability management system and other without a thermal stability management system. The importance of this block model is used to show that thermal stability is required for the proper working of the battery.

Figure 85.6 shows the measurement block which receives the parameters as voltage, current, SOC and temperature of the two cells and sends the output to the scope. The given output (Figure 85.7) shows how much the thermal stability is necessary to the cell and its management.

\section{Super-capacitor battery and relay modelling at motor load}

The block diagram in Figure 85.8 is to show the equal balancing of a prismatic cell and preventing it from going under deep discharge state The working of the system is such that if the SOC of the battery goes below $40 \%$ the relay will shut down the power supply from 


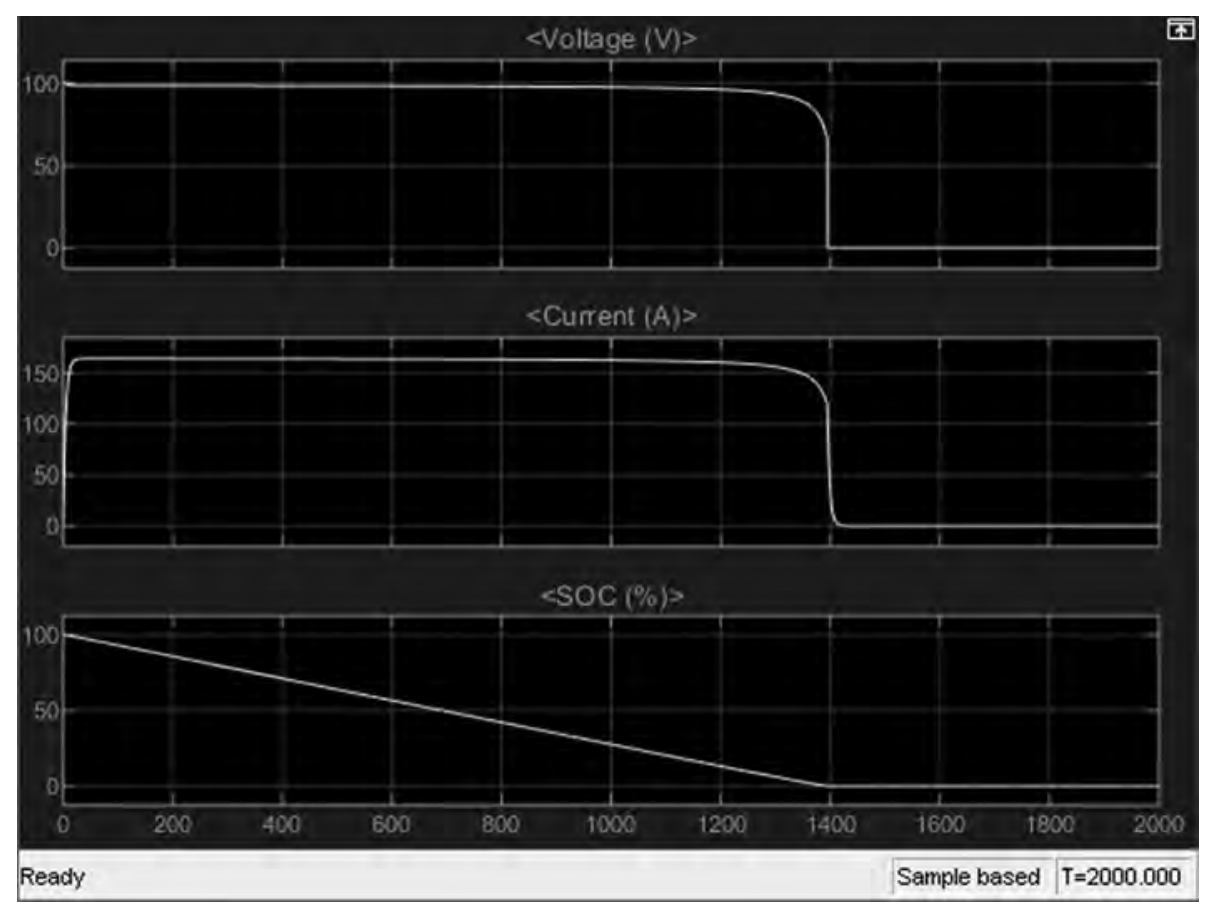

Figure 85.2 Voltage, current and SOC of battery cell.

the battery and the super-capacitor will come into action and will operate the load until and unless the battery again attains the SOC of $80 \%$.

The output in Figure 85.9 shows the SOC value going from $100 \%$ to $40 \%$. As soon as it reaches $40 \%$ the battery shuts down and goes under charging state until it again gets charged up to $80 \%$.

The output in Figure 85.10 shows the operation of the relay. When the relay is ON the value is 1 , otherwise 0 .

The output in Figure 85.11 shows the operation of a PV cell or solar cell. The parameters taken into account are PV cell voltage, PV cell current and the temperature of the cell while its operating.

\section{Conclusion}

In this paper various parameters have been taken into account while designing a battery management and monitoring system for electric vehicles. The simulations have been performed including its performance at constant load, motor load, under PV cell and at the time of occurrence of a fault. It has been concluded that the necessary parameters required for estimation of health of battery $(\mathrm{SOH})$ and to design its respective BMMS system are SOC, temperature, current, voltage, load value and capacity. The advantage of performing the simulation on MATLAB is that the simulation will give us a rough idea of the working of the battery under various conditions which can be kept in mind while designing the battery management and monitoring system for EVs. Moreover, one can make the system wireless without making it very costly after performing its simulation. 


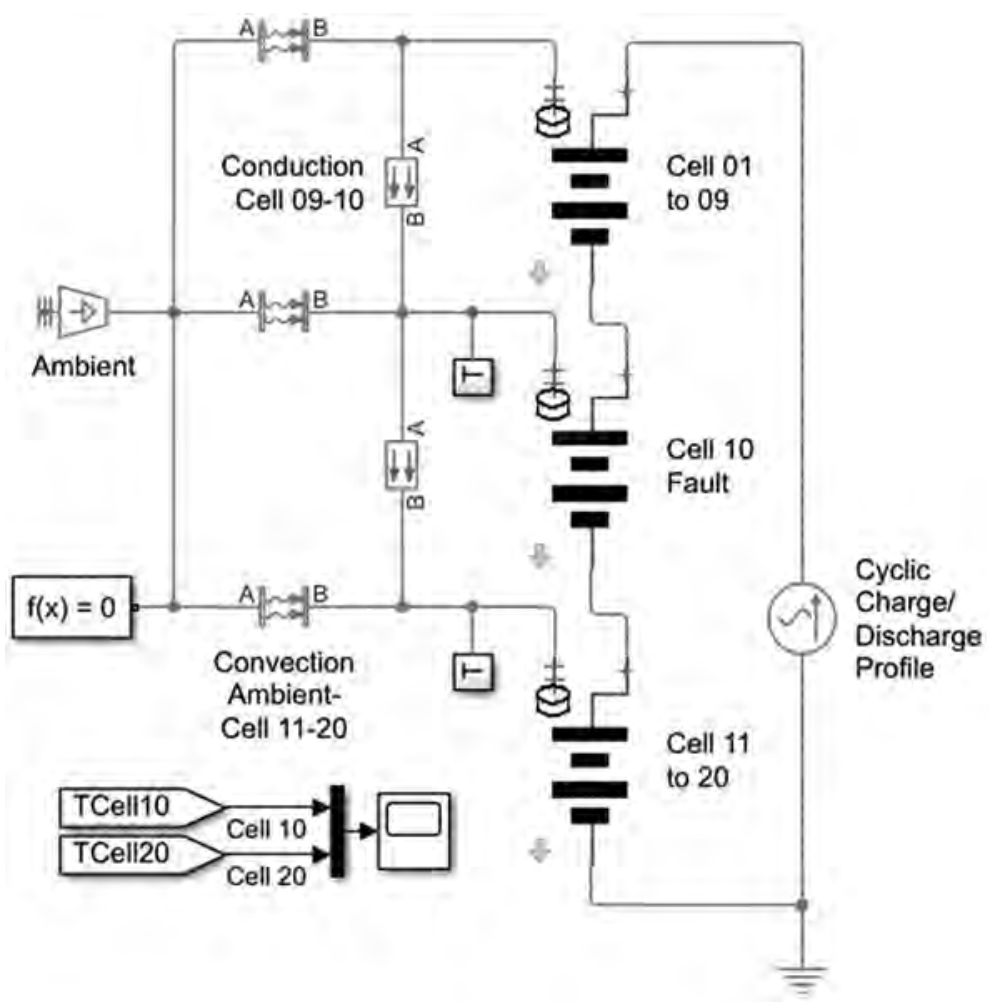

Figure 85.3 Lithium-ion battery pack with fault model.

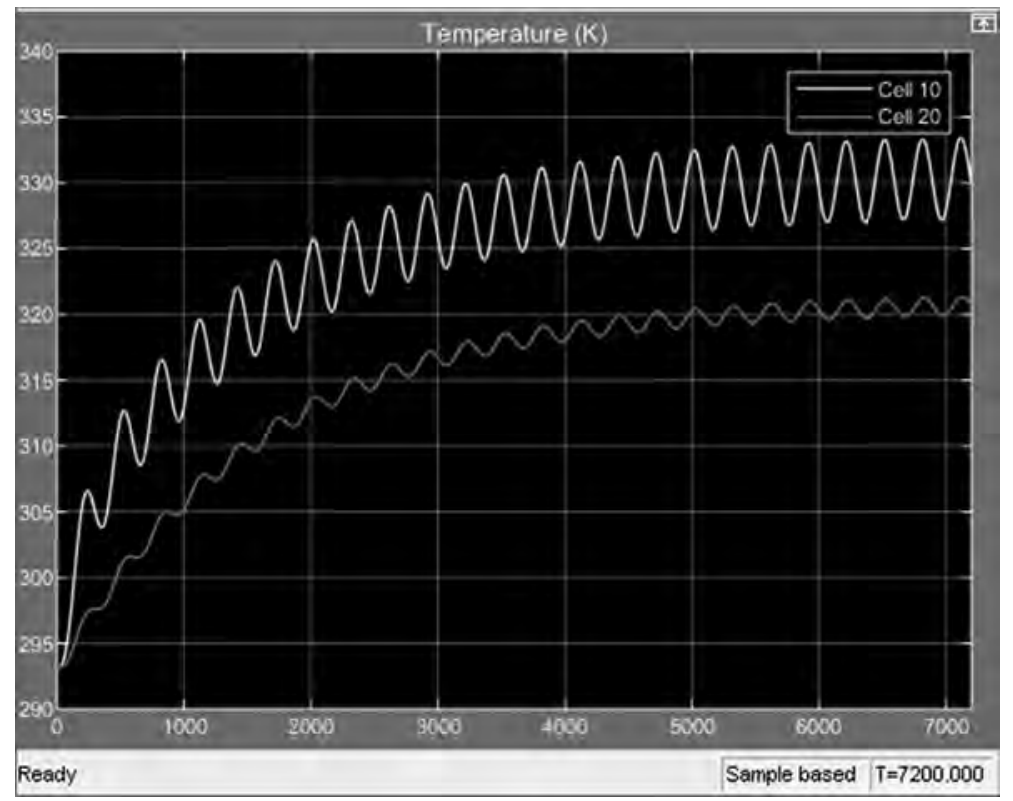

Figure 85.4 Temperature of healthy and faulty lithium cell. 


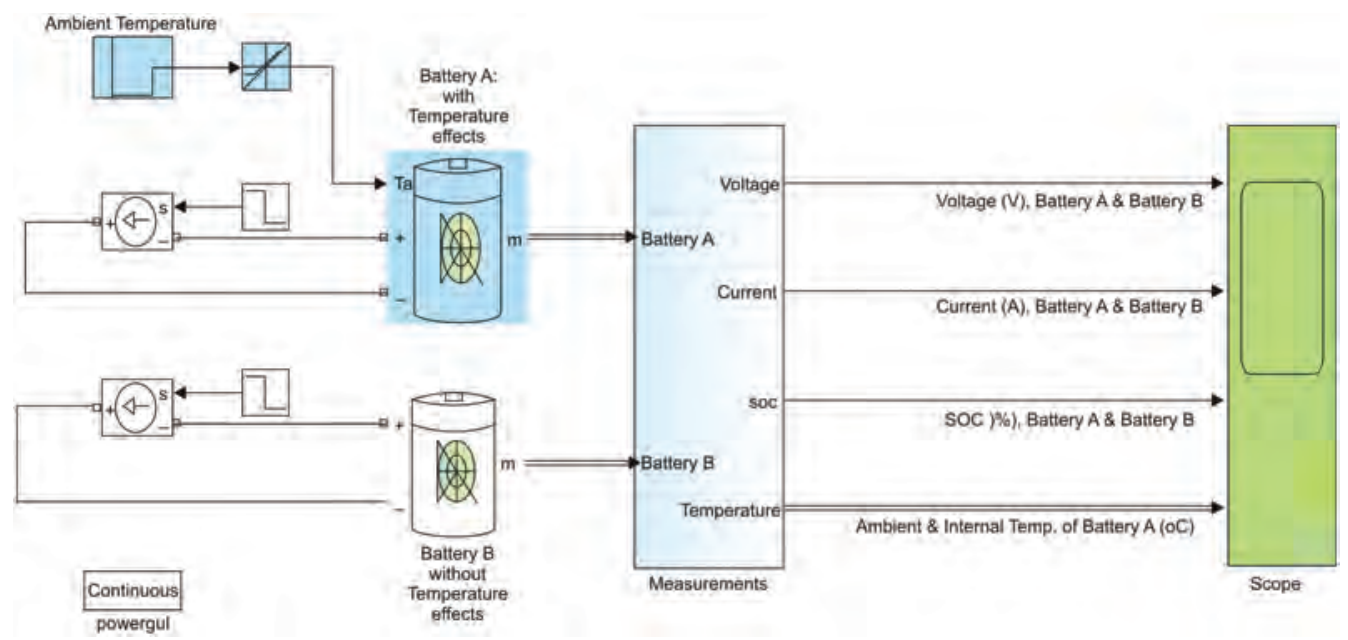

Figure 85.5 Lithium-ion temperature dependent battery model.

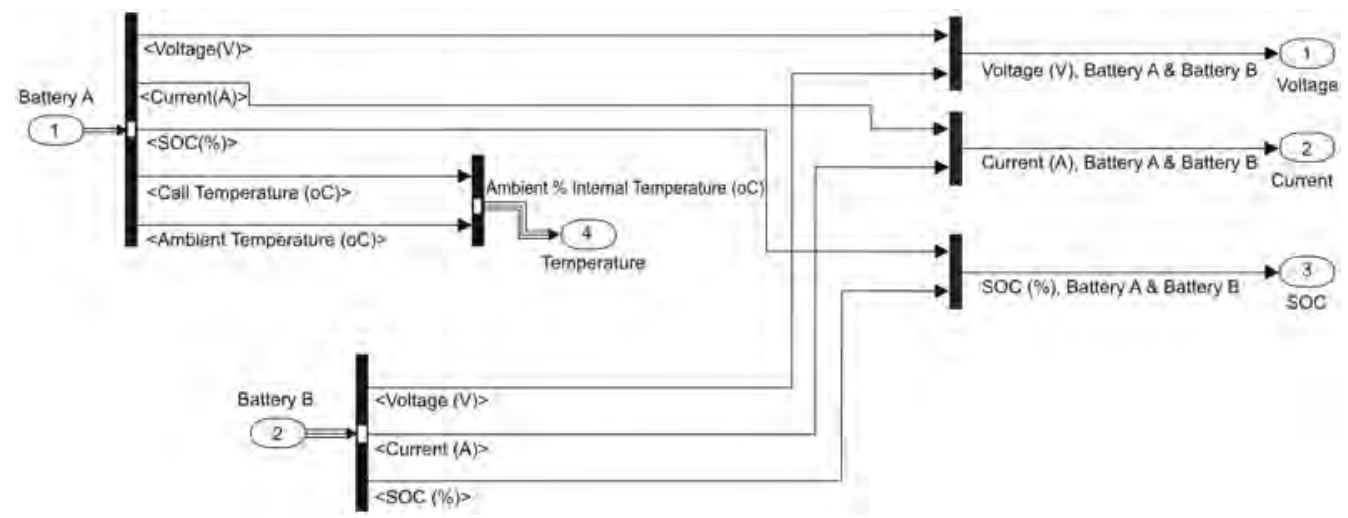

Figure 85.6 Measurement block.
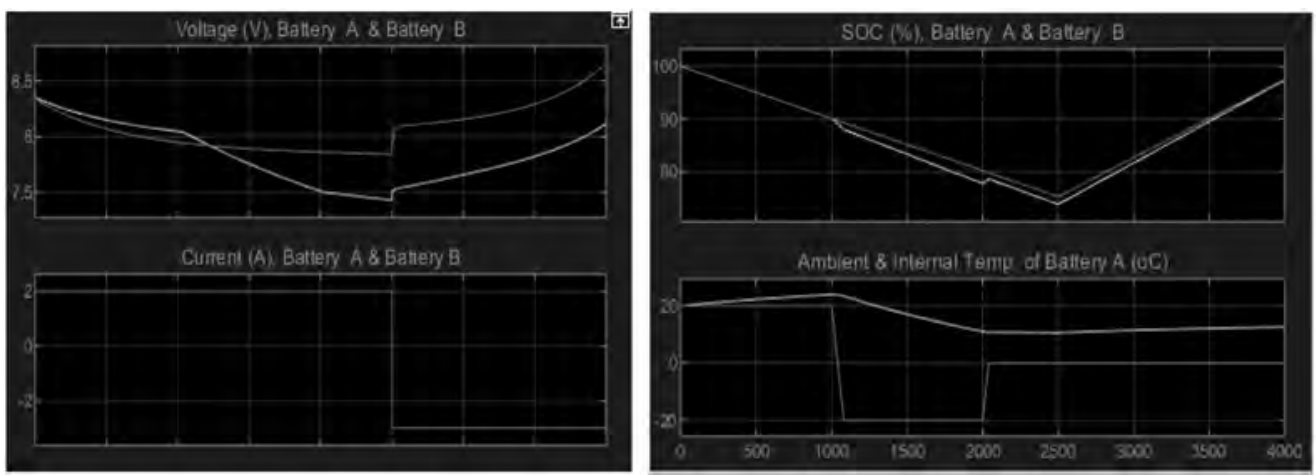

Figure 85.7 Voltage, current, SOC (batteries A,B), ambient and internal temperature of battery A. 


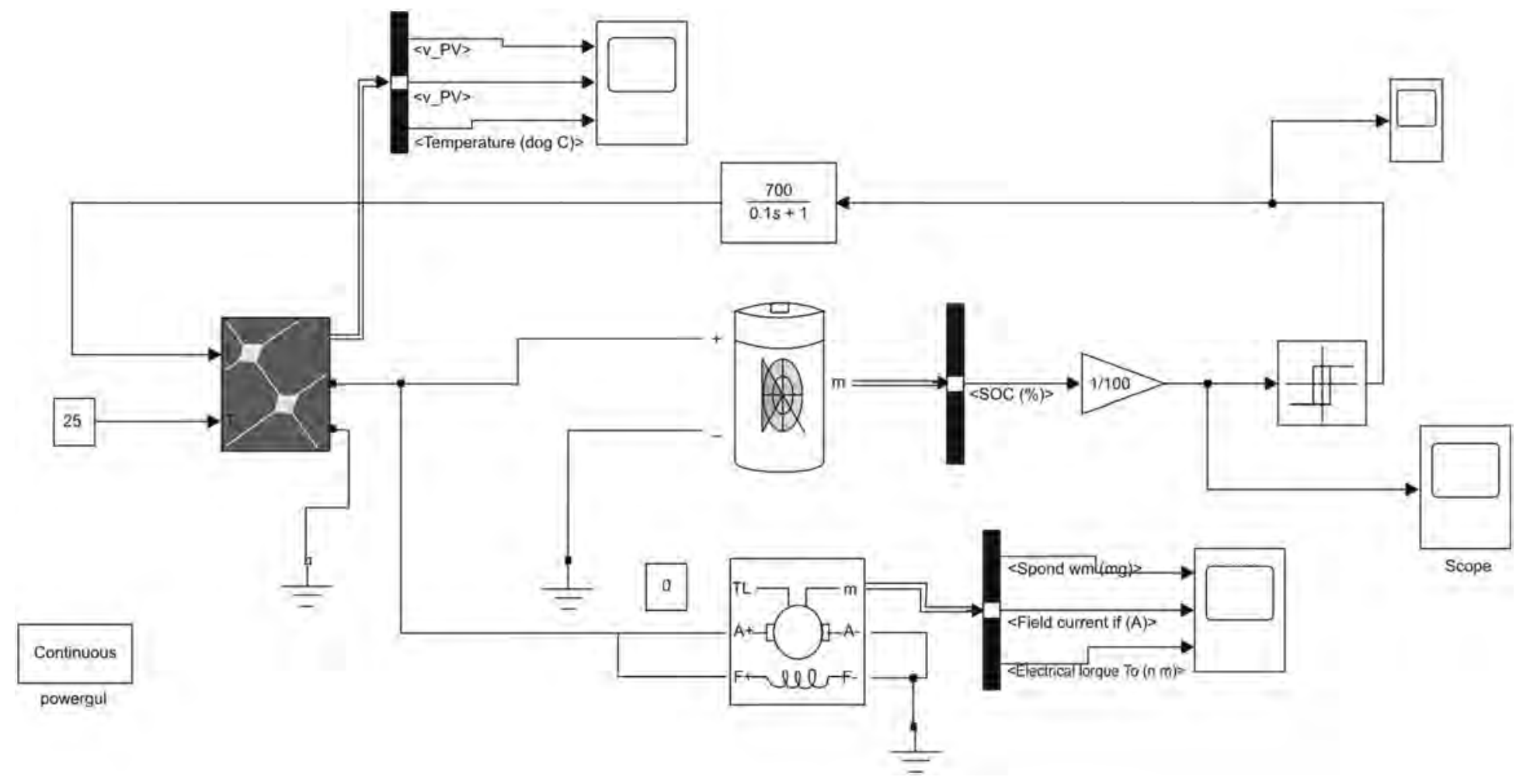

Figure 85.8 Battery with relay model. 


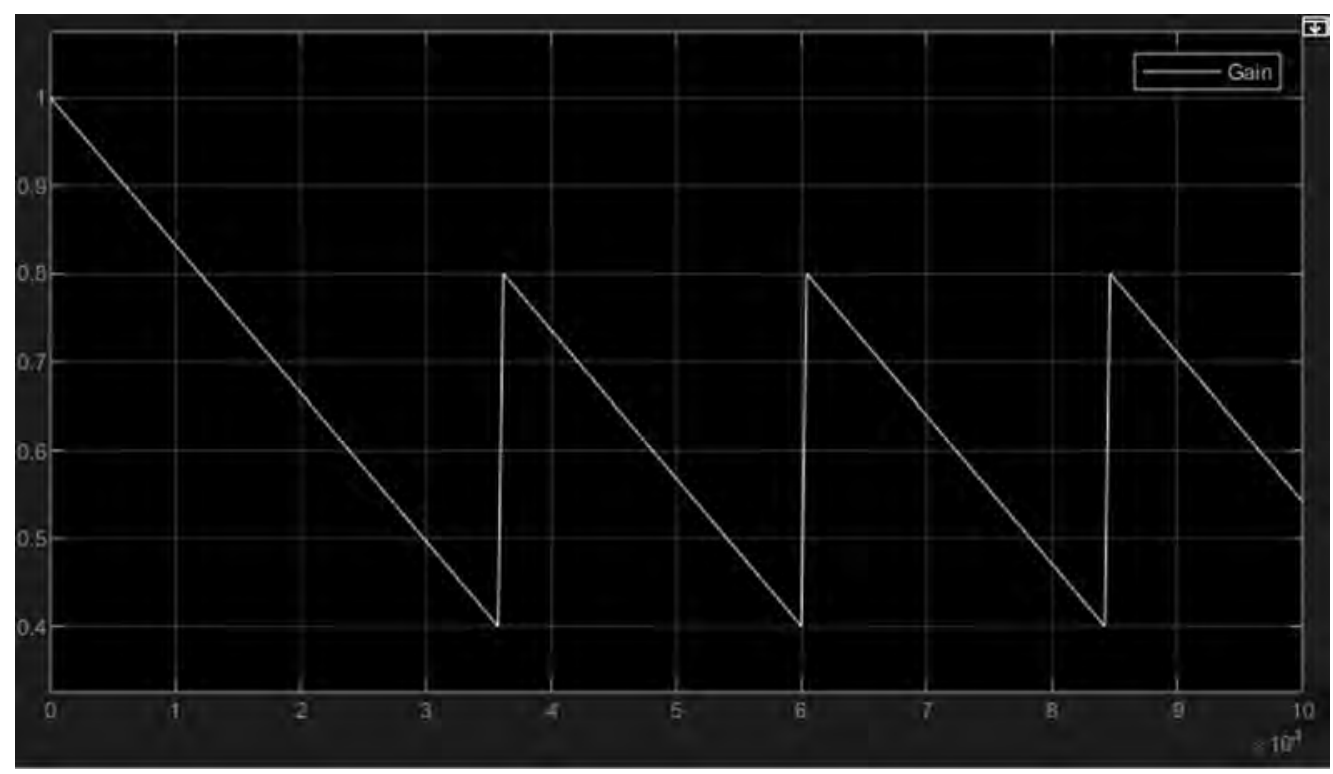

Figure 85.9 State of Charge (SOC) of the battery going through charging and discharging cycles.

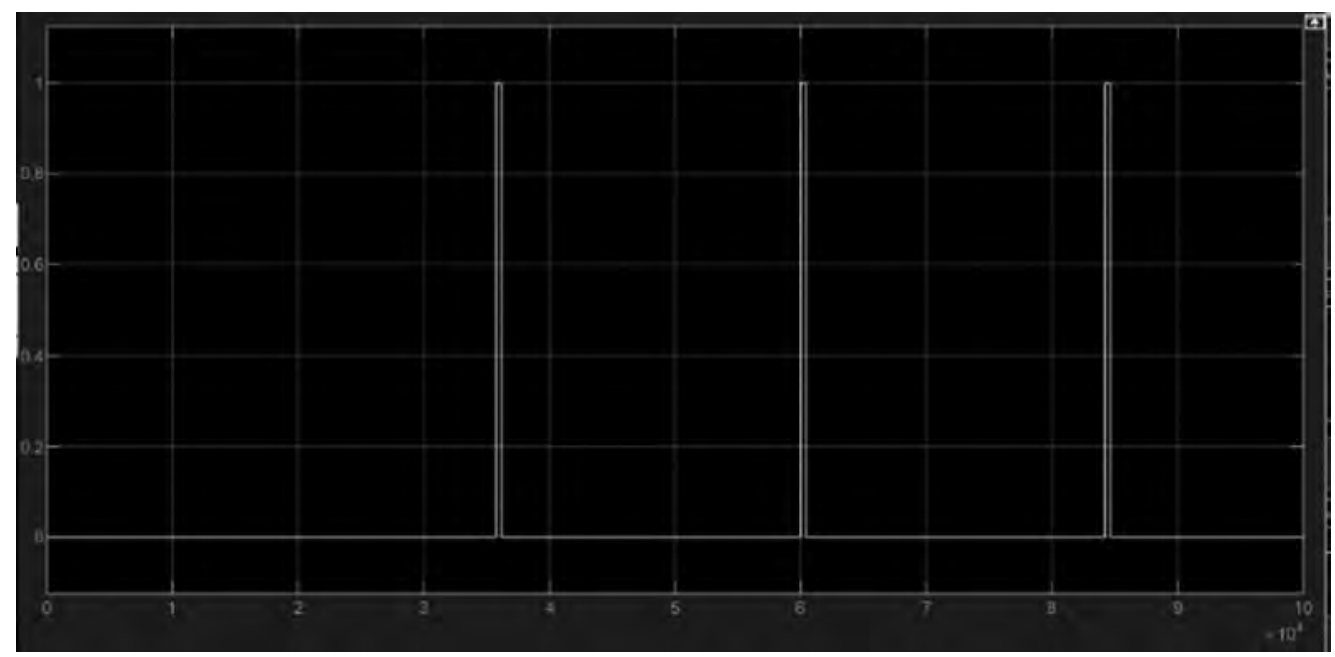

Figure 85.10 Relay giving signal based on SOC of the battery. 


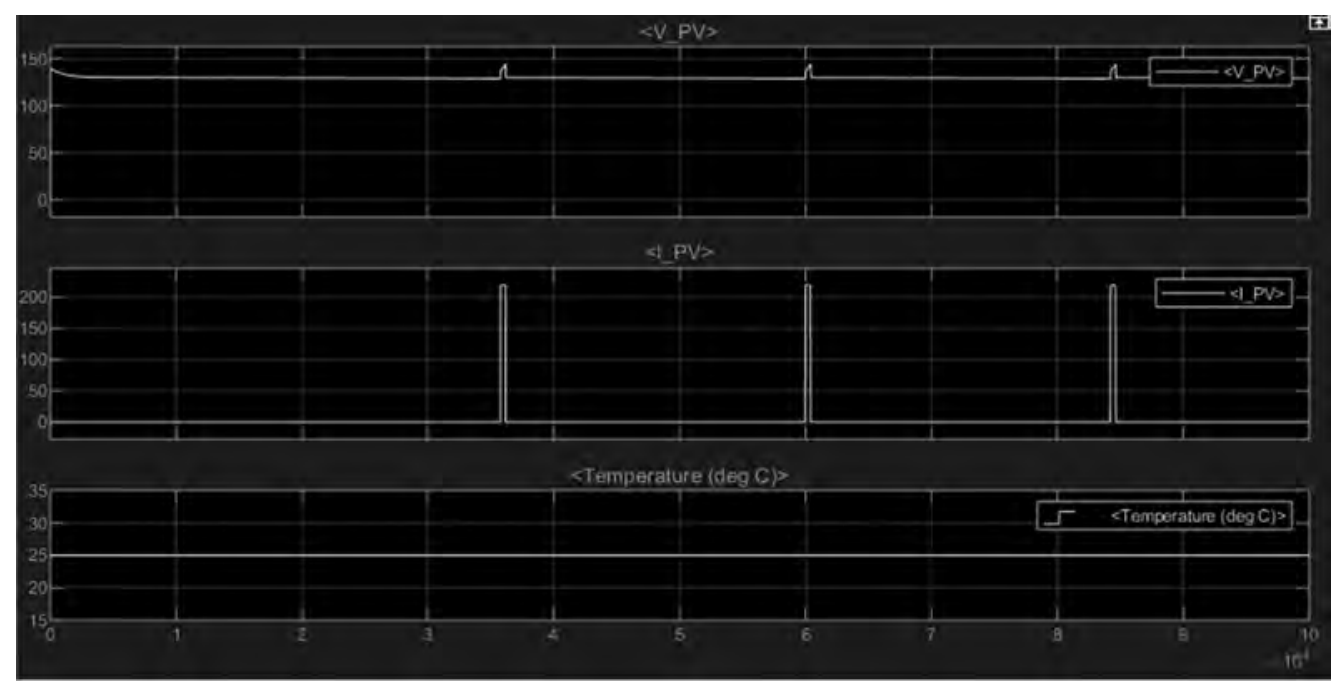

Figure 85.11 Voltage, current and temperature of PV cell.

\section{References}

[1] W. Menghua and X. Bing, "A real-time Android-based monitoring system for the power lithium-ion battery used on EVs," IEEE International Conference on Intelligent Computation Technology and Automation (ICICTA), Changsha, pp. 245-249, 2017.

[2] Huang, Shyh-Chin, Tseng, Kuo-Hsin, Liang, Jin-Wei, Chang, Chung-Liang, and Pecht, Michael G. 2017. "An online SOC and SOH estimation model for lithium-ion batteries," Energies 10, no. 4: 512, 2017

[3] D. Feng, J. Huang, P. Jin, H. Chen, A. Wang and M. Zheng, "Parameter identification and dynamic simulation of lithium-ion power battery based on DP model," IEEE Conference on Industrial Electronics and Applications (ICIEA), Xi'an, China, pp. 1285-1289, 2019.

[4] D. N. Tsyani, A. Kurniasari and C. Hudaya, "Battery monitoring system with LoRa technology," 3rd International Conference on Information Technology, Information System and Electrical Engineering (ICITISEE), Yogyakarta, Indonesia, 2018, pp. 125-129, 2018.

[5] J. Y. Yong, V. K. Ramachandaramurthy, K. M. Tan and N. Mithulananthan, "A review on the state-of-the-art technologies of electric vehicle, its impacts and prospects," Renew. Sustain. Energy Rev., vol. 49, pp. 365-385, 2015.

[6] N. Watrin, B. Blunier and A. Miraoui, "Review of adaptive systems for lithium batteries state-of-charge and state-of health estimation," in Proceedings of IEEE Transportation Electrification Conference and Expo, pp. 1-6, 2012.

[7] M. Luo, Y. Xiao, W. Sun and Z. Wang, "Online battery monitoring system based on GPRS for electric vehicles," 5th International Conference on Intelligent Human-Machine Systems and Cybernetics, Hangzhou, pp. 122-125, 2013.

[8] Rauniyar, Ashish, Irfan, Mohammad, Saputra, Oka D., Kim, Jin W., Lee, Ah R., Jang, Jae M., and Shin, Soo Y. 2017. "Design and development of a real-time monitoring system for multiple lead-acid batteries based on Internet of Things," Future Internet, 9, no. 3, p. 28, 2017.

[9] Song Yonghua, Y. Yang and H. U. Zechun, "Present status and development trend of batteries for electric vehicles," Power System Technology, vol. 35, no. 4, pp. 1-7, 2011.

[10] Languang Lu, Xuebing Han, Jianqiu Li, Jianfeng Hua and Minggao Ouyang, "A review on the key issues for Li-ion battery management in electric vehicles," Journal of Power Sources, vol. 226, pp. 272-288, 2013. 


\section{Intelligent Circuits and Systems}

[11] V. Sharma and V. K. Tayal, "Hardware implementation of sun tracking solar panel using 8051 micro-controller," IEEE International Conference on Reliability, Infocom Technologies and Optimization (Trends and Future Directions) (ICRITO), pp. 483-486, 2017.

[12] S. Singh, P. Rathore, V. K. Tayal and S. K. Sinha, "Improved design of automatic car battery charging system," IEEE International Conference on Power Energy, Environment and Intelligent Control (PEEIC), pp. 1-5, 2019. 


\title{
86 Stewart Platform The 6 DOF parallel manipulator
}

\author{
Manan Luthra*, S.K. Sinha* \\ Department of Electrical and Electronics Engineering, Amity University, \\ Noida, Uttar Pradesh, India
}

\section{Introduction}

A Stewart platform, formerly known as the Gough-Stewart platform, is a parallel manipulator having six prismatic actuators which are fixed to the baseplate in three positions in pairs, which cross over to three positions on the top plate. The platform is flexible enough to move in six different degrees of freedom which is similar to the motion of a freely suspended object or body. The Stewart platform is a one of a kind design which has a variety of applications which involve high precision and accuracy for a work having a limited workspace. Some of them have been studied in this paper. This paper has been divided into several parts. The first few parts give a brief about the basics regarding manipulators. The middle part explains about the Stewart platform, its applications and evolution. The last part describes the mathematics of the platform, which includes its inverse kinematics, followed by control of the platform.

\section{Manipulators}

There are several tasks assigned to an industry grade robot. The most common of them is picking and placing of objects from one place to another, imitating the movement of a fully functional human arm. Such types of robotic arms are called robotic manipulators. Their introductory use was in the inaccessible areas where radioactive or bio-hazardous materials were used. One such robotic manipulator is SCARA (Selective Compliance Assembly Robotic Arm) [1]. A manipulator can be divided into three parts, as shown in Figure 86.1: a base, arm and body and a wrist, each of which has different functions [2].

The technology and science of robotics is developed in such a manner as to develop the mechanical systems which would carry out the work that were initially assigned to human beings. Initially the main thrust was towards using the serial robot manipulators. These types of robots have the advantage of working like a human arm but they lack in their load carrying capability due to their cantilever structure. Hence the links become heavy on one hand and have the tendency to bend due to heavy loads. Therefore, one can always keep their approach inclined towards the use of parallel type manipulators where heavy loads are involved.

\footnotetext{
* Emails: manan894@gmail.com,sksinha6@amity.edu
} 


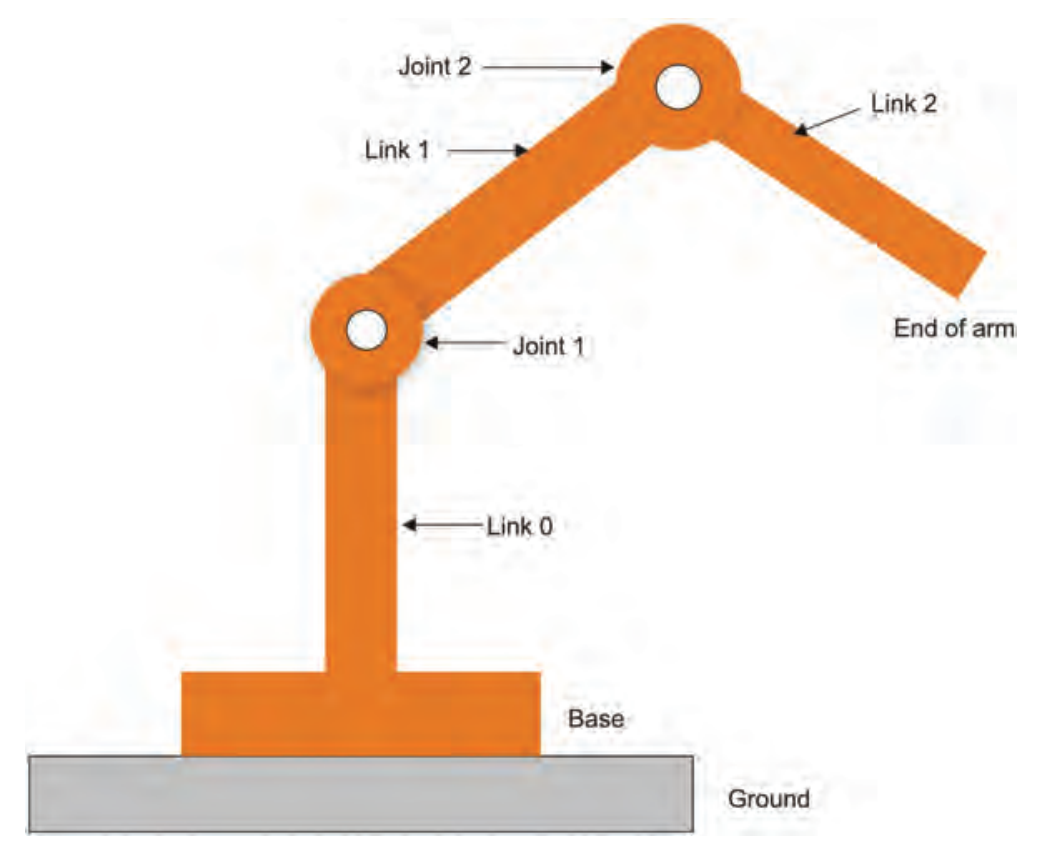

Figure 86.1 Design of a basic manipulator.

\section{Stewart platform}

The Stewart platform [3-5], otherwise known as the Gough-Stewart platform, is a parallel manipulator having six prismatic actuators (such as hydraulic jacks or electric actuators). These actuators are connected to the baseplate of the platform. The devices which are placed on top of the baseplate can have a movement of up to six degrees of freedom.

\subsection{Components of a Stewart platform}

A Stewart platform constitutes of a base plate (fixed) and a mobile plate which is supported with the help of upper legs and lower legs of adjustable length, cylindrical and universal joints.

Six linear actuators are configured in such a way to make it a fully parallel actuated mechanism as shown in Figure 86.2.

\subsection{Degree of freedom}

Degree of freedom refers to the extent to which a mechanical system or a solid inflexible body can move in a three-dimensional space. For example, the rigid train cars connected by the engine in series have only one degree of freedom because the movements of the cars behind the engines are restricted to the shape of the railway tracks.

A human arm is known to have 7 degrees of freedom. A shoulder allows for pitch, yaw and roll, which are given by the elbow and the wrist collectively.

A Stewart platform on the other hand has 6 degrees of freedom, which include 3 linear (translational) movements and 3 rotational movements. These are: up / down movement (heave), left / right movement (sway), forward / backward movement (surge), side to side tilt (roll), forward / backward tilts (pitch), left / right turning (yaw). 


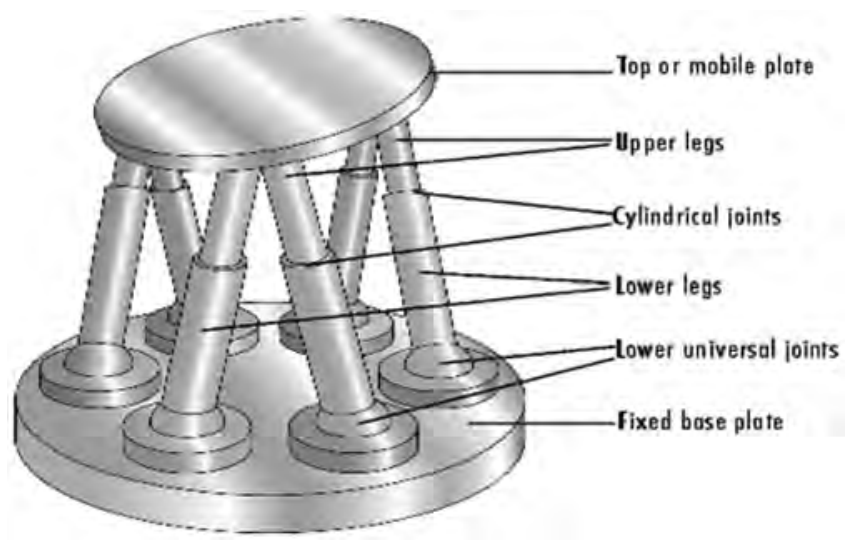

Figure 86.2 Components of Stewart platform.

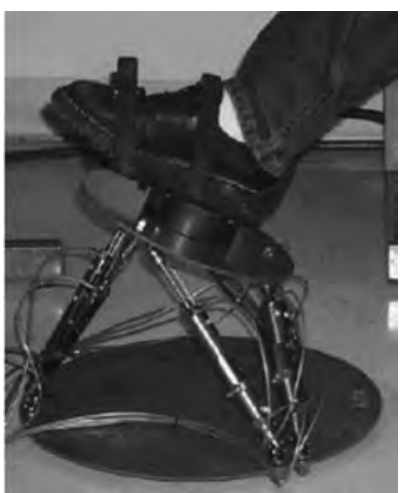

Figure 86.3 Rutgers ankle CP.

\section{Major applications}

\subsection{Rutgers ankle CP}

The ankle is one of the most important parts of the human body. Injuries related to ankles are most common during sports. Taking this into account, various researches have been done in order to build ankle rehabilitation devices that help in increasing the patient's strength and flexibility.

Apart from this, this device is also used to treat a common condition known as hemiplegic cerebral palsy which occurs due to perinatal brain injury. This leads to impaired muscle coordination due to partial paralysis of the body.

The Rutgers ankle CP, as shown in Figure 86.3, is a haptic interface based on the design of the Stewart platform. It aims at strengthening the damaged muscles and better control for children having cerebral palsy. The patient can make the board tilt by applying weight by adjusting their feet during rehabilitation exercises. Various data along with the health of the muscle tissues is collected.

This device at the same time applies forces to the patient's ankle which can be varied in six degrees of freedom according to the patient's condition. 


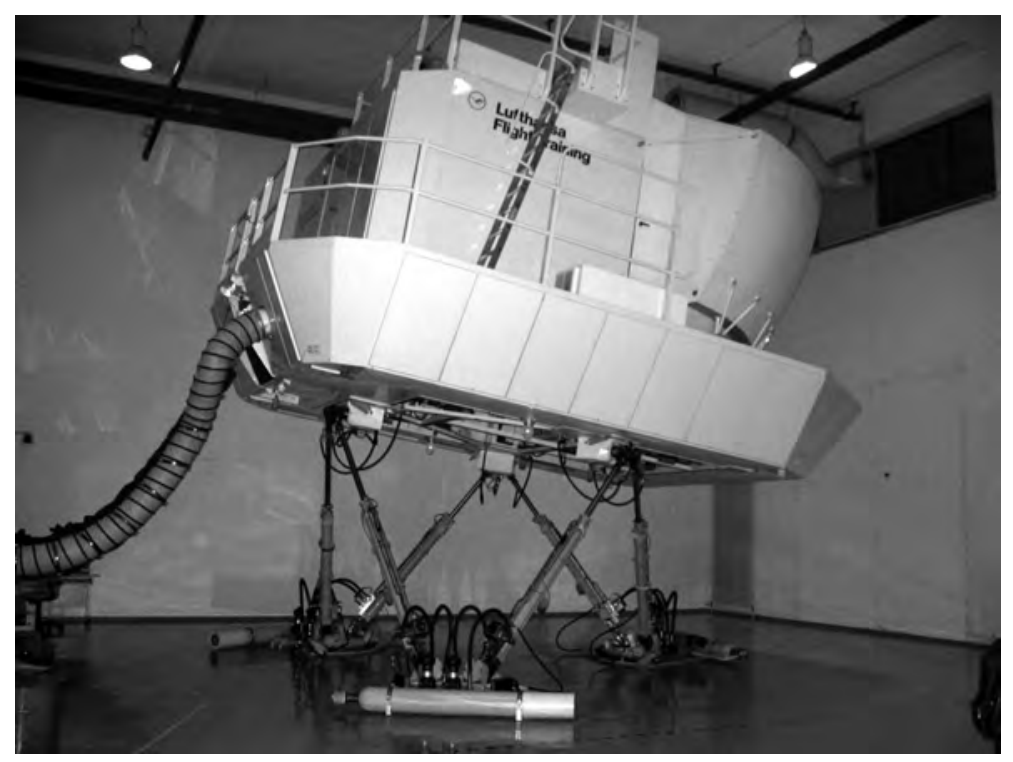

Figure 86.4 Flight simulator.

The treatment session is made a bit more interactive and interesting with the help of rehabilitation games, which helps the patient maintain focus for a longer time, thus enhancing the results.

A major challenge regarding the Rutgers ankle CP is to make its at-home rehabilitation capability a success for those who are unable to commute to the clinic $[6,7]$.

\subsection{Flight simulator}

A flight simulator is a device used to create an artificial environment in order to train a pilot to fly an aircraft, as shown in Figure 86.4. It also replicates how well an aircraft works under certain circumstances such as thunderstorm, precipitation etc. and also helps in analysing aircraft designs and how it reacts with the aircraft system.

Almost all flight simulators are mounted on a six-degree hexapod, or the Stewart platform. The Stewart platform provides six degrees of freedom, or a 360-degree movement to the flight simulator. This aids in creating a realistic design of a real-time aircraft and helps train the pilots without even flying a real aircraft, thus reducing the risk, the overall cost and the challenges which might have been faced otherwise [8].

\subsection{Endoscopy and medical surgeries}

Due to its high accuracy and a very rigid design, parallel manipulators are being used for various medical purposes. Two researchers, Jeff M. Wendlandt and S. Shankar Sastry, proposed a prototype having properties similar to that of the Stewart platform in order to implement it for endoscopy [9]. Endoscopy involves the insertion of a thin long tube inside the human body in order to examine specific internal organs and tissues with the help of a camera. This is usually done through the natural openings of the human body such as the mouth or the anus. 


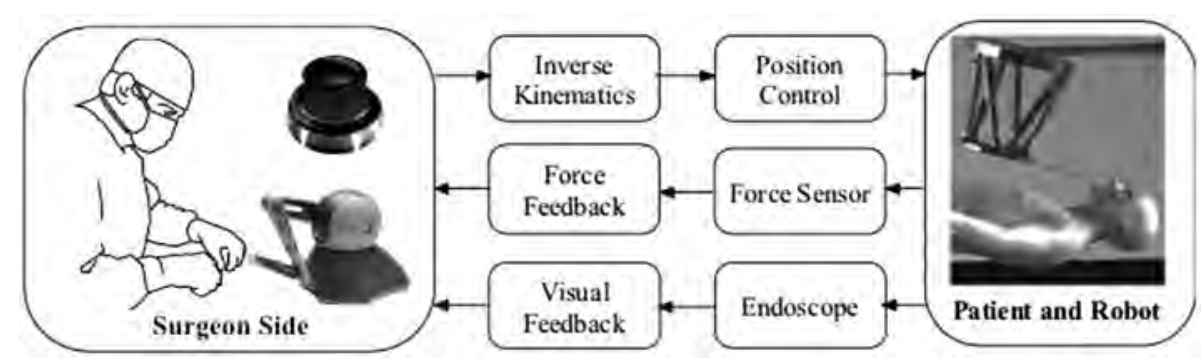

Figure 86.5 Block diagram depicting precision surgery based on Stewart platform design.

A lot of research is also going on in order to implement the Stewart platform design in medical surgeries [10]. It allows up to 6 DOF precision motion just like a surgeon's hand. As illustrated in Figure 86.5, the surgeon can control the robotic arm using a joystick in order to perform precision surgeries [11].

Another application which is a result of the Stewart platform design is in the construction of the upper torso of a spacesuit [12]. The spacesuit is a very important piece of equipment when it comes to performing extravehicular activities by the astronauts, which provides life protection as well as flexibility to do tasks. The design of the Stewart platform has also been used in applications such as telescopes [13], biomedical research, space optics, underwater research, tool technology etc.

\section{Evolution of Stewart platform}

The original purpose of the Stewart platform built in 1965 was its application as a flight simulator. The design by D. Stewart had three linear actuators in parallel. Gough on the other hand had suggested a similar model to that of Stewart's. It was a tire testing machine having six actuators in parallel. Both of their contributions led to the research on what is known today as the Stewart platform or sometimes the StewartGough platform.

The original proposal was devised and presented in 1965. No research was done till 15 years after this proposal, after which Hunt realized the advantages of parallel manipulators. The Stewart platform's high load capacity and precise positioning capabilities were acknowledged after the year 1983 when researchers analysed the advantages that the parallel manipulators possess over the serial ones [14]. Since then, the design of the Stewart platform has been used as a base for a lot of prototypes as well as in applications such as control of electrical devices, simulation of earthquakes, mechanical bulls, crane technology etc.

\section{Kinematics}

\subsection{Forward kinematics}

Forward kinematics involves determining the position as well as the orientation of the end connector with the help of joint angles. It's about moving in a forward direction starting from the base, towards the end connector of the robot. It has one distinct solution. 


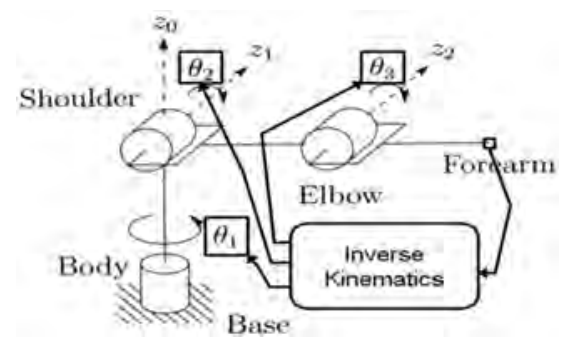

Figure 86.6 Reverse kinematics of a manipulator.

\subsection{Reverse kinematics}

Reverse kinematics works completely opposite from the forward kinematics and shows much more relevance to a majority of the real-world applications and tasks performed by the robots.

It is all about finding the angles of the joints such that the end connector is able to achieve a certain position and a certain orientation, as shown in Figure 86.6. This can be done in many different ways in a workspace.

The forward kinematics of serial manipulators is simpler while its reverse kinematics is quite complicated as it requires a system of non-linear equations.

On the other hand, the reverse kinematics of parallel manipulators is quite straightforward while its forward or direct kinematics is quite sophisticated [15-17].

\subsection{Inverse kinematics of Stewart platform}

In order to understand and solve the inverse kinematics of the Stewart platform, first it is necessary to derive the inverse kinematics for the Stewart platform in two dimensional space having 2 degrees of freedom. Then we will expand that to the three dimensional case having six degrees of freedom.

As you can see in Figure 86.7, we are considering two frames of reference; the "base frame" (or frame 0) and the "platform frame" (or frame 1).

In the inverse kinematics problem, we are going to mention the position as well as the orientation for the end-effector, i.e. for the platform frame (or frame 1). Now we want to find the joint variables, in this case the length of the two legs, to get that position and orientation that we have specified. This problem can be solved much more easily if we solve it simply through vector addition.

The vectors $\overrightarrow{a 1}, \overrightarrow{a 2}, \overrightarrow{s 1}$, and $\overrightarrow{s 2}$ are in the base frame. In order to achieve the solution of the inverse kinematics' problem of the Stewart platform, we need to find the vectors $\overrightarrow{s 1}$ and $\overrightarrow{s 2}$. The magnitude of these two vectors will give us the length of the two legs.

$\vec{p}$ is the position that we want this platform to have. This is an input to this problem, i.e. we already know the position. We also know the vectors $\vec{a} 1$ and $\vec{a} \overrightarrow{2}$ as they're a part of our robot design.

The vectors $\overrightarrow{b 1}$ and $\overrightarrow{b 2}$ are also a part of our robot design. They state where on the platform we have attached the joints relative to the centre of the platform frame (or frame 1). It is important to note that the vectors $\overrightarrow{b 1}$ and $\overrightarrow{b 2}$ are not expressed in the base frame. Rather, they are expressed in the platform frame (or frame 1). 


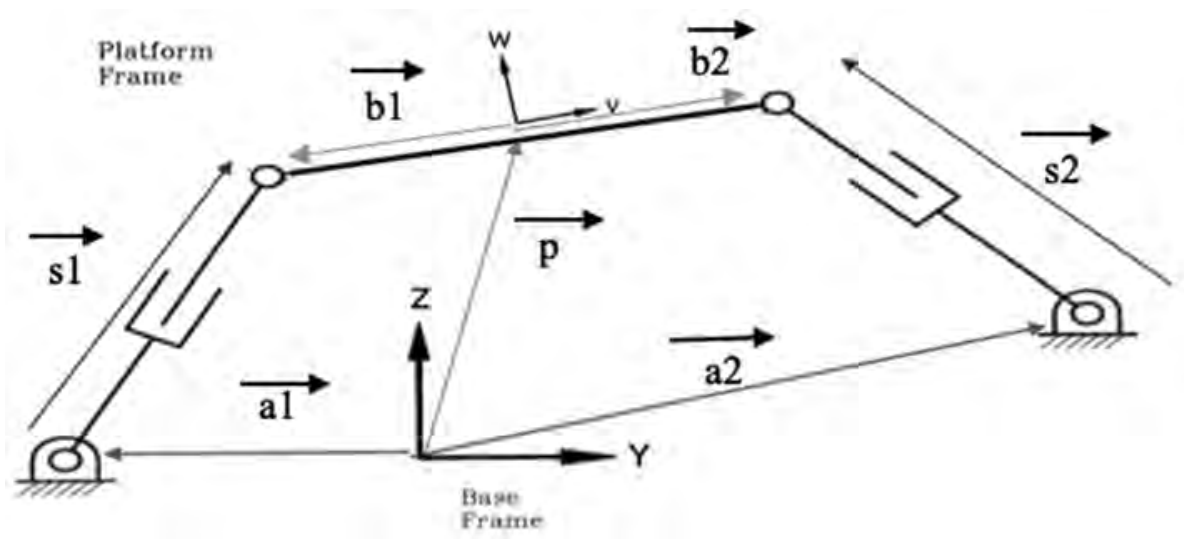

Figure 86.7 Vector depiction of inverse kinematics (Stewart platform).

From vector addition, we get

$$
\overrightarrow{a 2}+\overrightarrow{s 2}=\vec{p}+\overrightarrow{b 2}
$$

We know that $\overrightarrow{b 2}$ here is in frame 1 . In order to express it in frame 0 , we have to multiply $\overrightarrow{b 2}$ by the rotation frame $R_{1}^{0}$, which goes from frame 1 to frame 0 .

We get,

$$
R_{1}^{0} \overrightarrow{b 2}=\overrightarrow{b 2},
$$

where $\overrightarrow{b 2}$ is in frame 0 .

The final inverse kinematics equation,

$$
\overrightarrow{s 2}=\vec{p}+R \overrightarrow{b 2}-\overrightarrow{a 2}
$$

where $\vec{p}$ denotes the position, and $R$ denotes the rotation of the platform required.

$\overrightarrow{b 2}$ and $\overrightarrow{a 2}$ are the robot design parameters.

$\overrightarrow{s 2}$ is the length of the leg 2 in order to achieve the desired position and rotation of the platform.

Figure 86.8 depicts the generalized inverse kinematics equation for six degrees of freedom which is mathematically is given by

$$
\overrightarrow{s_{i}}=\vec{p}+R \overrightarrow{b_{i}}-\overrightarrow{a_{i}}
$$

where

$\overrightarrow{s_{i}}$ represents leg 'i' of the robot

$\vec{p}$ represents the position vector of the centre of the platform

$R$ represents the rotation matrix set for the platform 


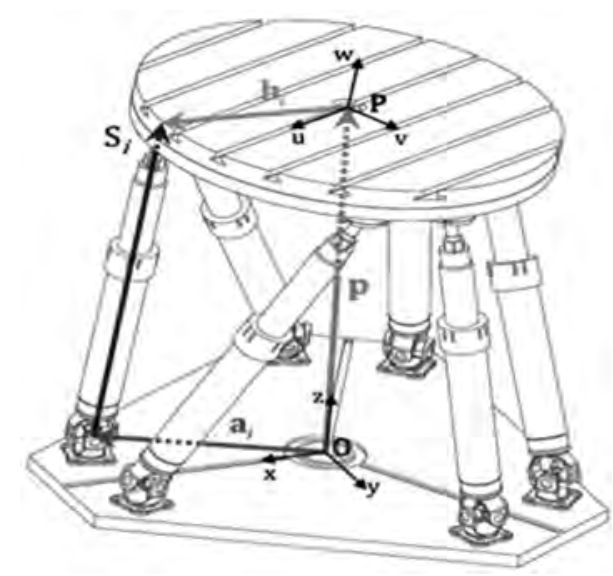

Figure 86.8 Generalised vector diagram (inverse kinematics) of Stewart platform.

$\overrightarrow{b_{i}}$ represents the vector between the centre of the platform and the position where the leg $\mathrm{i}$ is attached to the platform

$\overrightarrow{a_{i}}$ represents the vector between the centre of the base frame and the position where the leg joint is attached to the ground.

\section{Control}

Controlling a system has always been posed as a problem when there are uncertainties in various parameters due to operating condition changes, load changes etc. A system which is designed identical to the original one may even show differences due to different manufacturing processes. When it comes to the precision and accuracy of a machine, it is highly advisable that the same control specifications should meet the control specifications for all the machines of the same or similar type.

Therefore, finding a methodology that builds a robust controller that can be designed by considering only a specific type of system parameters is a necessity $[18,19]$.

This can be achieved with the help of variable structure control (VSC), which is also known as the sliding mode control (SMC) [20]. In a VSC system, the feedback control strategy applied is changed on purpose in the middle of the control process in accordance with some pre-set rules and regulations that depend upon the state of the system. Such a type of a control design may result in a very durable and efficient system and is quite precise and has a very fast response.

The main reason why VSC is highly preferred as a control system for the Stewart platform is because the closed loop feedback of the system is completely unaffected by the system uncertainties and external factors which gives the Stewart platform its high load capacity and precise positioning capabilities.

Other applications of VSC include control of electrical drives, power converters, robots, spacecraft and also in chemical engineering systems.

\section{Conclusion}

The basic structure of the Stewart platform has been studied along with its applications in the real world. It has come to our knowledge that the design of the Stewart platform is a 
masterpiece in technology which has aided a lot of new innovations in robotics. Though the main focus of this paper has been the Gough-Stewart platform itself, a comparison between serial and parallel manipulators has also been drawn. Another area which has been looked upon is the history and the origin of this platform.

Apart from this, a distinction between the forward kinematics and reverse kinematics of the Stewart platform was seen and it was clearly understood that its inverse kinematics is quite difficult and has a lot of complexities involved as compared to the forward kinematics. Efforts have been made in order to give a brief about the mathematical equations related to the same.

The paper lastly presents the method of control involved for the Gough-Stewart platform, which is the ultimate reason behind its high precision and reliability.

\section{References}

[1] Claudio Urrea, Juan Cortés and José Pascal, "Design, construction and control of a SCARA manipulator with 6 degrees of freedom", Journal of Applied Research and Technology, vol. 14, pp. 396-404, 2016.

[2] Pires, J. Norberto. "Robot manipulators and control systems" in Industrial Robots Programming: Building Applications for the Factories of the Future. Boston, MA: Springer, 2007, pp. 35-107

[3] Dasgupta, B., and Mruthyunjaya, T. S., "The Stewart platform manipulator: a review", Mechanism and Machine Theory, vol. 35, no. 1, pp. 15-40, 2000.

[4] Stewart, D. "A platform with six degrees of freedom", Proceedings of the Institution of Mechanical Engineers, vol. 180, no. 1, pp.371-386, 1965. doi:10.1243/PIME_PROC_1965_ 180_029_02.

[5] Furqan, M., Suhaib, M., and Ahmad, N. “Studies on Stewart platform manipulator: A review”, Journal of Mechanical Science and Technology, vol. 31, pp. 4459-4470, 2017. https://doi.org/ 10.1007/s12206-017-0846-1.

[6] M. Girone, G. Burdea, M. Bouzit, V.G. Popescu and J. Deutsch, "A Stewart Platform-based System for Ankle Telerehabilitation”, invited article, Special Issue on Personal Robotics, Autonomous Robots, vol. 10, pp. 203-212, 2001.

[7] Cioi, Daniel, Kale, Angad, Burdea, Grigore, Engsberg, Jack, Janes, William, and Ross, Sandy, Ankle control and strength training for children with cerebral palsy using the Rutgers Ankle CP, Proc. IEEE International Conference on Rehabilitation Robotics, 2011.

[8] Kai-Feng Liu, Dong Li, Xu Liu, Chen Zuo and Jian Zha "Study of 6-D of control of the Stewart platform”, 2018 International Conference on Energy, Power and Materials Engineering, 2018

[9] J. M. Wendlandt and S. S. Sastry, "Design and control of a simplified Stewart platform for endoscopy," Proceedings of 1994 33rd IEEE Conference on Decision and Control, Lake Buena Vista, FL, vol. 1, pp. 357-362, 1994.

[10] Kizir, Selçuk, and Bingul, Z., "Design and development of a Stewart platform assisted and navigated transsphenoidal surgery", Turkish Journal Of Electrical Engineering \& Computer Sciences, vol. 27, no. 2, pp. 961-972, 2019.

[11] Wapler, M., Urban, V., Weisener, T., Stallkamp, J., Dürr, M., and Hiller, A. (2003). “A Stewart platform for precision surgery", Transactions of the Institute of Measurement and Control, vol. 25, Ino. 4, pp. 329-334, 2003.

[12] Li, Yuan Feng, and Wan Xin Zhang. "The application of Stewart platform in spacesuit upper torso active fit design”, Applied Mechanics and Materials, vol. 798, pp. 571-575, 2015.

[13] Su, Y.X., and Duan, B.Y., "The application of the Stewart platform in large spherical radio telescopes", Journal of Robotic Systems, vol. 17, no. 7, pp. 375-383, 2000.

[14] Ilian Bonev, "The true origins of parallel robots", January 24, 2003. Available: www. parallemic.org/Reviews/Review007.html.

[15] Petrescu, Relly Victoria, Aversa, Raffaella, Apicella, Antonio, Kozaitis, Samuel, Abu-Lebdeh, Taher, and Petrescu, Florian Ion, "Inverse kinematics of a Stewart platform", Journal of Mechatronics and Robotics, vol. 2, no. 1, pp. 45-59, 2018. 


\section{Intelligent Circuits and Systems}

[16] K. Liu, J. M. Fitzgerald and F. L. Lewis, "Kinematic analysis of a Stewart platform manipulator," in IEEE Transactions on Industrial Electronics, vol. 40, no. 2, pp. 282-293, 1993.

[17] Stefan Staicu, "Dynamics of the 6-6 Stewart parallel manipulator", Robotics and ComputerIntegrated Manufacturing, vol. 27, no. 1, 2011

[18] Uluçay, Özer. "Design and control of Stewart platform." M.S. thesis, Sabancı University, Istanbul, Turkey, 2006.

[19] Azar, A.T., Zhu, Q., Khamis, A., and Zhao, D., "Control design approaches for parallel robot manipulators: a review", Int. J. Modelling, Identification and Control, vol. 28, no. 3, pp.199_ $211,2017$.

[20] H. Wang and Z. Zuo, "Adaptive sliding mode control of flexure jointed hexapods," 2019 Chinese Control Conference (CCC), Guangzhou, China, pp. 479-484, 2019. 


\title{
87 IoT security algorithms A performance comparison
}

\author{
Adnan Mukhtar*, P.M. Tiwari*, H.P. Singh*
}

Amity University Uttar Pradesh, Noida, India

\section{Introduction}

The term Internet of Things (IoT) is bifurcated into two terms: Internet and Things, the Internet is the backbone of affinity and Things are the embedded devices. IoT is a grid of embedded devices that gathers and interchanges data. It is an idea to connect any physical device to the internet. Initially, it used to be "Embedded Internet", but, in 1997, Kevin Ashton renamed it to the Internet of Things [1,2]. The main idea laid behind it is to link any device, anything, any service, through any path at any place and time with the Internet. There is a dire need to adopt such technologies that may serve fruitful for the users so that they can address various requirements of IoT like speed and security [1]. It is a way by which embedded devices talk to each other directly or indirectly. It is a heterogeneous mix of embedded technology (ET), network technology (NT) and information technology (IT) [1].

$$
\mathrm{IOT}=\mathrm{ET}+\mathrm{NT}+\mathrm{IT}
$$

This paper mainly emphasizes IoT security because security is an essential component of IoT. By performing security algorithms, it is possible to secure IoT devices [8]. Moreover, there are several ways to secure sensitive information, each having its pros and cons [10]. However, the main aim of the security algorithms is not only to ensure confidentiality but also to secure and stop the illegitimate users from accessing sensitive information. In this way, legitimate users can access their information, data and messages without any interruption [10] and it is possible to filter out the hacker's threat. These algorithms are categorized into two classes: symmetric and asymmetric. The algorithms performed in this paper are AES and RSA. AES comes under the symmetric class and RSA comes under the asymmetric class. The legitimate users can access sensitive information, data and messages by masking and un-masking processes and these processes serve fruitful to the users [12]. When users communicate over the networks, the sender executes the masking process with the help of a private key, then the receiver executes the unmasking [13].

This paper consists of five sections: Section 2 describes the architecture and security of IoT, Section 3 describes the security algorithms, Sections 4 describes the simulations carried out and Section 5 discusses the conclusion.

\footnotetext{
*Emails: adnan.mukhtar143@gmail.com,pmtiwari@amity.edu, hpsingh2@amity.edu
} 


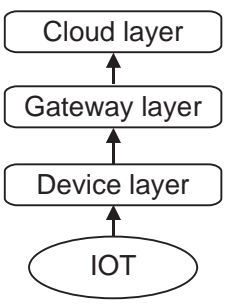

Figure 87.1 IoT architecture.

\section{IoT architecture and its security}

\subsection{IoT architecture}

Figure 87.1 represents the IoT architecture.

The IoT comprises a device layer, a gateway layer and a cloud layer.

\section{a. Device layer}

Mainly the device layer of IoT consists of embedded devices such as sensors, actuators, controllers, etc. [3]. These devices perform several functions.

1. They sense and record data.

2. They establish a connection with the internet.

Sensors transform the information attained from the outside world into data which is then analysed [3]. The food of IoT (data) originates from sensors. Actuators turn energy into motions.

\section{b. Gateway layer}

The gateway layer consists of the devices that link the devices of the device layer with the Internet. The devices of the gateway layer can establish the networks. This layer collects data from various sensors and then imparts it to the cloud [3]. This layer resembles that of the Wi-Fi router.

\section{c. Cloud layer}

The cloud layer stores the data captured from various sensors present in the actuators. The data is accepted, stored and then processed to analyse and to make decisions. This layer creates live dashboards that the decision-maker monitors.

Analytics of the IoT depends upon data collection to reveal actionable insight by making use of various tools and techniques so to attain intended outputs.

\subsection{IoT security}

IoT security is a technological platform and an important element linked with IoT which lays its emphasis in securing the components and the networks which have Internet 
connectivity. The main goal of IoT security is to secure and stop the illegitimate users from accessing the data, messages and files when sending over the networks [10]. IoT aims at maintaining the confidentiality and ensures the user in securing the devices, important data and messages [11]. Internet connectivity is incorporated into the components which serve the purpose of computation and to the components which are digitally driven. Various components are supplied with distinctive attributes and the capabilities to carry a huge pile of information automatically. Internet connectivity is provided to various components which in turn serve as a platform to various severe issues if the components are not properly secured. Security is an important and a major concern since IoT component can infiltrate and attack various wider networks. Devices that have Internet connectivity face very serious issues about their security and these issues are very hard to resolve if they are not properly secured. IoT users are linked to the Internet from everywhere, so they always lie under the threat of losing a huge pile of important data and their personal information. There is a threat from the hacker's side, who can hack IoT devices, IoT architecture and IoT networks [4]. Moreover, the addition of new components, upcoming updates and third party data protection is also a critical concern.

Lack of security measures will result in decreased adoption among users. IoT security is one of the major concerns related to IoT components, network architecture, user's data and personal information. There is a dire need to take safety precautions and it is very important and essential to emphasize the security of IoT devices that will filter out the hacker's threat.

Moreover, some other ways to secure Internet-enabled devices are as follows:

- By installing Norton Security software that can protect the internet-enabled devices from the viruses that already exist.

- By making use of special and robust passwords for Wi-Fi networks.

- When it is about using various applications, it is very important to be mindful of their privacy policies.

- It is very important to make some surveys before buying new devices because they might carry our information and data. It is also important to be mindful of what kind of data they collect, how they store and protect it.

- By making use of Norton Secure VPN that will protect the data and information transferred on home or public Wi-Fi security algorithms.

\section{IoT security algorithms}

Out of many existing security algorithms, this paper aims to highlight two algorithms:

1. Advanced Encryption Standards (AES).

2. Rivest Shamir Adleman (RSA).

These algorithms secure and stop the illegitimate users from accessing the data, messages and files when sending over the networks. Securing the data, messages and files involves masking and unmasking processes. The transformation of simple text into cipher text and cipher text into the plain text refers to masking and unmasking respectively.

\subsection{Advanced Encryption Standard (AES)}

AES is a symmetric technique by which messages, data and files can be masked and unmasked [8]. It operates on a private key. The private key is used for masking and 
unmasking messages, data and files. AES helps in securing the data that is sent by the sender to the receiver. The key size can be 128, 198 or 256 bits while the size of the block is 128 bits [9]. The time required for masking and unmasking files, messages and data is fast. This technique is well secured and was framed by Vincent Rijmen and Joan Daemen in the year 2000. For masking and unmasking processes, the private key is used.

\subsection{Rivest Shamir Adleman (RSA)}

RSA is an asymmetric technique by which messages, data and files can be masked and unmasked [8]. It operates on two keys, one is public and the other private. The masses can use the public key and no one has access to the private key. To mask and unmask messages, access to public and private keys is required. RSA helps in securing the data transferred by the sender to the user. The length of the keys can be greater than 1024 bits [9]. The heterogeneous mix of both the keys helps the users in masking the important data [6]. The basic steps involved are [7]:

1. Generation of public and private keys.

2. Masking.

3. Unmasking.

The public key and the private key are created as [7]:

- Select two prime numbers (L, M) randomly.

- Compute modulus (S) for both the keys

$\mathrm{S}=\mathrm{LM}$

- Compute Euler's totient

$$
\varphi^{\prime}(\mathrm{S})=(\mathrm{L}-1)(\mathrm{M}-1)
$$

- Select an exponent $\left(\mathrm{K}^{\prime}\right)$ for the public key such that

$$
1<\mathrm{K}^{\prime}<\varphi^{\prime}(\mathrm{S})
$$

- Compute an exponent $\left(\mathrm{D}^{\prime}\right)$ for the private key for an integer value $\mathrm{x}$ such that

$$
\mathrm{D}^{\prime}=\left[\left(1+\mathrm{X} \varphi^{\prime}(\mathrm{S})\right)\right] / \mathrm{K}^{\prime}
$$

The RSA flowchart is given in Figure 87.2.

Table 87.1 gives the comparison between AES and RSA algorithms.

\section{Simulations results for masking and unmasking a file hello.c}

The simulations for masking and unmasking a file named hello.c are carried out in Linux 16.04 LTS with the help of the security algorithms (AES and RSA). 


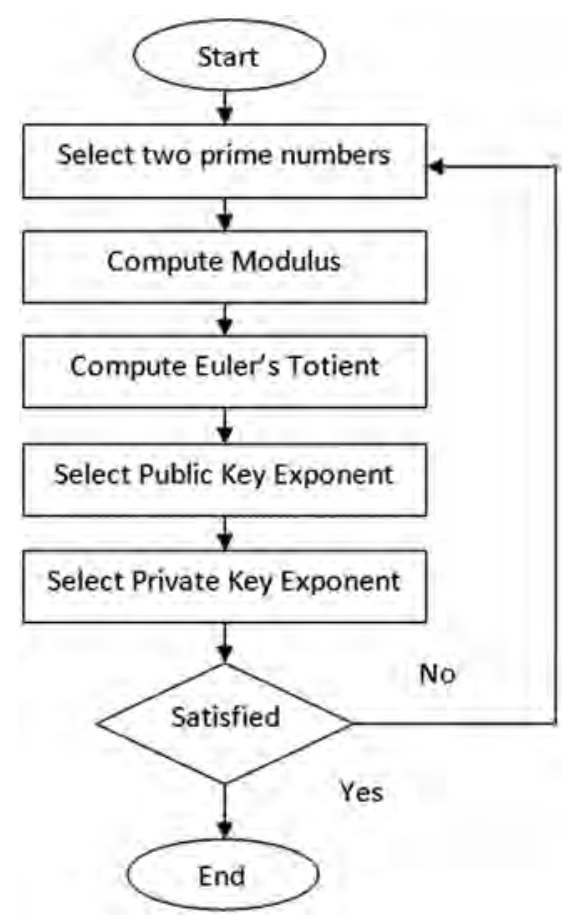

Figure 87.2 RSA flowchart.

Table 87.1 Comparison of AES and RSA algorithms

\begin{tabular}{|c|c|c|}
\hline S. no. & AES & $R S A$ \\
\hline 01 & It is a symmetric algorithm & It is an asymmetric algorithm \\
\hline 02 & The key size can be 128,192 or 256 bits & The key size is $\geq 1024$ bits \\
\hline 03 & The size of the block is 128 bits & The minimum size of the block is 512 bits \\
\hline 04 & The time required for masking a file is fast & $\begin{array}{l}\text { The time required for masking a file } \\
\text { is slow }\end{array}$ \\
\hline 05 & The time required for unmasking a file is fast & $\begin{array}{l}\text { The time required for unmasking a file } \\
\text { is slow }\end{array}$ \\
\hline 06 & This algorithm is well secured & This algorithm is least secured \\
\hline 07 & The private key is used for masking a file & The public key is used for masking a file \\
\hline 08 & The private key is used for unmasking a file & $\begin{array}{l}\text { The private key is used for unmasking } \\
\text { a file }\end{array}$ \\
\hline
\end{tabular}

\subsection{AES algorithm}

The command used for masking is OpenSSL aes-256-cbc -a -salt -in hello.c -out hello.enc. The content inside the file hello.c is shown as

\#include <stdio.h>

Void main ()

\{

Printf ("Kashmir the paradise on earth $\backslash n$ ”);

\} 


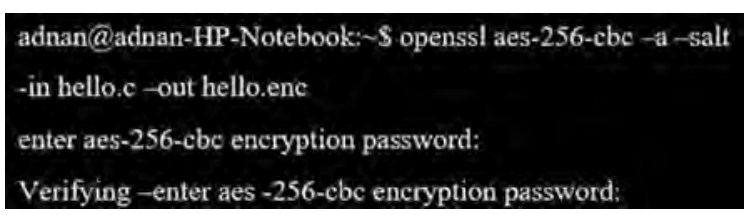

Figure 87.3 Command for masking.

\section{V2FdGVKX1/2Nf308MFyFdd6G0HqIX+KcLwJdTyfN 17TXv+S0Tm6BSd4FYuB5GiI3CLZGPKpv4TGVMWh \\ IQtb+WUKPMAnIM6upH6wMNUcTVAKwD/SqLWG}

Figure 87.4 Masked file.

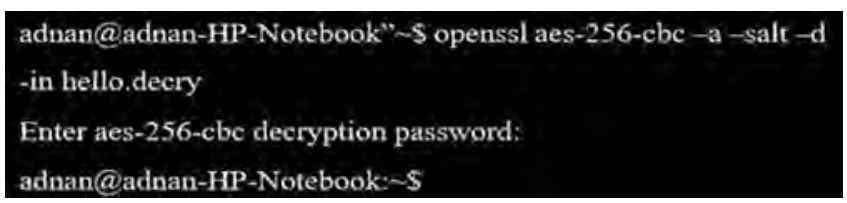

Figure 87.5 Command for unmasking.

The command used for masking the file hello.c in Linux 16.04 LTS is shown in Figure 87.3 and after masking the file, a password is required as a masking key. The masked file is shown in Figure 87.4.

The command used for unmasking is OpenSSL aes-256-cbc-a -salt - $\mathrm{d}$-in hello.enc -out hello.decry. The command used for unmasking the file hello.c is shown in Figure 87.5.

After unmasking the file, a password is required as an unmasking key. The content inside the unmasked file hello.c is shown as

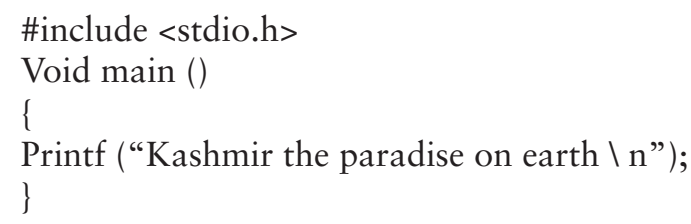

\subsection{RSA algorithm}

The command for generating the private key is Openssl genrsa -out private.pem 1024. The public key is extracted from the private key. The command for generating the public key from the private key is Openssl RSA -in private.pem -out public.pem -out form PEM -pub out. Figure 87.6 presents the generation of the private key.

To check the key file generated, the command used is cat private.pem. The key generated is shown in Figure 87.7.

The generation of the public key is given in Figure 87.8.

To check the key file generated, the command used is cat public.pem. The key generated is given in Figure 87.9. 


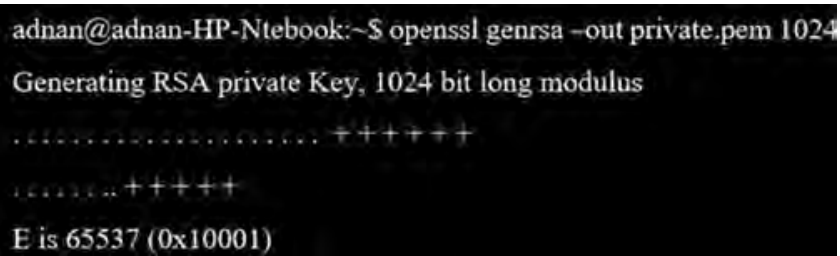

Figure 87.6 Generation of private key.

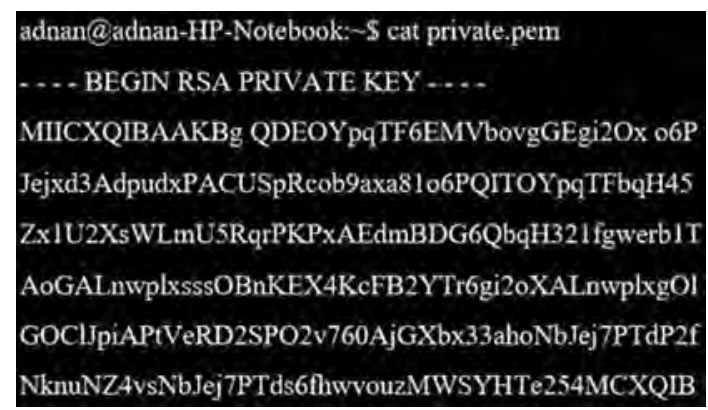

Figure 87.7 Generated private key.

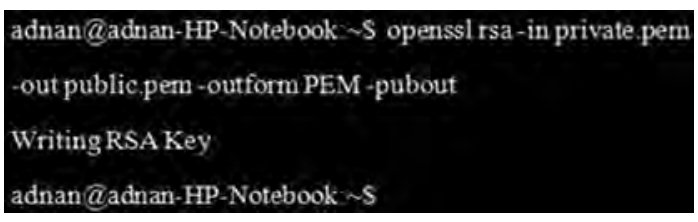

Figure 87.8 Generation of public key.

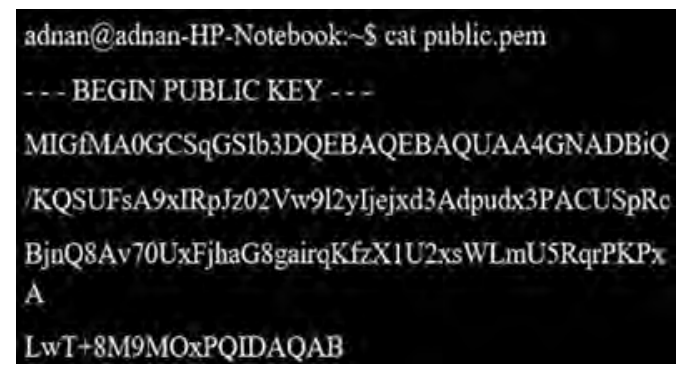

Figure 87.9 Generated public key.

For masking, the public key is used and for unmasking, the private key is used. The command used for masking is: OpenSSL rsautl -encrypt -inkey public.pem - pub in -in the filename. The command used for unmasking is OpenSSL rsautl -decrypt inkey private. pem -in new filename -out new filename [10]. For masking, first, the file name is entered 


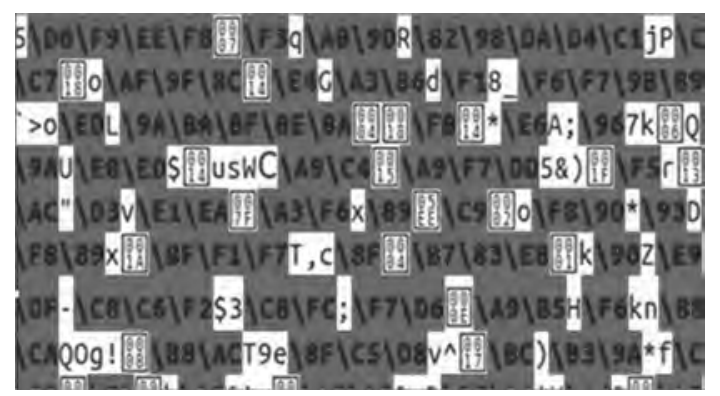

Figure 87.10 Masked data inside the file.

using the command: cat filename. In this paper, the file name used is hello.c. The content inside the file hello.c is shown as

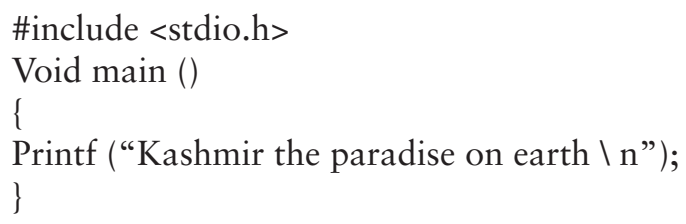

Now to check the masked file the command used is: cat hello.ssl. The masked file hello.c is given in Figure 87.10.

For unmasking, the command used is cat hello.txt. The content inside the unmasked file hello.ssl is shown as

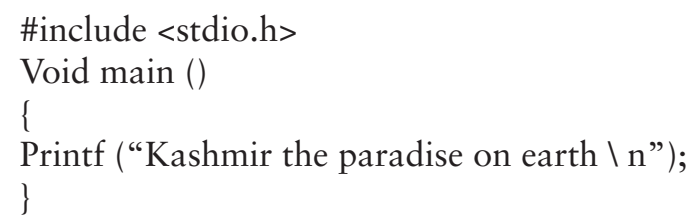

\section{Conclusion}

The security of IoT is a very crucial and critical concern. Security algorithms play a vital role in securing IoT. In this work, the AES and RSA algorithms are simulated and their performances are verified using simulations on Linux 16.04 LTS. The simulations show that the AES algorithm is faster and takes comparatively less masking and unmasking time. Additionally, simulations verified that with one key only the AES algorithm can perform masking and unmasking processes, whereas the RSA algorithm uses two separate keys for masking and unmasking. Moreover, simulations also verified that on increasing the key size above 1024 bits, the generation process of private and public keys became slow and the RSA algorithm took more time for masking and unmasking processes.

\section{References}

1. Keyur K. Patel and Sunil M. Patel. "Internet of Things-IoT: definition, characteristics, architecture, enabling technologies, application \& future challenges”, International Journal of Engineering Science and Computing, vol. 6, no. 5, pp. 6122-6131, 2016. 
2. Ashton, K., "The Internet of Things", Journal of Advances in the Internet of Things, vol. 5, no. 3, pp. 97-114, 2009.

3. Alex Grizhnevich, "IoT architecture: building blocks and how they work", Science Soft, pp. 1-3, 2018.

4. Margaret Rouse, Sharon Shea, and Ivy Wigmore, "IoT analytics guide: Understanding Internet of Things data".

5. Prashanti, G., Deepthi, S., and Sandhya, Rani. K., "A novel approach for data encryption standard algorithm", International Journal of Engineering and Advanced Technology, vol. 2, no. 5, pp. 589-598, 2013.

6. A. Padmapriya and P. Subhasri, "Cloud computing: security challenges \& encryption practices", International Journal of Advanced Research in Computer Science and Software Engineering, vol. 3, no. 3, pp. 255-259, 2013.

7. Sunitha, K., and Prashanth, K.S., "Enhancing privacy in cloud service provider using cryptographic algorithm", IOSR Journal of Computer Engineering, vol. 12, no. 5, pp. 98-103, 2013.

8. Idrizi, Florim, Dalipi, Fisnik, and Rustemi, Ejup, "Analyzing the speed of combined cryptographic algorithms with secret and public key", International Journal of Engineering Research and Development, vol. 8, no. 3, pp. 45-51, 2013.

9. Prerna Mahajan and Abhishek Sachdeva, "A study of encryption algorithms AES, DES, and RSA for security, "Global Journal of Computer Science and Technology”, Network, Web and Security” vol. 13, no. 15, pp. 15-22, 2013.

10. Namita George Gonsalves, Nootana G. Bhat, and Kiran K. Tangod, "Performance analysis of secured communication with cryptography using Fibonacci series", International Journal of Innovations in Engineering and Technology, vol. 4, no. 6, pp. 42-46, 2017.

11. Mardiana Binti Mohamad Noor and Wan Hassan, "Current research on Internet of Things (IoT): a survey, Computer Networks, vol. 148, pp. 283-294, 2019.

12. Md. Alam Hossain, Md. Biddut Hossain, Md. Shafin Uddin and Shariar Md. Imtiaz, "Performance analysis of different cryptography algorithms", International Journal of Advanced Research in Computer Science and Software Engineering, vol. 6, no. 3, pp. 659$665,2016$.

13. Sangeeta and Arpneek Kaur, "A review on symmetric key cryptography algorithms", International Journal of Advanced Research in Computer Science, vol. 8, no. 4, pp. 23582361, 2017. 


\title{
88 COVID-19 ramifications during pregnancy
}

\author{
Rydhm Beri ${ }^{1, *}$, Mithilesh Kr. Dubey ${ }^{2, *}$, Anita Geblot ${ }^{3, *}$, \\ Rajesh Singh",*
}

\author{
${ }^{1} \mathrm{PhD}$ Scholar, School of Computer Applications, Lovely Professional University, \\ Phagwara, India \\ ${ }^{2}$ Associate Professor, School of Computer Applications, Lovely Professional \\ University, Phagwara, India \\ ${ }^{3}$ Associate Professor, School of Electronics \& Communication Engineering, \\ Lovely Professional University, Phagwara, India \\ ${ }^{4}$ Professor, School of Electronics \& Communication Engineering, Lovely \\ Professional University, Phagwara, India
}

\section{Introduction}

Coronavirus $(\mathrm{CoV})$ is a group of viruses usually found in mammals or birds. It has crownlike particles so it got the name Coronavirus. The coronavirus ordination is +ssRNA, which is second highest sized RNA with $27-32 \mathrm{~kb}$ size [2]. It directly attacks the human respiratory which leads to minor health issues like cough or cold to major issue like failure of lungs that become the reason of death. Coronavirus was first found in human in 1960s when an adult's respiratory system was damaged with the mild cold. Later in 2003, five humans were detected with the same virus but had the severe health condition [1].

\subsection{Categories of coronaviruses}

The coronavirus is a danger disease and may ranges from mild cold to severe health conditions. Coronavirus for human beings is a subgrouping into two major groups. Some of them are further categorized into sub-groups which affect human health in various ways and have different symptoms and health issues as shown in Figure 88.1.

1.1.1. SARS CoV: Severe Acute Respiratory Syndrome (SARS CoV), first found in 2002 at Southern China. SARS CoV is considered an animal virus but in 2002 the first human was found infected with SARS CoV. During that period the virus spread across 26 countries and 8000 got infected. This virus transmits from animal to humans. The symptoms include malaise, headache, diarrhoea, fever and shivering, and breath shortness is found in the second week of illness [20]. To reduce transmission of virus it was advisable to reduce travelling and critical care was provided to patients. There is a severe category of SARS termed as COVID-19. This study focus on describing COVID-19 properties and its effects on pregnancy.

1.1.2. MERS CoV: Middle East Respiratory Syndrome (MERS CoV) was first identified in 2012 in Saudi Arabia. According to the report of the WHO [19], 35\% of patients suffering from MERS died. The typical symptoms of MERS include, cough, fever, breath shortness and diarrhoea and it transmits from person to

\footnotetext{
*Emails: rydhmberi@gmail.com, Mithilesh.21436@1pu.co.in, Eranita5@gmail.com, srajssssece@gmail.com
}

DOI: $10.1201 / 9781003129103-88$ 


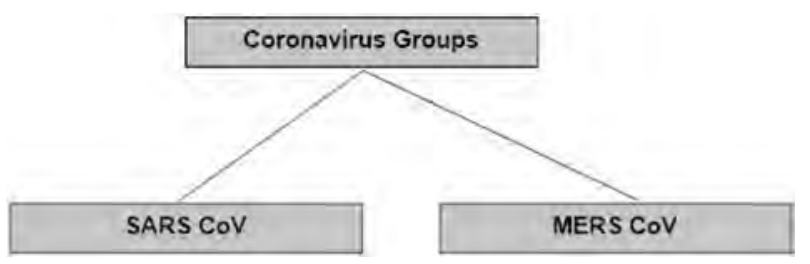

Figure 88.1 Groups of coronaviruses.

personby close contact and animal to person. People with diabetes, kidney disease, lung disease and low immunity are more vulnerable to MERS CoV infection. The social distancing was found to be useful for the person to stop virus spread to other persons.

\section{Literature review}

P.S. Masters [22] reviews the coronavirus in long-established and modern discoveries. The author discussed infectious agents, viral RNA synthesis and viral receptors. The author discussed the molecular complexity of gene expression due to the presence of SARS. Van der Hoek et al. [21], in their study, discussed virus discovery cDNA AFLP (VIDISCA) for detection of coronavirus. The VIDISCA technique improved the testing efficiency by the presence of restricted enzyme site for PCR amplification. The authors applied the techniques on the sample of a 7-month-old child suffering from coronavirus. It helps in easier detection of the presence of the virus in the human body.

Chen et al. $[23,30]$ highlights the features of coronavirus and the various reasons behind the spread of coronavirus in humans and animals. The authors started with an introduction related to coronavirus and moved towards its genome structure and replication. The authors also described the structural as well as non-structural protein replication in the human body as well as the possible treatment and preventive measures needing to be taken to reduce its impact.

Mehta et al. [24,25] represented the facts related to the mortality ratio across the world occur due to the increase in COVID-19 patients. The authors suggested that in hyperinflammation immunosuppression is beneficial and will reduce the mortality rate. Zheng et al. [26] discussed the impact of COVID-19 on the cardiovascular system. The authors discussed that COVID-19 is a more harmful virus as compared to SARS CoV and has a stronger transmission capability.

Dashraath et al. [27,31] reviewed the impact of COVID-19 in pregnancy. The authors discussed the challenges occurring with real-time diagnostic of pregnant women, intrauterine transmission and complications related to mother and infant. The study described the framework needing to be adopted for management of cross-infection to healthcare providers and other individuals.

Le et al. [28] presented a case study of a 3-month-old infant diagnosed positive for COVID-19 in Vietnam. Doctors didn't find any symptoms of COVID-19 in the baby during pregnancy or delivery. The baby got infected by having contact with her grandmother, who was suffering from the same disease. Liu et al. [29] discussed the difficulties in finding Covid-19 positive in pregnant women. They discussed that computed tomography $(\mathrm{CT})$ helps to detect early the symptoms associated with COVID-19 and also helps in health condition assessment. 


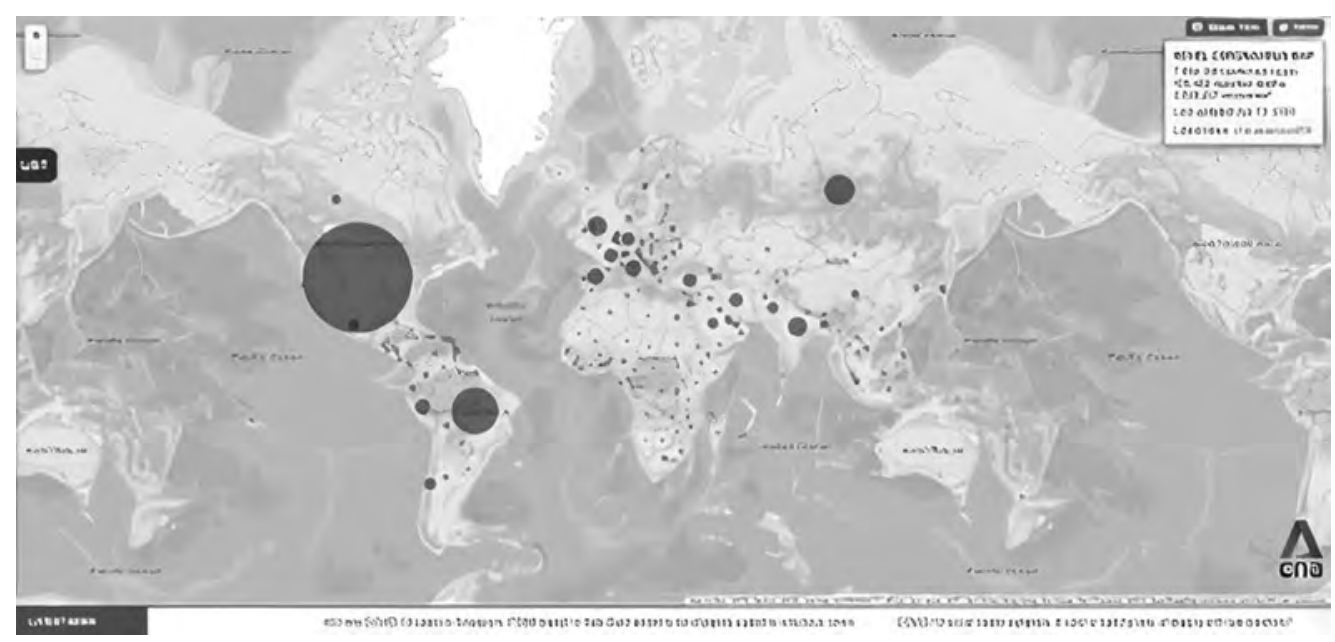

Figure 88.2 Cumulative confirmed cases of COVID-19 around the world June 2020 [3].

\section{Coronavirus disease 2019 (COVID-19)}

COVID-19 is a category of SARS CoV-2, and is a dangerous infectious communicable disease, which is spreading very fast across the world and is affecting many lives. It originated from China in December 2019, is rapidly spreading globally, and is considered as a pandemic disease. The latest report of confirmed cases of COVID-19 across the world is shown in Figure 88.2 [3].

Globally there are lacs of people suffering from COVID-19 with more than 190,988 deaths reported. In India, to date there are 23,867 positive COVID-19 cases with 758 deaths and 5271 cured cases reported [18].

Symptoms of COVID-19 vary from person to person and may include fever, cold, cough and difficulty in breathing. The symptoms of the virus may be detected within 2 to 14 days. This virus may transmit from person to person by shaking hands, coughing, sneezing, or having contact with an object touched by the viral person without sanitization. According to the National Health Institution [6] and Centers of Disease Control and Prevention [7], young children, people older than 65 years, asthmatic patients, diabetic patients, heart patients and pregnant woman are at more risk of catching the virus.

During pregnancy, a woman's body undergoes several changes which may have a high risk of the severe disease COVID-19. There may be the chances of vertical transmission of the virus from mother to infant. There are two cases reported in which a newly born child suffered from COVID-19 but it is not confirmed yet whether it is pre or after birth transmission. Also, in China four women positive with COVID-19 gave birth and there is no symptoms of the virus found in their children [5].

\section{Effect of SARS during pregnancy}

Severe acute respiratory syndrome (SARS) first appeared in 2003 in China, spread across 30 countries and found 8000 positive cases with 770 deaths [8]. This epidemic was controlled by reduction in direct contact of the infected persons and since 2004 no case has been reported. Typical symptoms like a cold, fever, hemicranias, body pain, restlessness and diarrhoea. 


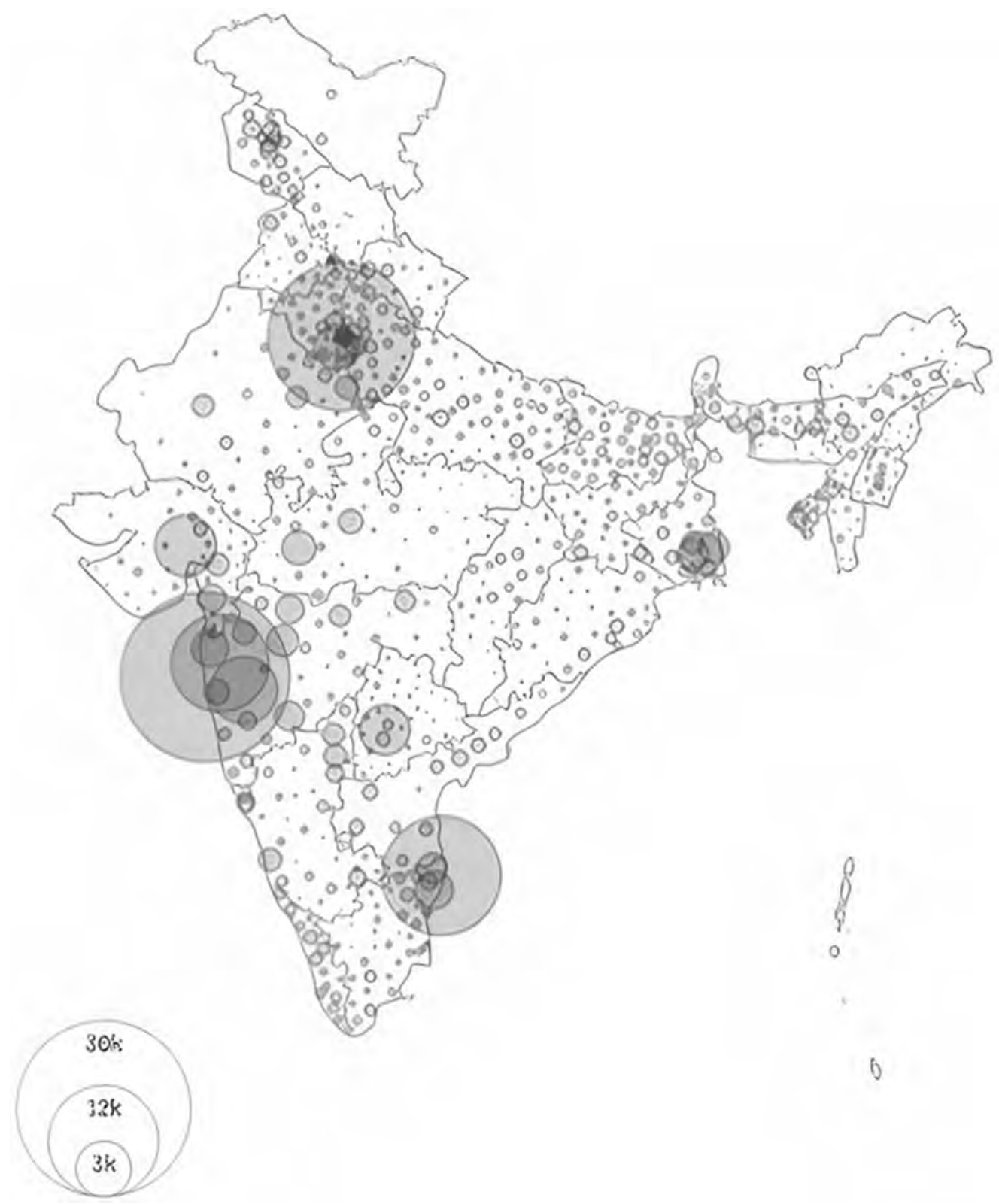

Figure 88.3 Cumulative confirmed cases of COVID-19 in India, June 2020 [18].

The transmission of SARS is carried out by close contact of the infected person's body fluid layers of the respiratory tract with respiratory beads formed when a contaminated individual hacks or sniffles. Airborne spread because of inward breathing of little aerosol products may likewise be conceivable. During this virus 4.6 days of mean period of incubation is required and with the range of 2-14 days. The transmission occurs within the second week of the virus entering the body and prior to this no side effects are shown in the person. 


\section{COVID-19 in pregnancy}

The immune system and hormones of pregnant women move to numerous changes in nine months and even some post-delivery changes occur in the body. Due to this pregnant woman are more vulnerable to infectious disease and respiratory illness. This may increase maternal mortality or infant death. Although humans are found with mild coronavirus disease, severe acute respiratory syndrome (SARS-CoV) and Middle East respiratory syndrome (MERS-CoV) produced a death rate of one-third of pregnant women $[9,10]$; whereas according to a case report [11], of 43 pregnant women in New York $86 \%$ of women found a mild effect of COVID-19 while $9.3 \%$ have a severe effect and $4.7 \%$ of women developed critical disease with no maternal or infant loss. Moreover according to the report [12], 100 women with COVID-19 delivered in March and one maternal death with 30 weeks of gestation was reported in April 2020 in Iran [13]. Also, in India, there were some cases of pregnant women found positive with COVID-19 but no data found concerning transfer of COVID-19 from mother to infant during labour [14]. Moreover, preterm delivery may occur in women positive with COVID-19 [15].

Apart from this it is still unknown what other loss such as fetal loss, distress, still-birth or tachycardia may be due to COVID-19. According to the latest statistics [16], globally there are 2,074,529 confirmed cases, so more spreading chances are there in pregnant women across the world. Therefore, it is advisable for pregnant women as well as other individuals to take the necessary precautions to save lives. This study offers some of the necessary practical clinical advice to manage COVID-19 during pregnancy.

\section{Preventive measures for COVID-19}

The person suffering or having a chance of suffering from COVID-19 usually has the symptoms of fever, dry cough, tiredness, sore throat, runny nose etc. The incubation period of 14-28 days is suggested to the person having such symptoms. Sometimes, before becoming symptomatic usually the person with strong immunity becomes a carrier of the disease and might be capable of transferring the disease to the less immune person with close contact or respiratory droplets. Pregnant women are more vulnerable to get infections due to some physiological and hormonal changes in their bodies. Scientists are consciously working on the preparation of vaccine or medicine for the individuals to guard them against the pandemic, but till now no such medicine or vaccine is available for the same. So, it is appropriate for pregnant women to keep themselves socially hygienic, avoid going to crowded areas, increase usage of sanitizers or wear masks $24 * 7$ (change it after interval of 5 hours) and try to have contact with a limited number of persons, 1 or 2. If some pregnant women have the symptoms of cough, breath shortness, fever or fatigue then they should take timely consultation with doctors or healthcare providers. It is also advisable to pregnant women to keep themselves busy in such activities which prevent them from psychological or physiological activities.

\section{Infant care during labour and post-delivery}

The transfer of COVID-19 from mother to child during pregnancy has not so far been found. However, the child may get infected after birth from having contact with an infected person. Only a few cases have been found in which new born babies have tested positive with COVID-19 and the reason for this infection is still unknown, i.e. whether they got infected from the mother or from some infected person contact [18]. According to 
the WHO (CDC) [17], COVID-19 infection is not found in breast milk or amniotic fluid. To minimize the transmission risk of COVID-19 to the infant it is advisable to have separation from the new-born for 2 weeks and avoid close contact with the infected mother. Moreover, in this case mother's breast milk is preferred via pump to feed the baby by healthcare providers.

\section{Conclusion}

During this pandemic situation everyone needs to prepare themselves for handling the situation caused by COVID-19. It is possible by providing proper clinical management and healthcare provider support to the patients with the integration of the individual's self-care and precautions. Several individuals are working together to face and guard humans against the issue but sometimes fail due to lack of facilities, PCR kits, shortage of medicine and blood. Due to this even many healthcare providers lost their lives while taking care of the patients. So, there is an urgent need to train and educate the individuals for self-care and social distancing. There is a critical need to analyse the effect of COVID-19 in pregnancy as well as maternal and infant health. Moreover, there is a need to improve the facilities to identify and resolve the problems occurring due to COVID-19 during pregnancy. Educating health professionals and individuals will improve the health conditions of mother and infant.

\section{References}

[1] Kahn, J. S., and McIntosh, K. (2005). History and recent advances in coronavirus discovery. The Pediatric Infectious Disease journal, 24(11), S223-S227.

[2] Fan, Y., Zhao, K., Shi, Z. L., and Zhou, P. (2019). Bat coronaviruses in China. Viruses, 11(3), 210.

[3] https://infographics.channelnewsasia.com/covid-19/map.html; www.mohfw.gov.in/.

[4] www.rcog.org.uk/en/guidelines-research-services/guidelines/coronavirus-pregnancy/covid-19virus-infection-and-pregnancy/.

[5] www.nih.gov/health-information/coronavirus?fbclid=IwAR0Gq1VTTMazxaMsuhKFifr QEzUclUFbQkmbeI4wGrZBu5SjyEE5eZNjn-k\#!/detail/403283.

[6] www.cdc.gov/coronavirus/2019-ncov/prepare/pregnancy-breastfeeding.html\#anchor_ 1584169983.

[7] Hui, D. S., and Zumla, A. (2019). Severe acute respiratory syndrome: historical, epidemiologic, and clinical features. Infectious Disease Clinics, 33(4), 869-889.

[8] Wong, S. F., Chow, K. M., Leung, T. N., Ng, W. F., Ng, T. K., Shek, C. C., ... and Lai, S. T. (2004). Pregnancy and perinatal outcomes of women with severe acute respiratory syndrome. American Journal of Obstetrics and Gynecology, 191(1), 292-297.

[9] Alfaraj, S. H., Al-Tawfiq, J. A., and Memish, Z. A. (2019). Middle East respiratory syndrome coronavirus (MERS-CoV) infection during pregnancy: Report of two cases and review of the literature. Journal of Microbiology, Immunology, and Infection = Wei mian yu gan ran za zhi, 52(3). doi:10.1016/j.jmii.2018.04.005

[10] Breslin, N., Baptiste, C., Gyamfi-Bannerman, C., Miller, R., Martinez, R., Bernstein, K., ... and Fuchs, K. (2020). COVID-19 infection among asymptomatic and symptomatic pregnant women: Two weeks of confirmed presentations to an affiliated pair of New York City hospitals. American Journal of Obstetrics \& Gynecology MFM, 100118.

[11] Liang, H., and Acharya, G. (2020). Novel corona virus disease (COVID-19) in pregnancy: What clinical recommendations to follow? Acta Obstetricia et Gynecologica Scandinavica, 99(4), 439-442.

[12] Karami, P., Naghavi, M., Feyzi, A., Aghamohammadi, M., Novin, M. S., Mobaien, A., ... and Norooznezhad, A. H. (2020). Mortality of a pregnant patient diagnosed with COVID-19: A 
case report with clinical, radiological, and histopathological findings. Travel Medicine and Infectious Disease, 101665.

[13] Mimouni, F., Lakshminrusimha, S., Pearlman, S. A., Raju, T., Gallagher, P. G., and Mendlovic, J. (2020). Perinatal aspects on the covid-19 pandemic: a practical resource for perinatalneonatal specialists. Journal of Perinatology, 1-7.

[14] Yang, H., Wang, C., and Poon, L. C. (2020). Novel coronavirus infection and pregnancy. Ultrasound Obstet Gynecol, 55(04), 435-437.

[15] World Health Organization. (2020). Coronavirus disease 2019 (COVID-19): situation report, 88 .

[16] www.cdc.gov/coronavirus/2019-ncov/need-extra-precautions/pregnancy-breastfeeding.html.

[17] Qiao, J. (2020). What are the risks of COVID-19 infection in pregnant women?. The Lancet, 395(10226), 760-762.

[18] www.covid19india.org/.

[19] www.who.int/news-room/fact-sheets/detail/middle-east-respiratory-syndrome-coronavirus(mers-cov).

[20] who.int/ith/diseases/sars/en/

[21] van der Hoek, L., Pyrc, K., Jebbink, M. F., Vermeulen-Oost, W., Berkhout, R. J., Wolthers, K. C., $\ldots$ and Berkhout, B. (2004). Identification of a new human coronavirus. Nature Medicine, 10(4), 368-373.

[22] Masters, P. S. (2006). The molecular biology of coronaviruses. Advances in Virus Research, 66, 193-292.

[23] Chen, Y., Liu, Q., and Guo, D. (2020). Emerging coronaviruses: genome structure, replication, and pathogenesis. Journal of Medical Virology, 92(4), 418-423.

[24] Mehta, P., McAuley, D. F., Brown, M., Sanchez, E., Tattersall, R. S., and Manson, J. J. (2020). COVID-19: consider cytokine storm syndromes and immunosuppression. The Lancet, 395(10229), 1033-1034.

[25] World Health Organization. (2020). Coronavirus disease 2019 (COVID-19): situation report, 72.

[26] Zheng, Y. Y., Ma, Y. T., Zhang, J. Y., and Xie, X. (2020). COVID-19 and the cardiovascular system. Nature Reviews Cardiology, 17(5), 259-260.

[27] Schnettler, W. T., Al Ahwel, Y., and Suhag, A. (2020). Severe ARDS in COVID-19-infected pregnancy: obstetric and intensive care considerations. American Journal of Obstetrics and Gynecology MFM, 100120.

[28] Le, H. T., Nguyen, L. V., Tran, D. M., Do, H. T., Tran, H. T., Le, Y. T., and Phan, P. H. (2020). The first infant case of COVID-19 acquired from a secondary transmission in Vietnam. The Lancet Child \& Adolescent Health.

[29] Liu, H., Liu, F., Li, J., Zhang, T., Wang, D., and Lan, W. (2020). Clinical and CT imaging features of the COVID-19 pneumonia: Focus on pregnant women and children. Journal of Infection.

[30] Baud, D., Giannoni, E., Pomar, L., Qi, X., Nielsen-Saines, K., Musso, D., and Favre, G. (2020). COVID-19 in pregnant women-Authors' reply. The Lancet Infectious Diseases.

[31] Dashraath, P., Jeslyn, W. J. L., Karen, L. M. X., Min, L. L., Sarah, L., Biswas, A., ... and Lin, S. L. (2020). Coronavirus disease 2019 (COVID-19) pandemic and pregnancy. American Journal of Obstetrics and Gynecology. 


\title{
89 Frame selection based video watermarking technique based on DWT, K-SVD, rail fence to address quality loss of data
}

\author{
Chirag Sharma ${ }^{1, *}$, Amandeep $p^{2, *}$ \\ ${ }^{1}$ Department of Computer Science and Engineering, Lovely Professional \\ University, Phagwara, India \\ ${ }^{2}$ Department of Computer Application, Lovely Professional University, \\ Phagwara, India
}

\section{Introduction}

The rapid growth of multimedia technologies, i.e., audios, videos and images, has led to the need for the development of secured techniques to transfer multimedia contents over the Internet. Many users are using the multimedia content without taking care of copyright protection and ownership identification. Unauthorized use of videos across the Internet has led to the development of covert security methods termed as watermarking. The same has been digital watermarking, which is a technique to embed secret information termed a watermark into digital data which is hidden from users such that if any user tries to manipulate the digital data without copyright protection, that user can be caught on the basis of the copyright protection act. The embedded information can be extracted so as to achieve copyright protection and broadcast monitoring. The major attributes of watermarking are imperceptibility, robustness, capacity and security [1]. Most of the research work is being carried out so far on images and videos and the latest work is being done on videos. The video watermarking poses a great deal of challenge while embedding a watermark to it. The proposed technique addresses the challenges imposed in [11-15] to get good results in terms of PSNR values. The video watermarking can be done in many ways listed in [1].

In spatial or pixel domain methods the watermark is embedded directly on pixel positions; the best example of this method is LSB (least significant bit) modification; although this is a fast method it lacks in achieving imperceptibility [1].

In transform domain methods, the host signal is converted into a different domain and the watermark is converted to the frequency domain then it is embedded in selective coefficients. The techniques under these domain methods are discrete wavelet transform (DWT), discrete cosine transform (DCT) and discrete Fourier transform (DFT) [1].

The proposed work deals with transform domain techniques applied with K-SVD (singular valued decomposition) and additional cipher (rail fence cipher) which is an extension of work done by Sharma [14]. The validity of the watermarking scheme is dependent upon values of PSNR (peak signal to noise ratio) after applying attacks such as frame averaging, cropping, additional noise attack on the proposed watermarking scheme. The next section, explains the research work done in this area followed by the proposed technique.

\footnotetext{
*Emails: chiragsharma1510@gmail.com, amandeep.bagga@gmail.com
}

DOI: $10.1201 / 9781003129103-89$ 


\section{Related work}

A number of research works related to watermark embedding using DWT as a transform domain have been done by $[1,2,5,6]$ that embed the watermark on selected frames. Identical frames are decomposed to 3-level DWT before the watermark is embedded on them. High frequency sub-bands are selected on the areas where the watermark embedding is. From the research the frequency domain achieves a higher level of robustness compared to the spatial domain but is not efficient in handling various attack scenarios.

The combination of discrete wavelet transform (DWT) and singular valued decomposition (SVD) was also applied by various researchers [11-14]. The improvement of results based on values of PSNR was obtained against image processing attack scenarios.

The particle swarm optimization methods [3] based on Dither modulation optimize the results of PSNR by optimizing various factors (optimal frequency set) for embedding the watermark.

Applying an optimization algorithm on the combination of DWT and SVD has also produced high values of PSNR. The same has been listed in work done by Agarwal et al. [10]. Here the combination of DWT and SVD is done with the Firefly algorithm where multiple scaling factors were optimized using the Firefly algorithm.

Rajpal [16] also extended work by using hybrid Fuzzy-BPN architecture to embed a binary watermark in grey-scale images; the proposed scheme achieves good results in real time scenarios using an extreme learning machine.

K-SVD, a modified version of SVD, is implemented in Song et al. [15] by combining with ELC for image recognition. The implemented algorithm has high performance when compared to other image processing techniques.

Tabassum [5] proposed a method of identical frame extraction and using a frame difference method, identification and selection of appropriate frames is done to embed the watermark inside them, The method is improved by Masoumi [7] where the motioned part of video is detected using a technique proposed in [6] by applying 3-D wavelet transformation coefficients of different sub-bands selected for embedding.

\section{Proposed work}

The proposed work will aim to insert a watermark in the video to address quality loss of data. The major task to perform watermarking on videos is to select frames from the video then put the watermark on selected frames then find the value of PSNR considering the watermarked video and the host video. The frame selection done in the proposed work supersedes the approach adopted in [5] and the scheme missing in [13,14] has been addressed in this research work. The proposed work deals with three phases.

\subsection{Frame extraction and selection}

The first step in phase 1 is taking a host video and converting into JPEG frames. It allows the process of frame extraction followed by pre-processing. The pre-processing includes frame identification, frame adjustment and frame selection. While most previous studies have used random frame selection using a key, this is not suitable and instead, the frames must be selected by grouping similar images and extracting the most suitable image from it. Edge detection algorithms can be used to group the images that are similar and randomly select a frame from that. The adjacent images are compared with one another to identify the amount of difference between the frames. If the difference is very large, then it can be considered to be a part of the next group. In addition, temporal sampling will 
be done to the video before splitting into frames to ensure that the frame selection algorithm is efficient. Once the images are grouped together, a random key generator is used to extract the images. Here, addition of edge detection improves the frame extraction process since the adjacent frames that are very similar are identified and grouped. The work gives better results in comparison to [5] where more time was consumed in selection of frames and gives better results than Sharma [14] where random frame selection was done. Frame difference can be represented as histogram difference that can be expressed as

$$
\mathrm{FD}_{\mathrm{k}}=\sum_{k=1}^{T}\left(H_{K}(\mathrm{~m})-H_{k}(m+1)\right)
$$

where $\mathrm{FD}_{\mathrm{k}}$ represents the frame difference and $\mathrm{H}_{\mathrm{k}}$ is the histogram value of $\mathrm{k}^{\text {th }}$ frame of level $\mathrm{m}$ and $\mathrm{T}$ is the number of levels of the histogram. The proposed work is dependent upon the histogram difference: frames with minimum difference can't be chosen but the frames representing the large histograms difference value are picked to be the part of next group. The threshold is maintained to detect intensity histogram difference to calculate sudden transition amongst frames (in order to find larger frame difference). This scenario is expressed as -

$$
M_{b}=\propto+\alpha \sigma
$$

$$
\mathrm{M}_{\mathrm{b}}=\text { threshold value }
$$

$\sigma$ and $\mu$ are the standard deviation and mean value of selected frame intensity histogram difference. $\alpha$ is the factor that varies from 2 to 6 ; in our research, we have taken this value as 4 . The criteria of frame selection depend upon the comparison of $\mathrm{FD}_{\mathrm{k}}$ with $\mathrm{M}_{\mathrm{b}}$. The algorithm is given below.

\section{Algorithm 1: Frame selection algorithm}

1: Initialize

$$
\begin{aligned}
& \mathrm{T}=\text { number of frames obtained from videos } \\
& \mathrm{M}=\text { mean of } \mathrm{T}, \mathrm{S}=\text { standard deviation of } \mathrm{T} \\
& \mathrm{M}_{\mathrm{b}}=\mathrm{M}_{+} \alpha S \\
& \mathrm{FD}_{\mathrm{k}}=\text { frame difference } \\
& \text { (Equation 2) }
\end{aligned}
$$

2: For $\mathrm{i}=1$ to $\mathrm{T}$ (number of frames)

3: Read the frames one after the other and store them in variables.

4: Compute the difference amongst frames and group them in different groups using a Sobel filter, store them in a variable $\mathrm{FD}_{\mathrm{k}}$.

5: If $\left(\mathrm{FD}_{\mathrm{k}}>\mathrm{M}_{\mathrm{b}}\right)$

6: Select frames and group them.

7: Apply random key amongst frames from different groups and write them to disk.

8: End If

9. End For

A video having format mp4 is taken and is converted into a set of frames that are stored in the directory, a Sobel filter is used to identify and group similar frames together, frames having larger frame difference become part of different groups where random frames are extracted from identical frames. The simple form is represented in the form of the diagram in Figure 89.1. 


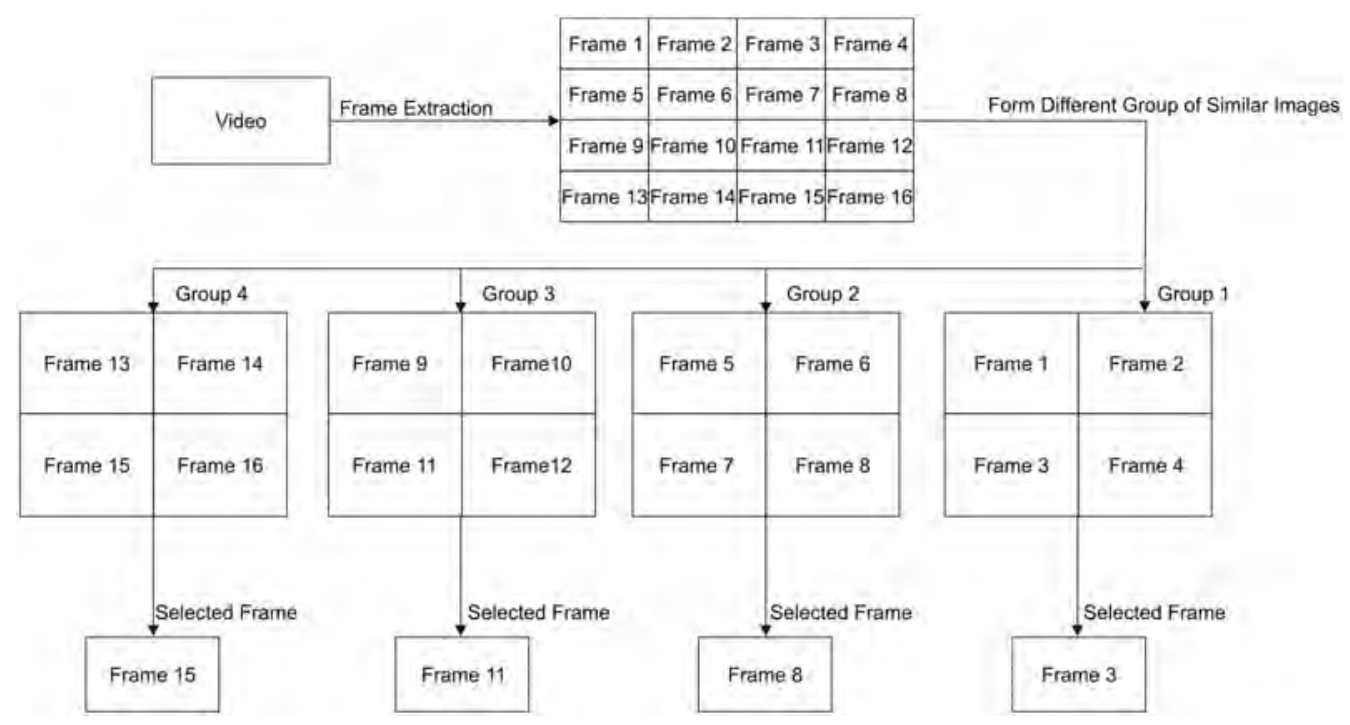

Figure 89.1 Frame selection process using proposed approach.

\subsection{Watermark embedding procedure}

Once the frames are selected, the watermark is embedded into the selected frames. Three-level DWT is an effective technique, however, it is old and may not work well with newer attacks, and hence it is combined with a newer technique referred as K-SVD. The proposed technique provides better results than [14]. Selected frames are converted to three-level DWT followed by division into non-overlapping blocks from the wavelet transform, further K-SVD is applied to enhance feature selection process, binary watermark in the form of image is ciphered using rail fence algorithm followed by conversion into DWT then embedding is done followed by inverse transform to get the watermarked video. The major focus of this step is to choose the area of watermarking. For this step the implementation is done using following the algorithm. The same is illustrated in Figure 89.2.

\section{Algorithm 2: Watermark embedding algorithm}

1. For selected frames 1 to $\mathrm{k}$

2. Use colour separation and pick blue channel of RGB frame and apply 3 level DWT using Haar filter to obtain LH3, LL3, LH2, LH1 sub-band coefficients for watermark embedding.

3: Select block of coefficients using block classifier.

4: Apply K-SVD to select

4.1 best column using OMP [15] of selected coefficients.

4.2 select coefficients which are close to original form to get sparse coefficients.

5: Take a binary watermark - text or image and apply rail fence cipher to them.

6: Apply three-level DWT to the media taken in step 5.

7. Embed the coefficients taken in step 6 on coefficients obtained from step 4.2. 


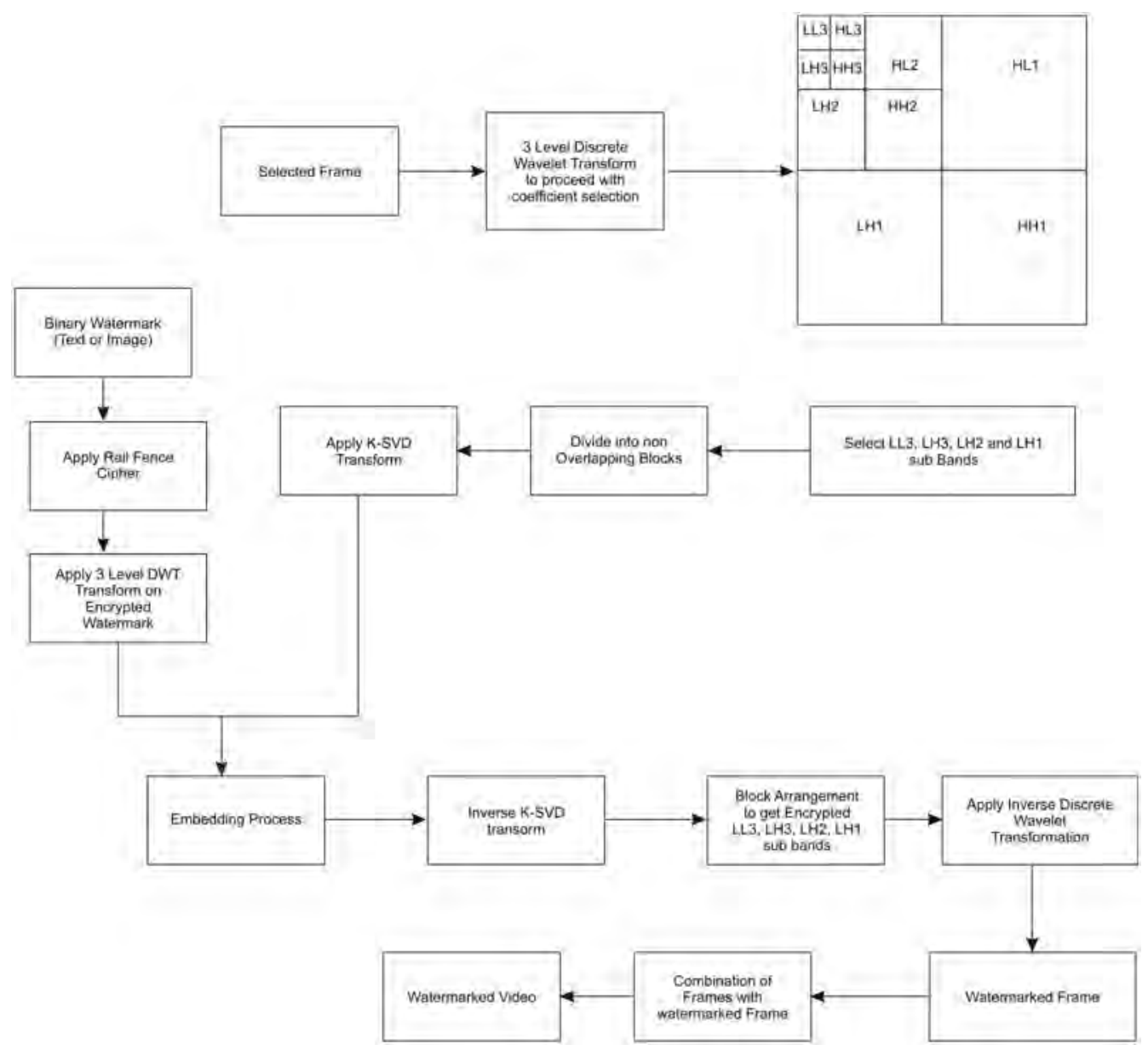

Figure 89.2 Watermark embedding process.

8. Apply inverse transform of K-SVD followed by inverse DWT and decryption to reconstruct the frames.

9. Combine colour channels.

10. Repeat steps from 1 to 9 till all the selected frames are processed.

11. End For.

12. For frames 1 to $\mathrm{m}$ in directory of extracted frames.

13. Combine selected watermark frames from a directory and replace them with same frame number.

14. Process all the extracted frames and selected frames to form watermarked video.

15. End For.

\subsection{Performance evaluation and comparison}

The performance of the proposed work depends upon measuring the loss of quality after insertion of the watermark and the quality metric to measure performance is PSNR which is peak signal to noise ratio which is inverse of MSE, known as mean square error. The 
results obtained in $[13,14]$ had high value of high values of PSNR but they were unable to select the best possible frame to embed the watermark, which was addressed in Section 3.1 of the proposed work. The third phase of the proposed work is to calculate results from phase 1 and phase 2 .

The validation of our research will be based on performance metrics PSNR and BER. The scheme will be tested when the original video is compared with two videos: watermarked video and watermarked video with attacks.

\section{Results and discussion}

The proposed technique will be tested when the original video is compared with two videos.

a. Watermarked video

b. Watermarked video with attacks

The result of phase 1 is illustrated in Table 89.1.

From Table 89.1, the analysis was done and the video of $1280 * 720$ is taken that contains motioned and motionless frames. The major focus is to select motioned frames and detect changes done in motioned frames. The videos are of two types in terms of domain-compressed or uncompressed. The compressed domain of the videos is selected here; that means the raw video is not taken. The video has codec H.264. The time taken for the process is 39.79 seconds. Since the extracted frames are of RGB channel where blue channel gets selected after colour separation and frame selection is done on the basis of Sobel filter and the major scene detection was done in frames number 33, 65, 95, 121, $149,174,320,386$. Hence 8 frames out of 541 frames get selected after phase 1 .

PSNR (peak signal to noise ratio) is a quality measurement parameter. Robustness is calculated by the value of PSNR and BER (bit error rate). Mean square error evaluates the mean absolute error between original and distorted frames.

$$
\mathrm{MSE}=\sum_{i=0 j=0}^{M-1 N-1} \frac{1}{M * N}([\mathrm{OI}(i, j)-D I(i, j)]) 2
$$

MSE is calculated as the difference between original and watermarked frames.

$$
P S N R=10 \log _{10} \frac{(255)^{2}}{\mathrm{MSE}}
$$

$$
\mathrm{BER}=1 / \mathrm{PSNR}
$$

The attacks are divided into various categories: salt and pepper noise, Gaussian noise, frame dropping and cropping, as mentioned in [13,14].

The watermark to be embedded is selected in the form of an image which is hidden in a frame to obtain a watermarked frame. The results of PSNR and BER were calculated by adding the watermark in the host video and after embedding watermark the comparison 
Table 89.1 Result: calculation of phase 1 (Section 3.1)

\begin{tabular}{ll}
\hline Video format & mp4 \\
Width & 1280 \\
Height & 720 \\
Video resolution & $1280 * 720$ \\
Video size & $1.57 \mathrm{MB}$ \\
Frame rate & 29.97 \\
Bits per pixel & 24 \\
Video format & RGB24 \\
Domain used & Compressed domain \\
Number of frames extracted & 541 \\
Number of frames selected using proposed technique & 8 \\
Time complexity (in seconds) & 39.792466 \\
Colour frames used & RGB \\
Channel used & Blue channel \\
Video codec & H.264 \\
\hline
\end{tabular}

Table 89.2 Results formulated by embedding watermark using proposed technique by applying different image watermarks

\begin{tabular}{llll}
\hline S. no. & PSNR & BER & Watermark used \\
\hline 1 & 42.678 & 0.0234 & \\
2 & 43.123 & 0.0231 & \\
\hline
\end{tabular}

Table 89.3 Results formulated by applying attacks on watermarked video

\begin{tabular}{llll}
\hline S. no. & Attacks & PSNR & BER \\
\hline 1 & Salt and pepper & 29.568 & 0.033 \\
2 & Gaussian noise & 29.123 & 0.034 \\
3 & Frame dropping & 30.145 & 0.033 \\
4 & Cropping & 32.156 & 0.031 \\
\hline
\end{tabular}

is done of the host video with the watermarked video, illustrated in Table 89.2, and comparison is done of host video with the watermarked video with attacks.

\section{Conclusion and future scope}

The proposed work presents a robust technique that focuses on embedding of a watermark in the best suitable frame and addressing the quality of the watermarked frame after insertion of the watermark. The proposed work was tested against various image watermarks on the selected frames mentioned in Section 3.1 and the embedding was done by using a hybrid technique involving DWT and K-SVD along with encryption of the watermark done using the rail fence cipher before embedding on selected frames. The proposed work did give optimal results compared to work done in [11-14] where the 
frame selection procedure was used (3.1) instead of random frames. The results can also be improved by using graph based transform instead of discrete wavelet transform, which will be a part of future work.

\section{References}

[1] S. Bhattacharya, T. Chattopadhyay, A. Pal, A survey on different video watermarking techniques and comparative analysis with reference to H. 264/AVC, in: Consumer Electronics, 2006. ISCE'06. 2006 IEEE Tenth International Symposium on, IEEE, 2006, pp. 1-6.

[2] D. Ye, C. Zou, Y. Dai, Z. Wang, A new adaptive watermarking for realtime MPEG videos, Applied Mathematics and Computation 185 (2) (2007) 907-918.

[3] C. Wu, Y. Zheng, W. Ip, C. Chan, K. Yung, Z. Lu, A flexible H. 264/AVC compressed video watermarking scheme using particle swarm optimization based dither modulation, AEUInternational Journal of Electronics and Communications 65 (1) (2011) 27-36.

[4] M.-J. Lee, D.-H. Im, H.-Y. Lee, K.-S. Kim, H.-K. Lee, Real-time video watermarking system on the compressed domain for high-definition video contents: Practical issues, Digital Signal Processing 22 (1) (2012) 190-198.

[5] T. Tabassum and S. M. M. Islam, A digital video watermarking technique based on identical frame extraction in 3-Level DWT, in 2012 15th International Conference on Computer and Information Technology (ICCIT), 2012, pp. 101-106.

[6] M. Masoumi, S. Amiri, A blind scene-based watermarking for video copyright protection, AEU-International Journal of Electronics and Communications 67 (6) (2013) 528-535.

[7] M. Masoumi, S. Amiri, Content protection in video data based on robust digital watermarking resistant to intentional and unintentional attacks, Int. Arab J. Inf. Technol. 11 (2) (2014) 204-212.

[8] O. S. Faragallah, Efficient video watermarking based on singular value decomposition in the discrete wavelet transform domain, AEU-International Journal of Electronics and Communications 67 (3) (2013) 189-196.

[9] P. Venugopala, H. Sarojadevi, N. N. Chiplunkar, V. Bhat, Video watermarking by adjusting the pixel values and using scene change detection, in: Signal and Image Processing (ICSIP), 2014 Fifth International Conference on, IEEE, 2014, pp. 259-264.

[10] A. Mishra, C. Agarwal, A. Sharma, P. Bedi, Optimized gray-scale image watermarking using DWT-SVD and Firefly algorithm, Expert Systems with Applications 41 (17) (2014) $7858-7867$.

[11] B. Sridhar, C. Arun, An approach in video watermarking with multiple watermarks using wavelet, Journal of Communications Technology and Electronics 61 (2) (2016) 165-175.

[12] D. Kaur Thind, S. Jindal, A semi blind DWT-SVD video watermarking, International Conference on Information and Communication Technologies, 2014.

[13] C. Sharma, G. Singh, and G. Singh Saini, Efficient video watermarking technique for quality loss of data, Indian J. Sci. Technol. 9 (47) (2016).

[14] C. Sharma, A. Bagga, Video watermarking scheme based on DWT, SVD, rail fence for quality loss of data, in 2018 4th International Conference on Computing Sciences (ICCS), 2018, pp. 84-87.

[15] Y. Song, Y. Liu, Q. Gao, X. Gao, F. Nie, R. Cui, Euler label consistent K- SVD for image classification and action recognition, Neuro Computing 310 (2018) 277-286.

[16] A. Rajpal, A. Mishra, R. Bala, A novel Fuzzy selection based watermarking scheme for MPEG-4 videos using bi-directional extreme learning machine, Applied Soft Computing Journal, 2018. 


\title{
90 Deep learning inspired continuous estimation framework for Scrum projects
}

\author{
Shivali Chopra, Arun Malik* \\ Lovely Professional University, Phagwara, Punjab, India
}

\section{Introduction}

Continuous is the new fuel for an uninterrupted software development. IT stakeholders are making use of Agile and DevOps in their organizations to make it ready for continuous development, integration, testing and deployment. Due to the volatile nature of requirements, effort estimation in Scrum becomes challenging (Arora et al. 2020). The major challenges are change and sprint-wise estimation. Most of the IT industries have adopted hybrid process models which are mostly driven by Agile umbrella methodologies (Gultekin Muaz and Oya 2020). Figure 90.1 shows user story classification.

\section{Related work}

Research in the field of Scrum estimation has been categorized and classified in two verticals, text classification and numerical estimation (Ali and Gravino 2019). Few studies have made use of deep learning architectures to predict the effort of issues aka user stories (Arora et al. 2018). Deep learning architectures like long short-term memory (LSTM) provides semantic analysis and vectorization of the user story text whereas recurrent highway networks (RHWN) provide continuous transformations to align and adjust the story point estimate with actual points. We have observed a significant amount of work in metric estimation but less evident research is available in classification (Kaushik et al. 2019; Pospieszny et al. 2018; Kocaguneli et al. 2013) given in Table 90.1.

\section{Proposed framework}

As per the aforementioned literature, we have proposed a framework which will not only assist IT managers in better decision-making but also foster continuous estimation. The dataset used for the proposed algorithm is taken from 16 open source projects of ten repositories: Apache, Duraspace, LsstCorp, Spring, Talenforge, Mulesoft, Atlassian, Appcelaerator, JIRA, Moodle. These repositories have been referred to extract user stories $a k a$ issues. The issue tracking system contains project title, its description, and actual story points. There are more than 20,000 issues extracted from these repositories. The inputs to the proposed algorithm will be 'title' and 'descriptions' of the issues extracted from the open source projects. The proposed algorithm will automatically extract features from issues with no manual feature engineering. The proposed framework is given in Figure 90.2 .

\footnotetext{
* Corresponding author email: arun.17442@lpu.co.in
} 


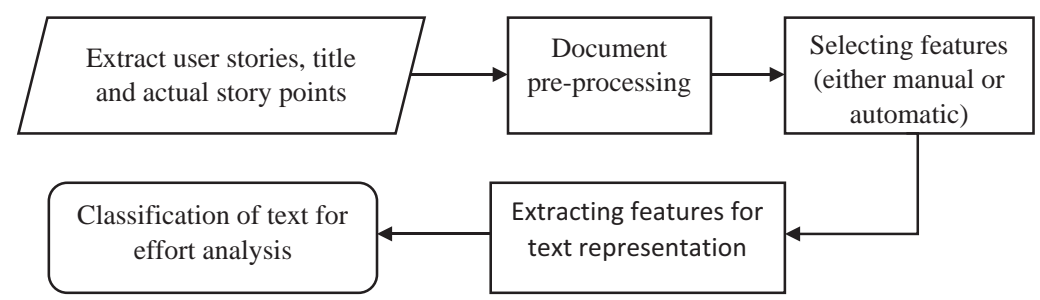

Figure 90.1 Scrum estimation as text classification. This figure presents the basic flow of classification of user stories. A pre-processing of the user story document will be carried out followed by feature selection, extraction and classification.

Table 90.1 Story point classification techniques

\begin{tabular}{|c|c|c|}
\hline Techniques used & Dataset & Findings \\
\hline $\begin{array}{l}\text { Long short memory with } \\
\text { recurrent highway network } \\
\text { (Choetkiertikul et al. 2019) }\end{array}$ & $\begin{array}{l}23313 \text { issues from } 16 \\
\text { open source repositories }\end{array}$ & $\begin{array}{l}\text { LSTM with RHNN performs } \\
\text { best as compared to LSTM } \\
\text { with other models such as } \\
\text { Doc } 2 \text { Vec, SVM etc. }\end{array}$ \\
\hline $\begin{array}{l}\text { Autoencoders with support vector } \\
\text { machines (Soares 2018) }\end{array}$ & 6 open source projects & $\begin{array}{l}\text { All autoencoders performed } \\
\text { well but SVM could better } \\
\text { predict than TF-IDF. }\end{array}$ \\
\hline $\begin{array}{l}\text { Term frequency inverse document } \\
\text { frequency, Doc2Vec, support } \\
\text { vector regression, Gaussian } \\
\text { naïve Bayes (Ionescu et al. } \\
\text { 2017) }\end{array}$ & $\begin{array}{l}9 \text { datasets provided by a } \\
\text { software company }\end{array}$ & $\begin{array}{l}\text { Using regressors with } \\
\text { traditional methods of text } \\
\text { vectorization such as TF- } \\
\text { IDF, Doc2Vec, fasttext yields } \\
\text { better results. }\end{array}$ \\
\hline $\begin{array}{l}\text { Natural language processing based } \\
\text { frameworks (Khan et al. 2012) }\end{array}$ & 10 software projects & $\begin{array}{l}\text { An easy to use tool for } \\
\text { beginners. Can be used in } \\
\text { different situations. Effort } \\
\text { can also be calculated. }\end{array}$ \\
\hline $\begin{array}{l}\text { Machine learning classifier } \\
\text { using term frequency inverse } \\
\text { document frequency for feature } \\
\text { selection (Porru et al. 2016) }\end{array}$ & $\begin{array}{l}\text { Industrial and open source } \\
\text { issue reports from } \\
\text { JIRA consisting of total } \\
16523 \text { issues }\end{array}$ & $\begin{array}{l}\text { Proposed technique can } \\
\text { automate effort estimation } \\
\text { process within a timeframe } \\
\text { of } 8 \mathrm{~s} \text { to } 15 \mathrm{~s} \text {. }\end{array}$ \\
\hline $\begin{array}{l}\text { Natural language processing using } \\
\text { parts-of-speech tagging and } \\
\text { naïve Bayes classifier (Ratke } \\
\text { et al. 2019) }\end{array}$ & 8 projects & $\begin{array}{l}\text { Keywords can be detected } \\
\text { dynamically with the feature } \\
\text { of log generation. }\end{array}$ \\
\hline
\end{tabular}

Model training includes an automatic calibration and tuning of proposed system parameters (Rijwani and Jain 2017) through a learning gain matrix. A learning gain refers to the gain in the experience of the Scrum team members after successive successful sprints. It depends on the team's velocity (Khuat and Le 2018). The pseudo code to calculate and incorporate the learning gain, $\mathrm{G}$, is given below.

Step 1: Calculate initial agile velocity, $V_{\text {initial }}$ for initial sprint $S_{1}$.

Step 2: Set initial value of learning gain, $\mathrm{G}$ to 0 for $S_{1}$.

Step 3: Determine $V_{i_{\text {current }}}$ and $V_{i_{\text {preuious }}}$, where $V_{i_{\text {current }}}$ is velocity of current sprint and $V_{i_{\text {previous }}}$ is velocity of previous sprint. 


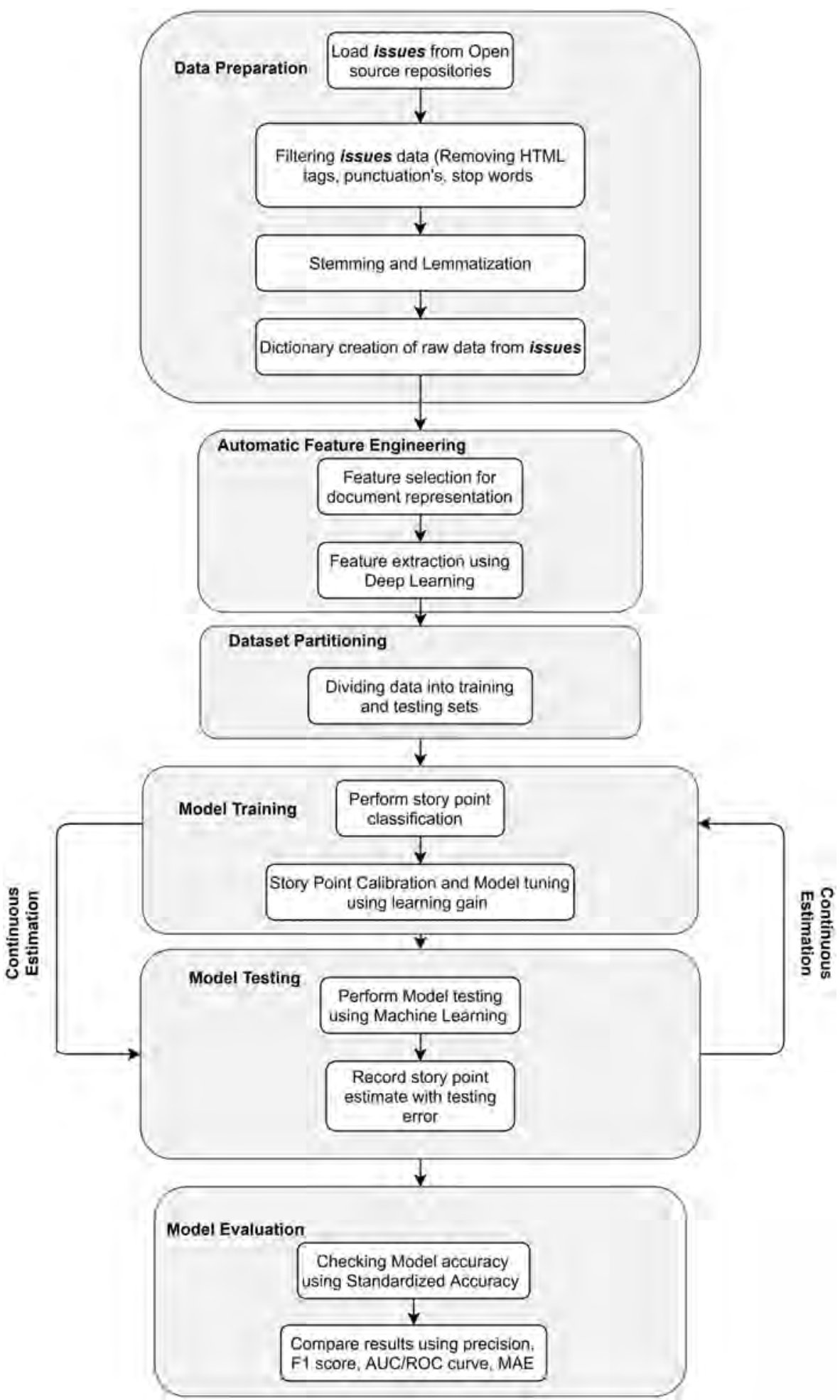

Figure 90.2 Proposed methodology framework. 
Step 4: Set $V_{i_{\text {previous }}}$ as $V_{\text {initial }}$.

Step 5: Determine velocity gain, $V_{G}$, for sprint $S_{2}$ using $V_{G}=V_{i_{\text {current }}}-V_{i_{p}}$

Step 6: Calculate velocity adjustment factor, VAF, assign 0 if no change is there.

Step 7: Adjust $V A d j_{G}$ by $V A d j_{G}=\left(V_{G}\right)^{V A F}$

Step 8: Observe the value of $V_{G}$ as per (3), to decide $G .=$

$V_{G}=\left\{\begin{array}{c}-v e, \text { for negative change in the velocity, negative } G \\ 0, \text { for no change in the velocity or no significant improvement, no } G \\ +v e, \text { for postive change in the velocity, positive } G\end{array}\right.$

Step9: Calculate $\mathrm{G}$ and adjust $\mathrm{G}$, as per (3)

Step10: Adjust effort, $\mathrm{E}_{2}$ of sprint 2 by $\mathrm{E}_{2}=\left(\mathrm{E}_{\text {initial }}\right)^{\mathrm{G}}$

Step11: Reiterate the process and adjust effort of all subsequent iterations/sprints.

The above calculated learning gain, $G$, will be used to adjust the magnitude of model coefficients. To determine the efficacy of the proposed system model, testing and evaluation can be performed using Standardized Accuracy (SA) (Murillo-Morera et al. 2017). The comparison can be carried out using F1 score, area under curve (AUC)/(receiver operating characteristics (ROC) (Choetkiertikul et al. 2017) curve and mean absolute error (MAE).

\section{Conclusion}

Scrum project estimation is an alarming issue raised by many IT stakeholders. A lot of solutions have been proposed to address inaccurate estimation in Scrum but their efficacy is limited to a specific project's data only. Our proposed framework provides an end-toend generic solution to handle Scrum projects of different contextual domains. The continuous estimation framework will provide real time and most recent value of the story points which will assist the Scrum team in making the right decisions related to project management.

\section{References}

Ali, Asad, and Carmine Gravino. 2019. "A systematic literature review of software effort prediction using machine learning methods." Journal of Software: Evolution and Process 31 (10): 1-25. doi:10.1002/smr.2211.

Arora, Mohit, Sahil Verma, and Shivali Chopra. 2020. "A systematic literature review of machine learning estimation approaches in Scrum projects." In Cognitive Informatics and Soft Computing, 573-586.

Arora, Mohit, Sahil Verma, and Kavita. 2018. "An efficient effort and cost estimation framework for Scrum based projects." International Journal of Engineering and Technology 7 (4.12): 52-57. www.sciencepubco.com/index.php/IJET.

Choetkiertikul, Morakot, Hoa Khanh Dam, Truyen Tran, and Aditya Ghose. 2017. "Predicting the delay of issues with due dates in software projects." Empirical Software Engineering 22 (3): 1223-1263. doi:10.1007/s10664-016-9496-7.

Gultekin Muaz and Kalipsiz Oya. 2020. "Story point-based effort estimation model with machine learning techniques." International Journal of Software Engineering and Knowledge Engineering 30 (1): 43-66. doi:10.1142/S0218194020500035.

Ionescu, Vlad-Sebastian, Horia Demian, and Istvan-Gergely Czibula. 2017. "Natural language processing and machine learning methods for software development effort estimation." Studies in Informatics and Control 26 (2): 219-228. doi:10.24846/v26i2y201710. 
Kaushik, Anupama, Devendra Kr Tayal, and Kalpana Yadav. 2019. "A comparative analysis on effort estimation for Agile and non-Agile software projects using DBN-ALO.” Arabian Journal for Science and Engineering. doi:10.1007/s13369-019-04250-6.

Khan, Khairuz Zaman, Shahid Kamal Tipu, and Shahrukh Zia. 2012. "An intelligent software effort estimation system." Journal of Expert Systems 1 (4): 91-98.

Khuat, Thanh Tung, and My Hanh Le. 2018. "A novel hybrid ABC-PSO algorithm for effort estimation of software projects using Agile methodologies." Journal of Intelligent Systems 27 (3): 489-506. doi:10.1515/jisys-2016-0294.

Kocaguneli, Ekrem, Tim Menzies, Jacky Keung, David Cok, and Ray Madachy. 2013. "Active learning and effort estimation: finding the essential content of software effort estimation data." IEEE Transactions on Software Engineering 39 (8): 1040-1053. doi:10.1109/TSE.2012.88.

Morakot Choetkiertikul, Hoa Khanh Dam, Truyen Tran, Trang Pham, Aditya Ghose, and Tim Menzies. 2019. "A deep learning model for estimating story points." IEEE Transactions on Software Engineering 45 (7). IEEE: 637-655. doi:10.1007/978-3-030-11928-7_88.

Murillo-Morera, Juan, Christian Quesada-López, Carlos Castro-Herrera, and Marcelo Jenkins. 2017. "A genetic algorithm based framework for software effort prediction." Journal of Software Engineering Research and Development 5 (1): 1-33. doi:10.1186/s40411-017-0037-x.

Porru, Simone, Alessandro Murgia, Serge Demeyer, Michele Marchesi, and Roberto Tonelli. 2016. "Estimating story points from issue reports." In Proceedings of the 12th International Conference on Predictive Models and Data Analytics in Software Engineering - PROMISE 2016, 1-10. doi:10.1145/2972958.2972959.

Pospieszny, Przemyslaw, Beata Czarnacka-Chrobot, and Andrzej Kobylinski. 2018. "An effective approach for software project effort and duration estimation with machine learning algorithms." Journal of Systems and Software 137: 184-196. doi:10.1016/j.jss.2017.11.066.

Ratke, Cláudio, Helcio Hermes Hoffmann, Tércio Gaspar, and Pedro Edmundo Floriani. 2019. "Effort estimation using Bayesian networks for Agile development." In 2nd International Conference on Computer Applications and Information Security, ICCAIS 2019, 1-4. IEEE. doi:10.1109/CAIS.2019.8769455.

Rijwani, Poonam, and Sonal Jain. 2017. "A preliminary performance evaluation of machine learning algorithms for software effort estimation.” India J. Sci. Res. 14 (2): 208-214.

Soares, Rodrigo G.F. 2018. "Effort estimation via text classification and autoencoders." In International Joint Conference on Neural Networks (IJCNN), 2018 July: 1-8. IEEE. doi:10.1109/IJCNN.2018.8489030. 